\title{
Journal of Cardiovascular Magnetic Resonance
}

Volume II Suppl I, 2009

Meeting abstracts

\section{Abstracts of the $12^{\text {th }}$ Annual SCMR Scientific Sessions - 2009}

\author{
Orlando, FL, USA \\ 29 January-I February 2009
}

Published: 28 January 2009

These abstracts are available online at http://jcmr-online.com/supplements/I I/S I

\section{ORAL PRESENTATIONS}

\section{OI}

Contrast enhanced cardiovascular magnetic resonance imaging prior to prophylactic implantation of a cardioverter/defibrillator identifies patients with increased risk for ventricular arrhythmias

Philipp Boyé, Hassan Abdel-Aty, Udo Zacharzowsky, Alexander Schirdewan, Rainer Dietz and Jeanette Schulz-Menger

Franz-Volhard Clinic, Charite Campus Buch, Berlin, Germany

Journal of Cardiovascular Magnetic Resonance 2009, I I (SuppI I):O I

Introduction: Prophylactic implantation of a cardioverter/ defibrillator (ICD) has been shown to reduce mortality in patients with chronic myocardial infarction (CMI) and an increased risk for life threatening ventricular arrhythmia (VA). The use of ICDs in this large patient population is still limited by high costs and possible adverse events including inappropriate discharges and progression of heart failure. VA is related to infarct size and seems to be related to infarct morphology. Contrast enhanced cardiovascular magnetic resonance imaging (ceCMR) can detect and quantify myocardial fibrosis in the setting of $\mathrm{CMI}$ and might therefore be a valuable tool for a more accurate risk stratification in this setting.

Hypothesis: ceCMR can identify the subgroup developing VA in patients with prophylactic ICD implantation following MADIT criteria.

Methods: We prospectively enrolled 52 patients (49 males, age $69 \pm 10$ years) with $\mathrm{CMI}$ and clinical indication for ICD therapy following MADIT criteria. Prior to implantation (36 \pm 78 days) patients were investigated on a $1.5 \mathrm{~T}$ clinical scanner (Siemens Avanto $^{\circ}$, Germany) to assess left ventricular function (LVEF), LV end-diastolic volume (LVEDV) and LV mass (sequence parameters: GRE SSFP, matrix $256 \times 192$, short axis stack; full LV coverage, no gap; slice thickness $6 \mathrm{~mm}$ ). For quantitative assessment of infarct morphology late gadolinium enhancement (LGE) was performed including measurement of total and relative infarct mass (related to LV mass) and the degree of transmurality (DT) as defined by the percentage of transmurality in each scar. (sequence parameters: inversion recovery gradient echo; matrix $256 \times 148$, imaging $10 \mathrm{~min}$ after $0.2 \mu \mathrm{g} / \mathrm{kg}$ gadolinium DTPA; slice orientation equal to SSFP). MRI images were analysed using dedicated software $\left(M^{\circ} S^{\odot}\right.$, Medis,
Netherlands). LGE was defined as myocardial areas with signal intensity above the average plus $5 \mathrm{SD}$ of the remote myocardium. After implantation, patients were followed up including ICD readout after 3 and than every 6 months for a mean of $945 \pm 344$ days. ICD data were evaluated by an experienced electrophysiologist. Primary endpoint was the occurrence of an appropriate discharge (DC), antitachycard pacing (ATP) or death from cardiac cause.

Results: The endpoint occurred in 10 patients (3 DC, 6 ATP, I death). These patients had a higher relative infarct mass $(28 \pm 7 \%$ vs. $22 \pm 11 \%, p=0.03)$ as well as high degree of transmurality $(64 \pm 22 \%$ vs. $44 \pm 25 \%, p=0.05)$. Their LVEF $(29 \pm 8 \%$ vs. $30 \pm 4 \%, p=0.75)$, LV mass $(I 48 \pm 29 \mathrm{~g}$ vs. I $54 \pm 42 \mathrm{~g}$, $p=0.60), \operatorname{LVEDV}(270 \pm 133 \mathrm{ml}$ vs. $275 \pm 83 \mathrm{ml}, \mathrm{p}=0.90)$ or total infarct mass $(43 \pm 19 \mathrm{~g}$ vs. $37 \pm 21 \mathrm{~g}, \mathrm{p}=0.43)$ were however not significant from the group with no events. In a cox proportional hazards regression model including LVEF, LVEDV, LV mass, DT and age, only degree of transmurality and relative infarct mass emerged as independent predictors of the primary end point ( $p=0.009)$.

Conclusion: In CMI-patients fulfilling MADIT criteria ceCMR could show that the extent and transmurality of myocardial scarring are independent predictors for life threatening ventricular arrhythmia or death. This additional information could lead to more precise risk stratification and might reduce adverse events and cost of ICD therapy in this patient population. Larger trials are needed to confirm this finding.

\section{$\mathrm{O2}$}

Cardiac T2* magnetic resonance for prediction of cardiac complications in thalassemia major Paul Kirk', Michael Roughton', John B Porter' John M Walker', Mark A Tanner', Junaid Patel', Dianne Wu', Jane Taylor', Mark A Westwood', Lisa J Anderson' and Dudley J Pennel'

'Royal Brompton Hospital, London, UK

${ }^{2}$ University College Hospital London, London, UK

Journal of Cardiovascular Magnetic Resonance 2009, I I (SuppI I):O2

Background: Myocardial siderosis is the main cause of morbidity and mortality in thalassaemia major. In the United Kingdom approximately $50 \%$ of patients die before reaching 35 years. The cardiomyopathy is reversible if chelation is commenced early but diagnosis is often delayed due to the late onset of symptoms. T2* CMR can now assess cardiac iron 
directly and this has profound implications for clinical management of iron overload and the assessment of chelation regimes. Left ventricular ejection fraction falls with increasing myocardial iron (reduced myocardial T2*; normal value $>20 \mathrm{~ms}$ ), and accordingly iron overloaded patients with symptomatic heart failure have a low T2*. Although data is available on the level of T2* in patients developing heart failure there is no published data on the incidence of heart failure and arrhythmia in patients during follow-up according to baseline myocardial T2*. The aim of this study therefore was to establish the risk of cardiac complications in patients with cardiac siderosis as measured by T2*.

Methods: A prospective database containing clinical data and T2* values on 652 thalassaemia major patients (I 442 scans) was maintained over a 6 year period with 1,285 patient years of prospective follow-up. Of these patients, 319 were male and 333 female with a mean age at time of first scan of $27.1 \pm 9.6$ years. The mean number of blood units transfused per year per patient was $32.6 \pm 11.5$.

Results: At I year of follow-up, there were 84 episodes of heart failure and 100 episodes of arrhythmia. There were 4 deaths, with 3 patients dying from sepsis following bone marrow transplant and I patient dying following an episode of ventricular tachycardia.

Heart failure: For the 84 heart failure episodes, 64 presented in New York Heart Association (NYHA) class two, 16 were NYHA class three, and 4 were NYHA class four. The mean ejection fraction of these patients was $43.1 \pm 7.2 \%$. In these heart failure patients, the preceding cardiac T2* was $6.7 \pm 1.8$ $\mathrm{ms}$, the liver T2*3.9 $\pm 3.7 \mathrm{~ms}$ and ferritin $2,7 \mathrm{I} 3 \pm \mathrm{I}, 686 \mu \mathrm{g} / \mathrm{L}$. In comparison with cardiac T2* values $>20 \mathrm{~ms}$, there was a significantly increased risk of heart failure associated with cardiac T2* values $<10 \mathrm{~ms}$ (Relative Risk 159, $\mathrm{P}<0.00 \mathrm{I}$ ) and $\mathrm{T} 2 *<6$ (RR 268, $P<0.00 I$ ). Serum ferritin using the conventional threshold was a significant but weaker predictor of heart failure (ferritin $>2500 \mu \mathrm{g} / \mathrm{L}, \mathrm{RR} 0.56, \mathrm{P}=0.02 \mathrm{I}$ ). Liver T2* $<0.96 \mathrm{~ms}$ (equivalent to the conventional threshold of $>15 \mathrm{mg} / \mathrm{g} / \mathrm{dw}$ iron) was not a significant predictor of heart failure (liver T2* $<0.96$ ms, RR I.25, $\mathrm{P}=0.76$ ). The Kaplan Meier curve of $\mathrm{T} 2 *$ vs heart failure is shown in Figure $I$.

Arrhythmia: For the 100 episodes of arrhythmia, 79 episodes were atrial fibrillation (AF), 14 episodes were supraventricular tachycardia (SVT), 6 episodes were ventricular tachycardia (VT),

\section{Figure I (abstract 02)}

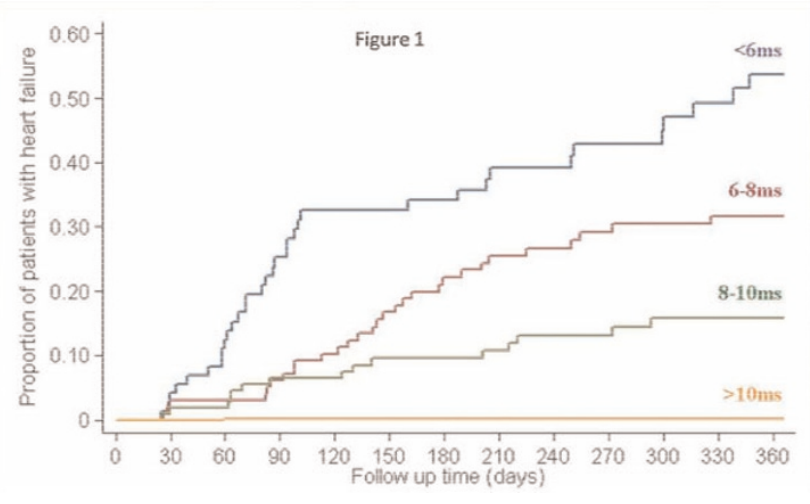

Figure 2 (abstract 02)

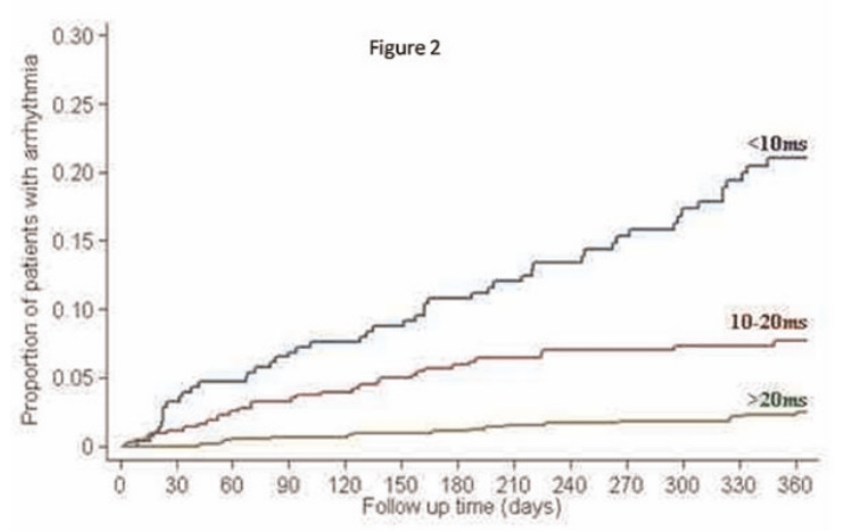

and I episode was ventricular fibrillation (VF). The mean cardiac T2* was $13.5 \pm 9 \mathrm{~ms}$, mean liver T2* $6.0 \pm 6.4 \mathrm{~ms}$, mean serum ferritin $2140 \pm 1540 \mu \mathrm{g} / \mathrm{L}$, and the mean ejection fraction was $60.7 \pm 9.3 \%$. In comparison with cardiac T2* values $>20 \mathrm{~ms}$, there was a significantly increased risk of arrhythmia associated with cardiac T2* values $<6 \mathrm{~ms}(\mathrm{RR} 8.65, \mathrm{P}<0.00 \mathrm{I})$ and $\mathrm{T} 2 *<20$ (RR 4.6, $P<0.00 I$ ). There was no significant predictive value using the conventional thresholds of ferritin (ferritin $>2500 \mu \mathrm{g} / \mathrm{L}$, RR 0.90, $\mathrm{P}=0.66)$ or liver $\mathrm{T} 2 *(\mathrm{~T} 2 *<0.96 \mathrm{~ms}$, RR 0.78 , $P=0.68$ ). (See Figure 2.).

Conclusion: These data provide strong evidence that a myocardial $\mathrm{T}^{*}<10 \mathrm{~ms}$ predicts a high risk of developing heart failure. It is clear that these patients should be aggressively chelated to reduce their high morbidity and mortality from cardiac siderosis.

\section{O3}

Sub-clinical systolic dysfunction with persistent myocardial edema and inflammation in elite high-endurance athletes with common colds: a cardiovascular magnetic resonance study Myra S Cocker', Oliver Strohm', David J Smith², Craig Butler', Israel Belenkie ${ }^{3}$, Willem Meeuwisse ${ }^{4}$ and Matthias G Friedrich'

'Stephenson CMR Centre at the Libin Cardiovascular Institute, University of Calgary, Calgary, AB, Canada

${ }^{2}$ Human Performance Lab, Faculty of Kinesiology,

University of Calgary, Calgary, $A B$, Canada

${ }^{3}$ Department of Cardiac Sciences at the Libin Cardiovascular Institute, University of Calgary, Calgary, $A B$, Canada

${ }^{4}$ Sports Medicine Centre, University of Calgary, Calgary, $A B$, Canada

\section{Journal of Cardiovascular Magnetic Resonance 2009, I I(SuppI I):O3}

Background: Basic research has demonstrated that myocardial inflammation may be a feature of systemic viral inflammation, resulting from agents such as influenza. Physical activity during exposure to pathogens has been shown to exacerbate the propensity to develop adverse cardiac events. As such, based upon empirical findings, current guidelines on athletic training 
deter athletes from participating in sport during common colds. Cardio-vascular Magnetic Resonance (CMR) allows for noninvasive visualization of myocardial inflammation, where it has emerged as the imaging modality of choice to assess the course of myocarditis. Thus, using CMR-based tissue characterization, we hypothesized that colds in elite high-endurance athletes would lead to depressed cardiac function and myocardial inflammation.

Methods: 62 (32 male, $31 \pm 13$ years) elite high-endurance athletes were prospectively recruited. CMR scans were performed at baseline, with an acute common cold, and 4 weeks after. Pre-defined symptoms were used to rule in an acute cold. LV function, edema, and myocardial inflammation were assessed using standard SSFP, T2-, and TI-weighted imaging, respectively, on a I.5 T MRI system.

Standard, previously described approaches for the quantification of $L V$ function, edema and myocardial inflammation were utilized. Statistical comparisons were performed with repeated measures ANOVA, at 2 levels of measurement.

Results: During the II-month period of recruitment, 21 athletes completed all 3 scans. During an acute cold, we observed a significant increase in LVESVI, with reduced LVSVI and LVEF ( $p<0.05$ ), while LVEDVI and LVMI did not differ (Table I). Moreover, there were no statistical differences between LV volumes at the 4-week follow-up to those at baseline or with an acute cold.

In terms of tissue characterization, 19\% of athletes had evidence for myocardial edema with an acute cold, and $24 \%$ at follow-up

\section{Figure I (abstract O3)}

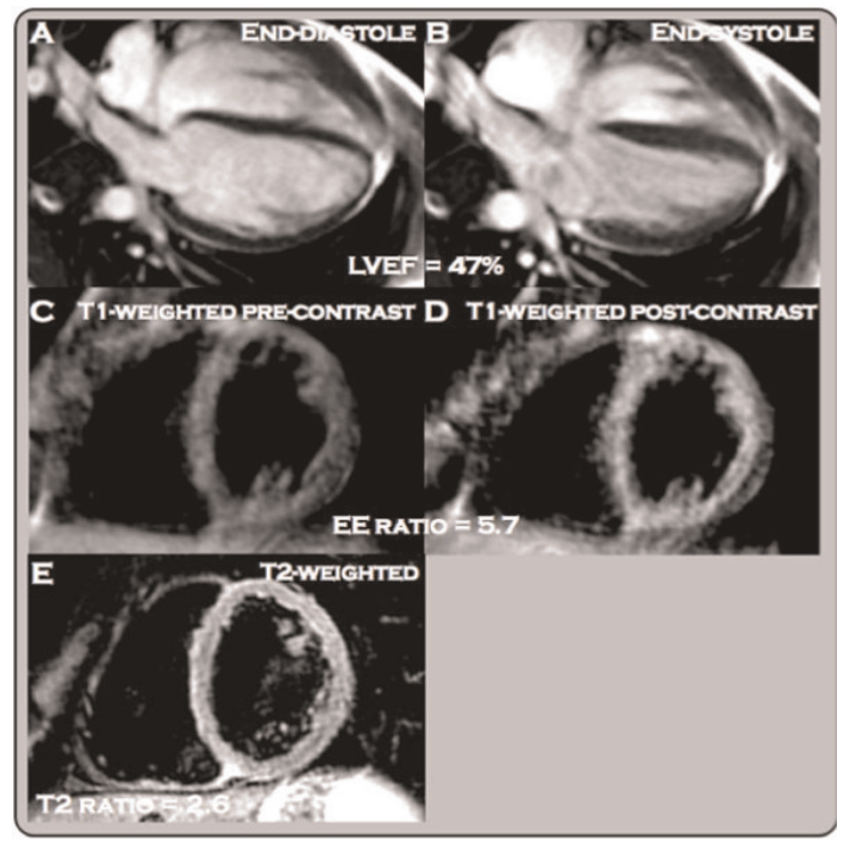

Reduced contractile function in an elite professional swimmer with a common cold ( $\operatorname{LVEF}_{\text {cold }} 47 \%, \mathrm{LVEF}_{\text {baseline }} 54 \%$ ) (A, B). Visually apparent increased early enhancement post-contrast in $\mathrm{TI}$-weighted images (C, D) suggestive of myocardial inflammation, with an early enhancement ratio of 5.7. Evidence of global myocardial edema (E, ratio 2.6).
Table I (abstract O3) LV volume and CMR markers for edema and inflammation at baseline, with a common cold and at a 4-week follow-up. Volumetric data are presented as mean standard deviation

\begin{tabular}{lccc}
\hline & $\begin{array}{l}\text { Baseline } \\
\text { visit }\end{array}$ & $\begin{array}{l}\text { Visit with } \\
\text { common cold }\end{array}$ & $\begin{array}{l}\text { 4-week } \\
\text { follow-up }\end{array}$ \\
\hline LVEDVI $(\mathrm{ml} / \mathrm{m})$ & $\mathrm{III} .4 \pm 20$ & $110.0 \pm 22$ & $109.9 \pm 21$ \\
LVESVI $(\mathrm{ml} / \mathrm{m})$ & $39.4 \pm 11$ & $41.7 \pm 1 I^{*}$ & $40.6 \pm 9$ \\
LVSVI $(\mathrm{ml} / \mathrm{m})$ & $72.0 \pm 12$ & $68.3 \pm 13^{*}$ & $69.3 \pm 15$ \\
LVEF $(\%)$ & $65.0 \pm 4.8$ & $62.5 \pm 4.9^{*}$ & $63.0 \pm 5.8$ \\
LVMI $(\mathrm{g} / \mathrm{m})$ & $58.8 \pm 15$ & $59.1 \pm 15$ & $60.1 \pm 16$ \\
Edema $(\mathrm{n})$ & 4 of 21 & 4 of 21 & 5 of 21 \\
Inflammation $(\mathrm{n})$ & 7 of 21 & 8 of 21 & 10 of 21 \\
\hline
\end{tabular}

$*_{p}<0.05$ baseline compared to visit with common cold.

(Figure I). $38 \%$ had myocardial inflammation during an acute cold; and this proportion increased to $48 \%$ at follow-up.

Conclusion: We provide first evidence of sub-clinical myocardial involvement with common colds in high-endurance athletes. Colds were associated with a small yet significant decrease of systolic function, and persisting myocardial inflammation visualized with CMR-derived markers for edema and inflammation. Further research is required to investigate the implications of these findings on athletic performance.

\section{4}

A T2-mapping method to quantitatively differentiate edema from normal myocardium Shivraman S Giri', Yiu-Cho Chung', Ali Merchant', Tam Tran', Subha Raman' and Orlando Simonetti' IThe Ohio State University, Columbus, OH, USA

${ }^{2}$ Siemens Medical Solutions, Columbus, OH, USA

Journal of Cardiovascular Magnetic Resonance 2009, I I(SuppI I):O4

Introduction: T2-Weighted (T2W) imaging sequences can detect myocardial edema associated with acute inflammation, infarction, and the area at risk, but these techniques suffer from several drawbacks [I]. In this work, we describe a rapid technique for quantitative myocardial T2 mapping. This method is expected to quantitatively differentiate edema from normal tissue, to be insensitive to tissue motion, to easily distinguish edema from stagnant blood, and to be immune to surface coil sensitivity variations. The proposed T2-mapping method can be performed with either a short breath-hold or respiratory navigator gating.

Purpose: To develop a rapid, quantitative method of myocardial T2-mapping to detect edema in patients with acute coronary syndrome.

Methods: Sequence: A single-shot T2-prepared SSFP acquisition was used to generate images with three T2-prep times: 0 (i.e., no T2 prep), 24, and $55 \mathrm{~ms}$ with parameters listed in Table I. The technique is relatively motion insensitive due to the SSFP readout and the non-selective T2-preparation pulse. T2 maps were produced by fitting pixel intensities to a twoparameter mono-exponential model (Signal $=M 0 * \exp (-\mathrm{TE} /$ T2)), and setting any pixel with T2 > $120 \mathrm{~ms}$ to zero.

Phantom: A two-compartment phantom was created to approximate the $\mathrm{TI}$ and $\mathrm{T} 2$ values of normal $(\mathrm{TI} / \mathrm{T} 2=897 / 52.3 \mathrm{~ms})$ and 
Table I (abstract 04) Imaging parameters

\begin{tabular}{lll}
\hline Parameter & T2Prep SSFP & DB-TSE \\
\hline TE $(\mathrm{ms})$ & $0,24,55$ & 62 \\
TR & $2 \times \mathrm{RR}$ & $2 \times \mathrm{RR}$ \\
Avg. FOV & $350 \times 400$ & $350 \times 400$ \\
Image Matrix & $128 \times 160$ & $144 \times 192$ \\
Flip angle & 40 & 90 \\
\hline
\end{tabular}

edematous myocardium (TI/T2 $=1119 / 101.8 \mathrm{~ms})$ [2]. Phantom TI and $\mathrm{T} 2$ values were verified using a standard spin echo sequence. In-vivo: T2 Maps were acquired in 9 healthy subjects to determine the normal range of T2 values. Three short-axis and two long-axis views were imaged during breath hold (duration $\sim 5$ $\mathrm{HB}$ ) and in free breathing using navigator gating. In 5 subjects, four averages were acquired with navigator gating to test the benefits of increased SNR. Average T2 values were calculated in 16 myocardial segments using both methods and compared. Measurements were pooled to obtain global mean and standard deviation to investigate inter-subject and inter-segment variability.

Signal intensity variability: In six healthy subjects, T2W images using conventional dark-blood STIR turbo spin echo (DBSTIR-TSE) were acquired and compared to the T2 maps generated using the proposed method. Only anterior coil elements were used to investigate signal variability due to surface coil intensity variation, as well as motion induced signal loss. Parameters are listed in Table I. In each subject, average signal was computed and normalized to the maximum segment. The standard deviation (SD) of this normalized mean was used as a measure of variability.

Animal studies: Three pigs underwent 90 minute LAD occlusion and were imaged with breath-hold within six hours of reperfusion.

Results: Phantom: T2 values were slightly overestimated (I07.9 ms vs. I0I.8 ms, and $60.7 \mathrm{~ms}$ vs. $52.3 \mathrm{~ms}$ ) in the two phantom compartments.

In-vivo: T2 values did not show significant variation among the 16 segments ( $P=0.277$, ANOVA) or between breathhold and free breathing techniques ( $p=0.76$, paired t-test). The mean T2 and standard deviation were $51.54+3.5 \mathrm{~ms}$ (range: 49.6 to $53 \mathrm{~ms}$ ). Figure I demonstrates the discrimination of static apical blood from myocardium in the T2 map.

Signal intensity variability: Signal in T2W DB-STIR-TSE showed high variability (36.7\%) while T2 maps showed no such variation (3\%) as seen in Figure 2.

\section{Figure I (abstract 04)}

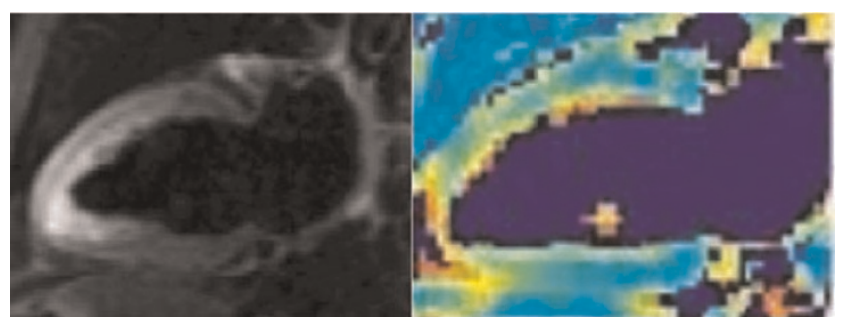

T2 Weighted image (DB-STIR-TSE) (I) showing high signal intensity due to stagnant blood in the apical regions. The corresponding T2 Map clearly distinguishes myocardium from stagnant blood.
Figure 2 (abstract 04)
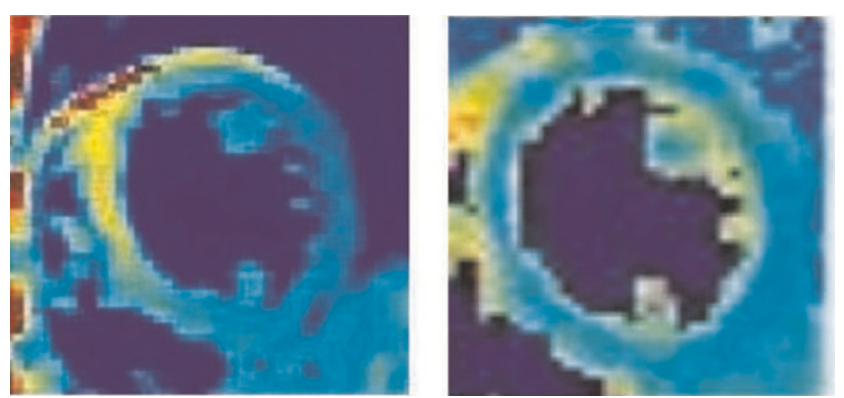

T2W image (left) shows a lot of signal variability due to surface coil sensitivity variations and motion as compared to the corresponding T2 Map (right). Similar color map used in both images for comparison.

\section{Figure 3 (abstract 04)}

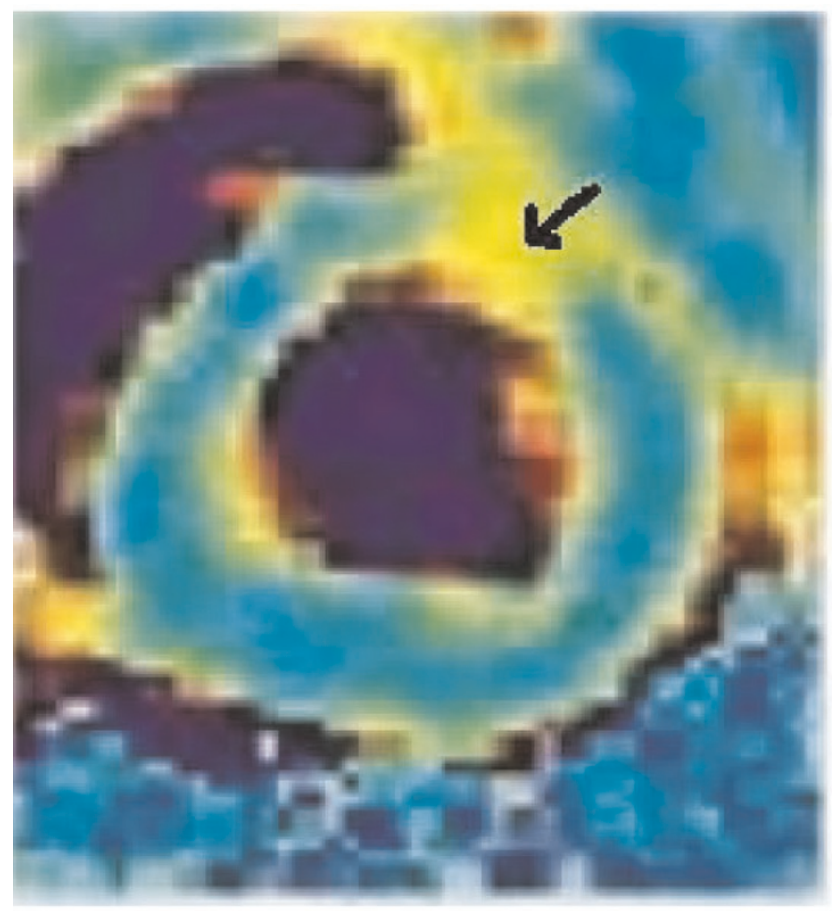

T2 Maps from a pig showing enhanced T2 in anterior segment (arrow). In this pig, T2 in anterior segment was $83.5+11 \mathrm{~ms}$ vs. $58.6+4.5 \mathrm{~ms}$ in inferior segment.

Table 2 (abstract 04) Results from pig studies.

\begin{tabular}{lll}
\hline Animal \# & Infarcted segment & Remote normal segment \\
\hline 1 & $83.5,11$ & $58.6,4.5$ \\
2 & $80.4,6.8$ & $51.1,5.6$ \\
3 & $83.6,10$ & $58.4,7.2$ \\
\hline
\end{tabular}

The values are mean, SD of $\mathrm{T} 2(\mathrm{~ms})$ in mid ventricular SAX slice. 
Animal study: Results from the pig study are shown in Figure 3 and Table 2 . In each animal, edematous segment showed a T2 value $>2$ SD of remote segment.

Conclusion: We have demonstrated a rapid method of T2-mapping for quantitative detection of myocardial edema. Direct quantification of T2 eliminates many unwanted sources of signal variation, and removes the subjectivity of observer interpretation of bright regions. Further studies with patients are required to assess sensitivity and specificity.

\section{References}

I. Arai AE: Circulation 2008, I I 8(8):795-6.

2. Aletras AH, et al: Magn Reson Med 2008, 59(2):229-35.

\section{5}

3T contrast-enhanced whole heart coronary

MRA using 32-channel cardiac coils for

the detection of coronary artery disease Qi Yang', Kuncheng $\mathrm{Li}^{\prime}$, Xiaoming $\mathrm{Bi}^{2}$, Jing $\mathrm{An}^{3}$, Renate Jerecic ${ }^{2}$ and Debiao $\mathrm{Li}^{4}$

'Xuanwu hospital, Beijing, PR China

${ }^{2}$ Siemens Medical Solutions, Chicago, IL, USA

${ }^{3}$ Siemens Mindit Magnetic Resonance Ltd, Shenzhen, PR China

${ }^{4}$ Department of Radiology, Northwestern University, Chicago, IL, USA

Journal of Cardiovascular Magnetic Resonance 2009, I I (SuppI I):O5

Introduction: In recent years, improved gradient performance and radiofrequency (RF) receiving coils and advanced data acquisition techniques including navigator gating and parallel imaging allowed non-invasive whole-heart coronary imaging. Previous studies have shown that $3.0 \mathrm{~T}$ is a promising platform for the detection of significant coronary artery stenoses with contrast-enhanced data acquisition. However, the imaging time $(\sim 10$ minutes $)$ and spatial resolution $\left(1.3 \times 1.3 \times 1.3 \mathrm{~mm}^{3}\right)$ remain major limitations [I]. Newly developed 32-channel cardiac coils allow greater acceleration factors and thus reduced imaging time and higher spatial resolution [2].

Purpose: To evaluate the feasibility and diagnostic accuracy of $3 \mathrm{~T}$ contrast-enhanced whole-heart coronary MRA using 32-channel cardiac coils. The imaging time, image quality score, and diagnostic accuracy were evaluated in consecutive patients with suspected coronary artery disease.

Methods: 20 patients with suspected coronary artery disease who were scheduled for $x$-ray coronary angiography (mean age $68 \pm 14$ years, II males) underwent MRA at 3 T (MAGNETOM Tim Trio, Siemens) after informed consent was obtained. Contrast-enhanced coronary MRA was also performed in 5 patients who were scheduled for 64-slice coronary CTA. The imaging technique was an ECG-triggered, navigator-gated, inversion-recovery, segmented gradient-echo sequence. A 32-channel matrix coil was used for data acquisition. To reduce imaging time, parallel acquisition (GRAPPA) was used in the phase-encoding direction with an acceleration factor of three. Imaging parameters included: voxel size $0.55 \times 0.55 \times 0.65 \mathrm{~mm}^{3}$ (interpolated from $\left.1.1 \times 1.1 \times 1.3 \mathrm{~mm}^{3}\right)$, TR/TE $=3.3 / 1.5 \mathrm{msec}$, flip angle $=20^{\circ}$, bandwidth $=700 \mathrm{~Hz} /$ pixel. Contrast agent $(0.15$ $\mathrm{mmol} / \mathrm{kg}$ body weight, Multihance, Bracco Imaging SpA, Italy) was intravenously administered at a rate of $0.3 \mathrm{ml} / \mathrm{sec}$. The diagnostic accuracy of MRA in detecting significant stenoses ( $\geq 50 \%$ ) with the intention to diagnose method was evaluated on a
Figure I (abstract 05)

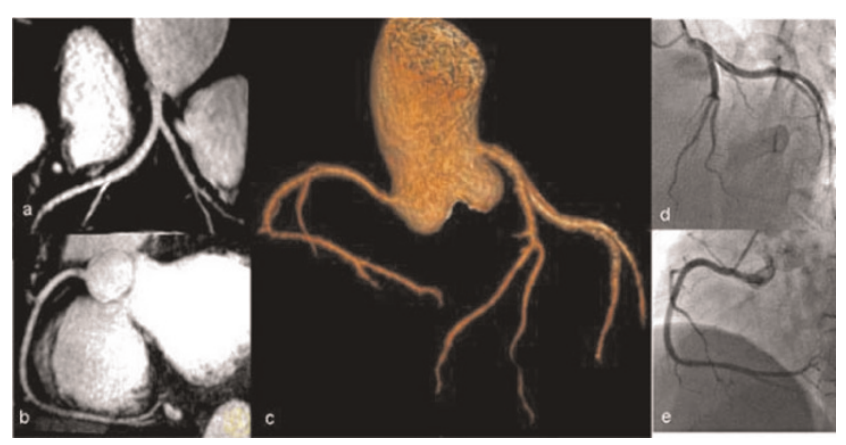

3 T Coronary MR image of a 50-year-old patient. Reformatted images (a, b) and volume rendering image demonstrates normal RCA, LM, LAD. Better visualization of the entire coronary artery tree after removing the background of myocardium, long segments of all major coronary arteries are well depicted and correlate well with X-ray angiography $(\mathbf{d}, \mathbf{e})$.

per-segment basis using $\mathrm{x}$-ray angiography as the reference, non-assessable segments were considered to be false-negative or false-positive, respectively.

Results: Whole-heart coronary MRA was successfully completed in 19 of 20 (95\%) patients who were scheduled for x-ray coronary angiography and in 5 patients who were scheduled for 64-slice coronary CTA. The averaged imaging time with 32 -channel cardiac coils was $6.2 \pm 1.3 \mathrm{~min}$. The sensitivity, specificity, positive predictive value, and negative predictive value of coronary MRA for detecting significant stenoses were $81 \%$ (62-94\%), 96\% (92-98\%), 71\% (52-86\%), 98\% (94-99\%), respectively, on a per-segment basis. Figure $\mathrm{I}$.

Conclusion: Combined with dedicated 32-channel cardiac coils, parallel imaging with higher acceleration factors allows improvements in imaging speed, study success rate, and reduced dose of the contrast agent when compared with conventional 12-channel coils. Higher study success rate achieved by 32-channel coils substantially improved overall accuracy of coronary MRA in detecting coronary artery disease when using the intention to diagnose method.

References

I. Qi Yang and Li DB, et al: \#29/7 Proceedings of 16th annual ISMRM, Toronto 2008.

2. Niendorf T, et al: Magn Reson Med 2006, 56:167-I76.

\section{O6}

Mt Everest trek causes impaired cardiac high energy phosphate metabolism and diastolic impairment

Cameorn J Holloway', Andrew Murray',

Lowri E Cochlin', Yaso Emmanuel', Denny ZH Levett ${ }^{2}$, Oliver J Rider', Damian J Tyler', Matthew Robson', Jane M Francis ${ }^{2}$, Hugh Montgomery ${ }^{2}$,

Michael PW Grocott' ${ }^{2}$, Stefan Neubauer'

and Kieran Clarke

'University of Oxford, Oxford, UK

${ }^{2}$ UCL, London, London, UK

Journal of Cardiovascular Magnetic Resonance 2009, I I(Suppl I):O6

Background: Cardiac function in normal subjects is altered by exposure to hypobaric hypoxia, yet the cellular mechanisms 
Figure I (abstract 06)

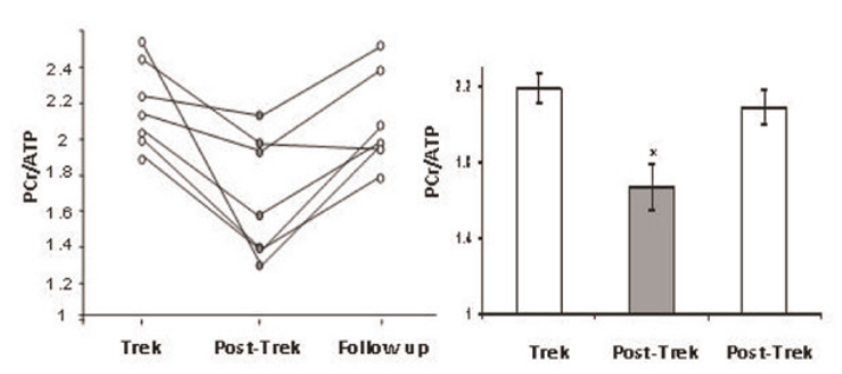

leading to such changes are unknown. We have examined the impact of sustained exposure to environmental hypobaric hypoxia, on cardiac function and energetics.

Methods and results: Healthy normal volunteers $(n=7)$ were studied immediately before, and within four days of return from 17 days exposure to environmental hypobaric hypoxia whilst trekking to Mount Everest Base Camp (I7388 feet, $5300 \mathrm{~m}$ ) and back. 3 IP magnetic resonance (MR) spectroscopy was used to measure cardiac phosphocreatine (PCr)/ATP, and $M R$ imaging and echocardiography were used to assess cardiac function. All measurements were repeated six months after return from Everest. Immediately after their return from Everest, subjects showed a $24 \%$ decrease in cardiac PCr/ATP, from $2.19 \pm 0.09$ to $1.67 \pm 0.13(p<0.01)$ (see Figure I). Peak left ventricular filling rates had declined from $832 \pm 64 \mathrm{ml} / \mathrm{sec}$ to $691 \pm 56 \mathrm{ml} / \mathrm{sec}(p<0.05)$ and transmitral E/A was reduced from $1.56 \pm 0.11$ to $1.16 \pm 0.08(p<0.05)$. Left and right ventricular stroke volumes had fallen by $13 \%$. No change on cardiac mass was observed. Six months later, all measures had returned to baseline values.

Conclusion: Exposure to prolonged hypobaric hypoxia is associated with significant, but reversible, energetic and functional abnormalities in the human heart.

\section{7}

Multifunctional perfluorooctylbromide alginate microcapsules for monitoring of mesenchymal stem cell delivery using CT and MRI

Yingli Fu', Dorota Kedziorek', Ronald Ouwerkerk', Veronica Crisostomo ${ }^{2}$, Wesley Gilson ${ }^{3}$,

Nicole Azene', Aravind Arepally', Christine Lorenz ${ }^{3}$, Steven Shea ${ }^{3}$, Robert Krieg ${ }^{4}$, Jeff WM Bulte' and Dara L Kraitchman'

IJohns Hopkins University, Baltimore, MD, USA

${ }^{2}$ El Centro de Cirugía de Mínima Invasión Jesús Usón, Caceres, Spain

${ }^{3}$ Siemens Corporate Research, Baltimore, MD, USA

${ }^{4}$ Siemens Healthcare, Erlangen, Germany

Journal of Cardiovascular Magnetic Resonance 2009, I I (SuppI I):O7

Background and objectives: Many patients with peripheral arterial disease (PAD) cannot undergo conventional medical or surgical therapy due to the extent or severity of atherosclerotic disease. Stem cell therapy has shown promising results as an angiogenic therapy in PAD patients. However, the poor survival of transplanted cells due to early immunodestruction and the inability to noninvasively monitor and track the distribution and proliferation of transplanted cells hinders stem cell therapeutic efficacy. We present here a multifunctional mesenchymal stem cell (MSC) microencapsulation and trafficking method utilizing perfluorooctylbromide (PFOB) incorporated alginate-poly-Llysine-alginate microcapsules (PFOB Caps) for MSC delivery and noninvasive engraftment tracking using clinical $X$-ray and $M R$ imaging equipment.

Methods: Microencapsulation of bone marrow-derived rabbit or human MSCs $\left(1.5 \times 10^{6} \mathrm{cells} / \mathrm{ml}\right)$ were performed by extruding a PFOB-impregnated $2 \%(\mathrm{w} / \mathrm{v})$ alginate solution from a syringe pump in conjunction with an electrostatic droplet generator, followed by cross linking with poly-L-lysine to form X-ray- and MRI-visible microcapsules. MSCs viability was examined and compared between unlabeled capsules and PFOB Caps. Using ${ }^{19} \mathrm{~F} \mathrm{MRI}$ and rotational angiograms reconstructed into CT-like images, the minimum detectable concentration was determined in phantoms using standard clinical imaging systems. X-ray delivery and tracking of intramuscular injections of PFOB Caps ( 5000 capsules/injection) was assessed in a rabbit PAD model.

Results: The viability of rabbit MSCs encapsulated with PFOB was $90 \pm 3 \%$ immediately after encapsulation and remained high ( $88 \pm 5 \%$ at 4 weeks post-encapsulation). PFOB Caps containing human MSCs had enhanced cell viability relative to unlabeled capsules ( $83 \pm 3 \%$ for PFOB vs. $50 \pm 1 \%$ for control at 65 days post-encapsulation, $P<0.00$ I). Viability of human MSCs in PFOB Caps was maintained up to 100 days, while it decreased sharply to $<10 \%$ in unlabeled capsules at 80 days post-encapsulation. In vitro $\mathrm{CT}$ and ${ }^{19} \mathrm{~F}$ MRI imaging of PFOB Caps demonstrated the ability to detect as few as 2 and 25 capsules (Figure I), respectively. In vivo, PFOB visibility on CT images was demonstrated relative to unlabeled capsules with persistence of intact microcapsules up to 5 weeks post delivery in PAD rabbits.

Conclusion: By adding PFOB, a dual contrast agent and oxygen carrier, to alginate microcapsules, we have demonstrated the enhanced viability of MSCs within PFOB Caps, and the ability to deliver and track engraftment of stem cells using multiple conventional clinical imaging systems in vivo.

Figure I (abstract 07)

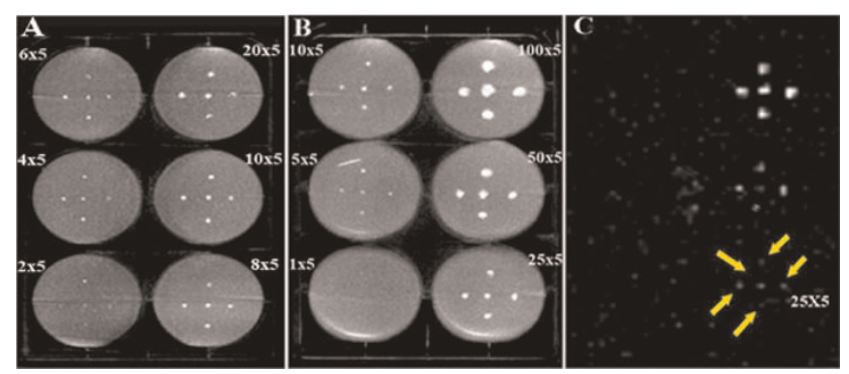

In vito DynaCT and ${ }^{19} \mathrm{~F}$ MR images of PFOB Caps phantoms. (A, B) DynaCT images of PFOM Caps phantoms demonstrated the ability to detect as few as 2 capsules. (C) ${ }^{19} \mathrm{~F} \mathrm{MRI} \mathrm{(3D-TrueFISP,} \mathrm{BW}=1500 \mathrm{~Hz} /$ $\mathrm{px}, \mathrm{TR} / \mathrm{TE}=3.0 / 1.5 \mathrm{~ms}, 2.0 \times 2.0 \times 5.0 \mathrm{~mm}^{3}, 24$ partitions, 4 avgs, $62 \mathrm{~s}$ acquisition) of the same phantom as B showed as few as 25 PFOB Caps were identifiable. 


\section{8}

Cardiac magnetic resonance of targeted annexin-iron oxide labeling detects cardiac cell death in vivo after doxorubicin and myocardial infarction Rajesh Dash', Trevor Chan ${ }^{2}$, Mayumi Yamada ${ }^{2}$, Marietta Paningbatan², Bat-Erdene Myagmar', Philip Swigart', Paul C Simpsonjr ${ }^{1}$ and Phillip $C$ Yang $^{2}$ 'San Francisco Veterans Affairs Hospital, San Francisco, CA, USA

${ }^{2}$ Stanford University, Stanford, CA, USA

Journal of Cardiovascular Magnetic Resonance 2009, I I (SuppI I):O8

Background: Heart failure from myocardial infarction (MI) or doxorubicin (DOX), used in cancer therapy, is preceded by significant cell apoptosis. Real-time, non-invasive detection of early cardiac apoptosis might impact patient treatment and outcomes. Early apoptosis is detected by Annexin $\mathrm{V}$ protein (ANX) binding to externalized membrane phosphatidylserine. To this end, we previously conjugated ANX to superparamagnetic iron oxide (ANX-SPIO). This conjugate specifically binds to early apoptotic cardiac cells in culture and is detectable by in vitro magnetic resonance imaging (MRI).

Hypothesis: We tested whether ANX-SPIO could detect cardiac apoptosis, in vivo, via MRI (3 Tesla, GE Excite, WI) after ischemic or oxidative injury.

Methods: Mice underwent LAD ligation or intraperitoneal, cardiotoxic DOX (25 mg/kg) injection. After 24-48 hours, ANX-SPIO was given by tail vein, and mice were imaged by T2-weighted cardiac MRI (3 Tesla, GE Excite).

Results: After $\mathrm{MI}$ and DOX, myocardial T2 MRI signal was detectable within 30 minutes of ANX-SPIO delivery, exhibiting either a focal (MI) or diffuse (DOX) signal distribution (see Figure I). Peak signal was evident 24 hours after ANX-SPIO delivery, and decreased over the next 2 weeks.

Conclusion: Cardiac MRI using ANX-SPIO can accurately detect myocardial apoptosis in vivo. Distinct MRI signal distributions were noted following ischemic (MI) versus oxidative (DOX) injury. This molecular imaging strategy may help identify 'at risk' cardiac cell populations.

Figure I (abstract 08)
A)

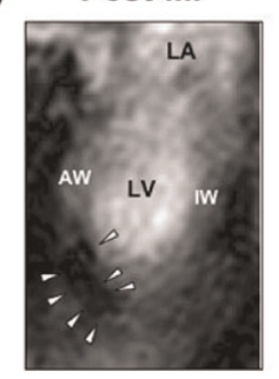

B) Post-DoX

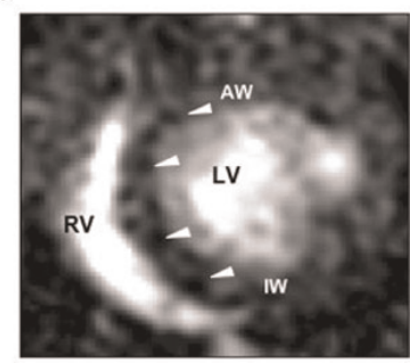

ECG- and respiratory-gated T2-weighted cardiac MRI of mice post-MI (A, 2-chamber view) and post-DOX (B, short axis view), 30 minutes after tail vein infection of $100 \mu \mathrm{l}$ ANX-SPIO. Note focal T2 signal void (white arrows) of ANX-SPIO in antero-apex of post-MI heart, and diffuse septal, anterior and inferior T2 signal void in post-DOX heart (LA, left atrium; LV, left ventricle; RV, right ventricle; AW, anterior wall; IW, inferior wall).
09

Integrated analysis of diastolic, systolic and pulmonary vascular function using MRI guided catheterization

Boris Schmitt', Paul Steendjik², Karsten Lunze', Stanislav Ovrouski', Jan Falkenberg',

Pedram Rahmanzadeh', Nizar Maarouf', Peter Ewert', Felix Berger' and Titus Kuehne'

'Unit of Cardiovascular Imaging - Congenital Heart Diseases, Deutsches Herzzentrum Berlin, Berlin, Germany

${ }^{2}$ Cardiology, Medical University Leiden, The Netherlands, Leiden, Netherlands

Journal of Cardiovascular Magnetic Resonance 2009, I I (SuppI I):09

Indroduction and purpose: An integrated approach for assessing ventricular pump function, diastolic compliance (EDPVR), myocontractility and pulmonary vascular resistance would be of clinical interest. In addition to pump function, MRI guided catheterization was demonstrated to accurately measure myocontractility and vascular resistance. We now extended this method for acquisition of the EDPVR. Subsequently, this approach was applied in patients with Fontan hemodynamic in which abnormalities in pulmonary vascular, myocontractile and diastolic properties are debated.

Methods: The EDPVR was determined by synchronizing invasive ventricular pressures with cine and real-time MRI derived ventricular volumes and pulmonary/aortic blood flow measurements.

Validation part: In 7 pigs the MRI and conductance-catheter method (gold standard) were compared for measuring the EDPVR at rest and during dobutamine.

Clinical part: Parameters of global function, myocontractility (ESPVR), vascular resistance and EDPVR were measured with MRI at rest and under dobutamine in 14 patients with Fontan circulation. Results: Bland-Altman test showed agreement between the conductance-catheter and MRI method. In the pigs, there was in both ventricles during dobutamine a right/bottom shift of the EDPVR, the stiffness co efficient decreased slightly $(P<0.05 I)$. In the patients during dobutamine we noted failure to increase stroke volumes despite increased contractility and evidenced diastolic dysfunction. Active relaxation was inconspicuous but the EDPVR shifted towards the left/top, the stiffness constant remained unchanged. Pulmonary resistance decreased slightly $(p=0.058)$ and thus showed adequate response to augmented cardiac outputs.

Conclusion: This novel MRI method provides differential information about diastolic, systolic and vascular function. The method evidenced that in Fontan patients diastolic dysfunction is an important pathophysiologic cause of heart failure.

\section{0}

Diffusion-prepared dark blood delayed enhancement imaging for improved detection of subendocardial infarcts Michael Salerno, Frederick H Epstein and Christopher M Kramer University of Virginia, Charlottesville, VA, USA

Journal of Cardiovascular Magnetic Resonance 2009, I I (SuppI I):O I0

Introduction: Delayed enhancement MRI enables detection of non-viable myocardium with high spatial resolution and has 
Figure I (abstract OI0)

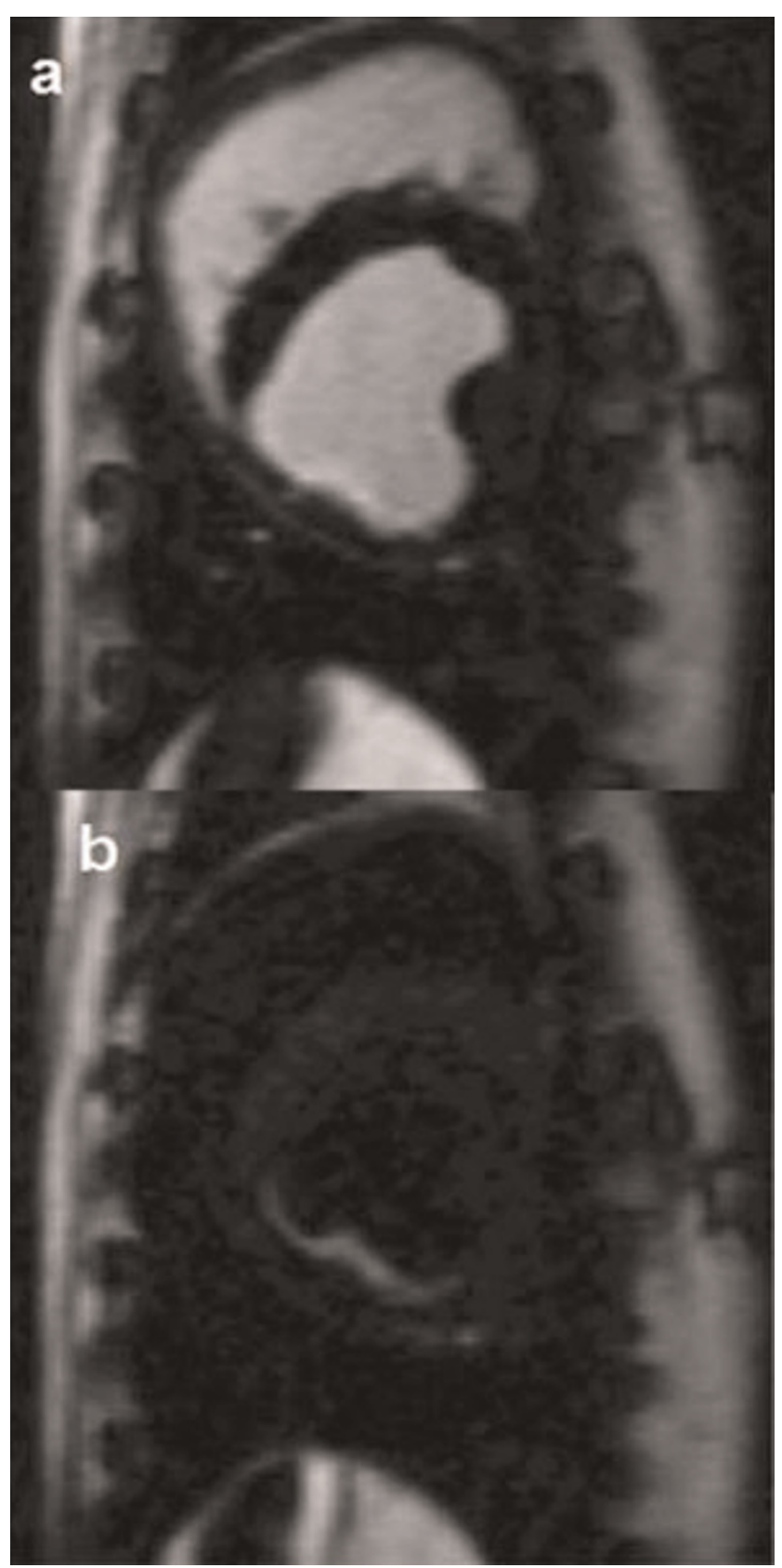

become the gold standard for imaging myocardial infarction. However, subendocardial infarcts are sometimes difficult to detect as they may demonstrate similar image intensity as the ventricular cavity. A double inversion dark-blood pulse sequence to create black blood delayed enhancement images of myocardial infarction has been previously described [I, 2, 3]. The sequence relies on precise timing of non-selective and selective inversion pulses, and it is sensitive to incomplete exchange of blood and changes in TI relaxation of the blood and myocardium. The technique significantly improves blood-infarct contrast at the expense of some reduction in SNR and infarct-myocardial contrast. Diffusion preparation-gradients have been used to create dark-blood vessel wall images [4], but have never been applied post-contrast for infarct imaging.

Purpose: To develop a dark-blood delayed enhancement pulse sequence based on diffusion preparation which would not rely on complete blood exchange and would be relatively insensitive to changes in relaxation times.

Methods: A diffusion-prepared inversion recovery (IR) pulse sequence was developed by adding a driven equilibrium module using a BIR-4 0 degree radiofrequency pulse with motion sensitization gradients between its components prior to a segmented FLASH readout. The timing of this preparation was optimized to minimize the effects of strain-induced signal loss. The technique was tested in a canine model of chronic infarction using a I.5 T MR scanner (Magnetom Avanto, Siemens Medical Solutions). Images were obtained 5-10 minutes after injection of $0.15 \mathrm{mg} / \mathrm{kg}$ of Magnevist. Sequence parameters included field of view $300 \mathrm{~mm}$, matrix $192 \times 1 \mathrm{I} 4$, TE $2.7 \mathrm{~ms}$, spatial resolution $1.6 \times 2.3 \times 10 \mathrm{~mm}$, lines per segment 12 , bandwidth $400 \mathrm{~Hz} /$ pixel, acquisition duration 16 heartbeats, effective b-value $0.25 \mathrm{~s} /$ $\mathrm{mm}^{2}$.

Results: Figure I(a) shows a standard bright-blood IR-FLASH delayed enhancement image. There is an infarct in the inferior wall which is difficult to distinguish from the blood pool. Figure I (b) displays an image of the same slice that demonstrates the utility of the diffusion-prepared IR sequence. Here, the diffusion preparation causes suppression of the blood pool, improving the ability to detect the subendocardial region of infarction.

Conclusion: We have developed a new dark-blood delayed enhancement pulse sequence which attenuates the blood pool based on motion sensitization. In preliminary studies, this technique improves delineation of subendocardial infarcts.

\section{References}

I. Rehwald WG, et al: J Cardiovasc Magn Reson 2007, 9:I0I-2.

2. Salerno M, et al: Proc $15^{\text {th }}$ ISMRM 2007.

3. Rehwald WG, et al: Proc $15^{\text {th }} 2007$.

4. Koktzoglou I, et al: J Magn Reson Imaging 2006, 23:699-705.

\section{Ol I}

\section{Phase-sensitive black-blood coronary vessel wall imaging}

Khaled Z Abd-Elmoniem and Matthias Stuber

Department of Radiology, School of Medicine, Johns Hopkins University, Baltimore, MD, USA

\section{Journal of Cardiovascular Magnetic Resonance 2009, I I(SuppI I): O I I}

Introduction: Black-blood coronary vessel wall imaging is a powerful non-invasive tool for the quantitative assessment of positive arterial remodeling [I]. Although dual-inversion-recovery [2] (DIR) is the gold standard for vessel wall imaging, optimal lumen-vessel wall contrast is sometimes difficult to obtain and the time-window available for imaging is limited due to the competing requirements between $\mathrm{TI}^{*}$ (blood signal nulling time) and TD (period of minimal myocardial motion). In addition, atherosclerosis is a spatially heterogeneous disease and therefore imaging at multiple anatomical levels of the coronary circulation is mandatory. However, this requirement of enhanced volumetric coverage typically comes at the expense of increased 
scanning time. Phase-sensitive IR [3, 4, 5] (PS-IR) has shown to be valuable for enhancing tissue-tissue contrast and for making IR imaging less sensitive to TI*. This work extends PS-IR to PS-DIR and combined with spiral-imaging, multi-slice black-blood coronary vessel wall imaging is enabled in a single breath-hold.

Purpose: To develop, and test a phase-sensitive DIR (PS-DIR) single-breath-hold multi-slice spiral black-blood coronary vessel wall imaging method.

Methods: Concept: After DIR (Fig. I), the inversion time TI* allows for signal-nulling of the in-flowing blood-pool at the anatomical level of interest. Blood-tissue contrast therefore depends on the accurate determination of TI*. Although the MR signal is complex (magnitude and phase), DIR images only show the magnitude of the signal with a suboptimal blood-tissue contrast if $\mathrm{TI}$ does not equal TI*. However, by additionally using the MR signal phase, a signed (positive/negative) black-blood image can be acquired at TI less than TI* and reconstructed with a blood-tissue contrast higher than that obtained at $\mathrm{TI}^{*}$. Simultaneously, competing constraints related to TI* and TD are avoided (Fig. I). Consequently, single-breath-hold multislice black-blood coronary vessel wall imaging is enabled using PS-DIR.

Reconstruction: A local region-growing reconstruction algorithm was developed and is summarized in Fig. 2. Pixels with high
Figure I (abstract OII)

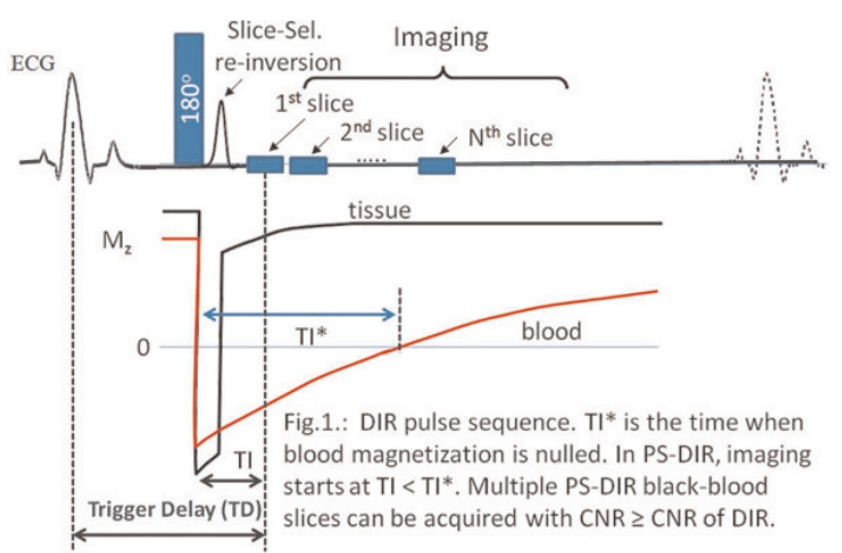

DIR pulse sequence. TI* is the time when blood magnetization is nulled. In PS-DIR, imaging starts at TI < TI*. Multiple PS-DIR black-blood slices can be acquired with $C N R \geq C N R$ of DIR.

signal near the cross-sectional coronary artery are selected as seed points. The phase values of these points are used to estimate the local signal phase inhomogeneity which is needed for local signed-magnitude image reconstruction [4].

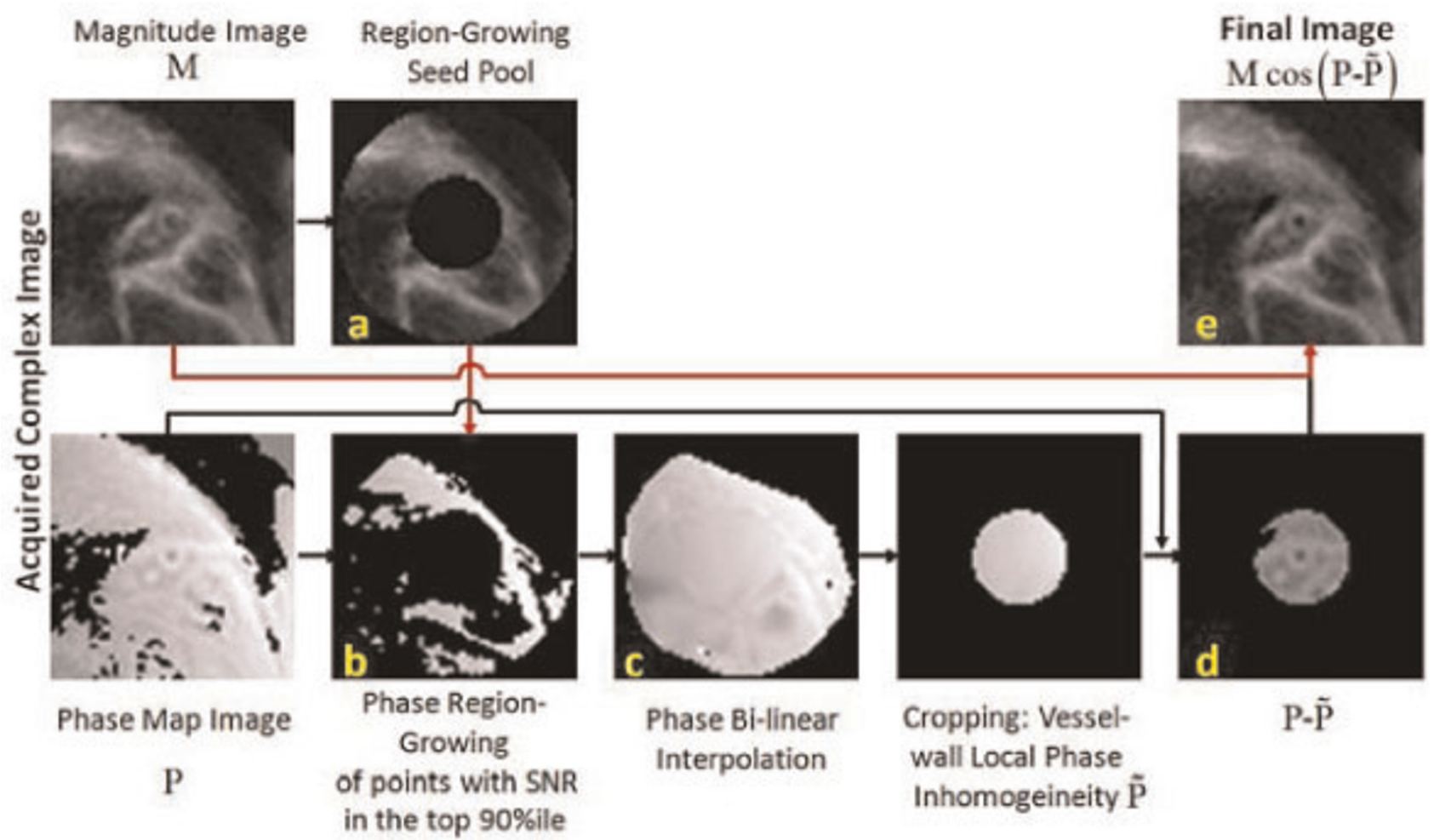

Summary of the PS-DIR Coronary wall signed-image reconstruction algorithm. A ring-shaped region-of-interest is selected around the coronary wall (a). Phase points with high magnitude SNR are selected for region-growing (b). Map of local phase inhomogeneity is created using bi-linear interpolation (c). Inhomogeneity is removed from the phase image (d) and a final signed image is calculated (e). 
Figure 3 (abstract OII)
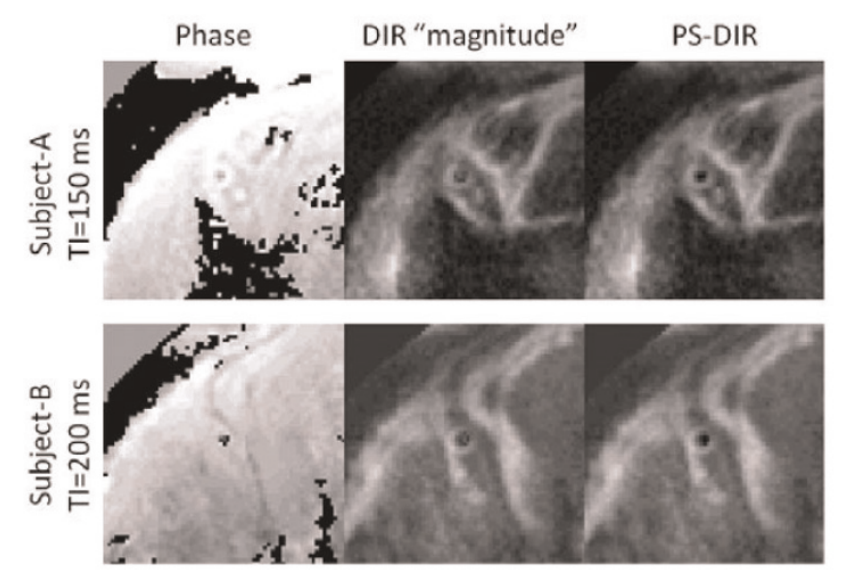

PS-DIR Coronary vessel wall imaging at different $\mathrm{TI}<\mathrm{TI}$ in two separate subjects.

Implementation: A single breathhold DIR sequence was implemented (Fig. I) on a clinical 3 T Philips-Achieva MRIsystem. Data were acquired using a segmented k-space spiral acquisition with spectral spatial excitation [6]. Image processing was performed off-line on a personal computer.

Experiments: Anatomical slices perpendicular to the proximal part of the right coronary artery (RCA) at end-systole were planned similar to a previously published methodology [7]. First, serial single-slice multi-phase PS-DIR images were acquired with incremental $\mathrm{TI}$ ranging from $50 \mathrm{~ms}-500 \mathrm{~ms}$ in 15 healthy adult subjects (slice-thickness $=8 \mathrm{~mm}, F O V=190 \times 190 \mathrm{~mm}$, matrix $=320 \times 320$, interleaves $=20$, acq. window $=18 \mathrm{~ms} /$ interleaf). CNR was calculated on the signed-magnitude images reconstructed with the above algorithm. Mean vessel wall thickness was measured manually on the images obtained with incremental $\mathrm{TI}$ and was compared to that from TI*. Finally, a dualslice rather than a multi-phase version of the sequence (Fig. I) was tested in four subjects.

Results: Using $\mathrm{TI}$ less than TI*, Fig. 3 shows that PS-DIR enables delineation of the coronary artery vessel wall and supports an increased wall-lumen contrast when compared with

Figure 5 (abstract OII)

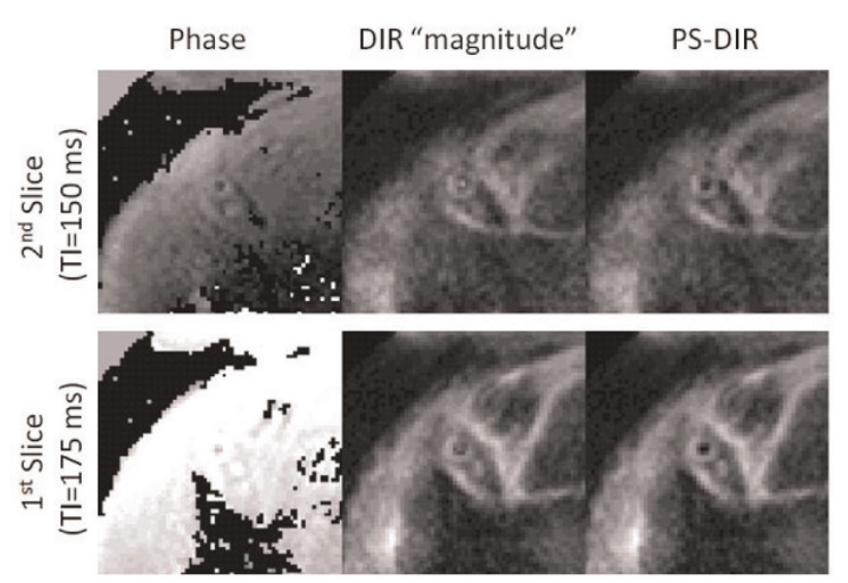

Dual-slice single breath-hold PS-DIR Coronary vessel wall black-blook imaging

Figure 4 (abstract OII)
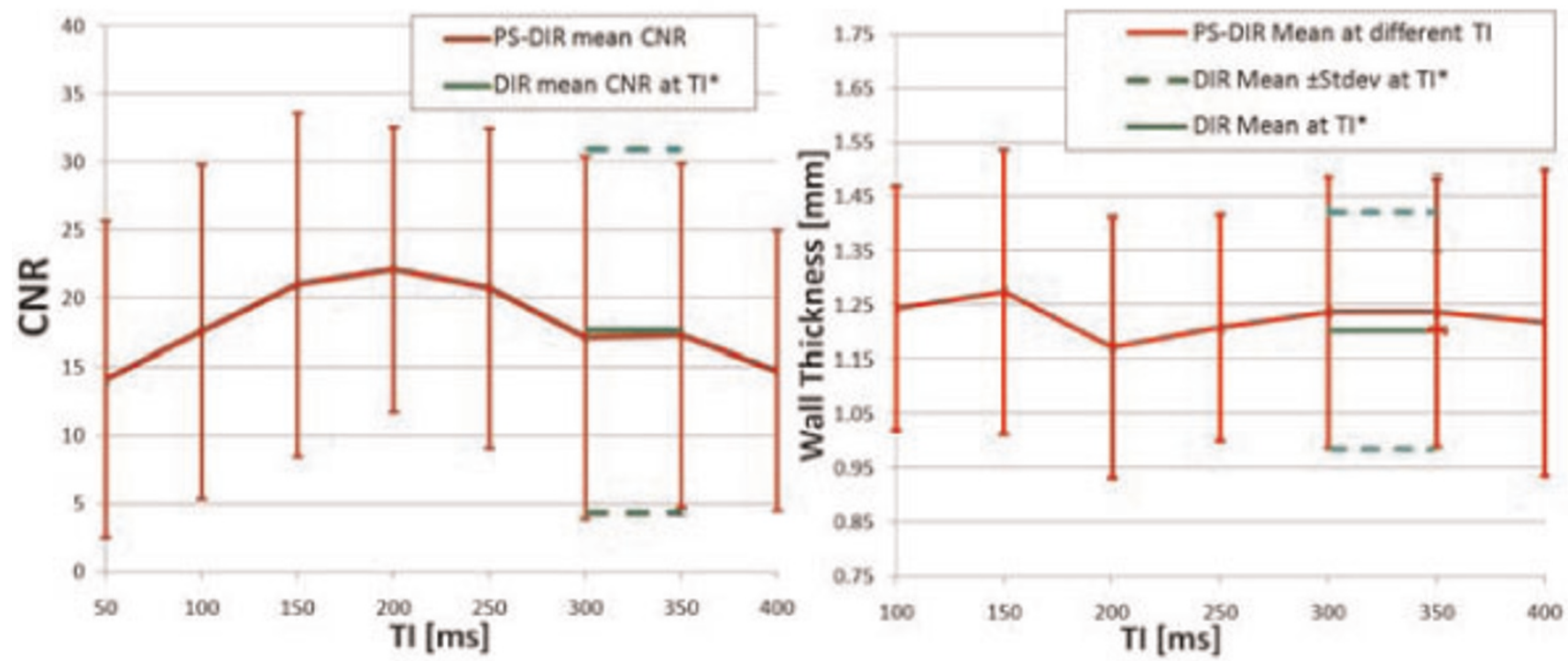

Left: CNR (mean \pm stdev) at different TI values in PS-DIR signed images (red) and in DIR magnitude images at TI* (green). Right: Wall thickness at different TI using PS-DIR images (red) and using DIR images at TI*. Note the agreement with the measurements using TI*. 
the conventional DIR in which TI was too short for adequate blood signal-nulling. Consistent with the visual findings, Fig. 4 shows that the CNR significantly increased in PS-DIR over a broad range of $\mathrm{TI}(150 \mathrm{~ms}-300 \mathrm{~ms})$. Wall thickness measurements using PS-DIR at different TI values were consistent with those from DIR at TI*. Since the PS-DIR method permits image data collection over a broad range of TI (Fig. 4), multiple slices rather than multiple phases can be obtained at no extra cost in scanning time (Fig 5).

Discussion: PS-DIR provides a Tl-insensitive higher CNR alternative to conventional DIR for coronary vessel wall imaging. TI-insensitivity can be traded for enhanced volumetric coverage at no extra-cost in imaging time.

\section{References}

I. Kim WY, et al: Circulation 2002, I06(3):296-9.

2. Edelman RR, et al: Radiology |99|, I 8 I (3):655-60.

3. Borrello JA, et al: Magn Reson Med 1990, I4(I):56-67.

4. Xiang QS: J Magn Reson Imaging 1996, 6(5):775-82.

5. Kellman P, et al: Magn Reson Med 2002, 47(2):372-383.

6. Meyer CH, et al: Magn Reson Med 1990, I 5(2):287-304.

7. Botnar RM, et al: Circulation 2000, I 02(I 02)2 I:2582-7.

\section{OI 2}

Imaging the vessel wall in major peripheral arteries using susceptibility weighted imaging: visualizing calcifications

Qi Yang', Kuncheng Li', Jiangtao Liu', S Barnes², Z Wu ${ }^{3}$, $J$ Neelavalli $^{2}$, J Hu ${ }^{2}$ and EM Haacke ${ }^{2}$

${ }^{I}$ Xuanwu Hospital, Beijing, PR China

${ }^{2}$ Wayne State University, Detroit, MI, USA

${ }^{3}$ McMaster University, Hamilton, ON, Canada

Journal of Cardiovascular Magnetic Resonance 2009, I I(SuppI I):OI2

Introduction: Magnetic resonance imaging (MRI) has been used for many years to study atherosclerosis [I]. Black blood techniques are the most ubiquitous and are used to suppress the signal from flowing blood, making the vessel wall more conspicuous. The purpose of this study was to demonstrate a novel approach to imaging the vessel wall and vessel wall calcification using susceptibility weighted imaging [2] (SWI) with no need to suppress the signal from the blood.

Methods: Optimizing the imaging parameters: The SWI sequence parameters were optimized to allow for the best visualization of the femoral artery lumen in the magnitude images and the arterial wall in the phase images. Parameters such as resolution (for time considerations), flip angle (for contrast in the magnitude images) and echo time (for phase contrast) were considered.

Vessel wall magnitude and phase measurements: ROls from the top to the bottom of the visible portions of the femoral artery were taken. The lumen SNR and muscle SNR were calculated on both magnitude and phase images. The contrast-tonoise ratio of vessel wall/lumen and vessel wall/muscle was also calculated.

Patients study: A series of 18 subjects were imaged with multi-detector computed tomography (MDCT) and high resolution susceptibility weighted imaging $(\mathrm{SWI})$ at $3 \mathrm{~T}$.
Figure I (abstract OI2)
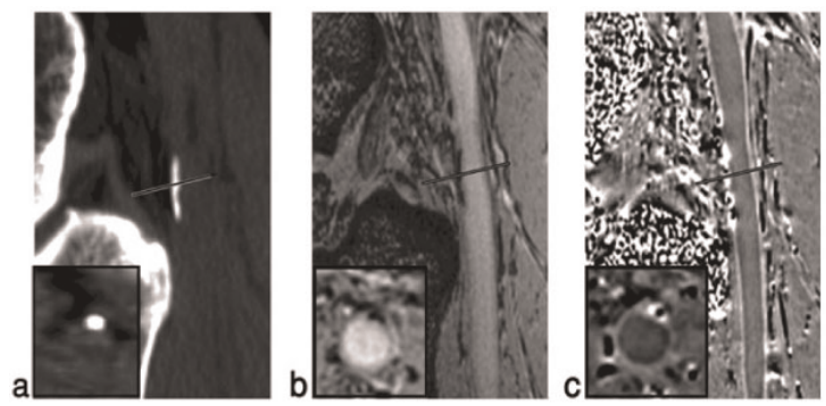

(a) CT scan showing calcification at the edge of the popliteal artery just behind the knee. (b) Magniture gradient echo image showing the signal loss from the calcification of the same area. (c) Phase image showing the diamagnetic effect from the calcification. Note the simliar shape and extent of the calcification in both the CT and MR results. Inserts are zoomed images of the cross-section of the vessels in reformatted transverse images.

Calcification Measurements: The area of calcification was manually measured on CT images and MR images (both magnitude and phase images) by an experienced radiologist. SPIN software (Detroit, MI) was used to interpolate the images by a factor of 4 and measure the calcifications. The correlation of calcification area (CA) between $C T$ and MR images was performed and a Pearson correlation coefficient calculated. The agreement of CA measurements by MR and CT was assessed by using the Bland and Altman plot.

Results: The optimal choice of imaging parameters was found to be: $\mathrm{TE}=15.6 \mathrm{~ms}$ (in-phase for fat); $\mathrm{TR}=25 \mathrm{~ms}, \mathrm{FA}=10^{\circ}$, $\mathrm{BW}=80 \mathrm{~Hz} /$ pixel, resolution $=0.5 \mathrm{~mm} \times 0.5 \mathrm{~mm}$ in-plane and I.0 $\mathrm{mm}$ through-plane, with an acquisition matrix of $512 \times 384 \times 64$ (for read, phase and slice-select direction) and a total scan time of 8 minutes. The magnitude contrast-to-noise ratio (CNR) between artery and vessel wall was 12:I. The phase CNR between the arterial wall and the lumen was 7:I. A total of 19 calcifications in the femoral vessel wall were identified with SWI in 8 subjects. The mean area of calcification measured on $\mathrm{CT}$, magnitude and phase images was $0.37 \pm 0.17 \mathrm{~cm}^{2}$, $0.29 \pm 0.13 \mathrm{~cm}^{2}, 0.38 \pm 0.18 \mathrm{~cm}^{2}$ respectively. The Pearson correlation coefficient of the measured lesion area between CT and magnitude image is $0.85(\mathrm{p}<0.00 \mathrm{I})$; between CT and phase image is $0.92(p<0.001)$. A typical case having popliteal artery calcification is shown in Figure I. Both magnitude and phase images show the calcifications clearly in the popliteal artery wall and correlate well with the CT image.

Conclusion: SWI offers a means to image a large field-of-view over which the arterial wall can be clearly seen in both magnitude and SWI filtered phase images. These lesions were seen in CT and SWI and correlated well in both size and position with both methods. We anticipate that SWI will play a complementary role to the current multi-contrast approach in studying atherosclerosis.

\section{References}

I. Yuan C and Kerwin WS: JMRI 2004, 19:710-719.

2. Haacke EM, Xu Y, Cheng YC and Reichenbach JR: MRM 2004, 52:6|2-618. 
013

European cardiovascular magnetic resonance (EUROCMR) registry - preliminary results of the German pilot phase

Oliver Bruder', Steffen Schneider ${ }^{2}$, Detlev Nothnagel ${ }^{3}$, Thorsten Dill ${ }^{4}$, Eike Nagel ${ }^{5}$, Massimo Lombardi ${ }^{6}$, Albert C van Rossum ${ }^{7}$, Anja Wagner ${ }^{8}$, Juerg Schwitter", Jochen Senges ${ }^{2}$, Georg V Sabin', Udo Sechtem ${ }^{10}$ and Heiko Mahrholdt ${ }^{\text {Po }}$

'Department of Cardiology and Angiology, Elisabeth Hospital, Essen, Germany

${ }^{2}$ Institut für Herzinfarktforschung, Ludwigshafen, Germany

${ }^{3}$ Department of Cardiology, Klinikum Ludwigsburg,

Ludwigsburg, Germany

${ }^{4}$ Department of Cardiology, Kerkhoff-Klinik, Bad Nauheim, Germany

${ }^{5}$ Division of Imaging Sciences, King's College, London, UK

${ }^{6}$ Clinical Physiology Institute, CNR National Research Council, Pisa, Italy

${ }^{7}$ Department of Cardiology, VU Medical Center, Amsterdam, Netherlands

${ }^{8}$ Duke Cardiovascular Magnetic Resonance Center, Duke University Medical Center, Durham, NC, USA

${ }^{9}$ Clinic of Cardiology and Cardiac MR Center, University of Zurich, Zurich, Switzerland

${ }^{10}$ Department of Cardiology, Robert Bosch Medical Center, Stuttgart, Germany

Journal of Cardiovascular Magnetic Resonance 2009, I I(Suppl I):OI3

Background: Cardiovascular magnetic resonance (CMR) has a broad range of clinical applications and is increasingly used in daily clinical practice in many European countries.

During its German pilot phase the EUROCMR Registry sought to evaluate indications, image quality, safety and impact on patient management of CMR imaging in clinical routine in a large number of cases.

Methods: Multicenter registry with consecutive enrolment of patients scanned in 29 German CMR centers using web based online case record forms.

Results: 6530 consecutive patients were enrolled from April 2007 to September 2008 (66\% male, median age 6I years [quartiles 49-70]). Ninety-three percent of patients received a gadolinium based contrast agent. Twenty percent of patients underwent adenosine perfusion, and I3\% high-dose dobutamine stress CMR. The indications for CMR can be viewed in Figure I. Case reading and reporting was mostly done by cardiologists (70\%), or a team of cardiologists and radiologists (26\%). Image quality was found to be good or excellent in $91 \%$, moderate in $8 \%$, and inadequate in $1 \%$ of cases.

Severe complications occurred in a minority of patients $(0.07 \%)$ and were all associated with stress testing. We reported NSVT $(n=I)$, VF $(n=1)$ during dobutamine infusion, as well as overt heart failure $(n=2)$ and unstable angina $(n=I)$ related to adenosine stress CMR. No patient died during or due to the CMR procedure.

In nearly half the patients included (48\%), CMR findings resulted in a change of patient management. Importantly, in $16 \%$ of cases the final diagnosis based on CMR was different to the diagnosis before CMR, leading to a complete change in patient management. In more than $70 \%$ of cases CMR was capable of satisfying all imaging needs so that no further imaging procedure was required after completion of CMR.
Figure I (abstract OI3)

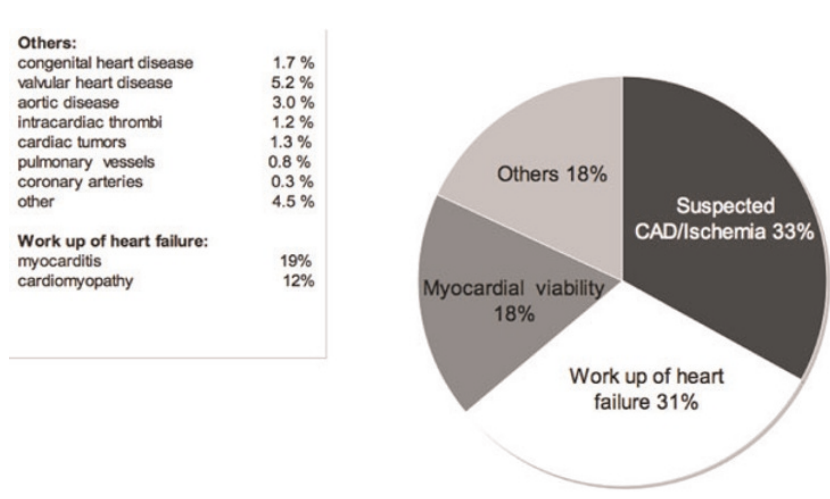

Initial indications for CMR $(n=6530)$.

Conclusion: CMR is a frequently performed in German clinical practice. The most important indications are risk stratification in suspected CAD/lschemia, workup of heart failure, and assessment of myocardial viability. CMR imaging is a safe procedure, has diagnostic image quality in $99 \%$ of cases, and its results have strong impact on patient management.

\section{4}

Sudden cardiac death with normal coronaries: cardiac MRI in the differential diagnosis of underlying disease in survivors

Peter Hunold', Thomas Schlosser', Kai Nassenstein ${ }^{2}$, Oliver Bruder ${ }^{3}$, Holger Eggebrecht ${ }^{4}$, Peter W Radke and Jörg Barkhausen

'University Hospital Schleswig-Holstein, Campus Lübeck, Lübeck, Germany

${ }^{2}$ University Hospital Essen, University of Duisburg-Essen,

Essen, Germany

${ }^{3}$ Elisabeth Hospital, Essen, Germany

${ }^{4}$ West German Heart Center, University of Duisburg-Essen, Essen, Germany

Journal of Cardiovascular Magnetic Resonance 2009, I I(SuppI I):OI4

Introduction: Sudden cardiac death (SCD) is most commonly caused by acute myocardial infarction as a correlate of coronary artery disease. Therefore, survivors of SCD undergo cardiac catheter to treat or rule out CAD. However, in cases with normal coronary arteries SCD often remains unexplained. Diagnostic work-up in this collective is important to adjust and optimize therapy.

Purpose: Aim of this study was to evaluate the use of contrastenhanced cardiac MRI (CMR) in defining the underlying pathology of survived SCD in patients without coronary artery occlusion.

Methods: More than 6.000 contrast-enhanced CMR studies from 3 different hospitals were reviewed for cases of survived SCD with angiographic rule out of obstructive coronary artery disease. The CMR protocol (I.5 T) consisted of a functional left ventricular study using a segmented SSFP sequence (TrueFISP, balancedFFE) in long and short axes. Data sets for late gadolinium enhancement detection were acquired 8-15 min after $0.2 \mathrm{mmol} /$ $\mathrm{kg}$ BW of Gd-based contrast material using a segmented inversion-recovery TurboFLASH/FGRE sequence (TI, 200-260 ms; 
slice thickness, $8 \mathrm{~mm}, 2 \mathrm{D}$ or 3D). All cases of non-coronary SCD were reviewed and the different underlying pathologies as defined by MRI were collected.

Results: In total, 18 cases of unclear SCD were identified. In I4 patients thereof, MRI could state the diagnosis based on the typical imaging features of myocardial disease: Primary cardiomyopathy was found in 7 patients (arrhythmogenic right ventricular cardiomyopathy, 2; dilated cardiomyopathy, 3; hypertrophic cardiomyopathy, I; isolated left ventricular non-compaction, I). Acute myocarditis and acute cardiac sarcoidosis were found in 3 patients each. Chronic aneurysm of the anterior wall most probably due to cardiac contusion was found in I patient. In 4 patients, CMR could clarify the etiology of SCD.

Conclusion: Contrast-enhanced CMR has unique features in detecting and differentiating myocardial disease with possibly fatal outcome. It has proven to be an utmost valuable tool for the diagnostic work-up of survivors of unclear SCD. This underlines the role of $\mathrm{CMR}$ as the first-line technique in myocardial disease.

\section{OI5}

Predictors of subclinical diastolic dysfunction measured by MRI: multi-ethnic study of atherosclerosis (MESA)

Sadia Qadir', Wendy S Post ${ }^{2}$, Gregory W Hundley ${ }^{3}$, Gregory DN Pearson ${ }^{4}$, Shantanu Sinha ${ }^{5}$, Joao Lima ${ }^{2}$ and David A Bluemke ${ }^{6}$

'Massachusetts General Hospital, Boston, MA, USA

2 Johns Hopkins Hospital, Baltimore, MD, USA

${ }^{3}$ Wake Forest University School of Medicine, Winston-Salem, NC, USA

${ }^{4}$ Columbia University, New York, NY, USA

${ }^{5}$ UCLA School of Medicine, Los Angeles, CA, USA

${ }^{6}$ National Institute of Health, Bethesda, MD, USA

Journal of Cardiovascular Magnetic Resonance 2009, I I (SuppI I):O I5

Introduction: Diastolic dysfunction, often preclinical with no recognized CHF diagnosis, is associated with marked increases in all-cause mortality. Current data on diastolic dysfunction have limitations due to retrospective study designs and/or exclusive reliance on echocardiography.
Figure I (abstract OI5)

\section{Females $\square$ Males}

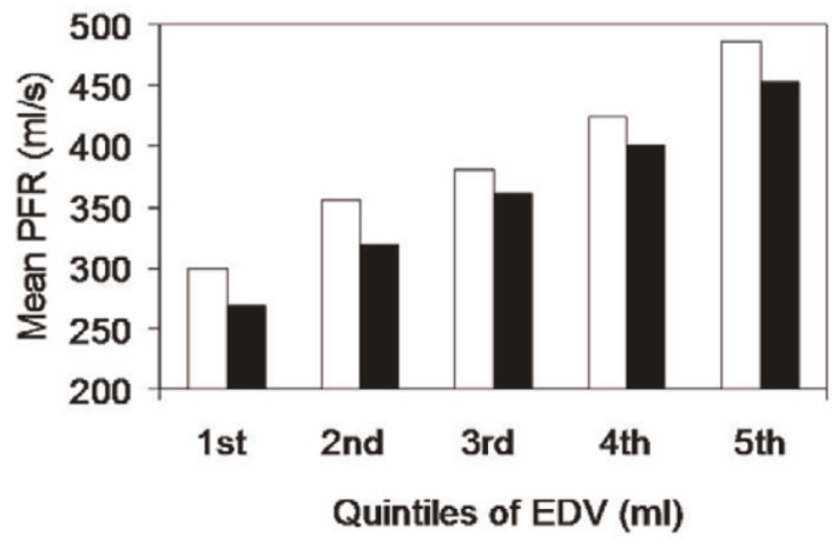

Purpose: The purpose of this study was to analyze the predictors of diastolic dysfunction in the MESA population using cardiac MRI.

Methods: We studied peak filling rate (PFR $\mathrm{ml} / \mathrm{s}$ ) and time to peak filling (TPFR msec) in a subclinical population $(n=4465$, males $47 \%$, mean age $62 \pm 10$ years). Mean (SD), correlation coefficients and multivariable regression coefficients were determined.

Results: Table I illustrates associations between risk factors with diastolic LV function. End diastolic volume (EDV) modified the relationship of gender and PFR. Mean PFR was therefore analyzed across quintiles of EDV and was found to be higher in females 386.35 , [ $95 \% \mathrm{Cl} 382.53$ to 390.17 ] compared to males $359.1 \mathrm{l} \mathrm{ml} / \mathrm{s}$ [95\% Cl 355.05 to 363. I7] (Fig I). Comapred to nonsmokers smokers had lower peak filling rates. Compared to Whites, Hispanics were at a higher risk for diastolic dysfunction, Chinese ethnicity showed a relative protective effect after adjusting for all other risk factors.

Table I (abstract OI5) Multivariable regressions analysis of predictors of diastolic LV dysfunction

\begin{tabular}{|c|c|c|c|c|c|c|}
\hline \multirow[t]{2}{*}{ Independent Predictors } & \multicolumn{3}{|c|}{ Peak Filling Rate $(\mathrm{ml} / \mathrm{s})$} & \multicolumn{3}{|c|}{ Time to Peak Filling (msec) } \\
\hline & $\begin{array}{l}\text { Regression } \\
\text { Coefficient }\end{array}$ & $95 \% \mathrm{Cl}$ & P-Value & $\begin{array}{l}\text { Regression } \\
\text { Coefficient }\end{array}$ & $95 \% \mathrm{Cl}$ & P-Value \\
\hline Age (years) & -1.70 & -1.20 to -1.40 & $<0.001$ & 1.94 & 1.53 to 2.36 & $<0.001$ \\
\hline $\mathrm{DBP}(\mathrm{mmHg})$ & -0.42 & -0.78 to -0.05 & 0.02 & 0.48 & 0.14 to 0.82 & 0.005 \\
\hline HTN meds & -5.92 & -11.18 to -0.62 & 0.03 & 8.86 & 3.01 to $|4.7|$ & 0.003 \\
\hline $\mathrm{BMI}\left(\mathrm{kg} / \mathrm{m}^{2}\right)$ & -0.67 & -1.32 to -0.05 & 0.04 & 1.37 & 0.62 to 2.11 & $<0.001$ \\
\hline Former Smokers & -6.52 & -12.30 to 0.65 & 0.03 & 1.00 & -4.56606 .58 & NS \\
\hline Impaired Fasting Glucose & 2.63 & -2.74 to 8.01 & NS & 9.06 & 3.21 to 17.38 & 0.03 \\
\hline Chinese & 8.14 & -0.25 to 16.53 & NS & -8.19 & -14.27 to -1.87 & 0.01 \\
\hline Hispanics & 8.33 & 1.88 to 14.88 & 0.006 & 4.67 & -1.76 to 11.1 & NS \\
\hline
\end{tabular}


Conclusion: Impaired LV relaxation is associated with increasing age, male gender, obesity, diastolic hypertension, smoking, and varied by ethnicity.

\section{6}

\author{
Correlation of pericardial and mediastinal \\ fat with coronary artery disease, metabolic \\ syndrome, and cardiac risk factors \\ Onn Chenn', ljaz Ahmad', Betty Hua', \\ Joshua A Sockolow', Igor Klem², Terrence Sacchi' \\ and John F Heitner' \\ ${ }^{I}$ New York Methodist Hospital, Brooklyn, NY, USA \\ ${ }^{2}$ Duke University Medical Center, Durham, NC, USA
}

Journal of Cardiovascular Magnetic Resonance 2009, I I(SuppI I):OI6

Background: Obesity and abdominal fat have been shown to correlate with coronary artery disease (CAD) and may play a role in development of metabolic syndrome (MS). The significance of pericardial adipose tissue (PAT) and mediastinal adipose tissue (MAT) is less clearly defined.

Objective: To study the association between PAT and MAT measured by cardiac magnetic resonance with: I) severity of CAD, 2) MS and 3) cardiac risk factors (CRF) for CAD.

Methods: We enrolled 100 consecutive patients, 63 male, who underwent CMR for cardiac evaluation and had coronary angiogram performed within 12 months. The baseline characteristics of these patients were as follows: Eighty had hypertension (HTN), 42 had diabetes mellitus (DM), 37 had hyperlipidemia and 4 were smoker. We measured PAT and MAT on 4-chamber cine view. The surface area of fat was measured by computer analysis from free-hand region of interest (ROI) curves. The presence and the extent of CAD were measured using Duke Jeopardy Score. MS was considered positive if the patient had 3 or more of the 5 criteria. The CRF included HTN, DM, hyperlipidemia, smoking, peripheral vascular disease (PVD) and a family history of premature CAD (FH).

Results: PAT had significant correlation with MS and HTN, but not with CAD. MAT did not have a significant correlation with either CAD or MS, but did correlate with DM, hyperlipidemia, smoking and FH. The combination of PAT and MAT correlated with MS and all the risk factors, except PVD, but did not correlate with CAD. (Data in Table I.).
Conclusion: The combination of MAT and PAT correlates with MS and a number of CRFs but does not correlate with CAD.

\section{OI7}

Prognostic significance of magnetic resonance imaging parameters in patients with idiopathic dilated cardiomyopathy

Nico Merkle, Jan Torzewski, Georg Grossmann, Volker Rasche, Matthias Kochs, Jochen Woehrle and Vinzenz Hombach University Ulm, Ulm, Germany

Journal of Cardiovascular Magnetic Resonance 2009, I I(SuppI I):OI7

Background: Patients with idiopathic dilated cardiomyopathy (IDC) have a limited prognosis. Aim of this study was to evaluate the prognostic significance of novel magnetic resonance imaging (CMR) parameters in IDC patients.

Methods and results: $16 \mathrm{I}$ patients with IDC were studied by CMR for hemodynamic and late enhancement (LE) analysis and followed for a mean of 933,8 $\pm 529,2$ days. QRS and QTc intervals were measured from I2-lead ECGs. LV and RV enddiastolic and endsystolic volume indexes were increased and ejection fractions (EF) decreased (LV-EF $33 \pm 14 \%$, RV-EF $50 \pm 16 \%$ ). LE was seen in 43 patients (27\%). Interventricular dyssynchrony $(50.9 \pm 67.2 \mathrm{~ms})$ was present in 68 patients $(42 \%)$. Primary endpoint was cardiac death, sudden death (SCD) and rehospitalization for pump failure, secondary endpoint cardiac death and SCD. 3 patients died from non-cardiac, 10 patients from cardiac death, 2 patients from SCD and additional 3 patients had ICD shock for ventricular flutter/fibrillation, and 35 patients were rehospitalized. Multivariate analysis revealed depressed left ventricular $\mathrm{EF}(<25 \%)$ right ventricular $\mathrm{EF}(<30 \%)$, as well as the presence of LE as independent prognostic parameters. KaplanMeier survival analysis displayed low LV $(<25 \%)$ and RV $(<30 \%)$ ejection fraction and the presence of LE as significant parameters for a worse outcome.

Conclusion: In addition to impaired left ventricular ejection fraction $(<25-30 \%)$ a depressed right ventricular function $(E F<30 \%)$ and the presence of late enhancement derived from CMR are novel prognostic parameters in patients with IDCM.

Table I (abstract 016)

\begin{tabular}{|c|c|c|c|}
\hline & Pericardial Fat $\left(\mathrm{cm}^{2}\right)$ & Mediastinal Fat $\left(\mathrm{cm}^{2}\right)$ & Total Fat $\left(\mathrm{cm}^{2}\right)$ \\
\hline CAD & $8.3\{p$-value $=0.317\}$ & $19.5\{p$-value $=0.16\}$ & $27.7\{\mathrm{p}$-value $=0.03\}$ \\
\hline Metabolic Syndrome & $9.3\{\mathrm{p}$-value $=0.0005\}$ & $18.9\{\mathrm{p}$-value $=0.19\}$ & $26.8\{\mathrm{p}$-value $=0.02\}$ \\
\hline \multicolumn{4}{|l|}{ Cardica Risk Factors } \\
\hline HTN & $9.5\{\mathrm{p}$. value $=0.008\}$ & $19.6\{\mathrm{p}$-value $=0.08\}$ & $29.1\{\mathrm{p}$-value $=0.03\}$ \\
\hline DM & $9.9\{\mathrm{p}$-value $=0.08\}$ & $21.2\{\mathrm{p}$-value $=0.05\}$ & $31.2\{\mathrm{p}$-value $=0.03\}$ \\
\hline Hyperlipidemia & $9.0\{\mathrm{p}$-value $=0.56\}$ & $20.4\{\mathrm{p}$-value $=0.01\}$ & $29.4\{\mathrm{p}$-value $=0.02$ \\
\hline Smoking & $9.2\{\mathrm{p}$-value $=0.86\}$ & I3. $\mid\{\mathrm{p}$-value $=0.05\}$ & $22.2\{\mathrm{p}$-value $=0.27\}$ \\
\hline PVD & $11.9\{\mathrm{p}$-value $=0.008\}$ & $19.5\{\mathrm{p}$-value $=0.79\}$ & $32.3\{\mathrm{p}$-value $=0.05\}$ \\
\hline $\mathrm{FH}$ & $9.0\{\mathrm{p}$-value $=0.74\}$ & $23.2\{\mathrm{p}$-value $=0.02\}$ & $31.2\{\mathrm{p}$-value $=0.58\}$ \\
\hline
\end{tabular}




\section{8}

Complementary prognostic values of stress myocardial perfusion and late gadolinium enhancement imaging by cardiac magnetic resonance in patients with suspected myocardial ischemia

Kevin Steel', Ryan Broderick², Vijay Gandla ${ }^{2}$,

Eric Larose ${ }^{3}$, Frederick Resnic ${ }^{2}$,

Michael Jerosch-Herold ${ }^{2}$, Kenneth Brown ${ }^{4}$

and Raymond Y Kwong ${ }^{2}$

${ }^{I}$ Wilford Hall Medical Center, San Antonio, TX, USA

${ }^{2}$ Brigham and Women's Hospital, Boston, MA, USA

${ }^{3}$ Quebec Heart Institute, Quebec City, QC, Canada

${ }^{4}$ University of Vermont College of Medicine,

Burlington, VT, USA

Journal of Cardiovascular Magnetic Resonance 2009, I I(SuppI I):O I8

Background: Recent studies have demonstrated the prognostic implication of CMR myocardial perfusion imaging (CMRMPI) in a clinical setting. Apart from detecting reversible perfusion defect from flow-limiting coronary stenosis, CMR late enhancement imaging (LGE) is currently the most sensitive method in detecting clinically unrecognized subendocardial infarction (UMI) from prior ischemic injury. We therefore tested the hypothesis that, characterization of these 2 processes from coronary artery disease (CAD) by CMR can provide complementary patient prognostic values.

Methods and Results: We performed CMR on 254 patients referred with symptoms suspicious of myocardial ischemia. Rest
Figure 2 (abstract 018 )

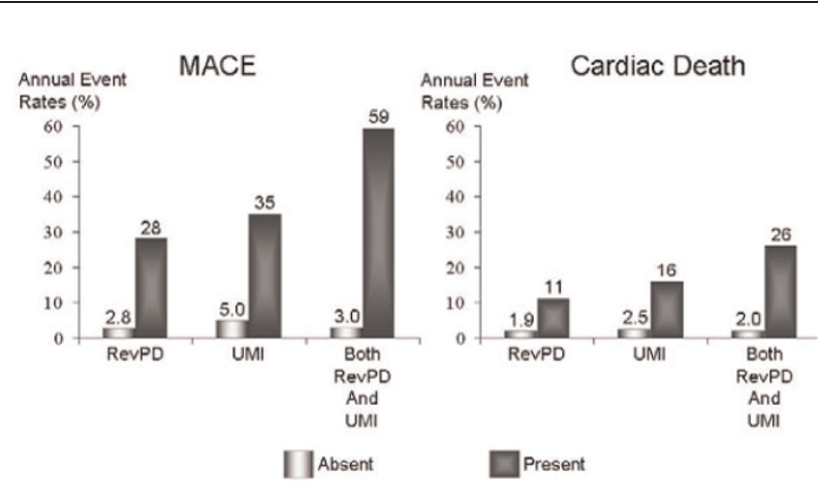

Annual event rates of MACE and cardiac death by RevPD, UMI, and both RevPD and UMI.

and vasodilator (adenosine or dipyridamole) stress first-pass CMRMPI images were obtained and followed by LGE imaging. All CMRMPI images were interpreted for reversible perfusion defects (RevPD) using the 16-segment nomenclature and graded segmental LGE in a separate session. The readers were blinded to any clinical outcome in either session. At a median follow up of I 5 months, 13 cardiac deaths and 26 nonfatal events occurred. RevPD was the strongest multivariable predictor to MACE, demonstrating a $>8$-fold hazard increase to MACE $(P<0.000 \mathrm{I})$

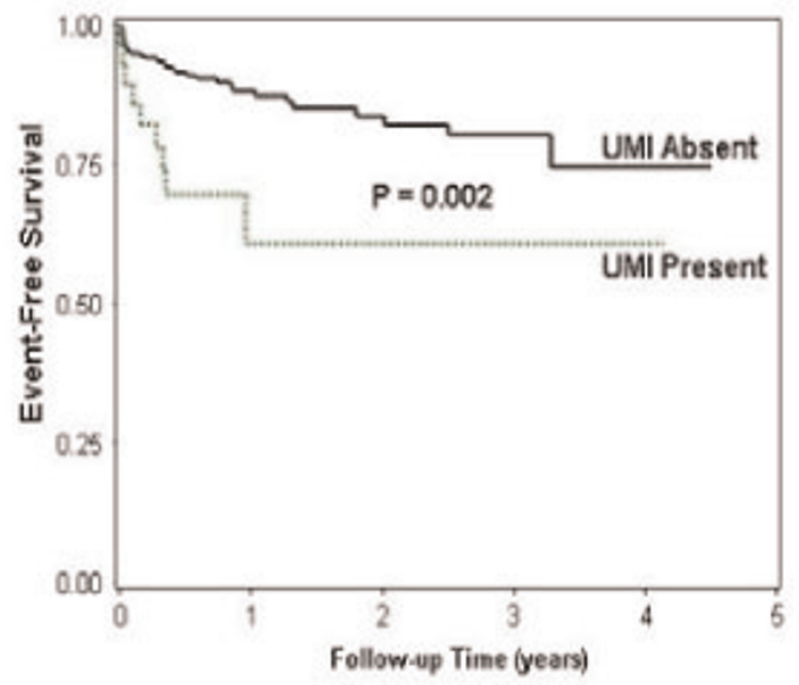

A

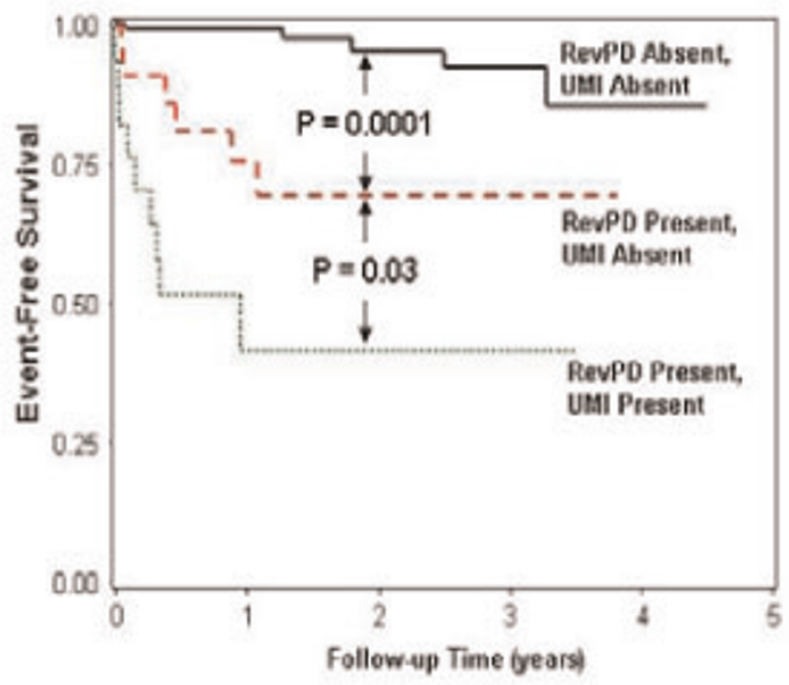

B

Kaplan-Meier curves illustrating. A) the prognostic implications of UMI alone and B) the complementary prediction of MACE by RevPD and UMI, in patients without any history of MI. 
and a $>4$-fold increase to cardiac death $(P=0.02)$. Adjusted to the effects of RevPD, LGE maintained a > 2-fold adjusted hazards with MACE (adjusted HR 2.38, P = 0.03). In 198 patients without any history of MI, presence of RevPD and UMI by LGE provided complementary prognostic information after adjusting to each other's effects. Figures I and 2.

Conclusion: Reversible myocardial perfusion and evidence of MI, assessed by CMRMPI and LGE, respectively, provide incremental long-term patient prognostic information.

\section{9}

Initiation of statin therapy halts progression of atherosclerotic plaque burden in peripheral arterial disease

Amy M West, Justin D Anderson, Craig D Meyer,

Frederick H Epstein, Klaus D Hagspiel,

Stuart S Berr, Nancy L Harthun,

Joseph M DiMaria, Jennifer R Hunter,

John M Christopher, Gabriel B Winberry

and Christopher M Kramer

University of Virginia, Charlottesville, VA, USA

Journal of Cardiovascular Magnetic Resonance 2009, I I(SuppI I):O 19

Introduction: Studies suggest lipid lowering therapy improves symptoms and exercise performance in patients with peripheral arterial disease (PAD); however, the mechanism of action is unclear.

Purpose: We sought to use CMR to study the relationship between LDL reduction and superficial femoral artery (SFA) plaque burden in patients with PAD treated with lipid lowering therapy over the course of I year.

Methods: 63 patients with mild-to-moderate symptomatic PAD (mean age $63 \pm 10$ years, mean ankle brachial index (ABI) $0.69 \pm 0.15$ ) had their most symptomatic leg studied with MRI to assess atherosclerotic plaque burden before and I year after being started on lipid lowering therapy. At study entry, statinnaïve patients were randomized to either simvastatin $40 \mathrm{mg}$ or simvastatin $40 \mathrm{mg}$ plus ezetimibe $10 \mathrm{mg}(\mathrm{R}$ group, $\mathrm{n}=3 \mathrm{I})$ while patients already on a statin were given open-label ezetimibe $10 \mathrm{mg}$ ( $Z$ group, $\mathrm{n}=32$ ). Lipid measurements were obtained as part of the VAP test. CMR was performed using fat-suppressed multi-slice turbo-spin-echo pulse sequence on a Siemens Avanto I.5 T scanner. A custom-built flexible, linear four-element ( $10 \mathrm{~cm} \times 10 \mathrm{~cm}$ square element) surface coil array was placed over the SFA to image $15-20 \mathrm{~cm}$ along the vessel beginning

Table I (abstract O I9) Changes in plaque and vessel wall volume over time

\begin{tabular}{llll}
\hline & Baseline & One Year & $\%$ Change \\
\hline Plaque volume $(\mathrm{cm} 3)-\mathrm{R}$ & $8.64 \pm 4.72$ & $8.50 \pm 4.70$ & $-2 \pm 8 \% *$ \\
Plaque volume $(\mathrm{cm} 3)-\mathrm{Z}$ & $9.16 \pm 4.94$ & $9.53 \pm 4.82$ & $+3 \pm 11 \%$ \\
TVV $(\mathrm{cm} 3)-\mathrm{R}$ & $13.61 \pm 7.99$ & $13.34 \pm 7.93$ & $-2 \pm 8 \%$ \\
TVV $(\mathrm{cm} 3)-\mathrm{Z}$ & $14.27 \pm 6.88$ & $14.64 \pm 6.83$ & $+1 \pm 8 \%$ \\
\hline
\end{tabular}

$*_{p}<0.04$ vs. $Z$ group.
Figure I (abstract O19)

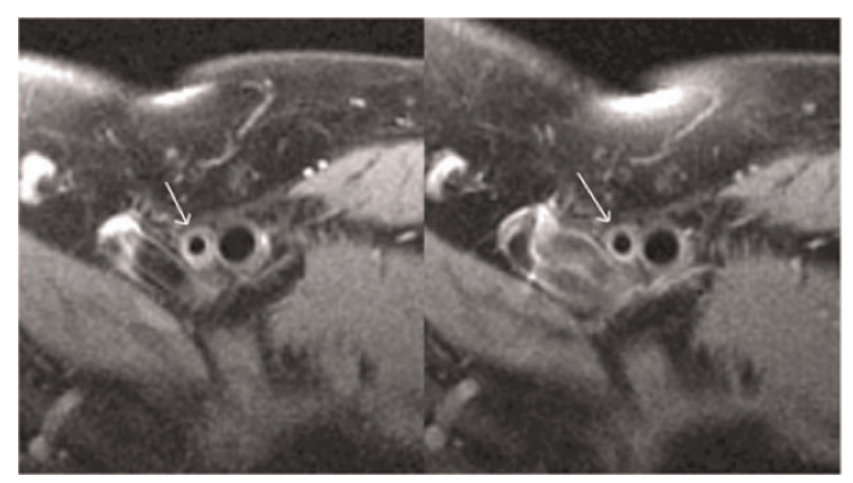

Left - Representative black blood image of SFA from R group patient at baseline. Right - same location imaged one year later. Notice plaque regression in the SFA.

below the bifurcation of the common femoral. Blood was suppressed through the multislice data set using spatial presaturation, with periodic excitation of upstream slices. Imaging parameters included: repetition time $1100 \mathrm{~ms}$, echo time $7.6 \mathrm{~ms}$, echo spacing $7.5 \mathrm{~ms}$, turbo factor (9), voxel size $0.5 \times 0.5 \times 3 \mathrm{~mm}, 4$ signal averages, with interleaved image sets. Plaque volume (PV) defined as total vessel volume (TVV) minus lumen volume (LV) was measured with VesselMass software. Changes in all parameters between groups from baseline to year one were compared by unpaired t-test. Changes in LDL were compared with changes in plaque parameters by linear regression.

Results: LDL at baseline was higher in the R group (120 \pm 37 ) than the $Z$ group $(100 \pm 27) \mathrm{mg} / \mathrm{dl}, \mathrm{p}=0.02$. The decrease in $\mathrm{LDL}$ at one year was significantly greater in $\mathrm{R}(-4 I \pm 37)$ than $Z$ $(-21 \pm 30) \mathrm{mg} / \mathrm{dl}, \mathrm{p}<0.03$, such that final LDL was similar between groups $(79 \pm 36$ in the $R$ group and $79 \pm 33$ in the $Z$ group, $\mathrm{mg} / \mathrm{dl}$ ). The total cholesterol at baseline was higher in the $R$ group $(195 \pm 42)$ than the $Z$ group $(I 7 I \pm 39) \mathrm{mg} / \mathrm{dl}$, $p<0.03$. There was a trend towards a greater fall in total cholesterol in $R(48 \pm 42)$ compared to $Z(27 \pm 42) \mathrm{mg} / \mathrm{dl}$, $p=0.07$. The final total cholesterol was similar between groups. No between group changes in HDL or triglycerides were seen. See Table I for changes in vessel wall parameters in the 2 groups. Plaque volume regressed in $R$ while it progressed in $Z$ and total vessel volume followed the same trend $(p=0.1 I)$. No between group differences in lumen volume or change in lumen volume over time was noted. No correlation was found between change in LDL and plaque volume in the SFA. Figure I.

Conclusion: Statin-naive patients with PAD who were begun on either simvastatin or simvastatin plus ezetimibe for one year had a halting of progression of atherosclerotic plaque volume in the superficial femoral artery when compared to those already treated with statin given ezetimibe. Reverse vessel wall remodeling was noted among PAD patients newly treated with statins compared to those with ezetimibe added to preexisting statin therapy. Thus the degree and/or mechanism of LDL lowering rather than the final LDL achieved may be important in halting atherosclerotic plaque progression. In 
addition, this study demonstrates that CMR can show differences in plaque progression in PAD with small numbers per patient group.

\section{O20}

Characterization of tissue heterogeneity

by contrast-enhanced cardiovascular

magnetic resonance imaging is a powerful

predictor of ventricular tachyarrhythmias on

ambulatory holter ECG in hypertrophic cardiomyopathy

Caitlin J Harrigan ${ }^{1}$, A Selcuk Adabag ${ }^{2}$,

Evan Appelbaum ${ }^{3}$, Kevin S Heffernan ${ }^{\text {, }}$

John R Lesser ${ }^{2}$, James E Udelson', Warren J Manning ${ }^{4}$,

Barry J Maron ${ }^{2}$ and Martin S Maron'

${ }^{I}$ Tufts Medical Center, Boston, MA, USA

${ }^{2}$ Minneapolis Heart Institute Foundation,

Minneapolis, MN, USA

${ }^{3}$ Perfuse Core Lab, Boston, MA, USA

${ }^{4}$ Beth Isreal Deaconess Medical Center,

Boston, MA, USA

Journal of Cardiovascular Magnetic Resonance 2009, I I(Suppl I):O20

Background: Cardiovascular magnetic resonance with late gadolinium enhancement (LGE-CMR) identifies areas of myocardial scarring in patients with hypertrophic cardiomypathy (HCM). The presence of LGE identifies HCM patients at risk for ventricular tachyarrhythmias, which is an independent predictor of sudden death in this disease. In this study, we sought to determine whether regions of abnormal tissue at the confluence of both viable myocardium and fibrosis ("border zone") (BZ) are more predictive of ventricular tachyarrhythmias than areas of myocardial scarring alone.

Methods: Cine CMR and LGE-CMR were performed in 145 HCM patients ( $42 \pm 15$ years; $74 \%$ male) from two HCM referral centers using standard techniques $(0.2 \mathrm{mmol} / \mathrm{kg}$ gadoliniumDTPA). LGE was determined by a blinded, independent reader using grayscale thresholds of both 4 and 6 standard deviations (SD) above the mean of normal, remote myocardium. BZ was determined as the difference between the 4 SD and 6 SD thresholds. All subjects underwent 24-h ambulatory Holter electrocardiogram (ECG) within $7.8 \pm 8.3$ weeks of the CMR

Results: The amount of myocardial scarring (6 SD) alone was $5.5 \pm 7.2 \%$ of the LV myocardium, while the amount of BZ was $6.5 \pm 4.6 \%$ of the LV myocardium. Runs of non-sustained ventricular tachycardias (NSVT) were present in 23 patients (I6\%), ventricular couplets were present 39 patients (27\%), while premature ventricular couplets (PVCs) were present in 119 patients (82\%). There was no significant association with the extent of myocardial scarring and the occurrence of NSVTs, couplets, and PVCs (overall $p=0.05$ ). However the extent of BZ was significantly associated with the occurrence of NSVTs, couplets and PVCs (overall $p<0.001$ ).

Conclusion: These data demonstrate that in HCM patients, the extent of BZ may be a superior predictor of ventricular tachyarrhythmias than myocardial scarring alone (using a grayscale thresholding technique of 6 SD above normal myocardium). This study indicates the need for follow-up studies evaluating the independent prognostic value of $B Z$ in risk stratification strategies in HCM patients.
O2 I

Late enhancement in 39 cardiac transplant patients: prevalence, pattern, and extent Craig RL Butler', Andreas Kumar', Mustafa Toma', Richard Thompson', Matthias Friedrich ${ }^{3}$ and David Ian Paterson'

${ }^{I}$ University of Alberta, Edmonton, AB, Canada

${ }^{2}$ University of Calgary, Calgary, $A B$, Canada

${ }^{3}$ Stephenson Cardiovascular MR Center, Calgary, AB, Canada

Journal of Cardiovascular Magnetic Resonance 2009, I I(SuppI I):O2 I

Aim: To better characterize delayed enhancement patterns in the cardiac transplant population.

Background: Cardiac transplant patients experience significant morbidity related to transplant vasculopathy and acute transplant rejection, both of which can cause scarring of the myocardium. Contrast enhanced cardiovascular magnetic resonance (CMR) has the unique ability to visualize and quantify myocardial scarring. It is well understood that myocardial infarctions resulting from transplant vasculopathy adversely affect prognosis and modify therapy. There is a growing body of evidence from non-transplant disease states, that the presence of non-infarct myocardial scar is also correlated to poor prognosis. Currently there is very little data on the scarring patterns present in the cardiac transplant population and it is our goal to better describe this pathology.

Methods: Thirty-nine transplant patients underwent contrast enhancement imaging at the time of routine myocardial biopsy at two hospital centers in Alberta, Canada. Standard phase sensitive inversion recovery sequences were used on commercially available scanners (Siemens Avanto and Sonata, Siemens, Erlangen, Germany). Delayed enhancement (DE) was evaluated visually using $\mathrm{CMR}^{42}$ (Circle Canada Inc, Calgary, Canada) software analysis package by two independent readers. DE had to be cross-referenced in two orthogonal views. Disagreements were settled by consensus. The extent of DE was assessed semiquantitatively by scoring each of the 17 myocardial segments according to the proportion of DE in each segment ( $I=75 \%)$. The scores of the 17 individual myocardial segments were added together to give an aggregate DE burden.

Results: Three (8\%) out of 39 patients scanned had to be excluded due to poor image quality. There were seven women (I8\%) and thirty two men (82\%). Fifteen (45\%) patients had grade IR cellular rejection rejection, and two $(6 \%)$ had grade $2 R$

Figure I (abstract O2I)

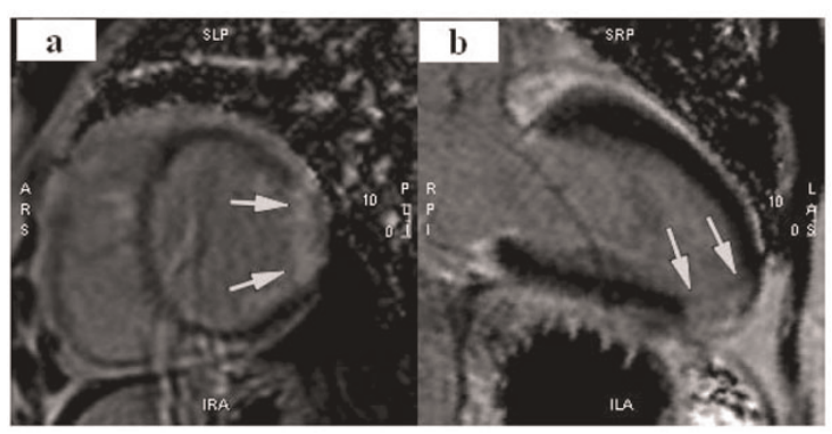

Transmural lateral wall infarction (a) and Inferoapical infarction (b). 
Figure 2 (abstract O2I)

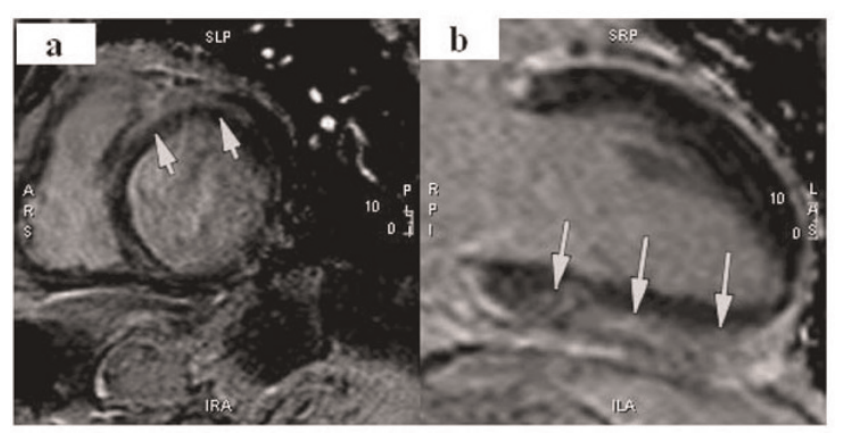

Example of non-ischemic fibrosis. Subepicardial delayed enhancement of the anteroseptal and anterior walls (a) and inferior wall (b).

rejection. Mean time since transplant was 37 months (standard deviation $=55$ months). Eighteen $(50 \%)$ of 36 patients had DE. Among patients with DE, four patients $(22 \%)$ had a subendocardial or transmural pattern consistent with myocardial infarction (Figure I), and I4 (78\%) had a midwall or subepicardial pattern (Figure 2) consistent with non-ischemic injury. Overall, patients with DE had scores ranging from I to 19, with a mean of 5.4 (standard deviation $=4.8$ ). Non-ischemic DE was most commonly seen in the anterolateral and inferior walls (Figure 3). There was no significant association between the presence of DE and time since transplant or current biopsy result.

Conclusion: DE is a common feature in the transplant population. Most DE observed is in a non-ischemic pattern;

\section{Figure 3 (abstract O2I)}

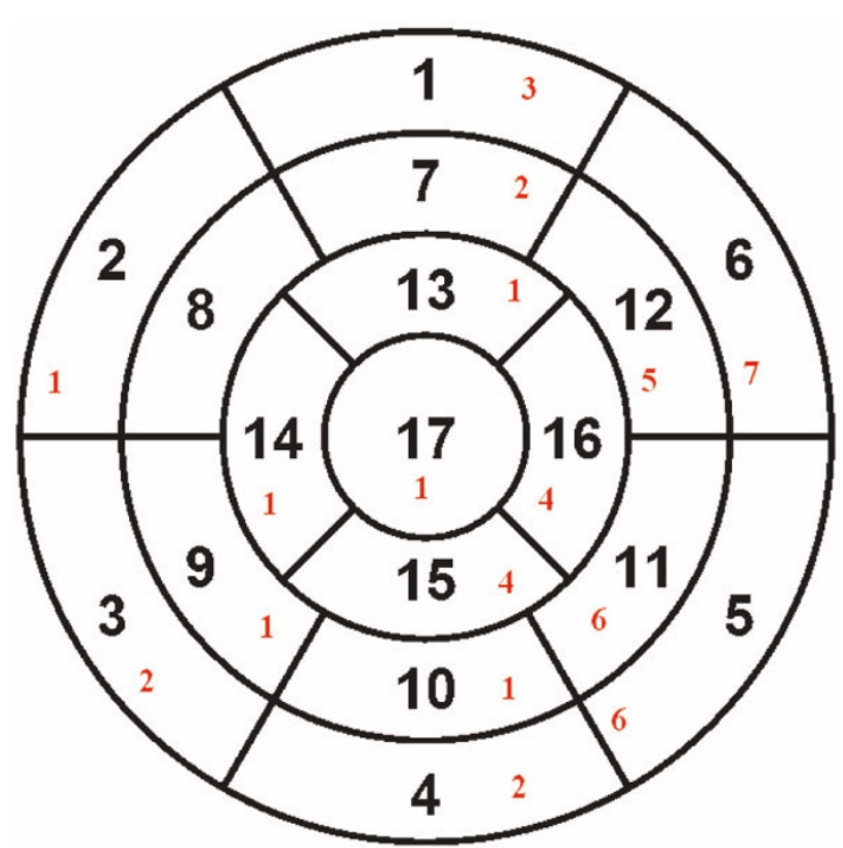

Frequency of any delayed enhancement by myocardial segment. however a significant proportion had DE patterns consistent with infarction. The relationship between DE and cumulative episodes of rejection, hospitalization, and long term prognosis needs to be explored in more detail.

\section{2}

Comparison of a rapid visual algorithm for quantification of infarct size with direct planimetry of infarct size by delayed enhancement-CMR Omar M Cheema, Ankit A Patel and Dipan J Shah Methodist DeBakey Heart \& Vascular Center, Houston, TX, USA

Journal of Cardiovascular Magnetic Resonance 2009, I I(SuppI I):O22

Introduction: Direct planimetry of myocardial infarct size by delayed enhancement CMR (DE-CMR) has been well validated as a technique for quantification of infarct size with both a high degree of accuracy and reproducibility. Direct planimetry however requires extensive post processing and is time consuming and therefore not ideal for performance in a routine clinical service. We propose a visual algorithm to quantify total infarct size that would be rapid and easily incorporated into routine clinical practice. In this study we sought to: I) compare the level of agreement between our visual scoring algorithm and direct planimetry of infarct size; 2) compare the time required for each method of quantifying infarct size.

Methods: We enrolled IOI consecutive patients with known chronic myocardial infarction (MI) who underwent CMR viability assessment. Direct planimetry was performed by segmentation of hyperenhanced $(\mathrm{HE})$ regions (signal intensity $>2$ standard deviations above remote myocardium) in all short axis DE-CMR slices. The total volume of the HE zone was then divided by the total volume of myocardium within the left ventricle (calculated by planimetry of endocardial and epicardial borders on all DECMR images so as to include both $\mathrm{HE}$ and non-HE myocardium). Visual scoring of all studies was performed using a 17 segment model with scores assigned based on the visual extent of $\mathrm{HE}$ myocardium in each segment (i.e. $0=$ no $\mathrm{HE}, \mathrm{I}=\mathrm{I}-25 \% \mathrm{HE}$, $2=26-50 \% \mathrm{HE}, 3=5 \mathrm{I}-75 \% \mathrm{HE}$, and $4=76-100 \% \mathrm{HE})$. Visual infarct size was then calculated by averaging the midpoint of the $\% \mathrm{HE}$ within each of the 17 segments (i.e. score of $0=0 \%$, $I=13 \%, 2=38 \%, 3=63 \%, 4=88 \%)$. Agreement between the visual algorithm and planimetry was compared using BlandAltman analysis.

Results: The study population consisted of 101 patients with chronic MI (infarct age $5.3 \pm 6.5$ years) of which 70 (69\%) were men, age $63.7 \pm 2.3$ years, left ventricular (LV) ejection fraction $52.4 \pm 15.5 \%$, and LV mass $154.7 \pm 5 \mathrm{I} .3 \mathrm{~g}$. Of the $\mathrm{I} 0 \mathrm{I}$ patients, $30(30 \%)$ had a history of congestive heart failure, $29(29 \%)$ had diabetes mellitus, 86 (85\%) had hypertension, and 94 (93\%) had hyperlipidemia. The total infarct size was not statistically different between the visual algorithm (II.4 $\pm 10.4 \%$ ) and directly planimetry $(9.3 \pm 8.5 \%, p=N S)$. The range of infarct sizes was $0-40 \%$ by direct planimetry. Bland Altman analysis revealed a nonsignificant bias of $2.1 \%$ of LV ( $p=N S)$ with $95 \%$ confidence interval between the visual algorithm and planimetry of $+11.3 \%$ to $-7.1 \%$, see figure. The visual algorithm required less time than direct planimetry $(5.5 \pm 1.6$ minutes vs. $22.4 \pm 4.5$ minutes respectively; $\mathrm{p}<0.00 \mathrm{I})$. 
Conclusion: The visual algorithm provides a rapid method to quantify total infarct size with good agreement to direct planimetry. This is a useful time-efficient alternative for quantifying chronic infarct size in a routine clinical setting.

\section{3}

The utility of cardiovascular magnetic resonance imaging in Takotsubo Cardiomyopathy (apical ballooning) for differential diagnosis, pathophysiological insights and additional findings Ingo Eitel, Florian Behrendt, Mahdi Sareban, Gerhard Schuler, Matthias Gutberlet and Holger Thiele Heart Center Leipzig, Leipzig, Germany

Journal of Cardiovascular Magnetic Resonance 2009, I I (SuppI I):O23

Introduction: Takotsubo cardiomyopathy (TTC) is an increasingly recognized acute cardiac syndrome (ACS), which underlying pathophysiological mechanisms are still controversially discussed. Cardiovascular magnetic resonance imaging (CMR) contributes to an understanding and differential diagnosis of this new entity by demonstrating the absence of irreversible injury (delayed enhancement) but oedema formation on T2-weighted images. However, clinical experience with CMR in this entity is still limited and is based mainly on relatively small case series.

Purpose: The aim of this study was therefore to evaluate CMR criteria for the diagnosis/differential diagnosis of TTC and the assessment of potential pathophysiological mechanisms as well as additional findings by using a comprehensive CMR approach.

Methods: Between January 2005 and October 2008 8I consecutive patients, showing a left ventricular dysfunction with apical ballooning not explainable by the coronary artery status and initially admitted with ACS underwent CMR using a I.5 T MRI scanner. Left ventricular function, T2-weighted spin echo sequence for oedema and delayed enhancement (DE) images after administration of Gadobutrol were assessed. Additionally, in the last 20 patients T2-weighted triple-inversion-recovery imaging to calculate the edema ratio (ER) and TIweighted imaging before and after contrast agent administration to calculate the myocardial global relative enhancement (gRE) were performed for detection of inflammation.

Results: CMR revealed diagnosis of myocardial infarction in 18 (22.2\%) patients and diagnosis of myocarditis in $9(10.8 \%)$ patients with typical patterns of DE. In all other 54 (67.0\%) patients ( $5 \mathrm{I}$ female, age $7 \mathrm{I} \pm 10$ years) no DE was detected, consistent with the diagnosis of TTC. Of these 20 patients (37\%) showed focal oedema, 17 (31\%) initial right ventricular involvement and 19 patients a pericardial effusion (PE) (35\%). Of the last 20 TTC patients 9 showed elevation of both inflammatory markers (ER and gRE) with concomitant PE indicating acute inflammation. Follow-up CMR after three months showed complete normalization of left ventricular function and inflammatory parameters in the absence of DE, oedema and PE.

Conclusion: CMR has incremental value for differential diagnosis, pathophysiological insights and detection of additional findings in TTC. Therefore CMR should be performed in all patients with suspected TTC for further differential diagnosis and guidance of medical therapy. Moreover, our results support the probable underlying cause of inflammation in TTC. If inflammation is the primary cause or secondary phenomena due to sympathetic overdrive needs further research.

\section{O24}

Potential of multidetector computed tomography and magnetic resonance imaging in quantifying left ventricular function, perfusion and viability of chronic microinfarction

Marcus Carlsson ', David Saloner², Alastair Martin², Loi Do ${ }^{2}$ and Maythem Saeed ${ }^{2}$

${ }^{I}$ Clinical Physiology, Lund University Hospital, Lund, Sweden

${ }^{2}$ Dep of Radiology and Biomedical Imaging, San Francisco, CA, USA

Journal of Cardiovascular Magnetic Resonance 2009, I I(SuppI I):O24

Introduction: Microinfarction is common following coronary interventions ( $\mathrm{PCl}$ and $\mathrm{CABG}$ ) and acute coronary syndromes. Magnetic resonance imaging (MRI) has become the reference method for non-invasive quantification of: I) left ventricular (LV) function using cine imaging and 2) myocardial perfusion deficits using first pass perfusion and 3) acute and chronic myocardial contiguous infarction using delayed contrast enhancement (DE) technique. Modern multidetector computed tomography (MDCT) has also been recently used in assessing the above parameters in contiguous infarction. To our knowledge both modalities have not been tested in the assessment of microinfarction caused by microembolic agents.

Purpose: This study aimed to examine the potential of 64-slice MDCT and MRI in assessing LV function, regional perfusion and viability in microinfarction.

Methods: An XMR-suite was used to catheterize the LAD coronary artery under X-ray and to define the LAD-territory using first-pass MRI during intracoronary injection of $10 \% \mathrm{Gd}$ DOTA. The perfusion territory was selectively microembolized in six pigs using a microembolic agent $(40-120 \mu \mathrm{m}, 250,000$

Figure I (abstract 024)

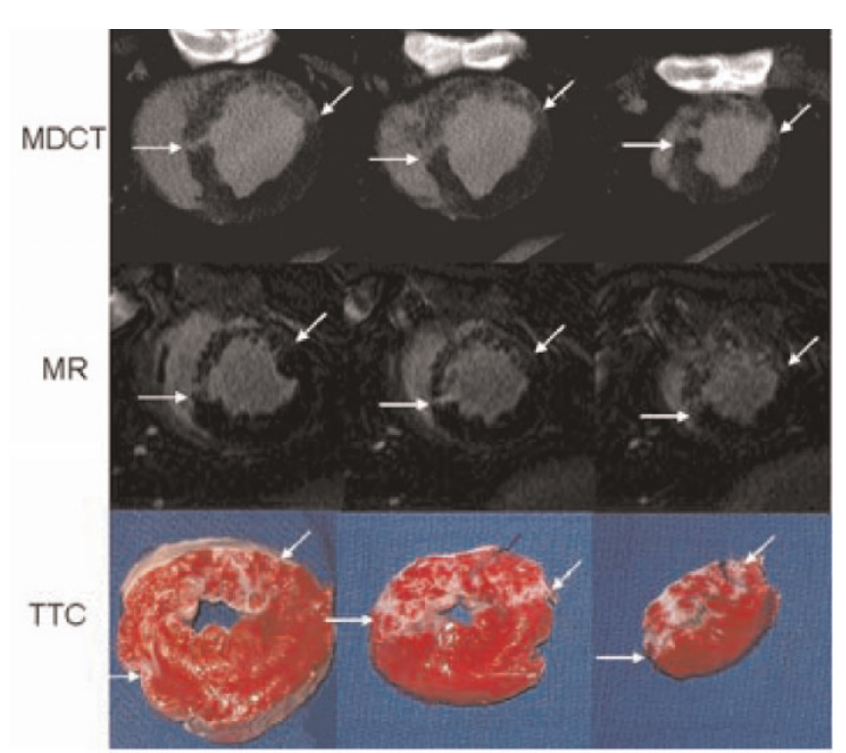

Multi-slice MDCT (top), MR delayed enhancement images (middle) and TTC slices (bottom) from a representative animal show good correspondence between modalities in defining patchy microinfarction embedded in viable myocardium (indicated by arrows). 
count). LV function (cine imaging), perfusion (first-pass imaging) and viability (DE imaging) were determined 7-8 weeks after microembolization using MRI followed by MDCT ( 5 days later). Histochemical staining (TTC) was used as golden standard for quantification of microinfarction. Figure I.

Results: The LAD-territory was $32.4 \pm 3.8 \%$ of the LV mass. There was no significant difference between MR and MDCT measurements of systolic wall thickening. Global LV function did not differ between MRI (end diastolic volume: $92 \pm 8 \mathrm{ml}$, endsystolic volume $48 \pm 5 \mathrm{ml}$ and ejection fraction: $47 \pm 3 \%$ ) and MDCT (96 $\pm 8 \mathrm{ml}, 49 \pm 3 \mathrm{ml}, 49 \pm 2 \%$, respectively). Both MRI and MDCT showed a decrease in function in the microembolized territory and an increased contractility in remote myocardium. There was no difference in the quantification of systolic wall thickening (radial strain) between the methods ( $P=\mathrm{ns}$ for all segments, bias $0.3 \pm 21.9 \%$ ). MRI, but not MDCT, detected a perfusion deficit in the microembolized territory (significant decrease in max upslope and maximum signal intensity). Microinfarction size did not differ between MDCT $(6.3 \pm 0.8 \%$ LV), MRI $(6.6 \pm 0.5 \% \mathrm{LV})$ or TTC $(7.0 \pm 0.6 \% \mathrm{LV})$. Bias (BlandAltman test) for quantifying microinfarction on MDCT was $0.6 \pm 1.9 \% \mathrm{LV}$ compared to TTC and for MRI $-0.4 \pm 1.3 \% \mathrm{LV}$ compared to TTC.

Conclusion: Modern MDCT and MRI techniques have the sensitivity to: I) visualize and quantify chronic microinfarction and 2) demonstrate regional LV dysfunction. MRI, but not MDCT, has the sensitivity for detecting small changes in regional perfusion of chronic microinfarction. Close agreements were found between MDCT and MRI in measuring regional and global LV function. The results of this study suggest that MDCT and MRI can be used for detecting the consequences of microinfarction following coronary interventions and evaluating the efficacy of new therapies and devices designed to prevent microembolization.

Acknowledgements

This study was supported by grant ROIHL07295 from NIH.

\section{$\mathbf{O 2 5}$}

Co-localization of areas of delayed mechanical activation and areas of myocardial scar Jana G Delfino', Brandon K Fornwalt', Calvin R Kurz², Jack A Talsma' and John N Oshinski ${ }^{3}$

${ }^{1}$ Emory University, Atlanta, GA, USA

${ }^{2}$ Georgia Institute of Technology, Atlanta, GA, USA

${ }^{3}$ Emory University/Georgia Institute of Technology, Atlanta, GA, USA

Journal of Cardiovascular Magnetic Resonance 2009, I I(SuppI I):O25

Objective: To determine the overlap between the regions of delayed mechanical activation and regions of myocardial scar tissue.

Introduction: The greatest benefit from Cardiac Resynchronization Therapy (CRT) is likely achieved when the LV pacing lead is placed in the area with the greatest mechanical activation delay [I]. However, in patients with ischemic heart failure, the region with the greatest mechanical activation delay may correspond to an area of myocardial scar. Placing the pacing leads in areas of scar tissue will result in nonresponse to CRT[2]. The colocalization of areas of delayed mechanical activation and myocardial scar in patients with previous $\mathrm{MI}$ is not known.
Methods: 16 patients with a prior history of myocardial infarction (MI) were studied six-months post infarct. Cine SSFP images were obtained in the 2-chamber, 4-chamber, and short axis orientations. Following a double-dose injection $(0.2 \mathrm{mmol} /$ $\mathrm{kg}$ ) of Gd-DPTA, delayed contrast enhancement (DCE) images were obtained at the same locations as the cine SSFP images. Mechanical activation delays were calculated from the cine SSFP images. Radial displacement curves showing movement of the endocardial border toward the LV center of mass were calculated for 360 chords around each short axis slice. Displacement curves were averaged throughout the entire myocardium to generate a global displacement curve. Cross correlation analysis between the global curve and the curve for each chord was done to determine the mechanical activation delay. A cross correlation delay of $31 \mathrm{msec}$ was used as the threshold to determine mechanical activation delay[3]. Mechanical activation delay information for the entire myocardium was mapped onto the standardized AHA Bullseye model.

Endocardial and epicardial borders were traced on the short axis DCE images. A separate region of interest was drawn around the area of infarct. Each slice of the myocardium was resampled at 360 chords around the heart, and each chord was assigned a value between 0 and $\mathrm{I}$ depending on the scar burden at that

\section{Figure I (abstract 025)}

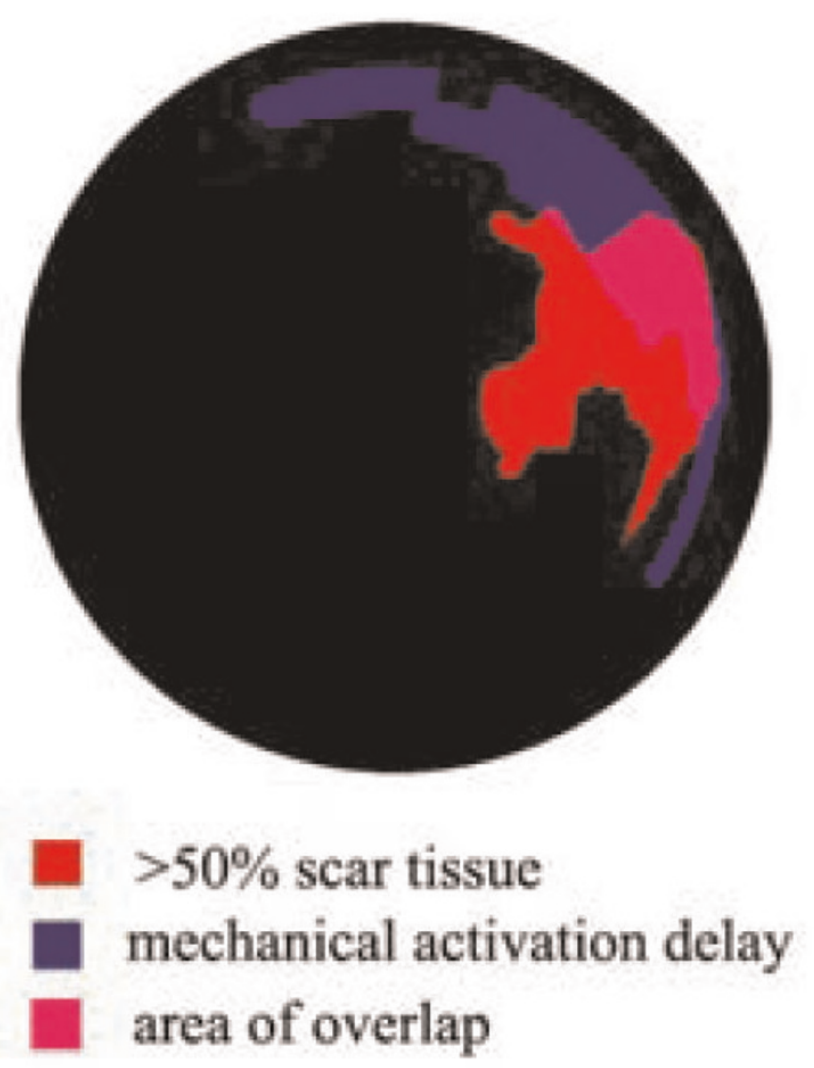

An example patient showing regions of $>50 \%$ scar tissue, regional mechanical activation delay, and regions of overlap. In this example, $32 \%$ of the region of delayed mechanical activation was also a region of $>50 \%$ scar tissue. 
location $(I=$ transmural infarct, $0=$ no infarct $)$. The analysis was repeated for each short axis slice. DCE information for the entire myocardium was displayed on the standardized AHA Bullseye model of the myocardium.

Areas of overlap between mechanical activation greater than 31 msec and scar burden $>50 \%$ transmurality were examined.

Results: A scar burden of $>50 \%$ was seen in $8.6+/-6.7 \%$ of the LV; mechanical activation delay greater than $31 \mathrm{msec}$ was seen in $8.0+/-4.0 \%$ of the LV. On average, only $14.6+/-14.7 \%$ of the region of greatest mechanical activation delay was also an area of $>50 \%$ scar tissue. However, this value varied greatly between patients and ranged from 0 to $40 \%$. In the two patients where there was no overlap, scar burden was $<5 \%$ of the myocardium. See Figure I.

Conclusion: The region of greatest mechanical activation delay does not necessarily correspond to the region of myocardial scar tissue.

\section{References}

I. Becker, et al: Am J Cardiol 2007, 100:167|-6.

2. Bleeker, et al: Circulation 2006, I I 3:969-76.

3. Fornwalt, et al: J Am Soc Echocardiogr 2007, 20:1330-7.

\section{O26}

Papillary muscle involvement in acute and chronic myocardial infarction: an MRI study using multi-contrast delayed enhancement pulse sequence Yuesong Yang, Kim Connelly, Jay Detsky, Gideon Paul, Graham A Wright and Alexander J Dick

Sunnybrook Health Sciences Centre, Toronto, ON, Canada

Journal of Cardiovascular Magnetic Resonance 2009, I I(SuppI I):O26

Introduction: The papillary muscle (PM) is an integral component of the mitral valve apparatus. Acute or chronic myocardial infarction (MI) with PM ischemia is a primary factor leading to the occurrence of mitral regurgitation, with associated substantial morbidity and mortality [I, 2]. PM-MI is also a source of ventricular arrhythmia in these patients [3]. Although DE-MRI using IR-FGRE can detect PM-MI, its accuracy is primarily limited by poor contrast between left ventricular (LV) blood pool and infarcted myocardium.

Hypothesis: We hypothesize that multi-contrast delayed enhancement (MCDE) imaging will improve the identification of
PM-MI in patients with acute and chronic MI, compared to conventional IR-FGRE imaging.

Methods: Cardiac DE-MRI studies using both MCDE and IRFGRE in patients with MI were reviewed. Twenty-three patients ( 2 I males, 2 females, average age of $62 \pm 10$ years old; 5 acute MI within 7 days, 18 chronic $M I>4$ weeks) met the diagnostic criteria of PM-MI, as outlined below. All studies were performed on a I.5 T GE Signa HDx system (GE Healthcare, Milwaukee, WI), which included a short-axis oblique (SAO) and two or four chamber SSFP studies. Both IR-FGRE and MCDE covering the whole LV in SAO were performed 10-20 minutes after doubledose bolus injection of Gd-DTPA. For IR-FGRE, the TI varied from 200 to $300 \mathrm{~ms}$, depending on the null point of healthy myocardium. For MCDE, a segmented SSFP readout is used following an inversion pulse, providing 20 cardiac-phase-resolved images at varying effective Tls [4]. The in-plane resolution was $1.5 \times 1.5 \mathrm{~mm}$ for both techniques.

PM-MI was considered if the following criteria were satisfied in the IR-FGRE or MCDE images: (I) the increased signal intensity of PM was similar to that of adjacent hyper-enhanced infarct segments; (2) the hyper-enhanced PM region was limited to the PM area defined by pre-contrast SSFP. The contrast between blood pool and hyper-enhanced LV infarct was rated as excellent (3), good (2) or fair (I) based on their differentiation.

Results: Based on the standard AHA 17-segment model [5], all patients with PM-MI demonstrated wall motion abnormalities and DHE involving multiple coronary artery territories. Of these 23 patients, 13 studies demonstrated primarily involvement of the territories of the RCA ( 8 patients) and/or LCX (5 patients) and 10 involved the territories of LAD with some LCX involvement. Although IR-FGRE and MCDE determined the presence and extent of LV MI equally well, better contrast scores were achieved in MCDE $(2.9 \pm 0.3)$ compared to IR-FGRE $(\mathrm{I} .6 \pm 0.8, \mathrm{P}<0.00 \mathrm{I})$. MCDE clearly demonstrated PM-MI in all cases $(100 \%, 23 / 23)$. However, only $39 \%(9 / 23)$ could be visualized in the corresponding IR-FGRE images (Figure I), which displayed poor contrast between blood pool and infarcted myocardium. The multi-contrast capability of MCDE facilitates an improved differentiation between blood pool and infarct. This is especially important for the detection of PM-MI since a large of portion of PM extends within the LV blood pool and does not attach to the solid LV wall [6]. Moreover, the MCDE sequence provided cine images that also facilitated the simultaneous

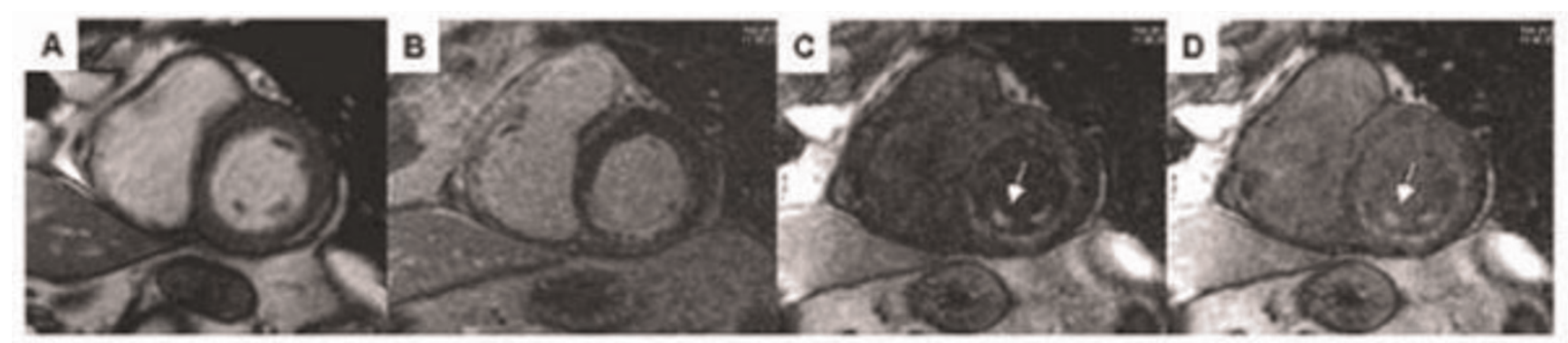

PM involvement in a subject with RCA territorial infarction. Pre-contrast SSFP image (A) depicted the morphology of PM. IR-FGRE image (B) did not show the PM-MI, while MCDE (C and D) clearly demonstrated the infarcted PM (arrows). 
appreciation of wall motion abnormalities in the region of MI. Mitral valve regurgitation with mild or moderate degree was noted on four- and/or two-chamber SSFP scans in $52 \%$ (I2/23) of patients.

Conclusion: MCDE imaging provides better contrast between blood pool and infarcted myocardium, thus improving the determination of PM-MI that may help identify patients in whom the significant mitral regurgitation may affect morbidity and mortality.

\section{References}

I. DePasquale NP: Annu Rev Med 1971, 22:327.

2. Han Y, et al: JACC Img 2008, I:294.

3. Bogun F: JACC 2008, $5 \mathrm{I}: 1794$.

4. Detsky JS, et al: MRM 2007, 58:365.

5. Cerqueira MD: Circulation 2002, 105:539.

6. Axel L: Circulation 2004, 109:3।45.

\section{O27}

Myocardial salvage in acute myocardial infarction assessed by magnetic resonance

imaging - influences by the antioxidative agent $\mathbf{N}$-acetylcystein

Holger Thiele', Ingo Eitel ${ }^{1,2}$, Lysann Hildebrand', Carmen Schirdewahn', Volker Adams', Georg Fürnau', Matthias Gutberlet' and Gerhard Schuler'

'University of Leipzig - Heart Center, Leipzig, Germany

${ }^{2}$ Internal Medicine - Cardiology, Leipzig, Germany

Journal of Cardiovascular Magnetic Resonance 2009, I I (SuppI I):O27

Introduction: Myocardial salvage can be assessed retrospectively by T2-weighted and delayed enhancement images as shown in animal studies [I]. Currently there are only limited data in humans with acquisition of T2-weighted images not covering the full ventricle [2]. Animal trials showed that the antioxidant effects of $\mathrm{N}$-Acetylcystein ( $\mathrm{N}$-ACC) reduce reperfusion injury and $\mathrm{N}$-ACC might prevent the occurrence of contrast-induced nephropathy $(\mathrm{CIN})$ in patients undergoing primary $\mathrm{PCl}$.

Purpose: Aim of this trial was to establish myocardial salvage imaging by $M R$ as a surrogate endpoint in a randomized singleblinded trial and to show that high-dose N-ACC reduces reperfusion injury by its antioxidative properties. Furthermore, $\mathrm{N}$-ACC might reduce the incidence of contrast-induced nephropathy (CIN).

Methods: Two hundred-fifty-one patients undergoing primary $\mathrm{PCl}$ were randomized to either high-dose N-ACC $(2 \times 1200 \mathrm{mg} /$ $\mathrm{d}$ for 48 hours) or placebo plus optimal hydratation. The two primary endpoints were: I) occurrence of $\mathrm{CIN}$ defined as an increase in the serum creatinine concentration of $>25 \%$ from the baseline value within $72 \mathrm{~h} ; 2$ ) Myocardial salvage measured by T2-weighted STIR-images (covering the left ventricle from base to apex) and delayed enhancement MRI at day 2-4 after primary $\mathrm{PCl}$. Myocardial salvage index was calculated as area at riskinfarct size/area at risk.

Secondary endpoints were infarct size and microvascular obstruction, ST-resolution at 90 minutes and occurrence of MACE at 30 day follow-up.

Results: Due to contraindications MRI could not be performed in $3 \mathrm{I}$ patients. All images were assessable for the calculation of the myocardial salvage index. The area at risk was $47.3 \%$ of the left ventricular mass (IQR 33.9; 58.8) in the N-ACC group versus
42.7\% (IQR 33.7; 53.0; $p=0.39$ ) in the placebo group. The primary endpoint reperfusion injury measured by myocardial salvage index was not different between both treatment groups (57.7; IQR 39.2; 78.0 versus 6I.I; IQR 40.6; 77.7; $p=0.32$ ). In addition, no differences in infarct size (I8.3\%; IQR 9.3; 26.4 versus I4.9\%; IQR 8.0; 26.4; $\mathrm{p}=0.48$ ) and microvascular obstruction (0.7\%; IQR 0.2 ; I.5 versus $0.6 \%$; IQR 0.0 ; I.2; $\mathrm{P}=0.25$ ) as well as in ST-segment resolution were observed. The median volume of an iso-osmolar contrast agent during $\mathrm{PCl}$ was $190 \mathrm{ml}$ (IQR I30, $250 \mathrm{ml}$ ) in the N-ACC and I80 (IQR I43; 228 $\mathrm{ml})$ in the placebo group $(\mathrm{p}=0.67)$. The primary endpoint $\mathrm{CIN}$ occurred in $14 \%$ in the N-ACC group and in $22 \%$ in the placebo group $(p=0.12)$.

Conclusion: MRI can reliably measure the area at risk and infarct size retrospectively and served as a surrogate endpoint in this randomized clinical trial which showed that high-dose $\mathrm{N}$ ACC does not provide an additional clinical benefit to placebo with respect to prevention of myocardial reperfusion injury and $\mathrm{CIN}$ and in patients undergoing primary $\mathrm{PCl}$.

\section{References}

I. Aletras AH, Tilak GS and Natanzon A, et al: Retrospective determination of the area at risk for reperfused acute myocardial infarction with T2-weighted cardiac magnetic resonance imaging: Histopathological and displacement encoding with stimulated echoes (DENSE) functional validations. Circulation 2006, I | 3: | 865-1870.

2. Friedrich MG, Abdel-Aty H, Taylor A, Schulz-Menger J, Messroghli $D$ and Dietz $R$ : The salvaged area at risk in reperfused acute myocardial infarction as visualized by cardiovascular magnetic resonance. J Am Coll Cardiol 2008, 5 I:| $158|-| 587$.

028

Clinical, angiographic, and electrocardiographic predictors of infarct size and microvascular obstruction sssessed by MRI

Holger Thiele, Josef Friedenberger, Kathrin Schindler, Ingo Eitel, Eigk Grebe, Matthias Gutberlet and Gerhard Schuler

University of Leipzig - Heart Center, Leipzig, Germany

Journal of Cardiovascular Magnetic Resonance 2009, I I (Suppl I):O28

Introduction: Infarct size (IS) and presence of microvascular obstruction (MO) assessed by delayed enhancement MRI are associated with major adverse events in ST-elevation myocardial infarction (STEMI). The time-to-reperfusion, electrocardiographic and angiographic parameters are also of prognostic relevance in STEMI patients. Predictors of IS and MO occurrence have not been assessed so far.

Purpose: To assess predictors of IS and $M O$ in a large consecutive series of patients with STEMI.

Methods: This study analyzed 358 consecutive STEMI patients reperfused by primary percutaneous coronary intervention within 12 hours after symptom onset. IS and MO were assessed by delayed enhancement MRI as percentage of left ventricular mass $(\% L V) 3 . I \pm 4$.I days after the index event. Reperfusion times, 90 min ST-segment resolution, TIMI-flow grades pre and post $\mathrm{PCI}$, TIMI risk score and multiple clinical parameters such as cardiovascular risk factors, Killip-class, and infarct location were assessed. 
Results: In patients with pre PCI TIMI flow 0-I IS was significantly higher with $24 \pm 14 \%$ versus $15 \pm 14 \%$ in TIMIflow $2-3(p<0.00 I)$. Similarly, the extent of $M O$ occurrence was affected by the pre PCI TIMI flow. The post PCI TIMI flow had no significant effect on final IS and $M O$ occurrence. In patients with TIMI flow $<3$ IS was $28 \pm 11 \%$ versus $20 \pm 12 \%$ in TIMI-flow $=3(p=0.05)$. The ST-segment resolution correlated inversely with final IS and presence of $M O$ (IS $r=-0.34$, $\mathrm{p}=0.003 ; \mathrm{MO} r=-0.31, \mathrm{p}=0.004)$. Anterior $\mathrm{MI}$ IS was $25 \pm 16 \%$ (MO $7.8 \pm 9.8 \%$ ) versus $17 \pm 12 \%$ (MO $3.8 \pm 4.7 \%$ ) in inferior $\mathrm{MI}(\mathrm{p}=0.002 \mathrm{IS} ; \mathrm{p}=0.003 \mathrm{MO})$. According to sixtiles of time-to-reperfusion, there was no interaction between timeto-reperfusion and IS and extent of $\mathrm{MO}$ even when restricted to patients with pre $\mathrm{PCI}$ TIMI flow $0-I$. In a multivariable model the strongest predictors of IS and $\mathrm{MO}$ were pre-PCI TIMI-flow, infarct location, Killip class, and 90 minute ST-segment resolution ( $p<0.05$ for all).

Conclusion: The pre-PCI TIMI flow, infarct location, Killip class and ST-segment resolution are the strongest predictors of IS and extent of MO. This may explain why these clinical, angiographic and electrocardiographic measures are associated with survival. In contrast to other studies the time-to-reperfusion did not affect IS and MO, which might be a selection bias, as patients with larger infarctions will be treated earlier.

\section{O29}

Myocardium at risk and myocardial salvage after acute infarction in humans; quantification by magnetic resonance imaging Marcus Carlsson', Joey Ubachs', Einar Heiberg', Erik Hedstrom', Stefan Jovinge ${ }^{2}$ and Hakan Arheden' ${ }^{l}$ Clinical Physiology, Lund University Hospital, Lund, Sweden

${ }^{2}$ Cardiology, Lund University Hospital, Lund, Sweden

Journal of Cardiovascular Magnetic Resonance 2009, I I(SuppI I):O29

Introduction: To assess reperfusion therapy, it is necessary to determine how much myocardium is salvaged by measuring the final infarct size in relation to the initial myocardium at risk (MaR) of the left ventricle (LV). T2 weighted MRI has recently been shown to be able to identify MaR but has not been validated against an independent method in humans.

Purpose: To validate myocardium at risk by T2 weighted MRI (T2-STIR) over time, compared to perfusion SPECT in patients with ST-elevation myocardial infarction (STEMI), and, to assess the amount of salvaged myocardium after I week.

Methods: Sixteen patients with first-time STEMI received ${ }^{99 \mathrm{~m}} \mathrm{Tc}$ tetrofosmin prior to primary percutaneous coronary intervention and SPECT was performed within 4 hours to identify MaR. MRI was performed on a I.5 T Siemens Vision (5 patients) or Philips Intera. T2-STIR was at I day, I week, 6 weeks and 6 months. Image parameters for T2-STIR were: TE, $43 \mathrm{~ms}$ (Siemens), or 100 ms (Philips); TR, 2 heart beats; NEX, 2; image resolution, $1.5 \times 1.5 \mathrm{~mm}$; slice thickness, $10 \mathrm{~mm}$ (Siemens) or 8 $\mathrm{mm}$ with a slice gap of $2 \mathrm{~mm}$ (Philips). At I week, patients were injected with a gadolinium-based contrast agent for quantification of infarct size on delayed contrast enhanced (DE) MRI. T2-STIR and DE MR images were acquired in the short-axis view, covering the left ventricle from the base to apex.

Results: MaR at occlusion on SPECT was $33 \pm 10 \%$ of the LV. MaR on T2-STIR did not differ from SPECT, at day I $(29 \pm 7 \%$, $\mathrm{P}=0.74)$, or week I $(3 \mathrm{I} \pm 6 \%, \mathrm{p}=0.23)$ but declined at week 6
Figure I (abstract O29)

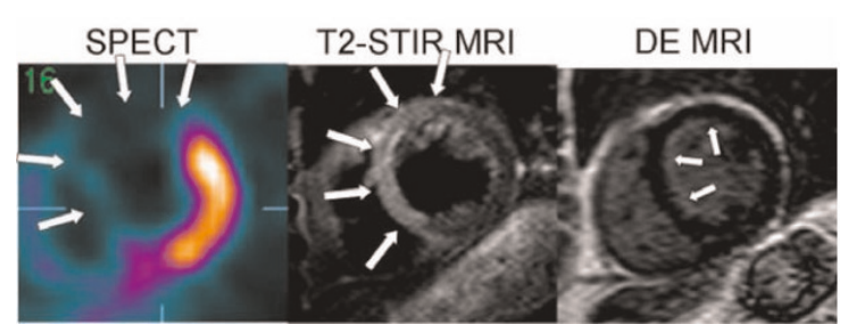

$(10 \pm 12 \%, p=0.03$ vs. I week) and month $6(4 \pm I I \%, p=0.02$ vs. I week). The difference between SPECT and T2-STIR at I week was $-2.3 \pm 5.7 \%$. Both modalities identified $M a R$ in the same perfusion territory and in concordance with coronary angiography. Final infarct size was $8 \pm 7 \%$ and salvage was $75 \pm 19 \%$ of MaR. Figure I.

Conclusion: This is the first study to validate T2-STIR for quantification of MaR against an independent method (SPECT) in patients with acute ST-elevation myocardial infarction after reperfusion therapy. The results demonstrate that T2-STIR performed up to one week after reperfusion can accurately determine myocardium at risk as it was before opening of the occluded artery. The result of reperfusion therapy can therefore be assessed clinically by calculating myocardial salvage as the difference between myocardium at risk and final infarct size using $M R$ imaging.

\section{0}

Impact of myocardial hemorrhage on left ventricular function and remodeling in patients with reperfused acute myocardial infarction Javier Ganame, Giancarlo Messalli, Steven Dymarkowski, Frank E Rademakers, Walter Desmet, Frans Van de Werf and Jan Bogaert

University Hospitals Leuven, Leuven, Belgium

Journal of Cardiovascular Magnetic Resonance 2009, I I(SuppI I):O30

Background: Myocardial hemorrhage is a common complication following reperfusion of ST-segment-elevation acute myocardial infarction (MI). Although its presence is clearly related to infarct size, at present it is unknown whether post-reperfusion hemorrhage affects left ventricular (LV) remodeling. Magnetic resonance imaging (MRI) can be used to identify myocardial infarction, myocardial hemorrhage and microvascular obstruction (MVO), as well as measure LV volumes, function and mass. Methods and results: Ninety-eight patients (I 4 females, 84 males, mean age: 57.7 years) with $\mathrm{Ml}$ reperfused with percutaneous coronary intervention $(\mathrm{PCl})$ were studied in the first week and at 4 months after the event. T2-weighted MRI was used to differentiate between hemorrhagic (i.e., hypo-intense core) and non-hemorrhagic infarcts (i.e., hyper-intense core). MVO and infarct size were determined on contrast-enhanced MRI, while cine MRI was used to quantify LV volumes, mass and function. Twenty-four patients $(25 \%)$ presented with a hemorrhagic MI. In the acute phase, presence of myocardial hemorrhage was related to larger LV end-diastolic and end-systolic volumes and infarct transmurality, lower LV ejection fraction as 
Table I (abstract 030) Results of multiple linear regression of left ventricular remodeling

\begin{tabular}{lllll}
\hline Predictors of end point & $\mathbf{9 5 \%} \mathbf{C l}$ & $\mathbf{R}^{\mathbf{2}}$ & $\mathbf{F}$ value & $\mathbf{p}$ value \\
\hline Hemorrhagic MI & $0.15-0.31$ & 0.17 & 20.19 & $<0.001$ \\
Infarct size at baseline & $20.8-27.7$ & 0.16 & 18.11 & 0.001 \\
Microvascular obstruction & $5.0-9.3$ & 0.12 & 13.13 & 0.001 \\
Maximum troponin I & $91.9-142.6$ & 0.10 & 10.75 & 0.001 \\
Size of area at risk & $37.0-46.5$ & 0.09 & 9.12 & 0.003 \\
LV mass at baseline & $118.6-131.4$ & 0.02 & 2.31 & 0.132 \\
Percent Ml transmurality & $78.6-87.2$ & 0.03 & 3.39 & 0.068 \\
Infarct location & $0.37-0.58$ & $<0.01$ & 0.41 & 0.892 \\
Time to PCl & $24 I . I-305.6$ & $<0.01$ & 0.02 & 0.874 \\
\hline
\end{tabular}

well as lower systolic wall thickening in the infarcted myocardium (all p-values $<0.001$ ). Infarct size, size of area at risk and size of MVO were significantly larger in patients with hermorrhagic MI. At 4 months, a significant improvement in LV ejection fraction in patients with non-hemorrhagic $\mathrm{Ml}$ was seen (baseline: $49.3 \pm 7.9 \%$ vs. 4 months: $52.9 \pm 8.1 \%$; $p<0.01$ ). LV ejection fraction did, however, not improve in patients with hemorrhagic MI (baseline: $42.8 \pm 6.5 \%$ vs. 4 months: $41.9 \pm 8.5 \%$; $p=0.68$ ). Multivariate analysis showed myocardial hemorrhage to be an independent predictor of adverse LV remodeling at 4 months (defined as an increase in LV end-systolic volume). This pattern was independent of initial infarct size (See Table I).

Conclusion: Myocardial hemorrhage, the presence of which can easily be detected with $\mathrm{T2}$-weighted MRI, is a frequent complication after successful myocardial reperfusion, and an independent predictor of adverse LV remodeling regardless of initial infarct size.

\section{O3I}

Strain-encoded imaging for prediction of functional recovery in patients after acute myocardial infarction

Mirja Neizel', Grigorios Korosoglou², Tim Schaeufele², Dirk Lossnitzer', Harald P Kuehl', Evangelos Giannitsis', Malte Kelm', Hugo A Katus ${ }^{2}$, Nael F Osman ${ }^{3}$ and Henning Steen ${ }^{2}$

'University Hospital Aachen, Aachen, Germany

${ }^{2}$ University Hospital Heidelberg, Heidelberg, Germany

3 Johns Hopkins University, Baltimore, MD, USA

Journal of Cardiovascular Magnetic Resonance 2009, I I(SuppI I):O3 I

Introduction: Evaluation of reversible dysfunction after acute myocardial infarction (AMI) has important therapeutic and prognostic implications. The role of impaired systolic function for evaluation of functional recovery has been extensively investigated. However, whether impaired regional diastolic function after $\mathrm{AMI}$ also has predictive implications has not been investigated so far in humans using magnetic resonance imaging (MRI). Recently, Strain-Encoded (SENC) Imaging was introduced as a new MR-technique to evaluate myocardial strain and strain rate. SENC, compared to MR tagging, is a method that does not suffer that much from diastolic fading. Therefore, SENC is an ideal MR-method to determine not only regional systolic but also regional diastolic function.
Figure I (abstract O3I)

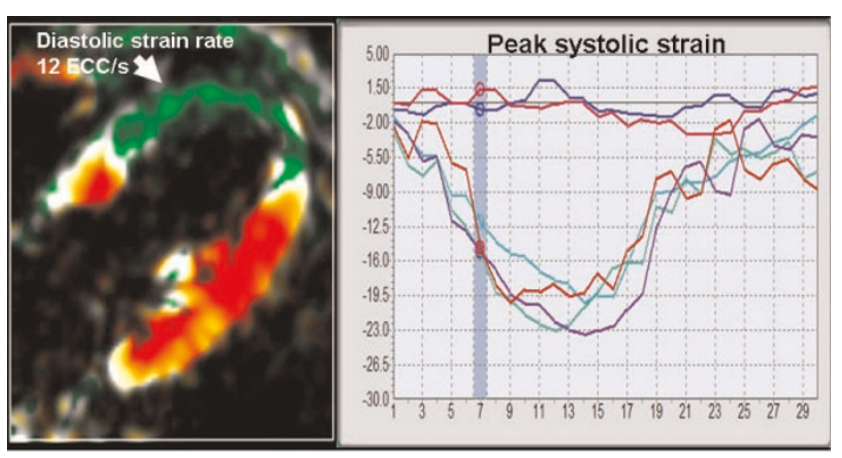

Colour coded SENC-image of a patient with transmural myocardial infarction with corresponding strain-curve.

Purpose: To evaluate the predictive value of regional systolic and diastolic function for improvement of regional myocardial function in patients after AMI.

Methods: MRI (I.5 T, Achieva, Philips, the Netherlands) was performed to 23 consecutive patients (mean age $57 \pm 10) 3 \pm 1$ days after successfully reperfused ST-elevation-myocardial infarction and at a follow-up of $6 \pm 2$ month. 10 age-matched volunteers served as controls. True cine sequences of 3 longaxis views (2-,3- and 4-chamber) and a short-axis (SA) view covering the ventricle from apex to basis were acquired using a Steady State Free Precession (SSFP) sequence. After that, SENC cine images were acquired on the same long-axis planes to measure circumferential strains. Finally, using the same plane

Figure 2 (abstract $03 \mathrm{I}$ )

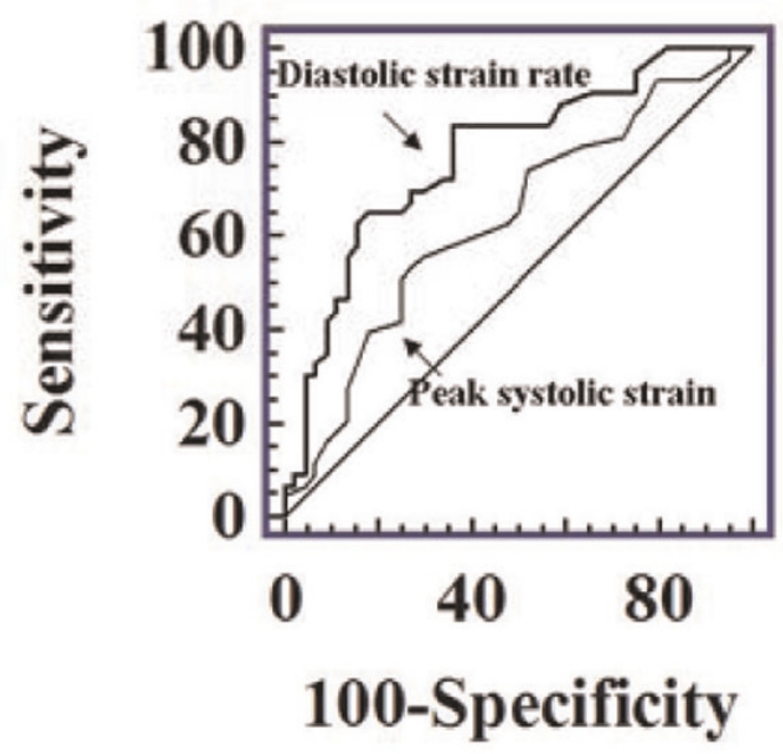

Receiver-operating characteristic (ROC) curve demonstrates that diastolic strain rate assessed with Strain-Encoded Imaging is more sensitive than peak systolic strain for prediction of functional recovery (diastolic strain rate AUC 0.77 (0.67-0.86); peak systolic strain AUC 0.64 (0.53-0.74); $p<0.05)$. 
orientations, multislice contrast enhanced MRI (CE-MRI) with an inversion-recovery (IR) sequence covering the whole ventricle was conducted after injection of 0.2 mmol Magnevist ${ }^{\circledR}$ (Bayer, Germany) and waiting for 10 minutes.

SENC-Data were analysed using a dedicated software (Diagnosoft, Paolo Alto, CA, USA). Peak systolic circumferential strain and early diastolic strain rate were measured at each segment in a modified 17 segment model. Early-diastolic strain rate $(\mathrm{ECC} / \mathrm{s})$ was defined as the slope over the duration from peak-systole to mid-diastole.

Regional wall motion was evaluated at baseline and at follow-up semi-quantitatively from the SSFP cine sequences by consensus reading of two blinded observers as normokinetic, hypokinetic or akinetic to evaluate functional recovery. CE-MR images were analyzed to quantify the size and transmurality of the scared myocardium using a regular workstation (EWS, Philips, the Netherlands).

Results: 276 segments were analyzed. In 6 segments (2,2\%) image quality did not allow adequate data analysis of SENCimages. 157 segments showed normal resting function and 119 segments showed wall motion abnormalities at baseline. 44 segments showed functional recovery at follow, 75 segments did not recover.

Peak systolic circumferential strain in healthy volunteers was $-22 \pm 3 \%$, early diastolic strain rate was calculated with $122.3 \pm 36 \mathrm{E}_{\mathrm{cc}} / \mathrm{s}$.

Peak systolic strain values and early diastolic strain rate (figure I) were significantly different in segments showing functional recovery and in segments without functional recovery (peak systolic strain - $10 \pm 1 \%$ versus $6 \pm 1 \%$, $p<0.01$; early diastolic strain rate $75 \pm 6 \mathrm{E}_{\mathrm{cc}} / \mathrm{s}$ versus $\left.38 \pm 5 \mathrm{E}_{\mathrm{cc}} / \mathrm{s}, \mathrm{p}<0.0 \mathrm{I}\right)$.

Diastolic strain rate was more sensitive for prediction of functional recovery than peak systolic strain (figure 2).

Interestingly, peak systolic strain and diastolic strain rate were even more impaired in segments showing microvascular obstruction compared to transmural infarcted segments without microvascular obstruction ( $p<0.05$ for both).

Conclusion: SENC allows mechanical characterization of regional myocardial injury. Diastolic function assessed with SENC is more precise in predicting functional recovery after AMI than peak systolic strain.

\section{O32}

Reperfusion hemorrhage following PCl quantification with $\mathrm{T}_{2} *$ imaging and impact on area at risk assessment

Declan P O'Regan', Rizwan Ahmed', Clare Neuwirth', Yvonne Tan', Giuliana Durighel', Jo V Hajnal', Imad Nadra ${ }^{2}$, Simon J Corbett ${ }^{3}$ and Stuart A Cook'

I Imperial College, London, UK

${ }^{2}$ Imperial College Healthcare NHS Trust, London, UK

${ }^{3}$ Southampton University Hospitals NHS Trust, Southampton, UK

Journal of Cardiovascular Magnetic Resonance 2009, I I(SuppI I):O32

Introduction: Occlusion of a coronary artery leads to myocardial tissue edema in the vascular bed downstream of the vessel. The extent of hyperintense edema on T2-weighted images allows the area at risk (AAR) from ischemic injury to be retrospectively determined. However, reperfusion of severely
Figure I (abstract 032)

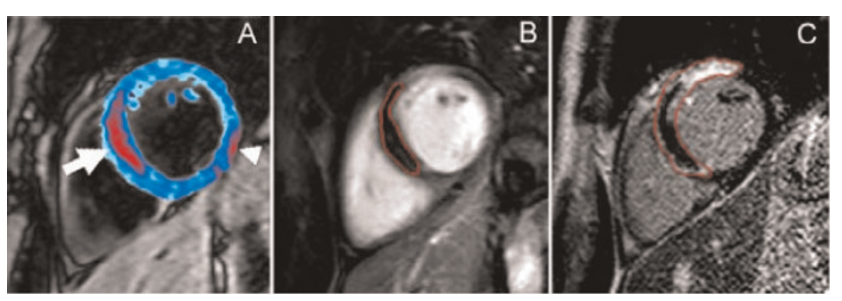

(A) T2* map acquired 2 days post-PCl. Pixels with a $\mathrm{T} 2 *<20 \mathrm{~ms}$ are shown in red and demonstrate the region of post-reperfusion hemorrhage (arrow). Susceptibility artifact (arrowhead). (B) The hemorrhage corresponds to the area of MVO (red contour) shown on the early enhancement image. (C) Myocardial necrosis (red contour), and residual MVO (black core), is shown on the late enhancement image.

ischemic myocardium also leads to interstitial hemorrhage and this may be an important marker for irreversible microvascular damage.

Purpose: We assessed the feasibility of using T2* mapping to quantify regions of myocardial hemorrhage following percutaneous primary coronary intervention (PPCl) for acute myocardial infarction. We also hypothesized that myocardial hemorrhage would lead to an underestimate of the AAR on T2-weighted imaging using conventional signal threshold criteria. Methods: Fifteen patients who had recently undergone $\mathrm{PPCl}$ within the previous 7 days were imaged. Left ventricular function was assessed with conventional cine sequences. Myocardial edema was imaged with a T2-weighted STIR sequence. Myocardial haemorrhage was imaged with a black-blood multiecho $\mathrm{T}^{*}$ sequence using navigator respiratory-gating. Microvascular obstruction (MVO) and late enhancement were imaged at $\mathrm{I}$ minute and 15 minute delays respectively using a 3 dimensional inversion-recovery sequence.

The area of myocardial edema on the T2 STIR images was measured with a boundary detection tool. This was compared to a conventional signal intensity threshold method using 2, 3 and 5 standard deviations (sd) above the mean of remote normal myocardium. A salvage index was calculated as the proportion of the AAR that did not show late enhancement. T2*-mapping of

Figure 2 (abstract 032)

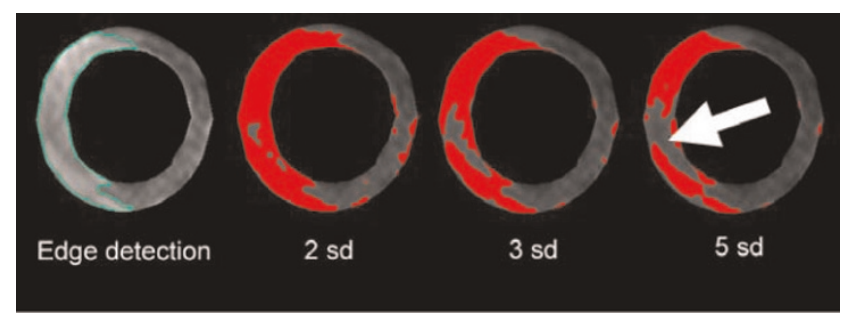

T2-STIR images in the same patient as in Figure I. Boundary detection identifies the myocardial edema (green line) which represents the AAR. The AAR determined at each signal threshold is shown in red. At low thresholds non-specific signal noise results in bright pixels in nonischemic territories causing an overestimation of the AAR. At higher thresholds the signal from the myocardial edema is masked by the presence of hemorrhage in the core of the infarct (arrow) and leads to an underestimation of the AAR. 
the left ventricle was performed using a threshold of $20 \mathrm{~ms}$ to define the presence of hemorrhage.

Results: The mean area of hemorrhage was $5.0 \%$ at the level of the infarct. There was a close correlation between hemorrhage and the MVO $\left(r^{2}=0.75, p<0.0 I\right)$ and infarct volumes $\left(r^{2}=0.76\right.$, $P<0.0 I$ ) (Figure I). When $\geq 5 \%$ hemorrhage is present the AAR was underestimated by $50 \%$ at a 5 standard deviation threshold compared to a boundary detection tool (21.8\% vs $44.0 \%$, $\mathrm{p}<0.05$ ) (Figure 2). Estimation of myocardial salvage at $3 \mathrm{sd}$ and 5 sd signal thresholds becomes unreliable in hemorrhagic infarcts as the apparent AAR becomes smaller than the actual infarct size.

Discussion: Our findings demonstrate the feasibility of using T2* mapping to quantify myocardial hemorrhage following infarct reperfusion. Hemorrhage is frequently observed and is associated with large infarcts where MVO is present and is an indicator of poor myocardial salvage. Hemorrhage in the core of the infarct causes signal loss on T2-weighted imaging and boundary-detection is required to reliably assess the AAR.

Conclusion: Studies using CMR to determine the AAR and myocardial salvage should use boundary detection methods for quantification as arbitrary signal thresholds are unreliable when hemorrhage is present. Post-reperfusion hemorrhage can be assessed with T2*-mapping and may provide an imaging marker of poor myocardial salvage.

\section{3}

T2-weighted MRI pulse sequences for imaging post-infarct edema in mice: comparison of spin echo and T2 preparation approaches Ronald J Beyers, Yaqin Xu, Frederick H Epstein and Brent A French

University of Virginia, Charlottesville, VA, USA

Journal of Cardiovascular Magnetic Resonance 2009, I I (Suppl I):O33

Introduction: An ongoing diagnostic challenge exists in reliably differentiating non-salvageable, acutely infarcted myocardium from surrounding stunned, yet viable, myocardium that defines the area at risk. T2w cardiac magnetic resonance (CMR) imaging has previously been used to image the edema characterizing the area-at-risk region in post myocardial infarcted (MI) canine, porcine and human hearts. Similar techniques would be valuable in basic research studies of $\mathrm{Ml}$ in mice, where they might be used jointly with gadolinium delayed enhancement (DE) imaging to non-invasively define infarct size as "\% area-at-risk" in transgenic/ knock-out mice. However, the rapid murine heart rate presents challenges to T2w CMR application in mice. The typical T2w echo time of $40-60 \mathrm{~ms}$ needed for the detection of edema occupies a significant portion of the murine cardiac cycle ( $100-$ $120 \mathrm{~ms}$ ) with significant periods of blood flow and cardiac motion.

Purpose: Develop an effective T2w CMR sequence for mice that exhibits high immunity to flow and tissue motion artifacts while maintaining sufficient and consistent signal-to-noise (SNR) and contrast-to-noise (CNR) performance.

Methods: We developed two T2w sequences for murine CMR: a flow and motion desensitized spin-echo (SE) sequence and a T2 preparation (T2prep) sequence. The SE sequence employed a slice-selective excitation RF pulse and a thicker slice-selective refocusing RF pulse, and applied readout and phase encoding
Figure I (abstract 033)

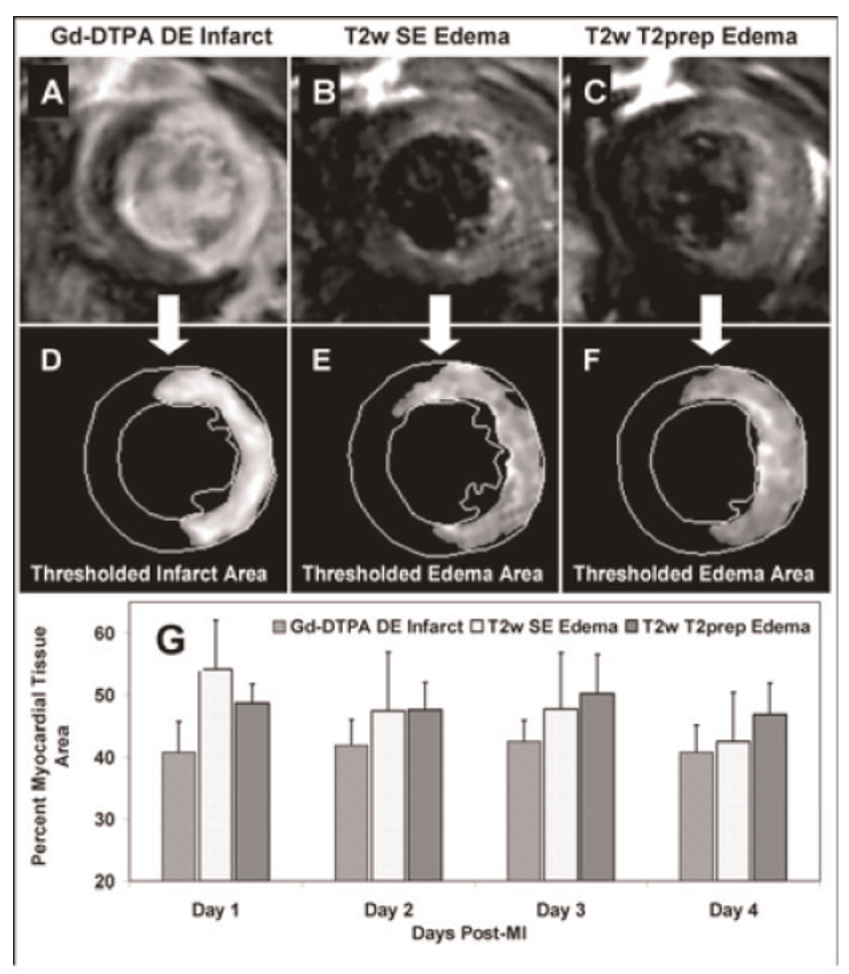

Gd-enhanced mid-ventricular image of the mouse heart I day after MI, showing enhanced infarct region (A) with corresponding thresholded infarct region (D). Same slice T2w spin echo image (B) with corresponding thresholded edema (E). Same slice T2w T2prep image (C) with corresponding thresholded edema (F). Comparison of detected infarct and edema region areas over Days I through 4 post-MI for each imaging method.

gradients after the refocusing pulse. The T2prep sequence employed non-selective MLEV-weighted composite RF pulses followed by a standard slice-selective gradient-echo readout. Each sequence was applied on an isoflurane-anesthetized mouse on Days I through 4 after reperfused $\mathrm{MI}$ induced by $60 \mathrm{~min}$ coronary occlusion, as described previously. Parameters for both sequences included $T R=1500 \mathrm{~ms}, T E=40-60 \mathrm{~ms}$, FOV $=$ $25 \times 25 \mathrm{~mm}$, slice thickness $=1 \mathrm{~mm}$, matrix $=128 \times 128$ and $\mathrm{BW}=520 \mathrm{~Hz} /$ pixel. In addition, gadolinium-DTPA DE CMR was performed each day to define infarct location. All scans of each sequence were performed consecutively at four identical contiguous slice positions from mid-ventricular toward the apex. All scans were performed on a $7 \mathrm{~T}$ Bruker/Siemens ClinScan.

Results: Panels A through C (Fig. I) show example sets of Gd-DTPA DE, T2w SE and T2w T2prep images (respectively) from the same LV slice position at Day I post-MI. Panels D through $\mathrm{F}$ show the respective hyperintense regions identified by threshold analysis performed after manual segmentation of the left ventricular (LV) myocardium. Threshold analysis for all images selected any pixels within the segmented myocardium with $>2$ standard deviation magnitude than a myocardial image sample chosen remote from the infarct area. Panel G tracks and compares the detected infarct and edema region areas over 
Days I through 4 post-Ml for each imaging method. Both T2w sequences detected regions of edema that corresponded to or surrounded the infarct as determined by DE CMR. The T2w SE sequence gave a consistently higher SNR of $104 \pm 6$ (mean \pm SEM) and CNR of $52 \pm 3$ over the T2prep SNR of $68 \pm 4$ and CNR of $34 \pm 2$, where CNR was measured between remote and threshold-selected myocardium. However, the SE sequence had a higher occurrence of flow and motion artifacts that degraded the consistency and accuracy of threshold selection.

Conclusion: As shown (panel G), the T2prep yielded a consistently higher edema area percentage of $48.2 \pm 2.5$ compared to the infarct area percentage of $41.6 \pm 1.8$, which correlates well with previous canine and human studies. Meanwhile, the SE gave a slightly lower mean with higher variance edema area percentage of $47.0 \pm 4.2$ that was attributed to flow and motion artifacts. To our knowledge, this is the first study to demonstrate the feasibility of performing T2w CMR edema imaging in mice, which opens a variety of potential basic research applications investigating the role of individual genes in acute and chronic settings post-MI.

\section{4}

First-pass myocardial perfusion assessment using eight-fold accelerated k-t BLAST stress DCE-MRI with rapid parametric mapping

Aleksandra Radjenovic', Sven Plein ${ }^{\uparrow}$, Neil Maredia', Sebastian Kozerke, , John Biglands', John Greenwood' and John Ridgway'

'University of Leeds, Leeds, UK

${ }^{2}$ University and ETH, Zurich, Switzerland

Journal of Cardiovascular Magnetic Resonance 2009, I I (SuppI I):O34

Background: First-pass myocardial perfusion assessment using dynamic contrast enhanced MRI (DCE-MRI) is still one of the
Table I (abstract 034) DCE-MRI sequence parameters

\begin{tabular}{ll}
\hline Saturation recovery pre-pulse delay & $150 \mathrm{~ms}$ \\
TFE readout & $\mathrm{TR} / \mathrm{TE} / \varphi=3.6 / 1.7 / 15^{\circ}$ \\
k-t acceleration factor & 8 \\
Image matrix & $192 \times 187$ \\
\hline
\end{tabular}

most challenging CMR applications. Image quality, spatial and temporal resolution are limited by the need to acquire multiple slices as single shot acquisitions within a single heart beat, as the process of interest is transient and very rapid, especially under pharmacologically induced stress hyperaemia. This is why accelerated acquisition methods, such as k-t BLAST [I], could provide a significant improvement in the assessment of myocardial perfusion by CMR.

An optimised DCE-MRI sequence with eight-fold k-t BLAST acceleration was shown to provide a significant improvement in spatial resolution without loss of image quality[2], making these datasets very suitable for parametric mapping.

Objective: To investigate the ability of eight-fold k-t BLAST accelerated stress perfusion DCE-MRI combined with a rapid parametric mapping algorithm to detect regions of ischaemia in a pilot cohort of patients with suspected coronary heart disease (CHD).

Methods: The regional ethics review board's permission was obtained and ten patients ( 9 male) were recruited into this study (age range 46-69, mean 59). First-pass stress myocardial perfusion DCE-MRI was performed on a whole body I.5 T MR scanner (Gyroscan Intera CV, Philips Medical Systems) with dedicated k-t BLAST acquisition and reconstruction software (GyroTools Ltd, Switzerland). An optimised DCE-MRI sequence (Table I) allowed three uniformly prepared slices to be acquired in every heart beat, for heart rates of up to $100 \mathrm{bpm}$ [2]. DCE-MRI was acquired under adenosine induced stress with

Figure I (abstract 034)

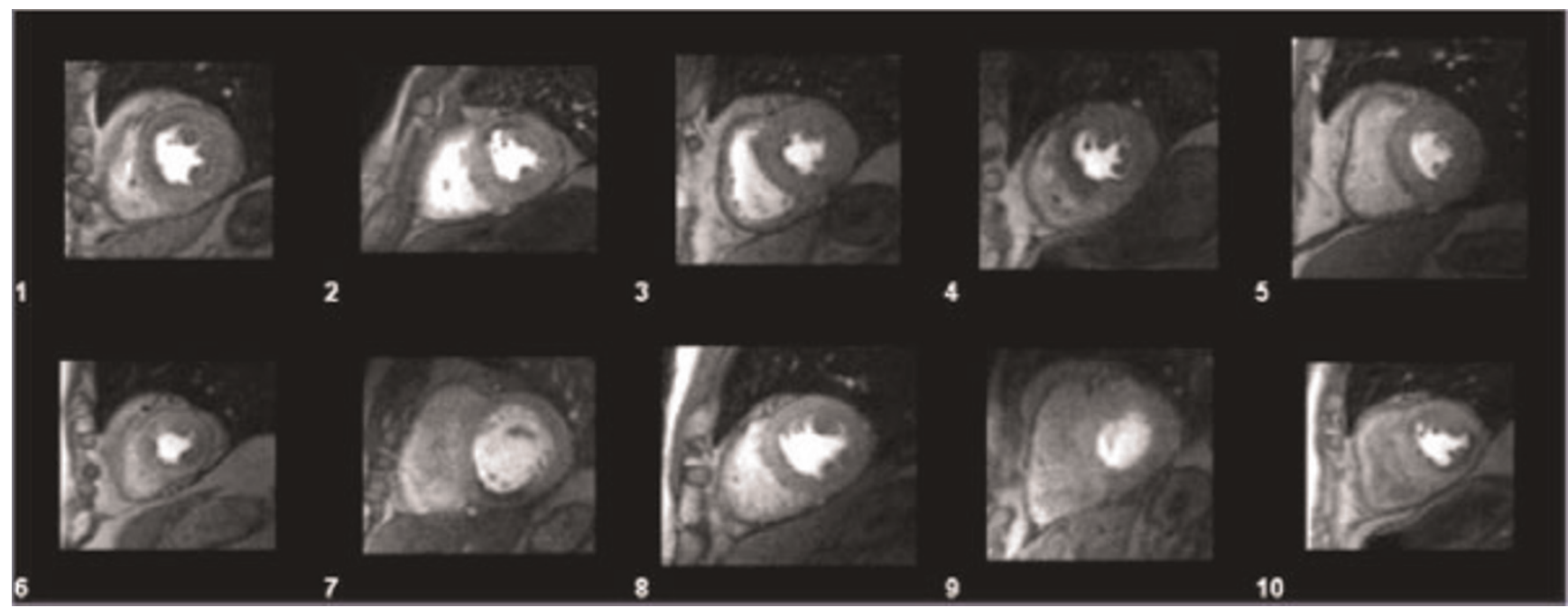

Representative dynamic frames from ten accelerated DCE-MRI datasets. 
Table 2 (abstract O34) Median Enhancement Ratios (ER\%)

\begin{tabular}{|c|c|c|c|c|c|c|c|c|c|c|}
\hline Patient & I & 2 & 3 & 4 & 5 & 6 & 7 & 8 & 9 & 10 \\
\hline Coronary artery X-ray (number of affected territories) & I & I & I & I & 0 & 2 & I & 0 & 1 & 3 \\
\hline Median Enhancement Ratio\% & 61.4 & 133 & 64.6 & 38.3 & 77.2 & 33.1 & 58.0 & 65.2 & 40.6 & 19.1 \\
\hline
\end{tabular}

Figure 2 (abstract 034)

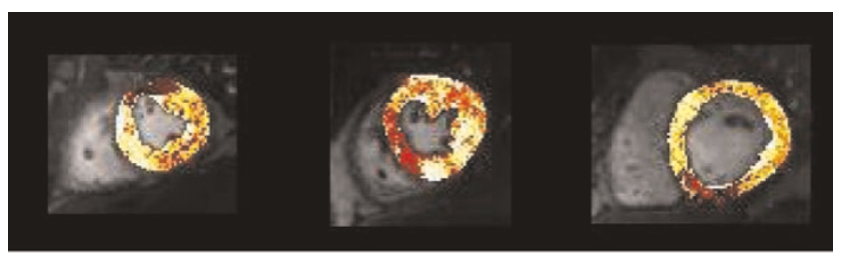

Examples of parametric maps of enhancement ratios in single-vessel disease (corresponding to images 2, 4 and 7 in Figure I). Dark coloured voxels belong to the lowest part of the individual study's frequency distribution.

peripheral venous administration of $0.1 \mathrm{mmol} / \mathrm{kg} \mathrm{Gd}-D T P A$. The algorithm for quantitative analysis and parametric mapping comprised the following steps: I) automated detection of the target post-contrast frame 2) endocardial border detection in the target post-contrast frame using automated region-growing algorithm and a single spline fitting to define epicardial border 3 ) automated registration of pre- and post-contrast frames using incremental rigid translation 4) computation of percentage enhancement ratios (ER) on voxel-by-voxel basis 5) histogram analysis of the ER datasets and creation of percentile-based colour maps.

Results: On coronary X-ray angiography, 8/10 patients had coronary stenosis $\geq 70 \%$, while two had no significant lesions (Table 2). Representative dynamic frames from all ten DCE-MRI studies are shown in Figure I. Example parametric maps of ER are presented in Figure 2. Global enhancement ratios were computed as a median percentage change over baseline (Table 2). Motion correction was not required in three datasets, and I-2 voxel displacement was applied in seven datasets. In patients with no significant coronary stenoses, the average ratio was $71.2 \%$ $(n=2)$, in single-vessel disease it was $66.0 \%(n=4)$. In a patient with two-vessel disease ER was $33.1 \%$ and in a patient with threevessel disease ER was $19.1 \%$.

Discussion: The results of this pilot study suggest that the proposed methods for acquisition and analysis of first-pass myocardial perfusion are robust and ready for use in clinical studies, where its diagnostic utility needs to be assessed formally in a larger patient cohort. The methods allow the assessment of regional differences in perfusion, as well as global changes in perfusion. There is scope for further improvement, notably in increasing resistance to motion artefact and reducing signal inhomogeneity, which can lead to the appearance of false positive lesions.

References

I. Tsao, Boesinger and Preussman : Magn Reson Med. I042, 50:1031-2003.

2. Radjenovic, et al: J Cardiovasc Magn Reson. 2007, 9(2):2I3214.

\section{5}

\section{k-t SENSE accelerated stress myocardial} perfusion MRI at 3 Tesla

Shingo Kato, Hajime Sakuma, Motonori Nagata, Nanaka Ishida, Kakuya Kitagawa, Masaki Ishida, Hiroshi Nakajima, Katsuya Onishi, Masaaki Ito and Kan Takeda Mie University Hosiptal, Tsu, Mie, Japan

Journal of Cardiovascular Magnetic Resonance 2009, I I(SuppI I):O35

Objective: The purpose of this study was to evaluate the feasibility and diagnostic accuracy of high spatial resolution stress myocardial perfusion MRI acquired at every heartbeat by using k-t SENSE and 3 Tesla MR imager.

Background: High spatial and temporal resolutions are required for the accurate assessment of myocardial ischemia using stress perfusion MRI.

Methods: Thirty-three patients with suspected coronary artery disease were studied. High spatial resolution $(<2 \mathrm{~mm})$ first-pass contrast enhanced $M R$ images were obtained at rest and during stress by using a 3.0 T MR imager (Achieva) and k-t SENSE acceleration factor of 5 . Saturation recovery TFE images were acquired with TR/TE of $2.9 \mathrm{~ms} / \mathrm{l} .5 \mathrm{~ms}$, FOV $=40 \times 30 \mathrm{~cm}$, matrix $=256 \times 192$, slice thickness $=8 \mathrm{~mm}$. Three short-axis sections of the left ventricle were imaged at every heart beat. Two observers determined the image quality score ( 1 :poor - 4 :excellent) and recorded the presence or absence of respiratory artifacts and endocardial dark rim artifacts using a 16-segment model.

Results: All studies were successfully completed, with the averaged image quality score of $3.8 \pm 0.4$. Endocardial dark rim artifacts were observed in $17(3.2 \%)$ of 528 segments, but there

\section{Figure I (abstract 035)}
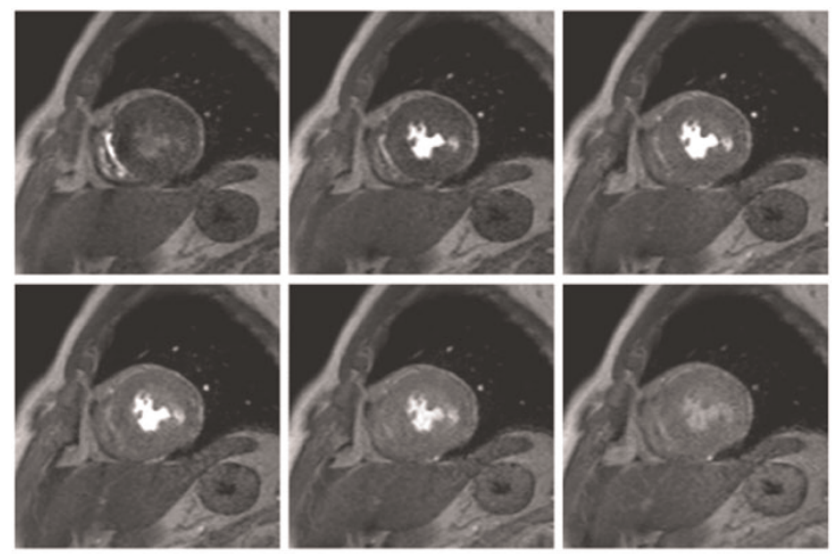

Rest perfusion MRI acquired with 3 T MR imager, 32 channel cardiac coils and $k-t$ SENSE in a patient with triple vessel disease. Rest perfusion MRI is normal and no endocardial banding artifact is observed. 
Figure 2 (abstract 035)
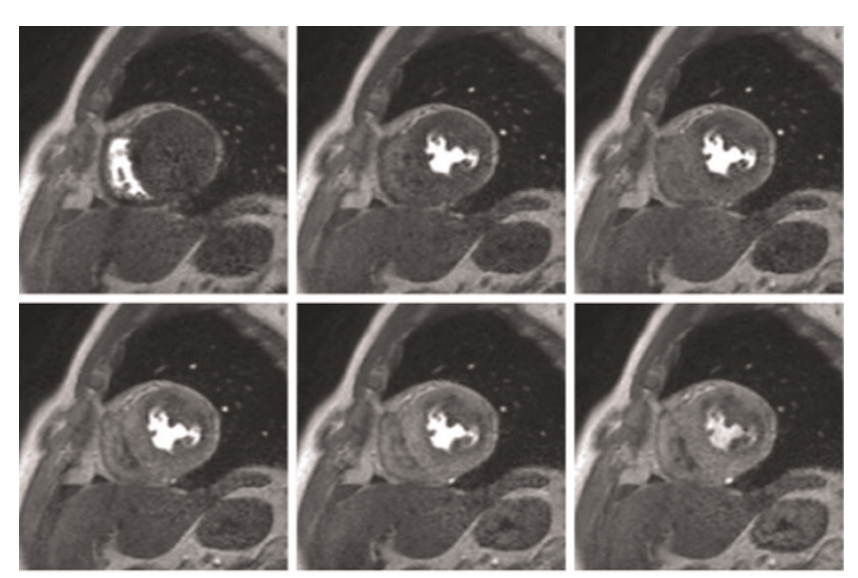

Stress perfusion MRI in the same patient with triple vessel disease. Subendocardial ischemia is clearly demonstrated in the anteroseptal well, lateral wall and inferior wall on high resolution images.

were no cases in which dark-rim artifacts influenced the diagnosis. Respiratory artifacts were found in II (2.1\%) of 528 segments. In 14 patients who underwent coronary angiography within 2 weeks from MR study, stress-rest perfusion MRI demonstrated the sensitivity, specificity, positive and negative predictive values and accuracy of were $90.9 \%(\mathrm{I} / \mathrm{III}), 96.7 \%(30 /$ $3 \mathrm{I}), 90.9 \%(10 / \mathrm{II}), 96.7 \%(30 / 3 \mathrm{I})$ and $95.2 \%(40 / 42)$ for detecting significant coronary artery disease. Figures $I$ and 2 .

Conclusion: Perfusion MR images with high spatial resolution can be acquired at every heart beat by using a 3 T MR imager and $k-t$ SENSE acceleration. This approach can substantially reduce endocardial dark rim artifacts and allows for an accurate detection of myocardial ischemia in patients with flow-limiting coronary artery disease.

\section{6}

Adenosine magnetic resonance imaging versus dobutamine stress echocardiography in patients with low probability for coronary artery disease Stamatios Lerakis, Athanasios V Anadiotis,

Elisa Zaragoza-Macias, Emir Veledar, John Oshinski,

Chris Vaccari, Akbar H Khan, Puneet Sharma, Irfan Shukrullah, Paolo Raggi and Arthur E Stillman Emory University, Atlanta, GA, USA

Journal of Cardiovascular Magnetic Resonance 2009, I I(SuppI I):O36

Introduction: Accurate assessment of patients with chest pain without electrocardiographic changes or elevation of serum cardiac enzymes is challenging. There is increased interest in the role of dobutamine stress echocardiography (DSE) and adenosine magnetic resonance imaging (AMRI) performed in the chest pain unit as a diagnostic method to rule out Coronary Artery Disease (CAD) as the cause of the chest pain in this population.

Purpose: The purpose of this study was to compare DSE and AMRI in patients with low probability of CAD.

Methods: Inclusion criteria for the study were patients with normal EKG (no signs of cardiac ischemia) and negative cardiac enzymes, who were admitted to the Cardiac Decision Unit
Table I (abstract 036)

\begin{tabular}{llll}
\hline Characteristics & MRI & DSE & p-value \\
\hline AGE & $52 \pm 12$ & $54 \pm 13$ & $0.057 \mid$ \\
Gender (males) & $144(70.9 \%)$ & $38(38 \%)$ & $<0.000 \mathrm{I}$ \\
CAD & $16(16 \%)$ & $13(6.4 \%)$ & 0.0076 \\
HTN & $66(64.7 \%)$ & $11 \mathrm{I}(54.7 \%)$ & $0.094 \mathrm{I}$ \\
DM & $30(29.7 \%)$ & $36(17.7 \%)$ & $0.017 \mathrm{I}$ \\
SMOKING & $20(19.6 \%)$ & $39(19.5 \%)$ & 0.2967 \\
Dyslipidemia & $40(39.2 \%)$ & $46(22.8 \%)$ & 0.0027 \\
Family_history_of_CAD & $52(51 \%)$ & $74(54.4 \%)$ & 0.6029 \\
EF & $65.0 \pm 10.4$ & $64.2 \pm 6.1$ & 0.4235 \\
Coronoary Artery Disease & $13(12.6 \%)$ & $3(1.5 \%)$ & $<0.000 \mathrm{I}$ \\
Beta Blocker use & $29(28.4 \%)$ & $25(12.4 \%)$ & 0.0005 \\
Ca_B & $19(18.6 \%)$ & $27(13.4 \%)$ & 0.2268 \\
ACEi & $21(20.6 \%)$ & $21(10.4 \%)$ & 0.015 \\
ARBs & $14(13.7 \%)$ & $20(9.9 \%)$ & 0.3178 \\
ASA & $27(26.5 \%)$ & $21(10.4 \%)$ & 0.0003 \\
& & & \\
\hline
\end{tabular}

(CDU) from 2006-2008 at Emory University Hospital. The diagnostic method used was chosen randomly by physician preference. T-test was used to assess differences in continuous variables, and $\mathrm{X}^{2}$ square to test differences in categorical variables between the two groups. Logistic regression was used to assess the likelihood of detecting CAD after adjusting for technique used and baseline characteristics.

Results: A total of 306 patients were included, 103 patients were evaluated with AMRI and 203 underwent DSE. Mean age was similar among groups ( 52 for AMRI vs. 54 for DSE). Patients in AMRI group were more likely to be males, had more risk factors for CAD, and used more Beta blockers or aspirin at baseline compared to patients evaluated by DSE. AMRI identified more patients as having CAD compared to DSE (I3 (I2.6\%) vs.3 (I.5\%), $\mathrm{P}=<0.000 \mathrm{I})$. This difference remained significant even after adjusting for baseline characteristics and risk factors [OR of CAD by AMRI vs DSE $=7.01, \mathrm{Cl}(1.48-33.16) \mathrm{p}=0.014]$. (Data in Table I.)

Conclusion: In this prospective study of patients with low probability of CAD, AMRI identified more cases of CAD than DSE even after adjusting for baseline characteristics. Although selection bias could account for part of these results, a higher sensitivity for AMRI is suggested.

037

Blood oxygen level-dependent MRI in patients with coronary artery disease and normal volunteers: a validation study against PET Theodoros D Karamitsos', Alejandro Recio-Mayoral ${ }^{2}$, Jayanth R Arnold', Lucia Leccisotti ${ }^{2}$, Paul Bhamra-Ariza ${ }^{2}$, Ruairidh K Howells', Nick Searle', Matthew D Robson!', Ornella E Rimoldi', Paolo G Camici ${ }^{2}$, Stefan Neubauer ${ }^{\prime}$ and Joseph B Selvanayagam '

'University of Oxford Centre for Clinical Magnetic Resonance Research (OCMR), Oxford, UK

${ }^{2}$ Medical Research Council (MRC) Clinical Sciences Centre, Imperial College of Science, Technology and Medicine, Hammersmith Hospital, London, UK

Journal of Cardiovascular Magnetic Resonance 2009, I I(SuppI I):O37

Background: Elevated deoxyhaemoglobin seen downstream in a territory subtended by a stenotic coronary artery can be 
Table I (abstract O37) Rest MBF, stress MBF, coronary flow reserve (CFR) and BOLD-signal intensity (SI) change of stenosed, remote to ischemia and normal segments

\begin{tabular}{|c|c|c|c|c|}
\hline & \multicolumn{2}{|c|}{ CAD patients } & \multicolumn{2}{|c|}{ Normal volunteers } \\
\hline & STENOSED N = 59 & REMOTE N = 73 & NORMAL N = 60 & P-value \\
\hline $\begin{array}{l}\text { REST MBF }(\mathrm{ml} / \mathrm{min} / \mathrm{g}) \\
\text { REST MBF corrected }\left[\mathrm{ml} / \mathrm{min} / \mathrm{g} /\left(\mathrm{mmH} \cdot \mathrm{bpm} / 10^{4}\right)\right] \\
\text { HYPEREMIC } \mathrm{MBF}(\mathrm{ml} / \mathrm{min} / \mathrm{g}) \\
\text { CFR }\end{array}$ & $\begin{array}{l}0.95 \pm 0.03(0.90-1.01) \\
1.43 \pm 0.05(1.34-1.52) \\
2.11(1.66-2.42) * \neq \\
2.31(1.70-2.91) * \neq\end{array}$ & $\begin{array}{l}0.94 \pm 0.03(0.88-0.99)^{\dagger} \\
1.42 \pm 0.04(1.34-1.51) \\
2.73(2.16-3.44)^{\dagger} \\
3.00(2.31-3.98)\end{array}$ & $\begin{array}{l}1.03 \pm 0.03(0.97-1.09) \\
1.39 \pm 0.04(1.31-1.46) \\
3.69(3.16-4.61) \\
3.62(2.89-4.93)\end{array}$ & $\begin{array}{l}0.04 \\
0.76 \\
<0.001 \\
<0.001\end{array}$ \\
\hline $\begin{array}{l}\text { CFR corrected } \\
\text { BOLD SI change (\%) }\end{array}$ & $\begin{array}{l}\text { I.4I }(1.06-2.00) * \ddagger \\
\mathrm{I} .25(-2.38-7.89) * \ddagger\end{array}$ & $\begin{array}{l}2.01(1.55-2.50)^{\dagger} \\
8.91(4.95-12.78)^{\dagger}\end{array}$ & $\begin{array}{l}2.68(2.32-3.45) \\
14.08(9.23-22.20)\end{array}$ & $\begin{array}{l}<0.001 \\
<0.001\end{array}$ \\
\hline
\end{tabular}

Data are presented as means \pm standard deviation ( $95 \%$ confidence intervals) or median (interquartile range) as appropriate Rest MBF corrected: rest $\mathrm{MBF}$ corrected for rate-pressure product (RPP), an index of myocardial oxygen consumption: $M B F=(M B F / R P P) \times 10^{4}$. Stenosed: myocardial segments subtended by a $>50 \%$ stenosed coronary artery. Remote: myocardial segments subtended by arteries with minimal or no CAD. Normal: myocardial segments in normal volunteers. * $p<0.05$ for comparison between stenosed and remote segments. ${ }^{\dagger} \mathrm{p}<0.05$ for comparison between remote to ischemia and normal segments. ${ }^{\ddagger} p<0.05$ for comparison between stenosed and normal segments.

assessed by blood oxygen level-dependent (BOLD) MRI. Deoxyhemoglobin is paramagnetic and acts as an intrinsic contrast agent leading to signal loss in T2- and T2*-weighted sequences. Previous animal and human BOLD studies at I.5 Tesla using $\mathrm{T} 2 *$-weighted sequences were fundamentally limited by the relatively small signal differences between normal and de-oxygenated myocardial regions. A new T2-prepared steadystate free precession (SSFP) BOLD sequence gave promising results in animal models at I.5 Tesla. [I] In theory, implementation of this sequence at the higher field strength of 3 Tesla would further improve the detection of BOLD signal intensity (SI) changes. We sought to apply a T2-prepared SSFP BOLD sequence at 3 Tesla in patients with coronary artery disease (CAD) and normal volunteers, and validated it against perfusion measurements by Positron Emission Tomography (PET).

Methods: Twenty-two patients (age $62 \pm 8$ yrs, 16 men) with CAD (at least I stenosis $>50 \%$ on quantitative coronary angiography-QCA) and 10 normal volunteers (age $52 \pm 7 \mathrm{yrs}$, 7 men) underwent 3 T BOLD MRI and PET. For BOLD-CMR, a single mid-ventricular slice was acquired at mid-diastole using a T2-prepared SSFP pulse sequence with the following parameters:
T2 preparation weighting $40 \mathrm{~ms}$, matrix $168 \times 192$, slice thickness $8 \mathrm{~mm}$, flip angle $44^{\circ}$. A set of 6 images was acquired at rest and at peak adenosine $(140 \mu \mathrm{g} / \mathrm{kg} / \mathrm{min})$ stress. Using PET with oxygen- 15 labeled water, myocardial blood flow (MBF) was measured at baseline and during adenosine hyperemia. The BOLD short-axis view was divided into 6 segments, according to the mid-ventricular segments of the I7-AHA segment model, and mean signal intensities (SI) were calculated using QMass (Medis) software. SI values were corrected for differences in TIweighting owing to heart rate changes at stress and rest. PET images were analyzed with home-built software under MATLAB (MathWorks Inc.) and registered with the BOLD short-axis image using anatomical landmarks.

Results: Based on the coronary anatomy, 59 myocardial segments were supplied by significantly stenosed vessels (stenosed segments) and 73 segments were supplied by vessels with minimal or no disease (remote to ischemia segments). A third group of myocardial segments $(n=60)$ from normal volunteers were labeled as 'normal' segments. Rest MBF, stress $\mathrm{MBF}$, coronary flow reserve and BOLD-SI change of stenosed, remote to ischemia and normal segments are shown in Table I.

\section{Figure I (abstract 037)}
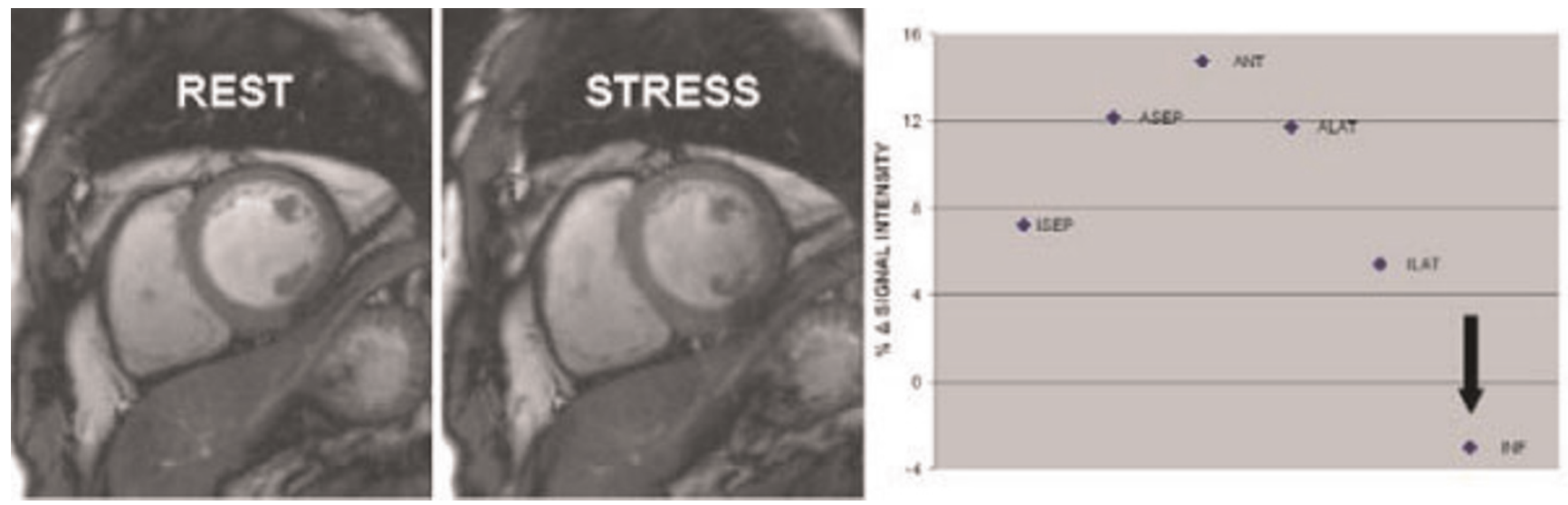

An example of a patient with significant disease in the right coronary artery. A SI drop was noted in the inferior wall (black arrow). All other myocardial segments showed a rise in SI during stress. 
Taking QCA as the gold standard, cut-off values for stress MBF $(\leq 2.45 \mathrm{ml} / \mathrm{min} / \mathrm{g}-\mathrm{AUC} 0.83)$ and BOLD SI change $(\leq 3.74 \%-$ AUC 0.78) were determined to define ischemic segments. BOLD $\mathrm{MRI}$ and PET agreed on the presence or absence of ischemia in 18 of the 22 patients (82\%), and in all normal subjects. With regards to per segment analysis: taking PET as the gold standard and by applying the cut-off values for stress MBF and BOLD SI, BOLD MRI had moderate sensitivity $(63 \%)$ but very good specificity $(88 \%)$ for the identification of ischemia. Minor off-resonance artifacts were found in 9 subjects (7 CAD patients and 2 normal volunteers). Figure I shows an example of a patient with significant disease in the right coronary artery.

Conclusion: T2-prepared SSFP 3 T BOLD imaging is feasible in the clinical setting and has good agreement with PET perfusion measurements for the detection of myocardial ischemia.

\section{Reference}

I. Fieno DS, et al: Circulation 2004, I I 0:1284-1290.

\section{8}

Assessment of stess and rest perfusion in patients early after first anterior STEMI patients treated successfully with $\mathrm{pPCl}$

Dorota Piotrowska-Kownacka, Lukasz Kownacki, Grzegorz Opolski, Leszek Krolicki and Olgierd Rowinski Medical University of Warsaw, Warsaw, Poland

Journal of Cardiovascular Magnetic Resonance 2009, I I(SuppI I):038

Introduction: Restoration of normal epicardial coronary flow in acute ST segment elevation myocardial infarction does not ensure adequate perfusion at the myocardial tissue level. In the era of primary $\mathrm{PCl}$, patients with acute myocardial infarction treated successfully with $\mathrm{PCl}$ are discharged mostly within first week. There are no studies available regarding blood flow at the tissue level in these patients during effort.

Purpose: Assessment of stess and rest perfusion defects early after STEMI successfully treated with PPCl.

Methods: 61 patients with first anterior STEMI $(57 \pm 10 \mathrm{yrs}$. $52 \mathrm{M}$ ) who underwent successful $\mathrm{PPCl}$ have been included into the study. CMR was performed on I.5 T scanner between 5 and 10 days after $\mathrm{PPCl}$. Myocardial perfusion was assessed at rest and in stress condition during infusion of adenosine ( $40 \mu \mathrm{g} / \mathrm{kg}$ b.w.l min., 3 minutes infusion) during first-pass perfusion imaging. Microvascular obstruction regions (MVO) were assessed on early enhancement images acquired I-2-minutes after stress perfusion. Delayed enhancement (DE) images were acquiered 15 minutes after Gd-DTPA. Transmurality of myocardial perfusion defecits at rest and in stress condition, MVO were evaluated using 5 point scale in 16 segments. DE was also evaluated in segment 17. The sum of scores were calculated for each variable. Scar size and MVO were additionally quantitatively analyzed using
MASS software. The results were given in $\mathrm{ml}$ and in $\%$ of LV volume.

Results: Only in 2 patients there was no evidence of at least subendocardial stress perfusion deficit. Stress perfusion sum of scores discriminated patients with normal EF (mean $59 \pm 1 \%$ ) and LV dysfunction (mean EF $=38 \pm 9,7$ ). Median stress perfusion sum of scores was 15 points (ranged 0 to 37). Median rest perfusion scores was significantly lower (3 points; ranged 0-27). Median MVO and DE sum of scores were 3 points (ranged 0-33) and $25(3-42)$ respectively. DE measured as a \% of $L V$ volume has discriminated patients with severe (mean EF $3 \mathrm{I}, 7 \pm 8,7$ ) vs moderate LV dysfunction (mean EF 4I,9 $\pm 9,5 ; \mathrm{p}=0,00 \mathrm{I}$ ) with a cut of point $40 \%$. Regression model revealed that only DE and stress perfusion sum of scores have significantly contributed to the LV EF model at discharge.

Conclusion: Despite TIMI 3 flow in coronary artery myocardial perfusion defects at the tissue level are very frequent. Only in patients with preserved LV function pharmacological stress have not induced or intensed perfusion deficits. All patients with STEMI anterior successfully treated with $\mathrm{PPCl}$, even with mild LV dysfunction should avoid effort which could induce ischemia. The pathogenesis remains unknown. Further studies are needed.

\section{O39}

Evaluation of contrast wash-in and peak enhancement in adenosine first pass perfusion in patients post bypass surgery Christoph Klein', Eike Nagel ${ }^{2}$, Kristof Graf', Stephan Dreysse ${ }^{i}$, Bernhard Schnackenburg ${ }^{3}$ and Eckart Fleck'

${ }^{I}$ German Heart Institute Berlin, Berlin, Germany

${ }^{2}$ King's College London, London, UK

${ }^{3}$ Philips Medical Systems, Hamburg, Germany

Journal of Cardiovascular Magnetic Resonance 2009, I I(SuppI I):O39

Introduction: CMR adenosine first pass perfusion yields excellent results for the detection of significant coronary artery disease. As in patients after coronary artery bypass grafts (CABG) myocardial perfusion is more complex and additionally the kinetic of a first pass bolus may by altered due to different distances through the bypasses and/or native vessels, this patient group has been excluded from most published studies. Parameters like speed of contrast wash-in (upslope, time to $50 \%$ or peak enhancement) and peak enhancement are indirectly used for visual analysis and may be altered post CABG imitating perfusion defects without significant stenosis. In this case, adenosine perfusion would be an inadequate diagnostic test.

Purpose: Aim of the study was to evaluate semiquantitative perfusion parameters in patients after CABG in order to evaluate

Table I (abstract 039) Semiquantitative parameters in native vessels and grafts

\begin{tabular}{|c|c|c|c|c|c|c|}
\hline & $\begin{array}{l}\text { Native } \\
\text { vessel }\end{array}$ & CABG & $\begin{array}{l}\text { p (native } \\
\text { vs. CABG) }\end{array}$ & $\begin{array}{l}\text { Correlation } \\
\text { (native vs. CABG) }\end{array}$ & $\begin{array}{l}\text { p (CABG } \\
\text { vs. CABG) }\end{array}$ & $\begin{array}{l}\text { Correlation } \\
\text { (CABG vs. CABG) }\end{array}$ \\
\hline Upslope & $17.7 \pm 7.8$ & $17.3 \pm 7.0$ & $>0.05$ & 0.92 & $>0.05$ & 0.89 \\
\hline Time to $50 \%$ & $8.2 \pm 1.8$ & $8.1 \pm 1.8$ & $>0.05$ & 0.94 & $>0.05$ & 0.90 \\
\hline Time to peak & $13.7 \pm 3.1$ & $13.7 \pm 3.1$ & $>0.05$ & 0.97 & $>0.05$ & 0.92 \\
\hline Relative peak enhancement & $22.9 \pm 9.2$ & $22.0 \pm 8.7$ & $>0.05$ & 0.91 & $>0.05$ & 0.86 \\
\hline
\end{tabular}


Figure I (abstract 039)

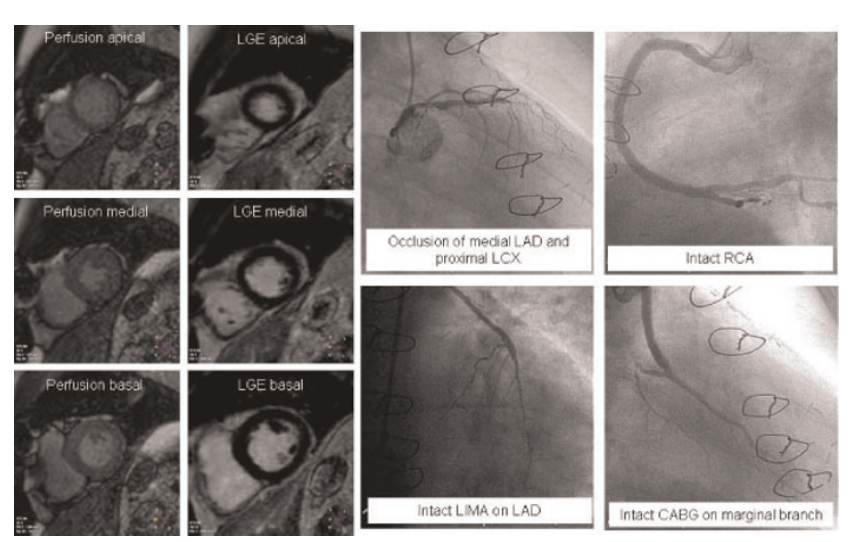

contrast kinetics in areas supplied by native coronaries and different bypass grafts.

Methods: 32 patients post CABG were included into the study consisting of adenosine first pass $(0.05 \mathrm{mmol} / \mathrm{kg}$ Gd-DTPA) perfusion ( 3 short axis views/heart beat) and late Gadolinium enhancement before undergoing invasive coronary angiography. In invasive angiography, areas perfused by native coronaries and the different bypasses were identified. In these areas upslope, time to $50 \%$ peak enhancement, time to peak enhancement and relative peak enhancement were calculated using the ViewForum (Philips Medical Systems, Best, Netherlands). Only segments without vessel stenosis and without LGE were used for final analysis.

Results: Results are displayed in Table I. No significant differences in any parameter comparing native vessels with $C A B G$ or $C A B G$ with $C A B G$ were found. Figure I shows homogenous perfusion enhancement in a patient post $C A B G$ with areas perfused by native RCA, LIMA on LAD and venous graft on a marginal branch.

Conclusion: Semiquantitaive parameters of first pass adenosine perfusion are similar in areas supplied by native vessels or by different bypass grafts. These parameters are indirectly used for visual analysis (speed of contrast wash-in and peak signal intensity). Therefore the possible different contrast kinetic through grafts and native vessel does not seem to be a limiting factor for the accuracy of first pass adenosine perfusion in patients post CABG.

\section{0}

In-room treadmill exercise stress cardiac magnetic resonance in patients with suspected ischemic heart disease

Subha V Raman, Mihaela Jekic, Jennifer A Dickerson,

Eric L Foster and Orlando P Simonetti

Ohio State University, Columbus, $\mathrm{OH}$, USA

Journal of Cardiovascular Magnetic Resonance 2009, I I(SuppI I):O40

Objective: To implement and demonstrate the feasibility of in-room treadmill exercise stress perfusion and cine CMR in patients with suspected coronary artery disease (CAD).
Figure I (abstract 040)

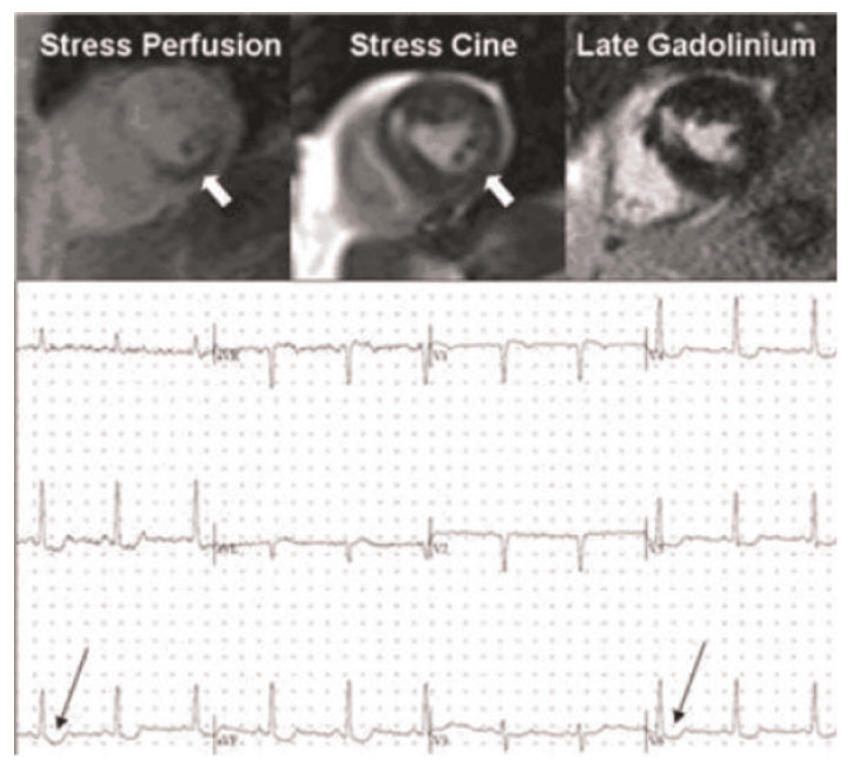

Background: Exercise is preferred to pharmacologic stress because it links physical activity to symptoms and ischemia and offers important information such as exercise capacity, blood pressure response, ECG changes, and the presence or absence of exercise-induced symptoms. The Bruce Treadmill Test is the most commonly-used protocol for cardiac stress testing in the US, with proven diagnostic and prognostic value. The lack of MRI-compatible exercise equipment has made pharmacological stress the only practical option for CMR stress testing to date. We implemented treadmill exercise stress cardiac magnetic resonance imaging (CMR) of both wall motion and perfusion in patients with suspected ischemic heart disease.

Methods: A treadmill was modified by replacing all ferromagnetic components except the motor with non-magnetic equivalents. This enabled safe placement of the treadmill in the corner of the MRI room, approximately $2 \mathrm{~m}$. from the patient table. Sixteen patients age $56 \pm 8$ years referred for stress SPECT were prospectively enrolled. Tc99m SPECT imaging was performed at rest; patients were then moved to the MRI suite for stress testing. Patients were positioned on the MRI table using a vacuum mattress to enable precise repositioning following treadmill exercise. Localizer scans followed by resting real-time cine CMR were performed, and then cine and perfusion scans were queued for rapid execution immediately following treadmill exercise. After removing the patient from the magnet, resting ECG was recorded and treadmill exercise commenced using the Bruce protocol. 12-lead ECG monitoring was performed throughout the treadmill test. At peak stress, Tc99m was injected and patients rapidly returned to their prior position in the magnet for post-exercise real-time cine followed immediately by multislice first-pass perfusion imaging with $0.1 \mathrm{mmol} / \mathrm{kg}$ IV gadoliniumbased contrast using GRE-EPI TR/TE 5.8/I.2 ms, ETL $4 \mathrm{~m}$ matrix $160 \times 96$ and TSENSE acceleration factor of 2 . The patient table was pulled out of the MRI system and patients remained supine 
on the patient table for 12 -lead ECG monitoring during 5-10 min of recovery. The table was then returned to magnet bore for recovery cine and resting perfusion followed by delayed postgadolinium imaging. Post-CMR, patients went to the adjacent SPECT lab for stress nuclear imaging. Five patients underwent coronary angiography. Images were reviewed blinded to other results.

Results: All patients completed the examination (Figure I: ischemia example). Mean time to completion of cine MRI postexercise was $73 \pm 9 \mathrm{sec}$, and to completion of perfusion imaging $91 \pm 6 \mathrm{sec}$. Accuracy in the five patients who underwent coronary angiography was $5 / 5$ for CMR and $3 / 5$ for SPECT. Follow-up at median of 60 days indicated freedom from cardiovascular events in 13/13 CMR-negative and I2/13 SPECTnegative patients.

Conclusion: Exercise stress CMR including wall motion and perfusion inside the MRI room is feasible in patients with suspected ischemic heart disease. Preliminary results indicate favorable accuracy and prognostic value of this new stress imaging system compared to nuclear perfusion imaging. Further technical modifications are required to facilitate completion of cine and perfusion imaging within I minute of peak stress.

\section{1}

Adenosine induced pulmonary vasodilation is blocked by active cigarette smoking, an evaluation by pulmonary transit time with first pass perfusion MRI

Jie Jane Cao, Sophie Wang, Marguerite Roth, William Schapiro, Yi Wang and Nathaniel Reichek St Francis Hospital, Roslyn, NY, USA

Journal of Cardiovascular Magnetic Resonance 2009, I I(SuppI I):O4 I

Introduction: Pulmonary transit time (PTT) measures the time that blood travels through the pulmonary vasculature. PTT correlates well with pulmonary vascular resistance. It is prolonged in patients with pulmonary hypertension and congestive heart failure. Similar to systemic and coronary vasculature, the pulmonary arteries dilate in response to purine nucleoside adenosine which has a direct endothelium independent effect on the $A 2 b$ receptor in vascular smooth muscle. Despite the detrimental effect of cigarette smoking on endothelium dependent systemic vascular function, little is known of its effect on endothelium independent function of the pulmonary vasculature.

Purpose: The objective of this study is to develop a noninvasive strategy using first pass perfusion MRI to measure PTT and to test the hypothesis that cigarette smoking inhibits endothelium independent pulmonary vasodilation.

Methods: The study included 63 prospectively recruited subjects (65\% women) without significant pulmonary disease. All subjects underwent first pass perfusion cardiac MRI in a I.5 Tesla Siemens scanner. Three long axis planes were acquired per heartbeat over 50 heartbeats using a partial Fourier saturationrecovery steady state free precession sequence during a breath hold. Dynamic perfusion imaging was first performed during adenosine infusion at $140 \mu \mathrm{g} / \mathrm{kg} / \mathrm{min}$ with gadolinium concentration at $0.05 \mathrm{mmol} / \mathrm{kg}$. Following a 20 minute washout period dynamic imaging was repeated without adenosine using the same parameters. Images were analyzed in commercial software (Argus, Siemens, Germany). PTT was measured between the times when signal intensity reached a peak in main pulmonary artery and in the left atrium.

Results: The mean age of the study cohort was $55.9 \pm 13.1$ years. The prevalence of never, past and current smoking was 42.4\%, 49.2\% and 8.5\%, respectively. Compared to rest PTT $(5.98 \pm \mathrm{I} .33 \mathrm{~s})$ there was a $25.4 \%$ reduction $(\mathrm{p}<0.00 \mathrm{I})$ in PTT during adenosine infusion $(4.46 \pm 0.94 \mathrm{~s})$. When the analysis was stratified by smoking status significant PTT reduction was seen in never smokers $(25.5 \%, \mathrm{p}<0.00 \mathrm{I})$ and in past smokers $(28.5 \%$, $\mathrm{P}<0.00 \mathrm{I})$ but not in the current smokers $(8.8 \%, \mathrm{P}=0.433)$. However, there was significant correlation between R-R interval and PTT with Spearman correlation coefficient 0.640 ( $p<0.00 \mathrm{I})$ for rest PTT and $0.55 \mathrm{I},(\mathrm{p}<0.00 \mathrm{I})$ for stress PTT. In view of a $27.5 \%$ heart rate increase $(p<0.00 I)$ the analysis was repeated using PTT values normalized to RR interval. In contrast to absolute PTT measurements the normalized values demonstrated minimal change between rest and stress PTT in never smokers $(0.06 \%, P=0.989)$ and in past smokers $(-1.55 \%$, $p=0.65 \mathrm{I})$. In contrast, there was an $18.7 \%$ increase $(p=0.029)$ in normalized stress PTT among current smokers.

Conclusion: Adenosine infusion was associated with significant PTT shortening in never and past smokers. Shortened PTT likely represented reduced pulmonary resistance in response to the endothelium independent vasodilatory effect of adenosine. However this response appeared to be blocked by active cigarette smoking. In the absence of active cigarette smoking PTT normalized to R-R interval remained constant at rest and during stress, suggesting an intact vasoreactivity to accommodate increased cardiac output during adenosine infusion. Conversely this value was significantly prolonged during adenosine infusion among current smokers, implying impaired vasoreactivity likely due to the detrimental effect of cigarette smoking, which resulted in delay of pulmonary blood transit in the presence of increased blood volume. Our findings suggest that CMR adenosine stress testing may be an important modality to evaluate pulmonary arterial function.

\section{2}

Coronary artery flow velocity reserve during the cold pressor test in overweight, healthy women using spiral imaging at $\mathbf{3} \mathbf{T}$

Alice Y Chang', Melanie Kotys ${ }^{2}$, Ivan Dimitrov ${ }^{2}$, Andrew Kontak', Hardik Yadav', Christopher Maroules', Tommy Tillery' and Ron Peshock'

'UT Southwestern Medical Center at Dallas, Dallas, TX, USA

${ }^{2}$ Philips Medical Systems, Cleveland, OH, USA

${ }^{3}$ University of Texas School of Public Health, Dallas, TX, USA

\section{Journal of Cardiovascular Magnetic Resonance 2009, I I(SuppI I):O42}

Introduction: Women with chest pain demonstrate coronary endothelial dysfunction before obstructive disease can be appreciated by angiography. Invasive measurements of coronary artery vasoreactivity have been shown to predict future cardiovascular events. However, there are significant risks associated with invasive studies. Thus, the ability to non- 
Figure I (abstract 042)

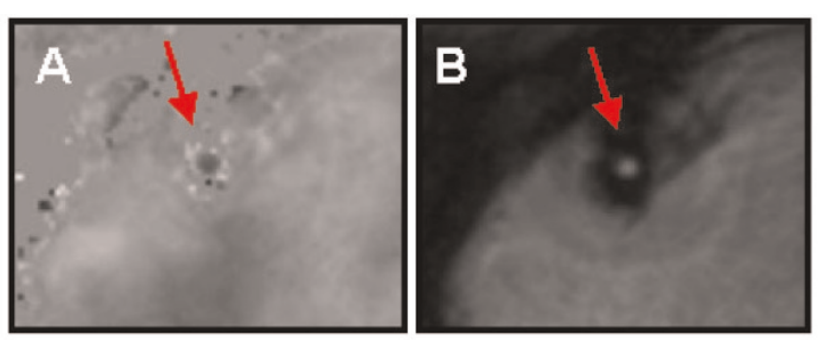

Cross-sectional images of the RCA at rest in a subject using velocityencoded MRI. (A) Phase image and accompanying (B) Magnitude image.

invasively assess coronary vasoreactivity would be especially useful in the early diagnosis and management of women with coronary artery disease.

Changes in coronary flow velocity have been successfully measured by magnetic resonance flow mapping in response to handgrip stress at $3 \mathrm{~T}$. The challenges for imaging women at risk for heart disease include the (I) smaller size of their arteries and (2) the high prevalence of overweight or obesity. In addition, we tested whether cold pressor stress, another widely used test of endothelial function that elicits a remarkable central sympathetic response, could provoke greater changes in coronary flow.

Purpose: We sought to determine the feasibility of coronary flow velocity measurements in response to the cold pressor test in overweight women using $3 \mathrm{~T}$ MRI.

Methods: Healthy, pre-menopausal women were recruited for this study and provided informed consent approved by the institutional review board. Subjects were placed supine in a $3 \mathrm{~T}$ MRI scanner (Achieva, Philips, Best, NL) using a 6 element cardiac receive coil. Scout scans were performed to determine the imaging plane orthogonal to the proximal right coronary

\section{Figure 2 (abstract 042)}

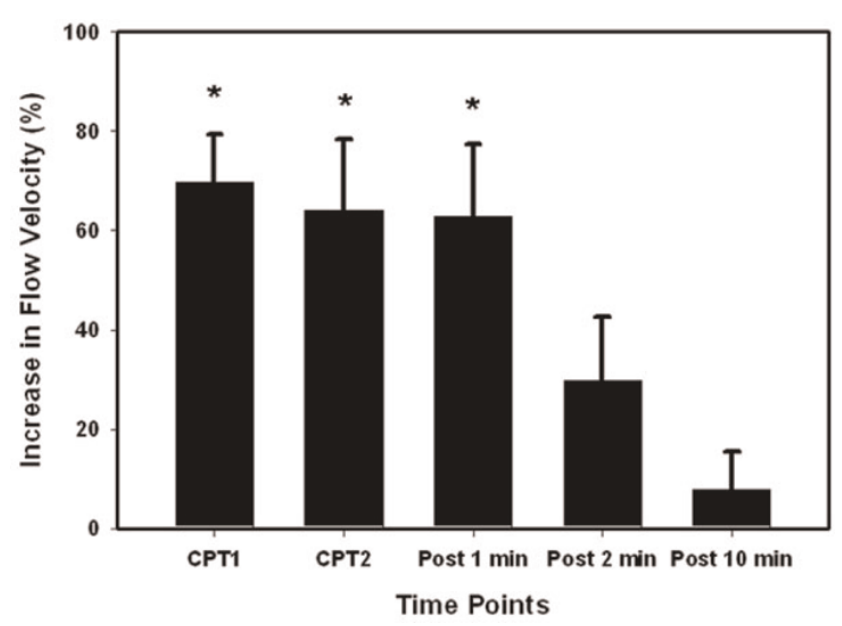

Percent changes in peak diastolic flow velocity during cold pressor test. $*_{p}<0.01$ compared to baseline and 10 minutes recovery. artery (RCA). Baseline coronary velocity measurements were obtained using a VCG triggered breath-hold ( 10 to 12 seconds) velocity-encoded spiral cine sequence perpendicular to the RCA $\left(\right.$ FOV $256 \times 256 \mathrm{~mm}^{2}$, matrix $=312 \times 312$, spatial resolution $=$ $0.8 \times 0.8 \times 7 \mathrm{~mm}^{3}, \mathrm{TR}=34 \mathrm{~ms}, \mathrm{TE}=3.5 \mathrm{~ms}$, RF excitation angle $=20^{\circ}$, spiral interleaves $=1 \mathrm{I}$, VENC $=35 \mathrm{~cm} / \mathrm{s}$, temporal resolution $=69 \mathrm{~ms}$ ). Heart rate and blood pressure were measured every 30 seconds during baseline imaging, stress and into recovery. After baseline imaging, the subject's left hand was placed in a half-water, half-ice bath for 3 minutes. Two successive velocity-encoded images of the RCA were acquired during the CPT. After the subject's hand was removed from the water, images were acquired at I, 2 and 10 minutes into recovery. Images were analyzed using Q Flow (version 4.l.6, Medis, Leesburg, Virginia), and peak diastolic coronary velocity was determined as the maximum velocity during the diastolic rest period. Coronary flow velocity reserve was calculated as peak diastolic velocity during stress divided by baseline velocity. Statistical analysis was performed using a one-way analysis of variance (ANOVA) with Tukey post-hoc tests of significance between specific time points.

Results: The mean age of the subjects $(n=7)$ was $35.1 \pm 6.5$ (mean \pm SD) and mean body mass index was $25.1 \pm 1.8$. Ratepressure product increased $46.0 \pm 23.0 \%$ from baseline to peak effect during CPT. Peak diastolic velocity increased significantly from baseline $(13.3 \pm 3.9 \mathrm{~cm} / \mathrm{s})$ to both CPT time points $(22.7 \pm 7.3 \mathrm{~cm} / \mathrm{s}$ and $21.6 \pm 7.5 \mathrm{~cm} / \mathrm{s})$ and one minute into recovery $(2 \mathrm{I} .8 \pm 8.6 \mathrm{~cm} / \mathrm{s})$ (each $\mathrm{p}<0.0 \mathrm{l})$. Recovery to baseline was achieved by 10 minutes. The average increase in peak diastolic flow velocity from baseline to peak cold pressor effect was $82.7 \pm 29.2 \%$. The mean coronary flow velocity reserve was $1.83 \pm 0.29$. Figures $I$ and 2 .

Conclusion: Cold pressor test provokes a significant increase in coronary flow velocity that is measurable at $3 \mathrm{~T}$ in overweight women. Compared to previous reports of hand-grip stress during $\mathrm{MRI}$, the CPT stimulated a greater increase in ratepressure product (46\% CPT v. $25.0 \%$ hand-grip) and coronary flow velocity $(82.7 \%$ CPT v. $39.6 \%$ hand-grip) which persists one minute after withdrawal of the stress. Future studies will examine if this greater potential for stress-provoked changes can better detect more subtle differences in cardiovascular risk factors or responses to treatments.

\section{3}

Association between aortic stiffness measured by cardiovascular magnetic resonance and sub-clinical carotid atherosclerosis in young adults Ilias Kylintireas, Colin Cunnington, Corinne Trevitt, Jonathan Diesch, Stefan Neubauer, Matthew Robson and Paul Leeson University Of Oxford, Oxford, UK

Journal of Cardiovascular Magnetic Resonance 2009, I I (SuppI I):O43

Introduction: Increased arterial stiffness is associated with increased cardiovascular risk in later life. Cardiovascular magnetic resonance (CMR) allows direct assessment of arterial stiffness by imaging the elastic properties of the aorta. This provides measures of both global (pulse wave velocity, PWV) and 
Figure I (abstract 043)

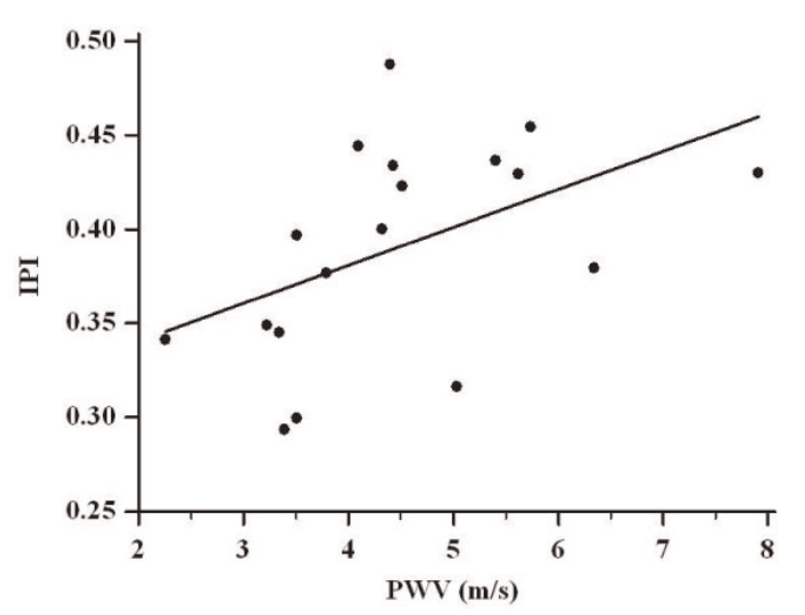

regional (aortic distensibility) aortic stiffness. Assessment can be combined with precise measurement of carotid atheroma burden, a marker of early sub-clinical atherosclerosis. We investigated whether aortic stiffness in early adult life is already associated with early changes in carotid structure.

Purpose: To determine whether aortic stiffness - quantified by cardiovascular magnetic resonance as pulse wave velocity (PWV) and aortic distensibility (AD) - is associated with early atherosclerosis-related structural changes in young adult life.

Methods: Thirty young healthy volunteers (aged 23-33) (without history of cardiovascular disease or classical risk factors for atherosclerosis) underwent CMR for measurement of aortic function and carotid wall imaging.

Aortic distensibility was measured from breath-hold ECG-gated, steady state free precession (SSFP) images. Distensibility was calculated as the relative change in area divided by the central pulse pressure. Pulse wave velocity was measured from an ECGgated, free breathing, spoiled gradient echo phase-encoded acquisition. The transit time method was used for the calculation of pulse wave velocity (PWV). TI weighted black blood turbo spin echo (TSE) cross-sectional images of both carotid arteries, centred at the lowest bifurcation were used for atheroma burden measurements (plaque index represented cross-sectional vessel wall area/total cross-sectional vascular area). Plaque index was averaged for the common carotid (CPI), the carotid bulb (BPI) and the internal carotid artery (IPI).

Results: CMR-derived PWV over the whole length of the aorta was correlated with carotid plaque index $(r=0.480, P<0.05)$ (Figure I.) particularly of the internal carotid artery. Regional measures of aortic distensibility and pulse wave velocity were unrelated to carotid atheroma burden. Applying a multiple regression analysis model (including applicable risk factors, demographics and anthropometric measurements) PWV was the sole independent predictor of IPI $[\beta=0.02( \pm 0.009)$, $\left.P<0.05, R^{2}=0.23\right]$.

Conclusion: Aortic stiffness assessed by CMR is associated with early atherosclerosis-related changes in carotid arteries in young adults.

\section{4}

Relationship between regional wall shear stress and carotid plaque composition using 3 T MRI and patient-specific computational fluid dynamics Raymond Q Migrino', Mark Bowers', Leanne Harmann', Robert Prost', Anil Doppalapudi', Tayyab Mohyuddin', Megan Bright', Jason Jurva', Osama Zaidat' and John LaDisa ${ }^{2}$

${ }^{I}$ Medical College of Wisconsin, Milwaukee, WI, USA

${ }^{2}$ Marquette University, Milwaukee, WI, USA

Journal of Cardiovascular Magnetic Resonance 2009, I I(SuppI I):O44

Introduction: Plaque vulnerability arises from the interplay among factors including plaque composition (PC) and wall shear stress (WSS). To date, the relationship between spatial WSS and $\mathrm{PC}$ is not established.

Purpose: Our aim is to determine the relationship between WSS and PC in established carotid atherosclerosis.

Methods: 5 subjects ( 4 males, $66 \pm 8$ years), with moderate to severe carotid plaque underwent $3 \mathrm{~T}$ MRI using 4-channel carotid coil. TI, T2, proton density and time of flight images were obtained $(0.47-0.55 \times 0.47-0.55 \times 2 \mathrm{~mm}$ spatial resolution) $12 \mathrm{~mm}$ above and below the bifurcation. Plaque composition (necrotic core and loose matrix) were quantified using Plaqueview software (VP Diagnostics). Subject-specific computational fluid dynamic models were created from MRI, B-mode ultrasound and blood pressure (BP) data using CVsim software. Outlet boundary conditions that replicated flow and BP were applied and simulations used a stabilized finite element solver. Each carotid slice were divided into 6 circumferential regions where WSS was correlated with PC.

Results: Please see figure I. WSS correlated significantly with necrotic core $(R=0.283$, $p<0.00 I)$ but not with loose matrix $(R=-0.03, p=0.6)$. The same relationship was seen in the common carotid, bifurcation or internal carotid artery. Carotid plaque regions with necrotic core had higher WSS than those without $\left(34.1 \pm 2.6\right.$ vs. $\left.17.3 \pm 4.6 \mathrm{dyn} / \mathrm{cm}^{2}, \mathrm{p}<0.00 \mathrm{I}\right)$. WSS in regions with and without loose matrix did not differ $(25.8 \pm 3.6$ vs. $22.5 \pm 3.3, p=0.7$ ).

Conclusion: In established carotid artery disease, regions with high WSS are associated with increased necrotic core, but not

Figure I (abstract 044)

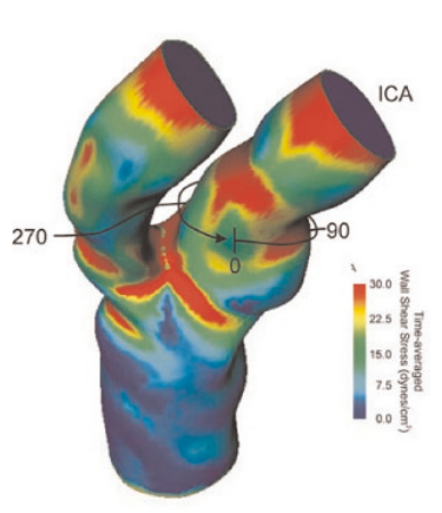

CFD Model and Regional WSS

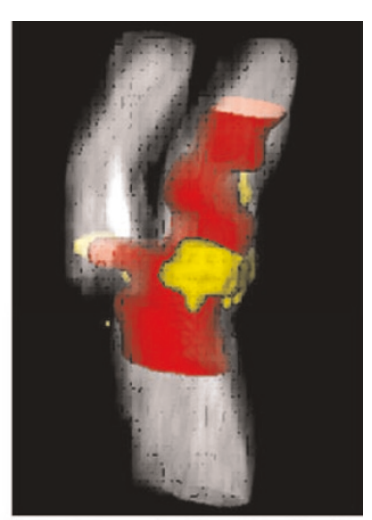

MRI: Necrotic Core (Yellow), Blood Pool (Red) 
loose matrix. This relationship between increased WSS and necrotic core content in the plaque may play an important role in plaque vulnerability.

\section{5}

A novel, dual-contrast in-vivo MR imaging method with principal component analysis reliably quantifies lipid-rich necrotic core and collagen in human carotid atherosclerotic plaques

Zhen Qian, Sarah Rinehart, Laura J Murrieta,

Gustavo Vasquez, Patrick M Battey and Szilard Voros

Piedmont Heart Institute, Atlanta, GA, USA

Journal of Cardiovascular Magnetic Resonance 2009, I I(SuppI I):O45

Background: In-vivo multi-spectral imaging of human carotid plaques using different pulse sequences, with and without exogenous contrast agents, has been implemented. However, an in-vivo dual-contrast approach with small paramagnetic iron oxide (SPIO) and gadolinium at multiple timepoints with principal component analysis (PCA) has not been previously performed.

Purpose: To develop a novel, PCA-based method for the detection of lipid-rich necrotic core (LRNC) and collagen in human carotid plaques utilizing a dual-contrast approach. We hypothesized that LRNC and collagen can be reliably identified based on different signal characteristics with different exogenous contrast agents and multiple different imaging pulse sequences. Methods: 10 pts scheduled for carotid endarterectomy (CEA) were imaged at I.5 T with a dedicated small surface coil. TI, T2 and inversion recovery delayed hyperenhancement (DHE) images with magnitude/phase reconstruction were obtained before contrast, immediately and 24 hours after $0.05 \mathrm{cc} / \mathrm{kg}$ of SPIO (Feridex) and after $30 \mathrm{cc}$ of Gd. Imaging parameters were: TI; TR: $1500 \mathrm{~ms}$, TE $10 \mathrm{~ms}$, slice $3 \mathrm{~mm}$, matrix $320 \times 320$, averages 3; T2; TR: $2500 \mathrm{~ms}$, TE $92 \mathrm{~ms}$, slice $3 \mathrm{~mm}$, matrix $320 \times 320$, averages 4; DHE; TR: $745 \mathrm{~ms}$, TE $3.5 \mathrm{~ms}$, slice $6 \mathrm{~mm}$, matrix $192 \times 192$, averages I. Corresponding histological sections from CEA specimens were stained with Movat's pentachrome for identification of LRNC and collagen.

For a training dataset, ROI in two pts were normalized to foreground median intensity and histopathological specimens were non-rigidly registered to the MR images using anatomical landmarks and a thin-plate spline-based image morphing algorithm (Panel C). Plaque composition masks consisting of 6 classes (LRNC [red], calcium [yellow], fibrous collagen [green], proteoglycans [light blue], elastin [grey], and fibrin [purple]) were created based on the registered histological images (Panel D). All 4 sequences at all 4 timepoints were independently tested for the identification of plaque composition. A more comprehensive PCA analysis utilizing all pulse sequences at all timepoints was also performed. Signal intensity (SI) statistics are expressed as mean \pm SD. The performance of composition identification was measured by comparing the predicted compositions with the 2-class mask, and was evaluated using the two-tailed t-test $P$ value, and the area under the ROC curve.

Results: See Figure I. SI was significantly higher in LRNC on T2 images immediately after SPIO (Panel A) compared to other tissues $(56.66 \pm 18.52$ vs. $26.21 \pm 12.74, p<0.0001)$. PCA showed significant difference between LRNC and non-LRNC tissue $(p<0.000 I)$ (Panel E). The percentage of correctly identified pixels was $80.4 \%$. Predicted total area for LRNC was 7I pixels $\left(22.22 \mathrm{~mm}^{2}\right)$, compared to 66 pixels $\left(20.65 \mathrm{~mm}^{2}\right)$ on
Figure I (abstract 045)

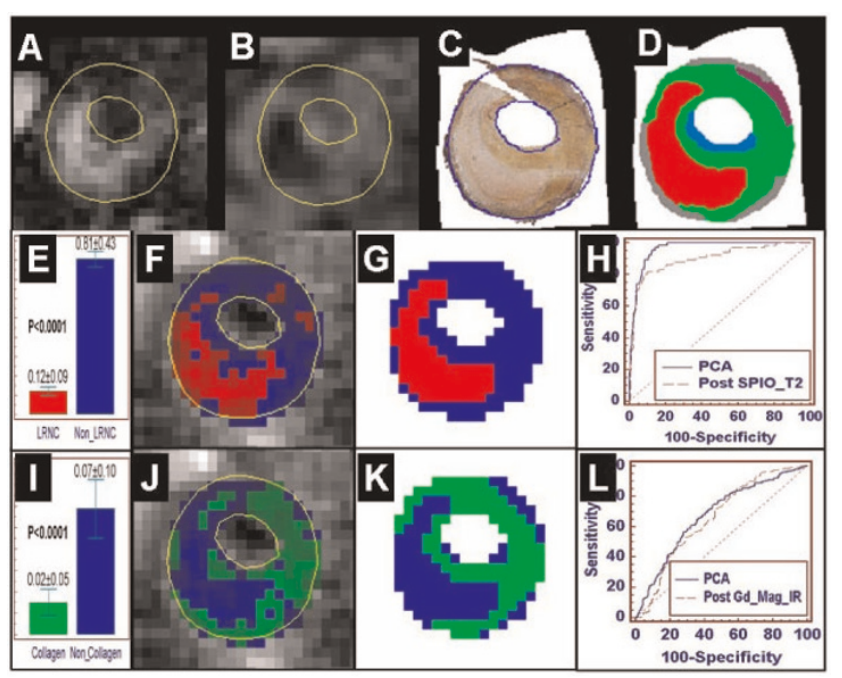

the manually identified mask (Panel F, G). ROC curve analysis showed that PCA (AUC: 0.96) was significantly better than the single best approach (T2 immediately post-SPIO; AUC: 0.90) ( $p=0.016)$ (Panel $\mathrm{H})$.

SI was significantly higher in collagen on the post-Gd DHE magnitude images (Panel B) $(36.56 \pm 8.97$ vs. $30.21 \pm 12.80$, $P<0.0001)$. PCA showed significant difference between collagen and non-collagen tissue $(p<0.000 I)$ (Panel I). The percentage of correctly identified pixels was $65.8 \%$. Predicted total area for collagen was 104 pixels $\left(32.54 \mathrm{~mm}^{2}\right)$, compared to 98 pixels $\left(30.67 \mathrm{~mm}^{2}\right)$ on the manually identified mask (Panel J, K). ROC curve analysis showed that PCA (AUC: 0.68 ) was similar to the single best approach (Post-Gd magnitude DHE; AUC: 0.66) ( $p=0.562$ ) (Panel L).

Conclusion: LRNC and collagen can be reliably identified in-vivo in human carotid atherosclerotic plaques using our novel, dual-contrast approach; a different pulse sequence with different exogenous contrast is optimal for the identification of different tissue components. A more sophisticated PCA analysis is significantly better than the evaluation of a single pulse sequence at a single timepoint for the identification of LRNC. Such sophisticated plaque analysis can now be applied to clinical outcomes studies and for the evaluation of the effects of pharmaceutical agents for the modification of atherosclerosis.

\section{6}

In vivo human coronary magnetic resonance angiography at $\mathbf{7}$ Tesla

Saskia GC van Elderen', Andrew G Webb', Maarten Versluis', Jos Westenberg', Joost Doornbos', Nadine B Smith', Albert de Roos and Matthias Stuber ${ }^{2}$ 'LUMC, Leiden, Netherlands

2John Hopkins University, Baltimore, MD, USA

Journal of Cardiovascular Magnetic Resonance 2009, I I(SuppI I):O46

Introduction: Coronary magnetic resonance angiography (MRA) is a promising technique for the non-invasive visualization 
Figure I (abstract 046)

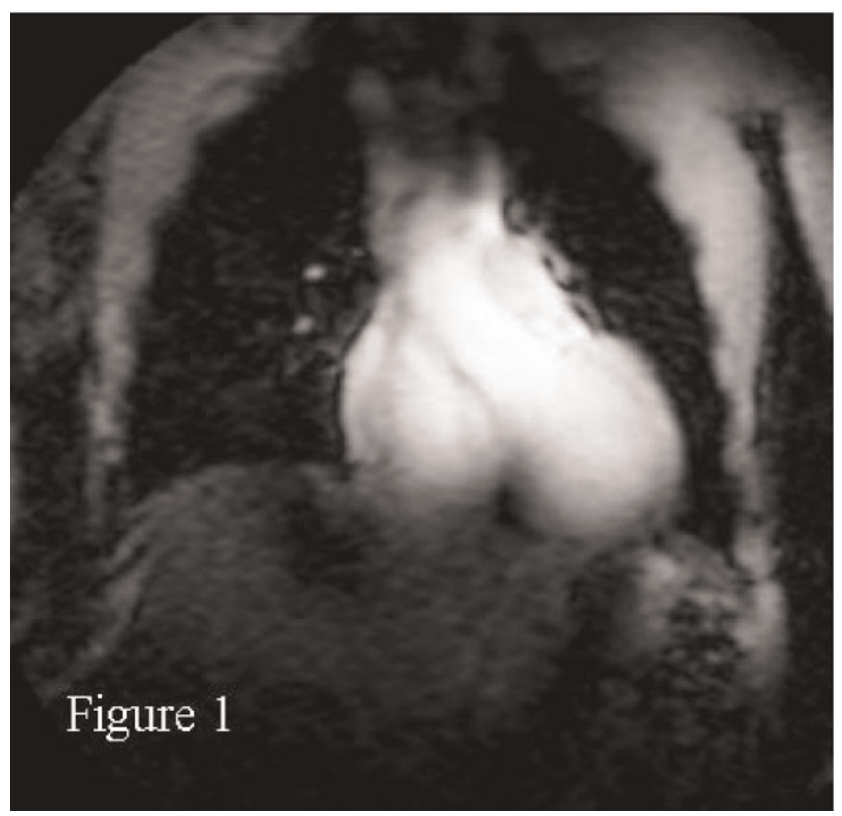

of the coronary anatomy. However, due to the small dimensions and tortuous nature of the coronary arteries, high spatial resolution and volumetric coverage are mandatory. This requirement is critically linked with prolonged scanning times. The use of a high magnetic field strength has several potential advantages since the higher signal-to-noise ratio (SNR) may support improved spatial resolution and/or shortened scanning times. For these reasons we tested the hypotheses that in vivo

Figure 2 (abstract 046)

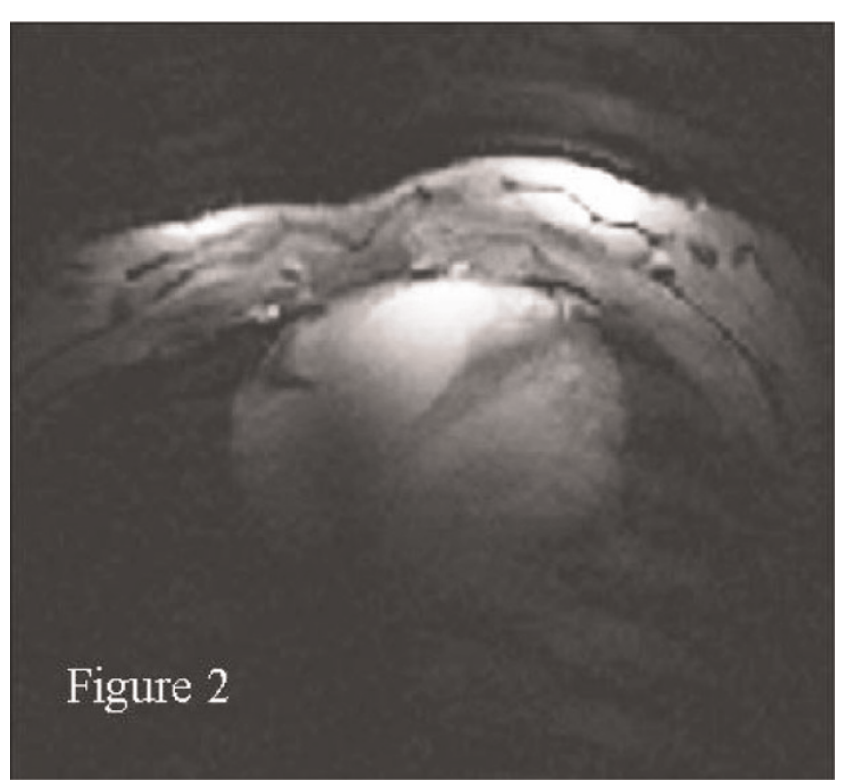

Figure 3 (abstract 046)

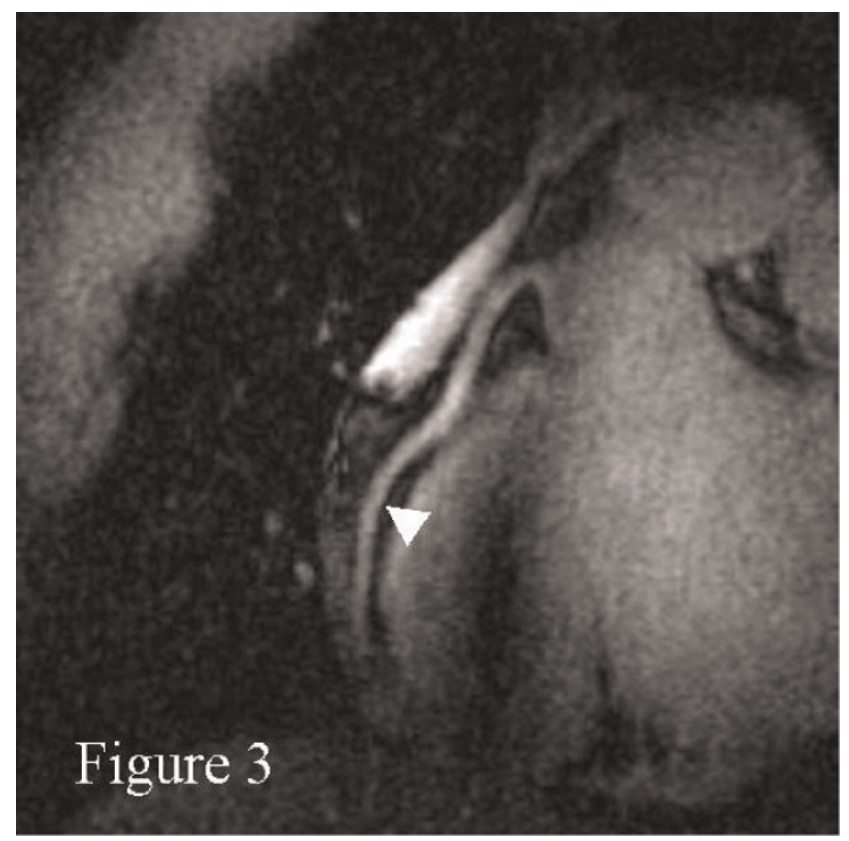

human coronary MRA technology is feasible and can be implemented on a commercial 7 Tesla $(T)$ system.

Methods: Eight healthy volunteers $(6 \mathrm{men}$, mean age 34 years \pm 8 ) were positioned in a $7 \mathrm{~T}$ MR system (Philips Healthcare, Best, NL). A I3-cm diameter anterior surface coil was designed and constructed and used in transmit/receive mode. The size was chosen to achieve adequate radio frequency (RF) penetration. Commercial vector ECG (VECG) technology was used for R-wave triggering. Volume selective RF power optimization and shimming were applied for each scan. A

\section{Figure 4 (abstract 046)}

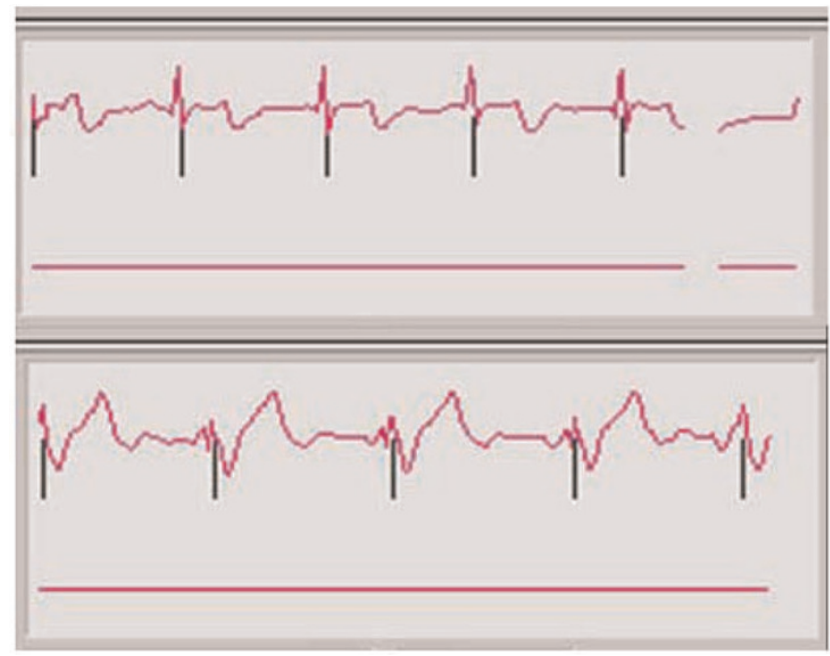

(a) ECG outside the magnet; (b) ECG inside the magnet. 
Figure 5 (abstract 046)

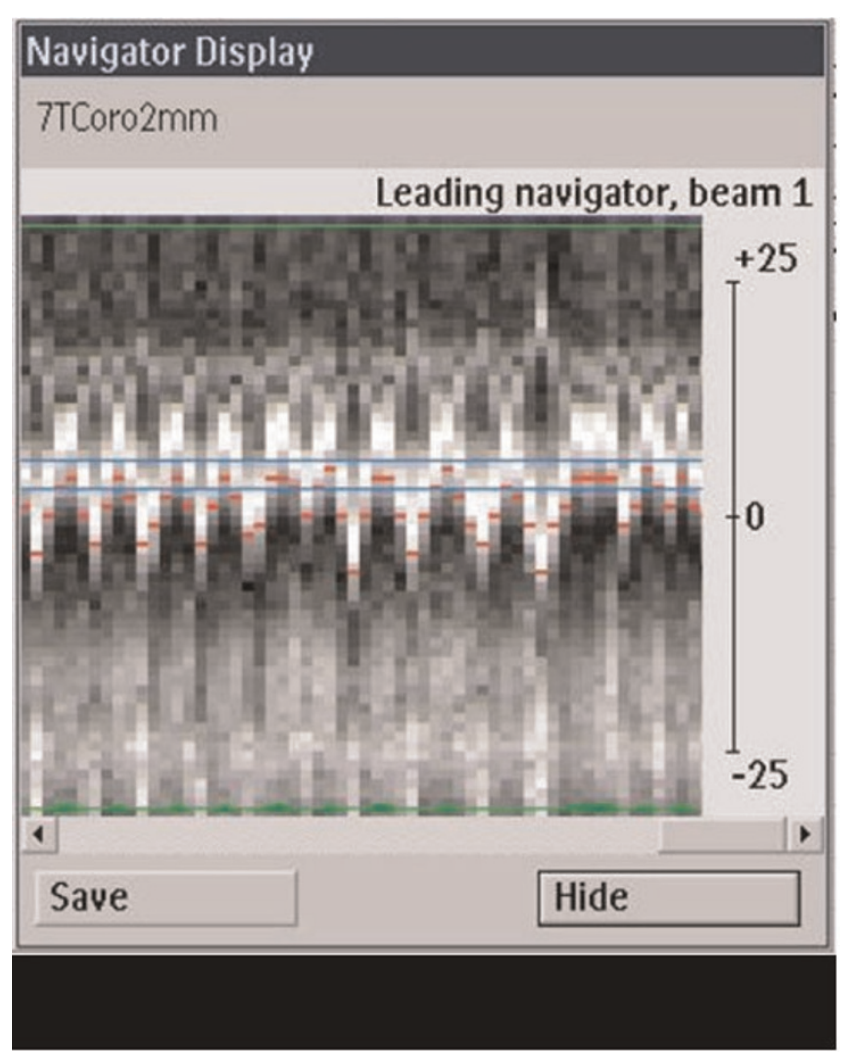

segmented k-space gradient echo sequence was used for scout scanning. Multi-slice cine scans were used for coronary artery localization and for the visual identification of the time period (Td) of minimal coronary motion. Scan plane localization parallel to the right coronary artery (RCA) was facilitated using a threepoint planscan tool. Double-oblique free-breathing 3D coronary MRA (segmented k-space gradient-echo imaging, TR $=4 \mathrm{~ms}$, $\mathrm{TE}=1.5 \mathrm{~ms}$, RF excitation angle $=15^{\circ}$, field-of-view $=320 \times$ $291 \mathrm{~mm}^{2}$, scan matrix $=392 \times 373,15$ slices, slice thickness $=$ $2 \mathrm{~mm}$, acquisition window $\sim 100 \mathrm{~ms}$, scan time $\sim 5 \mathrm{~min}$ ) was performed using prospective navigator gating with the $2 \mathrm{D}$ selective navigator localized at the heart-lung interface. Image data were collected in mid-diastole at the predetermined Td. An adiabatic spectrally selective inversion recovery pre-pulse ( $\mathrm{Tl}=200 \mathrm{~ms}$ ) was used for fat suppression and enhanced contrast between the coronary blood-pool and epicardial fat. Coronary MRAs were reformatted and length measurements were performed using the "Soapbubble" software tool.

Results: Right coronary MRAs were successfully obtained in all 8 healthy adult human subjects. Figures $I$ and 2 show scout images in the coronal and axial plane, respectively, illustrating that the RF penetration and signal uniformity is sufficient for RCA imaging. Figure 3 shows a long contiguous segment of the RCA with high contrast between the blood-pool and the epicardial fat. The average measured contiguous length of the RCA was $77 \pm 35 \mathrm{~mm}$. One potential concern is that, at the higher field strength, the magneto-hydrodynamic effect is amplified with an artificial augmentation of the T-wave of the
ECG (Figure 4a and 4b). Nevertheless, the VECG algorithm allowed reliable R-wave triggering. In Figure 5 the navigator signal from the heart-lung interface received by the surface coil can be seen.

Discussion: To our knowledge, this is the first report of human coronary MRA at $7 \mathrm{~T}$. With suitable adaptations of the scanning protocol (e.g. navigator localization and use of a spectrally selective adiabatic inversion recovery for fat saturation) and the use of a custom-built transmit-receive surface coil, coronary MRA technology has been successfully implemented at $7 \mathrm{~T}$ and long contiguous segments of the RCA can be obtained in vivo and in humans. Conventional T2-weighted preparation cannot currently be used for contrast generation because of conservative specific absorption rate (SAR) constraints. Future work will focus on optimizing contrast enhancement between the bloodpool and the myocardium within the SAR and BI homogeneity constraints. In order to improve volumetric coverage, the development of larger surface coils or coil arrays will be required. The latter will be most important in the trade-off between the expected SNR benefit vs. shorter scanning times. In conclusion, navigator gated free-breathing 3D coronary MRA has successfully been implemented in vivo and in humans on a commercial $7 \mathrm{~T}$ system.

\section{7}

Whole-heart contrast-enhanced coronary magnetic resonance angiography in less than 5 minutes using gradient echo interleaved EPI Himanshu Bhat ', Sven Zuehlsdorff', Xiaoming $\mathrm{Bi}^{2}$, Xin Liu', Renate Jerecic ${ }^{2}$ and Debiao $\mathrm{Li}^{\prime}$

${ }^{I}$ Northwestern University, Chicago, IL, USA

${ }^{2}$ Siemens Medical Solutions, Chicago, IL, USA

Journal of Cardiovascular Magnetic Resonance 2009, I I(SuppI I):O47

Introduction: Whole-heart coronary MRA is challenging due to the relatively long data acquisition time on the order of 10-15 minutes [I]. Interleaved EPI [2] is a method which can be exploited to provide significant speed gain for whole-heart coronary MRA and has previously been reported for volumetargeted imaging at I.5 T [3, 4]. The purpose of this work was to optimize an interleaved EPI acquisition scheme for reducing the imaging time of whole-heart contrast-enhanced coronary MRA.

Methods: Sequence design considerations: A schematic of the EPI-FLASH sequence is shown in Fig. Ia. Segmentation and interleaving were applied along the phase-encoding direction, eliminating discrete signal variations in the partition-encoding direction. The reordering scheme used in the phase-encoding direction is shown in Fig. Ib. The asymmetric k-space was divided into 6 regions corresponding to the echo train length, using the second region as central k-space region. The interleaved acquisition within a heartbeat initially samples the lower portion of each region (dashed line) and subsequently sequentially acquires the central k-space line and top of each region (dotted line). The signal varies within each heartbeat due to non steady-state conditions. In combination with the described reordering scheme, this results in amplitude modulations in k-space, leading to image ghosts [2]. These modulations were minimized by appropriate selection of the inversion time 
Figure I (abstract 047)

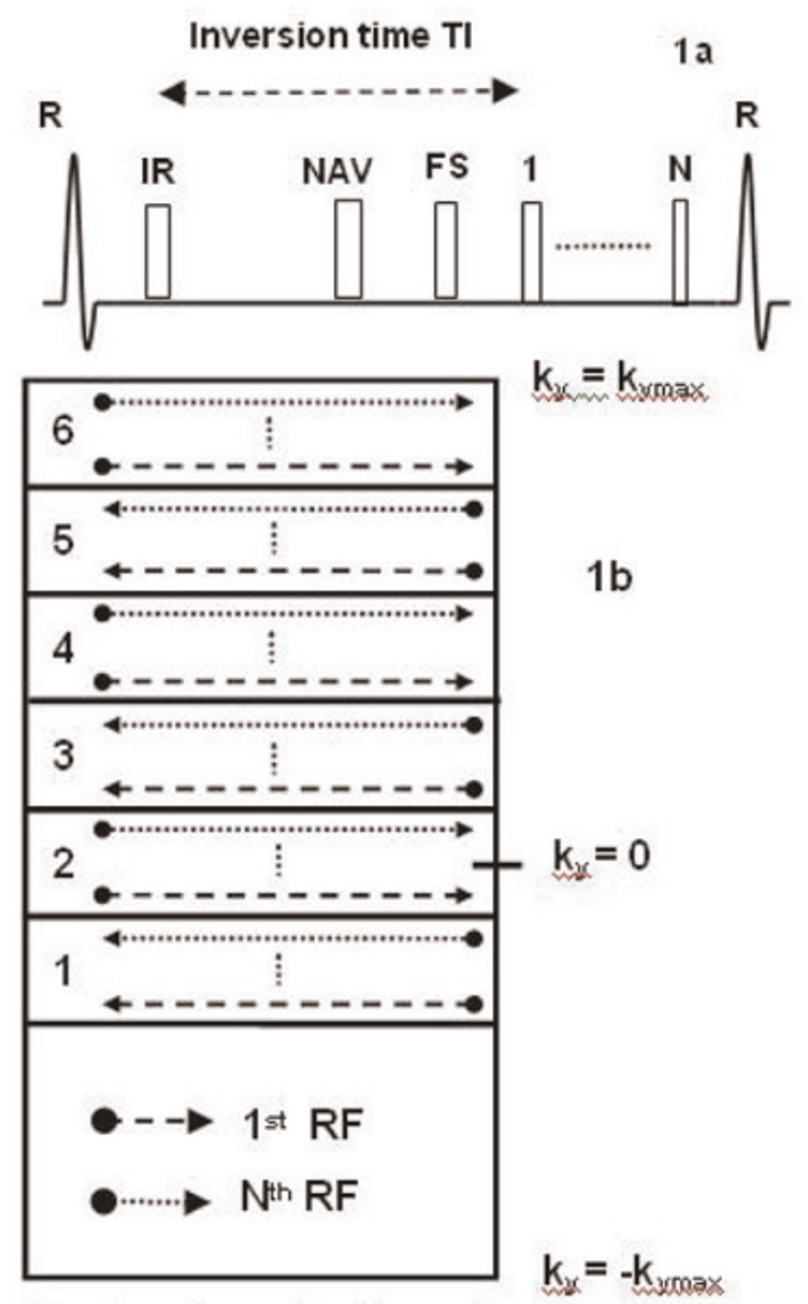

(a) schematic of the pulse sequence; (b) reordering scheme.

and flip angle, using simulations of the Bloch equations and phantom studies.

Volunteer imaging: 7 volunteers were scanned on a $1.5 \mathrm{~T}$ Espree scanner (Siemens Medical Solutions). Scan parameters were: $T R=11.3, T E=4.27$, flip angle $=25,66$ lines per heartbeat in a window of $124 \mathrm{~ms}$, acquired k-space lines $=132$, readout bandwidth $=977 \mathrm{~Hz} /$ pixel, $\mathrm{TI}=300 \mathrm{~ms}$, matrix: $256 \times 189 \times 60$, interpolated voxel size: $0.5 \times 0.55 \times 1 \mathrm{~mm}^{3}$. $0.2 \mathrm{mmol} / \mathrm{kg}$ body weight of Gd-DTPA was injected at $0.5 \mathrm{cc} / \mathrm{sec}$ [5]. The total imaging time for the whole-heart scan was
Figure 2 (abstract 047)

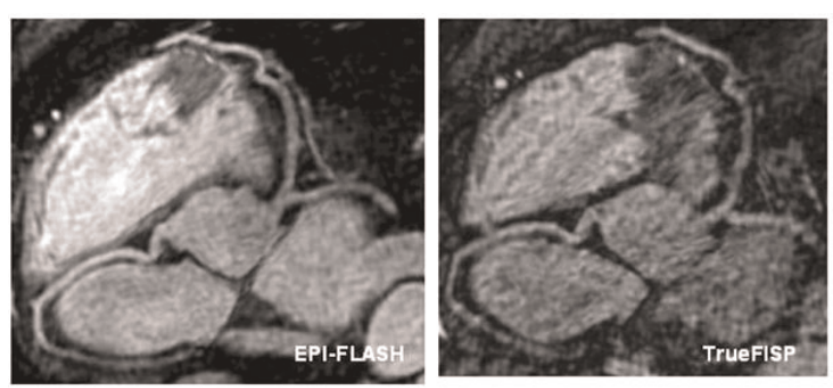

Coronary artery images using the EPI-FLASH and TrueFISP sequences with identical imaging times.

Figure 3 (abstract 047)
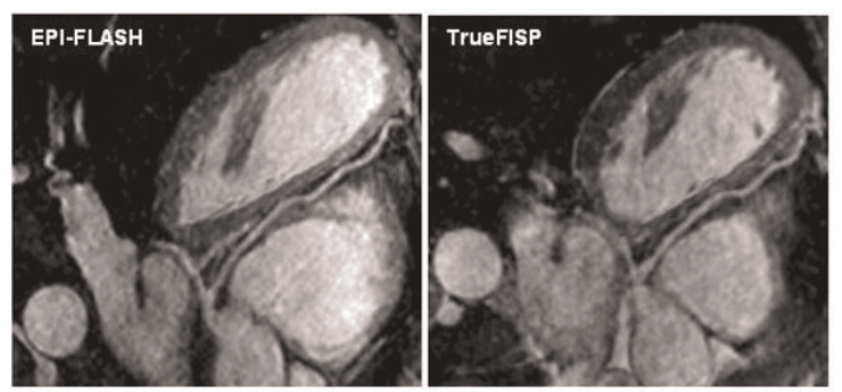

Coronary artery images using the EPI-FLASH sequence and a TrueFISP sequence with longer imaging time.

2 minutes (for a heart-rate of 60 without navigator gating). For comparison, a 3D TrueFISP whole-heart scan was acquired using matched data acquisition time and spatial resolution. The image quality scores (I, poor; 2, fair; 3, good; 4, excellent) and lengths of the coronary arteries visualized by the 2 techniques were compared. In addition, for a qualitative comparison, a wholeheart TrueFISP protocol [5] with longer scan time, representing the state of the art for coronary MRA at I.5 Twas performed on 2 of the volunteers.

Results: The average imaging time for contrast-enhanced whole-heart imaging was $4.7 \pm 0.7$ minutes with an average navigator efficiency of $44.7 \pm 6.2 \%$. Fig. 2 shows coronary artery images from 2 volunteers using the EPI-FLASH acquisition and the TrueFISP acquisition with identical imaging time. The EPIFLASH acquisition shows excellent depiction of all the coronary arteries. In comparison, the TrueFISP acquisition is very noise due to the high acceleration factor used. Quantitative compar-

Table I (abstract 047) Comparison between EPI-FLASH and TrueFISP with identical resolution and imaging time

\begin{tabular}{llllll}
\hline Sequence & Imaging time & Navigator efficiency & Image quality score & RCA length & LAD length \\
\hline EPI-FLASH & $4.7 \pm 0.7$ & $44.7 \pm 6.2$ & $3 \pm 0.3$ & $10.8 \pm 2.1$ & $11.5 \pm 3.2$ \\
TrueFISP & $4.9 \pm 0.9$ & $45.5 \pm 7.9$ & $2.2 \pm 0.3$ & $9.1 \pm 1.72$ & $11.0 \pm 4.1$ \\
P value $(n=7)$ & 0.5 & 0.8 & $\mathbf{0 . 0 0 I *}$ & $\mathbf{0 . 0 I *}$ & 0.4 \\
\hline
\end{tabular}


ison between the two sequences is shown in Table I. Fig. 3 shows coronary artery images from a volunteer using the EPI-FLASH acquisition (imaging time $=5.6$ minutes, navigator efficiency $=45 \%$ ) and a TrueFISP acquisition (imaging time $=$ I 3.2 minutes, navigator efficiency $=38 \%$ ). Both the sequences show similar depiction of the coronary arteries, but the imaging time for the EPI-FLASH technique is reduced by more than a factor of 2 .

Conclusion: An EPI-FLASH sequence was optimized for contrast-enhanced whole-heart coronary MRA at I.5 T. In volunteers, all the major coronary arteries were clearly depicted in a scan time under 5 minutes. Clinical utility of the technique needs to be tested on a patient population.

\section{References}

I. Magn Reson Med. 50:1223-1228.

2. Magn Reson Med. 30:609-616.

3. JMRI I3:676-68I.

4. JMRI I 0:82I-825.

5. Magn Reson Med. 58:1-7.

\section{8}

Right ventricular ejection fraction, measured during inter-stage cardiac magnetic resonance imaging, predicts outcome for patients with hypoplastic left heart syndrome

Marina L Hughes', Katherine Brown', Vivek Muthurangu ${ }^{2}$, Victor Tsang and Andrew Taylor ${ }^{2}$

'Great Ormond Street Hospital for Children, London, UK

${ }^{2}$ Institute of Child Health and Great Ormond Street Hospital for Children, London, UK

Journal of Cardiovascular Magnetic Resonance 2009, I I(SuppI I):O48

Background: Since 2003 our unit has adopted an imaging protocol for all infants with hypoplastic left heart syndrome (HLHS), which includes CMR imaging for inter-stage assessment prior to the formation of a bidirectional cavo-pulmonary shunt. The aim of this study was to assess whether the CMR data acquired during this protocolised follow-up could help to stratify the risk for these patients.

Methods: We assessed all locally followed patients, who had undergone the Norwood procedure for HLHS between January 2003 and May 2008, and who had undergone CMR imaging according to unit protocol.

Imaging was performed under general anaesthetic, using a $1.5 \mathrm{~T}$ $M R$ scanner, and a combination of cine sequences, phase contrast flow sequences and gadolinium-enhanced MR angiography. From short axis cine images, manual segmentation of the ventricles was completed, giving ventricular volumes, ejection fraction and cardiac output (CO). Arterial measurements were made from the isotropic angiographic data using 3D analysis software. The pulmonary artery (PA) measurements were made at the proximal native vessel and distally, just prior to the Ist lobar branch. Aortic measurements were made at the narrowest point of the proximal descending aorta (CoA) and at the diaphragmatic-level descending aorta. At each site the shortest and orthogonal cross-sectional diameters were averaged, to correspond with conventional 2D measurement methods. Additionally, the exact cross-sectional area of the vessel at each point was measured using manual planimetry. The
Figure I (abstract 048)

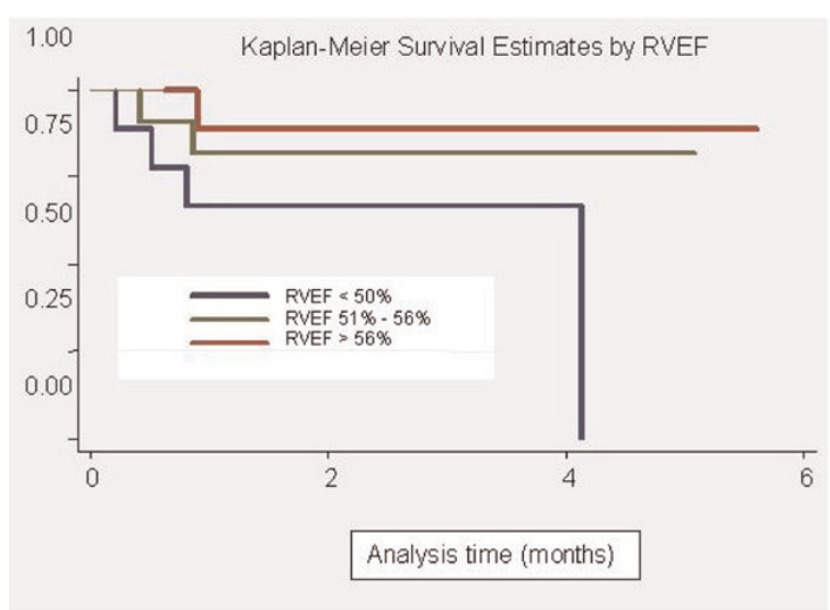

Kaplan-Meier survival estimates by RVEF.

coarctation (CoA) index was defined as the (CoA measurement/diaphragmatic aorta measurement), for vessel diameter and planimetered area respectively.

The primary outcome measure was survival to analysis date $\left(I^{\text {st }}\right.$ October 2008). Secondary, functional outcome measures were $\mathrm{RV}$ ejection fraction (RVEF), and CO.

Results: A total of 30 patients comprised the cohort of survivors of the first stage Norwood procedure, undergoing protocolised CMR. Of these 30 patients, 15 had a Sano-type, and I 5 a conventional Norwood. The median age and weight at CMR scan was 9I (33-29I) days, and $5(3.2-$ II) kg respectively. The planimetered area index of vessels was smaller than averaged orthogonal diameter index. The mean ratio of the CoA diameter index to the CoA area index was $1.4(95 \% \mathrm{Cl}$ I.25 - I.55), indicating that diameter measurements may underestimate true narrowing. The PA size and indices of proximal stenosis did not correlate with outcome. The median CoA area index for all patients was $0.52(0.22-1.0)$. Twenty-one $(70 \%)$ patients had a CoA area index $<0.7$, with no difference between the Sano-type and conventional Norwood surgery. There was a significant correlation between the CoA diameter index and cardiac output $-1.09(95 \% \mathrm{Cl}-2.17--0.02)(p=0.04)$, but the CoA size indices were not correlated with RVEF. The median RVEF for this cohort was $53 \%(30-81 \%)$. Eight patients had RVEF $<50 \%$. There were 7 deaths in this cohort, during a total follow-up time of 67 person years. The RVEF was strongly predictive of death, with a hazard ratio $0.92(95 \% \mathrm{Cl} 0.86-0.99)(p=0.02)$. Figure I demonstrates a Kaplan Meier survival curve with patients stratified by RVEF. Other factors, such as the age at time of MRI, the type of Norwood, the CO, the CoA and the PA indices did not predict death.

Conclusion: This study shows that death is a more likely outcome in HLHS patients with a lower RV ejection fraction at inter-stage CMR. Other CMR-measured factors such as CoA and PA size indices did not predict outcome. Measures to preserve RV systolic function, and CMR assessment of this, should be paramount in the complex management of these patients. 
049

Teleradiologic network for central management and analysis of MR images: a three-years experience of the competence network for congenital heart disease

Samir Sarikouch', Philipp Beerbaum², Stefan Mueller ${ }^{3}$, Ulrich $\mathrm{Sax}^{3}$ and Titus Kuehne ${ }^{4}$

'Department for Heart-, Thoracic-, Transplantation- and Vascular Surgery, Hannover Medical School, Germany

${ }^{2}$ Division of Imaging Sciences, King's College,

Guy's \& St Thomas' Hospital, London, UK

${ }^{3}$ Department for Medical Informatics, University of Goettingen, Goettingen, Germany

${ }^{4}$ Unit of Cardiovascular Imaging - Congenital Heart Diseases, German Heart Center, Berlin, Germany

Journal of Cardiovascular Magnetic Resonance 2009, I I(SuppI I):O49

Background: Analysis of cardiovascular MRI requires increasingly high standards and produces costs for specially trained personal and technical equipment. At the same time clinical decision making relies, particulary in congenital heart diseases (CHD), more and more on MRI derived data. In the German Competence Network for CHD, a science-association was founded in which MRI data were acquired in peripheral institutes and than send by teleradiologic means to a core-labaratory for central data analysis and archiving. The first three years of operation of this network were evaluated regarding its technical course of activity, accuracy of MRI data analysis and costs.

Methods: 16 cardiovascular institutes form part of the network. MR images are acquired in these institutes (Philips, Siemens, GE scanners) using a standardized protocol. Images are send by teleradiologic means (pseudonomyzed via internet) to a central core-laboratory. There, images are analysed for parameters of cardiac function using custom-made software. Variability of the measured parameters is continuously determined (quality assessment). Thereafter, analysed data and original $M R$ images are archived and are accessible to the participating institutes by a remote-data-entry-system (RDE). Economic evaluation of the network was done by cost-analysis and ex post-contemplation under consideration of investments, laborand consumption-costs.

Results: Data transmission speed was approximately $300 \mathrm{kbit} / \mathrm{s}$ upstream. MR images arrive at the core-laboratory as pseudonymized DICOM-data. After the pilot phase, no errors in image transmission (e.g. incomplete data sets) were observed. Therefore, all MRI scans that were acquired using standardized protocols could be analysed in the core-labaratory, so far more than 1000 scans. Interobserver variability of quantitative function parameters was significantly lower when analysis was done in the core-labaratory, compared to individual analysis in the peripheral institutes. MR images were ubiquitously accessible in the RDEsystem and via the internet at any time. Ex-post evaluation showed an expenditure of I55 EUR per MRI data set when 552 sets per year are enrolled under scientific (non economic) conditions to the core-labaratory.

Conclusion: A teleradiologic network was successfully established in a science-association for congenital heart disease and optimized for primary health care and scientific purposes. The first three-year experiences showed that this network/core-lab is attractive by reducing labor-, investment- and consumption costs and at the same time improves quality of data. Finally, the data archives of the core-labs are a very large and invaluable source of data for current and future scientific studies.

\section{0}

Location, patterns, and quantification of myocardial fibrosis identified by cardiac magnetic resonance delayed enhancement late after fontan operation

Rahul H Rathod, Ashwin Prakash, Andrew J Powell and Tal Geva

Children's Hospital Boston, Boston, MA, USA

Journal of Cardiovascular Magnetic Resonance 2009, I I(SuppI I):O50

Objective: The objective of this study was to investigate the frequency, location, patterns, and quantification of myocardial fibrosis as identified by the CMR myocardial delayed enhancement (MDE) technique and describe its association with functional single ventricular ejection fraction (EF) and regional wall motion abnormalities (WMA) in patients late after the Fontan operation.

Background: MDE has been associated with adverse ventricular mechanics late after tetralogy of Fallot repair and in patients with systemic right ventricles. No studies have reported the frequency, patterns, or associations of MDE in patients late after the Fontan operation.

Methods: All patients at our center following a Fontan operation who had a CMR study with MDE from January 2002 to July 2008 were retrospectively identified. MDE was characterized by: I) spatial location; 2) pattern; and 3) MDE quantification expressed as the MDE percent of ventricular mass (MDE \%). MDE \% was calculated using the histologically verified, full-width at half-maximum (FWHM) technique (Amado et al. JACC 2004; 44: 2383). Patterns of MDE were categorized as transmural, subepicardial/intramural, subendocardial, circumferential endocardial fibroelastosis (EFE), and speckled (Figure I). Multivariate linear regression analysis with forward stepwise selection was used to investigate independent associations of functional single ventricular EF. Covariables included demographic data, cardiac diagnosis, ventricular morphology, Fontan type, surgical history, MDE locations, MDE patterns, and MDE \%.

Results: Of the 85 subjects included $(65 \%$ male; median age at Fontan 4.5 [2.0, I I.2] years; mean age at CMR 23.I \pm I I.2 years), 2 I (25\%) had positive MDE in the ventricular myocardium. MDE was seen in the following locations: dominant ventricle free wall $(n=13$, $62 \%)$, secondary ventricle free wall $(n=9,43 \%)$, septal insertion ( $n=5,24 \%)$, ventricular septum $(n=3,14 \%)$, apex $(n=2,10 \%)$, previous surgical sites $(n=2,10 \%)$, and papillary muscle $(n=2$, $10 \%$ ). MDE was seen in the following patterns: transmural lesion

Figure I (abstract 050)

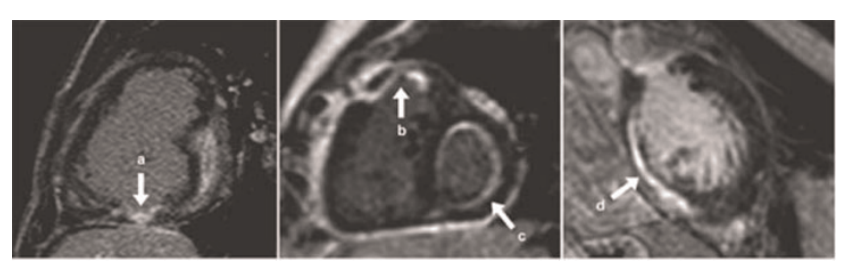

Arrows identify patterns of MDE. a) transmural; b) subendocardial; c) EFE; d) subepicardial/intramural. 
Table I (abstract O50) Univariate analysis of MDE location and pattern

\begin{tabular}{|c|c|c|c|c|c|c|}
\hline & \multicolumn{3}{|c|}{ MDE location in the dominant ventricle free wall } & \multicolumn{3}{|c|}{ Transmural MDE pattern } \\
\hline & MDE Pos $(n=13)$ & MDE Neg $(n=72)$ & p value & MDE Pos $(n=9)$ & MDE Neg $(n=76)$ & p value \\
\hline EF (\%) & $41 \pm 13$ & $56 \pm 11$ & $0.0001^{1}$ & $39 \pm 11$ & $55 \pm 12$ & $0.0001^{1}$ \\
\hline $\operatorname{EDVi}\left(\mathrm{ml} / \mathrm{m}^{2}\right)$ & $112[93,196]$ & $84[65,101]$ & $0.004^{2}$ & $144[93,196]$ & $83[65,101]$ & $0.006^{2}$ \\
\hline $\operatorname{ESVi}\left(\mathrm{ml} / \mathrm{m}^{2}\right)$ & $70[50,129]$ & $35[26,46]$ & $0.0001^{2}$ & $77[50,132]$ & $35[27,48]$ & $0.001^{2}$ \\
\hline MASSi $\left(g / \mathrm{m}^{2}\right)$ & $72[59,93]$ & $47[40,59]$ & $0.001^{2}$ & $63[49,80]$ & $49[40,64]$ & $0.05^{2}$ \\
\hline Regional WMA & $10(77 \%)$ & $18(25 \%)$ & $0.001^{3}$ & $9(100 \%)$ & $19(25 \%)$ & $0.0001^{3}$ \\
\hline Dyskinesis & $6(46 \%)$ & $3(4 \%)$ & $0.0001^{4}$ & $6(67 \%)$ & $3(4 \%)$ & $0.0001^{4}$ \\
\hline
\end{tabular}

Values are mean $\pm \mathrm{SD}$, median [25\%,75\%], or $\mathrm{n}(\%)$; 'Student $t$ test, ${ }^{2}$ Mann-Whitney $\mathrm{U}$ test, ${ }^{3}$ Fisher exact test, ${ }^{4}$ Chi-squared test of independence.

( $n=9,43 \%)$, subepicardial/intramural $(n=5,24 \%)$, subendocardial ( $n=5,24 \%)$, circumferential endocardial fibroelastosis $(n=4,19 \%)$, and speckled $(n=2,10 \%)$. Results of univariate analysis comparing patients with and without MDE, stratified by location and pattern are summarized in Table I. Multivariate linear regression analysis demonstrated that higher MDE \% (slope: $-1.7, \mathrm{Cl}:-2.5$ to -1.0 , $\mathrm{p}<0.000 \mathrm{I}$ ) and age at CMR (slope: $-0.6, \mathrm{Cl}:-\mathrm{I} .0$ to $-0.3, \mathrm{p}=0.002$ ) were independently and inversely associated with $E F\left(R^{2}=0.59\right)$.

Conclusion: In patients late after the Fontan operation, myocardial fibrosis was common and was associated with lower EF, higher ventricular volumes, WMA, and dyskinesis. Further studies are warranted to examine the mechanisms of myocardial fibrosis and their impact on ventricular performance.

\section{O5 I}

Left ventricular T2 distribution in Duchenne Muscular Dystrophy Janaka Wansapura', Robert Fleck', Kan N Hor', Wojciech Mazur', Woodrow Benson' and William M Gottliebson'

'Cincinnati Children's Hospital, Cincinnati, OH, USA

${ }^{2}$ Christ Hospital, Cincinnati, OH, USA

Journal of Cardiovascular Magnetic Resonance 2009, I I (SuppI I):O5 I

Background: The transverse relaxation time (T2) of water molecules differ between tissues. Because the molecular motion of water is significantly affected by macro molecules, tissue containing fibrous polymers such as collagen has shorter T2 values. Patients with Duchenne Muscular Dystrophy (DMD) develop myocardial fibrosis in the late stage of the disease. The goal of this study was to determine the association of myocardial T2 distribution to the severity of DMD.

Methods: Twenty six DMD patients and eight normal subjects were studied. T2 maps of the left ventricle were generated using a black blood dual spin echo method. ( $\mathrm{TE}_{1}=6 \mathrm{~ms}, \mathrm{TE}_{2}=34 \mathrm{~ms}$ ) DMD patients were grouped according to the ejection fraction (EF) and the circumferential strain $\left(\varepsilon_{\mathrm{cc}}\right)$ as: $\mathrm{A}: \mathrm{EF} \geq 56 \%, \varepsilon_{\mathrm{cc}} \geq$ $12 \%$, mean age $=10.5$ yrs, B: EF $\geq 56 \%, \varepsilon_{\mathrm{cc}} \leq 12 \%$, mean age $=17.5 \mathrm{yrs}, \mathrm{C}: \mathrm{EF} \leq 56 \%$, mean age $=17.5 \mathrm{yrs}$. T2 values were plotted for each subject as a histogram. The normalized mean histograms from each group were compared by the Full Width of Half Maximum (FWHM).

Results: The FWHM of the T2 histogram was significantly higher in group $B$ and $C$ compared to that of group $A$ and the normal group, indicating significantly high heterogeneity in $\mathrm{T} 2$ in Group B and C. FWHM/T2mean is significantly $(p<.000 \mathrm{I})$
Figure I (abstract 05I)

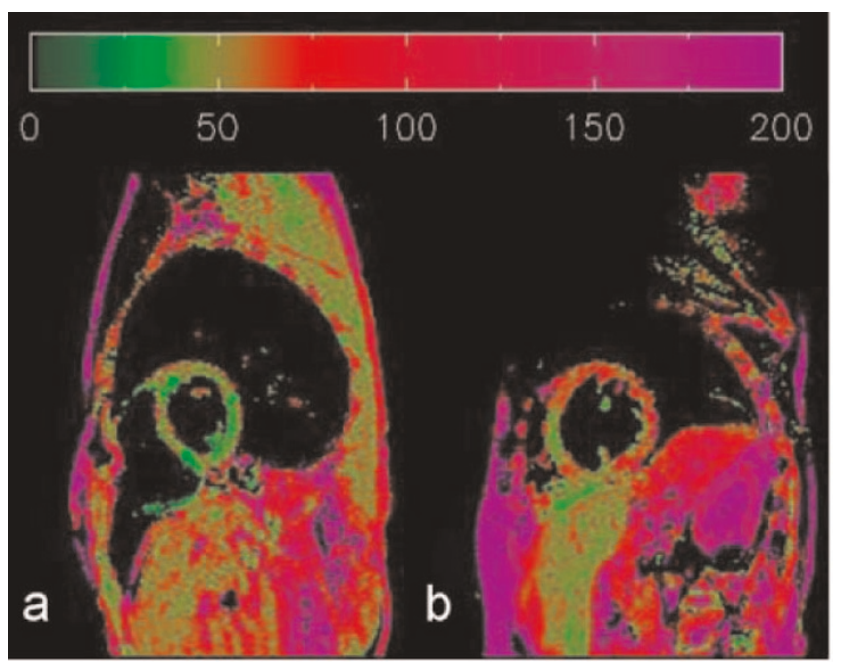

The T2 maps of a normal (a) and a DMD subject (b) showing the left ventricle in the short axis view.

Figure 2 (abstract $05 \mathrm{I}$ )

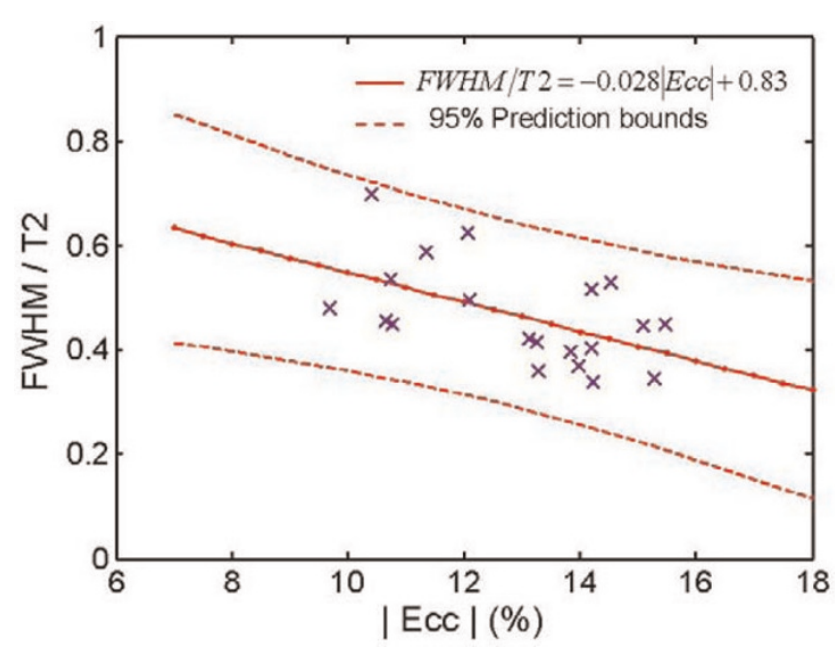

Association between FWHM/T2mean and $\varepsilon_{\mathrm{cc}}$ in group $\mathrm{A}$ and $\mathrm{B}$. Pearson correlation coefficient $r=.5 \mathrm{l}$. 
higher in group B $(0.42 \pm 0.06)$ compared to Group A $(0.54 \pm 0.08)$. Regression analysis show moderate association between FWHM/T2mean and $|\mathrm{Ecc}|$ in group $\mathrm{A}$ and $\mathrm{B}$. (Pearson correlation coefficient $r=.5 \mathrm{I}$ ).

Conclusion: The distribution of $\mathrm{T} 2$ in the LV of the DMD subjects were remarkably heterogeneous compared to that of normal subjects. These characteristics may suggest early signs of diffused collagen accumulation in the LV in DMD.

\section{2}

4D flow for accurate assessment of differential pulmonary arterial flow in patients with tetralogy of Fallot

Alison K Meadows, Michael D Hope, David Saloner and Charles B Higgins

University of California San Francisco, San Francisco, CA, USA

Journal of Cardiovascular Magnetic Resonance 2009, I I (SuppI I):O52

Objective: To demonstrate that 4D flow, in a single acquisition, can provide efficient, accurate, and complete data sets to determine differential pulmonary blood flow in patients with Tetralogy of Fallot (TOF).

Background: Patients with tetralogy of Fallot (TOF) often have branch pulmonary artery (PA) stenoses and abnormalities
Figure 2 (abstract 052)

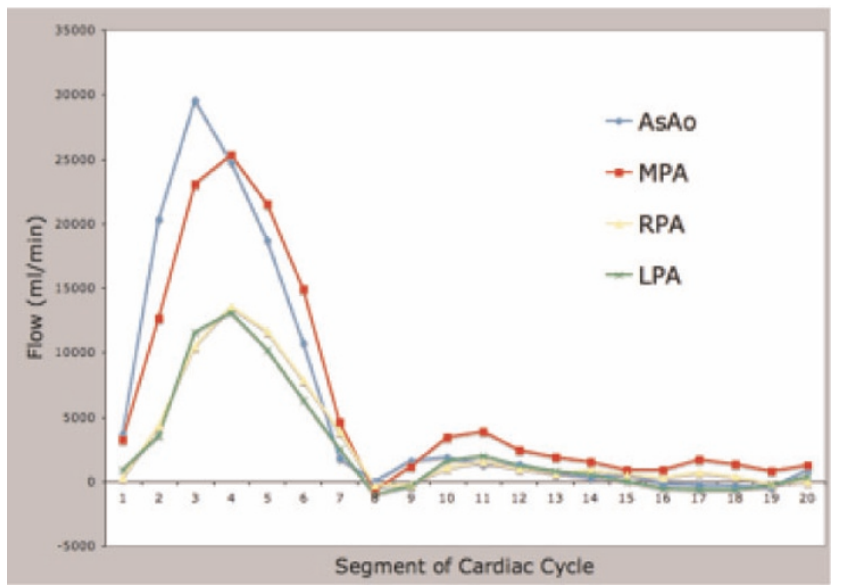

Healthy subject pulmonary flow.

in flow distribution. Such abnormalities can be addressed and have an impact on clinical management. Distorted anatomy, turbulent flow jets, and/or metallic stents often make the choice

\section{Figure I (abstract 052)}

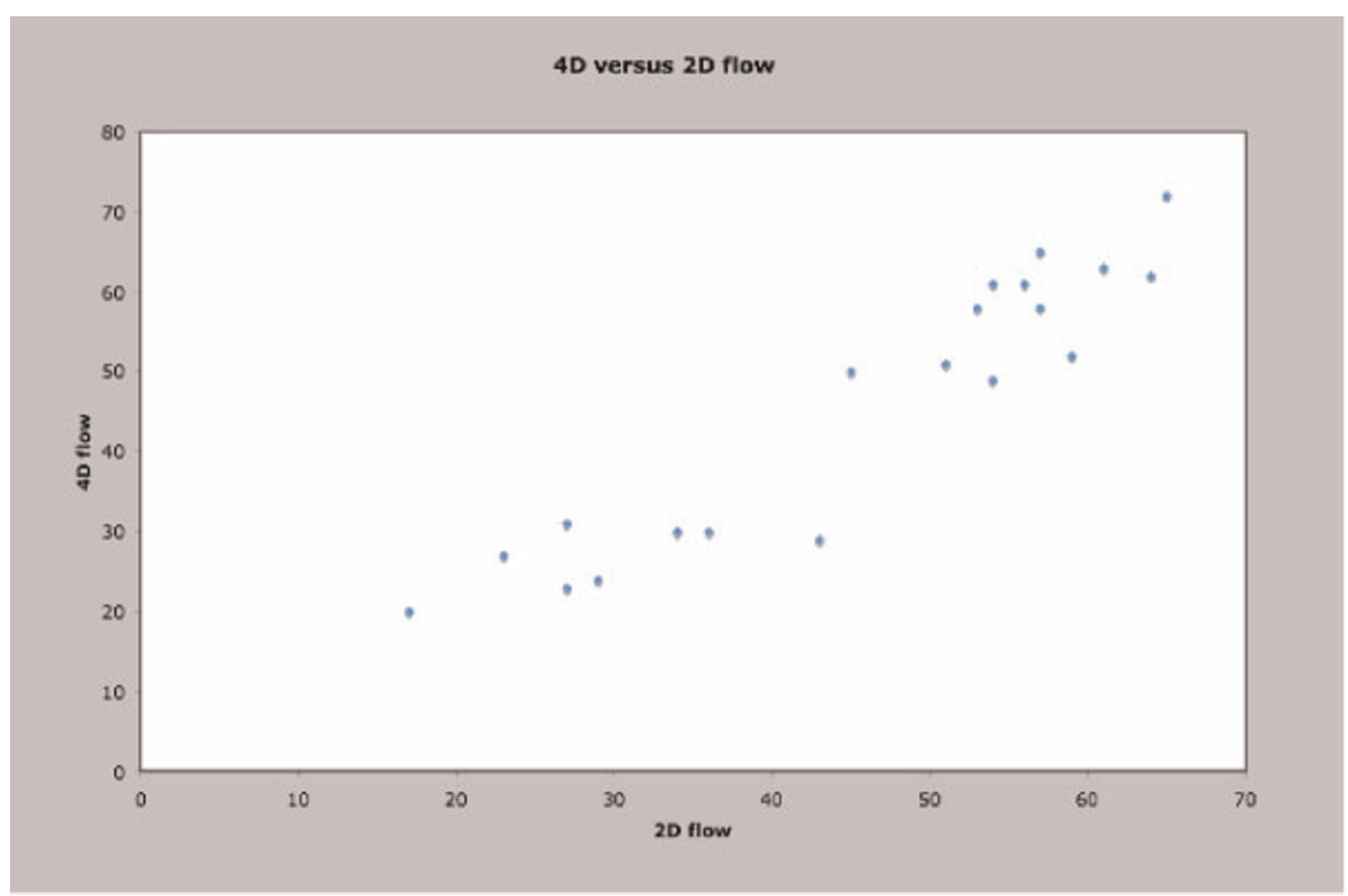

$4 D$ versus $2 D$ flow. 
Figure 3 (abstract 052)

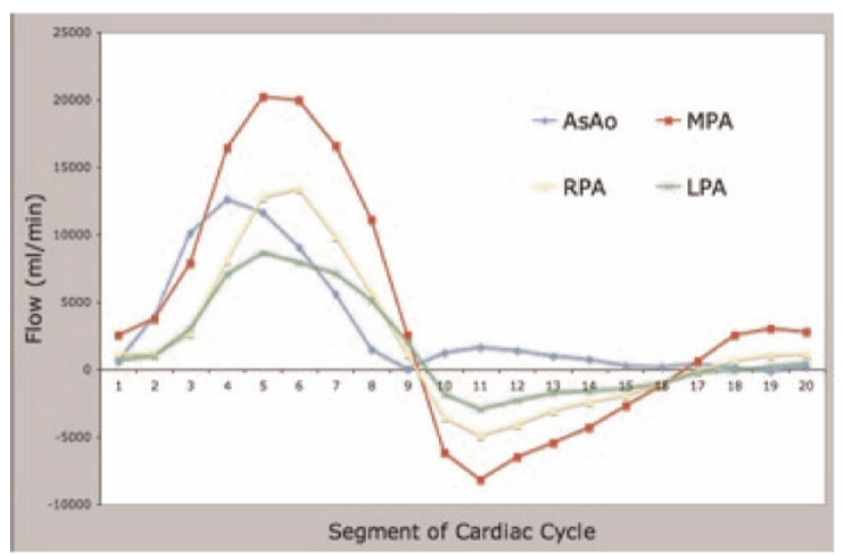

Flow profile.

of $2 \mathrm{D}$ phase contrast flow planes difficult, time consuming, and labor intensive. As a result, studies may be completed without collection of the data necessary to determine flow distribution. 4D flow techniques permit the collection of temporally-resolved 3D data sets of the central systemic and pulmonary vasculature in a single acquisition. Complete data acquisition is guaranteed and appropriate planes for flow quantification can be chosen during post processing.

Methods: Employed was a temporally-resolved, 3D phase contrast technique (4D flow), optimized for blood flow analysis in the thoracic vasculature. Data was acquired using an RFspoiled 3D gradient echo pulse sequence with velocity encoding in 3 spatial directions. All measurements were performed on a I.5 T clinical scanner (Signa CV/I, GE, Milwaukee, $\mathrm{WI}$ ) using an 8-channel cardiac coil. Scan parameters were as follows: VENC $=160-200 \mathrm{~cm} / \mathrm{s} ;$ fractional FOV $=300 \times 270 \mathrm{~mm}^{2}$, slab thickness $=78 \mathrm{~mm}$, and matrix $=256 \times 192 \times 30$ yielding a spatial resolution of $1.17 \times 1.56 \times 2.60 \mathrm{~mm}^{3}$. Within each

Figure 4 (abstract 052)

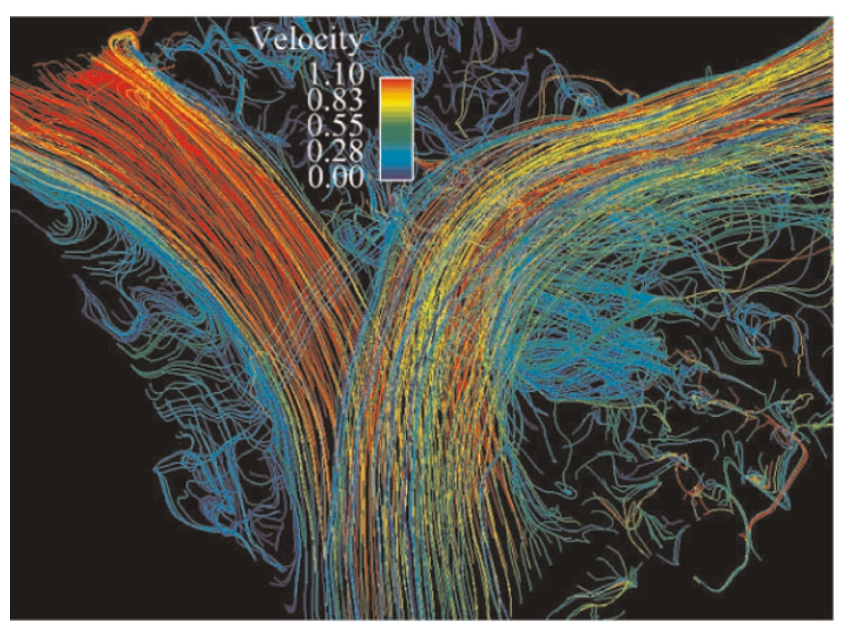

Figure 5 (abstract 052)

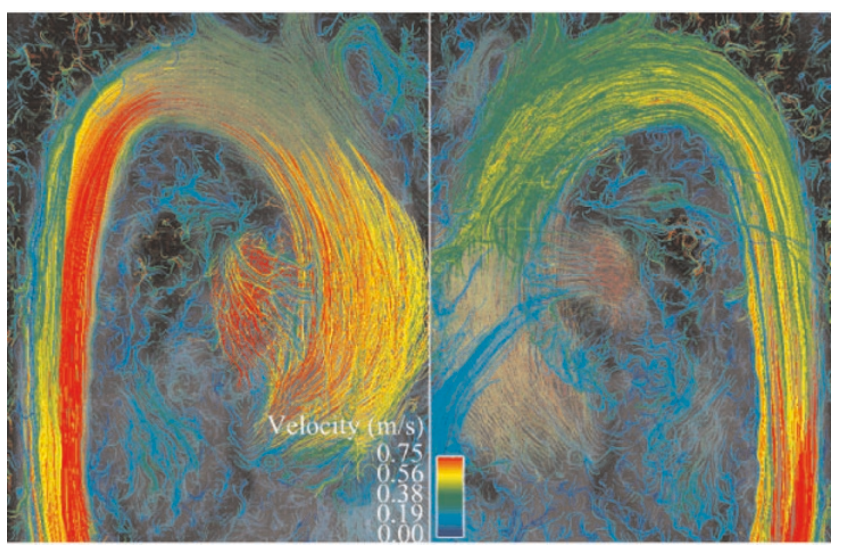

cardiac cycle, the in-plane phase encode value was held constant while 4 slice-encoding phase encodes are acquired to encode all flow directions. Parallel imaging (GRAPPA) with an acceleration factor of 2 was used. Scan times ranged from 12-16 minutes depending on heart rate. Retrospective EKG gating was used to resolve 20 time frames through the cardiac cycle yielding a temporal resolution of $50-80 \mathrm{msec}$. Respiratory compensation was employed. The raw data was reconfigured for EnSight visualization (CEI Inc., Apex, NC). Navigation within the 3D data set allows retrospective placement of planes perpendicular to the vessel of interest. 10 subjects with TOF were prospectively enrolled. For each subject, a 4D flow data set was obtained and an attempt was made to obtain 2D flow in 4 locations; the ascending aorta (AsAo), main pulmonary artery (MPA), and branch PA's (RPA and LPA).

Results: Of a total of 40 data points, there were 16 missing 2D data points and 7 missing 4D data points. Missing 2D data were secondary to aliasing and inappropriate prescription of 2D flow planes. Missing 4D data were secondary to aliasing. There were 20 total paired data points (Figure I). There was good correlation between the two techniques (Spearman's rho value of $0.9 \mathrm{I}, \mathrm{P}<0.000 \mathrm{I})$. The $4 \mathrm{D}$ flow data were internally consistent: AsAo flow equaled left ventricular stroke volume, MPA flow equaled right ventricular stroke volume, and the sum of the branch PA flows equaled MPA flows, all to within $10 \%$. This internal consistency is demonstrated in Figures 2 and 3, which display flows in the AsAo, MPA, RPA, and LPA over the cardiac cycle in a normal subject and a patient with TOF respectively. Figure 4 and 5 present streamlines in the pulmonary arteries at peak systole in the axial and sagittal planes respectively.

Conclusion: 4D flow provides complete and accurate assessment of differential pulmonary blood flow in patients with Tetralogy of Fallot (TOF) in a single acquisition. Good correlation between $4 D$ and 2D techniques for blood flow quantification in the central systemic and pulmonary vasculature is demonstrated. Navigation within 4D flow data sets allows placement of planes at will without being hindered by the prospective prescription of traditional 2D flow techniques. 


\section{3}

Effect of flow angle and flow profile on phase contrast flow measurements: overestimation at extreme angles and skewed profiles

Kevin K Whitehead, Ravi Doddasomayajula, Matthew A Harris, Matthew J Gillespie and Mark A Fogel Children's Hospital of Philadelphia, Philadlephia, PA, USA

Journal of Cardiovascular Magnetic Resonance 2009, I I (SuppI I):O53

Introduction: Flow measurements derived from phase contrast velocity mapping (PC-MRI) have become an important part of pediatric cardiology, allowing accurate quantification of shunts, valve regurgitation, and cardiac output. Clinicians generally try to align the imaging plane orthogonal to flow. However, while flow velocities decrease by the cosine of the angle from orthogonal, the area should ideally increase by the same rate. There are situations in which it may be desirable to quantify flow in a vessel on an image that was not intended during the acquisition. In addition, in some flow regimes with highly skewed flow it is sometimes difficult to align the imaging plane with flow. Understanding the settings in which PC-MRI is accurate is important to the evaluation of congenital heart disease.

Purpose: The purpose of this in vitro investigation is to assess the accuracy of PC-MRI flow measurements as a function of the angle of the imaging plane to the direction of flow. We hypothesize that the imaging angle does not have a significant effect on flow measurements.

Methods: Both steady and pulsatile flows $(0.7-10 \mathrm{~L} / \mathrm{min})$ were driven through an in vitro flow phantom consisting of tube diameters ranging from $10 \mathrm{~mm}$ to $19 \mathrm{~mm}$ both in a straight tube configuration and just distal to a 90 degree bend to simulate skewed flow. Through-plane PC-MRI velocity maps were obtained at each flow rate (I.5 T Siemens Avanto) with the imaging plane oriented from 0 to 75 degrees (I5 degree increments). Actual flows were measured for each condition using an MRI-compatible calibrated ultrasound flowmeter (Transonics) interfaced to a PC. Flow was measured using offline analysis (Argus, Siemens). Normalized flows (measured flow/ actual flow) were compared to the angle from orthogonal to flow

Figure I (abstract 053)

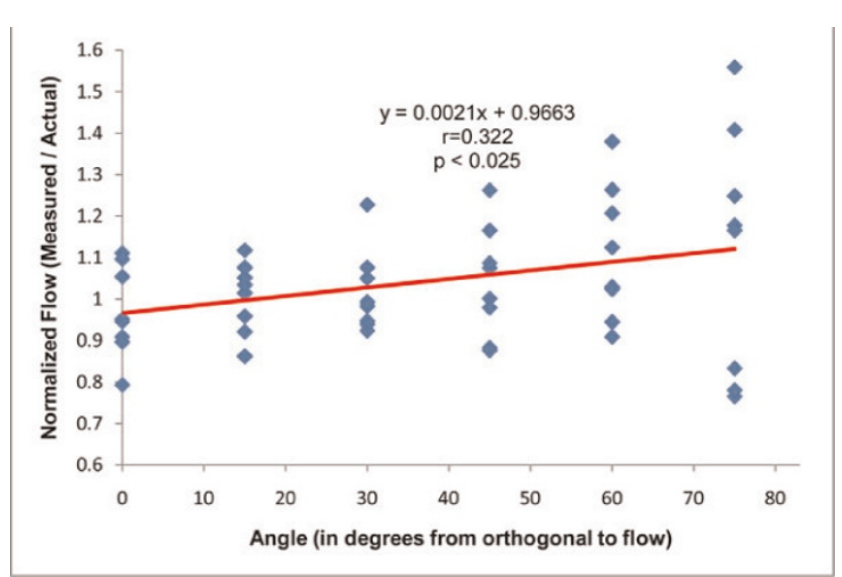

Normalized flow vs imaging angle. for all flow conditions. Variances in flow measurements between angles were compared using an F-test.

Results: Normalized flow demonstrated weak but significant correlation with flow angle for both steady and pulsatile flow conditions $(p<0.05)$. For pulsatile flow, mean normalized values ranged from 0.97 for 0 degrees to 1.12 for 75 degrees, and overestimation was as high as $11 \%$ for 0 degrees to as high as $56 \%$ for 75 degrees. Variability also tended to increase as angle increased, and the variance for 75 degrees was significantly greater than 0,15 , and 30 degrees. The flow estimation was not significantly different for skewed flow (distal to elbow) compared to flow in straight tubes. Figure I.

Conclusion: While PC-MRI is fairly robust with respect to flow angle, overestimation of flow increases significantly as a function of flow angle, to as high as a mean of $12 \%$ for the 75 degree angle. This is counterintuitive, and suggests that in general the measured velocity is not decreasing to the same degree that area is increasing. In addition, the variability of the measurements increases with increasing flow angle, especially at larger flow angles. The etiology of the error is likely related to partial volume effects. Care should be taken to be as orthogonal to flow as possible during PC-MRI to avoid overestimating flow.

\section{4}

CMR assessment of right ventricular function in patients with combined pulmonary stenosis and insufficiency after correction of tetralogy of Fallot Maureen Kohi, Karen G Ordovas and Charles B Higgins University of California, San Francisco, San Francisco, CA, USA

Journal of Cardiovascular Magnetic Resonance 2009, I I(SuppI I):O54

Introduction: Tetralogy of Fallot is the most common form of cyanotic congenital heart disease. Following surgical correction, most patients present with pulmonary insufficiency $(\mathrm{PI})$ which plays a pivotal role in right ventricular (RV) dysfunction. Multiple prior studies have demonstrated the accuracy of CMR for assessment of RV volumes, ejection fraction and pulmonary regurgitant fraction, especially in the setting of an enlarged right ventricle. The deleterious effect of chronic pulmonary regurgitation on RV function is well known and pulmonary valve replacement has been shown to improve ventricular function in these patients. However, the effects of residual pulmonary stenosis on RV function are not well understood. Using CMR and conductance catheter techniques, a study performed in a growing pig model demonstrated that chronic pulmonary stenosis and insufficiency (PSPI) results in improved RV myocardial contractility when compared to a group with isolated $\mathrm{PI}$, indicating a possible protective effect. The combined effect of PSPI on RV function has never been reported in a human model. Purpose: The purpose of this study was to compare CMR parameters for assessment of RV volumes and function between patients with combined PSPI and isolated PI following surgical repair of Tetralogy of Fallot.

Methods: A retrospective review of patients with corrected Tetralogy of Fallot who had undergone CMR and echocardiogram was performed. A total of 46 patients were included: 9 patients with PSPI and 37 patients with isolated PI. Cine MRI images in the short-axis plane were used to calculate the following parameters: pulmonary regurgitant fraction (PRF), RV end-diastolic volume (RVEDV), RV end-systolic volume (RVESV), RV stroke volume 
(RVSV) and RV ejection fraction (RVEF). RV end-diastolic volume and end-systolic volume indexes (RVEDVi, RVESVi) were calculated based on the body surface area. Peak pressure gradient across the pulmonary valve was obtained from echocardiogram performed within 3 months of the CMR. Means and standard deviations of the CMR parameters were compared between the combined PSPI and isolated PI groups using Student's t-test. A $\mathrm{p}<0.05$ was considered statistically significant.

Results: RVEF was significantly higher in combined PSPI patients $(47 \pm 9 \%)$ than in isolated $\mathrm{PI}$ patients $(40 \pm 10 \%)$ $(p=0.042)$. RVESVi was significantly lower in combined PSPI patients $\left(67.3 \pm 19 \mathrm{ml} / \mathrm{m}^{2}\right)$ than in patients with isolated $\mathrm{PI}$ $\left(92.1 \pm 43 \mathrm{ml} / \mathrm{m}^{2}\right) \quad(p=0.026)$. There was no significant difference between RVEDVi $(P=0.44)$ and PRF $(P=0.38)$ in the two groups.

Conclusion: RV function as assessed by RVEF and RVESVi was improved in patients with combined PSPI when compared to patients with isolated PI following surgical correction of Tetralogy of Fallot with similar degree of pulmonary regurgitation.

\section{5}

Chemotherapy induced abnormal aortic function assessed by magnetic resonance imaging

Narumol Chaosuwannakit, Ralph D'AgostinoJr,

Craig A Hamilton, Julia Lawrence, Frank M Torti,

William C Little and W Gregory Hundley

Wake Forest University School of Medicine, Winston-salem, NC, USA

Journal of Cardiovascular Magnetic Resonance 2009, I I(SuppI I):O55

Introduction: Abnormally increased cardiovascular stiffness is an independent predictor of cardiovascular events. Compared to age and gender matched healthy individuals, cancer survivors previously exposed to chemotherapy experience an elevated risk of cardiac events. We hypothesized that the administration of

Figure I (abstract 055)

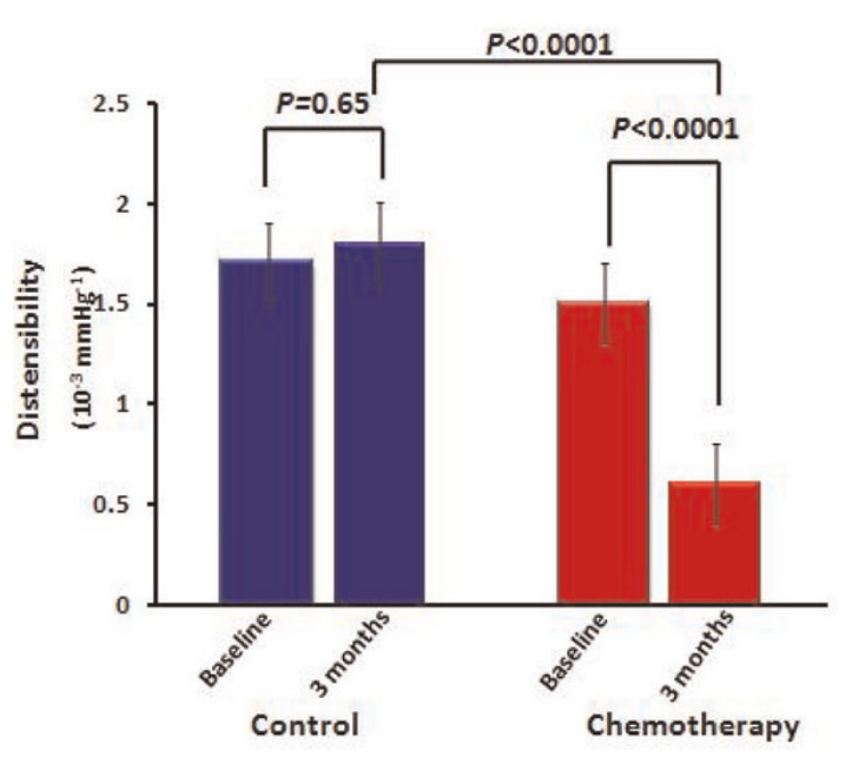

Figure 2 (abstract 055)

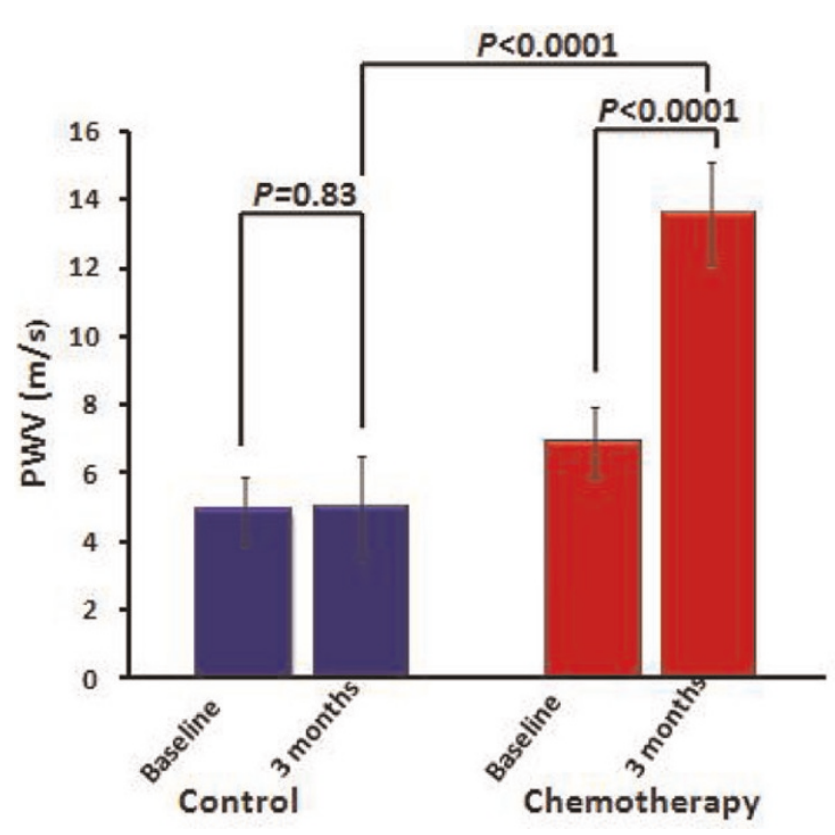

chemotherapy may increase arterial stiffness above that observed in age and gender matched controls.

Purpose: To determine if chemotherapy increases arterial stiffness within the thoracic aorta.

Methods: We performed a prospective, case-control study of 23 participants that received chemotherapy (cases) with 13 that did not (controls). For a variety of malignant neoplasms, 22 participants received anthracyclines and/or other therapies including trastuzumab or Herceptin $(n=5)$, paclitaxel $(n=7)$ or cyclophosphamide $(n=I I)$. Each participant underwent phase-contrast cardiovascular magnetic resonance imaging (PCCMR) at baseline (before chemotherapy administration in cases) and 3 to 4 months later. During CMR, thoracic aortic distensibility and pulse wave velocity (PWV) were determined according to the following formula:

Aortic distensibility $\left(10^{-3} \mathrm{mmHg}^{-1}\right)=[$ maximal aortic area minimal aortic area]/[pulse pressure $\times$ minimal aortic area] PWV $(\mathrm{m} / \mathrm{s})=$ Distance between ascending and descending thoracic aorta/Transit time of the flow wave

PC-CMR parameters included an $8 \mathrm{~mm}$ thick slice with a $192 \times 108$ matrix, a $36 \mathrm{~cm}$ FOV, a $15^{\circ}$ flip angle, a $76.5 \mathrm{~ms}$ TR, a $3.14 \mathrm{~ms}$ TE and a through-plane velocity encoding of $150 \mathrm{~cm} / \mathrm{sec}$. A nonferromagnetic brachial blood pressure cuff was applied to record heart rate and blood pressure noninvasively during the phase-contrast acquisition. To compare groups at their follow-up visit, four one-way analysis of covariance models (ANCOVA) were fit where factors known to influence aortic stiffness were included as covariates in the model.

Results: At 3 months, arterial stiffness (distensibility and PWV) remained similar in the control participants. However, in the participants receiving chemotherapy, aortic stiffness markedly increased as evidenced by a decrease in distensibility and an increase in PWV. When we compared the participants receiving chemotherapy with controls directly using an ANCOVA model 
that adjusted for baseline aortic stiffness, age, gender, body mass index, systolic blood pressure, heart rate, pulse pressure, medication use, and the presence of hypertension, diabetes and hyperlipidemia, distensibility and PWV were significantly different between controls and chemotherapy recipients $(p<0.000$ I; Figures I and 2).

Conclusion: Patients receiving relatively short courses of chemotherapy (3 months) experience a significant increase in vascular stiffness (manifest as both reduced aortic distensibility and increased PWV) compared to healthy controls. These results indicate that previously regarded cardiotoxic chemotherapy adversely increases cardiovascular stiffness, a known independent predictor of cardiovascular events.

\section{6}

Marfan's cardiomyopathy is associated with aortic annular and root dilatation in the absence of significant valvular regurgitation

Joyce Wong, Francisco Alpendurada, Elizabeth Burman and Raad Mohiaddin

Royal Brompton Hospital, London, UK

Journal of Cardiovascular Magnetic Resonance 2009, I I(SuppI I):O56

Introduction: Marfan syndrome is the commonest inherited disorder of connective tissue affecting multiple organ systems, caused by heterozygous mutations in the gene ( $F B N I)$ that encodes the extracellular matrix protein fibrillin-I. Limited evidence is available that Marfan syndrome is associated with a primary cardiomyopathy. Cardiovascular Magnetic Resonance (CMR) plays an important role in the identification and evaluation of cardiovascular disease in this population, the major source of morbidity and mortality. CMR was used to assess the prevalence and predictors of primary cardiomyopathy, in a Marfan population without evidence of significant valvular regurgitation or aortic disease.

Methods: 120 Marfan patients were consecutively referred to our centre for cardiovascular assessment with CMR between January 2003 and June 2007 (diagnosis based on Ghent criteria). Our study population consisted of 66 of these patients who had no significant valvular regurgitation, and no previous aortic or cardiac disease warranting surgery. This included 38 males (57.8\%) and 28 females (42.1\%), with a mean age of 33,2 years. Thoracic aortic dimensions were evaluated. Left ventricular (LV) volumes, ejection fraction, and mass were evaluated using semiautomated analysis software (CMRtools, Cardiovascular Imaging Solutions, London, UK). The volumes and mass were then indexed to the body surface area. Aortic (AV) and mitral valves (MV) anatomy and function were assessed, and thoracic musculoskeletal deformities identified. The final values were compared with previously published normal values with values being considered abnormal if they were outside the $95 \%$ confidence interval. We similarly compared our population to an age and sex matched cohort of normal individuals when assessing aortic dimensions.

Results: A significant proportion of this population demonstrated reductions in LV ejection fraction (27\%). LV indexed volumes were also significantly different. Annular and aortic root dimensions were increased in the population with reduced LV ejection fraction when compared with population with normal ejection fraction ( $r$ of 0.33 and 0.26 respectively, $p<0.05$ for both values, Pearson's coefficient). There was no significant difference in global aortopathy indices or incidence of musculoskeletal deformities between the two groups.

Conclusion: We confirm that Marfan syndrome is associated with a primary cardiomyopathy in a population without significant valvular disease. Moreover, annular and root dimensions are increased in patients with LV dysfunction when compared with patients with preserved LV systolic function, suggesting that aortopathy and cardiomyopathy could be related in this multi-systemic disorder. Recent advances in the molecular pathogenesis of Marfan syndrome suggest that modulation of abnormal fibrillin-I-TGF beta signalling by angiotensin-II receptor blockade in Marfan patients may reverse aortopathy. It remains to be determined whether angiotensin-II receptor blockade may reverse aortopathy and associated cardiomyopathy.

\section{7}

Prevalence and imaging features of nephrogenic systemic fibrosis at two large medical centers Martin R Prince', Honglei Zhang', Michael F Morris ${ }^{2}$, Jennifer L MacGregor ${ }^{2}$, Yang Zhang', Marc E Grossman², Jeffrey Silberzweig ${ }^{2}$, Robert L DeLapaz ${ }^{2}$, Henry J Lee', Cynthia M Magro , Anthony M Valeri' ${ }^{2}$, David N Silvers ${ }^{2}$ and Joan C Prowda ${ }^{2}$

${ }^{\prime}$ Weill Medical College of Cornell University, New York, NY, USA

${ }^{2}$ Columbia University, New York, NY, USA

Journal of Cardiovascular Magnetic Resonance 2009, I I(SuppI I):O57

Introduction: Nephrogenic Systemic Fibrosis (NSF) is a rare disease, seen in patients with severe renal impairment, that has garnered increased interest among radiologists due to reports of its association with gadolinium based contrast agents (GBCA).

Purpose: To determine the prevalence and illustrate the spectrum of imaging findings with photographic and histopathologic correlation of nephrogenic systemic fibrosis (NSF) in patients undergoing gadolinium based contrast agent (GBCA) enhanced MRI and associated risk factors.

Methods: With Institutional Review Board approval (informed consent not required) medical records from two hospitals were retrospectively reviewed to identify all cases of biopsy-confirmed NSF and all patients receiving GBCA administration from I/I/ 1997 to $6 / 30 / 2007$. Prevalence of NSF was calculated for patients receiving standard dose GBCA, high dose GBCA as well as in subgroups of patients with renal impairment. Imaging studies of these patients were reviewed to correlate with photographic and histopathologic findings.

Results: Fifteen patients had NSF following GBCA enhanced MR. All had an estimated glomerular filtration rate (eGFR) $<30$ $\mathrm{m} \mathrm{L} / \mathrm{min}$ and II had acute renal failure or acute deterioration of chronic renal failure. NSF prevalence following GBCA without screening for renal function was $0 / 74124$ for standard dose and $15 / 8997(0.17 \%)$ for high dose $(p<0.001)$. The high dose prevalence increased to $0.4 \%$ for chronic hemodialysis patients and $8.8 \%$ for patients with eGFR $<15 \mathrm{~mL} / \mathrm{min}$ but not on dialysis $(p<0.001)$. NSF prevalence in patients with acute renal failure given high dose GBCA during rising serum creatinine was $19 \%$ $(\mathrm{I}$ I/58) when dialysis was delayed by $>2$ days. More NSF patients 
had pro-inflammatory events, lower $\mathrm{pH}$, younger age, lower eGFR, elevated serum phosphate and increased delay between GBCA injection and dialysis compared to patients without NSF. NSF has a variable appearance on routine imaging studies. Plain radiographs can demonstrate joint contractures, skin thickening and possibly cutaneous calcinosis; ultrasound may show thickening and edema of the cutis, particularly in the breast; CT may show skin thickening and infiltration of subcutaneous tissues; MRI may show increased signal on fluid sensitive sequences in the skin, subcutaneous tissues, and extremity musculature; bone scintigraphy may show diffuse soft tissue uptake in the extremities.

Conclusion: For patients with eGFR $<15 \mathrm{~mL} / \mathrm{min}$, hemodialysis is protective. For patients with eGFR $<30 \mathrm{~mL} / \mathrm{min}$ receiving high dose GBCA, acute renal failure, delay in dialysis after GBCA injection, pro-inflammatory events and hyperphosphatemia were associated with increased NSF risk. Features of NSF may be evident on the patient's skin as well as on routine imaging studies although these imaging findings are nonspecific and more likely to occur with other diseases.

\section{8}

The role of peri-aortic fat in aortic atherosclerosis Ilias Kylintireas, Ikhlef Bechar, Cheerag Shirodaria, Alistair Lindsay, Justin Lee, Matthew Robson, Stefan Neubauer and Robin Choudhury University Of Oxford, Oxford, UK

Journal of Cardiovascular Magnetic Resonance 2009, I I(Suppl I):O58

Introduction: Recent data suggest that perivascular fat is metabolically active and may play a role in the initiation and progression of atherosclerosis mediated by the paracrine action of the adipokines it produces. MRI offers the unique capability for clear depiction and accurate quantification of adipose tissue alongside an effective non invasive and radiation free assessment of both early and advanced atherosclerotic effects on the vasculature.

Purpose: We investigated the relationship between perivascular adipose tissue and atherosclerosis-related structural and functional changes of the vasculature in an elderly population at high cardiovascular risk.

Methods: Fifty elderly subjects [mean age $=65( \pm 8), 16$ / $50=32 \%$ women] with at least one major cardiovascular risk factor (smoking, diabetes, hypertension, hyperlipidemia) underwent MRI (I.5 T Siemens Sonata) for abdominal and peri-aortic adipose tissue quantification and aortic atherosclerosis asses-

Figure I (abstract 058)

a.

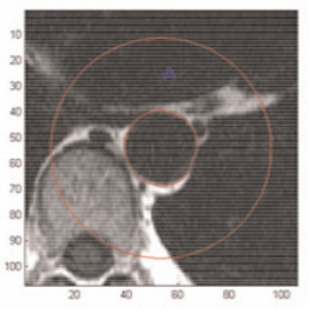

b.

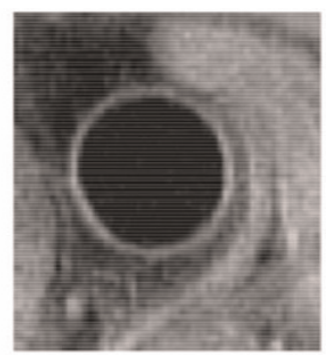

Figure 2 (abstract 058)

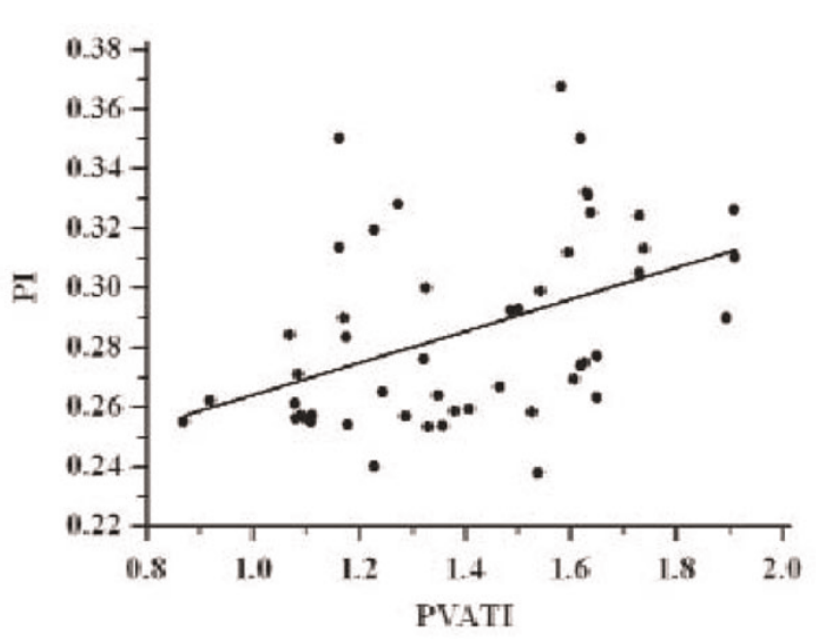

ment. A Water Suppression (WS) TI weighted (TIW) Turbo Spin Echo (TSE) multi-slice sequence was used for visceral adipose tissue (VAT) and abdominal subcutaneous adipose tissue (SCAT) measurement.

A modified version of the same sequence was used to produce WS cross-sectional images covering the descending thoracic aorta (figure la). On these images peri-vascular adipose tissue (PVAT) area was measured within a radius equal to three times the radius of the vessel cross-section. PVAT index (PVATI) was defined as the PVAT area divided by the cross-sectional area of the vessel (in order to normalise to the vessel size) and averaged for all the images along the aorta to produce a PVATI value per patient. Inter-scan and intra-observer variability of this method was very satisfactory (coefficient of variance was 0.06 and 0.03 respectively).

Proton density weighted fat saturation black blood turbo spin echo (TSE) cross-sectional images covering the descending thoracic aorta (figure la) were used for atheroma burden

Figure 3 (abstract 058)

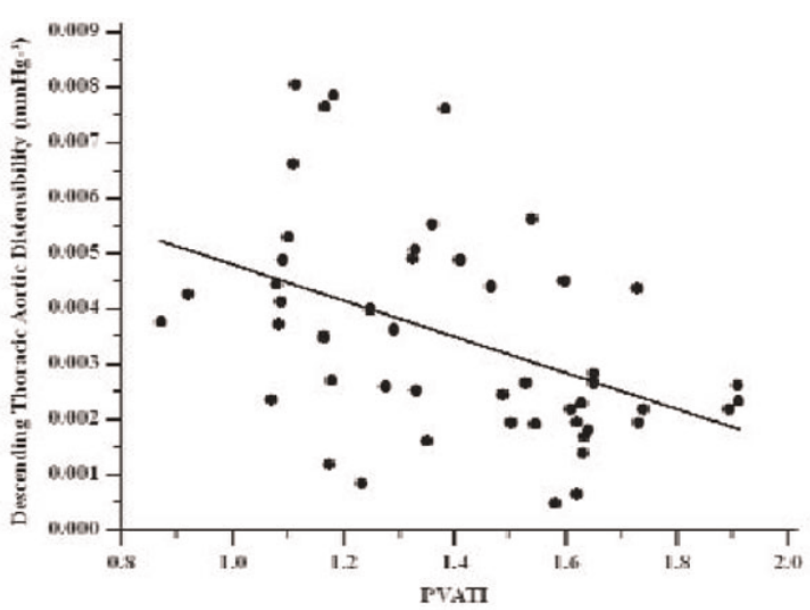


measurements (expressed as plaque index $(\mathrm{PI})=$ cross-sectional vessel wall area/total cross-sectional vascular area).

Aortic distensibility was assessed from breath-hold ECG-gated, steady state free precession (SSFP) images through the thoracic descending aorta. Distensibility was calculated as the relative change in area divided by peripheral pulse pressure.

Results: Aortic PVATI correlated with aortic atheroma burden $(r=0.44, P<0.005)$ (figure 2$)$ and inversely correlated with descending thoracic aortic distensibility $(r=-0.45, P<0.005)$ (figure 3). VAT and SCAT measurements did not correlate with thoracic aortic PI or descending thoracic aortic distensibility. Applying corresponding multiple regression analysis models (including classical risk factors, demographics and anthropometric measurements) PVATI emerged as an independent predictor of both aortic atheroma burden $[b=0.06( \pm 0.02)$, $\left.\mathrm{P}<0.05, \mathrm{R}^{2}=0.35\right]$ and descending thoracic aortic distensibility $\left[b=-0.00014( \pm 0.00005), P<0.0 I, R^{2}=0.42\right]$.

Conclusion: These results suggest a pathophysiological link between peri-vascular adiposity and the atherosclerotic process in the underlying vessel. MRI is an effective tool in depicting and quantifying PVAT. Further, interventional and analytical studies are required to elucidate this relationship.

\section{9 \\ MRI evaluation of right and left ventricular remodeling and surrogate markers of PH following acute pulmonary embolism Frederikus A Klok, Soha AH Romeih, Jos JM Westenberg, Lucia JM Kroft, Menno $V$ Huisman and Albert de Roos LUMC, Leiden, Netherlands}

Journal of Cardiovascular Magnetic Resonance 2009, I I(SuppI I):059

Introduction: It is difficult to identify patients at risk for chronic-thromboembolic pulmonary hypertension (CTEPH) in the clinical course of pulmonary embolism (PE). We evaluated ventricular remodeling and markers of pulmonary hypertension $(\mathrm{PH})$ using cardiac MRI in PE patients after 6 months treatment. Methods: Fifteen PE-patients and 10 controls, in whom PE was suspected but ruled out, were studied. A baseline CT scan was performed to diagnose PE and to assess dynamic right and left ventricular (RV; LV) function. After 200 days, a MRI scan was performed to assess bilateral ventricular function and several surrogate markers of $\mathrm{PH}$.

Results: Baseline characteristics of both controls and PEpatients were comparable. In the control cohort, end-systolic (ESV) and end-diastolic volume (EDV) in both ventricles did not change in time. PE patients with normal RV function at baseline had a significant improvement in RVEF $(+5.4 \pm 3.1 \%)$ due to a relative decrease in ESV $(-17 \pm 7.9 \%)$. Patients with abnormal RV function at baseline had a significant improvement in RVEF $(+14 \pm 15 \%)$ due to relative decrease in both ESV (-36 $\pm 23 \%)$ and EDV $(-22 \pm 16 \%)$. Furthermore, LVEDV increased significantly $(15 \pm 11 \%)$.

Pulmonary distensibility index $(0.033 \pm 0.0058)$ was significant decreased in patients with persistent RV dysfunction compared to patients with restored RV function $(0.22 \pm 0.18)$ and controls $(0.28 \pm 0.25)$. In addition, decreased stroke volume $(7 \mathrm{I} \pm 2 \mathrm{l} \mathrm{ml}$ versus $103 \pm 40 \mathrm{ml}$ and $94 \pm 19 \mathrm{ml}$ respectively) and $\mathrm{PH}$ specific alterations in the pulmonary flow curves were found in these patients compared to both other groups.

Conclusion: RV remodelling after PE is dependent on the degree of baseline RV dysfunction. RVEF improves due to decrease in RVESV and in lesser extent to RVEDV. Compared to controls and PE patients with normalized RV function, patients with persistent RV dysfunction have $\mathrm{PH}$ specific pulmonary flow alterations and a stiffened pulmonary artery. It remains to be studied whether these patients are at risk for developing CTEPH.

\section{0}

Effect of age on stress induced changes in aortic distensibility

Haroon L Chughtai, William G Hundley, Tim Morgan and Craig Hamilton

Wake Forest University Health Sciences, Winston Salem, NC, USA

Journal of Cardiovascular Magnetic Resonance 2009, I I (SuppI I):O60

Introduction: Aging increases aortic stiffness. We describe a novel method for assessing aortic distensibility throughout stress with dobutamine.

Purpose: Previous studies have shown aortic stiffness to be a predictor of adverse cardiovascular outcomes and mortality. Aging is one of the most significant factors associated with aortic stiffness. Aortic stiffness can be assessed by measuring aortic distensibility non-invasively with cardiac magnetic resonance. We undertook this study to assess and understand the changes in aortic distensibility with stress (induced by dobutamine) and find an association of these changes with advancing age.

Methods: We studied 128 patients (48\% women) aged 55-85 years in which aortic distensibility was determined during intravenous dobutamine infused to achieve $85 \%$ of predicted

Figure I (abstract 060)

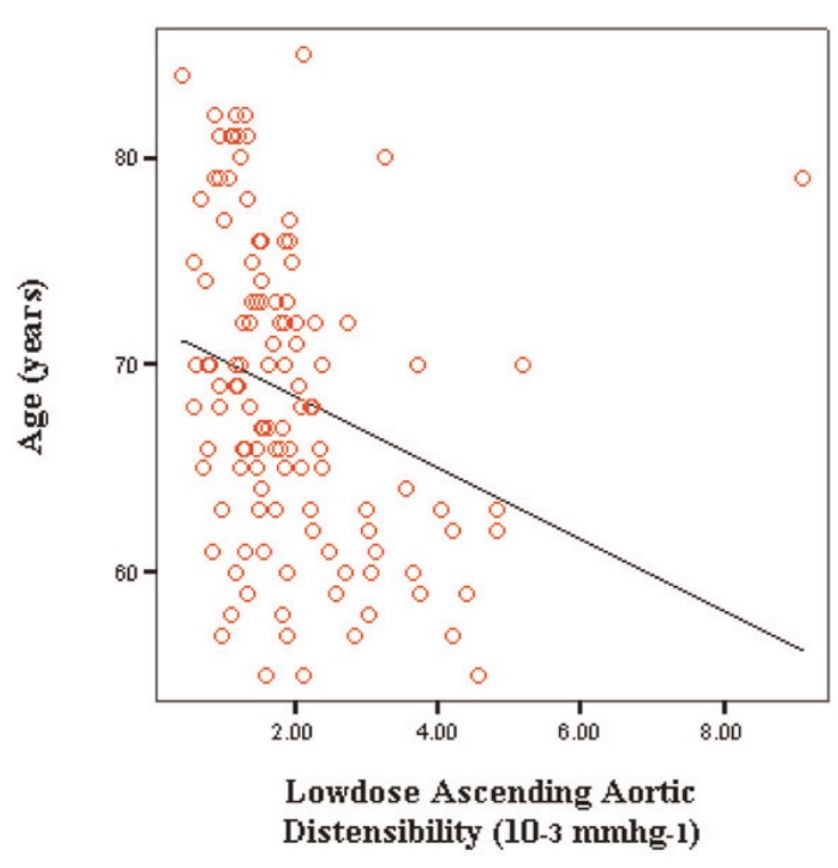

Page 49 of 316

(page number not for citation purposes) 
Figure 2 (abstract 060)

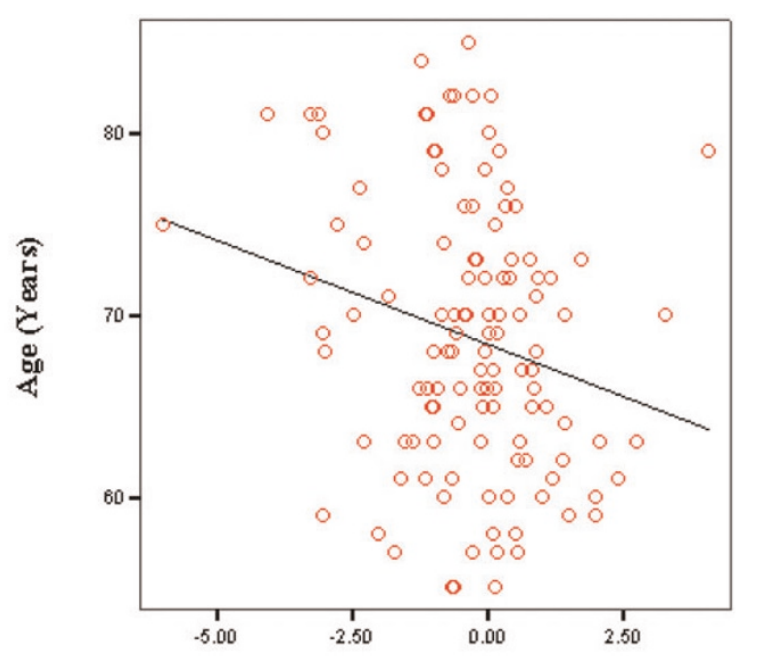

Change in Ascending Aortic Distensibility (10-3 mmhg-1) From Low Dose to Peak Dose

heart rate response for age. Image acquisition was accomplished with phase-contrast gradient echo cardiovascular magnetic resonance. Images were acquired perpendicular to the course of ascending aorta at the middle level of the left atrium. Aortic distensibility was determined by measuring cardiac cycle dependent changes in aortic area/\{brachial pulse pressure $x$ the end-diastolic area of the aorta\}. Changes between different stages of stress were calculated and correlated with age using linear regression.

Results: Resting baseline ascending aortic distentesibility was $2.0 \times 10^{-3} \mathrm{mmhg}^{-1}$. After infusion of low dose dobutamine (7.5 $\mu \mathrm{g} / \mathrm{kg} / \mathrm{min})$, mean ascending aortic distensibility was $1.9 \times 10^{-3} \mathrm{mmhg}^{-1}$. At peak stress, mean ascending aortic distensibility was $1.8 \times 10^{-3} \mathrm{mmhg}^{-1}$. On Pearson correlation, all the individual distensibilities (baseline, low dose and peak dose) correlated negatively with age (baseline and low dose significant at $p$ of .00). In the second step, differences between each of baseline to low dose and low dose to high dose distensibilities were calculated. From baseline to low dose, mean difference was $0.178 \times 10^{-3} \mathrm{mmhg}^{-1}$ which correlated with age at an $r$ of -0.24 (significant at a $p$ value of .00 ). The mean difference between low dose to peak dose was $-0.308 \times 10^{-3} \mathrm{mmhg}^{-1}$ which correlated with age at an $r$ of -0.22 (significant at a $p$ value of .01 ). A partial correlation was also done after adjustment for gender, hypertension and diabetes, which showed persistence of negative correlation of baseline, low dose and peak dose ascending aortic distensibilities with age (baseline and low dose were statistically significant). The adjusted analysis also showed the independent negative correlation of age with change in ascending aortic distensibility from low dose to peak dose $(r$ of -0.25 at a $p$ value of .0I). Figures I and 2.

Conclusion: Our study indicates that advanced age is an independent predictor of impaired ascending aortic distensibility and inability to adequately change distensibility during stress. These data imply that advanced aging may alter the relationship between aortic stiffness and left ventricular emptying during pharmacologic stress.
061

Does lipid lowering therapy improve calf muscle perfusion and cellular metabolism in peripheral arterial disease?

Amy M West, Justin D Anderson, Frederick H Epstein, Craig H Meyer, Klaus D Hagspiel, Stuart S Berr, Nancy L Harthun, Arthur L Weltman, Joseph M DiMaria, Jennifer R Hunter, John M Christopher and Christopher M Kramer University of Virginia, Charlottesville, VA, USA

Journal of Cardiovascular Magnetic Resonance 2009, I I(SuppI I):O6 I

Introduction: Previous studies suggest that lipid lowering therapy improves symptoms and walking performance in patients with peripheral arterial disease (PAD). We studied the relationship between LDL reduction and both tissue perfusion and cellular metabolism in PAD using magnetic resonance imaging (MRI) and spectroscopy (MRS).

Methods: 61 patients with mild-to-moderate symptomatic PAD (mean age $63 \pm 10$ years, ankle brachial index $0.69 \pm 0.15$ ) were studied before and I year after starting one of 3 lipid lowering therapies. Statin-naïve patients were randomized to simvastatin $40 \mathrm{mg}$ or simvastatin $40 \mathrm{mg}$ plus ezetimibe $10 \mathrm{mg}(\mathrm{n}=3 \mathrm{I})$ and patients already on a statin were given open-label ezetimbibe $10 \mathrm{mg}(\mathrm{n}=30)$. Lipid measurements were obtained as part of the VAP test. Patients with interval stenting of the leg studied $(n=4)$ or bypass surgery $(n=1)$ were excluded from analysis. All 56 remaining patients had calf muscle phosphocreatine recovery time constant $(\mathrm{PCr})$ measured using ${ }^{3 !}$ phosphorus (P) MRS immediately after symptom-limited calf muscle exercise using a MR compatible ergometer on a Siemens Sonata I.5 T scanner. Exercise time was recorded. ${ }^{31} \mathrm{P}$ MRS was obtained using a single-pulse, surface coil localized, $512 \mathrm{~ms}$ free induction decay acquisition with 20 averages centered on the mid-calf. PCr was then calculated using a monoexponential fit of phosphocreatine concentration versus time, beginning at cessation of exercise. Calf muscle tissue perfusion (TP) was measured in 50 patients using first-pass contrast-enhanced MRI at peak exercise. The remaining 6 patients were excluded due to compromised renal function. The patients pushed a MRcompatible foot pedal ergometer at a steady rate (10-12 bpm) until limiting symptoms or exhaustion while in a Siemens Avanto I.5 T scanner and gadolinium $(0.1 \mathrm{mM} / \mathrm{kg})$ was infused at peak exercise. Work expended during exercise was recorded. Time intensity curves were generated with ARGUS image analysis software from the region of calf muscle with the greatest intensity post contrast and the slope of this curve was defined as TP. To assess microvascular blood flow within the calf muscle, TP

Table I (abstract 06I) Changes in perfusion, metabolism, and exercise parameters over time

\begin{tabular}{lll}
\hline & Baseline & Follow up \\
\hline Perfusion Work, j & $225.6 \pm 156.1$ & $250.8 \pm 293.9$ \\
Tissue perfusion & $6.45 \pm 3.42$ & $6.32 \pm 3.44$ \\
Perfusion index & $0.57 \pm 0.39$ & $0.60 \pm 0.42$ \\
PCr Exercise, sec & $156.6 \pm 58.8$ & $173.1 \pm 57.9 *$ \\
PCr, sec & $85.9 \pm 59.3$ & $85.8 \pm 59.8$ \\
\hline
\end{tabular}

Page 50 of 316 (page number not for citation purposes) 
was indexed to macrovascular blood flow into the calf by dividing it by the slope of the popliteal arterial input curve to obtain a perfusion index $(\mathrm{PI})$. Changes in all parameters from baseline to year one were compared by paired t-test and between group differences by unpaired t-test.

Results: LDL at baseline was $110 \pm 34$ and was lower at one year $(79 \pm 34 \mathrm{mg} / \mathrm{dl}, \mathrm{p}<0.000 \mathrm{l})$. The total cholesterol at baseline was $183 \pm 42$ and decreased significantly at one year to $146 \pm 42 \mathrm{mg} / \mathrm{dl}, \mathrm{p}<0.000 \mathrm{I}$ as did triglycerides (I $65 \pm$ I I 9 tol $45 \pm 8 \mathrm{l} \mathrm{mg} / \mathrm{dl}, \mathrm{p}=0.05)$. There was no change in HDL from baseline to follow-up ( $44 \pm 14$ to $43 \pm 14 \mathrm{mg} / \mathrm{dl}, p=N S)$. See Table I for changes in TP, PI, PCr, exercise time and work expended between baseline and year one. A trend was noted towards an increase in exercise time for ${ }^{31} P$ MRS. There were no between group differences in any MR or exercise outcome parameter in patients treated with different lipid lowering regimens.

Conclusion: Lipid lowering therapy over the course of one year in PAD did not improve tissue perfusion as measured by first pass contrast-enhanced MRI or cellular metabolism as measured by phosphocreatine recovery kinetics, although exercise time tended to improve. Thus, the previously demonstrated increase in exercise capacity with LDL lowering in PAD is unlikely due to improvements in tissue perfusion or skeletal muscle metabolism, suggesting that other potential mechanisms of benefit must be at play.

\section{2}

Incremental value of cardiac magnetic resonance in the characterization of unselected patients referred to exclude arrhythmogenic right ventricular cardiomyopathy

Alberto Roghi, Stefano Pedretti, Patrizia Pedrotti and Santo Dellegrottaglie

Department of Cardiology, Niguarda Ca'Granda Hospital, Milan, Italy

Journal of Cardiovascular Magnetic Resonance 2009, I I(Suppl I):O62

Introduction: Cardiac magnetic resonance (CMR) may be efficiently applied to recognize morphologic and functional aspects employed in the diagnosis of arrhythmogenic right ventricular cardiomyopathy (ARVC). However, limited information is available regarding the actual impact of CMR findings in reaching a diagnosis of $A R V C$ in an unselected population of patients referred to a CMR laboratory.

Purpose: Aim of the study was to evaluate the incremental value of CMR over preliminary clinical/instrumental data in characterizing a group of consecutive patients referred for suspected ARVC.

Methods: From January 2006 to April 2008, a total of 91 consecutive patients $(60 \%$ males; mean age, $35 \pm 18$ years, range 8-72 years) underwent CMR (I.5 T, Magnetom Avanto, Siemens) to exclude ARVC. In each patient, CMR imaging protocol applied at the ventricular level included the acquisition of images for the identification of: (I) regional and/or global systolic dysfunction and chamber dilation (steady-state free precession sequences); (2) myocardial areas of fatty infiltration (TI-weighted fast spin-echo sequences with and without fatsaturation); myocardial areas of edema/flogosis (T2-weighted short-tau inversion recovery sequences); myocardial areas of delayed post-contrast enhancement (gradient-echo inversion recovery sequences). All patients had been previously evaluated by resting electrocardiogram, exercise stress test, 24-h Holter electrocardiogram monitoring and transthoracic echocardiography. Major and minor criteria for ARVC diagnosis were defined according to the standard Task Force criteria.

Results: The studied patients were referred for the CMR study mainly based on frequent premature ventricular contractions (52\%) or morphologic/functional alterations of the right ventricle (29\%). By considering pre-CMR clinical/instrumental data only, diagnosis of ARVC was already reached in $3(3 \%)$ patients, while the majority of patients $(n=62,68 \%)$ presented with a low pretest probability of disease. Inclusion of CMR data allowed to reach a diagnosis for ARVC in 4 (4\%) patients. In none of the patients in the subgroup at low pre-test probability, CMR information led to a diagnosis for ARVC. With the CMR protocol employed in this study, diagnosis other than ARVC were obtained in 17 patients (19\%), including: acute or chronic myocarditis $(n=5)$; chronic myocardial infarction $(n=2)$; partial anomalous pulmonary venous return $(n=2)$; other diagnoses $(n=8)$.

Conclusion: In an unselected population of patients with suspected ARVC, the proportion of cases with a confirmed diagnosis of ARVC is not significantly impacted by CMR information over preliminary clinical/instrumental data. In 19\% of patients referred to exclude ARVC, CMR allows the identification of previously unrecognized conditions other than ARVC.

\section{3}

Delayed hyper-enhancement cardiac magnetic resonance imaging is more accurate than other noninvasive parameters in diagnosis of patients with endomyocardial biopsy positive cardiac amyloidosis

Bethany A Austin, Scott D Flamm, E Rene Rodriguez, Carmela Tan, Randall C Starling and Milind Y Desai Cleveland Clinic, Cleveland, $\mathrm{OH}$, USA

Journal of Cardiovascular Magnetic Resonance 2009, I I(Suppl I):O63

Introduction: In patients with nonischemic cardiomyopathy and suspected cardiac amyloidosis (CA), endomyocardial biopsy $(E M B)$ provides a definitive diagnosis. A multitude of noninvasive parameters, including electrocardiography (ECG) and transthoracic echocardiography (TTE) have been utilized as potential markers of CA. Recently, it has been demonstrated that, in patients with $C A$, delayed hyper-enhancement cardiac magnetic resonance (DHE-CMR) reveals a characteristic diffuse enhancement of the entire subendocardium (arrows) with extension into the neighboring myocardium (Figure I).

Purpose: We sought to determine the diagnostic accuracy of DHE-CMR, as compared to standard noninvasive parameters, in patients with suspected $C A$ that underwent EMB.

Methods: A total of 38 patients (mean age $62 \pm 14$ years, 7I\% men, $57 \%$ with New York Heart Association class > 2) with suspected CA underwent electrocardiography (ECG), TTE (including tissue Doppler), DHE-CMR (Siemens I.5 T scanner, Erlangen, Germany) and EMB between I/05 and 4/08. Low voltage on ECG was defined as sum of $S$ wave in lead $V I+R$ wave in lead V5 or V6 < $15 \mathrm{~mm}$. Measured TTE parameters included left atrial size, interventricular septal thickness, speckled 
Figure I (abstract 063)

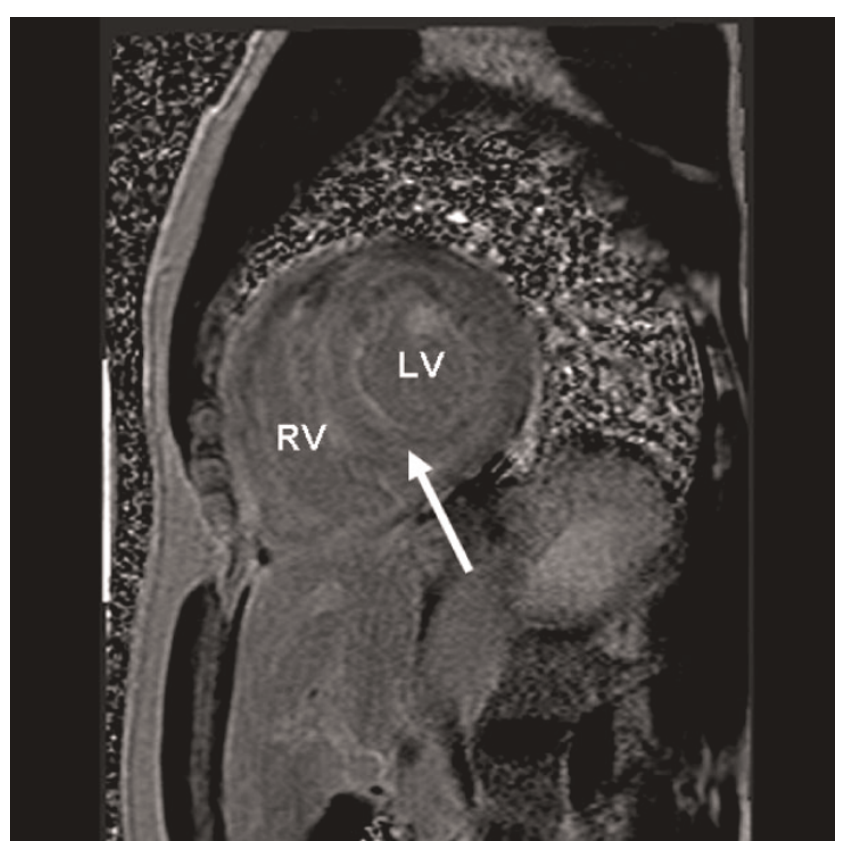

appearance, E/A ratio, E/E' ratio, stage of diastology, deceleration time (msec) and myocardial performance index [(isovolumic contraction time + isovolumic relaxation time)/ejection time]. DHE-MR images were obtained in standard long and short axis orientations (covering the entire LV), after injection of Gadolinium dimenglumine using an inversion recovery spoiled gradient echo sequence: TE $4 \mathrm{msec}$, TR $8 \mathrm{msec}$, flip angle $30^{\circ}$, bandwidth $140 \mathrm{~Hz} /$ pixel, $23 \mathrm{k}$-space lines acquired every other RR-interval, field of view (varied from 228-330 in the x-direction and $260-330$ in the $y$-direction) and matrix size (varied from 140-180 in the x-direction and 256 in the $y$-direction). CMR was considered positive in the presence of DHE of entire subendocardium with extension into the neighboring myocardium.
Results: There were 17 each with EMB-positive CA and CMRsuspected CA. Using EMB as gold-standard, there was 2 falsepositive and 2 false-negative CMR. Sensitivity, specificity, positive predictive value (PV) and negative PV of DHE-CMR in the diagnosis of CA were $88 \%, 90 \%, 88 \%$ and $90 \%$ respectively. Logistic regression analysis demonstrating the association between EMB-positive $\mathrm{CA}$ and various noninvasive parameters is shown in Table I.

Conclusion: DHE-CMR is highly accurate in noninvasive diagnosis of EMB-positive CA as compared to standard ECG and TTE criteria. Incremental prognostic value of DHE-CMR in CA for clinical outcomes needs to be determined.

\section{4}

Influence of late gadolinium enhancement on left ventricular morphology and function in patients with sarcoidosis

Amit R Patel, Nadera J Sweiss, Sonal Chandra, Lissa Sugeng, Kirk T Spencer, Jeanne M DeCara, Martin C Burke, Timothy B Niewold, Douglas K Hogarth, Stephen L Archer and John F Beshai University of Chicago, Chicago, IL, USA

Journal of Cardiovascular Magnetic Resonance 2009, I I(SuppI I):O64

Background: Cardiac involvement in sarcoidosis patients is associated with an increased risk of sudden death. However, conduction block and cardiomyopathy are late and therefore potentially insensitive markers of cardiac sarcoidosis. Prior publications have suggested that the detection of late gadolinium enhancement (LGE) by cardiac magnetic resonance (CMR) may be a more sensitive parameter for diagnosing cardiac sarcoidosis. Using CMR, in patients with known sarcoidosis, we sought I) to further define the incidence of cardiac involvement and 2) to determine the correlation between LGE and structural cardiac pathology.

Methods: We retrospectively evaluated 55 consecutive patients with known sarcoidosis who were referred for CMR. Imaging was performed on a I.5 Tesla MRI scanner with a flexible surface coil. Retrospectively gated cines of the left ventricular

Table I (abstract 063) Logistic univariate regression analysis testing the association between endomyocardial biopsy proven cardiac amyloidosis and various noninvasive imaging parameters

\begin{tabular}{|c|c|c|c|c|}
\hline \multirow[b]{2}{*}{ Noninvasive imaging parameters } & \multicolumn{2}{|l|}{ Univariate } & \multicolumn{2}{|l|}{ Multivariate } \\
\hline & Wald $\chi^{2}$ Statistic & p-value & Wald $\chi^{2}$ Statistic & p-value \\
\hline Carroll' Criteria on ECG & 2.23 & 0.13 & & \\
\hline Rahman's Criteria on ECG & 0.60 & 0.40 & & \\
\hline Dilated left atrium $\left(>20 \mathrm{~cm}^{2}\right)$ & 0.98 & 0.33 & & \\
\hline Interventricular septal thickness & 8.6 & 0.003 & 1.7 & 0.19 \\
\hline Left ventricular ejection fraction & 0.04 & 0.83 & & \\
\hline Speckled appearance on surface echocardiography & 0.0 & 0.9 & & \\
\hline Pseudonormal or restrictive physiology on Doppler echocardiography & 2.1 & 0.15 & & \\
\hline E/A ratio & 1.9 & 0.17 & & \\
\hline E/E' ratio $\geq 15$ & 1.06 & 0.30 & & \\
\hline Abnormal deceleration time $(\leq 150 \mathrm{msec})$ & 0.002 & 0.97 & & \\
\hline Myocardial performance index & 1.7 & 0.20 & & \\
\hline Positive delayed hyper-enhancement cardiac magnetic resonance & 16 & $<0.001$ & 9.6 & 0.002 \\
\hline
\end{tabular}


Figure I (abstract 064)

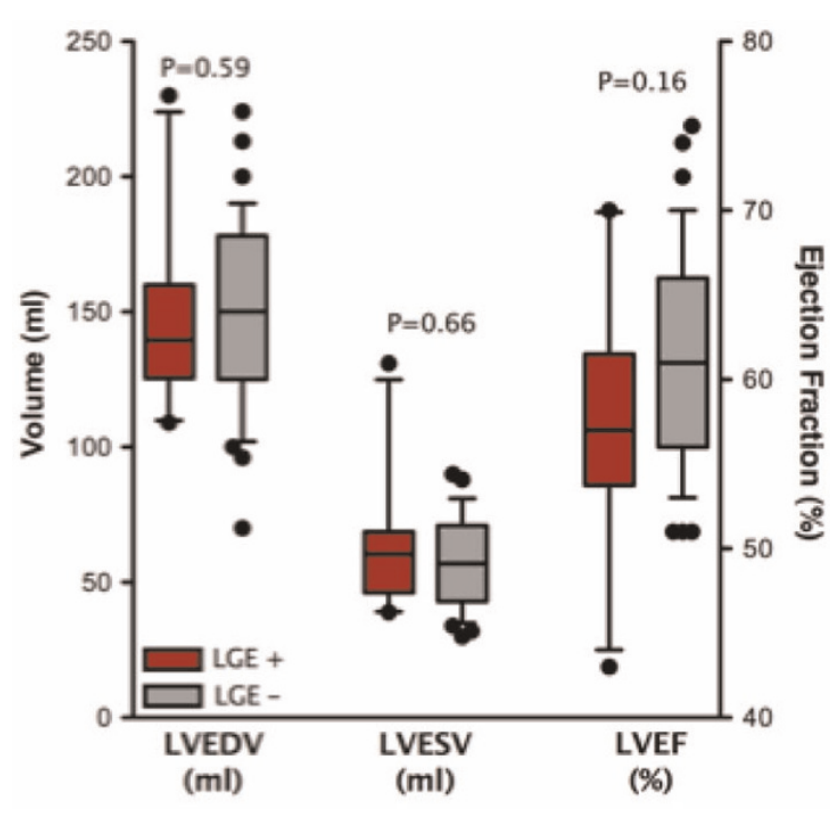

(LV) 2-, 3-, and 4-chambers, and a short-axis stack were obtained using steady state free precession imaging (TR $2.9 \mathrm{~ms}$, TE $1.5 \mathrm{~ms}$, flip angle $60^{\circ}$, temporal resolution $25-40 \mathrm{~ms}$ ). LGE images of the same views were obtained 10-20 minutes after infusion of GdDTPA (0.15-0.2 $\mathrm{mmol} / \mathrm{kg})$ using a TI-weighted inversion recovery GRE pulse sequence (TI based on optimal myocardial nulling, TR $3.9 \mathrm{~ms}$, TE $1.7 \mathrm{~ms}$, flip angle $=15-30^{\circ}$ ). The cines of the short-axis stack were used to determine LV and right ventricular end-diastolic volume (LVEDV and RVEDV), endsystolic volume (LVESV and RVESV), ejection fraction (LVEF and RVEF), and LV mass. The presence or absence of LGE was determined for each of the 17 segments of the American Heart Association left ventricular model. Continuous variables were reported as mean \pm standard deviation, groups (based on the presence or absence of LGE) were compared using a t-test, and linear regression analysis was used to investigate the correlation between LGE and LV size and function. A P value $<0.05$ was considered statistically significant.

Results: The patients were $48 \pm 13$ years old and the majority were women (73\%). Twenty-two percent of the patients had LGE involving on average $14 \pm 12 \%$ of the LV. In patients with or without LGE, there was no difference in LVEDV $(147 \pm 35 \mathrm{ml}$ vs. $150 \pm 35 \mathrm{ml}, \mathrm{p}=0.59)$, LVESV $(64 \pm 16 \mathrm{ml}$ vs. $57 \pm 16 \mathrm{ml}$, $p=0.66)$, LVEF $(58 \pm 8 \%$ vs. $62 \pm 7 \%, p=0.16)$, LV mass $(\mathrm{I} / 6 \pm 46 \mathrm{~g}$ vs. $99 \pm 24 \mathrm{~g}, \mathrm{p}=0.47)$, RVEDV $(172 \pm 35 \mathrm{ml}$ vs. I7I $\pm 46 \mathrm{ml}, \mathrm{p}=0.9 \mathrm{l}), \operatorname{RVESV}(85 \pm 26 \mathrm{ml}$ vs. $82 \pm 27 \mathrm{ml}$, $\mathrm{P}=0.78)$ and $\operatorname{RVEF}(52 \pm 6 \%$ vs. $53 \pm 7 \%, \mathrm{p}=0.70)$. See figure I. Moreover, based on linear regression analysis, in the patients with LGE, the extent of enhancement did not influence LVEDV $\left(r^{2}=0.14, p=0.29\right)$ and LV mass $\left(r^{2}=0.03, p=0.65\right)$. However, there was a trend that the extent of LGE correlated with both $\operatorname{LVESV}\left(r^{2}=0.27, p=0.13\right)$ and $\operatorname{LVEF}\left(r^{2}=0.29, p=0.11\right)$ (See figure 2). All of the above relationships persisted even after accounting for body surface area.
Figure 2 (abstract 064)
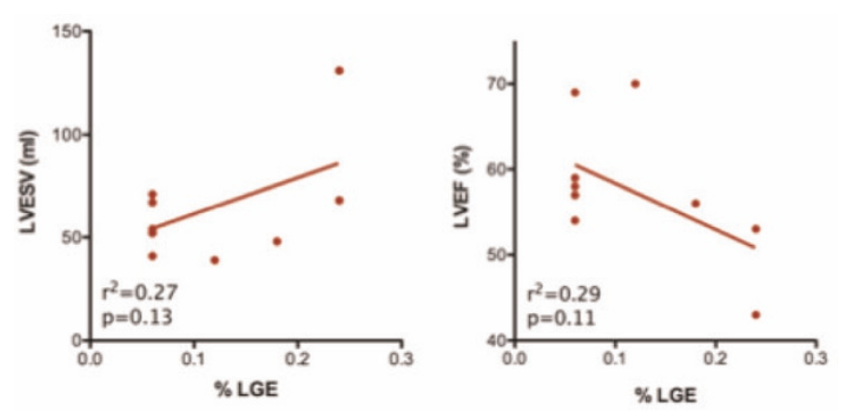

Conclusion: In patients with known sarcoidosis, nearly a quarter had evidence of cardiac involvement, as defined by the presence of LGE, despite the lack of any difference in traditional measures of cardiac structure and function. These preliminary findings suggest that volumetric and functional assessments alone are inadequate for the detection of cardiac sarcoidosis and that tissue characterization using LGE is essential. Further studies are needed to determine the long-term prognostic value of LGE and to further clarify the relationship between the extent of LGE and left ventricular morphology and physiology in patients with cardiac sarcoidosis.

\section{5}

Delayed hyper-enhancement cardiac magnetic resonance provides incremental prognostic value in patients with cardiac amyloidosis

Bethany A Austin, Scott Flamm, E Rene Rodriguez,

Carmela Tan, WH Wilson Tang, David O Taylor,

Randall $C$ Starling and Milind $Y$ Desai

Cleveland Clinic, Cleveland, $\mathrm{OH}$, USA

Journal of Cardiovascular Magnetic Resonance 2009, I I(Suppl I):O65

Introduction: Patients with cardiac amyloidosis (CA) have an unfavorable, albeit a variable prognosis. In patients with documented cardiac amyloidosis (CA), delayed hyper-enhancement-cardiac magnetic resonance (DHE-CMR) has been demonstrated to have a high diagnostic accuracy. However, its prognostic utility in CA has not been determined.

Purpose: We sought to determine the incremental prognostic value of DHE-CMR in CA.

Methods: We studied 47 consecutive patients with suspected CA (mean age $63 \pm 13$ years, $70 \%$ men, 55\% with NYHA class $>2$ ) that underwent electrocardiography (ECG), transthoracic echocardiography (TTE), DHE-CMR (Siemens I.5 T scanner, Erlangen, Germany) and biopsy (38 endomyocardial, 9 extracardiac) between 1/05 and 7/08. Low voltage on ECG was defined as sum of $S$ wave in lead $V I+R$ wave in lead V5 or V $6<15 \mathrm{~mm}$. Measured TTE parameters included left atrial size, interventricular septal thickness, speckled appearance, E/A ratio, $\mathrm{E} / \mathrm{E}$ ' ratio, stage of diastology, deceleration time (msec) and myocardial performance index [(isovolumic contraction time + isovolumic relaxation time)/ejection time]. DHE-CMR images were obtained in standard long and short axis orientations 
Table I (abstract 065) Cox proportional hazard analysis of various clinical and noninvasive imaging predictors of long-term mortality in patients with biopsy proven cardiac amyloidosis

\begin{tabular}{llll}
\hline & Univariate Analysis & & Multivariate Analysis \\
\cline { 2 - 3 } \cline { 3 - 4 } Variable & $\chi^{2}$ & p value & p value \\
\hline Age & 3.8 & 0.05 & 0.10 \\
Gender & 0.15 & 0.69 & 0.16 \\
New York Heart Association Class & 3.3 & 0.07 & \\
Low voltage on electrocardiogram & 0.67 & 0.41 & \\
Left atrial size > 20 cm2 & 0.76 & 0.39 & \\
Left ventricular ejection fraction & 2.21 & 0.14 & \\
Interventricular septal thickness & 1.7 & 0.19 & \\
E/E' > I5 on Doppler echocardiography & 1.12 & 0.29 & \\
Deceleration time on Doppler echocardiography $\leq 150$ msec & 1.41 & 0.23 & \\
Myocardial performance index & 2.10 & 0.15 & \\
Diastology grade & 0.35 & 0.55 & \\
Delayed hyperenhancement on cardiac magnetic resonance & 4.91 & 0.03 & 0.02 \\
\hline
\end{tabular}

Chi-square for multivariate model $=12.27, \mathrm{p}$-value $=0.007$.

(covering the entire LV), after injection of Gadolinium dimenglumine using an inversion recovery spoiled gradient echo sequence: TE $4 \mathrm{msec}$, TR $8 \mathrm{msec}$, flip angle $30^{\circ}$, bandwidth $140 \mathrm{~Hz} /$ pixel, 23 k-space lines acquired every other RR-interval, field of view (varied from 228-330 in the x-direction and 260330 in the $y$-direction) and matrix size (varied from I40-I80 in the $\mathrm{x}$-direction and 256 in the $y$-direction). CMR was considered positive in the presence of DHE of entire subendocardium with extension into the neighboring myocardium. All-cause mortality was ascertained.

Results: At baseline, 59\% patients had low voltage on ECG, while $67 \%$ had deceleration time < $150 \mathrm{msec}$ and $53 \%$ had E/E' > 15 (both on Doppler echocardiography). Mean MPI, left ventricular ejection fraction and interventricular septal thickness were $0.5 \mathrm{I} \pm 0.3,5 \mathrm{I} \% \pm \mathrm{I} 3$ and $\mathrm{I} .5 \mathrm{~cm} \pm 0.5$, respectively. At up to I-year after biopsy, there were 9 (I9\%) deaths. Results of Cox Proportional Hazard survival analysis are shown in Table I. On univariate Kaplan-Meier survival analysis, presence of DHE on

Figure I (abstract 065)

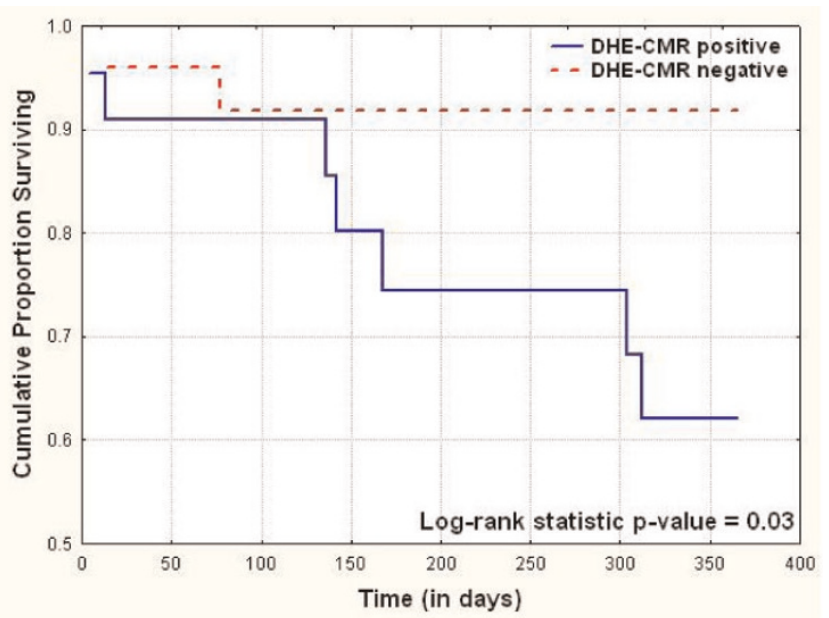

CMR was associated with worse I-year survival (log rank statistic $\mathrm{p}$-value $=0.03$, Figure I).

Conclusion: Presence of DHE on CMR is associated with worse I-year survival in CA. Along with a high diagnostic accuracy; DHE-CMR adds incremental prognostic value in CA, independent of other variables.

\section{6}

Delayed-enhanced magnetic resonance imaging for identifying the ventricular arrhythmia substrate in non-ischemic cardiomyopathy

Benoit Desjardins', Fred Morady ${ }^{2}$ and Frank Bogun ${ }^{2}$

'University of Pennsylvania, Philadelphia, PA, USA

${ }^{2}$ University of Michigan, Ann Arbor, MI, USA

Journal of Cardiovascular Magnetic Resonance 2009, I I(SuppI I):O66

Introduction: Scar tissue is often noted in the myocardium of patients with non-ischemic cardiomyopathy. Delayed-enhanced magnetic resonance imaging (DE-MRI) can precisely define the extension and distribution of this scar tissue. Such scar tissue can act as arrhythmogenic substrate and lead to ventricular arrhythmia. Patients with non-ischemic cardiomyopathy who present with ventricular arrhythmia can undergo ablation therapy to eliminate these arrhythmia.

Purpose: The purpose of the study is to determine if DE-MRI is useful to guide mapping of ventricular arrhythmias in patients with non-ischemic cardiomyopathy.

Methods: DE-MRI was performed in 28 consecutive patients (mean age $50 \pm 15$ years) with non-ischemic cardiomyopathy (mean ejection fraction $38 \pm 9 \%$ ) referred for catheter ablation of ventricular tachycardia (VT) or premature ventricular complexes (PVCs). If scar tissue was found on the DE-MRI, the myocardial contours and scar distribution was semi-automatically extracted, in order to generate 3-D maps of scar distribution. These maps were then integrated into the electroanatomic map using the CARTO Merge function (Figure I). This integration involved initial matching of fiducial markers (LV apex, center of mitral valve, aortic outflow tract) in both modalities, and then a 
Figure I (abstract 066)

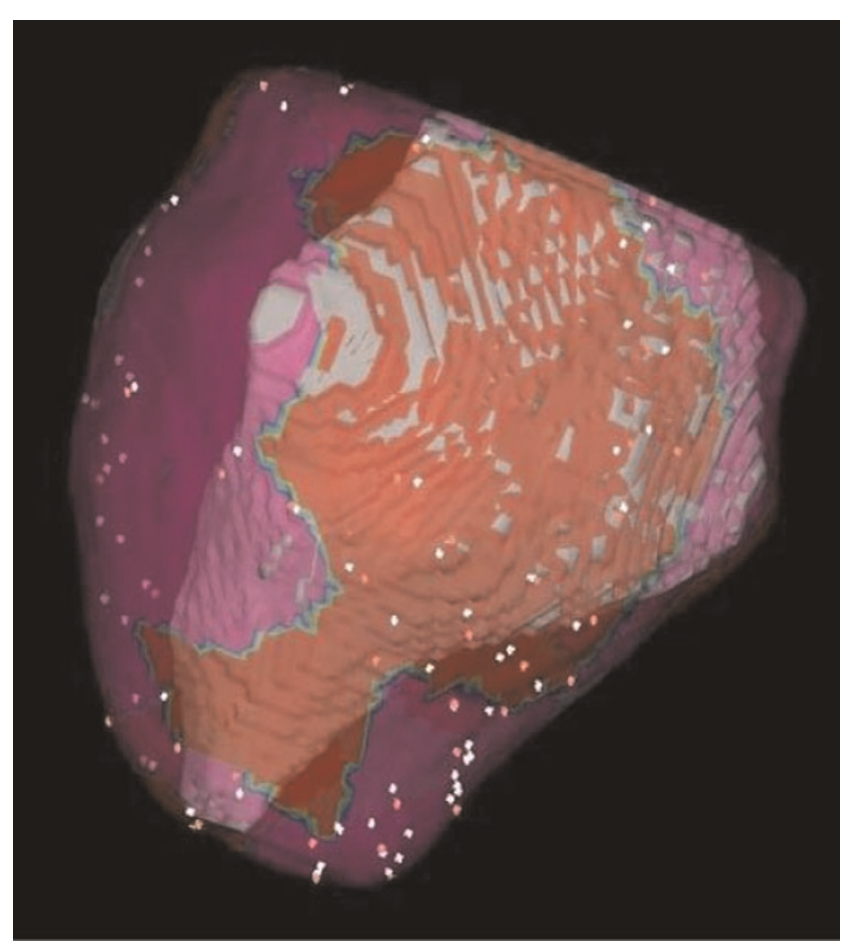

Fused DE-MRI and endocardial maps. The endocardial map is projected on the endocardial surface as determined by MRI. Areas with low voltages are illustrated in red, and areas of normal voltages are in purple. The 3-D distribution of DE on MRI (including both surface and transmural extent) are represented in gray. There is excellent correlation between the distribution of scar as represented by DE on MRI, and the areas of low voltage on the endocardial map.

surface based optimized registration within the CARTO Merge software. Mapping data were correlated with respect to the localization of scar tissue (right ventricular vs left ventricular and endocardial vs epicardial vs intramural).

Results: Scar tissue was identified by DE-MRI in 13 out of 28 patients. Characteristics of these 13 patients were as follow. Patients had either sarcoidosis $(n=3)$ or dilated cardiomyopathy $(n=10)$. They either had a single focus on DE-MRI $(n=5)$ or multifocal disease $(n=8)$. The ventricular arrhythmia were VT $(n=9)$ or PVC $(n=4)$. The distribution was predominantly endocardial $(n=5)$, midmyocardial $(n=4)$, epicardial $(n=2)$ and transmural $(n=2)$. On the electroanatomic map, there was always low voltage present and matching the endocardial or epicardial surface displaying DE on MRI. The size of the endocardial scar on DE-MRI correlated well with the size of the endocardial scar defined by voltage mapping $(45+-14 \mathrm{~cm} 2$, $R=0.94, p<0.000$ I with cutoff of $1.5 \mathrm{mV}$ ). All patients with inducible VT or sustained VT had evidence of DE on MRI. In all patients with DE on MRI where a critical site for the arrhythmia could be identified, this critical site was confined to the scar tissue.

Conclusion: DEMRI in patients without prior infarctions can help to identify the arrhythmogenic substrate; furthermore it helps to plan an appropriate mapping and treatment strategy.

\section{7}

\section{Quantification of myocardial fibrosis by delayed-enhanced MRI in patients with severe aortic valve disease: correlation with quantified histopathology}

Carlos E Rochitte, Clério F Azevedo, Marcelo Nigri, Flavio Tarasoutchi, Pablo M Pommerantzeff, Roney O Sampaio, Max Grinberg and Maria L Higuchi Heart Institute - InCor - University of São Paulo Medical School, São Paulo, Brazil

Journal of Cardiovascular Magnetic Resonance 2009, I I(SuppI I):O67

Introduction: Chronic aortic valve disease is characterized by progressive accumulation of interstitial myocardial fibrosis (MF) and impairment of myocyte ultrastructure. The amount of interstitial MF may play an important role in the transition from well-compensated hypertrophy to overt heart failure in the setting of chronic LV mechanical overload. However, assessment of interstitial MF and myocyte degeneration has only been possible through histological analyses of myocardial fragments from endomyocardial biopsies. Recently, delayed-enhancement magnetic resonance imaging (deMRI) has been shown to provide an accurate assessment of myocardial necrosis and fibrosis.

Purpose: We employed a semi-automatic algorithm for the quantification of MF using deMRI technique in patients with severe aortic valve disease. We sought to determine whether the amount of MF by deMRI demonstrated good correlation when compared with the gold-standard histopathological analyses. In addition, we investigated the relationship between the amount of MF and resting LV function before aortic valve replacement (AVR) surgery.

Methods: Fifty-four patients scheduled to undergo AVR surgery were examined by cine and deMRI in a I.5 T scanner. From the deMRI dataset, the regions of MF were automatically determined as the sum of pixels with signal intensity (SI) above a pre-determined threshold. The definition of this threshold was based on the mean SI of total myocardium, plus the SI variability we would expect from a non-diseased myocardium (free of focal regions of MF), and also taking into consideration the variability introduced by image noise. More specifically, the threshold was calculated as: mean $\mathrm{SI}$ of total myocardium +2 standard deviations (SD) of mean SI of a remote area +2 SD of mean SI of air. The remote area was manually delineated in a myocardial region free of hyper-enhancement. In addition, interstitial MF was quantified by histological analysis of myocardial samples obtained during AVR surgery and stained with picrosirius red. Eight subjects with no previous history and who died of noncardiac causes served as controls for the quantitative histopathology.

Results: The amount of interstitial MF determined by histopathology was higher in patients with aortic valve disease than in controls $(24.6 \pm 9.8 \%$ versus $6.0 \pm 1.8 \%, P<0.000 I)$. For each patient, the amount of interstitial MF was considered abnormally increased when it was higher than mean+2 SD of control interstitial MF, i.e., higher than $9.6 \%(6.0 \%+3.6 \%)$. The amount of MF measured by deMRI was $3.72 \pm 2.17 \%$ for all patients, $4.35 \pm 2.32 \%$ in aortic regurgitation and $3.15 \pm 1.87 \%$ in aortic stenosis subgroup ( $P=0.04$ between subgroups). Quantification of MF by de-MRI showed good correlation with measurements obtained by histopathology $(y=3.10 x+13.0 ; r=0.69$, $P<0.0001)$. Correlation was also good if considered only the 
subgroup of patients with aortic regurgitation $(y=3.09 x+12.3$; $r=0.70, P<0.000 I)$ or aortic stenosis $(y=3.34 x+12.9$; $r=0.67, P=0.000 \mathrm{I})$. Considering histopathology as the method of reference, ROC analysis revealed a good accuracy of deMRI in identifying patients with increased degrees of MF accumulation (area under the curve $=0.92 ; 95 \% \mathrm{Cl} 0.8 \mathrm{I}$ to I.00). In addition, the amount of MF by deMRI showed an inverse correlation with LV EF $(r=-0.67 ; p<0.0001)$ and direct correlations with LV $\operatorname{EDV}(r=0.46 ; p=0.0005)$, LV ESV $(r=0.62 ; p<0.000 I)$ and LV mass $(r=0.46 ; p=0.0005)$ before AVR surgery.

Conclusion: Delayed-enhanced MRI allows for the noninvasive quantification of MF with good accuracy when compared with histopathology in patients with severe aortic valve disease. In addition, the amount of MF by deMRI is associated with worse LV morphological and functional parameters before AVR surgery in this population.

\section{8}

Myocardial fibrosis is a prevalent finding in elite high-endurance athletes

Myra S Cocker', Oliver Strohm', David J Smith², Craig Butler', Israel Belenkie ${ }^{3}$, Willem Meeuwisse ${ }^{4}$ and Matthias G Friedrich'

'Stephenson CMR Centre at the Libin Cardiovascular Institute, University of Calgary, Calgary, AB, Canada

${ }^{2}$ Human Performance Lab, Faculty of Kinesiology, University of Calgary, Calgary, AB, Canada

${ }^{3}$ Department of Cardiac Sciences at the Libin Cardiovascular Institute, University of Calgary, Calgary, AB, Canada ${ }^{4}$ Sports Medicine Centre, University of Calgary, Calgary, $A B$, Canada

Journal of Cardiovascular Magnetic Resonance 2009, I I(SuppI I):O68

Background: Autopsies in athletes have shown that athletes have diffuse myocardial fibrosis, and epidemiological findings place athletes at a greater relative risk of sudden death due to cardiovascular causes. Electrocardiography abnormalities are well documented in athletes, where it is widely accepted that these are characteristic of the 'athlete's heart'. Furthermore, biochemical markers of collagen breakdown and myocardial fibrosis are elevated in athletes. As such, using CMR late Gadolinium enhancement (LE) imaging, we hypothesized that fibrosis is a feature of the athlete's heart and is associated with reduced cardiac function.

Methods: 48 elite athletes ( 25 male, age $32 \pm 13$ years), and a control group of 8 healthy individuals ( 4 male, $31 \pm 9$ years) were prospectively recruited. On a I.5 T MRI system, standard protocols for assessment for LV function and TI-weighted LE were performed.

Two experienced observers assessed LE images visually for the presence of fibrosis. The extent of fibrosis was assessed quantitatively, using semi-automated detection where regions that had signal enhancement above a threshold of 5 standard deviations from the mean signal of healthy myocardium, were considered to represent fibrosis. Areas that had a lack of contrast enhancement were used to define healthy myocardium.

Abbreviations utilized: LVEDVI - LV end-diastolic volume indexedto-height; LVESVI - LV end-systolic volume indexed-to-height; LVSVI LV stroke volume indexed-to-height; LVEF - LV ejection fraction

Results: 37 of 48 (77\%) athletes had non-ischemic diffuse LE, compared with I of 8 (13\%) of controls. The extent of myocardial fibrosis in athletes who had visual evidence for LE was $10.7 \pm 3.3 \%$.
Figure I (abstract 068)

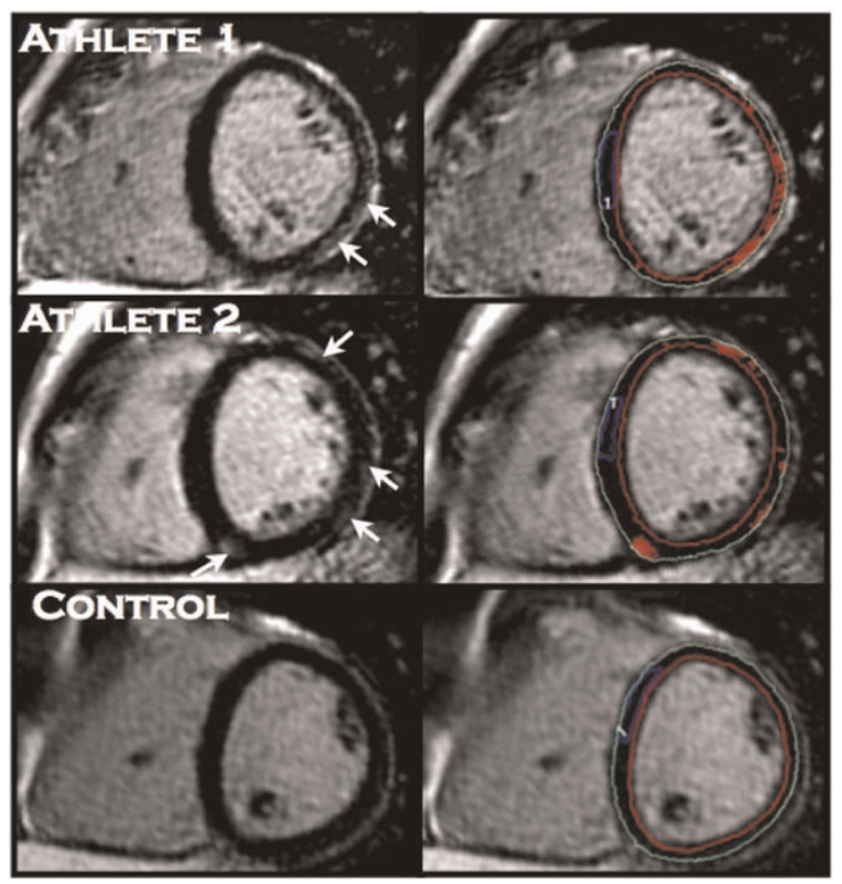

TI-weighted late gadolinium enhancement images. Left panels: Evidence of subepicardial non-ischemic myocardial fibrosis in athletes 1 and 2 (arrows), with none in healthy control. Right panels: Quantification of myocardial fibrosis by manually tracing a region of interest in health remote myocardium (blue outline). Automated computer thresholding set at 5 standard deviations above the mean signal intensity of remote myocardium detects fibrosis (red overlay). The detected extent of fibrosis for athlete $\mathrm{I}$ is $14.12 \%, 11.30 \%$ for athlete 2 and $0.20 \%$ for control.

Athletes with fibrosis had increased LVEDVI (1 $17 \pm 19$ vs. $98 \pm 15 \mathrm{ml} / \mathrm{m}, \mathrm{p}<0.05)$, LVESVI $(44 \pm 1 \mathrm{l}$ vs. $34 \pm 10 \mathrm{ml} / \mathrm{m}$, $p<0.05)$, and LVSVI $(73 \pm I I$ vs. $64 \pm 7 \mathrm{ml} / \mathrm{m}, p<0.05)$, while LVEF ( $63 \pm 5$ vs. $66 \pm 6 \%, p>0.05)$ did not differ, when compared to those who did not have fibrosis. Figure I.

Conclusion: This is the first evidence of myocardial fibrosis being a prevalent finding in elite athletes, and its relation to cardiac morphology with depressed cardiac function. Long-term follow-up is required to assess the impact of fibrosis on outcome and prognosis in the 'athlete's heart'.

\section{9}

Non-rheumatic streptococcal myocarditis mimicking acute ST-segment elevation myocardial infarction: characterization by cardiac magnetic resonance

Rasoul Mokabberi, Jamshid Shirani, Afsaneh Haftbaradaran Mohammadi and William Schiavone Geisinger Medical Center, Danville, PA, USA

Journal of Cardiovascular Magnetic Resonance 2009, I I(SuppI I):069

Introduction: Acute myocarditis may mimic acute ST-segment elevation myocardial infarction (STEMI) and result in unnecessary invasive coronary angiography. Cardiac magnetic resonance 
Table I (abstract 069) Clinical and Imaging Characteristic in 6 Patients

\begin{tabular}{|c|c|c|c|c|c|c|}
\hline Patient & I & 2 & 3 & 4 & 5 & 6 \\
\hline Age (years) & 27 & 35 & 33 & 32 & 29 & 22 \\
\hline Sex & $M$ & $M$ & $M$ & $M$ & $\mathrm{~F}$ & $M$ \\
\hline ECG ST-Elevation & Inferolateral & Inferolateral & Lateral & Lateral & Inferolateral & Inferolateral \\
\hline Echo RWMA & Inferolateral, lateral & Inferior, inferoseptal & Inferior, inferolateral & Inferior & Inferior, inferoseptal & Inferolateral \\
\hline Echo WMSI & 1.24 & 1.12 & 1.24 & 1.12 & 1.12 & 1.12 \\
\hline Echo LVEF (\%) & 40 & 45 & 45 & 57 & 52 & 51 \\
\hline Segments with LGE & NA & 4 & 3 & 3 & 5 & 9 \\
\hline
\end{tabular}

(CMR) can differentiate myocarditis from acute ischemic myocardial injury and provide a basis for adoption of an appropriate treatment strategy in such patients.

Purpose: The aim of this observation was to determine the diagnostic value of CMR in assessment of patients with acute non-rheumatic streptococcal myocarditis.

Methods and results: We evaluated 6 young adults [age 2235 (mean $29 \pm 5$ ) years, 5 men] with acute non-rheumatic streptococcal myocardits following recent (mean 4.8 days prior to presentation) pharyngitis (positive throat culture or rapid antigen test, elevated antistreptolysin antibody). All patients presented with non-pleuritic chest pain and focal ST segment elevation on admission electrocardiogram. Cardiac enzymes (creatine phosphokinase and troponin $\mathrm{T}$ ) were elevated and emergent coronary angiography revealed normal coronary arteries. Two-dimensional transthoracic echocardiography showed regional wall motion abnormality (RWMA) with wall motion score index (WMSI) ranging from I.I2-I.24 and left ventricular ejection fraction (LVEF) ranging from $40-57 \%$, and contrast-enhanced CMR demonstrated characteristic subepicardial late gadolinium enhancement (LGE) involving $4.8 \pm 2.4$ myocardial segments without an early perfusion defect (Table I). Patients were treated with antibiotics and anti-inflammatory agents and showed clinical recovery within 3 days.

Conclusion: Acute non-rheumatic streptococcal myocarditis may mimic STEMI. Clinical history (young individual, preceding pharyngeal infection), laboratory evidence of acute streptococcal infection and characteristic CMR findings can distinguish these patients from those with acute STEMI who benefit from primary coronary intervention.

\section{$\mathbf{O 7 0}$}

Screening for hypertrophic cardiomyopathy: a cost analysis of echocardiography, cardiac magnetic resonance and genetic testing

Thomas H Hauser, Martin S Maron and Warren J Manning Beth Israel Deaconess Medical Center, Boston, MA, USA

Journal of Cardiovascular Magnetic Resonance 2009, I I (SuppI I):O70

Introduction: Hypertrophic cardiomyopathy (HCM) is the most common heritable cardiomyopathy and is the leading cause of sudden cardiac death early in life. Screening of first degree relatives for HCM phenotype is recommended, typically with serial echocardiography (Echo). Recently, cardiovascular magnetic resonance (CMR) and genetic testing (GT) have emerged as additional screening strategies. Up to $5 \%$ of patients with HCM on CMR have normal Echo findings. GT has been proposed as a method for eliminating unnecessary diagnostic imaging tests in genotype negative family members in families identified to have a disease causing mutation, present approximately $50 \%$ of the time. Because large, long-term trials to compare these methods are not feasible, modeling of costs and outcomes can provide insight into the potential costs and benefits of various screening strategies.

Purpose: The objective of this study is to evaluate the costs and benefits of screening strategies of HCM using Echo, CMR and GT.

Methods: We developed a Markov model to evaluate 5 screening strategies: Echo, CMR, Echo and GT, CMR and GT, and Echo, CMR and GT. All screening strategies were designed based on standard guidelines with first testing at age 13, with imaging with Echo and/or CMR every 2 years until age 25 and every 7 years until age 50 . GT was performed once only at the start of screening. The model was tested using a theoretical cohort of 1000 probands with an average of 4 first degree relatives that required testing. The base case assumed an Echo cost of $\$ 650$, CMR cost of $\$ 800$, and GT cost of $\$ 3000$. The penetrance of HCM was modeled such that, of those destined to have $\mathrm{HCM}, 90 \%$ of screened first degree relatives would have phenotypic HCM by age 25 with the remaining $10 \%$ evident by age 50 . Echo was assumed to have a $5 \%$ error rate compared to CMR. The analysis was performed from the societal perspective using constant 2008 dollars. A 3\% discount rate was used.

Results: GT resulted in increased costs with no improvement in diagnostic accuracy when paired with either Echo or CMR. Echo was the least expensive strategy with a cost per patient screened of $\$ 3754$, but did not identify phenotypic HCM in $6 \%$.

Figure I (abstract 070)

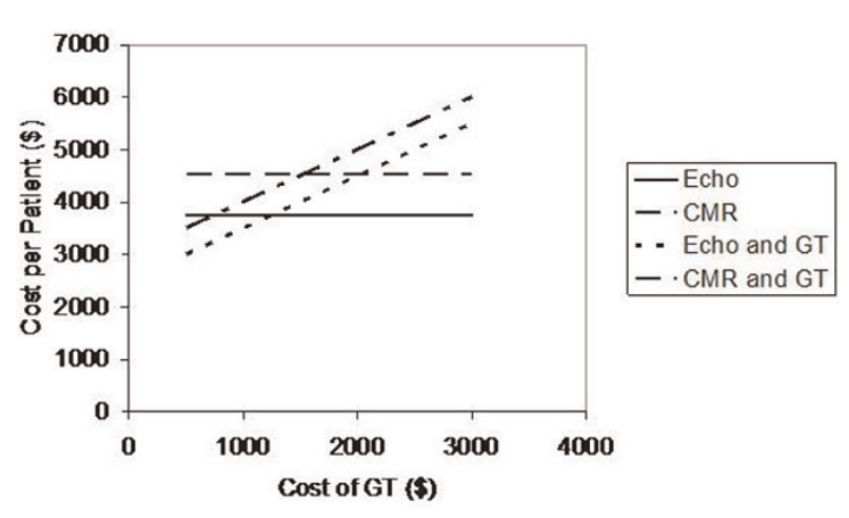

Cost per patient for HCM screening. 
CMR cost $\$ 4546$ per patient screened and did not identify I\% of patients with phenotypic HCM. The incremental cost for each additional diagnosis of HCM with CMR was $\$ 15,840$. The addition of Echo to CMR increases costs without any increase in diagnostic accuracy. Sensitivity analysis showed that the cost of GT had a large influence on the analysis. The break even cost for GT was \$I 243 when used with Echo and \$I530 when used with CMR (figure I).

Conclusion: In this model of screening for HCM, GT increased costs without improving outcome. Screening with CMR identified a larger proportion of patients with HCM with an associated increase in cost.

\section{O7I}

Impaired aortic distensibility determined by magnetic resonance imaging in patients with different bicuspid aortic valve phenotypes Thananya Boonyasirinant, Randolph M Setser, Milind $Y$ Desai and Scott D Flamm Cleveland Clinic, Cleveland, $\mathrm{OH}$, USA

Journal of Cardiovascular Magnetic Resonance 2009, I I(SuppI I):O7 I

Introduction: Beyond the morphologic and functional abnormalities of the bicuspid aortic valve (BAV) there is also intrinsic pathology of the aortic wall, manifested by potentially lethal complications such as aortic aneurysm or dissection. Aortic distensibility and compliance are impaired in atherosclerotic aortic aneurysms and Marfan syndrome. Similar abnormalities of compliance are felt to occur in the setting of $\mathrm{BAV}$, though this has been little studied with velocity-encoded magnetic resonance imaging (VENC-MRI), and further there is no data on the influence of BAV morphology on this abnormality. VENC-MRI is a potent non-invasive technique to determine aortic distensibility via aortic pulse wave velocity (PWV) measurements; in addition these measurements do not depend on knowledge of central arterial pressure or geometrical assumptions that may limit alternative measurement tools.

\section{Figure I (abstract 07I)}
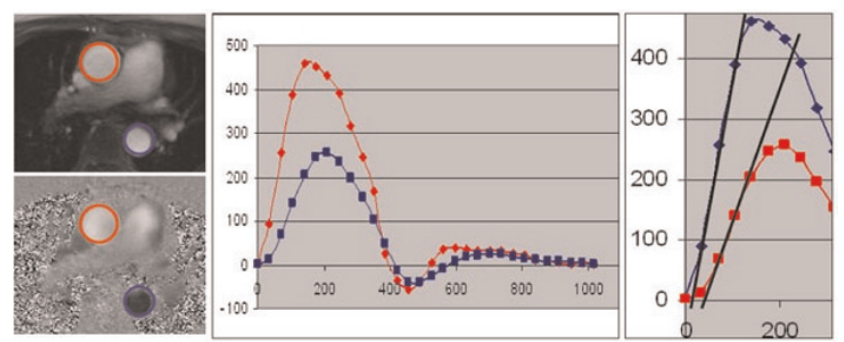

Left panel: Through-plane velocity-encoding magentic resonance imaging ascending (red circle) and proximal descending aorta (blue circle). Middle panel: Corresponding flow measurement at ascending (red line) and proximal descending aorta (blue line). Right panel: The measurement of arrival time at ascending and proximal descending aorta. The pulse wave velocity was calculated as the aortic path length between these 2 sites divided by the time delay between the arrival of the foot pulse wave at these 2 sites.
Figure 2 (abstract 07I)

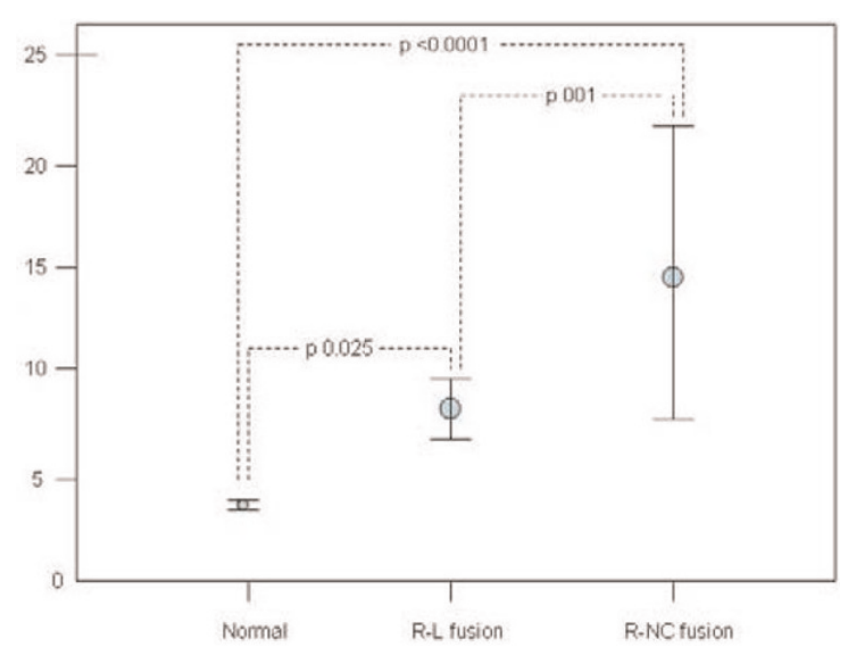

The pulse wave velocity (PWV) in normal patients, right and left (R-L) fusion, and right and non-coronary (R-NC) bicuspid aortic valve patients. Circal, mean and whiskers. $95 \%$ confidence intervals.

Purpose: We sought to assess thoracic aortic distensibility by PWV measurements from VENC-MRI in patients with bicuspid aortic valve, and to determine if differences exist between different BAV phenotypes.

Methods: VENC-MRI was performed in 100 BAV patients and 35 controls (trileaflet aortic valve without dysfunction, and no aortic aneurysm). The PWV was determined between the mid ascending and proximal descending aorta. Velocity measurements were made perpendicular to the long axis of the mid ascending and proximal descending thoracic aorta. The aortic path length between the two locations was directly measured from threedimensional reconstruction in the oblique sagittal orientation encompassing the aortic arch. The BAV phenotypes were imaged using a cine-SSFP or cine-GRE across the face of the aortic valve, and then classified as: right-left cusp fusion (R-L fusion), right and non-coronary cusp fusion (R-NC fusion), and left and noncoronary cusp fusion (L-NC fusion).

Results: BAV phenotypic classification: 76 R-L, 23 R-NC, and I L-NC fusion were identified. Mean age was not significantly different among patients with R-L fusion, R-NC fusion, and controls (49.0, 49.6, and 45.3 years, respectively; $p=N S)$. BAV patients revealed increased PWV compared to controls ( 9.8 vs. $3.8 \mathrm{~m} / \mathrm{s} ; \mathrm{P}<0.000 \mathrm{I})$. Furthermore, $\mathrm{PWV}$ was significantly different among patients with R-NC fusion, R-L fusion phenotypes, and controls (I4.9, 8.0, and $3.8 \mathrm{~m} / \mathrm{s}$, respectively; $\mathrm{p}<0.000 \mathrm{I})$. Figures I and 2 .

Conclusion: This study, in the largest cohort of BAV patients studied with MRI to date, has identified significantly diminished aortic distensibility (increased PWV from VENC-MRI) compared to controls. Further, this study is the first to demonstrate greater impairment of aortic distensibility in the phenotype of R-NC fusion as compared to R-L fusion. There is a clear association between impaired aortic distensibility and aortic valve configuration; the greater impairment in aortic distensibility in BAV with R-NC fusion phenotype raises concern for amplifica- 
tion of aortic pathology. This differentiation based on valvular phenotype suggests a potentially novel parameter for enhanced surveillance and potentially altered surgical triage in this high risk group.

\section{2}

A short-term, high fat diet impairs cardiac high energy phosphate metabolism, without change in cardiac function Cameorn J Holloway, Yaso Emmanuel, Lowri E Cochlinl, Cezary Szmigielski, Lindsay M Edwards, Jane M Francis, Stefan Neubauer and Kieran Clarke University of Oxford, Oxford, UK

Journal of Cardiovascular Magnetic Resonance 2009, I I(SuppI I):O72

Introduction: Heart failure patients have low cardiac phosphocreatine/ATP (PCr/ATP) ratios, which may be related to elevated circulating free fatty acids (FFAs).

Purpose: We tested whether raising plasma FFAs, using diet, causes abnormalities in cardiac energetics or function.

Methods: Healthy males ( $n=16$, age $22 \pm$ I years), recruited from the University of Oxford, were randomised to five days of a high fat diet (HFD) containing $75 \pm 1 \%$ of calorie intake through fat consumption, or an isocaloric control diet, providing $23 \pm 1 \%$ of calorie intake as fat. In a cross-over design, subjects undertook the alternate diet after a two week wash out period. Cardiac 3IP magnetic resonance (MR) spectroscopy was performed to assess PCr/ATP before and after the diets. MR imaging and echocardiography were performed to assess left ventricular function.

Results: Subjects on the HFD had a two-fold elevation in plasma FFAs, I2\% lower cardiac PCr/ATP with no change in cardiac systolic or diastolic function. Figure I.

Conclusion: We have shown a short term, high fat diet raised plasma FFA concentrations, impaired myocardial energetics without effect on systolic or diastolic function. This suggests that high plasma FFAs may be detrimental for heart in normal subjects and shows a potential mechanism of impairment in heart failure patients.

Figure I (abstract 072)

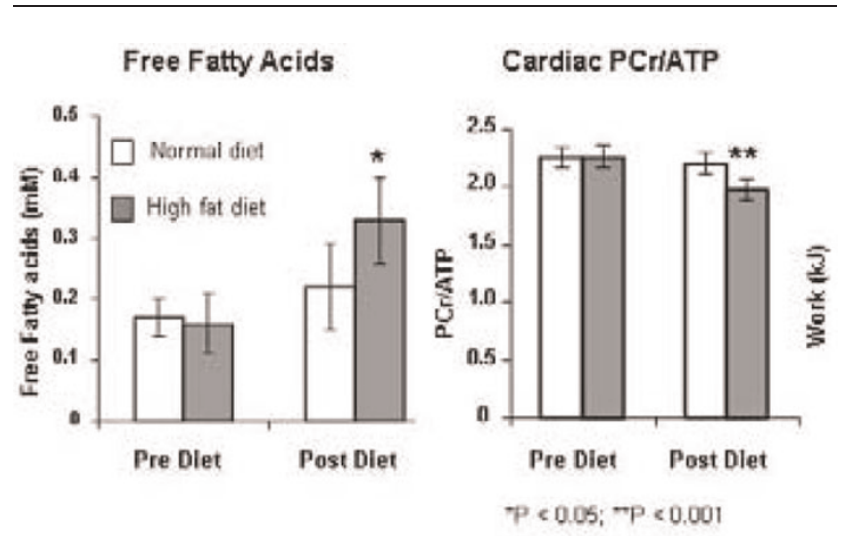

\section{3}

Relationship of ostial pulmonary vein scar with reduction in pulmonary vein size after atrial fibrillation ablation

Thomas H Hauser, Dana C Peters, John Wylie, Catherine Lau, Mark E Josephson and Warren J Manning Beth Israel Deaconess Medical Center, Boston, MA, USA

Journal of Cardiovascular Magnetic Resonance 2009, I I(SuppI I):O73

Introduction: Atrial fibrillation (AF) is the most common sustained arrhythmia. Ablation procedures to electrically isolate the pulmonary veins (PV) from the left atrium have become increasingly popular for the prevention of recurrent AF. PV stenosis is a rare but serious complication of the procedure, thought to be due to scarring of the PV. Previous studies have shown that the intensity of ablation is related to the reduction in PV size after the procedure, but direct assessment of scar in patients has not been performed.

Purpose: We sought to define the relationship of the change $\mathrm{PV}$ size after AF ablation with ostial PV scar as determined by late gadolinium enhancement (LGE) cardiovascular magnetic resonance (CMR).

Methods: We performed 3D breath-held contrast-enhanced CMR angiography of the PV before and after AF ablation using a I.5 T MR system. The diameter and cross sectional area (CSA) were determined in the sagittal plane using a previously published method. LGE CMR of the left atrium and PV was obtained after AF ablation using a high-resolution, 3D, navigator gated technique. The scar volume at the ostium of each PV was measured using a threshold technique. The scar volume was normalized to the PV CSA. The change in PV diameter and CSA was expressed as the percentage change compared to the pre-AF ablation PV measurement. The change in PV size before and after evaluation was evaluated using a paired $T$ test. The relationship of the change in PV diameter and CSA to ostial PV scar was evaluated with standard correlation and linear regression.

Figure I (abstract 073)

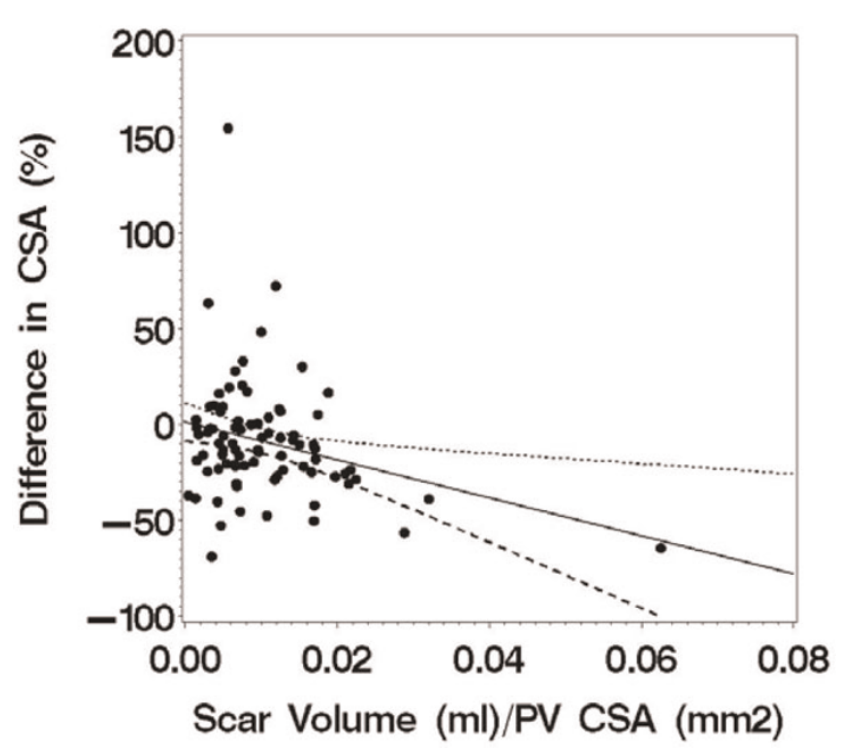


Results: The study cohort was comprised of 23 subjects ( 3 women, age $58 \pm 13$ years). CMR was performed $41 \pm 17$ days after $A F$ ablation. The left sided PV had a common origin in 5 subjects. Scar could not be assessed in one PV due to artifact. A total of 85 PV were available for analysis. Mean PV diameter was $22 \pm 7 \mathrm{~mm}$ before ablation and declined to $21 \pm 6 \mathrm{~mm}$ after $(p=0.001)$ while mean PV CSA declined from $285 \pm 141 \mathrm{~mm}^{2}$ before ablation to $246 \pm 110 \mathrm{~mm}^{2}$ after $(p<0.001)$. A significant correlation was found between ostial PV scar with both the change in PV diameter $(r=-0.21, P=0.049)$ and was even stronger with PV CSA $(r=-0.28, p=0.010$, figure I).

Conclusion: PV diameter and CSA significantly decrease after $\mathrm{AF}$ ablation. There is a linear relationship between these changes and the magnitude of PV scar as measured by LGE MR.

\section{4}

Right ventricular dysfunction and injury following marathon running: correlating biomarkers with cardiac MRI

Negareh Mousavi', Andrew Czarnecki', Kanwal Kumar', Nazanin Fallah-Rad', Matthew Lytwyn', Song-Yee Han', Andrew Francis', lain D Kirkpatrick', Tomas G Neilan², Sat Sharma' and Davinder S Jassal'

'St. Boniface General Hospital, University of Manitoba, Winnipeg, MB, Canada

${ }^{2}$ Massachusetts General Hospital, Harvard Medical School, Boston, MA, USA

Journal of Cardiovascular Magnetic Resonance 2009, I I (SuppI I):O74

Background: Although previous studies including endurance athletes following marathon running have demonstrated biochemical evidence of cardiac injury and have correlated these findings with echocardiographic evidence of cardiac dysfunction, in particular the right ventricle, a study of marathon athletes incorporating biomarkers, echocardiography and cardiac MRI (CMR) has not been performed to date.

Objective: To demonstrate the cardiac changes associated with participation in a marathon using serial cardiac biomarkers, echocardiography and CMR.

Results: Fourteen participants (mean age $33 \pm 6$ years; 8 males) completed the full marathon. Serum myoglobin, creatine kinase and troponin $\mathrm{T}$ were elevated in all athletes post-race. There was a strong linear correlation between right ventricular (RV) fractional area change (FAC) as assessed by echocardiography and RV ejection fraction as assessed by CMR $(r=0.96)$ post marathon (Figure I). RV function, using echocardiography, transiently decreased from pre- to post-race (RV FAC $43 \pm 5 \%$ vs. $34 \pm 7 \%, p<0.05)$. There were also post-race changes in LV and RV diastolic filling. While RV systolic changes were transient, both LV and RV diastolic abnormalities persisted up to one week post marathon. We did not find evidence of delayed enhancement of the LV myocardium on CMR suggesting that the increase in cardiac biomarkers post-marathon is not due to myocardial necrosis.

Conclusion: Right ventricular systolic dysfunction transiently occurs post marathon, and has been validated for the first time by CMR. The increase in cardiac troponin following marathon running is due to cytosolic release of the biomarker, and not due to true breakdown of the myocyte as confirmed by delayed enhancement CMR.
Figure I (abstract 074)

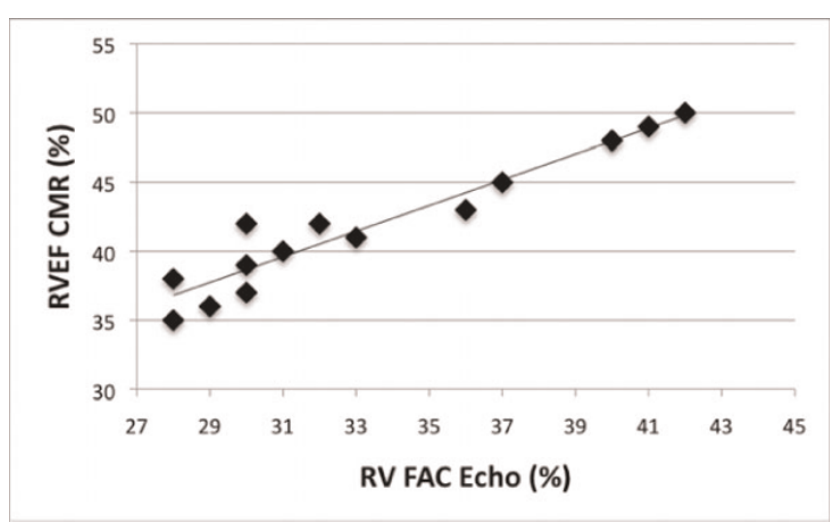

\section{$\mathbf{O 7 5}$}

Cardiovascular magnetic resonance imaging for the assessment of cardiac inflammation and injury following prolonged exercise Rory O' Hanlon ', Gregory P Whyte ${ }^{2}$, Gillan Smith', Francisco Alpendurada', Joyce Wong', Matthew Wilsonl ${ }^{3}$, David Oxborough ${ }^{4}$, Richard Godfrey, Keith George ${ }^{6}$, Annette Dahl', David Gaze ${ }^{7}$, Dudley J Pennell ${ }^{1}$ and Sanjay K Prasad'

${ }^{I}$ Royal Brompton Hospital, London, UK

${ }^{2}$ Institute for Sport and Exercise Science, Liverpool, UK

${ }^{3}$ University of Wolverhampton, Wolverhampton, UK

${ }^{4}$ University of Leeds, Leeds, UK

${ }^{5}$ Brunel University, London, UK

${ }^{6}$ Liverpool John Moores University, Liverpool, UK

${ }^{7}$ St George's Hospital, London, UK

Journal of Cardiovascular Magnetic Resonance 2009, I I(SuppI I):O75

Introduction: Acute bouts of ultra-endurance exercise may be deleterious for cardiac structure and function as demonstrated by a reduction in diastolic and systolic function concomitant, and elevations in humoral markers of cardiac myocyte damage above acute myocardial infarction cut-off levels - most notably reflected by an elevation in troponin levels. A possible mechanism of biomarker release may be secondary to acute myocardial inflammation. CMR is established as the imaging modality of choice to visualize myocardial inflammation and fibrosis using STIR (short tau inversion recovery) imaging and early/delayed enhancement imaging following intravenous contrast administration. The relationship between ultraendurance exercise, biomarker elevation and CMR imaging for inflammation and fibrosis has not been studied before.

Aims: We proposed that acute ultra endurance exercise in moderately trained athletes leading to elevation of cardiac troponin is associated with CMR detectable myocardial inflammation

Methods: We performed CMR in 18 male athletes of moderate to high fitness levels 24 hrs pre and 6 hours post a marathon run. Each scan was performed by the same operator on a $1.5 \mathrm{~T}$ Siemens Avanto scanner using a 4 channel body array coil. Myocardial structure and function was assessed using breathhold SSFP cine imaging in long and short axis views. The presence of 
myocardial inflammation and oedema was assessed using STIR imaging and a 3-4 minute spin echo sequence immediately post $0.1 \mathrm{mmol}$ intravenous gadolinium-DTPA (Magnavist, Schering, Germany) given using an automated injector, to assess relative gadolinium enhancement. Inversion recovery segmented-FLASH imaging with $\mathrm{TI}$ adjusted to null normal myocardium to highlight regions of fibrosis was used to image delayed enhancement. Each subject had bloods drawn for Tnl, NT-proBNP, and CRP at baseline, immediately post and 6 hours after the run. Each CMR scan was analyzed by a blinded observer for wall thickness per segment, volumes and function, myocardial oedema using customized software (CMRTools, London, UK). Delayed enhancement images were analysed using Medis software (MASS Medis, Leiden) using a full width half maximum technique. Results: All 18 subjects completed the study protocol and none complained of any cardiovascular symptoms. Biventricular volumes, stroke volume, ejection fraction, and mass were unchanged pre and 6 hrs post marathon. The majority of subjects were found to have a rise in BTproBNP levels immediately and 6 hours after the race as well as elevations in Tnl above the level of cut off for myocardial infarction $(P=0.00 I)$. There were no focal regions of visual signal increase on the STIR images in any of the 18 subjects. Global myocardial oedema was predefined using a cut off ratio of 1.9 comparing $\mathrm{SI}$ of myocardium to skeletal muscle on STIR imaging, and a relative $45 \%$ increase in the SI myocardium/skeletal muscle immediately post intravenous gadolinium on relative gadolinium enhancement imaging ( $r G E)$. No subject reached these cut off values. None had any visual myocardial fibrosis on late enhancement imaging or using automated software (MASS, Medis, Leiden).

Conclusion: Serum markers of myocardial cell damage post ultra endurance exercise are not associated with CMR detectable levels of myocardial oedema, inflammation or scarring. These findings suggest lower degrees of myocardial damage than that normally sustained in patients with acute myocardial infarction or myocarditis, inspite of similar levels of troponin elevation.

\section{6}

Molecular imaging of atherosclerotic plaque targeted to oxidized LDL receptor LOX-I using magnetic resonance

Dayuan Li, Amit R Patel, Alexander Klibanov, Christopher M Kramer, Rene J Roy, Mirta Ruiz, David K Glover, George A Beller and Craig H Meyer University of Virginia, Charlottesville, VA, USA

Journal of Cardiovascular Magnetic Resonance 2009, I I(SuppI I):O76

Background and objectives: Oxidized low-density lipoprotein and its receptor LOX-I play a crucial role in the initiation, progression, and destabilization of atherosclerotic lesions. A noninvasive tool to improve the clinical characterization of this pathological process is needed. The aim of this study was to assess the feasibility of CMR based molecular imaging targeted to LOX-I which is highly expressed on atherosclerotic lesions in mice.

Materials and methods: LDLR-/- mice on an atherogenic diet for $>16$ weeks were used. The imaging probe consisted of liposomes decorated with anti-LOX-I antibody (or nonspecific $\operatorname{lgG}$ ), gadolinium and Dil fluorescence markers. MRI at 7.0 T (Clinscan, Bruker/Siemens) was performed at baseline and $24 \mathrm{hrs}$ after intravenous injection of I50 $\mu \mathrm{l}$ of probe containing LOX-I
Figure I (abstract 076)
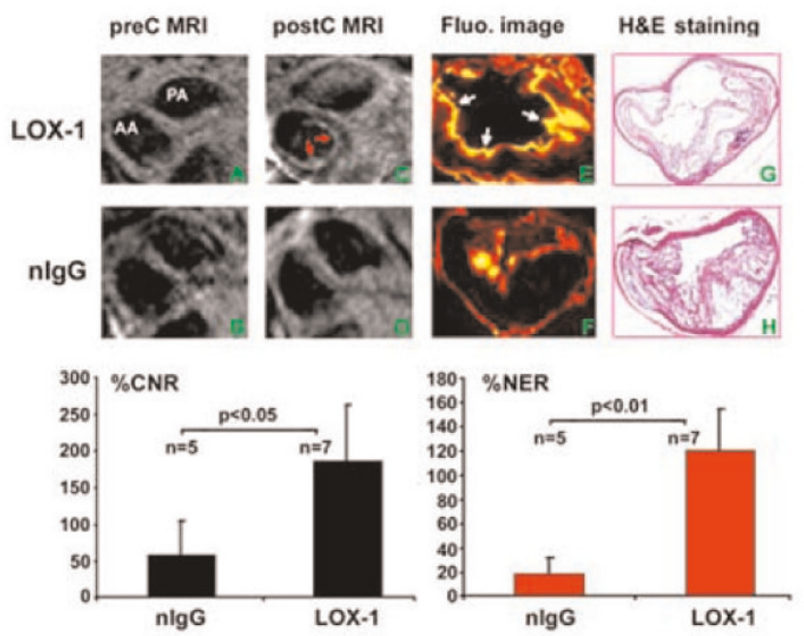

antibody $(n=7)$ or nonspecific $\lg G(n \lg G)(n=5)$ with $0.075 \mathrm{mmol} \mathrm{Gd} / \mathrm{kg}$, followed by excision of the aorta for frozen cross-sections. The fluorescence image used to indicate whether the probe bound to the plaque was examined under fluorescence microscopy.

MRI of the ascending aorta was performed with a TI-weighted black-blood spiral gradient-echo sequence (echo time, I.2 ms; flip angle, $90^{\circ}$; field of view, $3 \times 3 \mathrm{~cm}$; 135 interleaves; readout window, $4.1 \mathrm{~ms}$; spatial resolution, $67 \mu \mathrm{m})$ ) with II contiguous $0.5 \mathrm{~mm}$-thick slices. Four signal averages with cardiac and respiratory gating were used, for a total imaging time of 2.5 minutes per slice. For the post-injection scan, the slices were matched to the baseline preinjection scan by using the left main and LAD coronary artery as anatomic landmarks.

To quantitatively analyze the MRI results, signal intensity (SI) was measured in 4 regions of interest within the aortic wall as well as the aortic lumen and muscle on each slice at both time points. The standard deviation of noise was also recorded for each slice. These measurements were recorded for all slices at every time point imaged. The contrast-to-noise ratio (CNR) of aortic wall to lumen was calculated for each slice. \%CNR $=$ (CNRpostCNRprecontrast)/CNRprecontrast. The normalized enhancement ratio (NER) was defined as the average post-contrast $\mathrm{SI}$ from 4 regions of interest within the aortic wall divided by the muscle $\mathrm{SI}$ in the same slice and then divided by the pre-contrast SI. \%NER $=($ NER-I $) \times 100$.

Results: Fluorescence imaging found that the LDLR-/- mice injected with the LOX-I antibody probe showed significant uptake in atherosclerotic plaque (Fig. IE, white arrows). There was little fluorescence signal in atherosclerotic plaques in LDLR-/- mice that received the nlgG probe (Fig. IF). The MR images consistently showed strong post-contrast signal (red arrows, C) on atherosclerotic plaques at 24 hours in LDLR-Imice injected with LOX-I antibody probe, but not those injected with nlgG probe (Fig. ID). The \% CNR was significantly higher at 24 hours in LDLR-I- mice that received the probe with LOX-I antibody compared to nlgG (I87.4 $\pm 7 \mid .5 \%$ vs. $58.4 \pm 46.9 \%$, 
$P<0.05$, Fig I, bottom left panel). Accordingly, the \%NER was also significantly higher at 24 hours in LDLR-/- mice that received the probe with LOX-I antibody compared to nlgG $(|2| . \mid \pm 38.9 \%$ vs. $18.4 \pm 13.9 \%, p<0.01$, Fig I, bottom right panel). The atherosclerotic lesions were similar between the 2 groups as determined by H\&E staining of aortic cross-sections (G\&H).

Conclusion: MRI with liposomes containing gadolinium and LOX-I antibody demonstrates specific targeting of atherosclerotic plaques with high contrast to noise ratios. Spiral imaging produced high spatial resolution without motion artifacts and black blood imaging improved visualization of vessel wall. Further study on imaging of LOX-I may provide more detailed characterization of atherosclerotic plaque in vivo. This technique shows significant promise for molecular MR imaging of atherosclerosis.

\section{7}

Controlling ventricular preload using an MRI-compatible lower body negative pressure chamber: measuring changes in volumes, mechanical and hemodynamic function Richard Thompson', Ben Esch', Jessica Scott ${ }^{2}$, June Cheng Baron', Kelvin Chow', lan Paterson' and Mark Haykowsky'

'University of Alberta, Edmonton, AB, Canada

${ }^{2}$ University of British Columbia, Vancouver, BC, Canada

Journal of Cardiovascular Magnetic Resonance 2009, I I(SuppI I):O77

Introduction: Cardiac output is dependent, in part, on the ability of LV to accept preload at low filling pressure. Systematic modulation of preload is thus an important capability for the study of the preload dependence of any given aspect of cardiac performance in health and disease. Previously, LV (un)loading has been studied by control of lower body pressure, used to

Table I (abstract 077) Heart rate and volumes and function

\begin{tabular}{llllll}
\hline & HR* & EDV $(\mathbf{m L}) * *$ & ESV $(\mathbf{m L})$ & SV(mL)** & EF(\%)* \\
\hline 0 & $60.6(10.7)$ & $177.6(28.6)$ & $68.7(16.3)$ & $109.0(14.8)$ & $61.6(3.7)$ \\
$-30 \mathrm{mmHg}$ & $63.5(9.7)$ & $153.1(25.6)$ & $65.8(17.1)$ & $87.2(10.6)$ & $57.5(4.4)$ \\
\hline
\end{tabular}

modulated central blood volume, primarily in conjunction with echocardiographic or invasive measures of LV volumes and systolic function [I, 2, 3]. MRI offers the gold standard measures of LV volumes and a growing number of functional parameters based on tissue and blood dynamics, but has not previously been used in conjunction with lower body pressure control. Using a low-cost custom-made MRI-compatible lower body pressure chamber we illustrate controllable preload modulation of LV volumes and mechanical and hemodynamic functional parameters (several of which have not previously been measured with variable preload).

Methods: MRI-Compatible Pressure Chamber: A sealed and flexible pressure chamber was constructed from a waterbed mattress using a thin flexible veneer wood frame and a kayak water skirt for subject entry (Figure I). A compact $20 \mathrm{HP}$ vacuum was used to pressurize the system with a supine subject in place (tested for pressures of -50 to $50 \mathrm{mmHg}$ ). 10 healthy male subjects $(3 \mathrm{I} \pm 9 \mathrm{yrs})$ were studied at atmospheric and -30 $\mathrm{mmHg}$ box pressures (serially); the later is comparable the unloading provided by standing. A 5 element cardiac receiver was used in all subjects, who were studied with conventional cines, phase contrast (LAX and SAX at base) and tissue tagging (5 SAX, $3 \mathrm{LAX}$ slices) to measure a wide range of systolic and diastolic parameters; EDV, ESV, SV, EF, E and A filling waves (peak filling velocity $(\mathrm{cm} / \mathrm{s})$ and filling rate $(\mathrm{mL} / \mathrm{s})$ ), intraventricular (IVPG) and atrial (IAPG) pressure gradients (calculated from LAX phase contrast data), peak systolic (S') and diastolic (E') annular velocities $(\mathrm{cm} / \mathrm{s})$, peak torsion (deg) and rate of untwisting (deg/sec), peak diastolic radial velocity (ventricular average $-\mathrm{cm} /$ s), and peak diastolic circumferential strain rate (ventricular average, $\mathrm{s}^{-1}$ ). All studies were breath held with ECG gating (Siemens Sonata, I.5 T).

Results: All subjects were comfortable throughout volume unloading experiments ( 20 minutes). Tables I, 2 and 3 summarize the changes in physiologic parameters with volume unloading. All values are reported as mean (SD). A paired t-test was used to determine if changes in parameters are significant $(* p<0.05$, ** $\mathrm{p}<0.00 \mathrm{I})$.

Conclusion: We have shown that a simple MRI-compatible lower body pressure chamber can significantly unload the LV and that a comprehensive systolic and diastolic function study is feasible during this unloading (45 minute study duration for both atmospheric and $-30 \mathrm{mmHg}$ unloading). Our changes in EDV and

Table 2 (abstract 077) Hemodynamics

\begin{tabular}{lllllll}
\hline & $\mathbf{E}(\mathbf{c m} / \mathbf{s})^{* *}$ & $\mathbf{A}(\mathbf{c m} / \mathbf{s})^{*}$ & $\mathbf{E}_{\mathrm{Vol}}(\mathbf{m L} / \mathbf{s})^{* *}$ & $\mathbf{A}_{\mathrm{Vol}}(\mathbf{m L} / \mathbf{s})^{*}$ & IVPG $_{\text {peak }}(\mathbf{m m H g})^{* *}$ & IAPG $_{\text {peak }}(\mathbf{m m H g})^{* *}$ \\
\hline 0 & $61.6(7.7)$ & $34.0(9.4)$ & $567(109)$ & $258(50)$ & $2.9(1.2)$ & $1.9(0.4)$ \\
$-30 \mathrm{mmHg}$ & $46.5(10.4)$ & $25.9(11.6)$ & $371(57)$ & $208(47)$ & $1.8(0.7)$ & $1.3(0.4)$ \\
\hline
\end{tabular}

Table 3 (abstract 077) Tissue mechanics

\begin{tabular}{|c|c|c|c|c|c|c|}
\hline & $\begin{array}{l}\mathbf{E}^{\prime} \\
(\mathrm{cm} / \mathrm{s})^{* *}\end{array}$ & $\mathrm{~S}^{\prime}(\mathrm{cm} / \mathrm{s})$ & $\begin{array}{l}\text { Peak } \\
\text { Torsion(deg)* }\end{array}$ & $\begin{array}{l}\text { Peak } \\
\text { Untwisting } \\
\text { Rate (deg/sec) }\end{array}$ & $\begin{array}{l}\text { Radial } \\
\text { Velocity }(\mathrm{cm} / \mathrm{s}) * *\end{array}$ & $\begin{array}{l}\text { Circumferential } \\
\text { Strain rate }\left(\mathrm{s}^{-1}\right)^{* *}\end{array}$ \\
\hline 0 & $14.7(3.1)$ & $8.3(3.0)$ & II.I(2.I) & I57(28) & $4.4(0.9)$ & $1.60(0.24)$ \\
\hline$-30 \mathrm{mmHg}$ & $9.7(2.0)$ & $8.8(2.0)$ & $13.4(2.8)$ & $162(32)$ & $3.5(0.7)$ & $1.37(0.21)$ \\
\hline
\end{tabular}


Figure I (abstract 077)

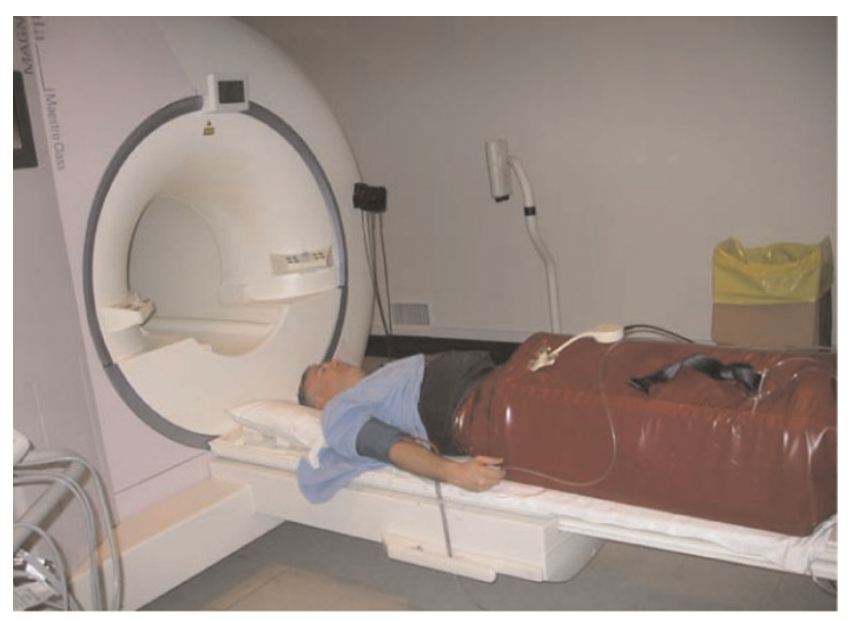

Volunteer in the lower body pressure chamber.

SV and standard measures of early filling (E, A and E') are comparable to previous echocardiography unloading studies[I, 2, 3] and we report significantly larger decreases in pressure gradients than previous studies[I]. To our knowledge, this is the first report of the unloading dependence of torsion (which is shown to increase with unloading), untwisting rate and radial and circumferential parameters. The superior quantitative functional imaging capabilities of MRI in combination with variable loading conditions enabled by the lower body pressure control will allow detailed physiological studies in controls and any patient group that can be studied using MRI.

References

I. Popovic ZB, et al: American journal of physiology 2006, 290: HI454-I459.

2. Firstenberg MS, et al: Journal of the American College of Cardiology 2000, 36: 1664-1669.

3. Levine BD, et al: Circulation 1991, 84:1016-1023.

\section{8}

The MR-stethoscope: safe cardiac gating free of interference with electro-magnetic fields at I.5 T, 3.0 T and 7.0 T

Frauenrath Tobias ${ }^{i}$, Sebastian Kozerke ${ }^{2}$, Fabian Henzel ${ }^{\prime}$ and Thoralf Niendorf'

${ }^{I}$ RWTH Aachen, Aachen, Germany

${ }^{2}$ Institute for Biomedical Engineering, University and ETH Zurich, Zurich, Switzerland

Journal of Cardiovascular Magnetic Resonance 2009, I I(SuppI I):O78

Introduction: In clinical CVMR, cardiac motion is commonly dealt with using ECG-gating. ECG, being an inherently electrical measurement, is prone to lead and patient burns. Furthermore, ECG is corrupted by interferences with electromagnetic fields and by magneto-hydrodynamic effects. Consequently, artifacts in the ECG trace and T-wave elevation might be mis-interpreted as $R$ waves resulting in erroneous triggering together with motion corrupted image quality - an issue which is pronounced at (ultra) high fields.
Figure I (abstract 078)
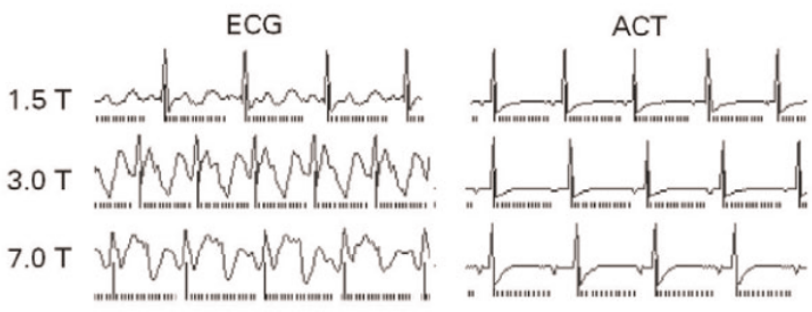

Purpose: Overcome the challenges of conventional ECGgating by developing an acoustic cardiac gating approach, which offers (i) no risk of high voltage induction and patient burns, (ii) immunity to electromagnetic interferences, (iii) suitability for all magnetic field strengths and ease of use for the pursuit of robust and safe clinical CVMR. For this purpose, this study examines and demonstrates the suitability, efficacy and robustness of acoustic cardiac triggering (ACT) in CVMR applications at I.5 T, 3.0 T and 7.0 $\mathrm{T}$ including prospective gating and retrospective triggering regimes.

Methods: The acoustic gating device consists of three main components: (i) an acoustic sensor, (ii) a signal processing unit and (iii) a coupler unit to the MRI system. An acoustic wave guide was used for signal transmission while accomplishing galvanic decoupling. Signal conditioning and conversion were conducted outside of the scanner room using dedicated electronic circuits. All scanner and gradient coil noise contributions to the acoustic signal were cancelled using a 3 rd order inverse Chebychev filter. The final waveform was delivered to the internal physiological signal controller circuitry of a clinical MR scanner. The current implementation connects the trigger signal with the MR-scanner's standard ECG-signal input. Hence, no changes to the MR system's hardware and software are required. Volunteer studies $(n=10)$ were performed on I.5 T, 3.0 T and 7.0 T whole body MR systems (Achieva, Philips, Best, The Netherlands). The acoustic sensor was positioned at the anterior left side of the torso to obtain acoustic cardiograms. For comparison, ECG was recorded for all subjects. A retrospectively triggered 2D CINE SSFP technique was used to examine acoustic gating for reliable tracking of myocardial contractions over entire R-R intervals. Black blood prepared gradient echo imaging, 3D phase contrast

Figure 2 (abstract 078)

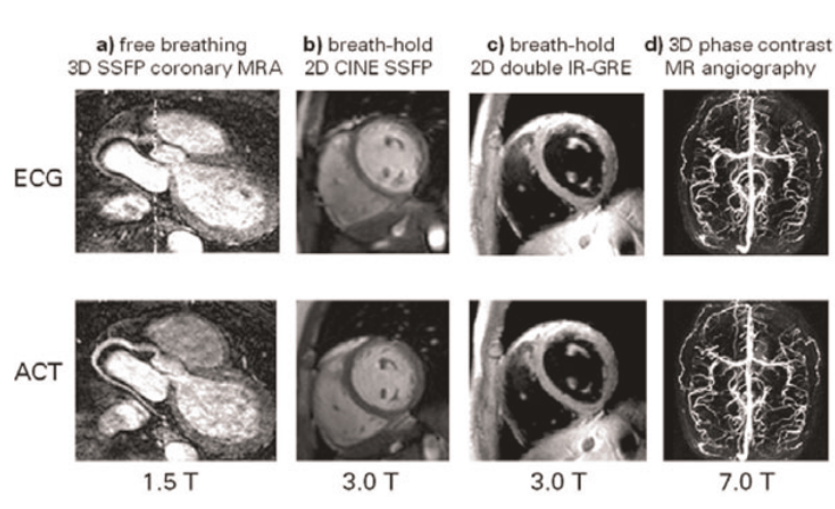


MRA and free breathing 3D coronary MRA were employed to evaluate acoustic triggering in a prospective gating regime.

Results: The acoustic MR-stethoscope provided cardiograms at I.5 T, 3.0 T and 7.0 T free of interferences from electromagnetic fields or magneto-hydraulic effects and hence is suitable for synchronization (Fig I). In comparison, ECG waveforms were susceptible to T-wave elevation and other distortions which were pronounced at (ultra)high fields (Fig I). Acoustically triggered 3D SSFP coronary MRA imaging produced images free of motion artifacts (Fig. 2a). Conversely, R-wave mis-registration occurred in ECG-triggered acquisitions due to T-wave elevation, which made 3D coronary MRA prone to motion artefacts (Fig. 2a). Motion artifacts were not present in full R-R interval coverage, acoustically triggered CINE imaging (Fig. 2b). The merits of acoustic triggering were further explored in prospectively gated, blood suppressed anatomic imaging, which provided image quality competitive or even superior to that obtained from the ECG-gated approach as indicated by Fig. 2c. Acoustically triggered 3D PC MRA acquisitions resulted in MR angiographies of superb quality free of motion artifacts even at (ultra)high magnetic field strengths, as shown in Fig. $2 d$.

Discussion and conclusion: The proposed acoustic approach was found to fully meet the demands of cardiac gated/triggered MRI. Its superior robustness has been demonstrated by eliminating the frequently-encountered difficulty of mis-triggering due to ECG-waveform distortions. ACT-MR substantially reduces the complexity of patient preparation by obviating the need to set up ECG-electrodes and position ECG-leads, and hence serves to streamline clinical CVMR.

\section{9}

Non-invasive monitoring allograft rejection by simultaneous cellular and functional cardiac MRI Yijen L Wu, Qing Ye, Kazuya Sato, Lesley M Foley, T Kevin Hitchens and Chien Ho

Pittsburgh NMR Center for Biomedical Research, Carnegie Mellon University, Pittsburgh, PA, USA

Journal of Cardiovascular Magnetic Resonance 2009, I I(SuppI I):O79

Inroduction: The current gold standard for diagnosing and staging rejection after organ transplantation is biopsy, which is not only invasive, but is also prone to sampling errors. The regimen for treating acute rejection after heart transplantation varies among treatment centers, partly due to lack of sensitive and reliable indices for assessing the status of myocardial rejection. The goal of this study is to establish sensitive and reliable indices using cellular and functional MRI for non-invasive detection of acute cardiac allograft rejection after heart transplantation. Using a rodent model of cardiac transplantation, we monitor both immune cell infiltration and cardiac dysfunction resulting from rejection in the same imaging session. Immune cells, mainly monocytes and macrophages, are labeled in situ with dextran-coated ultra-small superparamagnetic iron oxide (USPIO) nano-particles. $\mathrm{T}_{2}{ }^{*}$-weighted MRI and strain analysis of tagged $\mathrm{MRI}$ are used to correlate immune-cell infiltration and ventricular function with rejection grades.

Methods: I. Animal model: An abdominal heterotopic working heart and lung transplantation model with DA to BN transplantation rat pairs was used. The transplanted hearts exhibit cardiac outputs and ventricular pressure similar to those
Figure I (abstract 079)

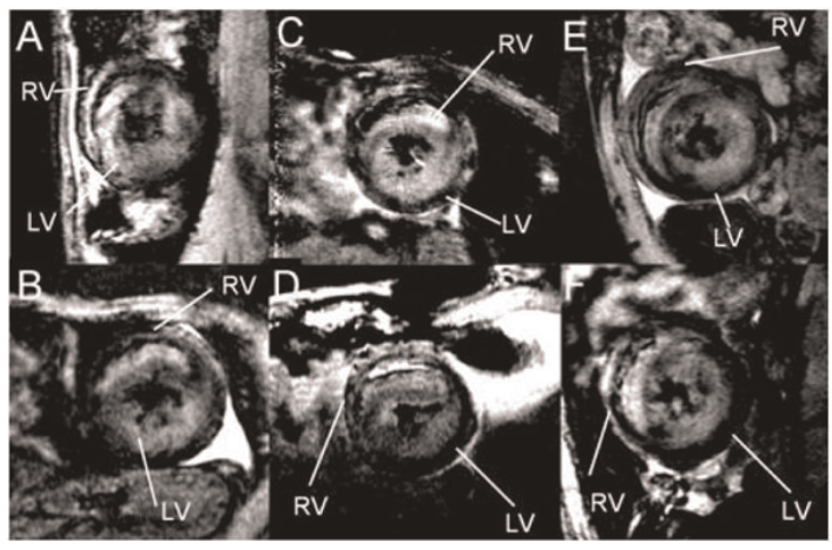

T2*-weighted in vivo MRI for different allografts after USPIO administration. Areas with UPSIO-labeled macrophage infiltration show signal loss in both LV and RV.

of native hearts. In this model, mild (Grade IA or B) rejection develops by post-operation day (POD) 2.5-3.5, Grade 2 rejection develops on POD 4.5-5.5, and moderate to the severe (Grade 3A) rejection develops after POD 6-7.

2. MRI methods: ECG and respiration gated $T_{2} *$-weighted cine imaging on Bruker AVANCE 4.7-T system was used for in-vivo imaging with an in-plane resolution of $156 \mu \mathrm{m}$. Tagged MRI is achieved with a modified DANTE sequence. Strains were analyzed by the HARP method with software obtained from Diagnosoft, Inc.

3. Iron-oxide particle labeling: Immune cells, mostly macrophages, are labeled in situ by direct intravenous injection of USPIO particles I day prior to MRI scans.

Results: Immune cells, mainly monocytes and macrophages, take-up circulating USPIO particles by endocytosis. Foci of labeled immune cells in the rejecting graft are observed by regions of hypointensity in $\mathrm{T}_{2}{ }^{*}$-weighted images (Fig. I). The

\section{Figure 2 (abstract 079)}

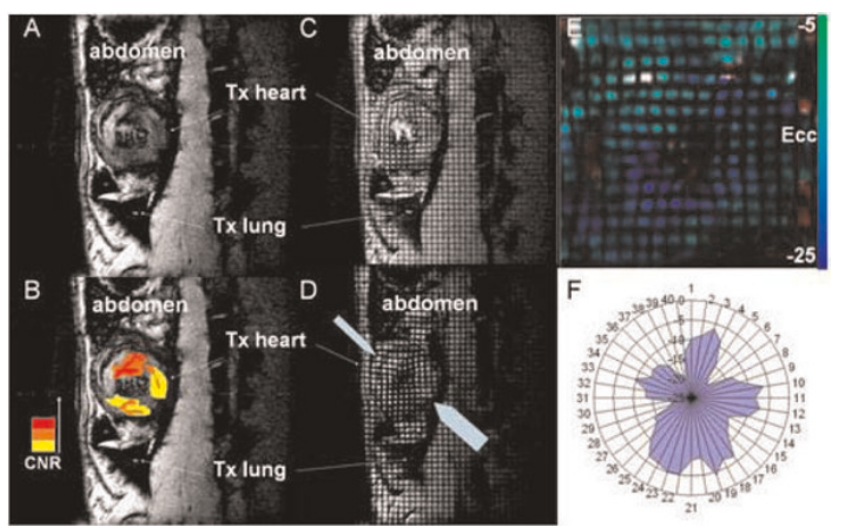

(A) T2*-weighted MRI for an allograft after USPIO administration. (B) Pseudo-coloring of different contrast-to-noise ratios (CNR) for easier visualization. (C, D) Tagged MRI of the same allograft heart at ED (C) and ES (D). The block arrows point to regions with compromised contractile functions. (E) Colored Ecc strain map of the allograft. (F) Ecc stran values of 48 probe-points throughout LV. 

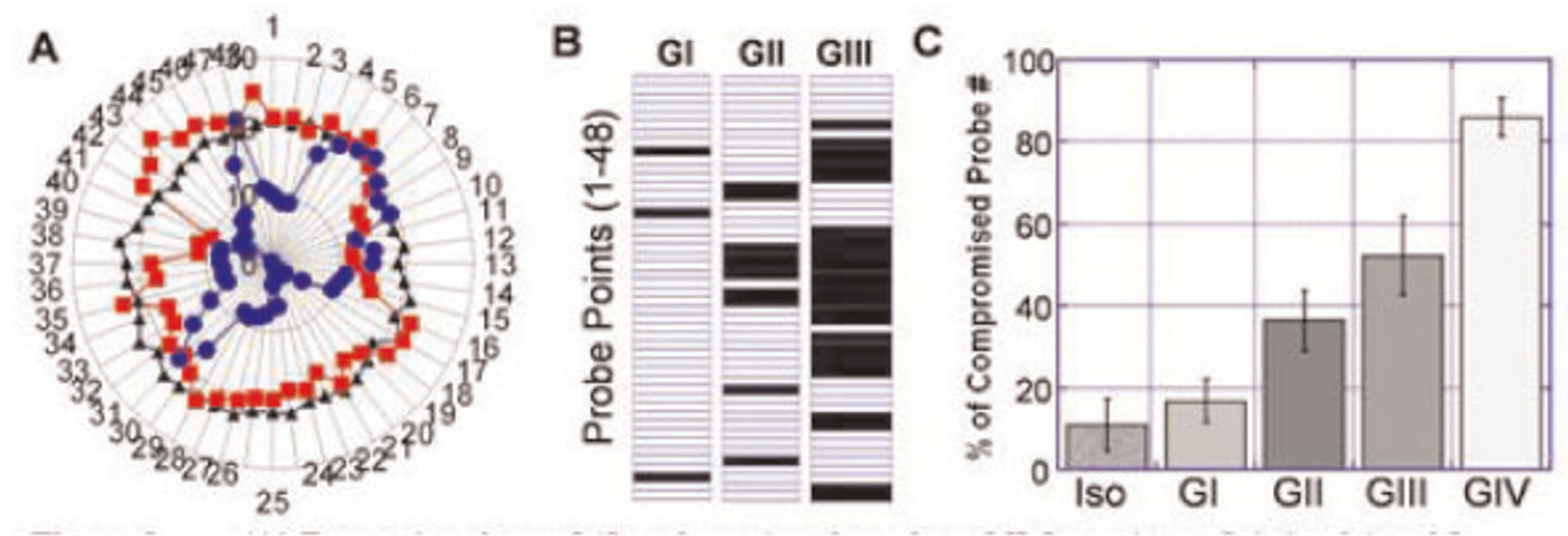

(A) Ecc strain values of 48 probe-points throughout LV for an isograft (triangle) and 2 allografts with Grade II (square) and Grade IV (circle) rejection. (B) Status of probe-points for 3 allografts with different rejection grades. Compromised probe-points are black, whereas the normal probe-points are left blank. (C) Degrees of compromised probe-points for different rejection grades.

observed immune-cell infiltration is heterogeneous, thus it is not surprising to have incidences of false-negative results with biopsy. Monitoring immune-cell infiltration with $\mathrm{MRI}$ is not only non-invasive, but also provides whole-volume 3D perspective of rejection.

To discern if the heterogeneity of rejection is manifested in cardiac function, tagged MRI is used to monitor regional ventricular contractile function. Although global function may appear normal, the rejected allograft may show local hypokinesis (Fig. 2D). Strain analysis by HARP was used to quantify the local contractile function. Regions with compromised strains (Fig. 2E \&2F) largely correlate with the areas of higher macrophage infiltration.

For easier visualization of the regions with compromised strains, 48 probe-points were placed evenly throughout LV, starting at the anterior intercept of LV and RV (Fig. 2F \& Fig. 3A). The probe-point is considered "compromised" if the strain value of the particular location deviats from the mean isograft values by more than I standard deviation; otherwise the probe-point is scored as "normal" (Fig. 3B). The number of compromised probe-points is well correlated with rejection grades (Fig. 3C), which can potentially be useful clinical index for rejection.

Conclusion: In our model, acute allograft rejection after heart transplantation is spatially heterogeneous, which is manifested in both immune-cell infiltration and ventricular function. Cardiac $M R I$ is both non-invasive and provides 3D, whole-heart perspective of rejection status, which potentially allows more reliable detection of acute allograft rejection.

\section{0}

\section{Fat/water separation imaging shows fatty deposition in areas of chronic left ventricular myocardial infarction \\ James W Goldfarb, Margeurite Roth and Jing Han St Francis Hospital, Roslyn, NY, USA}

Journal of Cardiovascular Magnetic Resonance 2009, I I(SuppI I):080

Introduction: Several studies indicate that fatty replacement of myocardium occurs after left ventricular myocardial infarction.
Magnetic resonance imaging can readily identify the location and morphology of both fatty tissue and myocardial infarction and may have the ability to non-invasively identify fatty replacement within infarcted regions. Fat deposition may impair global and regional cardiac function as well as the electrical activation of the heart.

Methods: Twenty-five patients (patient age: $64 \pm$ II yrs, infarct age: $12 \pm 9 \mathrm{yrs}$ ) with documented prior myocardial infarctions and ten normal volunteers (age: $63 \pm 10 \mathrm{yrs}$ ) underwent MR imaging on a clinical I.5 T scanner using precontrast fat-water separated (Dixon) and late gadolinium-enhanced infarct imaging. Myocardial infarct location and size were assessed using the full width half maximum (FWHM) infarct sizing algorithm applied to the late gadolinium-enhanced images. Fat-water separation was performed using a three-point Dixon reconstruction from in- and opposedphase gradient-echo images (Echo times $(T E s)=4.8,7.2,9.6 \mathrm{~ms}$ ). Fat segmentation was performed on a slice by slice basis using a signal intensity threshold set two standard deviations above the noise level. Volumes of myocardial late gadolinium-enhancement and fat deposition were compared using a Student's t-test. Precontrast infarct location and detection was compared with late gadoliniumenhanced infarct imaging using a seventeen segment model.

Results: Subjects with prior myocardial infarction had a $68 \%$ prevalence of fat deposition (17/25). In subjects without a history of myocardial infarction, fat deposition and late gadoliniumenhancement were not detected $(0 / 10)$. In the patients with fat deposition, the fat volume was $19.6 \pm 19 \mathrm{ml}$ (range: I.7-62.6 ml) vs late gadolinium-enhancement volume of $30 \pm 15 \mathrm{ml}$ (range: $9.6-59.2 \mathrm{ml}$ ). The volumes of late gadolinium-enhancement and fat deposition were statistically different $(p=0.01)$. Of the 425 myocardial segments, I56 (37\%) had late gadoliniumenhancement and 76 (18\%) had fat deposition. 65 segments (I5\%) had both fat deposition and late gadolinium-enhancement, while 9 I segments (2I\%) had late gadolinium-enhancement and no fat deposition. I I segments $(2.6 \%)$ had fat deposition and no late gadolinium-enhancement, but all II segments had adjacent myocardial segments with late gadolinium-enhancement. Figure I. 
Figure I (abstract 080)

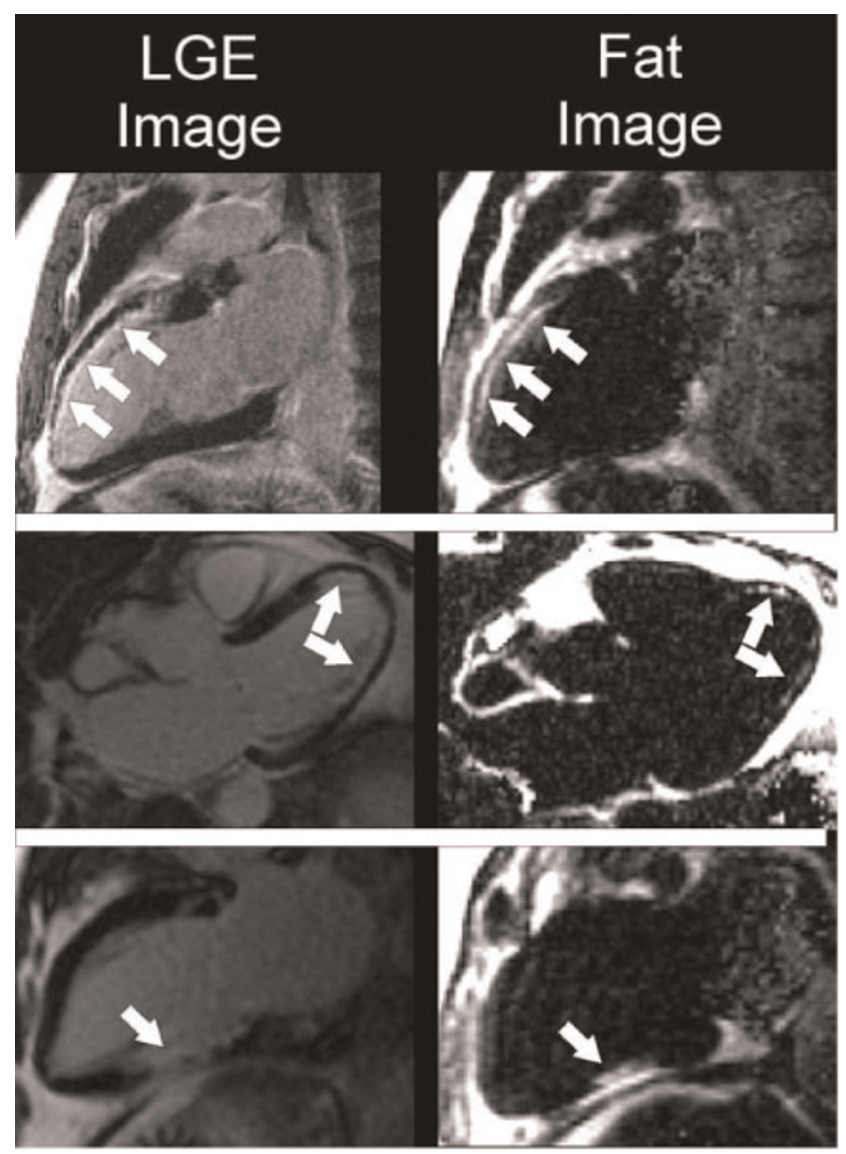

Results from three patients show fat deposition in areas of late gadolinium-enhancement (LGE).

Conclusion: Fatty replacement of myocardium after myocardial infarction is common and can be readily identified using fat/ water separation $M R$ imaging. The clinical significance and biologic mechanism of fatty deposition in infarction remain to be determined.

\section{O8I}

Shared velocity encoding (SVE): a new method for real-time velocity measurement with high temporal resolution Hung-Yu Lin', Yu Ding ', YiuCho Chung² and Orlando Simonetti ${ }^{i}$

${ }^{I}$ The Ohio State University, Columbus, OH, USA

${ }^{2}$ Siemens Healthcare, Inc, Malvern, PA, USA

Journal of Cardiovascular Magnetic Resonance 2009, I I(SuppI I):O8I

Objective: To develop and demonstrate a new method for rapid, real-time, phase-contrast velocity measurement using Shared Velocity Encoding (SVE) and gradient-echo planar imaging (GRE-EPI).

Introduction: Conventional ECG-triggered, segmented phasecontrast imaging (PC-MRI) is an accurate and clinically proven technique to characterize blood flow velocity. However, this method requires reliable cardiac gating, regular cardiac rhythm, and either signal-averaging, respiratory gating, or breath-holding to suppress respiratory motion artifacts. Furthermore, the resulting velocity information is a weighted temporal average of information acquired over multiple cardiac and respiratory cycles;short-term hemodynamic variations are lost. Real-time PC-MRI has been previously proposed using GRE-EPI [I] and spiral acquisitions [2], but limited performance has precluded routine clinical application. The aim of the present work is to design and demonstrate a novel method for rapid real-time velocity measurement with sufficient temporal resolution to eliminate the need for ECG synchronization and breath-holding, and to provide beat-to-beat hemodynamic information.

Methods: Sequence: SVE is a PC-MRI reconstruction technique designed to improve temporal resolution. Conventional real time PC-MRI works by alternating the polarity of velocity encoding gradients from one image frame to the next between positive $(+)$ and negative $(-)$ velocity encoding (i.e., $[+-],[+-]$ ). The velocity map is obtained by subtracting the negative velocity encoded image from the positive encoded image. The temporal resolution of the velocity map is therefore half the image frame rate. In SVE, images are acquired in the same way, but the velocity map is reconstructed by sliding the pair of images for subtraction one frame at a time (instead of two), resulting in a factor of 2 improvement in effective temporal resolution. This reconstruction technique was implemented to improve the temporal resolution of a GRE-EPI sequence for real-time PC-MRI on a I.5 T MR scanner (MAGNETOM Avanto, Siemens, Germany).

Imaging: Five healthy volunteers with no history of cardiovascular disease were scanned. Through-plane velocity measurements using segmented, spoiled gradient echo PC-MRI and the proposed real-time GRE-EPI with echo train length = 15 and SVE reconstruction were acquired for two slices: (i) cutting the ascending and proximal aorta and (ii) perpendicular to the distal descending aorta ( $2 \mathrm{~cm}$ superior to the renal arteries). Common acquisition parameters were: $\mathrm{FOV}=350 \times 262 \mathrm{~mm}$, matrix $=$

\section{Figure I (abstract O8I)}
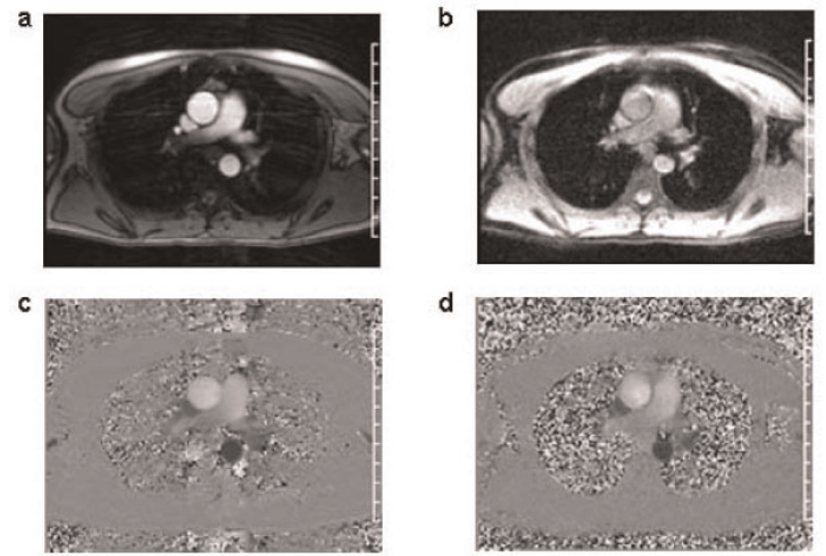

d

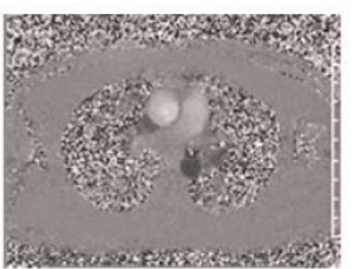

Magnitude images acquired using (a) conventional segmented, (b) realtime GRE-EPI SVE PC-MRI, and phase velocity maps from (c) conventional segmented PC-MRI, and (d) real-time GRE-EPI SVE PCMRI sequence in a slice cutting the ascending and descending aorta close to the aortic arch. 
Table I (abstract 08I) Peak velocities and RMSE) of the real-time SVE technique with respect to conventional PC-MRI

\begin{tabular}{llll}
\hline ROI & Peak Velocity (Segmented) & Peak Velocity (Real-time SVE) & RMSE \\
\hline Proximal Ascending Aorta & $83.7 \pm 4.23 \mathrm{~cm} / \mathrm{s}$ & $87.4 \pm 5.43 \mathrm{~cm} / \mathrm{s}$ & $3.6153 \mathrm{~cm} / \mathrm{s}$ \\
Proximal Descending Aorta & $24.4 \pm 5.61 \mathrm{~cm} / \mathrm{s}$ & $23.7 \pm 5.39 \mathrm{~cm} / \mathrm{s}$ & $1.1573 \mathrm{~cm} / \mathrm{s}$ \\
Distal Descending Aorta & $20.4 \pm 4.97 \mathrm{~cm} / \mathrm{s}$ & $20.4 \pm 4.77 \mathrm{~cm} / \mathrm{s}$ & $1.0744 \mathrm{~cm} / \mathrm{s}$ \\
\hline
\end{tabular}

$160 \times 120$, flip angle $=25^{\circ}$, spatial resolution $=2.18 \times$ $2.18 \mathrm{~mm}^{2}$, GRAPPA acceleration rate $=2$, and $V e n c=150 \mathrm{~cm} / \mathrm{s}$. The TE/TR/temporal resolution of the conventional gradient-echo and real-time GRE-EPI sequences were 3.5/7.0/42.0 $\mathrm{ms}$ and 2.9/14.6/58.4 ms, respectively.

Analysis: Peak velocity measurements were compared, and Root-Mean-Square Error (RMSE) was calculated between velocity curves obtained using the real-time SVE and the conventional sequence. Linear interpolation was used prior to RMSE calculation to compare curve values at exactly the same time points.

Results: In vivo images from one volunteer are shown in Figure I. Magnitude images clearly show vascular anatomy in both the segmented (Figure la) and real-time sequences (Figure Ib). In Table I, peak aortic velocity measurements show good agreement between conventional segmented PC-MRI and real-time PC-MRI with SVE reconstruction $(r=0.93, p<0.05)$. Insignificant RMS errors (Table I) were obtained in all regions-of-interest showing good agreement between velocity curves generated by conventional and real-time SVE techniques.

Conclusion: We have demonstrated the new SVE method that results in a factor of 2 improvement in effective temporal resolution in PC-MRI without sacrificing spatial resolution. With SVE reconstruction, real-time velocity measurement becomes practical with temporal resolution approaching that of conventional segmented PC-MRI.

\section{References}

I. Debatin JF, et al: J Magn Reson Imaging 1995, 5(6):656-662.

2. Park JB, et al: Magn Reson Med 2003, 49(2):322-328.

\section{2}

On the sensitivity of steady-state free precession myocardial blood-oxygen-level-dependent

MRI at I.5 T: theory and experiment

Xiangzhi Zhou', Richard Tang', Rachel Klein',

Debiao Li ${ }^{\prime}$ and Rohan Dharmakumar'

'Department of Radiology, Northwestern University, Chicago, IL, USA

${ }^{2}$ Northwestern University, Chicago, IL, USA

Journal of Cardiovascular Magnetic Resonance 2009, I I(Suppl I):082

Introduction: Through theoretical simulations and experimental studies, it has been shown in whole blood and in skeletal muscle that SSFP-based BOLD contrast is strongly dependent on repetition time(TR) and flip angle(FA). While these studies accounted for spin exchange effects between intra-(IV) and extra-vascular(EV) spaces, they have largely ignored the diffusion effects, particularly in the EV space. Since most of the blood present in the microvasculature of the heart is in the capillaries, we hypothesize that diffusion-mediated effects is an important mechanism allowing for the detection of oxygenation changes with SSFP-based myocardial BOLD imaging.
Figure I (abstract 082)

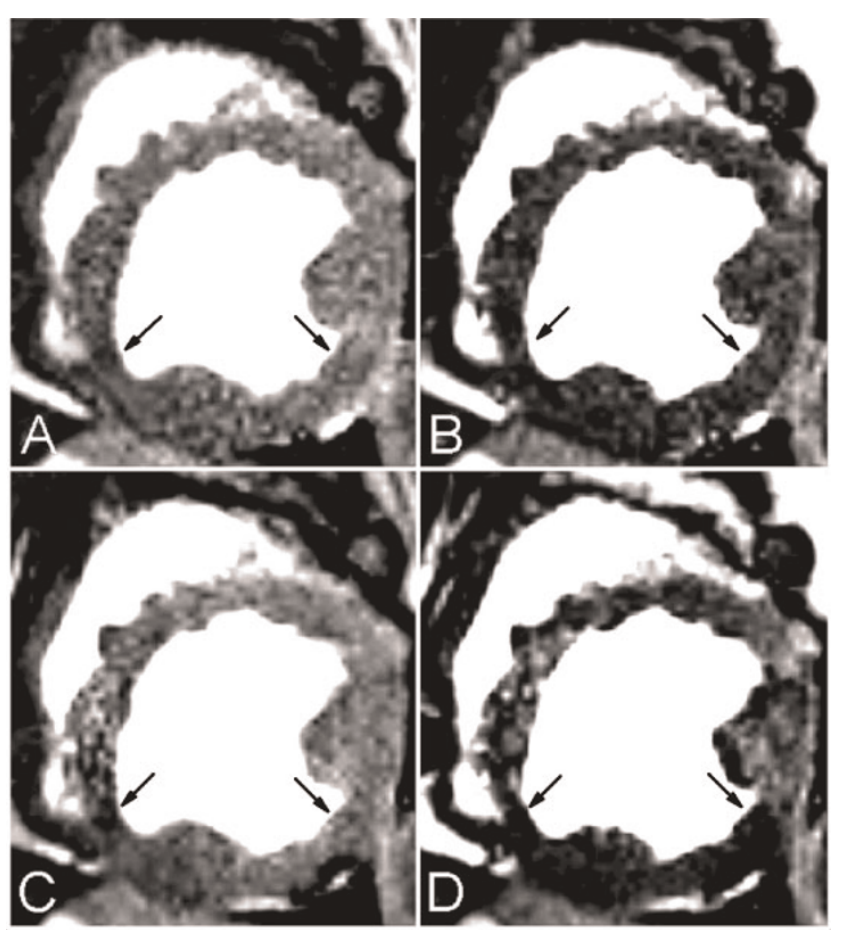

2D late diastole cine SSFP BOLD images of a dog with severe LCX stenosis and adenosine infusion obtained at different TR and FA: TR/FA = $3.5 \mathrm{~ms}, 30^{\circ}(\mathrm{A}) ; 3.5 \mathrm{~ms}, 70^{\circ}(\mathrm{B}) ; 6.0 \mathrm{~ms}, 30^{\circ}(\mathrm{C})$; and $6.0 \mathrm{~ms}$, $70^{\circ}(\mathrm{D})$. LCX territories are the shorter arcs subtended by arrows.

Purpose: To investigate the effect of TR and FA on SSFP-based myocardial BOLD sensitivity through a theoretical model accounting for diffusion effects and validate it using a controlled canine model.

Methods: Monte-Carlo simulations(MCS): Approximating capillaries as an infinite cylinder, the magnetic field variation $\left(\Delta B_{z}\right)$ outside the capillary vessel was computed as $B_{0} \Delta \chi(R / r)^{2}$ $\cos (2 \varphi) \sin ^{2} \theta / 2(E V)$ and $B_{0} \Delta \chi\left(\cos ^{2} \theta-I / 3\right) / 2(I V)$, where $R$ is the vessel radius, $r$ the distance from vessel axis, $\theta$ the angle between the main magnetic field $\left(B_{0}\right)$ and the vessel axis, and $\varphi$ the angle between $r$ and the projection of the main magnetic field onto the plane orthogonal to the vessel axis. The susceptibility difference $\Delta \chi$ between IV and EV space is: $\Delta \chi=\operatorname{Hct}(\mathrm{I}-\mathrm{Y}) \Delta \mathrm{X}$, where $\Delta \mathrm{X}=3.39 \mathrm{ppm}$ is the susceptibility difference between fully oxygenated and deoxygenated hemoglobin, Hct is hematocrit (0.4), and $Y$ is oxygen saturation( $20 \%$ and $80 \%)$. Spin diffusion was modeled as 3D Brownian motion of 5000 spins inside a cubic box with time steps of $50 \mu \mathrm{s}$ and diffusion coefficient of 
Figure 2 (abstract 082)

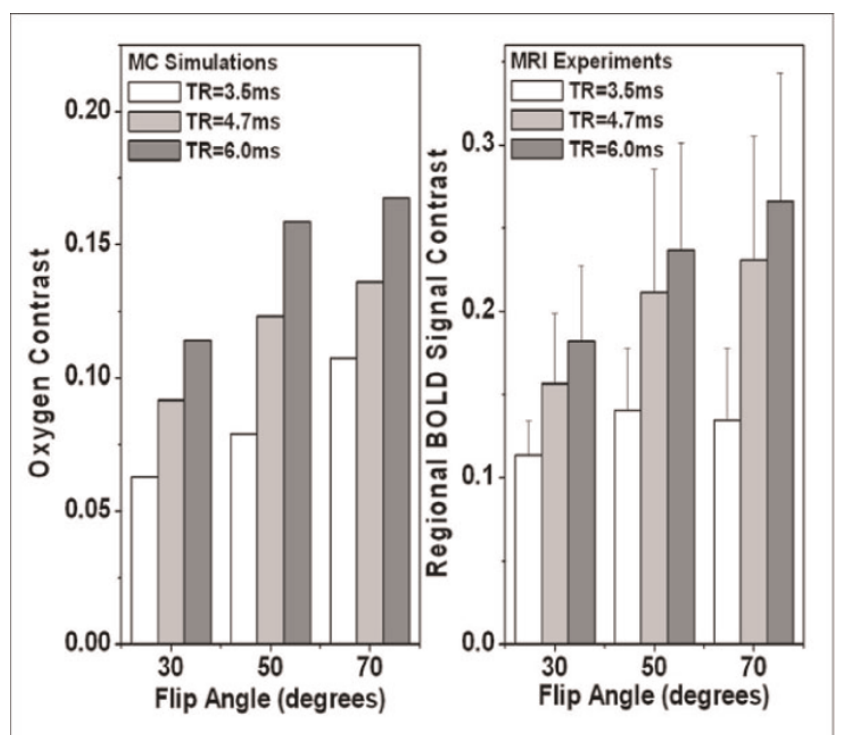

SSFP-baseed Myocardial BOLD contrast obtained from MCS and experimental studies with the same parameters $(T R=3.5 \mathrm{~ms}, 4.7 \mathrm{~ms}$, $6.0 \mathrm{~ms}$; Flip angle $=30^{\circ}, 50^{\circ}, 70^{\circ}$ ). For a given FA and TR, MCS oxygen contrasts were computed assuming myocardial oxygenation in the territory supplied by a stenotic vessl is $20 \%$, while that supplied by a healthy vessel is $80 \%$ under pharmacological stress, assuming myocardial blood volume fraction of $9 \%$.

$1.5 \times 10^{-9} \mathrm{~m}^{2} / \mathrm{s}$. Other parameters were: $\mathrm{R}=6 \times 10^{-6} \mathrm{~m}$, blood volume fractions $=9 \%$, number pulses to reach steady-state $=$ 2000; $\mathrm{TR}=3.5,4.7,6.0 \mathrm{~ms}$; and $\mathrm{FA}=30^{\circ}, 50^{\circ}, 70^{\circ}$.

Experimental studies: An external hydraulic occluder was placed around the left circumflex coronary (LCX) for the purpose of inducing reversible stenosis in 4 dogs. Following recovery (I week), animals were sedated, ventilated and placed in the scanner (I.5 T Siemens Espree). ECG-gated and multiple breath-held 2D-Cine SSFP sequences were prescribed under pharmacological stress with and without LCX stenosis over the LV. Three short-axis images with centre slice located on mid-LV were acquired for each study. Scan parameters: in-plane resolution $=1.2 \times 1.2 \mathrm{~mm}^{2}$, slice thickness $=5 \mathrm{~mm}, \mathrm{TR} /$ $\mathrm{TE}=6.0 \mathrm{~ms} / 3.0 \mathrm{~ms}, 4.7 \mathrm{~ms} / 2.35 \mathrm{~ms}, 3.5 \mathrm{~ms} / \mathrm{l} .75 \mathrm{~ms}$, flip angle $=70^{\circ}, 50^{\circ}, 30^{\circ}$, segments $/$ cardiac phase were adjusted to achieve an optimal temporal resolution $(10 \mathrm{~ms}-20 \mathrm{~ms})$ that minimize motion/flow artifacts.

Data analysis: Regional SSFP BOLD signal contrast, [I $L_{L A D^{-}}$ $\left.I_{L C X}\right] / I_{L A D}$, was used to evaluated the SSFP BOLD sensitivity, where $I_{L A D}$ and $I_{L C X}$ are average SSFP signal intensities measured within LAD and LCX territories.

Results: Figure I shows a typical set of 2D late diastole shortaxis cardiac images from SSFP cine scans of a dog with severe stenosis and adenosine infusion for different TR and FA. Note that the global and regional signal intensities are strongly dependent on imaging parameters. Figure 2 shows the SSFP BOLD signal contrast obtained from MCS and experimental studies. The regional BOLD signal contrast between LAD and LCX supplying regions match well with the MCS.
Discussion: MCS and experimental studies showed that TR and FA play a significant role in determining SSFP-based myocardial BOLD contrast. In particular, both MCS and experiments showed that increasing the FA or TR gave a concomitant increase in SSFP-based myocardial BOLD contrast. We found that a combination of TR/FA $=6.0 \mathrm{~ms} / 70^{\circ}$ gave the highest oxygen contrast among all the parameter sets studied, although more artifacts were observed in certain cardiac phases as TR was increased from $3.5 \mathrm{~ms}$. In order to enable the entire cine image set to provide reliable regional BOLD contrast at large TRs, robust artifact correction methods need to be developed.

\section{3}

Acute and chronic cardiac radio frequency ablation lesion visualisation using magnetic resonance imaging

Benjamin R Knowles , Dennis Caulfield', Michael Cooklin ${ }^{2}$, Aldo Rinaldi ${ }^{2}$, Reza Razavi', Tobias Schaeffter ${ }^{i}$ and Kawal S Rhode'

'King's College London, London, UK

${ }^{2}$ Guy's and St Thomas' NHS Foundation Trust, London, UK

Journal of Cardiovascular Magnetic Resonance 2009, I I (SuppI I):083

Introduction: The use of electro-anatomical mapping systems (EAMS) for catheter guidance and ablation point recording is widespread for the treatment of atrial arrhythmias using radio frequency ablation (RFA). Also, the evolution of RFA lesions over time may be an important factor in the reoccurrence of arrhythmias. In this study, we use late enhancement (LE) magnetic resonance imaging (MRI) as a tool to measure RFA lesions and present a novel visualisation method to represent this information in an intuitive way. This allows the validation of the in-vivo accuracy of EAMS and the examination of the evolution of RFA lesions from the acute to the chronic timescales.

Methods: Six patients with either atrial fibrillation (AF, 5 cases) or flutter (AFL, I case) underwent RFA. Prior to the procedure, each patient underwent a MRI examination that included administration of a double dose of Gd-DTPA contrast agent followed by MR angiography (MRA) and a $T_{2}$-prepared balancedSSFP (bSSFP) sequence. Approximately 20 minutes after contrast administration, a free-breathing, cardiac triggered, 3D LE scan was performed. The scan was inversion recovery-prepared with a resolution of $1.3 \times 1.3 \times 2 \mathrm{~mm}^{3}$ and TR/TE/á of $6.2 \mathrm{~ms} / 3.0 \mathrm{~ms} /$ $30^{\circ}$. Signal was acquired using Turbo Field Echo (TFE) with a $100 \mathrm{~ms}$ window and a low-high $\mathrm{K}$-space ordering. Inversion time was determined from a Look-Locker scan. All scans were performed using a I.5 T Philips Achieva MR scanner. During the procedure, ablation points from various EAMS (NavX, CARTO, XMR-EAMS [I]) were recorded. Post ablation, the patient returned to the MR scanner for a further examination including the bSSFP and LE scans. Approximately 6 weeks later, the patient returned for follow-up MR imaging. Offline, an atrial surface model was generated from the MRA scan, and was transformed into the coordinate system of each of the LE images. Integration of the LE image along the normal vector at each of the surface vertices was performed. This integral was used to colour code the surface model. Areas of LE were defined by integral values higher 
Figure I (abstract 083)

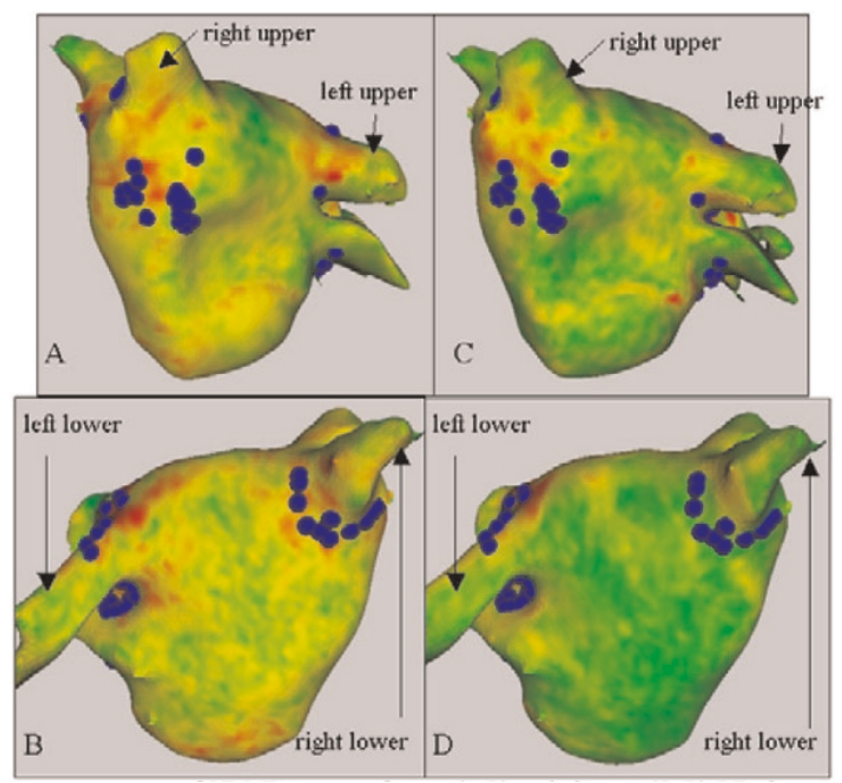

$3 D$ representation of $L E M R$ images of acute $(A-B)$ and chronic (C-D) RFA lesions. Overlaid in blue are the ablation points acquired with $\mathrm{NavX}$. It can be seen in the chronic image that an area of enhancement is no longer apparent around the right lower PV.

than the mean of the healthy myocardium plus three standard deviations. EAMS data from NavX and CARTO were exported. The EAMS-derived and the MR-derived cardiac surfaces were registered using a landmark-based registration. Using this registration, the EAMS lesions were transformed onto the MRderived cardiac surface and repositioned at the nearest vertex.

Results: Enhancement was present in all acute and chronic LE images. However, on inspection of the lesion patterns in three dimensions it could be ascertained that from the five patients undergoing PV isolation, one had isolation of both the left and right PVs, three had isolation of the left PVs, and one had isolation of neither. On comparing with the points acquired from NavX, on average $50 \%$ of the points were found in the MRdefined area of enhancement. In the patient after AFL ablation, a line of enhancement between the inferior vena cava and the tricuspid valve was visible, and $100 \%$ of ablation points acquired from the XMR-EAMS were found in an area of LE. The change in scarring between acute and chronic lesions was found to be considerable in all cases. Enhancing areas were either greatly reduced or non-existent, as shown in figure $\mathrm{I}$.

Conclusion: We have presented a technique based on LE MRI coupled to a novel visualisation method to measure RFA lesions. This technique has been applied to six patients undergoing RFA and used to assess the accuracy of EAMS and to monitor the evolution of lesions over time. We envisage that such an approach will have potential benefit in understanding the causes of arrhythmia reoccurrence and also in the guidance of redo ablations.

\section{Reference}

I. Rhode, et al: IEEE Trans Med Imaging 2005, 24(I I ): I 428-40.

\section{4}

\section{Towards MR-guided EP interventions using} an RF-safe approach

Sascha Krueger', Oliver Lips', Bernd David',

Daniel Wirtz', Steffen Weiss', Steen F Pedersen², Dennis Caulfield ${ }^{3}$, Julian Bostock ${ }^{3}$, Reza Razavi ${ }^{3}$ and Tobias Schaeffter ${ }^{3}$

'Philips Research Europe, Hamburg, Germany

${ }^{2}$ MR Research Centre, University Hospital, Aarhus, Denmark

${ }^{3}$ Division of Imaging Sciences, King's College, London, UK Journal of Cardiovascular Magnetic Resonance 2009, I I(Suppl I):O84

Introduction: Various cardiac arrhythmias, e.g. atrial fibrillation and ventricular tachycardia, can be treated by electrophysiological (EP) interventions [I]. Applying MR for guiding these interventions offers advantages like 3D visualization of the cardiac soft tissue in relation to the catheter and absence of ionizing radiation [2]. In this work, a prototype MR-EP system and catheter for diagnostic EP-interventions is described, which integrates concepts for RF-safe MR-tracking [3] and EP diagnostics [4]. The operation of the system is demonstrated in MR-guided EP experiments in pigs including mapping and pacing. RF-safety of the diagnostic MR-EP catheter prototype is shown and signal quality is compared to conventional EP catheters.

Materials and methods: System setup: All experiments were performed on a clinical whole-body I.5 T MR scanner (Achieva I/T, Philips Healthcare, Netherlands) equipped with an in-room display and an additional MR-EP-workstation (MR-EPWS) including a standard EP-recorder (EP Tracer, CardioTek, Netherlands). This workstation, located next to the scanner, combines and displays incoming real-time 2D and 3D images and real-time tracking positions from the MR scanner as well as realtime EP-data from the EP-recorder.

A 7F diagnostic EP catheter (Fig. I) with two ring electrodes and a tracking coil was used. Intracardiac and tracking signals are transferred via RF-safe high resistance wires [2] and a transformer-based transmission line [3], respectively.

\section{Figure I (abstract 084)}

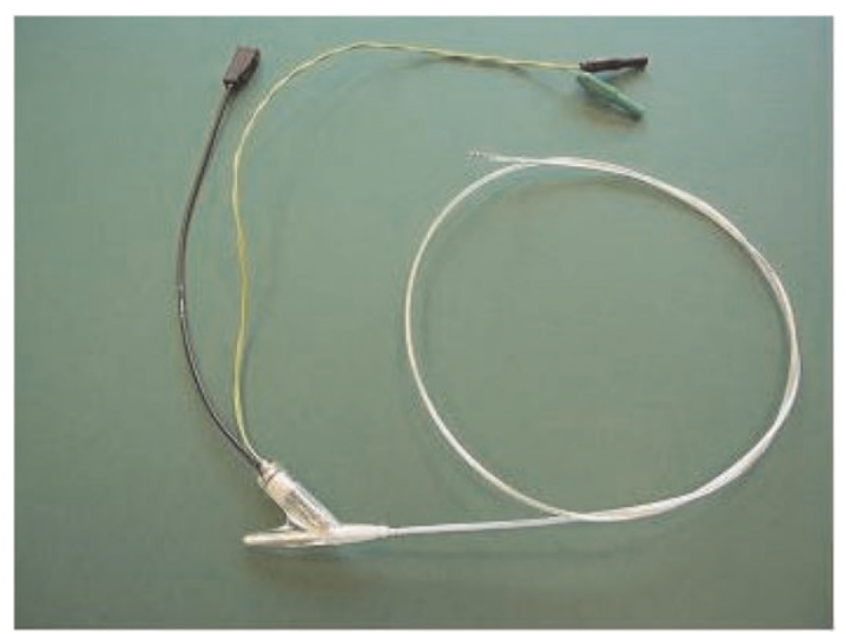

Diagnostic RF-safe MR-EP catheter. 
Figure 2 (abstract 084)

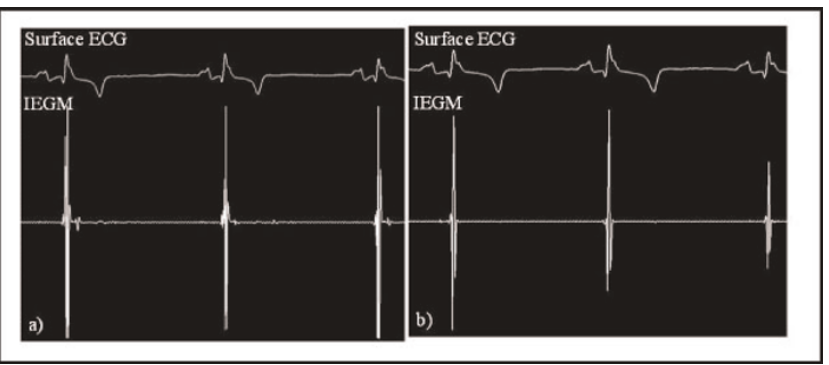

EP signals acquired in the RV (a) with the MR-EP catheter equipped with highly resistive wires and (b) with the conventional catheter.

Comparison MR-EP/conventional EP catheter: Conventional diagnostic EP catheters (Supreme Quad, JSN, 5F, St Jude, $M N$ ) and MR-EP catheters were compared under X-ray. Bipolar intracardiac electrograms (IEGM) were acquired with both catheters at corresponding locations (RA lateral wall, RV apex, TV ring, and HIS).

In-vivo proof of RF-safety: Temperature recordings during a typical real-time bFFE sequence (TR $2.4 \mathrm{~ms}$, flip $65^{\circ}$, global SAR $4 \mathrm{~W} / \mathrm{kg}$ ) were performed for the MR-EP and the conventional EP catheter. The catheters were equipped with fiber optic temperature probes and were inserted into the RA.

EP-Mapping procedure: The RA and RV were mapped using the MR-EP system and catheters. 3D bFFE and 3D CE-MRA datasets were acquired prior to catheterization of the animals.
Figure 4 (abstract 084)

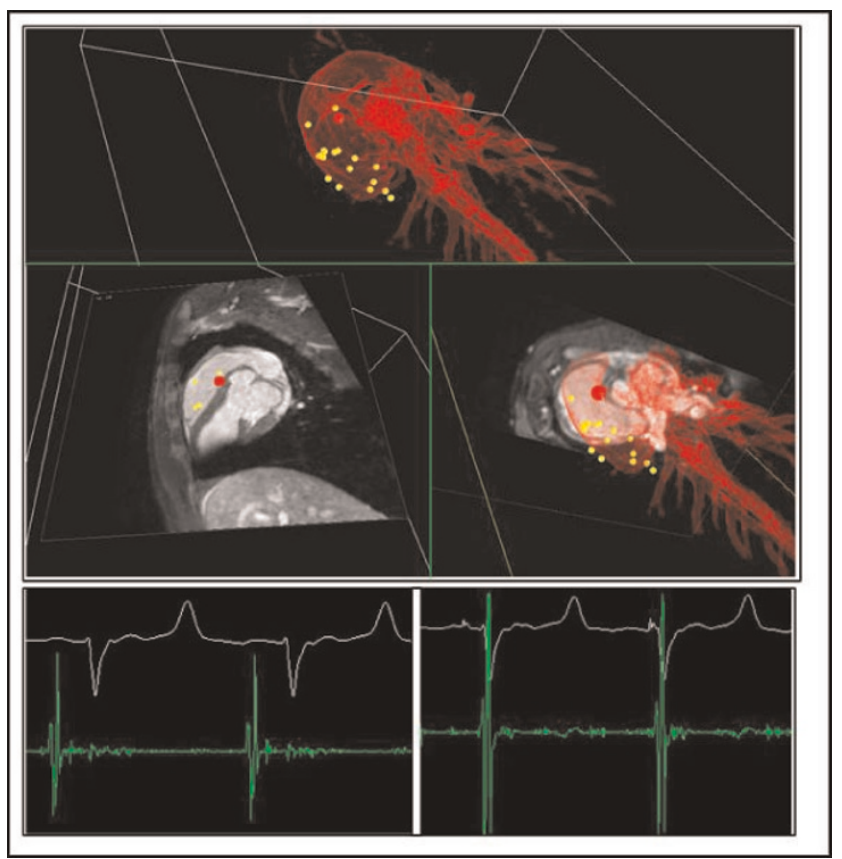

Top: Roadmap-based real-time 3D-visualization of the catheter position during recording (red dot) on the MR-EP workstation. The yellow dots in the $3 D$ rendering of the heart indicate previous mapping positions. Bottom: In-bore EP recordings at two selected positions showing an atrial signal (left) and a ventricular signal (right).

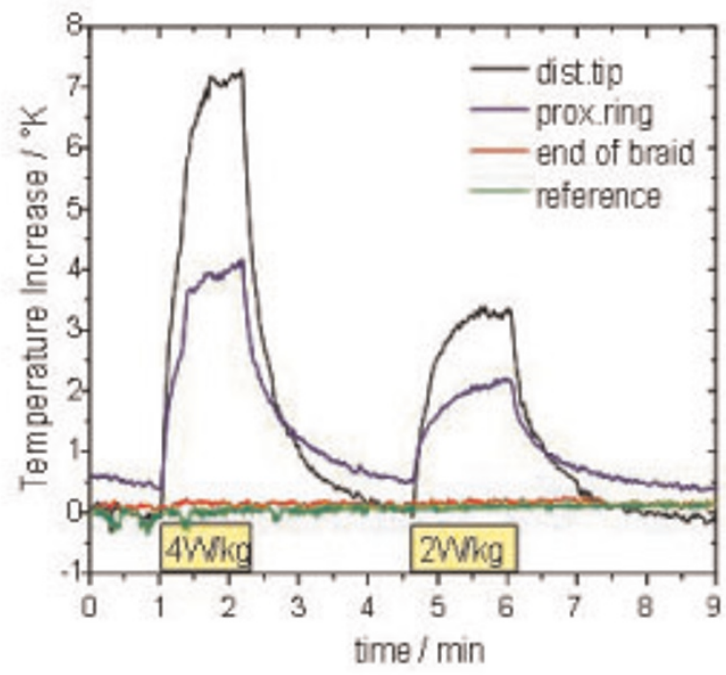

(a)

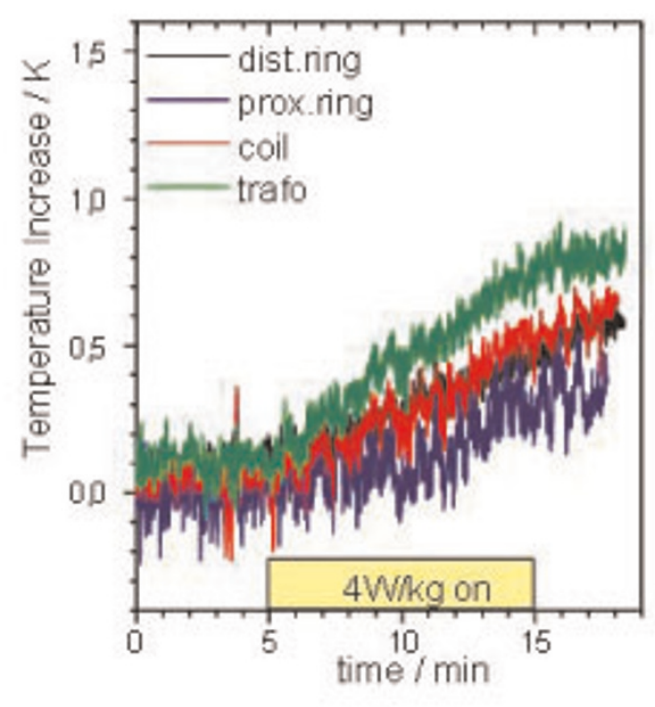

(b)

In-vivo temperature increase (a) with the MR-EP catheter and (b) with the conventional catheter. 
All MR and EP data can be combined and displayed on the MR-EPWS for guidance, including a surface model of the cardiac vessels, reformatted slices at the catheter position either manually angulated or using the real-time MR imaging geometry.

Results: Comparison with conventional EP catheter: IEGMs acquired with the MR-EP catheter were equivalent in quality to those acquired with the conventional EP catheter (Fig. 2).

In-vivo proof of RF-safety: The MR-EP catheter's maximal temperature increase after $10 \mathrm{~min}$ of RF transmission at $4 \mathrm{~W} / \mathrm{kg}$ was $0.7 \mathrm{~K}$ (Fig. 3a) almost corresponding to the expected increase in global body temperature $(0.6 \mathrm{~K})$. Hence, devicerelated local heating effects are negligible.

In contrast, an increase of up to $7.5 \mathrm{~K}$ in only $80 \mathrm{~s}$ was observed at the tip of the conventional catheter (Fig. 3b).

EP recording under MRI: The MR-EP-WS enabled a fast mapping, e.g. 40 points in $\mathrm{RV}$ in $20 \mathrm{~min}$. The in-bore IEGM recordings were comparable to those under X-ray (Fig. 4).

Furthermore, atrial and ventricular pacing was achieved via the MR-EP catheters. Successful stimulation was confirmed by a second MR-EP catheter and was also clearly visible in the surface ECG.

Conclusion: Recording of intracardiac electrograms is feasible with the MR-EP catheter. EP data quality is equivalent to conventional EP catheters. The combined use of highly resistive wires and a transformer-based transmission line for active tip tracking effectively suppresses RF-heating even during high SAR MRI.

The prototype setup of the MR-EP system provided excellent guidance and an efficient workflow for diagnostic MR-EP interventions.

\section{References}

I. Singer I and (ed): Interv Electrophys Lippincott Williams \& Wilkins; $2200 \mathrm{I}$.

2. Lardo AC: Pediatr Cardiol 2000, $21: 80-98$.

3. Weiss S, et al: Magn Reson Med. 2005, 54:182-189.

4. Wirtz D, et al: ISMRM 2007, 738.

\section{5}

\section{Assessment of myocardial perfusion reserve with blood oxygen level-dependent cardiovascular magnetic resonance imaging} Jacqueline A Flewitt', Matthias Vöhringer ${ }^{2}$, Jordin Green ${ }^{3}$ and Matthias Friedrich ${ }^{\prime}$

'Stephenson CMR Centre, Calgary, AB, Canada

${ }^{2}$ Robert-Bosch-Krankenhaus, Stuttgart, Germany

${ }^{3}$ Seimens Healthcare Canada, Calgary, AB, Canada

Journal of Cardiovascular Magnetic Resonance 2009, I I (SuppI I):O85

Background: New Blood Oxygen Level-Dependent Cardiovascular Magnetic Resonance Imaging (BOLD-CMR) sequences show a high sensitivity and consistent image quality that allows for assessing tissue oxygenation. We hypothesized that BOLDCMR can quantitatively assess myocardial blood flow changes using myocardial oxygenation as a biomarker.

Objective: To test whether a BOLD-CMR sequence accurately estimates myocardial perfusion changes.

Methods: Six anesthetized mongrel dogs were instrumented with a coronary infusion catheter in the circumflex coronary artery (LCX), an MR-compatible epivascular flow probe around the LCX and a catheter in the coronary sinus. Using a clinical
Figure I (abstract 085)

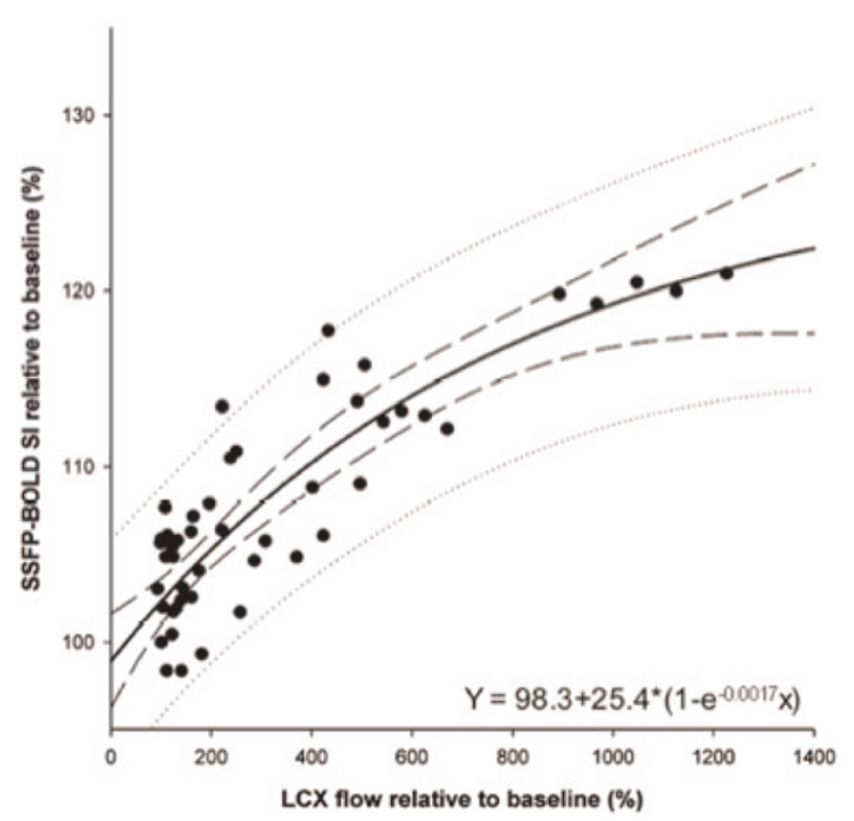

Blood flow changes and BOLD-SI in canine model under adenosis infusion.

I.5 T MRI system (MAGNETOM Avanto, Siemens Healthcare, Germany), SSFP BOLD-CMR was performed during graded intracoronary infusion of adenosine in the LCX. Typical scan parameters were: Field-of view (FOV) $190 \times 280 \mathrm{~mm}$; matrix size $106 \times 192$; slice thickness $10 \mathrm{~mm} ; \mathrm{T}_{\mathrm{R}} / \mathrm{T}_{\mathrm{E}} 5.8 / 2.9 \mathrm{~ms}$; flip angle $90^{\circ}$; typical breath-hold duration $14 \mathrm{~s}$. Images were analyzed using clinically validated software $\left(\mathrm{cmr}^{42}\right.$, Circle Cardiovascular Imaging Inc., Calgary, Canada) and the BOLD signal intensity (SI) for each was calculated. Correlations of coronary flow, oxygen saturation in the coronary sinus and myocardial BOLD-CMR signal intensity (BOLD-SI) changes were calculated by regression analysis. The same CMR imaging protocol was used in II healthy volunteers (6 male, 5 female) before, during and after intravenous adenosine infusion (140 micro-g/kg). Myocardial perfusion reserve in the human volunteers was calculated from flow measurement in the coronary sinus using velocity-encoded CMR.

Results: In dogs, adenosine-induced blood flow changes in the LCX agreed very well with changes in coronary venous saturation (logarithmic scale, $r^{2}=0.94, p<0.001$ ). Furthermore, coronary venous saturation showed a strong yet linear correlation with BOLD-SI changes $\left(r^{2}=0.80, p<0.00 I\right)$. Consequently, as shown in Figure $\mathrm{I}$, blood flow changes correlated very well with the BOLD-SI $\left(r^{2}=0.84, p<0.001\right)$. The exponential correlation is described by the equation $(y)=98.3+25.4$ $*\left(I-e^{-0.0017 x}\right) \quad(x=$ flow, $y=$ BOLD-SI). In the volunteers, adenosine infusion resulted in a significant myocardial perfusion increase $(416 \pm 69 \%$ of baseline, $p<0.001)$. BOLD SI increased significantly by $20.1 \pm 9.5 \%$ ( $p<0.00 \mathrm{I}$ as compared to baseline). The reproducibility of the BOLD-SI in the two baseline measurements before and after adenosine infusion was excellent (mean difference $0.1 \pm 2.6 \%, p=0.97$ ). 
Conclusion: State-of-the-art BOLD-sensitive MRI sequences detect changes of myocardial perfusion in an experimental animal model and in humans in vivo. This technique may allow for an accurate, non-invasive assessment of myocardial perfusion reserve in humans.

\section{6}

Feasibility of noninvasive 3 T MRI-guided myocardial ablation with high intensity focused ultrasound

Aravind Swaminathan', Viola Rieke², Randy L King ${ }^{2}$, John Pauly ${ }^{3}$, Kim Butts-Pauly ${ }^{2}$ and Michael McConnell ${ }^{1}$

IStanford Hospital \&Clinics, Stanford, CA, USA

${ }^{2}$ Stanford University Department of Radiology,

Stanford, CA, USA

${ }^{3}$ Stanford University Department of Electrical Engineering, Stanford, CA, USA

Journal of Cardiovascular Magnetic Resonance 2009, I I(SuppI I):086

Objective: This study sought to determine the feasibility of noninvasive myocardial HIFU ablation using real-time MRI guidance and thermometry.

Background: Invasive catheter-based myocardial ablation has become an important treatment of hypertrophic cardiomyopathy (HCM) and cardiac arrhythmias, but has known complications as well as the inability to actively visualize and control the extent of ablated tissue. High-intensity focused ultrasound (HIFU) can noninvasively create focal ablation lesions and has been

\section{Figure I (abstract 086)}

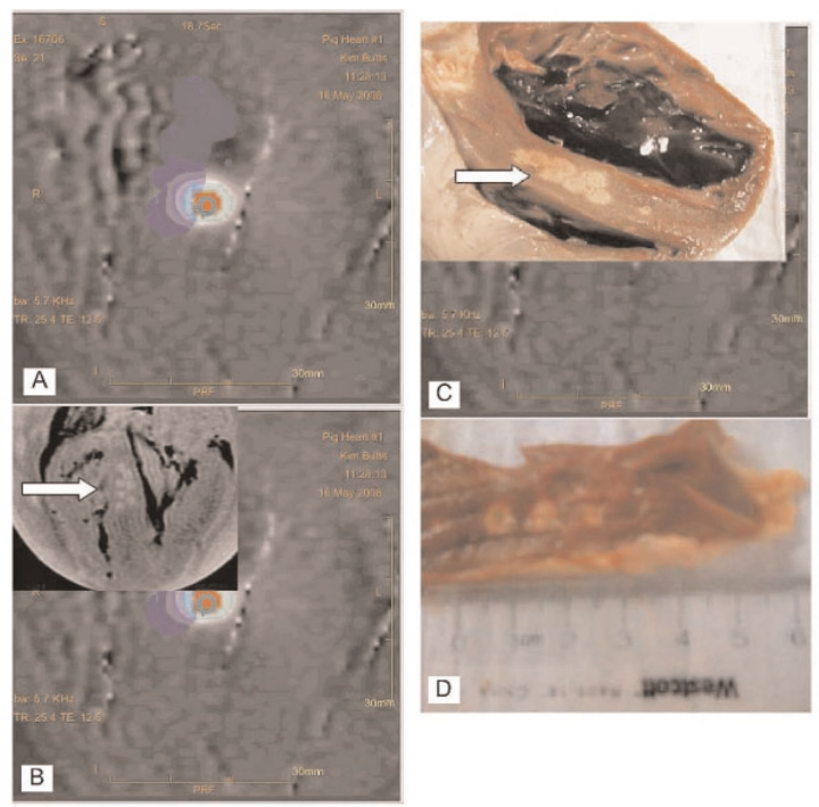

A) Real-time MR-temperature map during HIFU septal ablation (red zone on image), B) T2 MR image of HIFU lesions, C) Gross pathology of septal lesions from (B) using multiple 20s pulses, D) Lesions, each $4 \mathrm{~mm} \times 4 \mathrm{~mm}$ created with single $20 \mathrm{~s}$ HIFU pulses along the LV lateral wall.
Figure 2 (abstract 086)

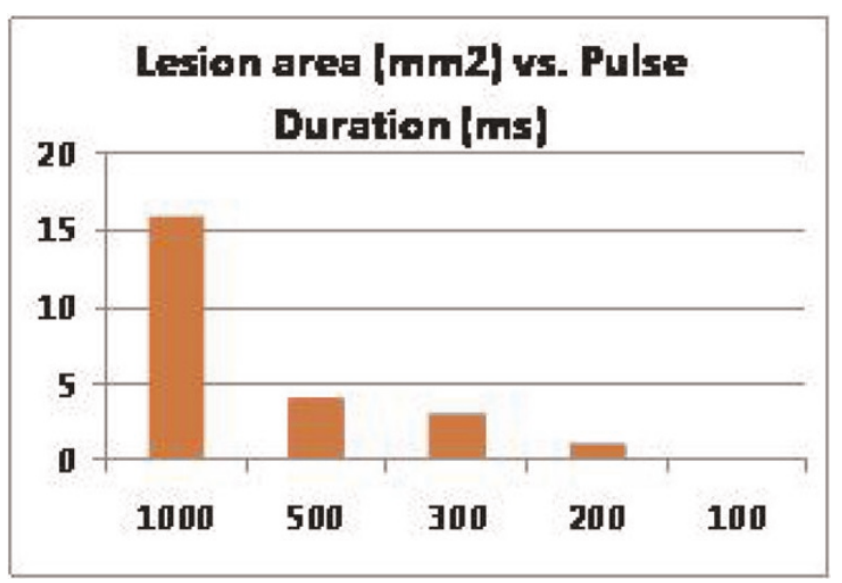

Lesion size $(\mathrm{mm} 2)$ as a function of pulse duration. Pulses were cycled on and off with a signal generator at $\mathrm{I} \mathrm{Hz}$.

developed for multiple non-cardiac clinical applications. MRI, in addition to imaging of myocardial pathology, can provide image guidance of HIFU targeting and then perform real-time monitoring of myocardial temperature during ablation. This study included preliminary feasibility work on ex-vivo MRI-guided myocardial HIFU with cardiac gating.

Methods: For ex vivo ablation, an existing MRI-guided HIFU ablation system (Insightec Ltd., Tirat Carmel, Israel) was used on a 3 T MRI scanner (GE Healthcare, Milwaukee, WI). Ex-vivo porcine hearts $(N=7)$ were immersed in water and degassed. MR scout imaging was performed to identify and guide the myocardial treatment areas to the septum. Multiple HIFU ablations lesions were formed using acoustic powers between 60-90 Watts and sonication duration of $20 \mathrm{~s}$ at a HIFU frequency of I.I MHz. MR thermometry was performed during lesion formation to verify correct ablation location and achievement of thermal ablation threshold $\left(>55^{\circ} \mathrm{C}\right)$. T2-weighted imaging was used to image lesions post-ablation. Lesion location and size was confirmed by pathology. Additional experiments were performed to simulate cardiac gating - HIFU pulses (acoustic power I50 W, duration $20 \mathrm{~s}$ ) were activated once per second (assuming heart rate of $60 \mathrm{bpm}$ ) with a range of pulse durations (100 ms-I s).

Results: Ablation lesions were formed in the ventricular septum of ex-vivo porcine hearts, with lesion size adjustable depending on the number of sonications used (each $20 \mathrm{~s}$ pulse created a $4 \mathrm{~mm} \times 4 \mathrm{~mm}$ lesion). (Figure la-d). Lesion size decreased at lower pulse duration. Pulse durations as low as 200 ms with interval cooling durations of $800 \mathrm{~ms}$ created HIFU lesions as small as I $\mathrm{mm} \times \mathrm{I} \mathrm{mm}$ with no visible lesion at lower duration (Figure 2). This cutoff is compatible with ablation during normal cardiac cycle lengths $(600-1000 \mathrm{~ms})$.

Conclusion: This study shows noninvasive MRI-guided HIFU is feasible on ex-vivo myocardium using a 3 T MRI-guided HIFU system with MR-based temperature monitoring. Furthermore, lesions could be created with HIFU pulses under physiologic cardiac gating intervals. Further work is needed based on these results to allow animal testing and ultimately clinical translation. 


\section{7}

XMR is a useful modality to guide, map and quantify the perfusion territories of coronary arteries Marcus Carlsson' and Maythem Saeed ${ }^{2}$

'Clinical Physiology, Lund, Sweden

${ }^{2}$ Dep of Radiology and Biomedical Imaging, San Francisco, CA, USA

Journal of Cardiovascular Magnetic Resonance 2009, I I(SuppI I):087

Introduction: The effects of locally delivered angiogenic factors or stem cells are not well defined. Obstacles to effective angiogenic treatment have been the difficulties in providing clear delineation of the status and extent of the injury and the coronary artery perfusion territory.

Purpose: This study aimed to determine the ability of selective injection of Gadolinium based MR contrast media (MR-CM) to map and quantify the territories of the major coronary arteries using first-pass perfusion (FPP) and early contrast enhanced (CE) MRI.

Methods: Selective coronary catheterization ( $n=16$ pigs) was performed under X-ray and MRI fluoroscopy in an XMR-suite. Catheters were placed in LAD, circumflex or right coronary artery. The coronary perfusion territories were mapped by intracoronary injection of $6-10 \mathrm{ml} 10 \%$ diluted MR-CM using a saturation-recovery gradient echo sequence for FPP images (TR/ $\mathrm{TE} / \mathrm{flip}=4.5 \mathrm{~ms} / 2.2 \mathrm{~ms} / 20^{\circ}$, slice thickness $\left.=10 \mathrm{~mm}\right)$ and an inversion recovery gradient echo sequence for early CE MRI (TR/ $\mathrm{TE} / \mathrm{flip}=5 \mathrm{~ms} / 2 \mathrm{~ms} / 15^{\circ}$, shot interval $=2 \mathrm{RR}$-intervals, slice thickness $=3-4 \mathrm{~mm}$ ). Cine MRI was used to quantify LV mass. In 12 animals, the LAD was occluded by embospheres to create infarction. Infarct size was measured on delayed enhanced (DE) MRI after intravenous injection of MR-CM. Figure I.

Results: Catheterization of the coronary arteries was successful in all animals under $X$-ray guidance $(n=13)$ and MRI guidance $(n=3)$ and took 15-20 min for X-ray and 30-45 min for MRI fluoroscopy. The perfusion territories of the coronary arteries were defined as hyperenhanced regions on FPP and CE-MRI. The LAD territory was $33.7 \pm 2.2 \%$ of LV-mass on FPP and $33.0 \pm 2.3 \%$ on CE-MRI $(P=0.63)$. Bland-Altman analysis

Figure I (abstract 087)

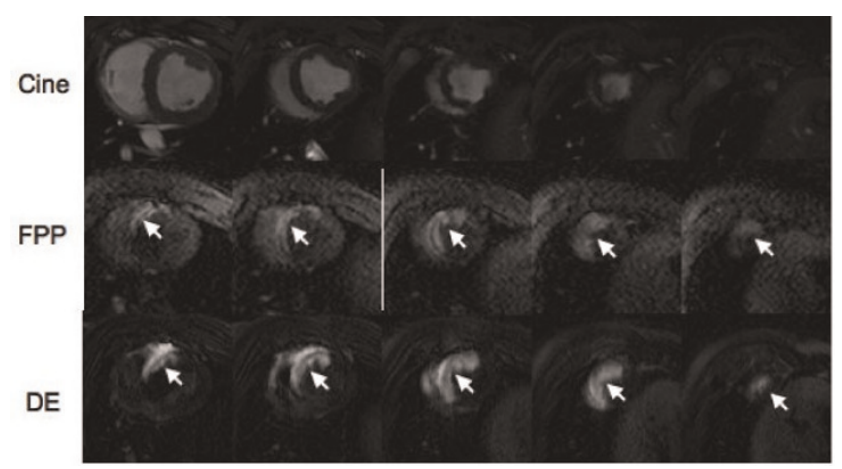

Multislice MR images showing the LV on cine (top) and the LAD perfusion territory on corresponding FPP (middle) and early CE (bottom) MRI as hyperenhanced regions. showed close agreement between the two methods $(0.7 \pm 5 \%)$. The signal intensity of LAD territory after injection of diluted Gd-based MR contrast media retuned to baseline after 6-8 min on CE MR imaging, suggesting complete washout of the contrast medium from normally perfused myocardium and lack of myocardial damage due to coronary catheterization. DE-MRI demonstrated the infarcted myocardium as hyperenhanced subregions of the perfusion territory $(7.5 \pm 1.2 \% \mathrm{LV}$ mass $)$ which did not differ from post mortem TTC size $(7.1 \pm 0.8 \%$ LV mass, $P=$ 0.99). Postmortem inspection revealed that there was no evidence of vascular or valvular injury caused by the endovascular catheter.

Conclusion: In this experimental study, we developed a method combining $X$-ray and MR fluoroscopy for selective mapping of the perfusion territories of the LAD, circumflex and right coronary arteries and quantifying the LAD perfusion territory. The extents of the LAD coronary artery perfusion territory measured on FPP and CE-MRI did not differ and neither did the infarct size on DE-MRI and TTC staining. This experimental method can be used prior to and after local delivery of angiogenic factors or stem cell therapy to determine their efficacy.

\section{Acknowledgements}

This study was supported by a grant from NIH (ROIHL07295).

\section{8}

CMR atrial angiography makes redo AF ablations faster and easier with less $x$-ray fluoroscopy Andrew S Flett, Don M Milliken, Syed S Ahsan, James McReady, Pier D Lambiase and James C Moon The Heart Hospital, London, UK

Journal of Cardiovascular Magnetic Resonance 2009, I I(SuppI I):088

Objective: To determine if merging CMR atrial angiograms with the fluoroscopy and ECG mapping during the ablation procedure make redo AF ablations easier.

Background: AF ablations have a relatively low success rate (52-74\%) and are long, complex procedures. CT merge into electroanatomic mapping in the catheter lab has been shown to produce better outcomes than using electroanatomic mapping alone. However, CT is associated with substantial radiation exposure. It is unclear whether CMR integration offers similar benefits. We hypothesised that CMR-derived 3D atrial anatomical merge would result in faster, easier procedures with less use of ionising radiation.

Methods: 64 patients (39 male, mean age: $57+/-12$ years) underwent repeat radiofrequency catheter ablation of atrial fibrillation. Twenty-two (34\%, the MERGE group) had a CMR merge, while 42 (66\%, NO MERGE) did not. All patients underwent their procedure using the Ensite NavX system (St Jude Medical). The CMR atrial angiogram was performed prior to the procedure (non-gated, 3D atrial angiogram, $0.1 \mathrm{mmol} / \mathrm{Kg}$ contrast, timed for atrial delineation), and was available (nonsubtracted) for importing into the cardiac catheterisation suite. Results: Compared to the NO MERGE group, the MERGE group demonstrated a substantial reduction in both total procedure time (34 minutes, II 9 vs. $153 \mathrm{~min} ; \mathrm{p}=0.0 \mathrm{I}$ ) and fluoroscopy time (19 minutes, 60 vs. $41 \mathrm{~min} ; p=0.01)$. In addition, there was a trend towards a reduction in left atrial 


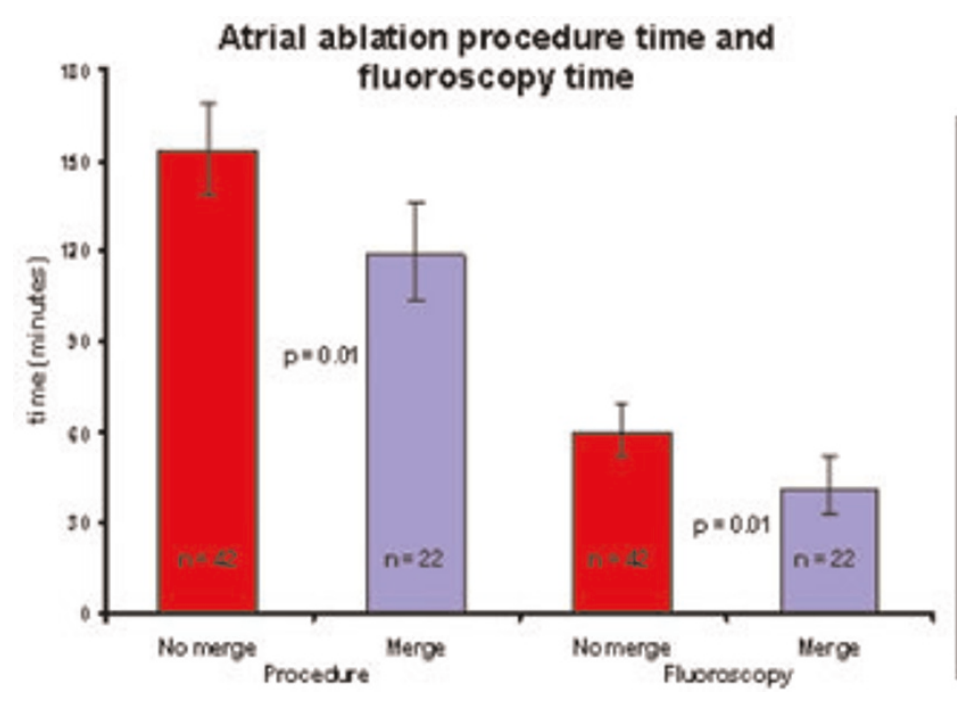

\section{A CMR atrial angiogram with PV isolation bums overlaid}

mapping time ( 27 vs. 33 minutes; $p=0.25)$ and fewer radiofrequency lesions being required (204 vs. 250; $p=0.3$ ). Figure I

Conclusion: CMR integration into electroanatomic mapping results in a reduction in procedure times and $x$-ray fluoroscopy in redo AF ablation compared to electroanatomic mapping alone. This is a potential benefit over CT image integration and electroanatomic mapping alone.

\section{9}

Magnetic resonance imaging for identification of myocardial injury during ablation for atrial fibrillation: first experiences with the Miyabi MRI system

Anil-Martin Sinha', Guido Ritscher', Christian Mahnkopf', Nathan Burgon ${ }^{2}$, Troy J Badger ${ }^{3}$, Martin Schmidt', Harald Marschang', Klaus J Gutleben',

Edward VR DiBella ${ }^{4}$, Nassir F Marrouche ${ }^{3}$ and Johannes Brachmann'

${ }^{I}$ Klinikum Coburg, Coburg, Germany

${ }^{2}$ School of Medicine, University Of Utah, Salt Lake City, UT, USA

${ }^{3}$ School of Medicine, University of Utah, Salt Lake City, UT, USA ${ }^{4}$ Center of Advanced Imaging Research, University of Utah, Salt Lake City, UT, USA

Journal of Cardiovascular Magnetic Resonance 2009, I I (SuppI I):O89

Introduction: Pulmonary vein antrum isolation (PVAI) has become an effective therapy in patients with paroxysmal atrial fibrillation (AF). Extension and location of ablation lesions often remain unclear during the procedure.

Purpose: We evaluated a new approach on visualization of myocardial injury using cardiac magnet resonance imaging (CMR) during PVAI procedure.

Methods: Patients who underwent PVAI, received CMR before and at the terminal phase of PVAI using the Miyabi-MRI system (Siemens, Germany). Delayed enhancement (DE-CMR) free breathing sequences were applied, and maximum intensity projections (MIP) obtained. Myocardial injury size was then measured on manually segmented 3D images by a computer algorithm using dynamic thresholding.

Results: 30 patients received PVAI from February to July 2008. In a subset of 14 patients, CMR was performed before and during the procedure. Using DE-CMR, the increase in average lesion to healthy myocardium ratio was $10.3 \pm 4.1 \%$ during PVAI. Figure I shows an example of MIP of a DE-CMR scan in 2D (A, B) and 3D segmentation $(C, D)$ in a anterior view pre $(A, C)$, and during PVAI

\section{Figure I (abstract 089)}

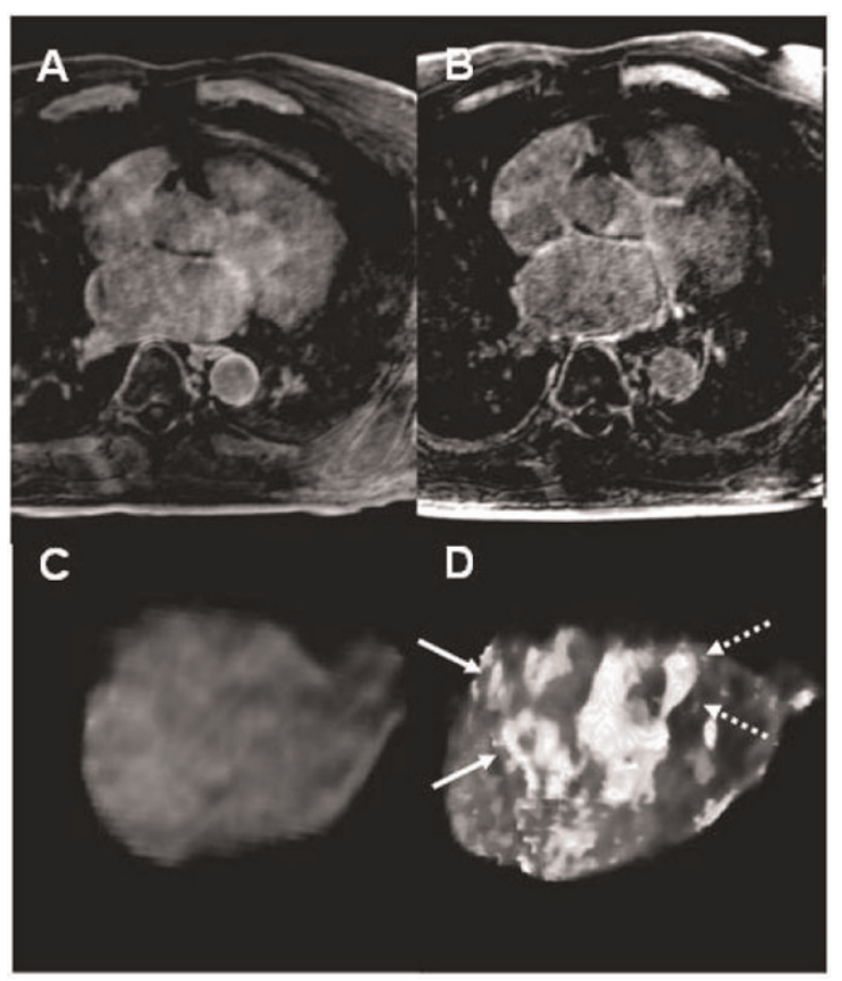


(B, D). Myocardial injury is identifiable as white tissue around PV single ostia (full arrows) and common trunk (dashed arrows).

Conclusion: Using CMR is feasible in the course of ablation procedures. In PVAI patients, DE-CMR allowed identification of location and extension of myocardial injury. Therefore, this new CMR approach might improve ablation techniques, and thus longterm success of PVAI.

\section{0}

Cine DENSE MRI for circumferential and radial dyssynchrony in patients referred for cardiac resynchronization therapy

Alexander B Jehle, Frederick H Epstein, Xiaodong Zhong, Robert L Janiczek, W Kevin Tsai, John M Christopher, Dale E Fowler, John D Ferguson, Christopher M Kramer and Kenneth $C$ Bilchick

University of Virginia, Charlottesville, VA, USA

Journal of Cardiovascular Magnetic Resonance 2009, I I(SuppI I):O90

Introduction: Cine DENSE (Displacement Encoding with Stimulated Echoes) MRI offers high resolution circumferential, radial, and longitudinal strain assessment. Dyssynchrony assessment using midwall circumferential strain corresponds to the predominant physiologic orientation of myofibers, and its effectiveness for cardiac resynchronization therapy (CRT) patient selection has recently been demonstrated [I]. Current echocardiographic dyssynchrony measures are based on radial or longitudinal velocity/strain.

Purpose: To test the hypothesis that cine DENSE circumferential strain analysis most effectively distinguishes between HF patients referred for CRT (HF/CRT) versus HF patients not candidates for CRT (HF/no CRT) and normal volunteers.

Methods: MRI and echocardiography were performed in 2 I subjects separated into 3 groups as defined above: HF/CRT patients $(\mathrm{N}=9)$, HF/no CRT patients $(\mathrm{N}=8)$, and normal volunteers $(\mathrm{N}=4)$. All MRI studies were performed on a I.5 T system (Avanto, Siemens, Germany). An ECG-gated spiral cine DENSE pulse sequence was used to acquire images with displacement encoding applied in two orthogonal in-plane directions. Separate 14-heartbeat breath-hold acquisitions were used for each displacement encoding direction. Short-axis images were acquired at basal, mid-ventricular, and apical levels. Additional imaging parameters included field of view $=340-$ $400 \mathrm{~mm}^{2}$, matrix $=128 \times 128$, slice thickness $=8 \mathrm{~mm}$, flip angle $=20^{\circ}, \mathrm{TR}=17 \mathrm{~ms}, \mathrm{TE}=\mathrm{I} \mathrm{ms}$, number of spiral interleaves $=6$, fat suppression, temporal resolution $=17 \mathrm{~ms}$, and displacement encoding frequency $=0.1$ cycles $/ \mathrm{mm}$. Images were exported to a PC and analyzed using custom-developed segmentation, tissue tracking, and strain analysis methods that have been described previously [2]. Echocardiography studies were performed on GE Vivid 7 Scanners and analyzed on an EchoPAC workstation. The standard deviations in timing in 12 segments were calculated for MR-based onset of circumferential contraction (MR Ecc Onset SD), peak circumferential contraction (MR Ecc Peak SD), onset of radial contraction (MR Err Onset SD), and peak radial contraction (MR Err Peak $\mathrm{SD})$, as well as for echo peak longitudinal velocity (Ts-SD), as described by Yu et al [3].

Results: Of the 21 patients (age $60+/-11$ years, $29 \%$ female), 17 had a cardiomyopathy, 6 (35\%) of which had an ischemic
Figure I (abstract 090)

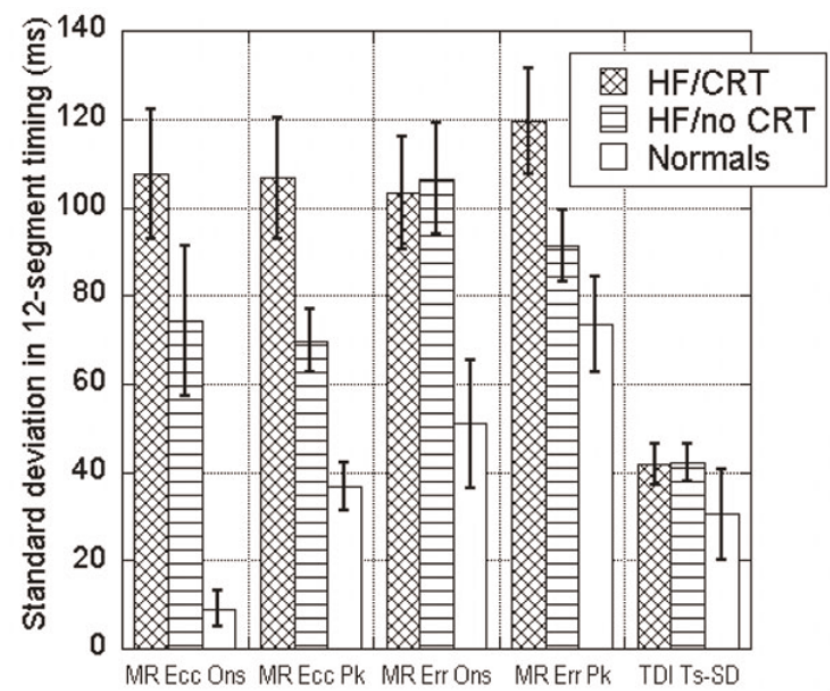

CMR DENSE dyssynchrony measures.

etiology. For all 252 segments, DENSE circumferential and radial peak timing correlated modestly $(R=0.43$; $p<0.000$ I). Patientlevel circumferential timing measures for onset of circumferential contraction and peak circumferential contraction correlated closely $(R=0.79 ; p<0.000 I)$. As shown in Figure I, MR Ecc Peak SD was significantly higher in HF/CRT patients versus HF/no CRT patients $(p=0.0 \mathrm{I})$ and normal patients $(p=0.008)$. MR Ecc Onset SD was markedly higher in HF/CRT patients than normals $(p=0.00 \mathrm{I})$. Although DENSE radial dyssynchrony measures effectively differentiated HF/CRT patients from normals, they were not as effective as the circumferential measures in distinguishing between patients in the HF/CRT and HF/no CRT groups ( $p=N S$ ). Ts-SD as assessed by echocardiography was similar in all 3 groups.

Conclusion: MR cine DENSE assessment of circumferential strain timing using contraction onset and peak contraction effectively distinguished CRT candidates from HF patients not candidates for CRT and normal volunteers. The better discrimination of circumferential versus radial strain dyssynchrony measures has important implications for identifying the optimal imaging modality to identify CRT candidates.

\section{References}

I. Bilchick KC, Dimaano $\mathrm{V}$ and $\mathrm{Wu}$ KC, et al: Magnetic resonance imaging analysis of dyssynchrony and myocardial scar predicts function class improvement following cardiac resynchronization therapy. JACC Cardiovascular Imaging 2008, 1:56I-8.

2. Spottiswoode BS, Zhong $X$ and Meintjes EM, et al: Tracking myocardial motion from cine-DENSE images using spatiotemporal phase unwrapping and temporal fitting. IEEE Trans Med Imag 2007, 26: I5-30.

3. Yu CM, Chau E and Sanderson JE, et al: Tissue Doppler echocardiographic evidence of reverse remodeling and improved synchronicity by simultaneously delaying regional contraction after biventricular pacing therapy in heart failure. Circulation 2002, 1 05:438-45. 


\section{1}

Spatiotemporal relationship between ventricular expansion and flow propagation during early filling Thompson Richard', June Cheng Baron', Kelvin Chow', Jessica Scott', Ben Esch², Mark Haykowsky' and lan Paterson'

'University of Alberta, Edmonton, $A B$, Canada

${ }^{2}$ University of British Columbia, Vancouver, BC, Canada

Journal of Cardiovascular Magnetic Resonance 2009, I I(SuppI I):O9I

Introduction: Flow propagation refers to the delay in the onset of blood flow during early filling at more apical ventricular locations. The velocity of flow propagation $\left(V_{p}\right)$ into the left ventricle (LV) provides a preload insensitive estimate of LV relaxation, confirmed invasively by a strong negative correlation with the relaxation time constant (tau) $[1,2]$. Clinically, $V_{p}<$ $50 \mathrm{~ms}$ is commonly taken as evidence of abnormal diastolic function. No direct physical relationship between muscle relaxation and flow propagation has been previously illustrated. By simultaneous measurement of myocardial mechanics (radial expansion) and blood patterns throughout the LV and during early filling we expect to illustrate a correlated spatial and temporal relationship between the mechanics which drive blood flow and the resulting blood flow patterns.

Methods: Phase contrast and tissue tagged MRI were used to measure the timing of blood and myocardial tissue dynamics during early diastole (Siemens Sonata I.5 T scanner). Experiments had a true temporal resolution of $\sim 20 \mathrm{~ms}$, interpolated to $10 \mathrm{~ms}$ for all analyses. The time of onset, t0 (time from QRS), of blood flow (phase contrast MRI) and radial expansion (tissue tagged MRI) were calculated at three $16 \mathrm{~mm}$ intervals from base to apex in 8 healthy volunteers. t0 was also evaluated as \% systole (normalized to the time of aortic valve closure). Figure I shows an example of the measurement of t0 for both radial tissue motion (B) and blood flow (C). To account for residual low velocities that are unrelated to the early filling impulse (which can obscure the time of onset) the early diastolic blood and tissue velocities were linearly extrapolated to determine $t 0$.

Results: Propagation of flow and of radial expansion from the base to the apex (cartoon in Figure IA) was observed in all subjects, as shown by the increasing delay in $t_{0}$ as one moves towards the apex (see Table I). The flow and radial expansion $t_{0}$ values for each spatial position have similar means and good correlation ( $R$ in table). The resulting $V_{p}$ values are also in general agreement.

$\mathrm{t}_{0}$ (ms/\% systole)

Conclusion: We demonstrated that spatiotemporal patterns of radial expansion (base to apex) are strongly correlated to conventional blood flow propagation during early filling, implying that muscle relaxation is related to $V_{p}$ via the propagation of strain in the relaxing ventricle. These preliminary results show
Figure I (abstract 09I)

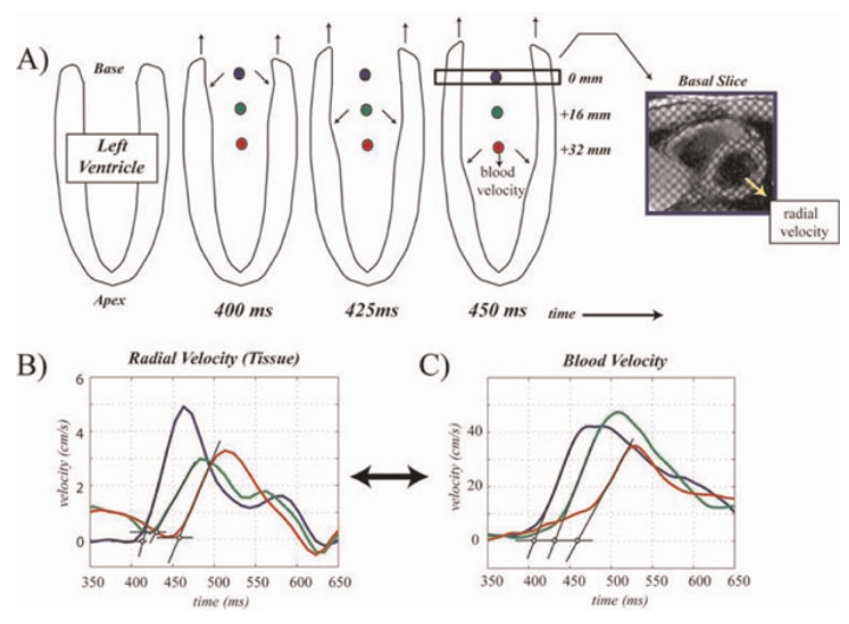

Measuring deformation of the LV and blood velocity during early filling.

that MRI can be used to simultaneously quantify mechanics and hemodynamics, with good spatial and temporal registration (which is a strength of MRI as compared to ultrasound). It remains to be shown that the many other aspects of ventricular mechanical function (longitudinal strain, rotation, endo vs. epicardial motion, for example) are as closely coupled to hemodynamics as was shown in this study.

\section{References}

I. Brun P, et al: Left ventricular flow propagation during early filling is related to wall relaxation: a color M-mode Doppler analysis. J Am Coll Cardiol 1992, 20:420-432.

2. Garcia MJ, et al: Color M-mode Doppler flow propagation velocity is a preload insensitive index of left ventricular relaxation: animal and human validation. J Am Coll Cardiol 2000, 35:20I-208.

\section{2}

Internal Flow Fraction discriminates patients with dyssynchronous heart failure from age and sex-matched controls Brandon K Fornwalt, Jana G Delfino, Calvin R Kurz, Patrick C Gonzales, Robert Eisner, Angel R León and John N Oshinski

Emory University School of Medicine, Atlanta, GA, USA

Journal of Cardiovascular Magnetic Resonance 2009, I I(Suppl I):092

Objective: Evaluate the ability of Internal Flow Fraction to diagnose left ventricular dyssynchrony using standard cine MRI.

Table I (abstract 09I)

\begin{tabular}{|c|c|c|c|c|}
\hline & Base & $+16 \mathrm{~mm}$ & $+32 \mathrm{~mm}$ & $V p(\mathrm{~cm} / \mathrm{s})$ \\
\hline Flow & $384 \pm 24 / 112 \pm 7$ & $4 I I \pm 24 / 123 \pm 5$ & $436 \pm 24 /|3| \pm 7$ & $66 \pm 13$ \\
\hline Radial Expansion & $386 \pm 31 / 114 \pm 5$ & $405 \pm 32 / \mid 21 \pm 7$ & $430 \pm 32 / 129 \pm 7$ & $73 \pm 10$ \\
\hline$R\left(t_{0}\right)$ & .78 & .73 & .72 & NS \\
\hline
\end{tabular}


Figure I (abstract 092)
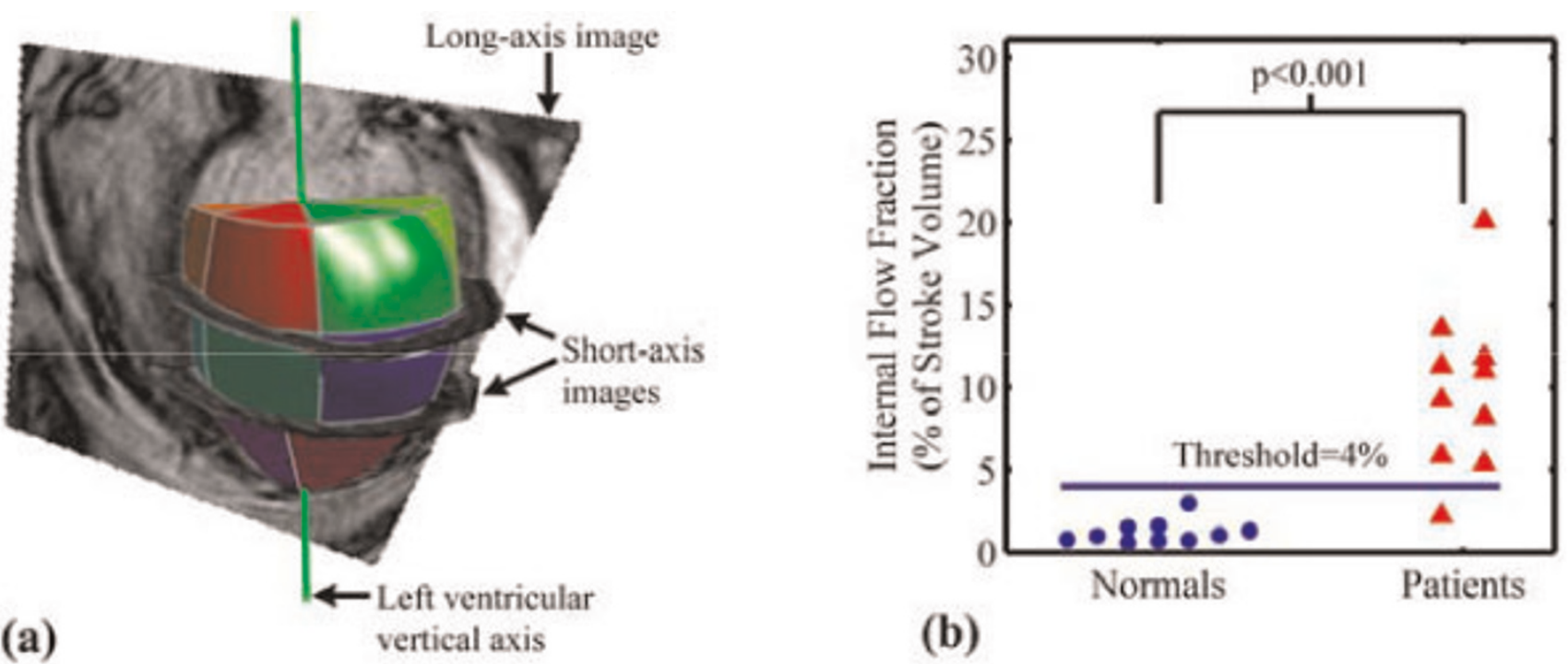

(b)

(a) The 3-dimensional left ventricular volume is superimposed on the 4-chamber long-axis MRI. Two of the short-axis images are also displayed to demonstrate the spatial arrangement of the images. The left ventricle was divided into 16 wedge-shaped regional volumes as shown. (b) Interal Flow Fraction (IFF) discriminates between patients and healthy controls with $95 \%$ accuracy.

Background: Better methods to quantify mechanical dyssynchrony in the heart may improve patient selection for cardiac resynchronization therapy. Dyssynchrony creates inefficient "sloshing" of blood volume internally within the left ventricle (LV). This "internal flow" represents wasted energy due to the dyssynchronous motion of the LV walls. We developed a new method to quantify internal flow from cine cardiac MRI which may provide a better, more physiologic measure of dyssynchrony than existing methods.

Hypothesis: LV internal flow will be significantly increased in patients with dyssynchronous heart failure compared to healthy, age and sex-matched volunteers.

Figure 2 (abstract 092)
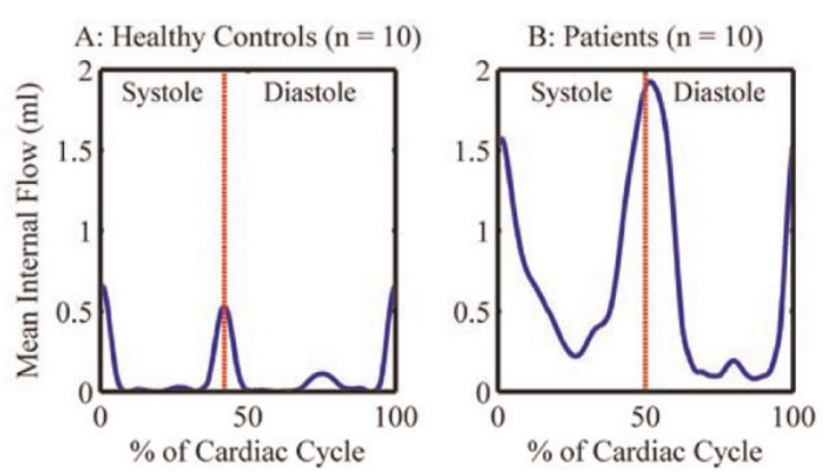

Internal flow is significanly increased thoughout the cardiac cycle in patients with dyssycnhronous heart failure compared to age and sexmatched healthy controls. Note the peaks in internal flow surrounding the periods of isovolumic contraction (time 0 ) and relaxation (vertical dashed line marking the end of systole).
Methods: Images were obtained with a I.5 T Philips Intera scanner using a 5-element phased array cardiac coil. Short-axis steady-state free-precession (SSFP) cines were acquired over the length of the LV during breath-holds $(8-10 \mathrm{~mm}$ slices with no gaps, 20 phases per cardiac cycle). Two and four-chamber longaxis cine images were also acquired. Ten patients with dyssynchronous heart failure (New York Heart Association class III/IV, LV ejection fraction < 35\%, QRS > I50 ms) and I 0 age and sex-matched healthy controls were imaged. The 3-dimensional LV volume was reconstructed and divided into 16 wedgeshaped volumes adjacent to the American Heart Association standardized myocardial segments (Fig IA). Internal flow was defined as the sum of the magnitude of the volume changes in the 16 regions minus the magnitude of the global volume change over each time step in the cardiac cycle: $I F(t)=\sum\left|\Delta V(t)_{\text {regional }}\right|$ $\mid \Delta \sum V(t)_{\text {regional }}$. This difference is zero if no internal flow has occurred. Internal Flow Fraction (IFF) was defined as the total internal flow as a percentage of stroke volume.

Results: IFF was significantly increased in the patients ( $10 \pm 5 \%$ vs $\mathrm{I} \pm 1 \%$ in the healthy controls, $p<0.00 \mathrm{I}$ ). An IFF threshold of $4 \%$ discriminated between patients and controls with $90 \%$ sensitivity and 100\% specificity (Fig IB). There were two large physiologic peaks of internal flow in the healthy controls: one during isovolumic contraction and another during isovolumic relaxation (Fig 2A). Internal flow occurred throughout the cardiac cycle in the patients, but peaked during the isovolumic periods (Fig 2B).

Conclusion: Left ventricular Internal Flow Fraction can be quantified from images acquired in a standard cine cardiac MRI exam. Internal flow during the isovolumic periods is a normal, physiologic component of left ventricular contraction and relaxation. A left ventricular Internal Flow Fraction of $4 \%$ discriminated patients with dyssynchronous heart failure from age and sex-matched healthy controls with $95 \%$ accuracy. 


\section{3}

Accurate quantification of heart valve regurgitation in all four heart valves simultaneously using 3D velocity-encoded MRI with retrospective valve tracking Jos JM Westenberg, Stijntje D Roes, Rob J van der Geest, Sebastiaan Hammer, Nina Ajmone Marsan, Jeroen J Bax, Albert de Roos and Johan HC Reiber Leiden University Medical Center, Leiden, Netherlands

Journal of Cardiovascular Magnetic Resonance 2009, I I(SuppI I):093

Introduction: In regurgitant heart valves, surgical decisionmaking is based on the severity of the regurgitation through the particular valve. Conventional two-dimensional (2D) one-directional velocity-encoded (VE) MRI is routinely used for flow assessment, but this technique has been shown to be inaccurate and correlation between the net flow volumes through the valves is weak, even in the absence of regurgitation. 2D one-directional VE MRI is limited because the position and angulation of the acquisition plane cannot be adapted to the valve motion and the direction of the inflow and regurgitant jet.

Figure 2 (abstract 093)
Figure I (abstract 093)

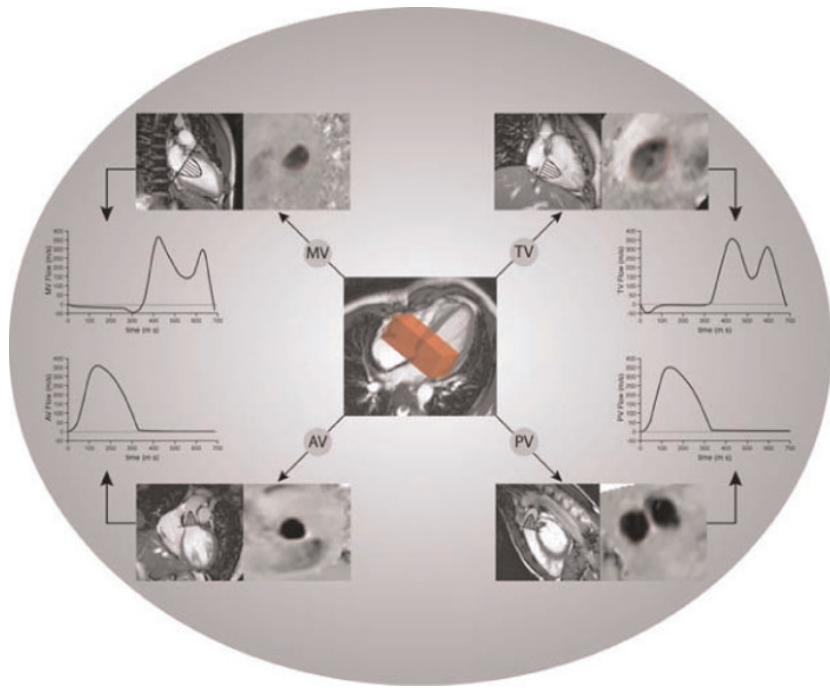

3D 3-directional velocity-encoded MRI is performed at the basal level of the heart. In offline analysis, retrospective valve tracking for each of the heart valves can be performed, resulting in flow at the mitral valve (MV), tricuspid valve (TV), aortic valve (AV) and pulmonary valve (PV).

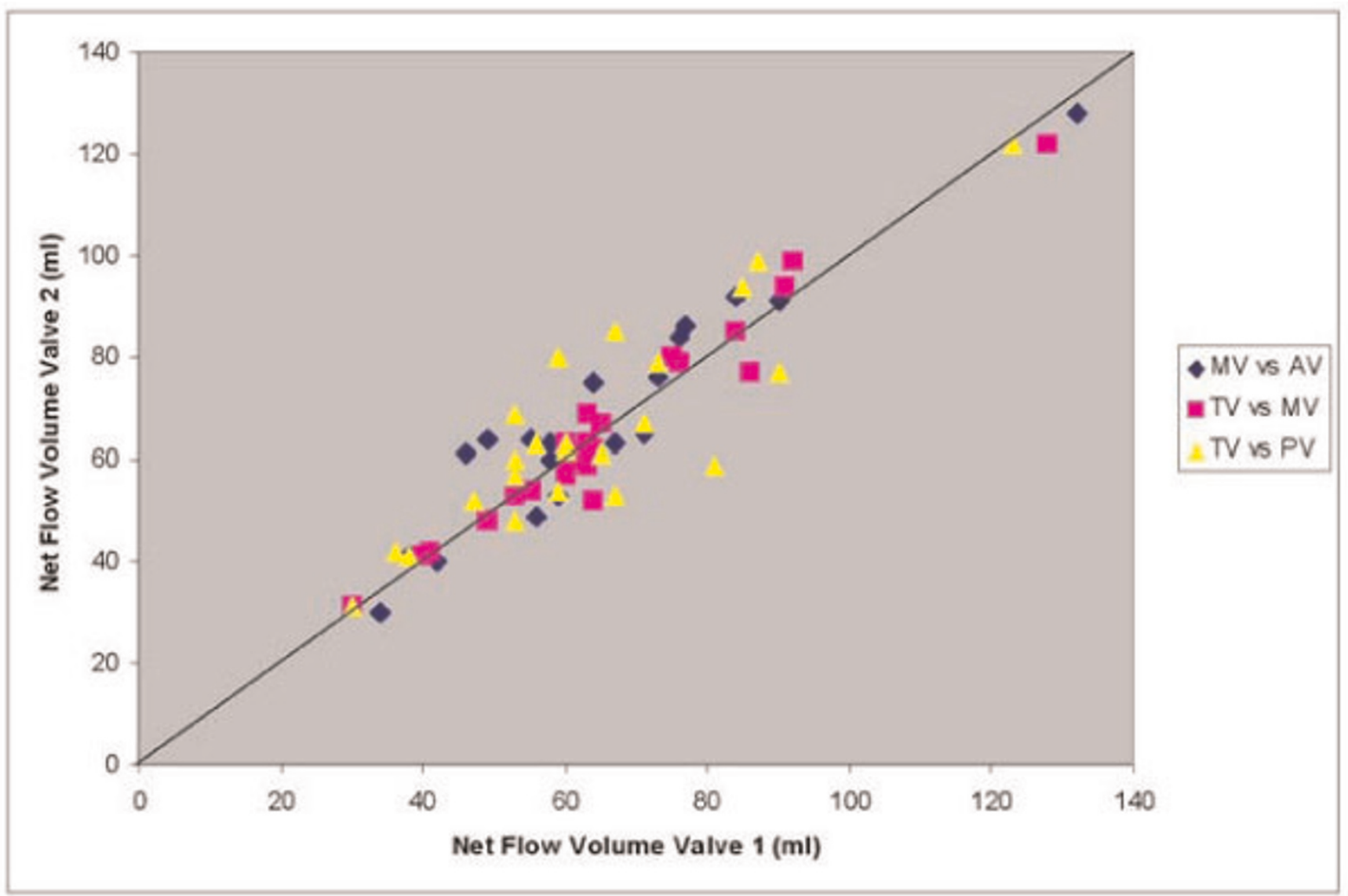

Net flow volumes for mitral valve (MV) vs. aortic valve (AV), tricuspid valve (TV) vs. MV and TV vs. pulmonary valve (PV) in 23 patients with valve regurgitation. 
Table I (abstract 093) Statistical results

\begin{tabular}{lllll}
\hline & & MV-AV & MV-TV & TV-PV \\
\hline Volunteers $(\mathrm{n}=14)$ & Pearson correlation & 0.96 & 0.97 & 0.96 \\
& P-value t-test & 0.01 & 0.31 & 0.01 \\
& Mean difference & $6 \pm 7 \mathrm{ml}$ & $-2 \pm 5 \mathrm{ml}$ & $6 \pm 6 \mathrm{ml}$ \\
Patients $(\mathrm{n}=23)$ & Confidence interval & $-7-19 \mathrm{ml}$ & $-12-9 \mathrm{ml}$ & $-6-17 \mathrm{ml}$ \\
& Pearson correlation & 0.95 & 0.98 & 0.88 \\
& P-value t-test & 0.07 & 0.75 & 0.30 \\
& Mean difference & $3 \pm 6 \mathrm{ml}$ & $0.3 \pm 4 \mathrm{ml}$ & $-10 \mathrm{ml}$ \\
& Confidence interval & $-10-16 \mathrm{ml}$ & $-8-9 \mathrm{ml}$ & $-17 \mathrm{ml}$
\end{tabular}

Purpose: Three-dimensional (3D) 3-directional VE MRI with retrospective valve tracking during offline analysis is introduced for flow assessment through all heart valves simultaneously. This technique is validated in phantoms and applied in 14 volunteers without and 23 patients with valve regurgitation.

Methods: MRI was performed on a I.5 T Gyroscan ACS/NTI5 MRI (Philips, Best, the Netherlands). A 3D 3-directional VE $M R I$ sequence was designed (3D volume scan with slab thickness $48 \mathrm{~mm}$, acquisition voxel size $2.9 \times 3.8 \times 4.0 \mathrm{~mm}^{3}$, threedirectional velocity sensitivity $150 \mathrm{~cm} / \mathrm{s}$, with 30 phases reconstructed during one average cardiac cycle, Echo Planar Imaging factor 5, with free-breathing in vivo) and tested in stationary flow phantoms and in a phantom simulating harmonic left ventricular filling. From the 3D velocity-data, through-plane velocity was reformatted offline for each valve plane using two orthogonal reformat-guides per plane (i.e., for mitral valve (MV): 2 - and 4-chamber of the left ventricle (LV); tricuspid valve (TV): 2- and 4-chamber of the right ventricle (RV); aortic valve (AV): two orthogonal views of LV outflow tract; pulmonary valve (PV): two orthogonal views of the RV outflow tract) (Figure I).

In 14 volunteers without regurgitation and in 23 patients with single or multiple valve regurgitation proven on echocardiography, trans-valvular flow was assessed at all four valves using 3D 3directional VE MRI. Regurgitation was quantified. Correlation between the net flow volumes per valve was examined and differences were studied.

Results: Validation in phantoms showed less than $5 \%$ error in flow. In vivo, mean scan time $=4.2 \pm 0.8 \mathrm{~min}$ at a mean heart rate of $67 \pm 12$ beats per minute. In volunteers, comparison of the net flow volumes through the four valves showed strong correlation with a only small differences between $A V$ and $M V$ and between PV and TV (statistically significant but clinically nonsignificant) and with small confidence intervals (Table I). In patients, also strong correlation between the net flow volumes per valve were found (Figure 2), with no significant biases. Mean regurgitant fraction for $M V=12 \pm 8 \%$ (range: 4-29\%), $\mathrm{TV}=$ $10 \pm 7 \%$ (range: $2-25 \%$ ), $A V=2 \pm 2 \%$ (range: $0-5 \%$ ) and $\mathrm{PV}=3 \pm 3 \%$ (range: $0-10 \%$ ).

Conclusion: 3D VE MRI provides the true trans-valvular flow for all four heart valves from a single acquisition in less than 5 minutes scan time. Regurgitation can be quantified accurately, providing essential information for surgical decisionmaking.

\section{4}

Navigator-gated 3D cine DENSE: development and initial evaluation

Xiaodong Zhong', Bruce S Spottiswoode ${ }^{2}$, Craig H Meyer', Christopher M Kramer'

and Frederick $\mathrm{H}$ Epstein ${ }^{\prime}$

'University of Virginia, Charlottesville, VA, USA

${ }^{2}$ University of Stellenbosch, Tygerberg, South Africa

Journal of Cardiovascular Magnetic Resonance 2009, I I(SuppI I):O94

Introduction: Ideally, imaging of cardiac function should cover the entire heart and completely quantify myocardial deformation in three dimensions.

Purpose: To (a) develop a free-breathing navigator-gated 3D cine DENSE (Displacement-encoding with Stimulated Echoes) pulse sequence to acquire such data and (b) implement postprocessing methods to quantify 3D myocardial strain throughout the left ventricle (LV).

Methods: An ECG-gated segmented 3D spiral cine DENSE pulse sequence with navigator gating and online image reconstruction was implemented on a I.5 T MRI scanner (Siemens Avanto, Erlangen, Germany). A 3D stack of spirals k-space trajectory was employed for rapid data acquisition. Three-point phase cycling was used for artifact suppression, a balanced four-point method was used for optimal displacement encoding, and field map acquisition and online spiral deblurring were employed. A navigator echo was placed at the end of the cardiac cycle, so as not to interfere with imaging of the onset of myocardial contraction. The navigator echo was used to accept or reject the DENSE data acquired in the subsequent heart beat. Five normal volunteers provided informed consent and were studied in accordance with protocols approved by our institutional review board. Imaging parameters included voxel size $=2.8 \times 2.8 \times 5.0 \mathrm{~mm}^{3}$, flip angle $=20^{\circ}, \mathrm{TR}=16 \mathrm{~ms}$, $\mathrm{TE}=1.3 \mathrm{~ms}$, number of spiral interleaves $=6$, temporal resolution $=32 \mathrm{~ms}$, and cardiac phases $=22$. A double-oblique 3D volume was aligned with the short and long axes of the LV. Fourteen $k$-space partitions were acquired and then zero-padded to reconstruct 28 slices. A $3 \mathrm{~mm}$ navigator acceptance window was placed at the end-expiration position. For displacement and strain analysis, images were exported to a PC and manually segmented. Tissue tracking and strain analysis were performed using 3D extensions of $2 \mathrm{D}$ methods that were described previously. To validate the $3 D$ measurements, separate $2 D$ cine DENSE MRI was also performed in multiple short- and long-axis planes. Nonnavigator-gated $3 \mathrm{D}$ data were also acquired in some volunteers. 
Figure I (abstract 094)

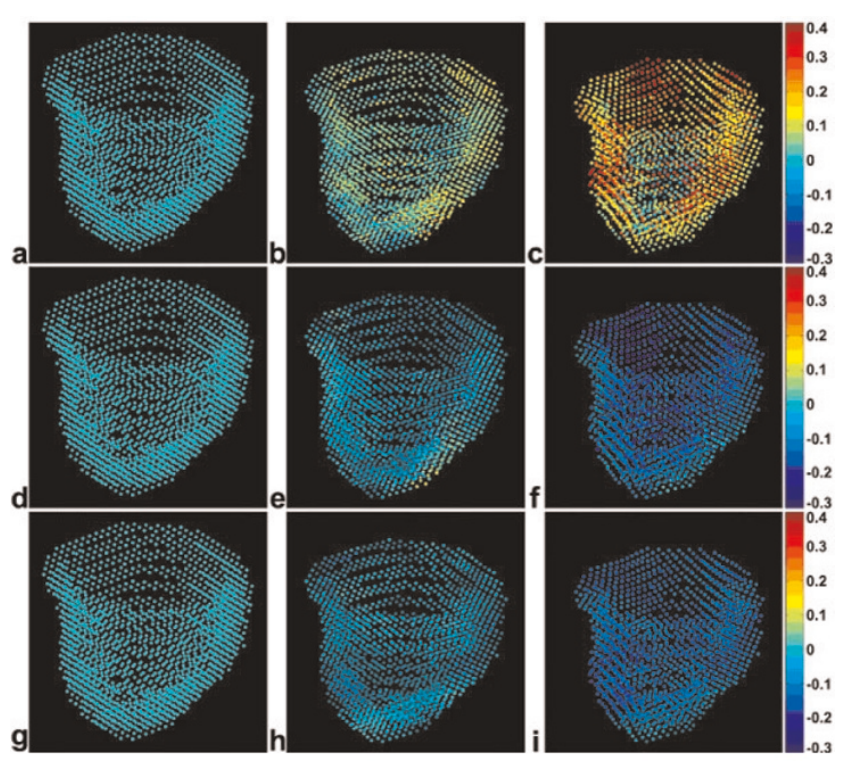

Radial (a-c), circumferential (d-f), and longitudinal (g-i) strains computed throughout the LV from 3D cine DENSE MRI of a normal volunteer. Data are shown at end diastole (a, d, g), mid systole (b, e, h), and end systole (c, $f, i)$. Three of 22 cardiac phases are displayed. Individual dots represent displacement, while color represents strain.

Results: Using a $3 \mathrm{~mm}$ navigator acceptance window, the acceptance rate was $48.0 \pm 15.7 \%$ and the total scan time was $20.5 \pm 5.7$ minutes. High-quality data were acquired from all volunteers, and comparisons with non-navigator-gated free breathing scans clearly demonstrated the reduction of respiratory artifacts provided by navigator gating. Typical 3D strain data from one subject are shown in Fig. I, where the development of radial (a-c), circumferential (d-f), and longitudinal strain (g-i) from end diastole $(a, d, g)$ through mid systole $(b, e, h)$ to end systole $(c, f, i)$ are displayed for the entire LV. Strain values and straintime curves were consistent with previous data from myocardial tagging and DENSE studies of normal volunteers. For the comparison with 2D cine DENSE, linear regression showed that radial, circumferential, and longitudinal strains from 3D cine DENSE correlated well with those from 2D cine DENSE, with a slope of 0.974 and $R=0.647$ for radial strain, a slope of 0.945 and $R=0.902$ for circumferential strain, and a slope of 0.888 and $R=0.772$ for longitudinal strain. Bland-Altman analysis also demonstrated good agreement between the 2D and 3D cine DENSE methods for all 3 strains.

Conclusion: A free-breathing navigator-gated 3D cine DENSE pulse sequence was developed that provides high spatial and temporal resolutions, coverage of the entire LV, and measurement of 3D strain with a scan time of approximately 20 minutes. In normal volunteers, the resulting strain data show good agreement with those from 2D cine DENSE. With additional development aimed at further shortening the scan time and automating image analysis, these methods may enable routine clinical imaging that completely quantifies contractile function throughout the LV in patients with contractile dysfunction.

\section{5}

\author{
Comprehensive evaluation of diastolic \\ function with MRI \\ Thompson Richard', June Cheng Baron', Kelvin Chow', \\ Jessica Scott ${ }^{2}$, Ben Esch ${ }^{2}$, Mark Haykowsky' \\ and lan Paterson' \\ 'University of Alberta, Edmonton, AB, Canada \\ ${ }^{2}$ University of British Columbia, Vancouver, BC, Canada
}

Journal of Cardiovascular Magnetic Resonance 2009, I I (SuppI I):O95

Introduction: Diastolic dysfunction is a contributing factor in most cardiovascular diseases. For example, from the ischemic cascade, it is well known that ventricular relaxation is impaired prior to changes in systolic function. Diastolic parameters are predictive of outcome in acute $\mathrm{MI}[\mathrm{I}]$, and a third to a half of all cases of heart failure have preserved LVEF (>50\%) (HFpEF) [2]. The importance of diastolic dysfunction in the many manifestations of HFpEF is not well characterized. Clinical evaluation of diastolic function is predominantly by echocardiography, for which several conventional and novel quantitative measures of function are available (the vast majority of which are not routinely acquired using MRI). With its increasing use in clinical cardiology, and improvements in temporal resolution, it is now practical for MRI to provide an equivalent or superior assessment of diastolic function. We illustrate the measurement of conventional and novel diastolic parameters using universally available clinical pulse sequences.

Methods: Diastolic parameters are measured in a controls $(n=10)$ and heart failure patients $(n=10)$ with diverse etiologies (ischemic and non-ischemic cardiomyopathies, $13 \%<\mathrm{EF}<67 \%$ ). MRI studies consisted of conventional volumetric cines (SAX and LAX) for the measurement of ESV, EDV, SV (normalized to body surface area) and EF, phase contrast (basal SAX through-plane with $\mathrm{V}_{\text {enc }}=120 \mathrm{~cm} / \mathrm{s}$ and $\mathrm{V}_{\text {enc }}=30$ $50 \mathrm{~cm} / \mathrm{s}, 3 \mathrm{ch}$ and $4 \mathrm{ch}$ with in-plane velocities) and tissue tagging (5 SAX and LAX slices). Conventional diastolic parameters: $\mathrm{E}$ and

Figure I (abstract 095)

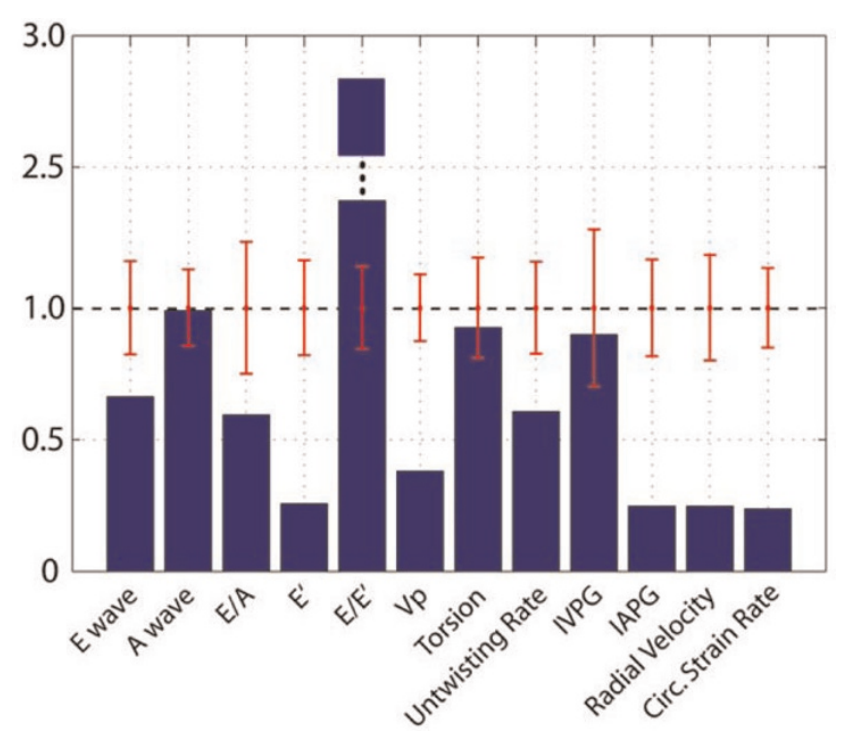

Normalized diastolic parameters (heart failure patient). 
Table I (abstract 095) Heart rate, volumes and function

\begin{tabular}{llllll}
\hline & HR & EDVi $\left(\mathbf{m L} / \mathbf{m}^{2}\right)$ & ESVi $\left(\mathbf{m L} / \mathbf{m}^{2}\right)$ & Svi $\left(\mathbf{m L} / \mathbf{m}^{2}\right)$ & EF(\%) \\
\hline Control & $67.1(14.0)$ & $92.3(16.3)$ & $35.7(9.0)$ & $56.6(8.4)$ & $61.6(3.7)$ \\
Patients & $74.9(20.9)$ & $126.4(88.5)$ & $80.8(41.4)$ & $45.6(21.7)$ & $38.5(17.6)$
\end{tabular}

Table 2 (abstract 095) Conventional diastolic parameters

\begin{tabular}{lllllll}
\hline & E(cm/s) & A(cm/s) & E/A & E' (cm/s) & E/E' & Vp (cm/s) \\
\hline Control & $64.6(11.4)$ & $34.6(5.0)$ & $1.9(0.5)$ & $14.4(2.6)$ & $4.5(0.7)$ & $57.8(7.3)$ \\
Patients & $63.5(22.5)$ & $40.8(13.4)$ & $1.5(0.9)$ & $9.7(6.5)$ & $8.6(4.5)$ & $32.5(13.7)$ \\
\hline
\end{tabular}

Table 3 (abstract 095) Novel diastolic parameters

\begin{tabular}{|c|c|c|c|c|c|c|}
\hline & $\begin{array}{l}\text { IVPG peak } \\
\text { (mmHg) }\end{array}$ & $\begin{array}{l}\text { IAPG } G_{\text {peak }} \\
\text { (mmHg) }\end{array}$ & $\begin{array}{l}\text { Peak } \\
\text { Torsion (deg) }\end{array}$ & $\begin{array}{l}\text { Peak Untwisting } \\
\text { Rate (deg/sec) }\end{array}$ & $\begin{array}{l}\text { Radial } \\
\text { Velocity (cm/s) }\end{array}$ & $\begin{array}{l}\text { Circumferential } \\
\text { Strain rate }\left(s^{-1}\right)\end{array}$ \\
\hline Control & $2.9(0.9)$ & $1.9(0.4)$ & II.I(2.I) & I57.2(27.6) & $4.4(0.9)$ & $1.60(0.24)$ \\
\hline Patients & $3.3(2.3)$ & $0.8(1.4)$ & $8.4(4.3)$ & $77.7(30.5)$ & $2.3(1.0)$ & $0.87(0.46)$ \\
\hline
\end{tabular}

A wave filling velocities $(\mathrm{cm} / \mathrm{s})$, mitral annular velocity $\left(E^{\prime}\right.$ in $\left.\mathrm{cm} / \mathrm{s}\right), E /$ $A$ ratio, E/E' ratio and inflow propagation velocity $\left(V_{p}\right.$ in $\left.\mathrm{cm} / \mathrm{s}\right)$. Additional parameters include the intraventricular (IVPG) and atrial (IAPG) pressure gradients (derived from in-plane blood velocities), peak torsion (deg) and rate of untwisting (deg/sec), peak diastolic radial velocity (ventricular average $-\mathrm{cm} / \mathrm{s}$ ), and peak diastolic circumferential strain rate (ventricular average, $s^{-1}$ ). All tagged images were analyzed using a user-independent morphing approach. All studies were breath held with ECG gating (Siemens Sonata I.5 T scanner).

Results: Tables I, 2 and 3 summarize the conventional volume and diastolic parameters (both conventional and novel measures) in the control and heart failure subjects. Figure I compares one failure case (ischemic cardiomyopathy with $\mathrm{EF}=26 \%$ ) with the control population using normalized diastolic parameters. The control population standard deviations for each parameter are shown, clearly illustrating that several diastolic parameters are abnormal, notably the conventional E' and E/E' values (most sensitive clinical measures of diastolic dysfunction [3]) and most of the novel measures in this subject. Similar striking patterns of abnormal diastolic function are seen in most heart failure patients in this study as indicated by Tables 2 and 3.

Conclusion: MRI can offer a comprehensive evaluation of diastolic function that is comparable or superior to echocardiography. In most heart failure patients the conventional and several novel measures could be measured using conventional pulse sequences, with arrhythmias being the most common technical limitation (2 of the 10 subjects were excluded due to arrhythmias). Using automated processing tools for tag and phase contrast data analysis, rapid and standardized processing is now feasible. In addition to superior LV volumes and function, MRI is the gold standard measure of LA volumes, which is sensitive to increased diastolic pressures, and delayed enhancement offers a measure of fibrosis, which is an important modulator of ventricular relaxation and stiffness and underlying cause of diastolic dysfunction.

\section{References}

I. Moller JE, et al: Circulation 2006, I I 4:438-444.

2. Paulus WJ, et al: Eur Heart J 2007.

3. Kasner M, et al: Circulation 2007, I | 6:637-647.

\section{6}

Evidence across CMR sites and systems

of background velocity offset errors

requiring correction before accurate

measurement of regurgitant and shunt flow

Peter D Gatehouse', Marijn P Rolf ${ }^{2}$, Martin J Graves ${ }^{3}$, John Totman ${ }^{4}$, Jochen von Spiczaki ${ }^{5}$, Maria-

Filomena Santarelli ${ }^{6}$, Yingmin Liu', Rebecca A Quest ${ }^{8}$,

Matthias Dieringer', Massimo Lombardi ${ }^{6}$, Jürg Schwitter ${ }^{10}$, Jeanette Schulz-Menger ${ }^{9}$, David N Firmin ${ }^{\text {, }}$

Mark BM Hofman ${ }^{2}$ and Philip J Kilner'

'Royal Brompton Hospital, London, UK

${ }^{2} \mathrm{VU}$ Medisch Centrum, Amsterdam, Netherlands

${ }^{3}$ Cambridge University Hospitals NHS Foundation Trust, Cambridge, UK

${ }^{4}$ King's College, London, UK

${ }^{5}$ Institute for Biomedical Engineering, University and ETH,

Zurich, Switzerland

${ }^{6}$ CNR Institute of Clinical Pharmacology, Pisa, Italy

${ }^{7}$ University of Auckland, Auckland, New Zealand

${ }^{8}$ Imperial College Healthcare NHS Trust, London, UK

${ }^{9}$ Franz-Volhard-Klinik, Charité Universitätsmedizin, Berlin, Germany

${ }^{10}$ University Hospital, Zurich, Switzerland

Journal of Cardiovascular Magnetic Resonance 2009, I I(SuppI I):096

Purpose: To assess velocity offsets in the background of phasecontrast acquisitions across CMR sites and systems.

Introduction: Phase-contrast CMR potentially provides accurate measurements of aortic or pulmonary regurgitation, cardiac output and shunt flow. Among several known errors (assuming 
Figure I (abstract 096)

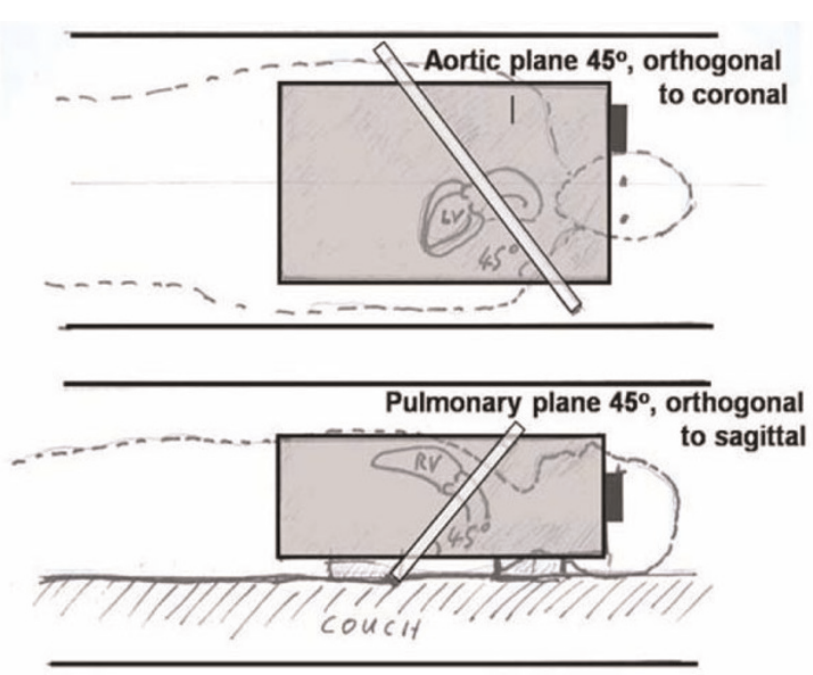

The planes used for veolicity offset acquisitions at all sites. The aortic (AO) plane at 45 degress between transaxial and sagittal used anteroposterior phase-encode. The main pulmonary artery (MPA) plane at 45 degrees between transaxial and coronal was repeated with head-foot and left-right phase-encoding. The grey bloc represents the gelatin phantom.

concomitant gradient correction [I]) we examined one: a $2 \mathrm{~cm} / \mathrm{s}$ velocity offset (i.e. around I\% of a typical VENC) can cause $>20 \%$ error in cardiac output [2, 3] with larger consequences for regurgitation and shunt flow, particularly in dilated vessels.

As a collaborative group, we measured velocity offsets across sites and scanner types, an initiative backed by the European Society of Cardiology CMR Working Group.

Methods: To eliminate slow flow, 10-15 litre uniform gelatine phantoms were used, containing 5 millimoles/litre Gd-DTPA for SNR. We used similar phase-contrast sequence parameters at each site, measuring $45^{\circ}$ oblique 'aortic' (Ao) and 'main pulmonary artery' (MPA) planes (Figure I). Constant sequence parameters were: retro-gated cine at $1000 \mathrm{~ms}$ RR-interval, through-plane Venc $=150 \mathrm{~cm} / \mathrm{s}, \mathrm{SLT}=6 \mathrm{~mm}, \mathrm{TE}=2.8-3.0 \mathrm{~ms}$, FOV $=320 \mathrm{~mm}$ square, uninterpolated pixels $1.25 \mathrm{~mm}(\mathrm{FE})$ by $2.5 \mathrm{~mm}(\mathrm{PE})$, bandwidth $355 \mathrm{~Hz} /$ pixel, 6 rawdata lines per cardiac cycle, no cine data-sharing or parallel imaging. Unless stated, velocity encoding was asymmetric (i.e. phasesubtraction of compensated and velocity-encoded). The gradient-echo was asymmetric (early for short TE); Philips applied partial-echo sampling which may explain its shorter TR. Slower machines were excluded because fast gradient performance was necessary. Three I.5 T scanner types were compared, and the sequences were reproduced exactly among the 4 sites of each scanner type by protocol file transfer (except I site below):

GE (4 sites) Signa Excite I4M5. Symmetric velocity-encoding (compulsory), flow analysis on, flow optimization off, TR5.9$6.0 \mathrm{~ms}$, TE2.9-3.0 ms (image orientation dependent) (I site on I2M5 TR5.7-5.8 ms TE2.9 ms)

Philips (4 sites) Achieva R2.53. TR5.5 ms, TE2.8 ms, asymmetric RF pulse (late centre), phase correction off.

Siemens (4 sites) Avanto VBI5 TR6.6 ms, TE2.8 ms.
Table I (abstract 096) Largest ROI mean offset $(\mathrm{cm} / \mathrm{s})$ within $50 \mathrm{~mm}$ of Isocentre, for aortic slice, MPA slice (HF phaseencoding) and MPA slice (LR phase-encoding). The four rows per slice are from the four sites using each scanner type. The column order is not specified, i.e. scanner types are not identified. ( $< \pm 0.3 \mathrm{~cm} / \mathrm{s}$ stdev total error as described above)

\begin{tabular}{|c|c|c|c|c|}
\hline $\mathrm{cm} / \mathrm{s}$ & Site & $\begin{array}{l}\text { Scanner } \\
\text { type I }\end{array}$ & $\begin{array}{l}\text { Scanner } \\
\text { type } 2\end{array}$ & $\begin{array}{c}\text { Scanner } \\
\text { type } 3\end{array}$ \\
\hline \multirow[t]{4}{*}{ Aorta } & I & 2.5 & 1.4 & 1.7 \\
\hline & 2 & 0.7 & 2.5 & 0.9 \\
\hline & 3 & 0.9 & 2.9 & 1.4 \\
\hline & 4 & 1.9 & 1.6 & 0.7 \\
\hline \multirow[t]{4}{*}{ MPA (HF phase-enc) } & I & 0.8 & 3.9 & 1.0 \\
\hline & 2 & I.I & 3.4 & 1.3 \\
\hline & 3 & 0.8 & 4.9 & 1.2 \\
\hline & 4 & 1.0 & 3.9 & 0.8 \\
\hline \multirow[t]{4}{*}{ MPA (LR phase-enc) } & I & I.2 & 3.3 & I.5 \\
\hline & 2 & 1.0 & 5.3 & 1.6 \\
\hline & 3 & 0.8 & 5.0 & 1.8 \\
\hline & 4 & 0.4 & 3.6 & 0.6 \\
\hline \multirow[t]{4}{*}{ Maximum per site } & I & 2.5 & 3.9 & 1.7 \\
\hline & 2 & 1.1 & 5.3 & 1.6 \\
\hline & 3 & 0.9 & 5.0 & 1.8 \\
\hline & 4 & 1.9 & 3.9 & 0.8 \\
\hline
\end{tabular}

Velocity images were reconstructed without offset correction (which performed unrealistically well in this large uniform phantom). Within $50 \mathrm{~mm}$ of isocentre, the largest offset in $\mathrm{cm} /$ $\mathrm{s}$ averaged over $300 \mathrm{~mm}^{2}$ circular ROI was recorded for each plane. All images were measured independently by two sites; total error was estimated between these, also from noise and cine frame variations.

Results: Please see data in Table I.

Discussion and conclusion: The results are believed reliable for 3 reasons: I) cine images were stable without ghosting, 2) similar results occurred in nearby parallel slices, 3) image analysis was repeated independently. Comparison of hardware is prevented by remaining differences between sequences; we emphasise that small sequence changes may alter these results. An offset of $1 \%$ of Venc ( $\pi / / 00)$ is impressive engineering, representing a residual gradient of $\sim 0.02 \%$ (for approx. TE/2, $50 \mathrm{~mm}$ from isocentre) of typical velocity-encoding gradients. This extreme sensitivity to adjustments such as pre-emphasis could explain variations between nominally identical sites.

Various automatic offset corrections are routinely installed. However, this study intentionally omitted them; their usefulness may depend on applications. Offset correction uses stationary tissue pixels which can be identified automatically based on their smaller temporal variation [2]), or identified by users during postprocessing. This approach is sometimes limited by insufficient stationary tissue, its low SNR in flow images, and possible spatial non-linearity. A more time-consuming approach repeats identical flow acquisitions on a static phantom, subtracting the corresponding apparent phantom velocities from the clinical acquisition [2].

We conclude that most systems require velocity offset correction of flow images for the most sensitive clinical applications. There is a general need for optimization of acquisition protocols or (if possible) system engineering and correction methods to minimise velocity offsets. 


\section{Acknowledgements}

Drs Jason Polzin (GE), Marc Kouwenhoven (Philips), Andreas Greiser (Siemens).

References

I. Bernstein and Zhou, et al: MRM 1998.

2. Lankhaar and Hofman, et al: JMRI 2005.

3. Chernobelsky and Shubayev, et al: JCMR 2007.

\section{7}

Should reference values for ventricular volumes and mass of children be indexed for body surface area, height or weight considering gender differences?

Samir Sarikouch', Titus Kuehne ${ }^{2}$, Matthias Gutberlet ${ }^{3}$ and Philipp Beerbaum ${ }^{4}$

'Department of Heart-, Thoracic-, Transplantation- and Vascular Surgery, Hannover Medical School, Germany

${ }^{2}$ Unit of Cardiovascular Imaging - Congenital Heart Diseases, Deutsches Herzzentrum, Berlin, Germany

${ }^{3}$ Department of Radiology, Heart Centre, Leipzig, Germany

${ }^{4}$ Division of Imaging Sciences, King's College, Guy's \& St

Thomas' Hospital, London, UK

Journal of Cardiovascular Magnetic Resonance 2009, I I(SuppI I):097

Background: Medical decision making in congenital and acquired heart disease is based increasingly on quantitative MRI assessment of ventricular volumes and myocardial mass. However, for children in different age groups, there is only very limited reference data available, yet. The objective of this study was therefore to provide statistically robust reference data for ventricular volumes and mass for healthy children.

Methods: A total of 114 healthy children and adolescents, uniformly distributed, were examined in a standard I.5 Tesla scanner in breathhold-technique using steady-state free precession and phase-contrast sequences according to a standardized pediatric CMR protocol. Transversal acquisition, 5-6 mm slicethickness, no gap, 25-35 phases, resolution 2.0-2.5 × I.5$1.8 \mathrm{~mm}^{2}$. Semiautomatic volumetric analysis as well as analysis of stroke volumes in the main pulmonary artery and the ascending aorta were performed by one observer to minimize observer error.

Results: II4 children and adolescents were examined, age ranged between 4 and 20 years (25. percentile 9,2 yrs, 50 . percentilelI,9 yrs, 75. percentile 16,I yrs). Reference centile curves were constructed using the lambda-mu-sigma (LMS) method for left and right enddiastolic and-endsystolic volumes, stroke volume, ejection fraction and ventricular mass. Figure I.

With increasing age there was a steady increase in left and right ventricular enddiastolic volumes indexed for body surface area, as the most common form of indexing in MRI, from 4 up to 14 years where the volumes reached a plateau. Ventricular volumes and masses indexed to the body surface area were in general $10 \%$ higher in boys than in girls. This statistically significant gender difference showed to be the same when indexing for height and disappeared after relating ventricular volume to weight. $2.5 \mathrm{ml} / \mathrm{kg}$ resulted in the enddiastolic volume of the ventricles and $\mathrm{I} \mathrm{ml} / \mathrm{kg}$ in the endsystolic volume.

Conclusion: Data for ventricular volumes and mass for children are provided which can serve as a reference tool for the assessment of pathologic changes in congenital and acquired
Figure I (abstract 097)

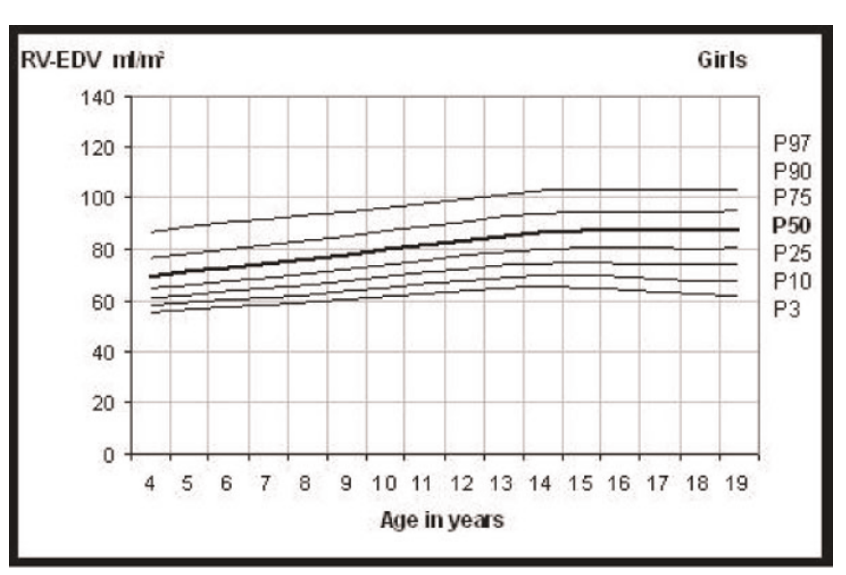

heart disease. Indexing of ventricular volumes to body weight instead of body surface area elimates the gender difference in children and should therefore be preferred.

\section{8}

Measuring right ventricular volume and ejection fraction with Simpson's method: which MRI axis is best? Comparison with a "gold standard" Shawn Haji-Momenian', Kevin J Chang', David J Grand', Florence H Sheehan ${ }^{2}$ and Michael K Atalay'

'Brown University, Providence, RI, USA

${ }^{2}$ University of Washington, Seattle, WA, USA

Journal of Cardiovascular Magnetic Resonance 2009, I I(SuppI I):098

Purpose: Because of its complex morphology, accurate and reliable quantification of right ventricular (RV) volume and function using MRI is challenging. This study had two aims: (I) to

Figure I (abstract 098)

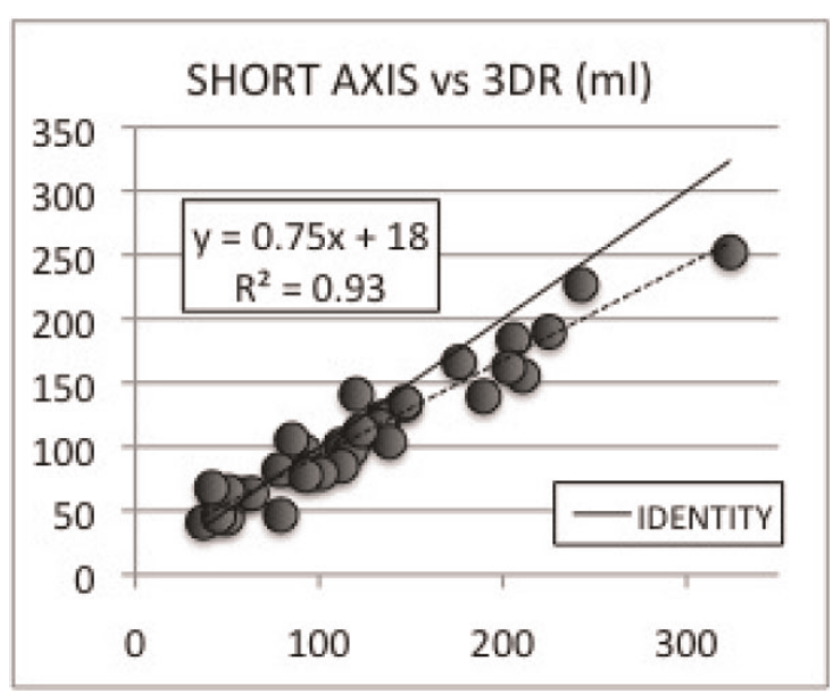

Short axis vs 3DR (ml). 
Figure 2 (abstract 098)

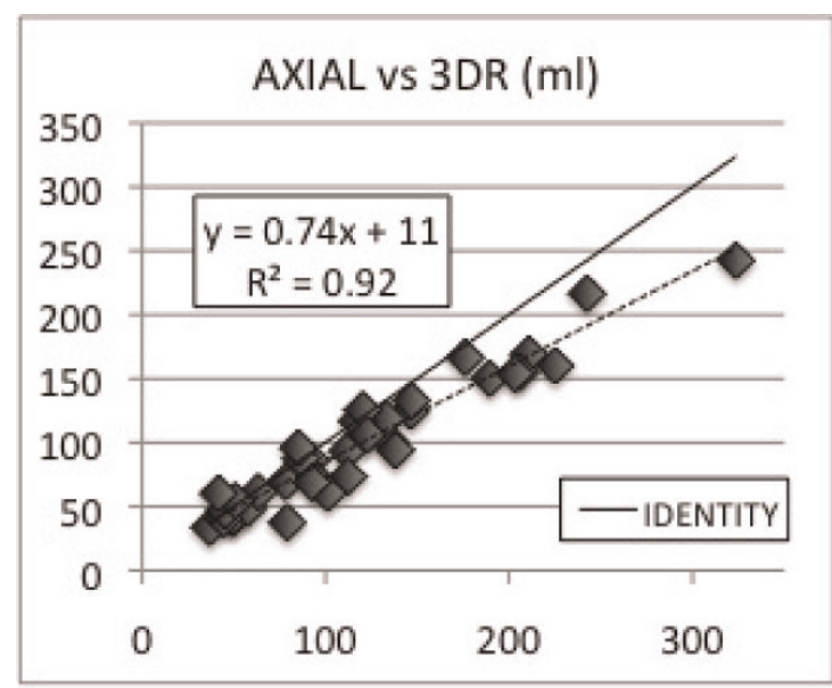

Axial vs 3DR (ml).

determine the interobserver reliability of RV volume and ejection fraction (EF\%) calculated using Simpson's method of slice summation applied to data acquired in three different orientations: short axis (SA), transaxial (TA), and parallel to the horizontal long axis (pHLA); and (2) to determine how RV volume and EF\% by each of the three orientations compared with values obtained using a validated "gold-standard" method with 3-D reconstructions (3DR).

Materials and methods: Twenty-three consecutive, consented patients referred for cardiac MRI were included in the study ( 10 males, 13 females; ave. age $43 \pm 19$ yrs; ave. ht: $66 \pm$ 4 in; ave. wt: $\mid 77 \pm 5 \mathrm{I}$ lbs). Steady-state free precession was used to generate stacked, bright-blood cine loops in 3 separate, randomly ordered orientations: SA, TA, and pHLA. Slice

Figure 3 (abstract 098)

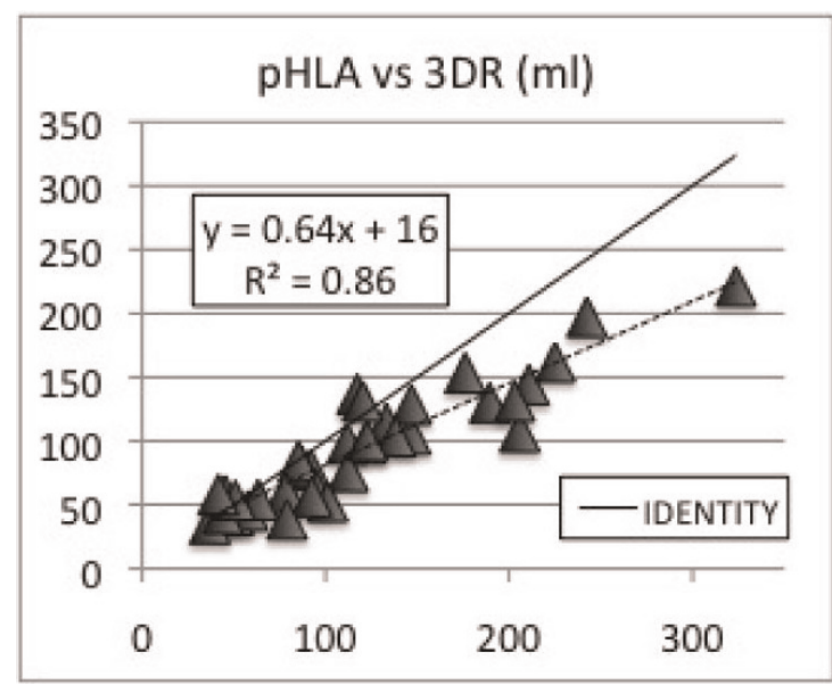

pHLA vs 3DR (ml). thickness was $8 \mathrm{~mm}$ and slice separation $2 \mathrm{~mm}$. Using Simpson's method and pre-determined end-diastolic (ED) and end-systolic (ES) time points, three experienced reviewers independently measured RV ED and ES volumes and, in turn, EF\% for each of the orientations. Volumes and EF\% were also calculated using a 3DR technique based on the piecewise smooth subdivision surface method [I,2], employing data from multiple orientations. Intraclass correlation was used to compare data from different observers. Paired t-test analysis was used to compare volumes and $\mathrm{EF} \%$.

Results: Interrater reliability (IRR) of RV ED and ES volumes and EF\% was determined for each of the axes. For the SA, IRR for all readers was $0.92,0.87$, and 0.33 , respectively; for TA, 0.95, 0.90 , and $0.7 \mathrm{I}$; for $\mathrm{PHLA}, 0.83,0.9 \mathrm{I}$, and 0.67 . (A higher ratio indicates greater reliability). For comparison, the same parameters were also determined for LV ED and ES volumes, and EF\% measured using the SA: $0.98,0.96$, and 0.84 .

A wide range of RV volumes (37-323 cc) and EF\%s (30-67\%) were observed. Average EDV volumes for 3DR, SA, TA, \& PHLA were: $161 \pm 60 \mathrm{cc}, \quad 140 \pm 45 \mathrm{cc}, \quad 136 \pm 44 \mathrm{cc}, \quad 126 \pm 37 \mathrm{cc}$. Average ESV volumes for 3DR, SA, TA, \& pHLA were: $80 \pm$ $44 \mathrm{cc}, 76 \pm 35,65 \pm 31 \mathrm{cc}, 62 \pm 30 \mathrm{cc}$. All ED and ES volumes were underestimated using Simpson's method $(p<.01)$. SA, TA, and $\mathrm{PHLA}$ volumes were linearly correlated with $3 \mathrm{DR}$ volumes with $R$ values 0.96, 0.96, and 0.93 (See Figures I, 2, 3). Correlations for EDVs were slightly better than those for ESVs for SA and TA orientations. Average EF\%s for 3DR, SA, TA, \& pHLA were: $53 \pm 9 \%, 47 \% \pm 8 \%(p<.05), 53 \pm 7 \%(P=N S), \&$ $52 \pm 9 \%(P=N S)$. SA, TA, and PHLA EF\%s were linearly correlated with $3 \mathrm{DR}$ EF\%s with $\mathrm{R}$ values $0.66,0.57$, and 0.65 .

Conclusion: Reliability of RV ED and ES volume measurements is comparable for all three axes evaluated. However, the reliability of the EF\% is best on TA imaging. Moreover, although TA offers slightly worse EF\% correlation with 3DR, our data suggest that for consistency - if Simpson's method is used for RV volume and EF\% quantification - the TA axis is preferred.

\section{References}

I. Hubka, et al: Int J Cardiovasc Imaging 2002, I 8: | | |- | | 8 .

2. Legget, et al: IEEE Trans Biomed Eng 1998, 45:494-504.

\section{9}

Assessment of left ventricular volumes and mass using single-breath-hold 3D k-t BLAST cine b-SSFP in comparison with multiple-breath-hold 2D cine b-SSFP

Alessandro Palumbo, Giancarlo Messalli, Erica Maffei, Chiara Martini, Cosetta Saccò and Filippo Cademartiri Ospedale Maggiore di Parma, Parma, Italy

Journal of Cardiovascular Magnetic Resonance 2009, I I(SuppI I):O99

Purpose: To compare performance of new single-breath-hold 3D k-t BLAST cine b-SSFP sequence for left ventricular volume and mass evaluation using multiple-breath-hold 2D cine b-SSFP as a reference standard. We also compared time-efficiency of the two sequences calculating scan time and reporting time.

Methods: On a commercially available I,5 T MR scan (Achieva, Philips Medical System), single-breath-hold 3D k-t BLAST cine b-SSFP sequence (3D-cine) and multiple-breath-hold 2D cine b-SSFP sequence (2D-cine) were performed in 46 patients referred to investigate different diseases. The global functional 
parameters, LV mass, scan time and report time were evaluated in each patient for both sequences. Differences between functional parameters and LV mass were made with a paired Student's T test; correlation between parameters was assessed with Pearson's correlation coefficient. A Bland-Altman analysis was used to investigate the limits of agreement between the measurements. Differences between time-efficiency related parameters were made with a paired Student's T test.

Results: Functional parameters and mass were significantly different in the two sequences $(p<0.05)$ but a strong correlation was found for LVejection fraction $(r=0.96)$ and good correlation for other functional parameters ( $r$ between 0.83 and 0.93 ). Scan time was significantly lower for 3D sequence, report time was significantly higher for 3D sequence.

Conclusion: 3D k-t BLAST sequence can be used to assess EF in patients who have poor compliance in performing multiple apnoeas and in patients who are not able to remain in the scanner for a long time. Conversely report time is significantly higher for $3 D$ sequence.

\section{0}

CMR T2* technique for segmental and global quantification of myocardial iron: multi-centre transfereability and healtcare impact evalaution Vincenzo Positano', Anna Ramazzotti', Antonella Meloni', Alessia Pepe', Giuseppe Rossi', Cristina Salvatori', Paolo Marcheschi', Maurizio Mangione', Luigi Natale ${ }^{2}$, Eliana Cracolici ${ }^{3}$, Gennaro Restaino ${ }^{4}$, Gianluca Valeri ${ }^{5}$, Antongiulio Luciani ${ }^{6}$, Calogera Gerardi ${ }^{7}$ and Massimo Lombardi

I "G Monasterio" Foundation and Institute of Clinical

Physiology, CNR, Pisa, Italy

${ }^{2}$ Policlinico "Gemelli", Roma, Italy

${ }^{3}$ Policlinico "Paolo Giaccone", Palermo, Italy
${ }^{4}$ Università Cattolica del Sacro Cuore, Campobasso, Italy

${ }^{5}$ Ospedali Riuniti di Ancona, Ancona, Italy

${ }^{6}$ Az. Osp. "Garibaldi”, Catania, Italy

${ }^{7}$ Ospedali Civili Riuniti, Sciacca (AG), Italy

Journal of Cardiovascular Magnetic Resonance 2009, I I(SuppI I):O I00

Introduction: Iron induced cardiomiopathy is the main cause of mortality in thalassemic population. Thus, the improvement of chelation regimens, to reduce cardiac disease, has the highest priority. Efficient evaluation of cardiac iron status and careful epidemiologic assessment of thalassemic patients play an important role in this matter. T2* cardiac magnetic resonance imaging (CMR) is a unique technique to quantify myocardial iron overload and useful to tailor the chelation therapy. In particular, effective and reproducible assessment of myocardial iron loading using the multislice multiecho T2* approach for segmental and global myocardial iron distribution has been demonstrated within a single CMR site. Thalassemia major (TM) patients require lifelong myocardial iron load monitoring to assess the effectiveness of chelation therapies. Hence, it is highly desirable that CMR be performed near the patients' locations, and that the patients be able to safely move between different CMR centers.

Purpose: Aim of this work is to build a reliable network of haematological and paediatric centers specializing in thalassemia care and MRI sites able to perform feasible and reproducible heart iron overload assessments for a consistent number of thalassemia patients in a standardized and robust manner.

Materials and methods: In order to assess the transferability of the multislice multiecho $\mathrm{T}^{*}$ technique, heart multislice multiecho T2* sequence was installed on I.5 T MRI scanners (GE Healthcare) at six different sites. Five healthy subjects at each site $(n=30)$ were scanned to verify the homogeneity of normal ranges (T2* lover limit of normal $20 \mathrm{~ms}$ ). Then, five TM patients were scanned at the reference site and were rescanned locally

\section{Figure I (abstract O 100)}
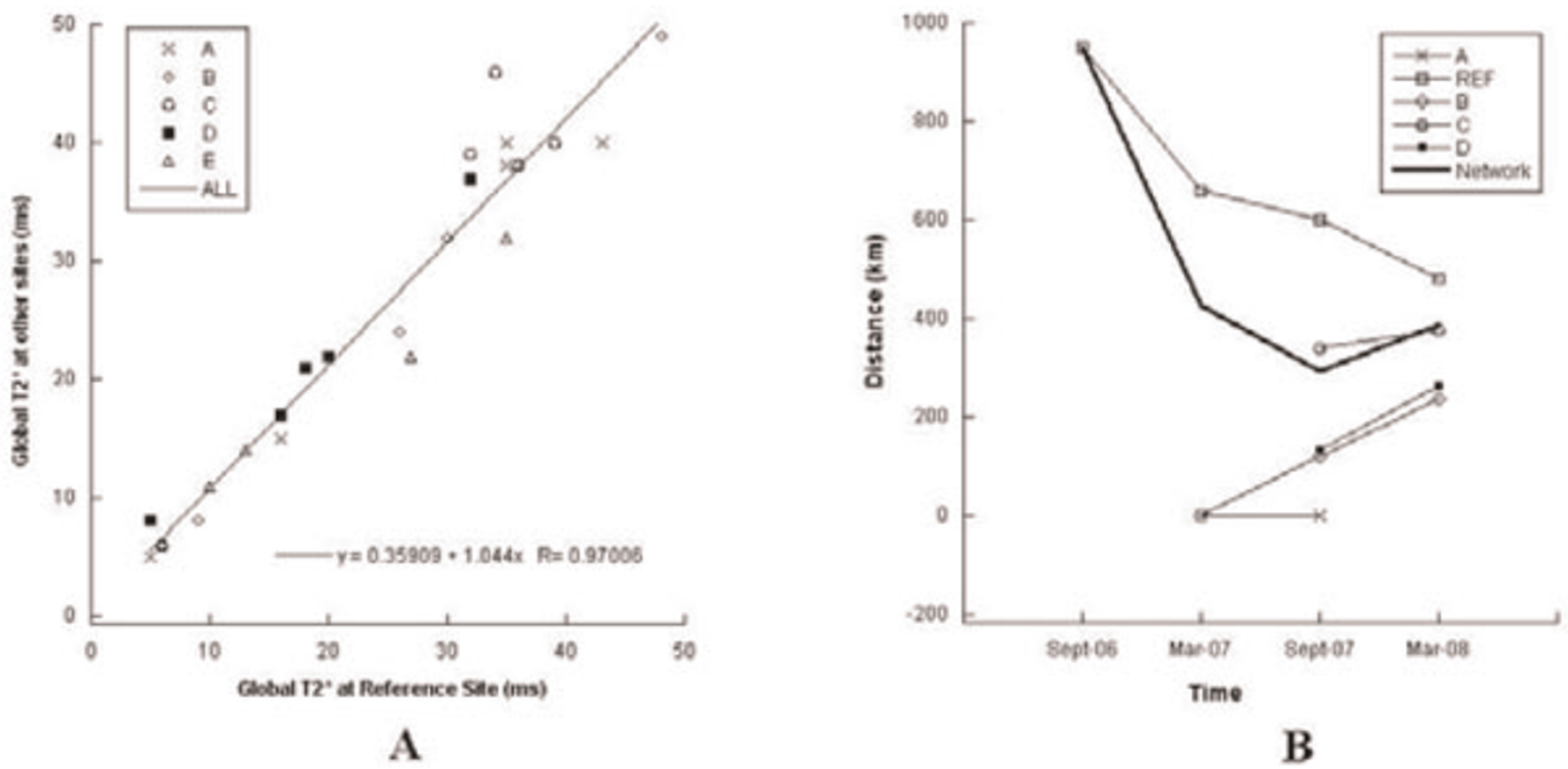

Page 85 of 316

(page number not for citation purposes) 
$(\mathrm{n}=25)$ within one month. T2* images were analysed using a previously validated software (HIPPO MIOT $^{\circledR}$ ).

After the assessment of CMR technique reproducibility, patients enrolling started in September 2006. A centralized data management system was made to share patient data between CMR and thalassemia sites. It allowed optimizing the TM patients care and favouring the creation of a clinical-instrumental database with data exchange facilities to develop diagnostic, prognostic and therapeutical evidence-based treatments for thalassemia patients. The study was approved by the local ethics committees and followed the principles outlined in the Declaration of Helsinki.

Results: Global and segmental T2* values of healthy subjects showed inter-sites homogeneity. On TM patients, for global heart T2* values the correlation coefficient was 0.97 , Coefficients of Variation (CoVs) ranged from 0.04 to 0.12 and Intraclass Coefficients (ICCs) ranged from 0.94 to 0.99 . The mean CoV and ICC for segmental T2* distribution were 0.198 and 88 , respectively. Figure IA shows linear regression of global heart T2* values obtained from $25(5 \times 5)$ patients who were scanned at the reference site and locally at each of the other five sites on the same conditions.

Since the project's beginning, 695 thalassemia patients have been involved in the network. 630 patients $(90 \%)$ successfully underwent CMR examination. Twenty patients $(3 \%)$ refused CMR, mainly due to claustrophobia. The remaining patients $(7 \%)$ have been scheduled for future examination. The mean distance from the patient home locations to the CMR site where the patients underwent the exams, which is considered an indicator of patient comfort, significantly decreased during the network's evolution. In Figure IB, the average distances from the thalassemia centers which sent the patients for CMR examination are plotted versus time.

Conclusion: The multislice multiecho T2* technique is transferable among scanners with good reproducibility. The network seems to be a robust and scalable system in which T2* CMRbased cardiac iron overload assessment is available, accessible and reachable for a significant and increasing number of thalassemia patients, reducing the mean distance from the patients' locations to the CMR sites.

\section{1}

$3 \mathrm{~T}$ cardiac magnetic resonance performs well as the primary scanner in a clinical setting: our initial experience at a tertiary care center Mahadevan Rajaram', Luciana F Seabra',

Shuaib M Abdullah', Sanjeev A Francis', Sofia C Masri', Renate Jerecic ${ }^{2}$, Michael Jerosch-Herold'

and Raymond Y Kwong'

'Brigham and Women's Hospital, Boston, MA, USA

${ }^{2}$ Siemens Medical Solutions, Chicago, IL, USA

Journal of Cardiovascular Magnetic Resonance 2009, I I(SuppI I): O IO I

Introduction: Despite the advantage of increased signal-noiseratio, skepticism exists regarding the use of $3 \mathrm{~T}$ as the primary scanner for routine clinical CMR examination due to potential for gating difficulties related to the increased magnetohydrodynamic effect, off-resonance artifacts, and patient heating. We quantified the diagnostic potential and artifacts based on our experience of the first 4 months of routine clinical 3 T CMR exams in a tertiary clinical center.
Purpose: To test the hypothesis that $3 \mathrm{~T} M R I$ is practical in serving a busy clinical CMR service as the primary routine cardiac scanner.

Methods: Two-hundred and eighty patients were referred for CMR for a broad range of clinical indications over a 4-month period and underwent a $3 \mathrm{~T}$ cardiac MRI scan (MAGNETOM Tim Trio, Siemens, Germany). Three experienced readers quantified total scan time, troubleshooting time for 3 T-related offresonance artifacts, image quality, and artifacts in all pulse sequences performed. Image quality was graded per accepted criteria ( $\mathrm{I}-\mathrm{Non}$ diagnostic, 2-diagnosis suspected but not established with severe blurring, 3-definite diagnosis despite moderate blurring, 4-definite diagnosis with only mild blurring, 5-definite diagnosis without visible blurring). Artifacts severity was graded in a 5-point scale (I-No artifacts, 2-minimal artifacts, good diagnostic quality images, 3-moderate artifact and diagnosis established, 4-considerable artifacts, diagnosis suspected but not established, 5 - severe artifacts, non diagnostic images). Excellent image quality was classified as a score $\geq 4$ and minimal or no artifact was classified as an artifact score of $\leq 2$. Forty-six I.5 T CMR studies performed at the same study period with a matched spread of indications were randomly selected as a control group for comparison.

Results: On average, 2.8 minutes (5\% of total scan time) were spent to eliminate off-resonance banding artifacts in $3 \mathrm{~T}$. This time is made up by more aggressive accelerated parallel imaging technique. As a result, average total scan time using $3 \mathrm{~T}$ was not different from I.5 T $(54 \pm 14$ vs. $54 \pm 12$ minutes, $P=0.47)$. No patients failed to complete the study due to SAR limit. There were no complications during any of the I.5 T or $3 \mathrm{~T} C M R$ studies. A significantly higher proportion of perfusion images were graded as being of excellent quality on $3 \mathrm{~T}$ when compared to $1.5 \mathrm{~T}(82.4 \%$ vs. $4 \mathrm{I} .4 \%, \mathrm{P}<0.000 \mathrm{I})$ (Figure I). A significantly higher number of perfusion images also had minimal or no artifact on $3 \mathrm{~T}$ when compared to $1.5 \mathrm{~T}(93.7 \%$ vs. $72.4 \%$, $\mathrm{p}=0.0016)$. When LGE images were analyzed, a significantly higher proportion of images on $3 \mathrm{~T}$ were graded as being excellent $(82.6 \%$ vs. $46.2 \%, \mathrm{p}<0.000 \mathrm{I})$ and the proportion of LGE images having minimal or no artifact was also significantly higher on $3 \mathrm{~T}(83.0 \%$ vs. $56.4 \%, \mathrm{p}=0.0042)$. The number of Cine SSFP, pulmonary vein MRA, and phase contrast images that were

Figure I (abstract OI0I)

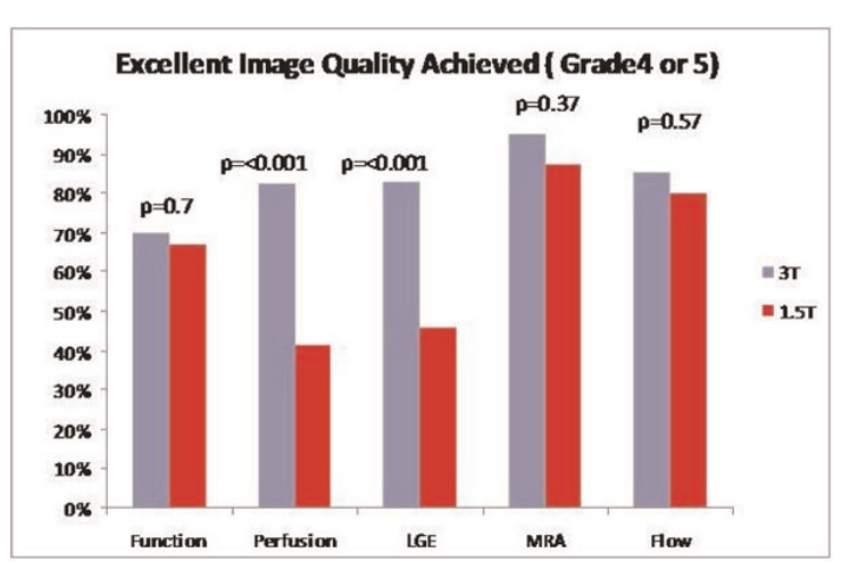

Excellent image quality achieved (grade 4 or 5 ). 
graded as being of excellent quality or with no or minimal artifact did not differ between $3 \mathrm{~T}$ and $1.5 \mathrm{~T}$.

Conclusion: $3 \mathrm{~T}$ cardiac MRI performs well serving as the primary scanner in a busy CMR service with comparable scan times to I.5 T cardiac MRI. 3 T has improved image quality and fewer artifacts especially for applications like perfusion and LGE which benefit from the increase in TI times at $3 \mathrm{~T}$. The high SNR leaves additional room to also decrease the overall scan time in the future using higher acceleration factors for parallel imaging techniques without sacrificing diagnostic image quality.

\section{2}

Three-dimensional measurement of LV and RV dimensions using prospective self-gating for simultaneous compensation of cardiac and respiratory motion

Robert Manka', Peter Boesiger², Martin Buehrer² and Sebastian Kozerke ${ }^{2}$

${ }^{\prime}$ German Heart Institute Berlin, Berlin, Germany

${ }^{2}$ Institute for Biomedical Engineering, Zürich, Switzerland

Journal of Cardiovascular Magnetic Resonance 2009, I I(SuppI I):O 102

Purpose: To compare three-dimensional (3D) balanced steadystate free precession (SSFP), prospective self-gating technique [I] without ECG triggering and breath-holding for the assessment of left ventricular (LV) and right ventricular (RV) function in the heart in comparison to standard 2D, multiple breath-hold SSFP cine imaging.

Methods: Data were acquired in 15 subjects ( 10 volunteers, 5 patients) using a I.5 T system with a five element cardiac array coil. In each subject a standard multi-slice, multi-breathhold 2D cine SSFP sequence was performed with complete ventricular coverage. Additionally, a three-dimensional cine sequence with prospective self-gating [I] with complete ventricular coverage was acquired during free breathing. LV and RV end-systolic volume (ESV) and end-diastolic volume (EDV) and LV mass were calculated for each method. With both imaging techniques, a patient-based analysis of image quality was performed with grading on a four-point scale, referring to the visibility of the endocardial border (excellent (4), good (3), moderate (2) and nondiagnostic (I)).

Results: Good agreement between LVEDV, LVESV, LV mass, LVEF, RVEDV, RVESV, and RVEF calculated for the standard 2D and the $3 D$ prospective self-gating method (concordance

Figure I (abstract O102)

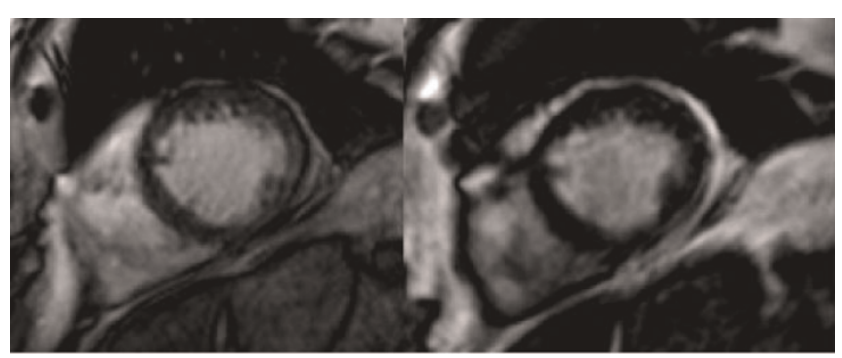

End-diastolic (ED) frame of the left and right ventricle (mid-ventricular slice in short-axis orientation); left: multi-breath-hold, standard SSFP, right: free breathing prospective self-gating. coefficients $0.99,0.99,0.99,0.90,0.95,0.95$ and 0.91 , respectively). The mean bias ( $95 \%$ confidence interval $(\mathrm{Cl})$ for each parameter was; LVEDV: $-0.3 \%(-5.2$ to 4.6$)$, LVESV: $0.3 \%$ (-5.4 to 6.0$)$, LV mass: $-0.8 \%$ ( -8.3 to 6.8$)$, LVEF: $-0.2 \%(-2.7$ to 2.4), RVEDV: $4.5 \%$ (-9.6 to I8.6), RVESV: $3.8 \%$ (-II.2 to I8.7), RVEF: $1.0 \%$ ( -4.7 to 6.7$)$. The overall image quality score for prospective self-gating $(2.7 \pm 0.8)$ was lower when compared to standard SSFP $(3.9 \pm 0.4 ; p<0.0$ I $)$. Figure I shows representative images from one patient.

Discussion: Three-dimensional, free-breathing, prospective self-gating MRI enabled accurate assessment of LV and RV quantitative parameters when compared to standard multi-slice, multi-breathhold SSFP cine imaging. Image quality with prospective self-gating was rated lower relative to the reference ECG triggered, multiple breathhold scans due to lower image contrast between blood and myocardium and residual motion artefacts.

Reference

I. Buehrer M, et al: Magn Reson Med 2008, 60(3):683-690.

\section{3}

Randomized comparison of observation unit plus stress cardiac MRI and hospital admission Chadwick D Miller, Wenke Hwang, James W Hoekstra, Cedric Lefebvre, Doug Case and W Gregory Hundley Wake Forest University School of Medicine, Winston-Salem, NC, USA

Journal of Cardiovascular Magnetic Resonance 2009, I I(SuppI I):OI 03

Introduction: Adoption of an observation unit (OU) strategy in patients with chest pain at intermediate-risk for ACS has been hampered by limitations of traditional cardiac testing. As a result, most intermediate risk patients are admitted to the hospital for their evaluation. CMR demonstrates superior accuracy compared to other testing modalities. Additionally, CMR is with highly sensitive to recent or ongoing infarction which may allow stress imaging to be performed without waiting for the results of serial cardiac markers. These advantages of CMR make it well suited for use in OUs. An OU-CMR strategy may be resource saving compared to hospital admission.

Purpose: To compare resource consumption between an observation unit stress cardiac MRI (OU-CMR) strategy and hospital admission when used to evaluate emergency department (ED) patients with chest pain at intermediate risk for acute coronary syndrome (ACS).

Methods: Patients meeting intermediate risk criteria (TIMI risk score $\geq 2$ or clinical impression of intermediate risk) underwent stratified blocked randomization to OU-CMR or hospital admission. OU-CMR participants underwent cardiac markers at 0,4 , and 8 hours with adenosine or dobutamine CMR imaging performed at the first available time after the return of the first two cardiac marker results. CMR imaging included resting wall motion, T2 weighted imaging, and perfusion, stress wall motion and perfusion, and delayed enhancement. Hospital admission participants underwent evaluations as determined by their treating physician. Participants were contacted at 30 days to determine events occurring after hospital discharge. Primary outcomes included direct cost of the index hospitalization and length of stay. Cost was calculated using cost:charge ratios and physician work-RVUs. The results of the first 50 participants are reported in this interim analysis. 
Results: Participants had a mean age of 56 years, a median TIMI risk score of $2(\mathrm{Q} I=2, \mathrm{Q} 3=3), 25(50 \%)$ were female, and 10 (20\%) reported prior coronary disease. Participants were equally randomized with 25 participants in each treatment group. Protocol adherence was high among both groups (24/25 [96\%] OU-CMR, 23/25 [92\%] standard care remained until hospital discharge). Stress CMR imaging was obtained in 24/25 (96\%) OUCMR participants during the index visit; II/25 (44\%) received imaging the same day as presentation. In the OU-CMR group, 20/ $25(80 \%)$ of participants were discharged from the OU without admission. Five participants were admitted from the $\mathrm{OU}$ and underwent continued monitoring $(n=1)$ or cardiac catheterization $(n=4)$. Of the 4 undergoing cardiac catheterization, I was diagnosed with a non-ischemic emergent cardiac condition and 3 were not found to have an emergent cause of chest pain. There were no complications related to CMR testing. Among standard care participants, all patients were admitted, 2 I/25 (84\%) had cardiac imaging with 14 undergoing stress echocardiography, 3 stress cardiac MRI, 3 cardiac catheterizations, and I resting echo exam.

ACS criteria were met in $3 / 50$ (6\%) participants due to revascularization $(n=2)$ and myocardial infarction $(n=1)$, all in the hospital admission arm during the index hospitalization. Two participants had repeat hospitalizations for chest pain within 30 days, both in the hospital admission arm, and neither met ACS criteria. The OU-CMR group had a trend towards same day discharge more frequently than standard care participants (7/25 $(28 \%)$ vs $3 / 25(12 \%), p=0.29)$. Cost of index hospitalization demonstrated a near significant trend towards favoring OU-CMR before adjustment (mean $\$ 2823$ vs $\$ 4342, p=0.12$ ) and after adjustment for covariates $(\$ 1537$ difference, $p=0.10)$. Length of stay demonstrated a trend towards favoring OU-CMR before (mean $27.5 \mathrm{~h}$ vs $3 \mathrm{I} .4 \mathrm{~h}, \mathrm{p}=0.38)$ and after adjustment $(5.5 \mathrm{~h}$ difference, $p=0.24$ ).

Conclusion: An OU-CMR approach among ED patients with chest pain at intermediate risk for ACS is feasible. OU-CMR decreases hospital admissions and has demonstrated a strong near-significant trend towards decreasing index hospitalization cost.

\section{POSTER PRESENTATIONS}

\section{P I}

Ventricular function and volume assessment in children, adolescents and young adults with thalassemia major without myocardial iron overload

Juliano L Fernandes', Matheus Avelar ${ }^{1,2}$, Monica Verissimo ${ }^{3}$ and Otavio Coelho'

'University of Campinas (Unicamp), Campinas, Brazil

${ }^{2}$ Instituto Boldrini, Campinas, Brazil

${ }^{3}$ Centro Infantil Boldrini, Campinas, Brazil

Journal of Cardiovascular Magnetic Resonance 2009, II (SuppI I):PI

Introduction: Reference ranges for normal ventricular function and volumes in patients with thalassemia major without myocardial iron overload have been established before in European countries. However, these values might not be directly applicable to patients in most other countries where they tend to be younger and start chelation therapy later in life.
Purpose: To study children, adolescents and young adults with thalassemia major with normal $\mathrm{T}^{*}$ values and compare the results to matched normal volunteers as well as patients with high ferritin levels, normal myocardial iron and chronic anemia due to other etiologies.

Methods: We selected 25 patients ( $52 \%$ male) with thalassemia major (TM) and normal myocardial iron concentrations (T2* > $20 \mathrm{msec}$ ) and compared them to 17 age- and gender-matched normal (NL) volunteers (4I\% male) and 24 gender-matched $(58 \%$ male) patients with high ferritin levels from other etiologies (NT). All patients underwent a cardiovascular magnetic resonance (CMR) function study using a steady-state free precession sequence. Normalized data to body surface area was compared among the three groups.

Results: The mean age of TM patients was $18.8 \pm 2.3$ years (range 7-3I) with no significant differences from NL volunteers ( $16.2 \pm 2.8$ years, range $5-34, P=N S$ ), but younger than NT patients $(39.5 \pm 2.4$ years, range $\mathrm{II}-69, \mathrm{P}<0.00 \mathrm{I}) . \mathrm{T} 2 *$ in patients with TM were somewhat lower than NT patients $(27.5 \pm 4.3$ versus $31.7 \pm 6.8, P=0.06)$ but still within the normal range. Body surface area was similar in the three study groups (TM, I.5I $\pm 0.32 \mathrm{~m} 2 ; \mathrm{NL}, \quad \mathrm{I} .45 \pm 0.37 \mathrm{~m} 2 ; \mathrm{NT}$, $\mathrm{I} .6 \mathrm{I} \pm 0.33 \mathrm{~m} 2 ; \mathrm{P}=\mathrm{NS})$. Left ventricular ejection fraction was not different when comparing TM and NL patients [65.2 $\pm 6.0 \%$ (95\% Cl 62.7-67.7), versus $65.4 \pm 5.6 \%$ (95\% Cl 62.6-68.3), $\mathrm{P}=\mathrm{NS}]$, with both values non significantly lower than NT patients [68.5 $\pm 6.9 \%(95 \% \mathrm{Cl} 65.6-71.4), \mathrm{P}=0.15]$. Despite that, normalized diastolic and systolic volumes were higher in patients with TM compared to NL volunteers $(78.5 \pm 15.8 \times$ $27.4 \pm 8.3 \mathrm{ml} / \mathrm{m} 2$ versus $63.8 \pm 10.4 \times 21.8 \pm 4.6 \mathrm{ml} / \mathrm{m} 2$, $\mathrm{P}=0.007$ and $\mathrm{P}=0.05$ respectively) with no significant differences compared to NT patients $(75.6 \pm 16.3 \times 24.8 \pm 8.2 \mathrm{ml} /$ $\mathrm{m} 2, \mathrm{P}=\mathrm{NS}$ ). Normalized mass also showed similar characteristics with higher values in TM patients compared to NL $(56.9 \pm 10.6 \mathrm{~g} / \mathrm{m} 2$ versus $37.9 \pm 7.9 \mathrm{~g} / \mathrm{m} 2, \mathrm{P}<0.00 \mathrm{I})$ and no differences compared to NT individuals $(62.9 \pm 14.2 \mathrm{~g} / \mathrm{m} 2$, $P=N S)$. Male and female comparisons showed similar results although no differences were found in normalized volumes when looking only in female patients.

Conclusion: Younger patients with TM do not present different left ventricular function values compared to normal controls despite having increase ventricular volumes and mass. The parameters presented by these patients are similar to older individuals with comparable degrees of chronic anemia. Previously published reference ranges for TM may not be applicable to younger patients with different clinical settings.

\section{P2}

Phenotyping of tako tsubo

cardiomyopathy - structural comparison

to acute myocardial infarction

Andreas Rolf, Guido Conradi, Johannes Rixe, Holger Steiger, Holger Nef, Helge Möllmann, Katharina Beiring, Christian Hamm and Thorsten Dill Kerckhoff-Heart-Center, Bad Nauheim, Germany

Journal of Cardiovascular Magnetic Resonance 2009, I I(SuppI I):P2

Objective: The tako tsubo cardiomyopathy (TTC) is characterized by a transient contractile dysfunction after severe physical or emotional stress. Relevant coronary artery disease is absent. Clinically it mimics acute myocardial infarction (AMI). 
Therefore, the aim of this study was to characterize morphological differences between TTC and AMI using different MRI parameters.

Methods: 22 TTC and 35 AMI patients were examined within 48 hours upon admission. Ejection fraction (EF), myocardial mass (MM) enddiastolic (EDV) and endsystolic volumes (ESV) and regional contractility scores were computed on 10 contiguous CINE SSFP short axis slices. Myocardial edema (ME) was computed on T2 weighted TSE images on short axis orientations, late enhancement (LE) was evaluated on FLASH 3D GRE (both defined as signal intensity of more than 2 standard deviations from remote myocardium). Measurements are given as mean \pm SE. Differences were computed using an ANOVA model, a $P$ value $=<0.05$ was cosidered significant.

Results: All AMI patients and none of the TTC patients included had LE. TTC patients had a significantly lower EF (TTC $43.8 \pm 2.4 \% ;$ AMI $50.6 \pm 1.9 \% ; \quad p=0.03$ ) all other comparisons were therfore controlled for EF. TTC patients had significantly smaller ventricular volumes (EDV - TTC: $138 \pm 5.6$ $\mathrm{ml}$; AMl: $16 \mathrm{I} \pm 6.0 \mathrm{ml} ; \mathrm{p}=0.24$; ESV-TTC: $72.9 \pm 4.5 \mathrm{ml}$; AMl: $86.6 \pm 3.8 ; p=0.9)$ myocardial mass (TTC: $102.8 \pm 7.1 \mathrm{ml}$; AMI: $140 \pm 5.6 ; p=0.000 \mathrm{I}$ ) and edema (TTC: $19.5 \pm 4.2 \%$; AMI: $27.2 \pm 2.6 \% ; p=0.13$ ). In contrast regional contractility was more affected in TTC with a significantly larger number of dysfunctional segments (TTC: $7.5 \pm 0.6$ segments; AMI: $4.3 \pm 0.5$ segments; $P=0.000 \mathrm{I})$ and a significantly higher wall motion score (TTC: $1.9 \pm 0.1$; AMI: $1.4 \pm 0.1 ; p=0.000 I$ ).

Conclusion: In contrast to AMI, TTC patients show a marked regional dysfunction but no signs of early remodeling like ventricular enlargement and increased myocardial mass.

\section{P3}

4D flow of the whole heart and great vessels using real time self respiratory gating

Sergio A Uribe Arancibia ', Philipp Beerbaum', Allan Rasmusson 2, Thomas Sangild Sørensen²,

Reza Razavi' and Tobias Schaeffter'

'King's College London, London, UK

${ }^{2}$ University of Aarhus, Aarhus, Denmark

Journal of Cardiovascular Magnetic Resonance 2009, I I (SuppI I):P3

Objective: To evaluate the feasibility of a 4D-flow sequence of the whole heart and great vessel to retrospectively quantify blood flow within the entire heart.

Background: 4D-flow has been introduced as a means of acquiring anatomical and three-directional velocity information for all pixels within a 3D volume over different time points. The acquisition time of such data sets is long and respiratory compensation is thus required. However, navigator beams could disturb the steady state and are time consuming, which can be
Figure I (abstract P3)
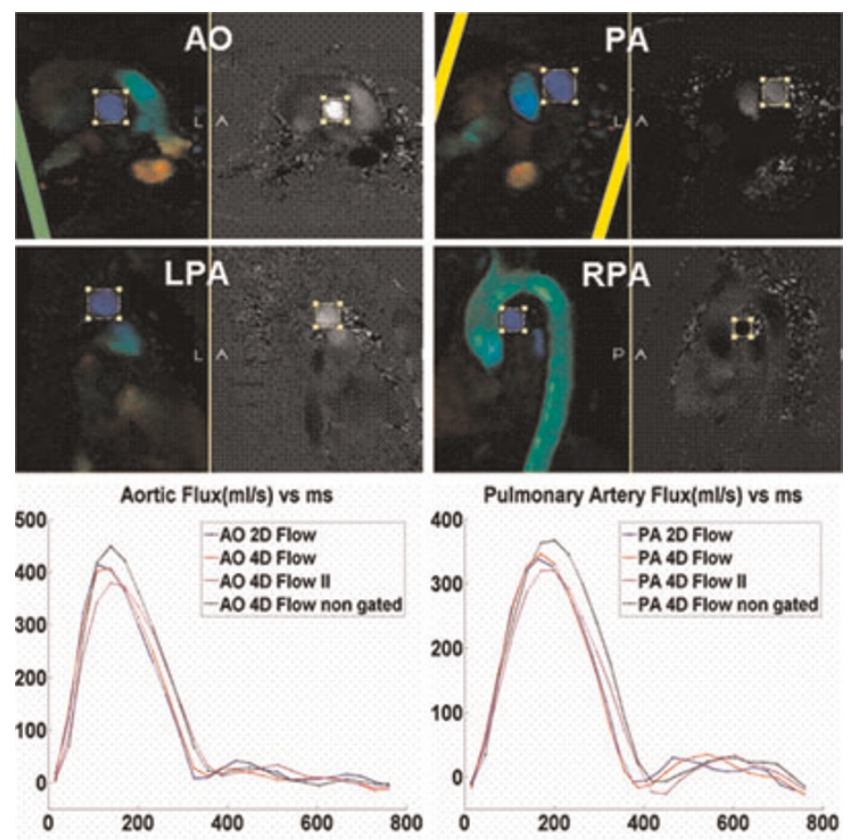

Pulmonary Artery Flux(ml/s) vs ms
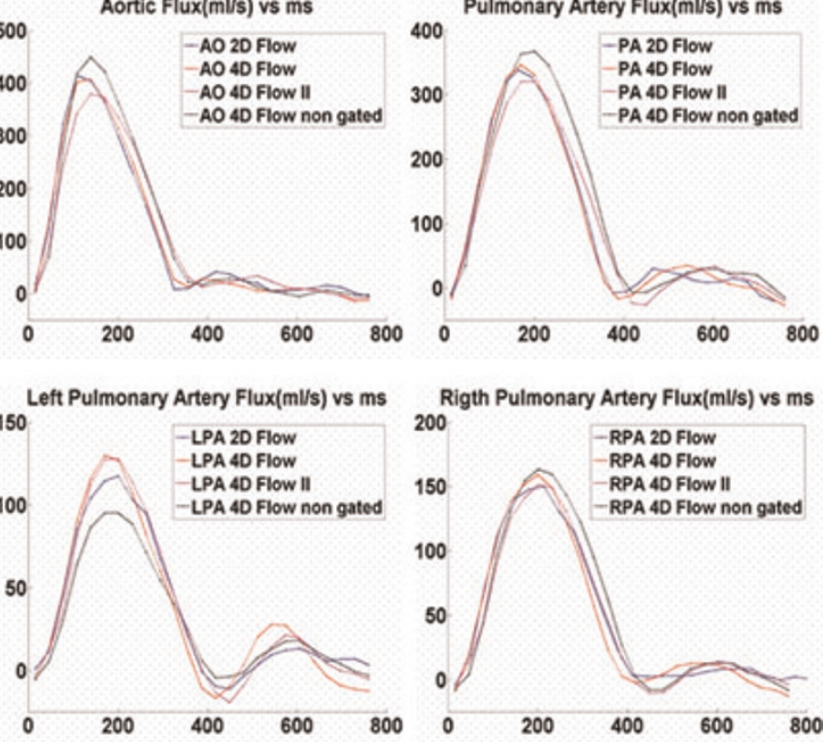

circumvented by using a self-navigation approach. Here, we present a new technique for the acquisition of 4D-flow data of an isotropic saggital volume using a real time self gating technique. The data can thereafter be reformatted in any clinical view allowing the quantification of flow in different vessels with arbitrary orientations. This is important in congenital heart $(\mathrm{CH})$ patients where scan planning can prolong the overall scan-time. Methods: Self navigation: A 3D Phase Contrast (PC) retrospective cardiac trigger sequence was used to acquire $4 D$ flow data. The sequence was modified to enable the acquisition of an extra $k_{0}$ profile at certain time intervals. These profiles were used to derive the breathing motion and to respiratory gate the acquisition in real-time. All the modifications were integrated

Table I (abstract P3) Stroke volume (mean and stdev) of the different acquisitions for all measured vessel

\begin{tabular}{llll}
\hline & $\begin{array}{l}\text { 4D gated tIMean } \\
{[\mathrm{ml}] \pm \mathbf{s d v}[\mathrm{ml}]}\end{array}$ & $\begin{array}{l}\text { 2DMean }[\mathrm{ml}] \pm \\
\mathbf{s d v}[\mathrm{ml}]\end{array}$ & $\begin{array}{l}\text { 4D non gatedMean } \\
{[\mathrm{ml}] \pm \mathbf{s d v}[\mathrm{ml}]}\end{array}$ \\
\hline AO & $85.86 \pm 18.54$ & $85.65 \pm 17.54$ & $81.78 \pm 21.03$ \\
PA & $81.38 \pm 17.67$ & $82.71 \pm 17.07$ & $78.65 \pm 23.76$ \\
LPA & $34.93 \pm 9.33$ & $36.15 \pm 9.14$ & $31.19 \pm 9.52$ \\
RPA & $40.74 \pm 9.07$ & $40.67 \pm 8.90$ & $38.05 \pm 10.99$
\end{tabular}


4D Gated vs 2D
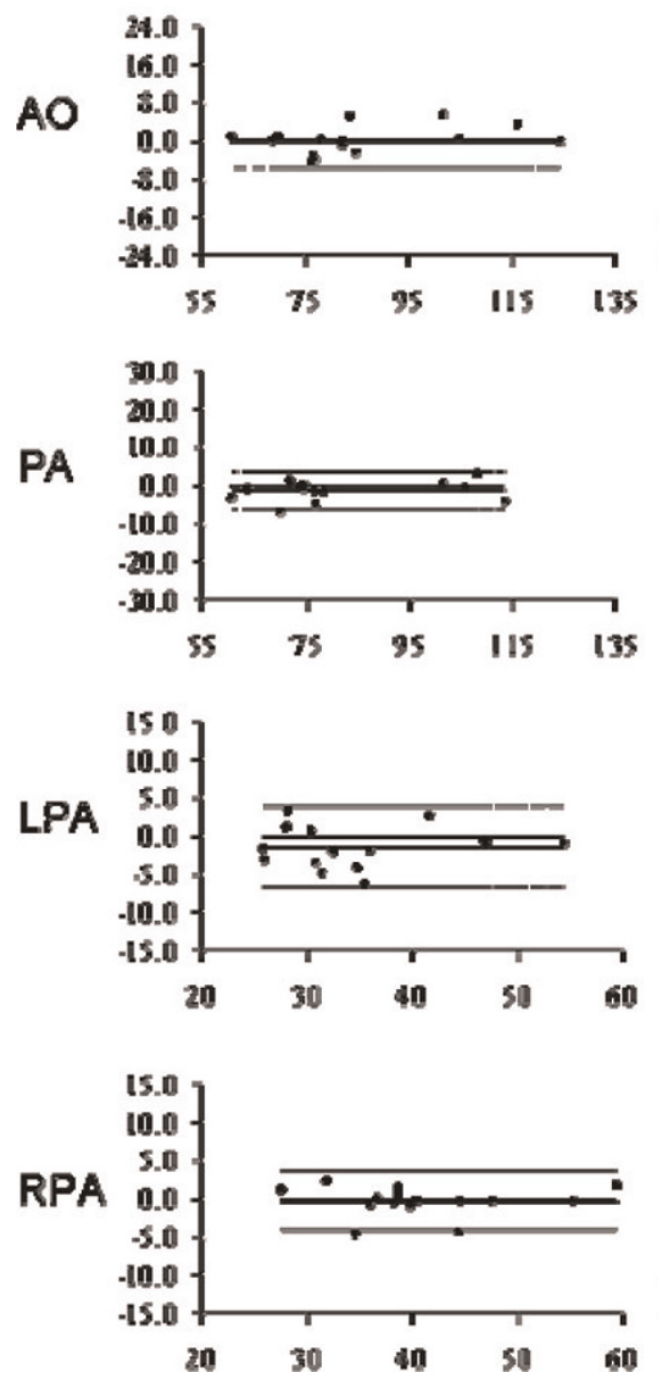

4D Non-Gated vs 2D
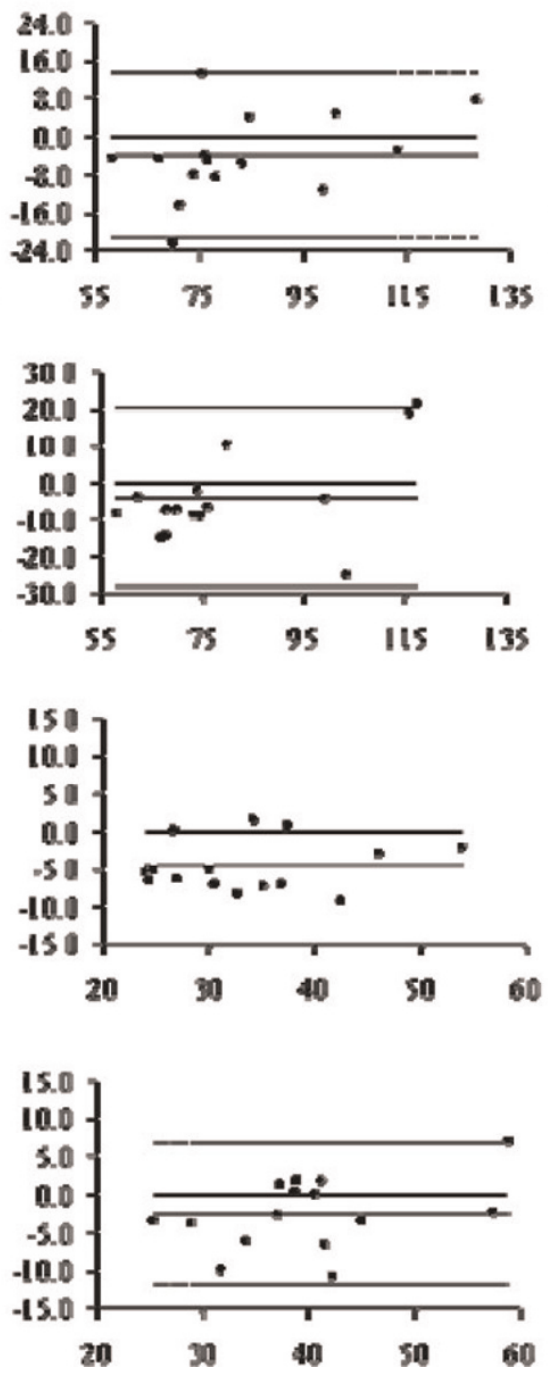

$4 D t_{1}$ vs $4 D t_{2}$
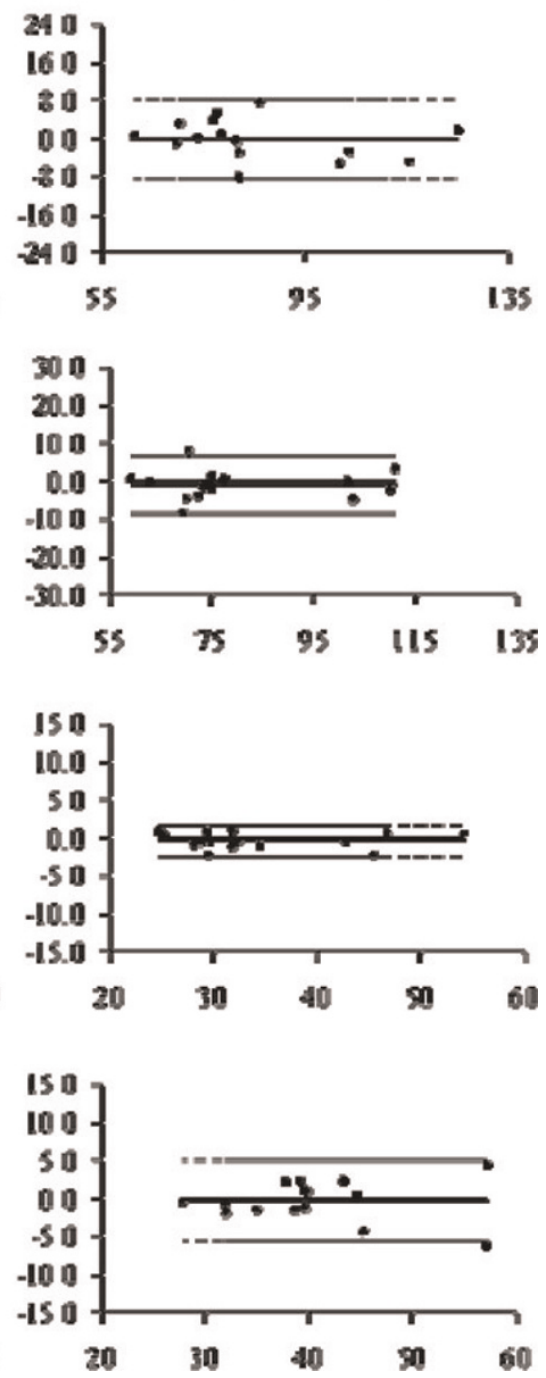

Figure 3 (abstract P3)

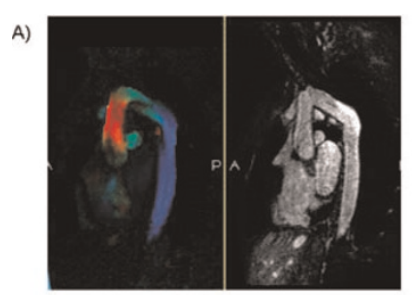
Flux [ml/ms]

C)

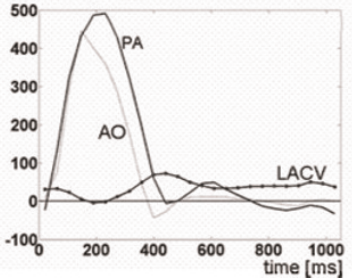

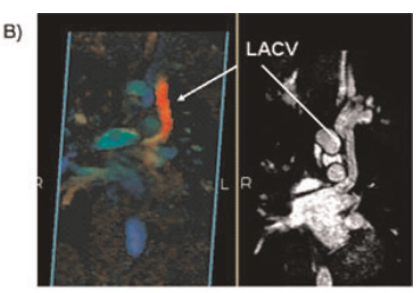

$$
\mathrm{SV}[\mathrm{ml}]
$$

D) ${ }^{150}$

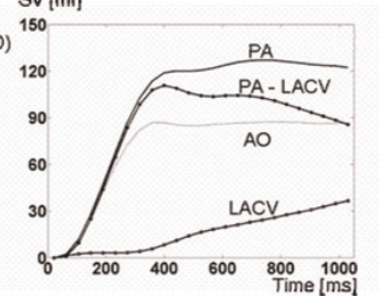

into the software of a clinical MR-scanner (Philips Healthcare, Best).

Experiments: 4D-flow data of the whole heart and great vessels was obtained in 15 volunteers on a $3 \mathrm{~T}$ scanner (resolution of $2.5 \mathrm{~mm}^{3}$ and 25 cardiac phases, acceptance window $=8[\mathrm{~mm}])$. To study the reproducibility of the technique, two 4D-flow data sets were acquired with self respiratory gating. For comparison one 4D flow with two averages were obtained without respiratory gating. Furthermore, 2D PC scans were obtained at the level of the AO, PA, LPA and RPA. Statistical analysis and Bland-Altman plots were used to compare the stroke volume (SV) derived from the $4 \mathrm{D}$ and $2 \mathrm{D}$ flow acquisitions.

Furthermore a 4D-flow data set was obtained on a $\mathrm{CH}$ patient with a repaired coarctation and a levo-atrial cardinal vein (LACV) connecting the left atrium with the brachiocephalic vein.

Results: The self-respiratory gated acquisition resulted in an overall scan time of $15 \pm 2.8 \mathrm{~min}$. Figure I shows reformatted 
slices of the 4D-flow data and an example of the flux in one volunteer. Table I shows the SV comparison for the different techniques. No statistically difference was found between the different pairs of data. However, Bland-Altman plots showed a larger standard deviation and bias for the pair " $4 D$ non-gated to 2D" (Figure 2). Therefore the 4D-flow gated scan showed to be more accurate than the 4D-flow data obtained during free breathing.

Figure 3 shows the data obtained in the congenital patient. This data set was used to visualize the flow pattern in the region of coarctation. Furthermore, it allows the accurate quantification of the flux in the AO, PA, and in the LACV (Figure 3C, D). We found the SV in the PA and AO was $127.91[\mathrm{ml}]$ and $90.68[\mathrm{ml}]$ respectively. The SV difference $(37.22[\mathrm{ml}])$ between the PA and AO was due to the connection caused by the LACV. Indeed the SV measured by the 4D flow in the LACV was $38.06[\mathrm{ml}]$, which matches the difference of the SV between the PA and AO.

Conclusion: We have demonstrated the feasibility of 4D-flow on the whole heart using a self respiratory gating technique. The method allows retrospectively flow quantification within the entire heart and great vessel from data obtained in a single free breathing scan. This method represents a practical advance for an easier cardiac MR examination and showed to be very valuable in congenital patients.

\section{P4}

Longitudinal versus cross-sectional studies of effects of aging on ventricular structure and function using cardiac magnetic resonance imaging Dipti Gupta', Mark Goldman ', Sunil T Mathew', Jing Han ', William Schapiro', Michael Passick', Katherine McGrath', Jie J Cao' and Nathaniel Reichek'

'St. Francis Hospital, Roslyn, NY, USA

${ }^{2}$ University of Oklahoma, Oklahoma city, OK, USA

Journal of Cardiovascular Magnetic Resonance 2009, I I(SuppI I):P4

Introduction: Cross-sectional studies of effects of aging on ventricular structure and function, including Framingham, MESA, the Dallas Heart Study and studies from our own laboratory have uniformly demonstrated lower ventricular chamber volumes in older normal subjects.

Purpose: We sought to assess aging effects on ventricular size and function prospectively in a carefully screened normal cohort. Methods: Normotensive, non-diabetic, non-obese (BMI < 28) volunteers ( $n=57,31$ females), aged $20-89$ at intake (59 \pm 13 yrs), were screened, including 2D echocardiography and CMR performed (I.5 T Siemens Sonata or Avanto) at baseline and 5 years. TrueFISP cine imaging was used to obtain contiguous $8 \mathrm{~mm}$ short axis slices of both left (LV) and right (RV) ventricles. LV and RV volumes at end-diastole and end-systole were determined (Medis, MASS) and indexed (i) to body surface area. Ejection fraction (EF) was also calculated.

Results: Systolic blood pressure increased (120 \pm 12 to $\mathrm{I} 30 \pm 20 \mathrm{~mm} \mathrm{Hg}, \mathrm{p}<.000 \mathrm{I}), \mathrm{LV}$ and RV end diastolic volumes increased $(|| 3 \pm 30$ to $|36 \pm 37|,|4 \pm 3|$ to $\mid 34 \pm 37 \mathrm{ml}$, $\mathrm{p}<.000 \mathrm{I}$ for both), as did end systolic volumes (45 \pm 17 to $59 \pm 23,55 \pm 2 \mathrm{l}$ to $60 \pm 22 \mathrm{ml}, \mathrm{P}<.000 \mathrm{l}$ and $\mathrm{p}=.0 \mathrm{l}$ respectively). LVEF decreased $(6 \mathrm{I} \pm 7$ to $58 \pm 7 \%, \mathrm{p}<.004)$, while RVEF increased slightly $(53 \pm 6$ to $56 \pm 7 \%, p=.0 \mathrm{I})$.
There were no major gender differences. Subjects with intake age $<50$ years and those $\geq 50$ years behaved similarly.

Conclusion: In contrast to cross-sectional studies, which demonstrate reduced LV and RV volumes in older subjects, this prospective study demonstrates that chamber volumes increase with age. This discordance may be due to generational differences which affect cross-sectional, but not longitudinal studies.

\section{P5}

\section{Coronary MRI with induced vasodilation using isosorbide dinitrate}

Peng Hu, Christian Stoeck, Dana C Peters,

Kraig V Kissinger, Beth Goddu, Lois Goepfert,

Warren J Manning and Reza Nezafat

Beth Israel Deaconess Medical Center, Boston, MA, USA

Journal of Cardiovascular Magnetic Resonance 2009, I I (SuppI I):P5

Introduction: Despite technical progress, coronary magnetic resonance imaging (MRI) still faces multiple challenges. Coronary vasodilators, such as sublingual nitroglycerin (NTG) or longer acting nitrates (e.g., isosorbide dinitrate), are commonly used to study coronary circulation. Terashima et al. [I] reported use of coronary MRI to evaluate the effect of sublingual NTG. Isosorbide dinitrate (Isordil) has been previously reported in a multi-center clinical coronary MRI trial [2], however no data has been provided to quantify coronary MRI image quality improvement and time course of Isordil.

Purpose: To investigate the impact of Isordil administration on SNR, vessel diameter and overall image quality in coronary MRI. Materials and methods: Coronary images were acquired on a cohort of healthy adult subjects before and after Isordil administration. Subjects were divided into four groups to investigate the impact of the imaging sequence and dose. In groups $A$ and $B$ the images were acquired using SSFP imaging sequence with either $2.5 \mathrm{mg}$ or $5 \mathrm{mg}$ Isordil dose. In groups $C$ and D images are acquired using GRE with either $2.5 \mathrm{mg}$ or $5 \mathrm{mg}$ Isordil dose. The impact of vasodilator during a time course was studied by repeated imaging. A free breathing, 3D VCG gated GRE sequence with typical imaging parameters of $T R=7.7 \mathrm{~ms}$, $\mathrm{TE}=2.2 \mathrm{~ms}, \mathrm{FOV}=270 \times 270 \times 30 \mathrm{~mm}^{3}$, flip angle $=30^{\circ}$,

\section{Figure I (abstract P5)}

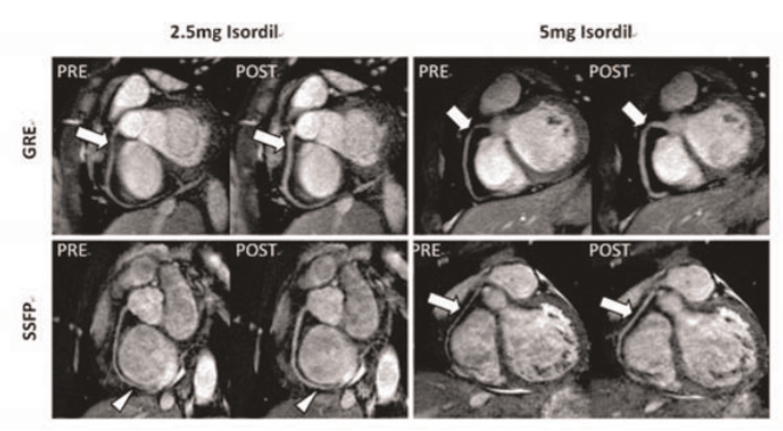

Examples of pre- and post-Isordil images from four different subjects using combinations of two different sequences (GRE and SSFP) and two different Isordil doses $(2.5 \mathrm{mg}$ and $5 \mathrm{mg}$ ) at $10-15$ minutes after Isordil. 
Figure 2 (abstract P5)

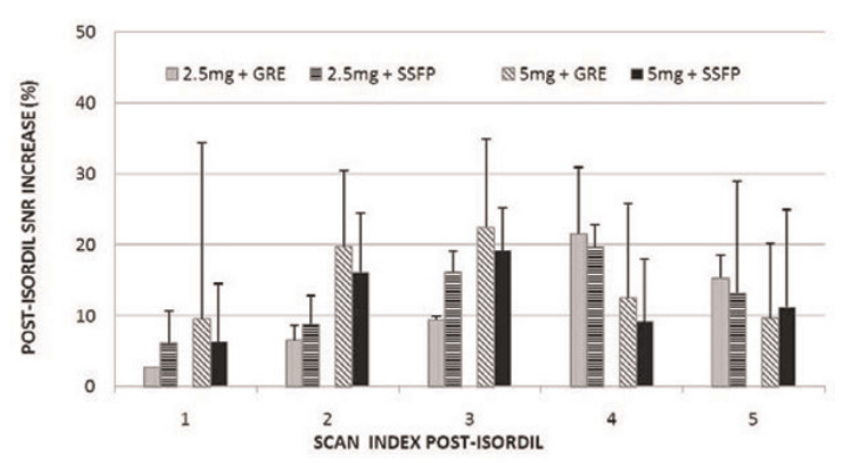

The time course of SNR increase after Isordil administration using the GRE and SSFP sequences. The first 5 post-Isordil scans were used to calculate the time course. The post-Isorfil times for the five scans were $1.3 \pm 0.7, \quad 10.4 \pm 4.2, \quad 17.7 \pm 6.7,26.2 \pm 7.3$ and $32.3 \pm$ 8.3 minutes.

spatial resolution of $0.7 \times 1 \times 1.5 \mathrm{~mm}^{3}$ reconstructed to $0.52 \times 0.52 \times 0.75 \mathrm{~mm}^{3}$ was used. The imaging parameters for SSFP imaging included: TR $=4.6 \mathrm{~ms}, \mathrm{TE}=2.3 \mathrm{~ms}, \mathrm{FOV}=270 \times$ $270 \times 30 \mathrm{~mm}^{3}$, flip angle $=90^{\circ}$, spatial resolution of $\mathrm{I} \times \mathrm{I} \times 1.5 \mathrm{~mm}^{3}$ reconstructed to $0.52 \times 0.52 \times 0.75 \mathrm{~mm}^{3}$ and a half $\alpha$ preparation pulse. $\mathrm{T}_{2}$ prep, fat saturation and navigator sequences were used in both sequences. In order to obtain a more accurate SNR measurement, no parallel imaging was used. SNR was measured with a previously published method [3]. To be consistent, the same noise and signal ROl's were used in all the GRE and balanced-SSFP scans during a study, unless motion is detected between the scans. The coronary cross-sectional diameter in the proximal right coronary artery was measured using the Soap Bubble tool (Philips Healthcare, Best, NL).

Results: Figure I demonstrates the improved coronary image quality 10-15 minutes after Isordil administration. The vasodilation and signal enhancement help better delineate and differentiate the right coronary artery (arrows). The visibility of distal branches of the coronaries was improved (arrow heads). Figure 2 shows the SNR enhancement during the time course of 5 post-Isordil scans. The maximum SNR increase was $21.5 \% \pm 9.3 \%$ for GRE with $2.5 \mathrm{mg}$ dose, $22.5 \% \pm 12.3 \%$ for GRE with $5 \mathrm{mg}, 19.7 \% \pm 3.1 \%$ for SSFP with $2.5 \mathrm{mg}$ and $19.1 \% \pm 6.0 \%$ for SSFP with $5 \mathrm{mg}$. The maximum SNR enhancement is earlier using $5 \mathrm{mg}$ dose than $2.5 \mathrm{mg}$. There were greater than $15 \%$ increase in vessel lumen diameter throughout the 5 post-Isordil scans, with a greater than $20 \%$ increase in all but the first time point.

Conclusion: Pre-scan Isordil administration improves coronary SNR by $20 \%$ for both GRE and SSFP imaging. $5 \mathrm{mg}$ and $2.5 \mathrm{mg}$ doses result in comparable vasodilation. For best SNR enhancement, imaging should be performed later post-Isordil if using $2.5 \mathrm{mg}$ dose than $5 \mathrm{mg}$.

\section{References}

I. Terashima, et al: JACC 2005, 45(I): 104-II0.

2. Kim, et al: N Engl J Med 200I, 345(26): I863-1869.

3. Botnar, et al: Circulation 1999, 99:3139-3148.

\section{P6}

Endocardial to epicardial perfusion ratios at rest and stress determined by perfusion-CMR Abdulghani M Larghat', Aleksandra Radjenovic', Neil Maredia', John P Greenwood', Sebastian Kozerke ${ }^{2}$ and Sven Plein ${ }^{1}$

'University of Leeds, Leeds, UK

${ }^{2} E T H$ and University of Zurich, Zurich, Switzerland

\section{Journal of Cardiovascular Magnetic Resonance 2009, I I(SuppI I):P6}

Introduction: Animal experiments using labelled microspheres have shown that at rest, blood flow to the subendocardial layer is higher than to the subepicardium. With increasing levels of stress this transmural gradient of myocardial blood flow is reduced, so that the endocardium has a lower perfusion reserve than the epicardium [I]. The causes for this observation include higher compressive forces and higher resting metabolic activity in the endocardium. If microvascular function is impaired, endocardial perfusion reserve is reduced further [2].

Myocardial perfusion-CMR is usually performed with coverage of several myocardial sections to allow detection of ischemic perfusion defects. Consequently, compromises regarding image quality and motion artefact are made. For the study of global physiological phenomena and diffuse myocardial disease, optimised acquisition of a single section may be more useful.

Purpose: I. To develop a first pass myocardial perfusion method optimised for acquisition of a single midventricular myocardial section at systole and diastole.

2. To compare rest and stress myocardial perfusion between the endocardium and epicardium.

3. To compare rest and stress myocardial perfusion at middiastole and mid-systole.

Methods: 10 volunteers ( 7 male, mean age 38 years) were studied on a I.5 T Philips Intera system during adenosine stress (1 $40 \mathrm{mcg} / \mathrm{kg} / \mathrm{min}$ for 3 minutes) and at rest. For each perfusion acquisition $0.05 \mathrm{mmol} / \mathrm{kg}$ Gd-DTPA was administered with a power injector followed by a $20 \mathrm{ml}$ Saline flush $(5 \mathrm{ml} / \mathrm{sec})$.

A saturation recovery segmented gradient echo perfusion method with twofold SENSE was optimised for imaging of a single cardiac section by timing the acquisition to a phase with minimal cardiac motion and by optimising the preparation pulse delay. Pulse sequence parameters were as follows: TR/TE/flip $2.7 \mathrm{~ms} / \mathrm{l} .0 / 15^{\circ}$, FOV $380 \times 380 \mathrm{~mm}$, matrix $160 \times 160$, slice thickness $10 \mathrm{~mm}$, preparation pulse delay (to middle of k-space)

\section{Figure I (abstract P6)}

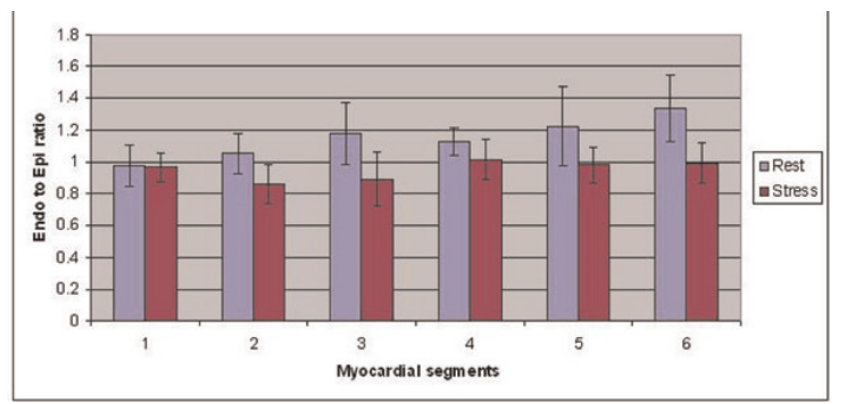

Endocardial to epicardial ratio of maximal SI upslope in systole at rest and adenosine stress. 
$150 \mathrm{~ms}$, shot duration $130 \mathrm{~ms}$. With the use of a software patch trigger delay for the acquisition of one midventricular slice could be individually to mid-systole, as determined on a high temporal resolution cine scout. A mid-diastolic phase in the same plane (adjusted for through-plane cardiac motion) was acquired if heart rate permitted.

Endo and epicardial contours were drawn (MASS, Medis, Leiden, The Netherlands) and the slice segmented into 6 equidistant sectors. These were further subdivided into a subepicardial, midmyocardial and subendocardial third. The maximal myocardial upslope of the signal-intensity time profiles for each sector and the three layers was calculated. Then the ratio of upslopes between the endocardial and epicardial layers ("endo-epi ratio") was computed.

Results: In systole, the mean (+/- SD) of the endo-epi ratio of all segments was I.I3 (+/-0.I) at rest and $0.94(+/-0.1)$ at stress. In all segments the endo-epi ratio was higher at rest than stress, albeit with regional differences (Fig I). In diastole, the mean endo-epi ratio of all segments was similar to systole at rest $(1.13+/-0.2, P>0.05$ versus systole), but at stress it was significantly higher than at systole $(I .08+/-0.1, p=0.005)$. The mean upslope for all three layers combined however was similar between systole and diastole at rest and stress (I7.9 vs 17.2 at stress and 8.0 vs 7.9 at rest, $p>0.05$ ).

Conclusion: I. Differences in endocardial and epicardial perfusion can be detected with CMR in vivo.

2. Perfusion to the endocardial layer is higher at rest, with a diminishing endo-epi ratio at stress, consistent with known physiology.

3. Perfusion to whole myocardial segments is similar in systole and diastole. In diastole differences between layers of the myocardium are less than in systole, probably because of partial volume effects.

\section{References}

I. Ball RM, et al: J Clin Invest 1975, 55:43-49.

2. Panting JR, et al: N Engl J Med 2002, 346:1948-1953.

\section{P7}

Correlation between T2-weighted CMR and Sestamibi-SPECT in acute myocardial infarction and acute coronary occlusion

Martin Hadamitzky, Nadine Kirchhartz, Eva Hendrich, Stefan Martinoff and Albert Schömig Deutsches Herzzentrum München, Munich, Germany

Journal of Cardiovascular Magnetic Resonance 2009, I I(SuppI I):P7

Background: Being a consistent finding in acute myocardial infarction, T2 weighted MRI imaging has recently been proposed as a marker for the area not perfused because of the underlying coronary occlusion. But data comparing T2 imaging with the gold standard for perfusion, acute Sestamibi SPECT, is very limited. In particular the interval between interruption of perfusion and onset of the cell edema in humans is unknown.

Methods: We therefore performed in 38 patients with acute myocardial infarction (interval between start of symptoms and intervention below $24 \mathrm{~h}$ ) and 10 patients with acute coronary occlusion as a complication during percutaneous coronary intervention both a myocardial single photon emission computed tomography (SPECT) and a cardiac MRI with fat suppressed T2-weighted turbo spin echo sequences. For SPECT
Figure I (abstract P7)
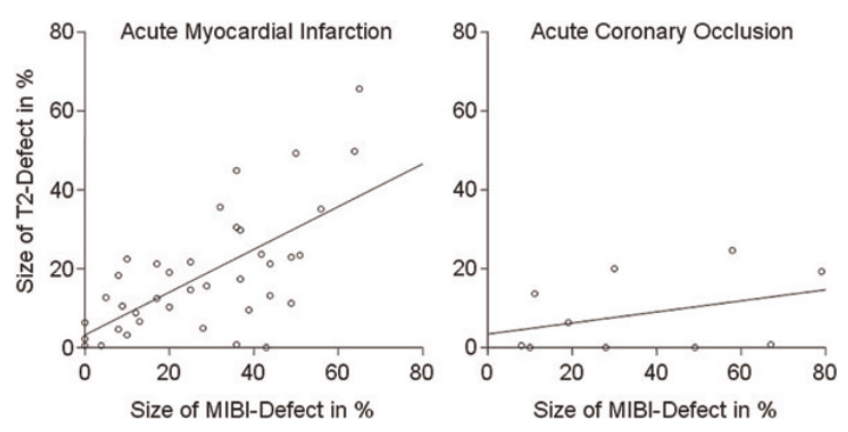

$99 \mathrm{mTc}$-Sestamibi applied before coronary revascularization, the measurement was done immediately after the intervention; the MRI was done 2 to 6 days after intervention. The area of increased T2 signal was quantified automatically using a cutoff of mean plus 2 standard deviations of the signal intensity in a remote myocardial region. In SPECT the area of risk was defined as area of intensity below $50 \%$ of maximum. Both values were expressed as fraction of left ventricular myocardial volume.

Results: In clinical AMI the defect size of T2 weighted MRI ranged between $0 \%$ and $66 \%$ (18\% mean), the defect size of SPECT between $0 \%$ and $65 \%$ ( $28 \%$ mean). There was a highly significant correlation between the two measurements with a correlation coefficient of 0.65 as depicted by the left image in Figure I.

In acute coronary occlusion the defect size of T2 weighted MRI (9\%) was only one fourth of the defect size of SPECT (36\%). There was no significant correlation between the two measurements $(r=0.14, p=0.31$, see also right image below).

Conclusion: T2-weighted CMR shows a good correlation to acute Sestamibi SPECT in depicting perfusion defects in clinical acute myocardial infarction, but it clearly underestimates shortlived perfusion defects as seen in acute coronary occlusions during $\mathrm{PCl}$.

P8

Diagnostic accuracy of half-contrast dose bSSFP vs full-contrast dose hEPI MR perfusion imaging in patients with known or suspected coronary artery disease

Chiara Bucciarelli-Ducci, Peter Gatehouse, Rory O’Hanlon, Jonathan Lyne, Agata Grasso, Joanna Petryka, Ricardo Wage, Winston Banya, Sanjay Prasad, David Firmin and Dudley Pennell CMR Unit, Royal Brompton Hospital, London, UK

\section{Journal of Cardiovascular Magnetic Resonance 2009, I I(SuppI I):P8}

Background: Non-invasive evaluation of myocardial perfusion with cardiovascular magnetic resonance (CMR) is clinically valuable in patients with known or suspected coronary artery disease CAD) but dark rim artifacts mimicking perfusion defects remain a diagnostic challenge. The perfusion protocol (pulse sequence and dose of contrast) giving highest diagnostic 
confidence is not yet standardised. Hybrid EPI (hEPI) is a fast sequence reducing motion artifact by its short imaging time, but with low SNR. Balanced SSFP (bSSFP) has greater SNR but previously has been reported as more prone to dark subendocardial rim artifacts: field distortion by the high-dose bolus is one suspected reason, and this distortion could potentially be reduced by using a half contrast dose, making use of bSSFP's abundant SNR. We therefore compared these 2 sequences.

Methods: Seventeen patients were scanned at I.5 T (Siemens, Avanto) with both protocols. All patients underwent coronary angiography (CA) and significant CAD (>50\% stenosis) was detected in $6 / 17$ patients (35\%).

The dose of gadolinium contrast agent was $0.1 \mathrm{mmol} / \mathrm{kg}$ for the hEPI sequence and $0.05 \mathrm{mmol} / \mathrm{kg}$ for bSSFP, with $15 \mathrm{ml}$ flush at $7 \mathrm{ml} / \mathrm{s}$. Centre-out hEPI (TR $5.8 \mathrm{~ms}, 30^{\circ}$, ETL 4, $1860 \mathrm{~Hz} /$ pixel) acquired $2.8 \times 2.8 \times 8 \mathrm{~mm}$ voxels over typically $360 \times 270 \mathrm{~mm}$ FOV (adapted per patient) at $\mathrm{TI}=110-160 \mathrm{~ms}$ for each of 3 fatsuppressed slices per cycle, using TSENSE (R2), typical image time 75 ms (i.e. excluding prepulses). Linear-ordered bSSFP (TR $2.6 \mathrm{~ms}, 70^{\circ}, 930 \mathrm{~Hz} /$ pixel) acquired the same voxel size at the same TI for central of k-space for each of 3 fat-suppressed slices per cycle, using TSENSE (R2), typical image time $125 \mathrm{~ms}$. The slice acquisition order was the same in hEPI and bSSFP, resulting in approximately similar image timings through the cardiac cycle in both. Slice positions in the heart were reproduced by viewing the first study while piloting the second.

The randomised scans were scored by 2 blinded experienced observers based on the 16-segment model. Perfusion scan myocardial SNR and diagnostic confidence were assessed subjectively (score from $0=$ unusable to $4=$ excellent). Severity of perfusion defects was graded based on its transmurality $(0=$ none, $I=<25 \% 2=25-50 \%, 3=5 I-75 \%, 4=>76 \%$, and $A=$ dark rim artefact). $A$ total of 272 segments were analyzed. Sensitivity and specificity of both sequences were calculated and comparison of agreement between observers and scan methods was assessed by kappa coefficients.

Results: The agreement between bSSFP and hEPI scans on the presence of normal perfusion, artefacts or genuine perfusion defects was $65 \%(k=0.25,95 \% \mathrm{Cl}: 0.17-0.33, p<0.000 \mathrm{I})$ for observer I and 53\% ( $\mathrm{k}=0.15,95 \% \mathrm{Cl}$ : 0.09-0.2I, $\mathrm{p}<0.000 \mathrm{I})$ for observer 2.

The agreement between observer 1 and 2 on the presence of normal perfusion, artefacts or genuine perfusion defects was $83 \%$ $(k=0.5 \mathrm{I}, 95 \% \mathrm{Cl}: 0.4 \mathrm{I}-0.6 \mathrm{I}, \mathrm{p}<0.000 \mathrm{I})$ for hEPI scans and $53 \%$ $(k=0.15,95 \% \mathrm{Cl}: 0.10-0.26, \mathrm{p}<0.000 \mathrm{I})$ for the bSSFP scans.

True artefacts (compared against CA) occurred more frequently with bSSFP (59 segments, $22 \%$ ) than with hEPI (I 4 segments, $5 \%$ ) $\left(\mathrm{X}^{2}, \mathrm{p}<0.00 \mathrm{I}\right)$.

Using CA as gold-standard, sensitivity and specificity of bSSFP scans was $17 \%$ and $88 \%$ (observer I), $25 \%$ and $72 \%$ (observer 2 ). Conversely, sensitivity and specificity of hEPI scans was $42 \%$ and 87\% (observer I), 33\% and 84\% (observer 2).

Observer I noted a significant lower diagnostic confidence for bSSFP vs hEPI scans $(p<0.03)$. Both observers reported a similar diagnostic confidence for hEPI scans. The SNR of the bSSFP and hEPI were similar for both observers.

Conclusion: Overall, bSSFP demonstrated lower diagnostic accuracy compared to hEPI. Dark-rim artifacts occurred more frequently in the bSSFP than hEPI, even when the half-contrast dose was used. Although two experienced observers were usually able to correctly identify dark-rim artefacts, using bSSFP in clinical practise in less experienced centers may not be ideal because of reduced diagnostic confidence.

\section{P9}
A 3D evaluation of dyssynchrony may offer an advantage over a 2D approach; a cardiovascular MRI method for dyssynchrony quantification Robert WW Biederman', Frank Grothues ${ }^{2}$, Helmut Klein 2', Ronald B 'Williams', June A Yamrozik', Geetha Rayarao', Diane A Vido', Christof Huth ${ }^{2}$ and Mark Doyle'

'Allegheny General Hospital, The Gerald McGinnis

Cardiovascular Institute, Pittsburgh, PA, USA

${ }^{2}$ University Hospital Magdeburg, Magdeburg, Germany

Journal of Cardiovascular Magnetic Resonance 2009, I I(SuppI I):P9

Introduction: Detection of dyssynchrony is primarily performed by EKG and/or tissue Doppler echocardiography, sampling the heart in selected basal segments. Limited approaches for dyssynchrony have been performed by cardiovascular MRI (CMR).

Hypothesis: We hypothesize that 3D global assessment of dyssynchrony may be more sensitive than conventional regional $2 \mathrm{D}$ analysis.

Methods: At baseline, 8 patients $(47 \pm 9$ yrs) with mean NYHA Class $2.3 \pm 0.5$ on optimal medical therapy (maintained throughout the study) underwent 3D CMR (I.5 T GE) to assess LV function. Using Medis Mass software (Leiden, The Netherlands), endo and epicardial boundaries were outlined in multiple contiguous short-axis slices. For each slice the myocardium was circumferentially divided into 16 equally spaced segments. Endsystolic (ES) time was automatically identified as time of maximal wall thickening and the dyssynchrony index taken as the dispersion of ES times. Patients underwent HeartNetTM (Paracor Medical Inc, Sunnyvale, CA) placement. A follow-up CMR was performed at 6 months. In total $>800$ data points were generated for each heart (as compared to 6 by standard echocardiography). The dispersion of the ES time was analyzed for pre to post treatment effect for the basal region separately using all measured points and for 4,6 , or 8 equally spaced circumferential regions, each set starting at several offset values, resulting in II regional data sets.

Results: All patients survived HeartNet ${ }^{\mathrm{TM}}$ placement and were available for 6 month follow-up. When using all segments to describe the dispersion of ES time pre to post, a statistically significant change in the dyssynchrony index was observed (254 vs. $220 \mathrm{~ms}, \mathrm{p}<0.00 \mathrm{I})$. When assessed using the separate $2 \mathrm{D}$ analysis, a pre-post change in dyssynchrony was only detected in 3 out of II data series (36\%).

Conclusion: By its nature, dyssynchrony is a heterogeneous phenomenon. When assessed using a 3D CMR approach, a pre to post treatment effect was detectable. However, when restricting measurements to 4,6 , or 8 equally spaced regions (analagous to a 2D echocardiographic approach), the chance of detecting the change in dyssynchrony substantially dropped, indicating that the phenomenon of Dyssynchrony should be assessed using a global 3D as opposed to regional 2D approach, irrespective of assessment modality. 


\section{PIO}

Quantitative evaluation of normal myocardial stress perfusion using Fermi and MMID4

Yi Wang, Paphael Hazel, Bin Luo, Jing Han

and Nathaniel Reichek

St. Francis Hospital, Roslyn, NY, USA

Journal of Cardiovascular Magnetic Resonance 2009, I I (SuppI I):PI0

Background: MR first pass perfusion quantification has been demonstrated to be a powerful technique for diagnosing cardiac perfusion deficits, since its introduction in the early 1990s. Model based MR perfusion quantification is feasible and may provide a sensitive absolute perfusion evaluation tool alternative to PET. However, MR perfusion quantification results vary depending on the model used, imaging sequences, and contrast dosage etc.

Purpose: We compared MMID4 and Fermi model-based quantitative methods for myocardial stress perfusion imaging on 16 normal volunteers. The goals of our study were to define normal quantification results and the relationship between Fermi and MMID4 methods, as well as the impact of age on normal myocardial perfusion.

Methods: To ensure normality of the volunteers ( $n=16$, ages: $43.9 \pm 15.8$ years, 5 males) exclusions included: hypertension, diabetes, smoking, family or personal history of cardiac disease and total coronary calcium score $<20$ by either EBCT or MDCT. Contrast first pass perfusion studies under adenosine stress and at rest were performed on a I.5 T scanner. After 3 minutes of adenosine infusion, long axis perfusion imaging was obtained with Gadodiamide injection at a dose of $0.05 \mathrm{mmol} / \mathrm{kg}$ bodyweight. A saturation recovery SSFP sequence was used with $160 \mathrm{~ms}$ per slice. $\mathrm{TR} / \mathrm{TE} / \mathrm{TI}=2.9 \mathrm{~ms} / \mathrm{l} .3 \mathrm{~ms} / 90 \mathrm{~ms}$ and voxel size $1.9 \times 2.8 \times 8 \mathrm{~mm}^{3}$. Using MASS (Medis, Leiden, the Netherlands) software, the myocardial contours were drawn, divided into 6 equal segments, and then propagated through all time point. Mean signal intensities in each myocardial segment at every time point were saved in text files. A custom developed program read the data file and calculated absolute perfusion based on both the Fermi deconvolution algorithm and the MMID4 algorithm.

A total 288 segmental perfusion values were evaluated for differences between subjects and algorithms using mixed effect ANOVA. The coefficient of variation (CV) of each algorithm was calculated as $\mathrm{SD} /$ mean $\times 100 \%$.

Results: The mean heart rates at rest and under stress were $61.9 \pm 5.9$ and $86.8 \pm 16.4$ beats per minute, respectively $(p<0.0001)$. The effect of adenosine infusion was a $40.3 \pm 22.5 \%$ increase in mean heart rate and no significant decrease in both systolic and diastolic blood pressure. The mean and SD of perfusion reserve for Fermi was $2.6 \mathrm{I} \pm 1.02$, and for MMID4 was $2.77 \pm 2.08$. There were significant differences between Fermi and MMID4 adjusted by slice and segment $(F=28.00, P<0.0001)$. However, MMID4 perfusion reserve showed a much greater variations (CV of I19.25) than Fermi $(C V=38.87)(p<0.0001)$.

There was a gradual decline with age of MPR quantified by the Fermi model with a linear regression, $M P R=3.6433$ $0.022 \times$ age, and a coefficient of determination of 0.4258 $(p=0.006)$. The MMID4 results did not demonstrate a significant age effect.

Conclusion: Both MMID4 and Fermi algorithms can be used for perfusion quantification and their perfusion reserve results in normals are very similar to that published in PET literature. However, MMID4 results are more variable, obscuring the effect of age, and seem to be more susceptible to artifacts.

\section{PII}

Comparison of visual scoring and planimetry methods for estimation of global infarct size on delayed contrast enhanced MRI and confrontation with biochemical markers of infarction Nathan Mewton', Pierre Croisille ${ }^{1,2}$, Michel Ovize' and Didier Revel ${ }^{i}$

${ }^{\prime}$ Hopital Cardiovasculaire Louis Pradel, Lyon, France ${ }^{2}$ Hôpital Cardiovasculaire Louis Pradel, Lyon, France

Journal of Cardiovascular Magnetic Resonance 2009, I I(SuppI I):PI I

Introduction: Infarct size assessment by delayed enhanced CMR is of critical importance for the patient's prognosis after an acute myocardial infarction (AMI) and to assess the efficiency of new reperfusion therapies. However infarct size quantification by planimetry is time-consuming and difficult to do on a daily clinical practice.

Purpose: By summing all the segmental scores using a 17segment model, a global index of the size of the infarcted myocardium is easily obtained and we compared it to infarct size obtained by visual planimetry.

Materials and methods: $10 \mathrm{I}$ patients admitted with reperfused AMI to our intensive care unit were prospectively scanned using an ECG-gated gradient echo sequence after injection of gadolinium contrast agent. The global score was defined as the sum of the scores on each segment, and expressed as a percentage of the maximum possible score. This index was compared with a planimetric evaluation of hyperenhancement, expressed as a percentage of the left ventricle myocardial volume. The area under the curve (AUC) and peak values of serum troponin I (Tnl) and creatine kinase (CK) release were measured in each patient.

Results: There was an excellent correlation between visual planimetry and visual global scoring for the hyperenhancement extent's measurement $(r=0.91 ; y=1.07 x+2.3 ;$ SEE $=1.2$; $P<0.00 I$ ) The Bland-Altman plot shows a good concordance between the two approaches (mean of the differences $=-3.9 \%$ with a standard deviation of 6.6). The mean percentage of hyperenhanced myocardium determined by the visual planimetry method was $21.2 \pm 14.1 \%$ (median of 18.7\%).

Mean post-processing time for visual planimetry was significantly longer than visual scoring post-processing time $(23.7 \pm 5.7$ minutes VS $5.0 \pm \mathrm{I} . \mathrm{I}$ minutes respectively, $\mathrm{P}<0.00 \mathrm{I}$ ).

Correlation between CK AUC and visual planimetry was $r=0.73(P<0.001)$ and $r=0.77(P<0.001)$ with visual global scoring. Correlation between peak $\mathrm{CK}$ and visual planimetry was $r=0.72(P<0.00 I)$ and $r=0.77(P<0.00 I)$ with visual global scoring. Correlation between troponin I AUC and visual planimetry was $r=0.72(P<0.00 I)$ and between peak troponin $\mathrm{I}$ and visual planimetry was $r=0.56(\mathrm{P}<0.00 \mathrm{I})$. Correlation between troponin I AUC and visual global scoring was $r=0.73$ $(P<0.00 \mathrm{I})$ and between peak troponin I and visual global scoring was $r=0.59(P<0.00 I)$.

Conclusion: A visual approach based on a 17-segment model can be used to evaluate the global myocardial extent of the hyperenhancement with similar results to planimetry and with shorter post-processing times. 
PI2

Increased susceptibility of the left lateral

free wall to myocardial delayed enhancement in Duchenne Muscular Dystrophy: progressive systolic dysfunction demonstrable by CMR regional strain analysis Narayan Kissoon', Kan N Hor ${ }^{2}$, janaka P Wansapura ${ }^{2}$, Wojciech Mazur ${ }^{3}$, Robert J Fleck ${ }^{2}$, Micheal D Puchalski ${ }^{4}$, D Woodrow Benson ${ }^{2}$ and William M Gottliebson ${ }^{2}$

'University of Minnesota, Minneapolis, MN, USA

${ }^{2}$ Cincinnati Childrens Hospital Medical Center, Cincinnati, $\mathrm{OH}$, USA

${ }^{3}$ Christ Hospital Medical Center, Cincinnati, OH, USA

${ }^{4}$ Primary Childrens Hospital, Salt Lake City, UT, USA

Journal of Cardiovascular Magnetic Resonance 2009, I I (SuppI I):PI2

Background: Cardiac magnetic resonance imaging (CMR) has demonstrated reductions in peak LV myocardial circumferential strain $\left(\varepsilon_{\mathrm{cc}}\right)$ despite normal ejection fraction (EF) in Duchenne Muscular Dystrophy (DMD) patients. We hypothesized that the increased initial contractility of the lateral LV free wall makes that region more susceptible to myocardial injury with subsequent fibrosis than the septum in DMD patients.

Methods: We analyzed regional $\varepsilon_{\mathrm{cc}}$ from myocardial tagged CMR images on the mid-papillary level LV slice (using HARPTM software) from I4 DMD males with global cardiac dysfunction (LV EF $<55 \%$ ) and myocardial delayed enhancement (MDE, a marker of myocardial fibrosis), as well as from 13 age-matched control males with normal cardiac function. Regions were assigned based on standard coronary perfusion regions. Regional $\Delta \varepsilon_{\mathrm{cc}}$ was computed as the difference between normal and DMD subject $\varepsilon_{\mathrm{cc}}$ per region.

Figure I (abstract PI2)
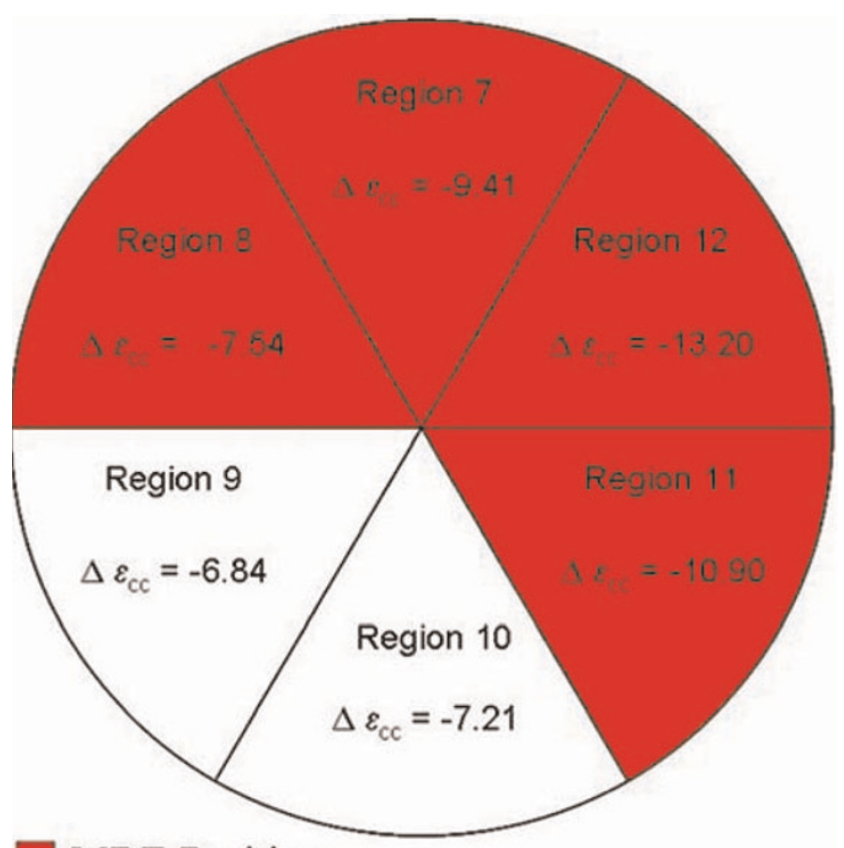

\section{MDE Positive}

Regional distribution of MDE and corresponding $\Delta \varepsilon_{\mathrm{cc}}$.
Results: In controls, the lateral free wall regions had a greater baseline $\varepsilon_{\mathrm{cc}}$ than the septal regions. In DMD patients, $\Delta \varepsilon_{\mathrm{cc}}$ was consistently greater in magnitude in the lateral free wall regions when compared to the $\Delta \varepsilon_{\mathrm{cc}}$ of the septal regions (Figure I). MDE was consistently detected in these same lateral free wall regions. Conclusion: Changes in $\varepsilon_{\mathrm{cc}}$ show that the regions with greatest contractility in control subjects (the lateral free wall) are the most susceptible to injury in DMD patients, as exemplified both by the greatest reduction in regional $\varepsilon_{\mathrm{cc}}$ and the development of MDE in those regions.

\section{PI3}

Pulse wave velocity in the aortic arch is the strongest predictor of left ventricular concentric remodeling in subjects with different levels of cardiovascular risk Alban Redheuil', Wen Chung Yu', Raymond T Yan', Elie Mousseaux ${ }^{2}$, Alain De Cesare ${ }^{2}$, David A Bluemke ${ }^{3}$ and Joao AC Lima'

Johns Hopkins University, Baltimore, MD, USA

INSERM U678, Paris, France

${ }^{3} \mathrm{NIH}$, Bethesda, MD, USA

Journal of Cardiovascular Magnetic Resonance 2009, I I (SuppI I):PI 3

Introduction: Increased systolic blood pressure (SBP), pulse pressure (PP) and left ventricular (LV) concentric remodeling are associated with aging and increased cardiovascular risk. The aortic arch accounts for most of the vascular buffering function and is primarily involved in arterial stiffening and complications. Mechanisms of heart failure during aging and the role of aorticventricular stiffening and interaction need clarification. In particular, the importance of the aortic arch on central arterial stiffness remains unclear.

Purpose: In this study, we evaluated the contribution of aortic arch stiffness to LV concentric remodeling

Methods: We studied 52 subjects: 28 men; mean age: 41 yrs [20-79], 40 without and 12 with risk factors (hypertension in all and diabetes in 6) and normal LV ejection fraction. Global aortic stiffness was determined from carotid-femoral pulse wave velocity (cfPWV) using tonometry and transit surface distances. Aortic stiffness in the aortic arch was determined by MRI from as regional pulse wave velocity (PWV): ratio of aortic length to flow transit time. Augmentation index was calculated from tonometry as the ratio of the pressure above the inflection point and PP. SBP, MBP and PP were averages of 6 brachial measurements. End diastolic LV mass was measured on cine MRI. LV mass to volume ratio $(M / V)$ was calculated as end diastolic volume over mass. Correlations are given and multivariate regression was used to study the determinants of concentric LV remodeling (M/V)

Results: $M / V$ ratio increased with age: $r=0.35(p=0.01)$; mean $M / V$ in subjects $<50$ years is 0.97 vs. 1.18 in subjects $>=$ 50 years $(p=0.006)$. Aortic arch PWV increased with age: $r=0.77(p<0.00 I)$, SBP: $r=0.62(p<0.00 I), P P: r=0.69$ $(p<0.00 I)$ and was well correlated with cfPWV: $r=0.83$ $(p<0.001)$. However, PWV in the aortic arch was a stronger determinant of $M / V$ ratio than global cfPWV in subjects $>50$ years. Moreover, in multivariate analysis using age, BMI, SBP, PP, augmentation index and cfPWV, PWV in the aortic arch was the 
strongest independent determinant of $M / V$ ratio $(R=0.43$; $\mathrm{p}<0.001$ )

Conclusion: Stiffness of the aortic arch directly measured by $\mathrm{MRI}$ as regional PWV was a stronger predictor of LV concentric remodeling than global cfPWV, age or blood pressure in a population sample with varied cardiovascular risk factors.

\section{P /4}

\section{Circumferential and radial myocardial strain in cardiomyopathy patients with and without left bundle branch block}

Yuchi Han', Jonathan Chan', Idith Haber², Dana C Peters', Peter J Zimetbaum', Warren J Manning' and Susan B Yeon

'BIDMC, Boston, MA, USA

${ }^{2}$ University of Chicago, Chicago, IL, USA

Journal of Cardiovascular Magnetic Resonance 2009, I I (SuppI I):PI4

Introduction: Electrical dyssynchrony associated with prolonged QRS duration is a commonly used criterion to select symptomatic heart failure patients for cardiac resynchronization therapy (CRT). However, there is concern that electrical dyssynchrony criterion is inadequate as $30-40 \%$ of patients do not respond to CRT [I]. Studies have shown that assessment of mechanical dyssynchrony may be a better predictor of response [2]. Most of the studies have assessed mechanical dyssynchrony in the longitudinal axis of myocardial motion [3]. There is limited data on the assessment of short axis mechanical dyssynchrony in humans. We sought to examine the relationship between electrical and mechanical dyssynchrony in mid-ventricular short axis using CMR tagging in patients with depressed left ventricular function.

Methods: 22 patients with NYHA class II to III heart failure were studied, including 12 patients with dilated cardiomyopathy (DCM) (age $60 \pm 9$ years, 83\% male, ejection fraction (EF) $28 \pm 9 \%$ ) and 10 patients with ischemic cardiomyopathy (ICM) (age $63 \pm 8$ years, $80 \%$ male, EF $30 \pm 5 \%$ ). Ten healthy adult subjects (age $37 \pm 12$ years, $50 \%$ male, EF $60 \pm 4 \%$ ) served as controls. CMR studies were performed on a $1.5 \mathrm{~T}$ Philips Achieva MR scanner (Philips HealthCare, Best, NL), equipped with a 5-element cardiac coil. Breath-hold ECG-gated tagged CSPAMM cine images at the mid-papillary muscle level were obtained. Scan parameters include spiral readout with 8 interleaves, $9 \mathrm{~ms}$ acquisition window, TR/TE/flip angle $=$ $25 \mathrm{~ms} / 3.6 \mathrm{~ms} / 25^{\circ}, \mathrm{FOV}=320 \mathrm{~mm}, 10 \mathrm{~mm}$ slice thickness with $5 \mathrm{~mm}$ tag spacing, temporal resolution 25-35 ms, spatial resolution $2.5 \times 2.5 \times 10 \mathrm{~mm}$. A customized software program (Cardiotool), written in MATLAB (MathWorks, Natick, MA), was used for semi-automated analysis of peak circumferential $(\varepsilon c)$ and peak radial strain $(\varepsilon r)$ [4].

Results: Thirteen patients with DCM $(n=8)$ and ICM $(n=5)$ had a left bundle branch block (LBBB) with a mean QRS duration of $161 \pm 15 \mathrm{~ms}$. The remaining nine patients had either normal QRS duration, non-specific interventricular conduction delay, or right bundle branch block with a mean QRS duration of $108 \pm$ $18 \mathrm{~ms}$. The control subjects had a mean QRS duration of $94 \pm$ I I ms. All patients with LBBB showed an initial negative $\varepsilon c$ in the anteroseptal segment, quickly followed by positive $\varepsilon c$ reflecting dyskinesis of the septum (as shown in Figure I). In patients with
Figure I (abstract P |4)
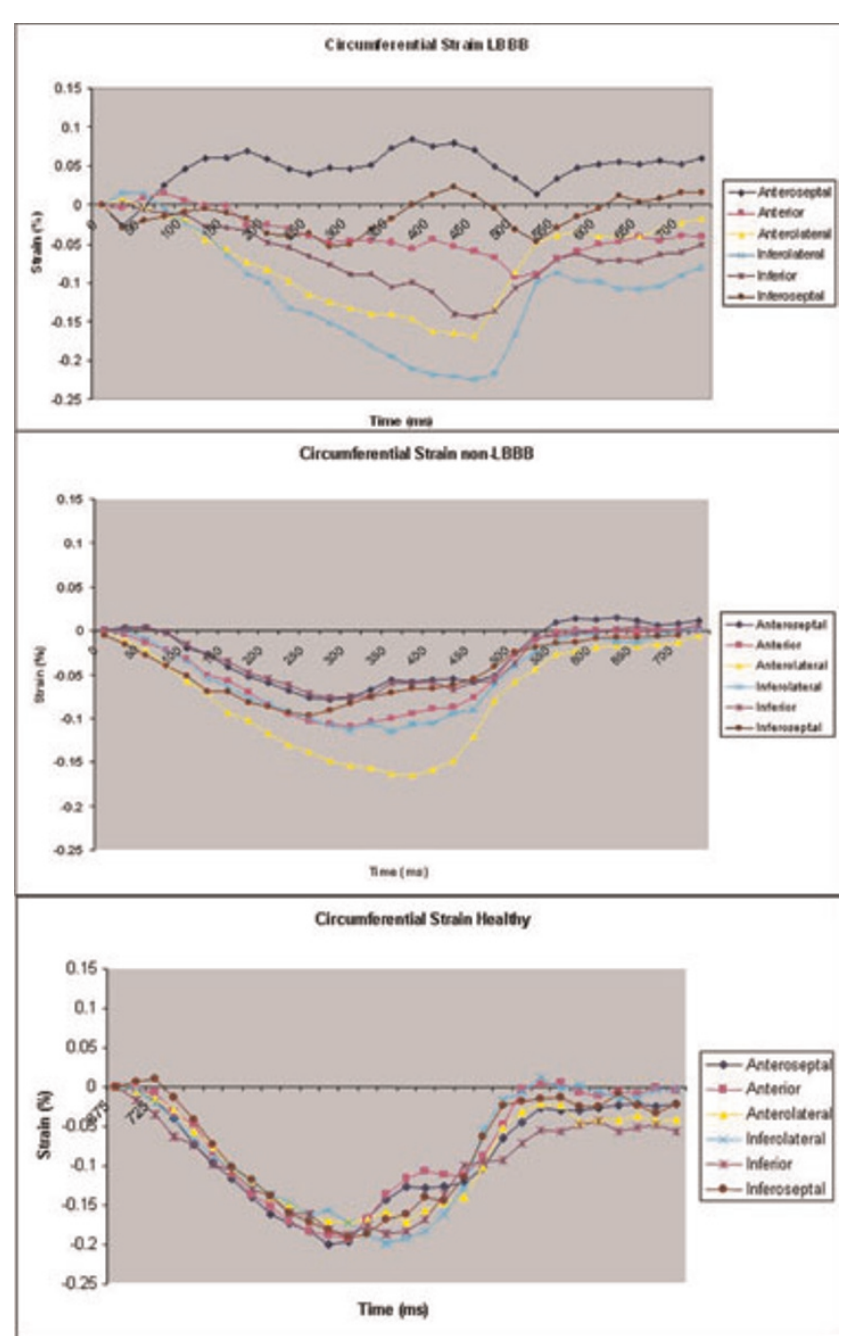

Representative circumferential myocardial strain in cardiomyopathy patients with LBBB, non-LBBB, and healthy controls.

LBBB, there was significant dysynchrony of contraction as indicated by greater opposing wall (inferolateral wall to anteroseptum) delays and standard deviations (SD) in time to peak $\varepsilon c$ and $\varepsilon r$ for all six wall segments $(p<0.00 I)$ (Figure 2$)$. All patients without LBBB and all normal subjects showed negative $\varepsilon c$ in all segments throughout systolic contraction (Figure I). Patients with cardiomyopathy showed reduced magnitude of $\varepsilon c$ and $\varepsilon r(p<0.002)$.

Conclusion: In patients with heart failure, a LBBB pattern with marked increase in QRS duration manifests as a specific contractile pattern with marked dyskinesis of the interventricular septum. Mechanical dyssynchrony and reduction in myocardial circumferential and radial strain are identified and quantified using a semi-automated method. This study identifies the relationship between electrical and mechanical circumferential and radial dyssynchrony in patients with dilated as well as ischemic cardiomyopathy. 
Figure 2 (abstract P|4)

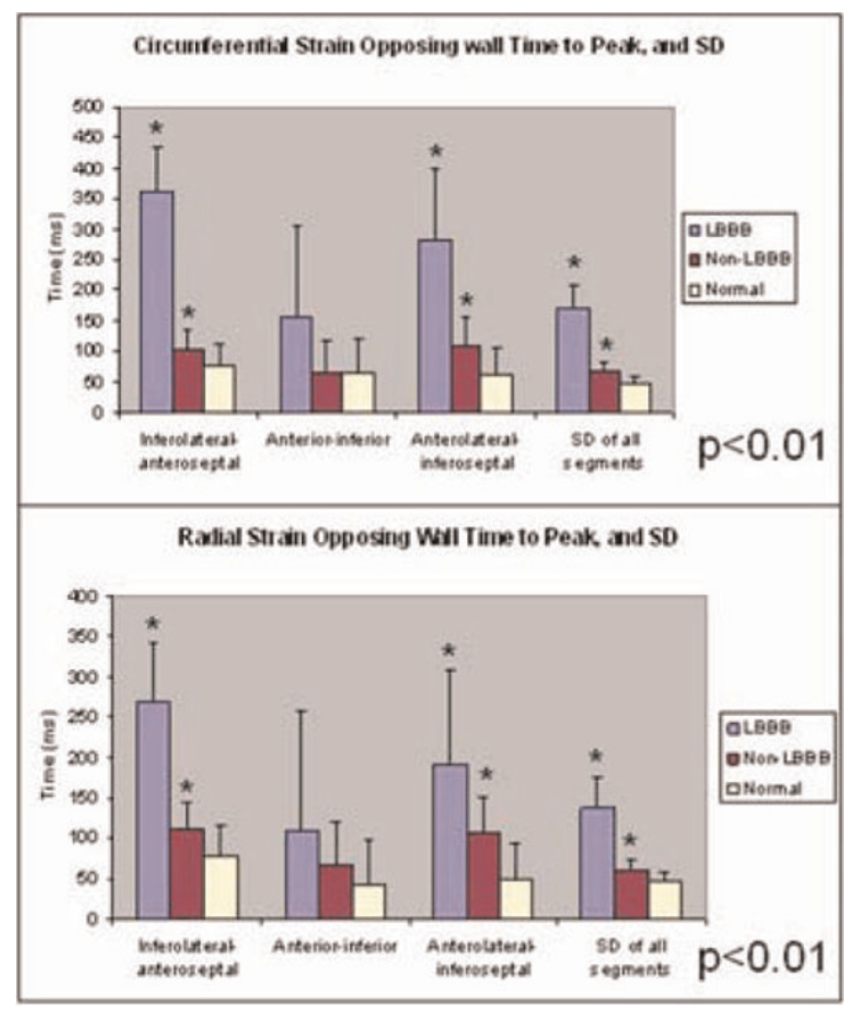

LBBB patients have more mechanical dyssynchrony than nonLBBB patients.

\section{References}

I. Yu CM, Fung JWH and Zhang Q, et al: J Cardiovasc Electrophysiol 2005, 16: I I 17-II24.

2. Nelson GS, Curry CW and Wyman BT, et al: Circulation 2000, I 01:2703-2709.

3. Yu CM, Chau E and Sanderson JE, et al: Circulation 2002, I 05:438-445.

4. Haber I, Metaxas DN and Axel L: Med Image Anal 2000, 4:335-355.

\section{PI5}

The use of multiparametric CMR to predict impaired exercise capacity in hypertrophic cardiomyopathy Andrew S Flett, Caroline J Coats, Brian A Mist, Giovanni Quarta, Ferdinando Pasquale, Perry M Elliott and James $C$ Moon

The Heart Hospital, London, UK

Journal of Cardiovascular Magnetic Resonance 2009, I I (SuppI I):PI5

Objective: To understand the role of CMR measured parameters including late gadolinium enhancement (LGE) on exercise capacity in patients with hypertrophic cardiomyopathy (HCM).

Background: Exercise intolerance in HCM is complex, depending on multiple factors including diastolic dysfunction, outflow tract obstruction, ischemia, left atrial pressure, hypertrophy and fibrosis. We sought to determine the relative
Figure I (abstract PI5)

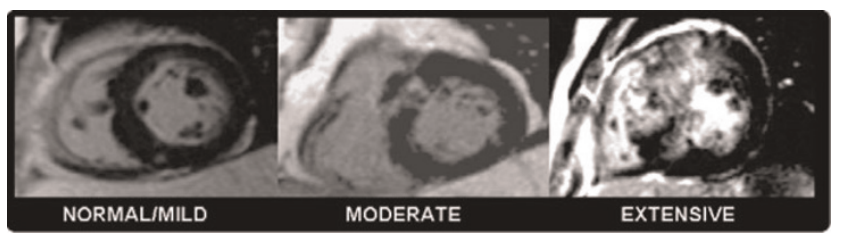

The extent of late gadolinium enhancement assigned to 3 groups.

contributions of 5 CMR measured variables on exercise capacity in HCM: function, mass, left atrial area, resting LVOT obstruction and LGE.

Methods: 135 consecutive patients with HCM (median age 46.5 years, $21 \%$ female, $67 \%$ Caucasian) underwent cardiopulmonary metabolic exercise testing (measuring percent predicted peak oxygen consumption $\left(\% \mathrm{pVO}_{2}\right)$ and contrast CMR (for function, volumes, mass, left atrial area, presence of rest LVOT obstruction and LGE). Two independent investigators blinded to results of the opposing dataset performed analysis of exercise and CMR data. The extent of LGE was categorised as normal/ minimal, moderate or extensive (3 point scale, 0 , I or 2 ). Univariate and multivariate analysis was used to assess correlations of $\% \mathrm{pVO}_{2}$ with $\mathrm{CMR}$ derived variables.

Results: Normal/minimal, moderate and extensive LGE was present in $67(50 \%), 58(43 \%)$ and 10 (7\%) respectively. 3 I (23\%) had resting LVOT obstruction. On univariate analysis, $\% \mathrm{pVO}_{2}$ was associated the presence of resting outflow tract obstruction $(p=0.03)$ and inversely asscociated with extensive LGE $(p=0.02)$. On multivariate analysis, the single significant factor associated with $\% \mathrm{pVO}_{2}$ was LGE $(p=0.01)$. Although LGE and EF were associated $(r=0.232, p=0.007)$, LGE was the independent predictor of $\% \mathrm{pVO}_{2}$. If significant exercise impairment is defined as a $\% \mathrm{pVO}_{2}<70 \%$, moderate and extensive LGE

\section{Figure 2 (abstract PI5)}

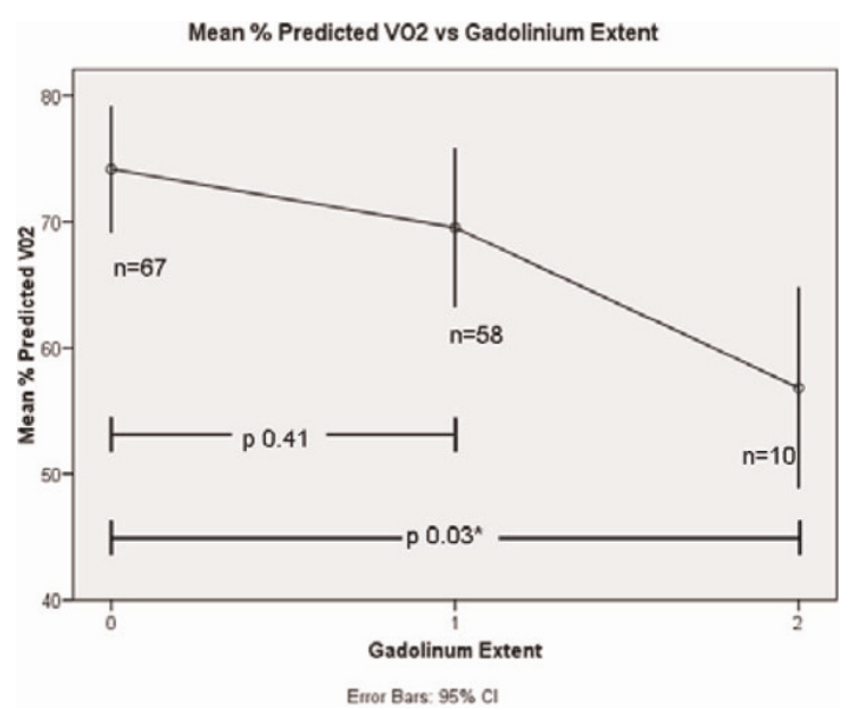

Peak oxygen consumption falls with extent of LGE in patients with HCM. 
independently predicts it with odds ratios of $2.2(95 \% \mathrm{Cl} \mathrm{I.0,}$ $4.5 \mathrm{p}=0.04)$ and I8.9 $(95 \% \mathrm{Cl} I .9,188.2, \mathrm{p}=0.0 \mathrm{I})$ respectively. Extensive LGE has low sensitivity (13\%) but high specificity (98\%) for predicting poor exercise capacity. Figures $I$ and 2

Conclusion: The main determinant of significant exercise limitation measured by multiparametric CMR in HCM is the presence of extensive LGE. Lesser amounts of LGE do not predict exercise capacity, highlighting the mulitfactorial nature of functional limitation in this condition.

\section{P I 6}

Cardiac magnetic resonance findings in asymptomatic patients with Brugada syndrome Christoph J Jensen', Holger C Eberle ${ }^{\text {,2, }}$, Dinh Q Nguyen', Thomas Schlosser ${ }^{3}$, Jan Hluchy', Christoph K Naber', Georg $\bigvee$ Sabin' and Oliver Bruder'

'Elisabeth Hospital Essen, Essen, Germany

${ }^{2}$ Elisabeth Hospital, Department of Cardiology and Angiology, Essen, Germany

${ }^{3}$ University Hospital Essen, Essen, Germany

Journal of Cardiovascular Magnetic Resonance 2009, I I (SuppI I):PI6

Introduction: The Brugada syndrome (BS) is characterized by distinctive ST-segment abnormalities, malignant ventricular arrhythmias, and sudden cardiac death and is attributed to a sodium channelopathy. Additionally, right ventricular wall motion abnormalities have been described by previous studies.

Purpose: To evaluate cardiac magnetic resonance (CMR) findings in asymptomatic patients with Brugada syndrome compared to matched controls.

Methods: CMR was performed in 24 asymptomatic patients ( 3 males; mean age $42 . \pm 7$ years) with proven Brugada syndrome on a I.5 Tesla MR System. The imaging protocol included steady-state free precession (SSFP) cine sequences (TrueFISP, TR $3 \mathrm{~ms}$, TE $1.5 \mathrm{~ms}$, FA $60^{\circ}$, slice thickness $5 \mathrm{~mm}$ ) in long axis views and contiguous short-axis views covering the entire left (LV) and right ventricle (RV) including the right ventricular outflow tract (RVOT). Additionally, TI weighted turbo spin-echo sequences with and without fat suppression (TR $700 \mathrm{~ms}$, TE I $4 \mathrm{~ms}$, FA $180^{\circ}$, slice thickness $5 \mathrm{~mm}$ ) were acquired, and delayed enhancement imaging was performed following gadolinium contrast administration using segmented 2D inversion-recovery fast low angle shot sequences (TR $8 \mathrm{~ms}$, TE $4 \mathrm{~ms}$, FA $25^{\circ}$, slice thickness $5 \mathrm{~mm}$ ) in corresponding slice orientation. Functional analysis and the area of the RVOT were calculated offline by semi-automatic post-processing software. CMR parameters were compared by Mann-Whitney test to age and sex matched controls $(n=24)$.

Results: Patients with BS had statistically significant larger RV end-diastolic ( $167 \pm 43 \mathrm{ml}$ vs. II $\pm 24 \mathrm{ml}, \mathrm{p}<0.00 \mathrm{I})$ and endsystolic volumes $(87 \pm 33 \mathrm{ml}$ vs. $5 \mathrm{I} \pm 16 \mathrm{ml}, \mathrm{p}<0.00 \mathrm{I})$, lower RV ejection fraction ( $49 \pm 8 \%$ vs. $58 \pm 4 \%, \mathrm{p}<0.00 \mathrm{I})$ and dilated RVOT $\left(\mathrm{II} \pm 2 \mathrm{~cm}^{2}\right.$ vs. $\left.8 \pm \mathrm{I} \mathrm{cm}^{2}, \mathrm{p}<0.00 \mathrm{I}\right)$ compared to controls. There was no statistically significant difference in LV volumes and function between patients and controls. Local RV myocardial signal enhancement in the TI weighted turbo spinecho images was observed in three (12.5\%) patients and RV delayed enhancement in four (17\%) of the 24 patients, as compared to no patient in the control group. Five (21\%) patients showed localised right ventricular wall motion abnormalities,
Figure I (abstract PI6)

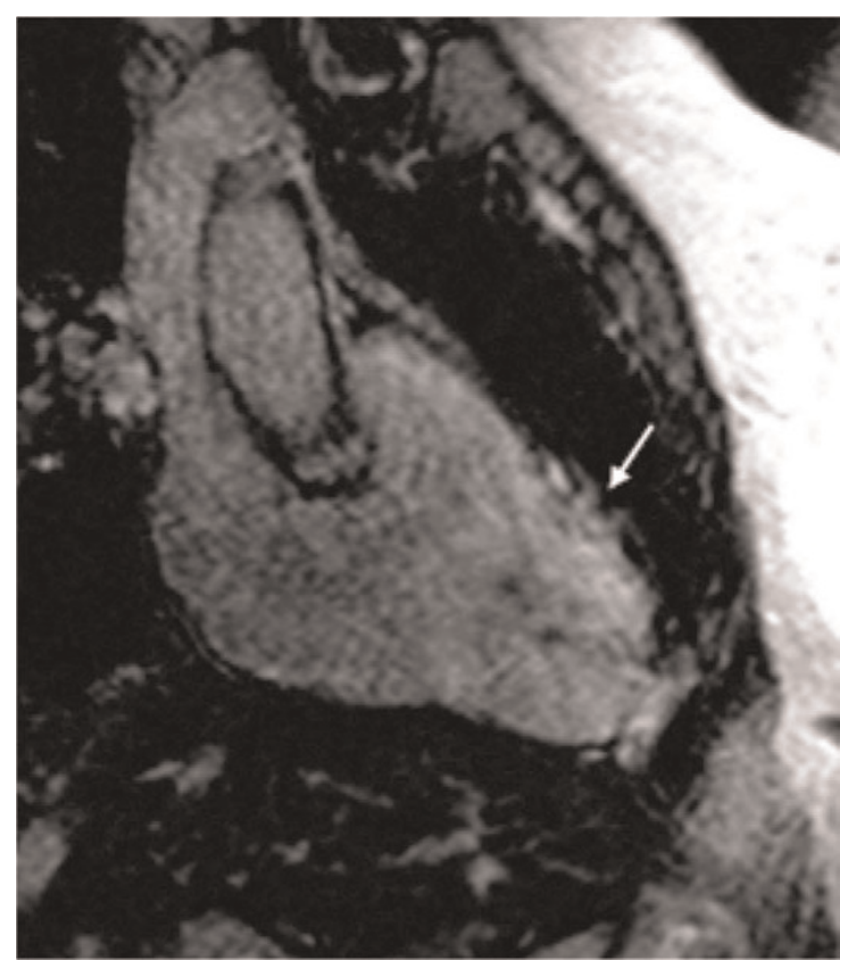

whereof in 2 patients no delayed enhancement or intramyocardial TI signal suggesting fat deposits was present. Figure I.

Conclusion: Patients with proven Brugada syndrome have larger right ventricular volumes, impaired RV function and dilated RVOT compared to matched controls. Right ventricular wall motion abnormalities, localized fat deposits and delayed enhancement can be found in some of the Brugada syndrome patients indicating subtle structural heart disease.

\section{PI7}

Focal myocardial fibrosis detected with magnetic resonance late Gadolinium enhancement imaging and diffuse interstitial fibrosis determined with histological staining of endomyocardial biopsy specimens in patients with non-ischemic left ventricular systolic dysfunction: two distinct entities?

Simon Schalla, Sebastiaan Bekkers, Robert Dennert, Robert J van Suylen, Johannes Waltenberger, Tim Leiner and Stephane Heymans

University Hospital Maastricht, Maastricht, Netherlands

Journal of Cardiovascular Magnetic Resonance 2009, I I(SuppI I):PI7

Introduction: Focal myocardial fibrosis detected with magnetic resonance late gadolinium enhancement imaging (LGE) as well as diffuse interstitial fibrosis seen on endomyocardial biopsy specimens are associated with an adverse prognosis in patients with non-ischemic left ventricular dysfunction.

Purpose: We tested the hypothesis if these two forms of fibrosis are linked to each other and whether focal fibrosis becomes visible if a certain amount of diffuse fibrosis is present. 
Thus, the aim of this study was to determine whether focal fibrosis detected non-invasively with LGE is related to the degree of diffuse fibrosis seen on histologic specimens obtained from endomyocardial biopsy in patients with idiopathic cardiomyopathy.

Methods and results: Fifty-five patients ( 30 males, 25 females, age $47 \pm 14$ years, range $18-73$ years) with impaired systolic function on intitial echocardiography underwent magnetic resonance cine and LGE imaging (I.5 T Gyroscan Intera, Philips Medical Systems, Best, The Netherlands) and endomyocardial biopsy. The scanparameters were I) steady-state free precession sequence for cine imaging with slice thickness $6 \mathrm{~mm}$, slice gap $4 \mathrm{~mm}, \mathrm{TR} / \mathrm{TE} 3.8 / \mathrm{l} .9 \mathrm{~ms}$, flip angle $50^{\circ}$, FOV $350 \mathrm{~mm}$, matrix $256 \times 256,22-25$ phases per cardiac cycle, 2) Look-Locker sequence to determine the inversion time for the subsequent late enhancement scan to optimally "null" left ventricular myocardium (typical range 200-280 ms) with slice thickness $10 \mathrm{~mm}, \mathrm{TR} / \mathrm{TE} 3.8 / 1.9 \mathrm{~ms}$, flip angle $8^{\circ}$, FOV $370 \mathrm{~mm}$, resolution $256 \times 256,39$ phases, phase interval 20-21 ms and 3) multislice TI-weighted 3D inversion-recovery gradient-echo sequence for LGE imaging ( 10 minutes after intravenous administration of $0.2 \mathrm{mmol} / \mathrm{kg}$ Gd-DTPA with an injection rate of $3 \mathrm{ml} / \mathrm{sec}$ ) with slice thickness $12 \mathrm{~mm}$, slice gap $6 \mathrm{~mm}$, TR/TE 4.2/l.3 ms, flip angle $15^{\circ}$, FOV $400 \mathrm{~mm}$, resolution $256 \times 256$.

The mean LVEF determined with cine MR-imaging was $37 \pm 13 \%$, EDV $234 \pm 122 \mathrm{ml}$ and LV massa $135 \pm 47 \mathrm{~g}$. Twenty patients had LGE, the majority of patients did not show focal fibrosis on MR imaging. On biopsies, the mean collagen volume fraction was $6.2 \pm 5.3 \%$. No correlation was found between LGE and histology. Furthermore, no correlation was found between the grade of fibrosis on histological examination and LVEDV or LV mass.

Conclusion: Focal fibrosis was uncommon in this group of patients with impaired systolic function. No correlation was found between diffuse and focal fibrosis and, thus, focal fibrosis as seen with LGE is not related to a manifestation of a certain degree of diffuse interstitial fibrosis. The underlying pathophysiologic process for the development of focal fibrosis remains to be investigated.

\section{P $\mid 8$}

Cardiovascular magnetic resonance imaging in early anthracycline cardiotoxicity

Gillian Smith', Paul Kotwinski², John Paul Carpenter', Montgomery Hugh ${ }^{2}$ and Dudley Pennell ${ }^{1}$

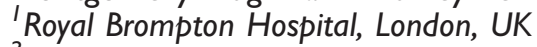

${ }^{2}$ University College London, London, UK

Journal of Cardiovascular Magnetic Resonance 2009, I I (SuppI I):PI8

Introduction: Anthracyclines are the mainstay of adjuvant chemotherapy for patients with breast cancer. This therapy is known to be cardiotoxic with population risk rising with dosage but individual susceptibility is idiosyncratic thus optimal dosage is denied to many. Cardiovascular magnetic resonance (CMR) offers an accurate means by which to assess ventricular function prior to and following chemotherapy. It also offers excellent intrinsic contrast which allows the detection of change at a tissue level which may indicate inflammatory changes without the need for a contrast agent and therefore cannulation. This would be of particular value for monitoring patients undergoing courses of chemotherapy who frequently develop problems with venous access or become needle phobic.

Purpose: As part of a prospective gene environment study in breast cancer, this study attempts to identify early markers of anthracycline mediated toxicity which may predict later cardiac dysfunction.

Methods: 276 female patients with early breast cancer were scanned before commencement of chemotherapy and one year post completion. CMR was performed using a I.5 T Siemens Avanto scanner with a 6 channel phased array cardiac coil. Patients were scanned 3 days post first cycle. Cine images are acquired using a retrospectively gated high temporal resolution SSFP sequence. Left ventricular ejection fraction (LVEF) and mass (LVM) are measure using a semi-automated analysis package (LVtools, Cardiovascular Imaging Solutions, London, UK). T2 weighted STIR images were also acquired and signal intensity compared.

Results: To date, 28 patients have had day 3 scans and one year follow-up studies. Mean ejection fraction (EF) changed from $72.9 \pm 5.0 \%$ to $71.0 \pm 4.6 \%(p=0.06)$. I4 patients showed a significant reduction in ejection fraction (mean $75.5 \pm 4.6 \%$ to $70.4 \pm 4.9 \%$ ). In these patients, the LVM increased from $1 \mid 5.7 \pm 14.6 \mathrm{~g}$ to $125.1 \pm 16.7 \mathrm{~g}(\mathrm{p}=0.0002)$, and this was associated with an increase in STIR image intensity (62.7 \pm 19.2 to $71.7 \pm 26.9 ; p=0.05)$. Of the 14 patients who showed no reduction in LVEF $(70.0 \pm 3.9$ at baseline vs $71.8 \pm 4.4$ at follow-up), there was no significant change in either LVM $(\mathrm{II} .8 \pm 13.0 \mathrm{~g}$ vs $1 \mid \mathrm{l} . \mathrm{I} \pm \mathrm{II} .4 \mathrm{~g}, \mathrm{p}=0.2)$ or Stir $(64.7 \pm$ 20.6 vs $63 \pm 17.1, p=0.6)$ at day 3 .

Conclusion: CMR with Stir and left ventricular mass measurements, which reflect myocardial edema, offers a sensitive predictor at day 3 post chemotherapy of myocardial dysfunction after one year secondary to anthracycline cardiotoxicity.

\section{P 19}

Utility of cardiac MRI in detecting diastolic dysfunction: comparison with Doppler echocardiography and tissue Doppler imaging Anthony F Tramontano, Cuilin Miao, Alison Hays, Robert Donnino, Ruth Lim, Leon Axel, Danny Kim, Hersh Chandarana and Monvadi B Srichai NYU Medical Center, New York, NY, USA

Journal of Cardiovascular Magnetic Resonance 2009, I I(SuppI I):PI 9

Background: Left ventricular diastolic dysfunction (LVDD) serves as a prognostic indicator of adverse cardiovascular events. Cardiac magnetic resonance (CMR) imaging is the gold standard for evaluating ventricular size and systolic function, but there is limited data on the utility of CMR in the evaluation of diastolic function. To assess the accuracy of CMR in assessing LVDD we compared quantitative CMR to Doppler echocardiography (ECHO).

Methods: 20 patients with cardiomyopathy underwent both $\mathrm{CMR}$ and ECHO studies. Through plane phase contrast CMR and Doppler ECHO was performed for assessment of early $(E)$ and late (A) mitral inflow velocities, early mitral deceleration time (DT), and early (e') myocardial tissue velocities of the basal lateral wall. The two imaging methods were correlated for measurement of several parameters of diastolic function including E/A ratio, E DT, and e'. Diastolic function was assessed based on a combination of mitral inflow and myocardial tissue velocities 
and was classified as normal, abnormal relaxation, pseudonormal or restrictive.

Results: There was a statistically significant correlation for measurement of E/A ratio with both techniques $(r=0.827$, $P<0.05$ ), but not for any of the other parameters evaluated (E DT, e', E/e' ratio). Patients were classified by ECHO into diastolic functional grades as: 4 (20\%) normal, 7 (35\%) abnormal relaxation, $4(20 \%)$ pseudo-normal, and $5(25 \%)$ restrictive. Diastolic function grade was identical in $10(50 \%)$ patients, differed by I grade in 5 (25\%), differed by 2 grades in 3 (I5\%), and differed by 3 grades in 2 (10\%). CMR tended to underestimate the degree of diastolic dysfunction in $70 \%$ of patients compared to $\mathrm{ECHO}$, and there were 3 patients (I5\%) who were classified as normal by CMR, but who demonstrated some evidence of diastolic dysfunction on ECHO.

Conclusion: CMR can detect the presence of LVDD with reasonable accuracy, although it tends to underestimate the degree when compared with Doppler ECHO.

\section{P20}

Contrast enhanced delayed myocardial enhancement in patients with end stage liver cirrhosis - further evidence for cirrhotic cardiomyopathy

Dirk Lossnitzer, Daniel Gotthardt, Stephanie Lehrke, Grigorios Korosoglou, Henning Steen, Peter Sauer, Evangelos Giannitsis and Hugo A Katus University of Heidelberg, Heidelberg, Germany

Journal of Cardiovascular Magnetic Resonance 2009, I I (SuppI I):P20

Introduction: Cirrhotic cardiomyopathy was first described in 1969 by Regan TJ et al. and was found in all kinds of liver cirrhosis since 1990. Nevertheless the disease is still not well understood due to the hyperdynamic LV function, the lack of reliable diagnostic tools as well as multimorbidity of patients with end stage liver disease. However there is evidence for a reduced cardiac response to stress factors, QT-prolongation as well as myocardial fibrosis, subendocardial edema and vacuolization of myocytes.

Purpose: The aim of this study was to further investigate the evidence of cirrhotic cardiomyopathy in patients with end stage liver disease by cardiac MRI.

Methods: We examined 20 patients (8 females, age $54 \pm 9$ ) who are/were listed for liver transplantation (MELD score $15 \pm 6$, CHILD score $8.7 \pm 2$ ) because of alcoholic liver cirrhosis on a I.5 T Philips Achieva scanner using a 5-element phased array cardiac synergy coil in 2007. Multiple short axes (SAX) cine images using a SSFP sequence with parallel imaging were acquired for the assessment of left ventricular ejection fraction (LVEF \%) followed by a standardized high-dose dobutamine stress protocol up to $40 \mu \mathrm{g}$ per kilogram of body weight per minute. A 3D FFE SAX multi-slice inversion recovery sequence 10 minutes post-Gd $(\mathrm{Gd}=0.2 \mathrm{mmol} / \mathrm{kg}$ of gadopentetate dimeglumine (Schering, Berlin, Germany)) was employed for the measurement of LE. TNT and NT-proBNP were measured according to clinical routine procedures (Roche Diagnostics, Penzberg, Germany).

Results: All patients showed normal to hyperdynamic LVEF at rest $(71,3 \pm 8 \%)$ as described before with other imaging methods and no wall motion abnormalities at rest or during dobutamine stress. However relevant LE $(27,4 \pm 17,4 \%$ of myocardial mass (see figure I), was detected in all patients. NTproBNP levels were increased $(846 \pm 1042 \mathrm{pg} / \mathrm{ml})$, but there was no correlation between the amount of LE and the NTproBNP levels $(r=-0.04)$. TNT levels were within normal range $(0,0225 \pm 0,008 \mathrm{ng} / \mathrm{ml}) .8$ patients underwent liver transplantation and 5 patients ( 3 of them after liver transplantation) died during the study period.

Conclusion: This is the first description of myocardial delayed contrast enhancement in patients with end stage liver disease. We assume that these findings might be of diagnostic and prognostic value and might be helpful to detect patients at risk for cardiac complications on the waiting list for liver transplantation. However further studies with bigger sample sizes have to be done to fortify our results.

\section{P2I}

MRI signs of carotid plaque inflammation in patients with unstable angina: a possible sign of widespread plaque activity Carlo Liguori', Luigi Natale', Agostino Meduri', Antonio Bernardini ${ }^{2}$, Antonella Lombardo' and Lorenzo Bonomo'

'Università Cattolica del Sacro Cuore - Policlinico A. Gemelli, Rome, Italy

2“G. Mazzini” Hospital - ASL Teramo, Teramo, Italy

Journal of Cardiovascular Magnetic Resonance 2009, I I (SuppI I):P2 I

Purpose: Inflammation may contribute to destabilize vulnerable plaques in acute coronary syndromes by promoting rupture and erosion; systemic inflammatory factors could be related with widespread plaque activity in many vascular districts.

The purpose of this study was to evaluate with contrast enhanced-MRI(CE-MRI) plaque inflammation signs in carotid arteries of pts with unstable angina (UA) and to relate them to serum levels of C-reactive protein (CRP), a marker of systemic inflammation.

Methods: 27 pts with carotid plaques, 16 with UA and II with stable angina(SA) underwent carotid arteries MRI with SE, FSE and bb-FSE sequences, before and after Gd iv. administration (0.2 mmol $/ \mathrm{Kg}$ ). We considered 3 markers: I) wall thickening, index of arterial wall edema or infiltration; 2) increased T2 or FSE-STIR signal intensity (SI), index of arterial wall or plaque edema; 3) arterial wall or plaque enhancement, index of increased capillary permeability. Totally 37 plaques were evaluated (I7 patients with monolateral and 10 with bilateral stenosis). CRP levels were determined with ELISA essay.

Results: 19 plaques showed wall thickening and/or increased T2 and/or FSE-STIR SI and Gd enhancement, 5 plaques showed only contrast enhancement, whereas 13 plaques had no inflammation signs. CRP levels of patients with enhanced plaques were significantly higher than those of patients without enhancement (median values: 12.5 vs 3.3 , $\mathrm{p}<0.05$ ).

Conclusion: Pts with UA showed plaque inflammation signs more frequently than controls; patients with UA and inflammation signs showed higher CRP levels than pts with UA and no inflammation signs. These results suggest a widespread plaque activity, possibly mediated by systemic inflammation, in acute coronary syndromes. 
P22

Does 3D volumetric carotid plaque imaging by cardiovascular MRI solve the mystery of the 'percent stenosis paradox'?

Robert WW Biederman', Ronald B Williams', Saundra B Grant', Geetha Rayarao', June A Yamrozik', Vikas K Rathi ${ }^{1}$, Diane A Vido , David R Neff ${ }^{2}$ and Mark Doyle'

'Allegheny General Hospital, The Gerald McGinnis

Cardiovascular Institute, Pittsburgh, PA, USA

${ }^{2}$ Merck/Schering-Plough Pharmaceuticals, Inc., North Wales, PA, USA

Journal of Cardiovascular Magnetic Resonance 2009, I I (SuppI I):P22

Introduction: Aggressive pharmacologic, dietary and exercise strategies have been employed in recent years to dramatically lower incidence of CAD as evidenced by striking reductions in $\mathrm{MI}$ and CVA and other cardiovascular complications. Yet, when examined at the pathologic source, namely the arterial lumen, little if any changes are seen. Typically, luminal \% stenosis changes are nominal after aggressive statin intervention, often $<5 \%$, yet translate clinically into substantial reductions in morbidity and mortality. The explanation for this dichotomy remains unclear. Recently, we and others have noted that morphologic characteristics of plaque composition help to explain the apparent paradox of improved clinical events despite no or minimal reduction in $\%$ stenosis with statins. In that CMR can distinguish underlying features that determine plaque 'vulnerability', we sought to define the role of composition vs. \%stenosis.

Hypothesis: We hypothesize that in statin-naive pts with highgrade carotid artery stenosis, there will be a high degree of correlation in the relationship between the 'unstable' lipid pool and 'stable' fibrous plaque by 3D CMR, yet may be independent of $2 \mathrm{D}$ luminal \%stenosis.

Methods: Representing 530-two mm contiguous CMR (I.5 T $\mathrm{GE}, \mathrm{EXCITE}$, Milwaukee, $\mathrm{WI}$ ) in vivo slices of advanced (> $50 \%$; mean $61 \pm 24 \%$ stenosis) carotid artery disease, 26 complete bilateral human (age: $66 \pm 14$ yrs) plaques were analyzed for 3D volumetric extent of vascular wall: lipid pool, fibrous cap, matrix and minima/maxima of each. All were related to fasting lipid levels relative to \%stenosis via QPlaque (Medis, The Netherlands). The plaque morphology was determined by TI, T2/PD CMR, chiefly reliant on the former.

Results: In all, 25/26 in vivo plaques were successfully imaged and quantified volumetrically. Mean resolution: I $\times 1 \times 2 \mathrm{~mm}$. The $\mathrm{mg} / \mathrm{dL}$ range of LDL-C was 63-I86, HDL-C: $28-59$ and TG: 8I-213. Lipid pool represented $15 \pm 4 \%$, while fibrous plaque represented $5 \pm 15 \%$ of total vessel wall. Total Cholesterol $\left(\mathrm{Chol}_{\mathrm{T}}\right)$ and LDL-C were inversely related to minimum vessel wall thickness $(r=-0.5$ and -0.6, respectively, $\mathrm{P}<0.05$ for both) while only $\mathrm{Chol}_{\mathrm{T}}$ was related to fibrous cap $(r=0.6, \mathrm{p}<0.0 \mathrm{I})$. The $\mathrm{Chol}_{\mathrm{T}} / \mathrm{LDL}-\mathrm{C}$ ratio was highly related to minimum fibrous plaque thickness $(r=0.8, p<0.00 \mathrm{I})$. The 3D lipid pool was the only fraction highly correlated $(>0.6)$ with triglycerides $(r=0.6, p<0.0 \mathrm{I})$. A linear regression relating fibrous cap: vessel wall ratio to non-HDL cholesterol and $\mathrm{Chol}_{\mathrm{T}}$ was highly correlated ( $r=0.6,0.7$, respectively, $p<0.01$ for both) but was independent of in vivo \%stenosis $(r=0.1)$. Relating \% luminal stenosis to any lipid fraction, subfraction or to any vessel wall component or its ratio revealed no statistically significant relationship.

Conclusion: Percent stenosis provides relatively little information about vulnerability of de novo, statin-naive carotid plaques in high-grade carotid artery disease. As most current imaging based clinical studies (IVUS, MRA, CTA and contrast angiography) monotonously concentrate on plaque stenosis for identification of timing for intervention, a more appropriate focus on underlying plaque composition may provide a more robust quantifiable volumetric metric potentially more indicative of the underlying pathology and vulnerability by high-resolution 3D CMR.

\section{P23}

MRI-assessed end-organ damage in hypertension: association between aortic stiffness, cardiac function and lacunar brain infarcts

Anne Brandts, Saskia GC van Elderen, Jos JM Westenberg, Jeroen van der Grond, Mark A van Buchem,

Menno V Huisman, Lucia JM Kroft, Jouke T Tamsma and Albert de Roos

Leiden University Medical Center, Leiden, Netherlands

Journal of Cardiovascular Magnetic Resonance 2009, I I(SuppI I):P23

Introduction: Hypertension is a major risk factor for cardiovascular morbidity and mortality through its effects on target organs including the heart, the blood vessels and the brain. Early detection of cardiac and cerebral outcome, before irreversible damage has occurred, can contribute to further decline in hypertension-related death.

Pulse Wave Velocity (PWV), defined as the propagation speed of the systolic pressure or flow wave through the aorta, is a surrogate marker for aortic stiffness. PWV has been shown to be an independent predictor of stroke and cardiac mortality in hypertensive patients. Gold standard for PWV-assessment comes from intravascular pressure measurement but this requires an invasive procedure, not suitable for screening. The current method-of-choice is non-invasive echo Doppler. Magnetic resonance imaging (MRI) provides an alternative, with the advantage over echo Doppler that image acquisition does not

\section{Figure I (abstract P23)}
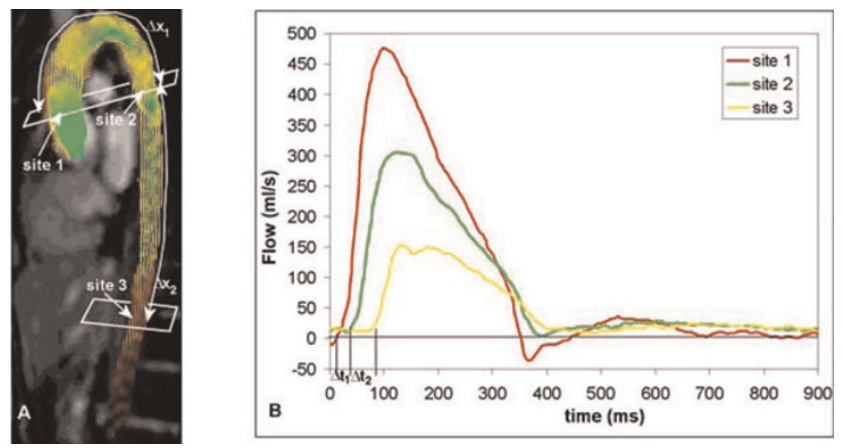

PWV determination. A, a sagittal gradient-echo image of the aorta is shown. Velocity-encoded MRI is performed perpendicular to the aorta at two levels: site one is positioned at the level of the pulmonary trunk; site two is positioned at the distal descending abdominal aorta $7.5 \mathrm{~cm}$ below the diaphragm; $\Delta \mathrm{x}_{1}$ : path length of the aortic arch $\Delta \mathrm{x}_{2}$ : path length of the descending aorta. $\mathrm{B}$, the flow curves of the ascending, proximal and distal descending aorta are shown; $\Delta \mathrm{t}_{1}$ and $\Delta \mathrm{t}_{2}$ denote the transit-time between the arrival of the pulse wave at subsequent measurement sites. Aortic $\mathrm{PWV}$ is defined as $\Delta \mathrm{x} / \Delta \mathrm{t}(\mathrm{m} / \mathrm{s})$. 
have a limited acoustic window and the aorta can be visualized along its full length, allowing for more accurate assessment of the path lengths of pulse waves. Also, MRI is the gold standard for assessment of cardiac left ventricular (LV) function and mass and cerebral lacunar infarcts and white matter hyperintensities (WMHs). An integrated MRI-approach for the assessment of aortic stiffness, cardiac function and brain examination is performed in hypertensive patients. Our hypothesis is that early end-organ damage (i.e. increased aortic stiffness, decreased cardiac function and cerebral lacunar infarcts and WMHs) are interrelated in hypertensive patients.

Purpose: To assess the association between aortic PWV, cardiac LV function and mass as well as cerebral lacunar infarcts and WMHs in hypertensive patients using MRI.

Methods: MRI of the aorta, heart and brain was performed in 50 consecutive hypertensive patients ( 19 male, 3I female; mean age $49 \pm 12$ years). Aortic PWV was determined from onedirectional through-plane velocity-encoded (VE) MRI at the ascending aorta, proximal and distal descending aorta (Figure I). From VE MRI, flow graphs were obtained. High temporal resolution $(6-10 \mathrm{~ms})$ allows for accurate assessment of the transit-time between the arrival of the pulse wave at subsequent measurement sites. A value for PWV was obtained for the aortic arch and the descending aorta.

Short-axis MRI using steady-state free precession was used to measure LV systolic function and mass. An ECG-gated gradientecho sequence with VE was performed to measure blood flow across the mitral valve to determine LV diastolic function. For detection of lacunar infarcts and WMHs, patients underwent T2 and Fluid Attenuated Inversion Recovery (FLAIR) acquisitions. The association between aortic PWV, cardiac and cerebral parameters was calculated using univariate and multivariate linear and logistic regression analyses.

Results: Mean systolic BP was $152 \pm 22 \mathrm{mmHg}$, mean diastolic BP $88 \pm 13 \mathrm{mmHg}$. Mean aortic arch PWV was $7.3 \pm 2.5 \mathrm{~m} / \mathrm{s}$, mean descending aorta PWV $6.9 \pm 2.7 \mathrm{~m} / \mathrm{s}$.

After adjustment for age, gender and hypertension duration, aortic PWV was shown to be independently associated with LV mass $(r=0.4 \mathrm{I}, \mathrm{P}=0.03)$ and lacunar brain infarcts $(O R=1.8$, $95 \% \mathrm{Cl}$ I.0-3.0, $\mathrm{p}=0.02$ ) and not with LV function and WMHs. Conclusion: Aortic PWV is independently associated with LV mass and lacunar brain infarcts, both indicators of early endorgan damage of hypertension.

\section{P24}

Initial validation of a multi-station

non-contrast dark blood approach for

the diagnosis of peripheral arterial disease Georgeta Mihai ', YiuCho Chung', Mbabazi Kariisa', Orlando P Simonetti ' and Sanjay Rajagopalan'

'The Ohio State University, Columbus, OH, USA

${ }^{2}$ Siemens Healthcare USA, Inc, Columbus, OH, USA

Journal of Cardiovascular Magnetic Resonance 2009, I I(SuppI I):P24

Introduction: Current magnetic resonance angiographic (MRA) diagnosis of peripheral arterial disease (PAD) is based on contrast-enhanced angiography (ce-MRA), a luminographic technique lacking information on atherosclerotic plaque burden. Moreover, the recent links between nephrogenic systemic
Figure I (abstract P24)

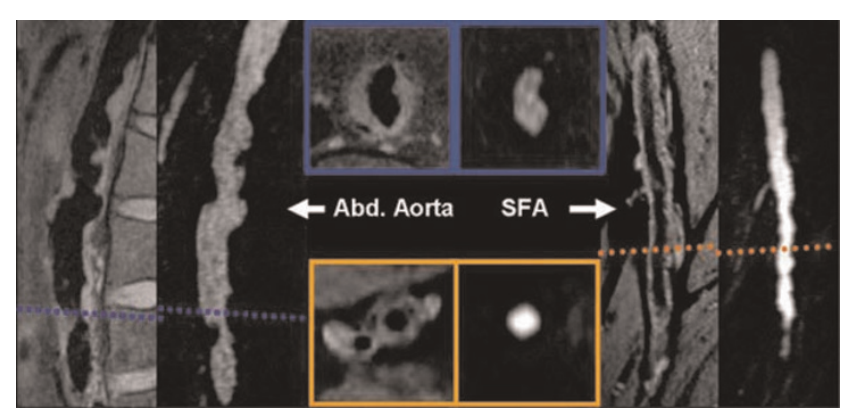

TIw-SPACE (left) and ce-MRA (right) images of segments of abdominal aorta (left group) and SFA (right group) showing the dark and bright lumen, as well as the arterosclerotic plaque in TI w-Space (left middle group).

fibrosis disease and gadolinium based contrasts limits use of this technique in PAD patients with impaired renal function.

Purpose: This study aims to evaluate the feasibility and accuracy of a multistationTIw-SPACE [I] dark blood MRA sequence to assess disease in the aorto-iliac and superficial femoral artery (SFA) (inflow vessels) by comparing it to a multistation ce-MRA protocol with identical spatial resolution.

Methods: Sequences: TIw-SPACE [I] is a highly efficient 3D TSE technique due to its long echo train and very short echo spacing. Blood signal is suppressed in TIw-SPACE by dephasing due to flow in the readout direction. Both TIw-SPACE and ceMRA were acquired with isotropic I $\mathrm{mm}$ resolution. Imaging parameters are listed in Table I.

Imaging: The study was approved by the Institutional Review Board. Two normal volunteers and six patients (with normal kidney function) diagnosed with PAD and symptoms of intermittent claudication were included in the study. Imaging was performed on a $1.5 \mathrm{~T}$ system (MAGNETOM Avanto,

\section{Figure 2 (abstract P24)}

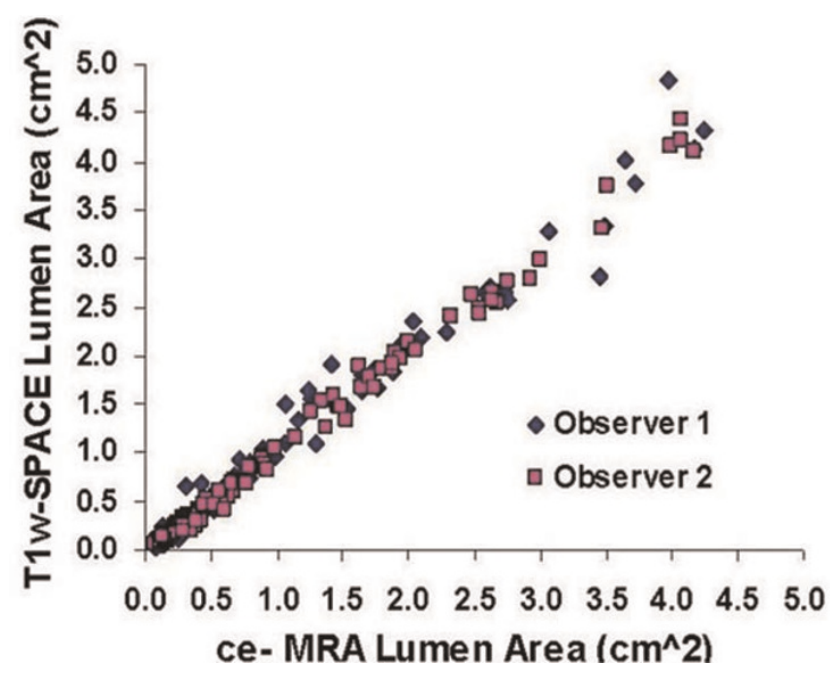

Graph showing the strong correlation between TIw-SPACE and ce-MRA lumen area, and strong inter-observer agreement. 
Table I (abstract P24) Acquisition parameters for TIw-SPACE and ce-MRA

\begin{tabular}{|c|c|c|c|c|c|c|c|c|}
\hline TIw-SPACE & ECG trg. & TR/TE (ms) & Echo train & Slices & Scan time (s) & BW (Hz/pixel) & iPAT & NEX \\
\hline Abd. Aorta & Yes & $\mathrm{RR} / 25$ & 180 & 80 & $14: 30-22: 30$ & 587 & 2 & 3.5 \\
\hline Thighs (SFA) & No & $700 / 23$ & 72 & 60 & I1:30 & 587 & 2 & 1.4 \\
\hline ce-MRA & ECG trg. & TR/TE (ms) & Echo train & Slices & Scan time (s) & BW (Hz/pixel) & iPAT & NEX \\
\hline Abd. aorta & NA & $3 / 1$ & NA & 88 & $0: 14$ & 445 & 3 & NA \\
\hline SFA/Lower legs & NA & $3 / 1$ & NA & 66 & $0: 15$ & 445 & 2 & NA \\
\hline
\end{tabular}

Siemens, Erlangen) using two body matrix coils for the abdominal/pelvic area and a peripheral coil for thighs and lower legs. The imaging protocol included localizers, coronal acquisition of abdominal aorta and SFA using TIw-SPACE, followed by ce-MRA covering the abdomen, thighs and the lower legs.

Analysis: TIw-SPACE and ce-MRA acquisitions for each subject were co-registered (abdominal aorta, and then SFA) on a workstation (FUSION, Siemens Healthcare, Inc.). Three, I mm multiplanar images (with $3 \mathrm{~mm}$ gap) perpendicular to the arteries were created at each of the following locations: celiac, superior mesenteric, renal, halfway between renal and bifurcation, iliac bifurcation, and at both right and left iliac, internal and external iliac, proximal, medial and distal SFA levels. Two experienced observers measured lumen area at each of these 17 locations on both TIw-SPACE and ce-MRA images, as well as the wall thickness area in TIw-SPACE for plaque burden quantification.

Results: Figure I shows an example of TIw-SPACE versus ceMRA images depicting the lumen area and the vessel wall atherosclerotic plaque. Quantitative comparison of lumen areas with ce-MRA and TIw-SPACE revealed strong correlation between the two techniques $(r>0.9)$ and strong inter-observer agreement between the two imaging methods (see Figure 2 ).

Conclusion: TIw SPACE imaging of inflow vessels (aorto-iliac and SFA) is feasible and an attractive alternative to ce-MRA in patients with PAD. The ability to assess atherosclerostic plaque and vascular remodeling is an additional significant improvement over other non-contrast [2] and ce-MRA techniques.

\section{References}

I. Park J, et al: Magn Reson Med 2007, 58(5):982.

2. Miyazaki M, et al: J Magn Reson Imaging 2000, I 2(5):776. and bulk head motion during the long 3D acquisition time. Respiratory movement artefact is controversial [4], but Boussel [5] demonstrated its detrimental effect on carotid wall imaging, using real-time transaxial cines. However, during quiet supine respiration, breathing is predominantly diaphragmatic resulting in the greatest carotid movement in the head-foot direction. We used a novel high temporal resolution interleaved approach to study carotid artery movement in all directions, over the typical 3D scan duration, for a true representation of the potential problem for 3D imaging.

Purpose: This study aimed to measure bulk respiratory and swallowing motion affecting carotid artery imaging and investigates optimal head support for carotid work.

Methods: Nineteen healthy volunteers were scanned at I.5 T (Siemens Avanto) with phased array carotid coils (Machnet). They had high-resolution bSSFP scans of the right carotid artery in the oblique-sagittal plane centered about the bifurcation and in the transverse plane to measure movement in the Head-Foot, Antero-Posterior and Left-Right directions. Each volunteer was scanned twice: with a vac-lok TM fixation pillow (CIVCO Medical Solutions) followed by a standard Siemens foam head support. Subjects were all asked "to lie still and relax" during scans. For 500 cardiac cycles, ECG-triggered diastolic oblique-sagittal and transverse single-shot bSSFP images were alternately acquired (one image/cardiac cycle) with pixel size $0.6 \times 0.6 \times 6 \mathrm{~mm}$, ETL I, flip angle $70^{\circ}$, TR $395 \mathrm{~ms}$, TE $1.6 \mathrm{~ms}$. Image post-processing using $2 \mathrm{D}$-correlation tracked an ROI with MATLAB ${ }^{\circledR}$ (The MathWorks) (figure I). For this, image pixels were 2D-interpolated to $0.31 \times 0.31 \mathrm{~mm}$.

Figure I (abstract P25)

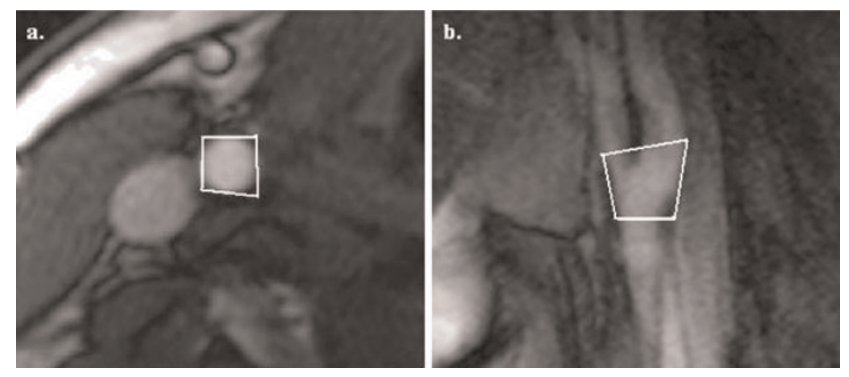

(a) Regions of interest are drawn around the common carotid artery in the transverse plane and (b) in the oblique-sagittal plane allowing for ROI automatic tracking using MATLAB after image acquisition.

\section{P25}

Do we need to consider motion artefacts other than swallowing in carotid artery imaging? Cheuk F Chan, Peter Gatehouse, Dudley J Pennell and David N Firmin

Royal Brompton Hospital, London, UK

Journal of Cardiovascular Magnetic Resonance 2009, I I(SuppI I):P25

Introduction: Recent advances in carotid artery wall imaging have seen a transition from 2D to more efficient 3D imaging [I] without sacrificing image quality [2]. A disadvantage of 3D scans is their longer time to complete the acquisition, making them more susceptible to motion artefacts, particularly swallowing [3] 


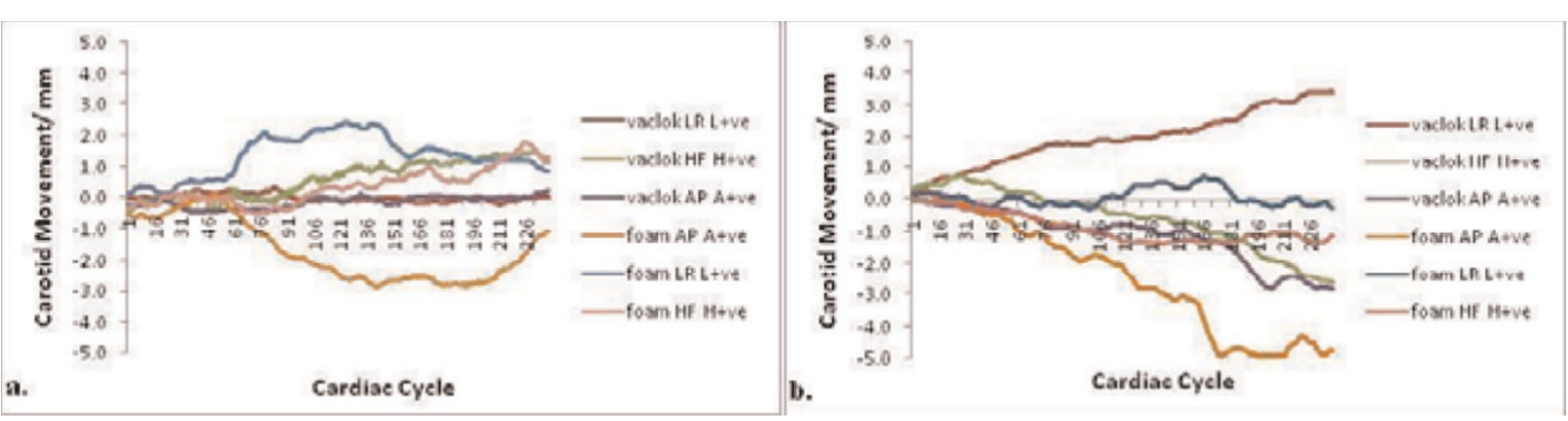

(a) Demonstrates the "drift" phenomenon in all three perpendicular directions with both types of head support after smoothing. The "drift" appears to increase as the scan progresses but then seems to decrease towards the end. This could be due to the individual falling asleep during the scan but becoming more alert towards the end. Whereas, in (b), there is a gradual "drift" away from baseline until the end of the scan. In this example, the phenomenon may be explained by increased muscle tension at the start; individuals are generally tense and anxious prior to the scan but once the scan has commenced, they start to relax and so muscle tension decreases resulting in the observed "drift".

Results: There was no obvious bulk head motion during scans with either pillow. In 30/38 scans, there was "drift" of the carotid artery compared to the baseline reference image with no clear pattern. "Drift" was seen in all three directions with both types of head support (figure 2).

During a swallow event, there was variable movement of carotid artery in all 3 directions. To measure swallowing motion the position measurements were compared between smoothed (20-cycle running average, swallows filtered out) and unsmoothed motion traces. Examining all the scans, the greatest movement was predominantly in the head-foot direction with average movement of $3.5 \mathrm{~mm}$ (SD I.8, range $1.6-6.2 \mathrm{~mm}$ ). LeftRight movement was greater in comparison to Antero-Posterior, $2.8 \mathrm{~mm}$ (SD I.4, range 0.9-6.2 $\mathrm{mm}$ ) and $1.7 \mathrm{~mm}$ (SD I.5, range $0.6-5.1 \mathrm{~mm})$, respectively.

The amount of movement during quiet respiration was calculated between smoothed and unsmoothed traces excluding swallow events. The largest average movement was in the head-foot direction $1.4 \mathrm{~mm}$ (SD 0.6, range $0.6-3.2 \mathrm{~mm}$ ), with substantially less movement in the LR $0.9 \mathrm{~mm}$ (SD 0.5, range 0.3-2.4 mm) and AP $0.5 \mathrm{~mm}$ (SD 0.3, range $0.1-1.1 \mathrm{~mm}$ ).

Although no obvious difference in head motion was measured between the different head supports, individuals reported that the vac-lok ${ }^{\mathrm{TM}}$ pillow was more comfortable and offered greater neck support.

Conclusion: We have shown that respiratory motion previously regarded as insignificant may be large enough to contribute to blurring and ghosting of 3D carotid images. Involuntary head motion even in motivated volunteers during 3D imaging times is clearly an issue for 3D imaging reliability, as is swallowing motion in some subjects. These motion artefacts can corrupt the entire 3D sequence and affect wall imaging and plaque characterisation.

\section{References}

I. Fayad ZA: Magn Reson Med. 200I, 89(4):305-316.

2. Chung YC: Proc ISMRM683.

3. Chan CF: Proceedings ISMRM 2008.

4. Aoki S: AJNR 2000, $21: 381-385$.

5. Boussel L: JMRI 2006, 23:413-4I5.
P26

Aortic pulse wave velocity in obesity as assessed by magnetic resonance imaging Oliver J Rider', Upasana Tayal', Jane M Francis ', Monique Robinson', James P Byrne ${ }^{2}$, Kieran Clarke' and Stefan Neubauer ${ }^{1}$

'University of Oxford, Oxford, UK

${ }^{2}$ Southampton General Hospital, Southampton, UK

Journal of Cardiovascular Magnetic Resonance 2009, I I(SuppI I):P26

Objective: Our aim was to investigate the effects of obesity and weight loss on aortic Pulse Wave Velocity as assessed by vascular magnetic resonance imaging.

Introduction: Obesity is an escalating global health problem associated with both an increased risk of death and an increased risk of cardiovascular events. The various mechanisms by which obesity mediates cardiovascular risk are not well understood but may be related to aortic stiffness, both present in obesity and linked to increased mortality. Our goal was to determine the effect of obesity, in the absence of the traditional cardiovascular risk factors on aortic pulse wave velocity (PWV) as assessed by vascular magnetic resonance imaging, a reliable, reproducible and accurate clinical measure of aortic stiffness.

Methods: Fifty obese (BMI $38.3 \pm 6.8 \mathrm{~kg} / \mathrm{m}^{2}$ ) and eighteen normal weight controls (BMI $22.0 \pm 1.7 \mathrm{~kg} / \mathrm{m}^{2}$ ) with no identifiable cardiovascular risk factors underwent vascular magnetic resonance imaging at I.5 T to assess of PWV at three points, the ascending (Ao) and proximal descending aorta (PDA) at the level of the pulmonary artery and the abdominal aorta (AA) $12 \mathrm{~cm}$ below the pulmonary artery. Aortic flow/time curves, at these three points, were used to calculate the arrival times of aortic pulse waveform. The time of arrival of the pulse wave $(\Delta \mathrm{t}(\mathrm{ms}))$ was determined to be the intercept of the tangent to the curve at the half maximal point of flow. The distance over which the pulse wave had travelled $(\Delta x)$ was measured from oblique sagittal aortic images. PWV was then calculated as $\Delta \mathrm{x} / \Delta \mathrm{t}$. 28 subjects underwent repeat imaging after weight loss, 16 obese subjects underwent a supervised low glycaemic index diet, while twelve underwent bariatric surgery. 


\begin{tabular}{|c|c|c|c|}
\hline & Control $(n=18)$ & Obese $(n=50)$ & p value \\
\hline PWV: Aortic Arch (m/s) & $9.80 \pm 8.26$ & $9.86 \pm 6.26$ & 0.34 \\
\hline PWV: Descending Aorta (m/s) & $4.34 \pm 0.82$ & $5.52 \pm 6.12$ & 0.25 \\
\hline \multirow[t]{2}{*}{ PWV: Ascending Aorta to Abdominal Aorta (m/s) } & $5.0 \pm 0.6$ & $5.7 \pm 1.5$ & 0.02 \\
\hline & Obese Pre Weight Loss( $\mathbf{N}=28)$ & Obese Post Weight Loss $(\mathbf{N}=\mathbf{2 8})$ & p value \\
\hline PWV: Aortic Arch (m/s) & $10.3 \pm 7.2$ & $10.1 \pm 7.7$ & 0.92 \\
\hline PWV: Descending Aorta (m/s) & $6.2 \pm 8.3$ & $4.3 \pm 1.4$ & 0.25 \\
\hline PWV: Ascending Aorta to Abdominal Aorta $(\mathrm{m} / \mathrm{s})$ & $5.8 \pm 2.0$ & $5.0 \pm 1.0$ & 0.03 \\
\hline
\end{tabular}

Results: Obese subjects and normal weight controls were well matched for age, height, systolic blood pressure, fasting plasma glucose and total serum cholesterol. concentration compared to controls $(p<0.005)$. Obesity was associated with a $14 \%$ increase in PWV when compared to the normal weight controls $(p=0.02 I)$ and furthermore was associated with significantly higher leptin $(p<0.00 \mathrm{I})$ and $\mathrm{C}$-reactive protein levels $(p<0.01)$, factors known to affect aortic mechanical function. After a one year period of weight loss (Average $21 \mathrm{~kg}, 50 \%$ of excess weight loss) there was a significant I $4 \%$ decrease in aortic pulse wave velocity when measured between the ascending and abdominal aorta $(p=0.03)$. In contrast, while showing a trend, aortic pulse wave velocity improvements in proximal sections did not reach statistical significance. Both leptin and C-reactive protein levels were also reduced with weight loss. See Table I.

Conclusion: Obesity, in the absence of the traditional risk factors of hypertension, diabetes and hypercholesterolaemia, is associated with significant increases in aortic PWV, a non invasive clinical measure of aortic stiffness known to be independently predictive of cardiovascular mortality. Furthermore, a period of one year of weight loss is associated with a reduction in pulse wave velocity. This may provide a potential explanation for both the link between obesity and increased mortality, and the link between weight loss and increased survival.

\section{P27}

Safety and accuracy of cardiac magnetic resonance imaging combined with low-dose dobutamine stress-testing in patients with congenital heart disease

Saskia E Luijnenburg, Daniëlle Robbers-Visser, Jochem van den Berg, Jolien W Roos-Hesselink, Adriaan Moelker and Willem A Helbing

Erasmus MC, Rotterdam, Netherlands

Journal of Cardiovascular Magnetic Resonance 2009, I I (SuppI I):P27

Objective: to report our experiences with low-dose dobutamine stress cardiovascular magnetic resonance (DCMR) imaging in patients with congenital heart disease (CHD) and to assess the intra-observer and interobserver variability of biventricular function, volumes, and mass.

Background: Stress-testing is an important tool to obtain additional information on ventricular and vascular function in patients with acquired or congenital heart disease. Low-dose
DCMR imaging in small groups of patients with $\mathrm{CHD}$ revealed ventricular and vascular dysfunction that is not apparent at rest. There are no reports in a large series of patients on the safety and accuracy of low-dose DCMR imaging.

Methods: inclusion of all patients who underwent low-dose DCMR in our institution. Acquisition of a short axis set and flow measurements at rest, and during dobutamine administered at $7.5 \mu \mathrm{g} / \mathrm{kg} / \mathrm{min}$ maximum. Intra-observer and interobserver variability (coefficient of variation) was determined for biventricular function, volumes, and mass.

Results: In 9I patients II0 studies were performed (63 male subjects, 54 tetralogy of Fallot, 37 Fontan patients, youngest age at study 6.8 years). In 3 patients minor side effects occurred (vertigo, headache, bigeminy). In 10 patients dobutamine was lowered to $5 \mu \mathrm{g} / \mathrm{kg} / \mathrm{min}$ because of an increase in heart rate of $>150 \%$ baseline, although well tolerated. Intra-observer variability was between 2.3 and $8.7 \%$ for rest and stress measurements. Interobserver variability was between 3.6 and $10.5 \%$ at rest. With stress-testing, the coefficient of variation for biventricular ESV increased significantly to $\geq 15 \%$, while it remained $<10 \%$ for the other variables.

Conclusion: In patients with various types of CHD low-dose DCMR imaging is feasible, safe, and can be performed from 7 years of age. Intra-observer variability is low for rest and stress measurements. With stress-testing, interobserver variability of biventricular ESV increases significantly.

Acknowledgements

Supported by NHF grant 2006B095.

\section{P28}

The right ventricle shows distinct wall motion characteristics in L-TGA vs. D-TGA/atrial switch Michelle Savacool', Philip Kilner", David Sahn ${ }^{3}$, Willem Helbing ${ }^{4}$, Harold Litt ${ }^{5}$, Emanuela Valsangiacomo ${ }^{6}$ and Florence Sheehan'

'University of Washington, Seattle, WA, USA

${ }^{2}$ Royal Brompton Hospital, London, UK

${ }^{3}$ Oregon Health \& Science University, Portland, OR, USA

${ }^{4}$ Erasmus Medical Center, Rotterdam, Netherlands

${ }^{5}$ University of Pennsylvania, Philadelphia, PA, USA

${ }^{6}$ University Children's Hospital, Zurich, Switzerland

Journal of Cardiovascular Magnetic Resonance 2009, I I(SuppI I):P28

Background: Patients whose right ventricles (RV) support the systemic load may develop heart failure. However it is unknown 
Figure I (abstract P28)

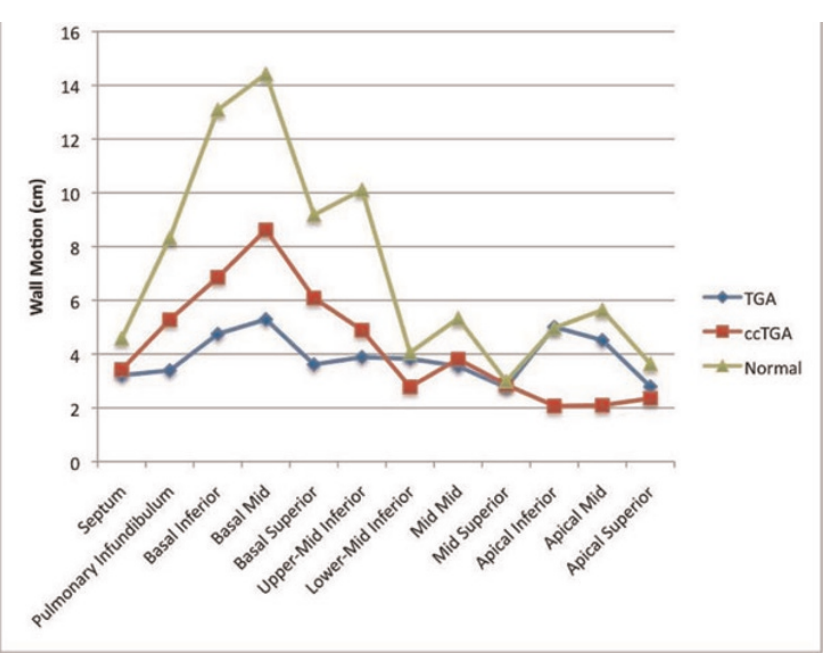

Regional wall motion $(\mathrm{cm})$.

whether the RV remodels similarly in different etiologies of systemic RV.

Methods: RV wall motion and shape were measured from 3D reconstructions generated from magnetic resonance images in 25 patients with transposition of the great arteries after atrial switch (D-TGA), 17 patients with L-TGA and 9 normal subjects. Wall motion was measured in II regions of the RV free wall and septum and normalized by dividing by the square root of body surface area. Regional RV Shape was measured as eccentricity (range from 0 for a line to I for a circle) in 20 short-axis slices of the RV.

Results: Systemic RV patients had severe RV dilatation (end diastolic volume index $137 \pm 37$ vs. $84 \pm 22 \mathrm{ml} / \mathrm{m}^{2}$ in normals, $\mathrm{P}<.00 \mathrm{I}$ ), poor RV function (ejection fraction (EF) $32 \pm 7$ vs. $55 \pm 5 \%, p<.00 \mathrm{I}$ ), and more circular cross sections (eccentricity .83 to .93 vs. below .66 for normals, $p<.001$ for all slices). L-TGA patients had better RV function than DTGA patients (EF $35 \pm 7$ vs. $30 \pm 7 \%, p<.05$ ). Both groups had significantly depressed wall motion in most regions compared to normal. However L-TGA had better wall motion at the base than D-TGA but contracted more poorly at the apex. Figure I.

Conclusion: Systemic RVs are dilated, spherical, and poorly functioning. L-TGA and D-TGA systemic RVs have distinctive wall motion and remodeling patterns. The functional and morphological response to systemic pressure in the RV may differ depending on the anomaly.
P29

Does a selective non-peptide angiotensin II type 2 receptor agonist reduce post-infarction left ventricular remodeling?

Alexander B Jehle, Yaqin Xu, Joseph D DiMaria, Frederick H Epstein, Brent A French, R Jack Roy, Robert M Carey and Christopher M Kramer University of Virginia, Charlottesville, VA, USA

Journal of Cardiovascular Magnetic Resonance 2009, I I(SuppI I):P29

Purpose: Cardiac overexpression of the angiotensin II type 2 receptor $\left(A T_{2}-R\right)$ attenuates left ventricular (LV) remodeling following myocardial infarction (MI). We hypothesized that the use of a novel non-peptide $A T_{2}-R$ agonist Compound $2 \mathrm{I}$ (C2I) would significantly reduce adverse post-Ml remodeling in mice as measured by cardiac magnetic resonance imaging (CMR). Such a compound could offer significant translational potential.

Methods: Forty nine C57/BL6 mice underwent 60-minute occlusion of the left anterior descending artery, followed by reperfusion. Nine died early post-MI, leaving 40 for further study. Twenty three received $0.3 \mathrm{mg} / \mathrm{Kg} /$ day $\mathrm{C} 2 \mathrm{I} \mathrm{SQ}$ by Alzet minipump and 17 were untreated. CMR images were obtained at baseline and post-infarct (days I and 28) using a Clinscan $7 \mathrm{~T}$ MRI system (Siemens/Bruker). Using cine gradient echo black blood imaging, six to 8 continuous short-axis slices were acquired, each I mm thick, from the apex to the base. Images were both electrocardiographic and respiratory gated, with echo time of $1.9 \mathrm{~ms}$, I5 degree flip angle and field of view $25.6 \mathrm{~mm}$, giving a spatial resolution of $200 \times 200 \times 1000 \mu \mathrm{m}^{3}$. The TR was adjusted continuously to obtain 16 equally spaced phases during each cardiac cycle. Three signal averages were used, resulting in an acquisition time of approximately 4 minutes per slice and total imaging time of 30 to 45 minutes per mouse. For infarct sizing on day I post-MI, late gadolinium enhancement was performed using an inversion recovery sequence with an initial 180 degree inversion pulse, TR of 5.4, TE of $0.67 \mathrm{~ms}$, TI $500 \mathrm{~ms}, 20$ degree flip angle and three averages. The FOV was $25.6 \mathrm{~mm}$, with a spatial resolution of $200 \times 200 \times 1000 \mu^{3}$. The ARGUS (Siemens Medical Systems) image analysis program was used to define LV end-diastolic (LVEDV) and systolic volumes (LVESV), stroke volume (SV), ejection fraction (LVEF) and LV mass at baseline and day 28 post-MI as well as infarct size on day I post$\mathrm{MI}$ as \% of total LV mass.

Results: At baseline, LV end-systolic volume, mass and EF were similar between groups. Baseline LV end-diastolic volume in the treated group was larger (mean \pm S.D., $48.6 \pm 12.8$ vs. $39.9 \pm 10.6, p<0.05)$. Infarct size was similar between groups $(4 \mathrm{I} \pm 9$ vs. $38 \pm 13 \%, p=0.32)$. By CMR at day 28 post-MI, enddiastolic volume, end-systolic volume, stroke volume, and LV

Table I (abstract P29) Post-MI day 28 LV parameters

\begin{tabular}{llllll}
\hline & LVEF $(\%)$ & LVEDV $(\mu \mathrm{l})$ & LVESV $(\mu \mathrm{l})$ & SV $(\mu \mathrm{l})$ & MASS $(\mathbf{m g})$ \\
\hline Control $(\mathrm{n}=17)$ & $27.5 \pm 10.5$ & $74.1 \pm 26.7$ & $54.8 \pm 24.8$ & $19.2 \pm 6.4$ & $47.5 \pm 13.9$ \\
C2I $(\mathrm{n}=23)$ & $27.3 \pm 11.8$ & $91.6 \pm 23.8$ & $66.9 \pm 21.5$ & $24.7 \pm 13.3$ & $56.7 \pm 15.2$ \\
P value & 0.98 & $<0.01$ & $<0.05$ & $<0.05$ & $<0.05$ \\
\hline
\end{tabular}


Figure I (abstract P29)

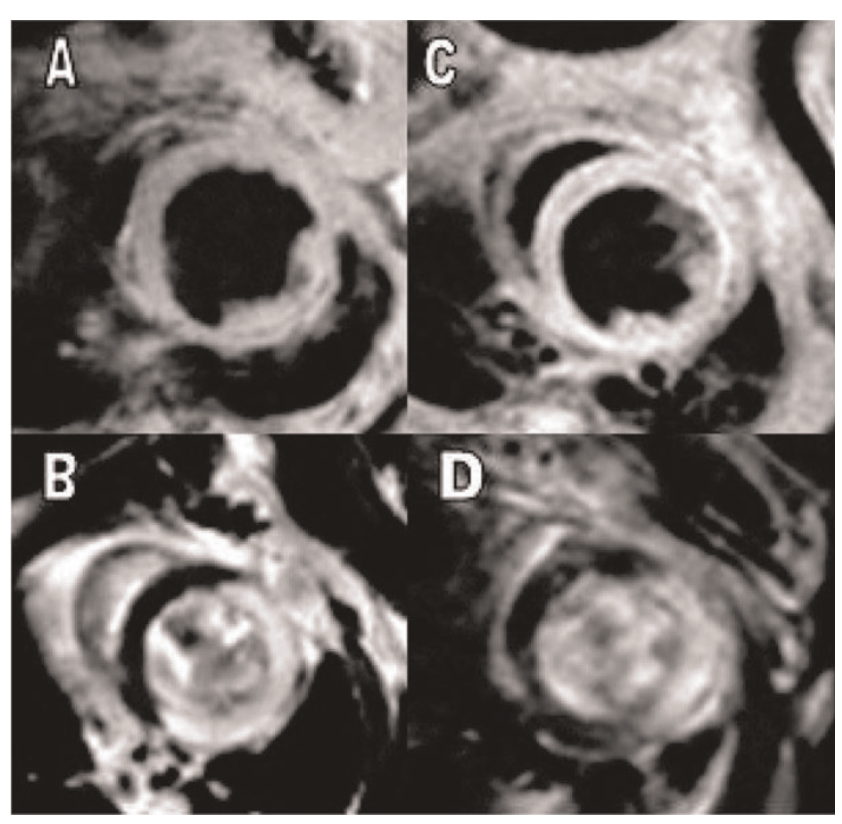

Day 28 end systolic black blood images of C2I (A) and control (C) mice, with corresponding Day I DHE images shown in B and D.

mass were higher in the treated group, although ejection fraction was similar. (See Table I and Figure I.)

Conclusion: Treatment of WT C57BL/6 mice with $0.3 \mathrm{mg} / \mathrm{Kg} /$ day of the novel non-peptide angiotensin II $\mathrm{AT}_{2}$ receptor agonist Compound $\mathbf{2 I}$ in the post-infarction period appears to cause LV hypertrophy and an increase in LV volumes without a decrement in ejection fraction. This finding counters the previously demonstrated protective benefit of transgenic overexpression of the receptor. Potential mechanisms for the lack of protective benefit of the receptor agonist include angiotensin II $\mathrm{AT}_{2}$ receptor down-regulation or an inadequate dose used in this preliminary study.

\section{P30}

Pulmonary vein stenoses are reversible early after plumonary vein isolation in patients with paroxysmal atrial fibrillation - a cardiac mri analysis

Christian Mahnkopf', Guido Ritscher', Nathan Burgon², Troy J Badger ${ }^{2}$, Martin Schmidt', Harald Marschang Klaus Gutleben', Edward DiBella ${ }^{3}$, Nassir F Marrouche ${ }^{2}$, Johannes Brachmann' and Anil M Sinha'

${ }^{I}$ Klinikum Coburg, Coburg, Germany

${ }^{2}$ University of Utah School of Medicine, Salt Lake City, UT, USA

${ }^{3}$ Utah Center for Advanced Imaging Research, Salt Lake City, UT, USA

Journal of Cardiovascular Magnetic Resonance 2009, I I (SuppI I):P30

Purpose: To evaluate pulmonary vein diameter and blood flow in patients with PVI using CMR.

Background: Pulmonary vein isolation (PVI) has become a efficient therapie in patients with paroxysmal atrial fibrillation $(\mathrm{AF})$. A serious complication of $\mathrm{PVI}$ is the pulmonary vein
Figure I (abstract P30)

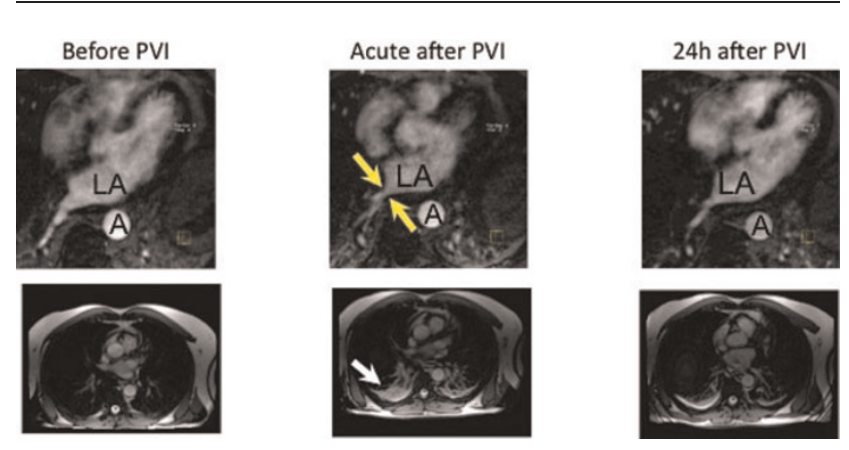

LA: left atrium; A: aorta.

stenosis, which is mostly diagnosed several weeks after intervention. The cardiac magnetic resonance tomography (CMR) allows analysis of heart function and pulmonary veins after ablation therapy.

Methods: Patients with paroxysmal AF were scheduled for CMR (Siemens Espree I.5 T, Siemens, Germany) before, immediately after, and 24 hours after PVI. For pulmonary vein diameter and blood flow analysis, angiography of the pulmonary veins and flow measurements of the right inferior pulmonary vein (RIPV) were performed. Maximum intensity projections (MIP) were created for offline analysis.

Results: 15 patients (9 male, $63 \pm 9$ years) were included in the study. RIPV diameter signifcantly decreased (I I.7 $\pm 1.8 \mathrm{~mm}$ vs $9.1 \pm 2.4 \mathrm{~mm},-22,2 \%, \mathrm{p}<0.05)$ during acute, and increased (I I. $2 \pm 2.7 \mathrm{~mm},+18,8 \%, \mathrm{p}<0.05)$ during $24 \mathrm{~h}$ measurements as compared to acute values. RIPV blood flow significantly increased $(34.3 \pm 14.0$ vs. $42.5 \pm 14.0 \mathrm{~cm} / \mathrm{s} ;+35 \% ; p<0.05)$ during acute, and slightly decreased $(40.7 \pm 13.9 ; p=n . s$. $)$ during $24 \mathrm{~h}$ measurements. Also, pulmonary congestion and pleural effusion, which occurred acutely after PVI, were recurrent after $24 \mathrm{~h}$. Figure I shows an example of CMR pre, acutely, and $24 \mathrm{~h}$ post PVI; arrows indicate pleural effusion (lower row), or RIPV stenosis (upper row).

Summary: Patients with paroxysmal atrial fibrillation might suffer from pulmonary vein stenosis, pulmonary congestion and pleural effusion early after PVI. As symptoms were regressive within 24 hours after ablation, edema of the vessel tissue might be one the main reasons for the deterioration. Further studies are needed to specify these findings.

\section{P3I}

Real-time free-breathing strategy for tracking labeled cells with in-vivo vardiac MRI Yijen LWu', Cornelius Brinegar ${ }^{2}$, Zhi-Pei Liang ${ }^{2}$, Qing Ye', T Kevin Hitchens', Lesley M Foley' and Chien Ho'

'Pittsburgh NMR Center for Biomedical Research, Carnegie Mellon University, Pittsburgh, PA, USA

${ }^{2}$ Electric and Computer Engineering, University of Illinois at Urbana-Champaign, Urbana-Champaign, IL, USA

Journal of Cardiovascular Magnetic Resonance 2009, I I(SuppI I):P3 I

Introduction: Non-invasive in vivo cellular imaging with MRI has greatly broadened our understanding in many cellular and molecular processes, as well as in monitoring stem cell therapy. 
Figure I (abstract P3I)

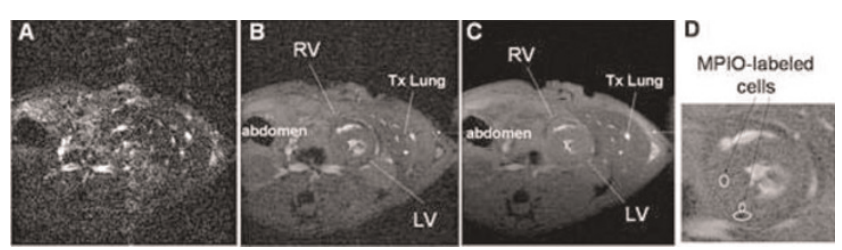

(A) Fourier reconstruction of free-breathing MRI. (B) Model-based reconstruction of the same free-breathing MRI. (C) ECG-and-respirationgated cine imaging for comparison. (D) Enlarged view of the heart showing the infiltrated MPIO-labeled cells.

The target cells can be made MRI-detectable by labeling them with iron-oxide contrast agents, such as micrometer-sized-ironoxide (MPIO) particles. With sufficient iron loading per cell, it is possible to detect single cells in vivo with MRI. However, motion artifacts and the need for gated acquisition have made imaging cells in the heart more challenging and time-consuming. The goal of this study is to develop a strategy to enable real-time imaging of individual labeled cells without the need for a gated or breathhold protocol. To this end, we have implemented a new modelbased imaging scheme for high-resolution free-breathing imaging, using the Partially Separable Function (PSF) model.

Methods: I. Model system: We use a rodent abdominal heterotopic working heart and lung transplantation model with DA to $\mathrm{BN}$ rat pair, using $\mathrm{BN}$-to-BN transplantation as isograft controls. 2. Iron oxide particle labeling: Immune cells, mostly macrophages, were labeled in vivo by direct intravenous injection of $3 \mathrm{mg}$ micrometer-sized-iron-oxide (MPIO) particles I day prior to MRI scans.

3. MRI protocols: Real-time free-breathing imaging, and ECG and respiration gated $\mathrm{T}_{2} *$ and cine imaging were performed using a Bruker AVANCE 4.7-T system with a home-built $5.5 \mathrm{~cm}$ surface coil and an in-plane resolution of $195 \mu \mathrm{m}$.

4. Partially Separable Function (PSF) method and theory: The PSF model approximates the hypersurface, $d(k, t)$, formed by the time varying data as a summation of $M$ simpler functions that are each the product of one-dimensional functions $\alpha_{m}(k)$ and $\emptyset_{m}(t)$, and recent results have shown that cardiac imaging with breathing exists in a low rank space where $M=16$. Data collection consists of interleaving two scans: (i) a high temporal resolution data set for estimating $\left\{\sigma_{m}(t)\right\}^{M}{ }_{m}=1$ and (ii) a high spatial resolution scan used to estimate $\left\{\alpha_{m}(k)\right\}_{m=1}^{M}$. The low rank of the model allows for temporal interpolation over time intervals failing the Nyquist criterion thereby avoiding the need to time data collection with the cardiac and respiratory motions.

Results: We have used a rodent heterotopic transplantation model for tracking labeled immune cells in vivo. In this model, immune cells, mostly macrophages, can be labeled in vivo by direct intravenous administration of MPIO particles; labeled immune cells then migrate to the rejecting transplanted hearts and are detected by $\mathrm{T}_{2}{ }^{*}$-weighted imaging. Low-dosage MPIO particles are used in this study for sparse labeling of macrophages to promote the visualization of single cells, even though the images lack the necessary spatial resolution by at least a factor of 4 .

Without gating, real-time free-breathing MRI results in blurring due to motion artifacts (Fig. IA). The model-based data collection and reconstruction scheme (Fig. IB) yields a high- quality image comparable to ECG-and-respiration-gated cine imaging (Fig IC), however in a much shorter acquisition time. The model-based method enables identification of MPIO-labeled cells in vivo (Fig. ID) in real-time data acquisition. The modelbased method acquires data continuously whereas the gated scheme collects data intermittently, depending upon the relationship of the ECG and respiratory signals. In addition to a shorter acquisition time, the continuous model-based scheme preserves all information in the $k$ - $t$ space, which is especially important in functional evaluation of the diseased conditions with arrhythmia or compromised breath-holding.

Conclusion: The model-based imaging scheme allows realtime free-breathing imaging with resolution and image quality sufficient for tracking labeled cells in vivo in beating hearts.

\section{References}

I. Magn Reson Med 2006, 55:242-249.

2. IEEE International Symposium on Bioimaging 2007, 988-99I.

3. Proc Natl Acad Sci 2006, 103:1852-1857.

4. Brinegar, et al: Engineering in Medicine and Biology Conference 2008.

\section{P32}

Assessment of myocardial oxygenation changes in the presence of coronary artery stenosis with three dimensional cardiac phase-resolved SSFP BOLD imaging in canines Xiangzhi Zhou', Sven Zuehlsdorff', Saurabh Shah" ${ }^{2}$, Richard Tang', Rachel Klein', Debiao Li' and Rohan Dharmakumar'

'Department of Radiology, Northwestern University, Chicago, IL, USA

${ }^{2}$ Siemens MED US, Chicago, IL, USA

Journal of Cardiovascular Magnetic Resonance 2009, I I(SuppI I):P32

Introduction: Recent studies have shown that regional myocardial oxygenation changes secondary to coronary artery

\section{Figure I (abstract P32)}

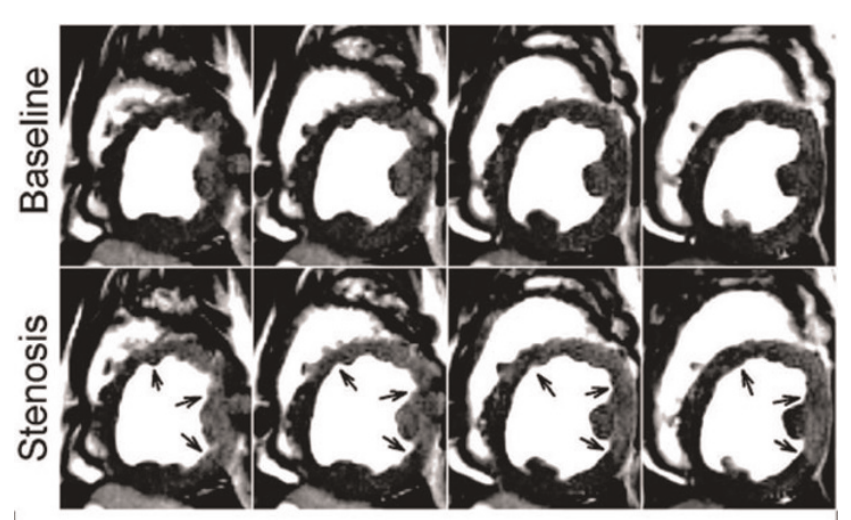

A representative set of end-diastolic 3D SSFP BOLD images (4 contiguous slices from apex to base (obtained from a dog under baseline condition (upper row) and with moderate stenosis (lower row) under adenomise stress is shown. Note the signal enhancement observed in the non-LCX supplying territories (arrows) uder stress, while no signal enhancement is observed within the LCX supplying region in the presence of adenosine. 
Figure 2 (abstract P32)

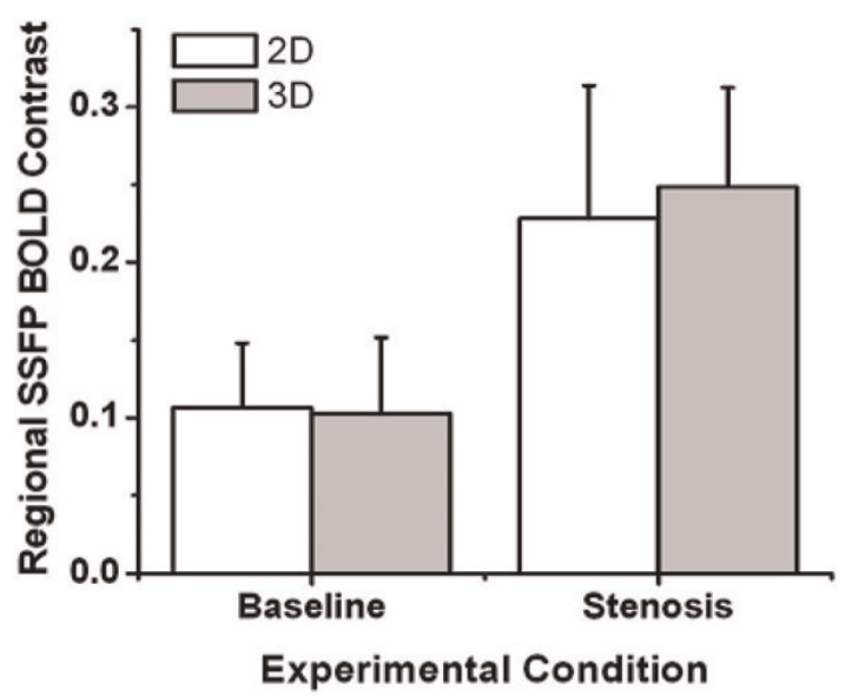

Regional 2D and 3D SSFP BOLD signal contrast between LAD and LCX regions under baseline conditions and steosis (with adenosine). There were no significant differences in regional signal intensity variations at baseline and under stress (with stenosis) between $2 D$ and 3D images. However, statistically significant differences were found for regional SSFP BOLD contrast between LAD and LCX territories in the presence of stenosis compated to baseline conditions with both $2 \mathrm{D}$ and $3 \mathrm{D}$ approaches. $(\mathrm{P}<0.0 \mathrm{I})$.

stenosis may be detected with $2 \mathrm{D}$ steady-state free precession (SSFP) blood-oxygen level-dependent(BOLD) imaging. One potential limitation of the 2D SSFP BOLD approach is the disruption of steady-state myocardial signal due to through-plane cardiac motion in the presence of slice-selective excitation. A consequence of breaking the dynamic steady state of SSFP BOLD signal is that it may lead to a reduction in myocardial oxygen sensitivity.

Purpose: To investigate whether there are any observable differences in myocardial BOLD contrast between 2D and 3D approaches using a canine model with adjustable coronary artery stenosis

Methods: Four dogs were operated and studied under institutional approval. For each dog, following thoracotomy, a hydraulic occluder was placed around the left circumflex coronary artery (LCX) to induce reversible LCX stenoses. A Doppler flow probe was placed distal to the LCX occluder to assess the fidelity of the occlusion during the MRI studies. Following recovery (I week), animals were sedated, ventilated and placed on the scanner table (I.5 T Siemens Espree). ECGgated and multiple breath-held $2 \mathrm{D}$ and $3 \mathrm{D}$ cine SSFP sequences were prescribed under basal and adenosine stress with and without LCX stenosis over the LV. Typically 2-3 stenosis levels (on the basis of Doppler flow) were assessed and each animal was studied 2-3 times. Scan parameters for 2D acquisitions: inplane resolution $=1.2 \times 1.2 \mathrm{~mm}^{2}$, slice thickness $=5 \mathrm{~mm}$, iPAT factor $=2$, TR/TE $=4.7 / 2.35 \mathrm{~ms}$, flip Angle $=70^{\circ} ; 3 \mathrm{D}$ acquisitions were the same, except for slab thickness $=30 \mathrm{~mm}$ (6 partitions). Both $2 \mathrm{D}$ ( 3 slices, $5 \mathrm{~mm}$ gap) and 3D short-axis images were acquired with center slice positioned over mid leftventricle. TR and segments/cardiac phase were adjusted to achieve the optimal temporal resolution ( $10 \mathrm{~ms}$ to $20 \mathrm{~ms}$ ) and minimize motion and flow artifacts in $2 D$ and $3 D$ acquisitions, while maximizing oxygen sensitivity.

Data analysis: Regional SSFP BOLD Contrast, defined as $\left[I_{L A D}-I_{L C X}\right] / I_{L A D}$, where $I_{L A D}$ and $I_{L C X}$ are average SSFP signal intensity of region $L A D$ and $L C X$, respectively, was used to evaluate the SSFP BOLD images at end diastole. Contrast values computed from $2 D$ and $3 D$ acquisitions were averaged separately for each study under basal and stenotic conditions. Two sample ttests were performed to test whether $(I)$ there were regional signal differences between basal and stenotic conditions for 3D BOLD SSFP and (2) to test where there were differences in regional SSFP BOLD Contrast between $2 D$ and $3 D$ acquisitions under stenotic conditions. Statistical significance was set at $\mathrm{P}<0.01$.

Results: Figure I shows a representative set of end diastolic 3D SSFP BOLD images (4 contiguous slices from apex to base) obtained from a dog under basal condition (upper row) and with moderate stenosis under adenosine stress (lower row). Note that under adenosine stress, the signal intensities within myocardial territories not supplied by the LCX enhance while that supplied by LCX remain isointense with the rest images. Results from quantitative analysis are shown in Figure 2. No statistically significant regional signal differences were found between $2 D$ and $3 D$ acquisition under baseline or LCX stenosis under adenosine stress $(p>0.2)$. There were statistically significant differences between baseline and LCX stenosis under adenosine stress for both 2D and $3 D$ acquisitions.

Conclusion: While 2D SSFP BOLD have shown promising results, reduction in oxygen sensitivity due to through-plane motion was a notable potential limitation. Results from this study showed that oxygen sensitivity between $2 D$ and $3 D$ acquisitions are not significantly different, demonstrating that through-plane motion does not significantly alter myocardial SSFP BOLD contrast. Further extension of 3D cine BOLD imaging with improved artifact reduction permitting the use of higher TR for improved oxygen sensitivity, faster data acquisition strategies, and free-breathing approaches may be necessary prior to successful clinical translation of 3D SSFP BOLD MRI.

\section{P33}

Novel technique of strain assessment utilizing feature tracking in nontagged SSFP images: validation with tagged strain analysis Kan N Hor', Erin Wash', Robert J Fleck', Janaka P Wansapura', James F Cnota', D Woodrow Benson', William M Gottliebson' and Wojciech Mazur ${ }^{2}$

${ }^{I} \mathrm{CCHMC}$, Cincinnati, $\mathrm{OH}$, USA

${ }^{2}$ Ohio Heart and Vascular Center, Christ Hospital, Cincinnati, $\mathrm{OH}$, USA

Journal of Cardiovascular Magnetic Resonance 2009, I I(SuppI I):P33

Introduction: Recent cardiac MRI (CMR) studies have demonstrated decline in left ventricular peak circumferential strain $\left(\varepsilon_{\mathrm{cc}}\right)$ despite normal ejection fraction in Duchenne muscular dystrophy (DMD) patients. However, these analyses used CMR tagging, a technique limited by tag fading and complicated analysis requirements. Feature tracking software has recently been 
Spearmen Correlation Coefficient $=0.812(\mathrm{p}<0.01)$

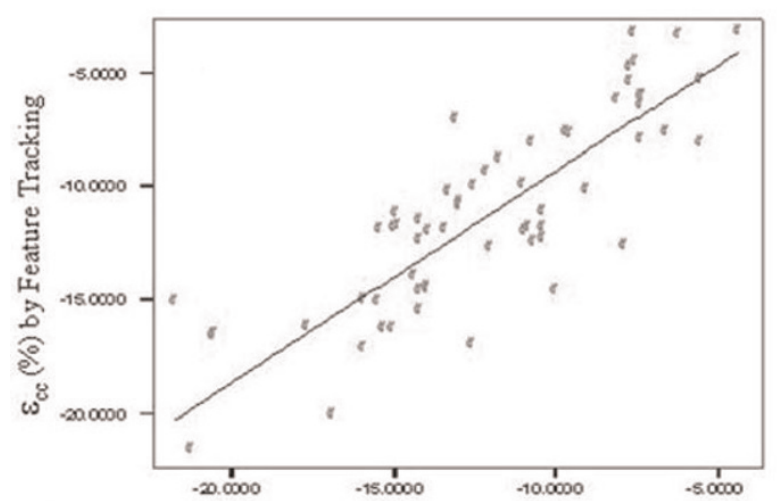

A

$$
\varepsilon_{c c}(\%) \text { by Tagged Analysis }
$$

Tagged $\varepsilon_{\mathrm{ce}} \mathrm{v} s$ Feature Tracking CMR $\varepsilon_{\mathrm{ce}}$

Bland-Altman Comparison Method

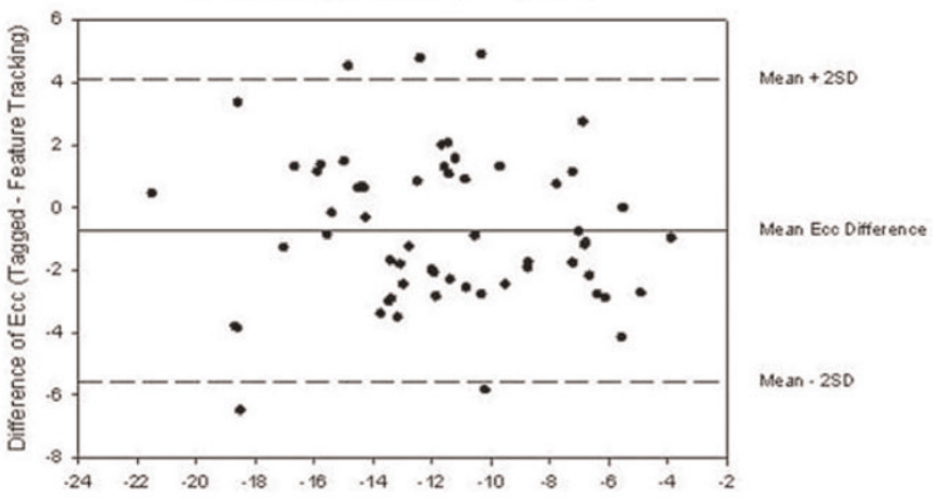

B developed for analysis of $\varepsilon_{\mathrm{cc}}$ from non-tagged standard steadystate free precession (SSFP) cine CMR images.

Purpose: The purpose of this study was to compare Mid-LV slice $\varepsilon_{\mathrm{cc}}$ by feature tracking of SSFP cine CMR images to HARP analysis of tagged images.

Methods: $\varepsilon_{\mathrm{cc}}$ was assessed from CMR SSFP short-axis cine stack images and cine myocardial tagged image data of 54 DMD patients and 6 aged-matched control subjects, utilizing both tagged analysis (via HARP ${ }^{\circledR}$ software, Diagnosoft Inc) and SSFP feature tracking (via DIOGENES ${ }^{\circledR}$ software, TomTec Inc) methods. Analyses were performed on identical location midpapillary LV slices (both tagged and standard SSFP). Average $\varepsilon_{\mathrm{cc}}$ was tabulated and compared via Spearmen rank correlation and Bland-Altman comparison of methods.

Results: Feature tracking $\varepsilon_{\mathrm{cc}}$ analysis correlated favorably with tagged HARP analysis (fig Ia). In addition, the techniques do not demonstrate systematic over or underestimation of one another, though the limits of agreement are relatively wide (fig Ib).

Conclusion: CMR feature tracking is a feasible method for assessment of $\varepsilon_{\mathrm{cc}}$. Further study on larger subject groups is warranted to determine efficacy and accuracy of feature tracking versus tagged analysis.

\section{P34}

Successful integration of MRI derived scar distribution during VT ablation procedures. Initial experience in 12 patients

Rob J van der Geest, Adrianus P Wijnmaalen, Lucia JM Kroft, Hans-Marc J Siebelink, Jeroen J Bax, Albert de Roos, Martin J Schalij, Johan HC Reiber and Katja Zeppenfeld Leiden University Medical Center, Leiden, Netherlands Journal of Cardiovascular Magnetic Resonance 2009, I I (SuppI I):P34

Introduction: Substrate based ventricular tachycardia (VT) ablation requires extensive electroanatomical voltage mapping (EAM) to delineate the myocardial scar and its border zone.

\section{Figure I (abstract P34)}

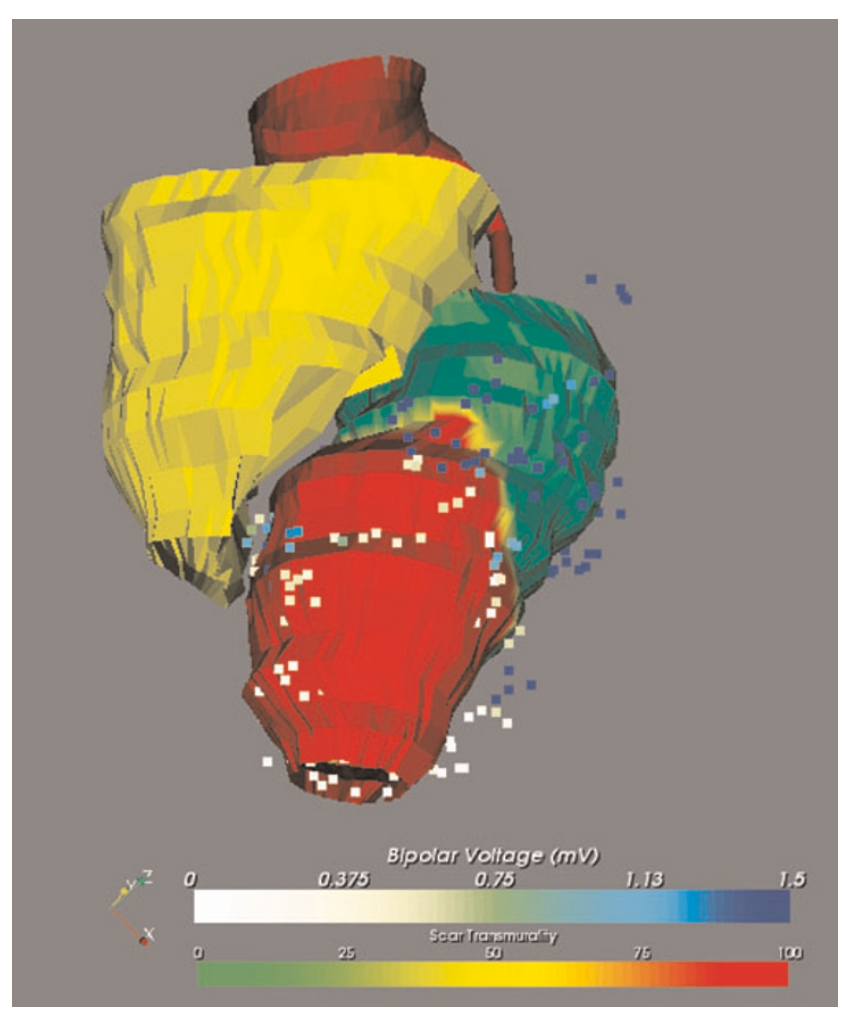

MRI derived surface mesh depicting the LV endocardial (color coded), $\mathrm{RV}$ endocardial (in yellow), aorta (in red) and RCA (in red) anatomy. Coloring of the LV endocardial surface is used to indicate the transmural extent of scar in the endocardial layer of the myocardium (green $=0 \%$, red $=100 \%$ ). The square dots indicate the mapping locations obtained during the VT ablation procedure. Color coding is used to indicate the bipolar electrogram amplitude (white $\leq 0 \mathrm{mV}$, dark blue $\geq 1.5 \mathrm{mV}$ ). 
Figure 2 (abstract P34)

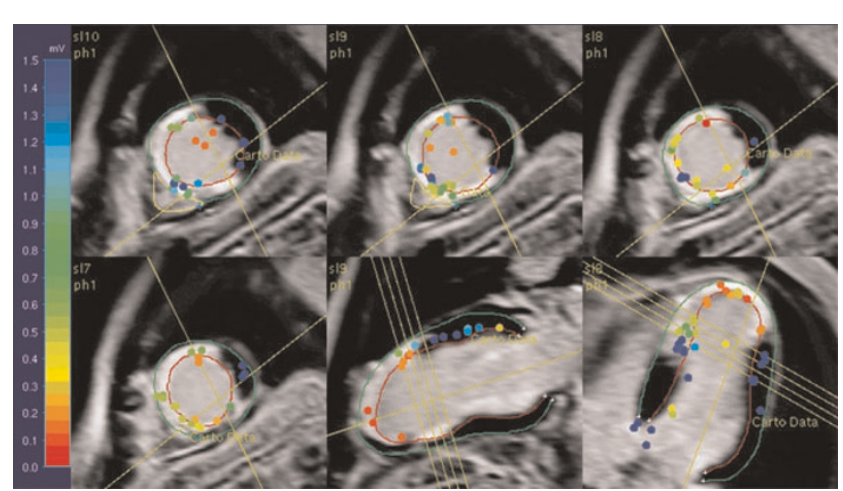

Late enhancement MRI of the same patient showing transmural scar in the septal-apical region with results of EAM superimposed. Bipolar voltages are low $(<1.5 \mathrm{mV})$ in the scar region and normal $(>1.5 \mathrm{mV})$ in the healthy myocardium.

Integration of three-dimensional (3D) information from late enhancement (LE) MRI may be feasible and may provide additional information on VT substrate mapping. This study was conducted to test the feasibility of merging 3D MR derived scar information during the VT ablation procedure.

Methods: In 12 patients (all men; age $64 \pm 10$ ) referred for catheter ablation of VT late after myocardial infarction CMR was performed 2-3 days prior to VTablation. LE MR was performed in multiple slices covering the complete LV in short-axis (slice thickness $10 \mathrm{~mm}, 5 \mathrm{~mm}$ overlap) and long-axis views (two-chamber, four-chamber views, slice thickness $12 \mathrm{~mm}, 6 \mathrm{~mm}$ overlap). In addition, a black-blood imaging sequence was used to image the proximal aorta and the origin of the left main (LM) and right coronary artery (RCA). In the short-axis LE scan, endocardial and epicardial boundaries were derived semi-automatically. The myocardium was divided in an endocardial and epicardial layer and for each layer, at each location, the extent of scar was computed. Subsequently, 3D endocardial and epicardial surface meshes were constructed. Mesh points were color coded based on the extent of scar at the respective myocardial location and layer. In addition, 3D meshes were constructed of the ascending aorta and origin of the LM and RCA.

Prior to VT ablation the generated meshes were imported into the 3D mapping system (CARTO, research version). Based on the $3 D$ catheter tracking capabilities of this system, the MRI derived meshes were registered with the endocardial EAM using the LM position and the endocardial surface as landmarks. After the procedure, 3D mapping point positions and the corresponding bipolar electrogram voltages were saved and superimposed on the original LE MRI data for comparison. Presence of scar from EAM was based on a cut off value of $1.5 \mathrm{mV}$.

Results: Average LV EF was $43 \pm 10 \%$; average MRI derived scar size was II \pm I I\% of the LV myocardium. Integration of MRI derived scar information with EAM was successful in all cases. The average number of endocardial mapping points was $264 \pm 37$. The mean registration error between the MRI derived endocardial surface and EAM was $4.4 \pm 3.0 \mathrm{~mm}$. In all patients, regions with transmural scar as seen on $M R I$, were also identified by EAM (bipolar electrogram amplitude $<1.5 \mathrm{mV}$ ). In the majority of regions without scar, bipolar voltages were $>1.5 \mathrm{mV}$. However, in regions with non-transmural scar as derived by MRI, EAM derived bipolar voltages were either $<1.5 \mathrm{mV}$ or $>1.5 \mathrm{mV}$. Figures I and 2 .

Conclusion: It was demonstrated that integration of MRI derived scar information into a 3D mapping system is feasible. The observed registration errors are small compared to the expected amount of cardiac and respiratory motion and the slice thickness of the LE MRI acquisition. The additional information provided by MRI, especially in regions of non-transmural scar, may be helpful to assess the anatomical substrate of VT and to improve the efficiency and outcome of VT ablation procedures.

\section{P35}

\section{Comparison of coronary endothelial function and brachial flow mediated vasodilatation using cardiac magnetic resonance imaging Dipti Gupta, Lynette Duncanson, Danielle Janosevic, William Schapiro, Jing Han, Katherine McGrath and Nathaniel Reichek St. Francis Hospital, Roslyn, NY, USA}

Journal of Cardiovascular Magnetic Resonance 2009, I I(SuppI I):P35

Introduction: Brachial artery flow-mediated dilatation (FMD) is used as a surrogate for invasive assessment of coronary artery endothelial function, concordance with invasive assessment of coronary endothelial function is controversial.

Purpose: We assessed both brachial FMD and coronary CPT noninvasively at the same sitting using cardiac MRI (CMR) and compared the two in a population with and without coronary risk factors.

Methods: Endothelium-dependent vasodilator function was assessed in the brachial and coronary arteries in 39 subjects. Using a Siemens I.5 T scanner, the left anterior descending (LAD) coronary artery was localized using a navigator angiographic sequence and a proximal cross-section imaged using TrueFISP bright blood imaging and T2 weighted turbo spin echo imaging. Images were obtained at rest and during cold pressor testing(CPT) and evaluated in ARGUS (Siemens). The \% change in lumen cross-sectional area in mid diastole was determined. FMD was assessed by inflating a blood pressure cuff to a reading $50 \mathrm{mmHg}$ higher than the subject's resting systolic pressure, and deflating after I min. Imaging was done using TrueFISP cine bright blood imaging at rest, inflation and at 5 sequential time points during deflation. Images were evaluated in ARGUS (Siemens). The $\%$ change in lumen cross-sectional area pre and post inflation was determined.

Results: Of 39 subjects, 16 were females and the mean age was $52 \pm 8$ yrs. Coronary risk factors (hypertension, hyperlipidemia, smoking, diabetes) were present in 19 while 20 had no risk factors.

The mean brachial FMD of the pooled cohort was 13\% and the mean coronary response to CPT was $16 \%$. There was no significant relationship between the brachial FMD and coronary CPT response $(r=0.03, P=0.8)$.

Conclusion: When determined with similar noninvasive methods at the same sitting, brachial FMD does not correlate closely with coronary endothelial response to CPT in a population with and without coronary risk factors. Use of brachial FMD as a surrogate for coronary endothelial function should be reconsidered. 
P36

The evaluation of right and left ventricular morphology by CMR with comparison to recipient heart after heart transplant: a surgical perspective Nicholas Farber, Mark Doyle, Ronald B Williams, June A Yamrozik, Perry Bruno, George J Magovern, James A Magovern, Srinivas Murali

and Robert WW Biederman

Allegheny General Hospital, The Gerald McGinnis Cardiovascular Institute, Pittsburgh, PA, USA

Journal of Cardiovascular Magnetic Resonance 2009, I I(SuppI I):P36

Introduction: Cardiovascular MRI (CMR) is considered the "gold standard" for non-invasive left and right ventricular mass quantitation. To our knowledge, this information is only based on animal and phantom data and has never been prospectively or retrospectively validated in humans undermining the credibility of the 'gold standard'. This issue is particularly important for the right ventricle having complex geometry ill suited for mathematical modeling, placing increased importance on accurate mass quantitation. The surest way to validate the accuracy and thus the true gold standard of CMR derived mass is through autopsy.

Purpose: To establish a correlation between CMR derived ventricular mass and autopsy mass of ex vivo hearts from heart transplants.

Methods: Over a 10-week period, five ex vivo hearts donor hearts were obtained immediately upon orthotopic heart transplantation from the operating room. They were quickly cleaned and suspended in a saline-filled container and scanned using a steady state free precession (SSFP) sequence and 2D short-axis slices to measure the CMR defined weight ( $g$ ), (GE I.5 T, Excite. Milwaukee, WI). Semiautomated endocardial and epicardial contouring was performed via Mass Plus, Medis (The Leiden, The Netherlands). The donor hearts were then dissected to shave the atria off at the atrioventricular plane and ventricles separated at the interventricular septum. The actual weight of the LV and RV was measured via high-fidelity scale giving the true mass of each ventricle for comparison with the weighed mass.

Results: The CMR measured LV mass (mean $=329.2 \mathrm{~g}$, $\mathrm{SD}=78.9 \mathrm{~g})$ significantly predicted the actual measured LV mass $($ mean $=329.4 \mathrm{~g}, \mathrm{SD}=83.7 \mathrm{~g}), \mathrm{P}=\mathrm{NS}$. The Pearson product-moment correlation for this sample was $0.94(p=0.02)$. The CMR measured RV mass (mean $=151.0 \mathrm{~g}, S D=50.5 \mathrm{~g}$ ) significantly predicted the actual measured RV mass (mean = $126.8 \mathrm{~g}$, SD = $44.6 \mathrm{~g}$ ). The Pearson product-moment correlation for this sample was $0.92(p=0.03)$. The CMR measured $L V+R V$ mass $($ mean $=240.1 \mathrm{~g}$, SD $=112.8 \mathrm{~g}$ ) significantly predicted the actual measured LV + RV mass (mean $=228.1 \mathrm{~g}, \mathrm{SD}=124.1 \mathrm{~g}$ ). The Pearson product-moment correlation for this sample was $0.98(\mathrm{p}<0.00 \mathrm{I})$.

Conclusion: CMR accurately determined both left and right ventricular masses as compared to weighed explanted hearts, despite variable surgical removal of instrumentation (LVAD/ RVAD and AICD's) in the majority and the complexities of the right ventricle in all. To our knowledge, albeit a small sample size, it represents a 'first' in human CMR vs. autopsy comparison, similar to the intrepid days of the initial validation of echocardiography vs. autopsy report by Reichek and Devereux in 1976. Future work will include sampling of additional ex vivo hearts and a correlation between in vivo CMR derived cardiac mass pre-heart transplant and following explantation.
P37

Correlation between velocity encoded cine magnetic resonance imaging and Doppler echocardiography for the evaluation of diastolic dysfunction

ljaz Ahmad', Mitra Sahebazamai', Lesan Banko', Joshua A Sockolow', Igor Klem², Terrence Sacchi' and John F Heitner'

${ }^{\prime}$ New York Methodist Hospital, Brooklyn, NY, USA

${ }^{2}$ Duke University Medical Center, Durham, NC, USA

Journal of Cardiovascular Magnetic Resonance 2009, I I(SuppI I):P37

Background: Doppler echocardiographic (Echo) measurements of the mitral and pulmonary venous flow have been used to assess diastolic dysfunction (DD). Velocity-encoded cardiac magnetic resonance (Ve-CMR) can measure similar parameters and may potentially be used for the assessment of DD.

Objective: To assess the correlation of Ve-CMR with Echo in assessing mitral inflow E/A ratio, left atrial (LA) size, and pulmonary vein (PV) flow.

Methods: We prospectively enrolled 22 outpatients with a mitral inflow pattern by Echo that was normal (8), impaired relaxation (7), pseudo-normal (2), or restrictive (5). The mean left ventricular ejection fraction (LVEF) was 60.8 and two patients had left ventricular hypertrophy on echo. Ve-CMR was performed within 2 hours of the Echo. The following indices were measured: LA size, mitral inflow E-velocity (E), A-velocity (A), E/A ratio, $P V$ waves (S, D and $A$ ).

Results: The mean age of the patients was 51 years. The average Echo mitral inflow absolute velocities were close to double the Ve-CMR \{Echo E-velocity $=85.8 \mathrm{~cm} / \mathrm{sec}$ vs. Ve-CMR E velocity $=41.4 \mathrm{~cm} / \mathrm{sec}$, Echo A-velocity $70 \mathrm{~cm} / \mathrm{sec}$ vs. Ve-CMR

Figure I (abstract P37)

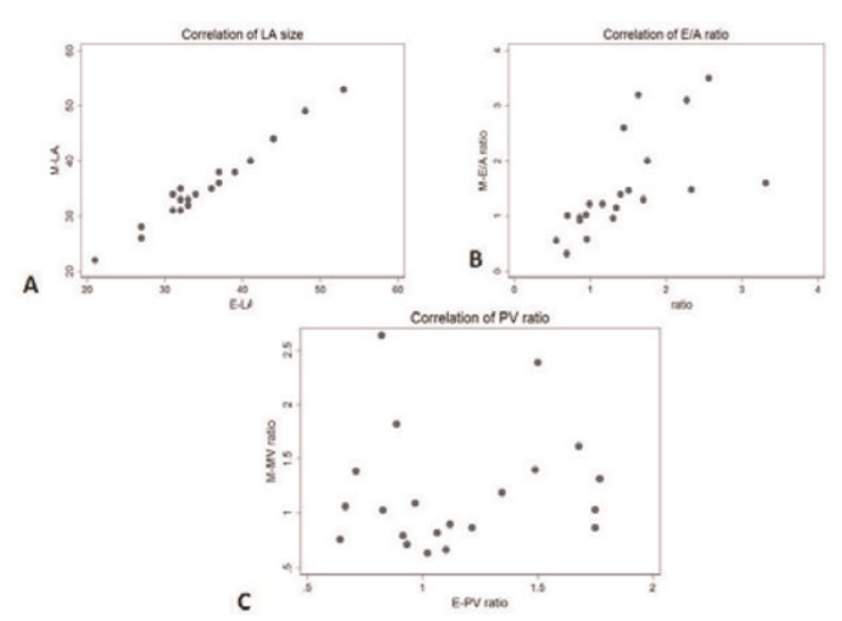

LA; left atrium, PV, pulmonary vein

Correlation between Ve-CMR and Doppler echo derived values was tested using Spearman's rank correlation coefficients. There was significant correlation between left atrial size and E/A ratio. A: LA size $(R=0.9$, $p$-value $<0.05)$, B: E/A ratio $(R=0.88$, $p$-value $<0.05)$, but not C: $(R=0.12, P=0.58)$. LA; left atrium, $P V$, pulmonary vein. 
A-velocity $32.5 \mathrm{~cm} / \mathrm{sec}$. The average E/A ratio was $1.4 \mathrm{I}$ by echocardiogram and $\mathrm{I} .48$ by Ve-CMR. There was a significant correlation in LA size and Mitral inflow E/A ratio between VeCMR and Echo (Rho $=0.9, p<0.01$; Rho $=0.88, p<0.01$, respectively). There were no significant correlations in $\mathrm{PV}$ waves (Rho $=0.12, p=0.58)$. Figure I

Conclusion: Ve-CMR has a very high correlation with Echo in the assessment of mitral inflow E/A ratio and LA size. Ve-CMR maybe useful for the assessment of diastolic dysfunction.

\section{P38}

Non-invasive assessment of coronary artery distensibility by 3.0 T cardiac MRI

Sebastian Kelle, Allison G Hays, Glenn A Hirsch, Gary Gerstenblith, Robert G Weiss and Matthias Stuber Johns Hopkins University, Baltimore, MD, USA

Journal of Cardiovascular Magnetic Resonance 2009, I I (SuppI I):P38

Introduction: Atherosclerotic changes in the coronary artery are associated with impaired coronary vessel wall distensibility [I]. Though non-invasive measures of central aortic distensibility are possible, only intravascular ultrasound (IVUS) invasive measurements of coronary distensibility have been reported until now [I, 2].

Purpose: High-field coronary magnetic resonance imaging (MRI) offers high temporal and spatial resolution, important for assessing distensibility-related changes in coronary dimensions during the cardiac cycle. We sought to test the hypothesis that coronary artery distensibility can be evaluated non-invasively with $3.0 \mathrm{~T}$ cardiac MRI and that distensibility differences can be detected between healthy (control group) and coronary artery disease (CAD) subjects.

Methods: Twenty healthy, adult subjects (II male, age 19-60 years, mean $32 \pm 12$ years) and twelve patients with coronary catheterization documented CAD ( 8 male, age 50-69 years, mean $58 \pm 8$ years) were studied on a commercial whole-body MR imaging system (Achieva 3.0 T; Philips, Best, The Netherlands). MR angiography of the right coronary artery (RCA) was performed with a navigator-gated free-breathing and ECGtriggered, T2-prepared, three-dimensional, segmented k-space, gradient-echo imaging sequence. In each subject, the proximal segment of the RCA was then imaged in cross-section using cine spiral MRI for area measurements. Imaging was performed at a constant room temperature and after at least 20 minutes of rest in the magnet. MRI parameters were: echo time $(T E)=1.5 \mathrm{~ms}$, radiofrequency (RF) excitation angle $=20^{\circ}$ and spectral spatial excitation, breath-hold duration $\sim \mid 4-24 \mathrm{sec}$, acquisition window $=10 \mathrm{~ms}$, repetition time $(T R)=14 \mathrm{~ms}, 21$ spiral interleaves, spatial resolution (acquired/reconstructed) $=$ $0.89 \times 0.89 \times 8.00 \mathrm{~mm}^{3} / 0.69 \times 0.69 \times 8.00 \mathrm{~mm}^{3}$. Both the blood pressure and the heart rate were recorded. Images were analyzed for cross-sectional area changes using full width half maximum (Cine version 3.15.17, General Electric, Milwaukee, WI, USA), and distensibility $\left(\mathrm{mmHg}^{-1}\right)$ was determined as: [(systolic lumen area - diastolic lumen area)]/(pulse pressure multiplied with the diastolic lumen area) [3]. Pulse pressure was calculated as pressure change during a cardiac cycle [2].

Results: Nineteen volunteers and eleven patients had adequate image quality for RCA area measurements (figure IA). The mean heart-rate pressure product (heart-rate multiplied by systolic
Figure I (abstract P38)
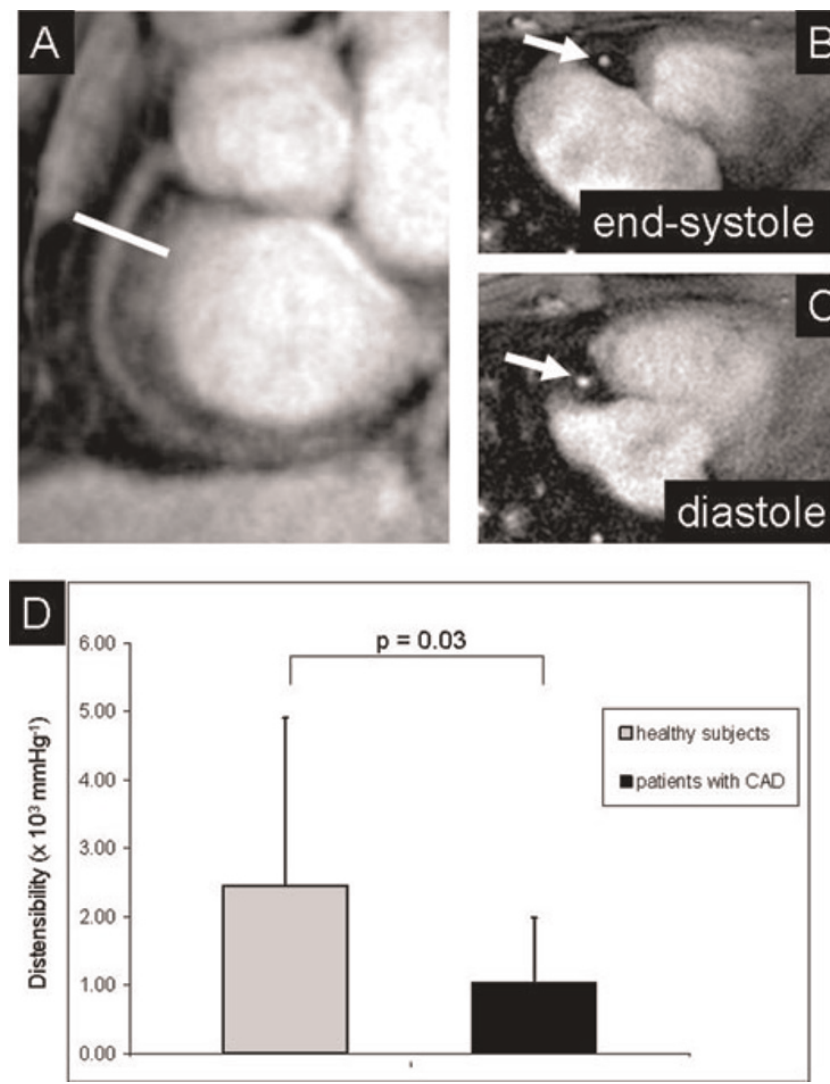

(A): The MRA of the right coronary artery (RCA) is used to select a cross-sectional plane (indicated by white line). Images of the proximal RCA (white arrow) in end-systole (B) and at diastole (C) in a patient with coronary artery disease (CAD). (D): Distensibility measurements in healthy subjects $(N=19)$ and patients with $\operatorname{CAD}(N=I I)$.

blood pressure) in healthy adults (8289 $\pm 1427 \mathrm{mmHg}$ *beats/ minute) was not significantly different from that in CAD patients (9048 $\pm 1878 \mathrm{mmH} \mathrm{g}^{*}$ beats/minute), $(p=0.18)$. The luminal area in healthy subjects was $9.60 \pm 2.02 \mathrm{~mm}^{2}$ during diastole and $12.90 \pm 4.78 \mathrm{~mm}^{2}$ for CAD patients $(p=0.01)$. At end-systole, the luminal area in healthy subjects was $10.60 \pm 2.47 \mathrm{~mm}^{2}$ and $13.55 \pm 5.39 \mathrm{~mm}^{2}$ for CAD patients respectively $(p=0.049)$. Coronary vessel area changed significantly between systole and diastole in healthy controls $(p<0.01)$, but not in CAD patients $(p=0.12)$. In healthy subjects coronary artery distensibility $\left(2.46 \pm 2.45 \mathrm{mmHg}^{-1} * 10^{3}\right)$ was significantly higher than that in CAD patients $\left(1.04 \pm 0.94 \mathrm{~mm} \mathrm{H}^{-1} * 10^{3}\right)$ (figure IB) $(p=0.03)$. We found no significant correlation between coronary artery distensibility and coronary vessel area at either systole or diastole in healthy adults or CAD patients.

Discussion: Non-invasive assessment of coronary artery vessel wall distensibility with $3.0 \mathrm{~T}$ is feasible and the findings are similar to those from invasive IVUS studies [I, 2]. Coronary artery distensibility measured by $3.0 \mathrm{~T} \mathrm{MRI}$ is significantly higher in healthy controls than it is in patients with documented CAD. The number of subjects needed to detect a difference is relatively small. This methodology may support the characterization of 
vascular anatomy and function in healthy and diseased states, as well as the response to interventions in patients with, or at increased risk for CAD.

\section{References}

I. Jeremias A, Spies C and Herity NA, et al: Coronary artery distensibility and compensatory vessel enlargement a novel parameter influencing vascular remodeling? Basic Res Cardiol 2001, 96:506-512.

2. Nakatani S, Yamagishi M and Tamai J, et al: Assessment of coronary artery distensibility by intravascular ultrasound. Application of simultaneous measurements of luminal area and pressure. Circulation 1995, 91:29042910.

3. Oliver JJ and Webb DJ: Noninvasive assessment of arterial stiffness and risk of atherosclerotic events. Arterioscler Thromb Vasc Biol 2003, 23:554-566.

\section{P39}

\section{Coronary sinus flow reserve in response to cold pressor stress in healthy women using velocity- encoded cine (VEC) spiral 3 T MRI}

Christopher D Maroules, Alice Y Chang, Andrew Kontak, Hardik Yadav, Tommy Tillery and Ron M Peshock UT Southwestern Medical Center, Dallas, TX, USA

Journal of Cardiovascular Magnetic Resonance 2009, I I (SuppI I):P39

Introduction: Despite the overwhelming focus on obstructive coronary artery disease over the last few decades, considerable cardiovascular morbidity and mortality is caused by diffuse, nonobstructive disease for which traditional cardiovascular imaging techniques are not suitable. Novel noninvasive techniques that can detect changes in global myocardial perfusion and function might improve the clinical management of patients with diffuse cardiac disease. Coronary sinus flow imaging by velocityencoding cine MRI has previously been described as a useful measure of global left ventricular perfusion in patients with heart failure, hypertensive heart disease, and cardiac transplants. To our knowledge, this technique has not been investigated using a

\section{Figure I (abstract P39)}

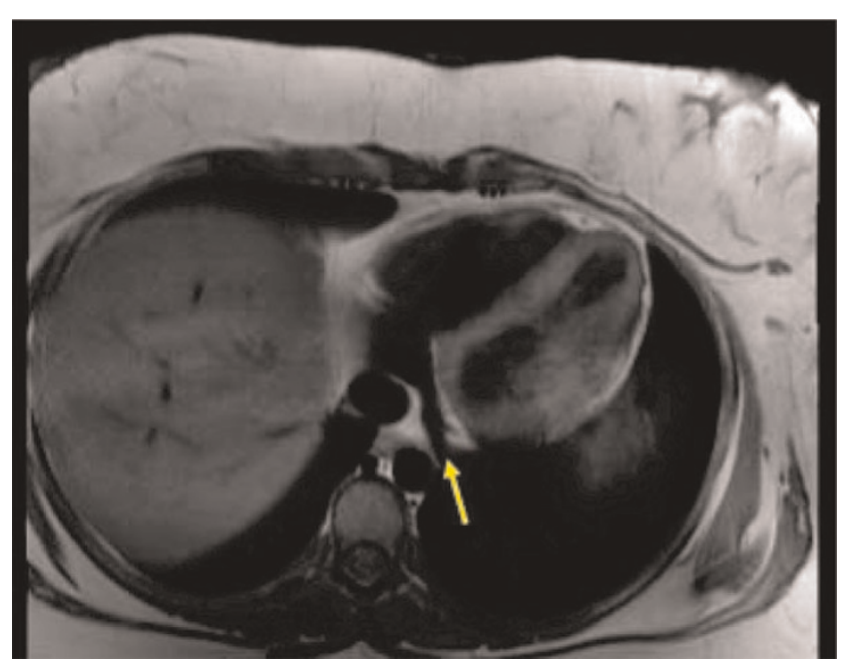

Figure 2 (abstract P39)

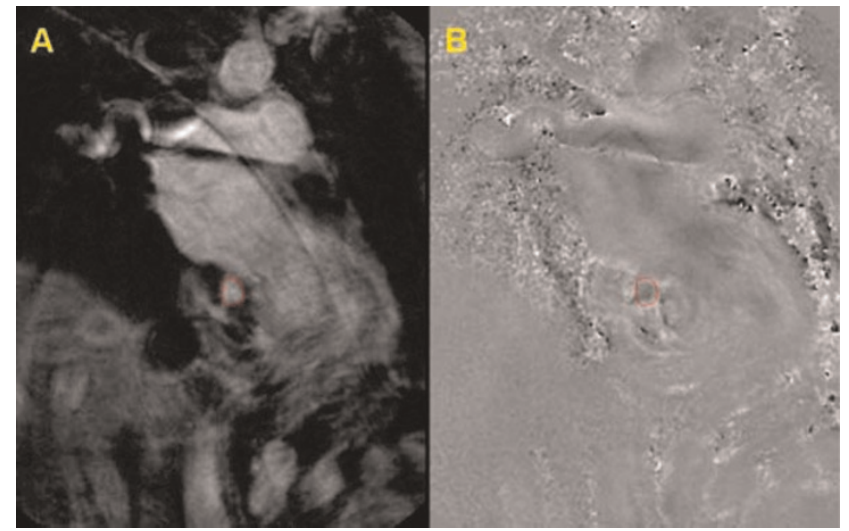

spiral 3 T MRI technique, which could potentially enhance vessel wall delineation and accelerate scan time.

Prior studies have demonstrated an increase in coronary sinus flow in response to pharmagologic stress. This study tested whether the cold pressor test (CPT), a more specific test of endothelial reactivity could provoke measurable changes in coronary sinus flow.

Purpose: To determine the feasibility of coronary sinus flow imaging in healthy women in response to the cold pressor test (CPT) using velocity-encoding cine (VEC) spiral 3 T MRI.

Methods: Five healthy women $29 \pm 9$ years old were enrolled in this study. MR imaging was performed using a $3 \mathrm{~T}$ clinical MR scanner (Achieva, Philips, Netherlands) using a 6 element cardiac receiver coil and ECG-gating. Scout images were obtained in the axial plane to localize the coronary sinus (Figure I). Oblique coronal slices perpendicular to the coronary sinus were aligned $2-\mathrm{cm}$ from the sinus ostium. Velocity-encoded spiral cine sequences were then acquired during end-expiratory breathholds (8-10 seconds) to measure coronary sinus flow. The following imaging parameters were used: FOV, $25 \times 25 \mathrm{~cm}$; matrix, $312 \times 312$; pixel size, $0.8 \times 0.8 \mathrm{~mm}$; slice thickness,

Figure 3 (abstract P39)

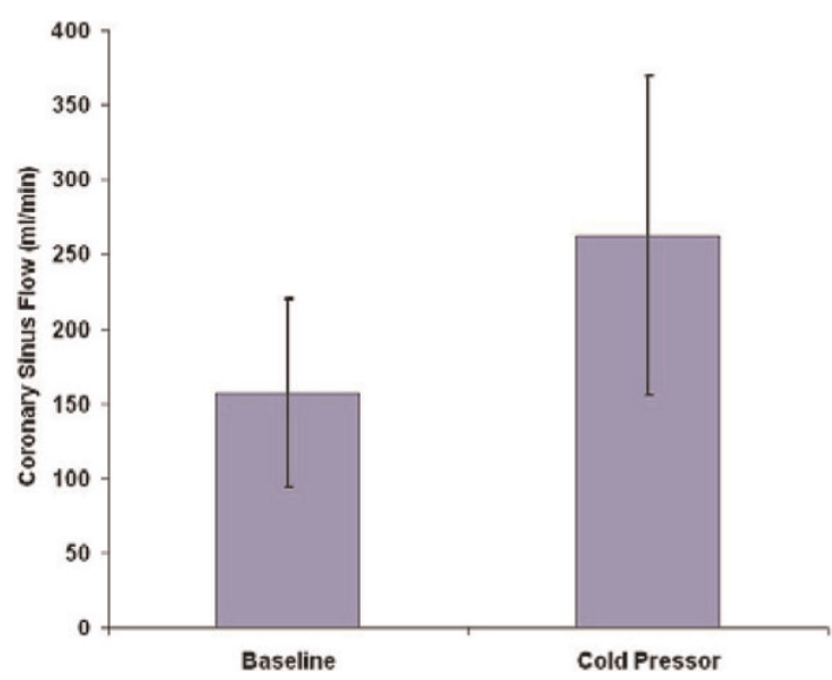


$7 \mathrm{~mm}$; TR, $34 \mathrm{msec}$; TE, $3.5 \mathrm{msec}$; flip angle 20; temporal resolution, $69 \mathrm{msec}$; spiral interleaves, II; VENC, $80 \mathrm{~cm} / \mathrm{sec}$. Heart rate and blood pressure were measured every 30 seconds. After baseline flow data acquisition, the participant's left hand was placed in an ice water bath (50\% ice, $50 \%$ water) for 3 minutes. Repeat flow images were acquired I-minute following CPT, and again at 2-minutes and 10-minutes. Images were transferred to a remote workstation and analyzed using QFlow (v. 4.I.6, Medis, Leesburg). The contour of the coronary sinus was manually traced on each magnitude image. Identical tracings were applied to the corresponding phase images so coronary sinus flow velocity and volume flow could be calculated. Coronary sinus flow was determined by integrating phasic flow over time. Coronary sinus flow velocity was determined by averaging flow velocity across all cardiac phases. Statistical significance was determined using the paired $t$-test assuming an $\alpha$-error of 0.05 .

Results: Coronary sinus flow was successfully measured in each subject throughout the cardiac cycle (Figure 2). All subjects tolerated the CPT. Rate-pressure product increased $41.2 \pm 15.8 \%$ from baseline to peak effect during CPT. Similarly, coronary sinus flow increased from $157 \pm 63 \mathrm{ml} /$ minute at baseline to $262 \pm 107 \mathrm{ml} /$ minute at peak effect during CPT, representing a $67 \%$ increase $(P<0.05$, Figure 3$)$. Coronary sinus flow velocity increased from $7.9 \pm 3.4 \mathrm{~cm} / \mathrm{second}$ at baseline to $12.6 \pm 6.3 \mathrm{~cm} / \mathrm{second}$ at peak effect during CPT, representing a $61 \%$ increase $(P<0.05)$.

Conclusion: Velocity-encoding cine spiral $3 \mathrm{~T} M R I$ is a promising tool for evaluating coronary sinus flow reserve. The CPT provokes a significant increase in myocardial blood flow which can be used to specifically evaluate endothelial function. Future studies will examine the ability of this technique to detect meaningful changes in myocardial flow reserve.

\section{P40}

Comparison of two novel methods of measuring the blood velocity in the deep veins of the lower leg using phase contrast MR imaging

lain Pierce, Peter D Gatehouse, Xiao Yun Xu, Jenny Keegan, Andrew D Scott and David N Firmin Imperial College London, London, UK

Journal of Cardiovascular Magnetic Resonance 2009, I I(SuppI I):P40

Aim: To compare the effectiveness of two novel methods developed to measure the velocity of the blood in the deep veins in the lower leg.

Background: To investigate the causes and treatment of deep vein thrombosis, there is a need for blood flow measurements in the lower limb veins. The work of Downie et al into the effects of compression stockings on geometry [I] and wall shear stress [2] concludes that MR imaging should be utilised for continued research of the haemodynamics of the lower limb venous system as it can supply structural and velocity data from the same acquisition. Venous velocity is low and more dependent on the respiratory than cardiac cycle, requiring new methods. Also the muscle pump can cause large spikes in the velocity requiring realtime measurement.

Methods: Two sequences have been developed using PhaseContrast velocity mapping: gated SEGmented GRadient Echo (SEG-GRE) sequence and an Interleaved SPiral (ISPLASH)
Figure I (abstract P40)
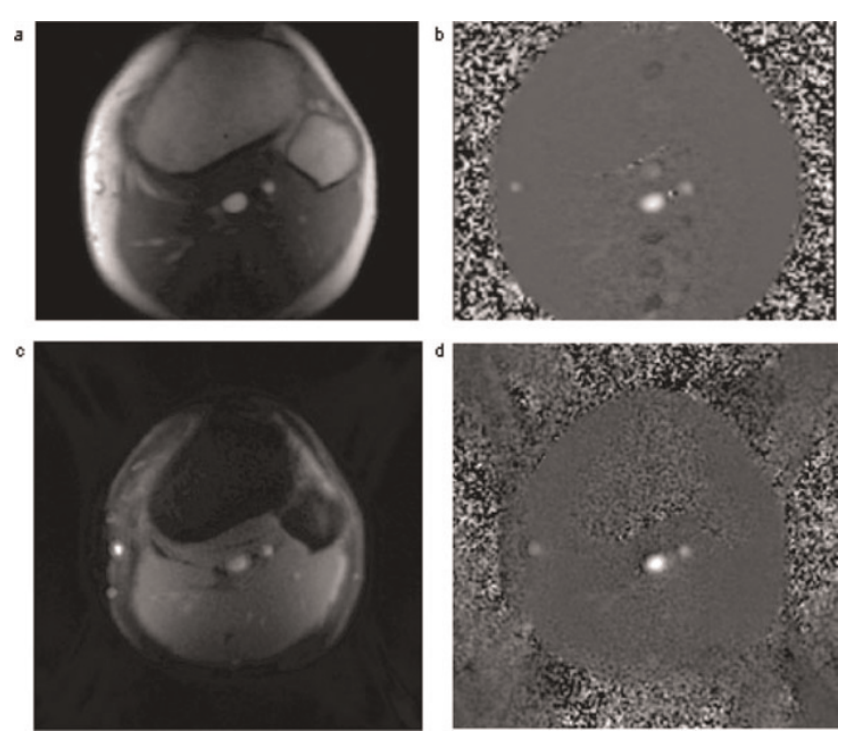

SEG-GRE magnitude image at peak mean velocity (a) and corresponding PC image (b). ISPLASH magnitude image at peak mean velocity (c) and corresponding PC image.

sequence. Both use VElocity ENCoding (VENC) of $10 \mathrm{~cm} / \mathrm{s}$, arterial flow saturation upstream, slice thickness of $7 \mathrm{~mm}$ and pixel size of $\mathrm{I} \times \mathrm{I} \mathrm{mm}$.

Seven normal volunteers were asked to lie prone (I.5 T Siemens Avanto) with left leg raised $10-15 \mathrm{~cm}$, resting the ankle on a foam support, with a pair of carotid surface coils (Machnet) placed around the calf. The volunteers were asked to breathe deeply, in time with an LED driven by a 7 second/cycle square wave input [3]. The logfiles for the subject's respiratory belt sensor were recorded for each spiral scan.

SEG-GRE: Triggered by the square wave input with 7 seconds cine acquisition window (23 respiratory-cine frames) covering one respiratory cycle, acquiring II rawdata lines per cine frame per cycle. The velocity encoding gradients are applied every other excitation (REF - VENC) within each cine frame. The number of averages was 2 , acquisition time 2.5 minutes, time per frame $285 \mathrm{~ms}$.

\section{Figure 2 (abstract P40)}

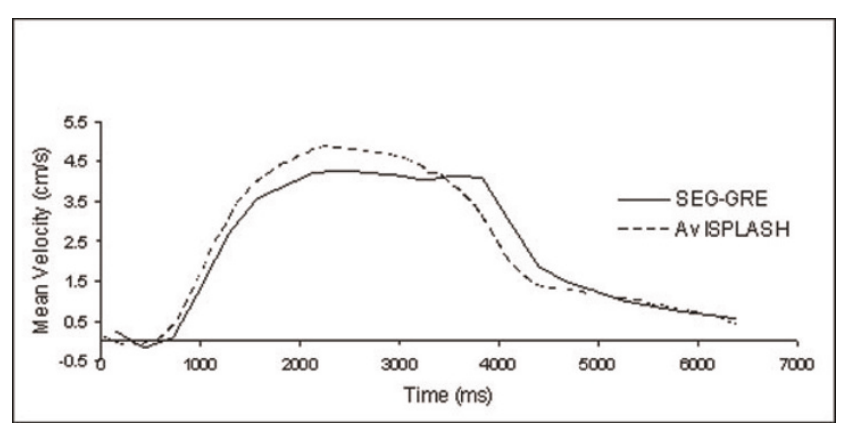

Mean velocity measured from SEG-GRE and the averaged mean velocity, 4 respiratory cycles, from ISPLASH. 


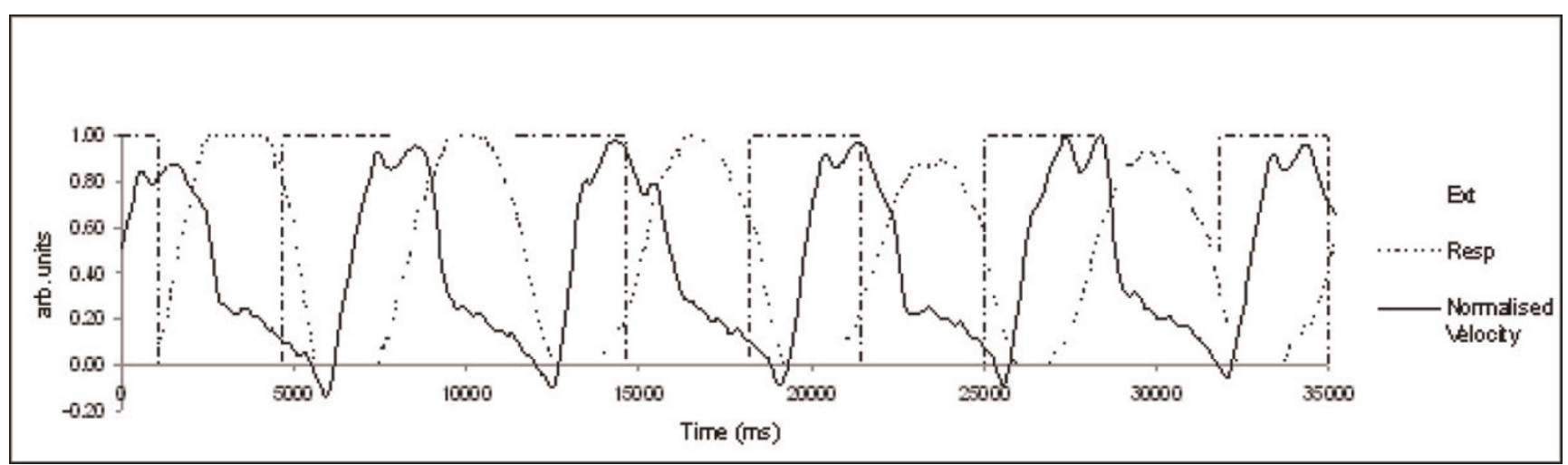

Full 'real-time' plot of normalised mean velocty with the respiratory (Resp) and the LED square wave used for breathing regulation (Ext).

ISPLASH: The ISPLASH sequence uses water excitation and balanced velocity encoding using alternate excitations (VENC (+ve) - VENC (-ve)). It was run with 4 interleaves repeated continuously covering 35 seconds. The spiral data was re-gridded and by sliding reconstruction (In-house Matlab programs) allowed interpolation between repetitions. Time per frame is $320 \mathrm{~ms}$, with interpolation $80 \mathrm{~ms}$ between frames.

The deep vessels were manually segmented (CMR Tools) from the magnitude images and the regions of interest transferred to the phase images for a mean velocity measurement. For comparison, an average from 4 cycles was taken from the ISPLASH velocities. Figure I.

Results and discussion: The SEG-GRE and 4-cycle averaged ISPLASH velocity waveforms (Fig. 2) show that both methods yield similar waveforms, similar to those previously seen using Doppler [2, 3]. The peak velocities were taken for each subject from the SEG-GRE, the averaged and real-time ISPLASH waveforms (Table I). While the average values agree closely, the results also show that the ISPLASH method reveals faster peaks of venous flow. These are averaged down in the SEG-GRE scan due to the number of cycles per image and can be duplicated with $30 \mathrm{sec}$ of ISPLASH data. Also the 'real-time' data (Fig. 3) probably shows cardiac pulsations on top of the respiratory based cycle.

Table I (abstract P40) The peak mean velocity measured from SEG-GRE averaged ISPLASH (4 respiratory cycles) and the full ISPLASH

\begin{tabular}{lccc}
\hline & \multicolumn{3}{c}{ Peak mean velocity (cm/s) } \\
\cline { 2 - 4 } Subject & SEG-GRE & Av ISPLASH & ISPLASH \\
\hline 1 & 2.06 & 2.39 & 2.89 \\
2 & 5.94 & 6.66 & 8.64 \\
3 & 4.94 & 5.88 & 6.81 \\
4 & 8.17 & 8.15 & 10.37 \\
5 & 6.43 & 7.24 & 8.98 \\
6 & 4.3 & 4.93 & 5.26 \\
7 & 6.79 & 6.63 & 7.09 \\
\hline
\end{tabular}

Conclusion: Two methods for measuring the venous blood velocities in the lower leg have been compared and show good agreement. The first method produces an averaged velocity profile over the respiratory cycle but relies on sustained regular breathing. The second method, ISPLASH, allows for more 'real time' imaging allowing further investigation into areas such as the muscle pump and graduated compression devices.

\section{References}

I. Downie S, et al: JMRI 2007, 26:80-85.

2. Downie S, et al: AJP - Heart Circ Physiol 2007, 294:21I22120.

3. Miller J, et al: J Physiol 2005, 563:925-943.

\section{P4I}

Comparison of MRI-derived pulmonary edema measures with LVEDP and serum BNP

Kelvin Chow', Mustafa Toma', Ben Esch', Jessica Scott ${ }^{2}$, Mark Haykowsky', Richard Thompson ' and lan Paterson' 'University of Alberta, Edmonton, AB, Canada

${ }^{2}$ University of British Columbia, Vancouver, BC, Canada

Journal of Cardiovascular Magnetic Resonance 2009, I I (SuppI I):P4 I

Introduction: Cardiogenic pulmonary edema is the accumulation of fluid in the interstitial and often alveolar space of the lungs, caused by increased hydrostatic pressure leading to extravasation of fluid. Currently, assessment of edema is limited to qualitative chest $\mathrm{x}$-ray and occasionally CT. It is known that $M R I$ can measure lung water content [I], but systematic sources of error such as $B_{1}$ field heterogeneity and breath-hold variability have not previously been accounted for, and MRI-derived lung water has not been compared with invasively measured heart pressures.

Purpose: To compare lung water content measured using freebreathing $\mathrm{MRI}$ in populations of healthy volunteers and heart failure patients to left ventricular end-diastolic pressure (LVEDP) and blood serum b-type natriuretic peptide (BNP) concentration. Methods: 7 healthy male volunteers $(31 \pm 9$ yrs $)$ and 10 heart failure patients ( $53 \pm 14$ yrs; 8 male) were imaged on a Siemens Sonata I.5 T MRI scanner with informed consent and IRB approval. A half-Fourier single-shot turbo spin-echo (HASTE) pulse sequence was used with typical parameters: excitation flip 
Figure I (abstract P4I)

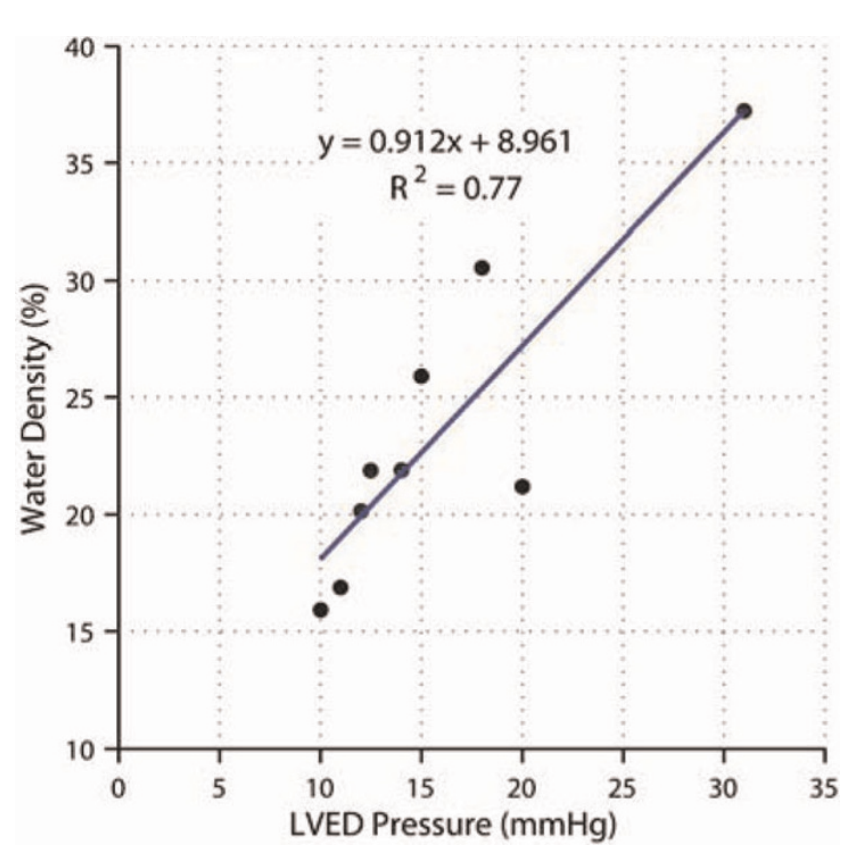

MRI-derived lung water as a function of LVEDP in heart failure.

angles $60^{\circ} / 120^{\circ}, \quad 1.4 \times 1.4 \times 8.0 \mathrm{~mm}$ resolution, ECG gating (end-diastolic imaging), $12 \mathrm{~ms}$ effective TE, $>5 \mathrm{~s}$ TR, 7 repetitions during free breathing, 6 minute total acquisition time for 10 sagittal slices across both lungs.

An automated image morphing algorithm was used to deform each image to a reference respiratory phase (end expiration) and signal intensity corrections were applied to account for normal changes during respiration. The lung was manually traced once per sagittal slice and an automated algorithm segmented the lung into smaller regions $\left(\sim 3.5 \mathrm{~mm}^{2}\right)$. Bright blood vessel contributions were removed with regional thresholding.

Images were acquired with two different flip angles and a modified double angle method [2] was used to measure and

Figure 2 (abstract P4I)

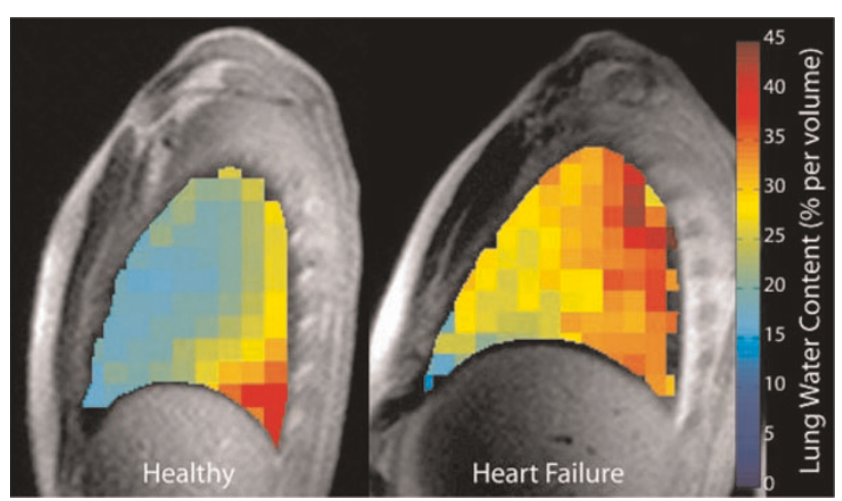

MRI-derived lung water (mid-sagittal right lung) for a healthy subject (total average lung water $21.7 \%$ ) and a heart failure patient (37.2\%). correct for a heterogeneous $B_{\text {I }}$ radiofrequency field. A large region of the liver, visible in a mid-sagittal slice of the right lung, was used as a source of known water density ( 70\%). For each heart failure patient, LVEDP was measured with an invasive catheter immediately before the MRI scan and BNP levels were collected immediately after.

Results: Average lung water content of lungs in the healthy population was $22.5 \pm 2.4 \%$ (percentage water content per unit volume), similar to previous reports [3]. Lung water content in the heart failure group is considerably more variable, ranging from $16 \%$ to $37 \%$, with good correlation to both LVEDP $\left(R^{2}=\right.$ 0.77, Fig. I) and BNP concentrations $\left(R^{2}=0.75\right)$. LVEDP and BNP also correlated well $\left(R^{2}=0.57\right)$. Lung water maps for a midsagittal slice through the right lung are shown for both a healthy individual and a heart transplant patient (Fig. 2).

For a prescribed $90^{\circ}$ tip angle, the computed $B_{\text {I }}$ field maps indicate that the average flip angle achieved within the left lung is $77.2 \pm 2.3^{\circ}$ and only $61.1 \pm 1.8^{\circ}$ in the right lung. Correcting for this $B_{1}$ heterogeneity resulted in an absolute increase in lung water density in the right lung of $9.6 \pm 1.5 \%$, with negligible correction in the left lung.

Conclusion: A free-breathing MRI approach to quantifying pulmonary edema incorporating corrections for heterogeneous

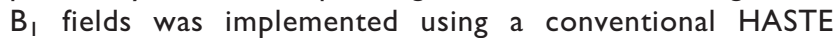
sequence. Lung water density was found to be tightly grouped within a population of healthy individuals, with good correlation to both gold standard LVEDP and BNP concentrations in heart failure patients. MRI-derived lung water, measured as part of comprehensive cardiovascular exam, is thus a potential surrogate for LVEDP.

\section{References}

I. Hayes CE, et al: Science 1982, 216:1313-1315.

2. Chow K, et al: Proc Intl Soc Mag Reson Med 2008, 16:1244.

3. Hatabu H, et al: Eur J Radiol 1999, 29:245-245.

\section{P42}

Myocardial vasculature: the third significant contributor to MR diffusion signals in the isolated rabbit heart

Min-Sig Hwang, Melvin Clark and John R Forder

Mcknight Brain Institute, University of Florida,

Gainesville, FL, USA

Journal of Cardiovascular Magnetic Resonance 2009, I I(SuppI I):P42

Introduction: The coronary circulation is a significant component of the myocardial mass, due to the high energetic demand of cardiac tissue - the capillary surface area in the heart occupies $14-22 \%$ by volume [I]. Thus, vascular spaces need to be considered in addition to interstitial and intracellular spaces for a comprehensive understanding of the physiological compartments responsible for the diffusion signals measured by magnetic resonance. Understanding the origin of MR diffusion signals may reveal changes that precede pathophysiological changes, electrical conductivity and congenital abnormalities, as well as the viability of the cardiac tissue non-invasively.

Purpose: In this experimental study, we investigated the contribution of the static vasculature to the observed multiexponential diffusion of water in isolated rabbit heart by replacing the vascular space with perfluorocarbon-emulsion (PFC). We examined the effect of flow by changing the 
Figure I (abstract P42)
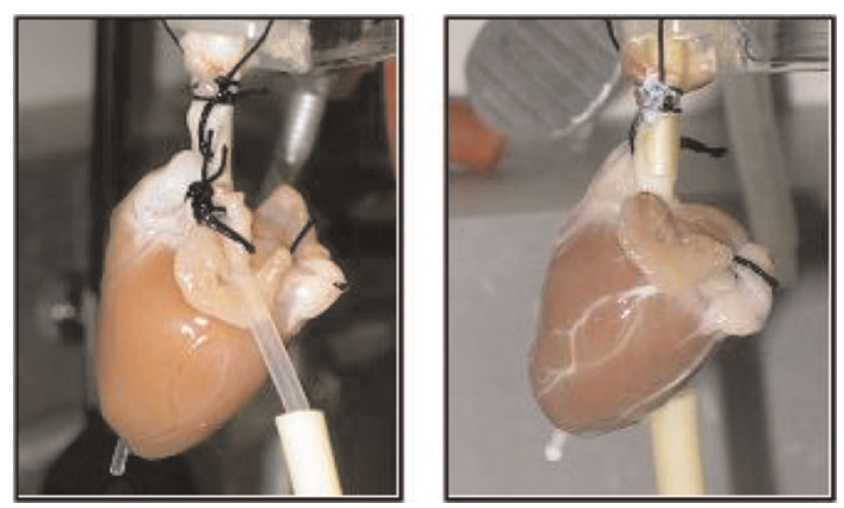

Experimental setup of isolated heart with the aorta cannulated. Left: perfused with STH, right: replaced with PFC emulsion. Coronary arteries shown are filled with the emulsion.

global flow rate of a modified St. Thomas' Hospital cardioplegic solution (STH).

Methods: Isolated perfused hearts $(n=9)$ of New Zealand White male rabbits $(2-4 \mathrm{~kg})$ were prepared according to the approved animal protocol by the UF Institutional Animal Care and Use Committee [2]. The hearts were arrested prior to imaging by switching perfusate to the STH. MR experiments were performed on an II.I T/40 cm clear bore magnet (Magnex Instrument Inc. UK, Bruker Instrument console). Diffusion weighted images were acquired by applying the gradients to give $14 \mathrm{~b}$ values up to $6500 \mathrm{~s} / \mathrm{mm}^{2}$ in 6 directions with a spin echo sequence. Imaging parameters were TR $=1.5 \mathrm{~s}, \mathrm{TE}=27.3 \mathrm{~ms}$,

\section{Figure 2 (abstract P42)}

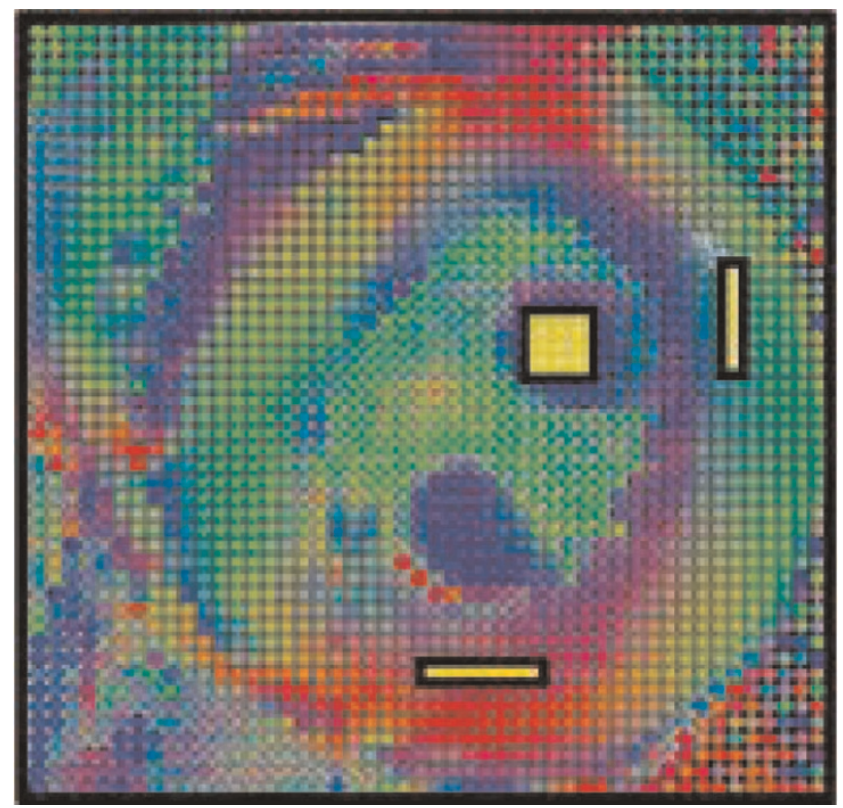

Manual selection of ROIs. (diffusion weighted: left to right). Right: free wall I, bottom: free wall 2, and middle: papillary muscle.
Figure 3 (abstract P42)

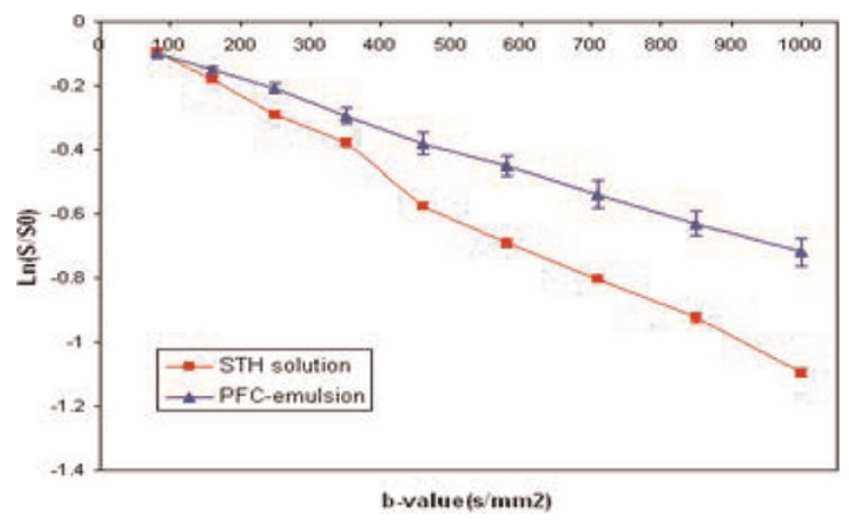

representative logarithmic normalized signal attenuation of STH and PFC. Each point corresponds to the mean and SD of selected ROls.

$\mathrm{NA}=\mathrm{I}, \Delta=11.59 \mathrm{~ms}, \delta=3.8 \mathrm{~ms}$. Slice thickness was $2 \mathrm{~mm}$ with an in-plane resolution of $0.5 \times 0.5 \mathrm{~mm}^{2}$. Five hearts underwent substitution of the STH in the coronary vascular space with $15 \mathrm{cc}$ of PFC and the DTI measurement repeated (Fig. I). Four hearts underwent variation of flow from 0 to $5 \mathrm{~mL} / \mathrm{min}$ and diffusion imaging was repeated after each flow rate change. Pixel by pixel analysis was conducted on a map of the primary eigen vectors of diffusion tensor using FLTView ${ }^{\mathrm{TM}}$ ( ${ }^{\odot} 2007$, Barmpoutis) (Fig 2). The normalized signal intensity with $b$ values within the manually selected ROls was plotted to derive apparent diffusion coefficients (ADCs).

Results: Replacement with the PFC: When the vascular space was replaced with PFC particles ( Ø $450 \mathrm{~nm})$, ADC decreased significantly by $23.1 \pm 8.5 \%$ compared to initial measurements $(p<0.01)$ as b-values approached $1000 \mathrm{~s} / \mathrm{mm}^{2}$ (Fig. 3). Based on the previous reports that the PFC emulsion stays inside vasculature when administered intravenously, this result suggests that the ADC decrease mostly came from the vascular replacement of STH with PFC, whose isotropic ADCs were $2.23 \pm 0.24 \times 10^{-3} \mathrm{~mm}^{2} / \mathrm{s}$, and $1.39 \pm 0.15 \times 10^{-3} \mathrm{~mm}^{2} / \mathrm{s}$, respectively.

Change of vascular flow rate: Figure 4 demonstrates the influence of flow upon the bi-exponential decline in signal intensity plotted as a function of $b$ values in the selected ROls. Free wall I (where fibers are parallel to diffusion encoded direction) shows the significant changes in the ADC over the bvalues with flow change, especially I to $2 \mathrm{ml} / \mathrm{min}$. Those appear to correlate with relative change in the two water pools (fast and slow components), which results from changes in the size of the tissue compartments (extracellular/intracellular). On the other hand, free wall 2 and papillary muscle don't exhibit the same degree of change in ADC or contribution of the slow component of diffusion.

Conclusion: This study demonstrates a significant contribution of vascular compartment to the myocardial MR diffusion characteristics. First, vascular compartment appears to be a significant contributor to the fast component of MR water diffusion. Second, attenuation of signal intensity with $b$ values up to $6500 \mathrm{~s} / \mathrm{mm}^{2}$ appears to be modulated by vascular flow. 
Figure 4 (abstract P42)
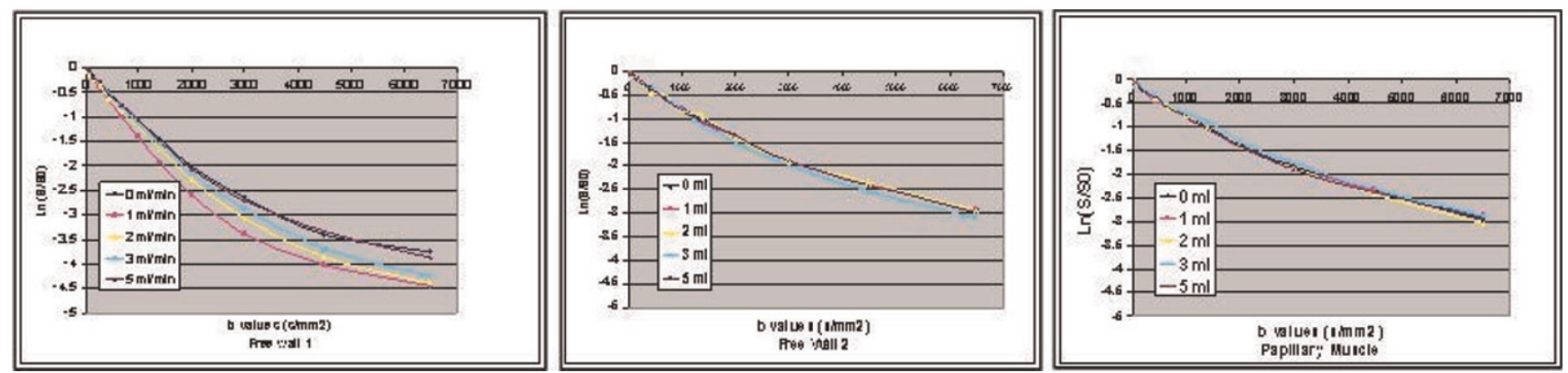

Logarithmic normalized signal attenuation with changes in perfusate flow. Free wall I (left panel) is parallel to the diffusion encoded direction. On the other hand, Free wall 2 (middle panel) and Papillary muscle (right panel) is oriented orthogonal to the diffusion encoding direction.

\section{P43}

T2-imaging of area-at-risk predicts recovery of cardiac function in a canine model of acute myocardial infarction

Jamieson M Bourque, David K Glover, Craig C Goodman, Mirta R Herrera, Fred Epstein, George A Beller and Christopher M Kramer University of Virginia, Charlottesville, VA, USA

Journal of Cardiovascular Magnetic Resonance 2009, I I (SuppI I):P43

Introduction: Infarct size is the strongest predictor of subsequent outcomes and remains an important therapeutic target. Myocardial edema measured by T2-weighted magnetic resonance (MR) imaging identifies the ischemic area-at-risk and predicts infarct size, allows more accurate evaluation of novel anti-ischemic therapies, and may be associated with stunning and long-term recovery of function.

Purpose: We sought to identify the extent of infarcted and atrisk myocardium and correlate these markers with post-infarct recovery of myocardial function.

Methods: Five dogs (mean weight $18.9 \mathrm{kgs}$ ) underwent successful 2-hour complete occlusion of the left-circumflex artery and collaterals after baseline functional cine imaging. They subsequently underwent 48-hour and 4-week complete leftventricular (LV) short-axis MR imaging on a 1.5 Tesla scanner using SSFP-cine (TE I.4 ms, TR $35.3 \mathrm{~ms}$, voxel size $0.6 \times 1 \times 7 \mathrm{~mm}^{3}$ ), ACUTE T2-weighted (TE $1.9 \mathrm{~ms}$, TR $168.1 \mathrm{~ms}$, voxel size

\section{Figure I (abstract P43)}

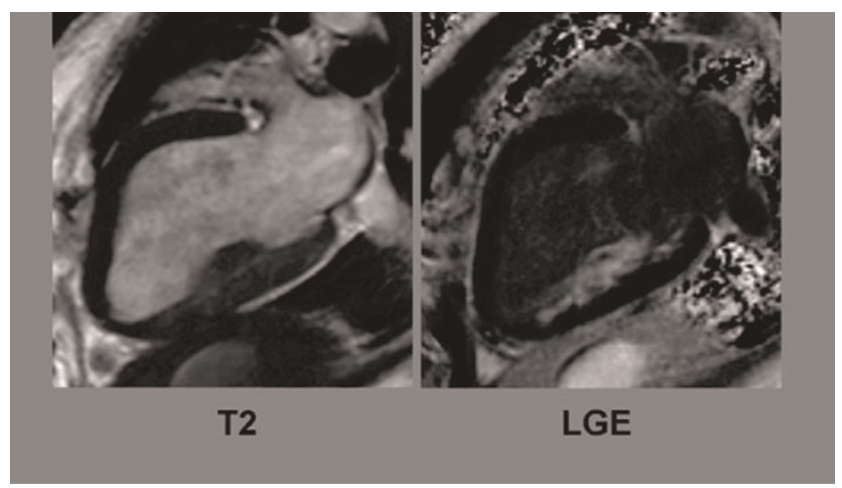

$\left.0.6 \times 0.9 \times 7 \mathrm{~mm}^{3}\right)$, and phase-sensitive, inversion recovery late Gadolinium $(0.15 \mathrm{mmol} / \mathrm{kg}$ Magnevist) enhancement sequences (LGE, TE $3.4 \mathrm{~ms}$, TR $548 \mathrm{~ms}$, voxel size $0.5 \times 0.8 \times 7 \mathrm{~mm}^{3}$ ). The percentages of LGE and T2 hyperenhacement were identified through intensity threshold testing, 5 standard devitations (SDs) and 2 SDs above the reference myocardium respectively. All outcome variables were given as mean percentages \pm SDs.

Results: The mean percentage LGE was extensive at 44.I $\pm 5.9 \%$. T2-imaging identified a large area-at-risk measuring $68.5 \pm 7.1 \%$ of the myocardium. The mean LV ejection fraction (LVEF) at 48 hours was $23.7 \pm 2.8 \%$. Significant recovery of function was seen at four weeks (mean LVEF increased to $42.7 \pm 7.3 \%$ ), likely in large part due to the significant stunning present and low LGE/T2 hyperenhancement ratio $(0.65 \pm 0.13)$ at 48 hours. This ratio was found to moderately correlate negatively with the percent LVEF improvement $(r=-0.57)$. Figure I.

Conclusion: The area-at-risk estimated by T2-weighted MR imaging correlates with recovery of myocardial infarction in an acute-MI canine model. Further evaluation of this method will maximize our ability to prognosticate and guide therapy postinfarction. Increasing the proportion of myocardial salvage from the initial area-at-risk should serve as an important therapeutic target. This imaging technique may become a significant marker of treatment efficacy and prognosis.

\section{P44}

Subendocardial reversible perfusion defects on adenosine stress MRI in ER patients with chest pain: relationship to cardiovascular risk factors Jan Skrok', David Dombroski', Steven M Shea ${ }^{2}$, Mark Bohlman', Christine H Lorenz ${ }^{2}$, Joao A Lima ${ }^{3}$, David A Bluemke' and Jens Vogel-Claussen'

'Johns Hopkins University, Department of Radiology, Baltimore, MD, USA

${ }^{2}$ Siemens Corporate Research Inc., Baltimore, MD, USA

3 Johns Hopkins University, Department of Cardiology, Baltimore, MD, USA

\section{Journal of Cardiovascular Magnetic Resonance 2009, I I (SuppI I):P44}

Background: Many patients with angina-type chest pain have normal coronary angiograms and nuclear cardiac SPECT exams. This is thought to be a result of microvascular disease. 
Purpose: To evaluate patients presenting to the ER with chest pain and negative cardiac enzymes by stress perfusion MRI and to correlate the findings with future cardiovascular events over a 9 month follow-up period.

Methods: 27 patients (I5 male, 12 female, mean age $56.3 \pm 13.2$ years) underwent a comprehensive stress cardiac adenosine MRI and a nuclear cardiac SPECT exam within $24 \mathrm{~h}$ after presenting to the emergency room with chest pain. MRI at I.5 T (Siemens Avanto, Erlangen, Germany) included steady state free precession (SSFP) cine MR imaging in short and long axis planes, adenosine stress and rest perfusion imaging, as well as delayed enhancement MR imaging. For the stress perfusion MR images, adenosine was injected i.v. at a rate of I $40 \mu \mathrm{g} / \mathrm{kg} / \mathrm{min}$ over six minutes. Four minutes into the adenosine injection, saturation recovery (SR) SSFP $M R$ images were obtained. Scan parameters were: TR/TE $2.4 / 1.0 \mathrm{~ms}$, TI $180 \mathrm{~ms}, 50^{\circ}$ flip angle, bandwidth $1000 \mathrm{~Hz} /$ pixel, FOV $36 \times 27 \mathrm{~cm}$, matrix $192 \times 115$, acquisition duration $150 \mathrm{~ms}$, slice thickness $8 \mathrm{~mm}$, acceleration

Figure I (abstract P44)

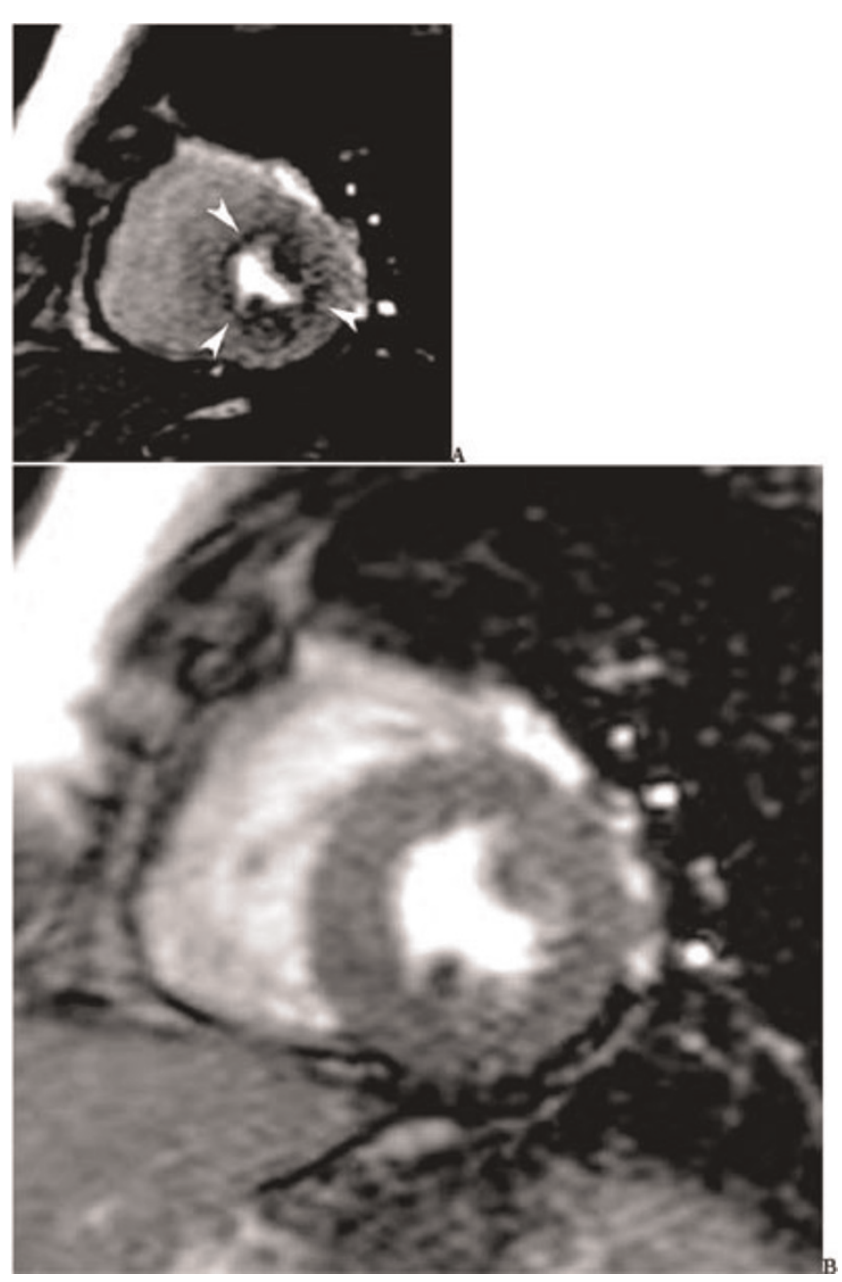

53 year old female patient with diabetes and hypertension, presenting to the emergency room with chest pain. (A) Diffuse subendocardial perfusion defect on adenosine stress MRI which was reversible at rest (B). The nuclear cardiac SPECT exam was normal, and there was no myocardial scar on delayed enhancement MRI. factor 2 (GRAPPA). Gadopentetate dimeglumine was injected at $5 \mathrm{cc} / \mathrm{sec}(0.075 \mathrm{mmol} / \mathrm{kg})$, immediately followed by a $20 \mathrm{cc}$ normal saline flush at $5 \mathrm{cc} / \mathrm{sec}$ for both rest and stress perfusion. Three short axis slices and one horizontal long axis slice were acquired with a temporal resolution of 2 RR. Resting perfusion images were obtained $10 \mathrm{~min}$ after the stress perfusion. Two experienced investigators evaluated all images in consensus. Patients were then followed for an average of $9.1 \pm 3.9$ months for subsequent cardiovascular events. Figure I.

Results: On MRI, 8/27 patients (30\%) had diffuse subendocardial perfusion defects on stress imaging in more than I coronary artery territory, which were reversible at rest. All of these patients had a normal SPECT cardiac stress exam and no myocardial delayed enhancement on MRI. Fifteen percent (4/27 patients) had focal reversible perfusion defects on MRI in a single coronary artery territory that correlated with $\geq 70 \%$ stenosis on conventional angiography. One patient had both small and large vessel disease (history of coronary artery bypass graft and stress induced diffuse subendocardial perfusion defects on MRI). Fiftysix percent (15/27 patients) had neither small nor large vessel disease on MRI; all were normal on SPECT and did not show any scar on MRI. Patients with diffuse perfusion defects had a significantly higher rate of diabetes ( $p=0.03$, two tailed Fisher's) and hypertension ( $p=0.05$, two tailed Fisher's) compared to patients without perfusion defects. Patients with diffuse perfusion defects had significantly more risk factors for cardiovascular disease (mean 4.4) compared to patients without diffuse subendocardial perfusion defects or significant large vessel disease (mean 2.9, $\mathrm{p}=0.02$ two-sided Wilcoxon test). Over a 9 month period, 3 patients presented again with angina-like chest pain, all of whom had shown diffuse subendocardial perfusion defects on adenosine stress MRI at baseline.

Conclusion: Diffuse subendocardial perfusion defects on stress MRI in the emergency room setting were frequent $(30 \%)$ in our patient population in the absence of positive findings on cardiac SPECT or myocardial scar on MRI. These findings were more frequent in patients with hypertension and diabetes and may relate to underlying microvascular disease. This could account for chronic recurrent chest pain in this patient population.

\section{P45}

The association of C-reactive protein with left ventricular systolic function and left ventricular myocardial fibrosis

Mohammed Ahmed', ljaz Ahmad', Munawar Hayat', Jasmin Ullah', Nadeem Asad', Joshua A Socolow', Igor Klem², Matt Briggs', Terrence Sacchi' and John F Heitner'

${ }^{I}$ New York Methodist Hospital, Brooklyn, NY, USA

${ }^{2}$ Duke University Medical Center, Durham, NC, USA

Journal of Cardiovascular Magnetic Resonance 2009, I I(SuppI I):P45

Background: C-reactive Protein (CRP) is a serologic marker that has been shown to predict adverse cardiovascular outcomes. In addition, CRP has been shown to be elevated in patients with congestive heart failure (CHF).

Objective: To evaluate if CRP is associated with the severity of left ventricular dysfunction, independent of the degree of myocardial fibrosis. 
Methods: We prospectively evaluated 46 outpatients referred to our cardiac MRI (CMR) center for the evaluation of ischemic cardiomyopathy (LVEF < 45\%). Patients were excluded if they were discharged from the hospital within 7 days of the CMR study or were in acute CHF by clinical exam on the day of the study. Blood samples were drawn for the measurement of CRP on the day of the CMR. All patients had left ventricular function and amount of scar tissue calculated by computer analysis after ROI curves were performed.

Results: The average age of the patients was 67.7 years (36 males and 10 females). The prevalence of the following risk factors was as follows: hyperlipidemia $(80 \%)$, hypertension $(77 \%)$, diabetes $(50 \%)$, tobacco $(14 \%)$, renal disease $(11 \%)$, and peripheral vascular disease $(7 \%)$. The mean left ventricular ejection fraction (LVEF) was $36.7 \%$. The mean left ventricular myocardial fibrosis was $16 \mathrm{~g}$. Separate regression models were applied for CRP predicting left ventricular myocardial fibrosis and LVEF. The log CRP was associated with both left ventricular myocardial fibrosis $(p=0.05)$ and $\operatorname{LVEF}(p=0.03)$. In addition, the log CRP was associated with a low ejection fraction, independent of the amount of left ventricular myocardial fibrosis $(p=0.03)$.

Conclusion: CRP is an independent predictor of left ventricular ejection fraction in patients with ischemic cardiomyopathy.

\section{P46}

\section{k-t SENSE-accelerated myocardial perfusion MR} imaging at 3.0 Tesla - comparison with pressure wire measurement of fractional flow reserve Tim Lockie', Divaka Perera', Simon Redwood', Sebastian Kozerke', Michael Marber', Eike Nagel' and Sven Plein ${ }^{3}$

'Rayne Institute, St Thomas' Hospital, London, UK ${ }^{2}$ ETH, Zurich, Swizerland \& Rayne Institute, St Thomas' Hospital, London, UK

${ }^{3}$ Academic Unit of Cardiovascular Medicine, University of Leeds \& Rayne Institute, St Thomas' Hospital, London, UK

Journal of Cardiovascular Magnetic Resonance 2009, I I (SuppI I):P46

Introduction: $k$-space and time sensitivity encoding ( $k-t$ SENSE) exploits coil encoding and spatiotemporal correlations and thus allows substantial acceleration of CMR data acquisition. $k-t$ SENSE has been used to improve temporal or spatial resolution of perfusion CMR at I.5 Tesla. In this prospective study high spatial resolution $k-t$ SENSE CMR perfusion at 3 Tesla was compared to fractional flow reserve (FFR), as the reference method for detection of flow-limiting coronary stenoses in the catheter laboratory.

Purpose: To determine the diagnostic accuracy of high spatial resolution $k$ - $t$ SENSE CMR perfusion at 3 Tesla to detect flowlimiting coronary stenoses in patients with stable coronary disease.

Methods: $k-t$ SENSE accelerated perfusion CMR was performed on a 3 Tesla Philips Achieva system. A saturation recovery gradient echo pulse sequence was implemented with a repetition time/echo time $3.0 \mathrm{~ms} / \mathrm{I} .0 \mathrm{~ms}$, flip angle $15^{\circ}, 5 \times \mathrm{k}-\mathrm{t}$ SENSE acceleration, II interleaved training profiles, spatial resolution $\mathrm{I} . \mathrm{I} \times \mathrm{I} . \mathrm{I} \times 10 \mathrm{~mm}^{3}, 3$ slices acquired at each RR interval. Patients with known or suspected coronary artery disease were studied during adenosine stress (an intravenous infusion of adenosine at $140 \mu \mathrm{g} / \mathrm{kg} / \mathrm{min}$, Adenoscan, Sanofi-
Figure I (abstract P46)

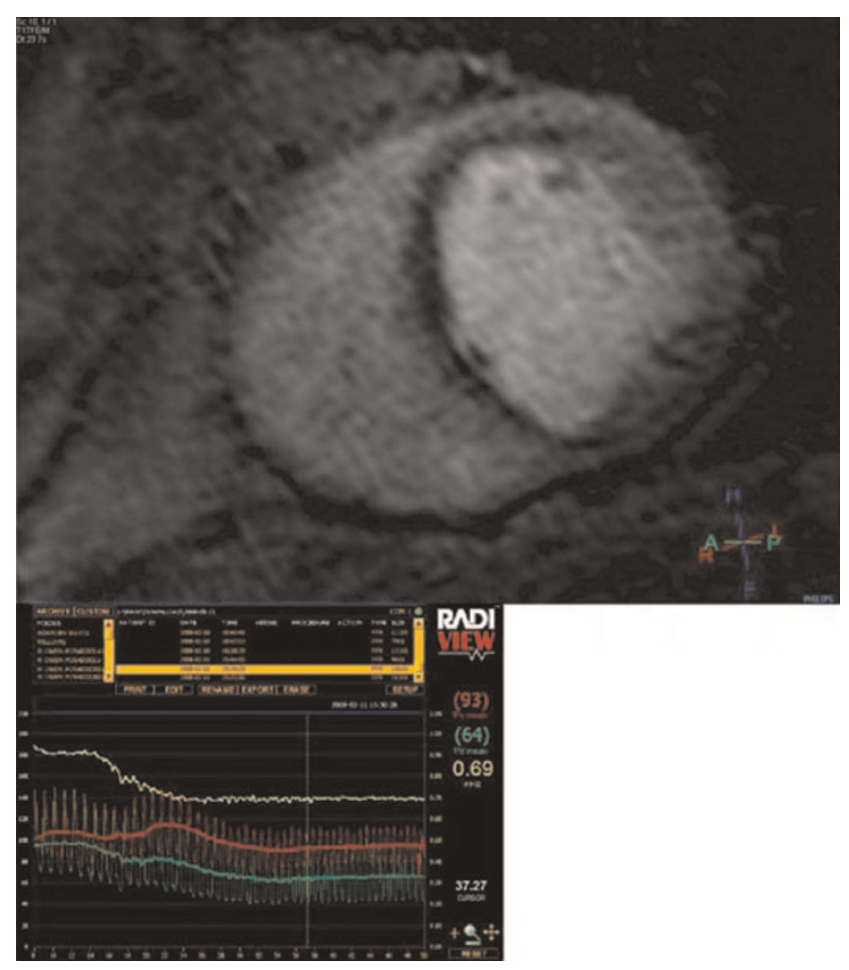

An anterior wall perfusion defect with FFR trace below showing a significant lesion (FFR 0.69) in the proximal left anterior descending artery. An FFR $<0.75$ is considered to be haemodynamically significant.

Synthelabo, Guildford, United Kingdom) and at rest. Main exclusion criteria were previous MI, previous CABG, renal failure, severe LV dysfunction or significant co-morbidities. All patients underwent clinically indicated cardiac catheterisation within 48 hours of CMR. Invasive measurements of FFR were performed in the catheter laboratory on all lesions of more than $40 \%$ severity. FFR was calculated as $\left(P_{d}-P_{v}\right) /\left(P_{a}-P_{v}\right)$, where $P_{a}, P_{v}$ and $P_{d}$ are simultaneous aortic, right atrial and distal coronary pressures measured during an intravenous infusion of adenosine at $140 \mu \mathrm{g} / \mathrm{kg} / \mathrm{min}$. A vessel with $<40 \%$ stenosis angiographically was assumed to be non-flow limiting. The ability of visual analysis of CMR to detect the presence of myocardial ischaemia per coronary territory was determined, blinded to the results of the angiogram and the FFR result.

Results: Seventeen patients were recruited (12 male, age $64.1 \pm 7.2$ years). One patient was excluded from the analysis because of technical problems with the FFR measurement, so that 48 coronary territories were studied. There were no complications with the adenosine infusion in either the CMR scan or during the pressure wire studies. Mean scanning time was $54 \pm 15$ minutes. Coronary stenosis of $>40 \%$ was seen in 20 vessels which underwent successful pressure wire assessment. Of these, 10 lesions had an FFR $<0.75$ (mean $0.50 \pm 0.14$ ) and 10 lesions had an FFR $\geq 0.75$ (mean $0.87 \pm 0.94$ ). Sensitivity and specificity of CMR perfusion to detect coronary stenoses at a threshold of FFR $<0.75$ was $84 \%$ and $97 \%$, respectively. An 
example of an anterior-septal wall perfusion defect with the corresponding FFR trace is shown in figure I.

Conclusion: k-t SENSE accelerated high-resolution perfusion MR at 3.0 Tesla accurately detects flow-limiting coronary artery disease as defined by FFR. Compared with previous reports, these results demonstrate very high specificity of perfusion CMR, possibly as a result of the high spatial resolution at which endocardial dark rim artefacts are reduced.

\section{P47}

Detection of coronary artery disease at 3 Tesla using a visual interpretation algorithm combining perfusion and delayed enhancement imaging Jayanth R Arnold, Theodoros D Karamitsos, Jane M Francis, Tammy J Pegg, Nick Searle, Stefan Neubauer and Joseph B Selvanayagam John Radcliffe Hospital, Oxford, UK

Journal of Cardiovascular Magnetic Resonance 2009, I I(SuppI I):P47

Introduction: Myocardial stress perfusion imaging with cardiovascular magnetic resonance (CMR) is increasingly used in the assessment of coronary artery disease (CAD). It has been demonstrated that a multiparametric approach combining perfusion and infarction imaging at I.5 Tesla further augments the diagnostic performance of CMR. Recent studies indicate that 3 Tesla is the preferred field strength for perfusion imaging, with increased signal-to-noise and contrast-to-noise ratios compared with I.5 Tesla. We sought to assess the diagnostic performance of a visual interpretation algorithm combining perfusion and infarction imaging at 3 Tesla.

Methods: Subjects scheduled for elective diagnostic angiography for investigation of exertional chest pain were studied prior to angiography. Patients were studied with first-pass perfusion at 3 Tesla (Trio, Siemens Medical Solutions), at stress (140 mcg/kg/ min intravenous adenosine) and at rest. Three short-axis images were acquired every heartbeat using a saturation recovery fast gradient echo sequence and $0.05 \mathrm{mmol} / \mathrm{kg}$ contrast agent (Gadodiamide, Omniscan $^{\mathrm{TM}}$, GE Healthcare) bolus injection.
Perfusion images were acquired every cardiac cycle during the first pass of contrast, using a $T_{1}$-weighted fast gradient echo sequence (echo time I.04 ms, repetition time $2 \mathrm{~ms}$, voxel size $\left.2.1 \times 2.6 \times 8 \mathrm{~mm}^{3}\right)$. After rest perfusion, following a further bolus of Gadodiamide $(0.045 \mathrm{mmol} / \mathrm{kg})$, delayed enhancement CMR was performed with a TI-weighted segmented inversionrecovery turbo fast low-angle shot $(\mathrm{FLASH})$ sequence (echo time $4.8 \mathrm{~ms}$, voxel size $1.4 \times 2.4 \times 8 \mathrm{~mm}^{3}$, flip angle $20^{\circ}$ ). Resting cine, stress and rest perfusion, and delayed enhancement images were interpreted visually by a single observer blinded to clinical and angiographic data. The diagnosis of CAD was determined by the presence of delayed hyperenhancement or reversible perfusion defects. Matched stress-rest perfusion defects in the absence of delayed enhancement were considered artifactual. To determine interobserver variability, all scans were interpreted by a second blinded observer. Quantitative coronary angiography, performed by a third operator blinded to CMR results, served as the reference standard. Significant CAD was defined angiographically as the presence of $>=\mid$ stenosis of $>=50 \%$ diameter in any of the main epicardial coronary arteries or their branches with a diameter of $>=2 \mathrm{~mm}$.

Results: Sixty-five subjects were prospectively recruited. Two individuals did not complete the CMR examination owing to claustrophobia and one withdrew consent, so 62 subjects were included in the final analysis. The prevalence of CAD was $66 \%$. All CMR images were visually interpretable. For the diagnosis of significant CAD, the combination of perfusion and delayed enhancement CMR had a sensitivity, specificity and diagnostic accuracy of $90 \%, 81 \%$, and $87 \%$ respectively, compared with $95 \%, 62 \%$, and $84 \%$ for perfusion alone. The combined algorithm had higher specificity and accuracy owing to the high specificity (95\%) of delayed enhancement imaging. The addition of resting wall motion analysis did not improve the diagnostic yield further. Receiver operating characteristic curve analysis demonstrated that the combination of perfusion and delayed enhancement imaging provided better performance than perfusion, delayed enhancement or wall motion assessment alone (figure I). Diagnostic accuracy was high in single-vessel and multivessel disease $(84 \%$ and $81 \%$, respectively), and independent of CAD location (LAD $82 \%$, LCx $81 \%$ and RCA

Figure I (abstract P47)
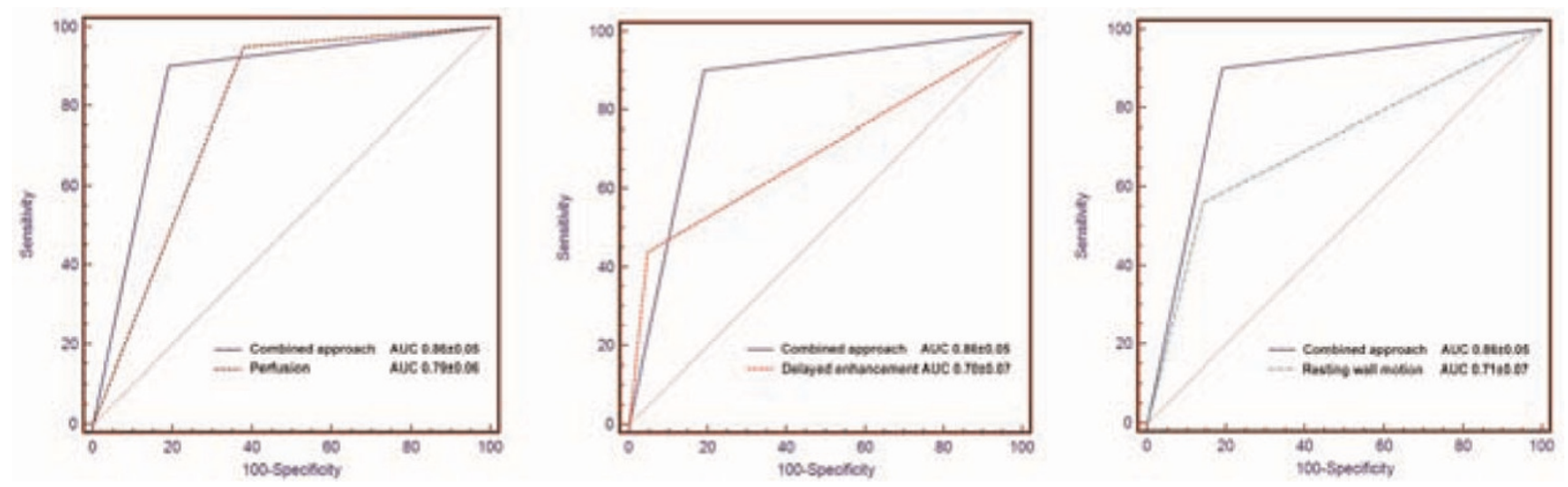

ROC curves comparing the combined visual interpretation algorithm and individual components for the detection of significant CAD. 
$81 \%$ ). There was excellent interobserver agreement for the overall determination of CAD using the combined algorithm (kappa $=0.82)$.

Conclusion: CMR imaging at 3 Tesla has high diagnostic accuracy for the identification of significant CAD. Combining perfusion and infarction imaging is superior to perfusion imaging alone and further augments diagnostic performance.

\section{P48 \\ Prevalence of non-cardiac incidental findings during routine clinical $C M R$ assessment Thomas R Burchell, Didier Locca, Anthony Mathur, Ceri Davies and Mark A Westwood London Chest Hospital, UK, London, UK}

Journal of Cardiovascular Magnetic Resonance 2009, I I (SuppI I):P48

Introduction: Over the last few years there has been a substantial increase in the use of cross sectional imaging modalities to assess the heart. Cardiac MRI (CMR) is now routinely used in the assessment of a variety of cardiac pathologies. It is not uncommon to identify non-cardiac abnormalities during image review. The frequency of non-cardiac findings has been previously addressed using cardiac CT, with 7 published studies totalling 2,495 patients. The prevalence of noncardiac findings was 529 (21\%) with a range of $5 \%$ to $69 \%$. The majority of the abnormalities were pulmonary, most commonly small isolated nodules $(<10 \mathrm{~mm})$. The diversity and significance of these incidental findings has to date not been assessed with CMR.

Objectives: The purpose of our study was to assess the prevalence of incidental non-cardiac findings found during routine CMR assessment.

Methods: The reports of $7 / 4$ consecutive adult CMR studies, performed for all current clinical indications with the exception of adult congenital heart disease, were reviewed retrospectively. These were performed between February and August 2008 on a Philips Achieva CV I.5 Tesla MRI scanner (Philips Medical Systems, Best, The Netherlands). The studies were performed and reported by consultant cardiologists, in accordance with SCMR standardised acquisition protocols.

A multislice single shot steady state sequence was used as an initial scout scan. Three orthogonal planes were acquired (transverse, coronal and sagittal) with a large field-of-view coverage. Axial black blood images were also obtained ( 10 to 16 slices) using turbo spin-echo and a double inversion prepulse (TE/TR $=20 / 1500 \mathrm{~ms}$ ).

Results: The mean patient age was $61.3+/-15$ years (range 16 to 86 years), and $476(67 \%)$ were male. $218(24 \%)$ of the patients had previously undiagnosed non-cardiac abnormalities. These included, renal cysts ( $9 \%)$, aortic pathology (3\%), lung collapse/ consolidation ( $2 \%)$, liver cysts $(2 \%)$, lung mass (I\%). There were also 2 cases of mediastinal masses, I mediastinal lympahadenopathy, 6 increased signal from the hila, I renal mass and one large hiatus hernia.

Conclusion: The detection rate of non-cardiac findings on routine CMR scans is relatively high and broadly similar to that of cardiac CT. Unlike CT, CMR detects very few incidental lung findings. The CMR scout scan has a larger field of view than cardiac $\mathrm{CT}$, allowing routine imaging of the abdomen, which explains the high number of intra-abdominal abnormalities. Further work is needed to assess the clinical significance of these findings.

\section{P49}

Prevalence and potential mechanisms leading to persistent elevation of high sensitive troponin $T$ in patients 6 month after acute myocardial infarction Mirja Neizel', Tim Schaeufele ${ }^{2,3}$, Grigorios Korosoglou', Dirk Lossnitzer², Harald P Kühl', Malte Kelm', Hugo A Katus², Evangelos Giannitsis ${ }^{2}$ and Henning Steen ${ }^{2}$ 'University Hospital Aachen, Aachen, Germany ${ }^{2}$ University Hospital Heidelberg, Heidelberg, Germany ${ }^{3}$ Department of Cardiology, Heidelberg, Germany

Journal of Cardiovascular Magnetic Resonance 2009, I I(SuppI I):P49

Introduction: Persistent minor troponin elevation in patients with coronary heart disease is associated with adverse outcome and prognosis. However, the mechanism is not yet clearly understood.

Purpose: Our objectives were to examine the prevalence and range of cardiac troponin $\mathrm{T}$ (cTnT) in stable patients six months after acute myocardial infarction (AMI) using a new high sensitive $\mathrm{cTnT}$ assay and to investigate the association of persistent minor cTnT-elevation to clinical variables, NT-pro BNP and cardiac MRfindings.

Methods: cTnT was measured in 98 patients at 6 months after AMI. Patients were investigated in a clinical I.5 Tesla whole body MR-scanner (Achieva, Philips, Best, the Netherlands) $3 \pm$ I days after successful mechanical reperfusion of the infarct related vessel and at a follow-up of $6 \pm \mathrm{I}$ month using a 5-element phased array cardiac synergy coil.

Assessment of resting left ventricular function was determined by cine images using a steady-state-free precession sequence in 10-12 $8 \mathrm{~mm}$-thick slices covering the whole left ventricle from base to apex as well as long axis 2,-3 and 4-chamber views.

Ten minutes after gadolinium contrast injection $(0.2 \mathrm{mmol} / \mathrm{kg}$ body weight of Gadolinium-DTPA (Magnevist ${ }^{\circledR}$, Bayer, Germany) late enhancement-imaging was performed using an inversionrecovery gradient-echo technique triggered to end-diastole. Data analysis of MRI-images was performed using a usual Philips work station.

cTnT was measured using a precommercial assay by electrochemiluminescence methods (Roche Diagnostics, Mannheim, Germany.

Results: Minor cTnT-concentrations were detectable in $90 \%$ of the entire cohort, of whom $16 \%$ had cTnT-values above the $99^{\text {th }}$ percentile (> $12 \mathrm{ng} / \mathrm{L})$. These patients were also significantly older, suffered more frequently from hypertension, had a higher New York Heart Association class and received more often diuretics at follow-up. Patients with cTnT-elevation had a more impaired left ventricular ejection fraction $(p=0.02)$ but did not have an increased infarct size $(p=0.73)$.

Conclusion: Elevated minor cTnT levels are frequently detectable in patients 6 month after AMI. Increased cTnT-level were associated with clinical parameter for heart failure, impaired 
ejection fraction and higher NT-pro-BNP levels suggesting that myocardial dysfunction leads to persistent cTnT-elevation.

\section{P50 \\ Relationship between infarct gray zone and characteristics of ventricular tachycardia using multi-contrast delayed enhancement: preliminary results \\ Gideon A Paul', Jay S Detsky², Kim A Connelly', Eugene Crystal', Alexander J Dick' and Graham A Wright ${ }^{2}$ \\ ISunnybrook Health Sciences Centre, Toronto, ON, Canada \\ ${ }^{2}$ University of Toronto, Toronto, ON, Canada}

Journal of Cardiovascular Magnetic Resonance 2009, I I (SuppI I):P50

Introduction: Myocardial infarction (MI) consists of a central fibrous scar surrounded by a heterogeneous region of viable and non-viable myocytes. The peri-infarct gray zone provides the potential substrate for lethal ventricular arrhythmias via the reentry phenomena. The extent of the gray zone, characterised by delayed-enhancement MRI (DE-MRI), has been shown to be a strong predictor of mortality [I] and to the susceptibility for ventricular tachycardia (VT) [2] in patients with a history of MI. We have recently shown that quantification of the gray zone using conventional inversion recovery gradient echo (IR-GRE) images is sensitive to both image noise and the accuracy of the required manual contours of the blood pool [3]. A new multicontrast delayed enhancement (MCDE) sequence [4] is an alternative approach to DE-MRI for which an automated data analysis procedure has been developed that provides more robust gray zone quantification [3].

Purpose: In this work, preliminary results are presented examining the relationship between the extent of gray zone and inducibility of VT as well as VT cycle length.

Methods and results: Eight patients (mean age $=67$, all men) with an ischemic cardiomyopathy referred for implantable cardioverter defibrillator (ICD) implantation (according to MADIT II criteria [5] or for secondary prevention of VT) underwent a cardiac magnetic resonance (CMR) examination. Five of the eight patients had documented evidence of prior VT. All patients underwent assessment of cardiac function (cine SSFP), followed by viability imaging ten minutes after administration of $0.2 \mathrm{mmol} / \mathrm{kg}$ Gd-DTPA using both IR-GRE and MCDE sequences. The MCDE sequence uses a segmented SSFP

Table I (abstract P50) CMR-derived infarct characteristics for patients with inducible and non-inducible VT

\begin{tabular}{llccl}
\hline & & Inducible & Non-inducible & P \\
\hline MCDE & Infarct core, g & $18.3 \pm 10.4$ & $11.9 \pm 14.8$ & 0.50 \\
MCDE & Gray zone, g & $16.3 \pm 0.7$ & $6.6 \pm 0.7$ & 0.046 \\
MCDE & Total, g & $34.7 \pm 11.0$ & $18.5 \pm 22.4$ & 0.22 \\
IR-GRE & Infarct core, g & $17.3 \pm 7.1$ & $8.7 \pm 10.9$ & 0.22 \\
IR-GRE & Gray zone, g & $10.4 \pm 4.6$ & $7.0 \pm 8.2$ & 0.48 \\
IR-GRE & Total, g & $27.7 \pm 11.7$ & $15.7 \pm 19.0$ & 0.31 \\
SSFP & EF (\%) & $25 \pm 5$ & $31 \pm 19$ & 0.55 \\
\hline
\end{tabular}

Figure I (abstract P50)

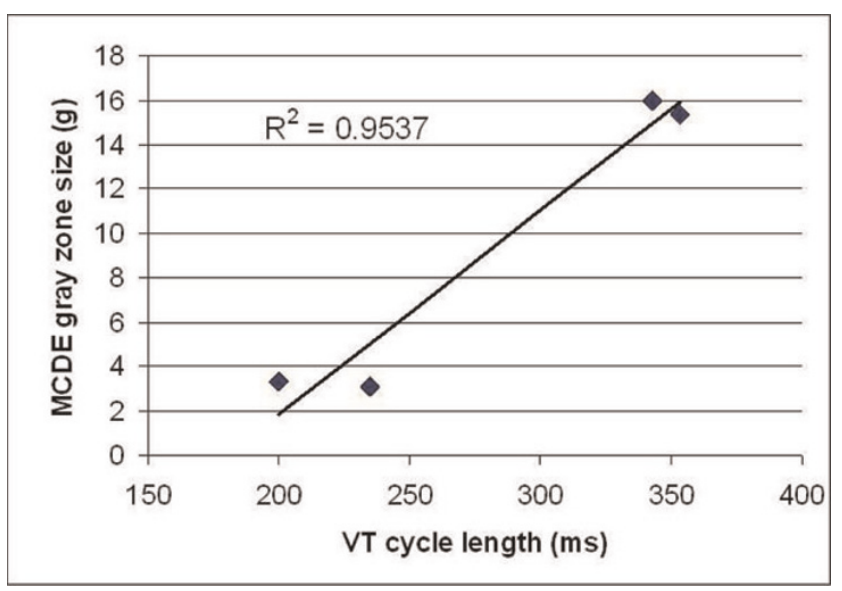

Correlation between VT cycle length and MCDE-derived gray zone.

acquisition following an inversion pulse, yielding 20 images at various effective inversion times. The IR-GRE gray zone analysis was performed using methods described by Schmidt et al [2] whilst for MCDE it was performed using an automated data clustering algorithm previously described [3].

VTwas inducible at the time of ICD implantation in three patients (two of whom did not have any prior episodes of VT) and noninducible in five patients. Imaging results within the two patient groups are shown in Table I. There was a statistically significant difference in the size of the gray zone in inducible (16.3 $\pm 0.7 \mathrm{~g})$ compared to non-inducible $(6.6 \pm 7.7 \mathrm{~g})$ patients using MCDE $(p=0.046)$ which was not observed with IR-GRE $(10.4 \pm 4.6 \mathrm{~g}$ versus $7.0 \pm 8.2 \mathrm{~g}, \mathrm{p}=0.48)$. There was no statistical difference between the two groups with regards to infarct core and total infarct size measured by either DE-MRI sequence or in the ejection fraction calculated using cine SSFP images. There was a strong correlation between the size of the gray zone as measured by MCDE and the VT cycle length $\left(R^{2}=0.95\right.$, Fig I). Note that in one patient with a prior occurrence of VT, the cycle length is currently unknown.

Conclusion: These results confirm that inducibility for VT is correlated to the size of the peri-infarct gray zone as assessed by MCDE. The higher variability in the IR-GRE analysis may contribute to the lack of a significant difference in the gray zone size between inducible and non-inducible patients in this small group of patients. We have also shown that the VT cycle length is correlated to the size of the MCDE-derived gray zone, consistent with previous results showing a positive correlation between VT cycle length and isthmus size measured during programmed electrical stimulation [6].

\section{References}

I. Yan A: Circ 2006, II4.

2. Schmidt A: Circ 2007, 115.

3. Detsky JS: SCMR 2008, 25.

4. Detsky JS: MRM 2007, 58.

5. Moss AJ: NEJM 2002, 346.

6. Ciaccio : Circ 200I, 104. 
P5 I

Assessment of cardiac oedema in patients with acute myocardial infarction by manual planimetry and computerised segmentation of triple inversion recovery prepared turbo spin echo images

Robert I Johnstone', John P Ridgway', John D Biglands', John P Greenwood ${ }^{2}$ and Aleksandra Radjenovic ${ }^{2}$

${ }^{\prime}$ Leeds Teaching Hospitals Trust, Leeds, UK

${ }^{2}$ University of Leeds, Leeds, UK

Journal of Cardiovascular Magnetic Resonance 2009, I I (SuppI I):P5 I

Introduction: The presence of oedema in human tissue increases the observed longitudinal and transverse magnetisation relaxation times ( $\mathrm{TI}$ and $\mathrm{T} 2$ ). $\mathrm{MRI}$ can therefore be used to assess the extent of cardiac oedema after a myocardial infarction (MI). The oedematous myocardium is considered to be at risk of becoming infarcted. The oedema mass in the left ventricle (LV) may therefore be a useful indicator of prognosis and of the effectiveness of treatment. Current methods of quantifying cardiac oedema are essentially visual in nature, involving either contouring and planimetry or the rating of oedema severity in cardiac segments. Inter- and intra-observer variability reduces the sensitivity of these techniques to small changes in mass.

Purpose: To establish an automated method for the quantification of cardiac oedema and to compare the results with a visual method.

Methods: Twenty patients ( 12 male, aged 4I-78 [mean 6I]) were scanned within 48 hours of the onset of Ml symptoms and seven were rescanned four weeks later. A triple inversion recovery prepared turbo spin echo sequence was used on a Philips Intera CV I.5 T system. 2D short-axis images of thickness $10 \mathrm{~mm}$ were acquired to cover the entire LV with no inter-slice gaps. Epicardial and endocardial contours were added manually to each slice image.

Oedematous regions apparent in the images were manually contoured by a cardiologist who also had access to steady state free precession cine images and late enhancement images. An automated computer segmentation was carried out using the LV contours and triple inversion recovery prepared images. The voxel intensity histogram for the entire LV was clustered by fitting two Gaussian functions to it. An initial segmentation was performed on the basis of a signal intensity threshold set at two standard deviations above the mean of the lower intensity Gaussian. Three-dimensionally connected regions with a mass less than I g were discarded, as were those regions that lay

Figure I (abstract P5I)
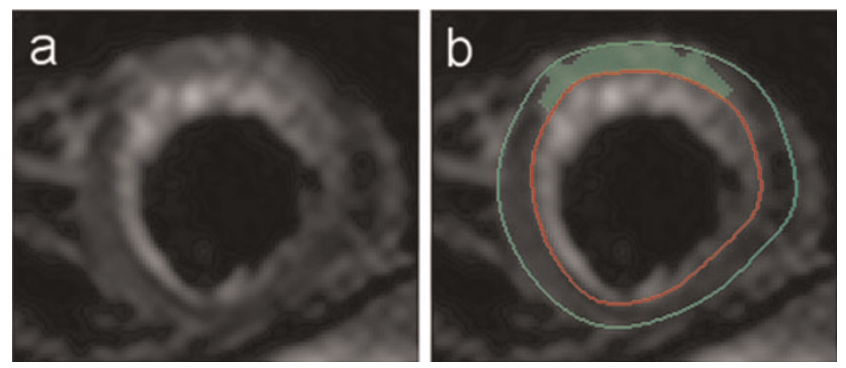

(a) Raw slice image, (b) Slice image with LV contours added and computer segmentation shaded.
Figure 2 (abstract P5I)

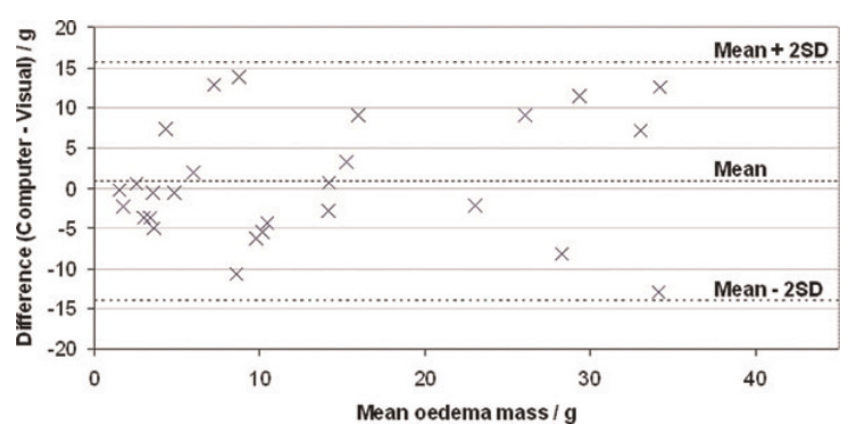

Bland-Altman plot of differences between computer and visual segmentation.

further than $2 \mathrm{~mm}$ from the endocardium. Finally, the surfaces of the regions were smoothed and holes within them were filled.

The oedema masses for the visual and computer segmentations were calculated assuming a density of $1.05 \mathrm{~g} \mathrm{~cm}^{-3}$.

Results: An example of a triple inversion recovery prepared turbo spin echo image is shown in Figure la. The same image slice is shown in Figure Ib with the LV contours and computer segmentation overlaid. A Bland-Altman analysis did not show statistically significant bias between the computer segmentation and the visual segmentation $(+0.8 \pm 2.9 \mathrm{~g}$, with a $95 \%$ confidence interval). The standard deviation of the differences (SDD) was $7.4 \mathrm{~g}$. The differences from the mean of the two segmentation methods are plotted against the mean in Figure 2. The mean oedema mass measured visually was $13.6 \mathrm{~g}$ compared to $12.8 \mathrm{~g}$ for the computer segmentation.

Conclusion: The computer algorithm described has demonstrated the segmentation of cardiac oedema within images having a T2 weighting. A comparison with a visual segmentation shows no significant bias. The performance of the visual segmentation is limited by the performance of the human visual system, while the computer segmentation is hindered by its lack of anatomical and pathological knowledge. Further work is required to assess whether the computer algorithm can improve scan-rescan variability and make clinically accurate assessments of the severity and progression of cardiac oedema.

\section{P52}

The relationship of left ventricular function to infarct surface area and volume Al-Hakim Ramsey', Michael C Fishbein', Thomas O'Donnell ${ }^{2}$, James Sayre', Kalyanam Shivkumar', Alan Kadish ${ }^{3}$, Paul Finn' and Carissa G Fonseca' 'University of California Los Angeles, Los Angeles, CA, USA

${ }^{2}$ Siemens Corporate Research, Princeton, NJ, USA

${ }^{3}$ Northwestern University, Chicago, IL, USA

Journal of Cardiovascular Magnetic Resonance 2009, I I(SuppI I):P52

Objective: To examine the relationship between left ventricular (LV) myocardial function and myocardial infarct (MI) surface area and volume.

Background: Delayed-enhancement MRI (DE-MRI) has been used successfully to describe the relationship between the volume of scar tissue and LV function [I]. Due to volume 
Figure I (abstract P52)

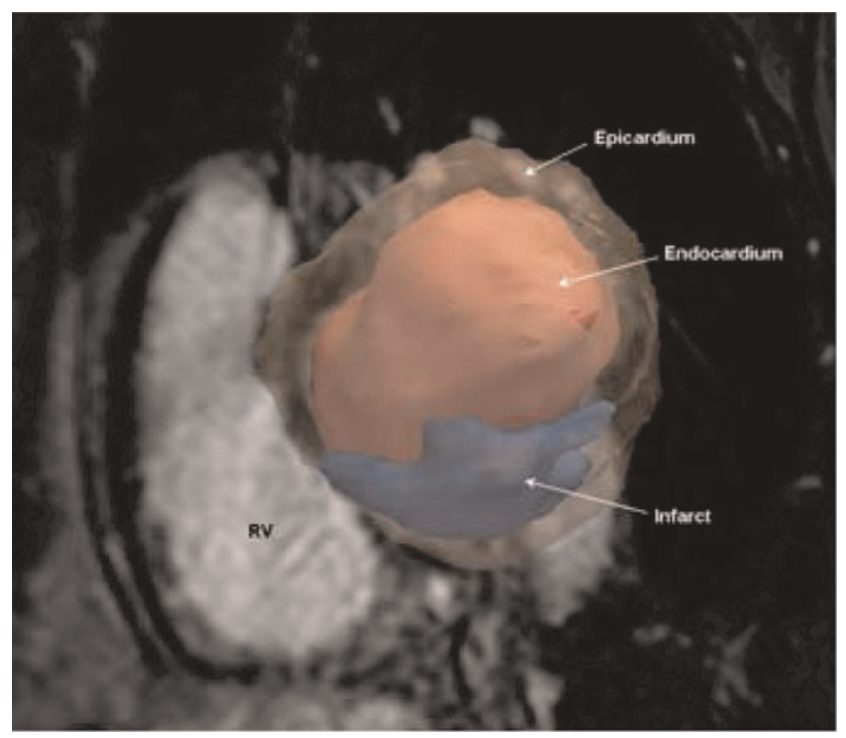

$3 D$ rendering of $L V$ and infarct (infarct mass: $14.1 \mathrm{~g}$, iSA: $52.9 \mathrm{~cm}^{2}$, iSA/V: $3.9 \mathrm{~cm}^{2} / \mathrm{ml}$ ) superimposed on a basal LV short-axis DE-MR image.
Figure 2 (abstract P52)

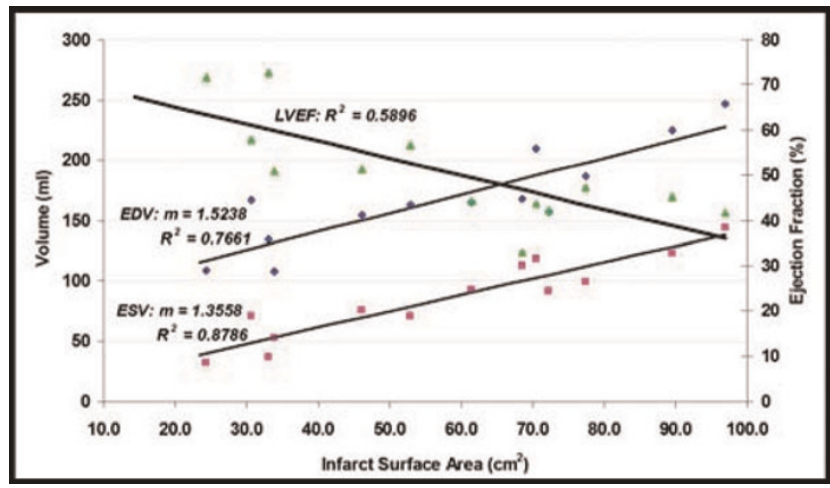

Relationshop of LV volumetric measurements to infarct surface area.

shrinkage of hyperenhanced tissue over time, DE-MRI derived infarct surface area (iSA) and the iSA to infarct volume ratio (iSA/ V) may provide more stable indices of the extent of infarction than does scar volume and may better predict LV functional impairment.

Figure 3 (abstract P52)

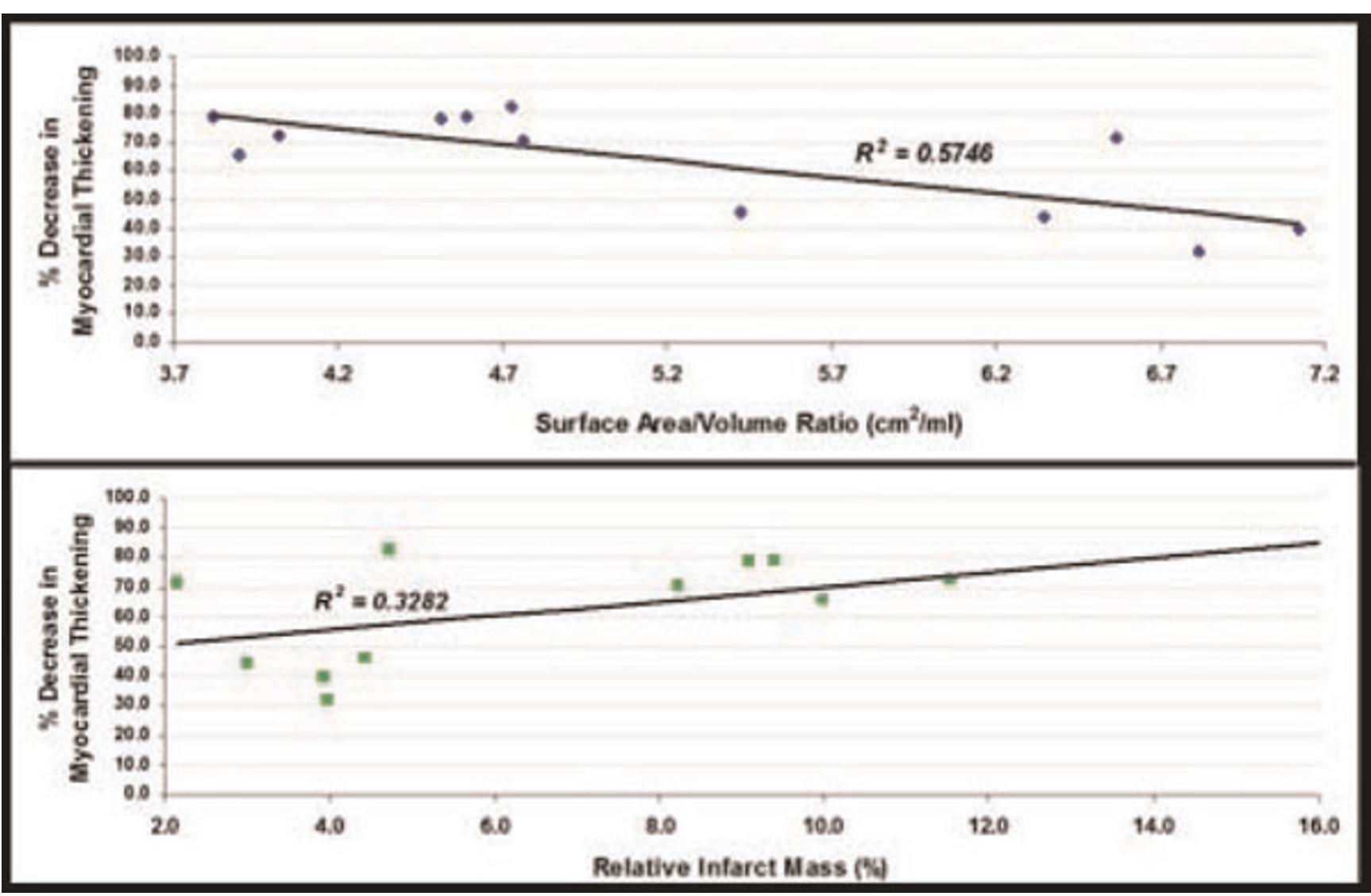

Decrease in LV wall thickening in relation to infarct surface area to volume (iSA/V) ratio and relative infarct mass. 
Methods: Segmented SSFP cardiac cine and multi-shot inversion recovery spoiled gradient echo DE-MR images were obtained at I.5 T, in I3 patients (I I M, 2 F; aged 40-82 years, mean $60.3 \pm 12.3$ years) with documented myocardial infarction from the Defibrillators To Reduce Risk by Magnetic Resonance Imaging Evaluation (DETERMINE) study. Cine and DE images were acquired in corresponding short-axis planes (spatial resolution $\leq 2.5 \times 2.0 \times 10.0 \mathrm{~mm}$; slice gap $\leq 2 \mathrm{~mm}$ ) from the base of the heart to the apex. 3DSlicer v2.6, free open source image analysis software (slicer.org), was used to manually segment and create a three-dimensional model of the infarct from which volume and iSA were derived (Fig I).

LV function was assessed using CIM software [2], which provided the following global measurements: LV mass, LVEF, LV enddiastolic volume (EDV), and LV end-systolic volume (ESV). CIM also provided segmental data based on a 16 segment LV model: myocardial thickening (peak thickness - minimum thickness), EDV, ESV, and EF. Infarct and LV mass were calculated from their respective volumes assuming a constant density of $1.04 \mathrm{~g} / \mathrm{ml}$. Midventricular segments that visually contained greater than $50 \%$ hyper-enhancement on DE-MRI were considered infarcted. Segments which were non-infarcted and not adjacent to infarcted segments were considered remote. Statistical comparisons between infarct and remote regions were made using a paired, two-tailed student's t-test. For linear relationship comparisons, the standard error of the estimate was calculated to examine goodness-of-fit.

Results: Infarct mass correlated with iSA ( $r=0.958)$. EDV and ESV both increased with iSA $(r=0.875$ and 0.937 respectively; Fig 2). ESV correlated more strongly with iSA than with relative infarct mass (100*infarct mass/LV mass; $r=0.499 ; p<0.05)$. LVEF decreased with relative infarct mass $(r=-0.526)$ and showed a higher correlation with iSA $(r=-0.768, p<0.01)$. Relative infarct mass correlated with iSA/V $(r=-0.845)$. A relationship was not observed between iSA/V and LVEF $(r=0.298)$.

Thickening of the infarcted region was reduced compared with the remote region $(3.47 \mathrm{~mm} \pm \mathrm{I} .95$ vs. $9.79 \mathrm{~mm} \pm 2.47, \mathrm{p}<0.00 \mathrm{I})$. The percent reduction in thickening of infarcted myocardium showed a negative correlation with iSA $N(r=-0.758)$ and a positive correlation with relative infarct mass $(r=0.573$; Fig 3$)$, the correlation with iSA $/ V$ being stronger $(P<0.000 I)$.

Conclusion: Impaired LV function is strongly related to infarct surface area. Relative infarct mass may underestimate the extent of damage post-MI because of thinning of infarcted myocardium during remodeling. Infarct surface area is less affected by volume shrinkage, and may be more stable over time than infarct mass in measuring the loss of functioning myocardium.

A greater decrease in LV wall thickening is associated with a larger relative infarct mass and surface area but a smaller iSA/V. A transmural increase in infarct size may increase infarct volume with little change in iSA. Infarct transmurality greater than $50 \%$ is associated with severe loss of myocardial function and mortality [3]. Thus, iSA/V can provide an objective quantitative index of transmurality, superior to relative infarct mass for predicting decrease in myocardial function.

\section{References}

I. Kolipaka A, Charzimavroudis GP, White RD, Lieber ML and Setser RM: Relationship between the extent of nonviable myocardium and regional left ventricular function in chronic ischemic heart disease. J Cardiovasc Magn Reson 2005, 7:573-579.

2. Chan J, Khafagi F, Young AA, Cowan BR, Thompson $C$ and Marwick TH: Impact of coronary revascularization and transmural extent of scar on regional left ventricular remodelling. Eur Heart J 2008, 29:1608-1617.

3. Roes SD, Kelle S, Kaandorp TA, Kokocinski T, Poldermans D, Lamb HJ, Boersma E, van der Wall EE, Fleck E, de Roos A, Nagel $E$ and $B a x ~ J$ : Comparison of myocardial infarct size assessed with contrast-enhanced magnetic resonance imaging and left ventricular function and volumes to predict mortality in patients with with healed myocardial infarction. Am J Cardiol 2007, 100:930-936.

\section{P53}

Contribution of the base of the heart to MR measures of left ventricular mass, volumes and scar in patients with myocardial infarction

Carissa G Fonseca, Tariq Balawi,

James Sayre and Paul Finn

UCLA, Los Angeles, CA, USA

Journal of Cardiovascular Magnetic Resonance 2009, I I (SuppI I):P53

Introduction: In planimetric measurement of left ventricular (LV) mass and volumes, selection of the most basal short-axis cine MR image plane is generally not standardized and may affect calculation of relative infarct size in post-myocardial infarct (MI) patients.

Purpose: To study the effect of LV base-plane selection on MR measures of LV mass, volumes, ejection fraction (EF) and relative infarct size in post-Ml patients.

Methods: SSFP cardiac cine and multi-shot inversion recovery spoiled gradient echo delayed enhancement (DE) images were acquired in 14 patients with documented $\mathrm{Ml}$ (3 F; mean age $59.6 \pm 10.4$ years), at I.5 T. Cine and DE images were acquired in corresponding short-axis planes (voxel size $\leq 2.5 \times 2.0 \times 10.0$ $\mathrm{mm}$; slice gap $\leq 2 \mathrm{~mm}$ ) from LV base (mitral valve plane) to apex. An expert observer used Argus software (Siemens Medical Solutions) to manually outline LV epi- and endomyocardial contours on cine images at end-diastole (ED) and end-systole (ES) using each of 3 methods for choosing the most basal LV slice: \#I base-plane showing $100 \%$ of myocardium at ED and corresponding ES plane, \#2 base-plane selected separately for ED and ES, with $100 \%$ of myocardium visible at both phases, \#3 base-plane showing 100\% of myocardium at ES and corresponding ED plane. LV mass was averaged over ED and ES for each method. EF, and ED and ES-volumes were also calculated. Infarct borders were manually outlined on DE images. Infarct size (Infarct\%) was expressed as a percentage of LV mass measured on the cines (in each method). Comparisons were made using repeated measures ANOVA, with Bonferroni-corrected posthoc P-values.

Results: LV mass was largest in method \#I and smallest in \#3 ( I $38.5 \pm 3 \mid .2$ vs. $\mid 33.0 \pm 29.3$ vs. $|24.8 \pm 27.3 \mathrm{~g}, \mathrm{P}<0.0|$ for all comparisons). Method \#2 produced a larger EF (5I.3 $\pm 8.9 \%)$ vs. \#I $(43.8 \pm 9.4 \%)$ or \#3 (43.9 $\pm 10.8 \%) ; P<0.01$. Infarct $\%$ was smallest in method $\# \mathrm{I}$ and differed significantly between all methods $(9.7 \pm 4.9$ vs. $10.2 \pm 5.3$ vs. $10.9 \pm 6.0 \%, P<0.05)$. ED 
volume was lower in \#3 than in \#I or \#2 $(P<0.01)$ and ES volume was higher in \#I than in \#2 or \#3 $(P<0.01)$.

Conclusion: Selecting the base-plane based on the anatomy visible in only one phase, will lead to miscalculation of MRI measures of LV mass, volumes and relative infarct size, due to through-plane motion of the heart over the cycle. To standardize measurements and to ensure that the same tissue is being measured at both phases, the most basal slice should be selected independently for ED and ES based on the visible anatomy.

\section{P54}

Comparison of right ventricular

involvement in $A L$ and transthyretin-type

cardiac amyloidosis by cardiovascular

magnetic resonance

Evan Appelbaum', Caitlin Harrigan',

Leah Biller', Warren J Manning

Frederick L Ruberg ${ }^{2}$, Kenneth Tripp ${ }^{3}$, Jeff Packman ${ }^{3}$ and Donna Grogan

'PERFUSE CMR Core Lab, Beth Israel Deaconess Medical

Center, Boston, MA, USA

${ }^{2}$ Amyloid Treatment and Research Program

and Section of Cardiology, Department of Medicine,

Boston University School of Medicine, Boston, MA, USA

${ }^{3}$ FoldRx Pharmaceuticals, Inc, Cambridge, MA, USA

Journal of Cardiovascular Magnetic Resonance 2009, I I (SuppI I):P54

Background: Increased right ventricular (RV) wall thickness and RV dysfunction are adverse prognostic signs in primary (AL) cardiac amyloidosis and suggest more extensive disease. RV involvement in other forms of cardiac amyloidosis remains poorly understood. We sought to determine differences in RV involvement for those with transthyretin (TTR) type and lightchain $(\mathrm{AL})$ type cardiac amyloidosis using cardiovascular magnetic resonance (CMR).

Methods: We assessed a multi-center cohort of subjects with proven cardiac amyloidosis due to $\mathrm{AL}(\mathrm{n}=2 \mathrm{I}, 14 \mathrm{M}$, age $6 \mathrm{I}+/-$ II.5 yr) and TTR ( $\mathrm{n}=14 \mathrm{I} 2 \mathrm{M}$, age $73+/-4.8 \mathrm{yr})$ using conventional cine SSFP CMR. RV thickening was defined as a wall thickness $=6 \mathrm{~mm}$. Volumetric assessments of RV free wall mass, volumes and ejection fraction (EF) were measured by an independent core lab blinded to patient data.

Results: The prevalence of RVH was greater in the TTR group (7I\% vs. $48 \% A L$ group, $p=0.01$ ). Similarly, RV free wall mass was increased in the TTR subjects $(55.4 \pm 20.6 \mathrm{~g}$ vs $37.3 \pm \mathrm{I} 5.4 \mathrm{~g}$; $\mathrm{P}<0.0 \mathrm{I})$. In addition, RV end-diastolic volume was greater in TTR amyloidosis ( $190 \pm 39 \mathrm{~g}$ vs I $38 \pm 48 \mathrm{~g}$; $\mathrm{p}<0.0 \mathrm{I})$ while RVEF was lower in the TTR subjects $(37 \pm 11 \%$ vs $53 \pm 13 \%$; $p<0.001)$. There were no differences in the degree of heart failure symptoms in the TTR and AL groups: NHYA Class I: 4 vs 5; Class II/III: 10 vs I5; Class IV: 0 vs I, respectively $(P=0.69)$.

Conclusion: These data suggest that, while similar in clinical heart failure symptoms, TTR amyloidosis appears to have more prevalent and extensive RV involvement than AL. The clinical impact of RV involvement in TTR amyloidosis remains to be determined.
P55

Abnormal myocardial perfusion in hypertrophic cardiomyopathy: preliminary findings of a cardiovascular MRI study

James A White', Sarah Armstrong ${ }^{2}$, Mohammed Al-Admawi ${ }^{2}$, Sherryn Rambihar ${ }^{2}$, Gerald Wisenberg' ${ }^{2}$, Ivonna Verschuur ${ }^{\prime}$, Anna MacDonald ${ }^{2}$, Cyndi Harper-Little ${ }^{3}$, Aaron So ${ }^{3}$, Ting-Yim Lee ${ }^{4}$, Frank Prato ${ }^{4}$ and Terry Thomspon ${ }^{4}$

'Dept. of Medicine and Lawson Health

Research Institute and Robarts Research Institute, University of Western Ontario, London, ON,

Canada

${ }^{2}$ Dept. of Medicine, University of Western Ontario, London, ON, Canada

${ }^{3}$ Robarts Research Institute, University of Western Ontario, London, ON, Canada

${ }^{4}$ Lawson Health Research Institute, University of Western Ontario, London, ON, Canada

Journal of Cardiovascular Magnetic Resonance 2009, I I(SuppI I):P55

Background: Reduced myocardial perfusion has been speculated as a potential mechanism for the development and/or propagation of myocardial fibrosis in hypertrophic cardiomyopathy (HCM). This study aims to evaluate the prevalence, distribution and extent of stress-induced perfusion abnormalities and their relationship to underlying fibrosis in patients with HCM using magnetic resonance imaging.

Methods: 15 patients with echocardiographically diagnosed HCM have been enrolled. Cine imaging, first-pass stress perfusion imaging using vasodilator stress (Dipyridamole), and delayed gadolinium enhancement imaging were performed. Stress hypoperfusion and delayed enhancement images were assessed both quantitatively and visually using a 16-segment model. Conversion of segmental visual scoring to \% of LV by volume was achieved for both hypoperfusion (HP) and late enhancement (LE) using a standardized scoring system. For quantitative assessment prospectively defined cut-offs for LE and HP were used.

Results: Maximal wall thickness ranged from 13 to $22 \mathrm{~mm}$ (mean $17 \pm 2.6 \mathrm{~mm}$ ). Non-ischemic pattern LE was present in $70 \%$ of patients. Perfusion abnormalities were identified on stress perfusion images in $80 \%$ of patients using visual analysis and $87 \%$ of patients using quantitative analysis. Perfusion abnormalities were predominantly subendocardial, and were regionally associated with segments containing LE $(p<0.0 \mathrm{I})$. Mean percent HP and mean percent LE were $17 \pm 8.4 \%$ and $10 \pm 9.3 \%$, respectively by visual estimation and $20.0 \pm$ $12.1 \%$ and $14.0 \pm 7.4 \%$, respectively by quantitative assessment. Figure I.

Conclusion: These preliminary results suggest that patients with HCM have a high prevalence of stress-induced myocardial hypoperfusion as represented by reduced first-pass gadolinium enhancement during vasodilator stress. This hypoperfusion appears to extend beyond regions of established LE suggesting a potential contribution of ischemia in the development and/or propagation of myocardial fibrosis in patients with HCM. 
Figure I (abstract P55)
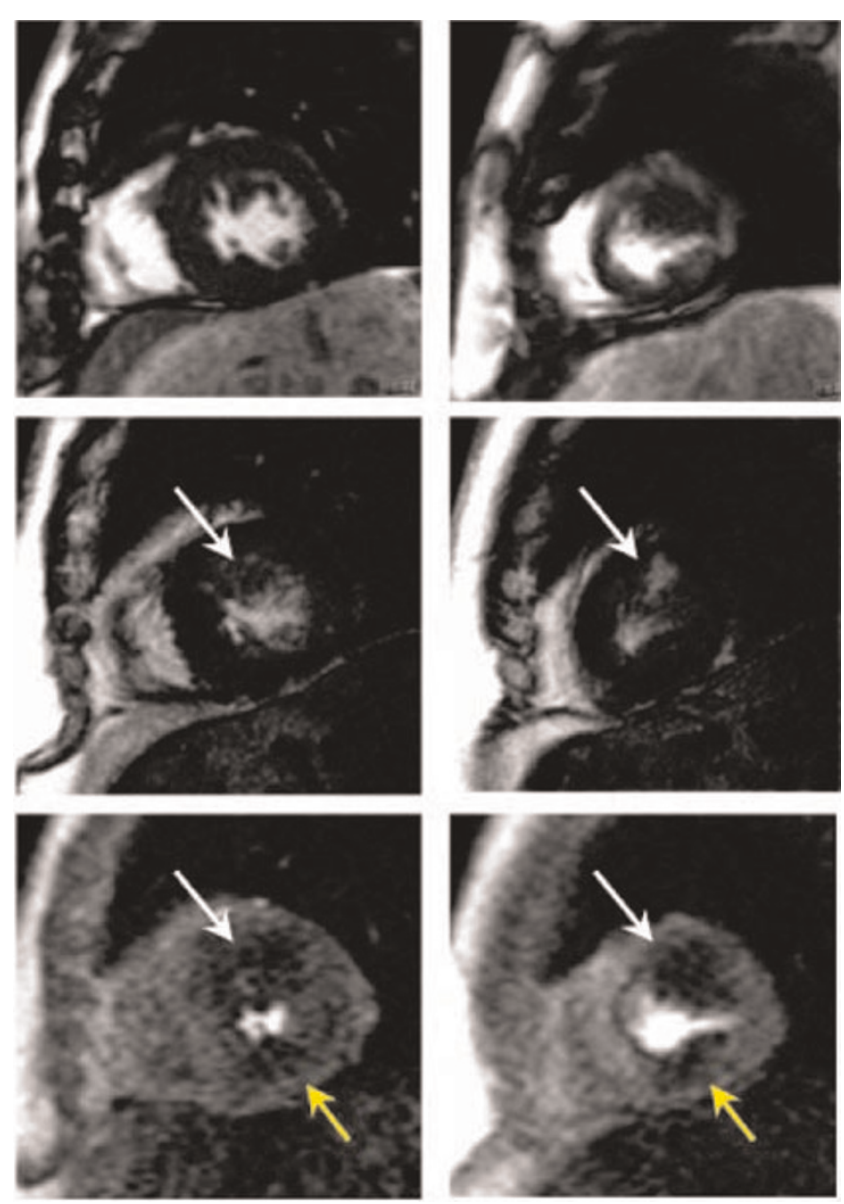

Short axis cine (top), delayed contrast (middle row) and stress perfusion (bottom row) images in a patient with apical hypertrophic cardiomyopathy. Stress perfusion abnormalities seen corresponding to (white arrows) and distinct from (yellow arrows) established fibrosis.

\section{P56}

Prognostic value of normal cardiac MRI in patients with suspected arrhythmogenic right ventricular dysplasia

Mario Njeim, Gurjit Singh, Leonidas Tzogias,

Claudio Schuger, Milan V Pantelic, Khalid Nour

and Mouaz $\mathrm{H}$ Al-Mallah

Henry Ford Hospital, Detroit, MI, USA

Journal of Cardiovascular Magnetic Resonance 2009, I I (SuppI I):P56

Background: Arrhythmogenic right ventricular dysplasia (ARVD) is an inherited cardiomyopathy characterized by ventricular arrhythmias and structural abnormalities of the right ventricle (RV). The aim of this study is to investigate the prognostic value of normal cardiac magnetic resonance imaging (CMR) in patients with suspected ARVD.

Methods: We included patients with clinically suspected ARVD who underwent CMR between I/I/2003 and I2/3I/2007.
Imaging protocol included steady-state acquisition cine imaging, $\mathrm{T}(\mathrm{I})$-weighted black blood imaging with and without fat suppression and post-contrast delayed enhancement on a I.5-T scanner to evaluate ventricular function and morphology, fatty infiltration and regional myocardial fibrosis. Patient who had CMR major criteria for ARVD were excluded from this analysis. In addition, patients in whom the clinical workup revealed alternative diagnosis were excluded. Included patients were followed up till 9/2008 for the incidence of all cause mortality, rehospitalization for arrhythmic causes and (implantable defibrillator) ICD implantation.

Results: A total of 66 patients (mean age 44.4 years, 56\% males) met the inclusion criteria. After a mean follow-up duration of 3.2 years, no patients died. None of the included patients developed ARVD or arrhythmogenic right ventricular cardiomyopathy. However, 12 (I8\%) patients were re-hospitalized for arrhythmic causes including 4 patients with supraventricular tachycardia and 4 patients with right ventricular outflow tachycardia, three of which required ICD implantation.

Conclusion: In patients with suspected ARVD, a normal CMR has a high negative predictive value for the development of death or future ARVD. However, an alternative diagnosis may be found in up to $18 \%$ of these patients on follow-up. Serial reevaluation for other arrhythmic causes may be warranted in symptomatic patients.

\section{P57}

Training-induced enlargement of the heart is balanced between the left and right atria and ventricles

Katarina Steding, Henrik Mosén and Håkan Arheden Dept. of Clinical Physiology, Lund, Sweden

Journal of Cardiovascular Magnetic Resonance 2009, I I(SuppI I):P57

Introduction: It is well known that long term endurance training induces morphological changes of the left ventricle. Few studies have investigated the effects of training on the volumes of the right and left atria and the right ventricle. Different pathologies can cause an enlargement of the chambers of the heart, e.g. dilated cardiomyopathy. We hypothesized that an enlargement of a healthy heart caused by long term endurance training should be well balanced between the left and right side. Purpose: The aim of this study was to investigate if long tem endurance training can cause a balanced enlargement between the left and right atrium and the left and right ventricle.

Methods: Eighteen elite triathletes (6 female) and 27 healthy normal subjects (12 female) underwent cardiac magnetic resonance imaging (CMR). Left and right end-diastolic volume (LVEDV, RVEDV) and left and right atrial volumes (LA, RA) were calculated from short-axis images using planimetry. MannWhitney non-parametric test was used to compare atrial and ventricular volumes between groups. Linear regression was performed to assess the relationship between LVEDV and RVEDV and between LA and RA.

Results: Male triathletes had significantly larger LA and RA volumes $(p<0.000 I, p<0.01)$ and LV and RV EDV $(p<0.000 I$, $p<0.001$ ) when compared to male normals. Female triathletes had significantly larger LV and RV EDV $(p=0.015, p<0.01)$. There were no significant differences in atrial volumes between female triathletes and female normal subjects (LA $p=0.247$ and $R A P=0.147)$. Linear regression showed a strong correlation 
Figure I (abstract P57)
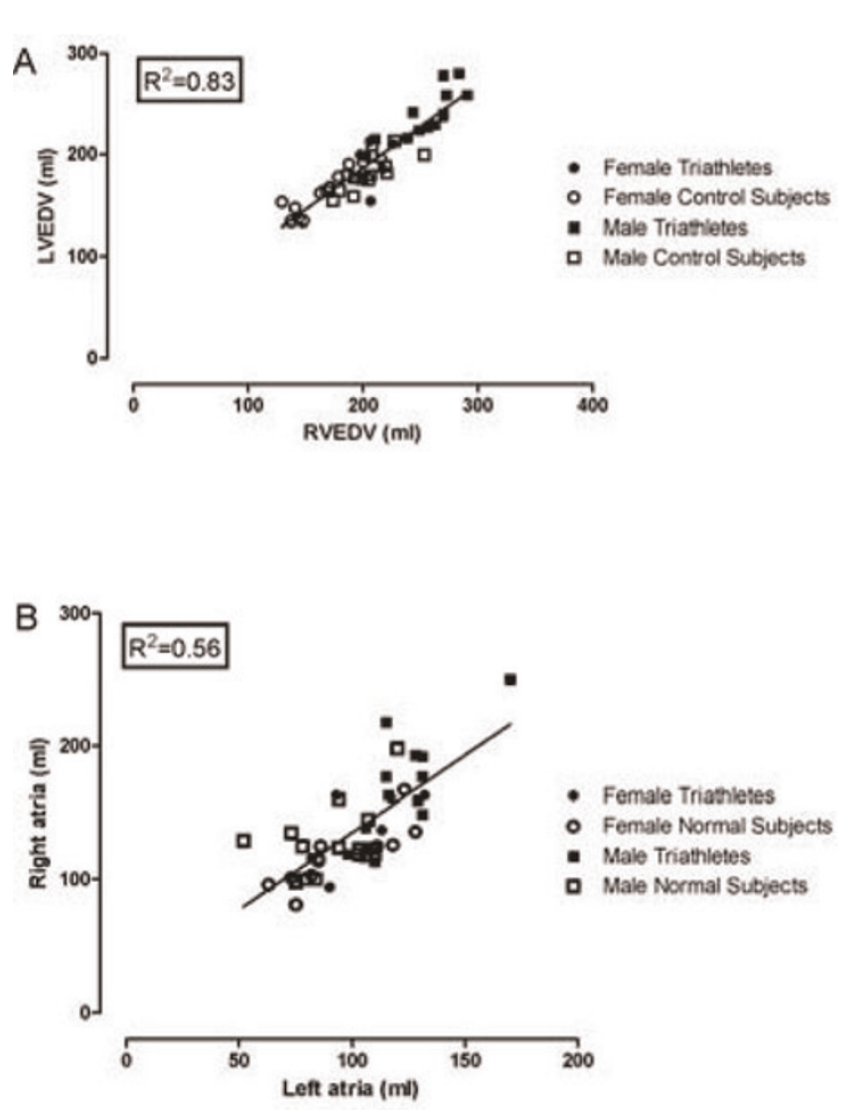

between LVEDV and RVEDV $\left(R^{2}=0.83, p<0.000 I\right)$ (Fig. IA). The correlation between LA and RA was somewhat weaker, yet significant $\left(R^{2}=0.56, p<0.000 \mathrm{I}\right)$ (Fig IB).

Conclusion: This study has shown that long term endurance training induces a balanced enlargement of the left and right atrial and ventricular dimensions in both males and females.

\section{P58}

Fragmented QRS complexes on a I 2-lead ECG as a marker of non-coronary artery disease related myocardial disease by gadolinium delayed enhancement cardiac magnetic resonance imaging Mohamed Homsi, Lamaan Alsayed, Anas Safadi, Mithilesh K Das and Jo Mahenthiran Indiana University School of Medicine, Krannert Institute of Cardiology, Indianapolis, IN, USA

Journal of Cardiovascular Magnetic Resonance 2009, I I (SuppI I):P58

Background: We have demonstrated that fragmented QRS complexes (fQRS) on a I2-lead ECG correlate with the presence of myocardial scar in coronary artery disease (CAD). However, the extent of $\mathrm{fQRS}$ as a marker of non-CAD related myocardial pathology as seen on delayed gadolinium enhancement (GDE) cardiac magnetic resonance imaging (CMR) is unknown.

Methods: The fQRS on 12-lead ECG was defined as the presence of fragmented QRS, notched R or S wave, or RSR'
Table I (abstract P58)

\begin{tabular}{llll}
\hline & Sensitivity & Specificity & P value \\
\hline FQRS & $88 \%$ & $80 \%$ & $<0.00 \mathrm{I}$ \\
Anterior fQRS & $50 \%$ & $94 \%$ & 0.01 \\
Lateral fQRS & $67 \%$ & $98 \%$ & $<0.00 \mathrm{I}$ \\
Inferior fQRS & $80 \%$ & $81 \%$ & 0.01 \\
\hline
\end{tabular}

Figure I (abstract P58)

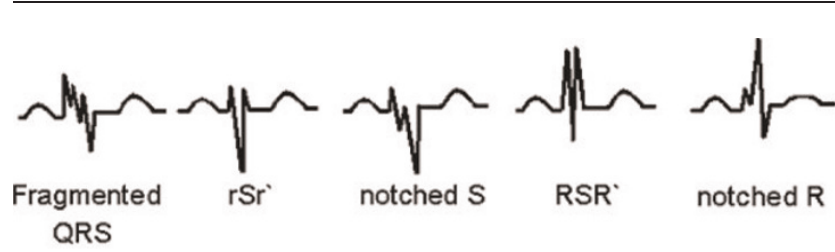

Different morphologies of fQRS on 12-lead ECG.

pattern in at least 2 contiguous leads corresponding to an individual coronary artery region (anterior: VI to V6 leads, lateral: I, aVL and V5, V6 leads, and inferior: II, III and aVF leads, respectively) in absence of a typical bundle branch block pattern (Figure I). CMR studies of 91 patients (pts) with GDE $(0.1 \mathrm{mmol} /$ $\mathrm{kg}$ Gadolinium) and ECG were studied. Presence of nonsubendocardial and patchy or diffuse mid to subepicardial GDE patterns was considered abnormal. Pts with CAD, prior myocardial infarction, and transmural/subendocardial GDE were excluded $(n=34)$.

Results: Of 57 pts (mean age $41 \pm 15$ years, $41 \%$ male), 17 (30\%) pts had fQRS on their 12-lead ECG and 8(14\%) pts had abnormal non-CAD-related GDE. The CMR exams were performed for dilated cardiomyopthy $(n=9)$, myocarditis $(n=5)$, constrictive pericarditis $(n=6)$, arrhythmia $(n=14)$, intracardiac mass $(n=7)$, sarcoidosis $(n=11)$, and suspected amyloidosis $(n=2)$ or hemochromatosis $(n=3)$. Sensitivity and specificity of detecting non-CAD related myocardial pathology by fQRS on ECG were $88 \%$ and $80 \%$ respectively $p<0.00$ I. Results with analysis of anterior, lateral and inferior fQRS are summarized in Table I.

Conclusion: Fragmented QRS complexes on a 12-lead ECG is reliable marker of both $C A D$ and non-CAD related myocardial disease with a high degree of specificity to localize the region of the involved myocardium.

\section{P59}

Is there a prognostic value of CMR derived 3D right ventricular geometry and function?

Vikas K Rathi, Vinayak Hegde, Srinivas Murali,

Madhavi Akkineni, June A Yamrozik, Ronald B Williams, Geetha Rayarao, Diane A Vido, Mark Doyle and Robert WW Biederman Allegheny General Hospital, The Gerald McGinnis Cardiovascular Institute, Pittsburgh, PA, USA

Journal of Cardiovascular Magnetic Resonance 2009, I I(SuppI I):P59

Introduction: Pulmonary hypertension $(\mathrm{PH})$ accounts for substantial morbidity and mortality. Advances in cardiac imaging have enabled the 3 Dimensional (3D) study of right ventricular 
(RV) geometry and functional parameters; however, their impact on patient outcomes in various groups of patients (pts) such as idiopathic pulmonary arterial hypertension (IPAH, WHO Group I) when compared to $\mathrm{PH}$ secondary to other causes $(\mathrm{SPH}, \mathrm{WHO}$ Group II-V) has not been fully studied.

Aim: We propose that the RV volume and geometry in IPAH and $\mathrm{SPH}$ pt groups will correlate with a clinically important prognostic parameter such as 6-minute walk distance (6 MWD). Methods: Pts $(n=32)$ with severe $\mathrm{PH}$ were divided into 2 groups: 15 pts with IPAH and 17 pts with SPH. All pts underwent cardiac MRI (CMR) (I.5 T GE, Milwaukee, WI) and measurements of 3D RV end-diastolic and end-systolic volumes (EDV, ESV), ejection fraction (EF), mass and relative wall thickness (RWT), all indexed to BSA and were compared to 6 MWD.

Results: There were no differences in the RV geometry or function between the two groups. The mean RV mass index, RWT and RVEF were $34.8 \pm 29,0.19 \pm 0.11$ and $34 \pm 14 \%$ in the IPAH group vs $27 \pm 10,0.20 \pm 0.1 \mathrm{I}$ and $44 \pm 15 \%$, in the SPAH group ( $p=n s$ ). The RWT and RV mass index demonstrated that the RV was eccentrically hypertrophied in both groups despite severe $\mathrm{PH}$. The IPAH pts had slightly higher PA systolic pressure when compared to SPH (84 \pm 16 vs $64 \pm 24 \mathrm{mmHg}, \mathrm{P}=0.04)$ but had lower pulmonary wedge pressures ( $10 \pm 7$ vs. $17 \pm$ $8 \mathrm{mmHg}$; $<$ 0.05). The mean $6 \mathrm{MWD}$ was similar between the IPAH vs. SPH group ( $344 \pm 88$ vs. $302 \pm 98$ meters, $p=N S)$. The RVEDVI, RVESVI and RVMI positively correlated with 6 MWD ( $R=0.52,0.48$ and 0.50, $\mathrm{p}<0.05$ ) of IPAH patients but did not demonstrate significant correlation for SPH pts. Furthermore, the 3D RVEF, 2D TAPSE and ratio of RVMI to RV volume did not demonstrate correlation with the $6 \mathrm{MWD}$.

Conclusion: Although IPAH and SPH patients have very similar RV geometry, structure and function, noninvasive CMR metrics only predict 6 MWD for IPAH patients. Clinically important predictors of 6 MWD by CMR metrics for the SPH group, a potentially more heterogeneous group, is not beneficial. These observations highlight the key pathophysiologic differences in the compensatory response of the RV to vastly different pathophysiology despite a more homogeneous response at the level of the RV chamber. This finding may account for the observed differences in treatment response and clinical outcomes in these patients.

\section{P60}

Right and left sided cardiac function in HIV patients on anti-retroviral therapy: a cine magnetic resonance imaging study

Ulrik S Kristoffersen , Anne-Mette Lebech ${ }^{2}$, Claus Leth Petersen ${ }^{3}$, Henrik Gutte', Jan Gerstoft' and Andreas Kjaer'

'Rigshospitalet, Copenhagen, Denmark

${ }^{2}$ Hvidovre Hospital, Copenhagen, Denmark

${ }^{3}$ Frederiksberg Hospital, Copenhagen, Denmark

Journal of Cardiovascular Magnetic Resonance 2009, I I (SuppI I):P60

Background: Numerous studies have found impaired right and left ventricular function in HIV patients. However, most studies are from the era before the introduction of highly active antiretroviral therapy (HAART) and only a few studies have been performed on patients on HAART. In these studies, predominantly echocardiography has been used to assess cardiac function. The aim of the present study was to determine right and left ventricular function in HIV patients on HAART with cine magnetic resonance imaging (MRI) which is a more precise method than echocardiography.

Methods: Thirty-nine well-treated HIV-I infected patients on HAART with no clinical evidence of cardiac disease were included. Mean age was $48.4+/-9$ years and mean HIV duration was $179.4+/-78.6$ months. All patients underwent MRI and had right (RVEF) and left ventricle ejection fraction (LVEF), as well as right (RVEDVI) and left ventricle end diastolic volume index (LVEDVI) measured. Thirty-three age and sex matched controls were included to establish reference values of the MRI measurements. The reference values were defined as mean +/- two standard deviations. When comparing the study group and the control group, independent samples t-test was used. Background parameters are presented as mean $+/-$ s.d. and results are presented as mean $+/-$ SEM.

Results: Compared with controls, the HIV group on average had reduced LVEF $(64.9+/-1.1 \%$ vs. $70.4+/-1.5 \%$; $p<0.01)$ and increased RVEDVI $\left(75.5+/-2.2 \mathrm{~mL} / \mathrm{m}^{2}\right.$ vs. $68.1+/-3.0 \mathrm{~mL} /$ $\left.\mathrm{m}^{2} ; \mathrm{p}<0.05\right)$. RVEF was not significantly reduced $(56.5+/-1.1 \%$ vs. $59.3+/-$ I.I\%; NS) nor was LVEDVI significantly increased $\left(64.6+/-2.1 \mathrm{~mL} / \mathrm{m}^{2}\right.$ vs. $58.3+/-2.8 \mathrm{~mL} / \mathrm{m}^{2}$; NS). Two HIV patients $(5 \%)$ had marginally reduced LVEF compared to the lower reference value of $53.3 \%$, and one patient $(3 \%)$ had marginally reduced RVEF compared to the lower reference value of $47.4 \%$. Four patients (10\%) had increased LVEDVI compared to the upper reference value of $87.9 \mathrm{~mL} / \mathrm{m}^{2}$, and two patients (5\%) had increased RVEDVI compared to the upper reference value of $98.8 \mathrm{~mL} / \mathrm{m}^{2}$.

Conclusion: No major cardiac dysfunction is present in well treated HIV patients. However, subclinical functional abnormalities are present in both right and left ventricle in HIV patients, even though they are well-treated on HAART.

\section{P6 I}

Normal values for wall thickening by magnetic resonance imaging

Joey Ubachs, Einar Heiberg, Katarina Steding and Håkan Arheden

Cardiac MR Group, Dept. Clinical Physiology, Lund University Hospital, Lund, Sweden

Journal of Cardiovascular Magnetic Resonance 2009, I I (SuppI I):P6 I

Purpose: The aim of this study was therefore to describe normal values for myocardial wall motion using steady state free precession MRI.

Introduction: Regional left ventricular dysfunction is a major consequence of myocardial ischemia. Accurate quantitative analysis of wall motion is therefore essential for risk area assessment. In order to assess wall motion abnormalities, normal values need to be established. Although previous studies exist, to our knowledge however, no absolute regional values for wall thickening have been published using MRI steady state free precession pulse sequence (SSFP). It has been shown that there is a statistical difference in left ventricular mass between SSFP and the older gradient echo pulse sequences.

Methods: Fifty healthy, non-athletic subjects (age: $35 \pm \mathrm{II}$, M/F: 30/20) were included in this study. Short-axis SSFP cine images were taken and absolute wall thickening and fractional wall thickening were assessed after manual tracing of the endocardial and epicardial borders. As an internal quality 
Figure I (abstract P6I)

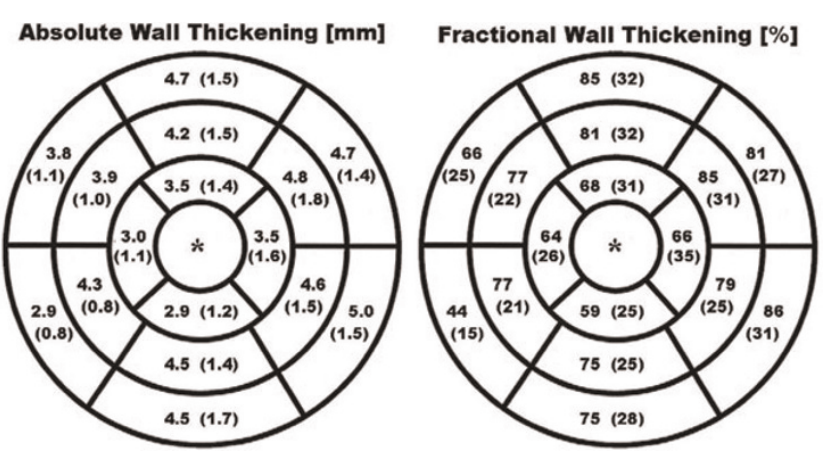

Measurements of absolute wall thickening (left panel) and fractional wall thickening (right panel) are displayed in a 17-segment model. Wall thickening measurements in the most apical slice is not meaningful in short-axis images and were therefore left out*. Absolute wall thickening is expressed in millimetre (standard deviation), fractional wall thickening in percentage (standard deviation).

check, tracings were considered correct when difference in left ventricular mass between end-diastolic and end-systolic tracings was less than $2 \%$. Normal values for wall thickening were calculated and converted into the American Heart Association 17-segment model. The most basal slice included in the analysis, was the first slice below the membranous septum in end-systole. The most apical slice was excluded.

Results: Mean normal values for absolute wall thickening were $4.3 \pm 1.4 \mathrm{~mm}$ (basal slices), $4.4 \pm 1.4 \mathrm{~mm}$ (mid-ventricular slices), and $3.2 \pm 1.4 \mathrm{~mm}$ (apical slices). Mean normal values for fractional wall thickening were $73 \pm 31 \%$ (basal slices), $79 \pm 26 \%$ (mid-ventricular slices), and $64 \pm 30 \%$ (apical slices). Between men and women, there was no significant difference in absolute wall thickening $(p=0.06)$, but a significant difference in fractional wall thickening $(p=0.003)$. Figure I.

Conclusion: The present study presents normal values for wall thickening derived from short-axis MRI. These normal values can be used as reference for quantitative assessment of wall motion abnormalities in various cardiac disease states.
P62

\section{The effects of continued obesity on the cardiovascular system}

Oliver J Rider, Jane M Francis, Steffen E Petersen

and Stefan Neubauer

University of Oxford, Oxford, UK

Journal of Cardiovascular Magnetic Resonance 2009, I I(SuppI I):P62

Objective: To determine the effects of a one year period of sustained obesity on the cardiovascular system

Background: Although the effects of obesity and weight loss on the cardiovascular system have attracted a lot of interest, the effects of continued obesity are not well understood. Obesity is characterized by left and right ventricular hypertrophy and cavity dilatation, left ventricular diastolic dysfunction and reduced aortic elastic function, and weight loss is associated with at least partial reversal of these adaptive changes. Here, we present data looking at the effects of one year of continued obesity in seven obese subjects who were unsuccessful in losing weight over a dietary trial period.

Methods: Seven obese subjects with no identifiable cardiovascular risk factors underwent CMR scanning before and after a period of one year of sustained obesity. All subjects underwent cardiac MR imaging at I.5 T for the assessment of left ventricular mass (g), left ventricular end-diastolic volume (EDV; $\mathrm{ml}$ ), stroke volume (SV; $\mathrm{ml}$ ) and LV EF (\%). Aortic distensibility was assessed at three levels; the ascending (Ao) and proximal descending aorta (PDA) at the level of the pulmonary artery and the abdominal aorta (AA). The abdominal cine images were piloted perpendicular to the orientation of the abdominal aorta. In addition to this, left ventricular diastolic function was assessed using volume time curve analysis.

Results: After one year there was no significant change in body mass index, weight, visceral fat mass or total fat mass. There were no significant differences in fasting serum glucose, serum cholesterol or systolic blood pressure. After one year of continued obesity, there was a significant $7 \%$ increase in left ventricular mass, and left ventricular mass when indexed to both height and height ${ }^{2.7}$. Right ventricular mass was unchanged over the year period. Both left and right ventricular end-diastolic

\section{Figure I (abstract P62)}

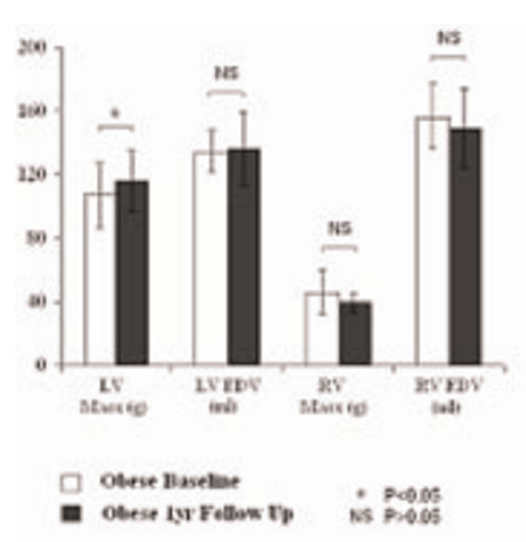

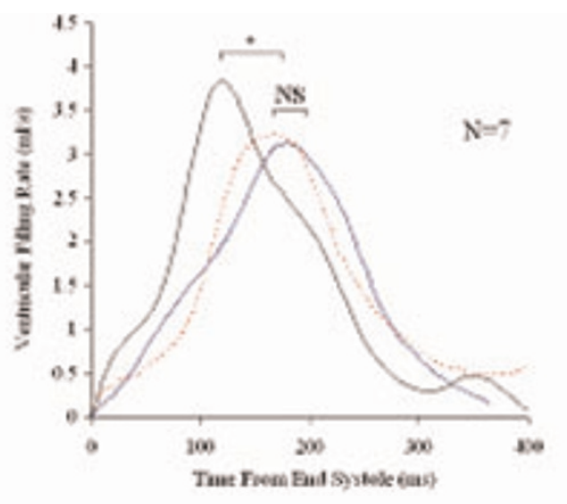

$$
\begin{aligned}
& \text { Nermal Weight } \\
& \text { Obeie Pre } \\
& \text { Obre Pest }
\end{aligned}
$$

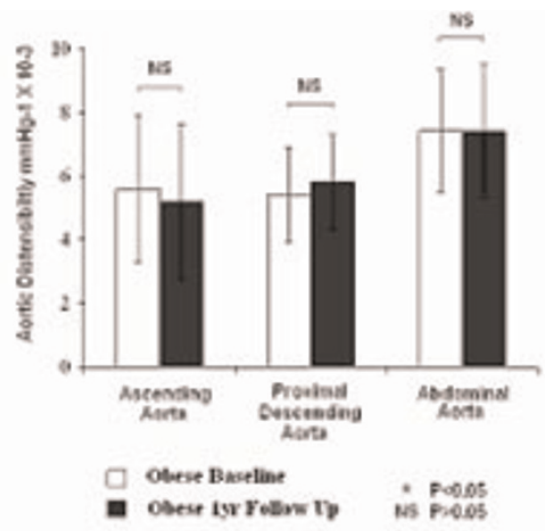

Page 133 of 316

(page number not for citation purposes) 
volume, end-systolic volume and stroke volume were unchanged with one year of continued obesity. Left ventricular ejection fraction was also unchanged over the one year period. There was no significant difference between left ventricular diastolic function or aortic distensibility measurements taken at any level of the aorta before and after one year of continued obesity. Figure I.

Conclusion: In this small sub analysis we have shown that continued obesity over a period of one year results in increases in left ventricular mass without any other change in ventricular or aortic function. This again points to the fact that left ventricular mass is very sensitive to body mass index and, in the setting of obesity, can increase or decrease over a period of one year.

\section{P63}

Contrast-enhanced MR imaging of pulmonary arteries: new imaging strategies using different contrast agents

Marcus R Makowski', Andrea J Wiethoff ${ }^{1,2}$, Vicky Parish ${ }^{1,2}$, Aaron Bell ${ }^{1,2}$, Rene M Botnar, ${ }^{1,2}$, Christian Jansen', Sergio Uribe', Martin Rohrer ${ }^{3}$, Reza Razavi ', Tobias Schaeffter' and Gerald F Greil'

'Imaging Sciences Division, King's College London, St Thomas' Hospital, London, UK

${ }^{2}$ NIHR Biomedical Research Centre at Guy's \& St Thomas' Hospital and King's College London, London, UK

${ }^{3}$ European Business Unit Diagnostic Imaging, Bayer Schering Pharma AG, Berlin, Germany

Journal of Cardiovascular Magnetic Resonance 2009, I I (SuppI I):P63

Objective: First pass breath hold non ECG triggered 3D GdDTPA contrast enhanced magnetic resonance angiography (CEMRA) is commonly used for morphologic assessment of the pulmonary arteries. However, image resolution is limited due to time constraints and vascular borders are blurred due to vascular motion and insufficient breath holds.

Background: Pulmonary vascular imaging using a respiratory gated and ECG triggered 3D contrast enhanced IR prepulse sequence in combination with Gadofosveset (mean intravascular $\mathrm{tl} / 2 \alpha=0.48 \pm 0.1 \mathrm{l} \mathrm{h}$ ) and 32 channel coil technology yields significant higher pulmonary vascular detail compared to breath hold 3D CEMRA.

Methods: In eight subjects ( $29 \pm 6 \mathrm{yrs}$ ) with normal pulmonary vasculature CEMRA was performed on a $1.5 \mathrm{~T}$ clinical scanner (Philips Medical Systems). Patients were investigated twice within 7 days using Gd-DTPA (day I, $0.10-0.17 \mathrm{mmol} / \mathrm{kg}$ ) and Gadofosveset (day 2, $0.03 \mathrm{mmol} / \mathrm{kg}$ ). 3D CEMRA as well as a respiratory navigator gated and ECG triggered 3D steady-state
Figure I (abstract P63)

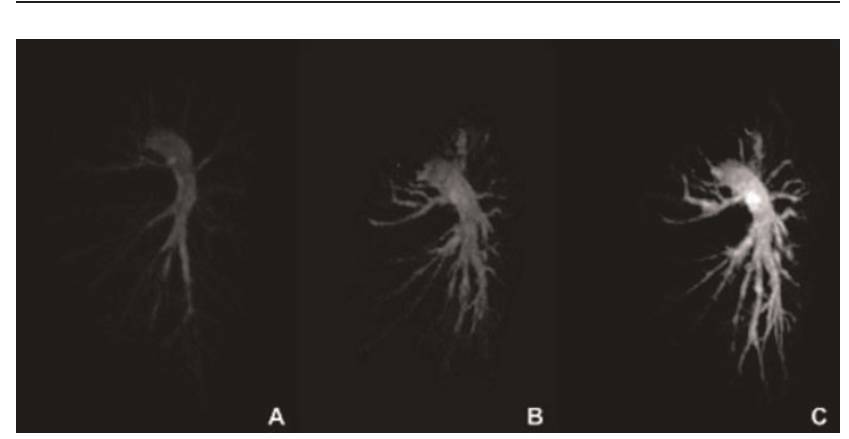

free precession (SSFP) sequence with a T2 prepulse were used on both days. An inversion recovery (IR) prepulse to suppress surrounding tissue signal was applied with Gadofosveset. Results were quantitatively and qualitatively (image quality: I = non diagnostic, 2 = diagnostic, 3 = good 4 = excellent) compared.

Results: Best results were achieved for the high resolution navigator gated and ECG triggered 3D IR SSFP sequence using Gadofosveset (Figure IC) regarding CNR, vessel length, vessel wall sharpness and image quality (Table I). Gadofosveset did not improve image quality in the 3D SSFP technique without IR (Figure IB) compared to Gd-DTPA and 3D first pass CEMRA (Figure IA).

Conclusion: Pulmonary vascular imaging using a navigator gated and ECG triggered 3D IR SSFP sequence with Gadofosveset and 32 channel coil technology yielded significantly higher morphologic detail compared to breath hold CEMRA and 3D SSFP without IR. This technique has the potential to improve diagnostic imaging of the pulmonary vasculature.

\section{P64}

Cardiovascular magnetic resonance assessment of abdominal adiposity predicts early sub-clinical atherosclerosis in young adults: importance of relative adiposity

Ilias Kylintireas, Jonathan Diesch, Corinne Trevitt,

Merzaka Lazdam, Colin Cunnington, Robin Choudhury,

Stefan Neubauer and Paul Leeson

University Of Oxford, Oxford, UK

Journal of Cardiovascular Magnetic Resonance 2009, I I(SuppI I):P64

Introduction: Gross obesity has a significant adverse impact on the cardiovascular system. The clinical importance of the

Table I (abstract P63) Comparison of contrast agents and imaging sequences

\begin{tabular}{|c|c|c|c|c|c|c|}
\hline $\begin{array}{l}\text { Contrast } \\
\text { Agent }\end{array}$ & Sequence & $\begin{array}{l}\text { Vessel } \\
\text { length }(\mathrm{cm})\end{array}$ & $\begin{array}{l}\text { Vessel } \\
\text { sharpness (\%) }\end{array}$ & $\begin{array}{l}\text { Contrast to } \\
\text { Noise Ratio (CNR) }\end{array}$ & $\begin{array}{l}\text { Image quality } \\
\text { (mean } \pm S D)\end{array}$ & $\begin{array}{l}\text { Isotropic spatial } \\
\text { resolution }\left(\mathrm{mm}^{3}\right)\end{array}$ \\
\hline \multirow[t]{2}{*}{ Gd-DTPA } & CEMRA & $14 \pm 4 \ddagger$ & $33 \pm 6 \ddagger$ & $89 \pm 37 \ddagger$ & $2.1 \pm 1.2 \ddagger$ & 1.77 \\
\hline & SSFP & $17 \pm 2^{*}$ & $41 \pm 4^{*}$ & $134 \pm 22 *$ & $3.2 \pm 1.1^{*}$ & 1.49 \\
\hline \multirow[t]{3}{*}{ Gadofosveset } & CEMRA & $13 \pm 4 \ddagger$ & $34 \pm 7 \ddagger$ & $99 \pm 39 \ddagger$ & $2.3 \pm 1.1 \ddagger$ & 1.77 \\
\hline & SSFP & $17 \pm 2 *$ & $40 \pm 4^{*}$ & $136 \pm 17 *$ & $3.1 \pm 1.5^{*}$ & 1.49 \\
\hline & SSFP+IR & $19 \pm 3^{\dagger}$ & $47 \pm 5^{\dagger}$ & $153 \pm 24^{\dagger}$ & $3.6 \pm 1.3^{\dagger}$ & 1.49 \\
\hline
\end{tabular}

$*_{p}<0.05$ vs CEMRA, ${ }^{\dagger} \mathrm{p}<0.05$ vs SSFP (same CA); no significant differences between corresponding sequences (Gd-DTPA vs Gadofosveset) 
Figure I (abstract P64)

$>$ TFI $($ Total Fat Index $)=($ VAT + SCAT $) /$ Total abdominal volume $>$ SFI (Subcutaneous Fat Index $)=($ VAT + SCAT $) /$ Total abdominal volume
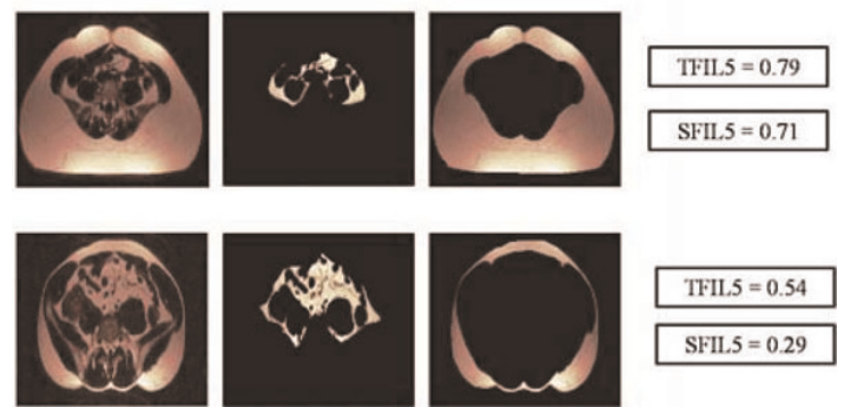

Relative abdominal adiposity indices.

normal variation in body fat seen in the general population during early adulthood is unknown. Cardiovascular magnetic resonance imaging offers the unique capability for clear depiction and accurate quantification of adipose tissue alongside an effective non invasive and radiation free assessment of both early and advanced atherosclerotic disease. We investigated the relation between abdominal adiposity and early sub-clinical carotid atherosclerosis.

Purpose: We related CMR assessment of abdominal adiposity to sub-clinical carotid atherosclerosis in a young non-obese population, free of classical cardiovascular risk factors.

Methods: Forty young volunteers (aged 23-33) (without any history of cardiovascular disease, gross obesity [mean $\left.\mathrm{BMI}=24.1 \mathrm{~kg} / \mathrm{m}^{2}( \pm 4.3)\right]$ or classical cardiovascular risk factors) underwent CMR for abdominal adipose tissue quantification and carotid atheroma burden measurements. A Water Suppression (WS) TI weighted (TIW) Turbo Spin Echo (TSE) multi-slice sequence was used for visceral adipose tissue (VAT) and abdominal subcutaneous adipose tissue (SCAT) measurement. We acquired 2 sets of 5 transverse slices ( $6 \mathrm{~mm}$ thick/I $\mathrm{mm}$ gap), one positioned at the level of the fifth lumbar vertebrae (L5) and one at the level of the second lumbar (L2) vertebra. A semiautomated volumetric method was used to measure VAT and SCAT volumes for each set of slices (VATL2 and SCATL2 for the L2 level, and VATL5 and SCATL5 for the L5 level). To obtain an index of relative adiposity we derived a Total Fat Index (TFI) and Subcutaneous Fat Index (SFI) at each level. TFI was defined as the sum of VAT and SCAT volumes divided by overall abdominal volume and SFI as the SCAT volume divided by abdominal volume (Figure I).

TI weighted black blood turbo spin echo (TSE) cross-sectional images of both carotid arteries, centred at the lowest bifurcation were used for atheroma burden measurements (plaque index represented cross-sectional vessel wall area/total cross-sectional vascular area). Plaque index was averaged for the common carotid (CPI), the carotid bulb (BPI) and the internal carotid artery (IPI).

Results: VAT and SCAT absolute measurements at both levels, TFIL2 and SFIL2 as well as body mass index (BMI), waist hip ratio (WHR), waist and hip circumferences, age and sex did not correlate with carotid atheroma burden. However, both TFIL5 and SFIL5 correlated with CPI $(r=0.359, P<0.05$ and $r=0.397, P<0.05$ respectively) and strongly correlated with BPI $(r=0.435, P<0.05$ and $r=0.428, P<0.05$ respectively). Applying respective multiple regression analysis models (including applicable risk factors, demographics and anthropometric measurements) TFIL5 and SFIL5 emerged as sole independent predictors of atheroma burden both at the common carotid $\left[\beta=0.1905( \pm 0.09), P<0.05, R^{2}=0.20\right.$ and $\beta=0.1584( \pm 0.06)$, $\mathrm{P}<0.05, \mathrm{R}^{2}=0.23$ respectively] and the carotid bulb $\left[\beta=0.1862( \pm 0.09), P<0.05, R^{2}=0.19\right.$ and $\beta=0.0 .1294( \pm$ $0.06), P<0.05, R^{2}=0.23$ respectively].

Conclusion: Relative increases in both total and subcutaneous abdominal adiposity across the normal ranges in a young adult population are associated with early sub-clinical atherosclerosis. Our observations illustrate the role of CMR in accurate evaluation of abdominal adiposity. Variation in abdominal fat relative to abdominal size may be of particular importance and abdominal adiposity at the fifth lumbar vertebral level may be the most relevant position to evaluate cardiovascular risk.

\section{P65}

Is hemorrhage in acute reperfused myocardial infarction a new marker for the severity of tissue injury?

Andreas Kumar', Jordin D Green', Jane M Sykes², Andrea Mitchell' ${ }^{2}$, Gerald Wisenberg ${ }^{2}$

and Matthias G Friedrich'

'University of Calgary, Calgary, AB, Canada

${ }^{2}$ University of Western Ontario, London, ON, Canada

Journal of Cardiovascular Magnetic Resonance 2009, I I(SuppI I):P65

Introduction: Reperfusion injury in myocardial infarction leads to microvascular obstruction, which can occur with or without gross reperfusion hemorrhage. The incidence and implications of reperfusion hemorrhage are not well investigated. A recently described in vivo imaging approach using T2*-weighted cardiovascular magnetic resonance can help investigate the pathophysiology of reperfusion hemorrhage in vivo.

Hypothesis: We hypothesized that hemorrhage reflects a severer from of reperfusion injury and therefore occurs with larger infarct size and worse LV function as compared to reperfusion injury without hemorrhage.

Methods: In 14 female mongrel dogs, myocardial infarction was induced by ligation of the left anterior descending coronary artery for $2-4$ hours, followed by reperfusion. On day $3 \pm 1$, a cardiovascular magnetic resonance study was performed in vivo to (I) assess presence of microvascular obstruction, defining reperfusion injury (2) assess presence of reperfusion hemorrhage (3) quantify left ventricular end-diastolic volume, ejection fraction and cardiac output and (4) quantify infarct size with late enhancement. An independent-samples t-test was performed to compare these parameters in dogs with and without hemorrhage in reperfusion injury.

Results: From 14 dogs, 9 had microvascular obstruction, and $4 / 9$ had reperfusion hemorrhage in addition to microvascular obstruction.

Dogs with hemorrhagic infarcts had significantly larger infarct size $(26.1 \pm 6.6 \mathrm{~g}$ vs. $5.5 \pm 3.9 \mathrm{~g}, \mathrm{p}<0.05)$, lower LV ejection fraction $(28 \pm 7 \%$ vs. $53 \pm 12 \%, p<0.05)$, and lower cardiac output $(1.9 \pm 0.2 \mathrm{l} / \mathrm{min}$ vs. $2.8 \pm 0.5 \mathrm{l} / \mathrm{min}, \mathrm{p}<0.05)$. There were no differences in LV end-diastolic volume and LV mass. 
Discussion and conclusion: In this dog model of ischemia/ reperfusion injury, gross hemorrhage was associated with significantly larger infarct size and worse LV functional parameters. This supports the hypothesis that hemorrhage may occur with advanced, severe ischemic tissue injury only.

\section{P66}

Early diastolic leftward septal motion in Tetrology of Fallot at MRI: analysis and comparison to patients with constrictive/restrictive physiology Jeremy D Collins, Karen Ordovas, Allison Meadows, Maureen Kohi, Brett Elicker, WR Webb and Charlie Higgins

UCSF, San Francisco, CA, USA

Journal of Cardiovascular Magnetic Resonance 2009, I I (SuppI I):P66

Background/purpose: Early diastolic leftward septal motion (EDSM) is a nonspecific finding at diagnostic cardiac imaging. Although touted as a sensitive marker for constrictive or restrictive physiology, EDSM can also be seen in volume overload and rhythm disturbances. The purpose of this study was to retrospectively evaluate early leftward diastolic septal motion in patients with Tetrology of Fallot (TOF) and right ventricular enlargement.

Methods: The UCSF Committee on Human Research approved this study. Medical records and cardiac MR images from II6 consecutive patients with suspected constrictive/ restrictive physiology (64 pts, mean age $49 \mathrm{yrs}, 34$ men), history of surgical repair of TOF with pulmonary insufficiency (PI) (3I pts, mean age 27 yrs, 18 men) or both PI and stenosis (PS) (2I pts, mean age 32 yrs, 10 men) referred for cardiac MRI between January 1997 and September 2008 were reviewed. Images from 9 volunteers (4 men, mean age $29 \mathrm{yrs}$ ) were also included in the analysis.

Cinegraphic cardiac MRI images were reviewed for the presence of EDSM, defined as $>2 \mathrm{~mm}$ leftward deviation on two consecutive short axis images immediately following isovolumetric relaxation. Ventricular chamber volumes were obtained from contoured short axis images and adjusted to body surface area. The extent of pulmonary insufficiency was assessed with velocity encoding sequences orthogonal to the right ventricular outflow tract. Images were reviewed for pericardial thickening, enhancement, effusions, and coupling of the parietal and visceral pericardial layers as well as evidence for impaired diastolic filling. QRS complex duration, pulmonary valve gradient, and systemic venous and pulmonary wedge pressures were obtained from the medical record, when available. An analysis of variance (ANOVA) was performed on TOF patients with $P>0.05$ considered statistically significant. Results from pathology, right heart

Table I (abstract P66)

\begin{tabular}{lll}
\hline & TOF Patients c EDSM \\
\hline RV EDVI & PI only & PI and PS \\
Normal & $0 \%(0$ of 4$)$ & $0 \%(0$ of 2$)$ \\
Mildly Enlarged & $63 \%(5$ of 8$)$ & $30 \%(3$ of 10$)$ \\
Moderately Enlarged & $63 \%(7$ of II) & $66 \%(4$ of 6$)$ \\
Severely Enlarged & $75 \%(6$ of 8$)$ & $100 \%(3$ of 3$)$ \\
\hline
\end{tabular}

catheterization, echocardiography and surgical procedures provided the reference standard for analysis.

Results: EDSM was identified in $58 \%$ (I8 of 31 ) TOF patients with $\mathrm{PI}$ and $48 \%$ ( $\mathrm{I} 0$ of $2 \mathrm{I}$ ) patients with $\mathrm{PI}$ and $\mathrm{PS}$. Grouping all TOF patients with PI for ANOVA, right ventricular end diastolic volume index was strongly associated with EDSM $(p=0.00 \mathrm{I})$; QRS complex duration $(p=0.18)$, pulmonary regurgitant fraction $(p=0.6 \mathrm{I})$, and left ventricular end diastolic volume index (LVEDVI) $(p=0.32)$ demonstrated weaker associations. Right atrial and pulmonary wedge pressures were available in 16 TOF patients, precluding evaluation with ANOVA. See Table I. EDSM was identified in 18 of 21 patients $(86 \%)$ with suspected (5 patients) or proven ( 16 patients) constrictive pericarditis and in 6 of 12 patients (50\%) with suspected (3 patients) or proven restrictive cardiomyopathy ( 9 patients). Eight individuals out of 39 (30 patients, 9 volunteers) without MR or corroborative evidence of constrictive pericarditis or myocardial pathology demonstrated EDSM; 7 patients were subsequently diagnosed with a volume overload state.

Conclusion: A substantial proportion of patients with TOF and enlarged right ventricular volumes demonstrated EDSM at CINE cardiac MRI, controlling for effects from QRS complex duration, LVEDVI and pulmonary regurgitation. This finding, although somewhat less prevalent in our TOF sample population than in constrictive pericarditis, suggests that progressive enlargement of the right ventricle may lead to an appearance mimicking constrictive physiology. We hypothesize that in TOF patients, development of EDSM is due to reduced compliance of the stretched pericardium. Careful prospective assessment of volume status in TOF patients will be necessary to validate these findings and enable correlation of EDSM with development of clinical symptoms.

\section{P67}

Evaluation of complex congenital heart disease and associated complications in newborns, infants and small children using multi-detector $\mathbf{C T}$ Joachim G Eichhorn', Sebastian Ley ${ }^{2}$, Christian Fink ${ }^{3}$, John Cheatham ${ }^{4}$, Wim Helbing ${ }^{5}$ and Frederick Long ${ }^{6}$

'University Children's Hospital, Pediatric Cardiology,

Heidelberg, Germany

2University Children's Hospital, Pediatric Radiology, Heidelberg, Germany

${ }^{3}$ German Cancer Research Center (DKFZ), Radiology,

Heidelberg, Germany

${ }^{4}$ Nationwide Children's Hospital, Pediatric Cardiology, Columbus, $\mathrm{OH}$, USA

${ }^{5}$ Erasmus MC, Sophia University Children's Hospital, Pediatric Cardiology, Rotterdam, Netherlands

${ }^{6}$ Nationwide Children's Hospital, Pediatric Radiology, Columbus, OH, USA

Journal of Cardiovascular Magnetic Resonance 2009, I I(SuppI I):P67

Introduction: Multi-detector CT (MDCT) with multiplanar and three-dimensional (3D) reconstruction has become an important first-line imaging tool in diagnosis of congenital vascular anomalies (VA) and airway diseases in children.

Purpose: To assess the diagnostic value of MDCT for evaluation of VA and associated complications in newborns and infants. 
Figure I (abstract P67)

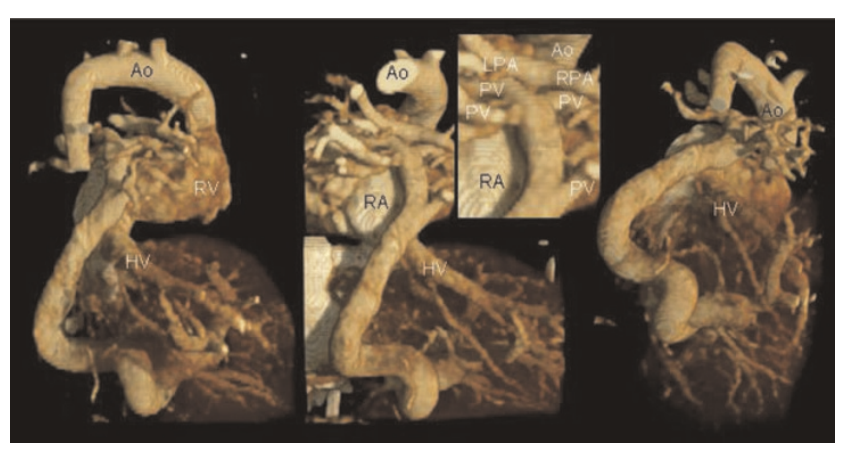

Newborn (body weight $3 \mathrm{~kg}$ ) with heterotaxia, tetralogy of Fallot with pulmonary atresia and total anomalous pulmonary venous drainage to the portal vein. 3D volume rendered images obtained on a 64 slice MDCT scanner following hand injection of contrast in the umbilical venous catheter. Images show the pulmonary veins (PV, diameter $<\mathrm{I} \mathrm{mm}$ ) draining to a confluens which crosses the diaphragm supplying the hepar veins (HV) in posteriorlateral and inferior projections. Aorta (Ao), right atrium and ventricel (RA and RV), left and right pulmonary artery (LPA and RPA).

Methods: At 4 centers, 262 children (mean age: $7 \pm 7$ months, range: 6 hours to 24 months) were examed. The diagnoses included: suspected of having in-stent stenosis after stent placement to treat vascular stenoses $(n=72)$, pulmonary atresia (32) and stenosis (48) with collateral arteries, arterial rings and slings (28), aortic arch anomalies (I5), bronchoscopy revealed stenosis(23), abnormal pulmonary venous return (I5) and others (29). The CT exams were performed on varying scanners (4 up to 64 slices, collimation isotrope $0.4-1.25 \mathrm{~mm}$; scan-time $2-20 \mathrm{~s}$, usage of low dose protocols: $80-120 \mathrm{kVp}, 60-80 \mathrm{~mA}$ ) under controlled ventilation or free breathing, mostly without ECGgating to minimize radiation exposure. The image quality was rated using a 5-point score. Image findings were correlated to echocardiography, conventional digital catheter angiography (DA), bronchoscopy, and intraoperativ findings. The effects of dose on image quality were also evaluated, retrospectively.

Results: High quality MDCT data were almost free of cardiac and respiratory motion. Images were scored for vascular contrast and for delineation of the tracheobronchial tree in $>95 \%$ of all cases as excellent or good, showing a significant improving with increasing number of detectors. Significant differences between higher and lower radiation settings were not found. The radiation exposure was mostly less than $2 \mathrm{mSv}$ (range I.I-3.2). High radiation exposure settings did not improve image quality. VA morphology and topography in relation to adjacent structures, e.g. tracheal and esophageal compression caused by an arterial ring or sling, could be assessed exactly and allowed the final diagnosis. Even smallest vessels with a diameter of less than I mm (see figure I), could be identified and excellently visualized. Eighty-two percent (215/262) of all patients had benefited from MDCT: Digital angiography was neither necessary to perform surgical or interventional planning nor to exclude a VA, or radiation doses and sedation time due to interventional procedures could be reduced markedly.

Conclusion: A 3D submillimeter evaluation of the heart and great vessels can be achieved routinely in a matter of seconds with little motion artifacts, without general anesthesia and with much less radiation exposure than previously thought. Nevertheless, the optimal radiation dose settings for performing cardiac CT in infants and pediatric patients are still being worked out. The use of CT for screening may result in a net decrease in overall radiation decreasing the number of diagnostic cardiac catherizations. CT can now be regarded as the modality of choice as a minimally invasive, robust, and accurate technique for the diagnosis of complex VA even in the group of newborns and infants or critically ill patients. Its accuracy for detecting VA appears at least equivalent to catherizations while it is more accurate in delineating potential life-threatening complications. This advance should have the greatest impact in the smallest, youngest, and most critically ill children with congenital heart disease.

\section{P68}

Coronary MR angiography in children during systole and diastole using a dual cardiac phase scan of the whole heart

Sergio A Uribe Arancibia', Israel Valverde²,

Philipp Beerbaum', Aaron Bell', Rene Botnar',

Reza Razavi', Tobias Schaeffter' and Gerald Greil'

'King's College London, London, UK

${ }^{2}$ Hospital Virgen del Rocio, Seville, Spain

Journal of Cardiovascular Magnetic Resonance 2009, I I(SuppI I):P68

Objective: To investigate the feasibility of dual cardiac phase whole heart MRI to obtain Coronary Magnetic Resonance Angiography (CMRA) at end-systole and mid-diastole in children with Congenital Heart Diseases (CHD).

Background: In children the visualization of the course of the coronary arteries is highly desirable for surgical planning and for follow up in CHD and Kawasaki disease. CMRA has been successful applied in adults and recently has been demonstrated in young children and infants. In children with CHD a whole heart CMR approach is of great clinical advantage, since the anatomy of the heart and the great vessels can be evaluated in addition to the coronary arteries. Usually, a 3D volume is obtained either at end-systole or mid-diastole. However, in children with high heart-rates and/or with $R R$ variability it is unclear which phase of the cardiac cycle results in better image quality. Therefore, the simultaneous acquisition of the

\section{Figure I (abstract P68)}
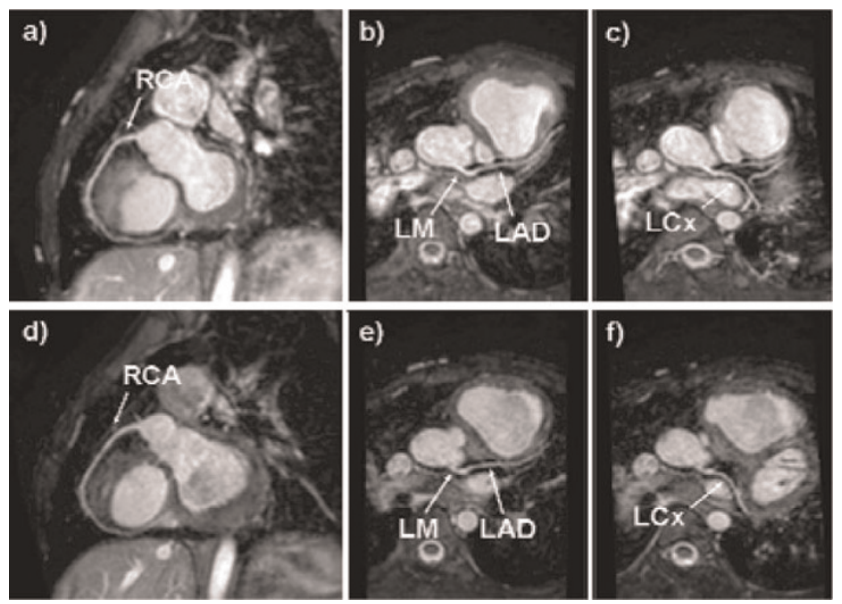


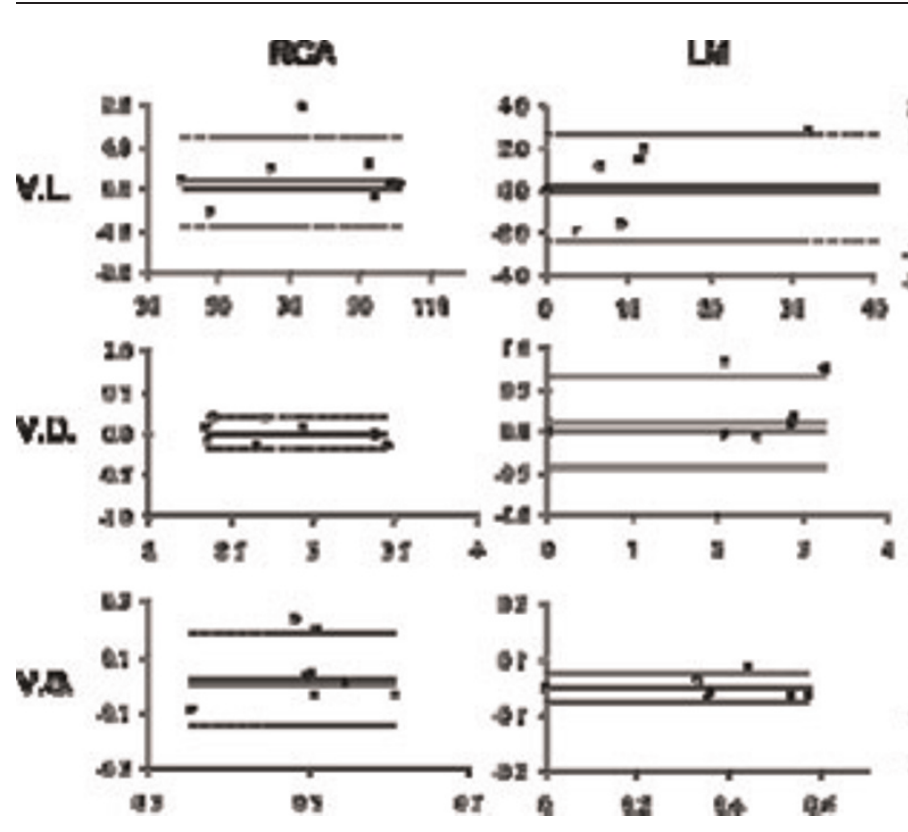

end-systolic and mid-diastolic phase in conjunction with the whole heart approach allows retrospectively selection of the best image phase for coronary artery visualization without any scan time penalty. Methods: Eight children (age $=6.38 \pm 4.27$, height $=116.57 \pm$ 30.77 , weight $=23.57 \pm 14.25$, Heart-rate $=85.02 \pm 8.59$ ) with CHD were scanned under general anesthesia on a I.5 T MR system (Achieva, Philips Healthcare). The cardiac rest period for end-systole and mid-diastole was determined from a 2D SSFP cine scan with high temporal resolution (TR/TE $=3.1 / 1.6 \mathrm{~ms}$, flip angle $60^{\circ}$, slice thickness $6 \mathrm{~mm}, 60$ to 80 cardiac phases). A previously developed free-breathing navigator gated 3D SSFP dual cardiac phase sequence [I] was then applied in sagittal orientation for imaging of the whole heart including the coronary arteries and great vessels $\left(\mathrm{TR} / \mathrm{TE}=3.4 / 1.7 \mathrm{~ms}\right.$, flip angle $90^{\circ}$, 60-120 slice, isotropic resolution of $1-1.5 \mathrm{~mm}^{3}$, temporal resolution of $60-75 \mathrm{~ms}$, Sense of 2 in AP direction). Data was obtained during end-systole and mid-diastole and the acquisition window of the 3D scan was adapted accordingly to the shortest rest period. Images were then reformatted along the major axes of the left and right coronary artery for both cardiac phases. Vessel length, diameter and sharpness of the visualized coronary arteries (RCA, LM, LAD, LCx) were measured using the "SoapBubble" software. Image quality was assessed by two independent observers. Statistical analysis and Bland Altman plots were used to compare the different data sets.
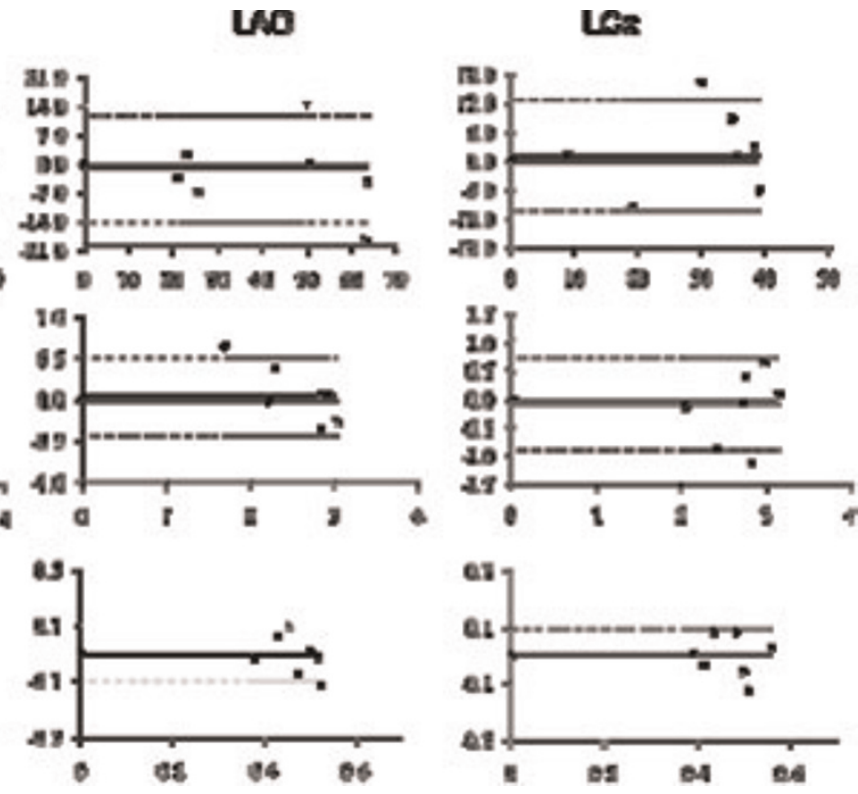

Results: The dual cardiac phase whole heart scan was applied successfully in all patients. An example of each coronary segment is shown in figure I. The vessel length, diameter, sharpness and consensus score for each segment during systole and diastole are shown in Table I. Bland Altman plots of the systolic versus diastolic data from each coronary segment are shown in figure 2 . No statistically difference was found comparing vessel length, diameter, sharpness for all vessels between systole and diastole. Moreover, no statistically difference was found in image quality. Although, there was no difference for the mean vessel length, diameter, and sharpness, it was found that on a patient level, those parameters and image quality showed differences either favoring systolic or diastolic image acquisition for different coronary segments within the same patient.

Conclusion: A 3D SSFP dual cardiac phase scan of the whole heart was capable of identifying the origin and proximal course of the coronary arteries in children in two cardiac phases. The ability to show the 3D relationship of the heart, great vessels and coronary arteries, and also the possibility to retrospectively select the heart phase with best coronary artery visualization make this technique a clinically valuable tool for surgical planning.

Reference

I. Uribe, et al: Radiology 2008.

Table I (abstract P68) Mean \pm SDV values for different parameters

\begin{tabular}{|c|c|c|c|c|c|c|c|c|}
\hline & RCA & RCA & LM & LM & LAD & LAD & LCx & LCx \\
\hline & Systole & Diastole & Systole & Diastole & Systole & Diastole & Systole & Diastole \\
\hline Vessel Length & $76.94 \pm 24.15$ & $75.54 \pm 23.96$ & $21.26 \pm 31.06$ & $20.93 \pm 31.41$ & $41.02 \pm 018.44$ & $43.36 \pm 20.24$ & $30.35 \pm 12.8$ & $28.21 \pm 11.48$ \\
\hline Vessel Diameter & $2.78 \pm 0.42$ & $2.76 \pm 0.47$ & $2.34 \pm 1.15$ & $2.09 \pm 1.02$ & $2.58 \pm 0.37$ & $2.5 \pm 0.64$ & $2.6 I \pm 0.56$ & $2.77 \pm 0.4$ \\
\hline Vessel Sharpness & $0.52 \pm 0.1$ & $0.48 \pm 0.09$ & $0.37 \pm 0.19$ & $0.37 \pm 0.19$ & $0.46 \pm 0.05$ & $0.47 \pm 0.07$ & $0.47 \pm 0.06$ & $0.47 \pm 0.07$ \\
\hline $\begin{array}{l}\text { Consensus Image } \\
\text { Ouality Score (I to } 4 \text { ) }\end{array}$ & $3.88 \pm 0.44$ & $3.19 \pm 0.75$ & $3 \pm 0.6$ & $2.5 \pm 0.53$ & $3 \pm 0.6$ & $2.5 \pm 0.53$ & $3.14 \pm 0.56$ & $3 \pm 1$ \\
\hline
\end{tabular}




\section{P69}

Non-invasive quantification of myocardial fibrosis in diabetic mice using in-vivo high-resolution MRI Sok Sithikun', Frank Kober', Alexis Jacquier', Jérôme Kalifa', Francis Kopp', Marie-France Bonzi², JeanClaude Deharo ${ }^{3}$, Patrick J Cozzone' and Monique Bernard

${ }^{I}$ CRMBM, Marseille, France

${ }^{2}$ Laboratory of Histology, Marseille, France

${ }^{3}$ Department of Cardiology, Arrhythmias unit, Marseille, France

Journal of Cardiovascular Magnetic Resonance 2009, I I (SuppI I):P69

Introduction: There is an established role for magnetic resonance imaging in the assessment of myocardial fibrosis, in ischaemic and non-ischaemic cardiomyopathies. The amount of fibrosis is of importance because of its prognostic value, in terms of arrhythmias occurrence, and haemodynamic consequences. $\mathrm{T} 2$ relaxation time directly depends on physico-chemical properties of each tissue. Thus, myocardial T2 time determination can help in quantifying fibrosis. Diabetic cardiomyopathy is characterized by myocardial structural modifications including hypertrophy, microcirculation impairment and interstitial fibrosis. Purpose: To describe a non-invasive MRI method using highresolution myocardial T2 time measurement to assess myocardial fibrosis in a model of diabetic mice in vivo.

Methods: Two multi-slice spin-echo sequences were performed for T2 time assessment respectively at 20 and $9 \mathrm{~ms}$ echo time (resolution $85 \times 85 \mu \mathrm{m} 2$, slice thickness $1.0 \mathrm{~mm}$, imaging time 15 minutes), in ten 16 -week old $\mathrm{C} 57 \mathrm{BI} / 6 \mathrm{~J}$ after 8 weeks of streptozotocin-induced diabetes, and ten control mice, under isoflurane anesthesia using a II.75 T vertical scanner (Bruker, Rheinstetten, Germany). All images were acquired in short-axis view. MRI measurements were then compared with histological quantification of fibrosis using picrosirius red staining. Results: T2 relaxation time was significantly lower in diabetic mice $(13.8+/-2.8 \mathrm{~ms}$ versus $18.9+/-2.3 \mathrm{~ms}$ in the control group, $p<0.05)$. This was associated with a significant increase of myocardial fibrosis as evaluated by picro-sirius red staining, in diabetic mice.

Conclusion: We describe here a non-invasive and accurate MRI method to quantify myocardial fibrosis in vivo in diabetic mice, using T2 relaxation time measurement. This method can be applied in many ischaemic or non-ischaemic cardiomyopathies in which a fibrotic phenomenon is involved.

\section{P70}

Cardiac MR for ventricular scar evaluation in patients with implanted defibrillators: technical and safety issues, and initial results

Harold Litt and Francis Marchlinski

University of Pennsylvania School of Medicine, Philadelphia, PA, USA

\section{Journal of Cardiovascular Magnetic Resonance 2009, I I (SuppI I):P70}

Introduction: Catheter based RF ablation is becoming an increasingly accepted treatment for refractory ventricular arrhythmias and late enhancement MRI can assist by providing anatomic maps of regions of scar which may form the basis for the arrhythmia. Unfortunately, many patients with ventricular arrhythmias have defibrillators (ICDs) implanted early in the
Figure I (abstract P70)

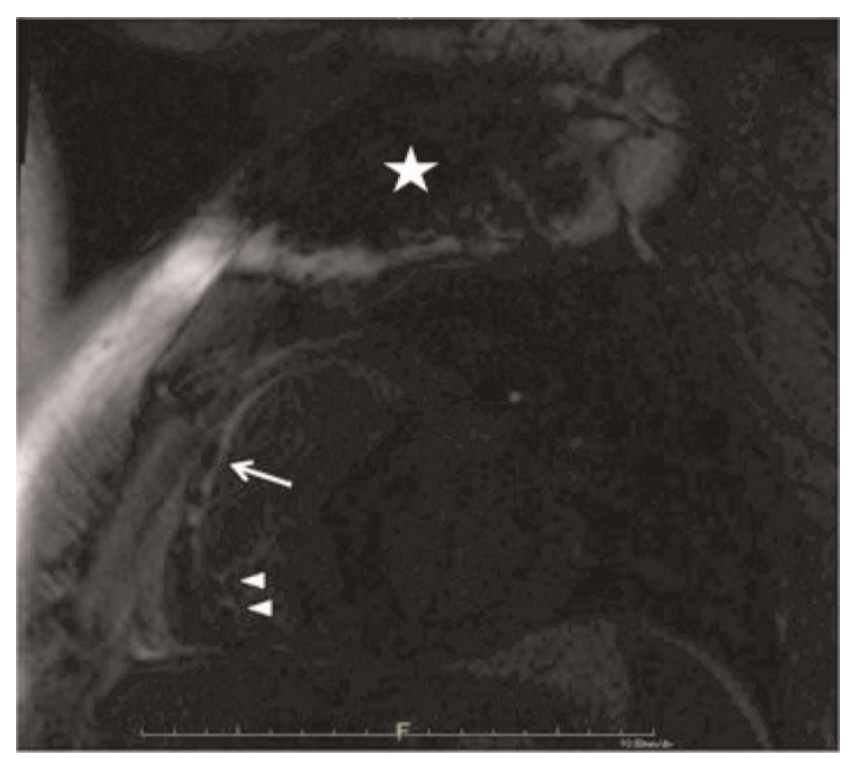

course of their disease, prior to MR imaging assessment of cardiac structure, function, and scar burden. As ICDs are generally considered a contraindication to $\mathrm{MRI}$, and the presence of a defibrillator may cause significant artifacts in the chest even if a study is performed, the use and utility of cardiac MR has not been studied in these patients.

Purpose: To evaluate the feasibility and safety of performing cardiac MR in patients with ICDs and refractory ventricular arrhythmias and provide an initial assessment of its utility for scar mapping related to RF ablation procedures.

Methods: Six patients with ICDs suffering from refractory ventricular tachyarrhythmias were imaged within several days of catheter ablation procedures. All patients gave informed consent for performing MRI in the presence of an ICD. The devices were interrogated prior to and after the study; tachycardia detection and therapies were turned off, and the pacemakers were set to VVI mode at a rate of $40 \mathrm{bpm}$ for the duration of the MRI.

All studies were performed at I.5 T. When possible, low SAR non-balanced gradient-echo cine and IR-prepped late-enhancement sequences were used, however real-time SSFP sequences were used in the presence of frequent ventricular ectopic beats. Imaging parameters were adjusted to minimize artifacts from the device.

Results: All studies were completed safely, no patients reported any discomfort, and there were no episodes of tachycardia requiring treatment. Post-procedure device parameters, including thresholds, lead impedances, and battery voltage were unchanged in all patients, and all devices could be reprogrammed to previous settings. Real time SSFP cine sequences were needed in two patients with frequent ventricular ectopic beats. As expected, spin echo sequences were less affected by artifact from the ICD generator than gradient echo sequences, with SSFP cine sequences showing greater artifact than non-balanced gradient echo cine sequences. Late enhancement images were particularly affected, with significant difficulties encountered in nulling the myocardium in regions affected by artifact. Increasing bandwidth, with resultant decrease in TE, 
decreased the size of the device related artifact but also impacted SNR. The degree to which cardiac evaluation was limited by artifact was most influenced by the size of the patient and distance from the generator to the left ventricle.

Two patients were diagnosed with right ventricular cardiomyopathy with good visualization of RV structure, function, and scar. One patient had hypertrophic cardiomyopathy, and three nonspecific LV cardiomyopathies, with variable limitations in visualization of the LV antero-lateral wall and apex. All patients did have evidence of focal myocardial scar in regions not affected by artifact; imaging information was consistent with electrophysiologic observations and was considered clinically valuable in all cases. Figure I shows a large region of scar in the RV free wall and RVOT (arrow) in a patient with RV cardiomyopathy; note pacemaker/ICD leads (arrowheads) and artifact from generator (star).

Conclusion: The results of this initial study suggest that cardiac MR for scar mapping may be performed safely in patients with ICDs under certain circumstances, giving clinically relevant results in those with refractory ventricular arrhythmias having RF ablation procedures.

\section{P7 I}

Altered right ventricular papillary muscle position and orientation in patients with dilated left ventricles

Erin M Spinner', Kartik Sundareswaran', Lakshimi Prasad Dasi ', Vinod H Thourani ${ }^{2}$, John Oshinski ${ }^{2}$ and Ajit P Yoganathan'

'Georgia Institute of Technology, Atlanta, GA, USA

${ }^{2}$ Emory University, Atlanta, GA, USA

Journal of Cardiovascular Magnetic Resonance 2009, I I (SuppI I):P7 I

Introduction: Displacement of papillary muscle (PM) positions in the right ventricle (RV) may be an important factor in inducing tricuspid regurgitation (TR). We hypothesize that left ventricular
Figure I (abstract P7I)

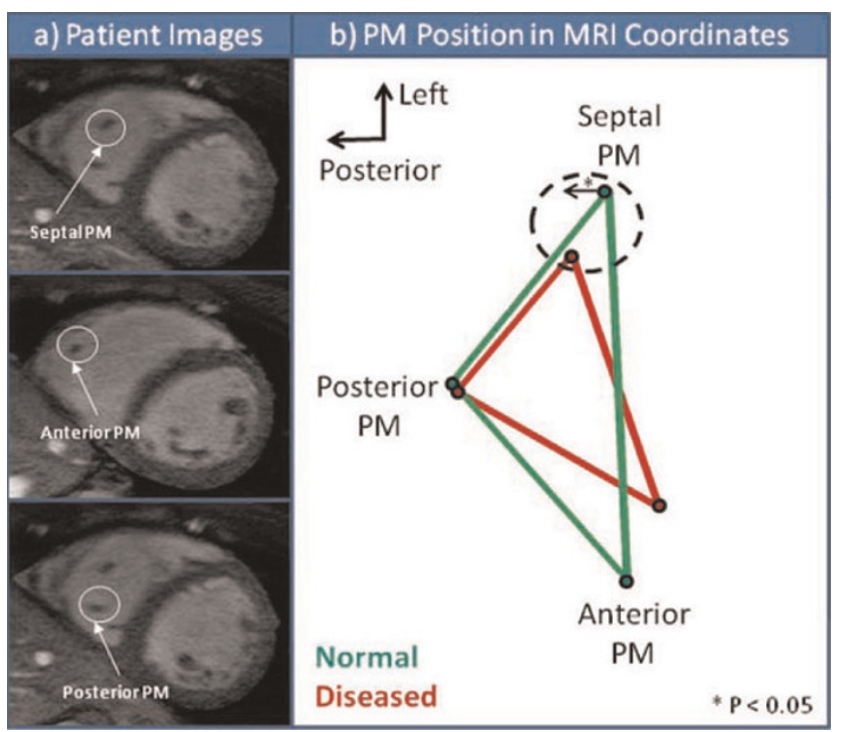

(LV) dilation is one possible mechanism for RV PM displacement; given the adjacent and proximal relative positions of the two ventricles.

Purpose: In this study, we identify and compare the position of RV PMs in normal subjects and patients with LV dilation to quantitatively establish RV PM positions and the influence of LV dilation on RV PM displacement.

Methods: 7 congestive heart failure patients with mild to severe mitral valve regurgitation and a dilated left ventricle (LVEDV $234.7 \pm 70.8 \mathrm{ml}$ ) were recruited at Emory University Hospital. 6 normal subjects were used as a control in this study. Scans were acquired on a Philips Intera CV I.5 T system using a cardiac coil. The MRI scan to assess PM position was a high

Table I (abstract P7I) Numerical measurements of PMs for normal and dilated LV patients

\begin{tabular}{|c|c|c|c|c|c|}
\hline & $\begin{array}{l}\text { Normal } \\
\text { Average }\end{array}$ & $\begin{array}{l}\text { Normal } \\
\text { Standard Error }\end{array}$ & $\begin{array}{l}\text { Dilated LV } \\
\text { Average }\end{array}$ & $\begin{array}{l}\text { Dilated LV } \\
\text { Standard Error }\end{array}$ & $\begin{array}{l}\text { T-Test } \\
\text { P value }(* p<0.05)\end{array}$ \\
\hline \multicolumn{6}{|l|}{ Anterior PM } \\
\hline$X$ dist & 0.61 & 0.09 & 0.38 & 0.04 & 0.06 \\
\hline Y dist & 0.20 & 0.04 & 0.30 & 0.04 & 0.11 \\
\hline$Z$ dist & 0.20 & 0.05 & 0.15 & 0.03 & 0.44 \\
\hline \multicolumn{6}{|l|}{ Posterior PM } \\
\hline$X$ dist & 0.00 & 0.07 & 0.03 & 0.06 & 0.76 \\
\hline$Y$ dist & -0.35 & 0.04 & -0.33 & 0.06 & 0.83 \\
\hline$Z$ dist & 0.65 & 0.03 & 0.62 & 0.03 & 0.48 \\
\hline \multicolumn{6}{|l|}{ Septal PM } \\
\hline$X$ dist & -0.61 & 0.08 & $-0.4 \mid$ & 0.07 & 0.09 \\
\hline$Y$ dist & 0.14 & 0.03 & 0.03 & 0.04 & $0.03 *$ \\
\hline$Z$ dist & 0.85 & 0.07 & 0.74 & 0.05 & 0.24 \\
\hline Dist Ant PMDist Post PM & 0.690 .76 & 0.080 .04 & 0.490 .65 & 0.060 .10 & 0.070 .32 \\
\hline Dist Sept PM & 1.07 & 0.08 & 0.76 & 0.11 & $0.05 *$ \\
\hline Triangle Area & 0.13 & 0.02 & 0.09 & 0.02 & 0.23 \\
\hline Sept - Ant PM dist & 1.40 & 0.15 & 1.04 & 0.08 & $\mathrm{I} .08$ \\
\hline Sept - Post PM dist & 0.84 & 0.10 & 0.63 & 0.11 & 0.19 \\
\hline Post - Ant PM dist & 0.99 & 0.07 & 0.90 & 0.07 & 0.35 \\
\hline
\end{tabular}


resolution, navigator-echo gated 'whole heart' sequence acquired in the short axis orientation. Pixel size ranged from $0.53-0.70$ $\mathrm{mm}$, slice thickness ranged from $1.5-2.0 \mathrm{~mm}$, and echo time ranged from I.3-2.I ms. Approximately 120 slices were acquired at mid-diastole to cover the entire LV and RV. Papillary muscle tip position was manually identified in the various images by identifying the regions of low intensity (PM) among regions of high intensity (blood) within the right ventricle (RV) (Fig la). Coordinates were recorded for all three papillary muscles, septal, posterior and anterior for all subjects. All images were registered to the MRI coordinate system for the purpose of position and orientation comparison. The centroid of the three PM coordinates was used as the reference point (Fig lb). All distances were normalized by ascending aortic diameter to minimize patient variability. Values for directional distances from the centroid $(x, y, z)$ where calculated for each PM as well as, area of the triangle formed by the three PMs, distance of each triangle side length, and distance from each PM to the centroid. Values for normal subjects and dilated LV patients were then analyzed for statistical significance using parametric unpaired t-test.

Results: Significant $(p<0.05)$ posterior displacement of $X Y Z$ $\mathrm{mm} /$ aortic size of the septal PM from its normal position was observed (Dilated $0.029 \pm 0.04 \mathrm{~mm} /$ aortic diameter, Normal $0.144 \pm 0.03 \mathrm{~mm} /$ aortic diameter). More specifically the septal PM significantly displaced from the septal wall (Dilated $0.38 \pm 0.04 \mathrm{~mm} /$ aortic diameter, Normal $0.6 \mathrm{I} \pm 0.09 \mathrm{~mm} /$ aortic diameter). The PM position in normal patients forms a symmetric triangle with sides of similar lengths. For the first time, specific location of all three RV PMs is reported for normal and dilated LV patients (Table I).

Conclusion: Dilatation of the LV has an impact on PMs in the RV. This may have implications in the development of tricuspid valve disease. This also demonstrates that diseases on the left side of the heart also affect the right side of the heart.

\section{P72}

Automatic delineation of myocardial contours in late-enhancement long-axis cardiac MR images Cybele Ciofolo', Maxim Fradkin', Gilion Hautvast ${ }^{2}$ and Marcel Breeuwer ${ }^{2}$

${ }^{I}$ Medisys Research Lab - Philips Healthcare, Suresnes Cedex, France

${ }^{2}$ Philips Medical Systems Nederland B.V., Best, Netherlands

Journal of Cardiovascular Magnetic Resonance 2009, I I (SuppI I):P72

Introduction: Viability assessment is essential for therapy planning following a myocardial infarction. In particular, the proportion of viable myocardium is a major factor in determining whether a patient may benefit from revascularization [I]. In addition to estimating the left ventricular myocardial thickness and thickening with functional imaging, it is possible to visualize normal and non-viable areas with high spatial resolution, using late-enhancement cardiac MR imaging (LECMR). To locate and quantify non-viable tissue, it is first necessary to delineate the endo- and epicardial contours on every available view of the LECMR acquisition. In particular long-axis (LA) views are useful because they provide a visualization of the apical area, which is often not well visible on or not covered well by short-axis slices. While manual delineation is a tedious and time-consuming task, its automation is challenging and, to our knowledge, not yet
Table I (abstract P72) Mean positioning error with respect to 3 manual contours and inter-observer variability. $D_{\text {Refi }}$ is the mean distance to reference contour number $i$

\begin{tabular}{llllll}
\hline Contour & Unit & $\mathbf{D}_{\text {Ref1 }}$ & $\mathbf{D}_{\text {Ref2 }}$ & $\mathbf{D}_{\text {Ref3 }}$ & $\begin{array}{l}\text { Inter-observer } \\
\text { variability }\end{array}$ \\
\hline Endocardium & $\mathrm{mm}$ & $2.4 \pm 0.9$ & $2.6 \pm 0.9$ & $2.3 \pm 0.8$ & $1.7 \pm 0.7$ \\
& pixels & $1.6 \pm 0.6$ & $1.7 \pm 0.6$ & $1.5 \pm 0.5$ & $1.1 \pm 0.5$ \\
Epicardium & $\mathrm{mm}$ & $2.3 \pm 1.0$ & $2.4 \pm 0.9$ & $2.4 \pm 1.1$ & $1.5 \pm 0.9$ \\
& pixels & $1.5 \pm 0.6$ & $1.6 \pm 0.6$ & $1.6 \pm 0.7$ & $1.0 \pm 0.6$ \\
\hline
\end{tabular}

addressed by any publication or commercial product. This is mainly due to the non-homogeneous intensity of the myocardium resulting from contrast agent accumulation in infarcted areas.

Purpose: We propose a novel method to delineate the endoand epicardial contours in late-enhancement long-axis cardiac $M R$ images with a minimal user-input in order to provide an accurate quantitative viability assessment.

Methods: Before running the automatic segmentation process, the user selects one enhancement type from four pre-defined ones (no enhancement, diffuse/small enhancement, large/transmural scar, sub-endocardial enhancement), depending on his own observation of the LA view.

Then the delineation of the myocardial contours is performed by alternating automatic deformation of a geometrical template and computation of a binary map of the enhanced areas. The template is ribbon-shaped with a variable width, its position is updated depending on image gray values. The map is a 2D binary image showing enhanced areas, it is updated by thresholding the image gray values in a region of interest centered on the endocardium, in order to include sub-endocardial scars. The segmentation is performed as follows: (I) automatic delineation of myocardial contours on short-axis LECMR slices [2],

\section{Figure I (abstract P72)}

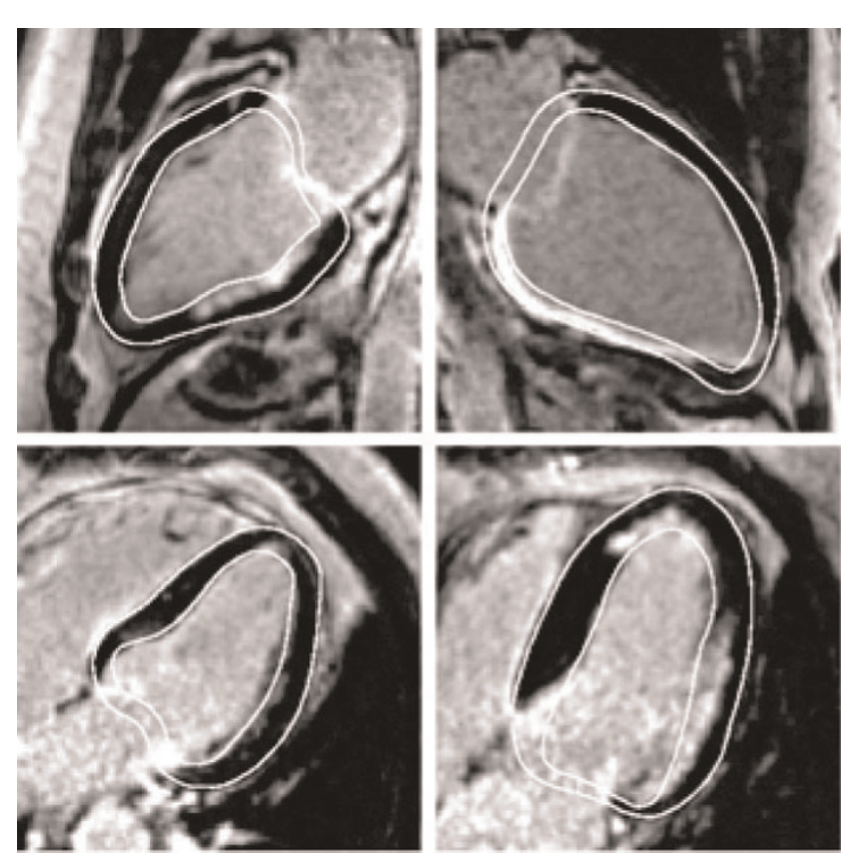

Final myocardium contours on 4 different patients. 
(2) initialization of the geometrical template position and binary map based on step (I) results, (3) iterative loop between geometrical template deformation and update of enhanced areas map: each new position of the template leads to a new map computation, which is then used to deform the template again.

Results: The method was tested on 20 LA LECMR images acquired in a multi-center study between 2004-2007 (Philips Intera scanner I.5 T, M FFE sequence, TE $=1.7 \mathrm{~ms}, \mathrm{TR}=4.5 \mathrm{~ms}$, flip angle $=15^{\circ}$ ). All images are $256 \times 256$, with pixel size around 1.5 $\mathrm{mm}$. Three experienced users manually delineated the myocardial contours to quantitatively assess the precision of the proposed method and evaluate inter-observer variability. The average error between the manual and automatic contours was $2.4+/-1.0 \mathrm{~mm}$ for the endocardium and $2.2+/-1.1 \mathrm{~mm}$ for the epicardium (see Table I for detailed results). The inter-observer variability was computed as an average distance from each manual contour to the other ones and was equal to $1.7+/-0.7 \mathrm{~mm}$ for the endocardium and $1.5+/-0.9 \mathrm{~mm}$ for the epicardium. As shown in Fig. I, the visual quality is good, the contours successfully surround both normal and abnormal parts of the myocardium, which allows a reliable assessment of the percentage of non-viable tissue. Moreover, the accuracy ( I.5 pixel) is in the same range as inter-observer variability (>I pixel). Remark: as accurate delineation of the valve plane is not required for viability assessment as long as myocardial contours are correct, it was not addressed in this study.

Conclusion: We presented a robust and efficient method for the automatic delineation of the myocardial contours in long-axis LECMR images.

References

I. Marshall, et al: Circulation 1983, 67:766-778.

2. Ciofolo, et al: Proc SCMR'08 2008, 203-204.

\section{P73}

\section{In vivo comparison of DENSE and CSPAMM for} cardiac motion analysis

Christian T Stoeck', Sebastian Kozerke', Neil Maredia², Andrew Crean ${ }^{2}$, John P Greenwood ${ }^{2}$ and Sven Plein ${ }^{2}$ 'Institute for Biomedical Engineering, University and ETH, Zurich, Switzerland

${ }^{2}$ Academic Unit of Cardiovascular Medicine, University of Leeds, Leeds, UK

Journal of Cardiovascular Magnetic Resonance 2009, I I (SuppI I):P73

Introduction: Tagging has shown great promise for analyzing cardiac motion patterns [I]. Two different methods have been proposed to utilize the phase associated with harmonic modulation of magnetization to enable tissue tracking. Using Displacement ENcoding with Stimulated Echoes (DENSE) [2] tagged magnetization is demodulated by decoding gradients permitting motion tracking from the signal phase. In contrast, HARmonic Phase (HARP) [3] analysis of Complementary SPAtial Modulation of Magnetization (CSPAMM) data decomposes tagged data into its harmonic components during post-processing. It has been argued in principle that the information content of DENSE and HARP should be identical [4]. However, a formal comparison of DENSE and HARP has not been undertaken to date. In this study, DENSE and HARP data were obtained consecutively in the same subjects and circumferential shortening, rotation and time to peak motion were evaluated.

Methods: Eight healthy volunteers were imaged using 2D CSPAMM [5] and 2D DENSE [6] with identical scan duration $(\sim 14 \mathrm{sec})$. Data were read out using an EPI sequence with the following parameters: TR/TE $/ \alpha=30 \mathrm{~ms} / 5.3 \mathrm{~ms} / 20^{\circ}$, acquisition matrix of $96 \xi 42$ (CSPAMM) or $48 \xi 40$ (DENSE) reconstructed to $192 \xi 192$, FOV of $320 \mathrm{~mm} \xi 253 \mathrm{~mm}$, slice thickness of $8 \mathrm{~mm}$. Tagging was applied using $2 \times$ lines (line distance: $8 \mathrm{~mm}$ ) employing two orthogonal imaging stacks.

Data were analyzed using TagTrack v.I.8 (GyroTools Ltd, Zurich, Switzerland). For DENSE the echo signal was shifted by $20.8 \%$ in $\mathrm{k}-$ space (corresponding to $8 \mathrm{~mm}$ tag line distance), to create a phase image for HARP processing. CSPAMM data were processed directly with the HARP method using peak combination, demodulated peak combination and conventional single peak method [7].

The mid-contour inside the left ventricular myocardium was tracked starting from an end-diastolic frame. Initial contours were identical for DENSE and CSPAMM data to reduce observer variability. The left ventricle was segmented into six equidistant sectors and resulting curves for circumferential length and rotation were fitted by a fourth order polynomial. Comparison of both methods was performed using a Bland Altman test.

Results: Figure I shows Bland Altman plots for the comparison of DENSE and single peak HARP analysis. The difference between two measurements is presented for the time to peak circumferential shortening $(A)$, the relative circumferential shortening $(B)$, the time to peak rotation $(C)$ and the amount of rotation $(D)$, relative to the average of both measurements. Relative motion measurements show a bigger variation than timing measurements. Table I gives a detailed overview of the levels of agreement, bias and the first standard deviation of a comparison between DENSE and peak combined HARP, demodulated peak combined HARP and single peak HARP.

Table I (abstract P73) The levels of agreement, bias and standard deviations from Bland Altman tests for the cardiac motion analysis are shown

\begin{tabular}{|c|c|c|c|c|c|c|c|c|}
\hline \multirow[b]{2}{*}{$\begin{array}{l}\text { DENSE vs. } \\
\text { peak combination HARP } \\
\text { demodulated peak combination HARP } \\
\text { single peak HARP }\end{array}$} & \multicolumn{4}{|c|}{ time to peak circ. shortening [\% of average] } & \multicolumn{4}{|c|}{ time to peak rotation [\% of average] } \\
\hline & $\begin{array}{l}95 \% \\
18.65 \\
9.75 \\
9.63 \\
\text { circ. s }\end{array}$ & $\begin{array}{c}-95 \% \\
-22.84 \\
-9.16 \\
-9.99 \\
\text { ning [\% }\end{array}$ & $\begin{array}{l}\text { bias } \\
-2.10 \\
0.30 \\
-0.18 \\
\text { rage] }\end{array}$ & $\begin{array}{l}\text { stdev } \\
10.58 \\
4.82 \\
5.00\end{array}$ & $\begin{array}{l}95 \% \\
42.15 \\
31.10 \\
30.89 \\
\text { rotati }\end{array}$ & $\begin{array}{c}-95 \% \\
-47.36 \\
-33.69 \\
-30.91 \\
\text { [\% of ave }\end{array}$ & $\begin{array}{l}\text { bias } \\
-2.60 \\
-1.30 \\
-0.01 \\
\end{array}$ & $\begin{array}{l}\text { stdev } \\
22.83 \\
16.53 \\
15.77\end{array}$ \\
\hline $\begin{array}{l}\text { DENSE vs. } \\
\text { peak combination HARP } \\
\text { demodulated peak combination HARP } \\
\text { single peak HARP }\end{array}$ & $\begin{array}{l}95 \% \\
45.18 \\
35.29 \\
41.73\end{array}$ & $\begin{array}{l}-95 \% \\
-47.23 \\
-44.54 \\
-38.81\end{array}$ & $\begin{array}{l}\text { bias } \\
-1.03 \\
-4.62 \\
1.46\end{array}$ & $\begin{array}{l}\text { stdev } \\
23.57 \\
20.36 \\
20.55\end{array}$ & $\begin{array}{l}95 \% \\
50.44 \\
84.44 \\
62.82\end{array}$ & $\begin{array}{l}-95 \% \\
-77.07 \\
-110.45 \\
-59.60\end{array}$ & $\begin{array}{l}\text { bias } \\
-13.31 \\
-13.00 \\
1.61\end{array}$ & $\begin{array}{l}\text { stdev } \\
32.53 \\
49.72 \\
31.23\end{array}$ \\
\hline
\end{tabular}




\section{Comparison of DENSE and CSPAMM (single peak HARP)}
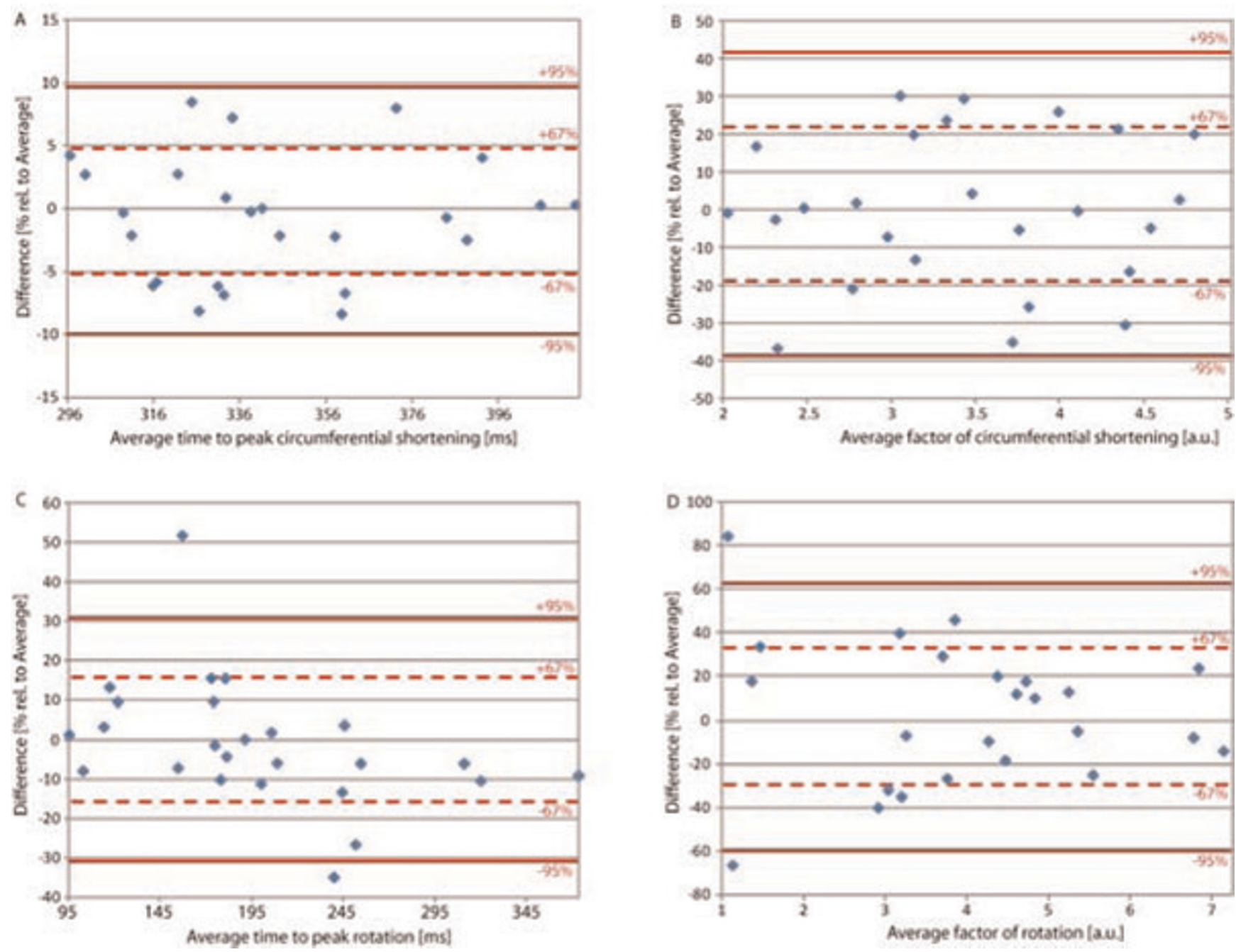

Bland Altman tests for the comparison of DENSE and single peak HARP analysis are shown for time to peak circ. shortening (A), relative circ. shortening (B), time to peak rotation (C) and amount of rotation (D).

Discussion: This work has presented a direct comparison of CSPAMM/HARP and DENSE. Results indicate that the both methods agree well when considering bias. However, considerable variation in individual values has been found which may partly be attributed to differences in breath hold position for the two different scans and phase unwrapping errors in demodulation of peak combination HARP. Future work is necessary to identify the cause of this variability.

\section{References}

I. Axel L: Radiology 1989.

2. Aletras AH: JMR 1999.

3. Osman NF: MRM 1999.

4. Kuijer JPA: JMRI 2006.

5. Fischer SF: MRM 1993.

6. Gilson WD: MRM 2004.

7. Ryf S: JMRI 2004.
P74

High-temporal resolution ( $<6 \mathrm{~ms}$ ) Cine Steady-State Free Precession (SSFP) imaging for assessing LV diastolic function

Ramkumar Krishnamurthy', Benjamin Cheong ${ }^{2}$,

Amol Pednekar ${ }^{3}$ and Raja Muthupillai ${ }^{2}$

${ }^{\prime}$ Rice University, Houston, TX, USA

${ }^{2}$ St. Luke's Episcopal Hospital, Houston, TX, USA

${ }^{3}$ Philips Medical Systems, Houston, TX, USA

Journal of Cardiovascular Magnetic Resonance 2009, I I(SuppI I):P74

Background: While a modest temporal resolution of conventional cine SSFP MRI $(30-50 \mathrm{~ms})$ is sufficient for calculating parameters characterizing LV systolic function, e.g., stroke volume (SV) or ejection fraction (EF), it is insufficient for characterizing diastolic relaxation phenomena via metrics such as 
Figure I (abstract P74)

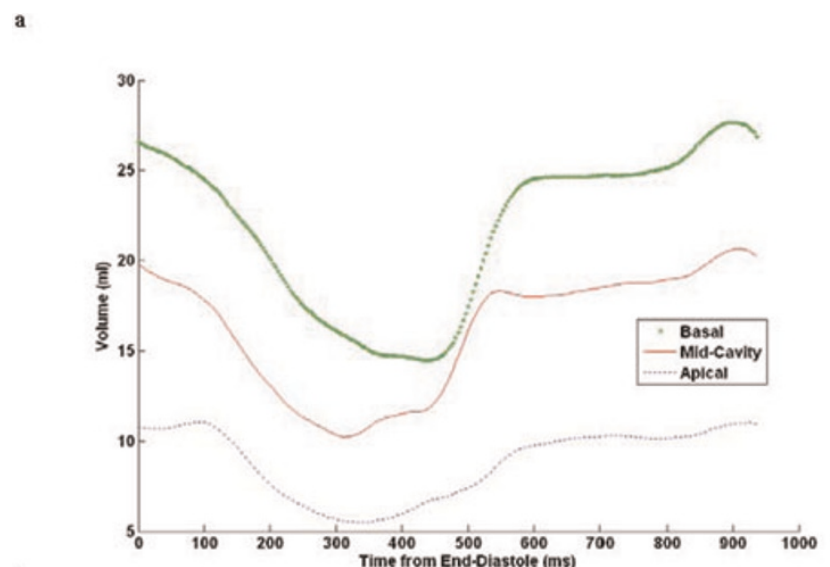

b

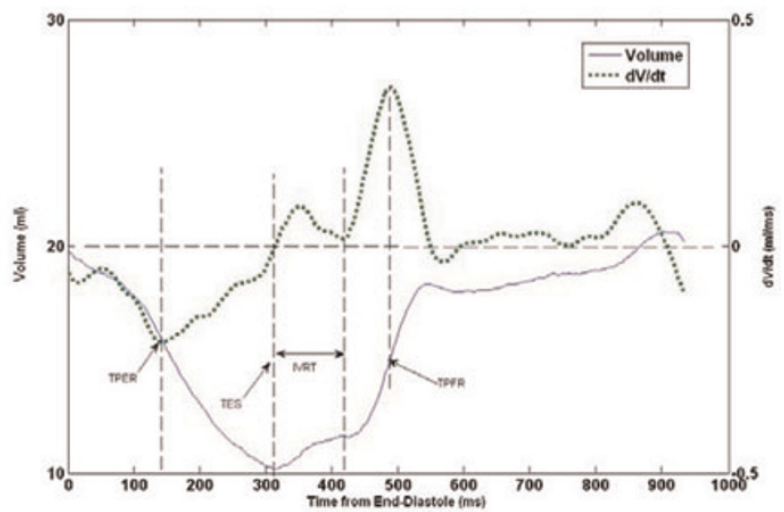

a) Volume-time curves of LV short axis slices at different locations. B) Time-Volume and dV/dt curves of mid-LV cavity short axis slice.

isovolumic relaxation time (IVRT) or Time to Peak Filling Rate (TPFR).

Purpose: We describe a fast cine MR imaging technique with a temporal resolution of under $6 \mathrm{~ms}$ using the acceleration techniques of Sensitivity Encoding (SENSE) and spatial-temporal frequency Broad-use Linear Acquisition Speed-up Technique ( $k-t$ BLAST).

Methods: MR acquisition: High temporal resolution cine SSFP images were acquired in 13 normal volunteers $(12 \mathrm{~m}$, $35 \pm 8$ years) using 32 channel// 6 channel cardiac coils at I.5 T (Achieva, Philips Medical Systems) using SENSE and k-t BLAST. The acquisition parameters were: acquired voxel size: $2 \times 2 \times 8$ $\mathrm{mm}^{3}$, temporal resolution $5.8-6 \mathrm{~ms} ; \mathrm{TR} / \mathrm{TE} / \mathrm{flip}=2.8-3 \mathrm{~ms} / \mathrm{l} .4-$ $1.5 \mathrm{~ms} / 55^{\circ}$; breath-hold time : 18 heart-beats/slice. The effective acceleration factors were: SENSE - 3; k-t BLAST - 3.8.

Table I (abstract P74) Bland-Altman Analysis: k-t BLAST and SENSE cine SSFP

\begin{tabular}{llll}
\hline & Bias \pm SD $(\mathrm{ms})$ & Mean $(\mathrm{ms})$ & Bias \pm SD $(\%)$ \\
\hline TES & $2.32 \pm 18.1$ & 296.2 & $0.76 \pm 6.1$ \\
TPFR & $5.8 \pm 8.7$ & 480.0 & $1.2 \pm 1.8$ \\
IVRT & $-0.08 \pm 16.6$ & 99.7 & $-0.08 \pm 16.6$ \\
\hline
\end{tabular}

Figure 2 (abstract P74)
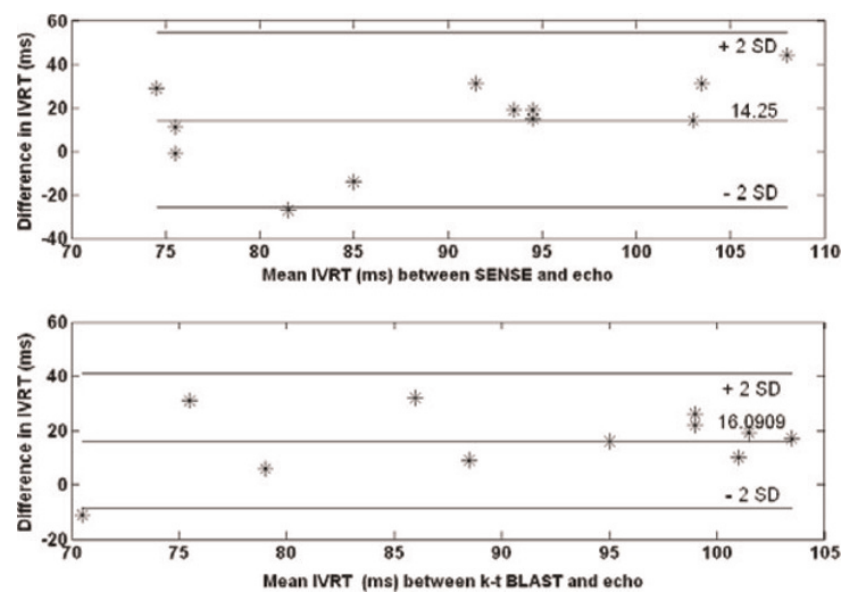

Bland-Altman analysis of IVRT between MRI and echo values.

Images were acquired along short-axis (basal, mid-cavity and apical) and long-axis (LVOT and 4-chamber) orientations. All subjects underwent echo immediately after MR.

Data analysis: The LV cavity was segmented from cine MR images using a custom-built algorithm, and the time-volume (T-V) curves were generated. From the T-V curve, diastolic parameters IVRT, PFR, and TPFR were determined.

Results: Representative T-V curves from the basal, mid, and apical LV slices are in Figure Ia. All relaxation metrics were calculated from the T-V curve of the mid LV cavity (Figure Ib). The iso-volumic period is marked by local minima (dip) in the $\mathrm{dV} /$ dt curve immediately after the occurrence of Time to End Systole (TES) (zero-crossing in $\mathrm{dV} / \mathrm{dt}$ ). The TPFR and Time to Peak Ejection Rate (TPER) values are obtained as the time of maxima and minima of $\mathrm{dV} / \mathrm{dt}$ values (Figure $\mathrm{Ib}$ ).

The Bland Altman analysis revealed close agreement in the values of TES, IVRT and TPFR between kt-BLAST and SENSE acquisitions (Table I). MR estimate of IVRT based on T-V curves was consistently higher (about $15 \mathrm{~ms}$ ) compared to echocardiography (Figure 2).

Discussion: Firstly, as shown in Figure Ia, the filling patterns at the basal, mid, and apical slices are different. The dV/dt curve of the mid-LV cavity (figure Ib) had a marked dip after TES, highlighting the region of IVRT. This feature was not always present in the basal and apical filling patterns across subjects. We hypothesize that this may be attributed to more pronounced shape changes in the base and apex of the LV compared to the mid-LV. A similar observation has also been made by Zwanenburg et al. who noted that the maximum radial strain of the mid LV cavity coincided more with aortic valve closure than for basal or apical slices[I].

Secondly, our results show that MRI estimates of IVRT are consistently higher by about $15 \mathrm{~ms}$ compared to echo. Unlike echo, MRI estimates use the LV volume curves, and as a result, the calculation of IVRT using MRI may also include the protodiastolic period.

Lastly, the ability to obtain high-temporal resolution cine MR images also allows for the estimation of hitherto unexploited parameters such as PFR, TPFR, PER, and TPER and can pave the way for a more comprehensive analysis of LV function. 
Conclusion: I. Cine SSFP images with a temporal resolution of 5-6 ms yield time-volume curves that can be used to estimate diastolic functional parameters such as TPFR, PFR, and IVRT.

2. Diastolic indices computed from high-temporal resolution $k-t$ BLAST and SENSE are in agreement.

3. IVRT estimated from MRI time-volume curves is longer than echocardiography (around 14-16 ms).

\section{Reference}

I. Zwanenburg, et al: Am J Physiol Heart Circ Physiol 2004, 286: $1872-1880$.

\section{P75}

Effective fat-suppression for late gadolinium enhancement combined with a sequential acquisition order

Dana C Peters', Basem Dokhan², Reza Nezafat', Yuchi Han', Evan Appelbaum' and Warren J Manning'

'Beth Israel Deaconess Medical Center and Harvard Medical School, Boston, MA, USA

${ }^{2}$ ETH, Biomedical Engineering, Zurich, Switzerland

Journal of Cardiovascular Magnetic Resonance 2009, I I (SuppI I):P75

Introduction: Fat suppression is valuable for late gadolinium enhancement (LGE) imaging, for situations in which an enhanced structure is adjacent to fat (e.g. myocardium at the apex of the left ventricle, vessel walls, and the thin atrial walls), where fat might be mistaken for fibrosis. However, the acquisition order of the segmented LGE sequence is also critical, and a sequential acquisition order is superior to a centric acquisition order when visualizing small areas of scar, in which the "edge-enhancement" (i.e. the stronger weighting of the edges of k-space due to signal regrowth during the acquisition window) effect is not desired. A fat-suppressed LGE sequence using a sequential acquisition order may be optimal, but is not feasible using the typical fatselective (spectrally-selective) RF pulse with a flip angle from $90^{\circ}$ to $180^{\circ}$ preceding the sequential acquisition. To address this, we developed a "fat restore" method for fat-suppressed LGE.

Methods: Without a "fat restore" pulse, the fat experiences two $180^{\circ}$ s. The $1^{\text {st }} 180^{\circ}$ (the non-selective inversion always used in LGE) results in greatly reduced $M_{z f a t}$ at the time of the $2^{\text {nd }}$ fatselective $180^{\circ}$. The result is that fat regrows very quickly after the $2^{\text {nd }}$ fat-selective $180^{\circ}$ and fat-suppression is not achievable for a sequentially ordered acquisition: fat crosses the null point too

\section{Figure I (abstract P75)}

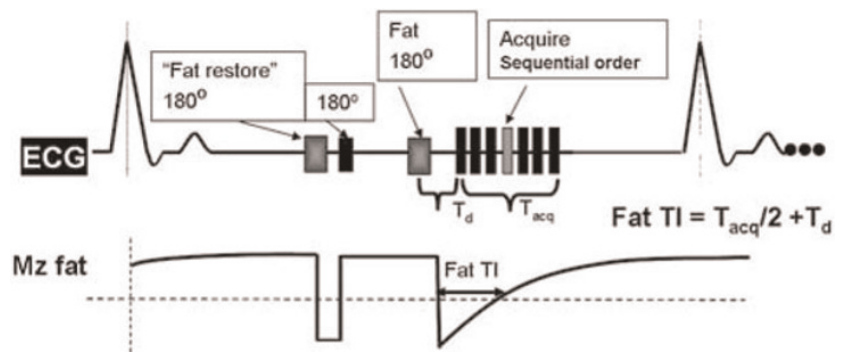

The LGE sequence is modified so that a "fat restore" pulse precedes the first inversion pulse, and fat is not inverted. This permits the $2^{\text {nd }}$ fatselective inversion pulse to be effective in providing fat-suppression for a sequential acquisition order.
Figure 2 (abstract P75)

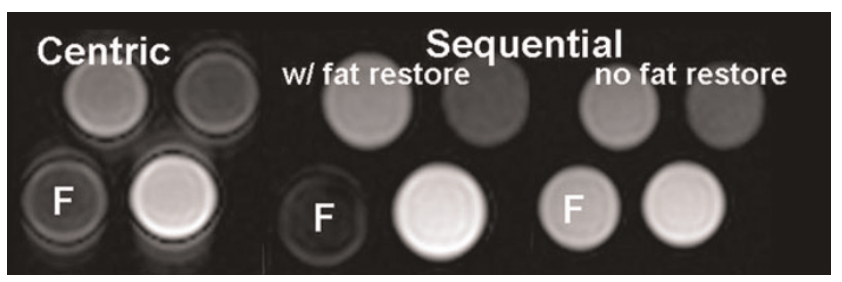

Phantom comparison of the fat suppression using standard method with centric order, and the sequential order with and without the fat restore pulse. Equivalent fat-suppression is provided by the fat restore technique. Edge enhancement is less using a sequential order. $F=$ fat.

early, requiring a very short fat $\mathrm{TI}$ (the time between the fat suppression pulse and the acquisition of the center of k-space, defined in Figure I). However, the minimum fat $\mathrm{TI}$ is $\sim \mathrm{Tacq} / 2 \mathrm{for}$ a sequential acquisition order. The "fat-restore" method uses an additional fat-selective $180^{\circ}$ before the standard non-selective $180^{\circ}$ used for LGE. The purpose of the "fat restore" pulse is to tip down the fat so that subsequent $180^{\circ}$ tips it back up to full magnetization. This will permit the later fat-selective $180^{\circ}$ to be effective (see schematic in Figure I). Phantoms including fat were imaged with and without the fat-restore pulse. Finally, the method was used in patient LGE studies. Scan parameters for phantoms and human studies were: $60 \mathrm{bpm}, \mathrm{TR} / \mathrm{TR} / \mathrm{flip}=4.3-$ $5 \mathrm{~ms} / \mathrm{l} .6-2.3 \mathrm{~ms} / 20^{\circ}, 32-40$ views per segment (Tacq $\sim 180 \mathrm{~ms}$ ), sequential order, I RR per inversion, TI set to null myocardium at the center of k-space ( $250 \mathrm{~ms}$ for phantoms). The fat TI was set to $100 \mathrm{~ms}$ using the fat-restore pulse. Without the fat-restore pulse, the minimum achievable fat $\mathrm{TI}(\sim 100 \mathrm{~ms}$ also) was used. For patients, $0.2 \mathrm{mmol} / \mathrm{kg}$ Gd-DTPA was injected, and scanning was performed 15-2 minutes post injection. All spectrally selective $180^{\circ}$ pulses were adiabatic pulses. The spatial resolution was $2 \times 2 \times 8 \mathrm{~mm}$ for 2D LGE.

Results: In phantom studies, the optimal fat TI (Figure I) was $\sim 120 \mathrm{~ms}$ and the ratio of $S N R_{\text {fat } w / f a t s a t}$ to $S N R_{\text {fat }}$ w/out fatsat was $8 \%$, using the fat restore pulse; without the additional pulse the ratio was $\sim 100 \%$. Figure 2 shows that the fat restore pulse with a sequential acquisition order provided similar fat-suppression as the standard fat-saturation using centric order with reduced edge enhancement. Furthermore, without a fat-restore pulse, fatsuppression was not good. Figure 3 compares a fat-suppressed 2D LGE scan with centric order and sequential order, with and

Figure 3 (abstract P75)

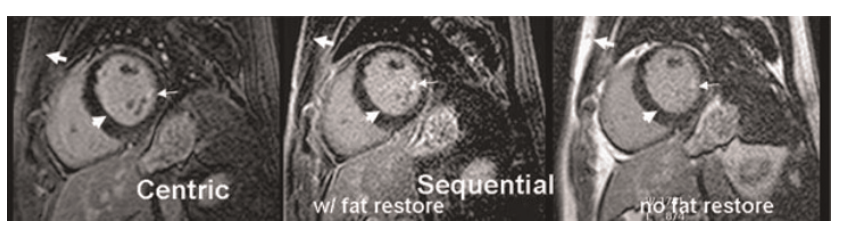

2D LGE comparing matched slices using centric, and sequential orders with and without the fat-restore pulse. The fat-restore pulse is needed for fat-suppression (thick arrows). Subtle edge-effects can be seen in the image with a centric acquisition (arrow heads). An area of enhancement at the tip of a papillary muscle (confirmed in other views) is also visualized in each image (thin arrows). 
without the fat restore pulse, in a patient with mitral valve prolapse. The subcutaneous fat is well suppressed even for the sequential order (thick arrows) using the fat restore pulse. A region of confirmed enhancement can be seen in all images (thin arrows), but a edge-enhancement effect is seen only in the image with centric acquisition order (arrow-heads).

Discussion and conclusion: We have demonstrated that the fat-restore pulse improves fat-suppression for sequentially acquired LGE scans. Studies with 3D LGE will likely improve with the linear acquisition order, improving the confidence for detecting small regions of scar.

\section{P76}

Registration and segmentation of cine and late enhancement cardiac magnetic resonance images Gilion Hautvast', Cybele Ciofolo-Veit ${ }^{2}$ and Marcel Breeuwer

IPhilips Healthcare, Best, Netherlands

${ }^{2}$ Philips Healthcare, Paris, France

Journal of Cardiovascular Magnetic Resonance 2009, I I (SuppI I):P76

Introduction: Cardiac Magnetic Resonance (CMR) can be used to assess myocardial function and viability by acquiring cine and late enhancement (LE) images. Quantitative assessment of myocardial function and viability requires delineation of the myocardial contours in both images. To overcome the burden of manual delineation, automatic delineation methods for cine CMR images exist. However, automatic delineation of the myocardium in LE CMR images is more challenging due to the lack of features between blood and scar tissue and variations in inversion times and contrast injection delays. Therefore, we propose to register the cine and LE images such that the LE images can be automatically delineated by transforming the cine contours, which may have been obtained automatically. In this respect, recently published SCMR guidelines for cardiac magnetic resonance (CMR) imaging [I] drastically reduce the complexity of the registration problem by stating that late enhancement (LE) CMR images have to be acquired in the same views as cine CMR images.

Purpose: The purpose of our work is to quantify the influence of different scanning procedures and cine CMR delineation methods on the accuracy of our automatic LE CMR delineation method.

Methods: We have used cine and LE CMR images from 32 patients, acquired between 2004-2007. The cine scans consisted of 10-14 slices and 20-25 phases, whereas the LE scans consisted of 10-12 slices. All images were $256 \times 256$ and covered fields of view of 320-460 mm (cine) and 344-494 mm (LE). Three experts delineated all images. Golden standard

Figure I (abstract P76)
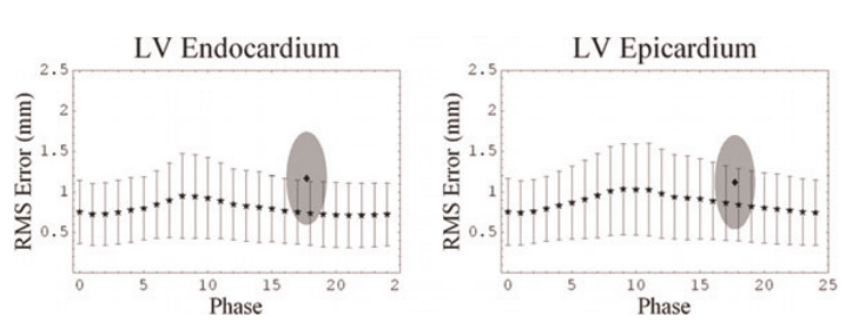

Variability of manual LV contours.
Table I (abstract P76) RMS positioning errors in $\mathrm{mm}$ for the LV endocardial and epicardial contours

\begin{tabular}{llll}
\hline & Manual & Propagated & Automatic \\
\hline LV Endocardium & & & \\
All cases & $2.20 \pm 1.15$ & $2.23 \pm 1.15$ & $2.54 \pm 1.38$ \\
Aligned cases & $1.25 \pm 0.65$ & $1.42 \pm 0.72$ & $1.97 \pm 0.83$ \\
Non-aligned cases & $2.34 \pm 1.15$ & $2.37 \pm 1.16$ & $2.64 \pm 1.43$ \\
& & & \\
LV Epicardium & & & \\
All cases & $2.08 \pm 1.02$ & $2.15 \pm 1.06$ & $2.53 \pm 1.11$ \\
Aligned cases & $1.41 \pm 0.35$ & $1.49 \pm 0.53$ & $1.72 \pm 0.68$ \\
Non-aligned cases & $2.18 \pm 1.05$ & $2.26 \pm 1.09$ & $2.76 \pm 1.11$ \\
\hline
\end{tabular}

contours were obtained by averaging contours. Inter-observer variability and contour accuracy were measured using RootMean-Square (RMS) positioning errors with respect to the golden standard.

The cine CMR images were delineated manually, semiautomatically and automatically. Semi-automatic delineation is performed using a contour propagation method. Automatic delineation is performed using a deformable template that is initialized using a ring detection procedure. The resulting contours were transformed to delineate the LE CMR images. The appropriate transformation was obtained by performing affine registration between cine and LE images that maximizes normalized mutual information in a coarse-to-fine approach using conjugate gradient optimization. Registration is performed between the LE slices and neighboring slices (to address through-plane motion) and phases (for accurate contour interpolation) in the cine scan. To anticipate on gross patient motion, initial translations are estimated by localizing the myocardium using a ring detection method.

Results: In cine CMR, the inter-observer variability over all phases was small, $0.80 \pm 0.43 \mathrm{~mm}$ and $0.89 \pm 0.48 \mathrm{~mm}$ for the endocardial and epicardial contours respectively, as compared to LE CMR, I. I $\pm 0.57 \mathrm{~mm}$ and I. $12 \pm 0.58 \mathrm{~mm}$ (figure I).

Our automatic segmentation method provided endocardial and epicardial contours at LE CMR with RMS errors of $2.20 \pm 1.15$ $\mathrm{mm}$ and $2.08 \pm 1.02 \mathrm{~mm}$ respectively given manual contours at cine CMR images. At the equivalent phase of the cine CMR images, contour propagation resulted in endocardial and epicardial contours with RMS errors of $0.32 \pm 0.42 \mathrm{~mm}$ and $0.19 \pm 0.29 \mathrm{~mm}$ respectively, whereas for automatic detection RMS errors of $1.87 \pm 2.35 \mathrm{~mm}$ and $1.64 \pm 2.17 \mathrm{~mm}$ were obtained. Table I shows the resulting RMS positioning errors after transforming cine contours, (either manually drawn, propagated or automatically detected) at all cases and divided between cases acquired according the recent SCMR guidelines $(6 / 32)$, at identical positions (aligned) and older cases (nonaligned).

Conclusion: We developed a new method for segmenting LE CMR images given delineated cine CMR images. Our new method provided very accurate contours for LE CMR images acquired according the recent SCMR guidelines [I], while maintaining reasonable accuracy in older, more difficult cases. Furthermore, the method is less sensitive to errors in initial contours if the images are acquired according recent guidelines. Reference

I. Kramer CM, et al: J Cardiovasc Magn Reson. 2008, I 0:35. 


\section{P77}

SNR improvement in GRE-EPI first-pass myocardial perfusion images with non-rigid body registration and $K L T$ filtering Georgeta Mihai ', Yu Ding', Hui Xue ${ }^{2}$, Yiucho Chung ${ }^{3}$, Jens Guehring ${ }^{2}$ and Orlando P Simonetti ${ }^{1}$

${ }^{\prime}$ The Ohio State University, Columbus, OH, USA

${ }^{2}$ Siemens Corporate Research, Princeton, NY, USA

${ }^{3}$ Siemens Healthcare USA, Inc, Columbus, OH, USA

Journal of Cardiovascular Magnetic Resonance 2009, I I (SuppI I):P77

Introduction: Signal-to-noise ratio (SNR) and contrast-tonoise ratio (CNR) are often limited in first-pass myocardial perfusion images due to the demands for ultra-fast image acquisition and the use of parallel imaging techniques. Spatial or temporal low pass filtering can enhance SNR but may blur boundaries and generate artifacts. The Karhunen-Loeve Transform (KLT) filter takes advantage of temporal correlation to remove random noise without compromising either spatial or temporal resolution, thereby enhancing SNR in dynamic images $[I, 2]$, but the performance of this filter has not been carefully evaluated for first-pass perfusion CMR. Image registration to compensate for respiration and variability in ECG triggering is a necessary step in quantitative evaluation of first-pass images. We hypothesize that registration can also increase the temporal correlation between images and reduce the potential for artifacts induced by KLT filtering.

Purpose: The purpose of this study is to demonstrate that the combination of non-rigid registration and KLT filtering significantly improves CNR between normal and abnormally perfused myocardium without introducing blurring or other image artifacts.

Methods: Ten consecutive first-pass perfusion datasets interpreted positive for ischemia or infarction were processed and analyzed retrospectively. All images were acquired using GRE-EPI with TSENSE acceleration rate 2 on a I.5 T MR system (MAGNETOM Avanto, Siemens Healthcare, Germany). For each subject, all slices showing clinically interpreted perfusion defect were included in the analysis. Non-rigid body registration [3] was first performed on each series to allow for semiquantitative analysis of signal enhancement, and to improve the

\section{Figure I (abstract P77)}

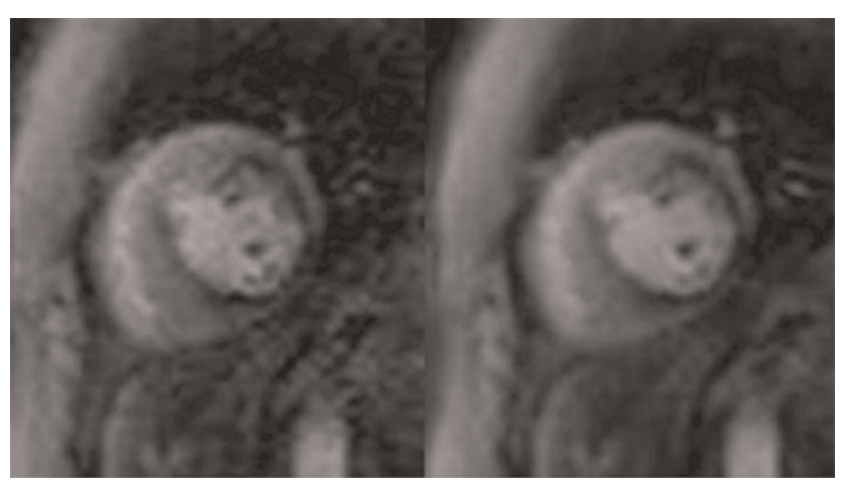

First-pass perfusion image before (left) and after (right) KLT filtering. KLT filter provides marked noise suppression without loss of contrast between normally and abnormally perfused regions. correlation of dynamic images prior to KLT filtering [2]. The image frame showing peak enhancement in normal tissue was selected and regions of interest (ROI) were manually drawn in abnormal and normal myocardium for CNR calculation according to the equation $C N R=\left(S_{\text {nornal }}-S_{\text {abnormal }}\right) / \sigma_{n}$, where $S_{\text {nornal }}$, $\mathrm{S}_{\mathrm{abnormal}}$ are the mean signal intensities of normal and abnormal myocardium and $\sigma_{\mathrm{n}}$ is the standard deviation of the noise from a region outside the body. Identical ROl's were drawn on filtered (registration + KLT) and unfiltered images for evaluation of CNR improvement. In order to assess blurring or other artifacts induced by filtering, KLT filtered images both with and without prior non-rigid registration were evaluated by two experienced observers. Artifact level relative to the unfiltered image series was scored as: (I) none, (2) mild, (3) moderate and (4) severe.

Results: The overall SNR of perfusion images increased with filtering. Figure I demonstrates the effect of the KLT filter on the registered perfusion images for one subject. There is a statistically significant increase in CNR with KLT filtering (mean $181.67 \pm 27.75, p<0.001$ ), ranging from $129 \%$ to $216 \%$ increase in CNR after filtering. Prior non-rigid registration reduced the occurrence of filter induced artifacts. Mild artifacts were detectable in the images filtered without prior registration (mean score $1.65 \pm 0.07$ ) as opposed to almost no detectable artifacts induced by KLT filtering with registration (mean score $1.08 \pm 0.08)$.

Conclusion: The combination of non-rigid registration and KLT filtering was shown to increase the SNR of GRE-EPI perfusion images, with a direct increase of CNR between normal and abnormal regions. Subjective evaluation of image artifacts revealed no significant blurring or other artifacts caused by filtering, provided non-rigid registration was performed first.

\section{References}

I. Ding Y, et al: ISMRM 2007, 3608.

2. Ding $Y$, et al: ISMRM 2008, 2858.

3. Xue $\mathrm{H}$, et al: MICCAI 2008, 35-43.

\section{P78}

Left ventricular dyssynchrony in patients with left bundle branch block and patients after myocardial infarction using 3D MR tagging

Robert Manka', Andrea Rutz ${ }^{2}$, Sebastian Kozerke²,

Peter Boesiger ${ }^{2}$ and Jürg Schwitter ${ }^{3}$

'German Heart Institute Berlin, Berlin, Germany

${ }^{2}$ Institute for Biomedical Engineering, University and ETH,

Zürich, Switzerland

${ }^{3}$ Clinic of Cardiology, University Hospital,

Zürich, Switzerland

Journal of Cardiovascular Magnetic Resonance 2009, I I(SuppI I):P78

Aims: To explore the value of accelerated 3D magnetic resonance (MR) tagging and delayed enhancement (DE) imaging to describe LV dyssynchrony in patients with left bundle branch block (LBBB) and in patients after myocardial infarction (MI).

Methods: 25 patients $(60.5 \pm 10.4$ years) after myocardial infarction, I5 patients $(62.9 \pm 10.1$ years $)$ with left bundle branch block and I5 healthy controls (53.3 \pm 9.7 years) underwent $3 \mathrm{D}$ tagging $[\mathrm{I}]$ and DE imaging at I.5 T. Spatial resolution was $3.0 \times 7.7 \times 7.7 \mathrm{~mm}^{3}$ with a temporal resolution of $27 \mathrm{~ms}$. 
Data acquisition was split into three breath-holds with heart position being monitored and corrected for by a navigator technique. The standard deviation (SD) of $T_{\max }$ of all segments, the CURE index, and a segmental-based systolic dyssynchrony index (SDI) were calculated as measures of LV dyssynchrony.

Results: All three parameters detected significantly increased dyssynchrony in patients compared to controls. The standard deviation of $T_{\max }$ was significantly higher in patients with $\mathrm{MI}$ $(78.1 \pm 12.6 \mathrm{~ms})$ and with $\operatorname{LBBB}(73.5 \pm 17.0 \mathrm{~ms})$ than in controls (47.2 $\pm 9.4 \mathrm{~ms}, \mathrm{p}<0.00 \mathrm{I}$ for both). The CURE and SDI showed similar characteristics and detected most dyssynchrony in LBBB-Patients. Bland-Altman plots demonstrate high interstudy and inter-observer reproducibility for CURE and intermediate reproducibility for the SDI and $\mathrm{T}_{\max } \mathrm{SD}$. Scar mass in the $25 \mathrm{MI}$ patients was $24.2 \pm 10.2 \%$ of the total LV mass.

Discussion: 3D tagging was successfully applied to provide detailed information on LV-dyssynchrony in patients with LBBB and in patients after myocardial infarction (MI). In combination with delayed enhancement imaging, this approach may show potential to improve patient selection and individual responsiveness for cardiac resynchronization therapy (CRT).

\section{Reference}

I. Rutz AK, et al: Magn Reson Med 2008, 59(4):755-63.

\section{P79}

Characterization of myocardial remodeling with diffusion tensor magnetic resonance imaging in chronic porcine model using the toroid-based representation Choukri Mekkaoui', Marcel P Jackowski ${ }^{2}$, Donald P Dione', Francis G Spinale ${ }^{3}$ and Albert J Sinusas' 'Yale University, New Haven, CT, USA

${ }^{2}$ University of São Paulo, São Paulo, Brazil

${ }^{3}$ Medical University of South Carolina, Charleston, SC, USA

Journal of Cardiovascular Magnetic Resonance 2009, I I (SuppI I):P79

Introduction: Diffusion Tensor Magnetic Resonance Imaging (DT-MRI) is a non-invasive technique capable of characterizing myocardial fiber architecture and structural properties [I], and may provide new insights about remodeling after myocardial infarction (MI). The helical organization and anisotropic nature of myofibers require efficient strategies for visualization and analysis. In this work, a toroid-based model of the diffusion tensor is used to create an improved depiction of myofiber orientation and derive a new diffusivity map, the toroidal volume (TV). This is applied to characterize regional tissue structure and fiber angle distribution in normal and post-MI remodeled porcine hearts.

Purpose: To evaluate the structure and fiber organization of normal porcine hearts and hearts 2- and 8-weeks post-MI using TV maps derived from the toroid-based representation of DT-MRI collected on a 3 T MR scanner.

Methods: LV remodeling was assed in 5 normal porcine hearts and hearts 2- and 8-weeks post surgical ligation of marginal branches of LCX coronary artery. Animals were euthanized and hearts were carefully excised and perfused with saline solution. Each heart was then placed in a container and filled with Fomblin (Ausimont, Thorofare, NJ). DT-MRI was performed on a $3.0 \mathrm{~T}$
Figure I (abstract P79)

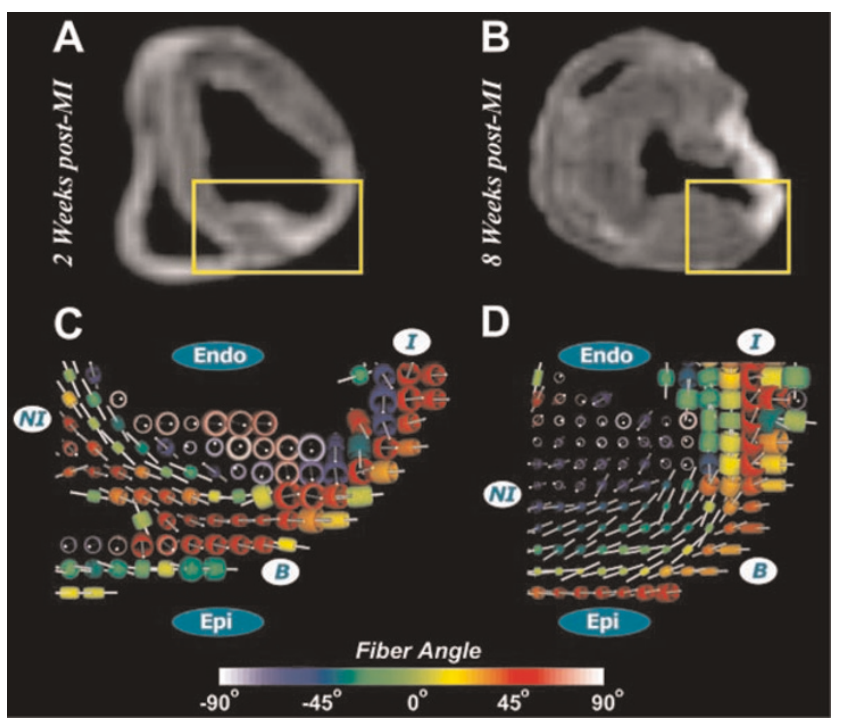

(A) and (B) mid-ventricular T2-weighted image of a 2- and 8-weeks post-MI pig hearts, respectively. The yellow rectangles represent the ROls used for toroidal glyph visualization, which includes $\mathbf{N I}, \mathbf{I}$ and B regions. Glyph are colored according to the fiber inclination angle as defined by Scollan et al. (1998, Am J Physiol). (C) Myocardial fiber angles in the infarct region at 2 weeks post-MI change from helical to more longitudinal with reorganization at 8 weeks post-MI (less longitudinal) (D).

scanner (Siemens, Erlangen, Germany) using a segmented EPI sequence, 6 gradient directions; b-values $=0$ (T2-weighted) and $600 \mathrm{~s} / \mathrm{mm}^{2}$; voxel-size $=2 \times 2 \times 2 \mathrm{~mm}^{3} ; 50$ short-axis slices; $\mathrm{TR}=5400 \mathrm{~ms} ; \mathrm{TE}=84 \mathrm{~ms} ; 40$ averages $(\mathrm{EPI}$ factor $=7)$. Based on T2-weighted images, tissue was classified into Infarct (I), Non-infarct $(\mathrm{NI})$, and Border $(\mathrm{B})$ regions. The toroid-based

Figure 2 (abstract P79)
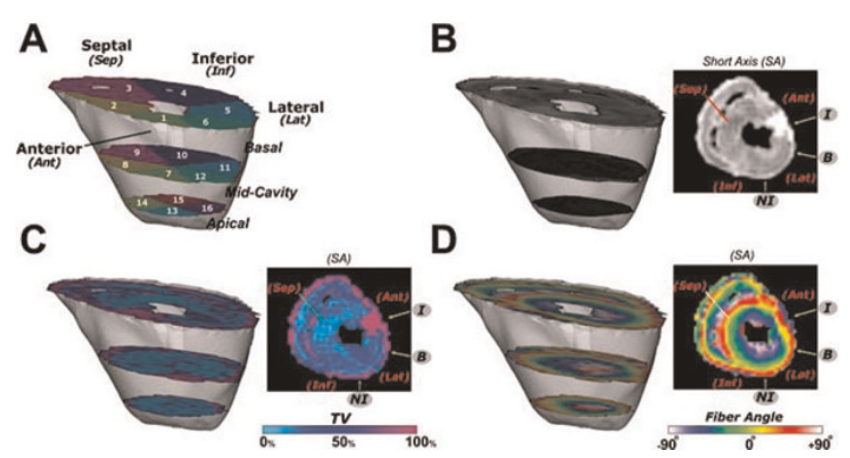

A three-dimensional view of an 8-weeks post-MI heart, with the myocardial segmentation is shown (A). The other frames show a midventricular slice of a T2-weighted image (B), a color-coded map of TV expressed as a percentage (C) and the color-coded fiber inclination angle map (D). The Infarct and Border regions are clearly distinguishable in the TV map and the laminar fiber architecture is altered in the Infarct region. 
Figure 3 (abstract P79)
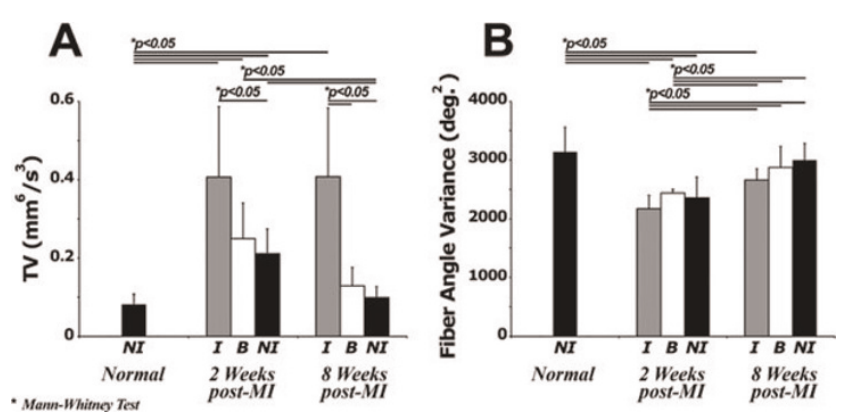

Mean TV (A) and fiber angle variance (B) computed from 16 regions of the standard cardiac polar map for normal, 2- and 8-weeks post-MI hearts. Myocardial regions were classified as NI, B or I based on the T2weighted image. TV and fiber angle variance for all categories is significantly elevated at 2 weeks post-MI and remains elevated only in the infarcted region at 8 weeks post-MI. These findings indicate the progress of the remodelling process post-MI.

representation of the DT is described by the modified parametric equation of an elliptical torus and TV is the volume of the toroid. Fiber angle variance (FAV) captures the degree of fiber angles spreading over the volume of interest. TV $\left(\mathrm{mm}^{6} / \mathrm{s}^{3}\right)$ and FAV (deg. ${ }^{2}$ ) indices were then quantified for 16 segments of the standard cardiac polar map.

Results: The toroidal representation revealed that myocardial fiber angles in the infarct region at 2-weeks post-Ml changed from helical to more uniform longitudinal orientation, with reorganization at 8-weeks post-MI (less longitudinal) (Figure I). This toroid-based approach exhibits the laminar architecture with the changing orientation of fiber angles from epicardium to endocardium and also reveals a change in the toroidal shape in the infarct region. The structural change is reflected by a substantial global increase in TV. Hence, the analysis of TV and FAV maps allows for a structural and a geometrical quantification of the myocardial remodeling (see Figure 2 for an example at 8 -weeks post-MI). TV and FAV were uniform in normal hearts, and significantly $(p<0.05)$ altered in the infarct regions at 2 - and 8 -weeks post-MI. TV and FAV were also altered $(p<0.05)$ in $\mathrm{NI}$ and $\mathrm{B}$ regions at 2-weeks post-MI with normalization by 8 -weeks (Figure 3). These changes in TV and FAV are in agreement with previously observed spatial and temporal changes in regional activation of matrix metalloproteinase's [2].

Conclusion: Results suggest that the DT-MRI indices of TV and FAV provide quantitative information that may enhance the understanding of the underlying myocardial structure properties and temporal changes involved in the post-MI remodeling process.

\section{References}

I. Helm PA, Younes L, Beg MF, Ennis DB, Leclercq C, Faris OP, McVeigh E, Kass D, Miller MI and Winslow RL: Evidence of structural remodeling in the dyssynchronous failing heart. Circ Res 2006, 98:125-32.

2. Vanhoutte D, Schellings $M$, Pinto $Y$ and Heymans $S$ : Relevance of matrix metalloproteinases and their inhibitors after myocardial infarction: a temporal and spatial window. Cardiovasc Res 2006, 69(3):604-13.
P80

Gadolinium enhanced magnetic resonance mean voxel signal intensity within the left ventricular myocardium changes prior to ejection fraction drop after receipt of cardiotoxic chemotherapy Jimmy C Lightfoot, Ralph B D'Agostino Jr, Craig A Hamilton, William C Little, Frank M Torti, Nancy D Kock, Mike Robbins and W Gregory Hundley Wake Forest University, Winston-Salem, NC, USA

Journal of Cardiovascular Magnetic Resonance 2009, I I(SuppI I):P80

Objective: To determine if serial measures of gadolinium (GD) signal intensity acquired with cardiac magnetic resonance (CMR) during receipt of cardiotoxic doxorubicin (DOX) chemotherapy are associated with left ventricular ejection fraction (LVEF).

Methods: 40 Sprague-Dawley rats were divided into 3 groups receiving weekly doses of: normal saline [NS] $(n=7), 1.5 \mathrm{mg} / \mathrm{kg}$ $\operatorname{DOX}(n=19)$, or $2.5 \mathrm{mg} / \mathrm{kg}$ DOX $(n=14)$. 1.5 T CMR images of LVEF and myocardial GD signal were acquired before and at 2 and 4 weeks after DOX treatment. LVEF was determined from a multi-slice short axis cine acquisition; signal intensity was obtained from an inversion recovery (IR) mid-LV slice. Analysis of variance models were fit with group or LVEF drop status (>10\% drop in LVEF from baseline yes/no, or NS) as factors. RESULTS: LVEF was similar among the 3 groups at baseline $(p=0.27)$, and at 2 weeks, $(73+5 \%, N S ; 74+5 \%, 1.5 \mathrm{mg} / \mathrm{kg}$ DOX; 7I + 6\%,2.5 mg/kg DOX). At 4 weeks, LVEF after NS was unchanged $(77 \pm 7 \%, p=0.93)$, but there was a $>10 \%$ drop in LVEF in $3 / 19$ in the $1.5 \mathrm{mg} / \mathrm{kg}$ DOX, and $10 / 14$ in the $2.5 \mathrm{mg} / \mathrm{kg}$ DOX groups: LVEF was $77 \pm 7 \%$ in non-dropped and $64 \pm 8 \%$ in dropped groups at 4 weeks $(p<0.001$ ). LVEF remained $\geq 70 \%$ in all animals at 2 weeks. In animals dropping their LVEF at 4 weeks, the GD signal within the LV myocardium was elevated relative to baseline at week 2 (2 weeks prior to LVEF drop - Figure I); in animals receiving NS or DOX without LVEF drop, GD was not elevated at 2 weeks.

Conclusion: These data suggest gadolinium CMR may be useful to monitor for doxorubicin chemotherapy cardiotoxicity: low

Figure I (abstract P80)

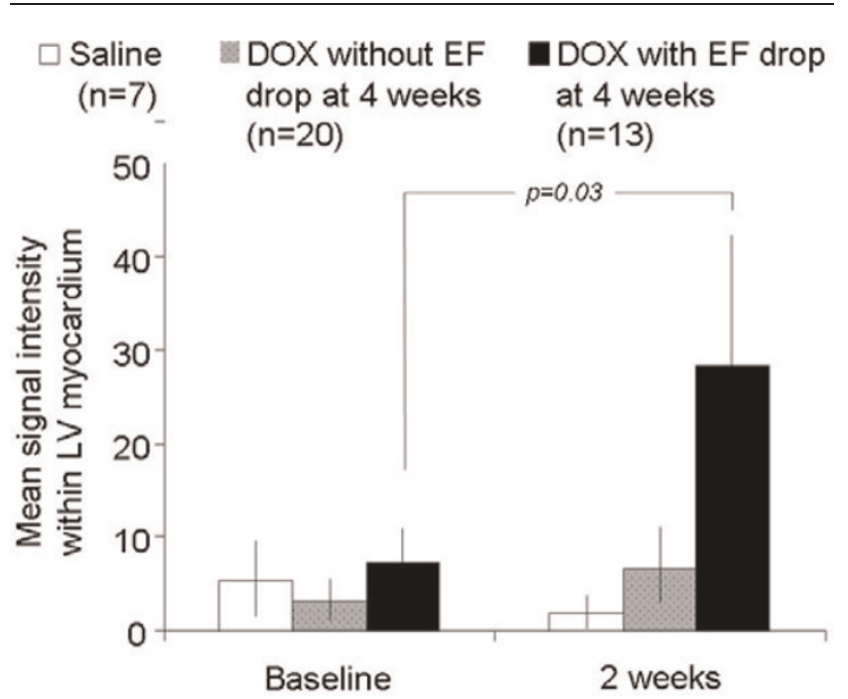


serial measures of GD intensity forecast no LVEF drop, whereas an increase in GD signal intensity forecasts a clinically important drop in LVEF.

\section{P8 I}

Limited plane analysis reduces accuracy of

3-dimensional reconstruction of the right ventricle Florence Sheehan', Ruslan Kazakov' and Mary-Pierre Waiss ${ }^{2}$

'University of Washington, Seattle, WA, USA

${ }^{2}$ VentriPoint, Inc, Seattle, WA, USA

Journal of Cardiovascular Magnetic Resonance 2009, I I (SuppI I):P8I

Background: Ventricular remodeling is a compensatory process that is little understood in the right ventricle (RV). We have previously reported an association between abnormalities in RV shape and function in patients with dilated RVs. In these studies we used the piecewise smooth subdivision surface (PSSS) reconstruction method because it provides accurate representation of the 3D shape of the RV. However the complexity of RV shape mandates manual tracing of RV borders from many images. Methods: We tested the error in RV volume, ejection fraction (EF), and shape accrued by reducing the number of borders traced from MRI studies of 18 patients with congenital heart disease and 2 normal subjects. The true volume and shape were defined by tracing RV borders from 17 to 33 (mean $23 \pm 4$ ) images recorded in short axis (SAX), long axis (LAX), oblique, and inflow-outflow track (IOT) views. LAX views could be radially oriented about the left ventricular LAX or parallel to the 4-chamber view, but always visualized the entire free wall from its inferior to superior aspect. Reduced data sets were created containing 10, 12, 14, or 16 image planes distributed between SAX, LAX, and IOT views. Because pilot studies suggested that 12 might suffice, 3 subsets of 12 views were created that emphasized SAX views (I2s), LAX views (I2I), or the combination $(12 c)$. The RV was reconstructed at end diastole and end systole from full border sets and from each subset of borders using the PSSS method. Volume was computed directly from the 3D surface and used to calculate EF. Shape was measured at 20 cross sections from apex to tricuspid valve as eccentricity (= 4 piArea/Perimeter ${ }^{2}$ ).

Results: RV volume at end systole was overestimated by $1.5 \pm 3.0 \%(p<0.05)$ using 10 and $12 c$ subsets. RV EF was underestimated by $2-3 \%(p<0.05)$ using 10,121 , and $12 c$ subsets. RV volume and EF did not differ significantly from true when measured using $12 \mathrm{~s}, 14$, or 16 view subsets. RV shape at end diastole but not at end systole was slightly but significantly more rounded in the two most apical slices when measured using 14 views. There was also small but significant underestimation at end diastole, although not at end systole, of other RV shape metrics: the ratio of septal area to total surface area, RV LAX length, and extent of bulging at the base. When reconstructed using 16 views, the only shape metric to show a significant difference from true was eccentricity at one slice.

Conclusion: Accurate measurement of RV volume and EF can be obtained from the PSSS method after tracing 14 views comprising 6 SAX slices mainly concentrated at the base, 6 LAX views distributed evenly from inferior to superior aspects of the free wall, and 2 IOT views. For the detailed 3D shape analysis that PSSS reconstruction enables, a minimum of 16 views is recommended.
P82

Quantification of interventricular dyssynchrony by phase contrast magnetic resonance angiography

Kai Muellerleile, Loant Baholli, Michael Groth, Katharina Koopmann, Achim Barmeyer, Ralf Koester, Gerhard Adam, Thomas Meinertz, Stephan Willems and Gunnar Lund University Medical Center Hamburg-Eppendorf, Hamburg, Germany

Journal of Cardiovascular Magnetic Resonance 2009, I I(SuppI I):P82

Objective: Interventricular dyssynchrony is typically assessed by pulsed-wave echocardiography (PW-Echo) as the delay between onset of aortic and pulmonary flow. Recent multicenter trials demonstrated the value of this interventricular dyssychrony to predict response to cardiac resynchronization therapy (CRT). In the present study, the ability of phase contrast magnetic resonance angiography (PC-MRA) was assessed to quantify interventricular dyssynchrony in comparison with PW-Echo.

Figure I (abstract P82)
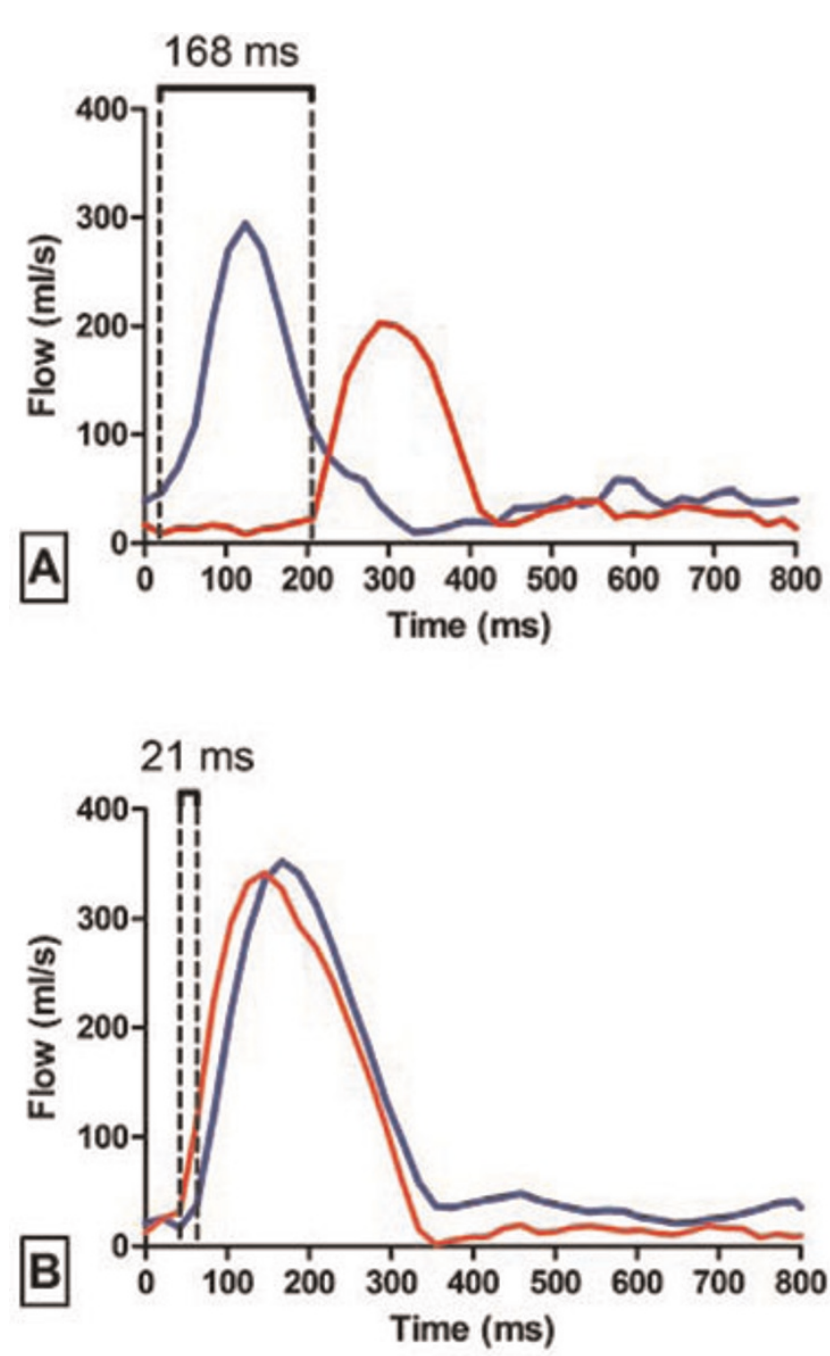
Methods: 40 patients with stable heart failure NYHA Class 2 to 3 , reduced ejection fraction $(28 \pm 11 \%)$, with $(n=21)$ or without $(n=19)$ complete left bundle branch block were prospectively included. Transvalvular flow curves of the aortic and pulmonary valve were acquired by PC-MRA and PW-Echo. Interventricular delay was calculated for PC-MRA as the delay between onset of aortic and pulmonary flow in analogy to PWEcho. Interventricular delays by PC-MRA were correlated with PW-Echo; agreement was assessed by Bland-Altman analysis.

Results: A strong correlation between interventricular delays by PW-Echo and PC-MRA was found $(r=0.89, P<0.000 \mathrm{I})$. Bland-Altman analysis demonstrated a good agreement between both methods (Mean difference $-6 \pm 15 \mathrm{~ms}$ ). An example of the assessment of interventricular delays by PC-MRA in one patient with $(A)$ and one patient without interventricular dyssynchrony (B) is illustrated by Figure I. Aortic (red) and pulmonary (blue) valve flow curves are plotted; interventricular delay by PC-MRA was $168 \mathrm{~ms}$ in patient $A$ (PW-Echo $=146 \mathrm{~ms}$ ) and $21 \mathrm{~ms}$ in patient $B$ (PW-Echo $=6 \mathrm{~ms}$ ).

Conclusion: PC-MRA quantifies interventricular dyssynchrony comparable with PW-Echo. PC-MRA has the potential to identify responders to CRT.

\section{P83}

MR imaging of human atherosclerosis using immunomicelles molecularly targeted to macrophages

Vardan Amirbekian', Michael J Lipinski ${ }^{2}$, Juan C Frias ${ }^{2}$, Smbat Amirbekian ${ }^{3}$, Karen C Briley-Saebo ${ }^{2}$, Venkatesh Mani², Daniel Samber ${ }^{2}$, Antonio Abbate ${ }^{2}$, Juan GS Aguinaldo ${ }^{2}$, David Masey ${ }^{2}$, Valentin Fuster², George Vetrovec ${ }^{2}$ and Zahi A Fayad ${ }^{2}$

'Brigham and Women's Hospital, Harvard Medical School Mount Sinai School of Medicine, Imaging Science Laboratories, New York, NY, USA

${ }^{2}$ Mount Sinai School of Medicine, Translational and Molecular Imaging Institute., New York, NY, USA

${ }^{3}$ Emory University School of Medicine., Atlanta, GA, USA

Journal of Cardiovascular Magnetic Resonance 2009, I I (SuppI I):P83

Introduction: Early assessment of atherosclerosis (leading cause of death in West and soon world) remains an elusive

\section{Figure I (abstract P83)}

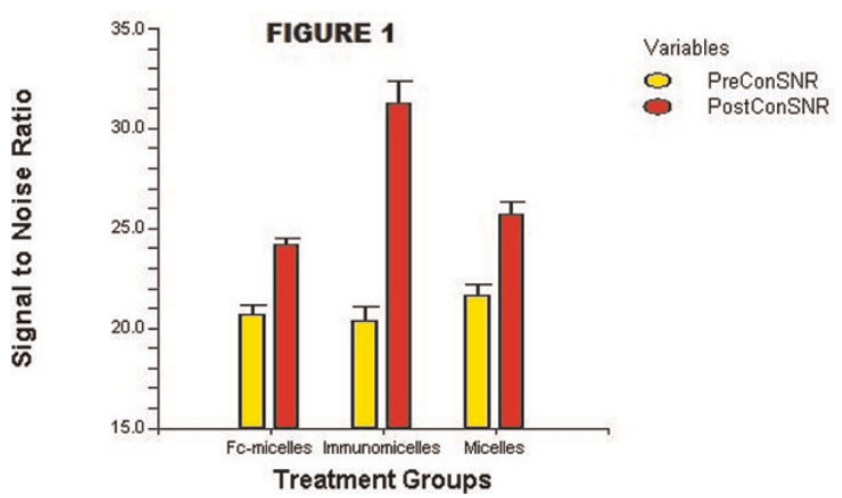

Figure 2 (abstract P83)

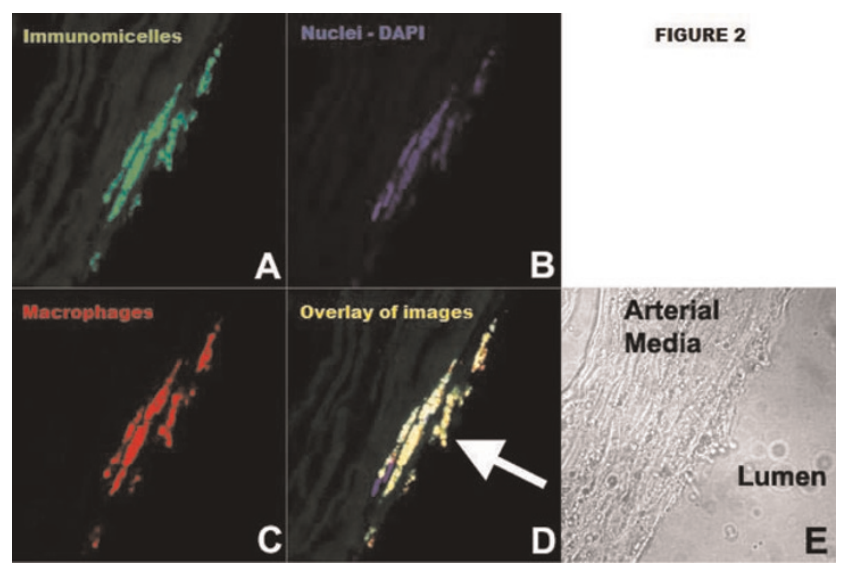

clinical goal, which if realized could lead to significant improvements in mortality and morbidity.

Purpose: Gadolinium (Gd)-containing immunomicelles targeting macrophages improved magnetic resonance (MR) detection of murine atherosclerosis. We sought to determine if immunomicelles targeting the macrophage scavenger receptor-B (CD36) improved ex-vivo MR detection and characterization of human aortic atherosclerosis.

Methods: Gd-containing micelles, anti-CD36 immunomicelles and Fc-micelles were created. Macrophages were incubated with fluorescent micelles and immunomielles to determine uptake via confocal microscopy and inductively coupled plasma mass spectroscopy (ICP-MS) was performed to quantify Gd uptake. Human aortic specimens with moderate to severe atherosclerosis were harvested at autopsy. Using a I.5 T Siemens clinical

Figure 3 (abstract P83)

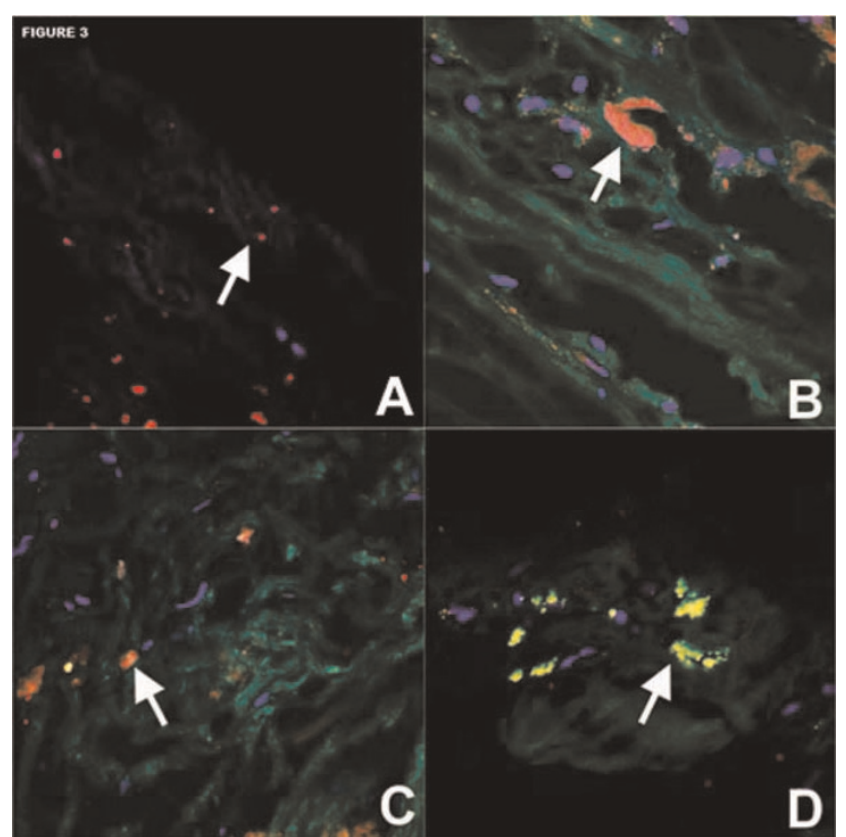


scanner, TI, T2, and PDW 3-dimensional scans were performed and post-contrast scans were repeated after $24 \mathrm{~h}$ incubation. TI analysis and cluster analysis were performed comparing immunohistopathology with $\mathrm{MR}$ images. $\mathrm{P}$-values $<0.05$ were considered significant.

Results: Micelles had a mean diameter of $125 \mathrm{~nm}$, average of I4,900 Gd-ions, and mean relaxivity was $37 \mathrm{mM}-\mathrm{I} \mathrm{s}-\mathrm{I}$ at I.5 T and $37^{\circ} \mathrm{C}$. Confocal microscopy and ICP-MS demonstrated significant in vitro uptake of immunomicelles by macrophages while non-targeted micelles had minimal uptake. On TI imaging, immunomicelles increased CNR by $52.5 \%(n=6, p<0.0001)$ while Fc-micelles increased CNR by $17.2 \%(n=4, p<0.000 I)$ and micelles increased CNR by $18.7 \%(n=6, p=0.0007)$. Please see Figure I. Immunomicelles increased CNR significantly greater than the Fcmicelles or micelles $(p=0.00 \mathrm{I})$. Confocal fluorescent microscopy showed that immunomicelles target macrophages in the aortic plaque while the micelles and Fc-micelles are found diffusely throughout the plaque. Please see Figures 2 and 3. Immunomicelles had a greater increase in post-contrast SNR in the fibrous cap compared with the lipid core $(p<0.00 I)$ while micelles and Fc-micelles had a greater increase in the lipid core $(p<0.01)$.

Conclusion: Macrophage-specific (CD36) immunomicelles bind to human macropages in vitro and improved MR detection and characterization of human aortic atherosclerosis. Thus, immunomicelles could help identify high-risk human plaque.

\section{P84}

Improved image quality in subtraction based non-contrast MRA using automated soft tissue motion correction with BRACE

Zhaoyang Fan', Peter Weale ${ }^{2}$, Xiaoming $\mathrm{Bi}^{2}$, James Carr', Saurabh Shah', John Sheehan ', Debiao Li ${ }^{1}$ and Renate Jerecic ${ }^{2}$

${ }^{\prime}$ Northwestern University, Chicago, IL, USA

${ }^{2}$ Siemens Medical Solutions, Chicago, IL, USA

Journal of Cardiovascular Magnetic Resonance 2009, I I (SuppI I):P84

Introduction: Recently several new approaches for noncontrast MRA have been proposed. Many of them are based on

Figure I (abstract P84)

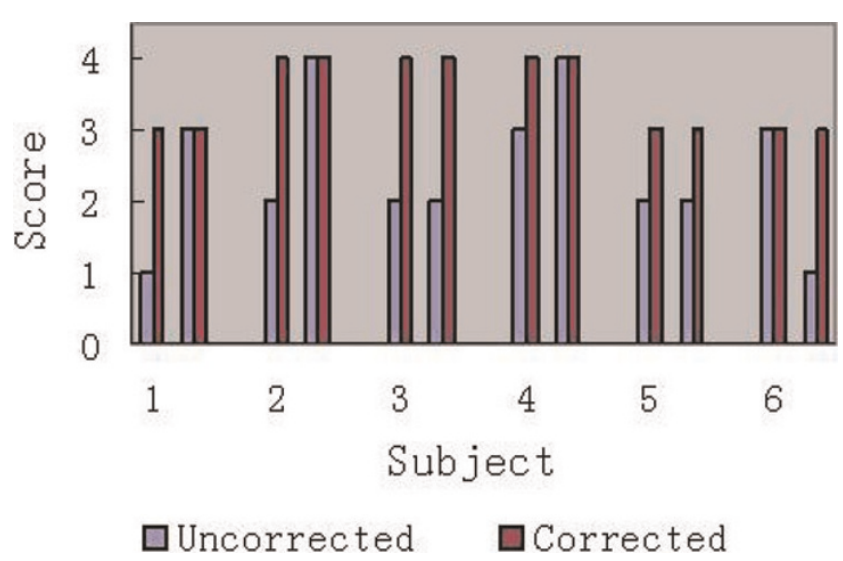

Scores of the anterior tibial artery on uncorrected images vs. corrected images in 6 subjects. Scores were improved in all legs with appreciable motion, but unchanged in all still.
Figure 2 (abstract P84)

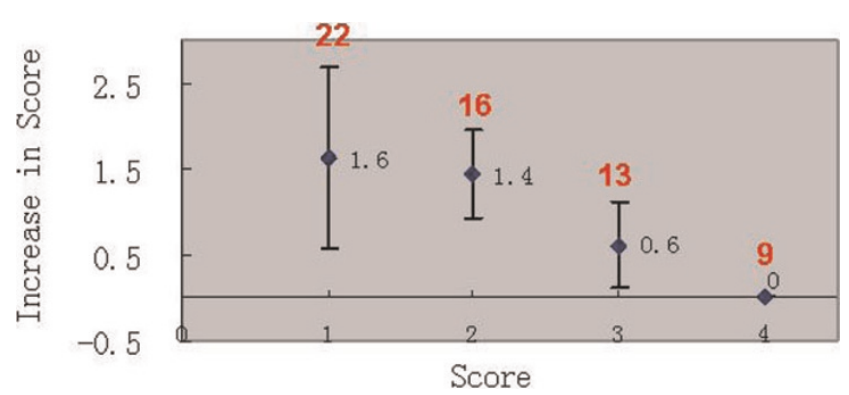

Absolute improvement in image scores for groups with initial score of I, 2, 3 and 4, respectively. All legs (60 segments) were pooled together. (Red figures: the total number of each group). Vessel depiction of segments with initial score of I or 2 (primarily due to motion) were substantially improved after motion correction.

subtraction of two 3D data sets, which are acquired either at two different points of the cardiac cycle, eg NATIVE SPACE or use different preparation mechnisms to alter the signal intensity in the arteries and veins, eg by flow sensitizing dephasing (FSD) gradients. As the acquisition of each dataset takes in the order of 2-3 minutes, motion can occur in between the two acquisitons and can impair therefore the resulting image quality. A common problem which is also known from contrast enhanced MRA, where the mask is subtracted form the contrast data set [I].

Purpose: To evaluate in volunteers and patients whether an automated soft tissue motion correction software can improve the image quality of subtraction based non-contrast MRA data.

Figure 3 (abstract P84)

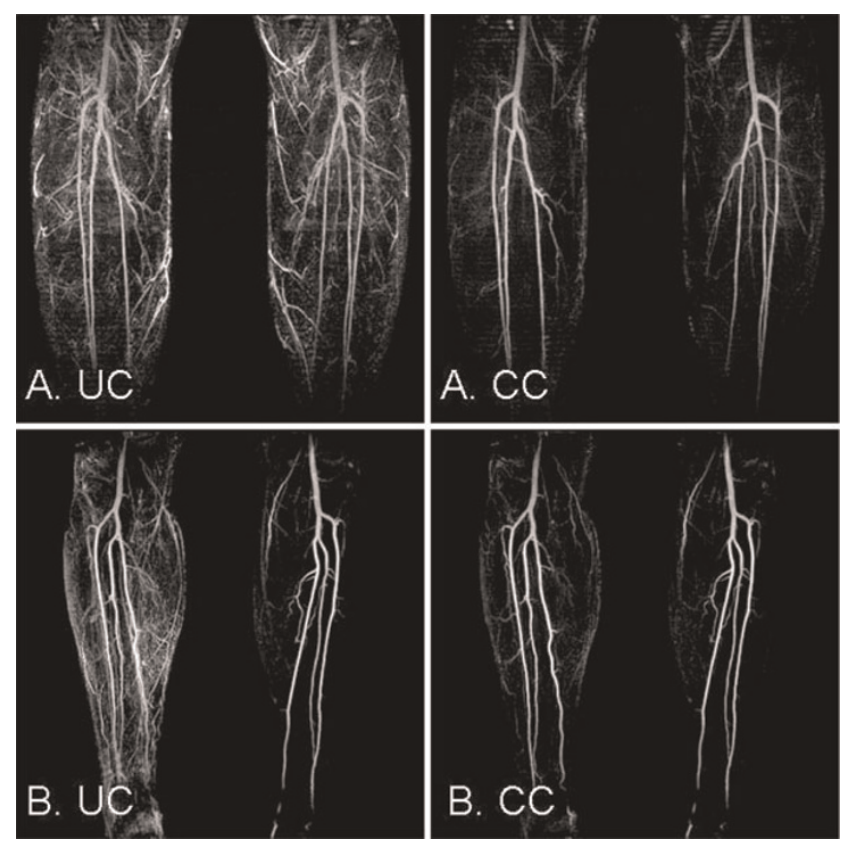

Image quality and artery depiction was improved after correction when motion was present in both legs (A) or only one leg in which case correction didn't affect the originally still leg (B). UC uncorrected; CC: corrected. 
Methods: Non contrast peripheral MRA data sets of the lower legs of 3 healthy volunteers and 3 patients where qualitatively scored before and after motion correction by an experienced radiologist. The commecially available software syngo BRACE (Siemens Healthcare, Germany) was used [I] to perform the automated motion correction of the two data sets.

Qualitative image quality was assed using a scale from I to 4 (I being poor, 2 fair, 3 good, 4 excellent vessel conspicuity) The conspicuity was scored for the popliteal, anterior tibial, posterior tibial and the peroneal branch as well as for the side branches. A score was given for each branch before and after motion correction.

Results: The results are shown in Figure I on the example of the anterior tibiaL branch assesment. The asessment of the other branches was comparable. Figure 2 shows the overall improvement based on the initial score before motion corredtion was applied. Figure 3 shows two typical case examples before and after BRACE was applied.

Conclusion: The motion correction software BRACE was able to improve the image quality in subtraction based non contrast MRA techniques when motion occued. Many cases showed that motion often occurs in only one leg. In those cases BRACE did not deteriorate the image quality of the leg without motion while at the same time improving the result in the leg affected by motion. No case was observed where the software did decrease the image quality compared to the uncorrected image. BRACE therefore provides a promising tool in the area of non contrast MRA.

Reference

I. Herrmann K-H, et al: Eur Radiol 2006.

\section{P85}

Stress cardiac magnetic resonance imaging in an outpatient setting

Amir-Ali Fassa', Alain Naïmi , Golmehr Ashrafpoor', Yannick Magliano ${ }^{2}$, Béatrice Veragut Davies ${ }^{3}$ and Juan Sztajzel ${ }^{2}$

${ }^{\prime}$ Geneva University Hospitals, Geneva, Switzerland

${ }^{2}$ Centre de Diagnostic Radiologique de Carouge, Geneva, Switzerland

${ }^{3}$ Centre Cardio-Pulmonaire de la Clinique de Carouge, Geneva, Switzerland

Journal of Cardiovascular Magnetic Resonance 2009, I I(SuppI I):P85

Introduction: Stress cardiac magnetic resonance (CMR) imaging is a non-invasive modality which is increasingly used for detection of myocardial ischemia, necrosis and viability.

Purpose: To assess the feasibility and safety of stress CMR in a non-hospital outpatient setting.

Methods: We reviewed the data of all patients who were referred for stress CMR (I.5 Tesla) from February I ${ }^{\text {st }} 2006$ to January $3 I^{\text {st }} 2008$ to the Centre de Diagnostic Radiologique de Carouge, an outpatient imaging centre. Standard protocol consisted of: I) assessment of myocardial function at rest; 2) pharmacological stress induced either by dobutamine (protocol of $10,20,30,40 \mu \mathrm{g} / \mathrm{kg} / \mathrm{min}$ during 3 minutes with atropine if necessary) until achieving submaximal heart rate ([220age] $\times 0.85$ ), or by adenosine (protocol of $140 \mu \mathrm{g} / \mathrm{kg} /$ minute during 3 minutes followed by a bolus of $10 \mathrm{ml}$ of gadolinium at
$4 \mathrm{ml} /$ second "first pass"); 3) assessment of myocardial scar and/or viability by delayed enhancement (DE) sequences.

Results: During the study period 472 patients were referred for stress CMR. The test was performed in 452 patients (96\%): 294 males (65\%), mean age $62 \pm$ II years, mean duration $55 \pm 10$ minutes, stress induction with dobutamine in $24 \mathrm{I}$ patients $(53 \%)$ and adenosine in $21 \mathrm{I}$ patients $(47 \%)$. The test could not be carried out in 23 patients (5\%) because of claustrophobia (18 patients), excessive thoracic diameter (4 patients) and excessive baseline arterial blood pressure (I patient). However, stress CMR could finally be performed during a second appointment in 3 patients. The remainder either underwent another non-invasive test (Thallium myocardial scintigraphy in 14 patients), or did not undergo further functional assessment at our centre (6 patients). No ischemia or infarction was found in 306 patients (68\%), while isolated ischemia was found in 18 patients (4\%) and ischemia in the presence of an infarction in 30 patients (7\%). Infarction without ischemia was found in 95 patients (2I\%). DE was found 146 patients (33\%), which was subendocardial in 124 patients $(28 \%$, involving $<50 \%$ of wall thickness in 53 patients ( $12 \%$ ) and $>50 \%$ of wall thickness in $7 I$ patients (I6\%)). DE sparing the subendocardium was found in 22 patients (5\%). The following complications occurred during 17 tests (4\%): supraventricular tachycardia or unsustained ventricular tachycardia (7 patients), anxiety attack ( 3 patients), chest pain ( 3 patients), suspected allergic reaction to gadolinium (I patient), dizziness (I patient), vomiting during dobutamine infusion (I patient) and hypotension (I patient). No other complications occurred.

Conclusion: Stress CMR is a well tolerated non-invasive assessment modality, which can be safely performed in a nonhospital outpatient setting.

\section{P86}

Evaluation of myocardial function after primary percutaneous intervention by cardial MRI:

5 years follow up

Tirza Springeling', Sharon W Kirschbaum², Timo Baks', Yusuf Karamermer', Gabriel K Krestin', Pim J de Feyter' and Robert-Jan M van Geuns'

'Erasmus MC, Rotterdam, Netherlands

${ }^{2}$ Erasmus MC, Thoraxcenter, Rotterdam, Netherlands

Journal of Cardiovascular Magnetic Resonance 2009, I I(SuppI I):P86

Background: We investigated the early and late effect of primary percutaneous coronary interventions $(\mathrm{PCl})$ for acute myocardial infarction on recovery of left ventricular ejection fraction (LVEF), end diastolic volume (EDV), end systolic volume (ESV) and segmental wall thickening (SWT) using Cardiac MRI.

Methods: This abstract includes preliminary analysis of the first 12 patients. All patients underwent cardial MRI within 5 days, at 5 months and at 5 years after successful primary PCI. LVEF, EDV, ESV and SWT quantified on cine-images, the transmural extent of the infarction (TEI) was quantified on delayed-enhancement images.

Results: EDV increased significantly between baseline and 5 months (from 193.5 \pm 45.4 to $216.4 \pm 61.5, p=.01$ ), with a trend for additional late remodelling at 5 years (from $216.4 \pm 61.5$ to $218.0 \pm 82.1, p=.27$ ). The same trend seems to be visible in ESV although also not significant (resp.l I4,2 \pm 33.9; I $22.0 \pm 5$ I.8; I $32.5 \pm 73.4 p=0.20$ ). LVEF showed some 
Figure I (abstract P86)

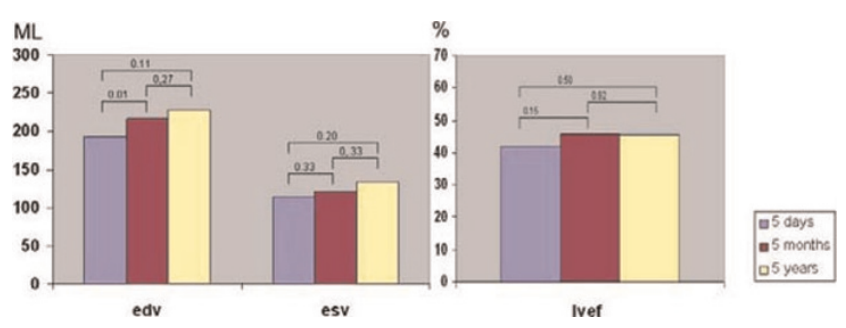

recovery between baseline and 5 months (from $41.60 \pm$ to $45,8 \pm 12.4, p=.15)$, with no further change after 5 months $(45,8 \pm 12.4 ; 45.56 \pm 10.7 p=.92)$ (see figure I).

SWT improved significantly between baseline and 5 months follow up (from $23 \%$ to $30 \%, p=0.04$ ), no additional improvement was seen at 5 years (SWT 33\%, $p=0.29$ ). TEl at baseline showed a good correlation with SWT at 5 months and 5 years follow up.

Conclusion: These interesting preliminary results of 12 patients indicates a tendency to late remodelling at 5 years follow up by which LVEF is preserved. TEI at baseline predicted regional SWT at follow up.

\section{P87}

Coronary risk factors for the increased of coronary wall thickness : multi-ethnic study of atherosclerosis (MESA)

Cuilian Miao', Shaoguan Chen', Robson Macedo', Shenghan Lai', Kiang Liu', Debiao Li ${ }^{2}$, Steven M Shea ${ }^{3}$, Moyses Szklo', Jens Vogel-Clausen', Joseph Polak ${ }^{4}$, Joao AC Lima and David A Bluemke ${ }^{5}$

Johns Hopkins Medicine Institutions, Baltimore, MD, USA

${ }_{2}$ Northwestern University Medical School, Chicago, IL, USA

${ }^{3}$ Siemens Corporate Research, Inc., Baltimore, MD, USA

${ }^{4}$ Tufts University School of Medicine, Boston, MA, USA

${ }^{5}$ National Institues of Health, Bethesda, MD, USA

Journal of Cardiovascular Magnetic Resonance 2009, I I(SuppI I):P87

Background: Coronary risk factors are established predictors of coronary atherosclerotic disease. The purpose of this study was to investigate the associations of conventional risk factors with increased of coronary artery wall thickness by MR imaging as a marker of atherosclerosis in an asymptomatic population.

Methods: Coronary wall MR imaging were performed in 194 participants in the Multi-Ethnic Study of Atherosclerosis free of clinical cardiovascular disease (98 men, age $6 \mathrm{I} \pm 9 \mathrm{yrs}$ ) using black-blood TSE technique. Cross-sectional coronary artery wall images were acquired and mean wall thickness was determined. Coronary calcium score (CAC) and carotid artery intimal-medial thickness (IMT) were obtained by standard protocols. Linear regression was used to determine the correlations of mean wall thickness and coronary risk factors, the number of risk factors, Framingham risk score, CAC and carotid IMT.

Results: Male gender, high density lipoprotein (HDL), CAC and number of risk factors were associated with coronary artery wall thickness $(p<0.05)$. In multivariable analyses, the number of risk factors remained predictive of increased arterial wall thickness
(0.0482 mm per risk factor $(0.0105,0.0859), P<0.05)$. Framingham risk score and the number of coronary artery disease risk factors were positively related to coronary artery wall thickness in both univariable and multivariable models $(p<0.05)$ for men only. Wall thickness was significantly greater in men than women $(p<0.05)$. Large patient size $(B M I \geq 30)$ limited the ability to detect coronary wall thickness changes by MRI.

Conclusion: Number of coronary risk factors and Framingham risk score were positively related to coronary artery wall thickness by MRI in asymptomatic men. Significant relationships were not observed in the women, possibly due to small vessel size and/or lower levels of atherosclerosis.

\section{P88}

Rapid assessment of myocardial scar and viability in ischemic heart disease: a cardiovascular magnetic resonance study

Didier Locca, Chiara Bucciarelli-Ducci, Jonhatan Lyne, Marinella Chapparo, Giuseppe Ferrante, Rory O'Hanlon, Sanjay Prasad, Peter Gatehouse, Peter Drivas, Rick Wage, David Firmin and Dudley Pennell Royal Brompton Hospital, London, UK

Journal of Cardiovascular Magnetic Resonance 2009, I I(SuppI I):P88

Introduction: One important use of Cardiovascular Magnetic Resonance (CMR) is to visualize the extent of myocardial infarction. Inversion recovery preparation with segmented gradient echo readout (IR-GRE) is considered the gold standard sequence for the detection of late gadolinium enhancement to visualise myocardial infarction. However this technique requires multiple breath-holds and is time consuming. A reliable single shot readout multislice inversion recovery imaging sequence would permit rapid viability imaging improving scanning efficiency.

Purpose: The aim of this study was to compare the diagnostic accuracy of the multislice single shot SSFP inversion recovery sequence (IR-SSFP) against multiple breath-hold IR-GRE images in detecting the presence or absence of late enhancement.

Methods: We studied 86 consecutive patients undergoing a CMR viability assessment. Images were acquired 10 minutes following intravenous injection of $0.1 \mathrm{mmol} / \mathrm{Kg}$ gadoliniumDTPA.

Results: Images were analyzed by two blinded observers for the presence or absence of late myocardial enhancement. Both techniques identified the presence of myocardial infarction in 21 patients. Interobserver variability was $-1.370(\mathrm{Cl}-1 \mathrm{I} .327$ to 8.588) for GRE and $-1.707(\mathrm{Cl}-9.849$ to 6.435$)$ for SSFP. The coefficient of repeatability was 42.54 fro GRE and 41.94 for SSFP. The interobserver agreement in the estimate of viability was 0.84 (0.II) for GRE and 0.95 (0.II) for SSPF technique.

Conclusion: Despite its lower spatial resolution, the IR-SSFP single shot multislice imaging sequence was equally capable of detecting the presence or absence of late enhancement compared to the single slice GRE sequence. Clinically, the IR-SSFP sequence can be used to perform a rapid assessment of the presence or absence of late myocardial enhancement to determine myocardial scar and viability. 


\section{P89}

The decisive role of cardiovascular MRI delayed hyperenhancement (DHE) pattern for risk stratification for dilated cardiomyopathy Jose V Venero, Srinivas Murali, Mark Doyle, Vikas K Rathi, Saundra B Grant, June Yamrozik, Ronald B Williams, Diane A Vido, Geetha Rayarao and Robert WW Biederman

Allegheny General Hospital, The Gerald McGinnis Cardiovascular Institute, Pittsburgh, PA, USA

Journal of Cardiovascular Magnetic Resonance 2009, I I(SuppI I):P89

Introduction: Risk stratification in dilated cardiomyopathy (CMX) patients with advanced heart failure remains a growing clinical challenge. A simple manner to non-invasively risk stratify this difficult cohort would have obvious advantages.

Hypothesis: Utilizing cardiovascular MRI (CMR), recently demonstrated to identify abnormal myocardial substrate, typically infarct or more recently infiltrative pathology via the delayed hyperenhancement technique (DHE), we hypothesize that $+\mathrm{DHE}$ will represent an adverse prognosis as defined by need for urgent cardiac transplantation (TX), LVAD or death in a dilated cardiomyopathy patients.

Methods: Over 24 consecutive months, 13 patients with a dilated CMX and NYHA class III-IV heart failure underwent standard 3D CMR (I.5 T GE, Excite, Milwaukee, WI) to interrogate the pattern, distribution and extent of DHE (MultiHance, Bracco Diagnostics, Princeton, N J, USA). Patients were categorized into: I) + DHE/+midwall Stripe 2) +DHE/-midwall Stripe and 3) -DHE/-Stripe. LVAD, Tx need, major adverse clinical events (MACE) and event free survival were evaluated over the next 6 months.

Results: All patients were alive at 6 months for folow-up while 5 required Tx. All pts completed the CMR exam in $50 \pm$ 10 minutes. Of I I/I3 pts (85\%) with +DHE, 9/II pts (82\%) had +Stripe and 2/I 3 pts were -DHE/-Stripe. DHE/Stripe categorization positively strongly correlated with NYHA class $(r=0.59$, $P<0.05)$ while only weakly correlating with $3 D$ LVEF $(r=0.14$, $p=n s)$ and EDV $(r=0.05, p=n s)$. However, DHE/Stripe categorization strongly predicted the need for LVAD and/or urgent Tx surgery over the ensuing 6 months $\left(X^{2}=5, p<0.05\right)$. Specifically, all 5 pts requiring LVAD and/or urgent $T x$ by 6 months had +DHE/+Stripe while no -DHE/-Stripe pts experienced the need for LVAD or urgent Tx. Similarly, a $+\mathrm{DHE} /+$ Stripe strongly predicted MACE $\left(\mathrm{X}^{2}=8, \mathrm{P}<0.005\right)$. Any +DHE pt with + or -Stripe, in general, predicted a more egregious course, meeting a MACE, worsening LVEF, or nonimproved/worsening NYHA class. No -DHE/-Stripe patient had a major adverse clinical event, see Table I.

Conclusion: CMX patients with advanced heart failure require an improved risk stratification policy. We believe this observation represents the first attempt to risk stratfiy systematically for those with a dilated CMX. Specifically, a simple observation of the binary nature of DHE/Stripe predicated early morbidity and mortality. Herein, using standard CMR, the presence of +DHEl +Stripe is highly predictive of LVAD and Tx need over the ensuing 6 months. Those +DHE/-Stripe patients have intermediate risk but no LVAD/Tx use, while those -DHE/-Stripe have a good prognosis. Thus, incorporating this approach into routine clinical practice may help manage CMX patients more expectantly and effectively.

\section{P90}

Comparison of myocardial infarct size measurements between noncontact mapping and cardiac contrast enhanced MRI

Henning Steen, Frederik Voss, Rüdiger Becker,

Hugo A Katus and Evangelos Giannitsis

University of Heidelberg, Heidelberg, Germany

Journal of Cardiovascular Magnetic Resonance 2009, I I(SuppI I):P90

Introduction: Once chamber geometry is determined, the EnSite 3000 noncontact mapping system can create a voltage map during a single cardiac cycle. The EnSite uses an inverse solution to the Laplace equation to process the amplified far-field signals from the noncontact catheter. This process creates a threedimensional endocardial potential map from a single cardiac cycle. Dynamic substrate mapping (DSM) is an algorithm designed to identify conduction boundaries, such as myocardial scars based on voltage distribution within the corresponding chamber.

Purpose: The purpose of this study was to investigate the correlation between DSM- and magnetic resonance imaging (MRI) - determined scar areas and to identify a suitable DSM voltage threshold.

Methods: A total of eight dogs were studied. Four healthy foxhounds underwent ligation of the left anterior descending coronary artery. Evidence of myocardial infarction, including ECG changes and elevated cardiac troponin $T$ levels, was noted in all animals. Cardiac MRI scan was performed $29 \pm 2$ days after ligation of the left anterior descending coronary artery. Subsequently, noncontact mapping of the left ventricle was obtained in each dog, and myocardial infarction size was determined using DSM at different filter settings. As a control group, another four foxhounds underwent sham thoracotomy/ pericardiotomy.

Results: A significant linear correlation of infarction size using DSM compared with MRI measurements was found at the filter setting "peak negative 34\%" $(P=0.00 I, r=0.99)$. Mean relative infarction size was $15.9 \% \pm 4.5 \%$ with DSM and $16.0 \% \pm 4.2 \%$ with MRI. Compared with the sham group, a significant reduction in left ventricular ejection fraction was found after ligation of the

Table I (abstract P89)

\begin{tabular}{lllll}
\hline DHE Status & $\begin{array}{l}\text { No. of } \\
\text { patients }\end{array}$ & $\begin{array}{l}\text { Heart } \\
\text { transplant }\end{array}$ & MACE & $\begin{array}{l}\text { Unchanged/ } \\
\text { worsening NYHA } \\
\text { EF }\end{array}$ \\
\hline +DHE/+Stripe & 9 & 5 & 7 & 8 \\
+DHE/-Stripe & 2 & 0 & 1 & 1 \\
-DHE/-Stripe & 2 & 0 & 0 & 0 \\
\hline
\end{tabular}


left anterior descending coronary artery $(51.0 \% \pm 3.8 \%$ vs $69.2 \% \pm 5.9 \%, P=0.002)$. Pathoanatomic studies were performed to confirm the measured infarct dimensions. No scars were detectable in shamoperated dogs using DSM or MRI.

Conclusion: Noncontact mapping allows identification of scar tissue within the left ventricle. An excellent correlation was observed between DSM-scar surface and MRI-determined scar size. Identifying and marking these areas can be useful when planning an ablation strategy in the clinical setting of ischemic heart disease.

\section{P9 I}

Pulmonary hypertension: role of septomarginal trabeculation and moderator band complex assessed by cardiac magnetic resonance imaging Monda L Shehata', Jan Skrok', Dirk Lossnitzer', Danielle Boyce', Joao AC Lima', Paul Hassoun', David A Bluemke and Jens Vogel-Claussen' 'Johns Hopkins University, Baltimore, MD, USA 2University of Heidelberg, Heidelberg, Germany ${ }^{3}$ National Institute of Health (NIH), Bethesda, MD, USA

Journal of Cardiovascular Magnetic Resonance 2009, I I(SuppI I):P9 I

Introduction: In the right ventricle (RV), the septomarginal trabecula (SMT) arises as a muscular band originating from the interventricular septum (IVS) at the lower segment of the crista supraventricularis. It forms a functional unit with the moderator band, which attaches to the lateral free wall of the RV [I, 2]. Strategically situated between the RV inflow and outflow tracts, the whole unit serves to help emptying blood into the pulmonary trunk during systole. Thus, it should be anticipated that the SMT may undergo changes in RV hypertrophy secondary to chronic pulmonary hypertension.

\section{Figure I (abstract P9I)}

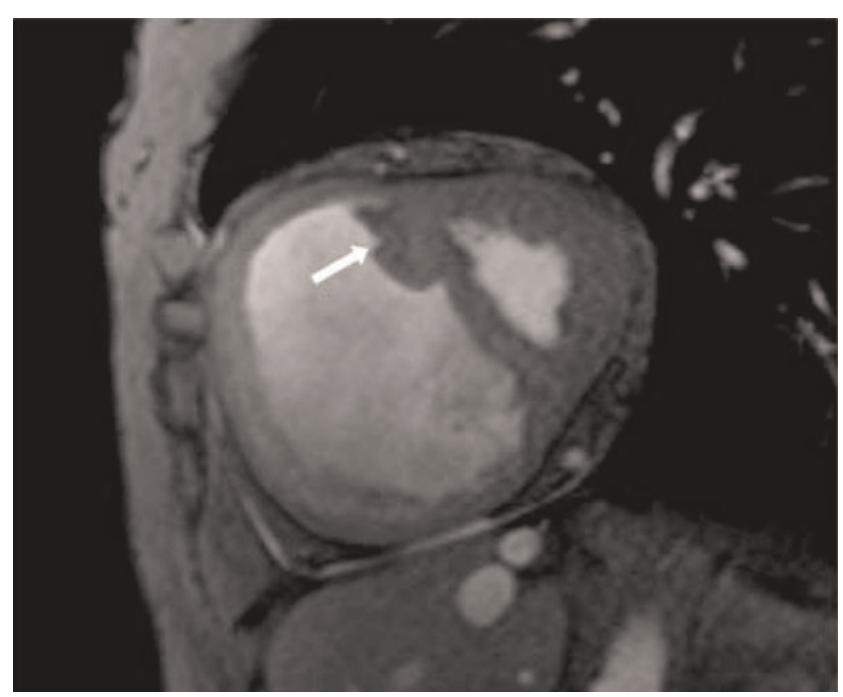

64-year-old female with mean PAP $=5 \mathrm{I} \mathrm{mmHg}$ demonstrates hypertrophy of SMT (septomarginal trabecula), white arrow, in a dilated right ventricle.
Figure 2 (abstract P9I)

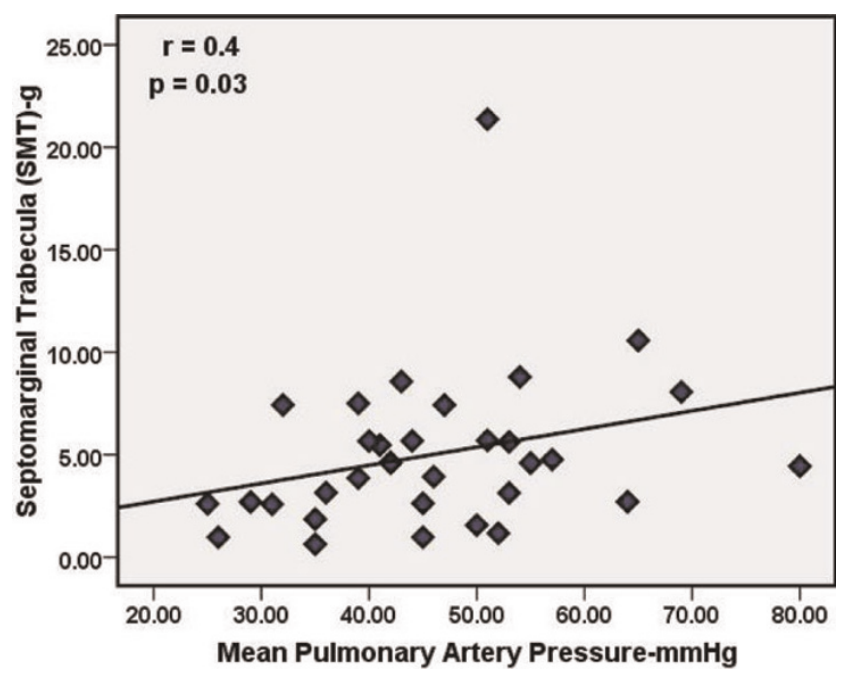

Purpose: To assess SMT mass in pulmonary arterial hypertension $(\mathrm{PAH})$ and its relationship with RV function and pulmonary hemodynamics using cardiac MRI.

Methods: Imaging was performed in two centers; one using $3 \mathrm{~T}$ and the other using I.5 T MR systems. 33 catheter proven PAH patients (mean age $=61.4 \pm 12.1$ years and $\operatorname{mPAP}=45.9 \pm$ $12.4 \mathrm{mmHg}$ ) were enrolled in the study [Idiopathic pulmonary arterial hypertension $($ IPAH) $=21$ and Scleroderma $(\mathrm{SSc})=12$ patients]. Similarly, 9 healthy volunteers (mean age $=45.56 \pm 8.6$ years) were included in the study for comparison. Short axis cine images were acquired using fast gradient echo technique. End diastolic frames were analyzed using MASS 6.2.I software (Medis, the Netherlands). Starting from the basal slices, the SMT was identified in patients and controls as the most anterior trabeculation arising from the IVS below the outflow tract level. Two independent observers manually contoured and traced the SMT from its origin towards the apex where the moderator

Figure 3 (abstract P91)

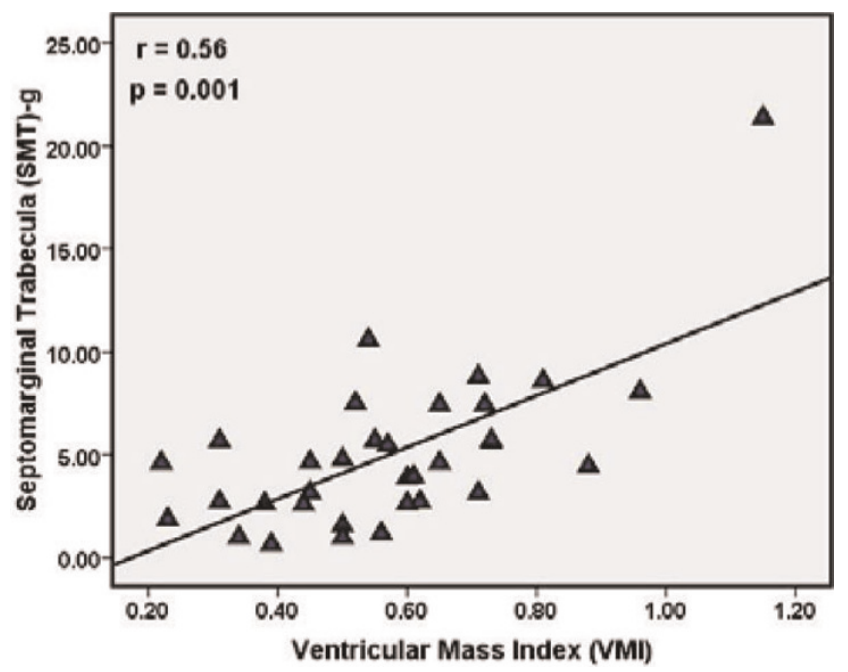


band and secondary trabeculation arise. SMT mass in grams was derived from the volume based on a myocardial density of $1.05 \mathrm{~g} / \mathrm{cm}^{3}$. Epicardial and endocardial ventricular borders were semiautomatically contoured for quantification of ventricular mass and functional indices. Ventricular mass index [ratio of RV over left ventricular (LV) mass] was derived for all groups. The MannWhitney test was used for direct comparisons. Correlation between SMT mass and MR derived cardiac functional indices as well as catheter derived hemodynamic parameters was tested using Spearman's rho correlation.

Results: In all subjects, the SMT was observed in the RV; however, the morphology was highly variable. The SMT mass was greater in PAH patients compared to controls, $5.0 \pm 3.9 \mathrm{~g}$ and $\mathrm{I} .2 \pm 0.7 \mathrm{~g}$ respectively $(\mathrm{P}=0.000 \mathrm{I})$. Similarly, significant differences in SMT mass were also noted between controls and each subgroup: IPAH $(p<0.00 I)$ and SSc $(p<0.0 I)$. In PAH patients, SMT mass showed significant correlations with catheter derived pulmonary hemodynamics mPAP $(r=0.4, p=0.032)$ and PA systolic pressure $(r=0.4, P=0.01)$. It also demonstrated good positive correlations with RV end diastolic mass indexed to body surface area (RVED mass/BSA $r=0.5, p=0.006)$, VMI $(r=0.56$, $p=0.00 I), R V$ end systolic volume/BSA $(r=0.4, p=0.0 I)$ whereas, it correlated inversely with RV stroke volume $(r=$ $-0.5, p=0.003)$ and RV ejection fraction $(r=-0.6, p=0.000 \mathrm{I})$. SMT mass displayed stronger correlations with IPAH RV structural and functional indices compared to patients with Scleroderma. Interobserver concordance for SMT mass quantification was $r=0.95,(p=0.000 \mathrm{I})$. Figures I, 2 and 3

Conclusion: SMT/moderator band complex hypertrophy was seen in all PAH patients studied. Degree of SMT hypertrophy correlated with RV systolic function and pulmonary hemodynamics. The SMT is a unique RV anatomical structure which contributes to RV adaptation mechanisms in chronic PAH. It is readily assessed with cardiac $M R I$ and aides in the diagnosis of PAH.

References

I. James TN: J Am Coll Cardiol 1985, 6(5): 1083-1095.

2. Kilner PJ, et al: J Cardiovasc Magn Reson 2002, 4(3):373-379.

\section{P92}

Late gadolinium enhancement and T2

$M R$ imaging features of cardiac sarcoidosis involving the left and right ventricle Varghese Cherian, Hersh Chandarana, Ruth P Lim, Leon Axel, Danny Kim and Monvadi B Srichai NYU Langone Medical Center, New York, NY, USA

Journal of Cardiovascular Magnetic Resonance 2009, I I (SuppI I):P92

Objective: To evaluate the presence and location of late gadolinium enhancement (LGE), increased T2 signal abnormality, right ventricular involvement and cardiac function by cardiac magnetic resonance (CMR) imaging in patients with suspected cardiac sarcoidosis.

Materials and methods: 46 patients with known extracardiac sarcoidosis underwent CMR with T2-weighted, LGE and cine imaging for evaluation of cardiac sarcoidosis. Images were evaluated by 2 expert reviewers in consensus using the 17segment model of the left ventricle (LV) for presence and location (subepicardial, midwall, subendocardial or transmural) of LGE, presence of increased T2-signal abnormalities, and regional wall motion abnormalities. In addition, right ventricular (RV) involvement was evaluated.

Results: 13 of 46 (28\%) patients demonstrated evidence of segmental LGE suggestive of cardiac sarcoidosis. In these I3 patients, LGE involvement was noted in 5 I of 22 I $(23 \%)$ segments, and was predominantly transmural (35\%), followed by midwall $(27 \%)$, subepicardial $(25 \%)$ and subendocardial $(20 \%)$. LGE was most frequently located in the basal segments (36\%) compared to mid (23\%) and apical (22\%) segments, and more commonly involved the septal (37\%) followed by the lateral (29\%), inferior $(23 \%)$ and anterior (10\%) segments. T2-weighted images were evaluable in II of the 13 patients, and 24 of 187 segments (13\%) demonstrated evidence of increased T2signal abnormality and was significantly associated with the LGE segmental involvement $(p<0.005) .4$ patients $(31 \%)$ also demonstrated evidence of RV involvement. There was no significant correlation between presence of LGE or increased T2-signal intensity with regional function.

Conclusion: Late gadolinium enhancement and increased T2signal intensity are common findings in the left ventricle in patients with cardiac sarcoidosis, but not necessarily correlated with overall ventricular size and function. Although not previously reported, similar findings are frequently present in the right ventricle as well.

\section{P93}

Fatty liver in uncomplicated type $2 \mathrm{DM}$ is associated with impaired myocardial HEP metabolism, modulated by myocardial glucose uptake

Jacqueline T Jonker', Rutger W van der Meer', Luuk J Rijzewijk ${ }^{2}$, Adriaan A Lammertsma ${ }^{3}$, Michaela Diamant ${ }^{2}$, Johannes WA Smit', Albert de Roos', Johannes A Romijn' and Hildo J Lamb'

'Leiden University Medical Centre, Leiden, Netherlands

${ }^{2}$ Diabetes Centre, VU University Medical Centre, Amsterdam, Netherlands

${ }^{3}$ VU University Medical Centre, Amsterdam, Netherlands

Journal of Cardiovascular Magnetic Resonance 2009, I I (SuppI I):P93

Purpose: To study the associations between fatty liver (FL), insulin resistance, myocardial high-energy-phosphate (HEP) and glucose metabolism, and heart function in patients with uncomplicated type 2 diabetes mellitus (T2DM).

Materials and methods: We studied 35 T2DM patients (Mean $\pm \mathrm{SD} \mathrm{Hbalc}=7.0 \pm 0.8 \%$ ) without coronary artery disease or heart failure, as determined by echocardiography. 'H-MRS of the liver for the assessment of liver fat, myocardial ${ }^{3}$ I P-MRS for assessment of myocardial HEP metabolism and MRI to determine left ventricular function were performed. Furthermore, a hyperinsulinemic, euglycemic clamp was performed to establish whole body insulin sensitivity. Moreover, PET with $\mathrm{H}_{2}{ }^{15} \mathrm{O}$ (fasting conditions) and $\left[{ }^{18} \mathrm{~F}\right]$-2-fluoro-2-deoxy-D-glucose (clamp conditions) were used to determine myocardial blood flow (MBF) and myocardial metabolic rate of glucose uptake (MMRglu) in a subgroup of 28 patients.

Results: Patients with FL (liver: fat/water ratio $>5 \%, n=17$ ) showed increased body mass index $\left(29.5 \pm 3.2 \mathrm{vs} 27.2 \pm 2.8 \mathrm{~kg} / \mathrm{m}^{2}\right.$, 
Figure I (abstract P93)

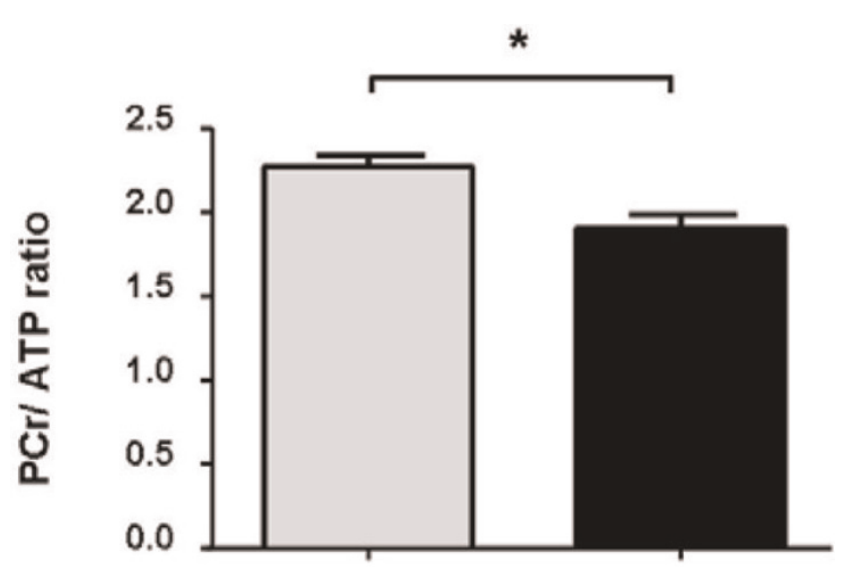

\section{Non fatty liver Fatty liver}

Myocardial HEP metabolism in patients with DM2. PCr/ATP ratio = ratio of phosphocreatine over adenosine triphosphate. $* \mathrm{P}<0.05$.

$\mathrm{P}<0.05)$, reduced whole body insulin sensitivity $(0.45 \pm 0.48$ vs $0.74 \pm 0.44(\mathrm{mg} / \mathrm{kg} \cdot \mathrm{min}) /(\mathrm{pmol} / \mathrm{L}), \mathrm{P}<0.05)$, and reduced MMRglu $(0.21 \pm 0.13$ vs $0.34 \pm 0.14 \mathrm{mmol} / \mathrm{mL} / \mathrm{min}, \mathrm{p}<0.05)$, as compared with patients without FL, while MBF was not different. The ratio of phosphocreatine over adenosine triphosphate, a marker of myocardial HEP metabolism, was reduced in patients with $\mathrm{FL}(1.90 \pm 0.35$ versus $2.27 \pm 0.29 ; \mathrm{p}<0.05)$, also after adjustment for BMI, and correlated to MMRglu ( $r=0.43$, $P<0.05)$. LV systolic and diastolic function were not statistically significantly different. Figure I

Conclusion: Fatty liver in patients with uncomplicated T2DM is associated with decreased myocardial HEP metabolism. In addition myocardial HEP metabolism is modulated by myocardial glucose uptake.

\section{P94}

Incongruity of LVH regression with persistent inopportune diastolic dysfunction; results following AVR for severe aortic stenosis. Sponsored by the American Heart Association Robert WW Biederman, Ronald B Williams, Saundra B Grant, Wadih Nadour, Vikas K Rathi, Diane A Vido, June Yamrozik, Geetha Rayarao, James A Magovern and Mark Doyle Allegheny General Hospital, The Gerald McGinnis Cardiovascular Institute, Pittsburgh, PA, USA

Journal of Cardiovascular Magnetic Resonance 2009, I I (SuppI I):P94

Background: Very elegant invasive animal and human studies have demonstrated that in subjects with pressure overload due to severe aortic stenosis (AS), following aortic valve replacement (AVR), while myocyte regression is quite rapid, interstitial collagen content regression lags.

We hypothesize that this discord manifests as a thwarted improvement in diastolic function as related to $\mathrm{LVH}$ regression after AVR for AS.
Figure I (abstract P94)

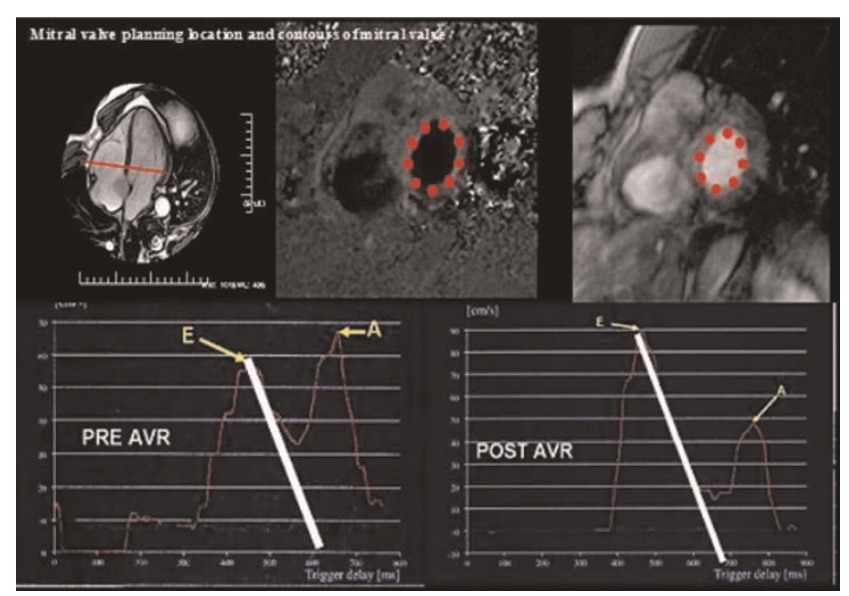

Methods: Ten patients with severe, but compensated AS underwent 4 serial 3D cardiac MRIs (CMR) with I.5 T EXCITE (GE Milwaukee, $\mathrm{WI}$ ) out to 4 years (40 time-points: baseline, 6 months, I year and up to 4 years). 3D LV volumetrics were measured. LV diastolic function was assessed by a phase velocity mapping slice placed at the tips of the mitral valve leaflets, acquired in a through-plane manner with temporal resolution of $25 \pm 5 \mathrm{~ms}$. Interrogation of resultant time-velocity curves was performed to resolve: I) E:A ratio as mean and peak absolute and relative velocities 2) deceleration time. Figure I.

Results: All patients survived AVR and were available for serial follow-up over 4 time periods (mean $3 \pm \mathrm{I}$ ) out to 4 years. E:A ratios and/or morphology almost uniformly improved ( $9 / 10 \mathrm{pts})$ from 0.9:I to I.7:I ( $p<0.005)$, including 4 patients improving one complete grade. This was moderately well correlated with LV mass index regression $(r=0.55, p=<0.05)$. Deceleration time also improved (233 vs. $192 \mathrm{~ms}, \mathrm{p}<0.005)$. While EF improved (55 \pm 22 to $65 \pm 1 \mathrm{I} \%, \mathrm{p}<0.05)$, as did LV geometry $\left(\mathrm{l} .07 \pm 0.2\right.$ to $\left.0.94 \pm 0.24 \mathrm{~g} / \mathrm{m}^{2}, \mathrm{p}<0.05\right)$, neither were tightly correlated with improvements in diastolic function. However, using predictive modeling from historic controls, the improvement seen in diastolic function was incomplete and tardy. Specifically, while there were 4 patients that returned to normal diastolic function as per morphologic criteria, even these patients did not return to pre-morbid e:a ratios or deceleration times of the normal historic cohort. It followed that the remaining patients, except for one, improved only in arithmatic metrics. Importantly, while the LVH regresssed quickly, most occured within the first 6 months, there was a lag in diastolic function improvement by $>12$ months.

Conclusion: Following AVR for severe AS, as expected, marked improvements in LV mass regression occur; however, improvements in diastolic physiology are incomplete and quite tardy, not paralleling structural changes. Pathophysiologically, it is likely that the interstitium, failing to be as heavily modulated by relief of pressure overload as are the myocytes, contributes to a paradoxical increase in diastolic stiffness. This interplay between collagen content and myocyte results in a less than expected improvement in myocardial diastolic properties following afterload relief contributing to residual dyspnea. 


\section{P95}

Characterisation of the long-term effects of anthracycline-associated myocardial toxicity using cardiac MRI; a pilot study

Marina L Hughes', Frederique Bailliard ${ }^{2}$, Andrew Taylor' and Gill Levitt'

${ }^{I}$ Great Ormond Street Hospital for Children, London, UK

${ }^{2}$ North Carolina Children's Hospital, Chapel Hill, NC, USA

Journal of Cardiovascular Magnetic Resonance 2009, I I (SuppI I):P95

Cardiac MR (CMR) is the imaging modality with the best potential to characterise the myocardial effects of anthracycline therapy in the short and long term. We sought to assess this potential in adult long-term survivors of childhood cancer. Subjects were identified from our hospital's Oncology database, and were specifically selected if they had had acute leukaemia or Wilms tumour, had received anthracycline treatment for childhood cancer without radiotherapy more than ten years prior, and had suffered cardiotoxicity at any time, recognised by an echocardiographic fractional shortening $<25 \%$.

Of a total of 43 patients fitting these criteria, I 8 (II female) were contactable and all agreed to be scanned. Their median (range) age at cancer diagnosis was $4(1-12)$ years, the total anthracycline dose ranged from $180-360 \mathrm{~g} / \mathrm{m}^{2}$, and the median time since cessation of chemotherapy was 16 (I I-26) years. All patients scanned claimed to be asymptomatic.

Four male patients had normal LV volumes and systolic function. The remaining 14 patients all had elevated LV end-systolic volumes, and 5 of these had elevated LV end-diastolic volumes indexed for body size. The median (range) LV ejection fraction (LVEF) was 55\% (33-7I\%), with LVEF < 50\% in 3/17 (all female) patients. The indexed LV mass was low for the whole group, with median (range) mass being $62(54-79) \mathrm{g} / \mathrm{m}^{2}$ for males and 53 (43-7I) $\mathrm{g} / \mathrm{m}^{2}$ for females. Following gadolinium, and using conventional techniques, there was no evidence of late enhancement of the myocardium.

Figure I (abstract P95)

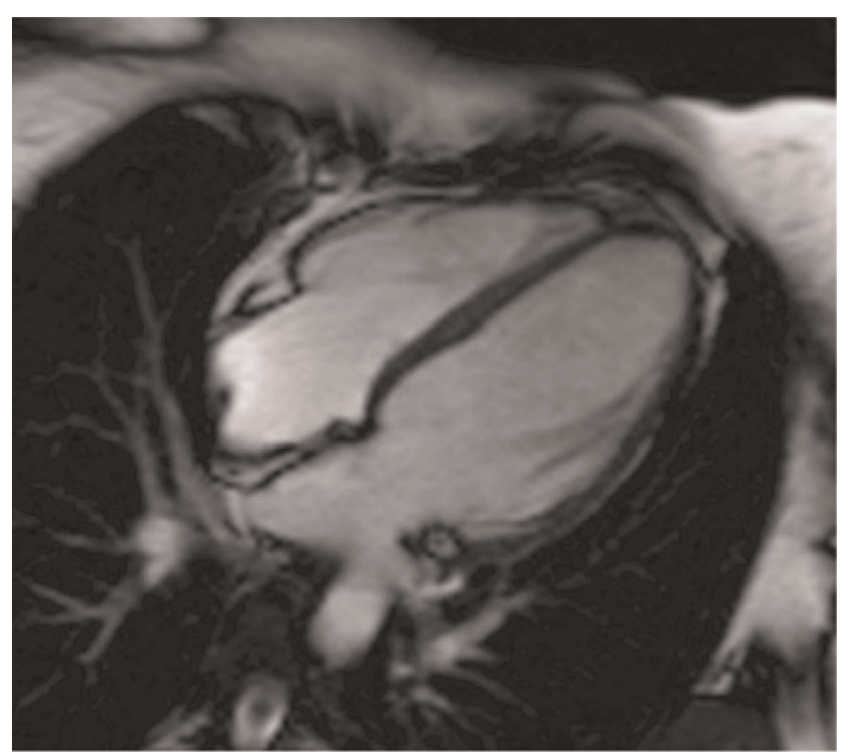

Figure 2 (abstract P95)

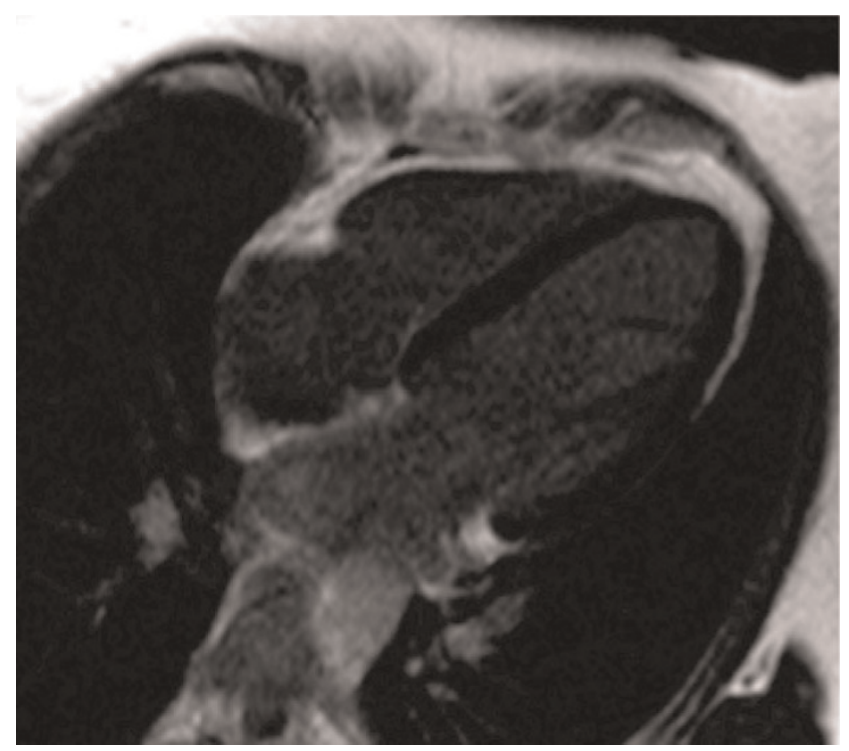

This pilot study specifically assessed patients identified on echocardiography to have had anthracycline-induced cardiac damage. A minority of these patients were found to have normal CMR findings. Most patients had poorly contractile LV myocardium, with low LV mass. Fibrosis or scarring, sought using conventional late gadolinium imaging techniques, was not evident in any patient. Further work is needed to find discriminative CMR indices to describe the long-term effects of myocardial toxicity and to predict prognosis in this patient group. See Figures $\mathrm{I}$ and 2.

\section{P96}

Selection of percutaneous aortic valve replacement candidates: CMR assessment of aortic valve stenosis and aortic root morphology in comparison with echocardiography and cardiac catheterization

Bernard P Paelinck, Delphine Petillot, Christiaan J Vrints and Johan M Bosmans

University Hospital Antwerp, Edegem, Belgium

Journal of Cardiovascular Magnetic Resonance 2009, I I(SuppI I):P96

Introduction: Percutaneous aortic valve replacement in patients presenting high risk for surgery is a promising new interventional treatment modality. The potential role of noninvasive imaging techniques in patient selection needs further validation.

Purpose: We aimed to compare I. planimetry of aortic valve area (AVA) by CMR with 3D echocardiography and calculated AVA by Doppler and cardiac catheterization 2. aortic root dimensions by CMR with echocardiography and angiography.

Methods: Twenty-eight high risk elderly symptomatic patients with severe aortic stenosis scheduled for potential percutaneous aortic valve replacement, were studied. AVA was determined using steady state free precession CMR and direct planimetry using 3D echocardiography. AVA was also calculated by cardiac catheterization using the Gorlin equation and by Doppler using 
Table I (abstract P96) Aortic root dimensions

\begin{tabular}{lll}
\hline & \multicolumn{2}{l}{$\begin{array}{l}\text { Mean difference 2D } \\
\text { echocardiography versus }\end{array}$} \\
\cline { 2 - 3 } & invasive & CMR \\
\hline Ring $(\mathrm{cm})$ & $0.42(0.29,0.55)^{*}$ & $-0.05(-0.12,0.02)$ \\
Sinus $(\mathrm{cm})$ & $0.03(-0.10,0.17)$ & $-0.10(-0.20,0)^{*}$ \\
Sinotubular junction $(\mathrm{cm})$ & $0.03(-0.09,0.15)$ & $-0.13(-0.23,-0.03)^{*}$
\end{tabular}

the continuity equation. Diameter of aortic ring, sinus and sinotubular junction were measured using steady state free precession CMR, 2D echocardiography and invasive aortography. Results: Mean differences and $95 \% \mathrm{Cl}$ in AVA were $0.02 \mathrm{~cm}^{2}$ $(-0.04,0.08) \quad(p=N S)$ for catheterization versus Doppler echocardiography, $-0.01 \mathrm{~cm}^{2}(-0.08,0.06)$ for catheterization versus $3 D$ echocardiography $(p=N S)$ and $0.01 \mathrm{~cm}^{2}(-0.07,0.08)$ for catheterization versus CMR $(p=N S)$.

Mean differences and $95 \% \mathrm{Cl}$ for diameter aortic ring, sinotubular junction and aortic sinus are displayed in table $\mathrm{I}$ $\left({ }^{*} \mathrm{p}<0.05\right)$.
Conclusion: I. CMR planimetry, Doppler and 3D echocardiography provided an accurate estimate of AVA in comparison with catheterization.

2. Catheterization underestimates aortic ring dimensions, while CMR overestimates aortic sinus and sinotubular junction dimensions in comparison with echocardiography.

\section{P97}

Oxidative stress and inflammatory markers are determinants of carotid artery disease quantified by magnetic resonance imaging

Saurabh S Dhawan, Asad Ghafoor, Hamid S Syed, Christina Niessner, Konstantinos Aznaouridis,

Muhammad Ali, Ibhar Al Mheid, Irina Uphoff,

Charles B Kitchen, John Oshinski, Dean P Jones and Arshed A Quyyumi

Emory University School Of Medicine, Atlanta, GA, USA

Journal of Cardiovascular Magnetic Resonance 2009, I I(SuppI I):P97

Introduction: Magnetic Resonance Imaging (MRI) can be used to measure common carotid artery maximum wall thickness

Figure I (abstract P97)
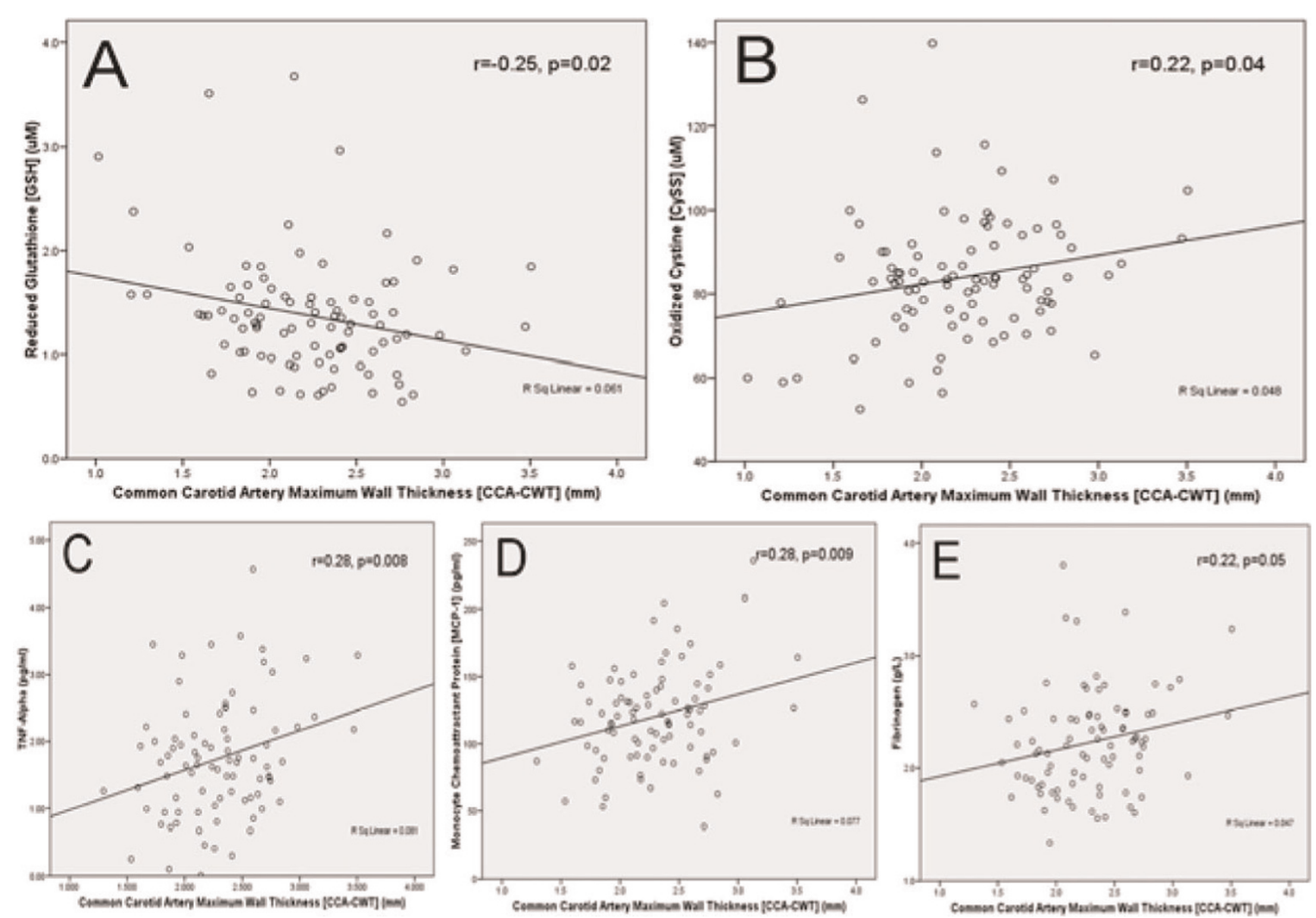
(CCA-CWT) that incorporates the adventitia to intima-media thickness (IMT). Whether serologic markers of oxidative stress or inflammation are better predictors of wall thickness than conventional risk factors is unknown.

Purpose: We hypothesized that patients with greater CCACWT have higher systemic levels of oxidative stress and inflammation.

Methods: CCA-CWT was measured using MRI based T2weighted black-blood sequence on transaxial slices in 92 subjects (6I \pm 9 years) with IMT $>0.65 \mathrm{~mm}$. Markers of oxidative stress included serum glutathione (GSH) and cystine (CySS), which are measures of reduced and oxidized thiols, respectively. Markers of inflammation included high-sensitivity c-reactive protein (HsCRP), tumor necrosis factor-alpha (TNF- $\alpha$ ), monocyte chemoattractant protein-I (MCP-I) and fibrinogen.

Results: CCA-CWT correlated negatively with GSH $(r=$ $-0.25, p=0.02$ ) [Figure IA] and positively with CySS $(r=0.22, p=0.04)$ [Figure IB], suggesting that oxidative stress was higher in those with more severe thickness. CCA-CWT also correlated with TNF- $\alpha(r=0.28, p=0.008)$ [Figure IC], MCP-I $(r=0.28, P=0.009)$ [Figure ID] and fibrinogen $(r=0.22$, $P=0.05$ ) [Figure IE], but not HsCRP. These associations were independent of age, gender, BMI, hypertension, hyperlipidemia, diabetes and smoking.

Conclusion: Biomarkers of oxidative stress and inflammation are better predictors of MRI quantified carotid artery disease than conventional risk factors. Whether progression of disease will also be predicted by these markers needs further study.
P98

Late enhancement findings in a prospective study concerning late functional outcomes following a Ross Procedure

Rod Jones ', Raj Puranik ${ }^{2}$, Wendy Norman', Victor Tsang ${ }^{2}$, Vivek Muthurangu', Johannes Nordmeyer², Philip Lurz ${ }^{2}$, Graham Derrick ${ }^{2}$, Fiona Walker ${ }^{3}$, Shay Cullen ${ }^{3}$, Philip Bonhoeffer ${ }^{2}$ and Andrew M Taylor'

'UCL Institute of Child Health, London, UK

${ }^{2}$ Great Ormond Street Hospital, London, UK

${ }^{3}$ The Heart Hospital, UCLH, London, UK

Journal of Cardiovascular Magnetic Resonance 2009, I I(SuppI I):P98

Background: In patients with aortic valve disease, long-term autograft and/or homograft durability post Ross procedure, remains controversial. Recent studies indicate a late reintervention rate of up to $40 \%$, compared to that of less than $25 \%$ published in earlier studies.

The long-term prospective data regarding functional outcomes in this patient group is also limited. We also aim to describe the extent of scar burden within the myocardium using late gadolinium MR imaging after the Ross procedure.

Methods: 60 subjects ( $22.9 \mathrm{y}$, range $6.4-52.2 \mathrm{y})$ who are in active clinical follow-up and underwent the Ross procedure between 1994 and 2006 (8.I y post Ross operation, range I.814.0 y) were prospectively assessed.

A multi-modality approach was employed, with subjects performimg cardiopulmonary exercise testing, 2D-echocardiography and cardiac MR imaging (including late enhancement).

Figure I (abstract P98)
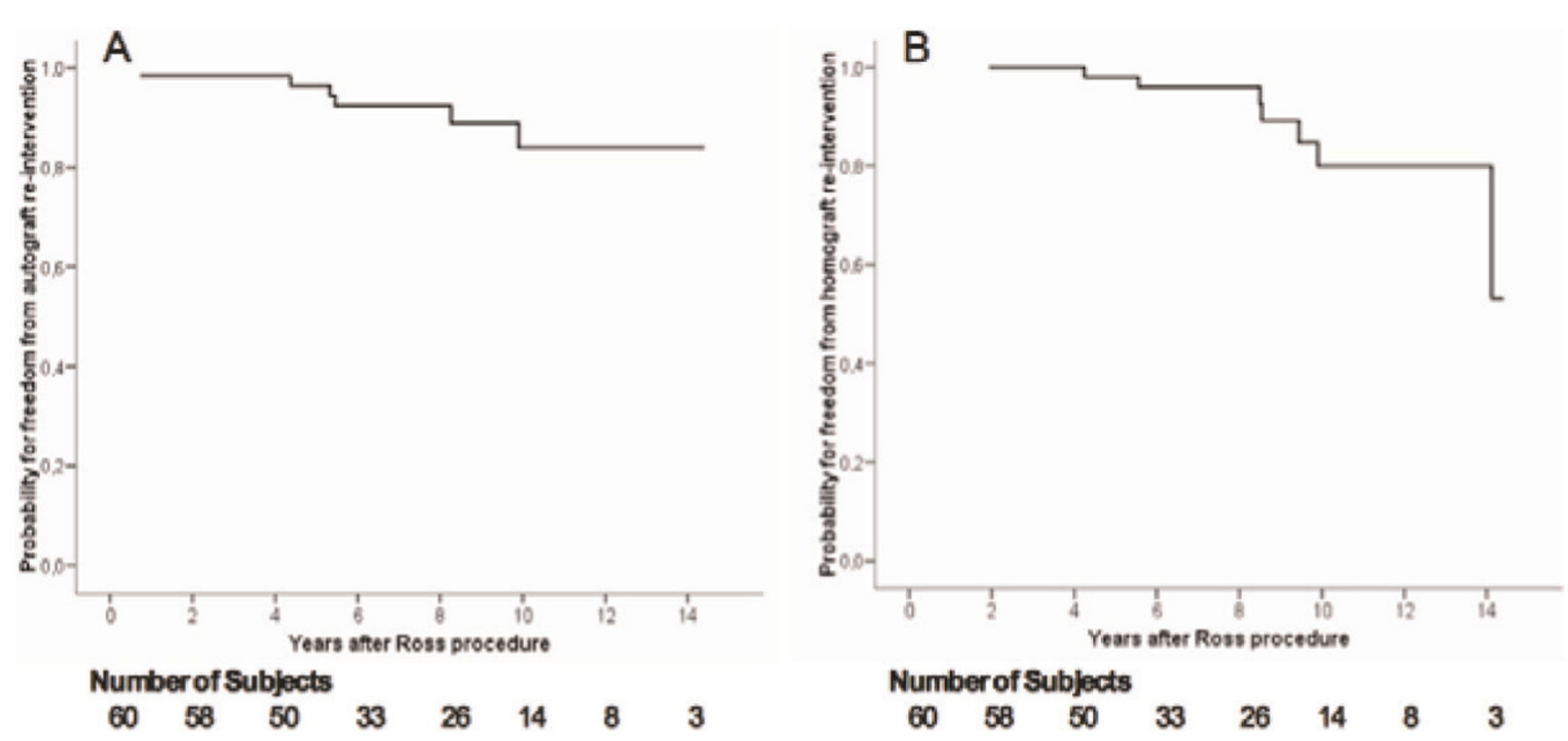

Probability of freedom of re-intervention at 10 years after the Ross Procedure. Data presented as Kaplan Meier plots demonstrating the probability of freedom from re-intervention for the autograft (Panel A) and the homograft (Panel B). The total number of subjects relative to the years after the Ross procedure is shown. 
Table I (abstract P98)

\begin{tabular}{|c|c|c|c|c|c|c|c|}
\hline Pts & Gender & Age & $\begin{array}{l}\text { Years } \\
\text { post Ross }\end{array}$ & $\begin{array}{l}\text { Scar } \\
\text { Vol }\left(\mathrm{cm}^{3}\right)\end{array}$ & $\begin{array}{l}\text { Scan as } \% \text { of } \\
\text { LV volume }\end{array}$ & Pattern within LV & Surgical aspects \\
\hline I & $\mathrm{F}$ & 48.4 & 5.2 & 1.9 & 1.8 & Apex (SE) & $\mathrm{N} / \mathrm{A}$ \\
\hline 2 & $M$ & 52.1 & 8.8 & 9.1 & 9.9 & Mid to distal septum (SE), Apex (SE) & $\mathrm{N} / \mathrm{A}$ \\
\hline 3 & $M$ & 19.3 & 5.8 & 3.3 & 1.9 & Basal septum (FT) & Bleeding at autograft harvest \\
\hline 4 & $\mathrm{~F}$ & 22.3 & 5.7 & 3.1 & 3.0 & Basal septum (SE) & $\mathrm{N} / \mathrm{A}$ \\
\hline 5 & $M$ & 16.7 & 8.2 & 1.9 & 1.4 & Apex (FT) & $\mathrm{N} / \mathrm{A}$ \\
\hline 6 & $M$ & 20.7 & 5.6 & 7.8 & 4.8 & Basal septum (FT) & Bleeding at autograft harvest \\
\hline 7 & $\mathrm{~F}$ & 33.8 & 5.5 & 6.3 & 4.5 & Basal septum (FT) and Apex (FT) & $\mathrm{N} / \mathrm{A}$ \\
\hline 8 & $\mathrm{~F}$ & 32.8 & 8.7 & 2.9 & 1.4 & Apex (FT) & $\mathrm{N} / \mathrm{A}$ \\
\hline 9 & $M$ & 34.6 & 6.2 & 0.9 & 0.5 & Mid septum (SE) & $\begin{array}{l}\text { Anomolous coronary anatomy, no } \\
\text { bleeding }\end{array}$ \\
\hline 10 & $M$ & 6.4 & 5.8 & 17.3 & 37.6 & Complete ring enhancement of LV SE & Endocardial fibroelastosis \\
\hline II & $M$ & 22.3 & 6.2 & 2.5 & 1.9 & Basal lateral wall (FT) & $\mathrm{N} / \mathrm{A}$ \\
\hline 12 & $M$ & 24.7 & 10.3 & 19.0 & 10.2 & Antero-septal (FT) & $\begin{array}{l}\text { Small branch coronary vessel divided } \\
\text { at autograft harvest }\end{array}$ \\
\hline 13 & $M$ & 26.7 & 12.2 & 6.9 & 3.9 & Basal septum (FT) and Apex (SE) & Bleeding at autograft harvest \\
\hline 14 & $M$ & 27.1 & 13.4 & 1.9 & I.I & Apex (SE) & Minor bleeding at autograft harvest \\
\hline 15 & $M$ & 25.2 & 13.0 & 4.0 & 2.8 & Apex (SE) & N/A \\
\hline 16 & $M$ & 23.3 & 12.0 & 7.2 & 4.4 & Antero-septal (FT) & $\begin{array}{l}\text { Small branch coronary vessel divided } \\
\text { at autograft harvest }\end{array}$ \\
\hline 17 & $M$ & 38.5 & 13.9 & 4.9 & 3.1 & Inferior-posterior (SE) & N/A \\
\hline 18 & $M$ & 24.7 & 13.9 & 6.7 & 3.5 & Inferior (SE) & $\mathrm{N} / \mathrm{A}$ \\
\hline
\end{tabular}

SE = subendocardium, $\mathrm{FT}=$ full thickness.

Late enhancement scar imaging within the myocardium was performed with segmented phase-sensitive inversion recovery sequences (Image parameters $-\mathrm{TR}=2 \times \mathrm{RR}$ interval; $\mathrm{TE}=$ $3.4 \mathrm{~ms}$; flip angle $=25^{\circ}$; slice thickness $=10 \mathrm{~mm}$; matrix $=$ $144 \times 256$; field of view $=300-380 \mathrm{~mm}$, acquired during a single breath-hold) 10 minutes post administration of intravenous contrast $(0.1 \mathrm{mmol} / \mathrm{kg}$ of gadolinium pentatate, Magnevist). Imaging included the entire short-axis and long axis planes. A 'Look-locker' sequence was used to determine the inversion time, which reflected the null point of the normal myocardium. The volume of scar within the myocardium was expressed as a \% of the total LV volume.

Results: $80 \%$ of subjects had aortic stenosis as their original diagnosis ( $43 \%$ biscuspid aortic valves). The probability of freedom from re-intervention on the autograft and the homograft at 10 years was $84 \%$ and $80 \%$ respectively (Figure I). On exercise testing, the mean exercise capacity achieved was $87 \pm 23 \%$ of predicted. On 2D-echocardiography the peak velocity across the autograft and homograft was $1.3 \pm 0.4 \mathrm{~m} / \mathrm{sec}$ and $2.6 \pm 0.6 \mathrm{~m} / \mathrm{sec}$ respectively. Cardiac MR imaging identified only trivial mean autograft and homograft regurgitation (5.9 $\pm 7.6 \%$ and $6.4 \pm 9.3 \%$ respectively). Biventricular systolic function was normal (LV EF $63 \pm 6 \%$ and RV EF $61 \pm 7 \%$ ).

$55 / 60$ subjects underwent delayed enhancement studies with intravenous gadolinium. 18/55 subjects (33\%) had evidence of myocardial scar within the LV (Table I). The mean scar volume within the LV was $6.0 \pm 5.0 \mathrm{~cm} 3$ and mean \% of scar within the left ventricle was $5.4 \pm 8.5 \%$. In $5 / 9$ cases of septal wall scar the surgeon had commented on bleeding at the time of autograft harvest. There was no significant relationship between LV scar and baseline ECG abnormalities ( $p=1.0$ ).

Conclusion: Results show, many years after the Ross procedure, there is excellent autograft and homograft function, translating into high functional capacity. Institution based re-intervention rates of less than $20 \%$ are in agreement with earlier published work.

We provide the first description of the extent of scar burden within the LV myocardium using late gadolinium MR imaging after the Ross procedure. The predominant distribution of LV scar was identified within the LV septum (50\% of cases). This is likely mainly related to technical aspects surrounding the autograft harvest, site's proximity to septal coronary vessels.

Although in our study we found no association between LV scar burden and ECG abnormalities, the prognostic significance of these findings may only be truly deciphered after longer-term follow-up.

\section{P99}

Enlarged right ventricular size at I I years follow-up after closure of secundum type atrial septal defect in children

Wilfred B de Koning, Saskia E Luijnenburg,

Lennie $M$ van Osch-Gevers, Derk Jan ADJ ten Harkel, Daniëlle Robbers-Visser, Ron T van Domburg,

Ad JJC Bogers and Willem A Helbing

Erasmus MC, Rotterdam, Netherlands

Journal of Cardiovascular Magnetic Resonance 2009, I I(SuppI I):P99

Objective: to assess ventricular dimensions, exercise capacity and rhythm-status of patients operated for secundum type atrial septal defect (ASD-II) and compare the results with those in healthy references.

Background: Long-term prognosis of children operated for ASD-II is excellent. It remains unclear whether right ventricular (RV) dimensions completely normalize after surgery and whether this may have implications for (timing of) treatment strategies.

Methods: Seventy-eight consecutive patients underwent surgical closure for ASD-II between 1990 and 1995. Forty-two 
patients were included in this study and underwent a crosssectional evaluation including echocardiography, cardiovascular magnetic resonance (CMR) imaging, exercise testing, and 24-hours Holter monitoring. For comparison of the results, ASD-II patients were matched with healthy controls for gender, body surface area (BSA) and age.

Results: Mean age at surgery was $4.6 \pm 2.8$ years and mean age at follow-up $16 \pm 3$ years. There were no residual intracardiac lesions. Mean right ventricular end-systolic volume (RVESV) was significantly larger in patients $(142 \pm 26 \mathrm{ml})$ than in references $(I 37 \pm 28 \mathrm{ml})(p=0.04)$. In $25 \%$ of the patients, RVESV was larger than the $95^{\text {th }}$ percentile for healthy controls. No relevant arrhythmias were detected. Exercise testing did not reveal differences between patients and healthy controls. Maximal workload (Watt) was $169 \pm 43$ for patients vs. $172 \pm 53$ for controls $(p=0.8)$, maximal oxygen uptake $(\mathrm{ml} / \mathrm{kg} / \mathrm{min})$ was $38 \pm 8$ for patients vs. $4 \mathrm{I} \pm 13$ for controls $(p=0.1)$.

Conclusion: After surgical closure of ASD-II, RVESV is increased. These findings have no impact on rhythm-status or exercise capacity at this stage of follow-up, but may have implications for the timing of surgery or the technique of closure if confirmed in longer follow-up.

\section{PIO0}

Intra-observer and interobserver variability of biventricular function, volumes and mass in patients with congenital heart disease measured by CMR imaging

Saskia E Luijnenburg', Daniëlle Robbers-Visser', Adriaan Moelker', Hubert W Vliegen², Barbara JM Mulder ${ }^{3}$ and Willem A Helbing'

'Erasmus MC, Rotterdam, Netherlands

${ }^{2}$ Leiden University Medical Center, Leiden, Netherlands

${ }^{3}$ Amsterdam Medical Center, Amsterdam, Netherlands

Journal of Cardiovascular Magnetic Resonance 2009, I I(SuppI I):PI 00

Objective: The aim of this study was to assess intra-observer and interobserver variability of biventricular function, volumes and mass in a heterogeneous group of patients with congenital heart disease (CHD) using cardiovascular magnetic resonance (CMR) imaging.

Background: CMR imaging provides highly accurate measurements of biventricular volumes and mass and is frequently used in the follow-up of patients with acquired and congenital heart disease. Data on reproducibility are limited in patients with CHD, while measurements should be reproducible, since CMR imaging has a main contribution to decision making and timing of (re) interventions.

Methods: Thirty-five patients with CHD (26 males, 9 females; age range $7-62$ years) were included in this study. A short axis set of contiguous slices was acquired with CMR imaging using a steady-state free precession (SSFP) pulse sequence. Intraobserver and interobserver variability was assessed for left ventricular (LV) and right ventricular (RV) volumes, function and mass by calculating the coefficient of variability.

Results: Intra-observer variability was between $2.9 \%$ and $6.8 \%$, with the smallest variations in LV and RV end-diastolic volume (EDV) and the highest variations in LV end-systolic volume (ESV) and RV mass. Interobserver variability was between $3.9 \%$ and $10.2 \%$, with the smallest variations in LV ejection fraction (EF) and RV EDV and the highest variations in biventricular ESV and mass.

Conclusion: Intra-observer and interobserver variability of biventricular parameters assessed by CMR imaging is good for a heterogeneous group of patients with CHD. CMR imaging is an accurate and reliable method for follow-up of biventricular function, volumes and mass and should allow adequate assessment of changes in ventricular size and global ventricular function.

Supported by NHF grant 2006B095

\section{PIOI \\ CMR tagging for measurement of the long axis function and deformation rate of the systemic right ventricular free wall \\ Sylvia SM Chen', Jenny Keegan 2,3, Andrew W Dowsey ${ }^{4,5}$, Rick Wage ${ }^{1,6}$, David N Firmin ${ }^{2,3}$ and Philip J Kilner ${ }^{2}$ \\ ${ }^{\prime}$ Royal Brompton Hospital, London, UK \\ ${ }^{2}$ Royal Brompton Hospital and Imperial College, London, UK \\ ${ }^{3}$ The Brompton Hospital and Imperial College London, \\ London, UK \\ ${ }^{4}$ Imperial College, London, UK \\ ${ }^{5}$ Imperial College London, London, UK \\ ${ }^{6}$ The Brompton Hospital, London, UK}

Journal of Cardiovascular Magnetic Resonance 2009, I I(SuppI I):PIO I

Introduction: Although the systemic right ventricle (RV) in transposition of the great arteries generally functions well through childhood, it typically shows signs of failure later in life. Ejection fraction has been used as a cardiac magnetic resonance imaging (CMR) measurement of systemic RV function, but it is not easy to measure reproducibly due to complex geometry and prominent trabeculations, and may fail to detect early changes. Early detection of dysfunction could be advantageous, for example for trial and eventual implementation of a prospective pharmacological therapy.

Purpose: We aimed to use CMR tagging of the free wall of the $\mathrm{RV}$, with semi-automated analysis, as a measurement of RV long axis function, comparing findings with multislice CMR measurements of ejection fraction, in patients with Mustard operation for transposition of the great arteries (OTGA), or with unoperated congenitally corrected transposition of the great arteries (CCTGA).

Methods: A breath hold four chamber steady state free precession cine dataset was acquired, followed by a breath hold cine gradient-echo echo-planar tagged acquisition in the same plane. Two pre-pulse labelled tag lines were applied immediately after the $\mathrm{R}$ wave, one across the basal RV myocardium and the other located $40 \mathrm{~mm}$ apical of the first. Motion of the tagged myocardium was tracked automatically by multi-resolution image registration so that the position of the tags, and their individual and relative displacements, could be derived throughout the cardiac cycle using semi-automated software. The RV free wall deformation rate was calculated by dividing the amount of deformation (the difference between the displacements of the basal and apical tag) by the time to maximum displacement of the basal tag $(\mathrm{m} / \mathrm{s})$.

Results: Twelve patients (6 OTGA, age $30 \pm 7$ years and 6 CCTGA, age $35 \pm 12$ years) were studied and compared to 6 control subjects (age $31 \pm 9$ years). Basal RV myocardial displacements in both OTGA and CCTGA were reduced 
compared to control subjects ( $12 \pm 5$ and $20 \pm 4$ vs $25 \pm 2 \mathrm{~mm}$, $p=0.0003$ and $p=0.03$ respectively). Basal myocardial displacement in OTGA was more impaired than CCTGA ( $p=0.007)$. The RV myocardial deformation rates in both OTGA and CCTGA were slower than in control subjects $(0.03 \pm 0.02$ and $0.03 \pm 0.02$ vs $0.07 \pm 0.02 \mathrm{~m} / \mathrm{s}, \mathrm{p}=0.03$ and $\mathrm{p}=0.02$ respectively). Ejection fractions did not differ significantly between patients and controls: OTGA $52 \pm 7 \%$ and CCTGA $63 \pm 12 \%$ vs control subjects $57 \pm 4 \%(p=0.2$ and $p=0.5$ respectively).

Conclusion: RV free wall tagging by CMR with semi-automated analysis showed reduction of RV free wall motion and deformation rate in the patients relative to the controls in the absence of significant differences of volumetric ejection fraction. The reduction is likely to result from pressure loading in both patient groups. Greater reduction in OTGA than CCTGA may be a consequence of surgery in patients who had undergone atrial switch repair. RV free wall tagging could provide a relatively quick and simple method for longitudinal comparison of systemic RV myocardial function.

\section{PIO2}

Improved accuracy in flow mapping of congenital heart disease using stationary phantom technique Thomas A Miller and Adrian M Moran

Maine Medical Center, Portland, ME, USA

Journal of Cardiovascular Magnetic Resonance 2009, I I(SuppI I):PI02

Objective: We aimed to define the differences between flow mapping with and without baseline correction using a stationary phantom when evaluating congenital heart disease (CHD).

Background: Flow mapping by cardiovascular magnetic resonance (CMR) has become the gold standard for defining cardiac output, shunt flow and regurgitation. Previous reports have highlighted the presence of inherent errors in flow mapping by CMR secondary to eddy currents and concomitant gradients. The use of a stationary phantom to control for these errors can improve predicted Qp/Qs ratios in normal volunteers. To our knowledge, no one has investigated the effect of phantom correction in CHD.

Methods: All patients were imaged using GE I.5 T Signa HDX systems. Phase velocity flow mapping by CMR was performed when clinically indicated on 20 patients with repaired Tetrology of Fallot (ToF), 7 patients with atrial level shunting (ASD/PAPVR), and $\mathrm{I} 3$ patients with bicuspid aortic valves (BAV). Flow velocities through the ascending aorta, main pulmonary artery (MPA) and proximal branch pulmonary arteries (BPA) were calculated using commercially available software (ReportCard) by placing a region of interest (ROI) around the vessel lumen. Flow velocities were then corrected by measuring baseline velocities in a ROI of the same size and location on a corresponding stationary phantom image. Comparison was then made between the uncorrected and corrected flows, degree of shunting, and regurgitant fraction. $\mathrm{Qp} / \mathrm{Q}$ was calculated as the ratio of net BPA flow to aortic flow. Results: Marked variation in net flow was seen in all vessels (Table I). The variation in net flow was largest when analyzing the MPA/RVOT with a range of 0.2 to $70 \mathrm{ml} / \mathrm{min}(0.3-107.5 \%$ difference). In repaired ToF patients, the average $Q p: Q s$ ratio was 1.03 with phantom correction and 1.15 without. Based on previously published criteria, $26 \%$ of the ToF group would have been incorrectly classified as having a residual shunt without performing phantom correction. In the ASD/PAPVR group $100 \%$ had significant shunting with phantom correction, while only $71 \%$ of uncorrected flows detected significant shunting. In the BAV group, the differences in the regurgitant fraction ranged from 0 to $31 \% .23 \%$ of the patients changed their classification between mild, moderate and severe regurgitation when the phantom was performed.

Conclusion: The impact of inherent errors in CMR flow mapping should not be underestimated. The range of variation, in common CHD, appears large and varied. Failure to correct for such variation can lead to clinically significant misinterpretation of flow data. The use of the stationary phantom correction technique appears to improve flow accuracy in patients with CHD. Further research is needed to elicit the causes and best control of such errors.

\section{PIO3}

Patient-specific coronary artery supply territory AHA diagrams

Maurice Termeer', Javier Oliván Bescós²,

Marcel Breeuwer', Anna Vilanova ${ }^{3}$, Frans Gerritsen ${ }^{2}$, Eduard Gröller ${ }^{1}$ and Eike Nagel ${ }^{4}$

${ }^{I}$ Vienna University of Technology, Wien (Vienna), Austria

${ }^{2}$ Philips Healthcare, Best, Netherlands

${ }^{3}$ Eindhoven University of Technology, Eindhoven, Netherlands ${ }^{4}$ King's College London, London, UK

Journal of Cardiovascular Magnetic Resonance 2009, I I(SuppI I):PI03

Introduction: The American Heart Association proposed a 17-segment model for the segmentation of the left ventricle together with a mapping from each segment to a supplying coronary artery. This proposal is based on population averages. Several studies have confirmed the inaccuracy of this mapping due to large anatomical variations of the coronary arteries among individuals. Several proposals have been made for a different mapping between the 17 segments and the coronary arteries.

Purpose: Due to the large variation in coronary anatomy there is a need for a patient-specific assignment of ventricular segments to supplying coronary arteries. We propose to use a segmentation of the coronary arteries and the ventricular epicardium to compute this patient-specific mapping.

Table I (abstract P 102) Differences in net flows between corrected and uncorrected flow mapping

\begin{tabular}{llll}
\hline & Aorta & MPA/RVOT & RPA \\
\hline Avg difference in net flow (ml/min) & 12.1 & 16.8 & 3.4 \\
Range of difference in net flow (ml/min) & $0.0-68.4$ & $0.2-70$ & $0.1-8.3$ \\
\% Difference in net flow (diff/corrected net flow) & $13 \%$ & $25 \%$ & $0.1-23.1$ \\
Range of \%difference & $0-62 \%$ & $0.3-108 \%$ & $0.3-24 \%$ \\
\hline
\end{tabular}




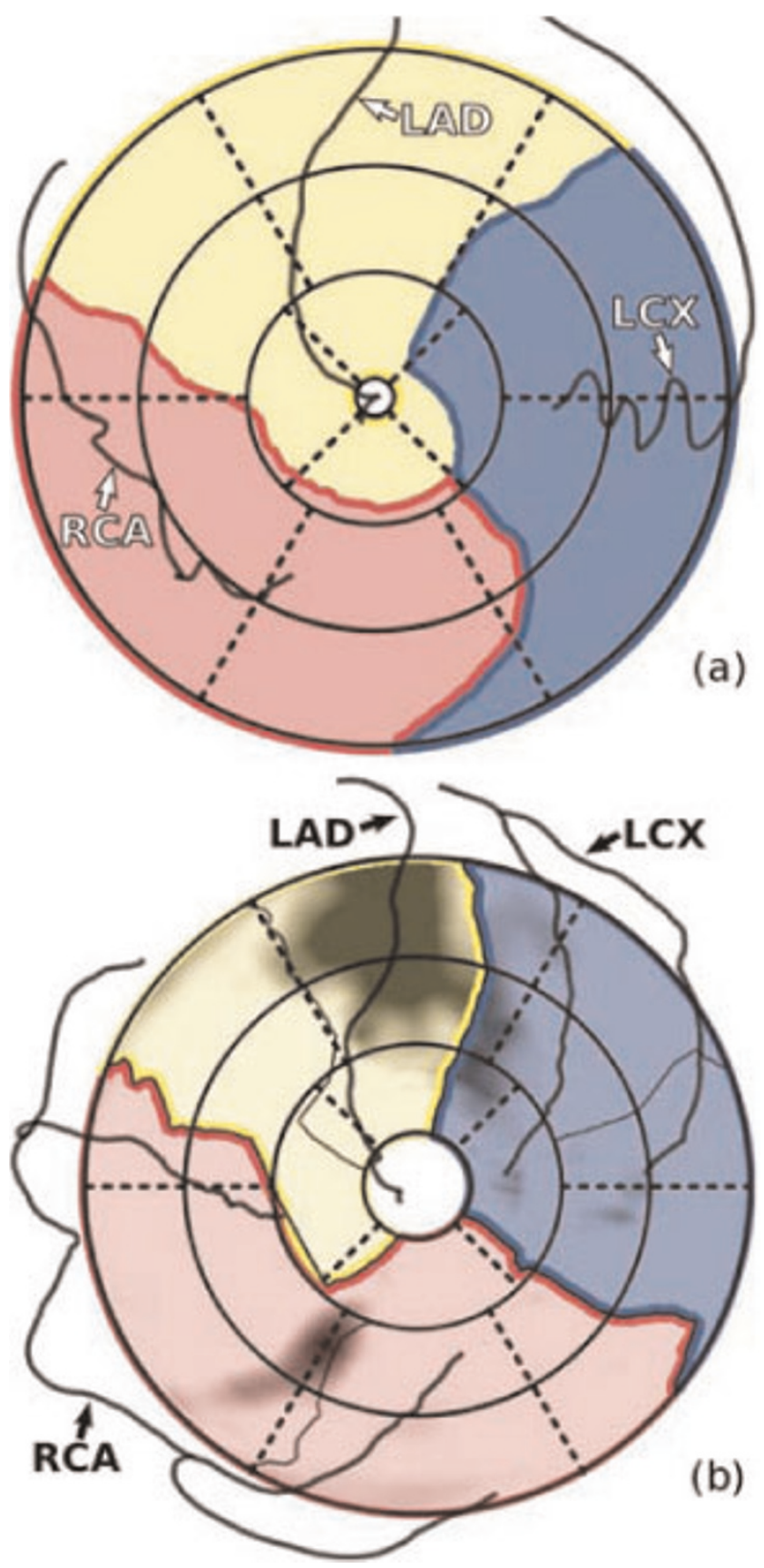

(a) Bull's eye plot showing patient-specific coronary supply territories. The dotted lines represent the 17-segment model. (b) Patient-specific coronary supply territories as an overlay on a bull's eye plot of a late enhancement scan.

Methods: The three primary coronary arteries (LAD, LCX and RCA) and the left ventricle are segmented in a whole-heart MRI (SSFP) or CT scan of at least I50 slices. For the coronary arteries we employ a semi-automatic vessel tracking algorithm. The left ventricle is segmented using a fully automatic approach. The epicardial surface of the resulting segmentation is represented as a quadrilateral mesh. The centerlines of the coronary arteries are projected on the epicardial surface. A Voronoi diagram of the projected arteries is computed using a geodesic distance metric. The patient-specific coronary supply territories are computed using a modified marching squares algorithm. The examples given here consist of three territories, but our approach is flexible enough to handle any amount of territories.

Both the coronary supply territories and the coronary arteries are projected onto a bull's eye plot using a parameterization of the left ventricle based on cylindrical coordinates, using the cardiac long axis as the primary axis of the cylinder (Figure la). The continuous nature of the epicardial surface is preserved in this projection. This means that the bull's eye plot does not consist of rings representing slices, but that the distance to the center is proportional to the distance to the apex. This bull's eye plot can for example be used as an overlay for the analysis of viability (Figure Ib).

Results: We evaluated our method on image data from five patients. For each patient we produced both a standard 17segment diagram and a diagram with the projection of the patient-specific coronary supply territories resulting from our approach. In both diagrams a projection of the segmented coronary arteries was shown. We then asked an experienced clinician to judge the correspondence between the coronary arteries and the suggested coronary supply territories for both diagrams. It was judged that our patient-specific coronary supply territories provide a better correlation with the position of the coronary arteries. The clinician expressed a preference to our method as compared to the standard 17-segment model.

The continuous relation between the distance to the center of the bull's eye plot and the distance to the apex caused some confusion with our clinician. Especially in combination with CMR data consisting of relatively few slices this relation should be clarified.

Conclusion: With our method the relation between coronary arteries and areas supplied by these arteries is better visualized. This will help to better correlate the location of infarcted or ischemic areas to the coronaries that have caused the respective infarction or ischemia.

\section{PIO4}

Accuracy of single breath-hold cine MRI analyzed by guide-point modeling for the assessment of Left Ventricular Function

Christina Heilmaier', Kai Nassenstein ${ }^{1,2}$,

Sonia Nielles-Vallespin ${ }^{3}$, Sven Zuehlsdorff ${ }^{4}$,

Peter Hunold' and Joerg Barkhausen'

'University Hospital Essen, Essen, Germany

${ }^{2}$ University Hospital, Essen, Germany

${ }^{3}$ Siemens AG Healthcare Sector, MED MR PLM AW

Cardiology, Erlangen, Germany

${ }^{4}$ Siemens AG Healthcare Sector, Cardiovascular MR

Research and Development, Chicago, IL, USA

Journal of Cardiovascular Magnetic Resonance 2009, I I(SuppI I):PI04

Purpose: To prospectively assess the performance of highly accelerated cine MRI in multi-orientations combined with a new guide-point modeling post-processing technique (GPMapproach) for the assessment of left ventricular (LV) function 
Table I (abstract PI04) Mean EDV, ESV and EF values \pm SD and ranges as determined by reader I and 2 with the SoS and GPM approach, respectively

\begin{tabular}{|c|c|c|}
\hline & Stack of short axis views + SoS & Multi-orientation sequences + GPM \\
\hline \multicolumn{3}{|l|}{ EF } \\
\hline$\overline{\mathrm{RI}}$ & $53.3 \% \pm 13.5 \%$ (range $15-73 \%)$ & $52.8 \% \pm 13.1 \%($ range $15-7 \mid \%)$ \\
\hline R2 & $52.8 \% \pm 12.5 \%$ (range $16-72 \%)$ & $53.2 \% \pm 12.6 \%$ (range $16-72 \%)$ \\
\hline \multicolumn{3}{|c|}{ (2) } \\
\hline$\overline{\mathrm{RI}}$ & $148.1 \mathrm{ml} \pm 57.2 \mathrm{ml}($ range $85-352 \mathrm{ml})$ & $163.8 \mathrm{ml} \pm 64.5 \mathrm{ml}($ range $93-409 \mathrm{ml})$ \\
\hline R2 & $\mid 47.4 \mathrm{ml} \pm 55.0 \mathrm{ml}($ range $79-33 \mathrm{I} \mathrm{ml})$ & $165.5 \mathrm{ml} \pm 63.0 \mathrm{ml}($ range $98-392 \mathrm{ml})$ \\
\hline \multicolumn{3}{|l|}{ ESV } \\
\hline$\overline{\mathrm{RI}}$ & $75.4 \mathrm{ml} \pm 58.0 \mathrm{ml}($ range $23-299 \mathrm{ml})$ & $84.8 \mathrm{ml} \pm 66.2 \mathrm{ml}($ range $28-335 \mathrm{ml})$ \\
\hline R2 & $75.5 \mathrm{ml} \pm 54.3 \mathrm{ml}($ range $22-274 \mathrm{ml})$ & $84.1 \mathrm{ml} \pm 63.0 \mathrm{ml}($ range $28-321 \mathrm{ml})$ \\
\hline
\end{tabular}

$\mathrm{R} 2=$ reader 2, $\mathrm{EDV}=$ end-diastolic volume, $\mathrm{ESV}=$ end-systolic volume, $\mathrm{EF}=$ ejection fraction, SoS = summation of slices, GPM = guide-point modeling.

compared to the standard summation of slices method based on a stack of short axis views (SoS-approach).

Materials and methods: 33 consecutive patients with sinus rhythm were examined on a $1.5 \mathrm{~T}$ scanner with a standard steady state free precession (SSFP) sequence (,trueFISP“, TR: $3.0 \mathrm{~ms}$, TE: $1.5 \mathrm{~ms}$, flip angle FA: $60^{\circ}$, matrix: $192 \times 156$, temporal resolution: $36 \mathrm{~ms}$; acceleration factor $A F=2$ ) in inspiratory breath-hold. End-diastolic volumes (EDV), end-systolic volumes (ESV) and ejection fractions (EF) were calculated from the standard stack of short axis using commercially available software (syngo ArgusVF, version VA80A; SiemensAG Healthcare Sector, Erlangen, Germany). Additionally, 2 long- and 3 short-axis views

\section{Figure I (abstract PI04)}

a.) R1

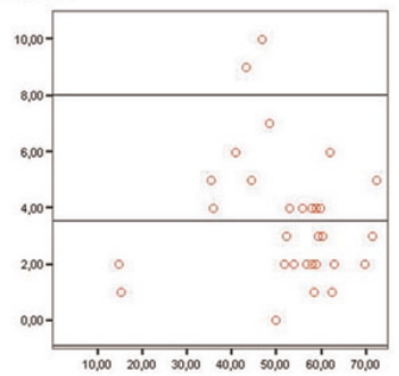

c.) SoS

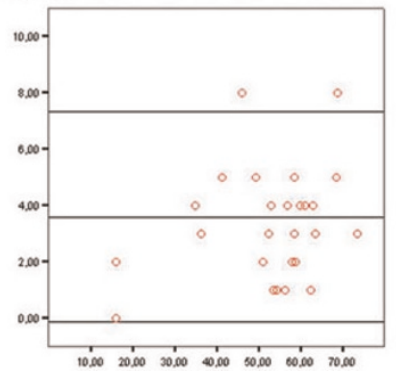

b.) R2

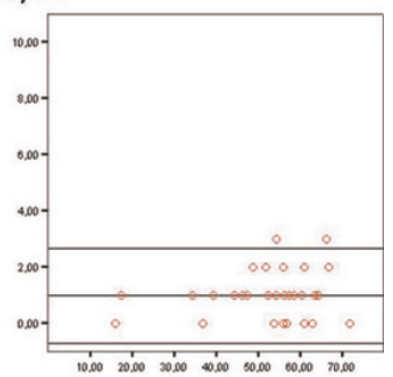

d.) GPM

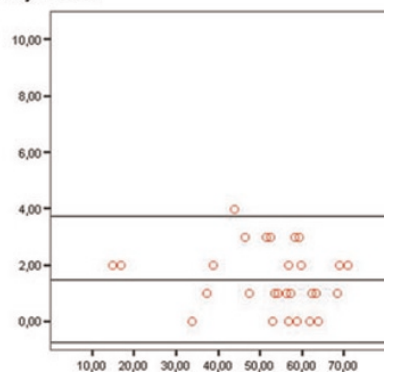

Bland-Altman-Plot for assessment of ejection fraction. (a) Reader I (RI): summation of slices (SoS) versus guide-point modeling (GPM) method. (b) Reader 2 (R2): SoS versus GPM approach. (c) Interobserver comparison of the SoS method based on a stack of short axis views. (d) Multi-orientation highly accelerated sequences analyzed with the GPM approach: RI versus R2. were measured using a highly accelerated, single breath-hold temporal parallel acquisition SSFP sequence (TPAT; TR: $4.6 \mathrm{~ms}$, TE: I.I ms, matrix: $192 \times 133$, temporal resolution: $40 \mathrm{~ms}$, $A F=3)$. This data set was analyzed by means of recently implemented software (syngo Argus 4 DVF, version VA80A; Siemens Healthcare Sector, Erlangen, Germany), which builds up a 4-dimensional (4D) model of the left ventricle and allows visualization of the model superimposed to anatomical images as references (guide-point modeling; GPM). In each patient volumetric measurements were performed twice by two independent readers ( 25 respectively more than 5000 volumetric measurements performed before study was started) using either the stack of short axis approach analyzed with the SoS method or the highly accelerated multi-orientation protocol combined with the GPM technique. For both sequences and post-processing techniques an intra- and interindividual comparison was performed by applying the Bland-Altman approach.

Results: Mean acquisition and post-processing time was significantly shorter with the GPM-approach (I5 seconds/ 3 minutes versus 360 seconds/6 minutes) when compared to the SoS-method.

End-Diastolic Volume: Due to an improved definition of the mitral valve plane using the long axis views volumes calculated by the highly accelerated protocol combined with the GPM approach were higher when compared to the stack of short axis views analyzed with the SoS method (mean difference: reader I, $15.7 \pm 11.0 \mathrm{ml}$; reader $2,18.6 \pm 10.7 \mathrm{ml}$ on average). Moreover, the statistical spread of the mean difference of both readers' EDV was less when measurements were performed with the GPM approach (mean difference $5.7 \pm 4.6 \mathrm{ml}$ ) compared to the SoS technique (mean difference $8.6 \pm 5.9 \mathrm{ml}$ ) (Table I).

End-Systolic Volume: As with the EDV mean ESV values were higher with the highly accelerated multi-orientation protocol post-processed with the GPM approach for both readers (reader I, I $0.5 \pm 10.2 \mathrm{ml}$; reader 2, $8.8 \pm 9.7 \mathrm{ml}$ ) and showed less statistical spread in comparison to the stack of short axis views analyzed with the SoS technique (mean difference $6.3 \pm 5.0 \mathrm{ml}$ versus $4.3 \pm 3.7 \mathrm{ml}$ ).

Ejection Fraction: EF determined by reader $I$ and 2 demonstrated less statistical spread if estimation was performed with the GPM approach (mean difference I.5 \pm I.I\%) compared with the SoS method based on the standard stack of short axis protocols (mean difference $3.6 \pm 1.9 \%$ ). Additionally, the agreement between the EF calculated by the SoS technique and the 
GPM method was excellent for reader 2 (more than 5000 volumetric measurements performed before study was started; mean difference I $.0 \pm 0.9 \%)$ and good for reader I ( 25 volumetric measurements done before; mean difference $3.6 \pm 2.2 \%$ ) (Figure I).

\section{PI05}

Effective saturation pulse for the whole heart at $3 \mathrm{~T}$ KellyAnne McGorty and Daniel Kim

New York University Langone Medical Center,

New York, NY, USA

Journal of Cardiovascular Magnetic Resonance 2009, I I(SuppI I):PI 05

Introduction: Cardiac MRI at $3 \mathrm{~T}$ is a promising modality to increase the contrast-to-noise ratio (CNR) in first-pass cardiac perfusion imaging. This CNR boost can improve the accuracy of perfusion estimation from dynamic contrast-enhanced images. However, radio-frequency $(R F)$ field $\left(B_{I}\right)$ variations and dielectric effects are comparatively higher at $3 \mathrm{~T}$ than at I.5 T. These challenging factors make it difficult to perform accurate $T_{1}$ weighting using a conventional rectangular saturation pulse. Previous studies have shown improved saturation of magnetization at $3 \mathrm{~T}$ using adiabatic $\mathrm{B}_{1}$-insensitive rotation (BIR-4) [I], rectangular RF pulse train [I], and specific absorption rate (SAR) constrained rectangular RF pulse train [2]. Unfortunately, these pulses did not achieve effective saturation of magnetization, which we shall define as residual magnetization after saturation $<5 \%$ of equilibrium magnetization $\left(M_{0}\right)$, within the whole heart, while remaining within clinically acceptable SAR limits. The purpose of this study was to develop an adiabatic-rectangular pulse train at $3 \mathrm{~T}$ that can achieve both of the aforementioned objectives.

Methods: Pulse sequence: Figure I shows pulse sequence diagrams of a conventional rectangular pulse and an adiabaticrectangular pulse train. This pulse train consists of a rectangular $130^{\circ}$ pulse with $0.8 \mathrm{~ms}$ duration, a rectangular $85^{\circ}$ pulse with $0.5 \mathrm{~ms}$ duration, and an adiabatic half-passage pulse with $1.5 \mathrm{~ms}$

Figure I (abstract PI05)

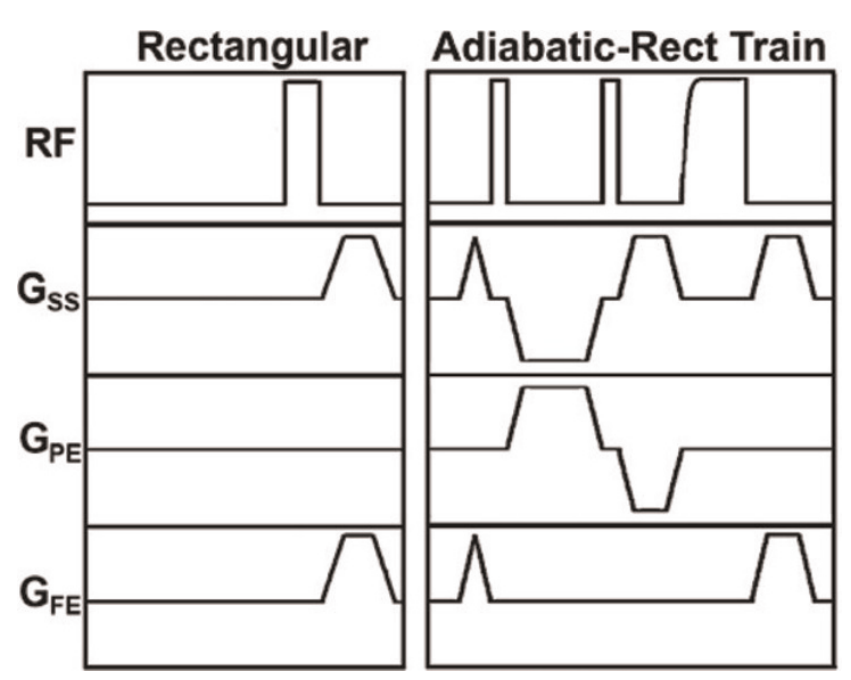

Pulse sequence diagrams of rectangular pulse and adiabatic-rectangular pulse train.
Figure 2 (abstract P 105)

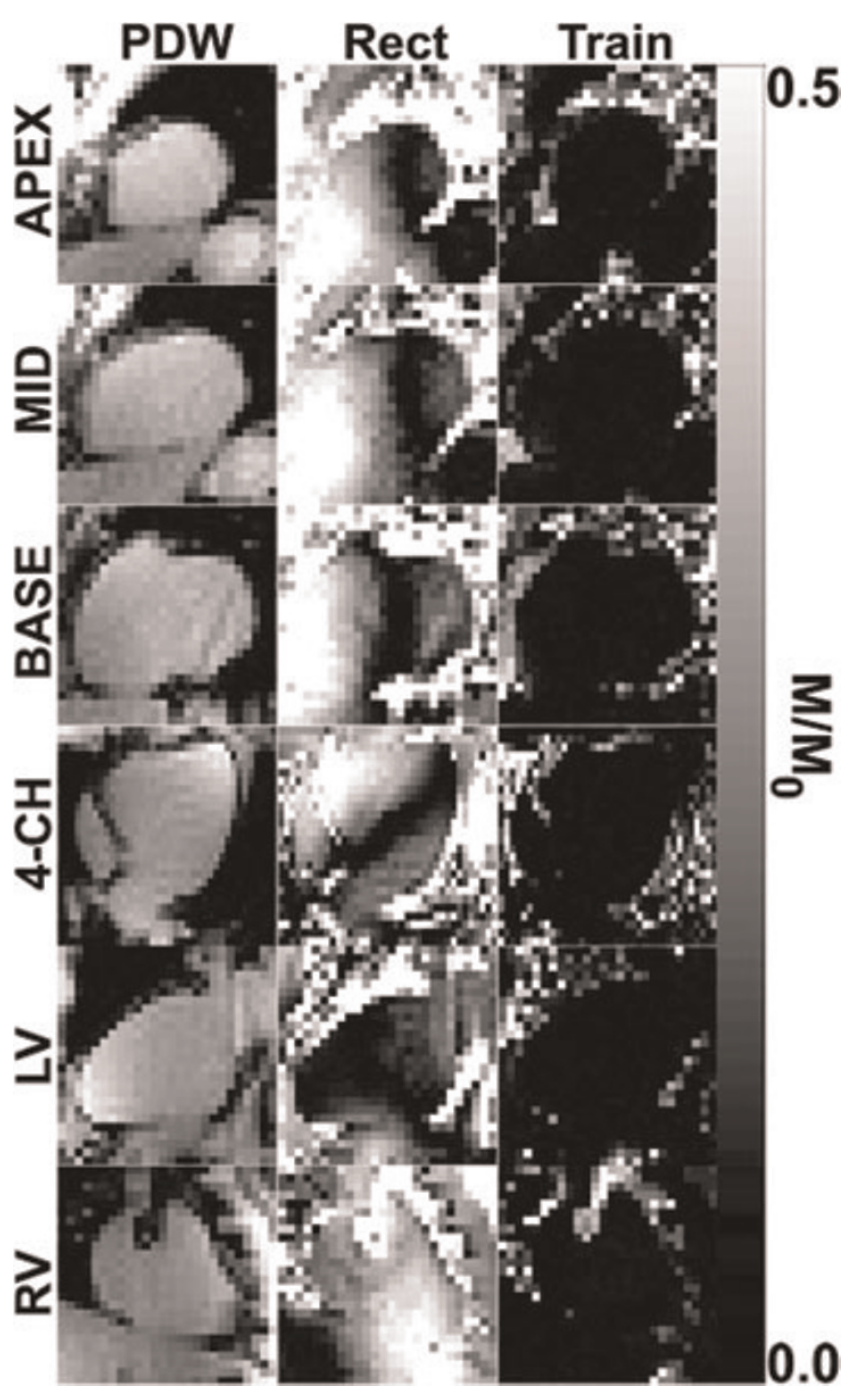

Representative normalized saturation images in six different views of the heart: PDW (left), rectangular pulse (middle), and adiabatic-rectangular pulse (right).

duration. Crusher gradients were played between successive RF pulses to suppress stimulated echoes. The total pulse durations, excluding the final spoiler gradients, for the rectangular and adiabatic-rectangular pulse train were I ms and $8.8 \mathrm{~ms}$, respectively. These two saturation pulses were implemented on a 3 Twhole-body MR scanner (Tim-Trio; Siemens) equipped with an 12-channel phased array RF coil.

The residual longitudinal magnetization left behind by the saturation pulse can be measured by performing a saturationno-recovery experiment using a TurboFLASH pulse sequence with centric k-space reordering, as previously described [I]. Normalization of the saturation image by the proton density weighted (PDW) image corrects for surface coil inhomogeneities and $M_{0}$. Relevant imaging parameters include: field of view = $320-400 \times 255-324 \mathrm{~mm}$, acquisition matrix $=64 \times 52$, slice thickness $=8 \mathrm{~mm}, \mathrm{TE} / \mathrm{TR}=1.1 / 2.3 \mathrm{~ms}, \mathrm{TD}=3 \mathrm{~ms}$, image 
acquisition time $=78 \mathrm{~ms}$, flip angle $=10^{\circ}$, parallel imaging acceleration factor $=1.5$, and bandwidth $=1002 \mathrm{~Hz} /$ pixel. PDW image was acquired using $3^{\circ}$ flip angle and without the saturation pulse. The RF and receiver scales were kept constant between the different acquisitions per subject.

Cardiac imaging: Nine volunteers ( 5 males; 4 females) were imaged in 3 short-axis (apical, mid-ventricular, basal) views of the left ventricle (LV), 4-chamber view, and 2-chamber view of each ventricle.

Image analysis: The LV and right ventricle (RV) were segmented manually. For each subject, the mean normalized signal within the whole heart was computed by averaging the normalized intensities from all five regions of interests (i.e. 3 short-axis and 2 long-axis views). Reported data represent the mean and standard deviation over subjects $(n=9)$. Student $t$-test was performed to compare the residual magnetization values between the two RF pulses.

Results: The energy of the adiabatic-rectangular pulse was 14.2 times larger than that of the rectangular pulse. Figure 2 shows representative saturation images that demonstrate their considerably different efficacies. Statistically, the adiabatic-rectangular pulse reduced the residual magnetization by $92 \%$ in the RV ( $0.03 \pm 0.0$ I vs. $0.38 \pm 0.08 ; p<0.00$ I, respectively) and $84 \%$ in the LV $(0.02 \pm 0.01$ vs. $0.13 \pm 0.04 ; \mathrm{P}<0.00 \mathrm{I}$, respectively $)$ compared with the rectangular pulse.

Discussion: This study demonstrates that the new adiabaticrectangular pulse train can effectively saturate the magnetization within the whole heart at $3 \mathrm{~T}$, while remaining within clinically acceptable SAR limits. This saturation pulse can be used to acquire multiple slices $(>5)$ with our typical clinical first-pass perfusion protocols. Effective saturation of magnetization is likely to produce more accurate estimation of cardiac perfusion.

References

I. Kim D, et al: Magn Reson Med 2008, 59:209-2I5.

2. Sung K and Nayak KS: ISM RM 2008, Prog. No. 228.

\section{PI06}

Cine-EPI can be used to detect coronary artery stenoses in canines

Jordin D Green', Matthias Voehringer²,

Jacqueline A Flewitt ${ }^{2}$, Sven Zuehlsdorff ${ }^{3}$, John $\vee$ Tyberg $^{2}$ and Matthias G Friedrich ${ }^{2}$

${ }^{I}$ Siemens Healthcare, Calgary, AB, Canada

${ }^{2}$ University of Calgary, Calgary, $A B$, Canada

${ }^{3}$ Siemens Medical Solutions USA, Inc., Chicago, IL, USA

Journal of Cardiovascular Magnetic Resonance 2009, I I(SuppI I):PI 06

Introduction: Non-invasive assessment of myocardial ischemia is challenging. Because the BOLD (Blood Oxygen Level Dependent) effect mainly relies on endogenous contrast to differentiate ischemic from non-ischemic tissue, BOLD has the potential to directly assess myocardial oxygenation.

Though $\mathrm{T}_{2}$-weighting is easily achieved using triggered, middiastolic echo planar imaging (EPI), it can be sensitive to artifacts. However, by using a cine-EPI approach, it may be possible to use a lower effective $T E$ ( $T E_{\text {eff }}$, thereby reducing artifacts but maintaining BOLD sensitivity by averaging several mid-diastolic phases during analysis.
Figure I (abstract PI06)

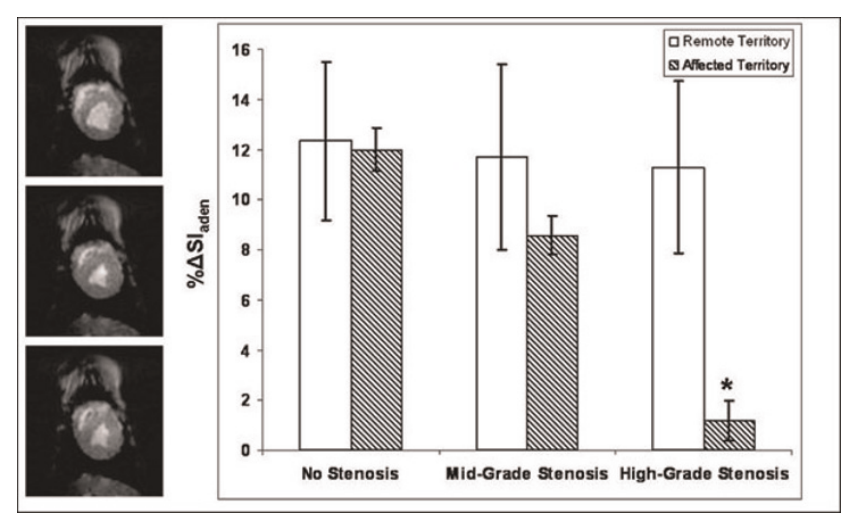

Left: Typical stress images from one study during the high-grade stenosis, taken at various points in the cardiac cycle using cine-EPI. Right: Plot of \% $\Delta \mathrm{SI}_{\text {aden }}$ (\%SI change from rest during adenosine infusion) for the affected territory and the remote territory for the three levels of coronary artery stenosis. Error bars represent \pm I SE. An * indicates statistically significant difference $(P<0.05)$.

Purpose: To demonstrate that a cine-EPI sequence can be used to detect adenosine-induced oxygenation changes in a stenosis dog model.

Methods: We developed a cine EPI sequence with the goal of obtaining strong BOLD-weighted imaging but without significant image artifacts. Cine phases were acquired using prospective ECGtriggering, a breath hold $(\sim 12 \mathrm{~s})$, a short echo train (4 echoes) with a segmented approach ( 8 lines/segment), and a bipolar readout. All studies were performed on a 1.5 T MAGNETOM Avanto (Siemens Healthcare, Germany) in canines $(n=4)$ with a balloon catheter fluoroscopically guided into the left circumflex or left anterior descending coronary artery, to create stenoses as validated by simultaneous fractional flow reserve.

We obtained cine-EPI images in a mid-ventricular slice, before and during an adenosine infusion of $140 \mu \mathrm{g} / \mathrm{kg}$ for two minutes. We repeated this process twice, once with a mid-grade stenosis and once with a high-grade stenosis. At the end of the study, we injected gadolinium (Gd) via our intracoronary catheter and ran a perfusion scan to verify the territory affected by the stenosed artery. We then injected Gd intravenously and performed late enhancement (LE) to verify the absence of infarct. Sequence parameters for cine-EPI: FOV $=300 \times 300 \mathrm{~mm}^{2}$; matrix $=123$ $\times 128$; thickness $=10 \mathrm{~mm}$; temporal resolution $=49 \mathrm{~ms}$; flip angle $=15^{\circ} ; \mathrm{TE}_{\text {eff }}=15 \mathrm{~ms}$.

We analyzed the data using a clinically validated software package $\left(\mathrm{cmr}^{42}\right.$, Circle Cardiovascular Imaging Inc., Canada). We used the intracoronary perfusion images to identify the affected and remote myocardium. By averaging 4 phases corresponding to mid-diastole, we measured mean signal intensity (meanSI) in the affected and remote territory during no stenosis and the two stenosis levels, when the subject was at rest and during adenosine infusion. For each territory, we calculated the adenosine response as a percent change in image meanSI going from rest to stress $\left(\% \Delta \mathrm{SI}_{\text {aden }}\right)$ for each of the three stenosis levels (no stenosis, mid-grade stenosis, high-grade stenosis). We then compared $\% \Delta \mathrm{SI}_{\text {aden }}$ in a particular territory for the different stenosis levels using a matched pairs t-test $(\alpha=0.05)$. 
Results: The results are summarized in the Figure I. In the remote territory, mean $\% \Delta \mathrm{SI}_{\text {aden }}$ at no, mid-grade, and high-grade stenosis ( \pm standard error) was $12.3 \% \pm 3 \%, 11.7 \% \pm 4 \%$, and $11.3 \% \pm 3 \%$ respectively. In the affected territory, the same measurements were $12.0 \% \pm 1 \%, 8.6 \% \pm 1 \%$, and $1.2 \% \pm 1 \%$. Looking at each stenosis level, there was only a statistically significant difference between the two territories for the high-grade stenosis. Looking at each territory, there was no statistically significant difference between the $\% \Delta \mathrm{SI}_{\text {aden }}$ observed for the different stenosis levels in the remote territory. For the affected myocardial territory, there was a statistically significant decrease in $\% \Delta \mathrm{Sl}_{\text {aden }}$ going from baseline to either stenosis level, as well as going from mid- to high-grade stenosis. No LE was observed.

Conclusion: We have shown that cine-EPI can accurately detect changes in adenosine response in myocardium affected by a high-grade stenosis. We could use a lower $\mathrm{TE}_{\text {eff }}$ than previously reported for EPI cardiac BOLD because we were able to signal average over several cardiac phases, which in turn reduced image artifacts. Cine-EPI shows promise for identifying regions of ischemia in CMR, simultaneous to functional assessment.

\section{PIO7}

Myocardial fat quantification using 2D Dixon MRI: feasibility study

Chia-Ying Liu', Alban Redheuil', Ronald Ouwerkerk', Joao Lima and David Bluemke ${ }^{2}$

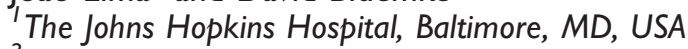

${ }^{2}$ National Institutes of Health, Bethesda, MD, USA

Journal of Cardiovascular Magnetic Resonance 2009, I I(SuppI I):PI 07

Introduction: The concept of fat contained within the myocardium, has recently received attention because of its potential role in diabetic myocardial disease, obesity, and human immunodeficiency virus (HIV) infected individuals [I]. Measurements of myocardial triglycerides in humans have been accessed using proton MR spectroscopy (IH MRS) [2]. IH MRS provides a precise and reproducible tool for in-vivo quantification of intracellular triglycerides within the sarcolemma. However, the spatial distribution of the fat deposition cannot be accessed by IH MRS due to its single voxel characteristics. We studied whether the dual-echo Dixon MRI could quantify the fatty content of the myocardium. The fraction of fat was also quantified directly with IH MRS as an independent method.

Methods: All MRI/MRS studies were performed using a $3.0 \mathrm{~T}$ MR scanner (TrioTim Imager, Siemens) on seven healthy individuals. Myocardial IH MRS was obtained with a 6-ml voxel positioned in the interventricular septum. Four chamber and

Figure I (abstract PI07)

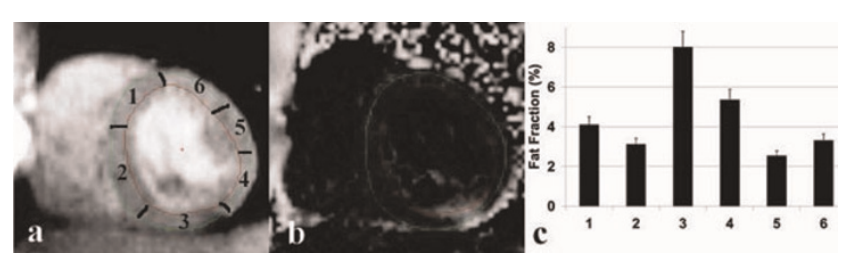

(a) Decomposed water and (b) Fat fraction images (c) Fat fraction from six segments (contoured on the water image). Fat deposition was observed on the inferior lateral wall (segment 3 ).
Figure 2 (abstract P I07)

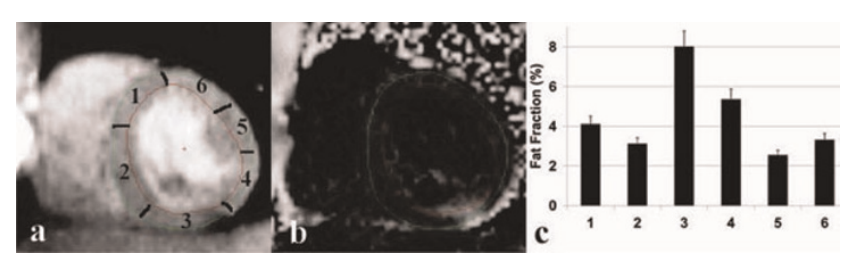

The fat fraction comparisons shown on the MRI is greater than those of MRS, likey due to noise bias contributing to the fat fraction calculation.

short-axis images were acquired by using a breath-hold dualecho spoiled gradient-recalled echo sequence with TR/TE (In, Out) $=6.3 / 2.46,3.69 \mathrm{~ms}$, flip angle $=15^{\circ}$ in late diastole. Fat $(F)$ and water $(\mathrm{W})$ images were reconstructed using Matlab. The short-axis water image was used as a reference to contour the epi- and endo-myocardial borders using Mass, and epi-cardial fat was carefully excluded. Contours were transposed to the fat fraction images (defined as $\mathrm{F} /(\mathrm{F}+\mathrm{W})$ [3]) to calculate the fat fraction of different sectors of the myocardium. MRS was performed with water suppressed ECG gated PRESS, TR/TE = I R-R/30 ms, with navigator across the liver-lung interface to reduce of breathing effects. Fat content was quantified with Amares/MRUI and related to water in unsuppressed spectra.

Results: Figure I(a) and I(b) demonstrate decomposed water and fat fraction images from a healthy participant. The fat fractions from six sectors of the myocardium are shown in Figure I(c). Note the $8 \%$ fat deposition on the inferior lateral wall (segment 3 ). Figure 2 shows the fat fraction based on the in- and out-phase images from the septum (average of section I and section 2) of the five volunteers and the comparison to those of IH MRS.

Discussion: The dual echo technique showed consistently higher estimates of fat fraction compared to the IH MRS technique. To accurately quantify the fat fraction, two major issues should be addressed: the effect of tissue relaxation ( $T$, bias) and image noise.

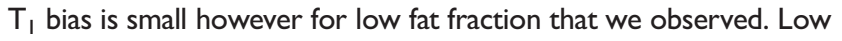
signal intensity/image noise may become the dominant source of bias since the magnitude image was reconstructed. Future work will focus on the noise reduction.

References

I. Szczepaniak LS, et al: Circ Res 2007, I 0 I:759-767.

2. Meer van der RW, et al: Diabetes 2007, 56:2849-2853.

3. Liu CY, et al: MRM 2007, 58:354-364.

\section{PI08}

Validation of left ventricular wall thickening in short-axis cine magnetic resonance imaging by correction of basal-descent through-plane movement

Jyh-Wen Chai', Wei-Hsun Chen', Chih-Ming Chiang' and Jachih $\mathrm{Fu}^{2}$

${ }^{I}$ Taichung Veterans General Hospital, Taichung, Taiwan

${ }^{2}$ Department of Industrial Engineering and Management, National Yunlin University of Science and Technology, Yunlin, Taiwan

Journal of Cardiovascular Magnetic Resonance 2009, I I(SuppI I):PI08

Introduction: The problem of the basal descent through-plane movement in quantitative measurement of regional left ventricular 
(LV) function has been recognized for a long time. But, it is a lack of sophisticated analysis to overcome the limitation.

Purpose: In this study, we tried to solve the limitation of the through-plane motion in quantification of the regional LV myocardial wall thickness and wall thickening (WT) in the short-axis cine MR imaging.

Methods: A series of the tagged cine MRI in the long-axis view of left ventricle was acquired to quantize the longitudinal translation of regional myocardium. Based on the quantified longitudinal translation of LV myocardium, a new data set of the LV end-systolic thickness (EST) was reconstructed from the original EST measured in multislice short-axis cine MRI by using a cubic spline interpolating algorithm. The wall thickening (WT) of left ventricle in young volunteers was calculated from the data of the reconstructed EST and the data without correction of the basal descent movement. The results were analyzed and compared to validate the effectiveness of the proposed method in quantification of LV WT.

Results: The linear correlation between the LVEF and the global LV WT raised from 0.57 before correction to 0.82 after correction of the basal descent through-plane movement. The variability in multislice WT of six segments after correction was significantly smaller than that before correction $(0.50 \pm 0.05$ vs. $0.64 \pm 0.09, p<0.001)$. The multislice WT of six segments in the basal sections after correction was significantly different from before correction $(p<0.05)$. The mean regional wall thickening of six radial segments in basal, mid-cavity and apical sections of $L V$ were $42.3 \pm 16.8 \%, 53.3 \pm 9.9 \%$ and $48.8 \pm 30.1 \%$ before correction, and $49.4 \pm 12.1 \%, 50.5 \pm 12.1 \%$ and $53.5 \pm 21.3 \%$ after correction. The WT in the basal section was much less than that in the mid-cavity section before correction, but was mildly increased and much approximate to that in the mid-cavity section after correction. The results might implicitly imply that the contribution of the myocardium in the basal section to LV function would be as important as that in the mid-cavity.

Discussion: The proposed technique may be potentially useful in quantification of regional WT of the same level of LV myocardium by correction of the basal descent through-plane movement in SA cine MRI.

\section{PI09}

\section{A novel prototype-based segmentation requiring only five training cases applied to $M R$ angiography}

Jane Sjögren ', Martin Ugander ${ }^{1,2}$, Håkan Arheden ${ }^{1,2}$ and Einar Heiberg ${ }^{1,2}$

${ }^{I}$ Cardiac MR Group, Dept. of Clinical Physiology, Lund, Sweden

${ }^{2}$ Lund University Hospital, Lund, Sweden

Journal of Cardiovascular Magnetic Resonance 2009, I I(SuppI I):PI09

Introduction: Image segmentation is an important pre-processing step and a prerequisite for visualization and quantification in medical imaging. In order to obtain accurate segmentation in MRI a priori information often needs to be used. Existing methods often construct a statistical model of the object to be segmented and this typically requires 50-100 manually segmented cases.
Figure I (abstract PI09)
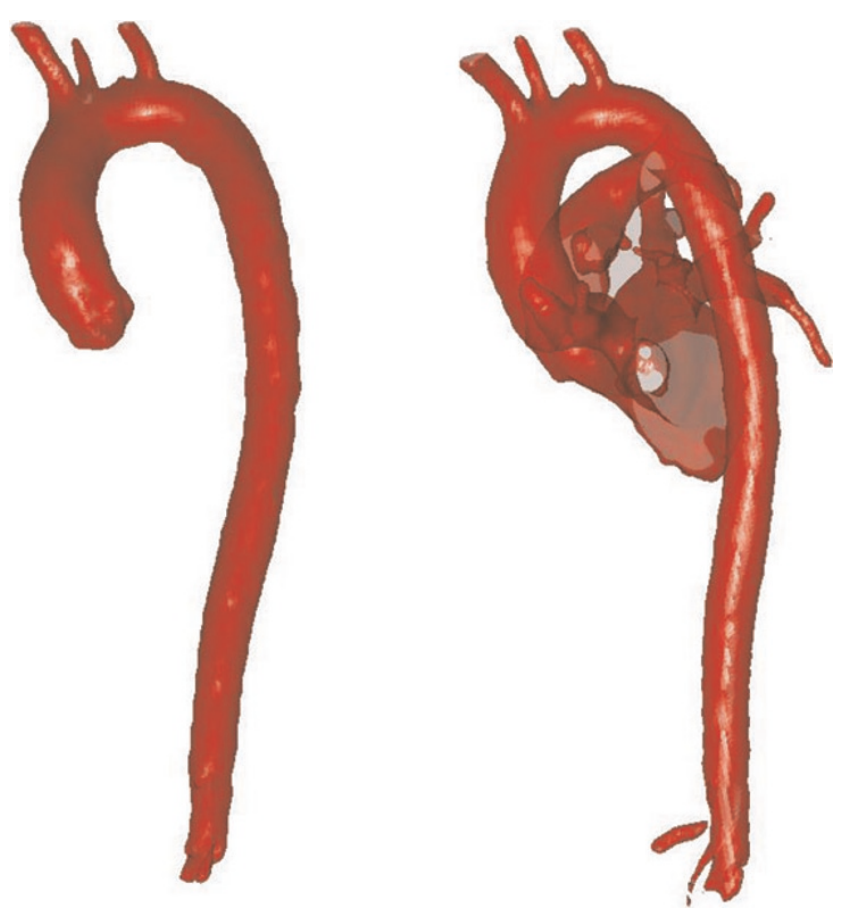

Purpose: To develop and assess the accuracy of a novel prototype-based segmentation method where few cases, typically five, are needed to introduce the required a priori information. Methods: Ten healthy volunteers underwent contrast enhanced MR imaging of the aorta at I.5 T (Philips). Imaging employed a steady state free precession sequence, resolution $1.6 \times 1.6 \times 1 \mathrm{~mm}$. The main idea behind the novel prototypebased segmentation algorithm is to use spatial a priori information to restrict the segmentation rather than to govern what to include. A priori information was extracted from five of the cases (training set) and stored into a prototype. Manual delineation of the aorta was performed in all ten cases and four anatomic landmarks were defined in each set of images. A standard level set segmentation was applied to the training set. The level set segmentation was compared to the manual delineation for each of the cases in the training set. The training set images were aligned to each other by the use of the four landmarks. The spatial information on where to restrict the segmentation, called a correction map, was calculated as the mean of the difference between the level set segmentations and the manual delineations for the training set. The correction map and intensity information from the training set were stored into a prototype. The remaining five cases were used as test set and the segmentation was done by the use of the prototype and a level set method. In the test set the segmentation error was calculated as both a volumetric error and a mean absolute distance between the manual delineation and the prototypebased segmentation.

Results: The segmentation error in the test set was (mean \pm SD) $4 \pm 5 \%$ when measured as volumetric error and $0.58 \pm 0.06 \mathrm{~mm}$ when measured as the error in mean absolute distance. Figure I shows 3D surface rendering of the results of 
the prototype-based segmentation (left) compared to the results of a purely intensity based level set segmentation (right).

Conclusion: The proposed segmentation algorithm is highly accurate, in particular considering the segmentation error expressed as mean absolute distance, which is on a sub pixel level. In the left panel of the figure it can clearly be seen that the prototype-based segmentation successfully constrains the segmentation to only include the aorta which can be compared to the purely intensity based segmentation in the right panel which includes part of the blood volume in the heart and in the pulmonary vessels. The greatest advantage with the prototypebased segmentation method is that only a small number of cases are needed to extract the necessary a priori information. Since only about five cases need to be manually segmented, a new prototype can easily be constructed when new MR pulse sequences are developed or new research fields arise. Future work includes applying the prototype-based segmentation to renal and carotid arteries.

\section{PIIO}

Right ventricular function and structure: results from the Multi-Ethnic Study of Atherosclerosis

Harjit Chahal', Craig Johnson ${ }^{2}$, Harikrishna Tandri', Aditya Jain ', Gregory Hundley ${ }^{3}$, Graham Barr ${ }^{4}$, Steven Kawut ${ }^{4}$, João Lima' and David Bluemke Johns Hopkins University, Baltimore, MD, USA

University of Washington, Seattle, WA, USA

${ }^{3}$ Wake Forest University Health Sciences,

Winston-Salem, NC, USA

${ }^{4}$ Columbia University, New York, NY, USA

Journal of Cardiovascular Magnetic Resonance 2009, I I(SuppI I):PI I0

Introduction: Recent studies of right ventricle (RV) structure and function have recognized the critical role of the RV in maintaining cardiac function. Previous studies on the RV have been limited by sample size and inadequate inclusion of participants from different races. The Multi-Ethnic Study of Atherosclerosis (MESA) is a large ongoing multi-center study that aims to investigate the subclinical progression of cardiovascular disease in an ethnically diverse clinically asymptomatic population.
Purpose: Traditional cardiovascular risk factors have been extensively studied in relationship to the left ventricle (LV). MRI has not been previously used to determine if the RV is independent of these traditional risk factors. The purpose of this study was to assess the relationships of traditional cardiovascular risk factors to RV morphology and function and their relationship to the LV.

Methods: Cardiac MRIs were performed on 5,004 participants without clinical cardiovascular disease at six field centers. 1572 randomly selected participants had complete interpretation of RV measures ( $46 \%$ men, mean age $61 \pm 10$ years). Endocardial margins of the RV were manually contoured on diastolic and systolic images. End-diastolic volume (EDV) and end-systolic volume (ESV) were calculated by using a summation of disks method ("Simpson's Rule"). Baseline variables included demographics and cardiac risk factors. Multivariate linear regression was performed for RV parameters versus traditional cardiovascular risk factors after adjusting for socio-demographic parameters. To evaluate intra-reader and inter-reader variability, $10 \%$ of the scans were selected randomly as re-reads (5\% intra-reader and $5 \%$ inter-reader). Intra-class correlation coefficients (ICC) and Pearson coefficients were used to describe the reproducibility in RV measures.

Results: All RV parameters (mass, diastolic and systolic volumes) showed a negative association with age $(p<0.000 \mathrm{I})$. After adjusting for body size, men had significantly higher RV mass and volumes than women. LV mass and volumes were positively associated with systolic blood pressure, BMI, smoking and diabetes and inversely to LDL cholesterol. Of these, BMI, age and gender were associated with RV mass ( $1.5 \mathrm{~g}$ per $5 \mathrm{~kg} / \mathrm{m}^{2} \mathrm{p}<0.000 \mathrm{I} ;-1.0 \mathrm{~g} / 10 \mathrm{yrs} \mathrm{p}<0.000 \mathrm{I}$ and $2.3 \mathrm{~g}$, $\mathrm{P}<0.000 \mathrm{I}$, respectively). Systolic blood pressure was positively associated with $\mathrm{RV}$ mass $(+0.3 \mathrm{~g}$ per $21 \mathrm{~mm} \mathrm{Hg}$, $\mathrm{p}<0.05)$ and stroke volume $(+2.0 \mathrm{ml}$ per $2 \mathrm{l} \mathrm{Hg}, \mathrm{p}<0.0 \mathrm{l})$. The ICC and Pearson coefficients for end diastolic and end systolic volume ranged from $0.93-0.96$ and $0.87-0.98$, respectively, and for RV mass $0.87-0.93$ and $0.78-0.87$, respectively. See Figure I.

Conclusion: Age, gender and BMI were related to RV mass and volumes. Other than blood pressure, traditional cardiovascular risk factors (diabetes mellitus, smoking, alcohol consumption, LDL cholesterol) had little relationship to RV mass and volume in the MESA cohort.

Figure I (abstract P I I0)

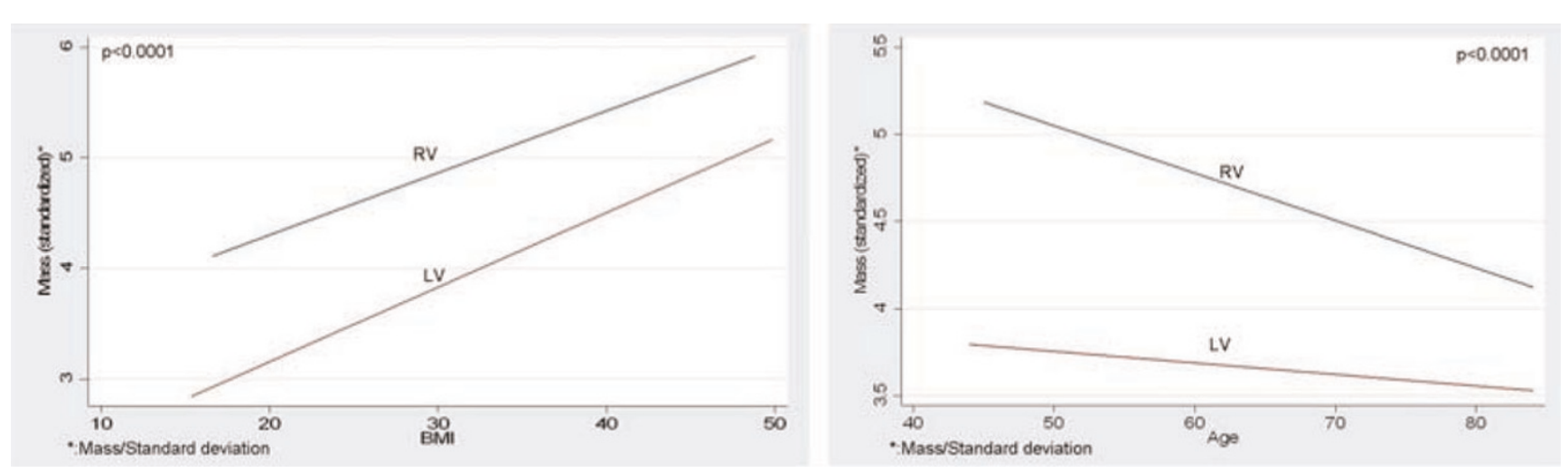


PIII

Comparison between single shot ir-steady state free precession and conventional ir-fast gradient echo sequence for automated quantification of the scar size in acute myocardial infarction in daily practice

Nadine Kirchhartz, Eva Hendrich, Stefan Martinoff, Albert Schömig and Martin Hadamitzky Deutsches Herzzentrum München, Munich, Germany

Journal of Cardiovascular Magnetic Resonance 2009, I I(SuppI I):PI I I

Background: For scar quantification of acute myocardial infarction traditionally an inversion recovery fast gradient echo sequence is used. During the last years a single shot IR steady state free precession sequence was introduced as an alternative. We performed this study to compare the robustness of both sequences on critically ill patients in daily practice.

Methods: 97 consecutive patients with acute myocardial infarction (interval between start of symptoms and intervention below $24 \mathrm{~h}$ ) undergoing cardiac MRI for quantification of the scar size between October 2006 and October 2007 were included into the study. After application of $0.2 \mathrm{mmol} / \mathrm{kg}$ body weight gadopentetate dimeglumine both a standard IR-fast gradient echo sequence and a single shot IR steady state free precession sequence were performed. Image quality was assessed semiquantitatively by two experienced readers using a score ranging from 0 (nondiagnostic) to 5 (excellent). On all images with a score of at least 2 (fair, suitable for automated analysis), the signal intensity of the scar and of a remote region was measured. In addition the scar size was quantified automatically using a cutoff of mean plus 4 standard deviations (sd) of the signal intensity in the remote region.

Results: The image quality score was significantly higher in the single shot sequence $(2.6$ vs. I.9, $\mathrm{p}<0.00 \mathrm{I})$. As a consequence there were significantly more single shot sequences suitable for automated analysis than standard sequences ( 91 vs. 80 studies, $p=0.009$ ), mainly due to less breathing/motion artifacts. Both signal to noise ratio and contrast to noise ratio were nearly identical in both sequences (I4.I vs. 13.8 and 13.0 vs. II.6, respectively, both $\mathrm{p}$ not significant). On automated analysis, both sequences showed a good correlation with a correlation factor of I.02 (indicating a marginal underestimation of the scar size by the single shot sequence) and a correlation coefficient $r$ of $0.8 \mathrm{I}$. Figure I.

Figure I (abstract PIII)

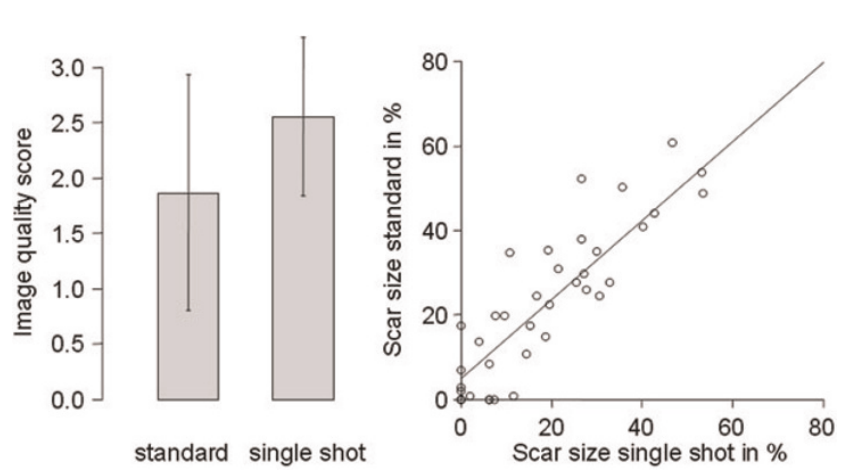

Conclusion: The new single shot IR-steady state free precession sequence is more robust against motion artifact, when compared with the standard IR-fast gradient echo sequence. Both sequences show a similar contrast to noise and signal to noise ratio. They correlate well on direct comparison.

\section{PII2 \\ Cardiac and pancreatic iron overload evaluation of sickle/ $\beta$-Thalassemia patients utilizing T2* MRI Carina Levine ${ }^{3}$, Alain Berebi ${ }^{4}$, Pinhas Stark ${ }^{5}$ \\ and Eliezer Rachmilewitz ${ }^{2}$ \\ ISheba Medical Center, Tel Hashomer, Israel \\ ${ }^{2}$ Edith Wolfson Medical Center, Holon, Israel \\ ${ }^{3} \mathrm{Ha}$ 'Emek Medical Center, Afula, Israel \\ ${ }^{4}$ Kaplan Medical Center, Rehovot, Israel \\ ${ }^{5}$ Rabin Medical Center, Petah Tikva, Israel}

Orly Goitein ', Eli Konen', Husam Ghoti ${ }^{2}$, Ariel Koren ${ }^{3}$,

Journal of Cardiovascular Magnetic Resonance 2009, I I(Suppl I):PI I 2

Introduction: Transfusion-dependent hemolytic anemias in particular thalassemia major and sickle cell disease require multiple transfusions in order to avoid chronic anemia sequelae. This regimen entails intrinsic deleterious effects, the majority of which are related to iron deposition in the reticuloendothelial system. Thus, iron is deposited in myocardial, hepatic and endocrine gland tissues. Both Ferrtin level and liver biopsies have no predictive values for cardiac iron deposition levles.

T2* MRI sequences have been used for reliable evaluation of iron load in the heart, liver and pancreas. Patients with no iron overload have normal cardiac function whereas those with cardiac iron deposition suffer from myocardial dysfunction and arrhythmias.

Purpose: To quantify iron content in the heart, liver and pancreas of sickle/ $\beta$-thalassemia patients.

Methods: Multicenter evaluation of eleven patients with sickle/ $\beta$-thalassemia was performed (3 males; 8 females, mean age 31 years). Mean hemoglobin: $9.0 \mathrm{gr} / \mathrm{dl}$; Serum ferritin: $3900 \mathrm{ng} / \mathrm{ml}$; Transferrin saturation: $80 \%$. All patients were multitranfused with an average of 97 packed cell units. One patient was treated with iron chelation for 10 months. Seven patients received daily Hydoxy-Urea for > 10 years.

MRI (I.5 T, GE MRI system) sequences included: Steady-state free precession (SSFP) for left ventricular ejection fraction (LVEF) evaluation; Breath-hold T2* multi echo gradient for iron load quantification, sampled across regions of interest in the LV septum, liver parenchyma and pancreatic tissue.

Results: All patients had normal cardiac and pancreatic T2* values ( $>20 \mathrm{~ms}$ and $>30 \mathrm{~ms}$, respectively). Normal cardiac function was demonstrated in all patients (LVEF, LV endsystolic and endiastolic volumes).

Seven patients demonstrated evidence of mild to moderate hepatic iron deposition (T2*<6.3 ms). In these patients mean serum ferritin $(5656 \mathrm{ng} / \mathrm{ml})$ and transferrin saturation $(92.4 \%)$ were significantly higher $(p=0.001)$ compared with the 4 patients with normal hepatic T2* levels.

Conclusion: T2* MRI sequences allow non invasive, repeated iron deposition quantification in multitransfused patients. In our cohort of eleven patients with sickle/ $\beta$-thalassemia, no cardiac or pancreatic iron deposition was demonstreted. Mild to moderate 
iron liver deposition was found in 7 of these patients, in which serum ferritin levels and transferrin saturation were significantly higher.

With respect to iron deposition, multitransfused sickle/ $\beta$ thalassemia are similar to patients with homozygous sickle cell disease and not to patients with thalassemia major. The reasons for this observation are still unclear. This similarity could be related in part to the relativly low number of transfusions, starting later in life, in these patients.

The liver is the dominant iron storage organ. Hepatic iron concentration correlates closely with the total body iron content. While iron uptake by hepatocytes is predominately mediated via transferrin and correlates well with serum ferritin levels, as confirmed in the present study, this is not the case in cardiac and endocrine iron uptake regulation. These organs might acquire the excess metal differently.

\section{PII3}

Pioglitazone alters fat distribution in patients with type 2 diabetes mellitus, in contrast to metformin Jacqueline $T$ Jonker', Rutger W van der Meer', Luuk J Rijzewijk ${ }^{2}$, Lisa M Menting', Michaela Diamant ${ }^{2}$, Johannes A Romijn ', Johannes WA Smit', Albert de Roos' and Hildo J Lamb ${ }^{1}$

'Leiden University Medical Centre, Leiden, Netherlands

${ }^{2}$ Diabetes Centre, VU University Medical Centre, Amsterdam, Netherlands

Journal of Cardiovascular Magnetic Resonance 2009, I I(SuppI I):PI I 3

Introduction: Diabetes mellitus is associated with an increased risk on cardiovascular disease and epicardial fat has been proposed as an additional cardiovascular risk factor. Treatment with pioglitazone leads to an improvement in glycemic control, but also increases body weight.

Purpose: The primary aim was to evaluate the effect of pioglitazone on weight and fat distribution in patients with type 2 diabetes mellitus (DM2). The secondary aim was to assess the relationship of epicardial fat to anthropometric measurements and fat distribution in these patients

\section{Figure I (abstract PII3)}

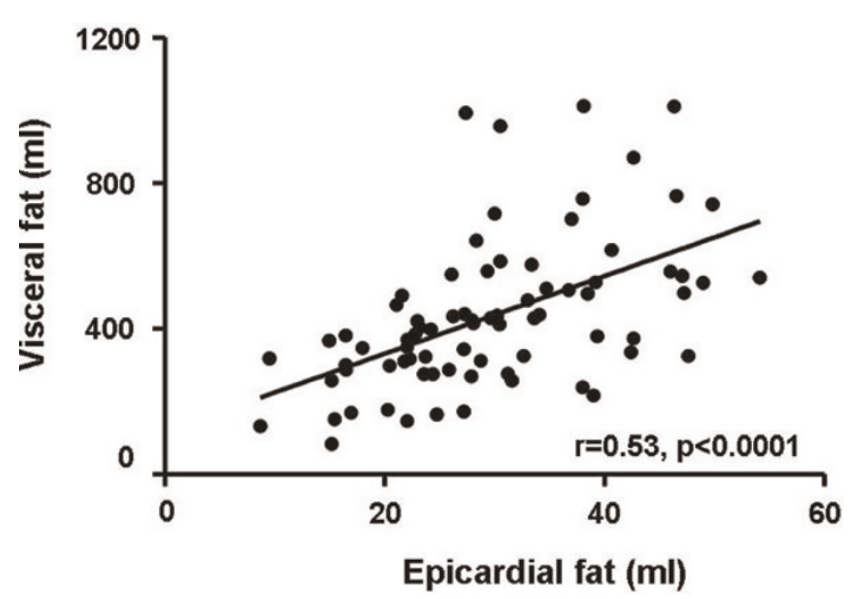

Correlation between epicardial and visceral fat at baseline.
Methods: Seventy-seven male patients with DM2 were included in this study (mean \pm SEM, age $56.5 \pm 0.6 \mathrm{yr}$; HbAlc $7.1 \pm 0.1 \%$ ), without cardiac ischemia. Patients were randomly assigned to pioglitazone $(30 \mathrm{mg} /$ day $)$ or metformin $(2000 \mathrm{mg} /$ day $)$ and matching placebo during 24 weeks. Epicardial fat and abdominal visceral and subcutaneous fat were measured by magnetic resonance imaging. Myocardial and hepatic triglyceride content (TG) were determined by ' $\mathrm{H}$ magnetic resonance spectroscopy.

Results: Epicardial fat correlated with abdominal visceral fat $(r=0.53, p<0.00 I)$, BMI $(r=0.42, p<0.00 I)$, abdominal subcutaneous fat $(r=0.35, P=0.002)$, myocardial TG $(r=0.24, p=0.04)$ and hepatic TG $(r=0.29, p=0.0 I)$. Both treatments improved glycemic control similarly. Patients treated with pioglitazone had an increased body weight $(p<0.00 \mathrm{I})$. In this group, epicardial fat increased by $9 \%(p=0.01)$ and abdominal subcutaneous fat by $18 \%(p<0.00 I)$, whereas metformin did not affect these fat compartments. In contrast, hepatic TG content significantly decreased in the pioglitazone group. Abdominal visceral fat did not change in both groups. See figure I.

Conclusion: In patients with DM2 there is a relation between epicardial fat mass, BMI and visceral fat mass. Pioglitazone increases body weight, epicardial fat and subcutaneous fat mass in patients with DM2 treated with pioglitazone, whereas hepatic triglyceride content decreases.

\section{P I 4}

Right ventricular assessment with cardiac magnetic resonance: usefulness in routine clinical practice compared to echocardiography Covadonga Fernández-Golfín, José Zamorano Gómez, Cecilia Corros Vicente, Tibisay Sánchez, Joaquin Ferreiros, Leopoldo Pérez de Isla, Ana Bustos, Betariz Cabeza and Carlos Macaya Hospital Universitario Clinico San Carlos, Madrid, Spain Journal of Cardiovascular Magnetic Resonance 2009, I I(SuppI I):PI I4

Introduction: Right ventricular (RV) morphology and function is essential in the evaluation of different cardiac diseases. It has prognosis significance and influence everyday clinical decision making. Routine RV assessment is done by means of echocardiography. Visual estimation of RV morphology and function is normally performed, using RV function echocardiographic indexes only in selected populations. This approach may lead to inaccurate RV assessment especially in cases with mild RV dilatation/impairment. Cardiac magnetic resonance (CMR) has emerged as the gold standard for biventricular volume and function quantiphication.

Purpose: The aim of the study was to analyze in a clinical setting, the utility of CMR in the evaluation of RV morphology and function, comparing it to routine echocardiography examinations reports.

Methods: 96 patients with RV volume and function assessed with CRM were initially included. Reports form echocardiography performed to these patients were reviewed. Patients in whom the echocardiography was performed more than one year from the CRM study were excluded, as well as patients in whom specific echocardiography RV information was lacking. Study population consisted of 72 patients, mean age 48 years, men 55 , 
6\%. Main indication for CRM study was as follows: viability assessment $7 \%$ dilated cardyomyopathy II, I\% and right ventricular dysplasia evaluation 7\%. All CMR exams were performed at I,5 T (Signa, General Electrics, Milwaukee, WI) scanner. Breath-hold, electrocardiographically triggered steady state free precession cine images were acquired in four chamber long axis view and short axis view from base to apex. Cine images were analyzed with CMR tools (Cardiac Report Card 2.0, General Electrics). RV end diastolic, end systolic volume and ejection fraction were calculated using Simpson method. Volumes and ejection fraction were then classified as normal/ abnormal in accordance to patient age and sex using published reference values. Based on echocardiography reports RV was considered dilated or not and RV ejection fraction normal or decreased in a binary manner. Statistical analysis was performed using SPSS program, version 12.

Results: 72 patients were included. Mean RVEDV was $133,21 \mathrm{ml}$, RVESV 56,68 $\mathrm{ml}$ and RVEF 58,94. According to CRM II, I\% patients had increased end diastolic RVV with $25 \%$ having increased end systolic RVV. Right ventricular dysfunction was present in $25 \%$. Echocardiography studies described RV dilatation in I5, 5 patients and RV dysfunction in 5, 6\% patients. Agreement index between both methods were 0,33 ( $p$ 0,004) for RV volume and 0,19 (p 0,019) for RV ejection fraction.

Conclusion: Routine echocardiography evaluation of RV in different cardiac diseases compared to gold standard CRM lack accuracy. Thus, in cases where RV assessment is essential other echo Doppler indexes or CRM should be considered in order to provide accurate RV evaluation.

\section{PII5}

Diagnostic quality of steady state free precession imaging of cardiac valve morphology in pediatric/congenital heart disease

Oscar J Benavidez, Ashwin Prakash, Kimberlee Gauvreau, Andrew J Powell and Tal Geva Childrens Hospital Boston/Harvard Medical School, Boston, MA, USA

Journal of Cardiovascular Magnetic Resonance 2009, I I(SuppI I):PI I5

Objective: To evaluate cine Cardiac Magnetic Resonance Steady State Free Precession (CMR-SSFP) imaging quality and diagnostic accuracy in the assessment of cardiac valve morphology.

Background: Evaluation of cardiac valve morphology has not been considered an indication for cardiac MRI due to suboptimal imaging quality; few studies have examined this objectively. MRI techniques utilizing CMR-SSFP may have overcome this limitation. Evaluation of CMR-SSFP diagnostic imaging quality in the assessment of valve morphology has not been performed for pediatric/congenital heart disease. Comparison of CMR-SSFP

Table I (abstract PII5) Diagnostic clarity score

\begin{tabular}{ll}
\hline 1 & No blurring, excellent diagnostic data \\
2 & Mild blurring, very good diagnostic data \\
3 & Moderate blurring, diagnosis possible \\
4 & Severe blurring, diagnosis uncertain \\
5 & Non-diagnostic, diagnosis not possible
\end{tabular}

Table 2 (abstract PI I5) Proportion of valve components with a high-quality diagnostic imaging (Diagnostic clarity scores I or 2)

\begin{tabular}{lllll}
\hline & Annulus & Leaflets & Chordae & $\begin{array}{l}\text { Papillary } \\
\text { muscles }\end{array}$ \\
\hline Tricuspid Valve & $80 \%$ & $64 \%$ & $30 \%$ & $53 \%$ \\
Mitral Valve & $74 \%$ & $69 \%$ & $36 \%$ & $77 \%$ \\
Aortic Valve & $72 \%$ & $80 \%$ & - & - \\
\hline
\end{tabular}

diagnostic accuracy of aortic valve morphology to echocardiography has not been previously performed.

Methods: We retrospectively reviewed 234 consecutive pediatric/congenital cardiac MRI cases. A diagnostic clarity score (Table I) was assigned to the tricuspid, mitral valve annulus, leaflets, chordae and papillary muscles. High-quality diagnostic imaging is defined as a clarity score of I or 2. The clarity score of the mitral (Figure I) and tricuspid valves was assessed by examination of standard cine CMR-SSFP imaging in two-chamber, four-chamber and short-axis views. Clarity score of the aortic valve annulus and leaflets was assessed when long axis imaging of the left ventricular outflow tract and cross-sectional imaging of the aortic root were performed. Among patients with aortic valve imaging, we compared morphologic diagnosis by CMR-SSFP to echocardiography.

Results: Patient age ranged from I month to 65.1 years (average 17.2 years). Table 2 illustrates the proportion of valve components with a high diagnostic clarity scores. There was no difference in weight or body surface area between those with an average mitral or tricuspid clarity score of $<2$ (excellent or very good clarity) vs. $\geq 2$ (fair to poor clarity). Among the 39 cases with aortic valve specific imaging, 27 (69\%) had an echocardio-

\section{Figure I (abstract PII5)}

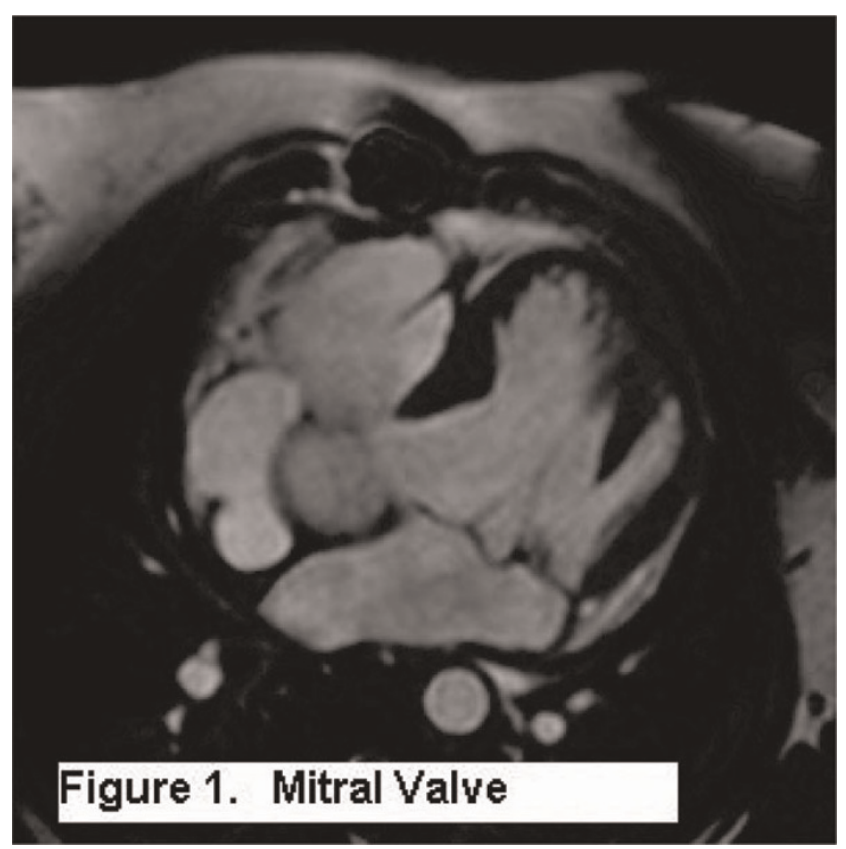

Mitral valve. 
Figure 2 (abstract PII5)

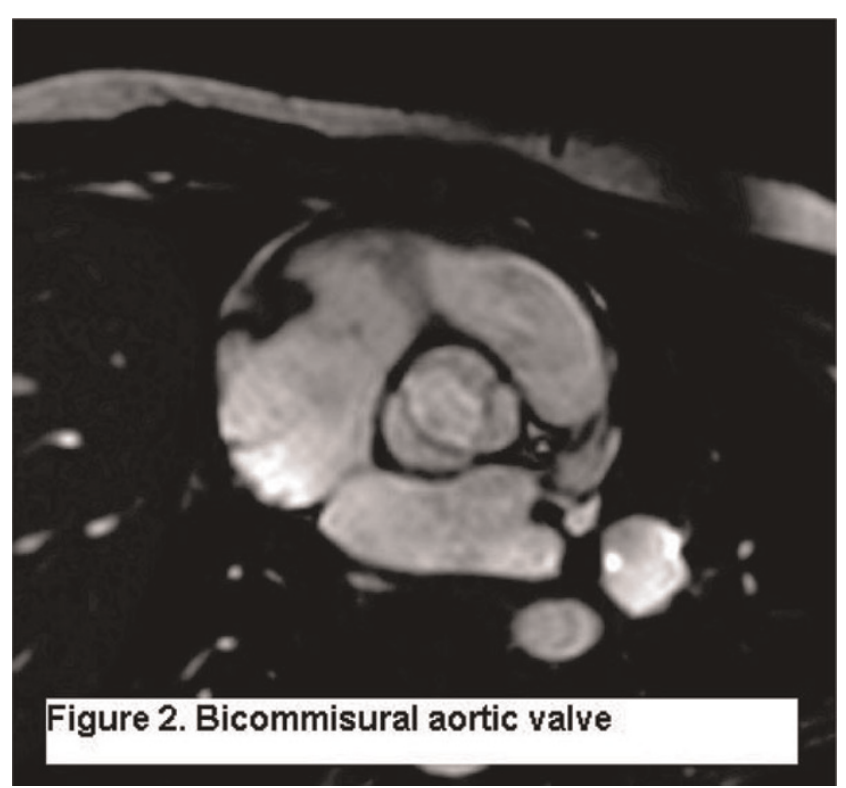

Biocommisural aortic valve.

gram for comparison. CMR-SSFP correctly identified the aortic valve morphology in all 27 cases. CMR-SSFP correctly identified the affected commissure in 13 of 13 bicommissural aortic valves (Figure 2) and correctly identified I4 of 14 tricommissural aortic valves.

Conclusion: In the majority of cases CMR-SSFP produces high diagnostic quality imaging of cardiac valve morphology in congenital/pediatric cardiac MRI. The valve components with the highest diagnostic clarity score are tricuspid and mitral valve annuli, leaflets and papillary muscles and aortic valve annuli and leaflets. CMR-SSFP however produced high clarity images of chordae in only a minority of cases. Aortic valve morphology can be diagnosed with a high degree of reliability.

\section{P I 16}

Differential branch pulmonary artery regurgitant fraction identifies patients with unilateral stenosis associated with relatively elevated pulmonary vascular resistance in the contralateral lung after repair of conotruncal anomalies

Matthew A Harris, Kevin K Whitehead,

Matthew J Gillespie, Timothy I Liu, Michael T Cosulich, Paul M Weinberg and Mark A Fogel

The Children's Hospital of Philadelphia, Philadelphia, PA, USA

Journal of Cardiovascular Magnetic Resonance 2009, I I(SuppI I):PI I6

Introduction: Repaired conotruncal anomaly pts may have residual branch pulmonary artery (BPA) stenosis or significant differences in the sizes of the BPAs. Phase-Contrast Magnetic Resonance (PCMR) can measure differential BPA regurgitation. Purpose: To determine if: (I) residual BPA stenosis or size discrepancy is associated with differential BPA regurgitation (2) differential BPA regurgitation correlates with differential pulmonary vascular resistance (PVR).
Figure I (abstract PI I6)

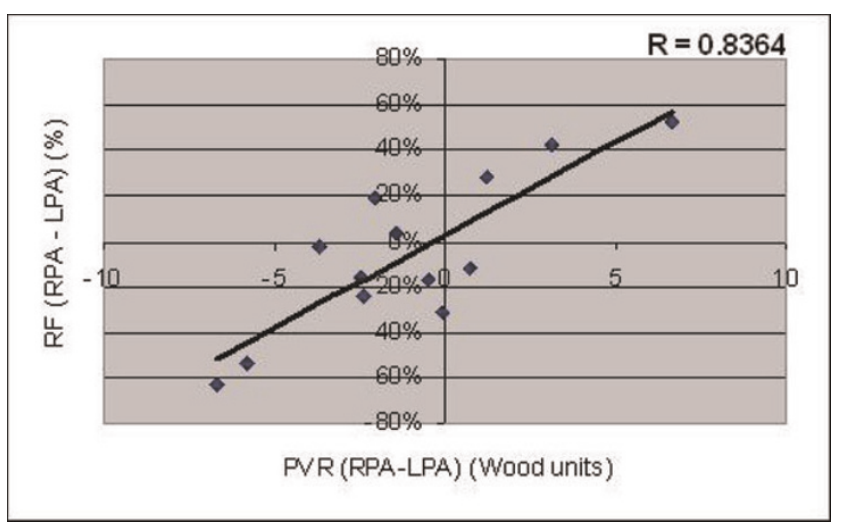

Methods: We retrospectively reviewed 7I consecutive cardiac magnetic resonance (CMR) studies for BPA size and PCMR data. We also reviewed $I 3$ consecutive pts who underwent both CMR and catheterization.

Results: 27 of the 7I pts had either BPA stenosis or one BPA cross-sectional area (CSA) comprise $<33 \%$ of the total BPA CSA. Among these $27 \mathrm{pts}$, there was no significant difference between RPA and LPA regurgitant fraction (RF) (28 vs $32 \%, p=0.49$ ), however, there was a significantly increased RF of the larger vs smaller BPA (39 vs $21 \%, \mathrm{p}<0.00 \mathrm{I})$. In contrast, the $44 \mathrm{pts}$ without BPA stenosis or size discrepancy showed a significant difference between RPA and LPA RF ( 3 I vs $38 \%, p<0.00 I$ ), without a significant difference in the larger vs smaller BPA RF (36 vs $33 \%, p=0.14$ ). Retrospective review of $p$ ts who underwent both CMR and catheterization demonstrates that differential BPA RF strongly correlates with differential PVR $(R=0.8364, p<0.001)$. See Figure I.

Conclusion: BPA stenosis or size discrepancy outweighs the increased LPA RF of pts without stenosis or size discrepancy. Since differential BPA RF correlates with differential PVR, PCMR can serve as an important screening tool for identifying pts with stenosis or size discrepancy who may have developed relatively increased PVR in the contralateral larger pulmonary artery.

\section{PII7}

Rapid automated quantification of left ventricular ejection fraction with LV-METRIC - a novel segmentation algorithm

Noel CF Codella, Matthew D Cham, Richard Wong, Christopher Chu, Kirsten Healy, Martin R Prince, Yi Wang and Jonathan W Weinsaft Weill Medical College of Cornell University, New York, NY, USA

Journal of Cardiovascular Magnetic Resonance 2009, I I(SuppI I):PI I 7

Objective: To evaluate diagnostic performance and time saved by LV-METRIC, a novel automated algorithm for LV quantification, among a broad unselected clinical patient population undergoing CMR.

Background: Cardiac magnetic resonance (CMR) is an imaging standard for quantification of LV ejection fraction (EF) and volume. CMR typically relies on manual tracing (MT). MT can be time consuming and operator-dependent. Automated segmentation holds potential for rapid LV quantification. Established 
Table I (abstract PII7) Comparison of LV-METRIC to manual tracing

\begin{tabular}{|c|c|c|c|c|c|}
\hline & Manual tracing & $\begin{array}{l}\text { LV-METRIC } \\
\text { (PV interpolation) }\end{array}$ & $\Delta$ & $\begin{array}{l}\text { LV-METRIC } \\
\text { (no PV interpolation) }\end{array}$ & $\Delta$ \\
\hline Ejection fraction & $57.1 \pm 17.0$ & $59.1 \pm 17.4$ & $-2.0 \pm 2.3$ & $56.5 \pm 16.7$ & $0.6 \pm 2.3$ \\
\hline End-diastolic volume (ml) & $157.8 \pm 67.9$ & $|32| \pm 60.5$. & $25.7 \pm 10.9$ & $153.8 \pm 65.9$ & $4.0 \pm 6.8$ \\
\hline End-systolic volume (ml) & $75.1 \pm 62.7$ & $60.9 \pm 55$ & $14.3 \pm 9.6$ & $73.7 \pm 60$ & $1.4 \pm 5.5$ \\
\hline Stroke volume (ml) & $82.6 \pm 26.8$ & $71.2 \pm 24.1$ & $11.4 \pm 6.6$ & $80 \pm 26.9$ & $2.6 \pm 5.3$ \\
\hline Processing time (minutes) & $5: 18 \pm 1: 56$ & $0: 22 \pm 0: 13$ & $4: 37 \pm 1: 51$ & & \\
\hline
\end{tabular}

automated algorithms often fail or require extensive user interface, possibly due to the fact that they employ assumptions regarding cavity shape or regional propagation. We developed an automated segmentation algorithm (LV-METRIC) that involves no geometric assumptions and instead quantifies LV EF and volume based on local per-pixel signal intensity while accounting for partial voxel effects. The purpose of the current study was threefold; (I) to evaluate LV-METRIC performance vs. MT among a broad unselected patient population; (2) to compare processing time by LV-METRIC to MT, and (3) to compare LV-METRIC and MT to an independent standard of LV flow quantification.

Methods: LV-METRIC and MT were independently applied for quantification of LV EF and volumes on consecutive patients that underwent CMR (I.5 T) between June-November, 2007. Processing times were recorded. Quantification was based on endocardial border delineation of consecutive short axis SSFP images throughout the LV (slice thickness $6 \mathrm{~mm}$, gap $4 \mathrm{~mm}$, typical spatial resolution $1.9 \times 1.4 \mathrm{~mm}$ ). Through-plane aortic valve phase contrast imaging was performed on a subset $(n=65)$ of patients for independent confirmation of LV stroke volume. No patients were excluded based on clinical characteristics; the only exclusion criteria were cardiac arrhythmias or image artifacts that prohibited MT (6.7\% patients).

Results: The study population was comprised of I5I patients (54 \pm I 4 yo, HTN 40\%, DM 23\%, CAD 34\%). LV-METRIC was successful in all patients; average processing time was 22 seconds for LV-METRIC and 4 minutes 59 seconds for MT ( $p<0.00 \mathrm{I})$. An example segmentation is shown in Figure I, where MT is depicted in Fig. Ia, and LV-METRIC in Fig. Ib. Voxel blood content color labels for LV-METRIC are also illustrated. LV EF based on LV-METRIC was, on average, within 2 percentage points of MT irrespective of whether partial voxel $(-2.0 \pm 2.3 \%)$ or full voxel $(0.6 \pm 2.3 \%)$ computation was used (Table I). LV volumes yielded by LV-METRIC were smaller than MT. Mean end-diastolic and end-systolic volumes in LV-METRIC measurements with partial voxel interpolation were $25.7 \mathrm{~mL}$ and $14.3 \mathrm{~mL}$ smaller

\section{Figure I (abstract PII7)}

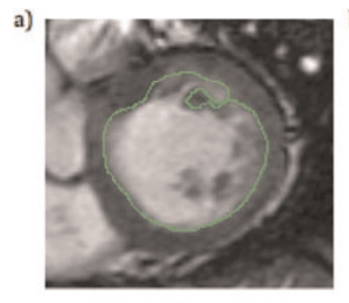

Manual Tracing

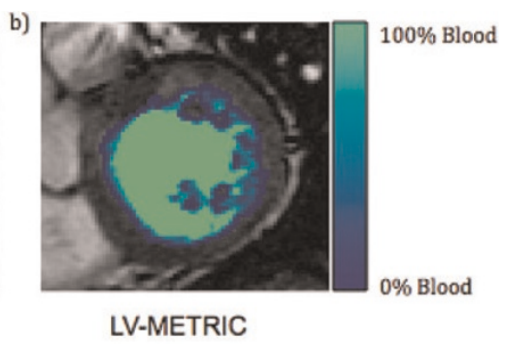

than MT measurements, respectively. Full voxel computation yielded less drastic differences, with diastolic volumes smaller by $4 \mathrm{~mL}$ and systolic volumes larger by $1.4 \mathrm{~mL}$. All EF and volumetric differences between LV-METRIC and MT were significant $(p<0.00 I)$. Correlations for cavity volumes, global stroke volume, and ejection fraction were high (all $\left.R^{2}>.95\right)$. Both LVMETRIC and MT similarly agreed with phase contrast (all differences $\mathrm{P}<0.05)$; Although volumetric stroke volume by MT was, on average, similar to PC $(\Delta 5.9 \mathrm{~mL})$, there was substantial variance between the two techniques (SD \pm I3.4 mL). Similar differences were evident when comparing PC and LV-METRIC with $(6.2 \pm \mathrm{l} 4.2 \mathrm{ml})$ or without $(-3.9 \pm 14.7 \mathrm{ml})$ partial voxel interpolation.

Conclusion: Among a broad population of consecutive patients, LV-METRIC, a novel automated CMR segmentation algorithm, provides highly accurate quantification of LV ejection fraction and volumes with a marked reduction in processing time vs. MT. Both LV-METRIC and MT provide similar agreement with an independent standard of phase contrast imaging.

\section{PII8}

Regional right ventricular wall motion in tetralogy of fallot: a three dimensional analysis

Michael Morcos, Christopher S Kirk and Florence Sheehan University of Washington, Seattle, WA, USA

Journal of Cardiovascular Magnetic Resonance 2009, I I(SuppI I):PI I8

Background: We previously reported an abnormal contraction pattern in the right ventricle (RV) in tetralogy of Fallot (TOF) patients by measuring the regional contribution to global stroke volume (rSV) at 20 cross sections. However the rSV method only measures regions spanning the RV from apex to base. Therefore we tested a second method that allows regions of interest to be defined for RV function.

Purpose: We wanted to develop a method that enables detailed regional RV wall motion analysis that is capable of producing a wide variety of user-specific regional designation.

Methods: We reconstructed the RV in 3D from manually traced borders in 20 repaired TOF patients and 9 normal (NL) subjects from magnetic resonance images. Wall motion was measured using the Centersurface (CS) method as the local orthogonal distance between the RV endocardium at end diastole and end systole in 10 regions on the RV free wall, the septum, and the outflow track. The free wall was divided into apical, middle, and basal levels; each level was further subdivided into inferior, middle, and superior regions. Regional function by both methods was compared between TOF and NL groups.

Results: Both methods showed that TOF patients have reduced function at the base and increased function at the apex compared 
Figure I (abstract PI I 8)

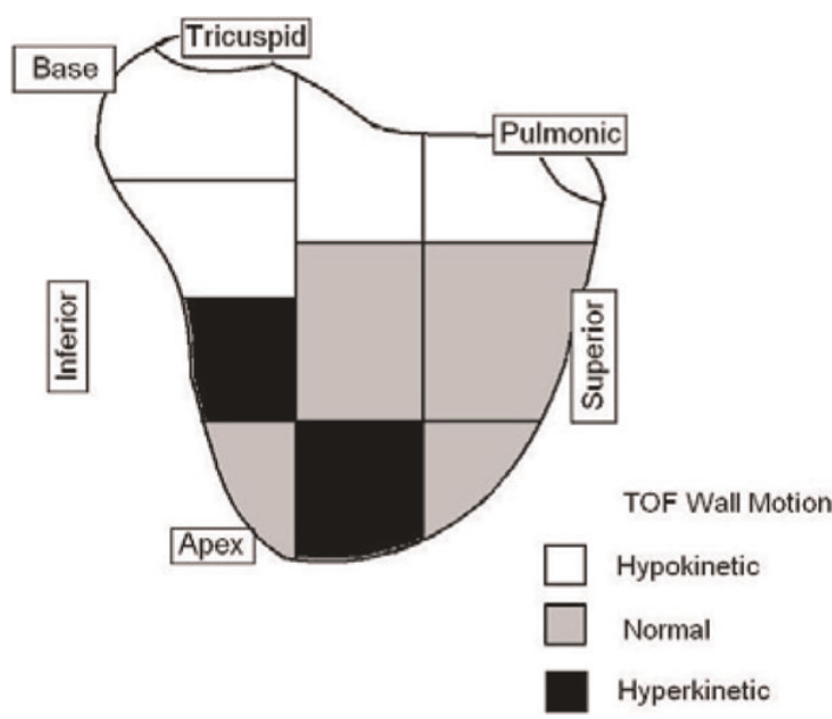

to $\mathrm{NL}$ (Figure I). In both groups motion was lower in superior than in mid or inferior regions $(p<0.00 \mathrm{I}$ for all).

Conclusion: The rSV and CS methods yielded similar results when comparing function in regions spanning the RV from apex to base. Only the CS method enabled comparison of function in the subdivided regions. CS provides a more comprehensive analysis of regional RV wall motion due to its flexibility in defining the region of interest.

\section{PII9}

MRI evaluation of the role of No-Reflow in left ventricular remodeling after Acute Myocardial Infarction (AMI)

Carlo Liguori', Luigi Natale', Agostino Meduri', Antonio Bernardini', Antonella Lombardo'

and Lorenzo Bonomo'

'Università Cattolica del Sacro Cuore - Policlinico A. Gemelli, Rome, Italy

2“G. Mazzini” Hospital - ASL Teramo, Teramo, Italy

Journal of Cardiovascular Magnetic Resonance 2009, I I(SuppI I):PI I 9

Purpose: LV remodeling represents the most important negative prognostic factor after myocardial infarction. Aim of the study is to assess by MRI the role of edema, no-reflow and myocardial viability in the remodeling process.

Materials and methods: 32 pts with $\mathrm{AMI}$ and primary $\mathrm{PCl}(25$ LAD, 3 CX, 4 RCA; 26: TIMI 3, 6: TIMI 2) were studied by MRI to measure end-diastolic (EDV) and end-systolic (ESV) volumes immediately and I-month later. A > 20\% increase of EDV and/or ESV was considered indicative of remodeling. Triple IR-FSE for edema evaluation, steady-state free precession cine (FIESTA) for contractile function, fast-gradient echo train (FGRET) for first-pass perfusion study and IR-prep fast GRE for delayed enhancement assessment were obtained. A score for edema, noreflow and hyperenhancement was calculated in each segment
(I7-segments LV model) based on number of segments and transmural extension (75\%).

Results: 14 pts showed remodeling. EDV and ESV increased from $106 \pm 30 \mathrm{ml}$ to $153 \pm 36 \mathrm{ml}$ and from $60 \pm 17 \mathrm{ml}$ to $91 \pm 23 \mathrm{ml}$ in pts with remodeling. No-reflow was detected in 26 pts $(81 \%)$. The scores for edema, no-reflow and hyperenhancement were $4.0 \pm$ I.6, $2.4 \pm \mathrm{I} . \mathrm{I}, 3.3 \pm \mathrm{I} .6$ respectively in pts with remodeling and $2.9 \pm 2.2$ (p:NS), I.4 \pm 0.9 (p:0.04), $2.6 \pm 1.7$ (p:NS) respectively in pts without remodeling.

Conclusion: First-pass MRI detects an high incidence of noreflow after $\mathrm{PCl}$. Its extension was more significantly related to remodeling if compared to edema and necrotic myocardium. No-reflow assessed by first pass imaging seems to be a stronger predictor of LV remodeling, compared to infarct size, in AMI.

\section{PI20}

Characterization of acute myocardial infarction by magnetic resonance imaging: correlation with enzymatic and angiographic findings

Aloha Meave, Erick Alexanderson, Gabriela Melendez and Ana L Mendizabal

Instituto Nacional de Cardiologia Ignacio Chavez, Mexico, Mexico

Journal of Cardiovascular Magnetic Resonance 2009, I I(SuppI I):PI 20

Introduction: Despite successful recanalization of the infarctrelated artery, perfusion of the ischemic myocardium is not or is incompletely restored in up to $30 \%$ of patients due to microvascular obstruction (MO). The presence of it has been found to be a predictor of adverse events.

CMR allows accurate assessment of function, transmural extent and total size of infarction, and MVO in all segments of the left ventricle.

Purpose: To explore the relation between enzymatic, angiographic and CMR findings in patients with treated acute myocardial infarction.

Methods: We included 23 patients with first acute myocardial infarction, all of them were sent to the cath laboratory. Functional imaging, first pass and delayed gadolinium enhancement was performed using a I.5 T scan (Sonata, Siemens) after the reperfusion treatment. The correlation between enzymatic, CMR (MO, ventricular function) and hemodynamic (TIMI, TMP) findings were evaluated.

Results: There were 22 males (95\%), average age $52 \pm 6$ years. Fifteen patients had OM. Ejection fraction was slightly better in patients without microvascular obstruction compared with patients with $\mathrm{MO}(37.55-50.08$ vs $31.85-42.7 \mathrm{p}=0.1 \mathrm{I})$. There was not correlation between MO with TIMI flow (pre or post procedure) neither TMP. Higher levels of creatine kinase and troponin I were founded in patients with microvascular obstruction, compared with group without MVO. $(p=0.04$ and 0.02).

Conclusion: Although it has been believed that TMP could be a good method to detect microvascular obstructions in patients with acute coronary syndromes, we don't found correlation between hymodinamics and resonance findings. There is correlation between enzymatic and the presence of $O M$ by CMRI, which is in accordance with more myocardial damage. 
PI2 I

Role of First Pass (FP) and delayed enhancement in functional recovery assessment after Acute Myocardial Infarction (AMI)

Antonio Bernardini ${ }^{1}$, Luigi Natale ${ }^{2}$, Agostino Meduri ${ }^{2}$, Carlo Liguori ${ }^{2}$, Antonella Lombardo ${ }^{2}$ and Lorenzo Bonomo ${ }^{2}$

I"G. Mazzini" Hospital - ASL Teramo, Teramo, Italy

${ }^{2}$ Università Cattolica del Sacro Cuore - Policlinico A. Gemelli, Rome, Italy

Journal of Cardiovascular Magnetic Resonance 2009, I I(SuppI I):PI 2 I

Purpose: To define contrast enhanced MRI role in functional recovery prediction after acute myocardial infarction.

Materials and methods: 46 patients with first AMI $(65 \pm$ 8 yrs., 39 anterior, 4 inferior, 3 lateral) underwent cardiac MRI after primary PTCA 5 to 7 days after onset. MRI Protocol: SA Cine (FIESTA), 8- 10 slices; SA first pass (FGRE-ET) (iv $0.1 \mathrm{mmol} / \mathrm{kg}$ Gd-DTPA, 3 mL/s), 3 slices; SA delayed imaging (IR-prep FGRE), 8-I0 slices, 15 mins after $0,2 \mathrm{mmol} / \mathrm{Kg}$ Gd-DTPA. FP and DE were evaluated together (736 segments) as: normal FP, DE absent or up to $50 \%$ of transmural extension (TME) (Pattern I); delayed hyperenhancement $>50 \%$ without FP hypoenhancement (Pattern $2 \mathrm{a}$ ); delayed hyperenhancement $>50 \%$ with FP hypoenhancement (Pattern 2b); hypoenhancement both at FP and DE(Pattern 3). 828 segments out of first-pass slices were classified on the basis of $D E$ as normal or hyperenhanced $50 \%$ TME (= pattern 2 ) and hypoenhanced (= pattern 3). Patterns 2, 2a, $2 \mathrm{~b}$ and 3 were considered non viable. Six months follow up MRI assessed functional recovery as improvement of segmental WMSI.

Results: Pattern I: I 167 segments, functional recovery in 1065 (91,3\%). Pattern 3: I 32 segments, recovery in 9 (6,8\%) Pattern 2: 265 segments, recovery in $78(29,4 \%)$. Pattern 2a showed recovery in 23 segments out of 42 (54,8\%); Pattern $2 b$ did not recover in 61 out of 80 (76,3\%).

Conclusion: Patterns $I$ and 3 respectively identify viable and non viable tissue. Pattern 2 is less specific early after AMI; the presence of FP hypoenhancement is often associated with failed recovery.

\section{P I 22}

Cardiac magnetic resonance imaging versus transesophageal echocardiography for the evaluation of mitral valve pathology prior to surgical intervention (The MagnaSound study) Jennifer D Cohen, Heather S Costa and Robert J Russo Scripps Clinic, La Jolla, CA, USA

Journal of Cardiovascular Magnetic Resonance 2009, I I(SuppI I):PI 22

Background: Transesophageal echocardiography (TEE) is the imaging modality of choice for the assessment of mitral valve (MV) pathology prior to valve repair or replacement surgery. However, TEE is a semi-invasive procedure requiring conscious sedation and may be limited by esophageal pathology and patient discomfort. Cardiac magnetic resonance (CMR) is a noninvasive imaging technique with excellent spatial resolution and is an attractive alternative for those unable or unwilling to undergo TEE. The purpose of the present MagnaSound pilot study is to assess the feasibility of a mitral valve specific CMR protocol to measure the parameters necessary for determining the appro-
Figure I (abstract PI22)

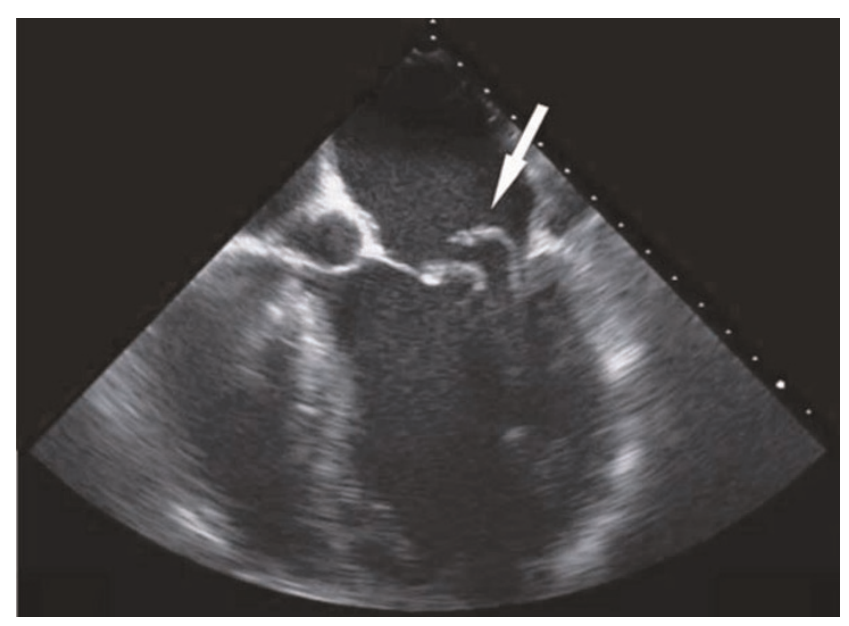

Transesophageal echocardiogram visualizing a flail posterior leaflet of the mitral valve.

priateness of valve repair or replacement surgery using TEE as a reference standard.

Methods: We performed CMR in 7 patients ( $57 \pm 18$ years) with sinus rhythm and severe MR who had undergone a clinically indicated TEE (Figure I). CMR exams were performed within II days of the TEE. Using a I.5 T Siemens Magnetom Symphony, balanced steady-state free precession (SSFP) cine images were obtained in the standard views. Additional views visualizing the papillary muscles and obtaining the maximum visualization of the regurgitant jet were performed (Figure 2). Evaluation of the maximum regurgitant jet was confirmed by gradient echo sequences with saturation bands placed across the left ventricle. Finally, phase contrast imaging sequences with velocity encoding

Figure 2 (abstract PI22)

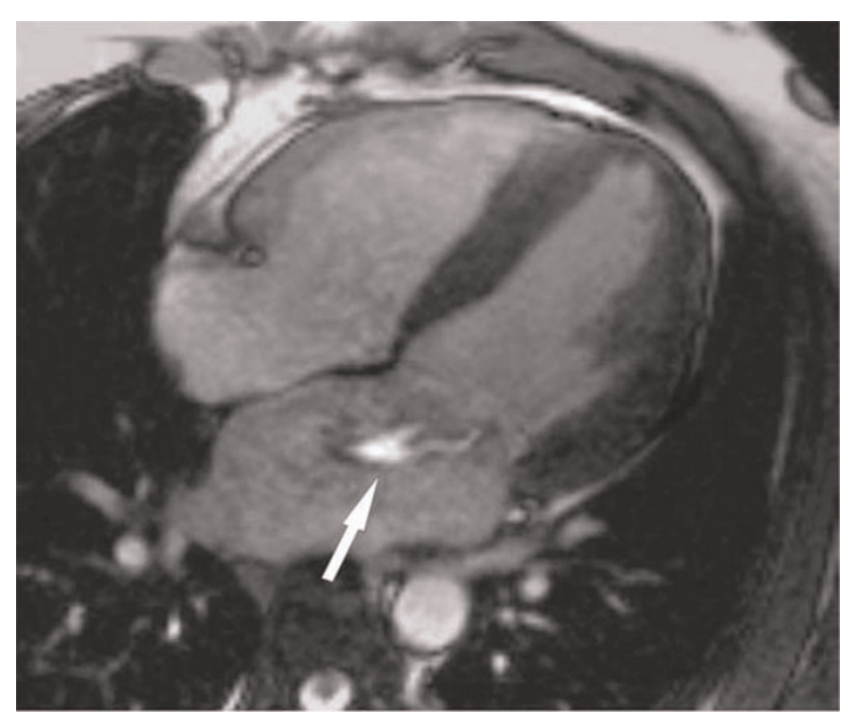

Breath-hold cine steady-state free precession imaging in a fourchamber view visualizing the mitral regurgitation jet (flow void) due to flail a posterior leaflet. 
demonstrated the mitral valve inflow patterns. Qualitative and quantitative image analysis was performed using QMass 7.0 (Medis, Inc.) and Argus (Siemens) software. The following measurements were made: regurgitant fraction, volume, and orifice area; the presence of prolapse versus flail leaflets; the length and thickness of MV leaflets; the MV inflow pattern; the number, direction and location of regurgitant jets; and threedimensional ejection fraction, and chamber dimensions.

Results: Data was successfully obtained in all 7 patients. Based on the results of the TEE, all 7 patients were deemed appropriate candidates for mitral valve repair surgery by the evaluating cardiac surgeon. The CMR images were interpreted by 2 physicians blinded to the TEE results. CMR correctly identified the involved leaflet and degree of impairment (prolapse versus flail) in all 7 patients compared with the TEE findings. Six of the 7 patients had a single leaflet involved. One patient had a flail posterior leaflet and a prolapsing anterior leaflet. The average maximum distance of the regurgitant jets was $4.8 \pm 1.3 \mathrm{~cm}$. The regurgitant fraction was $77 \pm 13 \%$ and the regurgitant orifice was $0.67 \pm 0.25 \mathrm{~cm}^{2}$ as measured by CMR. Three-dimensional ejection fraction and left ventricular dimensions were available for 5 of the 7 studies (ejection fraction $63.6 \pm 9 \%$; left ventricular end-diastolic dimension $59.9 \pm 4.2 \mathrm{~cm}$ ). CMR measurements (primary regurgitant jet, jet location and direction; leaflet lengths and thickness; MV inflow patterns; regurgitant fraction, volume and orifice area; and ejection fraction and LV dimensions) were used to assess the appropriateness of MV repair versus replacement. All 7 patients were deemed candidates for MV repair by CMR, independent of their TEE results. Both CMR and TEE correctly identified the leaflet and degree of involvement and the ability to repair the valve in the 3 patients who have since undergone surgery.

Conclusion: This pilot study confirms that a mitral valve specific CMR protocol can adequately visualize the severity of $M R$ and provide morphometric measurements necessary to determine a patient's candidacy for mitral valve repair versus replacement surgery. These results validate an approach to be adopted in the prospective MagnaSound study comparing TEE and CMR for the preoperative evaluation of mitral valve morphology prior to repair or replacement surgery.

\section{P I 23}

Moderate to severe renal impairment cannot be reliably detected from medical history alone: Implications for the use of Gadolinium-based contrast agents

John-Paul Carpenter, Elizabeth Burman

and Raad Mohiaddin

Royal Brompton and Harefield NHS Trust, London, UK

Journal of Cardiovascular Magnetic Resonance 2009, I I(SuppI I):PI 23

Introduction: Nephrogenic systemic fibrosis (NSF) is a rare condition characterised by thickening and tightening of the skin, hyperpigmentation and limitation of joint mobility. Autopsy data suggests that NSF may also be a more systemic disorder, affecting the heart, lungs, liver and other organs. An association with gadolinium-based contrast agents (GBCA) was first reported in 2006 [I] and therefore, the US Food and Drug Administration (FDA) recommends that GBCA 'should not be given in patients with known risks for developing NSF unless the diagnostic
Figure I (abstract PI23)

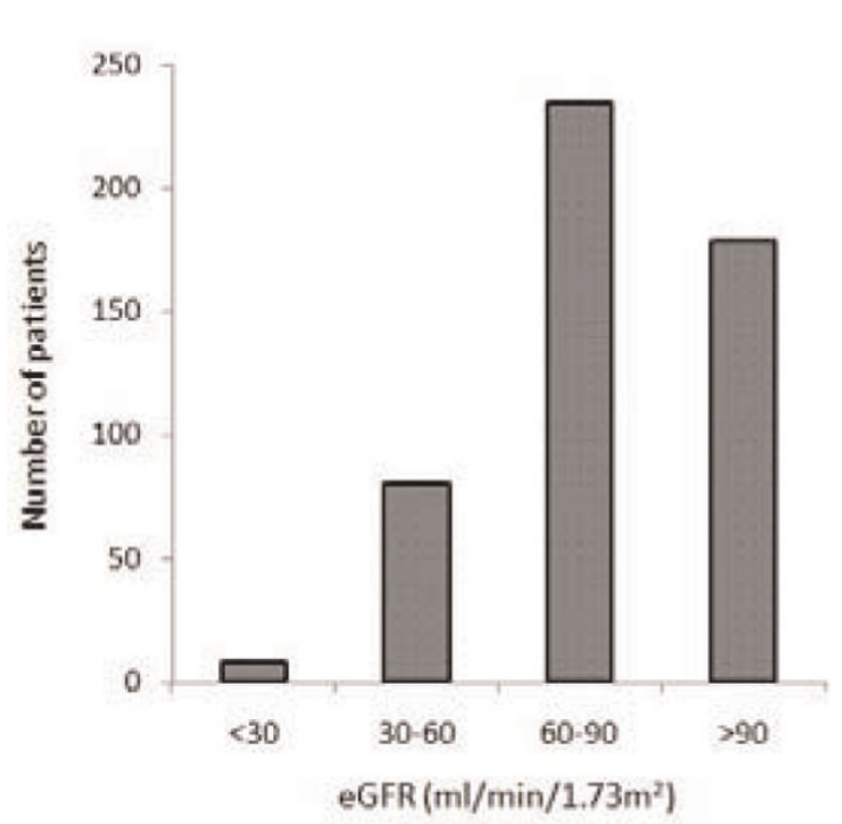

Distribution of eGFR in patients referred for contrast-enhanced CMR scans.

information is essential and cannot be obtained with noncontrast enhanced MRI or other diagnostic procedures'. The guidelines also state that patients should be evaluated for renal dysfunction either by obtaining a history or conducting laboratory tests [2]. Although NSF has only been reported in chronic kidney disease (CKD) patients with estimated glomerular filtration rate $(e G F R)<30 \mathrm{ml} / \mathrm{min} / 1.73 \mathrm{~m}^{2}$, it has been documented in acute renal failure patients with an intercurrent illness and eGFR of between 30 and $60 \mathrm{ml} / \mathrm{min} / 1.73 \mathrm{~m}^{2}$ [3].

Purpose: This study was designed to investigate whether patients with moderate to severe renal impairment could be easily identified from history alone or whether routine blood testing is required in every patient prior to the use of gadoliniumbased contrast agents.

Methods: Serum creatinine and eGFR is routinely measured prior to the use of gadolinium chelates in our unit. eGFR is calculated using the Modification of Diet in Renal Disease (MDRD) formula. As part of the pre-scan safety questionnaire, patients are asked if they are aware of any kidney problems.

Over a 3-month period from January 2008 to March 2008, all cases were reviewed to identify patients with moderate or severe renal impairment. This was defined as CKD Stage 3 (eGFR 30$\left.60 \mathrm{ml} / \mathrm{min} / 1.73 \mathrm{~m}^{2}\right)$, Stage $4\left(\right.$ eGFR $15-30 \mathrm{ml} / \mathrm{min} / 1.73 \mathrm{~m}^{2}$ ) or Stage 5 (End stage renal failure with eGFR $<15 \mathrm{ml} / \mathrm{min} / 1.73 \mathrm{~m}^{2}$ ). Documentation was then reviewed to see if renal impairment had been mentioned by the referring physician or was known by the patient themselves.

Results: Of 944 scans performed in the three months from January 2008 to March 2008, 593 patients (62.8\%) were given GBCA. $16 \%$ of patients with an indication for GBCA had an eGFR of $30-60 \mathrm{ml} / \mathrm{min} / 1.73 \mathrm{~m}^{2}$ and $1.6 \%$ had an eGFR of $<30 \mathrm{ml} /$ $\mathrm{min} / 1.73 \mathrm{~m}^{2}$. Only one patient had an eGFR of $<15 \mathrm{ml} / \mathrm{min} /$ $1.73 \mathrm{~m}^{2}$. The distribution is shown in Figure $\mathrm{I}$. 
Figure 2 (abstract PI23)

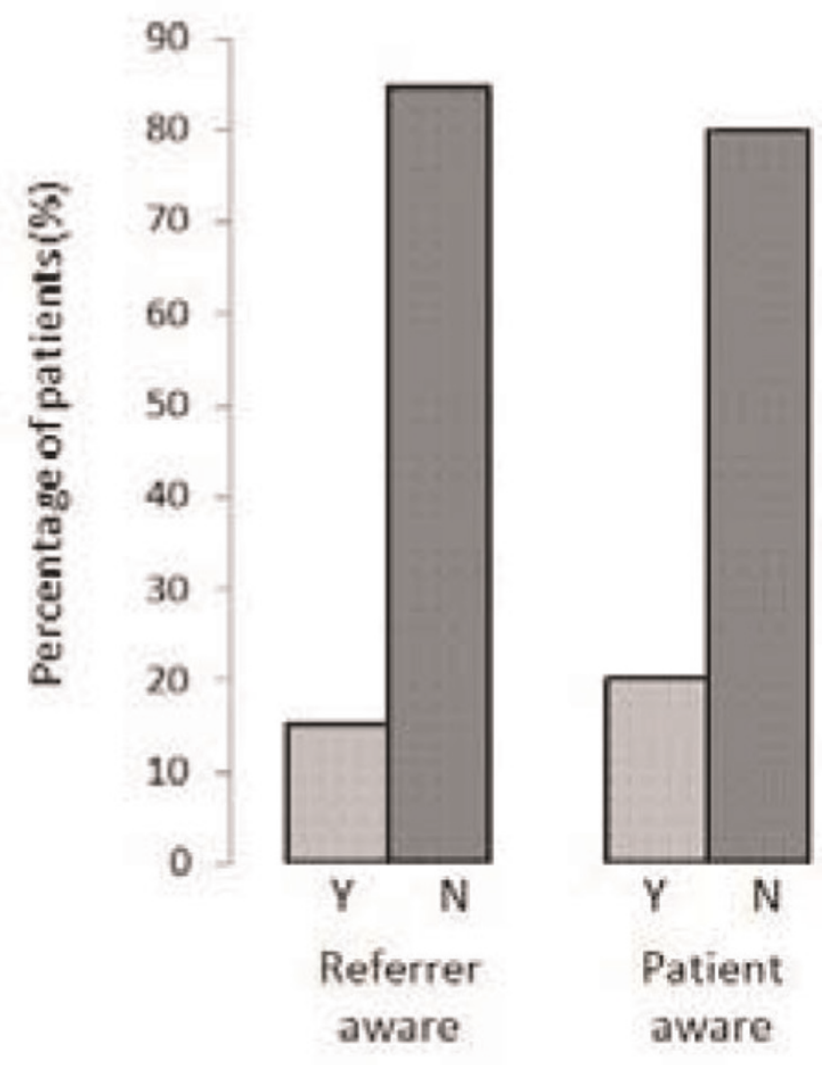

Awareness of moderate to severe renal impairment (eGFR 30-60 ml/min/ $1.73 \mathrm{~m}^{2}$ ) prior to blood results at time of CMR scan.

All patients with eGFR of $<30 \mathrm{ml} / \mathrm{min} / 1.73 \mathrm{~m}^{2}$ were identified either by the referring physician or by the safety questionnaire prior to the scan. The situation was very different for those with eGFR between 60 and 90 . Of the 79 patients with eGFR in this range, only a minority were identified by the referring physician or were aware that they had renal impairment (I5.2\% and $20.3 \%$ respectively - Figure 2).

Conclusion: This retrospective study highlights the significant numbers of patients with at least moderate renal impairment who are referred for CMR scans with an indication for GBCA. Our results suggest that most patients with moderate to severe renal impairment (eGFR $30-60 \mathrm{ml} / \mathrm{min} / \mathrm{l} .73 \mathrm{~m}^{2}$ ) will not be identified without blood testing. In view of the fact that NSF has been reported in these patients (albeit with a concurrent illness), this has important implications and supports the current practice of measuring renal function on all patients. Decisions about giving gadolinium-based contrast agents need to be made based on clinical need for the information which will be gained together with the informed consent of the patient concerned.

\section{References}

I. Grobner T: Gadolinium: a specific trigger for the development of nephrogenic fibrosing dermopathy and nephrogenic systemic fibrosis? Nephrol Dial Transplant 2006, 2 I (4): I I 04-1 I 08.
2. http://www.fda.gov/cder/drug/InfoSheets/HCP/gcca_200705. htm.

3. Sadowski EA, Bennett LK and Chan MR, et al: Nephrogenic systemic fibrosis: risk factors and incidence estimation. Radiology 2007, 243(I): |48-I57.

PI 24

Serum biomarkers and traditional risk factors as predictors of peripheral arterial disease assessed by magnetic resonance angiography

Yuliya B Goldsmith', ljaz Ahmad', Vishnu Singh', Marcus D'Ayala', Igor Klem², William M Briggs', Mohammed Ahmed', Munawar Hayat', Terrence J Sacchi' and John F Heitner'

${ }^{I}$ New York Methodist Hospital, Brooklyn, NY, USA

${ }^{2}$ Duke University Medical Center, Durham, NC, USA

Journal of Cardiovascular Magnetic Resonance 2009, I I(SuppI I):PI 24

Introduction: In recent studies, multiple serum biomarkers had been investigated for a relationship to peripheral arterial disease (PAD). PAD presence and severity have been shown to be associated with CRP and lipid levels. Although elevated proBNP has clearly been shown to be associated with left ventricular dysfunction and predict cardiovascular events, there is scant data showing its association with PAD. Prior small studies demonstrated elevated levels of NT-pro BNP in patients with PAD when compared to controls. The significance of the serum biomarkers as predictors of PAD in comparison with traditional risk factors is unclear.

Purpose: The aim of this study is to compare the role of serum pro-BNP, CRP, and lipid levels to the number of standard risk factors (nRF) as independent predictors of the presence and severity of PAD.

Methods: We prospectively evaluated 205 consecutive patients who underwent contrast-enhanced MRA for the evaluation of PAD. There were $7 \mid$ carotid (I0 segments), I3I abdominal ( 2 segments), I 15 renal ( 2 segment), 66 pelvic ( 6 segments), and 48 lower extremity studies ( 12 segments). We analyzed the studies for both the presence and the severity of PAD. Significant PAD was defined as any stenosis of $>50 \%$ in at least one vessel. PAD severity was assessed with fractional stenosis score (FSS): patients segment scores ( $0=$ no stenosis, $I=1-50 \%, 2=5 \mathrm{I}-$ $75 \%, 3=76-99 \%, 4=100 \%$ ) were summed and divided by the maximal possible sum score that would have resulted if all evaluated segments were occluded. nRF for each patient was calculated by assigning I point for the presence of each: age $>65$, male gender, $\mathrm{BMI}>25$, hypertension, diabetes, hyperlipidemia and smoking. Serum pro BNP, CRP and lipid profile values were collected on the day of the CMR for each patient.

Results: There were 124 pts with significant PAD. In a univariate model, serum log (pro BNP), total cholesterol (TC), LDL, triglycerides (TG), and nRF were associated with FSS. Log (pro BNP), TC, LDL, and $n R F$ were associated with presence of significant PAD. CRP was not predictive of either presence or severity of PAD. When a multivariate regression model was applied, only log (pro BNP) ( $p<0.027)$, nRF ( $<<0.007)$, and LDL $(P<0.004)$ were independently associated with FSS. When accounting for the presence of systolic and distolic dysfunction by echocardiogram ( 99 pts), log (pro BNP) remained a significant predictor of FSS. Only nRF was independently associated with 
the presence of significant PAD (OR $=1.09,95 \% \mathrm{Cl}$ I.04-I. 15 , $P<0.0008$ for each additional RF).

Conclusion: Although log (pro BNP) and LDL were associated with severity of PAD expressed as FSS, only nRF was independently predictive of both presence and severity of PAD. The role of serum pro BNP as a marker of PAD needs to be further investigated. In this study, serologic biomarkers do not add to traditional risk factors for the prediction of PAD diagnosed with MRA.

\section{PI25}

Whole heart 3 D MR coronary angiography with and without extracellular contrast agent

Dorota Piotrowska-Kownacka, Lukasz Kownacki,

Grzegorz Opolski, Leszek Krolicki and Olgierd Rowinski Medical University of Warsaw, Warsaw, Poland

Journal of Cardiovascular Magnetic Resonance 2009, I I(SuppI I):PI 25

Introduction: It is well known that extravascular contrast administration before whole heart $3 \mathrm{D} M R$ angiography improves image quality and distal coronary segments visibility. In opposite to extravascular, extracellular contrast agents are commonly used in cardiac MR. The influence on coronary angiography quality remains unknown.

Purpose: The aim of the study was quality assessment of coronary MR angiography performed after extracellular contrast administration (Gd-DTPA) in stable, consecutive patients referred to CMR lab for contrast enhanced cardiac examination in comparison to unenhanced coronary $M R$ angiography in healthy volunteers.

Methods: 69 patients and 16 volunteers were examined in I.5 T scanner with 3D navigator gated, inversion recovery, segmented gradient echo sequence. For data acquisition

\section{Figure I (abstract PI25)}

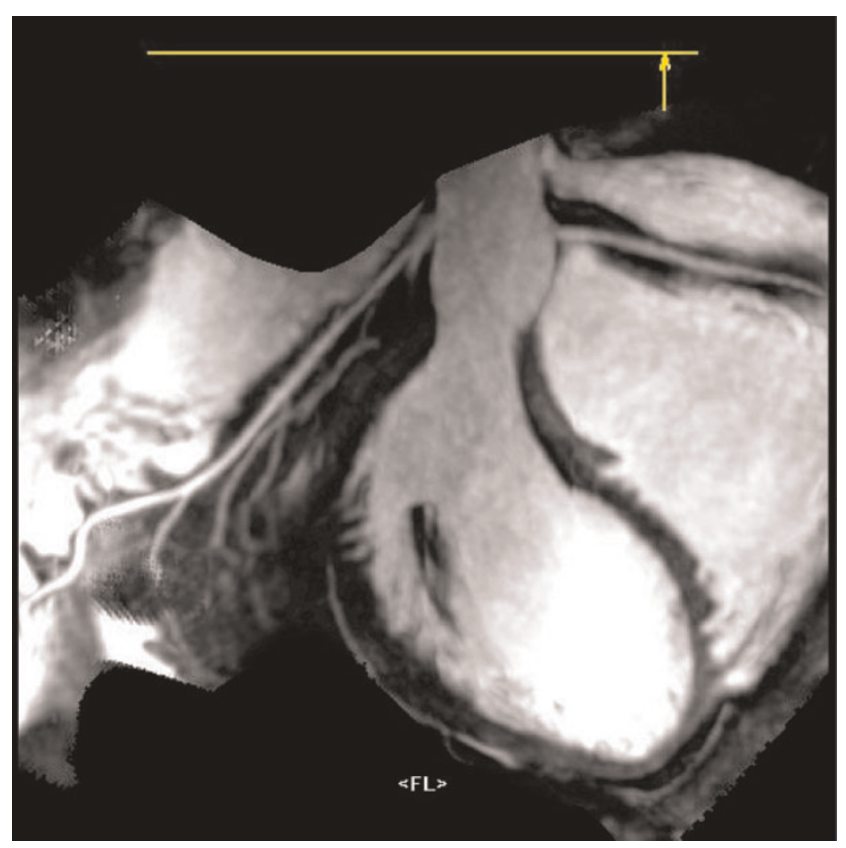

Enhanced MR coronary angiography - normal coronaries.
Figure 2 (abstract PI25)

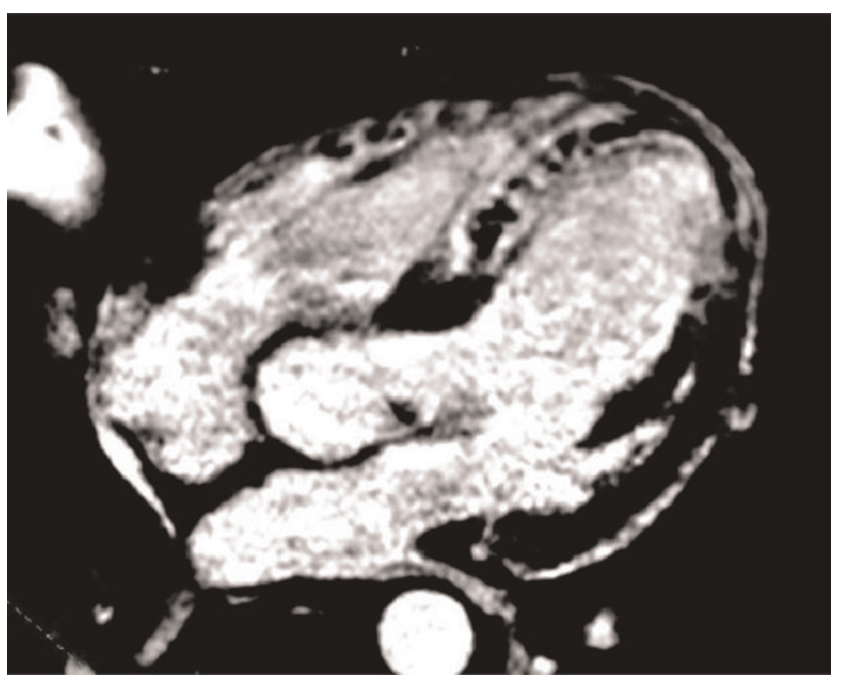

DE and MVO in STEMI.

32 Channel Cardiac Coil was used. In all patients MR angiography was the last sequence applied. In all patients extracellular contrast agent (Gd-DTPA, $0.1 \mathrm{mmol} / \mathrm{kg}$ b.w.) was injected. The time between contrast injection and whole heart MR angiography was monitored. The images were analyzed on MMWP Workstation. Quality of MR angiography was evaluated using 4 point scale: I-poor, 2-sufficient, 3-good, 4-very good. General image quality and visibility of coronary artery disease: left main, proximal and distal parts of LAD, Cx, RCA were evaluated separately. Differences between unenhanced and enhanced data

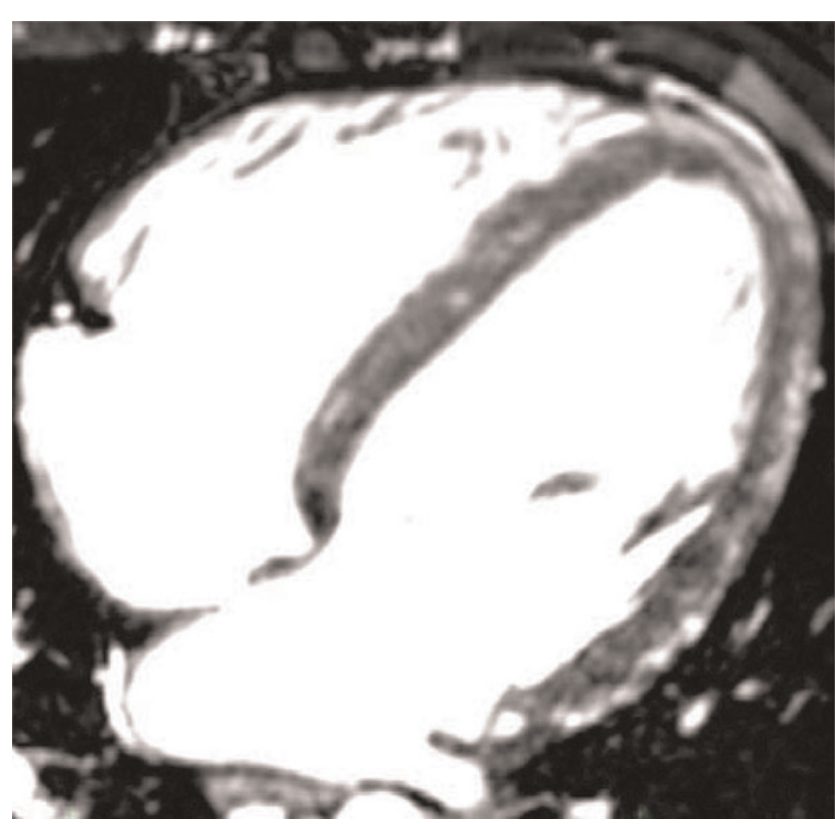

DE in myocarditis. 
were analyzed using Mann-Whitney test. $P$ value of below 0,05 was considered statistically significant.

Results: Mean time between contrast injection and sequence start was 12,8 \pm 7,7 min. Mean acquisition time was $14 \pm 5$,9. The median quality score was 3 . General MR angiography quality was good or very good in $76,6 \%$ of patient and $67,7 \%$ of volunteers. There was significant improvement of general quality as well as coronaries visibility in all evaluated arteries and parts on enhanced images. No relation between contrast injection time as well as acquisition time and image quality were found. Enhanced images have given additional information regarding viability in patients with acute myocarditis and viability/no-reflow regions in patients early after myocardial infarction.

Conclusion: Extracellular contrast agents, which are commonly used in cardiac MR, significantly improves image quality of the whole heart, navigator gated 3D MR coronary angiography. Preliminary data suggests that this sequence could be used as "one stop shop" alternative in patients in acute condition uncapable of breath-hold. MR angiography, morphology as well as viability and microvascular obstruction regions could be imagined. Further studies are needed. Please refer to Figures I, 2 , and 3 .

\section{PI 26}

\section{Analysis platform for hemodynamic function in congenital heart disease}

Elizabeth J Nett, Kevin M Johnson, Christopher J Francois and Oliver Wieben

University of Wisconsin, Madison, WI, USA

Journal of Cardiovascular Magnetic Resonance 2009, I I(Suppl I):PI 26

Introduction: Advanced Phase Contrast MR acquisition techniques such as PC VIPR [I] can simultaneously provide anatomical images and cine velocity fields with volumetric coverage. These data can be used for non-contrast enhanced MR Angiography, velocity and flow measurements, and the derivation of addition hemodynamic parameters such as trans-

\section{Figure I (abstract PI 26)}

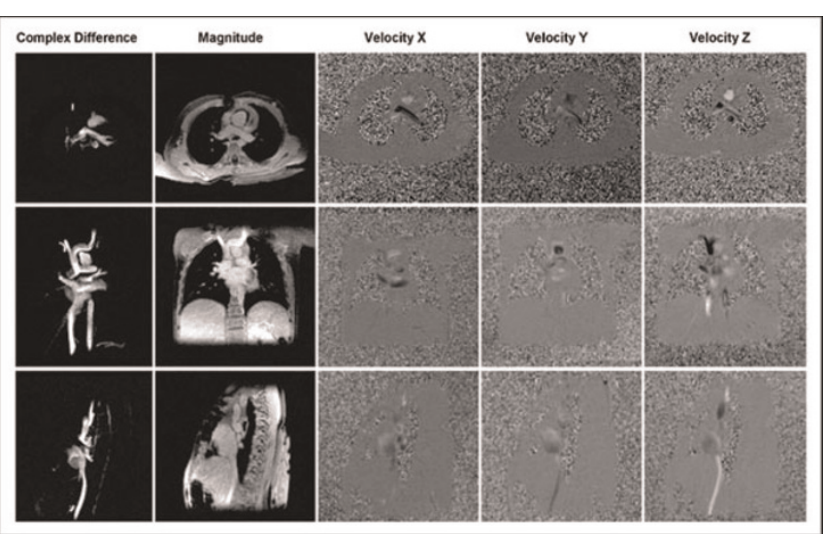

Time averaged complex difference, magnitude and velocity images in three orthogonal directions in the axial, saggital and coronal slices from a PC VIPR data set of a patient with pulmonary venolobar sydrome. The large data volume requires a new, efficient approach to data processing and visualization.
Figure 2 (abstract PI26)
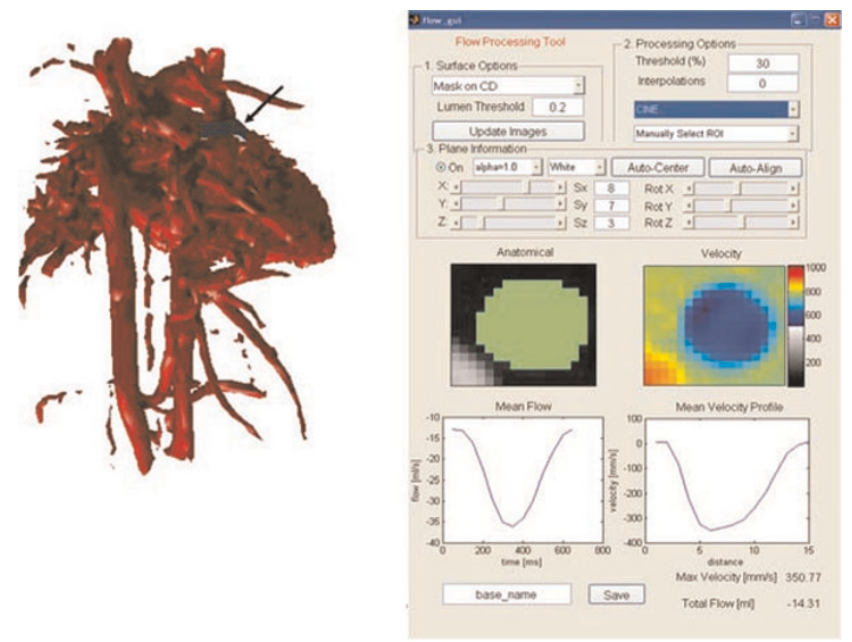

Matlab flow analysis software used on a PC VIPR data set of a patient with Scimitar syndrome. An arrow points to the ROI box (grey) that can be automatically centered and aligned with the vessel and the through plane velocities. Based on the RIO selection, the flow through the cardiac cycle and profile of the mean velocity can be derived.

stenotic pressure gradients and wall shear stress. While this comprehensive information can be very valuable, particularly in complex congenital heart disease, the large data volume with high spatial and temporal information requires new approaches for efficient visualization and extraction of clinically relevant parameters.

Purpose: To develop a software platform that streamlines hemodynamic analysis including flow measurements with automatic alignment with respect to the vessel orientation and the derivation of hemodynamic parameters such as wall shear stress and pressure gradients.

Methods: Data were acquired with a dual-echo PC VIPR trajectory with cardiac and respiratory gating on $1.5 \mathrm{~T}$ and $3 \mathrm{~T}$ clinical systems (GE Healthcare) [2]. Typical scan parameters were: $(1.0-1.25 \mathrm{~mm})^{3}$ isotropic spatial resolution in approximately 10 min scan time with $50 \%$ respiratory gating efficiency, imaging volume: $32 \times 32 \times 16 \mathrm{~cm}^{3}$, VENC of $50-100 \mathrm{~cm} / \mathrm{s}$ (application specific). Figure I displays representative axial, coronal, and sagittal reformats of the magnitude, three velocity components, and the derived angiogram. These data sets contain 320 slices $\times 20$ time frames $\times 5$ volumes $=32,000$ images.

We developed a software platform with a visual interface using Matlab (MathWorks) in order to visualize the results, enable quantitative measurements, and derive additional hemodynamic parameters. First, the reconstructed PC VIPR data are loaded into a segmentation tool in order to reduce the size of the data set for more efficient processing. The knowledge of vessel boundaries is also essential for the calculations of flow, wall shear stress, and pressure gradients, which are available processing tools. Next the data can be loaded into the analysis plug-ins to measure and visualize flow, derive pressure maps [3], or calculate wall shear stress [4] from the velocity fields with volumetric coverage. For improved flow measurements, the analysis plane can be automatically aligned perpendicular to the vessel path. Flow measurements are derived by integration of the velocity 
vectors over time and vessel area, which can be defined either automatically with a threshold algorithm or manual selection. Velocity and flow measurements as well as the time average and/ or cine volumetric maps of pressure gradients and wall shear stress can be exported for further analysis and visualization or comparisons with computational fluid dynamics (CFD) calculations. To validate the velocity and flow measurements, multiple PC VIPR data sets including flow phantom data and in vivo cardiac data were compared with standard 2D PC MR measurements prescribed perpendicular to the vessel orientation.

Results: Figure 2 shows the flow and velocity tool used in a patient with Scimitar syndrome. Among other parameters, the through-plane flow of the analyzed vessel segment is displayed as a function of time in the cardiac cycle and as net flow in addition to a velocity profile across the vessel diameter. For the validation of the flow measurements, good agreement was found between PC VIPR and 2D PC measurements.

Conclusion: A new software platform was implemented for the comprehensive analysis of hemodynamic information obtainable from high resolution PC MR datasets. Future studies using this platform will help to identify the clinically significant hemodynamic parameters for clinical applications such as early diagnosis, treatment monitoring, and understanding of disease development in congenital heart disease.

\section{References}

I. Gu TL, et al: AJNR 2005, 26(4):743-749.

2. Johnson KM, et al: PROC ISMRM 2008, 733.

3. Lum D, et al: Radiology 2007, 245(3):75I-760.

4. Moftakhar R, et al: AJNR 2007, 28(9):|7|10-17|4.

\section{P I 27}

Fast free-breathing planning in cardiac MR imaging Tamer A Basha, Monda L Shehata, Robert G Weiss and Nael F Osman

Johns Hopkins University, Baltimore, MD, USA

Journal of Cardiovascular Magnetic Resonance 2009, I I(SuppI I):PI 27

Introduction: Cardiac magnetic resonance imaging is an excellent tool for assessing global and regional function of the heart. It is now regarded as the gold standard for the analysis of ejection fraction, ventricular volumes and for quantification of myocardial wall-motion [I]. However, the initial planning step is

Figure I (abstract PI27)

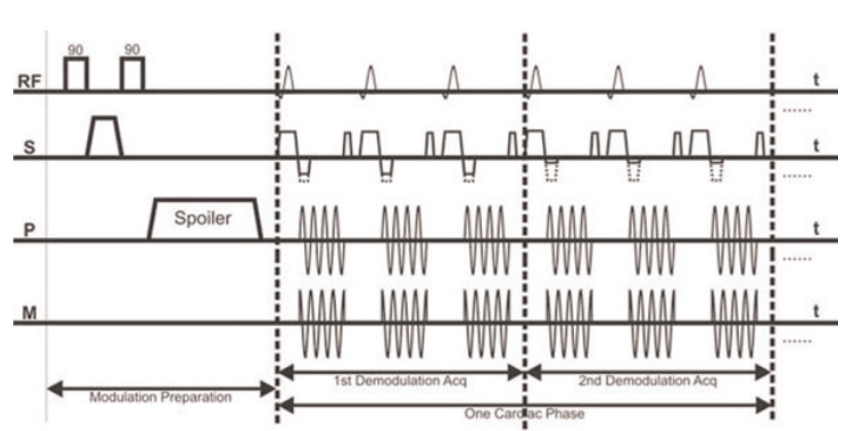

Pulse sequence diagram. Dotted lines in the gradient lobes represent the original gradient values without the demodulation time consuming and requires at least three or four breath-holds in order to get to the first targeted slices.

Purpose: In this work, we propose a fast black-blood pulse sequence which requires a scan duration as short as one heartbeat.

Methods: Pulse sequence: Based on the modified STEAM sequence proposed in [2], Figure I shows a diagram for the proposed sequence. First, modulation pulses are applied upon the detection of the QRS complex of the electrocardiogram (ECG). Then images are acquired at a demodulation frequency equal to the original one. This results in obtaining signal only from the tissues that maintained the original modulation and nulling the blood signal because of its flow. A reduced field of view (FOV) is achieved by localizing the modulation pulses such that only the region around the heart is modulated. Then, images are obtained using multi-shot spiral acquisition. The combination of reduced FOV and spiral acquisition allow for acquisition of a complete set of images in just one cardiac cycle.

In vivo experiments: During regular cardiac imaging exams on a 3 T MRI scanner (Philips Medical Systems, Best, the Netherlands), the proposed sequence was used for free-breathing image planning on fifteen healthy volunteers. All subjects were imaged in the supine position. Following standard survey images using a scout sequence, two series of planning acquisitions are acquired using regular breath-hold cine and the proposed free-breathing technique. Each planning series starts with pseudo 2-chamber then pseudo 4-chamber and ends with the true shortaxis plan. A true four-chamber plan was acquired depending on the need of this plan for the running exam.

The following acquisition parameters were used for the proposed technique: FOV, $256 \times 256 \mathrm{~mm}^{2}$; Excited region width, $200 \mathrm{~mm}$; matrix size, $64 \times 64$; last flip angle [2], $30^{\circ}$; slice thickness, $10 \mathrm{~mm}$; in-plane spatial resolution, 4-4 $\mathrm{mm}^{2}$. For each cardiac phase, two demodulation images were acquired, for each one 3 spirals were used with acquisition window of $7 \mathrm{msec}$. This results in a temporal resolution of $42 \mathrm{msec}$. Depending on the heart rate, 17-25 cardiac phases were acquired. The total scan time was $0.7-1.0 \mathrm{sec}$.

Results: Figure 2 shows representative results for the planning steps for one of the volunteers. While the regular cine images have better resolution and more detail, the images from the

Figure 2 (abstract P I27)

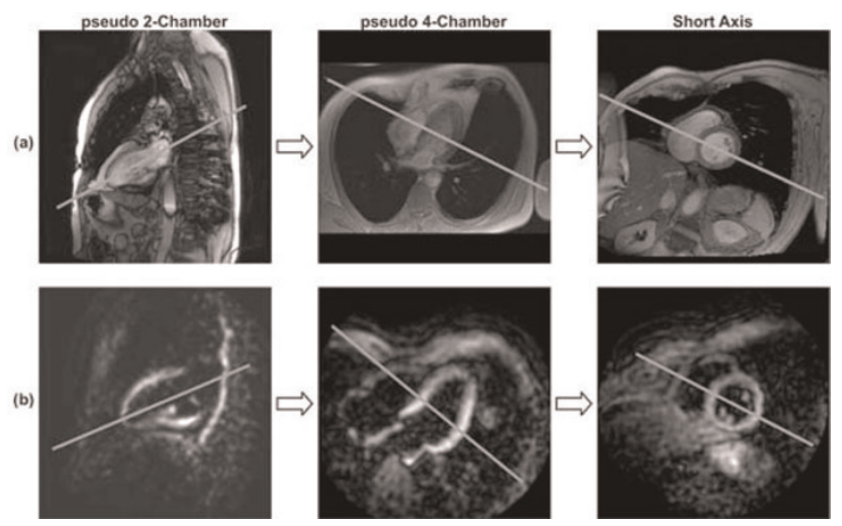

Planning steps using a) Regular Cine, and b) Proposed technique. 
Figure 3 (abstract PI27)

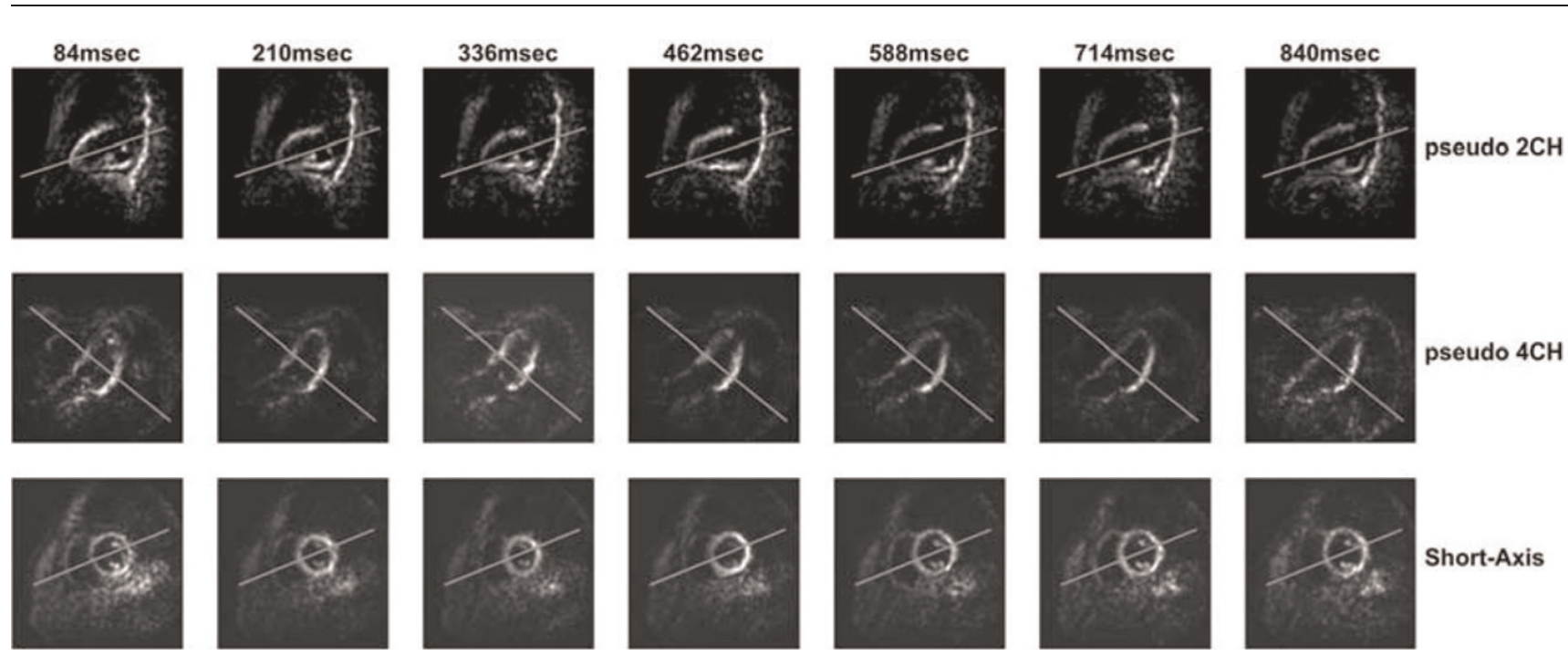

Representative cardiac phases for planning results using the proposed technique. Columns represent time frames while rows show different planning slices.

proposed technique have enough detail for adequate planning. The black blood feature allows for better recognition of the ventricles. Figure 3 shows the complete cardiac phases of each of these planning steps. The interleaving between the two demodulation images helps in getting clear images over the whole cardiac cycle, either during relaxation (where the first demodulation image has more information) or during contraction (where the second one has clearer ventricle view).

With the same operator, planning time was reduced from $5 \pm$ $2 \mathrm{~min}$ using regular cine images for planning to $2.2 \pm 0.7 \mathrm{~min}$ using the proposed protocol.

Conclusion: With a relatively low spatial resolution $(4 \mathrm{~mm})$, the proposed protocol can be used for cardiac imaging planning without any breath-holds. This helps to reduce the overall scan time and the number of breath-holds in during the exam which is especially important for dyspneic patients.

\section{Acknowledgements}

his work was supported in part by grants from the national institute of heart, lung and blood ROIHL072704 and ROIHL6I912, and Donald W. Reynolds Foundation grant.

References

I. Yang PC, et al: J Am Coll Cardiol 1998, 32:2049-2056.

2. Fahmy AS, et al: MRM 2006, 55:404-4I2.

\section{P I 28}

\section{Left atrial fat with cardiac MR}

Dana C Peters', James W Goldfarb², Reza Nezafat', Nisha I Parikh', Michael L Chuang' and Warren J Manning' 'Beth Israel Deaconess Medical Center and Harvard Medical School, Boston, MA, USA

${ }^{2}$ Department of Research and Education, Saint Francis Hospital, Roslyn, NY, USA

Journal of Cardiovascular Magnetic Resonance 2009, I I(SuppI I):PI 28

Introduction: It has been proposed that the ganglionated plexi (GP) residing in the fat pads surrounding the left atrium (LA) may be involved in the genesis of atrial fibrillation (AF) [I]. Appropriate ablation of these targets may be a treatment of $\mathrm{AF}$ [2]. The gross anatomy of the GPs embedded in peri-atrial fat has been described and classified into five regions [3]: the superior right atrial GP, the superior left atrial GP, the posterior right atrial GP, the posteromedial left atrial GP, and posterolateral left atrial GP. Cardiac MR (CMR) is unique among non-invasive imaging modalities, providing excellent depiction of fat, and can contribute by identifying these fat pads.

Methods: Six healthy adult subjects (age $=24+/-4,3 \mathrm{M}$ ) underwent CMR with two high spatial resolution 3D freebreathing sequences. Scan parameters in common for both sequences were: 3D gradient echo, axial, navigator-gated and ECG-gating to diastole, TR/TE $/ \theta=5.3 / 2.5 / 20^{\circ}, 32$ views per segment, $16 \mathrm{Nz}, 1.3 \times 1.3 \times 5 \mathrm{~mm}$ spatial resolution. The first sequence used an inversion pulse with a $\mathrm{Tl}$ of $400 \mathrm{~ms}$, to null blood and myocardium, while fat is bright (Figure IA). The second sequence used a T2-prep (20 ms), and fat-suppression to visualize the pulmonary veins (PVs) and the LA only (Figure IB). No exogenous contrast was used. These two free-breathing sequences can be fused, with color or non-color highlighting, to demonstrate fat in the context of anatomy (Figure IC).

Figure I (abstract PI28)

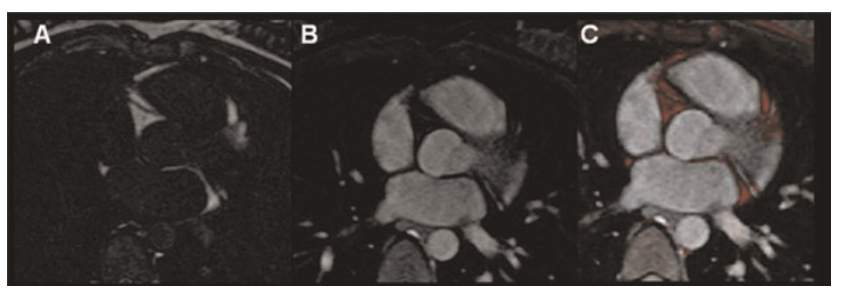

A) Fat image; B) blood pool image; C) fusion of fat and blood pool with fat highlighted in red. 
Figure 2 (abstract PI28)

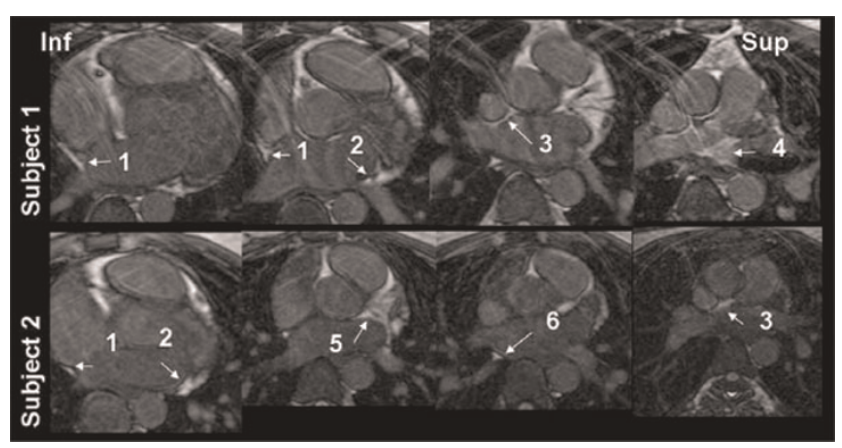

Four axial slices (inferior to superior) of the LA are shown in two subjects, with fat and blood pool images combined, and fat highlighted as brighter signal. Fat was present below and adjacent to the right inferior PV (labeled \#I in Figure 2, the posteromedial left atrial GP), below the left inferior PV (\#2 the posterolateral left atrial GP), adjacent to the right superior PV near the superior vena cava (\#3, the superior right atrial GP), above the left superior PV (\#4, the superior left atrial GP), along the anterior wall (\#5), and above the right inferior PV (\#6, the posterior right atrial GP).

Results: Figure 2 shows representative slices (from inferior to superior) from two healthy subjects, with arrows pointing to typical locations of fat. All subjects had fat in proximity to locations of the posterolateral left atrial GP, posterior right atrial GP, and superior right atrial GP, 4/6 subjects had fat around the superior left atrial GP, and 5/6 subjects had fat around the posteromedial left atrial GP.

Discussion and conclusion: This preliminary CMR study shows the ubiquity of fat around the LA and adjacent to the PV ostia, concentrated in previously described locations. Other CMR methods for detecting fat - from simple 2D balanced SSFP slices to 3-point Dixon techniques may also be useful. The ability to detect regional fat distribution may contribute to planning for ablation in AF patients.

References

I. Scherlag BJ, et al: J Electrocardiol 2006, 39(4 Suppl):SI80-3.

2. Pokushalov J: Current Opin Cardiology 2008, 23:55-59.

3. Armour JA, et al: Anatomical Record 1997, 247:289-298.

\section{PI29}

Volume tracking - a novel method for visualization and quantification of intracardiac blood flow from 3D time resolved phase contrast MRI

Johannes Töger, Martin Ugander, Håkan Arheden and Einar Heiberg

Cardiac MR Group, Dept. of Clinical Physiology, Lund, Sweden

Journal of Cardiovascular Magnetic Resonance 2009, I I(SuppI I):PI 29

Introduction: Three-dimensional time resolved flow measurement may provide greater insights into cardiovascular dynamics, as the detailed interactions of blood, myocardium, valves and vessels are not completely understood. Since three-dimensional flow data is highly complex, better methods providing both an intuitive visualization and quantification are needed. Existing methods have focused on tracking particles. However, following a volume may be more intuitive and easier to interpret visually.
Figure I (abstract PI29)
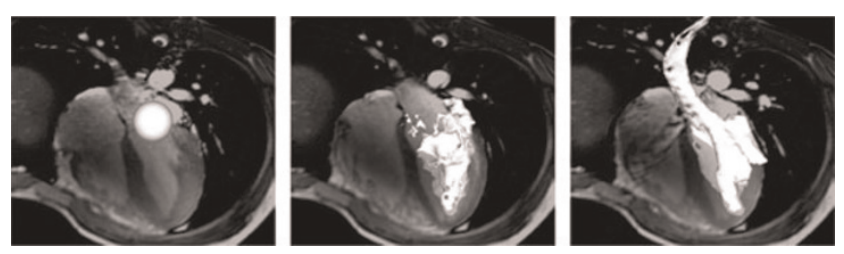

An example of Volume Tracking visualization in a healthy volunteer. Left: Early diastole, a white spherical volume of blood positioned near the mitral annulus. Middle: Late diastole, the same blood volume has now deformed as it has entered the left ventricle. Right: Early systole, additional deformation of the volume as it is ejected into the aorta.

More importantly, it may also enable quantification of physical parameters of the tracked volume, which may provide novel physiological insight.

Purpose: The purpose of the study was to develop and test the feasibility of Volume Tracking, a new method for visualization and quantification of three-dimensional intracardiac blood flow. Specifically, we sought to develop a method which offers the possibility of tracking volumes, as opposed to previous methods where only particles can be tracked.

Methods: Four healthy volunteers underwent acquisition of three-dimensional time resolved phase contrast velocity MRI data using a Philips $3 \mathrm{~T}$ scanner. State-of-the-art numerical methods were used to solve a novel representation of fluid transport which implicitly follows blood flow through the heart. Visualization was undertaken using the software Ensight (CEI, USA).

Results: The proposed Volume Tracking method is feasible. Volumes of arbitrary shapes and sizes can be interactively visualized and tracked over the cardiac cycle. Intuitive visualiza-

Figure 2 (abstract PI29)

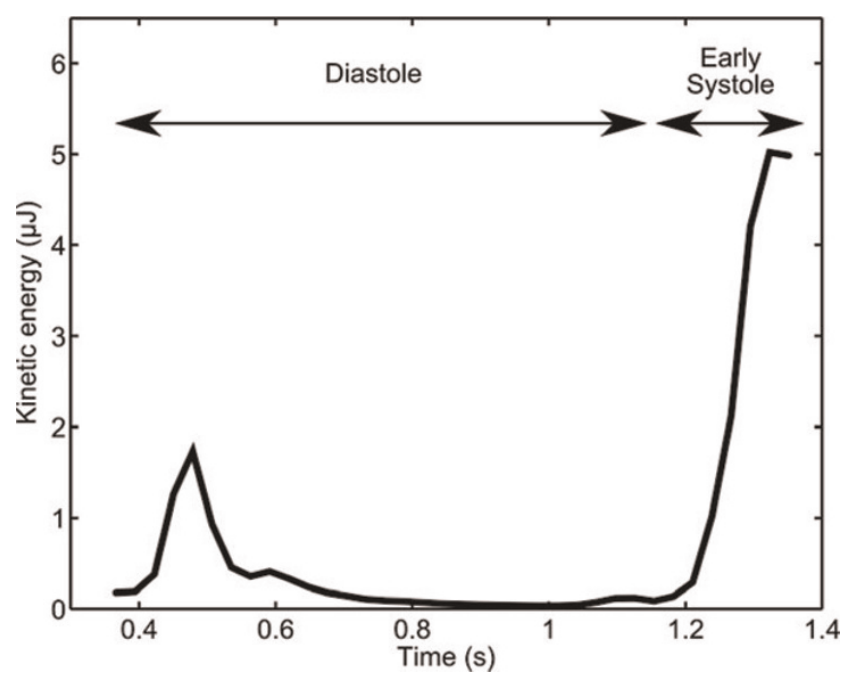

Results for quantification of the kinetic energy over time for the tracked volume from Figure I. Note the slight increase in kinetic energy in the volume during early diastole and the sizeable increase in kinetic energy during early systole. 
tion (Figure I) and quantification of physical parameters such as kinetic energy (Figure 2) was achieved.

Conclusion: A new method for visualization and quantification of intracardiac blood flow has been developed. The major advantage compared to the existing particle trace technique is the ability to quantify kinetic energy, momentum and other physical parameters of the blood flow. The method also offers real time interactivity and intuitive visualization of volumes moving through the heart. Once the data has been computed, exploring volumes of arbitrary shapes and sizes is straightforward. This new quantification and visualization method may facilitate the analysis of three dimensional flow and may bring additional physiological insight into cardiac blood flow.

\section{PI30}

Time-resolved MRA using sliding window reconstruction for evaluation of renal arterial anatomy and perfusion

Aoife N Keeling, Ravi K Singh, Cormac Farrelly, Hyun Jeong, Ty A Cashen, John Sheehan, Tim J Carroll and James $C$ Carr

Northwestern Memorial Hospital, Chicago, IL, USA

Journal of Cardiovascular Magnetic Resonance 2009, I I(SuppI I):PI 30

Introduction: Time-resolved contrast enhanced magnetic resonance angiography, if fast enough, can not only provide arterial anatomical and pathological detail, but can also follow the first pass of contrast through visceral parenchymal tissue in order to evaluate vascular flow dynamics or perfusion.

Purpose: To evaluate the feasibility of a new magnetic resonance imaging (MRI) technique that would provide unified anatomic and functional evaluation of the kidneys in a single scan with a single dose of Gadolinium contrast. We hypothesize that dynamic mask-mode subtraction yields perfusion weighted images of the renal parenchyma.

Methods: A new magnetic resonance angiography (MRA) pulse sequence, with a high frame rate, capable of simultaneous determination of renal arterial anatomy and pathology, and renal perfusion, was developed. Eleven healthy volunteers and one renal transplant patient were recruited to undergo MRI examination using a radial three-dimensional FLASH acquisition with sliding window view-share reconstruction (in-plane spatial resolution was $1.1 \times 1.1 \mathrm{~mm}^{2}$ ) on a $1.5 \mathrm{~T}$ Siemens MRI scanner. A single dose of Magnevist (gadopentetate dimeglumine, Berlex, Montville, NJ, USA) was administered intravenously. Images were processed with a dynamic mask-mode subtraction which has been shown to be beneficial in artery-vein separation. The raw data was reconstructed offline with a sliding window and a sliding mask subtraction technique generating sequential angiographic images at a rate of 3 frames per second. Perfusion analysis was also performed offline by two experienced diagnostic radiologists, implementing the upslope and deconvolution methods, as well as using the maximum value subtracted image. All reconstructions and analyses were performed using Matlab software. The maximum value subtraction image was compared on a pixel-by-pixel basis to the upslope and deconvolution methods using correlation and Bland-Altman plots.

Results: The technique produced diagnostic quality angiographic images and perfusion maps in all volunteers and the renal transplant patient. The maximum value subtracted image provided an accurate estimate of renal perfusion as compared to upslope and deconvolution methods using correlation plots $(r=0.98,0.94 ; m=0.96,0.89)$ and Bland-Altman plots (mean bias $=1.7 \%, 2.1 \% ; p<0.05)$.

Conclusion: This technique provides anatomic and functional evaluation of the kidneys with a single scan and single dose of contrast that would currently require multiple scans or examinations that require ionizing radiation. The maximum value subtracted image provides an accurate estimate of renal perfusion compared to the established upslope and deconvolution methods. This technique could be applied clinically to a population of potential renal transplant donors, providing arterial anatomic information for surgical planning as well as pretransplant functional evaluation.

\section{PI3 I}

Relationship between cardiac allograft vasculopathy and left ventricular diastolic dysfunction assessed by cardiac magnetic resonance imaging in heart transplant recipients Haruhiko Machida', Shinichi Nunoda', Kiyotaka Okajima', Kazunobu Shitakura', Akihiko Sekikawa', Yutaka Kubo', Kuniaki Otsuka', Masami Hirata', Shinya Kojima', Ai Masukawa', Satoru Morita', Kazufumi Suzuki', Mikihiko Fujimura', Eiko Ueno' and Yoshiaki Komori ${ }^{2}$ 'Tokyo Women's Medical University Medical Center East, Tokyo, Japan

${ }^{2}$ Siemens-Asahi Medical Technologies Ltd., Tokyo, Japan

Journal of Cardiovascular Magnetic Resonance 2009, I I(SuppI I):PI 3 I

Introduction: Cardiac allograft vasculopathy (CAV), a major late complication that limits long-term survival of heart transplant recipients, is typically characterized as a diffuse concentric intimal hyperplasia of the coronary artery. Invasive coronary angiography/intracoronary ultrasound (ICUS) are widely performed for CAV screening of patients with no ischemic symptoms from their denervated hearts before congestive heart failure, cardiac arrhythmia, or sudden death occurs. This vasculopathy usually accelerates left ventricular diastolic dysfunction before systolic dysfunction. Cine images on cardiac magnetic resonance (CMR) examination, the most accurate test for cardiac functional analysis in the clinical setting, easily quantify peak filling rate (PFR) as an index of left ventricular diastolic function. This measurement can noninvasively predict early-stage CAV and be useful for risk stratification and adequate patient management. On a single comprehensive CMR examination, we can also assess asymptomatic systolic dysfunction of the left ventricle from cine images and myocardial infarction or scar formation from late gadolinium-enhanced (LGE) images.

Table I (abstract PI3I) Relationship between cardiac allograft vasculopathy and parameters for left ventricular function

\begin{tabular}{llll}
\hline & CAV positive & CAV negative & P value \\
\hline PFR $(E D V / s e c)$ & $3.63 \pm 0.90$ & $4.43 \pm 0.84$ & 0.01 \\
EF $(\%)$ & $58.1 \pm 4.8$ & $58.8 \pm 7.1$ & 0.85 \\
SV $(\mathrm{mL})$ & $43.0 \pm 12.3$ & $49.4 \pm 17.6$ & 0.16 \\
$\mathrm{CO}(\mathrm{L} / \mathrm{min})$ & $3.41 \pm 0.81$ & $3.83 \pm 1.54$ & 0.46 \\
\hline
\end{tabular}


Purpose: We investigated the clinical feasibility of CMR imaging for noninvasively screening for CAV in asymptomatic recipients of heart transplants. Especially, we assessed the significance of left ventricular PFR value as a marker of early-stage CAV over several parameters of the systolic function and LGE by CMR imaging. Methods: Between June 2006 and June 2008, 38 asymptomatic recipients of heart transplants $(25$ men, 13 women, aged $37.2 \pm 14.9$ years) underwent both CMR and ICUS $8.5 \pm$ 4.4 years after heart transplantation. We measured PFR normalized to end-diastolic volume and several parameters for systolic function of the left ventricle, including ejection fraction (EF), stroke volume (SV), and cardiac output (CO), by steady-state free precession cine CMR imaging with 20 sampling phases during one cardiac cycle. We also evaluated intramyocardial LGE on the CMR examinations. According to Stanford classification based on intimal wall morphology assessed by ICUS [I], we classified recipients of grade $0-2$ as negative and grade $3-4$ as positive for CAV and compared the values of PFR, EF, SV, and CO between the 2 groups using Mann-Whitney $U$ test. $P<0.05$ was considered statistically significant. Furthermore, we calculated receiver operating characteristic (ROC) curve in the relationship between PFR value and CAV as defined by ICUS.

Results: Using ICUS, we classified 20 patients (53\%) positive and $18(47 \%)$ negative for CAV. There was no significant difference in the values for EF $(58.1 \pm 4.8 \%$ versus $58.8 \pm 7.1 \%$, $P=0.85) ; \mathrm{SV}(43.0 \pm 12.3 \mathrm{~mL}$ versus $49.4 \pm 17.6 \mathrm{~mL}, P=0.16)$; and $\mathrm{CO}(3.4 \mathrm{I} \pm 0.8 \mathrm{I} \mathrm{L} / \mathrm{min}$ versus $3.83 \pm \mathrm{I} .54 \mathrm{~L} / \mathrm{min}, P=0.46)$. No patient revealed intramyocardial LGE.

In contrast, the PFR value was significantly lower in the positive $(3.63 \pm 0.90 \mathrm{EDV} / \mathrm{sec})$ than negative group $(4.43 \pm 0.84 \mathrm{EDV} /$ sec, $P=0.01)$. Area under the ROC curve was $0.759(95 \%$ confidence interval: 0.587 to 0.882 ). When PFR cut-off value was 3.65, CAV sensitivity was $58.2 \%$ and specificity, $79.8 \%$. Table I.

Conclusion: The presence of CAV significantly enhances diastolic dysfunction of the left ventricle. For asymptomatic recipients of heart transplants, PFR measurement with CMR provides noninvasive prediction of CAV, which precedes systolic dysfunction and myocardial infarction or scar formation, and PFR is a feasible tool for decision-making in managing those patients. Reference

I. St Goar, et al: Circulation 1992, 85:979-987.

\section{P I 32}

Local coronary endothelial dysfunction varies with the extent of coronary disease: a 3 T MRI study Allison Hays, Sebastian Kelle, Glenn A Hirsch, Gary Gerstenblith, Robert G Weiss and Matthias Stuber Johns Hopkins, Baltimore, MD, USA

Journal of Cardiovascular Magnetic Resonance 2009, I I(SuppI I):PI 32

Introduction: Endothelial-dependent coronary artery vasoreactivity is an important indicator of vascular function and predicts cardiovascular events [I]. Non-invasive measures of endothelial dysfunction are typically obtained in the brachial arteries, which are exposed to systemic risk factors but which rarely develop severe atherosclerosis or plaque rupture. Endothelial injury plays a critical, causal role in the development and progression of local atherosclerosis, a regionally heterogeneous process in the coronary arteries. We therefore posit that local endothelial function varies throughout the coronary tree in patients with disease and may
Figure I (abstract PI32)

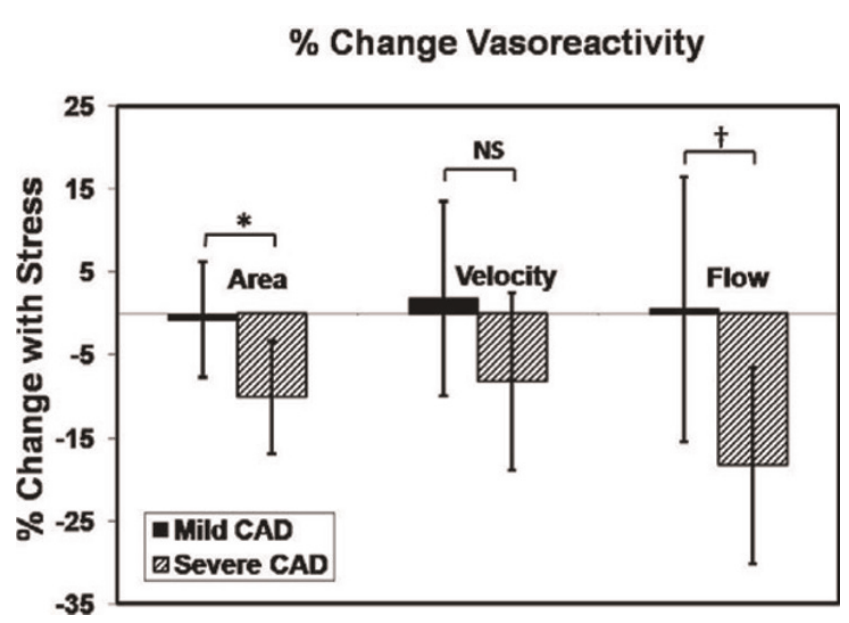

Percent change (mean \pm SD) from baseline in coronary artery area, peak diastolic coronary flow velocity and flow during isometric handgrip stress for arteries with mild vs. severe coronary disease. $\left({ }^{*} p=0.007, \dagger\right.$ $P=0.02$ mild vs. severe $C A D$ )

contribute to local atherosclerosis. By means of previously described non-invasive $3 \mathrm{~T} \mathrm{MRI}$ methods combined with isometric handgrip to assess endothelial-dependent coronary vasoreactivity [2], we therefore sought to test the hypothesis that local endothelial function varies within the coronary vasculature and is more deranged in regions with significant coronary atherosclerosis than in those with mild disease.

Methods: Eleven patients $(59 \pm 6.2$ years, mean \pm SD, 3 women) with $x$-ray-defined coronary artery disease (CAD) were recruited and imaged using a 3 T MRI scanner (Achieva, Philips, Best, NL). In each patient, two arteries were imaged in cross-section: one artery (LAD or RCA) with severe stenosis $(\geq 60 \%)$ and the contralateral artery with no significant stenosis $(<30 \%)$ by $x$-ray angiography. Baseline imaging at rest for crosssectional coronary artery area measurements was followed by coronary flow velocity-encoded MRI for flow velocity measurements. Alternating anatomical and velocity-encoded images were collected at baseline, and during 4 minutes of continuous isometric handgrip (at $30 \%$ of maximum grip strength). Three patients additionally received $0.4 \mathrm{mg}$ of sublingual nitroglycerin and images were collected after five minutes. MRI parameters for anatomical and flow imaging respectively were: echo time $(\mathrm{TE})=\mathrm{I} .5 \mathrm{~ms} / 3.5 \mathrm{~ms}$, radiofrequency $(\mathrm{RF})$ excitation angle $=$ $20^{\circ}$ and spectral spatial excitation (both), breath-hold duration $\sim 17-23 \mathrm{sec}$, acquisition window $=10 \mathrm{~ms} / 27 \mathrm{~ms}$, repetition time $(T R)=14 \mathrm{~ms} / 34 \mathrm{~ms}, 2 \mathrm{I} / \mathrm{I} \mathrm{I}$ spiral interleaves/cine frame, spatial resolution $=0.89 \times 0.89 \times 8.0 \mathrm{~mm}^{3} / 0.8 \times 0.8 \times 8 \mathrm{~mm}^{3}$ with velocity encoding $=35 \mathrm{~cm} / \mathrm{second}$. Blood pressure and heart rate were recorded at rest and during handgrip. Images were analyzed for cross-sectional area changes (Cine version 3.15.17, General Electric, Milwaukee, WI) and for peak diastolic coronary flow velocity (FLOW Version3.0, Medis, NL). Coronary flow $(\mathrm{mL} / \mathrm{min})$ was calculated as: coronary cross-sectional area $\times$ coronary artery peak diastolic velocity $\times 30$ [3].

Results: Nine patients had adequate image quality in both arteries for coronary area measurements and eight patients had sufficient image quality for flow velocity measurements. The 
mean percent increase in rate pressure product (heart rate*systolic blood pressure) with stress was $23 \pm 10.7 \%(p<0.00$ I vs. baseline). Isometric handgrip stress induced significant declines in cross-sectional area in arterial segments with severe coronary disease (baseline $15.8 \pm 3.3$ vs. stress $14.2 \pm 2.7 \mathrm{~mm}^{2}$, $P=0.005)$. There was no significant change in coronary area with stress in the arteries with minimal disease (baseline $12.5 \pm 2.8$ vs. stress $12.3 \pm 2.8 \mathrm{~mm}^{2}, \mathrm{p}=0.58$ ). All arterial segments dilated with nitroglycerin in the three patients that were studied. Although there was no significant change in peak diastolic coronary flow velocity with stress in both severe and mildly diseased arteries, there was a significant reduction in flow with stress in arteries with severe disease compared to the mildly diseased arteries (Figure I).

Conclusion: Using 3 T MRI combined with isometric handgrip exercise to quantify coronary endothelial-dependent vasoreactivity, we observe differences in local endothelial function throughout the coronary tree with more severe impairment of endothelial function in regions with more advanced coronary artery disease. This occurs despite an intact vasodilatory response to nitroglycerin. The present findings demonstrate local differences in coronary endothelial function related to disease severity and raise concerns about the ability of a single measure of endothelial function in a peripheral vessel to accurately reflect the spectrum of endothelial function present in diseased coronary arteries. This novel noninvasive approach may offer important insights into the pathobiology of atherosclerosis, the local progression of CAD and the identification of high risk lesions.

References

I. Schachinger V, Britten MB and Zeiher AM: Prognostic impact of coronary vasodilator dysfunction on adverse long-term outcome of coronary heart disease. Circulation 2000, I 0 I (16): I899-1906.

2. Hays AG, Kelle S, Hirsch GA, Gerstenblith G, Weiss RG and Stuber M: Non-invasive measurement of coronary artery flow velocity at rest and during handgrip stress in healthy subjects using 3 T MRI. Proceedings, Society of Cardiovascular Magnetic Resonance 2008, 103.

3. Doucette JW, Corl PD, Payne HM, Flynn AE, Goto M, Nassi $M$ and Segal J: Validation of a Doppler guide wire for intravascular measurement of coronary artery flow velocity. Circulation 1992, 85(5):|899-191।.

\section{P I 33}

Can we measure iron overload in the heart using in vivo MRI T2*?

Taigang $\mathrm{He}^{\mathrm{l}}$, John-Paul Carpenter ${ }^{\prime}$, Sutipong Jongjirasiri ${ }^{2}$, Jiraporn Laothamatas ${ }^{2}$, Suthat Fucharoen ${ }^{3}$, David Firmin ${ }^{i}$ and Dudley Pennell'

'CMR Unit, Royal Brompton Hospital and Imperial College London, London, UK

${ }^{2}$ Ramathibodi Hospital, Bangkok, Thailand

${ }^{3}$ Thalassemia Research Centre, Mahidol University, Bangkok, Thailand

Journal of Cardiovascular Magnetic Resonance 2009, I I(SuppI I):PI 33

Introduction: The measurement of cardiac iron is important for preventing heart failure and monitoring iron-chelating treatment in thalassemia patients. An MRI T2* technique has been developed and clinically validated for this purpose [I, 2, 3]. A recent advance of a black blood T2* sequence shows improved
Figure I (abstract PI33)

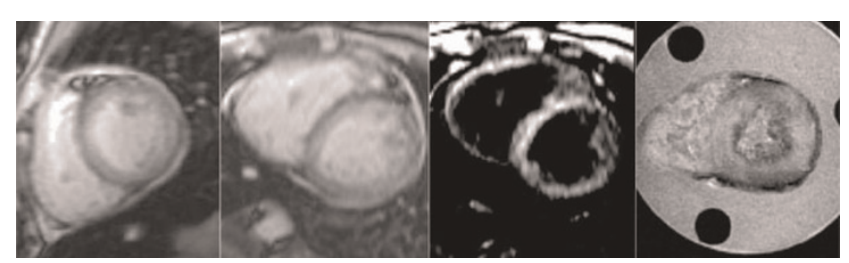

Example images (from left to right) acquired in Bangkok (TE = 2.I ms), London (bright and black preparation, TEs $=2.2 \mathrm{~ms}$ ), and ex vivo scan of a mid-ventricular slice $(\mathrm{TE}=2.5 \mathrm{~ms}$ ), respectively.

reproducibility [4] and accuracy [3]. However, this technique has not been calibrated against myocardial biopsy. Apart from ethical problems, biopsy is not ideal for assessment of myocardial iron overload because small samples of heterogeneous iron deposition may not accurately reflect the segmental iron content in the myocardium. An alternative approach would be an autopsy study in hearts donated after death or after cardiac transplantation in thalassemia patients. This autopsy study is ongoing aiming at calibration of MRI T2* against iron concentration in the heart [5]. There remains a particular issue as to, however, whether the in vivo T2* measurements equal ex vivo measurements.

A recent study [6] has suggested a significant difference between the in vivo and ex vivo measurement of $\mathrm{R} 2 *(\mathrm{I} / \mathrm{T} 2 *)$. The reasons for this discrepancy may not be clear, however, there existed body/tissue temperature difference in the reported study, which can contribute I.5\% per degree Celsius to this discrepancy [7]. In addition, the model used for T2* measurement may give rise to further errors [3]. Purpose: To compare in vivo and ex vivo myocardial T2* measurements at a comparable temperature and using the correct model.

Methods: A 23-year-old Thai female thalassemia majorpatient with longstanding cardiac and liver iron overload was studied. The patient underwent MRI scan using the bright blood T2* sequence [2] (twice for reproducibility) in Bangkok on a $1.5 \mathrm{~T}$ clinical scanner (GE Signa), again in London 9 days later on a I.5 T one (Siemens Sonata), using the same sequence. The patient was also scanned using the black blood T2* sequence [4]. The patient died two weeks later. With full ethical approval, the donated heart was formalin fixed before being sliced axially. A

Figure 2 (abstract PI33)
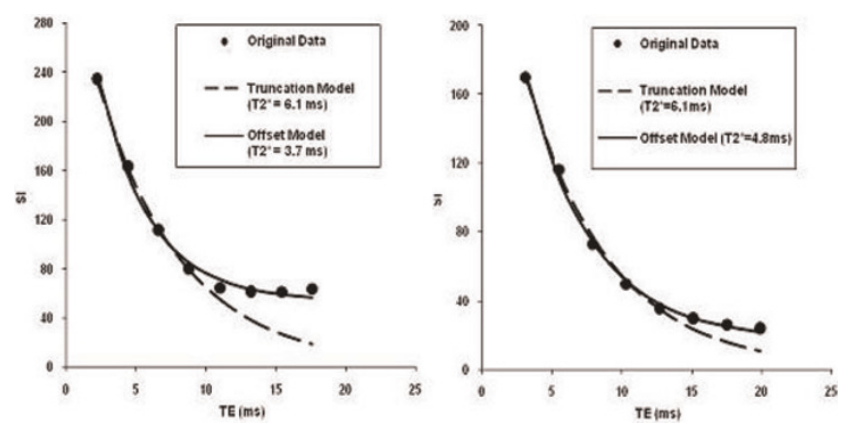

Examples of $\mathrm{T} 2 *$ curve fitting using both truncation and offset models. Left: In vivo scan data; Right: Ex vivo heart data. 
Table I (abstract PI33) Summary of T2* measurements using both truncation and offset models

\begin{tabular}{|c|c|c|c|c|c|c|c|}
\hline $\mathrm{T} 2 *(\mathrm{~ms})$ & Bangkok Baseline & Bangkok Repeat & London Bright Blood & London Black Blood & ex vivo & Mean & STD \\
\hline Truncation Model & 6.0 & 6.1 & 6.1 & 6.0 & 6.1 & 6.1 & 0.1 \\
\hline Offset Model & 4.6 & 6.1 & 3.7 & 4.6 & 4.8 & 4.8 & 0.9 \\
\hline
\end{tabular}

custom-made Perspex plinth was used to hold each of the slices which were then scanned at $37^{\circ} \mathrm{C}$. Main perameters [2] are TEs from $2.47 \mathrm{~ms}-16.9 \mathrm{~ms}$ (increment $2.01 \mathrm{~ms}$ ) and a resolution of $1.2 \times 1.2 \times 5 \mathrm{~mm}^{3}$.

Results: Figure I shows example images acquired at Bangkok and London including the ex vivo scan. Figure 2 shows two curve fitting examples demonstrating the discrepancy in $\mathrm{T} 2 *$ measurement using both the truncation and the offset model. The in vivo and ex vivo measurements, however, agree well using the truncation model. Table I summaries all T2* measurements using different models. The mean value and standard deviation are also calculated.

Conclusion: This study has demonstrated the good reproducibility of the myocardial T2* measurement using the truncation model, where an in vivo T2* measurement from the patient data agrees with ex vivo one of the fixed heart. These data, along with the calibration study [5], support the clinical use of myocardial T2* in iron overload syndromes.

\section{References}

I. Anderson I LJ, et al: Eur Heart J 2001, 22:2171-2179.

2. Westwood M, et al: J Magn Reson Imag 2003, 18:33-39.

3. He T, et al: Magn Reson Med 2008 in press.

4. He T, et al: J Magn Reson Imaging 2007, 25:1205-I209.

5. Carpenter JP, et al: Abstract in SCMR 2009.
6. Ghugre NR, et al: Magn Reson Med 2006, 56:68I-686.

7. He T, Smith GC, Carpenter J-P, Mohiaddin RH, Pennell DJ and Firmin DN: A phantom study of temperaturedependent MRI T2* measurement. J Cardiovasc Magn Reson 2009, I I(SuppI I):PI 47.

\section{PI34}

T2* heterogeneity detected by CMR could be related to myocardial iron distribution in Thalassemia patients

Vincenzo Positano', Alessia Pepe',

Maria Filomena Santarelli', Anna Ramazzotti', Daniele De Marchi', Antonella Meloni', Eliana Cracolici',

Domenico G D'Ascola ${ }^{3}$, Luigi Landini ${ }^{4}$

and Massimo Lombardi ${ }^{\prime}$

I "G Monasterio" Foundation and Institute of Clinical

Physiology, CNR, Pisa, Italy

${ }^{2}$ University of Palermo, Palermo, Italy

${ }^{3}$ A.O. "Bianchi-Melacrino-Morelli", Reggio Calabria, Italy

${ }^{4}$ University of Pisa, Pisa, Italy

Journal of Cardiovascular Magnetic Resonance 2009, I I(SuppI I):PI 34

Introduction: A large percentage of thalassemia major (TM) patients shows a significant heterogeneity of segmental distribu-

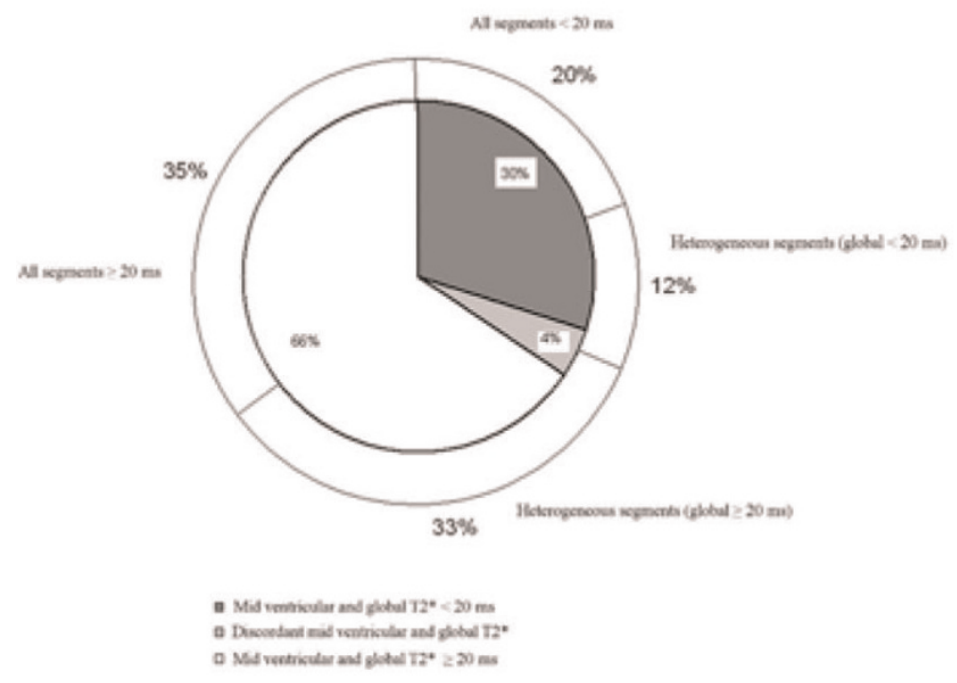

A

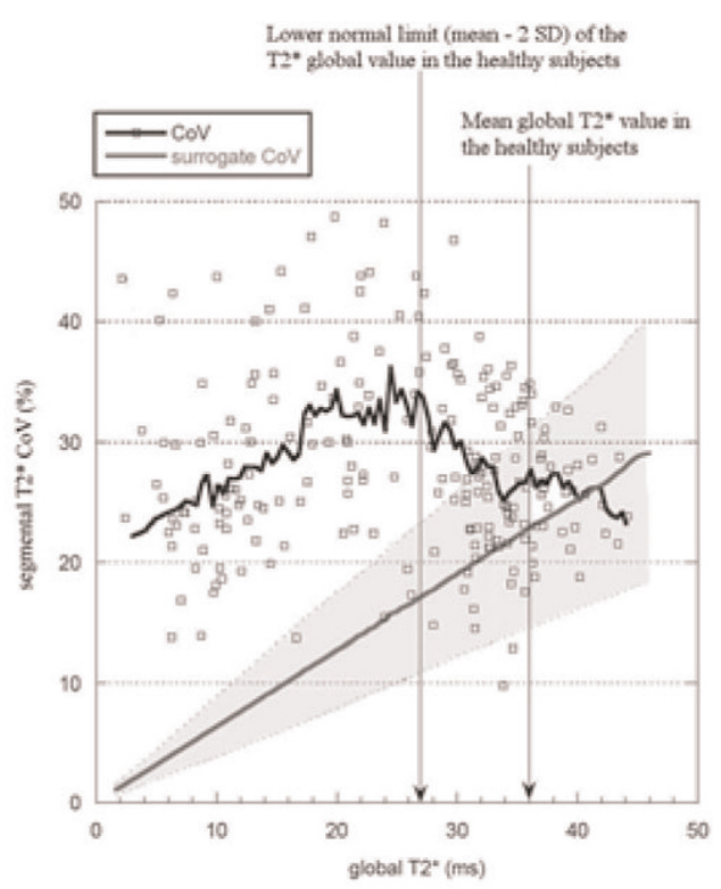

B 
tion of $\mathrm{T}^{*}$ values in myocardium, measured by multislice multiecho T2* cardiac magnetic resonance (CMR). This finding is in agreement with previous histological studies that have detected heterogeneous iron deposition in hemochromatotic hearts. However, it is not yet clear if that represented true heterogeneous iron density or if it could have been generated by geometric and susceptibility artefacts.

Purpose: The purpose of this study is to investigate myocardial T2* heterogeneity in TM patients by CMR, and to determine if it is related to inhomogeneous iron overload distribution.

Methods: 230 TM patients consecutively affered to our laboratory were retrospectively studied. Three short-axis views (basal, medium, and apical) of the left ventricle (LV) were obtained by multislice multiecho T2* CMR. T2* segmental distribution was mapped on a I6-segment LV model. The level of heterogeneity of the T2* segmental distribution on each patient was evaluated by the coefficient of variation. Measured heterogeneity was compared with that of a surrogate data set obtained from measurements of subjects without iron overload, to determine whether the inhomogeneous segmental distribution of T2* could be generated only by susceptibility artefacts.

Results: In 45 (20\%) TM patients, segmental T2* values were all below the lower limit of normal $(20 \mathrm{~ms})$. In 104 (45\%) patients, T2* values were heterogeneous with respect to the normal threshold. Of these patients, $74 \%$ showed a normal T2* global value. Eighty-one (35\%) patients had all normal segments (A). T2* value heterogeneity assessed by the CoV in TM patients and in the surrogate data is shown in Figure IB. The white squares and the black line represent single patient measurements and the CoV average on all patients, respectively. The grey line represents the mean CoV of the surrogate data with the mean \pm 2 SD limits (grey dotted lines). The mean and the normal lower limit of the T2* global value assessed in the healthy subjects are shown as well (vertical black arrows). T2* heterogeneity for patients without iron overload was compatible with the hypothesis that the heterogeneity was generated by susceptibility artefacts. Below the lower limit of normal for global T2*, the heterogeneity abruptly increased and could not be explained by artefactual effects.

Conclusion: A true heterogeneity in iron overload distribution may be present in TM patients. Heterogeneity seemingly appears in the borderline myocardial iron and stabilizes for moderate to severe iron burden.

\section{PI35}

Baseline correction of phase-contrast images in congenital Cardiac Magnetic Resonance Imaging Brian J Holland, Beth F Printz and Wyman W Lai Children's Hospital of New York, New York, NY, USA

Journal of Cardiovascular Magnetic Resonance 2009, I I(SuppI I):PI 35

Introduction: Phase-contrast flow measurements have been shown to accurately quantify blood flow in subjects with structurally normal hearts and those with congenital heart disease (CHD). There are a number of potential sources of error, however, in phase-contrast CMR flow measurements, including phase offset errors due to local non-compensated eddy currents. Wolff and colleagues recently reported on the clinical application of phantom correction of phase offset errors in adult volunteers with structurally normal hearts [I]. The effect of phantom correction on phase-contrast flow measurements in patients with known or suspected heart disease has not previously been reported.

Purpose: To assess whether phantom correction significantly changed flow measurements by phase-contrast imaging in patients referred to a congenital CMR program.

Methods: The clinical congenital cardiac magnetic resonance imaging database at a single institution was searched for patients who had examinations performed with phase contrast images using phantom correction. Examinations were performed on a GE Signa HDx I.5 T scanner using commercially available coils (GE Healthcare, Milwaukee, Wisconsin). Phase-contrast images were acquired perpendicular to the vessel of interest using orthogonal long-axis views of the vessel. Breathe-through images were obtained using the commercially resident FastCine PC pulse sequence with the Venc determined by clinical parameters. The phase-contrast sequences were each repeated on a stationary fluid phantom with an ECG simulator to establish a baseline of zero velocity. Based on the clinical protocol, phasecontrast images of flow were obtained in the ascending aorta (AAO), main pulmonary artery (MPA), right pulmonary artery (RPA), and/or left pulmonary artery (LPA) and analyzed with and without phantom correction using GE ReportCard software version 3.6. The ratio of pulmonary to systemic flow ( $Q p / Q s)$, percent flow to the RPA (QpR fraction), pulmonary regurgitation fraction (PR), and aortic regurgitation (AR) fraction were also calculated with and without phantom correction. Clinically significant changes in flow measurements with phantom correction were defined prior to analysis: a change in MPA or AAo flow $\geq 0.5 \mathrm{~L} / \mathrm{min} / \mathrm{m}^{2}$, change in RPA or LPA flow $\geq 0.25 \mathrm{~L} / \mathrm{min} / \mathrm{m}^{2}$, change in $Q_{p} / Q_{s} \geq 0.4$, change in $Q p R$ fraction $\geq 10 \%$, and change in $\mathrm{PR}$ or $\mathrm{AR}$ fraction $\geq 10 \%$. Marked changes in flow measurements were defined as double the amount of clinically significant change in each category - for example, a change in MPA flow $\geq 1.0 \mathrm{~L} / \mathrm{min} / \mathrm{m}^{2}$ or PR fraction $\geq 20 \%$.

Results: From May 2008 to September 2008, 89 patients (median age 17.5 years, range 0.3 to 58.7 years) were identified who had clinical CMR examinations using phase-contrast images with phantom correction. The patients were referred with the following diagnoses: 25 with tetralogy of Fallot (repaired), 17 miscellaneous, 12 other conotruncal diagnoses, II single ventricle, 9 shunt lesions, 9 cardiomyopathy/myocarditis, and 6 aortic coarctation. The number and percent of patients with clinically significant or marked changes in the phase-contrast measurements are listed in Table I.

Table I (abstract PI35) Phantom correction of phase-contrast measurements

\begin{tabular}{llll}
\hline Variable & N & $\begin{array}{l}\text { Clinically } \\
\text { Significant Change }\end{array}$ & $\begin{array}{l}\text { Marked } \\
\text { Change }\end{array}$ \\
\hline MPA flow (L/min/m2) & 67 & $29(43 \%)$ & $11(16 \%)$ \\
AAO flow (L/min/m2) & 85 & $32(38 \%)$ & $16(19 \%)$ \\
RPA flow (L/min/m2) & 38 & $9(24 \%)$ & $5(13 \%)$ \\
LPA flow (L/min/m2) & 33 & $17(52 \%)$ & $11(33 \%)$ \\
Qp/Qs & 64 & $12(19 \%)$ & $5(8 \%)$ \\
PR fraction (\%) & 28 & $7(25 \%)$ & $0(0 \%)$ \\
AR fraction (\%) & 8 & $3(38 \%)$ & $0(0 \%)$ \\
QpR fraction (\%) & 30 & $8(27 \%)$ & $3(10 \%)$ \\
\hline
\end{tabular}


Conclusion: Phantom correction in our study population resulted in clinically significant changes in 19\% to $52 \%$ of phasecontrast measurements in patients with known or suspected heart disease. There were marked changes in up to $33 \%$ of phasecontrast measurements. Clinically significant or marked changes were generally more common in measurements of output $\left(\mathrm{L} / \mathrm{min} / \mathrm{m}^{2}\right)$ as compared to regurgitant fractions or flow ratios. The potential effect of phantom correction of phase-contrast images may be important in clinical decision making for patients with congenital heart disease. The magnitude of change should be assessed on other CMR platforms and in additional patient groups.

\section{Reference}

I. Chernobelsy, et al: J Cardiovasc Magn Reson 2007, 9:68I-685.

\section{PI36}

\section{Perfusion MRI for monitoring therapy effects in experimental chronic limb ischemia Harald Kramer, Steven Sourbron, Rabea Hinkel, Franziska Globisch, Christian Kupatt-Jeremias, Maximilian F Reiser and Bernd J Wintersperger University Hospital of Munich, Munich, Germany}

Journal of Cardiovascular Magnetic Resonance 2009, I I(SuppI I):PI 36

Introduction: Until today, imaging of lower extremity blood supply and perfusion is limited to the display of the macroscopic vasculature by angiographic methods like DSA, CTA or MRA. However, all these methods are limited to high spatial resolution imaging of the arterial or venous vessels but do not display changes at the microvascular level in regard to tissue perfusion. MR perfusion imaging may overcome this limitation in experimental and clinical settings and may allow non-invasive monitoring of modern therapeutic options including angiogenisis.

Purpose: To implement and evaluate the use of perfusion MR in monitoring angiogenetic therapies and their effects based on a rabbit model of iatrogenic induced chronic lower limb ischemia. Methods: MR perfusion imaging was performed in 8 rabbits with chronic lower limb ischemia after unilateral SFA excision at 3 Tesla (Magnetom Verio, Siemens Healthcare) using a 32element coil. In 4 animals MRI was performed at day 7 and in 4 animals at day 7 and 35 (after angiogenesis therapy). Multi-slice coverage was provided using a 2D-TurboFLASH technique at I.5 $\mathrm{s}$ temporal resolution with repeated measurements over $10 \mathrm{~min}$ after injection of $0.1 \mathrm{mmol} / \mathrm{kg}$ gadobutrol (Gadovist, Bayer Schering Pharma). One slice was placed through the aorta for

Figure I (abstract PI36)

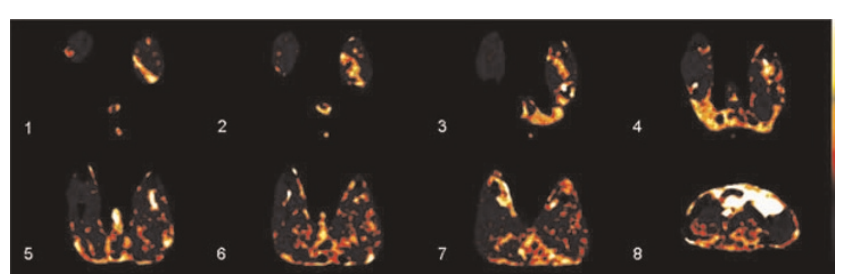

Colour coded plasma flow images over 8 slices $(I=$ distal, $8=$ central $)$ at day 7 after left side SFA excision. Images show significantly higher plasma flow on the ischemic side before angiogenetic therapy. measuring the Arterial Input Function (AIF) and 7 sliced covered the gluteal, thigh and knee musculature. Precontrast TI-mapping was performed using a variable TI GRE technique at identical slice locations (27 steps from $110 \mathrm{~ms}-5 \mathrm{~s}$ ). Data were postprocessed off-line using in-house written software PMI 0.3 including calculation of plasma flow (PF), plasma volume (PV) and extraction flow (EF) based on a 2-compartmental model. TI mapping data were used to convert signal-time courses to tracer concentration. Figure I.

Results: TI-maps produced robust results on with precontrast TI-values close to typical reference values at $3 \mathrm{~T}$. PF was significantly different between the non-ischemic and ischemic $\operatorname{limb}(|4.3 \pm 1| .5$ vs. $8.4 \pm 3.9 \mathrm{ml} / 100 \mathrm{ml} / \mathrm{min})$ on day 7 whereas there was no significant difference on day 35 after therapy $(9.3 \pm 2.3$ vs. $10.5 \pm 3.2 \mathrm{ml} / 100 \mathrm{ml} / \mathrm{min})$. EF showed similar findings with $2.17 \pm \mathrm{I} .7 \mathrm{I}$ vs. $1.7 \mathrm{I} \pm 1.6 \mathrm{ml} / 100 \mathrm{ml} / \mathrm{min}$ on day 7 and $2.3 \pm 1.98$ vs. $2.15 \pm 1.68 \mathrm{ml} / 100 \mathrm{ml} / \mathrm{min}$ on day 35 . PV did not show significant differences either before or after therapy. Conclusion: Chronic experimental limb ischemia results in significant changes of MR derived perfusion parameters, with reconstitution after experimental therapy. Initial data indicate that perfusion MRI provides a useful tool for the evaluation of muscle ischemia and of therapy effects.

\section{PI37}

Infarct size by cardiovascular magnetic resonance with delay enhancement as prognostic factor in the coronary artery disease: preliminary study

Leticia Castellanos Cainas, Sandra Graciela Rosales Uvera, Jaime Galindo Uribe, Jorge Vazquez La Madrid, Jorge Oseguera Moguel, Florencia Vargas Vorackova and Martha Morelos Guzman

National Institute of the Medical Science and Nutrition Salvador Zubiran, Mexico D.F., Mexico

Journal of Cardiovascular Magnetic Resonance 2009, I I(SuppI I):PI 37

Introduction: The magnetic resonance (MR) has taken nowadays a crucial role in the evaluation of ischemic heart disease, which is regarded as the technique of reference for the assessment of myocardial viability. Delay enhanced cardiovascular magnetic resonance (DE-CMR) is a specific marker of myocardial necrosis, using this technique can determine the presence of infarct localization, size and transmurality, parameters of great importance for determining treatment and prognosis.

Purpose: To determine if infarct size measured by DE-CMR is a prognostic factor for mortality in patients with ischemic heart disease.

Methods: Sixty eight patients were referred to cardiovascular magnetic resonance because of suspicion or knowledge of ischemic heart disease between September 2004 and September 2008. CMR imaging was performed using GE I.5 T system. Steady state free precession (SSFP) cine MR images were acquired in long and short axis orientation. Evaluation of functional parameters including end diastolic volume (EDV), end systolic volume (ESV), left ventricle ejection fraction (LVEF) and systolic volume (SV) indexed a body surface area (BSA).

Results: The average age of the study population was $65.6(+/-$ $10.7 \mathrm{SD})$ years, I2\% patients were in functional class III-IV NYHA, the mortality rate was $16.1 \%$. We evaluate 1156 segments and the $39.7 \%$ of this presented delay enhancement. 
Figure I (abstract PI37)

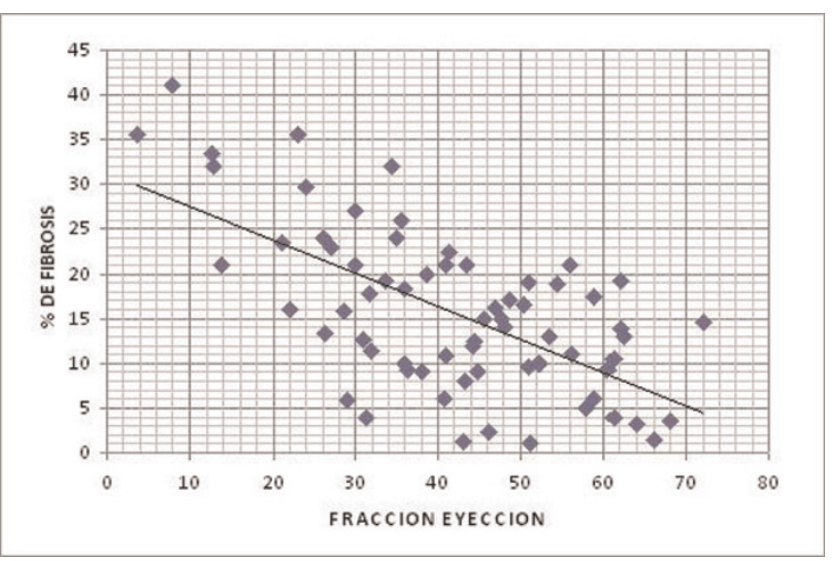

The myocardial infarction size was significantly higher in patients who died $(21.6 \%$ vs. $14.4 \% p=0.01)$. The relationship between infarct size, end-sistolic volume index, end-diastolic volume index, ejection fraction of left ventricle and systolic volume index, were statically significative $(p<0.001)$. The mayor adverse cardiac events (MACE) were presented in $90 \%$ of the group of death patients $(p=0.00 \mathrm{I})$. Figure $\mathrm{I}$.

Conclusion: Infarct size measured by delay enhanced cardiovascular magnetic resonance (DE-CMR) is a prognostic factor for mortality in patients with ischemic heart disease.

\section{P I 38}

Free-breathing steady-state free precession 3D coronary MRA: investigation of the dependency on the running direction of the vessel and the direction of the motion correction

Yuki Ohmoto', Rieko Ishimura ${ }^{2}$, Takashi Yoshida ${ }^{3}$, Yusuke Hamada ${ }^{3}$, Yoshinori Tsuji ${ }^{3}$, Junji Takahashi ', Shigehide Kuhara ${ }^{4}$, Sachiko Isono ${ }^{4}$, Ayako Ninomiya ${ }^{4}$, Hiroyuki Tsuji ', Yasuji Arase' and Shigeko Hara'

IToranomon Hospital Health management center, Minato-ku, Tokyo, Japan

${ }^{2}$ Toranomon Hospital Cardiology, Minato-ku, Tokyo, Japan

${ }^{3}$ Toranomon Hospital Radiology, Minato-ku, Tokyo, Japan

${ }^{4}$ Toshiba Medical Systems, Otawara, Tochigi, Japan

Journal of Cardiovascular Magnetic Resonance 2009, I I(SuppI I):PI 38

Introduction: Coronary magnetic resonance angiography (CMRA) is a very useful and safe technique for the screening of coronary artery disease.

However, the image quality of CMRA depends on the individual performing examination and has some dependency on the direction of the vessel. The CMRA is performed under free breathing while monitoring the position of the diaphragm and the motion correction is performed mainly in the superior inferior (SI) direction. Therefore there may be some dependency on the running direction of the vessel and the direction of motion correction. Our preliminary study suggested that the image quality obtained with oblique saggital acquisition (SA) is better than that by axial acquisition (AX) for evaluating lesions in the LMT to the left anterior descending artery (LAD).
Figure I (abstract PI38)
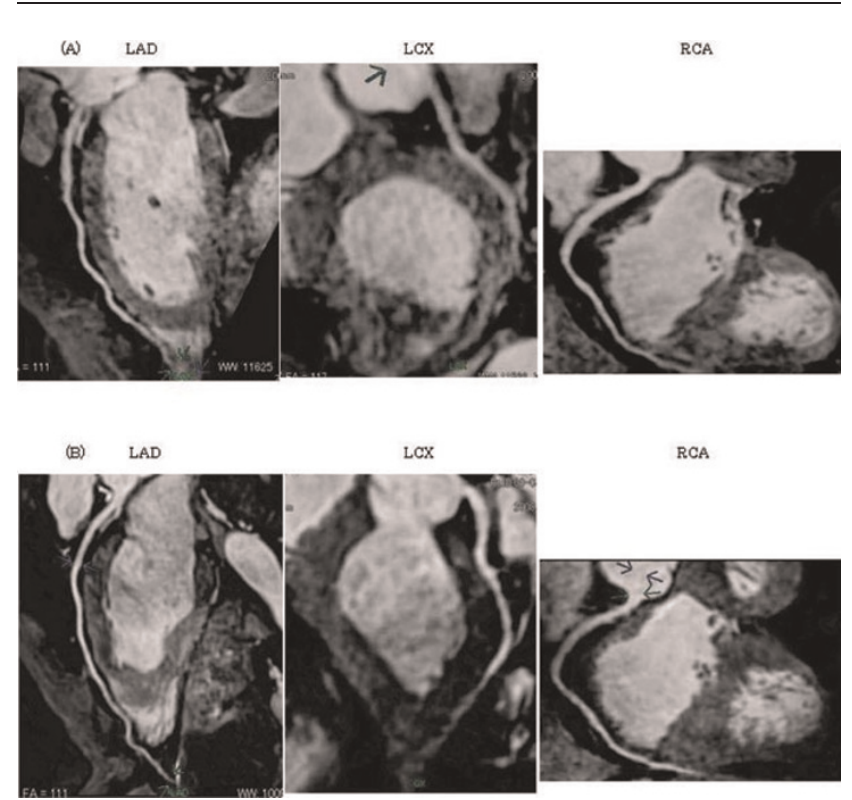

The images obtained by axial acquisition (A) and oblique sagittal acquisition (B).

Purpose: The purpose of this study was to evaluate I) the image quality, 2) the coronary length that can be evaluated, and 3) the scan time, between image acquisition by $A X$ and SA.

Methods: Eleven healthy volunteers ( 7 men, 4 women, mean age; $38 \pm$ II years) underwent CMRA at I.5 T (Toshiba Medical Systems Excelart Vantage ${ }^{\mathrm{TM}}$ powered by Atlas) using a l6-channel phased-array coil. The elements were arranged four-by-four at both the front and back, and the two element rows were used to cover the whole heart. Imaging was performed by applying RealTime Motion Correction (RMC) method to compensate the respiratory motion and corrected cardiac-triggered steady-statefree-precision (SSFP) sequence with fat suppression and T2 preparation. The imaging parameters were: TR/TE/FA $=4.3 \mathrm{~ms} /$ $2.2 \mathrm{~ms} / 120^{\circ}$, spatial resolution $=1.5 \times 1.5 \times 1.5 \mathrm{~mm}^{3}$. The $2 \mathrm{D}$ parallel imaging was applied with in phase and slice directions by a factor of 2.I and I.4. The data of the CMRA was transferred to the workstation with image reconstruction software (AZE VirtualPlace Fujin, AZE Ltd., Tokyo, Japan). The differences in the image quality were assessed by two experienced observers using randomized image pairs (four-point scale: $\mathrm{I}=$ poor, 2 = moderate, 3 = good, 4 = excellent). Motion artifacts and coronary delineations were used as criteria in the scoring of the quality. We also evaluated the available coronary lengths, that could be given a score of more than 2 by visual estimation, and the scan time.

Results: The image qualities of coronary arteries between $A X$ and SA were $3.5 \pm 0.5$ and $2.9 \pm 0.3$ for RCA $(p<0.05)$, $2.4 \pm 0.7$ and $3.6 \pm 0.5$ for LMT $(p<0.01), 2.6 \pm 0.7$ and $3.5 \pm 0.5$ for LAD ( $p<0.01$ ), $2.3 \pm 0.9$ and $3.2 \pm 0.9$ for LCX $(p<0.01)$ in each proximal lesion. The coronary lengths evaluable in the images acquired by $A X$ and $S A$ were | $36.4 \pm 42.0$ and $139.6 \pm 41.7 \mathrm{~mm}$ for RCA (n.s.), II $5.2 \pm 26.0$ and $124.4 \pm 25.4 \mathrm{~mm}$ for LAD (n.s.), and $79.5 \pm 30.5$ and $95.9 \pm 24.7 \mathrm{~mm}$ for $\operatorname{LCX}(\mathrm{p}<0.0 \mathrm{I})$. The scan 
time for image acquisition by $A X$ and SA were $680 \pm 159$ and $763 \pm 177$ seconds $(p<0.05)$, respectively. Figure I.

Conclusion: SA provides comparatively better image quality of coronary lesions in some arterial segments, especially the LMT and LAD. Although other lesions were not depicted as clearly as in the images acquired by $A X, S A$ provided valuable diagnostic information on lesions of the LMT. We suggest that SA and AX may be used to evaluate the coronary arteries in a complementary manner.

\section{PI39}

The beneficial cardiovascular effects of bariatric surgery are similar to dietary weight loss in obesity Oliver J Rider, Jane M Francis, Matthew D Robson, Monique Robinson, Steffen E Petersen, James P Byrne and Stefan Neubauer

University of Oxford, Oxford, UK

Journal of Cardiovascular Magnetic Resonance 2009, I I(SuppI I):PI 39

Objective: Our aim was to investigate the beneficial cardiovascular effects of bariatric surgery versus dietary weight loss in obesity.

Background: Bariatric surgery confers better long term weight management than dietary intervention, is an increasingly utilized method of weight management, and has been shown to reduce mortality. Despite this, no study to date has addressed the relative beneficial effects of these different weight loss approaches on cardiac and aortic structure and function. In view of this, we used cardiovascular magnetic resonance imaging (CMR) to compare the effect of either bariatric surgery or dietary weight loss on left (LV) and right ventricular (RV) structure, LV diastolic function and regional aortic elastic function in a group of healthy obese subjects.

Methods: 30 obese subjects, with no identifiable cardiovascular risk factors were recruited to the study. All subjects underwent cardiac MR imaging at I.5 T for the assessment of left ventricular mass $(\mathrm{g})$, left ventricular end-diastolic volume (EDV; $\mathrm{ml}$ ), stroke volume (SV; ml) and LV EF (\%). Aortic distensibility (AD) was assessed at three levels; the ascending (Ao) and proximal descending aorta (PDA) at the level of the pulmonary artery and the abdominal aorta (AA) The abdominal cine images were piloted perpendicular to the orientation of the abdominal aorta. In addition to this left ventricular diastolic function was assessed using volume time curve analysis.
CMR was performed at pre and post weight loss intervention, in 17 subjects on $\mathrm{Gl}$ index diet (BMI $35.2 \pm 5.5)$ and 13 subjects undergoing Bariatric surgery (BMI $45.6 \pm 6.0 \mathrm{~kg} / \mathrm{m}^{2}$ ).

Results: Both groups achieved significant weight loss, which tended to be greater after Bariatric surgery $(p=0.32)$. Fasting serum glucose, cholesterol, systolic \& diastolic blood remained within the normal range for both groups before and after intervention. Both groups had significant improvements in LV \& RV mass, LV \& RV EDV, diastolic filling rate and abdominal AD. When the improvements were normalized to percentage excess weight loss, there was no significant difference in the beneficial effects achieved in the surgical group to those seen in the dietary group for any of the cardiovascular parameters (Table I).

Conclusion: Irrespective of method, weight loss results in beneficial cardiovascular effects. Bariatric surgery and dietary weight loss provide similar levels of improvement in cardiovascular structure and function.

\section{PI40}

Left ventricular systolic dysfunction predicts incremental utility of delayed enhancement CMR vs. echocardiography for diagnosis of LV thrombus Kirsten $\mathrm{O} \mathrm{Healy}^{\top}$, Jason Chinitz , Raymond J Kim², Aqsa Shakoor', Michael I Ross', Matthew D Cham', James K Min', Michele Parker', Mary J Roman', Richard B Devereux' and Jonathan W Weinsaft' 'Weill Medical College of Cornell University, New York, NY, USA

${ }^{2}$ Duke Cardiovascular Magnetic Resonance Center, Durham, NC, USA

Journal of Cardiovascular Magnetic Resonance 2009, I I(SuppI I):PI40

Background: Delayed enhancement cardiac magnetic resonance (DE-CMR) imaging is well-validated as a highly accurate tool for left ventricular thrombus (LVT). In prior research studies, DE-CMR has been shown to provide improved diagnostic performance vs. echo for detection of LVT. However, in clinical practice, echo is widely used as an initial screening test for LVT. Identification of echo imaging variables that predict added utility of DE-CMR is important for optimization of clinical imaging strategies for LVT.

Methods: We studied markers that predicted improved LVT assessment by DE-CMR among patients with systolic heart failure or myocardial infarction enrolled in a multimodality imaging

Table I (abstract P I39)

\begin{tabular}{|c|c|c|c|}
\hline \multicolumn{2}{|c|}{ Absolute Values } & \multicolumn{2}{|c|}{ Normalized to $\%$ Excess Weight Loss $\left(\times 10^{-2}\right)$} \\
\hline Bariatric Cohort(N = I3) & Diet Cohort(N = I7) & Bariatric Cohort( $\mathbf{N}=13)$ & Diet Cohort( $\mathbf{N}=17)$ \\
\hline $61 \pm 22$ & $48 \pm 42$ & - & - \\
\hline $19 \pm 12^{*}$ & $10 \pm 11 *$ & $2.7 \pm 3.7$ & $2.9 \pm 2.5$ \\
\hline $13 \pm 14^{*}$ & $9 \pm 9 *$ & $1.7 \pm 3.2$ & $3.4 \pm 4.2$ \\
\hline $25 \pm 12^{*}$ & $19 \pm 8^{*}$ & $6.9 \pm 5.1$ & $4.8 \pm 3.4$ \\
\hline $4 \pm 17$ & $10 \pm 18$ & $0.3 \pm 3.2$ & $4.8 \pm 10$ \\
\hline $1.2 \pm 2.7$ & $0.7 \pm 2.3$ & $2.0 \pm 4.0$ & $3.0 \pm 11$ \\
\hline $1.9 \pm 1.9 *$ & $0.1 \pm 1.5^{* \#}$ & $3.0 \pm 3.0$ & $1.0 \pm 6.0$ \\
\hline $1.5 \pm 2.0 *$ & $1.8 \pm 1.8^{*}$ & $3.0 \pm 3.0$ & $7 \pm 10$ \\
\hline $0.78 \pm 0.77 *$ & $0.77 \pm 0.84 *$ & $0.14 \pm 1.0$ & $2.0 \pm 3.0$ \\
\hline
\end{tabular}

$*=p<0.05$ Pre vs Post Weight Loss \# = p $>0.05$ Surgical Vs Dietary Weight Loss. 
Table I (abstract P I40) Echo performance in relation to LV geometry

\begin{tabular}{|c|c|c|c|c|}
\hline & & $\begin{array}{l}\text { NC-Echo Concordance } \\
\text { with DE-CMR }\end{array}$ & $\begin{array}{l}\text { NC-Echo Discordance } \\
\text { with DE-CMR }\end{array}$ & $\mathbf{P}$ \\
\hline \multirow[t]{4}{*}{ Echo Parameters } & Ejection fraction (\%) & $41.2 \pm 13.6$ & $32.6 \pm 9.8$ & 0.003 \\
\hline & End-systolic diameter $(\mathrm{cm})$ & $4.7 \pm 0.9$ & $5.2 \pm 0.9$ & 0.03 \\
\hline & End-diastolic diameter $(\mathrm{cm})$ & $5.9 \pm 0.7$ & $6.1 \pm 0.8$ & .24 \\
\hline & Aneurysmal Dilation & $13.5 \%$ & $15.4 \%$ & .80 \\
\hline \multirow[t]{4}{*}{ Cine-CMR Parameters } & Ejection fraction (\%) & $40.8 \pm 13.8$ & $31.2 \pm 12.0$ & .002 \\
\hline & End-systolic volume (ml) & $113.0 \pm 68.6$ & $152.0 \pm 81.9$ & 0.02 \\
\hline & End-diastolic volume (ml) & $180.9 \pm 68.9$ & $211.1 \pm 82.9$ & 0.06 \\
\hline & Aneurysmal Dilation & $10.6 \%$ & $23.1 \%$ & 0.09 \\
\hline
\end{tabular}

study. CMR and echo were performed within a seven day interval $(\Delta 0.8 \pm .2$ days $)$ and interpreted blinded to results of the other modality. Echo included both non-contrast echo (NC-echo) and contrast echo (C-echo) imaging in all patients. CMR (I.5 T) included cine-CMR (SSFP) and DE-CMR (inversion recovery; standard $\mathrm{TI}=250-350 \mathrm{msec} /$ long $\mathrm{TI}=600 \mathrm{msec}$ ). DE-CMR was the reference standard for LVT. Left ventricular (LV) function and geometry were quantified by cine-CMR (planimetry) and echo (linear) methods. Infarct size was measured on DE-CMR. Echoes were graded for image quality on a uniform scale to account for endocardial definition, artifacts, and number of segments imaged. Results: 130 patients were studied (62 \pm 13 yo, 98\% CAD, NYHA class $2 \pm .8)$. LVT prevalence was higher by DE-CMR than C-echo $(21 \%$ vs. $14 \% p<0.05)$. Patients with LVT had larger infarcts (OR I.5 per I0\% LV $p=0.01$ ), lower ejection fraction (OR I.9 per I0\% EF $p=0.01$ ) and more aneurysms (OR 3.3 $P<0.05)$. Echo performance did not vary by clinical parameters or image quality, with similar reader-assigned quality scores between NC-echoes that did or did not detect LVT $(7.0 \pm 1.9$ vs. $6.8 \pm 1.7, \mathrm{P}=.8)$. Ejection fraction was lower among patients that derived improved LVT assessment by C-echo compared to those in whom NC-echo alone accurately assessed LVT (32.1 $\pm 10.7 \%$ vs. $39.9 .0 \pm 13.4 \%$, $p<0.05)$. Similarly, improved LVT assessment by DE-CMR was associated with lower ejection fraction and greater cavity dilation as measured by either echo or cine-CMR (Table I). In multivariate analysis, severity of EF impairment (OR I.8, p < 0.05) was an independent marker for added utility of DE-CMR after controlling for LV volume.

Conclusion: LV remodeling indices are useful for guiding imaging strategies for LVT. Patients in whom DE-CMR provides incremental utility for LVT detection vs. NC-echo have more advanced LV dysfunction than those in whom LVT is accurately assessed by $\mathrm{NC}$-echo alone.

\section{PI4I}

The effect of dobutamine stress on diastolic filling rates in obese subjects

Oliver J Rider', Jane M Francis', Tammy Peggy', James P Byrne', Kieran Clarke' and Stefan Neubauer'

'University of Oxford, Oxford, UK

${ }^{2}$ Southampton General Hospital, Southampton, UK

Journal of Cardiovascular Magnetic Resonance 2009, I I (SuppI I):PI4I

Objective: Our aim was to determine the effect of catecholamine stress on left ventricular filling rates in obesity and compare this to normal weight subjects.
Background: Obesity is associated with diastolic dysfunction at rest. However, it is unknown whether this is exacerbated during stress, i.e. whether the "relaxation reserve" of the heart is impaired in obesity. We aimed to determine the effect of simulated exercise on diastolic function in obese subjects without co-morbidity, and compared this to normal weight subjects.

Methods: 45 healthy subjects (28 obese, BMI $39.8 \pm 8.0$ \& 17 lean controls $21.6 \pm 1.6)$ were enrolled into the study. All subjects were screened for identifiable cardiac risk factors and excluded if present. All subjects underwent cardiac MR imaging at I.5 T for peak diastolic peak filling rate (PFR, derived from LV volume time curves, normalized to end-diastolic volume). II of the normal weight and 20 obese subjects underwent repeat assessment of diastolic function during dobutamine infusion.

Results: At rest, obesity was associated with a $20 \%$ reduction in LV peak filling rate $(3.6 \pm 0.8$ vs $4.6 \pm 1.0 \mathrm{ml} / \mathrm{s}, \mathrm{p}=0.0 \mathrm{I})$. As would be expected from the action of dobutamine, in normal weight subjects, with an increase in heart rate (HR) of $60 \%$ during stress (from $63 \pm 9$ to $99 \pm 15 \mathrm{bpm}$ ) there was a $66 \%$ increase in maximal PFR; furthermore, $H R$ and diastolic function showed a close linear correlation $\left(R^{2} 0.86, P<0.00 I\right)$. In contrast, in obese subjects, a $62 \%$ increase in HR (from $65 \pm 9$ to

Figure I (abstract PI4I)

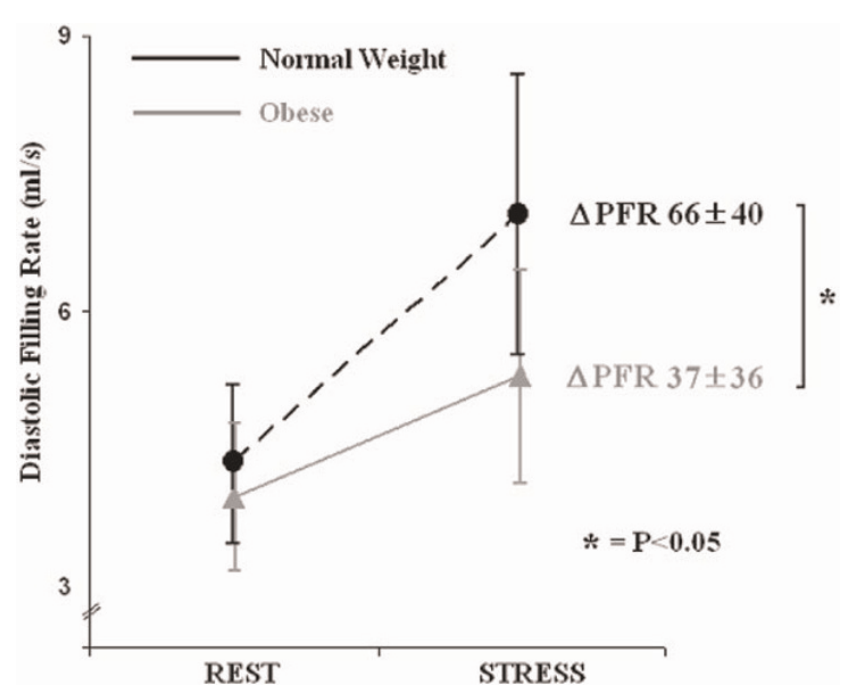

The effect of dobutamine infusion on peak filling rates in obese and normal weight subjects. 
$105 \pm 9$ ) resulted in a significantly smaller, $37 \%$ (vs $66 \%$, $\mathrm{P}<0.05$ ) increase in PFR, Figure I. Furthermore, the correlation between HR and diastolic function was no longer significant $(p=0.24)$.

Conclusion: Similar levels of stress result in significantly smaller increases in diastolic PFR in obese compared to lean controls. Thus, the "relaxation reserve" of the heart is impaired in obesity.

\section{P | 42}

Assessment of change in aortic distensibility in patients with left ventricular hypertrophy (LVH), before and after therapy, using Cardiac Magnetic Resonance (CMR) imaging

Kalliopi Negrou, Joanna Burns, Sanjay Gupta, Sven Plein, Aleksandra Radjenovic and John P Greenwood

Leeds University, Leeds, UK

Journal of Cardiovascular Magnetic Resonance 2009, I I(SuppI I):PI 42

Objectives: To determine whether two aggressive antihypertensive treatment strategies with opposing mechanistic effects, but the same effect on arterial blood pressure (BP) control, had different influences on aortic distensibility.

Background: Alteration in the structure and function of the aortic wall and its elastic properties are important in the pathogenesis of cardiovascular disease [I], and are associated with aging and diabetes mellitus. These changes can cause a decrease in aortic distensibility (AD) and as a consequence elevated pulse pressure [I]. This increased aortic stiffness can be a predictor of hypertension and may be both a cause and effect of sustained high BP [2]. Studies to date of antihypertensive therapy have been conflicting with regard to whether there is a treatment specific effect.

Methods: Thirty-four age matched patients (mean age $=56 \pm$ I I.6; 32-77 yrs) were randomly divided into two groups. All patients were hypertensive, non-diabetic and had CMR confirmed left ventricular hypertrophy (LVH). Both groups underwent treatment for 6 months; the first group (Group VM) was treated with Valsartan and Moxonidine and the second group (Group BA) with Bendroflumethiazide and Amlodipine. A third

Figure I (abstract P I42)

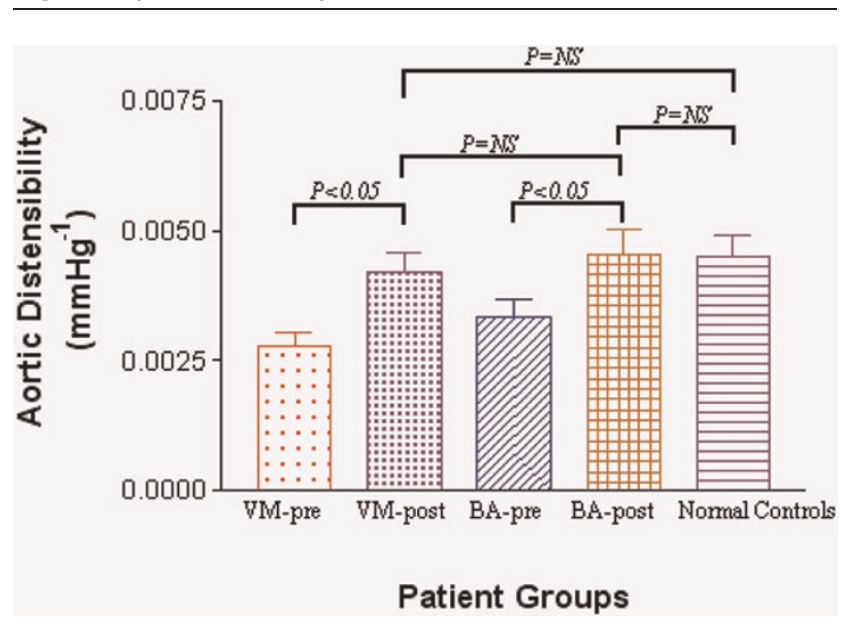

Figure 2 (abstract PI42)

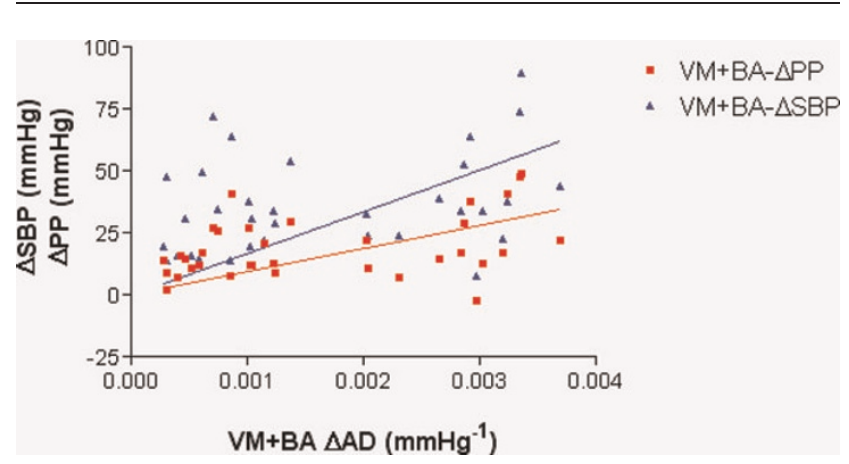

Data table -VM+BA linear regression $\triangle \mathrm{AD}$ vs $\triangle \mathrm{SBP}$ and $\triangle \mathrm{PP}$.

group (Group N) consisting of normal volunteers (I 5 volunteers; mean age $=49 \pm 10.5 ; 29-63$ years) was used as control. Both treatment groups (VM and $\mathrm{BA}$ ) were scanned using cine-MRI (SSFP, transverse to the aorta, 24 phases; Philips I.5 T Gyroscan Intera $C V$ ) before and after therapy. $A D$ was measured using QMASS 6.2.I. (Medis, The Netherlands) and a standard equation $\left(A D=\Delta D /\left(\Delta P^{*} D\right)\right.$; where $\Delta D$ is absolute difference in systolic and diastolic volume of the aorta, $\Delta \mathrm{P}$ the pulse pressure of the artery and $D$ the diastolic arterial volume [3]).

Results: No significant differences in age, arterial BP, LV mass and $A D$ were found between the two treatment groups at baseline $(P>0.05)$. There was a significant increase in $A D$ after treatment in both groups $(P<0.05)$. However, there was no statistical difference in AD between Group VM and Group BA after treatment $(P>0.05)$. In both treatment groups $A D$ returned to normal as compared to the normal control group $(P>0.05)$ (Figure $I)$. There was a significant positive linear relationship between the difference in pre- and post-AD ( $\triangle A D)$ and pre- and post-treatment systolic $B P(\triangle S B P)$ and also the $\triangle A D$ and pre- and post-treatment pulse pressure $(\triangle \mathrm{PP})$ (for $\Delta \mathrm{SBP} r=$ $0.3493, P=0.043$ and $r^{2}=0.122$; for $\Delta \mathrm{PP} r=0.402, P=0.018$ and $r^{2}=0.162$; figure 2 ). The intra-observer variability of measurements was $\leq 7 \%$.

Conclusion: Both groups improved their AD after six months of aggressive antihypertensive treatment, but there was no statistical difference between the two groups. The posttreatment $A D$ in both hypertensive groups was found to have returned to normal levels when compared to a control group. It therefore appears that the improvement in $A D$ is not treatment specific, but rather occurs as a consequence of lowering BP.

\section{References}

I. Metafrazi Z, Efremidis S, Skopelitou AN, Roos S and De A: The clinical significance of aortic compliance and its assessment with magnetic resonance imaging. Journal of Cardiovascular Magnetic Resonance 2002, 4(4):48I-49I.

2. Dernellis $J$ and Papetrou $M$ : Aortic stiffness is an independent predictor of progression to hypertension in nonhypertensive Subjects. Journal of the American Heart Association 2005, 45:426-43I.

3. Woodman R and Watts GF: Measurement and application of arterial stiffness in clinical research: focus on new methodologies and diabetes mellitus. Medical Scientific Monitoring 2003, 9(5):RA8I-89. 


\section{PI43}

Hypertrophic cardiomyopathy patients have a steep left ventricle to aortic root angle compared to normal as demonstrated on 3-D Tomographic Imaging: a case-control study

Deborah H Kwon, Nicholas G Smedira,

Zoran B Popovic, Bruce W Lytle, Randolph M Setser, Maran Thamilarasan, Paul Schoenhagen, Scott D Flamm, Harry Lever and Milind Y Desai

Cleveland Clinic Foundation, 9500 Euclid Avenue, Cleveland, $\mathrm{OH} 44195$, USA

Journal of Cardiovascular Magnetic Resonance 2009, I I(SuppI I):PI43

Introduction: Hypertrophic cardiomyopathy (HCM) is characterized by disproportionate left ventricular (LV) hypertrophy, which cannot be attributed to other concomitant cardiac or systemic diseases. HCM can result in accelerated cardiac remodelling; thus affecting the pathophysiology of the disease. Because of an increased utilization of cardiac magnetic resonance $(C M R)$ in the diagnosis and management of HCM, we have observed that a subgroup of HCM patients had a steep LV to aortic root angle (LVARA, Figure I).

Purpose: We conducted a case-control study to assess if HCM patients have a steeper LVARA as compared to normal subjects. Furthermore, we sought to discern the potential predictors of a steeper LVARA.

Methods: We studied 153 consecutive patients ( $\leq 65$ years) with echo-documented HCM that underwent standard CMR (I.5 T Siemens Avanto, Erlangen, Germany) along with whole-heart 3D MR angiogram which was a navigator-assisted free-breathing, ECG-triggered, fat saturated, T2-prepared, segmented 3D SSFP sequence. Imaging parameters were as follows: TR $=3.8 \mathrm{~ms}$, $\mathrm{TE}=1.9 \mathrm{~ms}$, flip angle $=70^{\circ}$, acquired matrix $=175-216$ (phase direction) by 256 (readout direction) points (no interpolation), Grappa factor of 2, 24 reference lines and sampling bandwidth $= \pm 125 \mathrm{kHz}$. Typically, $60-70$ slices were acquired at $1.5 \mathrm{~mm}$ thickness (interpolated) in order to cover the heart. The in-plane resolution was typically $1.3-1.6 \mathrm{~mm}$. Images were acquired during a $150 \mathrm{msec}$ data acquisition window in mid-to-late diastole. LVARA, LV volumes (indexed to body surface area) and basal septal thickness (BST) were measured on CMR. We also studied 42 controls ( $<65$ years of age, $<1.2 \mathrm{~cm}$ diastolic BST, without hypertension, valvular, myocardial or aortic diseases) that underwent contrast-enhanced 64-multi-detector computed
Figure I (abstract P I43)

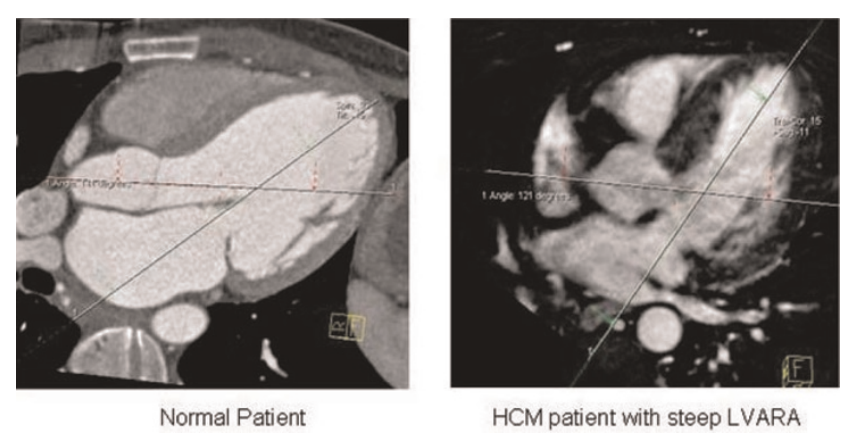

Quantification of LVARA using the 5 chamber view.

tomography (MDCT) coronary angiography (90 cc iodinated contrast). No controls had coronary artery disease or LV dilatation.

Results: The baseline characteristics of $\mathrm{HCM}$ patients were as follows: mean age $46 \pm 14$ years, $68 \%$ male, 36\% hypertensives, and $73 \%$ were on beta-blockers. The mean age of the controls was $43 \pm \mathrm{II}$ years and $38 \%$ women. There was a significant difference in BST $(1.98 \mathrm{~cm} \pm 0.64$ vs. $0.99 \mathrm{~cm} \pm 0.14)$ and LVARA between HCM patients and controls $\left(134^{\circ} \pm 10\right.$ vs. $\left.140^{\circ} \pm 7, P=0.001\right)$. There was a significant inverse correlation between LVARA and age in both, HCM (beta $=-0.56, \mathrm{p}<0.00 \mathrm{I}$ ) and control (beta $=-0.48, \mathrm{p}<0.00 \mathrm{I}$ ) groups. In the HCM group, the mean body surface area, end systolic and end diastolic volume indexes were $134^{\circ} \pm 1.98 \mathrm{~cm} \pm 0.6,32 \mathrm{ml} / \mathrm{m}^{2} \pm \mathrm{II}$ and $84 \mathrm{ml} / \mathrm{m}^{2} \pm 16$, respectively. Within the HCM group, the univariate and multivariate associations between LVARA and various potential predictors are shown in Table I.

Conclusion: As compared to normal subjects, patients with HCM have a significantly steeper LVARA. The strongest predictor of a steep LVARA is age, followed by BST and body surface area. In HCM patients, a steep LVARA, which likely represents remodelling of the $\mathrm{LV}$, might result in increased turbulence across the LV outflow tract. This finding may also contribute to the complex pathophysiology of dynamic LVOT obstruction. CMR can accurately discern the LV-aortic morphology and angulation.

Table I (abstract P I43) Univariate and multivariate regression analysis testing the association between LV-aortic root angle

\begin{tabular}{lllll}
\hline & $\begin{array}{l}\text { Univariate } \\
\text { Analysis Beta }\end{array}$ & $\begin{array}{l}\text { Univariate } \\
\text { Analysis p value }\end{array}$ & $\begin{array}{l}\text { Multivariate } \\
\text { Analysis Beta }\end{array}$ & $\begin{array}{l}\text { Multivariate } \\
\text { Analysis p value }\end{array}$ \\
\hline Age & $-\mathbf{0 . 5 6}$ & $\mathbf{0 . 0 0 0 I}$ & $-\mathbf{0 . 6 I}$ & $<\mathbf{0 . 0 0 0 I}$ \\
Basal septal thickness & $-\mathbf{0 . 1 8}$ & $\mathbf{0 . 0 2}$ & $-\mathbf{0 . 2 5}$ & $\mathbf{0 . 0 1}$ \\
Body surface area & $-\mathbf{0 . 2 0}$ & $\mathbf{0 . 0 2}$ & $-\mathbf{0 . 1 6}$ & $\mathbf{0 . 0 2}$ \\
Hypertension & -0.19 & 0.02 & 0.09 & 0.20 \\
Coronary artery disease & -0.17 & 0.04 & 0.05 & 0.4 \\
Betablockers & -0.16 & 0.05 & 0.002 & 0.98 \\
Gender & -0.16 & 0.05 & & \\
End-systolic volume index & 0.11 & 0.17 & & \\
End-diastolic volume index & -0.007 & 0.9 & & \\
\hline
\end{tabular}


PI44

GEISIR: gadolinium exposure induced systemic inflammatory response in dialysis patients Henning Steen, Constanze Merten, Dirk Lossnitzer, Stephanie Lehrke, Vedat Schwenger, Hugo A Katus and Evangelos Giannitsis

University of Heidelberg, Heidelberg, Germany

Journal of Cardiovascular Magnetic Resonance 2009, I I(SuppI I):PI44

Introduction and purpose: Several late sequelae of the administration of gadolinium (Gd)-containing MRI contrast agents have been described in patients with advanced renal failure. In an observational series we found a remarkable frequency of peracute reactions after administration of Gd-DTPA used for cardiovascular evaluation before renal transplantation.

Methods: In a 26 months observational period 13 of 136 hemodialysed or CAPD patients exhibited onset of fever, chills and nausea within hours after administration of Gd-DTPA peracute. A minority showed persistent cessation of residual diuresis. We performed blood cultures in most patients and evaluated white blood cell counts (WBC), eosinophils, CRP, heart rate and blood pressure.

Results: Within an average of $12 \mathrm{~h}$ (range 12-36 h) after Gdadministration, the $\mathrm{I} 3$ patients ( 9 male, 4 female; median age $6 \mathrm{I}$ years, range 47 - 79) developed consistent symptomatology with fever (median $39.0^{\circ} \mathrm{C}$, range 37.5 - 39.5), chills, malaise, hypotension, vomiting, dyspnea - initially raising suspicion of septicaemia. Subsequent blood cultures on bacterial contamination of the injected product remained negative throughout; bacterial or endotoxin contamination of the reagent was excluded. Steroids were tried in the first two patients without noticeable effect. In all subsequent patients symptoms were attenuated during the first $5 \mathrm{~h}$ dialysis (F60HPS with $280 \mathrm{ml} / \mathrm{min}$ blood flow) and disappeared within $72 \mathrm{~h}$. CRP levels remained markedly elevated up to I4 days. Lymphopenia was seen in all patients and polymorphic neutrophils (PMN) remained normal. Two polyuric patients developed persistent anuria. After a median of 16 months none of these patients developed nephrogenic systemic fibrosis.

Conclusion: This series with unusually severe acute phase reactions was caused by one specific preparation. Such peracute reactions may be relevant for the so far largely unresolved pathogenesis of the skin reaction to some Gd products in endstage renal disease (ESRD) patients. It remains unresolved, whether the reaction observed with Gd-DTPA do in principle also occur with other $\mathrm{Gd}$ reagents.

\section{P I 45}

Cardiovascular MRI derived mitral valve geometry predicts the surgical treatment of mitral regurgitation

Andre M Fernandes, Robert WW Biederman, Mark Doyle, June A Yamrozik, Ronald B Williams,

Electra Markopoulos, George Magovern and Vikas K Rathi Allegheny General Hospital, The Gerald McGinnis Cardiovascular Institute, Pittsburgh, PA, USA

Journal of Cardiovascular Magnetic Resonance 2009, I I(SuppI I):PI45

Background: Mitral regurgitation (MR) is the most common valvular heart disease worldwide with varying causes. The ultimate treatment of mitral regurgitation is surgical. The surgical decision making classically depends on the symptoms, severity of $M R$, left ventricular dimension and function. Common mitral apparatus descriptors now serve as a 'road map' to direct surgical options.

Aim: Distinguish mitral valvar/apparati geometric patterns in pts who undergo mitral valve (MV) surgery via cardiovascular MRI (CMR).

Methods: Thirty-seven (37) pts underwent CMR (I.5 T GE). The 3D MV and ventricular geometry was analyzed by multiplanar SSFP imaging without contrast administration. The following MV parameters were measured: tenting area, tenting angle, tenting height, anterior leaflet $(\mathrm{AL})$ and posterior leaflet $(\mathrm{PL})$ length and annulus diameter. Pts were divided into two groups: $M V$ surgery $(M V+)$ and no $M V$ surgery (MV-). The data was statically analyzed by t-test, linear regression and ANOVA. Results: Out of 37 pts, 18 had mild to moderate MR (I-2+) and 19 pts had moderate-severe MR (3-4+). Eight out of 37 pts underwent surgery ( 6 of 8 had severe 4+ MR and two were moderate 3-4+). As expected, MR severity correlated with surgical treatment $(r=0.6, p<0.00 I)$. The $A L$ length most strongly predicted the severity of $M R(r=0.7, p=0.007)$. Tenting area and annulus diameter were higher in $M V+$ than in MV- $\left(14 \mathrm{I} \pm 92\right.$ vs. $106 \pm 38 \mathrm{~mm}^{2}, 34 \pm 8$ vs. $27 \pm 4 \mathrm{~mm}$, $P<0.005)$. However, a composite of MR degree, annular diameter and the ventricular length most strongly predicted MV surgery $(r=0.8, p<0.00 \mathrm{I})$. Interestingly, in contradiction to current dogma, the tenting angle and tenting height did not predict the severity of MR or need for MV surgery. More importantly, the appearance and description of the MV on CMR study correlated well with the surgeon's description of the mitral apparatus at time of surgery (7/8 pts; $88 \%$ concordance). In I pt, the surgeon's description of the MV was sparse.

Conclusion: While many criteria have been well established to direct mitral valve surgical strategies, little has been defined by non-echocardiographic methods. Herein, CMR is shown to be concordant with the clinical need for MV surgery and independently predictive of etiology of $M R$ as confirmed at surgery.

\section{PI46}

Exploring the promise land of $7 \mathrm{~T}$ for CMR with T-PAT accelerated imaging techniques - first results for real time cardiac function and tagging in volunteers

Gregory Metzger', Peter Weale ${ }^{2}$, Lance DelaBarre', Patrick Bolan', Sven Zuehlsdorff', Sonia Nielles-Vallespin ${ }^{3}$, Pierre-Francois Van de Moortele', Carl J Snyder', Edward J Auerbach', J Thomas Vaughan ', Kamil Úgurbil' and Renate Jerecic ${ }^{2}$

${ }^{I}$ CMRR, Minneapolis, IL, USA

${ }^{2}$ Siemens Medical Solutions, Chicago, IL, USA

${ }^{3}$ Siemens Healthcare, Erlangen, Germany

Journal of Cardiovascular Magnetic Resonance 2009, I I(SuppI I):PI46

Introduction: CMR faces many challenges at $7 \mathrm{~T}$, including increased magnetic susceptibility, increased power deposition and non-uniform BI distribution. Particularly, non-uniformities in $\mathrm{BI}$ become a challenge beyond 3 Tesla, as the radio-frequency wavelength becomes smaller than the size of the imaged object. 
Figure I (abstract P|46)

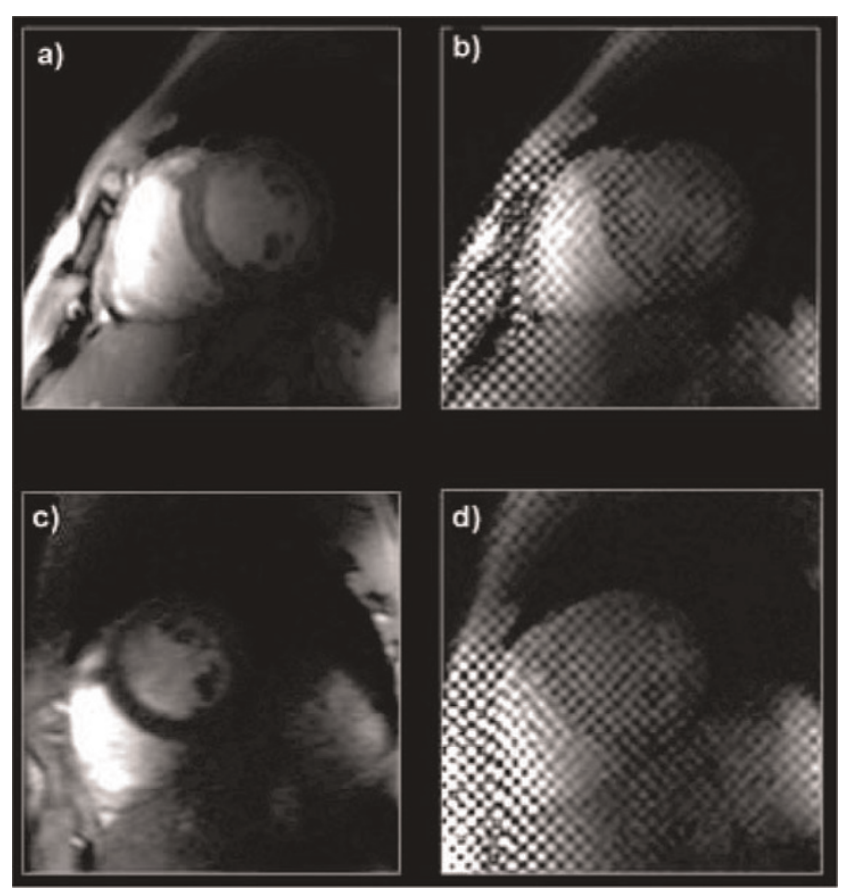

On the other hand the wish for higher spatial and temporal resolution, volumetric coverage and short scan times, ideally all at once, can only be satisfied to some extent, even at $3 \mathrm{~T}$, by using parallel imaging techniques until the SNR limit is reached. Therefore the increased SNR and prolonged TI relaxation times of $7 \mathrm{~T}$ are very tempting as a way to further push the limits of cardiac function and tissue characterization in combination with parallel imaging, especially as it could be shown recently, that the technical and physical challenges mentioned above can be overcome and first cardiac images of healthy volunteers were obtained at $7 \mathrm{~T}[\mathrm{I}]$.

Purpose: The purpose of this study was to explore the feasibility of increased temporal and spatial resolution for cardiac function and tagging using $k-t$ accelerated imaging techniques at $7 \mathrm{~T}$ in healthy volunteers

Methods: Hardware and adjustments: Three volunteers were imaged under a protocol approved by the University of Minnesota's IRB using a 7 T whole-body system (MAGNETOM 7 T, Siemens Healthcare, Germany) with the magnet provided by MAGNEX Scientific, UK. A home-built sixteen-channel, flexible, transceiver array coil was used, with 8 elements on the anterior and posterior part. Standard wireless VCG gating was used.

The $\mathrm{BI}$-shimming procedure was performed, to optimize the RFtransmit efficiency over the heart. The transmit Bl-field components of the independent coil elements were adjusted, to affect an approximate "constructive interference" of the short RF wavelengths over the region of the left ventricle as described in [2, 3]. SAR monitoring was done according to [4]

Cardiac sequences: As a reference a typical clinical protocol was acquired for cardiac function and tagging using a segmented, breath hold, VCG triggered, retro-gated 2D cine FLASH sequence with a GRAPPA factor of 2 , temporal resolution
$40 \mathrm{~ms}$, spatial resolution $2.3 \mathrm{~mm}$ inplane, slice thickness $3 \mathrm{~mm}$ for both sequences.

The tagging acquisition was then repeated using a T-PAT accelerated segmented 2D cine FLASH technique to maintain the spatial resolution and increase the temporal resolution to $19 \mathrm{~ms}$ using a T-GRAPPA factor of 3.

To show the potential of high spatio-temporal resolution cine imaging, real-time 2D cine FLASH images were acquired during free breathing, using T-PAT [5] with a T-GRAPPA factor 5, temporal resolution $68 \mathrm{~ms}$ and matched spatial resolution to the segmented acquisition.

All scans were acquired in short axis (sax) orientation. No surface coil normalization was used.

Results: All scans could be completed successfully. A typical result is shown in Figure $I$ a) and b) reference cine and tagging, $c$ ) rt cine, d) 19 ms tagging). The tag pattern remains persistent throughout the complete cardiac cycle, due to the increased TI. Image contrast between blood pool and myocardium is increased for FLASH compared to I.5 T for the same reason. None of the sequences was limited by SAR using the sequence parameters described above.

Conclusion: Real-time cine imaging and tagging with high temporal and spatial resolution is possible at $7 \mathrm{~T}$ with good image quality, without running into SAR limitations. This sets the basis for further protocol optimization going to even higher acceleration factors.

Further improvements in BI-shimming will allow a homogeneous excitation over a larger region-of-interest and therefore the extension of the protocol towards a whole heart coverage.

\section{References}

I. DelaBarre L, et al: ISMRM 2007.

2. Metzger GJ, et al: MRM 2008, 59(2):396-409.

3. Snyder C, et al: ISMRM 2007.

4. Vaughan, et al: in press.

5. Breuer FA, et al: MRM 2005, 53(4):98I-985.

\section{PI47}

A phantom study of temperature-dependent MRI T2* measurement

Taigang He, Gillian Smith, John-Paul Carpenter, Raad Mohiaddin, Dudley Pennell and David Firmin CMR Unit, Royal Brompton Hospital and Imperial College London, London, UK

Journal of Cardiovascular Magnetic Resonance 2009, I I(SuppI I):PI 47

Introduction: The measurement of cardiac iron is essential for preventing disease and managing iron-chelating treatment in thalassemia patients. A T2* technique has been developed and clinically validated for this purpose [I, 2]. This method has demonstrated reproducibility and accuracy [2, 3, 4] and been increasingly used in clinical practice. However, this technique has not been calibrated against myocardial biopsy because of the risk, the heterogeneity of myocardial iron distribution, and sampling errors. Although autopsy study in hearts donated after death or after cardiac transplantation in thalassemia patients can be performed for calibration, there remain concerns of postmortem changes in the myocardium and extrinsic variations. Another major issue is the effect of temperature difference from in-vivo to ex-vivo. In a recent post mortem study [5], the ex-vivo T2* value was shown to be significantly higher than that matched 
Figure I (abstract P|47)

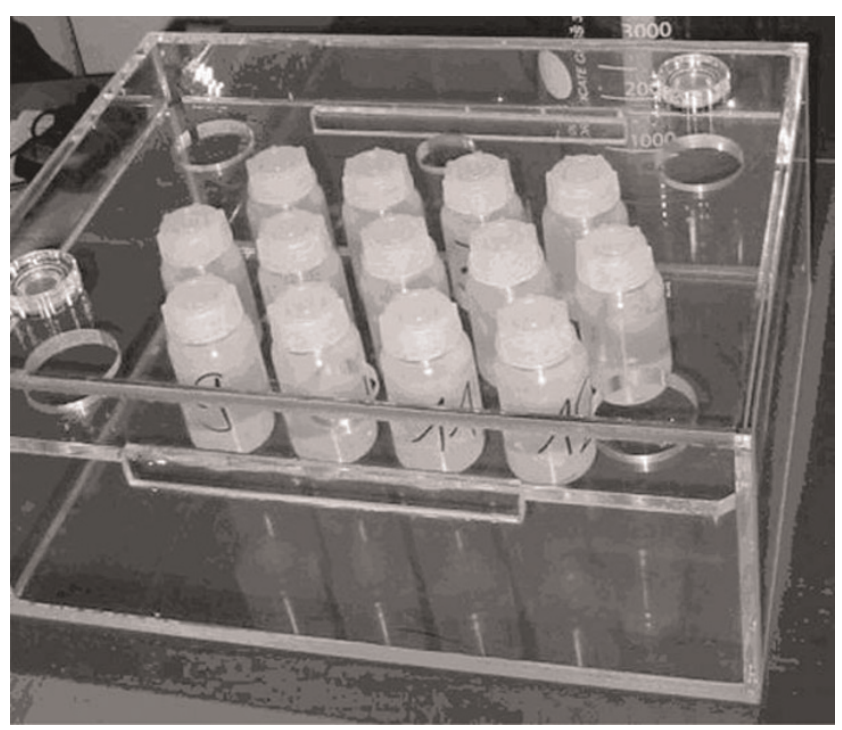

Photograph of the phantom.

in-vivo measurement. However, the ex-vivo measurement was performed at a room temperature of $25^{\circ} \mathrm{C}$. Since temperature can potentially affect the calibration, there is the need to investigate this issue. To date we are unaware of any reports regarding myocardium $\mathrm{T} 2 *$ measurements at different temperatures. The purpose of this study, therefore, was to use a carefully designed phantom to determine this relationship.

\section{Figure 2 (abstract P I47)}

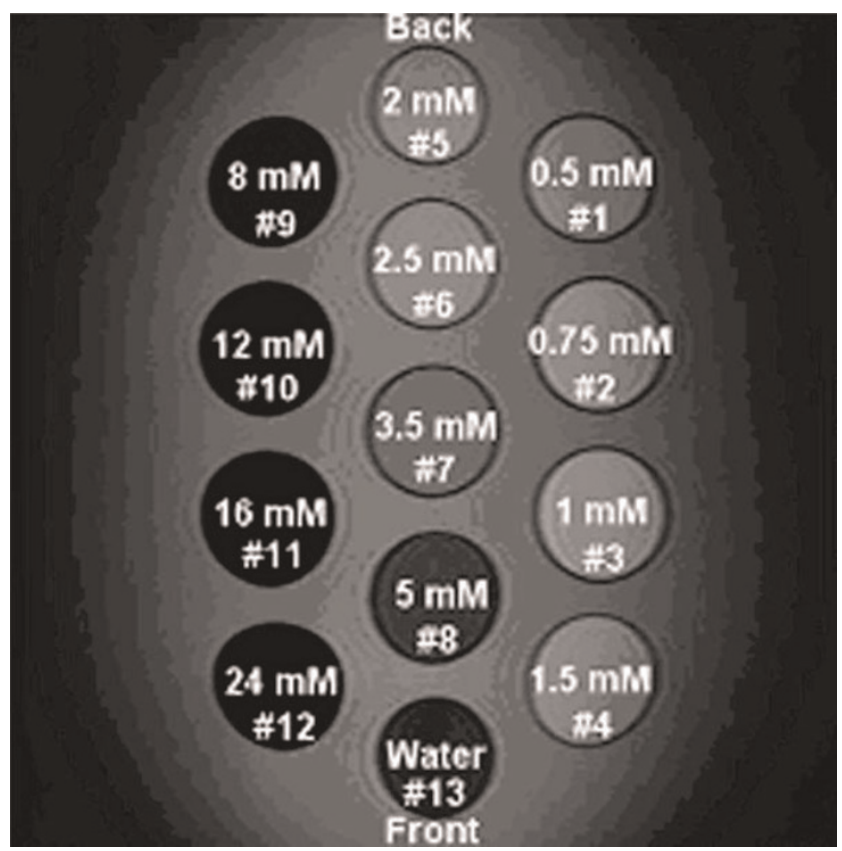

$\mathrm{MnCl} 2$ concentration.
Figure 3 (abstract PI47)

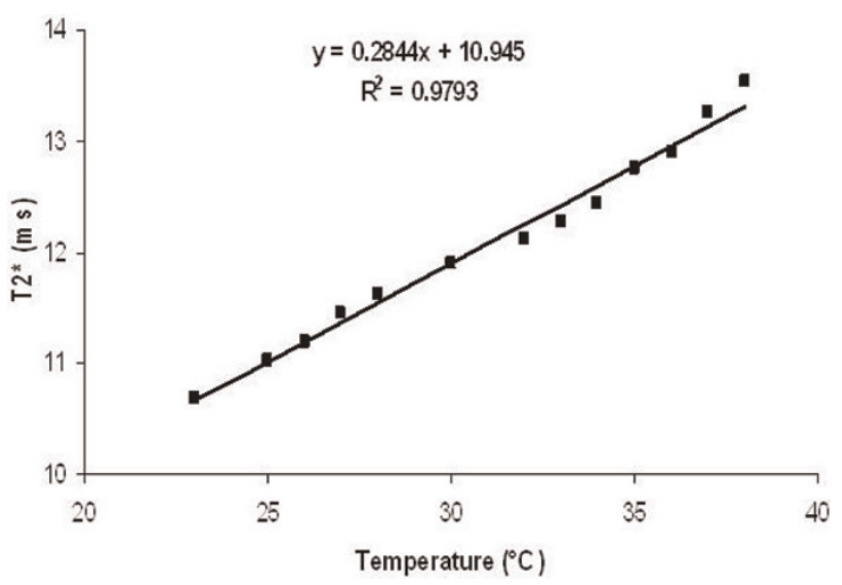

The temperature dependence of $\mathrm{T} 2 *$ measurements in the phantom.

Methods: This study was carried out on a I.5 T whole-body Siemens Sonata system equipped with high performance gradients having a maximum strength of $40 \mathrm{mT} / \mathrm{m}$ and maximum slew rate of $200 \mathrm{~T} / \mathrm{m} / \mathrm{s}$ on each axis independently. A fourelement cardiac phased array coil was used.

The phantom consists of a Plexiglas container holding 13 bottles (Figure I) with various concentrations of $\mathrm{MnCl}_{2}$ arranged as depicted in Figure 2. The $\mathrm{MnCl}_{2}$ concentrations range from 0 to $24 \mathrm{mM}$ according to the scheme in Figure 2. All solutions contain $0.03 \% \mathrm{NaN}_{3}$ (sodium acid) in order to prevent growth of bacteria or fungi.

The phantom was immersed in warmed water to reach the temperature of $38^{\circ} \mathrm{C}$ and then taken out of the water for the scan. The phantom was scanned using the $\mathrm{T} 2^{*}$ sequence and a coronal image was positioned near the middle of the bottle. During the scan, the phantom was monitored by a temperature probe with an accuracy of $0.1^{\circ} \mathrm{C}$ continuously until the phantom cooled down to a temperature of $23.0^{\circ} \mathrm{C}$. Eight bottles with $\mathrm{MnCl}_{2}$ concentrations from 0 to $5 \mathrm{mM}$ were selected for analysis in this study. For T2* measurement, a region of interest was chosen in the vicinity of the center for each bottle. The mean signal intensity of ROI was measured in the series of increasing TE images, and the data were plotted against the TEs to form an exponential decay curve. Nonlinear curve fitting was employed to obtain all T2* measurements of the phantom.

Results: There is clear temperature dependence of T2* measurements. Figure 3 demonstrates that T2* increases linearly with temperature. From $23.0^{\circ} \mathrm{C}$ to $38.0^{\circ} \mathrm{C}$, the maximum T2* change is $23 \%$, which is approximately $1.5 \%$ increase per degree Celsius.

Conclusion: This study has demonstrated that T2* measurement is highly dependent on temperature. In post mortem studies, therefore, the temperature should be set the same as body temperature to avoid significant errors. Further studies are necessary to investigate this relationship in human myocardium. Acknowledgements

NIH Grant: R0I DK66084-0I. 


\section{References}

I. Anderson I LJ, et al: Eur Heart J 200I, 22:2171-2179.

2. Westwood M, et al: J Magn Reson Imag 2003, 18:33-39.

3. He T, et al: Magn Reson Med 2008, 60:350-356.

4. He T, et al: Magn Reson Med 2008 in press.

5. Ghugre NR, et al: Magn Reson Med 2006, 56:68I-686.

\section{PI48}

Validation of myocardial perfusion and coronary flow reserve in rats using spin-labeling gradient echo imaging with a fluorescent microsphere technique as standard of reference

Alexis Jacquier, Franck Kober, Soksithikun Bun,

Patrick J Cozzone and Monique Bernard

CRMBM, UMR CRNS 66I2, Marseille, France

Journal of Cardiovascular Magnetic Resonance 2009, I I(SuppI I):PI48

Introduction: Cardiac arterial spin labeling (ASL) MRI might become an important technique for quantitative mapping of myocardial blood flow in humans. A previously proposed gradient echo ASL technique has provided high spatial resolution but without external validation against microspheres technique. Purpose: The purpose of this study was to validate in vivo myocardial perfusion measurements and coronary flow reserve by gradient echo arterial spin labeling (ASL) MRI in rats anesthetized with isoflurane against fluorescent microspheres technique as standard of reference.

Methods: Male Wistar rats (weight $=200-240 \mathrm{~g}, \mathrm{n}=21$ ) were anesthetized with $2.1 \%$ isoflurane added to $\mathrm{I} \mathrm{I} / \mathrm{min}$ of pure $\mathrm{O}_{2}$. Heart rate, breath rate, temperature, blood oxygen saturation and arterial blood pressure were recorded. In 7 rats, myocardial perfusion was assessed on a Bruker Biospec 4.7 T horizontal MRI system using an ECG- and respiration-gated IR gradient-echo technique (resolution $=234 \times 468 \mu \mathrm{m}^{2}, \mathrm{TE}=1.52 \mathrm{~ms}$, slice thickness $3 \mathrm{~mm}$, acquisition time $25 \mathrm{~min}$ at $350 \mathrm{bpm}$ ) at rest and during adenosine infusion $(140 \mu \mathrm{g} / \mathrm{kg} / \mathrm{min})$. In the 14 other animals, under the same physiologic conditions, a mixture containing 200,000 fluorescent microspheres (Yellow, $15 \pm 0.1$ $\mu \mathrm{m}$; Triton, San Diego, CA, USA) was injected into the left ventricle at rest in 7 animals and during adenosine infusion in 7 other animals. The animals were killed by instantaneaous injection of pentobarbital. Hearts were harvested and samples were processed for fluorescence spectroscopy. A two-tailed unpaired Student's t-test was used to compare groups, All values are given as mean $\pm \mathrm{SD}$.

Figure I (abstract P I48)

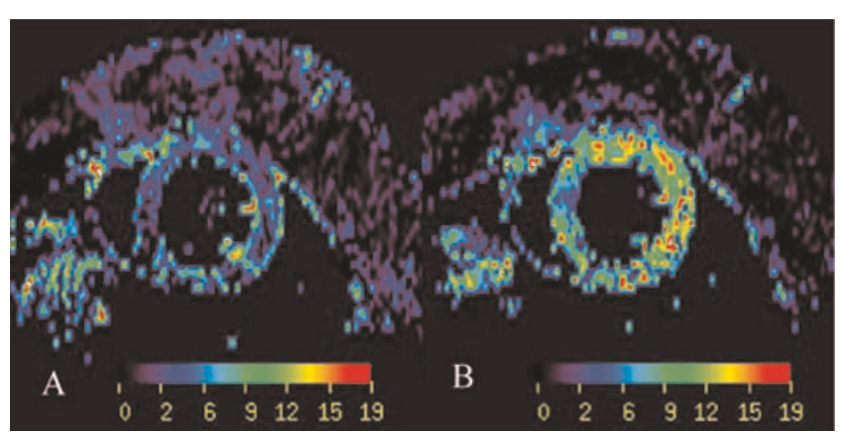

Results: There was no statistical difference between myocardial perfusion at rest assessed with ASL (6.5 $\pm 1.4 \mathrm{ml} / \mathrm{g} / \mathrm{min}$; Figure la) and with fluorescent microspheres $(5.9 \pm 2.3 \mathrm{ml} / \mathrm{g} / \mathrm{min} ; \mathrm{P}=0.5)$. During adenosine infusion there was no statistical difference between myocardial perfusion measured using ASL technique $(\mathrm{I} 1.9 \pm 1.6 \mathrm{ml} / \mathrm{g} / \mathrm{min}$; Figure $\mathrm{lb}$ ) and using fluorescent microsphere technique $(13.1 \pm 2.1 \mathrm{ml} / \mathrm{g} / \mathrm{min} ; \mathrm{P}=0.6)$. Coronary flow reserve was calculated as $2.3 \pm \mathrm{I}$.I using ASL. There was no significant difference between groups in terms of heart rate $(400 \pm 20 \mathrm{bpm})$, breath rate $(50 \pm 12 / \mathrm{min})$, temperature $\left(36.9 \pm 0.1^{\circ} \mathrm{C}\right), \mathrm{O} 2$ saturation $(98 \pm 1 \%)$ or mean blood pressure $(9.8 \pm 0.3 \mathrm{mmHg})$ at rest. Under adenosine infusion a tendency to heart rate increase was measured $(42 \mathrm{I} \pm 2 \mathrm{I}$ bpm; $\mathrm{P}=\mathrm{ns})$. Group standard deviation was lower with MRI than with microspheres. These values obtained under isoflurane anesthesia are higher than previously reported values under pentobarbital. They confirm capillary vasodilation by isoflurane.

Conclusion: Non-invasive gradient echo ASL MRI provides accurate and reliable myocardial perfusion maps and assessment of coronary flow reserve with high spatial resolution. This study has provided validation of gradient echo ASL against an external gold standard technique.

\section{PI49}

Comprehensive assessment of myocardial strain in post-infarct mice using 3D Cine DENSE

Xiaodong Zhong, Brent A French, Craig H Meyer,

Christopher M Kramer and Frederick $\mathrm{H}$ Epstein

University of Virginia, Charlottesville, VA, USA

Journal of Cardiovascular Magnetic Resonance 2009, I I(SuppI I):PI49

Introduction: Transgenic and knockout mice are widely used to study the roles of individual genes in cardiovascular disease. Quantitative MR imaging methods for assessing 3D strain at high resolution in mice are needed to elucidate the roles of individual genes in modulating cardiac contractile function.

Purpose: To develop 3D cine DENSE (Displacement Encoding with Stimulated Echoes) MRI for comprehensive myocardial strain imaging of the mouse heart.

Methods: An ECG-gated segmented 3D spiral cine DENSE pulse sequence with online image reconstruction was implemented on a $7 \mathrm{~T}$ MRI scanner (Bruker Clinscan, Germany). A stack of spirals k-space trajectory was employed for 3D spatial encoding, rapid data acquisition, and short echo time. Threepoint phase cycling was used for artifact suppression, 4-point displacement encoding was used to efficiently measure 3D motion, and a low resolution field map was acquired for online spiral deblurring. To further ensure minimal blurring, the spiral readout duration was limited to $3 \mathrm{~ms}$. In accordance with protocols approved by the animal care and use committee at our university, 3 mice were imaged before and I day after experimental myocardial infarction (MI). During MRI, mice were anesthetized with isoflurane and maintained at $37^{\circ}$. ECG and respiration were monitored using an MRI-compatible system for small animals (SAIl, Stony Brook, NY). Specific pulse sequence parameters included $T R=6 \mathrm{~ms}, T E=\mathrm{I} \mathrm{ms}$, matrix $=$ $128 \times 128 \times 8$, and displacement-encoding frequency $=2.5$ radians $/ \mathrm{mm}$. The field of view was $32 \times 32 \times 3.2 \mathrm{~mm}^{3}$, which covered the entire middle section of the mouse left ventricle (the longitudinal dimension of the mouse left ventricle is approximately 

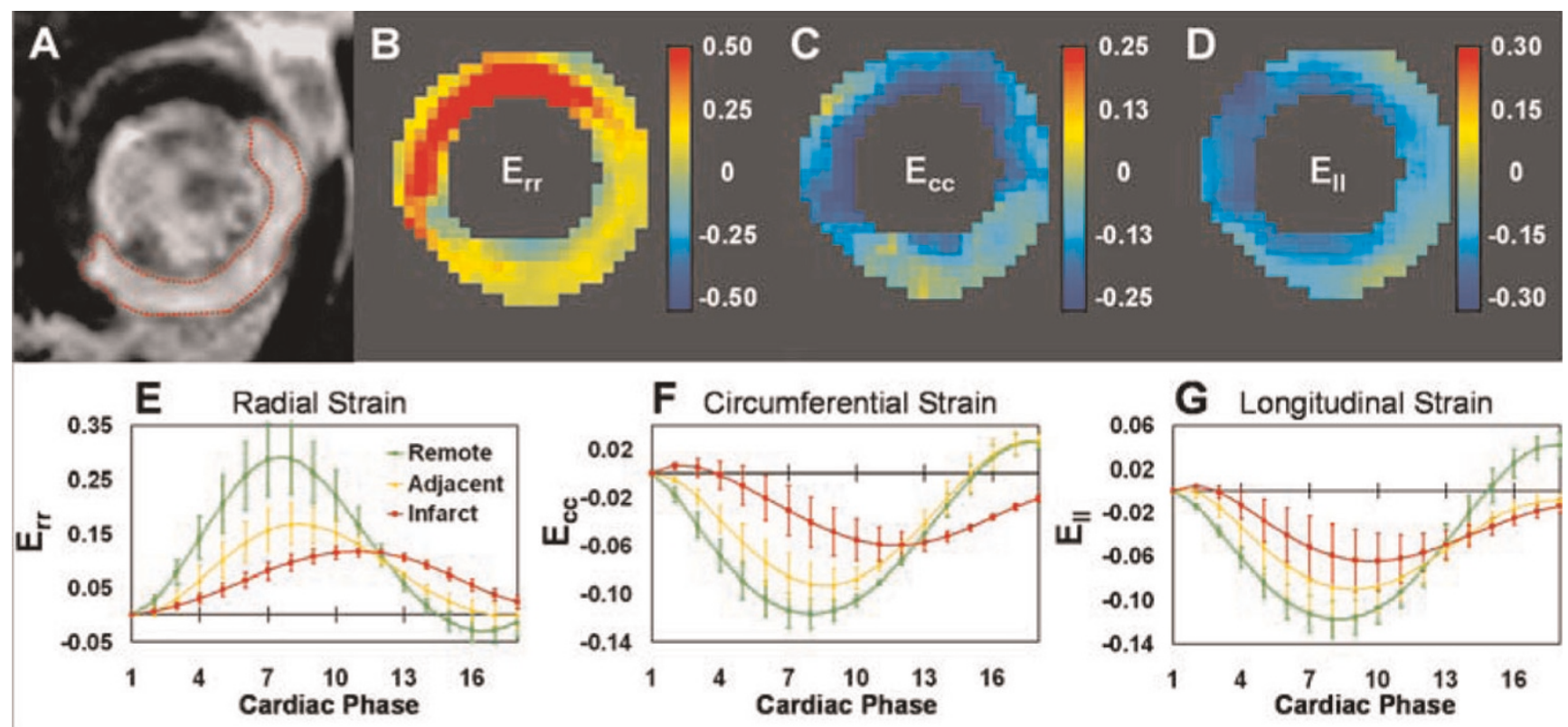

$6 \mathrm{~mm}$ ). These parameters provided voxel size $=0.25 \times 0.25 \times$ $0.4 \mathrm{~mm}^{3}$, temporal resolution $=6 \mathrm{~ms}$, typically 18 cardiac phases across the cardiac cycle, and a scan time of approximately 45 minutes, depending on heart and respiratory rates. Additionally, on day-I post-MI, delayed Gd-DTPA-enhanced inversionrecovery $M R I$ was performed to image the region of infarction. For quantitative analysis of DENSE data, images were exported to a PC and manually segmented. Tissue tracking and strain analysis were performed using $3 D$ extensions of $2 D$ methods that were described previously.

Results: Example images and strain maps are shown in Figure I for a mouse I day after MI. Specifically, the Gd-enhanced image (Panel A) defined the infarcted, adjacent (within I mm of infarction) and remote regions. Panels $B-D$ show the corresponding end-systolic radial $\left(E_{r r}\right)$, circumferential $\left(E_{c c}\right)$ and longitudinal $\left(E_{\| l}\right)$ strain maps for the same slice. Abnormal strain generation is evident in the infarcted and adjacent regions of each map. Panels $E$ through $G$ show strain-time curves (mean \pm SEM) from the mid-ventricle for the infarct, adjacent, and remote regions, where severely reduced strain was measured in the infarct region, intermediate strain characterized the adjacent region, and nearly normal strain was found in the remote region. In the day-I post $\mathrm{Ml}$ infarct region, the times to peak strain were also all significantly delayed. In contrast to data from post-MI mice, baseline data showed normal strains throughout the mid ventricle.

Conclusion: Cine DENSE methods were developed that provide a quantitative assessment of $3 \mathrm{D}$ strain throughout the mid-section of the mouse LV in a scan time of approximately 45 minutes. Using the parameters provided here, DENSE images had high signal-tonoise ratio and high temporal and spatial resolutions. The highquality displacement images lead to high resolution 3D strain data that clearly distinguished differences in function between infarcted, adjacent, and remote regions in post-infarct mice. When applied to genetically-engineered mice, these accurate high resolution data may help elucidate the roles of individual genes in modulating postinfarct regional function and LV remodeling.
PI50

Myocardial fat quantification using navigator $\mathbf{I H}$ MRS combined with MRI: myocardial fat fraction is inversely correlated with indexed left ventricular mass in healthy subjects

Alban Redheuil', Ronald Ouwerkerk', Chia-Ying Liu', Joao AC Lima' and David A Bluemke ${ }^{2}$

Johns Hopkins University, Baltimore, MD, USA

${ }^{2} \mathrm{NIH}$, Bethesda, MD, USA

Journal of Cardiovascular Magnetic Resonance 2009, I I(SuppI I):PI 50

Introduction: Myocardial fat content has recently been shown to be a potential indicator of myocardial involvement in diabetes or obesity. Myocardial triglycerides (TG) can be quantified noninvasively in humans with proton magnetic resonance spectroscopy (IH MRS). In this study, we quantified myocardial TG with an ECG and navigator gated IH MRS sequence in combination with a cardiac MRI measuring left ventricular (LV) volumes, mass and function. Our aim was to study the feasibility of this protocol and the relationship of myocardial fat to LV mass in healthy subjects.

Methods: All studies were performed on a 3.0 T MR scanner (Trio Tim, Siemens) on 20 healthy subjects ( 12 men, mean age: $42 \pm 18$ years), Myocardial IH MRS spectra were obtained with an average 6-ml voxel positioned in the interventricular septum prescribed on two chamber, four chamber and short-axis images of the LV. Two MRS spectra were recorded with ECG gated PRESS, $T R / T E=I R-R / 30 \mathrm{~ms}$, with navigator across the liver-lung interface to reduce of breathing effects. One spectrum (32 to I 28 averages) was recorded with WET water suppression. Another spectrum (4 averages) was recorded without water suppression. Fat content was quantified with Amares/MRUI and related to water in unsuppressed spectra. LV volumes and mass were measured on short axis cine MRI images using QMass (MEDIS). The correlation between left ventricular parameters and myocardial fat fraction were studied using Pearson's coefficients. 
Figure I (abstract PI50)

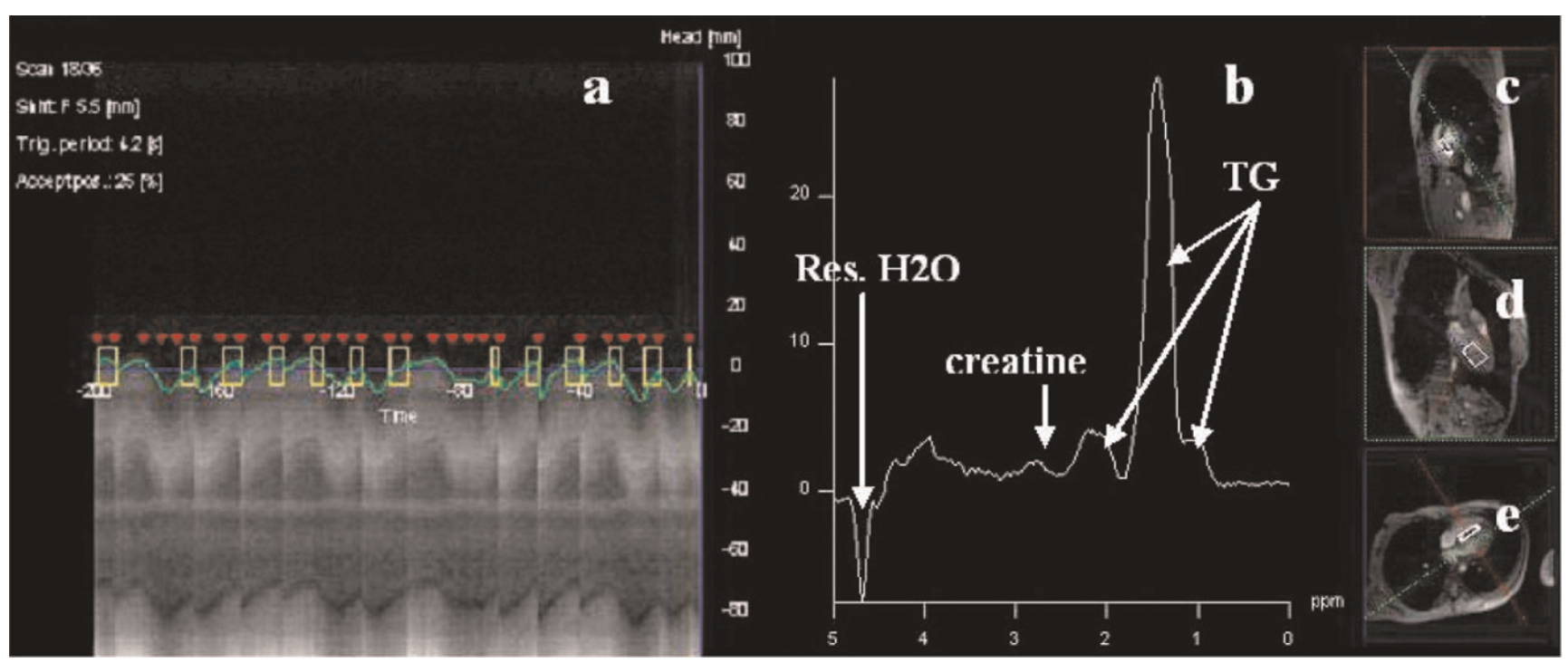

Navigator signal (a) and water suppressed single voxel PRESS spectrum (b) with the selected volume superimposed on short axis (c), 2 chamber (d) and 4 chamber (e) IH MR images from cine MRI.

Results: Figure I shows a water suppressed single voxel PRESS spectrum. The fat peak is clearly visible, but the creatine peak is relatively weak. In this subject fat/water ratio was found to be $0.8 \%$. Shimming was of variable quality because this step could not be performed with both navigator and ECG gating. Nevertheless a fat/water ratio could be determined in all subjects. The fat/water ratio was $0.89 \pm .9 \%$ (mean $\pm \mathrm{SD}, \mathrm{n}=20$ range 0.02 to 3.5). Mean ejection fraction was $67.5 \pm 5.3 \%$, mean systolic blood pressure: $125 \mathrm{mmHg}$, mean body mass index: 28 , mean body surface area (BSA): $1.87 \mathrm{~m}^{2}$ and mean LV mass was $129 \pm 5$ g. We found a negative correlation between LV mass and myocardial fat/water ratio $(r=-0.5 \mathrm{I}, \mathrm{p}=0.05)$ and a significant correlation between LV mass indexed by BSA and myocardial fat fraction $(r=-0.56, p=0.03)$.

Conclusion: Myocardial fat fraction quantification using ECG and navigator gated IH MRS was feasible within a comprehensive exam in healthy subjects and correlated significantly with indexed LV mass.

\section{PI5 I}

$7 \mathrm{~T}$ MRI of macrophages in mouse carotid atherosclerosis using novel nanoparticle platforms Hisanori Kosuge', Masahiro Terashima', Masaki Uchida ${ }^{2}$, Sarah Sherlock ${ }^{3}$, Philip S Tsao', Mark J Young ${ }^{2}$,

Trevor Douglas ${ }^{2}$, Hongjie Dai ${ }^{3}$ and Michael V McConnell ${ }^{\prime}$ ${ }^{\prime}$ Cardiovascular Medicine, Stanford University School of Medicine, Stanford, CA, USA

${ }^{2}$ Department of Chemistry and Biochemistry, Montana State University, Bozeman, MT, USA

${ }^{3}$ Department of Chemistry, Stanford University, Stanford, CA, USA

Journal of Cardiovascular Magnetic Resonance 2009, I I(SuppI I):PI 5 I

Introduction: Macrophages are important imaging targets for identifying and monitoring high-risk atherosclerotic plaque.

Human protein cages and graphite/FeCo core-shell nanocrystals are unique, multi-functional nanoparticle platforms for cellular imaging.

Purpose: To evaluate 7 T MRI using human ferritin protein cage nanoparticles (HFn) and graphite/FeCo core-shell nanocrystals (CN) for detecting macrophage accumulation in mouse carotid atherosclerosis in vivo.

Methods: A macrophage-rich carotid lesion in FVB mice $(N=6)$ was induced as follows: high fat diet for 4 weeks then diabetes induction by 5 daily intraperitoneal injections of streptozotocin. Two weeks later, we performed carotid ligation of the left common carotid artery. Either one of two different nanoparticles, HFn or CN, was injected into mice via tail vein 2 weeks after carotid ligation ( $\mathrm{N}=3$ for each nanoparticle).

I) HFn-Fe are human ferritin protein cages chemically modified to contain an iron oxide core (dose $-25 \mathrm{mgFe} / \mathrm{kg}$ ).

Figure I (abstract PI5I)

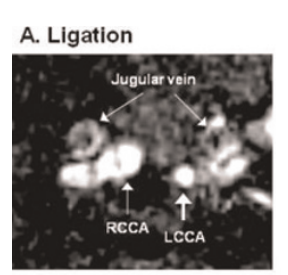

Pre

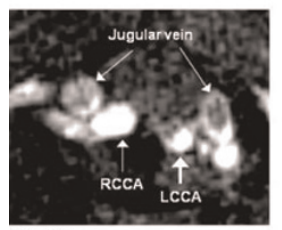

B. Sham

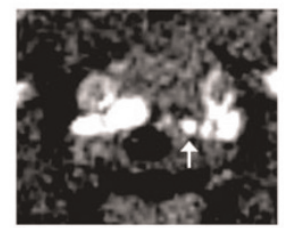

$24 \mathrm{H}$

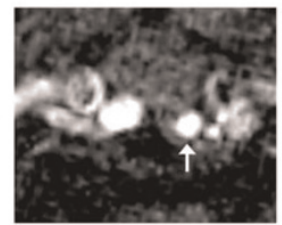

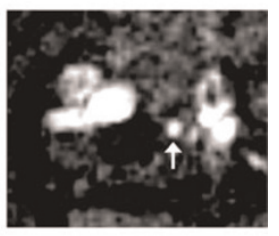

$48 \mathrm{H}$

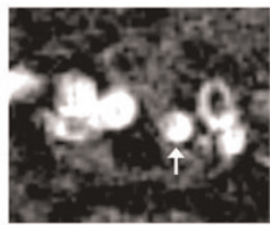



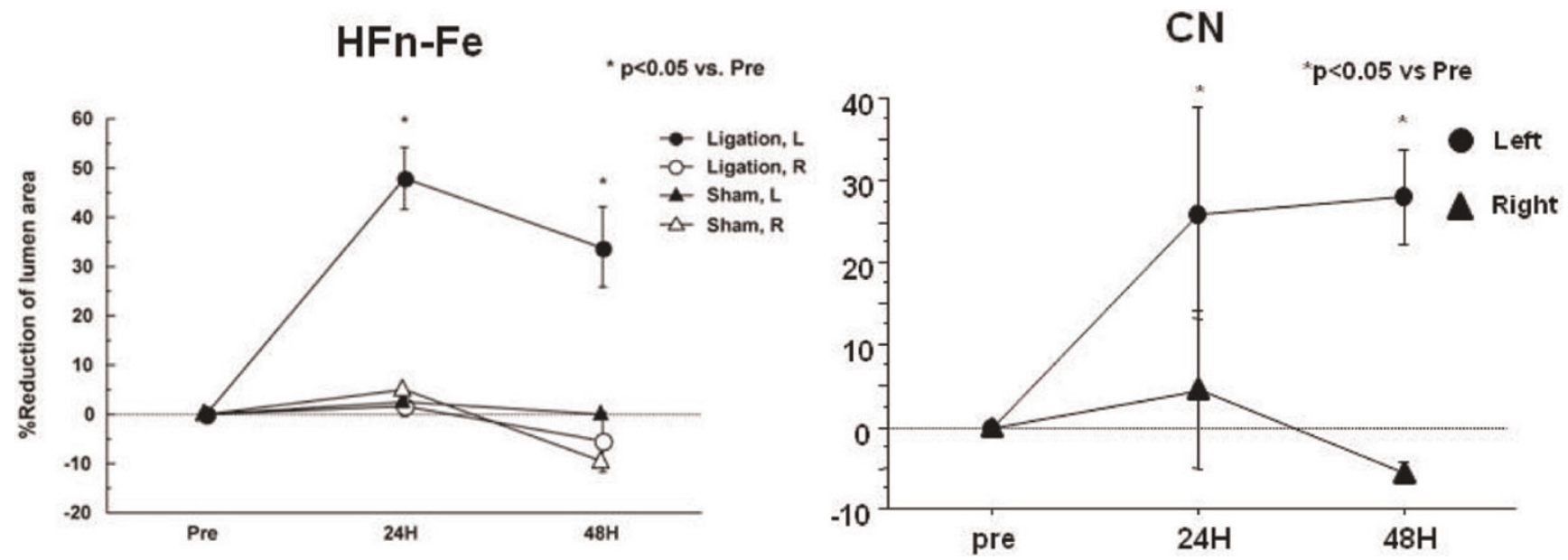

2) $\mathrm{CN}$ have a FeCo core with a graphite shell, with $\mathrm{Cy} 5.5$ also attached for fluorescence imaging (dose $-32.14 \mu \mathrm{gFe} / \mathrm{mouse}$, $8 \mathrm{nmol}$ Cy5.5/mouse).

$\mathrm{MRI}$ at 24 and 48 hours was performed on $7 \mathrm{~T}$ MRI scanner (Varian, Inc. Walnut Creek, CA) using a gradient echo sequence $(T R / T E=50 / 4.2$, slice thickness $=0.5 \mathrm{~mm}, F O V=3 \mathrm{~cm}$, matrix $=256 \times 256, \quad F A=50$, acquisition time $=9 \min 55$ $\mathrm{sec})$. Accumulation of nanoparticles on MRI was measured as the size of susceptibility artifacts (\% reduction of lumen area). After final in vivo MRI, both in situ and ex vivo fluorescence imaging was performed using Maestro ${ }^{\mathrm{TM}}$ in vivo imaging system (CRi, Woburn, MA). Perl's iron and immunofluorescence staining was also performed to confirm co-localization of nanoparticles with macrophages.

Results: In vivo MRI showed the accumulation of HFn-Fe and $\mathrm{CN}$ in the ligated left carotid arteries (Figure IA), with T2* signal loss causing significant reduction in luminal area (HFn-Fe; $24 \mathrm{H}$ $48 \pm 8.9 \%, 48 \mathrm{H} 33.9 \pm \mathrm{II} .3 \%, \mathrm{CN} ; 24 \mathrm{H}: 26.1 \% \pm 13.0 \% ; 48 \mathrm{H}$ : $28.0 \% \pm 5.7 \%$, Figure 2 ). There was no evidence of $\mathrm{T}_{2} *$ signal loss in the non-ligated right carotid arteries (HFn-Fe; $24 \mathrm{H}$ : I.6 $\pm 2.8 \%, 48 \mathrm{H}:-5.6 \pm 8.8 \%, \mathrm{CN} ; 24 \mathrm{H}: 4.6 \% \pm 9.6 \%, 48 \mathrm{H}:-$ $5.5 \% \pm 1.2 \%)$. No significant signal was seen in sham-operated mice (HFn-Fe; $24 \mathrm{H}: 2.7 \%$ and $48 \mathrm{H}:-0.2 \%$, Figure IB and 2). For confirmation, $\mathrm{CN}$ fluorescence imaging showed high signal intensity in the left carotid arteries (Figure 3) with no accumulationseen in either non-ligated right carotid arteries or sham-operated left carotid arteries. HFn-Fe uptake by macrophages was confirmed on histology (Figure 4).

Figure 3 (abstract PI5I)
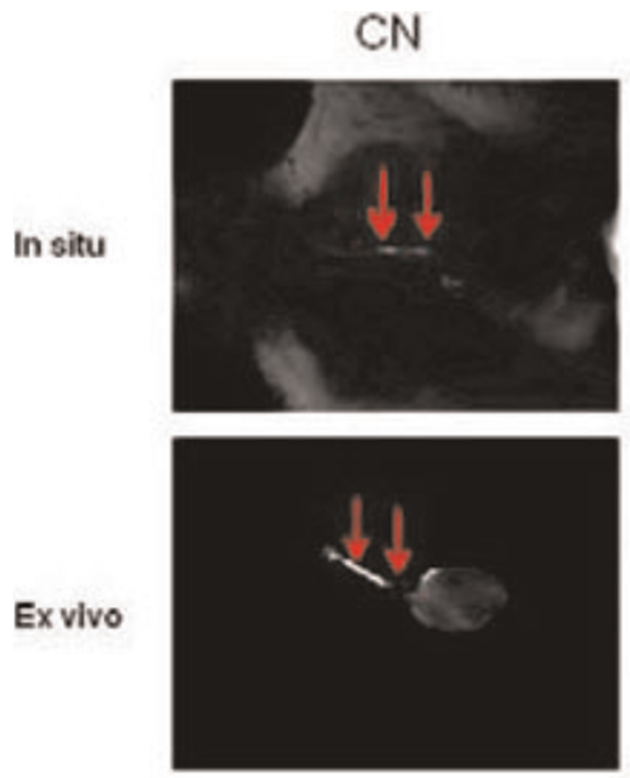

\section{Sham}
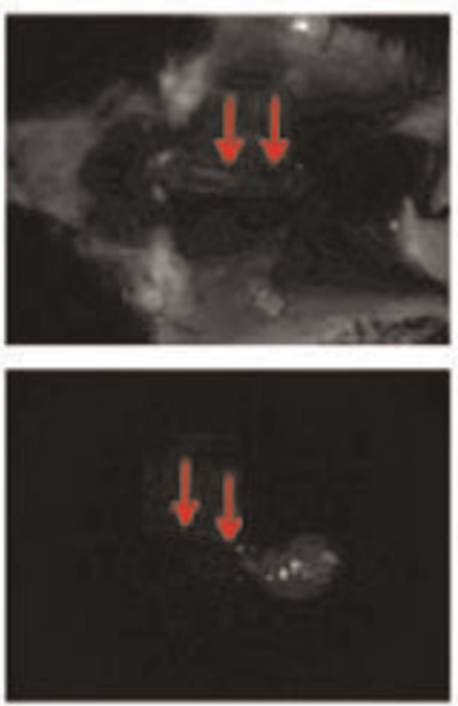
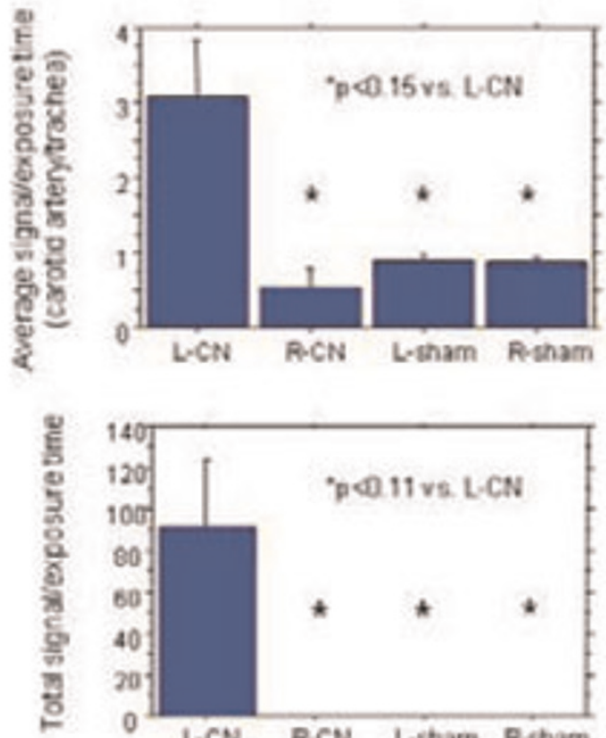
Figure 4 (abstract PI5I)

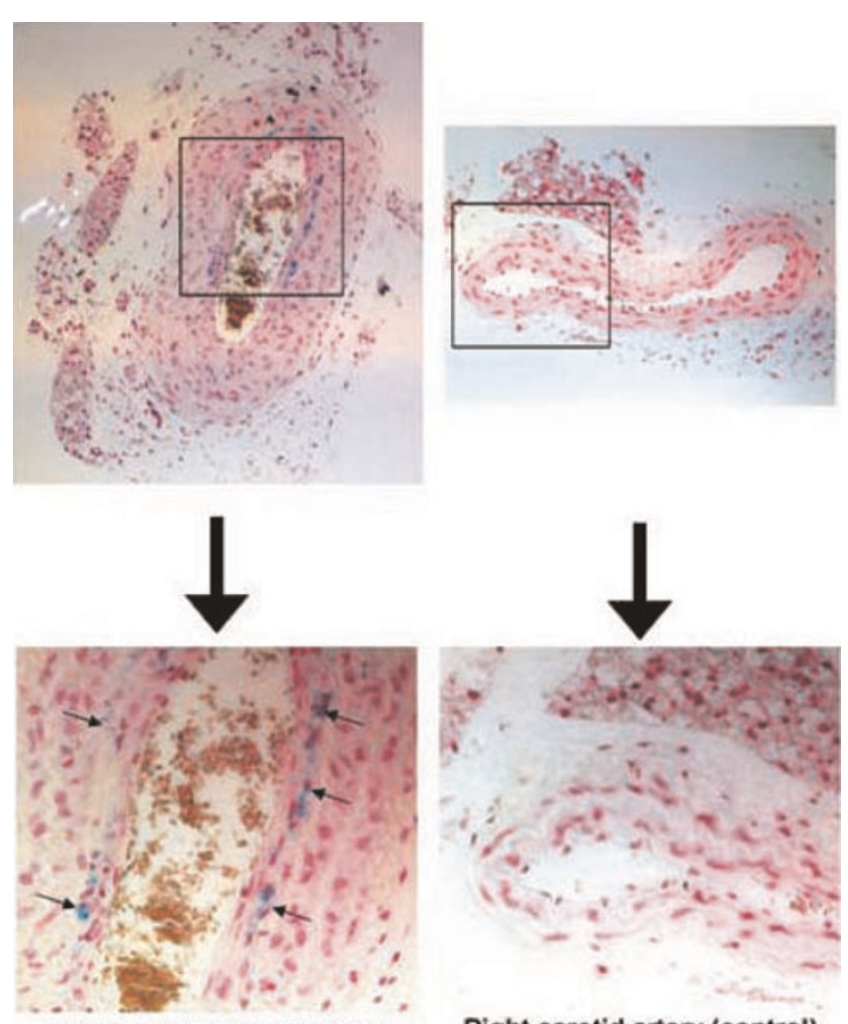

Left carotid artery (ligation)
Right carotid artery (control)

Conclusion: Both iron oxide protein cage nanoparticles and graphite/FeCo core-shell nanocrystals allow noninvasive MRI of macrophage accumulation in mouse atherosclerosis. These powerful, multi-functional nanoparticles may allow improved noninvasive characterization of vascular inflammation and atherosclerosis.

\section{P I52}

Myocardial perfusion and viability after percutaneous recanalization of coronary chronic total occlusions: a cardiovascular magnetic resonance study

Chiara Bucciarelli-Ducci', Didier Locca', Giuseppe Ferrante', Joanna Petryka', Agata Grasso', Rory O'Hanlon', Francesca Del Furia ${ }^{2}$, Christine Wright', Karen Symmonds', Peter Gatehouse', Sanjay Prasa', Carlo Di Mario and Dudley Pennell'

ICMR Unit, Royal Brompton Hospital, London, UK

${ }^{2}$ Interventional Cardiology, Royal Brompton Hospital, London, UK

Journal of Cardiovascular Magnetic Resonance 2009, I I(SuppI I):PI52

Background: Coronary angiography can assess the anatomy of collaterals but is of limited value for evaluating their functional significance. In clinical practise, the size of collateral connection correlates is assessed by 3 grades: $\mathrm{CCO}$, no continuous connection between donor and recipient artery; $\mathrm{CCl}$, contin-

uous, thread-like connection; and $\mathrm{CC} 2$, continuous, side branchlike connection. Its direct validation by myocardial perfusion imaging has not been established so far. The aim of this study was to assess the impact of collateral connection on infarct size and myocardial perfusion reserve by adenosine cardiovascular magnetic resonance (CMR) perfusion imaging in patients with single-vessel chronic total occlusion (CTO).

Methods: Forty-two consecutive patients with CTO (occlusions older > 3 months) underwent adenosine CMR perfusion prior to percutaneous coronary intervention. Grading of collateral connection (CCO-CC2) was carried out in multiple angiographic projections prior to recanalization. Myocardial perfusion reserve index (MPRI) (cut-off of normality $\geq 2$ ), the amount of infarcted area, and the number of non-viable segments were calculated and corrected for the dimension of area at risk. Data are expressed as mean \pm SD or median and range (minmax), as appropriate.

Results: The angiographic grading demonstrated that II patients had no continuous connection (CCO), 14 patients had continuous thread-like connection $(\mathrm{CCl})$ and 17 patients continuous side branch-like connection (CC2). In 75\% of the patients MPRI was reduced (<2). MPRI $>2$ was present in $25 \%$ of patients and its prevalence significantly increased from $0 \%$ to $23.1 \%$ to $43.7 \%$ in $\mathrm{CC}, \mathrm{CCl}$ and $\mathrm{CC2}$ respectively, $\mathrm{p}=0.03$.

The percentage of infarcted myocardium in the area at risk was significantly different among the three groups: $39 \%(0-91 \%)$ in $\mathrm{CC} 0,9 \%(0-79 \%)$ in $\mathrm{CCl}$ and $12 \%(0-75 \%)$ in $\mathrm{CC} 2, \mathrm{p}=0.038$ ( $p=0.0128$ for comparison CC0 vs CC2, $p=0.054$ for CC0 vs $\mathrm{CCl}, \mathrm{p}=0.7 \mathrm{I}$ for $\mathrm{CCl}$ vs $\mathrm{CC} 2$ ). (See figure I) Patients with $\mathrm{CCO}$ had a higher percentage of non-viable segments compared with patients with $\mathrm{CCl}$ and $\mathrm{CC} 2(48 \% \pm 4 \mathrm{I}$ vs $22 \% \pm 54$, $\mathrm{p}=0.04)$.

Conclusion: In patients with CTO the presence of CC2 (more developed collaterals) is more frequently associated with smaller infarct size and a lower number of non-viable segments in the risk area. More developed collaterals are also associated with a higher prevalence of normal myocardial perfusion reserve in the area at risk. CMR perfusion is a useful non-invasive method to assess the functional significance of collateral connections.

\section{PI53}

Reproducibility of myocardial salvage in acute myocardial infarction by the use of ContrastEnhanced Magnetic Resonance Imaging Mahdi Sareban, Hubertus Engelhardt, Ingo Eitel, Matthias Gutberlet, Gerhard Schuler and Holger Thiele University of Leipzig - Heart Center, Leipzig, Germany

Journal of Cardiovascular Magnetic Resonance 2009, I I(SuppI I):PI53

Introduction: Using mortality as an endpoint in studies comparing different reperfusion strategies in STEMI requires increasingly large sample sizes to test advances with existing therapy. Thus, there is rising interest in surrogate endpoints allowing a reduction in sample size. One of those measures is myocardial salvage which can be assessed retrospectively by T2weighted and delayed enhancement images as shown in animal studies [I]. Currently there are only limited data in humans with acquisition of T2-weighted images not covering the full ventricle [2]. In order to establish myocardial salvage $M R$ imaging as a surrogate endpoint there is the need of convincing reproduci- 
bility data. So far reproducibility has been established only for infarct size measurements [3].

Purpose: To assess the reproducibility of myocardial salvage measurements in a group of patients with acute myocardial infarction scanned at two subsequent days with a second contrast agent injection.

Methods: In 20 patients with acute STEMI transverse electrocardiographically gated T2-weighted triple-inversion-recovery images were acquired to assess the area at risk and delayed enhancement (DE) images using a 3D inversion recovery gradient echo sequence assessed the infarct size. All patients underwent a repeated measurement the subsequent day starting with new scout images and using the same dose of the contrast agent. Area at risk and infarct size were determined as the percentage of left ventricular mass (\%LV) by two independent observers and subsequently the myocardial salvage index was calculated as area at risk-infarct size/area at risk. In addition extent of microvascular obstruction was assessed. Inter-, intraobserver variabilities and reproducibility data were calculated according to standard definitions and compared by the method of Bland and Altman. Image quality was assessed by a score ranging from $0-4$ ( 0 = not assessable; 4 = optimal image quality).

Results: The mean difference between percutaneous coronary intervention and MR imaging was $2.8 \pm 1.3$ days. All images were suitable for analyses with a mean image quality of $3.2 \pm 0.6$ for DEimages and $2.5 \pm 0.6$ for T2-weighted-images. The area at risk was as high as $47.4 \pm 11.7 \% \mathrm{LV}$ and the infarct size $20.2 \pm 9.6 \% \mathrm{LV}$. The corresponding myocardial salvage index was $57.7 \pm 14.9$ (range 23.4-75.8). In 17 (85\%) patients there was evidence of microvascular obstruction. Area at risk difference (bias) between scan I and scan II was I.2\%LV and limits of agreement were $\pm 7.5 \% \mathrm{LV}$. The results for infarct size were $0.5 \% \mathrm{LV} \pm 2.6 \% \mathrm{LV}$ limits of agreement. The resulting bias for myocardial salvage index was -0.3 with limits of agreement of \pm 5 .0. Intra- and interobserver variability was low with a mean bias of -0.7 (limits of agreement \pm 4.6 ) and -0.0 (limits of agreement \pm 4.7 ), respectively.

Conclusion: The multimodal MRI approach is an excellent tool for myocardial salvage assessment with excellent reproducibility in acute STEMI. It has therefore the potential to serve as a surrogate endpoint to uncover advantages of new reperfusion strategies and allows a further reduction in the sample size of clinical trials. The previously shown excellent reproducibility for infarct size measurement could be confirmed in this trial.

\section{References}

I. Aletras AH, Tilak GS and Natanzon A, et al: Retrospective determination of the area at $r$ risk for reperfused acute myocardial infarction with T2-weighted cardiac magnetic resonance imaging: histopathological and displacement encoding with stimulated echoes (DENSE) functional validations. Circulation 2006, I I 3: | 865-70.

2. Friedrich MG, Abdel-Aty H, Taylor A, Schulz-Menger J, Messroghli D and Dietz R: The Salvaged Area at Risk in Reperfused Acute Myocardial Infarction as Visualized by Cardiovascular Magnetic Resonance. J Am Coll Cardiol 2008, 5 I: I58I-7.

3. Thiele H, Kappl MJ, Conradi S, Niebauer J, Hambrecht R and Schuler G: Reproducibility of chronic and acute infarct size measurement by delayed enhancement-magnetic resonance imaging. I Am Coll Cardiol 2006, 47: $|64|-5$.
PI54

Diagnostic accuracy of high-temporal resolution whole heart coronary magnetic resonance angiography at 3.0 Tesla

Nagata Motonori, Hajime Sakuma, Nanaka Ishida, Shingo Kato, Hiroshi Nakajima, Masaki Ishida, Kakuya Kitagawa, Katsuya Onishi, Masaaki Ito and Kan Takeda

Mie University hospital, Tsu, Mie, Japan

Journal of Cardiovascular Magnetic Resonance 2009, I I (SuppI I):PI54

Introduction: $30 \mathrm{~T}$ whole heart coronary $\mathrm{MR}$ angiography permits acquisition of coronary arterial images with improved signal to noise ratio and high spatial resolution. The use of a patient-specific, narrow acquisition window in the cardiac cycle can reduce motion blurring on coronary MR angiography, and may allow for improved delineation of distal coronary arteries and branch vessels.

Purpose: The purpose of this study was to determine the image quality and diagnostic accuracy of $3.0 \mathrm{~T}$ whole-heart coronary MR angiography acquired with a subject-specific, narrow acquisition window in the cardiac cycle.

Methods: Free breathing whole heart coronary MR angiograms were acquired in 32 patients with suspected coronary artery disease by using a $3.0 \mathrm{~T}$ MR imager and 6 channel cardiac coil system. After administration of gadolinium contrast medium, navigator-echo gated, 3-dementional TFE images were obtained with T2 preparation, fat saturation, TR/TE/FA of $4.2 \mathrm{~ms} / 2.1 \mathrm{~ms} /$ 20 degree and SENSE factor of 2. A narrow data acquisition window in the cardiac cycle was used by monitoring motion of the coronary arteries on cine MRI in each patient. MRA data were acquired during diastole in 22 patients (averaged heart rate $=65.5 \pm 8.2 \mathrm{beat} / \mathrm{min}$, acquisition window $=63 \pm 25 \mathrm{~ms}$ ), and during systole in 10 patients (averaged heart rate $=75.1 \pm$

\section{Figure I (abstract PI54)}

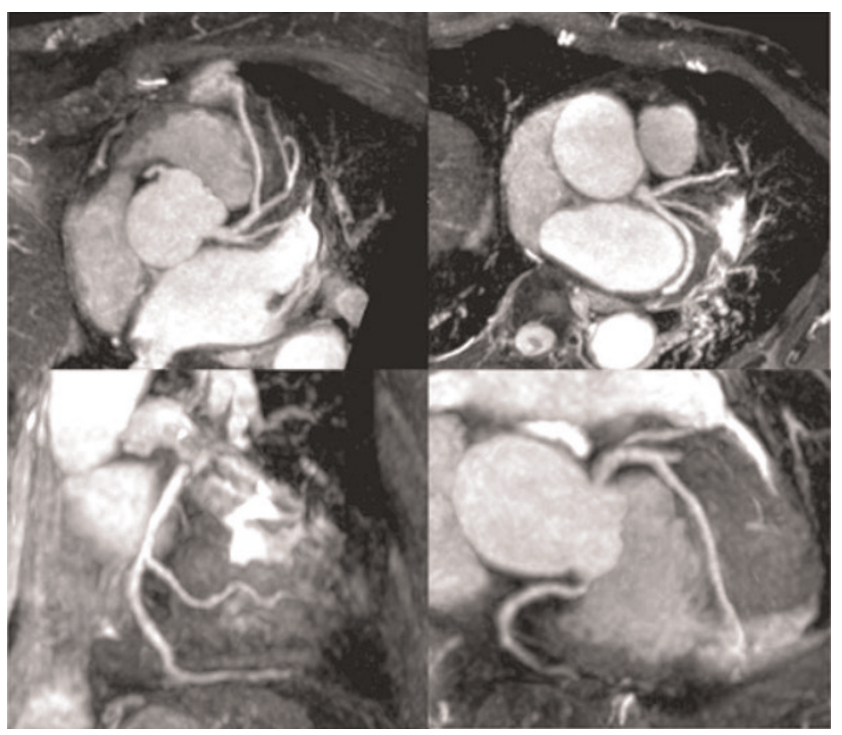

Sliding partial MIP images of $3 \mathrm{~T}$ whole heart coronary MRA acquired with a patient-specific narrow acquisition window (50 $\mathrm{ms}$ ) in the cardiac cycle. 
Figure 2 (abstract PI54)

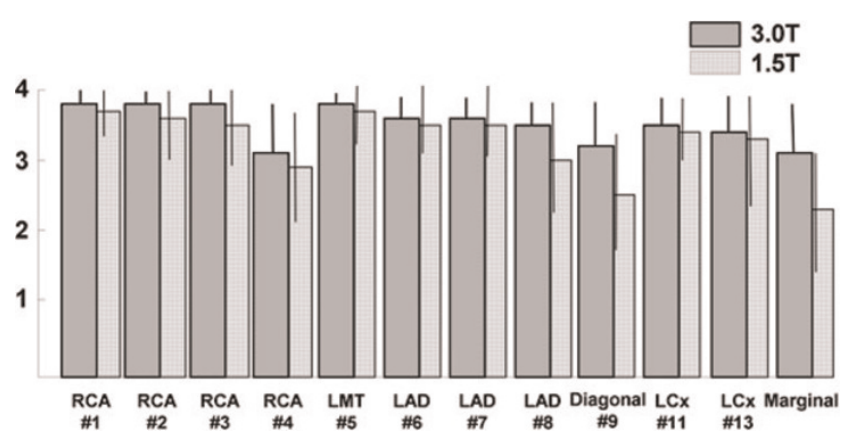

Averaged image quality score of 3 Twhole heart coronary MRA compared with I.5 MRA.

I0.3 beats $/ \mathrm{min}$, acquisition window $=36.2 \pm 6.2 \mathrm{~ms})$. Image quality was classified on a 4-point scale $(\mathrm{I}=$ poor, 2 = moderate, 3 = good, 4 = excellent). X-ray coronary angiograms were obtained in II patients within 2 weeks from MR study. Significant coronary artery disease was defined as a diameter reduction of $\geq$ $50 \%$ in coronary arteries with a reference of $\geq 2 \mathrm{~mm}$ on X-ray coronary angiography. MRA images were interpreted by 2 independent observers by using a sliding multiplanar reformat reconstruction method, and disagreement between 2 observers was settled by a consensus reading.

Results: Acquisition of whole heart coronary $M R$ angiography was completed in all 32 patients with averaged imaging time of II $.4 \pm 4.5$ minutes. Excellent average image quality scores $(\geq 3.8)$ were observed in the proximal and mid portion of the arteries (RCA \#I-3, LMT\#5, LAD \#6-7, LCX\#II) (Figure I). In addition, high image quality scores $(\geq 3.0)$ were achieved in the distal segments and branch vessels as well (RCA\#4, LAD\#8-9, LCX\#I213) (Figure 2). On a vessel-based analysis, the sensitivity, specificity, positive and negative predictive value and accuracy of whole heart coronary MR angiography for the detection of significant coronary artery disease was 83\% (95\% confidence interval [Cl] 36-99\%), 96\% (95\%Cl 79-100\%), 83\% (95\%Cl 36-99\%), 96\% (95\%Cl 79-100\%), and $94 \%(95 \% \mathrm{Cl} 86-100 \%)$, respectively.

Conclusion: $30 \mathrm{~T}$ free-breathing whole heart coronary MR angiography acquired with a subject-specific, narrow acquisition window in the cardiac cycle permits improved visualization of coronary arteries including distal segments and branch vessels. High negative predictive value found in this study indicates the value of 3.0 $\mathrm{T}$ coronary MRA for screening significant coronary artery disease.

\section{PI55}

Myocardial hypoperfusion due to hypoplastic coronary arteries: a cause for false-positive results during adenosine stress Cardiac Magnetic Resonance Imaging

Guenter Pilz, Maximilian Graw, Tobias Heer, Markus Klos, Eman Ali and Berthold Hoefling

Cardiac MRI, Clinic Agatharied, University of Munich, Hausham, Germany

Journal of Cardiovascular Magnetic Resonance 2009, I I(SuppI I):PI 55

Background: Magnetic resonance myocardial perfusion imaging (MRMPI) based on adenosine stress cardiac magnetic
Table I (abstract PI55)

\begin{tabular}{llll}
\hline CMR parameter & FP results & TP results & P value \\
\hline LV EF & $65.8 \%$ & $65.6 \%$ & 0.86 \\
LV EDV (ml) & 126 & 129 & 0.58 \\
LV mass (g) & 130 & 137 & 0.20 \\
LV wall stress & 35.7 & 34.7 & 0.62 \\
Transmural extent of ischemia & $41.1 \%$ & $41.3 \%$ & 0.64 \\
Temporal persistence of & 8.9 & 8.7 & 0.44 \\
ischemia (beats) & & & \\
CMR - CA correlation: & & & \\
Ischemia in area with & $51.2 \%$ & $0.6 \%$ & $<0.0001$ \\
hypoplastic vessel & & & \\
\hline
\end{tabular}

resonance (CMR) is increasingly proposed for non-invasive detection of relevant coronary artery disease (CAD). However, little is known about the impact of the normal variability of coronary anatomy and caliber on the diagnostic performance of adenosine stress CMR.

Purpose: Aim of our study was to examine whether hypoplastic coronary arteries found as normal variations in rightdominant or left-dominant circulation may account for visualization of hypoperfusion in the absence of coronary stenosis and thus may result in false-positive (FP) results of MRMPI in the assessment of CAD.

Methods: From 05/2007 until 01/2008, we enrolled 206 consecutive patients with suspected CAD undergoing MRMPI, who were diagnosed as having myocardial ischemia during adenosine-stress perfusion and subsequently underwent coronary angiography (CA). Patients were examined in a I.5 Twholebody scanner (GE Signa Excite). After three minutes of adenosine infusion ( $40 \mu \mathrm{g} / \mathrm{kg} / \mathrm{min})$, myocardial first-pass sequence in 4-5 continuous short-axis orientation using Gadolinium-based contrast agent was performed $(0.1 \mathrm{mmol} / \mathrm{kg}$, Omniscan, GE Healthcare Buchler). Images were visually analyzed in comparison to rest perfusion by two experienced investigators in consensus. A perfusion deficit was regarded relevant if affecting more than 1/3 of myocardial wall thickness in at least two neighbouring myocardial segments and persisting for more than five heart beats after maximal signal intensity in the LV cavity. Significant CAD was defined as luminal narrowing of $\geq 70 \%$ in $C A$.

Figure I (abstract PI55)

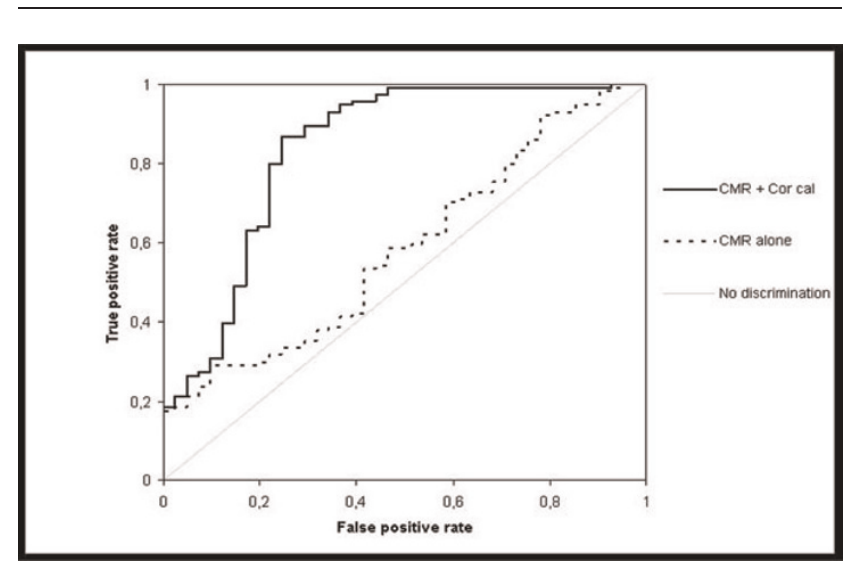


Results: Significant CAD was invasively confirmed in 165 out of 206 (CMR specificity: $80.1 \%=$ true positive (TP)) and ruled out in 4I / 206 patients with previous ischemia in MPMRI (= FP). TP and FP were comparable for pre-test risk (Morise scores 15.0 vs. I5.5, $\mathrm{p}=0.32$ ) and CMR findings (Table I). CA confirmed that the proximal diameters of the coronary vessels correlate well with the type of coronary dominance. As the main result we found a significant correlation between FP CMR results and presence of a hypoplastic coronary vessel in CA (mean proximal diameter $2.5 \pm 0.3 \mathrm{~mm}$ ) supplying the area of ischemia in CMR, opposite to the dominant vessel. Logistic regression analysis showed the presence of a hypoplastic vessel supplying the region of ischemia to be the most predictive parameter for discrimination of FP versus TP. By adding this information to CMR, diagnostic accuracy to avoid FP improved significantly (ROC analysis). Figure I compares ROC curves for identification of FP patients using standard CMR parameters (see table) alone (dotted line, AUC: $0.59 \pm 0.05$ ) and the combination of standard CMR parameters and coronary caliber (Cor cal) information (presence of a hypoplastic vessel in the area of ischemia (full line, AUC: $0.84 \pm 0.04, p<0.0001)$ ).

Conclusion: Hypoplastic coronary arteries found as normal variations in right-dominant or left-dominant circulation may account for visualization of hypoperfusion in the absence of coronary stenosis and thus may result in false-positive results of MRMPI in the assessment of CAD. Thus, noninvasive asessment of the proximal coronary diameter in the vessel supplying the area of ischemia could reduce the rate of false positive CMR results and the rate of subsequent superfluous diagnostic $C A$.

\section{PI56}

Sensitivity of resting magnetic resonance first-pass myocardial perfusion imaging for the detection of acute and chronic myocardial infarction

Alejandro Aquino, Edwin Wu, Thomas A Holly and Daniel C Lee

Northwestern University, 633 Clark Street Evanston, Chicago, 60208, IL, USA

Journal of Cardiovascular Magnetic Resonance 2009, I I(SuppI I):PI 56

Background: At rest, myocardial blood flow in an area of myocardial infarction is roughly $20 \%$ that of blood flow in remote myocardium [I]. Since magnetic resonance first-pass myocardial perfusion imaging has been shown to effectively identify 2:I differences in vasodilated blood flow in viable myocardium [2], one would expect that the $5: I$ difference in resting blood flow between infarcted and viable myocardium would be easily identified. However, the ability of resting first-pass perfusion imaging to detect areas of infarcted myocardium is unknown.

Objective: The purpose of this study was to determine the sensitivity of resting cardiac magnetic resonance (CMR) perfusion for evaluating the presence and extent of myocardial infarction, as defined by delayed contrast-enhanced (DE) CMR.

Methods: 59 patients were imaged serially following acute STsegment elevation myocardial infarction (MI) by CMR cine and $\mathrm{DE}$ as part of a study evaluating the ability of CMR indices to predict post-MI remodeling [3]. We retrospectively identified 83 studies in which resting CMR perfusion was performed within the same examination as DE. Short axis perfusion and DE images were obtained within 7 days (30 acute studies) and more than
Figure I (abstract PI56)

\section{$\mathrm{DE}$}

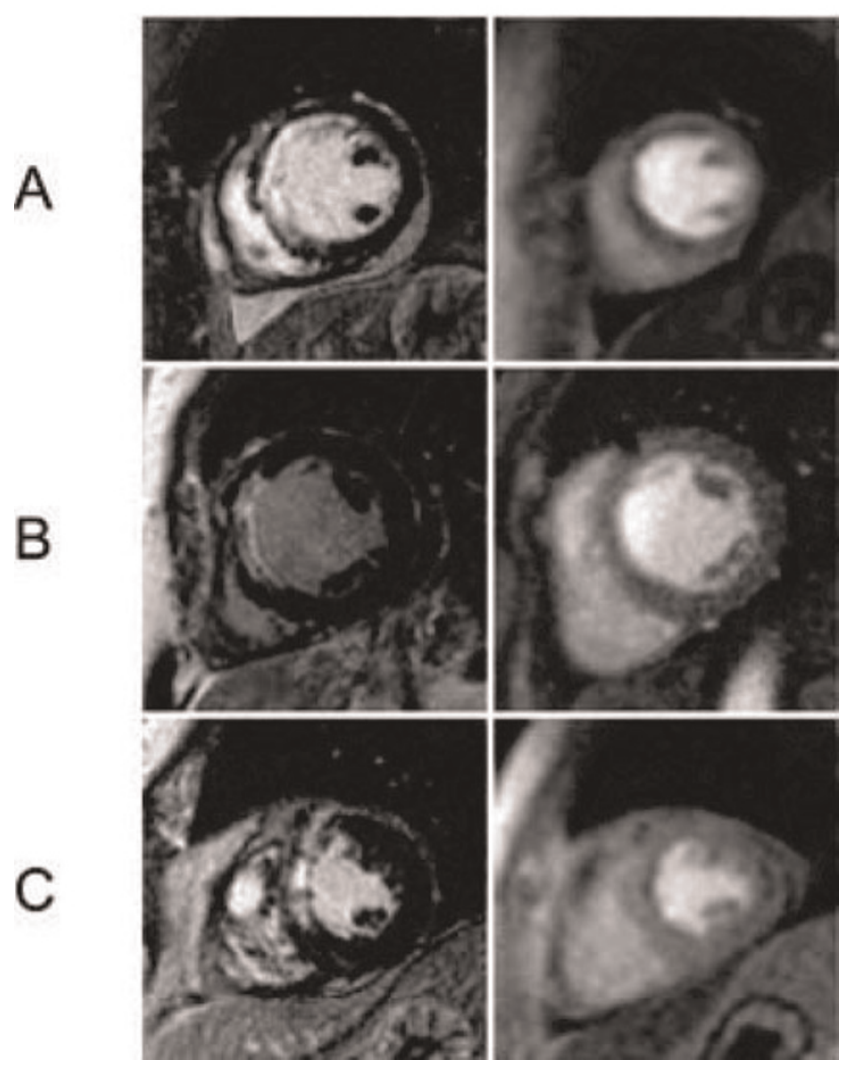

Panel A depicts a patient in whom the size and extent of perfusion defect and delayed contrast enhancement (DE) are matched. In Panel B, no perfusion defect is seen despite a large area of $D E$. In Panel $C$, the perfusion defect significantly underestimates the size of $D E$.

3 months (53 chronic studies) following MI. CMR studies were visually analyzed by two blinded readers on a 16-segment model. Each segment was scored independently for the presence and extent of DE $(0=0 \%, I=1-25 \%, 2=26-50 \%, 3=51-75 \%$, $4=76-100 \%)$ and resting perfusion defect $(0=$ no defect, $1=$ mild defect, $2=$ moderate defect, 3 = severe defect $)$. For sensitivity analysis, DE was considered the gold standard for presence of MI.

Results: For all studies with $\mathrm{MI}$ identified by $\mathrm{DE}$, the sensitivity of resting CMR perfusion for the detection of MI was 7I\% (59/ $83)$. The sensitivity was higher in patients with acute infarcts $(26 /$ $30,87 \%)$ than for patients with chronic infarcts $(33 / 53,62 \%)$. The mean number of segments demonstrating hyperenhancement was higher than the mean number of segments demonstrating resting perfusion defects $(4.7$ vs $2.5, P<0.0001)$. When comparing patients with true-positive perfusion studies to those with false-negative perfusion studies, the mean hyperenhancement score was higher (10.6 vs $6.0, \mathrm{p}<0.001)$. Figure I.

Conclusion: The sensitivity of resting magnetic resonance first-pass myocardial perfusion imaging for the detection of myocardial infarction is relatively low, and the extent of 
hyperenhancement is larger than the extent of perfusion defects. Sensitivity is higher for acute infarcts than for chronic infarcts, and smaller infarcts are more likely to be missed. When performing first-pass perfusion studies, delayed contrastenhanced imaging also needs to be performed for accurate assessment of the size and extent of myocardial infarction.

\section{References}

I. Selwyn AP, et al: Circulation 1986, 73(3):433-443.

2. Lee DC, et al: Circulation 2004, I I 0(I):58-65.

3. Wu E, et al: Heart 2008, 94(6):730-36.

\section{P I 57}

Multi-stage diastolic function classification algorithm by cardiac MRI demonstrates the relationship between severity of diastolic dysfunction and acute infarct size

Sean D Pokorney', Michael Andrew Morse ${ }^{2}$, Daniel C Lee', Kofo O Ogunyankin' and Edwin Wu'

'Northwestern University, Chicago, IL, USA

${ }^{2}$ University of North Carolina, Chapel Hill, NC, USA

Journal of Cardiovascular Magnetic Resonance 2009, I I(SuppI I):PI 57

Introduction: A previously validated multi-stage echocardiography (echo) classification algorithm for diastolic function (DF) showed that severe diastolic dysfunction independently predicts mortality after acute myocardial infarction. Currently, echo is the non-invasive imaging modality of choice for classifying diastolic dysfunction into four distinct groups based on a combination of echo parameters. However, a newer multi-stage echo algorithm has been developed that classifies patients into three diastolic function categories: Normal, Abnormal Relaxation, and Severe (which combines Pseudonormal and Restrictive patterns). This algorithm is a validated systematic approach that provides accurate diastolic function staging of patients, and it has been shown to correlate strongly with catheter derived left ventricular filling pressures. It is currently unknown whether CMR can accurately categorize the overall severity of diastolic dysfunction using a similar multi-stage approach.

Purpose: A CMR multi-stage diastolic function classification system, adapted by substitution of CMR-derived parameters for corresponding echo parameters, can accurately classify diastolic dysfunction severity. Furthermore, applying this diastolic dys-
Figure I (abstract PI57)

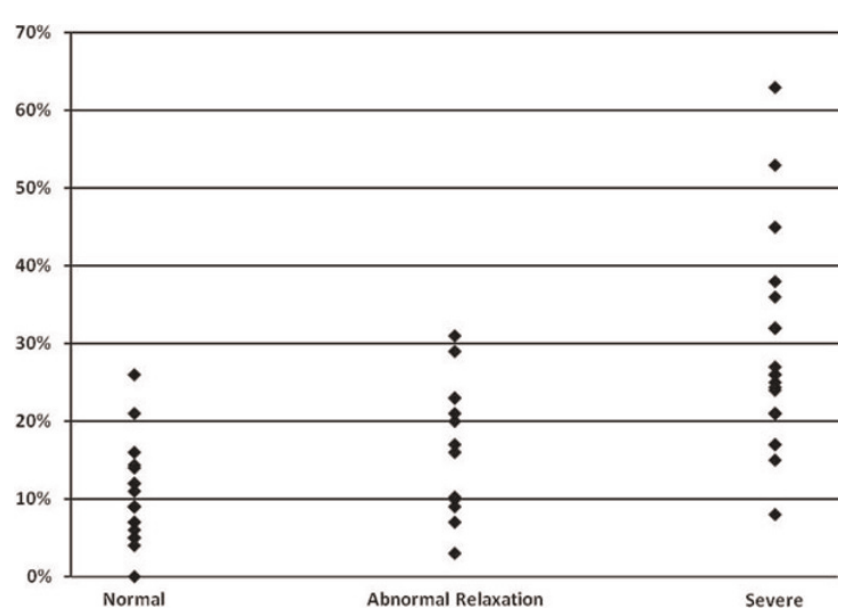

MRI infarct size (\% of LV) grouped by diastolic function.

function algorithm can facilitate evaluation of the relationship between diastolic function and CMR infarct size.

Methods: Subjects $(n=5 I)$ underwent CMR for characterization of left ventricular mass, left end diastolic volume, left atrial volume, ejection fraction, mitral inflow pattern, tissue velocities, and infarct size within $2.8 \pm 1.4$ days status post acute myocardial infarction. A subset of patients $(n=21)$ concurrently had echo within $2.3 \pm 1.6$ days of the CMR. CMR and echo data were used independently to classify subjects' diastolic function as Normal, Abnormal Relaxation, or Severe, using the multi-stage approach. The algorithm uses ejection fraction, pulmonary vein inflow, mitral inflow patterns, and deceleration time to initially categorize patients' diastolic function. If the initial parameters did not clearly delineate the appropriate classification, tissue velocity imaging, including E/E' ratios, was used to further differentiate between the three groups. Myocardial infarct sizes obtained by CMR were then compared between the resulting categories.

Results: The severity of diastolic dysfunction using echo $(n=2 I)$ and CMR $(n=5 I)$ showed a strong relationship with acute myocardial infarction size (ANOVA, $P=0.01$ and $\mathrm{P}<0.000$ I respectively). The correlation between individual

Table I (abstract P I57) Selected parameters and infarct sizes by diastolic function classification

\begin{tabular}{|c|c|c|c|c|c|c|c|c|}
\hline & \multicolumn{3}{|c|}{ Echo $(n=2 I)$} & \multicolumn{3}{|c|}{ CMR $(n=5 I)$} & \multirow[b]{2}{*}{$\begin{array}{l}\text { Echo vs CMR } \\
\text { Correlation (r) }\end{array}$} & \multirow[b]{2}{*}{ p-value } \\
\hline & $\begin{array}{l}\text { Normal } \\
(n=14)\end{array}$ & $\begin{array}{l}\text { Abnormal } \\
\text { Relaxation } \\
(n=4)\end{array}$ & $\begin{array}{l}\text { Severe } \\
(n=3)\end{array}$ & $\begin{array}{l}\text { Normal } \\
(n=18)\end{array}$ & $\begin{array}{l}\text { Abnormal } \\
\text { Relaxation } \\
(n=\mid 4)\end{array}$ & $\begin{array}{l}\text { Severe } \\
(n=19)\end{array}$ & & \\
\hline Ejection Fraction (\%) & $57.8 \pm 9.1$ & $50.9 \pm 5.5$ & $35.1 \pm 9.4$ & $51.2 \pm 5.9$ & $47.1 \pm 6.6$ & $36.4 \pm 10.8$ & 0.63 & 0.004 \\
\hline Mitral E/A & $1.29 \pm 0.48$ & $0.93 \pm 0.71$ & $1.62 \pm 1.45$ & $1.22 \pm 0.33$ & $1.20 \pm 0.72$ & $\mathrm{I} .43 \pm 0.77$ & 0.34 & 0.090 \\
\hline Mitral E DT (msec) & $|86.6 \pm 31|$. & $216.3 \pm 37.2$ & $|57.3 \pm 3| .7$ & $188.6 \pm 32.2$ & $215.2 \pm 53.0$ & $157.7 \pm 65.1$ & 0.08 & 0.702 \\
\hline TVI E' (lateral) mm/s & $10.9 \pm 3.3$ & $6.7 \pm 2.1$ & $7.3 \pm 1.5$ & $6.5 \pm 2.0$ & $6.4 \pm 1.3$ & $3.9 \pm 2.2$ & 0.83 & 0.002 \\
\hline E/E' (lateral) & $8.0 \pm 3.2$ & $7.8 \pm 3.3$ & $10.4 \pm 1.5$ & $7.4 \pm 2.8$ & $7.5 \pm 2.0$ & $12.3 \pm 6.4$ & 0.70 & 0.020 \\
\hline $\begin{array}{l}\text { LV Mass/Height } \\
\text { Index }\left(g / \mathrm{m}^{\wedge} 2.7\right)\end{array}$ & $42.0 \pm 10.8$ & $52.4 \pm 23.9$ & $51.9 \pm 8.7$ & $49.0 \pm 7.0$ & $62.1 \pm 17.9$ & $62.1 \pm 17.1$ & 0.36 & 0.060 \\
\hline
\end{tabular}

Values are mean \pm SD; Mitral E DT = Mitral inflow early diastolic deceleration time; TVI E' = Early diastolic tissue velocity; E/E' = ratio of early mitral inflow to early diastolic tissue velocity. 
parameters by echo and CMR was only fair (Table I). However, by using the multi-stage algorithm, the overall agreement between diastolic dysfunction classifications by both modalities was good (Kappa $=0.57, p<0.000 \mathrm{I})$. CMR had $80 \%$ sensitivity and $64 \%$ specificity in detecting any diastolic dysfunction when compared to echo. In identifying severe diastolic dysfunction, CMR had 100\% sensitivity and 63\% specificity.

Conclusion: Correlation of single parameters of diastolic dysfunction between echocardiography and CMR is only fair. This lack of agreement is likely related to the temporal separation between the imaging studies and the rapidly evolving changes following acute myocardial infarction. However, using the multistage classification algorithm provides improved agreement, and this system is very sensitive for diagnosing diastolic dysfunction, especially when severe. Lastly, there is strong association between acute myocardial infarct size and severity of diastolic dysfunction (Figure I).

\section{PI58}

Myocardial TI measurement in patients with thalassemia major

Taigang He', Maria Paes', John-Paul Carpenter', Andreas Greiser ${ }^{2}$, Daniel Messroghli ${ }^{3}$, Sanjay Prasad', Dudley Pennell and David Firmin

'CMR Unit, Royal Brompton Hospital and Imperail College London, London, UK

${ }^{2}$ Siemens Medical Solutions, Erlangen, Germany

${ }^{3}$ Cardiac MRI Unit, Franz-Volhard-Klinik Charité, Berlin, Germany

Journal of Cardiovascular Magnetic Resonance 2009, I I(SuppI I):PI 58

Introduction: Cardiac complications secondary to iron overload are the leading cause of death in thalassemia major (TM). Cardiovascular magnetic resonance (CMR) can provide a noninvasive means of measuring the amount of tissue iron. With CMR, tissue iron is detected indirectly by the effect on relaxation times of hydrogen nuclei in the presence of ferritin and hemosiderin iron. The iron results in shortening of proton relaxation times and both CMR T2* and T2 have been validated as non-invasive means for assessment of myocardial iron overload. [I, 2, 3, 4]. We have demonstrated a linear correlation between $\mathrm{T} 2$ and $\mathrm{T} 2 *$ in patients with iron overload [5]. However,

\section{Figure I (abstract PI58)}

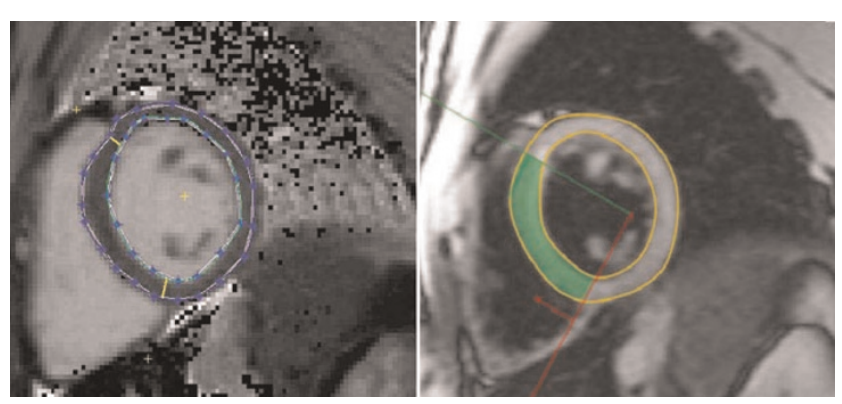

Drawing of ROIs in the left ventricular septum from the same subject. Left: TI mapping of the myocardium; Right: Black blood T2 image at TE of $4.8 \mathrm{~ms}$.
Figure 2 (abstract PI58)

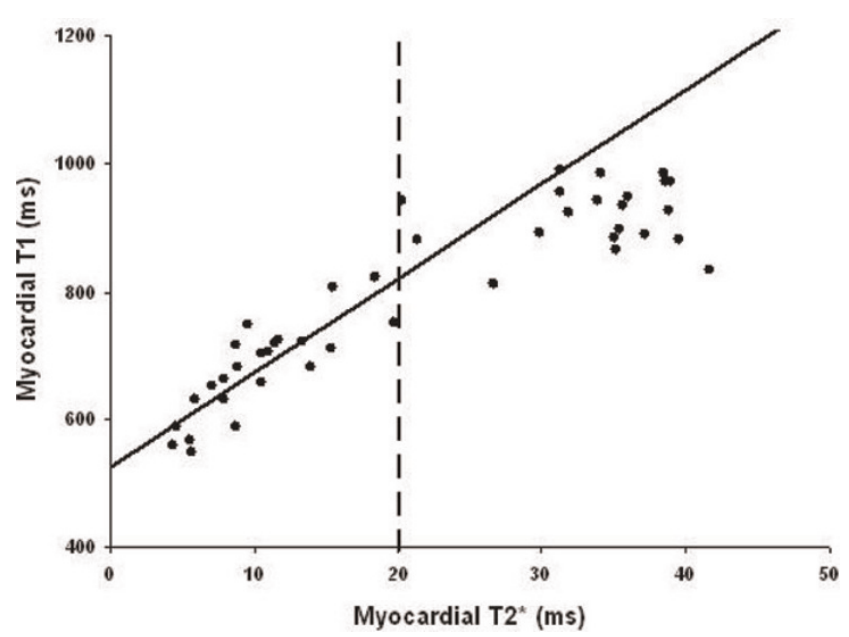

Correlation curve between $\mathrm{TI}$ and $\mathrm{T} 2 *$ measurements. The vertical broken line represents the cut-off myocardial T2* value to distinguish abnormal and normal patients. All data of $\mathrm{T} 2^{*} \geq 20 \mathrm{~ms}$ is fitted with a linear trend line $(R=0.84)$.

there is currently little data on myocardial iron assessment using the longitudinal relaxation time TI mainly due to the cardiac and respiratory problems in CMR.

A modified Look-Locker Inversion recovery (MOLLI) has been recently proposed for myocardial TI quantification [6]. It is of interest, therefore, to investigate whether TI measurement is affected by iron overload in TM patients. For quantitatively analysis, it is important to further investigate the relationship between TI and the more established T2* measurements in TM patient population.

In this study therefore, we aimed to compare myocardial TI [6] and T2* measurements [3]in vivo in order to establish the relationship between them. We hypothesised that TI would correlate with $\mathrm{T}_{2} *$ linearly when iron becomes dominant in the myocardium.

Methods: 43 TM patients (age $34 \pm 24$ years old, 25 males) were studied on a I.5 T MRI scanner (Siemens Sonata) using a cardiac phased array coil and with ECG gating. All patients were scanned using the black blood T2* [3] sequence and the TI sequence [6] subsequently, each within a breath-hold. A single mid-ventricular short axis slice was imaged with both T2* and TI measured in the left ventricular septum (Figure I). The monoexponential decay model and the nonlinear curve fitting algorithm were employed for the T2* analysis (CMRtools, Imperial College London). TI quantification was first acquired using MRMAP http://www.cmr-berlin.org/forschung/mrmapengl/ index.html and the septal analysis was done by use of a selfdeveloped software in Matlab.

Results: Figure I shows a T2 map (left) and a T2* (right) image from the same patient. Figure 2 shows the correlation curve between $\mathrm{TI}$ and $\mathrm{T} 2 *$ drawn from 43 TM patients. There is a linear correlation $(R=0.84)$ between myocardial $\mathrm{TI}$ and $\mathrm{T} 2 *$ measurements in the septum in iron overloaded patients (T2* $\leq 20 \mathrm{~ms}$ ). In the normal group of $\mathrm{T} 2 *>20 \mathrm{~ms}$, however, no clear correlation between these two measurements was found. 
Conclusion: T2* of $20 \mathrm{~ms}$ is a useful indicator to identify patient with cardiac iron. Myocardial TI correlated linearly with T2* in TM patients with cardiac iron overload, which is analogous to our previous finding regarding the relationship between T2 and T2* [5]. This study suggests myocardial TI may potentially be used for assessing iron overload in the heart for TM and other transfusion dependent patients.

References

I. Westwood M, et al: J Magn Reson Imaging 2003, I8:33-9.

2. Tanner MA, et al: Haematologica 2006, 9 I:|388-|39|.

3. He T, et al: J Magn Reson Imaging 2007, 25:1205-1209.

4. He T, et al: J Magn Reson Imaging 2006, 24:580-585.

5. He T, et al: Magn Reson Med 2008.

6. Messroghli DR, et al: Magn Reson Med 2004, 52:|4|-|46.

\section{PI59}

Prognostic value of cardiovascular magnetic resonance in patients with strong suspicion for myocarditis

Otavio R Coelho-Filho, Michael Jerosch-Herold, Sanjeev A Francis and Raymond $Y$ Kwong Brigham and Women's Hospital, Boston, MA, USA

Journal of Cardiovascular Magnetic Resonance 2009, I I(SuppI I):PI59

Background: While late gadolinium enhancement (LGE) imaging by Cardiovascular Magnetic Resonance (CMR) can detect evidence of myocarditis, the prognostic implication of this finding is still unknown.

Purpose: The objective of this study is to assess the prognostic value of CMR in patients with suspected myocarditis.

Methods: We performed gadolinium-enhanced CMR in 76 patients ( 27 females, mean age $4 I \pm 14$ years) with a strong clinical suspicion of myocarditis referred for CMR assessment. Patients were excluded if there was any clinical history of myocardial infarction or prior evidence of coronary artery disease. All enrolled patients had a recent normal coronary angiogram $(n=4 I)$ and/or a negative noninvasive stress test $(n=35)$. The CMR study was performed at a 1.5 Tesla scanner (GE Signa CVi) and a 3.0 Tesla scanner (Siemens Trio) and included short and long-axis function with cine SSFP imaging, and TI-weighted inversion recovery fast GRE imaging 10-15 minutes after a cumulative dose of $0.15 \mathrm{mmol} / \mathrm{kg}$ of gadolinium. In all CMR studies, we assessed the global and regional left ventricular function and presence of atypical (mid-wall or epicardial) LGE by CMR. The LGE mass was measured with a validated software (Pie Medical Imaging, CAAS, The Netherlands) using signal-intensity threshold ( $>3$ SDs above remote myocardium) (Figure I). In all patients, we obtained vital status and non-fatal MACE including heart failure admissions, significant ventricular arrhythmias requiring treatment, and recurrent myocarditis.

Results: After a median follow-up period of I.3 years (range 6 months to 48 months), only 7 patients experienced adverse events including I death, 2 heart failure admissions, 3 cases of significant ventricular arrhythmias, and I recurrent episode of acute myocarditis. Mean left ventricular ejection fraction of the study cohort was $54 \pm 14 \%$. LGE was detected in 23 patients $(30.2 \%)$ and was not significantly associated with MACE by Cox regression analysis. Presence of LGE in this study cohort was associated with an elevated left ventricular mass (65 \pm 17 vs. $\left.75 \pm 30 \mathrm{~g} / \mathrm{m}^{2}, \mathrm{p}=0.0 \mathrm{l}\right)$, regional wall motion abnormalities
Figure I (abstract PI59)

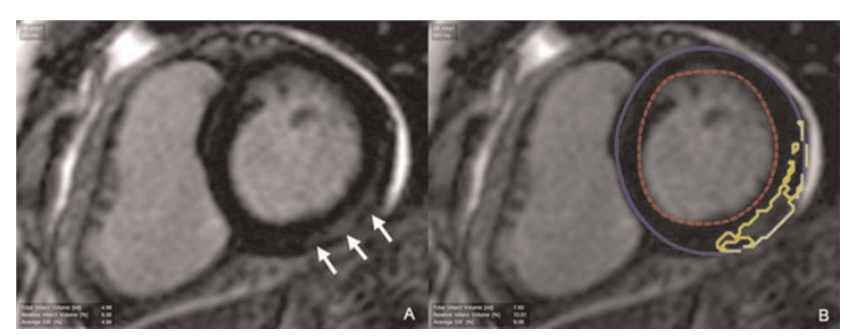

An example of mid-wall LGE present in the mid-inferior and lateral walls (arrows) in a patient with a strong clinical suspicion of acute myocarditis (A). The area confined represents the detected LGE (B).

$(O R=22, p=0.03)$, and with peak serum troponin $(2.4 \pm 6$ vs. $12 \pm 9, p=0.05$ ). The relationship between LGE and elevated left ventricular mass persisted after adjusting for comorbidities such as hypertension and diabetes. The LGE mass (mean $3.5 \pm 6.7 \mathrm{~g})$ was associated with CK levels $(r=0.02, p=0.02)$, with CK-MB levels $(r=0.4 \mathrm{I}, \mathrm{p}=0.008)$ and with troponin levels $(r=0.48, p=0.002)$.

Conclusion: While a high proportion of patients were seen to have evidence of small myocardial involvement by LGE imaging consistent with acute myocarditis, overall patient prognosis of this clinical cohort with small LGE myocardial extent and preserved left ventricular ejection fraction is benign. CMR is helpful in the diagnosis of acute myocarditis and provides quantifiable parameters of the disease activity.

\section{PI60}

Cardiovascular magnetic resonance measures of coronary artery blood flow improve after receipt of oral conjugated estrogen and/or high dose atorvastatin in early post-menopausal women without known coronary arteriosclerosis

Chirapa Puntawangkoon, Tim M Morgan,

David M Herrington, Craig A Hamilton

and William G Hundley

Wakeforest University, Winston Salem, NC, USA

Journal of Cardiovascular Magnetic Resonance 2009, I I(SuppI I):PI60

Objectives: To determine the effect of oral hormone replacement with conjugated equine estrogens (CEE, $0.625 \mathrm{mg}$ po qd) and/or high dose atorvastatin (ATORV, $80 \mathrm{mg}$ po qd) on submaximal exercise induced coronary artery blood flow $(\mathrm{CABF})$ in relatively early postmenopausal women without documented coronary artery disease (CAD).

Background: Oral conjugated equine estrogens and HMGCoA reductase inhibitor (statin) therapy have been shown to enhance arterial endothelial function, but the effect of these agents on submaximal coronary artery blood flow is unknown. Methods: We randomized 56 postmenopausal women aged $59 \pm 3$ years without evidence of coronary arteriosclerosis, into a randomized double blinded, cross-over study in which women were assigned to receive 8 weeks of oral CEE $0.625 \mathrm{mg}$ or placebo, with or without $80 \mathrm{mg}$ per day of ATORV. Each treatment period was separated by a 6 week "washout" period of no treatment. Prior to receipt of any therapy, and after each 
Figure I (abstract PI60)

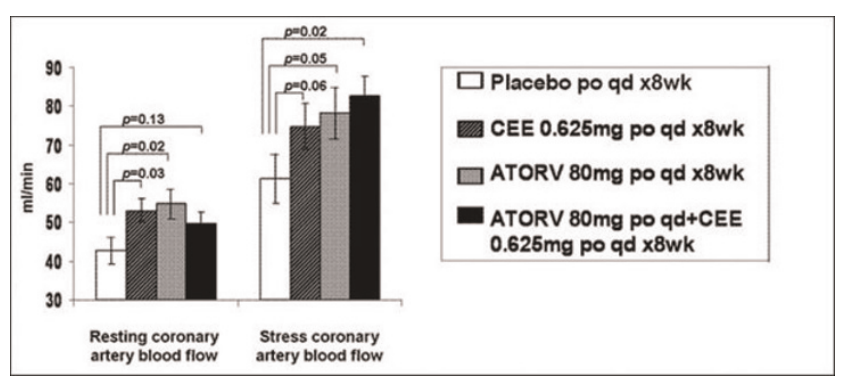

Left anterior descending coronary artery blood flow ( $\mathrm{ml} / \mathrm{min})$.

treatment period, each woman underwent rest/submaximal exercise measures of $\mathrm{CABF}$ in the left anterior descending coronary artery using phase contrast cardiac magnetic resonance imaging at I.5 T. Exercise was performed on an electronically braked bike (Lode, Netherlands). Scan parameters included an 8-mm-thick slice, a $256 \times 256$ matrix, a $20-\mathrm{cm}$ FOV, a throughplane velocity encoding of $150 \mathrm{~cm} / \mathrm{s}$, a $13.8-\mathrm{ms}$ TR, and a $6.7-\mathrm{ms}$ TE.

Results: Of 56 women, 47 were compliant with therapy (85\% of administrated medication) and their results are displayed below (least square mean \pm standard error).

Conclusion: In early postmenopausal women without coronary arteriosclerosis, the administration of conjugated equine estrogens $0.625 \mathrm{mg}$, Atorvastatin $80 \mathrm{mg}$, or their combination have favorable effects on resting and/or submaximal exerciseinduced coronary artery blood flow (Figure I).

\section{PI6I}

Individual versus combined diagnostic

performances of non-invasive CMR and invasive

EMB in troponin-positive patients without coronary artery disease

Hannibal Baccouche', Heiko Mahrholdt', Gabriel Meinhardt ${ }^{1}$, Karin Klingel ${ }^{2}$, Reinhard Kandolf ${ }^{2}$, Udo Sechtem' and Ali Yilmaz'

${ }^{\prime}$ Robert-Bosch-Krankenhaus, Stuttgart, Germany

${ }^{2}$ University of Tuebingen, Tuebingen, Germany

Journal of Cardiovascular Magnetic Resonance 2009, I I(SuppI I):PI6I

Introduction: Only few data is available regarding a direct comparison of both non-invasive CMR and invasive EMB with respect to conformity of procedure-derived diagnoses in the same patients.

Purpose: The aim of this study was to elucidate the diagnostic performance of non-invasive cardiovascular magnetic resonance imaging (CMR) and endomyocardial biopsy (EMB) in patients with troponin-I (Tnl) positive acute chest pain in the absence of significant coronary artery disease (CAD).

Methods: II 74 consecutive patients who were admitted with Tnl-positive acute chest pain between 03/2004 and 07/2007 underwent coronary angiography. In 1012 patients (86\%) significant CAD (stenosis $>50 \%$ ) was detected as underlying reason for the acute chest pain. In 82 out of the remaining 162 patients without significant CAD, further workup was performed including both CMR and EMB.
Results: CMR alone enabled a diagnosis in 66/82 (80\%) and EMB alone in $72 / 82(88 \%)$ patients $(P=0.31)$. Myocarditis was the most frequent diagnosis by both CMR and EMB in this cohort and was detected with a higher frequency by EMB ( $58 \%$ vs. $81 \%$; $P<0.001)$. With the combined approach comprising CMR and $E M B$, a final diagnosis could be established applying the "BelieveThe-Positive-Rule" in 78/82 patients (95\%). This combined approach turned out to yield more diagnoses than either CMR $(p<0.00 I)$ or EMB $(p=0.03)$ as single techniques, respectively. Comparison of diagnostic CMR procedures with the corresponding diagnostic EMBs demonstrated a substantial match of diagnoses (kappa $=0.70)$.

Conclusion: CMR and EMB have good diagnostic performances as single techniques in patients with Tnl-positive acute chest pain in the absence of CAD. The combined application of $C M R$ and $E M B$ yields a considerable diagnostic synergy by overcoming some limitations of $C M R$ and $E M B$ as individually applied techniques.

\section{PI 62}

Impact of myocardial iron loading on right ventricular function

Francisco Alpendurada, Monica Deac, John-

Paul Carpenter, Paul Kirk and Dudley Pennell

Royal Brompton Hospital, London, UK

Journal of Cardiovascular Magnetic Resonance 2009, I I(SuppI I):PI62

Introduction: Iron-overload cardiomyopathy is a potential complication of transfusion-dependent chronic anemic patients, resulting in significant morbidity and mortality. Beta-thalassaemia major is the commonest cause of iron-overload cardiomyopathy worldwide, representing a major health problem in endemic areas, where heart failure due to iron overload is the principal cause of death.

Cardiovascular magnetic resonance (CMR) has emerged as a non-invasive technique to quantify the amount of iron in the heart. T2-star (T2*) levels correlate with left ventricular (LV) ejection fraction and can predict patients who will develop heart failure. However, correlation between myocardial T2* and right ventricular (RV) function has not yet been established.

\section{Figure I (abstract P I62)}

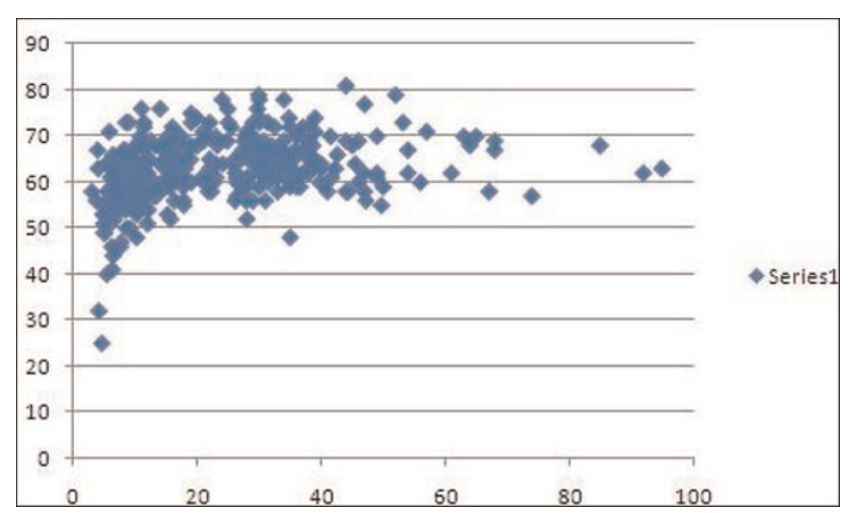


Purpose: To evaluate the relationship of myocardial T2* and RV ejection fraction (RVEF), as well as the relationship between RVEF and occurrence of heart failure within one year, in a population of patients with thalassemia major.

Methods: We studied 326 consecutive patients (average age: 26.5 years, $45 \%$ males) with beta-thalassemia major who were referred for their first scan for assessment of iron loading status by CMR. All patients were on Desferal ${ }^{\circledR}$ (deferoxamine) therapy only at presentation. Those taking other forms of chelation therapy were excluded. RV volumes and RV ejection fraction (RVEF) were calculated and compared with myocardial T2* measured within the interventricular septum. Other variables included LV volumes, left ventricular ejection fraction (LVEF), and the occurrence of heart failure within I year of the scan.

Results: There was a curvilinear relationship between heart T2* levels and RVEF (Figure I). As T2* decreased to critical levels (indicating significant myocardial iron loading), there was a decline in RV ejection fraction $(r=0.43, p<0.001)$. There was also a significant difference $(p=0.009 \mathrm{l})$ in RVEF between patients who developed heart failure within one year of the scan (RVEF: $55.8 \pm 11.8 \%$ ), compared to patients who did not subsequently suffer from episodes of heart failure (RVEF: $63.2 \pm 6.8 \%$ ). Furthermore, we noted a direct linear relationship between the LVEF and the RVEF $(r=0.69, p<0.001)$ in these patients.

Conclusion: Myocardial iron deposition as assessed by CMR is associated with RV dysfunction, which is related with the development of heart failure. This mirrors the decrease in LV function seen with worsening cardiac iron loading, which has previously been described.

Therefore, iron-overload cardiomyopathy not only affects the LV, but also involves the RV to a similar extent. These parameters can subsequently be used as a guide to the management of this condition.

\section{PI63}

Diagnostic and therapeutic impact of cardiac MRI in patients with cryptogenic ischemic stroke

John J Sheehan, George Lin, Jim Connors, Mark Alberts, Karin Dill, Reed Omary, Richard Bernstein and James Carr Northwestern Memorial Hospital, 25 I E. Huron Street, Chicago, IL 606 II, USA

Journal of Cardiovascular Magnetic Resonance 2009, I I(SuppI I):PI 63

Introduction: The aim of our study was to compare cardiac MR (CMR) and Echocardiography (TTE and TEE) used in the detection of intracardiac thrombi in patients with suspected cardioembolic stroke (CES). Over the years, cardiac imaging with different types of sequences has emerged as a non invasive alternative for the detection and chracterisation of intracardiac masses [I, 2, 3]. This study examined the utility of CMR for the detection of non-thrombotic additional findings. We will demonstrate the diagnostic and therapeutic impact of CMR.

Methods: Over a 12 month period between September 2005 and September 2006, 106 consecutive patients with a suspected CES had CMR for the detection of intracardiac thrombi. All CMR examinations were performed on a I.5 T MR scanner using CINE trueFISP, contrast enhanced MR angiography, delayed enhanced inversion recovery trueFISP and first pass imaging. The clinical
Table I (abstract PI63) Comparison of MR and echocardiography for thrombus positive studies with thrombus negative studies. *Two of the negative MRs were suboptimal studies due to motion and prothetic valve artifacts.

\begin{tabular}{lll}
\hline Modality & Number & $\%$ \\
\hline MR \& Echo & 93 & \\
MR+ & 9 & 9.6 \\
Echo- & 5 & 75 \\
Echo+ & 2 & 2.1 \\
MR- & 2 & 0 \\
\hline
\end{tabular}

information and study reports of echocardiography, CMR, MR Brain and Carotids was retrospectively reviewed.

Results \& discussion: 93 patients had CMR for suspected CES, revealing 9 thrombi in $n=9(9.7 \%)$ patients. The thrombi were located in the LAA $(n=3)$, left ventricle $(n=4)$ and right atrial appendage $(n=3)$. Of these 9 patients echocardiography was positive in $n=2(22 \%)$, indeterminate in $n=2(22 \%)$ and negative in $n=5(56 \%)$ (Figure I). No thombi were detected echocargraphy that were not seen on CMR. CMR reported 103 non thrombotic additional findings in $n=53$ (57\%) of patients compared to echocardiography. Sixty of these were considered significant in $n=38$ (40.9\%) of patients. Additional findings associated with thrombus formation (acute infarction, scarring and LV aneurysms) were $n=19(20 \%)$ for CMR and $n=7(7 \%)$ for echocardiography. In the $n=9$ patients with positive CMR and either false negative $(n=5)$ or indeterminate $(n=2)$ echocardiography, secondary preventive therapy changed from antiplatlet agents to anticoagulants (warfarin or heparin) in $n=$ 4 (44\%). Presumed stroke mechanism, changed in 3 out of 9 patients (33\%), from "cryptogenic" to "cardioembolic". See also Table I.

Conclusion: CMR is a non invasive method to detect intracardiac thrombi in stroke patients in whom echocardiograms are either negative or indeterminate. CMR identified intraventricular thrombi in a significant percentage of patients with cryptogenic strokes, leading to a change in secondary preventive therapy. These are complementary studies and when

Figure I (abstract P 163)

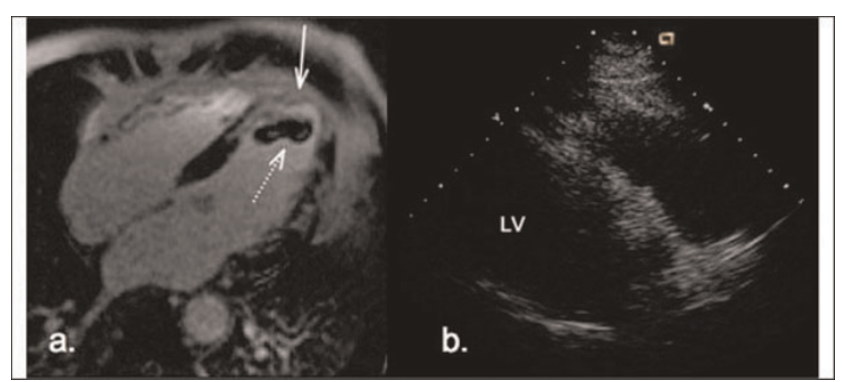

a. On PSIR Turbo FLASH there is a thrombus (dashed arrow) within the apical region of a mildly dilated left ventricle. This is located adjacent to a transmural infarct (arrow) b. No thrombus is identified on echocardiography. 
combined maximize the detection of the causes of cardioembolic stroke, may improve the effectiveness of secondary preventive medications, and may detect significant cardiovascular abnormalities that could be missed when only echocardiography is used. References

I. Jungehulsing M, et al: Radiology 1992, I 82:225-229.

2. Semelka RC, et al: J Magn Reson Imaging 1992, 2:4I5-420.

3. Fujita N, et al: Am J Card Imaging 1994, 8:69-80.

\section{P I 64}

\section{Differences in MR perfusion of malignant and} benign cardiac tumors

Kerstin U Bauner, Steven Sourbron, Michael Schmoeckel, Maximilian F Reiser and Armin M Huber Ludwig-Maximilian-University Munich, Campus Grosshadern, Munich, Germany

Journal of Cardiovascular Magnetic Resonance 2009, I I(SuppI I):PI64

Introduction: Primary cardiac tumors are a rare disease while secondary cardiac neoplasms are found more often. The localisation of the tumor, the patient's age and if present, primary malignancy or systemic disease, may give hints to the characterization of a particular cardiac neoplasm. Unenhanced TI- and T2- weighted MR-imaging and fat and suppression techniques allow for comprehensive tissue characterisation. However, additional information can be derived from first pass perfusion imaging and sometimes diagnosis can be made without biopsy and surgery.

Purpose: To determine whether dynamic contrast materialenhanced magnetic resonance (MR) imaging with use of kinetic parameters reveals statistically significant differences between benign and malignant cardiac tumors.

Methods: This study involved 23 patients with cardiac tumors (Myxoma $(n=7)$, Lipomatous hypertrophy of the interatrial septum $(n=2)$, Fibroma $(n=1)$, Rhabdomyoma $(n=2)$, Angiomyolipoma $(n=1)$, Angiosarcoma $(n=4)$, Rhabdomyosarcoma $(n=1)$, Hemeangiomyosarcoma $(n=1)$, Myoliposarcoma $(n=2)$, Lymphoma $(n=1)$, Metastasis $(n=1)$. The patients were examined with a TI weighted turboFLASH sequence. The data were transferred to an external workstation and postprocessing was performed by using software written in-house in IDL 5.4. Contrast enhancement ratios, maximum slope of contrast enhancement ratio curve $(\% / \mathrm{sec})$ and the area under

Figure I (abstract P 164)

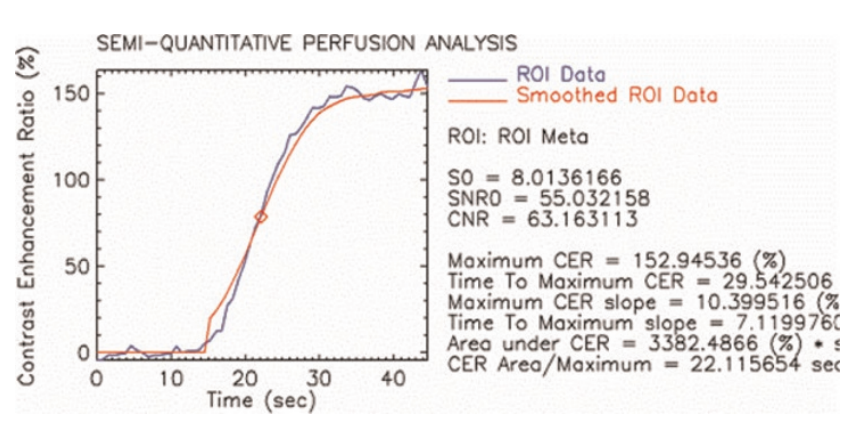

Figure 2 (abstract P 164)

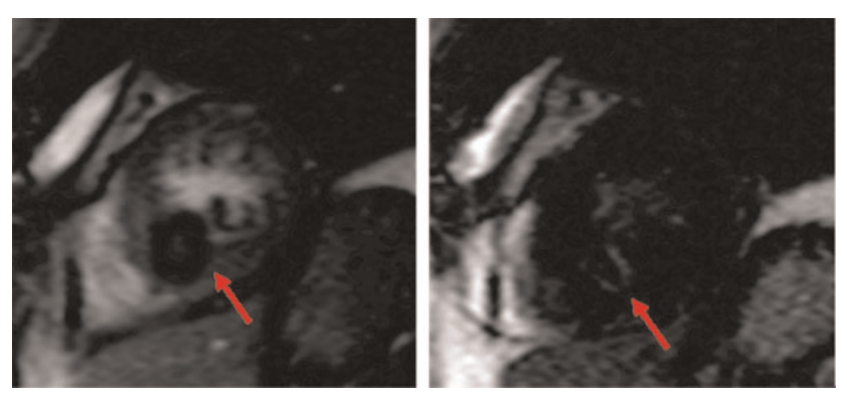

the contrast enhancement ratio curve $(\% * \mathrm{sec})$ were calculated. Statistical analysis were performed with the unpaired t-test. $\mathrm{P}$ values less than 0.05 were considered statistically significant. In addition sensitivities and specificities to differentiate benign from malignant masses were calculated for each parameter and receiver-operator-characteristics (ROC) were assessed.

Results: The contrast enhancement ratio (CER \%) was significantly higher $(p=0.0035)$ in malignant cardiac tumors ( $153.9 \pm 82.5)$ compared to benign lesions ( $57.8 \pm 52.5)$, as was the maximum slope of CER $(\% / \mathrm{sec})(p=0.01$; $10.5 \pm 5.9$ vs. $4.7 \pm 3.9)$. The values for the area under the curve $(\% * \mathrm{sec})$ were significantly higher $(p=0.00 \mathrm{II})$ in malignant cardiac lesions $(4204.5 \pm 2122.3)$ in comparison to benign lesions (I253.2 \pm I I35.2). The calculated sensitivity and specificity for the CER, the maximum slope of CER and the area under the curve resulted in $91 \%$ and $83 \%, 91 \%$ and $83 \%$ and $82 \%$ and $82 \%$ respectively with a cut-off value of $75 \%, 4 \% / \mathrm{sec}$ and $1985 \%$ *sec. The ROC analysis revealed identical values for the areas under the ROC curves for CER and the maximum slope of CER $(A \cup C=0.913)$. The ROC for the area under the contrast enhancement ratio curve was 0.803 . See Figures I, 2, 3.

Conclusion: Dynamic MR imaging delineates significant kinetic differences in perfusion between malignant and benign cardiac tumors. Semiquantitative assessment of perfusion parameters can help to discriminate these by determination of contrast enhancement ratios, calculation of the maximum slope of the CER curve and the area under the CER-curve.

Figure 3 (abstract P 164)

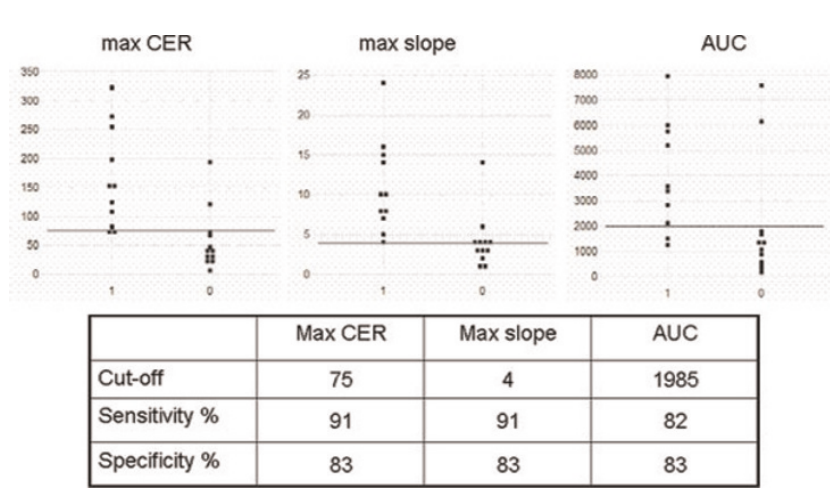




\section{PI65}

Can the outcome of post partum cardiomyopathy be predicted using late gadolinium enhancement CMR?

Isabelle Roussin, Chirine Parsai, Sanjay Prasad

and Dudley Pennell

Royal Brompton Hospital, London, UK

Journal of Cardiovascular Magnetic Resonance 2009, I I(SuppI I):PI 65

Introduction: Post partum cardiomyopathy (PPCM) can occur between the last trimester of pregnancy or within 5 months post partum, in the absence of any history of heart disease. Although rare it may become unresponsive to medical treatment and can in such cases require mechanical support, or even heart transplant. Predicting its evolution is unfortunately not possible. Late gadolinium enhancement CMR can identify myocardial fibrosis and has shown its prognostic significance in DCM. To our knowledge the role of late gadolinium enhancement in PPCM as a predictor of outcome has not been reported.

Purpose: To our knowledge the role of late gadolinium enhancement in PPCM as a predictor of outcome has not been reported.

Methods: From 2003 to 2008 , 9 women (mean age $38+/-5$ years) with suspected PPCM, clinically or by echocardiography, underwent standard CMR with late gadolinium enhancement images (LGE) following Gd-DTPA injection at the time of PPCM suspicion and after a follow up period of $27 \pm 15$ months. 2 patients had no long term follow up (one having a new pregnancy, and one lost to follow-up). Results: The initial study showed no LGE in all 9 patients, and this was confirmed at long-term follow-up (7/9 patients) indicating absence of detectable myocardial fibrosis, infiltration or infarction. CMR functional changes found at the time of diagnosis and at follow up were respectively: Left ventricular ejection fraction (LVEF) $53 \pm 8.1 \%$ versus $56 \pm 5.7 \%$ at follow-up. LVEDIV I $08.3 \pm 29.3 \mathrm{ml} / \mathrm{m}^{2}$ versus $99 \pm 19.3 \mathrm{ml} / \mathrm{m}^{2}$, LVESIV $68 \pm 44.2 \mathrm{ml} / \mathrm{m}^{2}$ versus $40 \pm 13 \mathrm{ml} / \mathrm{m}^{2}$, LV mass index $82.3 \pm 27 \mathrm{~g} / \mathrm{m}^{2}$ versus $87 \pm 37.7 \mathrm{~g} / \mathrm{m}^{2}$, RVEDV $159 \pm 43 \mathrm{ml}$ versus $149 \pm 22.4 \mathrm{ml}$, RVESV $75 \pm \mathrm{l} 4 \mathrm{ml}$ versus $64.1 \pm 17.8 \mathrm{ml}$, RVEF $49 \pm 1 \mathrm{I} \%$ versus $58 \pm 9 \%$. In addition, none of the patients showed active myocardial inflammation or oedema. At follow-up, $40 \%(n=2 / 5)$ with previously impaired LV function normalised their LVEF. In 3/5 pts with biventricular dysfunction (mean LVEF: $48 \pm 6 \%$, mean RVEF: $38 \pm 7 \%$ ), all but one showed a complete recovery of RVEF and LVEF. In I/7 pt, LVEF remained unchanged.

Conclusion: In our cohort, lack of myocardial late gadolinium enhancement was not predictive of LV function recovery in PPCM suggesting that mechanisms other than myocardial fibrosis are involved in the genesis of ventricular dysfunction.

\section{P I 66}

Cardio-metabolic profile is a determinant of carotid artery disease quantified by Magnetic Resonance Imaging

Saurabh S Dhawan, Asad Ghafoor, Hamid S Syed,

Christina Niessner, Konstantinos Aznaouridis,

Muhammad Ali, Ibhar Al Mheid, Irina Uphoff,

Charles B Kitchen, John N Oshinski, Dean P Jones

and Arshed A Quyyumi

Emory University School Of Medicine, Atlanta, GA, USA

Journal of Cardiovascular Magnetic Resonance 2009, I I(SuppI I):PI66

Introduction: Magnetic Resonance Imaging (MRI) can be used to measure common carotid artery wall volume (CCA-CWV) and maximum wall thickness (CCA-CWT) that incorporates adventitial thickness in addition to intima-media thickness (CCAIMT). Although, the predictors of CCA-IMT are well known, those of CCA-CWV and CCA-CWT have not been studied.

Purpose: We hypothesized that patients with higher CCACWV and CCA-CWT have lower high-density lipoprotein (HDL) levels and greater incidence of metabolic syndrome.

Methods: One hundred and three subjects with IMT $>0.65 \mathrm{~mm}$ underwent MRI of their carotid arteries using T2-weighted blackblood sequence transaxial slices to measure CCA-CWV and CCA-CWT. Blood pressure (BP), body mass index (BMI), fasting lipid profile and glucose were measured. Metabolic syndrome (MetS) was defined in patients as having $\geq 3$ risk factors, BMI $\geq$ $30, T G \geq 150 \mathrm{mg} / \mathrm{dl}, \mathrm{HDL} \leq 40 \mathrm{mg} / \mathrm{dl}$ in men and $\leq 50 \mathrm{mg} / \mathrm{dl}$ in women, BP $\geq 130 / 85$ and fasting glucose $\geq 110 \mathrm{mg} / \mathrm{dl}$.

Results: CCA-CWV correlated negatively with serum HDL levels $(r=-0.373, p<0.000 \mathrm{I})$, positively with BMI $(r=0.54$, $P<0.000 \mathrm{I})$ and diastolic BP $(r=0.28, P=0.009)$ independent of age, sex, the presence of diabetes, hypertension, hyperlipidemia and smoking. CCA-CWT correlated negatively with serum HDL levels (and $r=-0.255, P=0.01$ ) and positively with presence of MetS $(r=0.23, p=0.02)$ independent of age, gender and history of smoking.

Conclusion: Cardio-metabolic profile is a predictor of carotid artery disease as quantified by magnetic resonance imaging.

\section{PI67}

Assessing strain in arrhythmogenic right ventricular cardiomyopathy using cine DENSE MRI Joash N Ongori' , Neil Hendricks', Bruce S Spottiswoode', Ernesta M Meintjes', Frederick H Epstein ${ }^{3}$

and Bongani $M$ Mayosi $^{2}$

IMRC/UCT Medical Imaging Research Unit, University of Cape Town, Cape Town, South Africa

${ }^{2}$ Cardiac Clinic and Department of Medicine, University of Cape Town, Cape Town, South Africa

${ }^{3}$ Biomedical Engineering and Radiology, University of Virginia, Charlottesville, VA, USA

Journal of Cardiovascular Magnetic Resonance 2009, I I(SuppI I):PI67

Introduction: The kinematics of the right ventricle (RV) are not well understood due to its thin wall and asymmetric geometry [I]. Cine displacement encoding with stimulated echoes (cine DENSE), which measures intramyocardial displacement and strain [2], has been adapted for RV analysis with encouraging preliminary results in normal subjects [3]. This preliminary study evaluates cine DENSE MRI for detecting decreased myocardial strain in arrhythmogenic right ventricular cardiomyopathy (ARVC). ARVC is a unique heart muscle disease affecting predominantly the RV. The pathological hallmark of fibro-fatty replacement of the myocardium may result in localised aneurysms and wall motion abnormalities, detectable by cardiac magnetic resonance imaging [4].

Methods: In accordance with protocols approved by the UCT Research Ethics Committee, 3 confirmed, 6 suspected ARVC cases and 4 normal volunteers were scanned using cine DENSE. ARVC diagnosis was based on standard clinical criteria [4]. Cine DENSE images were acquired in basal, mid and apical short axis slices on a Siemens I.5 T Symphony scanner using segmented echo planar imaging for data acquisition [2]. Displacement encoding was applied in two orthogonal in-plane directions 
Table I (abstract PI67) Basal slice mean peak tangential strain values and standard deviation for three confirmed ARVC cases, six suspected cases, and four normal volunteers for the LV and RV segments

\begin{tabular}{|c|c|c|c|c|c|c|}
\hline \multicolumn{7}{|c|}{ Peak tangential strains } \\
\hline \multirow[b]{2}{*}{ Regions } & \multicolumn{2}{|c|}{ Confirmed ARVC cases } & \multicolumn{2}{|c|}{ Suspected ARVC cases } & \multicolumn{2}{|c|}{ Normals } \\
\hline & Mean & Std Dev & Mean & Std Dev & Mean & Std Dev \\
\hline Anterior LV wall & -0.11 & 0.04 & -0.11 & 0.05 & -0.16 & 0.03 \\
\hline Lateral LV wall & -0.15 & 0.04 & -0.14 & 0.05 & -0.17 & 0.03 \\
\hline Posterior Lvwall & -0.16 & 0.03 & -0.15 & 0.06 & -0.19 & 0.02 \\
\hline Inferior LV & -0.15 & 0.06 & -0.15 & 0.05 & -0.18 & 0.04 \\
\hline Inferior septum & -0.11 & 0.03 & -0.11 & 0.03 & -0.16 & 0.03 \\
\hline Anterior septum & -0.08 & 0.02 & -0.11 & 0.04 & -0.15 & 0.02 \\
\hline RV free wall & -0.15 & 0.03 & -0.13 & 0.04 & -0.18 & 0.02 \\
\hline Diaphragmatic RV & -0.13 & 0.01 & -0.10 & 0.02 & -0.13 & 0.04 \\
\hline
\end{tabular}

over two breath holds per slice. Imaging parameters include: FOV $=400 \mathrm{~mm}$, slice thickness $=7.0 \mathrm{~mm}$, TR $=24 \mathrm{~ms}$, FOV phase $=62.5 \%, E T L=9$, segments $=18$, cardiac phases $=10$ I6, and displacement encoding frequency $=0.1$ cycles $/ \mathrm{mm}$. Epicardial and endocardial contours were manually drawn for both the LV and RV on all cardiac phases. Phase unwrapping and tissue tracking were performed [5], and motion trajectories were estimated using temporal fitting with $5^{\text {th }}$ order Polynomial functions. Lagrangian strain taken tangential to the midwall (Ett) was computed from both the left ventricle (LV) and RV motion trajectories [3].

Results: Example basal slice cine DENSE magnitude images and corresponding end systolic tangential strain maps are shown for a normal volunteer (Figure I a) and a confirmed ARVC case (Figure Ib). The underlying colour represents Ett and the purple vectors represent displacement. The arrows in Figure Ib indicate regions

Figure I (abstract P I67)
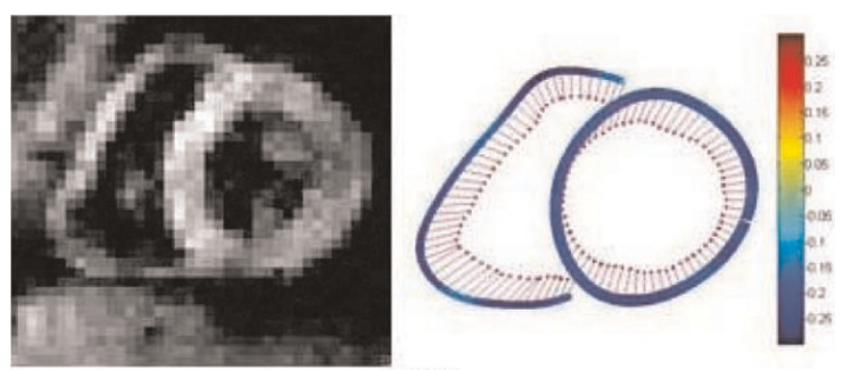

(a)
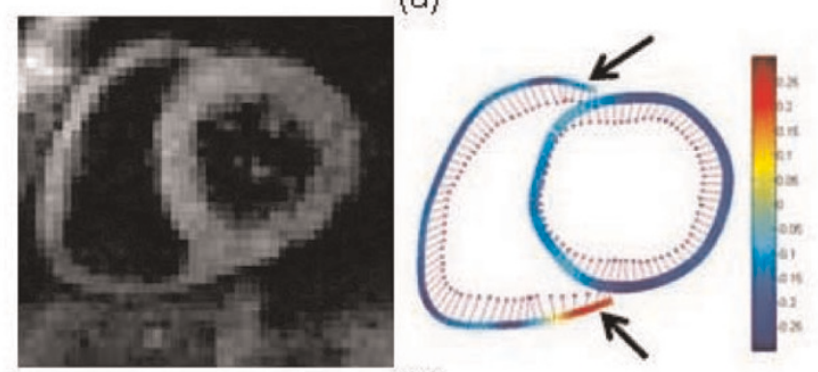

(b)

Cine DENSE magnitude (left) and tangential strain maps (right) for a normal volunteer (a), and one confirmed ARVC case (b). of abnormal strain and motion, which occur largely in the region of the RV insertion points and the LV inferior and anterior septum. Abnormal RV strain patterns were evident in 2 out of the 3 confirmed, and 3 out of the 6 suspected cases. The other confirmed case had a dilated RV but the strain pattern appeared normal. Table I presents regional peak tangential strains for the basal slice averaged for each group. A confidence measure for Ett was calculated based on the signal-to-noise ratio (SNR) of the magnitude images. Only Ett strain values with confidence greater than 0.5 (normalized to max confidence in entire heart) were used to compute regional peak Ett strain. More than half of the data points in the diaphragmatic RV had to be discarded because of low SNR. Both confirmed and suspected ARVC cases have low peak strain values in the inferior and anterior septum and the RV free wall. Peak Ett is significantly less in ARVC and suspected ARVC cases compared to normals in the anterior septum ( $p<0.005$ and $p<0.05$, respectively, student t-test).

Conclusion and discussion: Preliminary results indicate that cine DENSE CMR is useful in detecting abnormal myocardial strain in ARVC. Due to improved SNR and motion properties, cine DENSE using spiral data acquisition [6] may improve the ability to measure strain in the diaphragmatic RV segment.

\section{References}

I. Haber, et al: Am J Physiol Heart Circ Physiol 2005, HI826HI833.

2. Kim, et al: Radiology 2004, 230:862-87I.

3. Spottiswoode, et al: Proc $14^{\text {th }}$ ISMRM, USA 2006.

4. McKenna, et al: Br Heart J 1994, 71:2 I5-2 I8.

5. Spottiswoode, et al: IEEE Trans Med Imag 2007, 26(I):|15-30.

6. Zhong, et al: Proc $15^{\text {th }}$ ISMRM 2007.

\section{PI68}

Comprehensive assessment of of thoracic stent grafts after emergency implantation in multi trauma patients

Volker Rasche, Axel Bornstedt, Vinzent Hombach,

Nico Merkle, Alexander Oberhuber,

Ludger Sunder-Plassmann and Martin Hoffmann

University Ulm, Ulm, Germany

Journal of Cardiovascular Magnetic Resonance 2009, I I(SuppI I):PI68

Introduction: Thoracic Endovascular Aortic Repair (TEVAR) of various aortic pathologies has turned out as attractive 
Figure I (abstract P I 68)

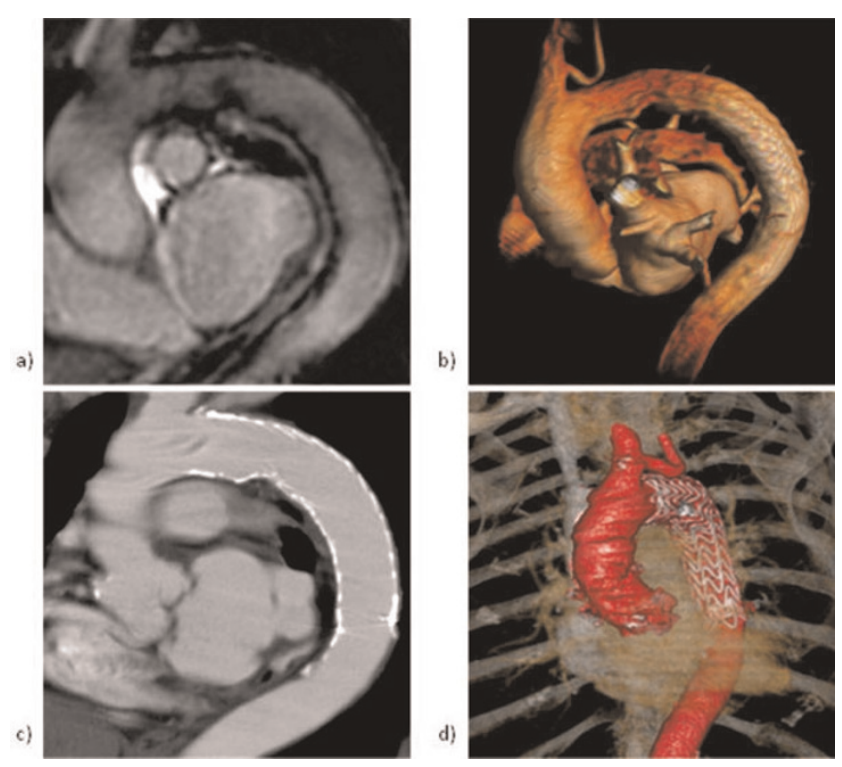

Comparison MRI and CT: a,c) parasagittal two-dimensional and b,d) volume-rendered view of the aortic arch and the thoracic descending aorta.

alternative option to conventional surgical approaches. TEVAR may be associated with graft related complications such as endoleaks, kinking, infolding, and stentgraft migration, disconformability and disattachment phenomena. Therefore lifelong regular follow up by tomographic imaging is required for closely monitoring of all patients treated with thoracic aortic stent grafts.

Multislice volumetric CT still represents the imaging gold standard for the assessment of the stent graft. However, its related X-ray dose and the nephrotoxicity of the required contrast agent, limits its frequent application especially in younger patients and in patients with renal insufficiency. The objective of this work was to proof the feasibility of MRI as a comprehensive imaging tool for the assessment of thoracic stent grafts, including disattachment phenomena, its dynamics over the cardiac cycle and its impact on vessel compliance.

Methods: Twelve consecutive patients ( 3 female, 9 male; mean age $38+1-18 ; 8-2417 d$ after intervention) were enrolled in this feasibility study. All patients initially presented with aortic rupture at the transition zone of the arch and descending segment of the thoracic aorta. The rupture site was treated with endovascular stent graft procedures in all patients. In six patients, a Medtronic Valiant stent graft and in six patients a Gore excluder TAG stent graft were implanted. All patients underwent conventional contrast agent (CA) enhanced routine CTA followed by the investigational MRI protocol, comprising a three-dimensional angiogram of the aorta with and without contrast enhancement.

The general image quality regarding appreciation of the stent graft geometry $Q_{G}$ and dynamics $Q_{D}$ were ranked on a I (poor) 5 (excellent) scale by three experienced MRI readers. Qualitative assessment of the stent graft motion $Q_{M}$ over the cardiac cycle was ranked on a I (no motion) to 3 (significant motion) scale independently for the proximal and distal segments of the stent. The deployment of the stent $Q_{d}$ against the vessel wall was qualitatively assessed on a I (attached to the vessel wall) to 3 (large areas of the stent not attached to the vessel wall) scale. Quantitative assessment of the vessel compliance was performed distal and proximal to the stent graft as well as in the distal and proximal section of the stent graft.

Results: The MRI imaging protocol could be completed in all patients. The average acquisition time for the entire MRI examination was $54+/-16$ minutes. In all patients, the geometry of the stent graft as well as its dynamic could be obtained with at least mediocre image quality (mean ranking $Q_{G}=4.2+/-0.75$; mean ranking $\left.Q_{D}=3.7+/-0.76\right)$. Stent graft motion could be assessed qualitatively and vessel compliances be quantitized in all patients. In direct comparison to CT (Figure I), no obvious loss of morphological information of the stented thoracic aorta was observed.

Discussion: MRI can provide a comprehensive assessment of thoracic aortic stents grafts after percutaneous implantation. The feasibility of assessing the stent graft geometry as well as motion without any need for ionizing radiation or nephrotoxic contrast agents, may MRI make a very attractive alternative to the current standard CTA as imaging modality of choice for regular follow up of thoracic stent grafts.

\section{PI69}

Aortic stiffness as an independent predictor of cardiac function and cerebral white matter hyperintensities in diabetes mellitus assessed by Magnetic Resonance Imaging Saskia GC van Elderen, Anne Brandts, Jos Westenberg, Jeroen van de Grond, Mark van Buchem, Jan Smit and Albert de Roos Leids Universitair Medisch Centrum, Leiden, Netherlands

Journal of Cardiovascular Magnetic Resonance 2009, I I(SuppI I):PI69

Introduction: Diabetes Mellitus (DM) causes damage in multiple organs and the vessels due to the metabolic state of hyperglycemia and insulin resistance. Increased arterial stiffness may be an important pathway linking DM to the increased cardiovascular (CV) risk by mechanisms of increased cardiac afterload, augmentation of pulse pressure, a subsequent transmission of high pulsatile flow to the end organs and furthermore as a representative of arteriosclerosis. Magnetic Resonance (MR) assessment of aortic pulse wave velocity (PWV) offers a good reproducible non-invasive tool for the detection of arterial stiffness. Furthermore MRI is an accurate tool for the detection of cardiac dysfunction and vascular cerebral lesions, like white matter hyperintensities (WMHs). To our knowledge, MRI has not previously been used to simultaneously determine end organ damage in the aorta, heart and the brain in patients with DM. We hypothesize that there is an important role for DM induced aortic stiffness in the underlying mechanism of cardiac failure and cerebral white matter hyperintensities in DM patients.

Purpose: Accordingly, the purpose of this study is to examine the possible association between aortic PWV, cardiac left ventricular (LV) function and cerebral white matter hyperintensities in DM patients using MRI.

Methods: MRI of the aorta, the heart and the brain was performed in $8 \mathrm{I}$ consecutively included subjects with proven DM 
(50 men; mean age $48 \pm 12$ years). PWV (defined as the propagation speed of the systolic wave front) was assessed using velocity-encoded MRI at two predefined levels in the aorta: in the aortic arch and the descending aorta. Short-axis MRI using a steady-state free precession sequence was performed to assess LV ejection fraction and LV mass. Transmitral flow measurements were performed by means of velocity-encoded MRI for the evaluation of LV diastolic function. WMHs on spin-echo T2weighted and FLAIR sequences were separately evaluated according to their two anatomical subtypes - periventricular (pv) and subcortical (sc) - and respectively quantified using the Fazekas classification. Spearman correlation, independent student t-test, multivariate linear and logistic regression models were applied for statistical analysis.

Results: PWV in the aortic arch was significantly associated with LV diastolic function $(r=-0.619, p=0.000)$, LV ejection fraction $(r=-0.227, P=0.042)$ and periventricular WMHs $(p=0.006)$. PWV in the descending aorta was significantly associated with LV diastolic function $(r=-0.567, p=0.000)$ and subcortical WMHs $(p=0.002)$. Furthermore, in multivariate regression analysis with confounding age, gender, smoking and duration of DM, PWV in the aortic arch was an independent predictor of LV diastolic function $(R=0.803, P=0.002)$, LV ejection fraction $(R=0.47 \mathrm{I}, P=0.00 \mathrm{I})$, $L V$ mass indexed for body surface area $(R=0.707, P=0.004)$ and $p v$ WMHs $(O R=1.329, p=0.023)$.

Conclusion: Stiffening of the aorta is associated with cardiac LV dysfunction and lesions of the cerebral white matter in DM. Moreover, PWV of the aortic arch is an independent predictor of cardiac LV diastolic and systolic function, LV mass and cerebral periventricular white matter hyperintensities in DM patients.

\section{P I 70}

Pulmonary distensibility and flow dynamics in systemic sclerosis using velocity encoded magnetic resonance imaging

Monda L Shehata', Sukhminder Singh', Joao AC Lima', Paul Hassoun', David A Bluemke ${ }^{2}$ and Jens VogelClaussen'

Johns Hopkins University, Baltimore, MD, USA

${ }^{2}$ National Institute of Health (NIH), Bethesda, MD, USA

Journal of Cardiovascular Magnetic Resonance 2009, I I(SuppI I):PI70

Introduction: Systemic sclerosis (SSc) is a progressive autoimmune disorder characterized by excessive collagen deposition leading to widespread fibrosis. Changes in distensibility of peripheral systemic arteries have been reported [I]. Involvement of pulmonary vasculature leads to pulmonary arterial hypertension (PAH). However, the role of the proximal pulmonary arteries remains unknown.

Purpose: Assessment of proximal pulmonary arterial distensibility and flow dynamics in systemic sclerosis with (SSc-PAH) and without PAH (SSc-Non PAH) using velocity encoded MR imaging. Methods: We enrolled 16 consecutive patients with systemic sclerosis (mean age $=62.8 \pm 9.8$ years). All patients underwent catheterization for quantification of pulmonary hemodynamics: mean pulmonary artery pressure (mPAP) and pulmonary vascular resistive index (PVRI). Patients were divided into 2 subgroups: SSc-PAH $(n=10), \quad(m P A P=42.8 \pm 9)$ and SSc-Non PAH $(n=6)$. 9 healthy subjects (mean age $=45.6 \pm 8$. 6) were enrolled for comparison. Imaging was performed using a $3 \mathrm{~T}$
Figure I (abstract PI70)

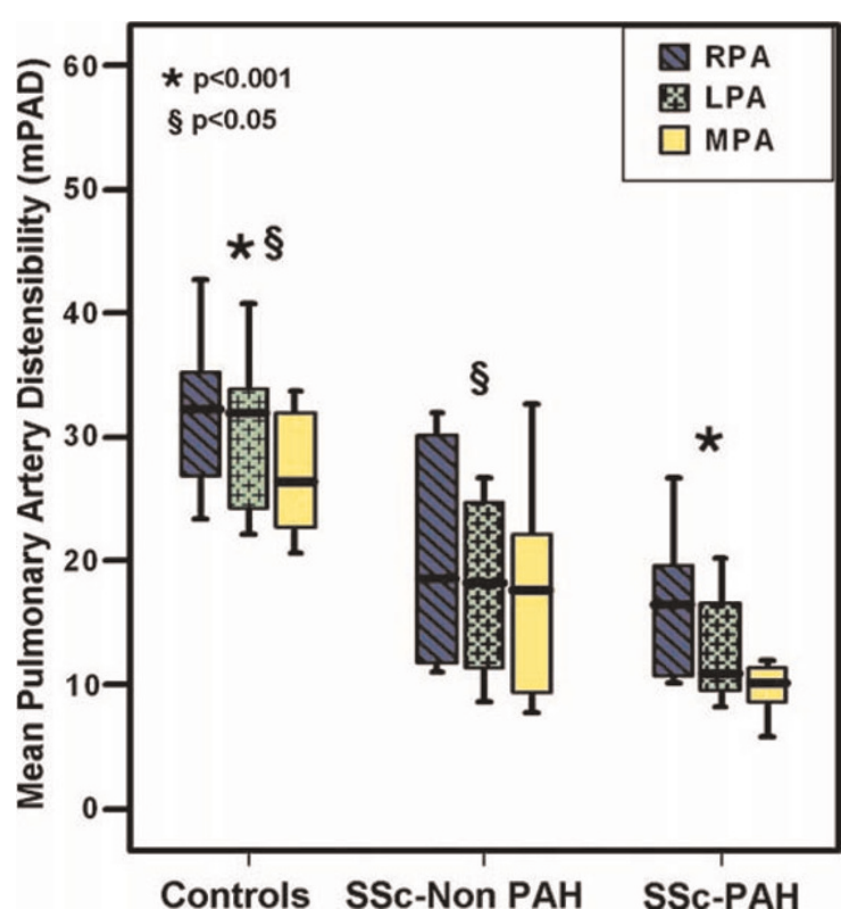

MRI system (Siemens, Erlangen, Germany). Phase contrast MR images were prescribed orthogonal to the long axis of the main pulmonary artery (MPA), right pulmonary artery (RPA) and left pulmonary artery (LPA), using a flash 2D gradient echo sequence with a predefined maximum velocity of $100 \mathrm{~cm} / \mathrm{second}(T R=$ 32 milliseconds, TE $=3.8$ milliseconds, Flip angle $=20^{\circ}, \mathrm{FOV}=$ $34 \times 34 \mathrm{~cm}$, matrix $=256 \times 256$, slice thickness $=8 \mathrm{~mm})$. Short axis fast gradient echo cine images were acquired for ventricular function quantification. Using dedicated flow software (MEDIS, Netherlands), magnitude images were semi-automatically contoured. Flow hemodynamics including: flow volume $(\mathrm{ml} / \mathrm{min})$, maximum velocity in $\mathrm{cm} / \mathrm{sec}\left(V_{\max }\right)$, systolic and diastolic crosssectional areas $\left(\mathrm{mm}^{2}\right)$ were computed for each vessel. Pulmonary arterial distensibility (PAD) was derived as (maximum systolic area - minimum diastolic area)/maximum systolic area) and expressed as \% change. Mann-Whitney test was used for intergroup comparisons. Spearman's rho correlations were performed.

Results: PAD was significantly reduced in SSc-PAH patients compared to control group in all pulmonary vessels examined: $\mathrm{MPA}=10 \% \pm 5$ vs $29 \% \pm 10, \mathrm{RPA}=17 \% \pm 6$ vs $31 \% \pm 6$, LPA $=13 \% \pm 4$ vs $32 \% \pm 9$, ( $P=0.000 \mid$ for all). Also, significant difference was found between SSc-Non PAH and control group: MPA $=18 \% \pm 9$ vs $29 \% \pm 10$, RPA $=20 \% \pm 9$ vs $31 \% \pm 6$, $L P A=18 \% \pm 8$ vs $32 \% \pm 9$, ( $P<0.05$ for all). However, comparing SSc-PAH to SSc-Non PAH, no significant difference in PAD was detected $(p>0.05)$. No significant relationship was found between PAD and catheter hemodynamics for all SSc patients $(p>0.05)$. See Figure $I$.

On phase contrast imaging, significant lower flow volume and lower Vmax were seen in the MPA in SSc-PAH vs SSc-non PAH group $(p=0.02, p=0.001$, respectively $)$ and controls ( $p=0.009, p<0.001$, respectively). 
MPA flow volume showed good inverse correlation with mPAP $(r=-0.76, p=0.00 I)$ and PVRI $(r=-0.79, p<0.00 I)$ for all SSc patients, however, no correlation was found between MPA flow volume and PAD ( $p>0.05)$.

$\mathrm{RV}$ ejection fraction (EF) and stroke volume indexed to body surface area were significantly reduced in SSc-PAH compared to controls and SSc-Non PAH patients $(p<0.05)$. No significant difference was found in RV function between SSc-Non PAH and control groups.

Conclusion: SSc patients have significantly lower PAD in the proximal pulmonary arteries, in presence or absence of $\mathrm{PAH}$, compared to normal controls, which is likely related to the patho-physiologic vascular change in systemic sclerosis. Main pulmonary artery flow volume, however, seems to be a better correlate for increased mPAP than PAD.

\section{Reference}

I. Moyssakis I, et al: Rheumatology (Oxford) 2005, 44(2):25 I-4.

\section{PI7I}

Underestimation of carotid plaque by ultrasound IMT and potential error in measurement of change in plaque burden: simultaneous comparison with 3 T MRI

Mark Bowers ', Leanne Harmann', Robert Prost', Megan Bright', Anil Doppalapudi', Tayyab Mohyuddin', John LaDisa ${ }^{2}$ and Raymond A Migrino

${ }^{1}$ Medical College of Wisconsin, Milwaukee, WI, USA

${ }^{2}$ Marquette University, Milwaukee, WI, USA

Journal of Cardiovascular Magnetic Resonance 2009, I I(SuppI I):PI 7 I

Introduction: Both ultrasound (intima-media thickness, IMT) and $\mathrm{MRI}$ are established methods of measuring carotid plaque burden. The recent ENHANCE trial demonstrated that despite greater reduction in LDL cholesterol with simvastatin-ezetimibe, carotid IMT did not decrease and in fact trended towards progression versus simvastatin control. This was a surprising finding and called into question whether measuring the change in posterior wall thickness by 2-dimensional IMT accurately reflects the change in plaque burden especially since plaque can be eccentric. Unlike IMT that by standard methodology quantifies posterior wall thickness, MRI allows measurement of plaque volume and is ideal for quantifying eccentric plaques.

Purpose: To compare carotid plaque volume calculated from ultrasound IMT and lumen radius (IMT-vol) versus plaque volume measured by MRI (MRI-vol) in patients with atherosclerotic disease and to compare \% change in IMT versus MRI-vol in patients taking statins for 6 months.

Methods: 16 patients (67 \pm 10 years, 3 females) with carotid atherosclerotic disease underwent sequential B-mode ultrasound and MRI of both carotid arteries at baseline, with II having 6-month follow up post-statin treatment. IMT of the right and left common carotid (CC, starting 2-4 mm below flow divider and $10 \mathrm{~mm}$ length going inferiorly) and internal carotid (IC, starting $2 \mathrm{~mm}$ above flow divider and $10 \mathrm{~mm}$ length going superiorly) arteries were measured from the posterior wall using standard ENHANCE trial methods. IMT-vol was calculated using the formula: $\left[\Pi(r+\mid M T)^{2}-\pi r^{2}\right] * 10 m m$ where $r$ is the lumen radius and IMT is the mean posterior plaque thickness. MRI-vol was derived from T2-weighted spin echo images using $3 \mathrm{~T}$ MRI (General Electric) and 4 channel carotid coil $(0.47 \times 0.47 \times 2 \mathrm{~mm}$ spatial resolution, $256 \times 256$ matrix). Plaque volume in $10 \mathrm{~mm}$ length of
Figure I (abstract PI7I)

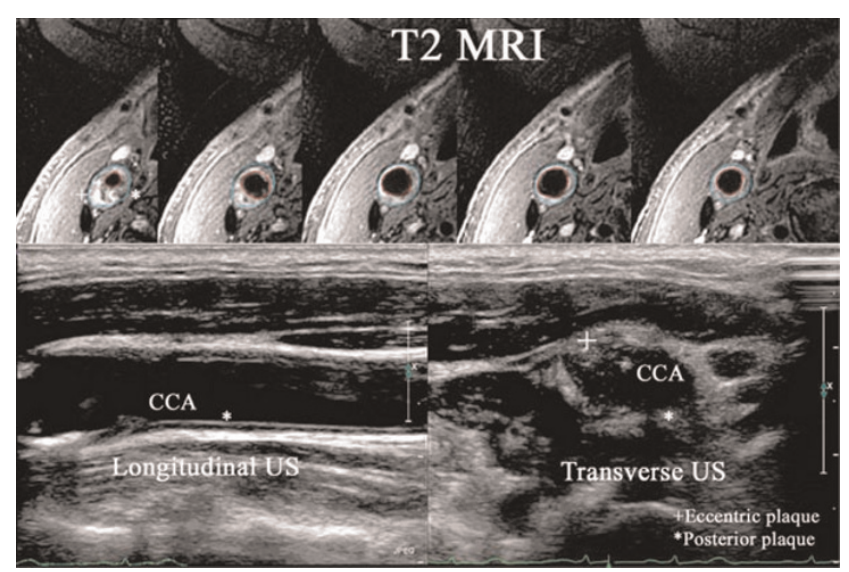

the corresponding CC and IC regions were derived using Simpson's formula from sequential $2 \mathrm{~mm}$ slice thickness images. IMT-vol and MRI-vol were compared in $6 \mathrm{I}$ evaluable carotid segments and $\%$ change in volume was compared in subjects with 6 month follow-up.

Results: Carotid plaques often have eccentric distribution (see Figure I). IMT-vol has significant correlation with MRI-vol $(R=0.5, p<0.00 I)$. However, IMT-vol is significantly less than MRI-vol (all, CC, IC: $227 \pm 159$ vs. $430 \pm 159 ; 21 \mathrm{I} \pm 63$ vs. $507 \pm 120 ; 232 \pm 21 \mathrm{l}$ vs. $405 \pm 164 \mathrm{~mm}^{3}$, all $\left.p<0.00 \mathrm{I}\right)$. Following 6-month statin treatment, IMT increased by $2.1 \pm 36 \%$, IMT-volume increased by $15 \pm 61 \%$ while MRI-vol decreased by $9.4 \pm 15 \%$. The $\%$ change in MRI-vol did not correlate with \% change in IMT $(R=0.2, p=0.3)$ or IMT-vol $(R=0.1, P=0.6)$. When \% change is categorized into 3 groups (greater than $+5 \%,+5$ to $-5 \%$ or greater than $-5 \%$ ), IMT-vol was concordant with MRI-vol change in only $8 / 21$ (38\%) of cases.

Conclusion: Carotid plaque burden derived from posterior wall IMT systematically underestimates plaque volume as measured by MRI, likely due to eccentric nature of carotid atherosclerosis. The change in plaque volume measured by MRI is discordant with change in IMT or IMT-vol suggesting non-uniform change in regional plaque burden that may not be reflected by simply measuring the posterior wall. This has important implications in utilization of ultrasound in serial studies of plaque burden and suggests need for 3-dimensional volumetric measurement of plaque burden.

\section{PI72}

4D flow for accurate assessment of complex flow distribution

Alison K Meadows, Michael D Hope, David Saloner and Charles B Higgins

University of California San Francisco, San Francisco, CA, USA

Journal of Cardiovascular Magnetic Resonance 2009, I I(SuppI I):PI 72

Objective: To demonstrate that $4 \mathrm{D}$ flow can provide fast, accurate, and complete data sets to determine differential blood flow in patients with complex distributions of flow.

Background: Patients with abnormal vasculature, particularly those with congenital heart disease, often have complex 
Figure I (abstract PI72)

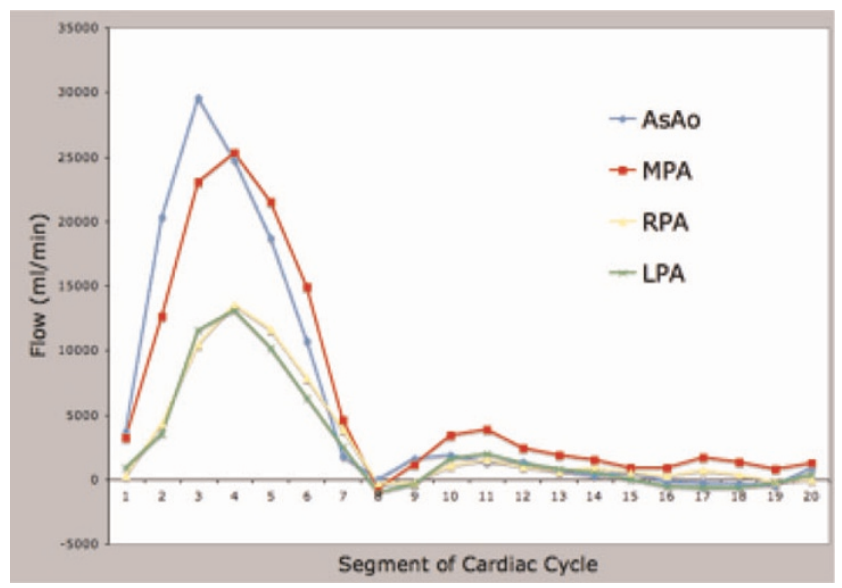

Healthy subject pulmonary flow.

distribution of flow. This distribution of flow is often clinically relavent. The choice of $2 \mathrm{D}$ phase contrast flow planes is often difficult and labor intensive. As a result, studies may be completed without collection of the data necessary to determine flow distribution. 4D flow techniques permit the collection of temporally-resolved 3D data sets of the central systemic and pulmonary vasculature in a single acquisition. Complete data acquisition is guaranteed and appropriate planes can be chosen during post processing.

Methods: Employed was a temporally-resolved, 3D-phase contrast technique (4D flow), optimized for blood flow analysis in the thoracic vasculature. Data was acquired using an RFspoiled 3D gradient echo pulse sequence with velocity encoding in 3 spatial directions. All measurements were performed on a I.5 T clinical scanner (Signa CV/I, GE, Milwaukee, WI) using an 8channel cardiac coil. Scan parameters were as follows: VENC = $160-200 \mathrm{~cm} / \mathrm{s}$; fractional FOV $=300 \times 270 \mathrm{~mm}^{2}$, slab thickness $=78 \mathrm{~mm}$, and matrix $=256 \times 192 \times 30$ yielding a spatial resolution of $1.17 \times 1.56 \times 2.60 \mathrm{~mm}^{3}$. Within each cardiac cycle, the in-plane phase encode value was held constant while

\section{Figure 2 (abstract PI72)}

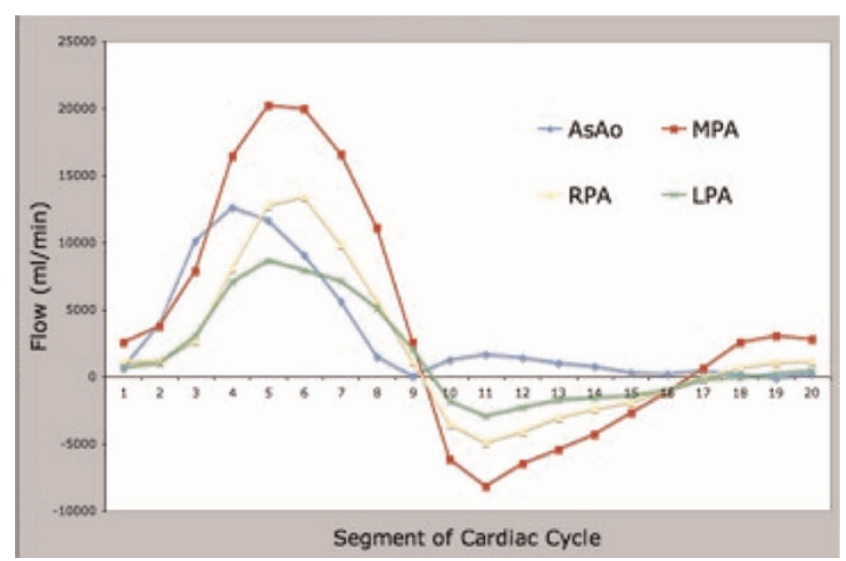

Flow profile.
Figure 3 (abstract P I72)

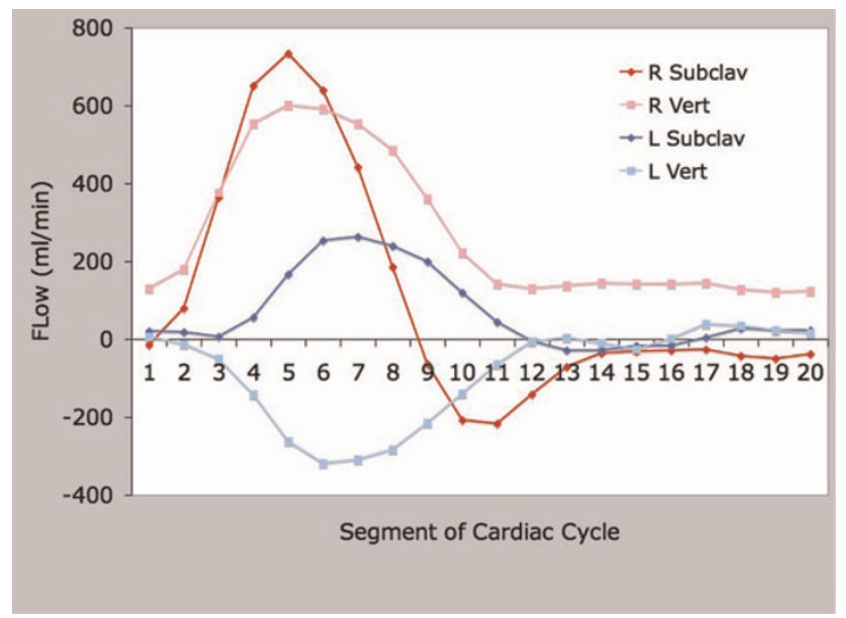

Left subclavian steal. Complex flow analysis in patient with bypass graft for interrupted aortic arch.

4 slice-encoding phase encodes are acquired to encode all flow directions. Parallel imaging (GRAPPA) with an acceleration factor of 2 was used. Scan times ranged from 12-16 minutes depending on heart rate. Retrospective EKG gating was used to resolve 20 time frames through the cardiac cycle yielding a temporal resolution of $50-80 \mathrm{msec}$. Respiratory compensation was employed. The raw data was reconfigured for EnSight visualization (CEI Inc., Apex, NC). Navigation within the 3D data set allows retrospective placement of $2 \mathrm{D}$ planes perpendicular to the vessel of interest. One normal control subject and 3 subjects with complex anatomy were enrolled. For each subject, a 4D flow data set was obtained.

Results: Presented are a series of curves that demonstrate differential flow patterns in the 4 enrolled subjects. Figure I presents flow in the ascending aorta (AsAo), main pulmonary artery (MPA), and branch pulmonary arteries (RPA and LPA) in a normal control. The AsAo and MPA flows are equal, and the sum of the branch PA flows equal the MPA flow, demonstrating internal consistency. Figure 2 presents the same flows in a patient with tetralogy of Fallot and LPA stenosis. Evident is diminished flow in the LPA. Again, internal consistency is demonstrated with equal AsAo and MPA flows, and the sum of the branch PA flows equalling the MPA flow. Figure 3 presents flow in a patient with

\section{Figure 4 (abstract PI72)}

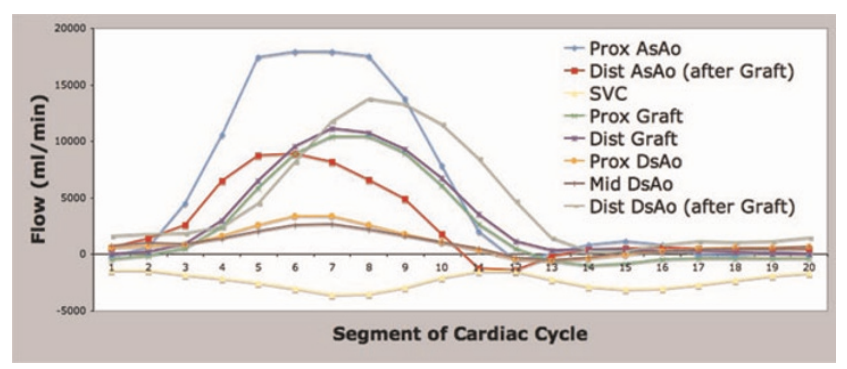

Complex flow analysis in patient with bypass graft for interrupted aortic arch. 
left subclavian artery steal. Note is made of the retrograde flow in the left vertebral artery supplying the left subclavian artery. Again, flows here are internally consistent. Figure 4 presents flow in a patient with interruption of the aorta status post placement of a graft from the AsAo to descending aorta (DsAo). Planes were selected to resolve flow in the AsAo proximal to the graft, AsAo distal to the graft, graft itself, and DsAo before and after the graft. The complex distribution is clearly shown.

Conclusion: 4D flow provides fast, accurate and complete assessment differential blood flow in patients with complex anatomy and physiology. Navigation within the 4D flow data set allows retrospective placement of cut planes without being hindered by the prospective prescription of traditional 2D flow techniques.

\section{P I 73}

\section{Etiology of diminished left ventricular function in} patients with Tetralogy of Fallot by cardiac magnetic resonance

David HF Gommans', Maureen J Van der Vlugt', Jeroen W Op den Akker², Erwin S Zegers' and Arie PJ Van Dijk'

'Heartlung Centre, Radboud University Medical Centre

Nijmegen, Nijmegen, Netherlands

${ }^{2}$ Department of Radiology, Radboud University Medical Centre Nijmegen, Nijmegen, Netherlands

Journal of Cardiovascular Magnetic Resonance 2009, I I(SuppI I):PI73

Introduction: Right ventricular (RV) function is impaired in patients with Tetralogy of Fallot due to right bundle branch block and volume overload by pulmonary regurgitation. As a consequence, paradoxical septal motion develops. Daily practice suggests left ventricular (LV) function is also reduced. Paradoxical septal motion could be one of the mechanisms causing diminished LV function.

Purpose: The purpose of this CMR study was to measure paradoxical septal motion and its effect on LV function in patients with Tetralogy of Fallot.

Methods: Twenty nine patients (17 males) with Tetralogy of Fallot with a mean age of $29 \pm 10$ years were included. MR imaging was performed on Siemens Avanto I.5 Tesla scanner. Data-analysis was performed with the MR Analytical Software System (Medis, Leiden, The Netherlands) to measure enddiastolic (EDV) and end-systolic (ESV) volumes and calculate ejection fraction (EF) for both ventricles. Paradoxical septal motion was quantified in end-diastole by the curvature of the septum, defined as I divided by the radius of the curvature and a negative value corresponds to septal motion towards the LV cavity. MR phase-contrast flow quantification was performed to assess pulmonary regurgitation. ECG analysis for QRS duration was performed to diagnose right bundle branch block. Pearson correlation tests were done to assess correlations between variables.

Results: RV EDV and ESV were $227.0 \pm 53.3$ and $148.4 \pm$ $47.7 \mathrm{ml}$, respectively. LV EDV and ESV were II $9.4 \pm 24.5$ and $55.9 \pm 17.9 \mathrm{ml}$, respectively. $\mathrm{RV}$ function was reduced $(\mathrm{EF}=$ $35.3 \pm 10.2 \%)$ and LV function was also mildly reduced (EF = $53.5 \pm 9.5 \%)$. All patients had reduced curvature $(0.14 \pm 0.08)$, but in only I case the septum moved towards the LV cavity. Mean pulmonary regurgitation fraction was $43.6 \pm 17.6 \%$. Mean QRS duration was I $44 \pm 30 \mathrm{msec}$. Significant correlations were found between curvature and pulmonary regurgitation fraction $(r=0.39, p<0.05)$, pulmonary regurgitation fraction and RV EDV $(r=0.49, P<0.01)$, RV ESV volumes and QRS duration $(r=0.56, p<0.0 I)$ and between QRS duration and RVEF $(r=$ $-0.676, p<0.01)$. No significant correlations could be found concerning LV function.

Conclusion: Patients with Tetralogy of Fallot have dilated right ventricles with impaired function due to pulmonary regurgitation and right bundle branch block leading to paradoxical septal motion. Paradoxical septal motion was seen in all patients with Tetralogy of Fallot. Although in this population with only mildly reduced LV function and mild paradoxical septal motion a correlation between both could not be demonstrated, further research in a more heterogeneous population is warranted.

\section{PI74}

Murine Es-derived cardiomyocytes form grafts and improve cardiac function in the infarcted myocardium

Hualei Zhang', Hui Qiao', Satoshi Yamanaka², Nataliya Petrenko', Vickas Patel', Bin Huang', Victor Ferrari', Kenneth Boheler ${ }^{2}$ and Rong Żhou' 'University of Pennsylvania, Philadelphia, PA, USA

${ }^{2}$ National Institute of Aging, NIH, Bethesda, MD, USA

Journal of Cardiovascular Magnetic Resonance 2009, I I(SuppI I):PI74

Background: embryonic stem cells (ESC) readily differentiate into cardiac lineage making them a potential source of transportable cells for myocardial regeneration. However the low yield of ESC-derived cardiomyocytes (ESC-CMs) using the conventional differentiation method makes it difficult to perform in vivo study and low enrichment of CMs leads to concerns of teratoma formation.

Methods: a murine ESC line containing puromycin resistance gene under control of a cardiac specific promoter, sodium calcium exchanger (NCX) was used to generate ESC-CMs. ESCCMs were labeled with Superparamagnetic iron-oxide nanopar-

Figure I (abstract PI74)

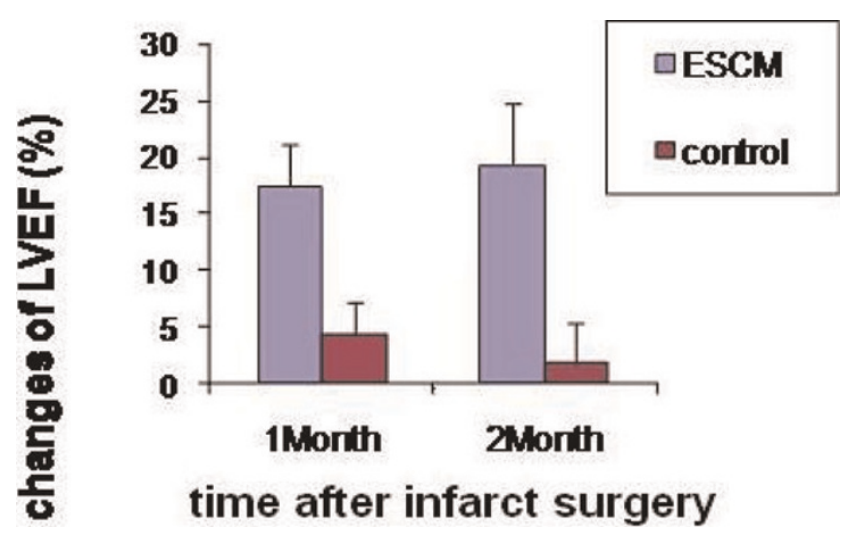

Left-ventricular ejection fraction LVEF changes. The rats treated with ESCM showed much higher the increase of LVEF change than the control rats both at I month $(p=0.0003)$ and 2 months $(p=0.000048)$, while the infarction size at the day I post-op was no significant difference between two groups $(20.02 \pm 3$. I I v.s $19.09 \pm 4.72$, $p=0.65)$. 
ticles (SPIO) for MRI detection. Reperfused myocardial infarction was induced in athymic rats. Infarction size was estimated by MRI post-op dayl to exclude animals with infarct size smaller than $10 \%$ or larger than $35 \%$ of the LV volume. At post-op day 7 , labeled ESC-CMs (5-10 millions) were injected into infarction region. Control group was injected with vehicle. MRI scan was performed at post-op day 8 to confirm successful CM cell transplantation. Global cardiac function in ESC-CM and vehicle treated animals was assessed by MRI for 2 months. Immunohistology staining and electrophysiology were performed on postmortem hearts and ESC-CMs, respectively.

Results: a high yield of ESC-CMs was achieved with positive cardiac specific alpha-actinin in more than $90 \%$ of cells. The low proliferative capacity of ESC-CMs allows them to retain SPIO for serial MRI tracking. LV ejection fraction in ESC-CM treated rats at I- and 2-month is significantly higher than that in the controls. IHC demonstrated formation of grafts in the host myocardium and gap junctions between grafted ESC-CMs and host CMs. See Figure I.

Conclusion: Large numbers of highly pure ESC-CMs were obtained. Preliminary results suggest that ESC-CMs form grafts and improve LV function in the infarcted hearts.

\section{PI75}

High-resolution diffusion tensor mr imaging for the evaluation of myocardial anisotropy and fiber tracking at 3 T: effect of the numbers of diffusionsensitizing gradient directions

Sang II Choi ', Joon-Won Kang', Eun Ju Chun ${ }^{2}$,

Seong Hoon Choi $^{3}$ and Tae-Hwan Lim '

'Department of Radiology, Asan Medical Center, University of Ulsan College of Medicine, Seoul, Republic of Korea

${ }^{2}$ Department of Radiology, Seoul National University Bundang Hospital, Seong-Nam, Republic of Korea

${ }^{3}$ Department of Radiology, Ulsan University Hospital, University of Ulsan College of Medicine, Ulsan, Republic of Korea

Journal of Cardiovascular Magnetic Resonance 2009, I I(SuppI I):PI75

Introduction: Diffusion tensor magnetic resonance imaging (DT-MRI) has provided a means for rapid and noninvasive evaluation of the cardiac geometry and three-dimensional fiber structure. However, most cardiac DT-MRI studies have used low diffusion resolution such as 6 directions in animal's fixed heart. To our knowledge, the effect of the numbers of diffusion-sensitizing gradient directions has not been studied for image quality of cardiac DT-MRI.

Purpose: To evaluate effect of numbers of diffusion-sensitizing gradient direction on image quality for the evaluation of myocardial anisotropy and fiber tracking by using ex vivo diffusion tensor MR imaging (DT-MRI).

Methods: DT-MRI using a SENSE-based echo-planar imaging technique was acquired by using a $3 T$ MR scanner from ten excised heart. With a b-value of $800 \mathrm{~s} / \mathrm{mm}^{2}$, the diffusion tensor images were obtained for 6, 15, 32 diffusion-sensitizing gradient directions at the midventricular level. For quantitative analysis, the numbers of tracked fibers, fractional anisotropy (FA), and the length of tracked fibers were measured. For qualitative analysis, two radiologists assessed the image quality of fiber tractography.
Figure I (abstract PI75)

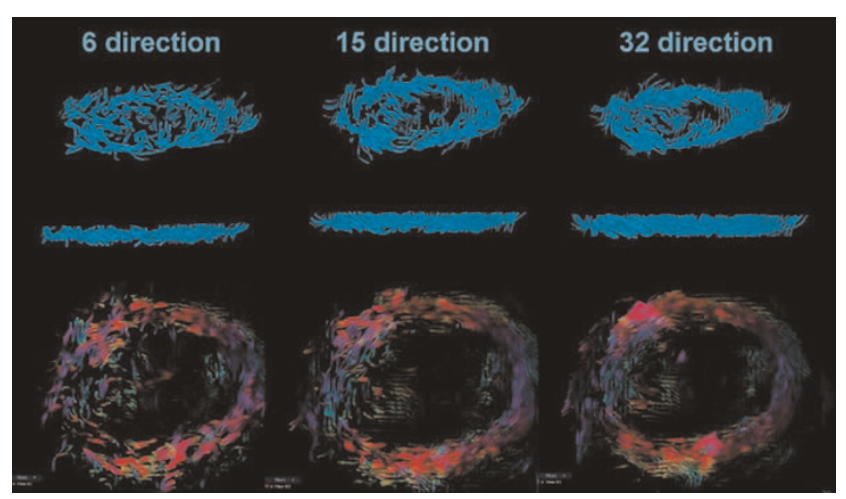

Results: By increasing the numbers of diffusion-sensitizing gradient directions from 6 to 15, and 32, FA and standard deviation were significantly reduced $(P=0.00 \mathrm{I})$, and the numbers of tracked fibers and the length of tracked fibers were significantly increased $(P=0.00 \mathrm{I})$. The image quality of fiber tractography was significantly increased as the increased numbers of diffusion-sensitizing gradient directions $(P<0.00 I)$. See Figure I.

Conclusion: Image quality of ex vivo DT-MRI is significantly improved as the numbers of diffusion-sensitizing gradient directions increased.

\section{PI76}

Three-dimensional changes in left atrial volumes and ejection fraction during dobutamine stress Cardiovascular Magnetic Resonance Vinodh Jeevanantham, William Ntim, Craig Hamilton, Tim Morgan, John Hoyle and Gregory Hundley Wake Forest University, Winston Salem, NC, USA

Journal of Cardiovascular Magnetic Resonance 2009, I I(SuppI I):PI76

Introduction: Left ventricular performance is routinely measured during dobutamine cardiovascular magnetic resonance (DCMR) and the stress induced left ventricular changes have been well described. Left atrial (LA) size and volume are associated with increased risk of developing atrial fibrillation, stroke and congestive heart failure; however changes in LA volume and LA ejection fraction (LAEF) during DCMR have not been described.

Purpose: To determine LA volumes and LAEF in patients undergoing DCMR.

Methods: We enrolled 128 consecutive participants referred for DCMR. Using white blood CINE steady-state free precession imaging, a 3 dimensional LA model (determined from a multislice, multi-phase stack of LA short axis views) was obtained to compute LA volumes and LAEF throughout dobutamine stress. Baseline clinical cardiac risk factors such as diabetes mellitus, hypertension, coronary artery disease (CAD), atrial fibrillation, age and gender were collected. Dobutamine stress protocol involved infusion of $7.5 \mu \mathrm{g} / \mathrm{kg} / \mathrm{min}$ for low dose stage and $20 \mu \mathrm{g} /$ $\mathrm{kg} / \mathrm{min} \pm$ atropine for peak dose stress stage. LA maximal volume (LAV max) was defined as maximal LA volume before the opening of mitral valves. LA minimal volume (LAV min) was defined as minimal LA volume at closure of mitral valve. LAEF 
Table I (abstract PI76) Mean differences in left atrial volumes and ejection fraction during dobutamine CMRI

\begin{tabular}{lllll}
\hline & $\begin{array}{l}\text { Baseline } \\
(\mathbf{N}=1 \mathbf{2 8})\end{array}$ & $\begin{array}{l}\text { Low dose } \\
(\mathbf{N}=\mathbf{I 2 6})\end{array}$ & $\begin{array}{l}\text { Peak dose } \\
(\mathbf{N}=1 \mathbf{0 5})\end{array}$ & P value \\
\hline LAV max & $33.1 \pm 8.7$ & $31.5 \pm 9.7$ & $26.3 \pm 8.1$ & $<0.00 \mathrm{I}$ \\
LAV min & $19.9 \pm 5.9$ & $17.8 \pm 6.3$ & $16.8 \pm 6.2$ & $<0.00 \mathrm{I}$ \\
LA EF & $40.1 \pm 6.9$ & $43.6 \pm 7.3$ & $36.6 \pm 10.2$ & $<0.00 \mathrm{I}$ \\
\hline
\end{tabular}

was defined as (LAV max-LAV min)/LAV max. All volumes were indexed to body surface area. One-way Analysis of variance (ANOVA) was used to detect the differences in LA volumes and LAEF across stages of DCMR. Linear regression analysis was used to find the independent predictors of LAEF and LA volumes.

Results: The age of our participants averaged $69 \pm 8$ years; $51 \%$ were men. Twenty seven percent of the participants had CAD, 33\% had diabetes, $88 \%$ had hypertension, and $7 \%$ had atrial fibrillation. Mean LA volumes and LAEF changed at each stage of DCMR (Table I). Cardiac risk factors influencing LA volumes and LAEF included: CAD $(p=0.04)$, male gender $(p=0.04)$, and atrial fibrillation $(p=0.02)$. Also, increasing age was inversely correlated with LAEF [baseline $(p=0.0 \mathrm{I})$, low dose $(p=0.004)$ and peak dose $(p=0.19)]$; and positively correlated with LA volumes; LAV Min [baseline $(p=0.0 \mathrm{I})$, low dose $(p=0.02)$ and peak dose $(p=0.0 \mathrm{I})$ ]. Regression analysis revealed that the significant predictors for baseline LAEF were atrial fibrillation ( $P 0.0 \mathrm{I})$, and CAD ( $\mathrm{p} 0.05$ ); for low dose LAEF were increasing age ( $p$ 0.02), and atrial fibrillation ( $p$ 0.003); and for peak dose LAFE was age $(p=0.01)$. After adjusting for all confounding variables, increasing age was the most significant predictor for LA volumes across all stages of stress [baseline ( $p$ 0.05), low dose ( $p$ 0.03) and peak dose ( $p$ 0.02)].

Conclusion: Left atrial volumes decrease during progressive stress levels of DCMR. Presence of underlying CAD or atrial fibrillation significantly decreased LA volumes and left atrial ejection fraction. Increasing age was associated with increased LA volumes but decreased LA ejection fraction. Further studies to understand the prognostic value of these changes are needed.

\section{PI77}

Quantitative assessment of intramyocardial function using Cine DENSE MRI: a validation study Li Feng', Robert M Donnino ${ }^{2}$, Leon Axel ${ }^{2}$ and Daniel Kim ${ }^{2}$ 'Polytechnic Institute of New York University, Brooklyn, NY, USA

${ }^{2}$ New York University Langone Medical Center, New York, NY, USA

Journal of Cardiovascular Magnetic Resonance 2009, I I(SuppI I):PI 77

Introduction: Non-invasive assessment of myocardial function plays a critical role in the management of heart disease. Conventional imaging modalities, such as echocardiography and cine MRI, are widely used for quantitative assessment of global cardiac function and qualitative assessment of regional cardiac function. Quantitative assessment of regional wall motion may additionally improve the accuracy of detecting wall motion abnormalities due to heart disease. Recently, fast cine displacement-encoded (DENSE) MRI was developed to produce relatively high quality strain maps within clinically acceptable breath-hold duration of 12 cardiac cycles [I]. While this pulse
Figure I (abstract PI77)

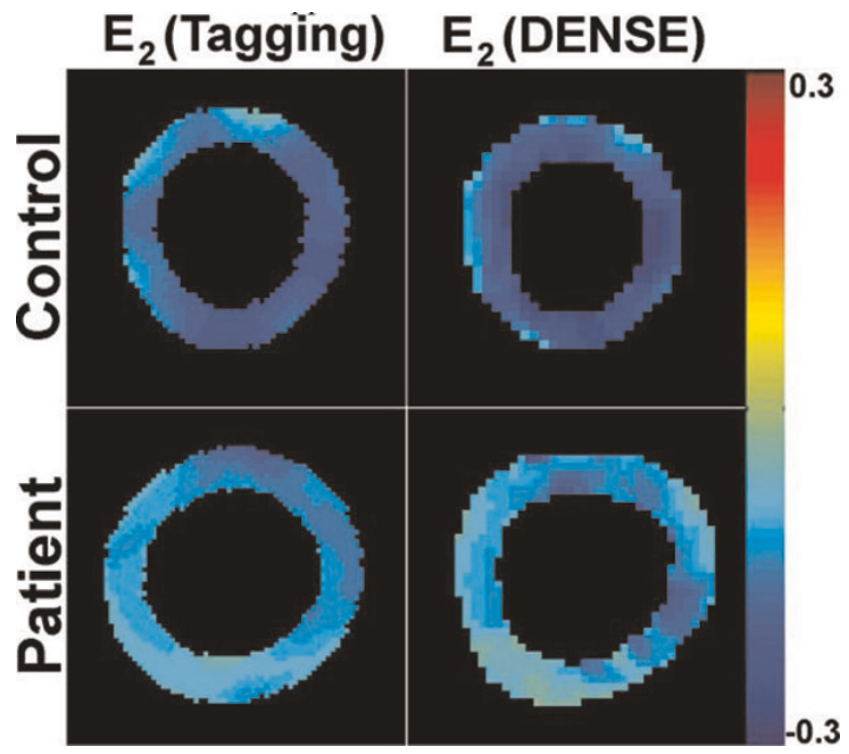

End-systolic E2 maps of (top row) control and (bottom row) patient comparing tagging and DENSE.

sequence is promising for clinical applications, it has not been validated. Therefore, the purpose of this study was to validate the relative accuracy of fast cine DENSE MRI in controls and patients with heart disease.

Methods: Pulse sequence: Myocardial tagged MRI was chosen as the reference method [2, 3, 4, 5]. Both the conventional tagged $M R$ and fast cine DENSE pulse sequences were implemented on a 3 T whole-body MR scanner (Tim-Trio, Siemens) equipped with a 12-channel phased array receive coil. Relevant imaging parameters for cine DENSE include: effective spatial resolution $=3.3 \mathrm{~mm} \times 3.3 \mathrm{~mm}$, slice thickness $=7 \mathrm{~mm}$, temporal resolution $=35 \mathrm{~ms}$, and breath-hold duration $=12$ heartbeats. Relevant imaging parameters for myocardial tagging include: spatial resolution $=1.3 \mathrm{~mm} \times 1.8 \mathrm{~mm}$, slice thickness $=$ $7 \mathrm{~mm}$, temporal resolution $=35 \mathrm{~ms}$, breath-hold duration $=$ 15 heartbeats, and grid tag spacing $=7 \mathrm{~mm}$.

Cardiac imaging: Twelve healthy human subjects $(7$ males; 5 females; mean age $=34.5 \pm 11.0$ years), with no history of heart disease and no risk factors for coronary artery disease, and thirteen

Figure 2 (abstract P I77)
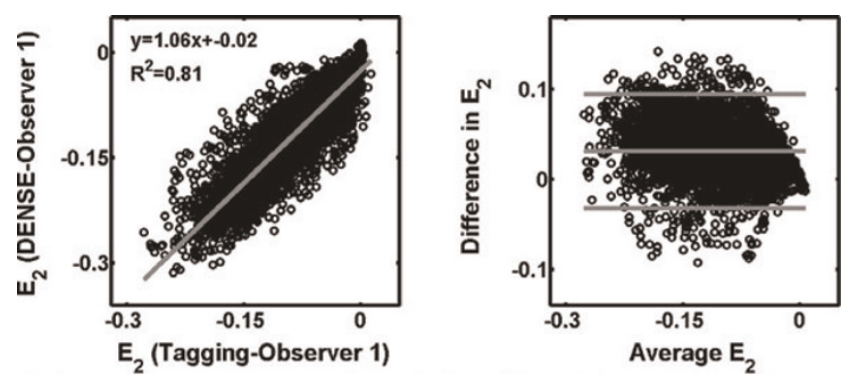

Plots showing linear correlation (left) and Bland-Altman (right) on E2 measurements $(n=4334)$. 
patients ( 12 males; I female; mean age $=55.3 \pm$ II.2 years) with prior history of heart disease were imaged in three short-axis (apical, mid-ventricular, basal) planes of the left ventricle (LV), using fast cine DENSE and myocardial tagged MRI pulse sequences.

Image analysis: For cine DENSE, cardiac contours were segmented manually by two observers through the first 15 cardiac phases. For myocardial tagging, contour segmentation and tag tracking were performed by observer I through the first I I cardiac phases. The resulting segmented functional data were further processed to calculate the second principal strain $\left(E_{2}\right)$. The principle direction of $E_{2}$ at end systole is primarily circumferential. The three short-axis images were subdivided into 16 segments according to the American Heart Association standardized model.

For cine DENSE, the intra-observer variability was assessed by calculating the mean difference and coefficient of repeatability (CR) of first and second calculations of $E_{2}$, which were made in a blinded fashion with a separation of at least two weeks. Interobserver variability was assessed by calculating the mean and $C R$ between the two observers.

The accuracy of fast cine DENSE was assessed relative to myocardial tagging by performing Bland-Altman and linear correlation analyses on their $E_{2}$ calculations. A $P<0.05$ was considered statistically significant.

Results: The intra- and inter-observer CR of DENSE for $E_{2}$ measurements were 0.03 and 0.04 , respectively $(n=5910)$. Their corresponding $95 \%$ limits of agreement were -0.03 to 0.03 and -0.04 to 0.04 . Figure I shows representative end-systolic $E_{2}$ maps that compare the results by DENSE and myocardial tagging. Both the control and patient examples show good agreement between the two methods. Statistically, as shown in Figure 2, the two methods were strongly correlated (slope $=1.06$; bias $=$ $\left.0.02 ; R^{2}=0.8 I ; n=4334\right)$ and in good agreement (mean $=0.03$; $95 \%$ limits of agreement $=-0.03$ and 0.09 ).

Discussion: This study demonstrated that fast cine DENSE MRI is highly reproducible and produces $E_{2}$ measurements that are strongly correlated and in good agreement with those produced by myocardial tagging. We conclude that cine DENSE MRI is a validated method for clinical applications.

\section{References}

I. Kim D and Kellman P: NMR Biomed 2007, 20:59I-60I.

2. Young AA, et al: Radiology 1993, I88:101-108.

3. Moore CC, et al: Radiology 1994, 190:765-769.

4. Lima JA, et al: JACC 1993, 21:1741-1751.

5. Yeon SB, et al: JACC 200I, 38:555-56I.

\section{PI78}

Impact of temporal resolution on cardiac phaseresolved oxygen-sensitive myocardial steady-state free precession imaging

Xiangzhi Zhou', Richard Tang', Rachel Klein',

Sotirios A Tsaftaris ${ }^{2}$, Debiao $\mathrm{Li}^{1}$

and Rohan Dharmakumar'

'Department of Radiology, Northwestern University, Chicago, IL, USA

${ }^{2}$ Department of Electrical Engineering and Computer Science, Northwestern University, Evanston, IL, USA

Journal of Cardiovascular Magnetic Resonance 2009, I I(SuppI I):PI 78

Introduction: Cardiac phase-resolved imaging studies that are used in the assessment of cardiac function are performed
Figure I (abstract PI78)

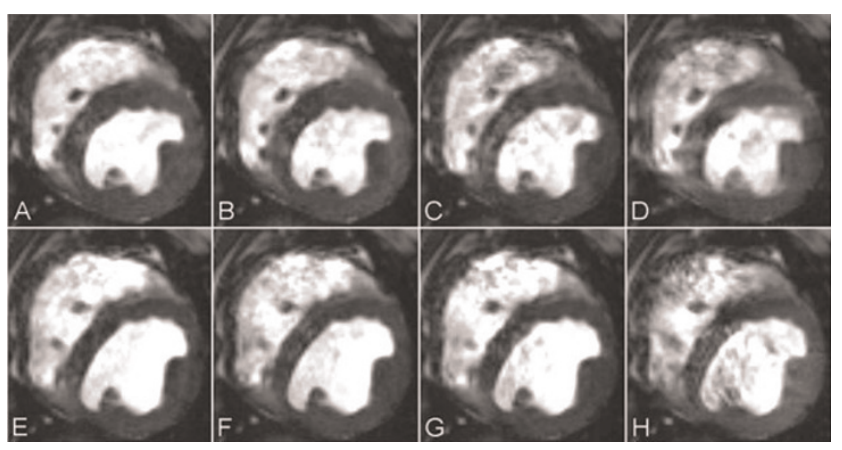

Short axis SSFP images $(T R=6.0 \mathrm{~ms})$ of med ventricle with different $T_{\text {RES }}$ A,E: 18 ms; B,F: 42 ms; C,G: 84 ms; and D,H: 204 ms (upper row from LS and lower row from LD). Note that as $T_{\text {RES }}$ increases the signal inhomogeneity increases and endocardial delineation becomes more difficult as signal from the blood pool blends in with the myocardial signal, likely due to motion.

with a temporal resolution ( $T_{\text {RES }}$ ) of approximately $50 \mathrm{~ms}$ to mitigate the effects from cardiac motion and flow. To date, there has been minimal interest on the characterization of myocardial signal intensities from cine images. Steady-state free precession based cardiac phase-resolved blood-oxygen-leveldependent (CP-SSFP BOLD) imaging is a relatively new method for identifying myocardial oxygen abnormalities on the basis of regional signal differences. For reliable assessment of oxygenation changes, it is imperative to ensure that acquisitions enable robust image quality. We hypothesize that $T_{\text {RES }}$ plays a significant role on CP-SSFP image quality and that, in particular, myocardial signal characteristics disintegrate with elevations in $\mathrm{T}_{\mathrm{RES}}$.

Figure 2 (abstract PI78)

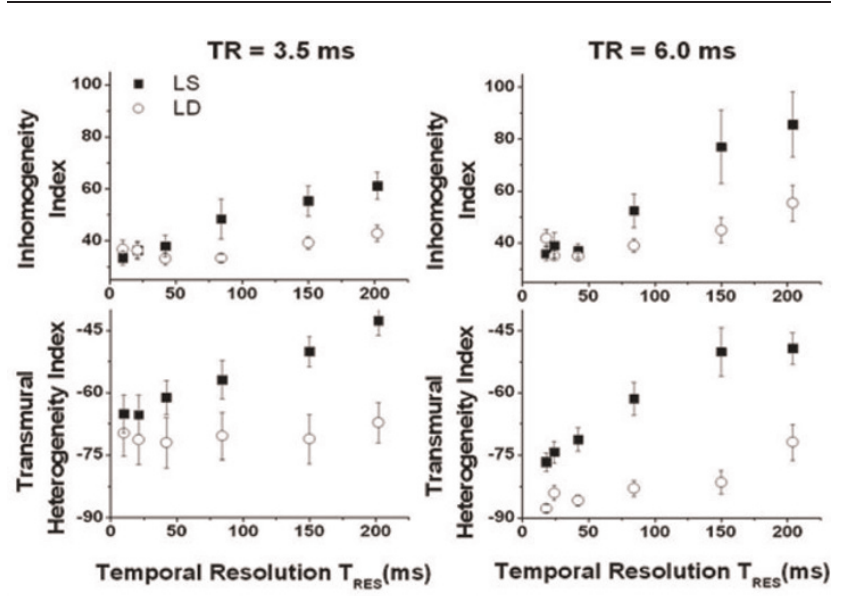

Mean MSI (upper plot) and THI (lower plot) computed from LS (squares) and LD (circles) images as a function of $T_{\text {RES }}$ for TR $=3.5 \mathrm{~ms}$ (left) and $6.0 \mathrm{~ms}$ (right). Note that both mean MSI and THI increase with increasing $\mathrm{T}_{\text {RES. }}$ 
Methods: Dogs were used to test the hypothesis under controlled conditions. Animals $(n=4)$ were anesthetized and their heart rate was monitored with ECG $(R-R$ interval $=$ $710 \mathrm{~ms}-780 \mathrm{~ms}$ ). Multiple breath-held acquisitions (20-40 s) were performed in each animal, interrupted by 2-3 minute rest, ensuring that the heart rate remained relatively constant between acquisitions. 2D balanced-SSFP imaging was prescribed in the cine mode to study the effects of $T_{\text {RES }}$ on short-axis mid-left-ventricular images of the myocardium in a clinical I.5 T scanner. The scan parameters were: in-plane resolution $=1.2 \times 1.2 \mathrm{~mm}^{2}, \mathrm{TR} / \mathrm{TE}=3.5 \mathrm{~ms} / 1.75 \mathrm{~ms}$ (conventional cine SSFP) and $6.0 \mathrm{~ms} / 3.0 \mathrm{~ms}$ (CP-SSFP BOLD), flip angle $=70^{\circ}, N E X=1$, segments/cardiac phase were changed to obtain different $\mathrm{T}_{\text {RES }}$ (10 ms to $200 \mathrm{~ms}$ ). This study was repeated 2-3 times with a two-day interval for each animal. In total 10 studies were performed. Two indices were used to quantify the myocardial signal characteristics obtained with cine SSFP images at different $\mathrm{T}_{\text {RES }}$ : (I) Myocardial Signal Inhomogeneity Index (MSI), defined as the standard deviation of the LV myocardial signal intensity; and (2): Transmural Heterogeneity Index (THI), defined as the minimum pixel intensity difference between pixels along a line perpendicular to the blood-muscle interface in the LV chamber. The global THI was calculated by sweeping the line along the interface for $360^{\circ}$ and averaging the THI for each $1^{\circ}$ increment. Results were averaged across all studies for late systole(LS) and late diastole(LD). Note that MSI measures the signal variation throughout the myocardium and $\mathrm{THI}$ measures the image quality permitting reliable delineation of the endocardial border from blood.

Results: Figure I shows short-axis SSFP images with different $T_{\text {RES }}(10 \mathrm{~ms}, 42 \mathrm{~ms}, 80 \mathrm{~ms}$ and $202 \mathrm{~ms}$ from left to right obtained at TR $=6.0 \mathrm{~ms}$ ). The upper row images are from LS and the lower row images are from LD. Numerical results from MSI (upper plot) and THI (lower plot) computations from the LS and LD images as a function of $T_{\text {RES }}$ are shown in Figure 2. On average, $M S I$ and $T H I$ are directly related to $T_{\text {RES }}$ at LS and LD (for TR = 3.5 and $6.0 \mathrm{~ms}$ ), albeit the rate of change of the indices at LS is much greater than at LD. In particular, results showed that, at $T R=6.0 \mathrm{~ms}$, for $\mathrm{T}_{\mathrm{RES}}>42 \mathrm{~ms}, \mathrm{MSI}$ and $\mathrm{THI}$ are significantly greater than with $T_{\text {RES }}=18$ ms (t-test, $p<0.0 \mathrm{I}$ ).

Conclusion: Reliable image quality is critical for accurate detection of changes in myocardial oxygenation. This study investigated the impact of $T_{\text {RES }}$ on two important features of CP-SSFP BOLD images: (I) myocardial signal variations, (2) endocardial blur. Findings show that $\mathrm{MSI}$ and $\mathrm{THI}$ are strongly influenced by $\mathrm{T}_{\text {RES. In }}$ particular, with both conventional cine SSFP (TR = $3.5 \mathrm{~ms}$ ) and CP-BOLD SSFP (TR = $6 \mathrm{~ms}$ ) imaging, the image quality diminishes with increasing $T_{\text {RES. Also, for any }}$ given $T_{\text {RES }}$, the reduction in image quality is significantly greater at systole than at diastole. We conclude that for reliable detection of myocardial oxygenation on the basis of CP-SSFP BOLD imaging, it is necessary to keep $T_{\text {RES }}$ as short as possible. These findings remain to be validated in humans.

\section{PI79}

\section{Abstract withdrawn by author}

Journal of Cardiovascular Magnetic Resonance 2009, I I(SuppI I):PI79
PI80

Rapid MR assessment of left ventricular systolic function early after acute myocardial infarction using single breath-hold cine imaging with temporal parallel acquisition technique (TPAT) and 4D guide-point modeling Holger C Eberle', Christoph J Jensen', Kai Nassenstein ${ }^{2}$, Thomas Schlosser ${ }^{2}$, Georg V Sabin', Christoph K Naber' and Oliver Bruder'

'Department of Cardiology and Angiology, Elisabeth Hospital Essen, Essen, Germany

${ }^{2}$ Department of Diagnostic and Interventional Radiology and Neuroradiology, University Hospital Essen, University of Duisburg-Essen, Essen, Germany

Journal of Cardiovascular Magnetic Resonance 2009, I I(SuppI I):PI80

Introduction: Systolic left ventricular ejection fraction (LVEF) is a strong prognosticator after acute myocardial infarction (AMI). Currently, section summation of contiguous cardiac magnetic resonance (CMR) short axis steady-state free precession (SSFP) images is the reference standard for measuring LVEF. However, the use of standard CMR LVEF measurement in the early postinfarction phase is limited by the time need of image acquisition in multiple breath-holds, which is frequently not well tolerated by critically ill patients. Additional time is needed for calculating LVEF by summation of discs by semi-automated postprocessing tools.

Cine imaging accelerated by Temporal Parallel Acquisition Technique (TPAT) allows for the acquisition of multiple cine images in a single breath-hold. LVEF calculation from TPAT images in short an long axis orientation can be performed by a novel software tool applying four dimensional guide point modeling ventricular function analysis (4DVF). As 4DFV is based on geometric assumptions of a normal heart, it is unclear whether 4DFV is applicable to patients with AMI.

Purpose: To compare 4D guide-point modeling LVEF analysis of TPAT images with standard LVEF analysis in patients with AMI. Methods: 27 consecutive patients (18 male, mean age $60 \pm$ 13 years) underwent CMR on a I.5 Tesla MRI scanner (Avanto ${ }^{\mathrm{TM}}$, Siemens Medical Solutions, Germany) within I to 15 (mean 4) days after AMI.

The CMR protocol included SSFP cine imaging (TrueFISP, TR $3 \mathrm{~ms}$, TE I.5 ms, FA $60^{\circ}$, sl $3 \mathrm{~mm}$ ) in 7-16 (mean l3) breathholds and inversion recovery (IR) delayed enhancement imaging following gadolinium contrast administration.

Table I (abstract p I80) LV ejection fraction, volumes and analysis time (Observer A).

\begin{tabular}{lccc}
\hline & Standard LVF 4DVF & $\begin{array}{c}\text { Mean Paired } \\
\text { Difference }\end{array}$ \\
\hline Ejection Fraction $(\%)$ & $5 \mathrm{I} . \mathrm{I} \pm 12.3$ & $5 \mathrm{I}, 6 \pm 13.3-0.54 \pm 5.3$ \\
LV Enddiastolic Volume $(\mathrm{ml}) 165.7 \pm 63.0$ & $157.5 \pm 64.4$ & $8.2 \pm 17.4$ \\
LV Endsystolic Volume $(\mathrm{ml})$ & $86.4 \pm 50.1$ & $81.8 \pm 51.2$ & $4.7 \pm 12.2$ \\
LV myocardial mass $(\mathrm{g})$ & $160.4 \pm 49$ & $173.5 \pm 54-13.1 \pm 44.9$ \\
Analysis Time $(\mathrm{s})$ & $305.7 \pm 117.2$ & $163.4 \pm 79.4 \mid 42.1 \pm 121.4^{*}$
\end{tabular}

Data are mean $\pm S D *_{p}<0.05$. 
Table 2 (abstract p 180) Interobserver variaion between stard LVF and 4DVR analysis.

\begin{tabular}{lcc}
\hline & Standard LVF & 4DVF \\
\hline Ejection Fraction (\%) & $-1.3 \pm 4.4$ & $-0.5 \pm 2.8$ \\
LV Enddiastolic Volume (ml) & $3.3 \pm 15.8$ & $1.4 \pm 11.0$ \\
LV Endsystolic Volume (ml) & $3.3 \pm 10.4$ & $0.9 \pm 11.1$ \\
LV Myocardial Mass $(\mathrm{g})$ & $4.6 \pm 17.9$ & $3.5 \pm 32.5$ \\
Analysis Time (s) & $59.2 \pm 68.2^{*}$ & $8.7 \pm 71.7$
\end{tabular}

$*_{p}<0.05$ for difference.

Contiguous short axis cine images were analyzed by standard left ventricular function (LVF) analysis and the disc summation method (Argus ${ }^{\mathrm{TM}}$, Siemens Medical Solutions, Germany).

Additionally, two long axis and four short axis cine images were acquired in a single breath-hold using a TPAT accelerated SSFP sequence (TR 2,7 ms, TE I, $12 \mathrm{~ms}$, FA 78 ${ }^{\circ}, \mathrm{sl} 6 \mathrm{~mm}$ ). The resulting cine images were used for 4-dimensional guide point modeling LVF analysis (Argus 4D TM, Siemens Medical Solutions, Germany). Both LVEF calculation methods were performed by two independent observers, and the duration of analysis was recorded.

Results: Mean infarct size was $17 \pm 12 \%$ of left ventricular mass. $6 \pm 3$ (range I to I2) LV myocardial segments were affected by wall motion abnormalities. II patients had anterior, 16 inferior wall myocardial infarction. A strong correlation of EF values from 4DVF with standard LVF analysis was observed $(r=0.92, P<0.01)$. The results for observer $A$ are shown in Table I. Interobserver variability was low for both methods, and there were no statistically significant differences for LVEF (4DVF: $\mathrm{I} .3 \pm 4.4 \%, \mathrm{p}=0.15$ vs. standard LVF: $-0.5 \pm 2.8 \%, \mathrm{p} 0.36)$ and

Figure I (abstract PI80)

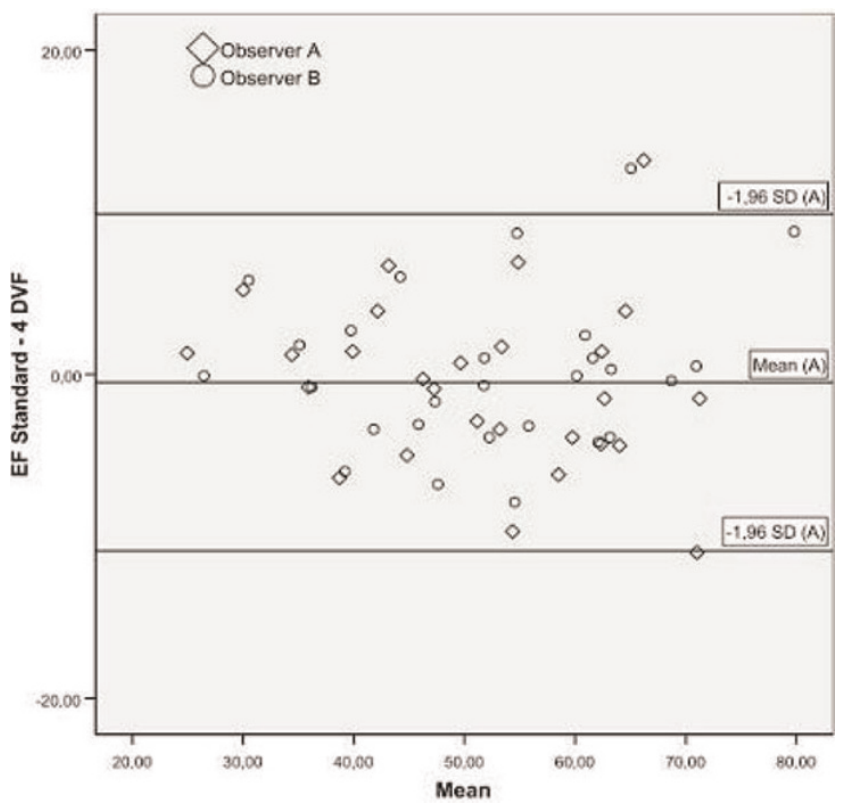

Bland-Altman plots shows good agreement of both methods for calculating left ventricular ejection fraction (LVEF) for both observers. Mean $\pm 1.96 *$ standard deviation is indicated for observer A.
LV Volumes (Table 2). For a Bland-Altman plot see figure I. LVF Analysis was faster with the 4DVF approach (I59 $\pm 84 \mathrm{~s})$ as compared to the standard Argus procedure $(276 \pm 107 \mathrm{~s}$, $\mathrm{p}<0.0 \mathrm{I})$.

Conclusion: 4DVF guide point modeling is a time saving alternative to standard LVF analysis. On the basis of images acquired in a single breath-hold, it yields results that are in good accordance with standard LVF analysis.

A protocol combining TPAT accelerated single breath-hold imaging and 4DVF image processing may be potentially useful for the rapid assessment of LV function in critically ill patients following acute myocardial infarction.

\section{PI8I}

Viability imaging of stem cell using a MRI reporter gene and MEMRI

Jaehoon Chung, Kehkooi Kee, Renee R Perra

and Phillip C Yang

Stanford University, Stanford, CA, USA

Journal of Cardiovascular Magnetic Resonance 2009, I I(SuppI I):PI8I

Introduction: Embryonic stem cells have demonstrated the potential to restore the myocardium. MRI is an ideal method to evaluate myocardial cell therapy. Superparamagnetic iron oxide nanoparticle (SPIO) has been widely used to monitor stem cell therapy. However, this technique does not provide any biologic information of cell viability.

Purpose: This novel reporter gene (RG) is designed to express antigenic epitopes on the surface of embryonic stem cell (ESC). Employing SPIO-conjugated monoclonal antibodies, MRI signal specific to hESC viability can be generated. $\mathrm{Mn}^{2+}$ is known to be able to enter viable cells through the voltage gated $\mathrm{Ca}^{2+}$ channel and subsequently can shorten $\mathrm{TI}$ relaxation time generating bright signal on $\mathrm{TI}$ weighted sequence.

Methods: MRI RG was constructed driven by EFI $\alpha$ promoter to express c-myc, HA epitopes and firefly luciferase (Luc) on the cell surface. This fusion protein has been designed to be anchored on the cell surface by PDGFR transmembrane domain. Both c-myc and HA epitopes are the molecular targets for MRI

Figure I (abstract PI8I)

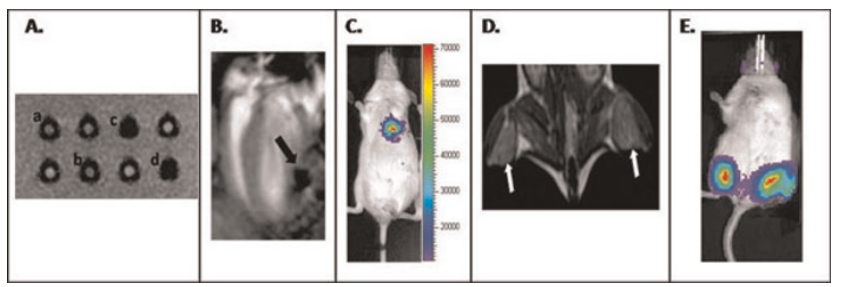

Functional confirmation of the MRI RG and MRI and BLI. A In vitro of hESC-RD with SPIO-conjugated anti-c-myc and HA antibody showed strong T2 weighted dephaing signal (c-d). Non-transduced mESCs showed no T2 weighted dephasing signal (a-b). MR image was taken with GRE sequence using the following parameters: $100 \mathrm{~ms}$ TR, $30 \mathrm{~ms}$ TE, FA 45, FOV 12, NEXI, $192 \times 192$. B. Long axis view of mouse heart after injection of $0.5 \times 10^{6}$ magnetically pre-labeled hESC onto the lateral left ventricular wall. C. Validation of viability of hESC using bioluminescence imaging (BLI). D. MEMRI of transplanted mESC-RG on bilateral mouse hindlimbs (indicated by white arrows). E. Viability of the injected $\mathrm{mESC}$ was confirmed with positive luciferase activity using BLI. 
viability signal using SPIO conjugated monoclonal antibodies. H9 hESC female line was tranduced with this RG using a p2K7 lentiviral vector. hESC-RG incubated with anti c-myc and HA microbeads were scanned by $3 \mathrm{~T} M R I$ using a high array knee coil. For MEMRI, $0.5 \mathrm{~mL}$ of $5 \mathrm{mM} \mathrm{MnCl}$, was injected intraperitoneally after transplanting $\mathrm{mESC}$ onto mouse hindlimbs. Mice were scanned using Spin echo sequence by 3 T MRI.

Results: Functional expression of hESC-RG was confirmed by FACS, bioluminescence and Prussian blue staining. In vitro MRI showed significant dephasing signal generated from SPIO conjugated antibody for c-myc and HA (Figure IA). Magnetically pre-labeled hESC were injected into the lateral wall of left ventricle (LV) and the robust dephasing signal was noted on MRI (Figure IB,C). In vivo MEMRI could show significant enhancement of viable mESC on the mouse hindlimbs using $\mathrm{TI}$ weighted sequence (Figure ID,E).

Conclusion: The novel MRI RG enabled viable embryonic stem cells to generate significant molecular MRI signal. In vivo molecular signal of hESC viability will be feasible using this innovative MRI RG. MEMRI enabled in vivo evaluation of viability of stem cells.

\section{PI 82}

"ART-FUN": an integrated software for functional analysis of the aorta

Alain De Cesare', Alban Redheuil', Anas Dogui ', Odile Jolivet Engineer', Alain Lalande ${ }^{2}$,

Frederique Frouin ', Elie Mousseaux ${ }^{1,3}$ and Alain Herment ${ }^{\prime}$

'Inserm, Paris, France

${ }^{2}$ Universite de Bourgogne, Dijon, France

${ }^{3}$ AP-HP, Paris, France

Journal of Cardiovascular Magnetic Resonance 2009, I I(SuppI I):PI82

A new software platform "ARTerial FUNction" was developed to analyse cine and phase contrast MR acquisitions in the ascending and descending aorta. It provides an exhaustive description and analysis of time velocity waveforms, as well as the accurate estimation of local and regional aortic function and stiffness indices.

The main functions implemented in this platform, running under MATLAB, include : I) import of DICOM cine and flow data (including the specific headers needed for further analysis of data); 2) segmentation (manual or automatic) of axial sections of the aorta; 3 ) analysis of flow data and waveforms recorded in the segmented arterial lumens; 4 ) advanced comparison of flow data between the ascending and descending aorta, including an automatic estimation of the propagation speed of the velocity wave; 5) export of results to Excel data sheets.

Here we describe specifically the automatic contouring of the aortic lumen integrated in this software. The method is based on a 3D snake deformable surface, modelling the evolution of aortic lumen with time; this allows the introduction of smoothness constraints both in the 2D image plan and the time axis. This cylindrical like surface is deformed under the influence of "external forces" including the classical attraction by the image gradient but also specific potentials to avoid the attraction of the contour by vascular structures surrounding the ascending aorta, such as the pulmonary artery and vena cava.

The duration of this segmentation ranges from 5 seconds (descending aorta, True-FISP sequence, breathhold, square pixel
Figure I (abstract PI82)

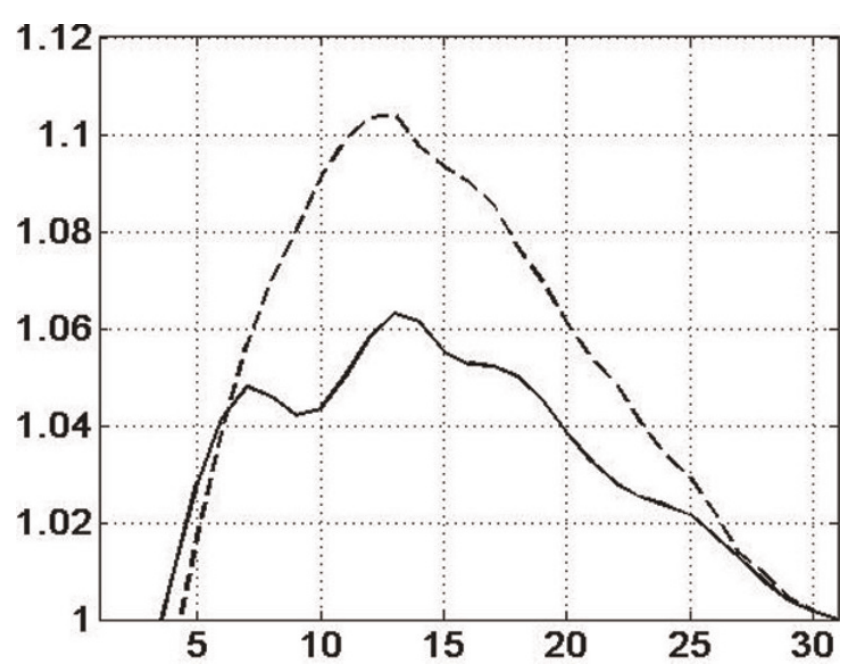

Horizontal axis: phases of the cardiac cycle (22.4 ms per sample). Vertical axis: area of the lumen normalised by the proto-diastolic area. Plain line: ascending aorta, dotted line: descending aorta. The propagation delay of the pulse wave in the aortic arch is visible and the larger distensibility of the aortic wall at the level of the descending aorta is visible as well.

size $=0.58 \mathrm{~mm}, 3 \mathrm{I}$ cardiac phases) to 35 seconds (ascending aorta, FISP-2D, free breathing, square pixel size $=0.37 \mathrm{~mm}$, 5 I cardiac phases) on a basic laptop. Figure I presents the evolution of the normalized area of the section in the ascending and descending aorta using the previously described True-FISP sequence.

The software was successfully tested on various MR sources, including General Electric, Siemens and Philips I.5 T and $3 \mathrm{~T}$ scanners, thus making possible the processing of multi vendor acquisitions in multi centric studies.

\section{PI83}

Pulmonary blood density in systemic sclerosis - a novel non-invasive measure of pulmonary arterial hypertension?

Mikael Kanski', Håkan Arheden', Roger Hesselstrand ${ }^{2}$ and Martin Ugander'

'Department for Clinical Physiology, Lund, Sweden

${ }^{2}$ Department for Rheumatology, Lund, Sweden

Journal of Cardiovascular Magnetic Resonance 2009, I I(SuppI I):PI83

Introduction: Patients suffering from systemic sclerosis (SSc) have a highly increased risk of developing pulmonary arterial hypertension (PAH). An early detection of PAH is important in order to halt the progress of disease. Currently, the assessment of pulmonary pressures lacks reliable quantitative non-invasive measures.

Purpose: The aim of this study was therefore to use magnetic resonance imaging (MRI) to measure the changes in pulmonary blood volume (PBV), the PBV variation (PBVV) throughout the cardiac cycle, and the pulmonary blood density (PBD) in earlystage SSc and to make a preliminary comparison to healthy individuals. 
Methods: Thirty-nine SSc patients (25 women and 14 men, $3|-8|$ years, mean 58 years) and healthy patients and healthy volunteers (three women and 10 men, $21-45$ years, mean 26) underwent cardiac MRI. PBV was calculated as the product of cardiac output determined by velocity encoded MRI, and the pulmonary transit time (PTT) determined as the time for a $2 \mathrm{ml}$ intravenously administered contrast bolus to pass from the pulmonary trunk to the left atrium. The lung volume was determined by planimetry using transversal MR images covering the lungs. The PBD was defined as the PBV divided by the lung volume. Also, the blood flow in the pulmonary artery and the pulmonary veins was measured using velocity encoded MRI. The PBVV was calculated by integration of the difference in arterial and venous pulmonary flow over the cardiac. PBV and PBD was measured in three patients (all men, 24, 34, and 45 years, respectively) who showed to be healthy. In 10 healthy volunteers (three women and 7 men, 2I-30 years, mean 24 years), the PBVV was assessed. The SSc patients were then compared to the healthy subjects considering PBV and PBD, and PBVV, respectively.

Results: Stroke volume, PBV and PBD in 36 SSc patients and three healthy subjects were respectively (mean \pm SD) $77 \pm 20 \mathrm{ml}$ vs $94 \pm \mathrm{ll} \mathrm{ml}, 467 \pm \mathrm{Ill} \mathrm{ml}$ vs $552 \pm 99 \mathrm{ml}$, and $17 \pm 5 \%$ vs $19 \pm 6 \%$. PBVV in 32 SSc patients and 10 healthy individuals were respectively $3 \mathrm{l} \pm 9 \mathrm{ml}$ vs $45 \pm \mathrm{l} 4 \mathrm{ml}$.

Conclusion: This study is the first to show the feasibility to assess the PBD using MRI. Preliminary data shows no difference between newly diagnosed SSc patients and healthy individuals considering PBD. The PBD may be a useful prognostic noninvasive measure of $\mathrm{PAH}$ and cardiac failure. Therefore, further studies are needed to reveal the importance of the PBD in these contexts.

\section{P I 84}

4D flow evaluation of abnormal flow patterns with bicuspid aortic valve

Michael D Hope', Alison K Meadows', Thomas A Hope', Karen G Ordovas', David Saloner', Gautham P Reddy', Marcus T Alley ${ }^{3}$ and Charles B Higgins ${ }^{\prime}$

'UCSF, San Francisco, CA, USA

${ }^{2}$ University of Washington, Seattle, WA, USA

${ }^{3}$ Stanford, Palo Alto, CA, USA

Journal of Cardiovascular Magnetic Resonance 2009, I I(SuppI I):PI84

Introduction: Bicuspid aortic valve (BAV) is the most common congenital heart defect and may account for more morbidity and mortality than all other congenital cardiac malformations combined. Two theories are commonly discussed for the elevated risk of aortic aneurysm and dissection in patients with BAV: I) an increased hemodynamic load placed on the proximal aorta results in progressive dilatation and 2) a genetic or developmental abnormality in the proximal aortic tissue leads to weakness of the aortic wall.

Purpose: This study utilizes 4D Flow to collect multidirectional blood flow velocity data in the thoracic aorta of patients with BAV. The goal of the study is to characterize altered flow patterns in these patients and uncover potential hemodynamic contributors to aneurysm and dissection formation.

Methods: Time-resolved, 3D phase contrast MRI (4D Flow) was employed to assess thoracic aortic blood flow in 12 individuals: 8 patients with bicuspid aortic valve and 4 healthy
Figure I (abstract PI84)

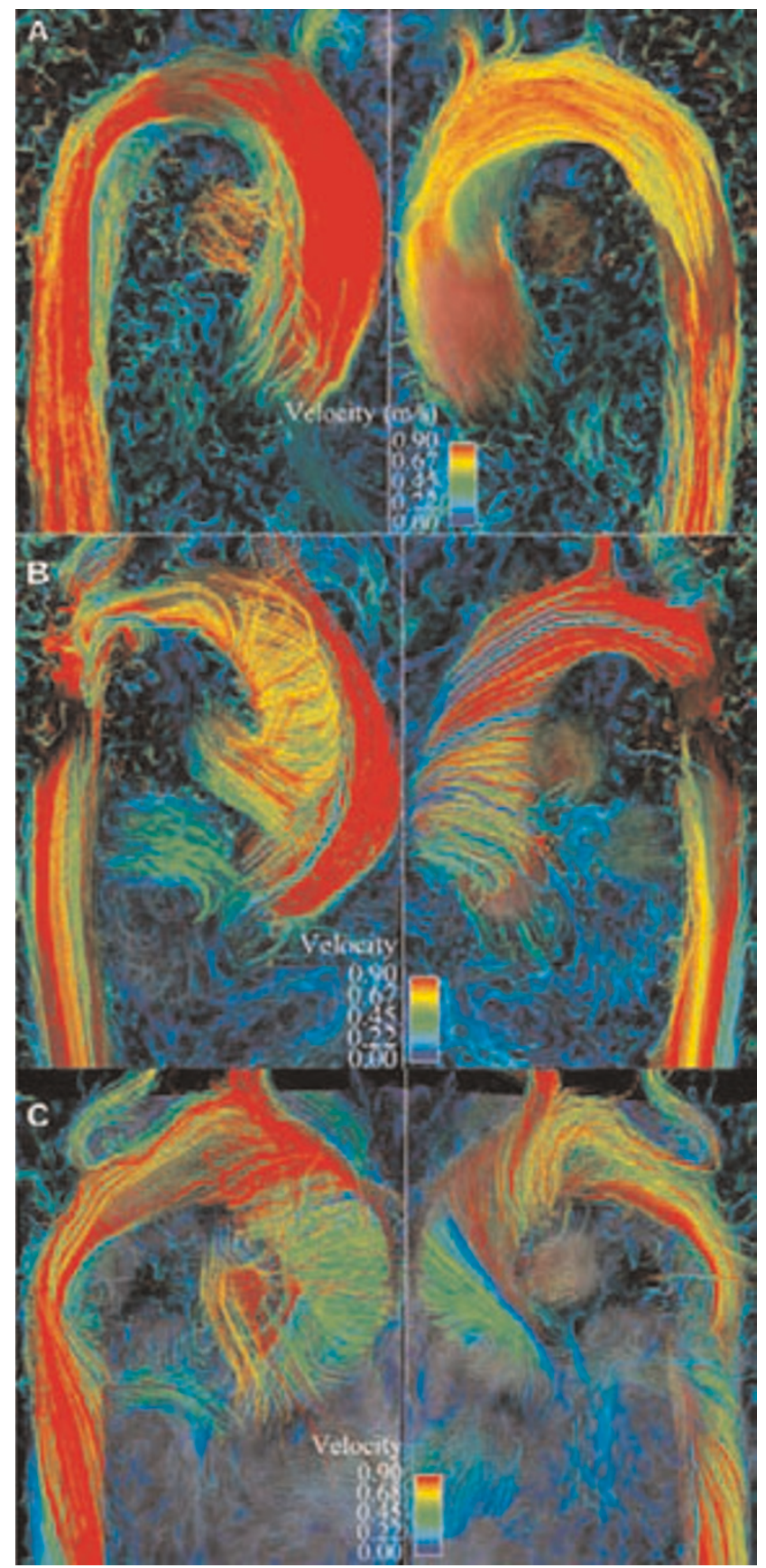

subjects. The technique, which has been previously validated, was performed with parallel imaging after the standard clinical MR evaluation.

Results: 4D Flow evaluation of the ascending thoracic aorta revealed markedly abnormal systolic helical flow in 6 of 8 patients with bicuspid aortic valve. Five of these patients demonstrated an eccentric right anterior systolic jet and right-handed helical flow; at least two of these patients had fusion of the right and left coronary cusps, and three had aneurysms of the ascending aorta along with aortic stenosis and/or regurgitation. The other patient 
Figure 2 (abstract PI84)

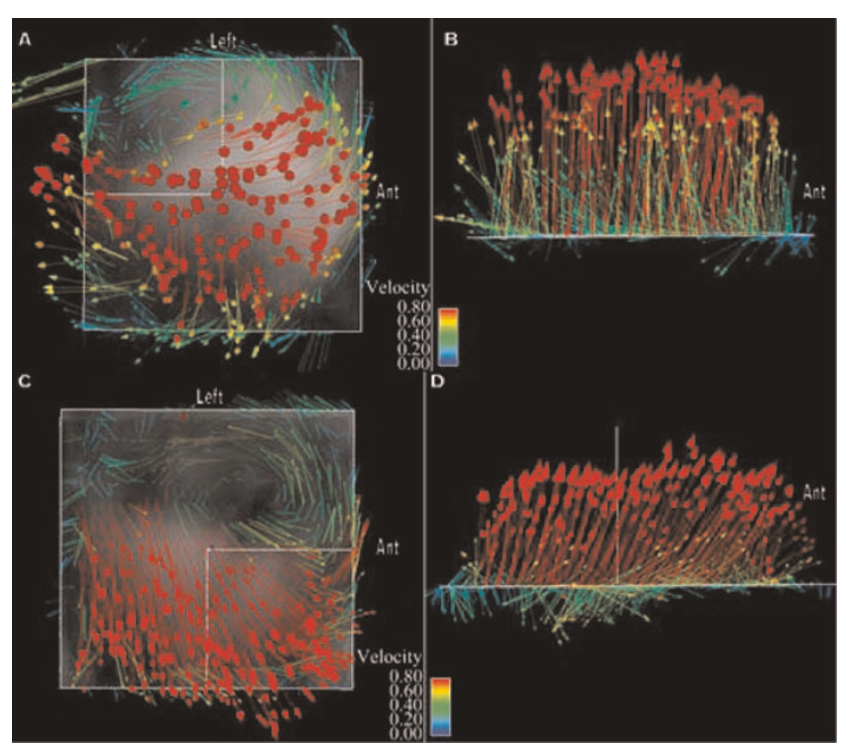

had a left posterior jet with left-handed helical flow, dilation of the aortic root and fusion of the right and noncoronary cusps. The two BAV patients with normal systolic flow in the thoracic aorta had central flow jets. Normal and abnormal systolic flow patterns are included as Figure I: I a demonstrates normal flow with streamlines mapped onto a sagittal oblique plane viewed from right and left orientations respectively, Ib shows an eccentric jet resulting in marked right-handed helical flow in a patient with BAV and aortic coarctation but without aneurysm, Ic shows left-handed helical flow in a BAV patient with fusion of the right and noncoronary cusps. Evaluation of aortic flow jets in BAV patients and healthy subjects is included as Figures 2 and 3:

\section{Figure 3 (abstract P I84)}

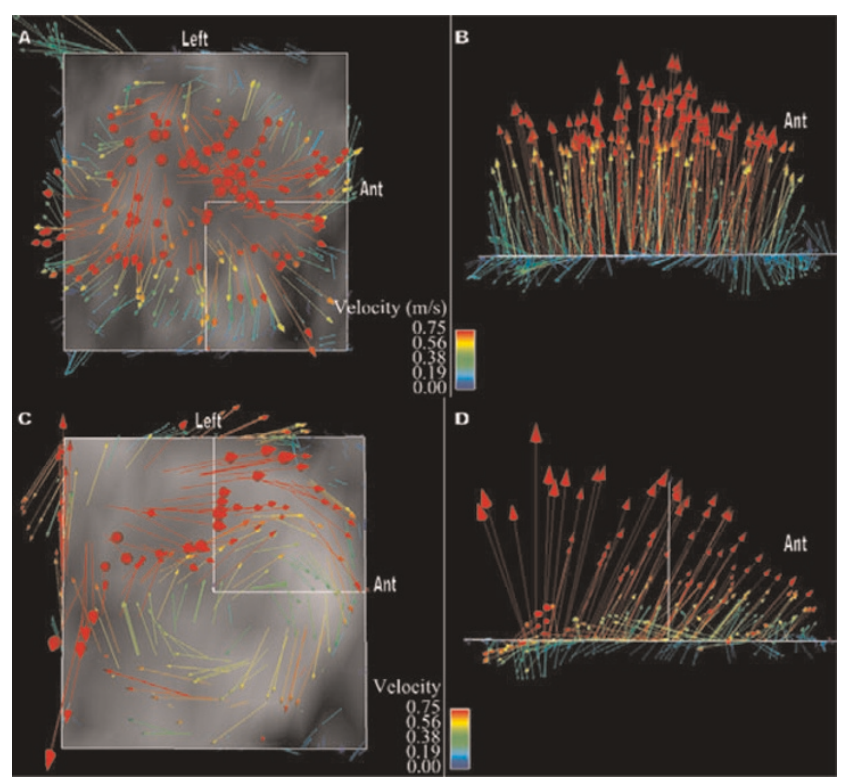

$\mathbf{2} \mathbf{a}$ and $\mathbf{b}$ represent systolic vectors from a plane orthogonal to the aorta just above the sinotubular junction in a healthy subject compared to $\mathbf{2 c}$ and $\mathbf{d}$ which demonstrate an eccentric, right anterior flow jet in a patient with fusion of the right and left coronary cusps; $\mathbf{3} \mathbf{a}$ and $\mathbf{b}$ show normal flow in a second healthy subject compared to $\mathbf{3 c}$ and $\mathbf{d}$ which show a left posterior flow jet in a patient with fusion of the right and noncoronary cusps.

Conclusion: Abnormal systolic helical flow is seen in the ascending thoracic aorta of patients with bicuspid aortic valve. Similar helical flow has been described in ascending aortic aneurysms associated with BAV, but we have demonstrated this flow pattern in two patients without aneurysm, suggesting that the pattern is not secondary to the dilated aorta, but may be implicated in the pathogenesis of aneurysm formation. The marked helical flow in the ascending aorta appears to be associated with eccentric flow jets in all 6 of our cases. In the single case of left-handed helical flow, fusion of the right and noncoronary cusps was found, a geometric configuration that may create a left posterior jet as we have demonstrated (Figure 3). Identification and characterization of eccentric flow jets in patients with BAV may help risk stratify for development of ascending aortic aneurysm and dissection.

\section{PI85}

Off-resonant pulmonary vein imaging

Peng Hu, Dana C Peters, Christian Stoeck,

Kraig V Kissinger, Beth Goddu, Lois Goepfert,

Warren J Manning and Reza Nezafat

Beth Israel Deaconess Medical Center, Boston, MA, USA

Journal of Cardiovascular Magnetic Resonance 2009, I I(SuppI I):PI85

Introduction: Non-contrast enhanced pulmonary vein (PV) MRA is a potential alternative to contrast enhanced methods for pre-procedural planning and post-procedural evaluation of pulmonary vein isolation (PVI) in treatment of atrial fibrillation (AF). In 2007, $12 \%$ of the AF patients referred to pulmonary

Figure I (abstract PI85)

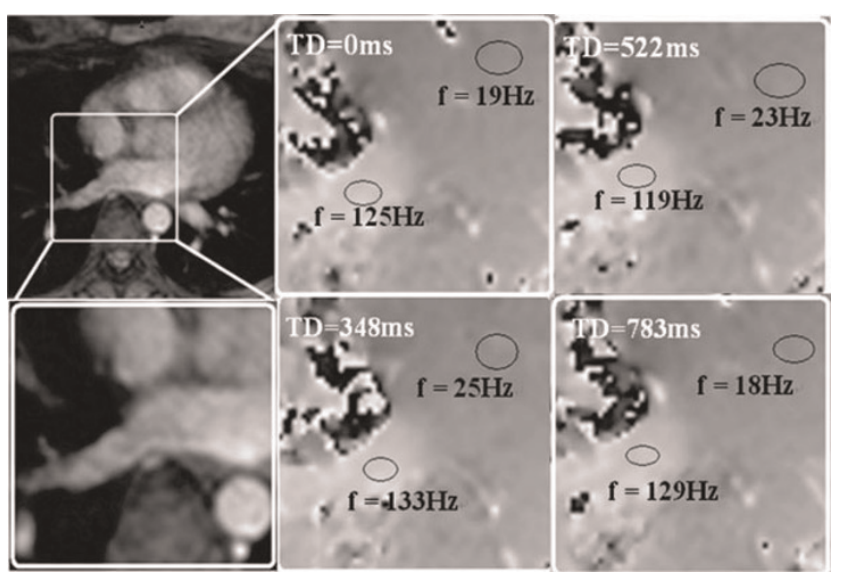

An example of right inferior PV frequency shift in a healthy subject through the cardiac cycle. PV images (left columns) and the corresponding off-resonance map (middle and right columns) at the same imaging plane are shown. The off-resonance at proximal PV and atrial blood are measured. TD = ECG trigger delay. 
Figure 2 (abstract PI85)

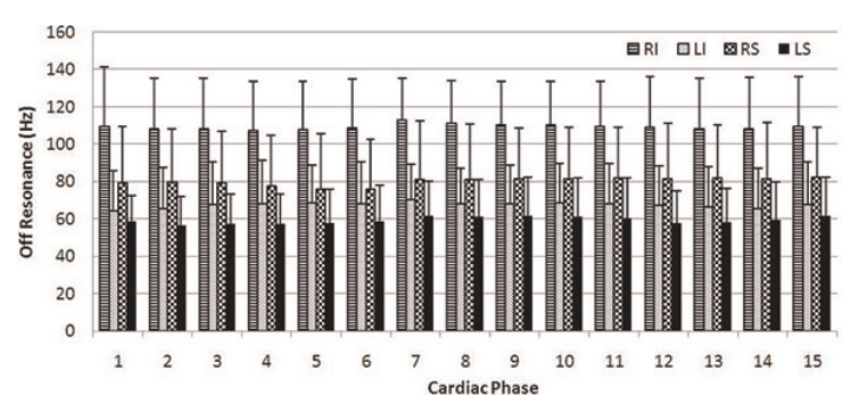

Variaions of PV off-resonance frequencies between subjects, PV branches and cardiac phase. $\mathrm{RI}=$ right inferior; $\mathrm{LI}=$ left inferior; $\mathrm{RS}=$ right superior; LS = left superior.

MRA in our center have impaired renal function. A major problem with non-contrast $\mathrm{PV}$ imaging is lack of contrast between PV and other great vessels (e.g. pulmonary artery) and cardiac chambers. Because the PV is in close proximity to the lungs, the PV blood could potentially experience significant offresonance due to susceptibility effects. This off-resonance could be used as a source of contrast to differentiate PV from other anatomical features [I].

Purpose: We sought to investigate variations of off-resonance frequency through the cardiac cycle for each PV branch and to investigate the use of off-resonance in PV SSFP imaging.

Methods: PV images were acquired on healthy volunteers in this study. We used a dual echo sequence based on gradient recalled echo (GRE) to measure the field map of the PV and the left atrium in cine mode. The off-resonance of a PV branch was measured by defining a region of interest (ROI) in the proximal $\mathrm{PV}$ of the generated field maps and calculating the mean offresonance frequency in the ROI. A 3D ECG gated free-breathing SSFP sequence with a navigator echo, which has been used in coronary imaging, was adapted to PV imaging. Balanced SSFP has a well-known signal profile modulated by frequency shift. By applying a linearly increasing radiofrequency (RF) excitation

Figure 3 (abstract PI85)

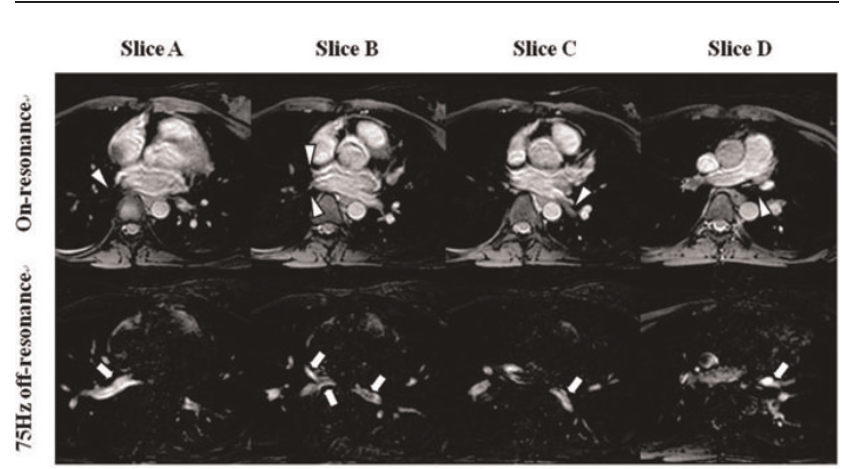

Example images from a healthy subject using on-resonance (top) and 75 $\mathrm{Hz}$ off-resonance (bottom) SSFP sequences. Slices A, B, C and D are part of a 3D data set. PV branches are highlighted in off-resonance SSFP (arrows) whereas they are suppressed in on-resonance SSFP (arrow heads). phase from TR to TR, we shift the frequency response of SSFP signal so that the off-resonant PV blood signal is enhanced.

Results: Figure I shows an example of off-resonance measurements in the right inferior PV from a healthy adult subject through the cardiac cycle. Figure 2 shows the variations of offresonance between different PV branches and different time in the cardiac cycle based on data from a cohort of 6 healthy subjects. On all six subjects, we observed the largest frequency shift in the right inferior PV among all branches. The mean frequency shifts for all PV branches stay within a narrow range of $10 \mathrm{~Hz}$ through the cardiac cycle. Figure 3 shows an example to use the frequency shift as a source of contrast for the PV's. Signal enhancement can be observed in all four major PV branches.

Conclusion: PV blood exhibits a mean off-resonance of $58 \sim 113 \mathrm{~Hz}$, with the right inferior PV having the largest frequency shift. We observed minimal RR variation in frequency off-set. By shifting the frequency response of SSFP sequence, we enhance the PV blood signal compared to on-resonance conditions. A combination of both on- and off-resonance SSFP acquisitions is a potential method to obtain coverage for both left atrium and PV.

Reference

I. Nezafat, et al: ISMRM 07.

\section{PI86}

Early prediction of infarct size by quantitative myocardial blush grade in patients with acute non-st-elevation and ST-elevation myocardial infarction treated with primary angioplasty and stent placement

Nina Riedle', Hartmut Dickhaus², Markus Erbacher², Henning Steen', Martin Andrassy', Dirk Lossnitzer', Stefan Hardt', Wolfgang Rottbauer', Christian Zugck', Hugo A Katus', Evangelos Giannitsis' and Grigorios Korosoglou'

'Department of Cardiology, University of Heidelberg, Heidelberg, Germany

${ }^{2}$ Department of Medical Informatics, University of Heidelberg, Heidelberg, Germany

Journal of Cardiovascular Magnetic Resonance 2009, I I(SuppI I):PI86

Introduction: The widespread use of percutaneous coronary interventions has resulted in a significant improvement of clinical outcomes in patients both with ST-elevation and in non-STelevation myocardial infarction (STEMI and NSTEMI). The restoration of epicardial artery blood flow in the revascularized coronary artery however, may not necessarily guarantee preserved microvascular integrity in the downstream myocardium. Because the latter is a principal predictor of clinical outcomes, methods that can provide objective assessment of infarct size early in acute infarction may be of great potential clinical utility.

Purpose: To determine whether quantification of myocardial blush grade (MBG) can aid the determination of infarct size during cardiac catheterization in patients with acute myocardial infarction.

Methods: We prospectively examined patients with first STEMI $(n=45)$ and NSTEMI $(n=50)$, all treated with primary angioplasty and stent placement. ECG-gated angiographic series were used to quantify MBG, by placing regions of interest (ROI) 


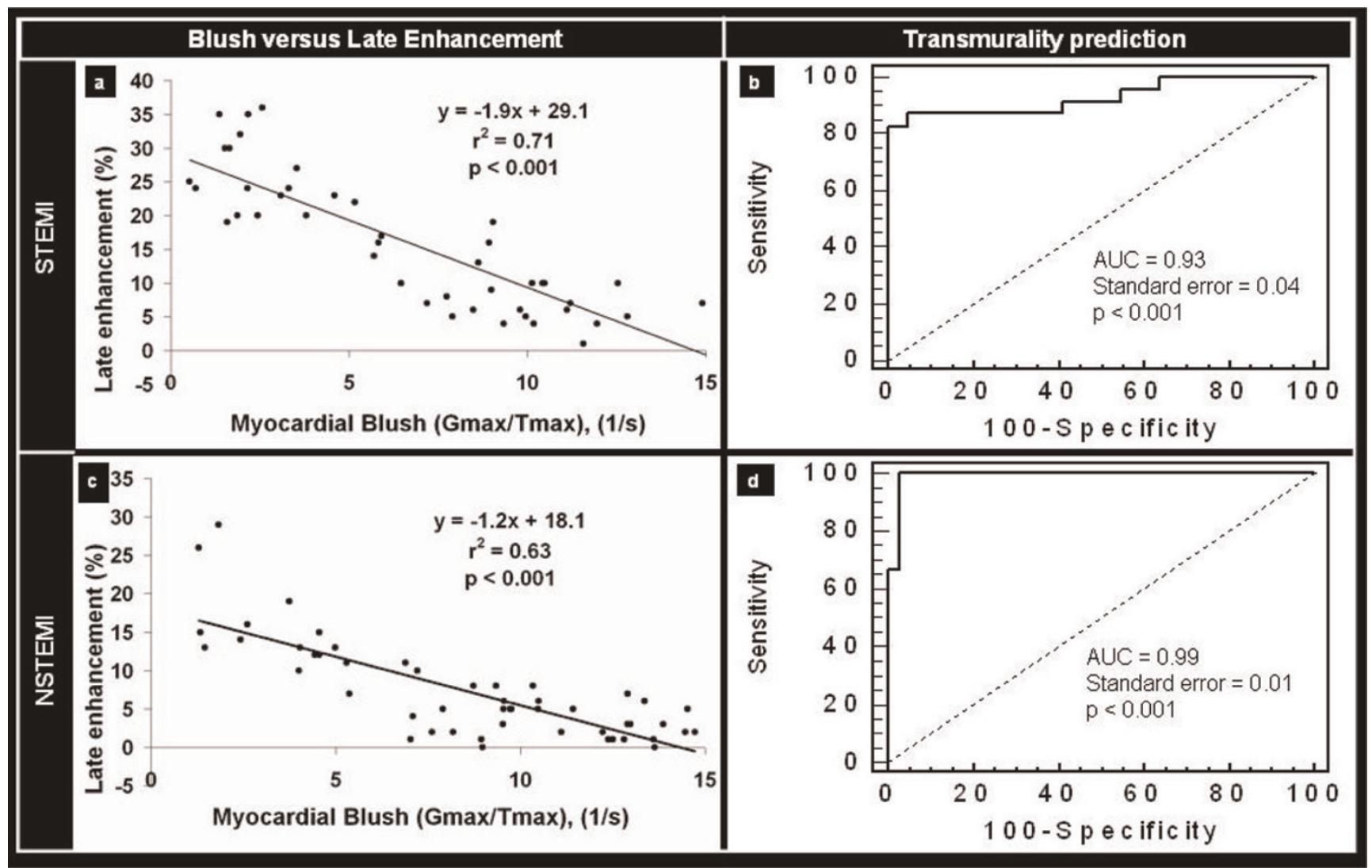

in the infarct related coronary territory, in order to estimate the time course of blush intensity rise. $G_{\max }$ was defined as the peak grey level intensity and $T_{\max }$ as the time to peak intensity rise. By this approach, we anticipated that an adequate and prompt filling of myocardial capillaries with contrast agent (high $\mathrm{G}_{\max }$ within a

Figure 2 (abstract P I86)

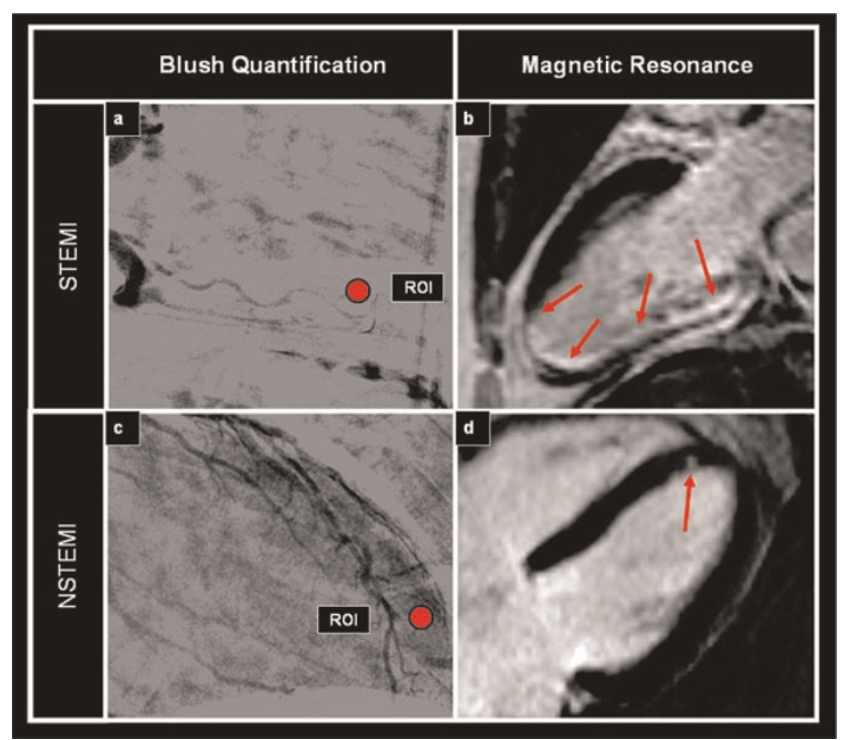

short $T_{\max }$ ) would be indicative of preserved microvascular integrity and predictive of small infarct size and vice versa. Assessment of myocardial scar, determined by contrastenhanced magnetic resonance imaging (MRI), 2 to 4 days after infarction, deemed as the standard reference for estimation of infarct size. Briefly, 10 minutes after $0.2 \mathrm{mmol} / \mathrm{kg}$ body weight gadolinium contrast injection, 2 volume stacks of inversionrecovery gradient-echo images covering the whole left ventricle were generated. Infarct size was calculated as the amount of hyperenhanced myocardium related to the total LV-mass. Infarct transmurality was assessed visually based on a 5 -grade scale (i.e. $\mathrm{I}=0-25 \%, 2=25-50 \%, 3=50-75 \%, 4 / 5=75-100 \%$ transmurality without/with microvascular obstruction).

Results: Patients with STEMI showed larger infarct size compared to patients with NSTEMI, both by cardiac enzymes (peak troponin T of $7.3 \pm 6.8 \mu \mathrm{g} / \mathrm{l}$ for STEMI vs. $2.3 \pm 2.5 \mu \mathrm{g} / \mathrm{l}$ for NSTEMI, $\mathrm{p}<0.0 \mathrm{I}$ ) and by delayed enhancement on MR-images (I5.7 $\pm 9.9 \%$ for STEMI vs. $7.5 \pm 6.7 \%$ for NSTEMI, $p<0.0 \mathrm{I}$ ). The ratio $G_{\max } / T_{\max }$ showed a significant inverse linear correlation with infarct size both in patients with STEMI and in those with NSTEMI $\left(r^{2}=0.71\right.$ vs. $\left.r^{2}=0.63, p<0.001\right)$, (Figure la,c). Furthermore, $\mathrm{G}_{\max } / \mathrm{T}_{\max }$ was significantly related with infarct transmurality $\left(\chi^{2}=42.7\right.$ for STEMI and $\chi^{2}=51.7$ for NSTEMI, respectively, $p<0.00 \mathrm{I})$ and cut-off values of $G_{\max } / T_{\max }=5.9 / \mathrm{s}$ and $5.0 / \mathrm{s}$, respectively were highly predictive for the presence of infarct transmurality $=75 \%$ in both groups $(A \cup C=0.93,95 \%$ $\mathrm{Cl}=0.8 \mathrm{I}-0.98$ for STEMI and $\mathrm{AUC}=0.99,95 \% \mathrm{Cl}=0.9 \mathrm{I}-0.99$ for NSTEMI, respectively, $p<0.00 I$ ), (Figure Ib,d). MR- and 
angiographic findings of a large transmural inferior wall STEMI with reduced MBG (Figure 2a,b) and of a small subendocardial anterior wall NSTEMI (Figure 2c,d) with higher MBG can be appreciated in corresponding figures.

Conclusion: Quantitative MBG is a valuable predictor of the total extent of myocardial infarction and infarct transmurality. This information can be easily acquired during clinically indicated cardiac catheterization, immediately after myocardial reperfusion, and can be utilized for tailoring appropriate pharmacological interventions and to support the early risk stratification of patients with acute ischemic syndromes.

\section{PI87}

Additional value of first pass magnetic resonance myocardial perfusion imaging to computed tomography coronary angiography for detection of significant coronary artery disease

Jan GJ Groothuis, Aernout M Beek, Stijn L Brinckman, Martijn R Meijerink, Simon C Koestner, Marco JW Götte, Mark BM Hofman and Albert $C$ van Rossum

VU Medical Center, Amsterdam, Netherlands

Journal of Cardiovascular Magnetic Resonance 2009, I I(SuppI I):PI87

Introduction: As computed tomography coronary angiography (CTCA) has a reported excellent negative predictive value for detection of significant coronary artery disease (CAD), it is increasingly used as first line technique in the evaluation of patients with suspected CAD. However, positive predictive value is low and CTCA lacks information about myocardial perfusion. As first pass magnetic resonance myocardial perfusion imaging (MRMPI) can accurately assess myocardial perfusion and does not involve ionizing radiation, it may be a valuable additional technique to CTCA in the evaluation of patients with suspected CAD. Subsequently, their combined use may lower the number of unnecessary, costly invasive coronary angiographies (CAG).

Purpose: The additional value of MRMPI to CTCA for detection of significant CAD was investigated using invasive coronary angiography as the standard of reference.

Methods: Patients with chest pain and intermediate pre-test probability CAD underwent both 64-slice CTCA (Sensation, Siemens, Erlangen) and adenosine stress and rest first pass MRMPI (I,5 Tesla MR scanner, Siemens, Erlangen). CTCA was scored per segment as: normal; non-obstructive CAD (0-50\% diameter stenosis) and abnormal ( $>50 \%$ stenosis). MRMPI was analyzed qualitatively and was assessed as abnormal in case of any segment with a perfusion defect. In case of abnormal CTCA and/ or abnormal MRMPI, CAG was performed. Significant CAD was defined as $>70 \%$ diameter stenosis on CAG.

\section{Figure I (abstract P I87)}

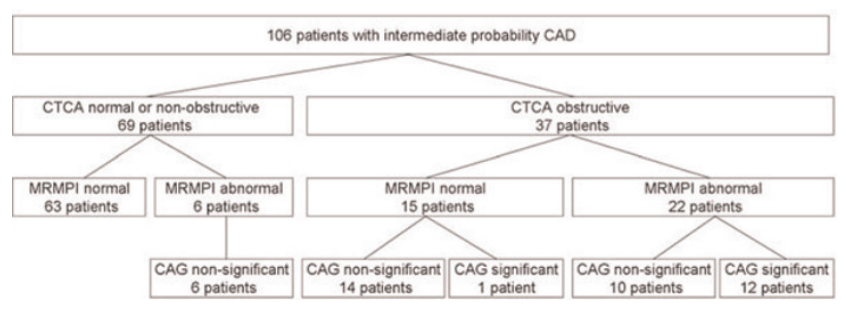

Results: A total of 106 patients (mean age $57 \pm 10 ; 53$ males) underwent both CTCA and MRMPI within 2 weeks. 43 Patients underwent CAG. Of 69 patients with normal or non-obstructive CTCA, 6 patients had abnormal MRMPI. None of these patients had significant CAD on CAG. Of 37 patients with abnormal CTCA, 13 patients had significant CAD on CAG (positive predictive value $35 \%$ ). Of 37 patients with abnormal CTCA, I 5 (4I\%) patients had normal MRMPI. In 14 of these I 5 patients absence of significant CAD was confirmed by CAG. Figure I.

Conclusion: By using MRMPI as additional technique in case of abnormal CTCA findings, significant CAD could be ruled out in I 4 of 37 (38\%) patients with abnormal CTCA. In case of normal CTCA however, the additional value of MRMPI for detection of significant $C A D$ is low.

\section{PI88}

Cardiovascular magnetic resonance imaging as a gold standard for diagnosis of true aborted myocardial infarction

Ingo Eitel, Georg Fuernau, Josef Friedenberger, Mahdi Sareban, Gerhard Schuler, Matthias Gutberlet and Holger Thiele

Heart Center Leipzig, Leipzig, Germany

Journal of Cardiovascular Magnetic Resonance 2009, I I(SuppI I):PI88

Introduction: For detection and quantification of myocardial infarction, delayed enhancement cardiovascular magnetic resonance imaging (CMR) has emerged as the new gold standard. Furthermore, CMR has the unique advantage of visualization of even microinfarctions at very high spatial resolution.

Some patients who receive prompt reperfusion do not have a significant enzyme rise, but do exhibit typical ECG changes, which are consistent with an aborted myocardial infarction (MI). These patients are presumed that myocardial necrosis has been avoided. However, this has not been studied systematically with CMR.

Purpose: The aim of this research was therefore to study CMR-parameters for the identification of aborted MI.

Methods: To investigate the extent of aborted MI we examined 421 consecutive patients undergoing primary percutaneous coronary intervention (PCl) in acute STEMI within $12 \mathrm{~h}$ after symptom onset. Aborted $\mathrm{Ml}$ was defined as maximal creatine kinase $\geq 2$ upper limit of normal coupled with typical evolutionary electrocardiographic changes (ST-segment resolution > 50\% within $2 \mathrm{~h}$ ). All patients with aborted Ml underwent CMR within 2-4 days using a I.5 T MRI scanner. Left ventricular function was assessed by a standard steady-state free precession technique. For acute infarct determination, 3 short-axis slices using a T-2 weighted turbo spin-echo sequence were obtained. Early and delayed enhancement images covering the whole ventricle were acquired $\approx I$ and 15 minutes after intravenous administration of $0.15 \mathrm{mmol} / \mathrm{kg}$ body weighted gadobutrol (Gadovist, Schering, Germany) with an inversion recovery gradient echo sequence.

Results: Of the 42 I STEMI patients 53 patients (12.6\%) fulfilled aborted MI criteria, with the highest frequency $(22.4 \%)$ occuring in patients treated with primary $\mathrm{PCl}<2 \mathrm{~h}$ after symptom onset. In patients with aborted MI, CMR detected no delayed enhancement (DE) in 22 patients (4I.5\%), consistent with the absence of myocardial necrosis and infarction. In 31 (58.5\%) patients CMR revealed transmural $(27.3 \%)$ or non-transmural 
(3I.2\%) DE in the distribution of a coronary artery compatible with Ml. As compared with true Ml patients, patients with aborted $\mathrm{Ml}$ had a significant lower infarct size $(30.9 \pm 19.6 \mathrm{ml}$ vs. $8.5 \pm 12.3 \mathrm{ml} ; 22.2 \pm 12.7 \%$ vs. $5.9 \pm 7.1 \%$; $p 0.00 \mathrm{I}$ respectively) shorter pain-to-balloon time and a significant better left ventricular ejection fraction ( $46 \%$ vs. $60 \%, \mathrm{p}<0.00 \mathrm{I})$.

Conclusion: CMR can distinguish between patients with true aborted MI with absence of myocardial scar and patients with fulfilled criteria of aborted MI but detected scar formation. Therefore CMR should be established as a diagnostic criteria for true aborted MI.

\section{PI 89}

TI nulling and contrast conditions with a $0.15 \mathrm{mmol} / \mathrm{kg}$ dosage of MultiHance as compared to the standard $0.2 \mathrm{mmol} / \mathrm{kg}$ gadolinium dosage in patients with myocardial infarcts June A Yamrozik, Mark Doyle, Vikas K Rathi, Diane A Vido, Ronald B Williams, Geetha Rayarao

and Robert WW Biederman

Allegheny General Hospital, The Gerald McGinnis

Cardiovascular Institute, Pittsburgh, PA, USA

Journal of Cardiovascular Magnetic Resonance 2009, I I(SuppI I):PI89

Introduction: It is known that utilizing the proper inversion time ( $\mathrm{TI})$ is essential when diagnosing myocardial infarction. Many factors play a role in acquiring optimal nulling of the myocardium. Recently, higher relaxivity contrast agents have been advocated for use in infarct imaging. Specifically, MultiHance ${ }^{\circledR}$ (gadobenate dimeglumine) has higher relaxivities $\left(r_{1}\right.$ and $r_{2}, 9.7^{\prime}$ and $\left.12.5^{\prime}\right)$ compared to standard gadolinium (gadopentetate dimeglumine) $\left(r_{1}\right.$ and $r_{2}, 4.9^{\prime}$ and $\left.6.3^{\prime}\right)$. Will this higher relaxivity allow a lower dosage of contrast to be used to maintain a comparable TI null time compared to the conventional gadolinium dosage and how does it affect contrast?

Purpose: We hypothesize that a $0.15 \mathrm{mmol} / \mathrm{kg}$ dosage of MultiHance with its higher relaxivity will produce a lower TI null time in infarct patients compared to patients imaged with a standard dosage of $0.2 \mathrm{mmol} / \mathrm{kg}$ of gadolinium at 10 and 20 minutes post-contrast.

Methods: A total of 52 patients with GFR $>60 \mathrm{~mL} / \mathrm{min} / 1.73 \mathrm{~m}^{2}$ were imaged.Twenty-six (26) patients (I9 M, $6 \mathrm{~F}$ ), age 42-8I years, post-myocardial infarction underwent a standard cardiac MRI (CMR), utilizing a total of $0.2 \mathrm{mmol} / \mathrm{kg}$ gadolinium dosage (Magnevist-Berlex, New Jersey, USA). This was administered as a $0.5 \mathrm{mmol} / \mathrm{kg}$ for perfusion imaging, followed by an additional $0.15 \mathrm{mmol} / \mathrm{kg}$ for viability (DHE) imaging. Twenty-six (26) patients (2I M, $5 \mathrm{~F}$ ), age 43-83 years, post-myocardial infarction underwent a standard cardiac MRI (CMR), utilizing a total of $0.15 \mathrm{mmol} / \mathrm{kg}$ MultiHance dosage (Bracco Diagnostics, Princeton, $\mathrm{N} \mathrm{J}$, USA). This was administered as a $0.5 \mathrm{mmol} / \mathrm{kg}$ dosage for perfusion imaging, followed by an additional $0.10 \mathrm{mmol} / \mathrm{kg}$ for viability imaging. The scans were acquired on a GE CV/i Excite Version 12, I.5 T system (GE, Milwaukee, WI). The sequence utilized for optimum myocardial nulling was a standard 2D Gradient Echo IRP (FGR with inversion recovery prep). Manual selection of $\mathrm{TI}$ was performed independent of contrast media. Contrast of infarct to myocardium and blood pool was measured in a subset of 14 patients ( 7 per group) with large infarcts. An 8-channel or 4-channel cardiac coil was used, again independent of the contrast agent. The sequence parameters were as follows:
TE: min, FA: 20, NEX: 2 trigger delay: adjusted to onset of diastole, I RR interval and TI adjusted to null the myocardium. This sequence was performed at 10 and 20 minutes postcontrast administration.

Results: All the patients successfully completed the CMR examination without difficulty, or impairment in infarct detection. Particular attention was focused on the contrast medium utilized, dosage and its effects on the $\mathrm{TI}$ at 10 and 20 minutes post-contrast. At 10 minutes post-contrast, the inversion time with Magnevist was $158.7 \pm$ 17.2, which was not different from that obtained with MultiHance $(154.3 \pm 13.6, P=0.3)$. At 20 minutes post-contrast, the TI with Magnevist was $200.4 \pm$ 28.8, and again was not different from that obtained with MultiHance $(193.0 \pm 15.6, p=0.3)$.

The signal intensity contrast between the infarct and myocardium at 10 minutes is greatest with the MultiHance a ratio of $(7.6 \pm 2.5)$ compared to $(4.9 \pm 1.2)$ with gadolinium.

Conclusion: While the relaxivity of MultiHance compared to standard gadolinium is almost a factor of two higher, similar contrast null times are achieved by using a $75 \%$ dosage of MultiHance as compared to gadolinium. Relaxivity alone does not predict arithmetic decreases in TI times. Despite this, MultiHance administration at the lower dose did result in a higher infarct-myocardium contrast. Further, the higher relaxivity agent is potentially beneficial in those situations when additional imaging might be necessary, since an additional $25 \%$ of contrast is held in reserve for further administration.

\section{PI90 \\ Does DHE imaging shed insight into the inaccuracy of EKGs? \\ Wadih Nadour and Robert W Biederman \\ Allegheny General Hospital, The Gerald McGinnis \\ Cardiovascular Institute, Pittsburgh, PA, USA}

Journal of Cardiovascular Magnetic Resonance 2009, I I(SuppI I):PI 90

Background: The presence of $Q$ wave on EKG remains in wide clinical use to identify people with prior myocardial infarct (MI), in particular transmural infarct. We hypothesized that standard assessment of infarct presence relying on EKG is both insensitive and inaccurate, especially as compared to CMR DHE assessment, regardless of the DHE pattern (ischemic versus nonischemic).

Objectives: The purpose of the study is to relate EKG defined scar ( $Q$ waves) as compared to late gadolinium enhancement (DHE) cardiovascular magnetic resonance (CMR) defined scar independent of the underlying pathology.

Methods: We reviewed a total of 106 unselected, consecutive patients who underwent CMR with DHE and had an EKG performed within I day of CMR between 2006 and 2008 at our institution. Non-ischemic, infiltrative and other cardiomyopathic patients were not excluded. EKGs performed on the same day or the day prior to the test were screened for the presence of a $Q$ wave and compared to those with a positive DHE regardless of the myocardial post-gadolinium pattern. Then we divided the patients into three subgroups: I) one group with only positive $\mathrm{DHE}(\mathrm{DHE}+)$ pattern of ischemia 2) the other group with other DHE (DHE+/-) patterns or 3) negative DHE (DHE-). Furthermore, we divided the ischemic group (DHE+) into transmural vs. endocardial post-gadolinium patterns. 
Results: While 58/106 (45\%) patients were either DHE+ orDHE+/-, Q waves were present in only 24/106 patients (22\%). However, of the DHE+ or $\mathrm{DHE}+/$ - patients, only 22 (20\%) had an ischemic DHE pattern, representing little overlap between presence of DHE+ and finding of $Q$ waves on EKG. The sensitivity and specificity of EKG to detect infarct were $63 \%$ and $90 \%$, respectively. The positive predictive value was $73 \%$ and negative predictive value was $85 \%$. In the subgroup of patients divided into transmural infarcts (15) and endocardial infarcts (7), the sensitivity was $60 \%$ and $57 \%$, respectively. However, of $10 / 24$ (4I\%) patients with Q waves on EKG, $50 \%$ were either DHE- (5/ 10) or $\mathrm{DHE}+/-(5 / 10)$. The latter group included 3 viral cardiomyopathy, I infiltrative disease and I hypertrophic cardiomyopathy.

Conclusion: EKG remains the most widely used method in clinical practice to identify patients with prior infarcts based on the presence of $Q$ waves; however it has a low sensitivity compared to CMR DHE defined scar. This sensitivity does not appreciably change with the presence of transmural versus subendocardial infarcts. Importantly, a significant number of $Q$ waves have no ischemic etiology, indicating a high percentage of false positive results by EKG.

\section{PI9I}

Evaluation by MRI of left ventricular remodeling and global functional recovery in patients treated with granulocyte-colony stimulating factor (G-CSF) after acute myocardial infarction (AMI) Antonio Bernardini', Luigi Natale ${ }^{2}$, Agostino Meduri', Carlo Liguori ${ }^{2}$, Antonio Maria Leone ${ }^{2}$ and Lorenzo Bonomo ${ }^{2}$

I'“G. Mazzini” Hospital - ASL Teramo, Teramo, Italy ${ }^{2}$ Università Cattolica del Sacro Cuore - Policlinico A. Gemelli, Rome, Italy

\section{Journal of Cardiovascular Magnetic Resonance 2009, I I(SuppI I):PI9I}

Purpose: To evaluate by MRI the effect of G-CSF on ventricular remodeling after AMI treated with PTCA.

Materials and methods: I4 patients with AMI (TIMI 3), 7 tretaed with G-CSF administration ( $5 \mathrm{mcg} / \mathrm{kg}$ sc for 5 days) studied by MRI (GE I.5 T) within 7 days and after 4-6 months to evaluate end systolic (ESV) and end diastolic (EDV) volumes and ejection ffraction (EF). B-SSFP sequences for functional study, FGRET forFirst Pass study (FP) and IR-prep FGRE for Delayed Enhancement (DE). 0,I mmol/Kg of Gd-DTPA were administered with a $3 \mathrm{ml} / \mathrm{sec}$ flow both for FP and DE (total dose $0,2 \mathrm{mmol} / \mathrm{kg}$ ). FP and DE were visually scored accounting for transmural and circumferential extent.

FP and DE correlation with functional recovery and differences between groups were evaluated by linear regression analysis and covariance analysis (ANCOVA).

Results: A statistically significant correlation between $D E$ and functional recovery at follow up is present (for ESV $p=0,05$; for $E F p=0,03$ ); there was no significant linear correlation with FP. ANCOVA showed a positive trend for patients treated with GCSF, for any infarct size, especially for EDV and ESV (mean G-CSF effect: EDV $-29,4 \mathrm{ml}, \mathrm{p}=0,045$; ESV $-28 \mathrm{ml} \mathrm{p}=0,06$ ).

Conclusion: MRI allows to correctly evaluate differences in functional recovery between different groups of patients, accounting for infarct size. We found a positive trend with partial statistical significance regarding global functional recovery in patients treated with G-CSF after AMI. These data need however to be confirmed in larger series, as other studies (REVIVAL2) did not show significant correlations.

\section{PI92}

Multi-contrast delayed enhancement imaging (MCDE): accuracy and reproducibility compared to conventional SSFP and delayed

\section{hyperenhancement imaging}

Kim A Connelly, Jay S Detsky, John J Graham, Gideon Paul, Ram Vijayaraghavan, Rhonda Walcarius, Graham A Wright and Alexander J Dick

Sunnybrook HSC, Toronto, ON, Canada

Journal of Cardiovascular Magnetic Resonance 2009, I I(SuppI I):PI 92

Introduction: Cardiac magnetic resonance (CMR) imaging is an important tool in the assessment of cardiac function and viability, with prognostic implications for patients with ischemic heart disease [ $\mathrm{I}]$. Multi-contrast delayed enhancement imaging (MCDE) allows myocardial viability and wall motion to be assessed simultaneously by producing cardiac-phase-resolved images at multiple inversion times [2].

Purpose: This study compared MCDE imaging to the conventional wall motion and viability CMR imaging for the evaluation of ejection fraction (EF), LV mass, LV end-diastolic volume (EDV) and infarct mass.

Methods: Forty-one patients with suspected myocardial infarction were studied. All patients underwent assessment of cardiac function (cine SSFP), followed by viability imaging ten minutes after administration of $0.2 \mathrm{mmol} / \mathrm{kg}$ Gd-DTPA using the inversion recovery gradient echo (IR-GRE) and MCDE sequences. The MCDE sequence uses a segmented SSFP acquisition following an inversion pulse; one inversion pulse is played out per heartbeat, and twenty images each at a different effective inversion time and cardiac phase are reconstructed. The position of the inversion pulse was placed just prior to diastole to produce infarct-

Figure I (abstract P |92)
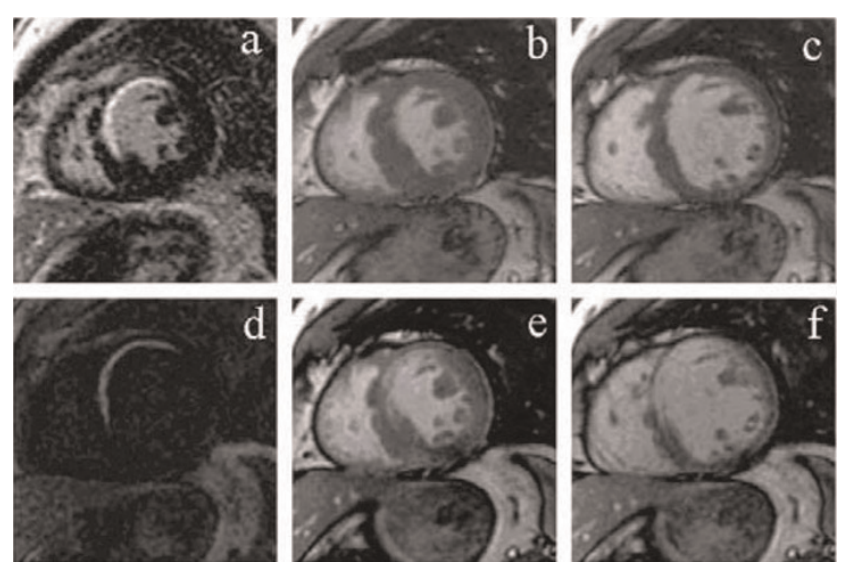

A comparison between (a) IR-GRE delayed enhancement image, (b) conventional SSFP end-systolic wall motion image, (c) conventional SSFP end-diastolic wall motion image with (d) MCDE infarct-enhanced image, (e) MCDE end-systolic image, and (f) MCDE end-diastolic image. Note that $(d-f)$ are three of the 20 MCDE images acquired during a single breath-hold. 
enhanced images in diastole and systolic images with normal SSFP contrast in systole. MCDE therefore produces wall motion images in systole and viability images in diastole using a single breath-hold (see Figure I).

Results: CMR imaging was well tolerated in all subjects. Images from two patients were excluded from the final analysis as an incorrect placement of the inversion pulse for the MCDE sequence prevented systolic frame identification. Myocardial infarct was detected on delayed enhancement images in 24 patients, with associated wall motion abnormalities and a reduced EF. MCDE, IRGRE, and SSFP imaging demonstrated excellent correlation for EF, LV infarct size, LV mass and LV EDV (all $r>0.9, p<0.001$ ). BlandAltman analysis demonstrated excellent agreement in the assessment of EF (bias $=-2 \%(95 \% \mathrm{Cl}:-8 \%$ to $4 \%)$ ), LV infarct size (bias $=0.2 \mathrm{~g}(-\mathrm{I} .5 \mathrm{~g}$ to $2.0 \mathrm{~g}))$ and $\mathrm{LV}$ mass (bias $=0.2 \mathrm{~g}(-\mathrm{I} 8 \mathrm{~g}$ to $18 \mathrm{~g})$ ). Agreement was clinically acceptable for LV EDV (bias = $-7 \mathrm{~mL}(-30 \mathrm{~mL}$ to $16 \mathrm{~mL})$ ). Inter and intra-observer variability was low between SSFP / IR-GRE and MCDE imaging.

Conclusion: MCDE demonstrated excellent clinical agreement with conventional SSFP and IR-GRE imaging in the assessment of cardiac function and viability. MCDE provides wall motion and viability information during a single breath-hold with inherent spatial registration, as opposed to the cine SSFP and IR-GRE acquisitions which require separate breath-holds. MCDE imaging may be considered an alternative to conventional imaging.

References

I. Wong M: JACC 2004, 43.

2. Detsky JS: MRM 2007, 58.

\section{PI93}

Co-registration of CTA coronary artery/vein maps and MR myocardial viability/scar maps for optimized revascularization or resynchronization therapy planning

Thomas P O'Donnell', Engin Dikici ${ }^{2}$, Teresa Swenson ${ }^{2}$, Jean Shaffer ${ }^{2}$ and Richard D White ${ }^{2}$

ISiemens Corporate Research, Princeton, NJ, USA

${ }^{2}$ University of Florida College of Medicine, Jacksonville, FL, USA

Journal of Cardiovascular Magnetic Resonance 2009, I I(SuppI I):PI 93

For optimized therapeutic yield from revascularization in patients with coronary artery/ischemic heart disease, establishment of the direct anatomic relationship between coronary arterial branches and segments of left ventricular (LV) myocardium under consideration would be helpful; intervention on a branch supplying nonviable myocardium could be better avoided. Similarly, in ischemic heart failure patients being considered for cardiac resynchronization therapy, lead placement into a coronary vein tributary overlying scarred myocardium could be better avoided with prior demonstration of the relationship between coronary venous anatomy and myocardial scar distribution. Currently, the most robust modalities for noninvasive coronary artery/vein mapping and myocardial viability/scar mapping are CT angiography (CTA) and MR, respectively. We report a methodology involving the segmentation of coronary vessels and the co-registration of CTA coronary artery and/or vein maps with MR myocardial viability or scar maps.

Analysis is composed of three parts: (I) segmentation of the vessels in CTA, (2) segmentation of the myocardial scar in the DEMR, and the (3) co-registration of the two modalities.
Figure I (abstract P I93)

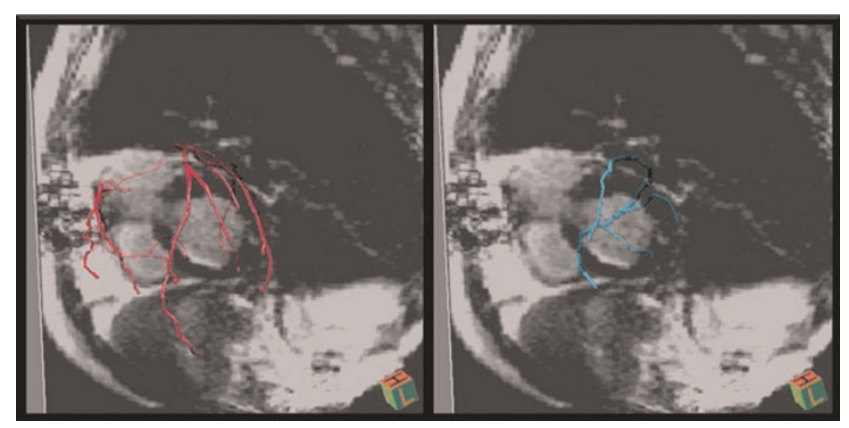

(I) To segment the vessels we employ a novel semi-automatic technique. Firstly, high radial symmetry positions (HSPs) in the image, which include the vascular structures' centerlines and the lobe shaped structures' centerpoints, are detected by vote-based Hough-like approach. Secondly, shape tensors for each of these HSPs are calculated via Principal Component Analysis. As the tensors expose local shape properties, HSPs that do not have vessel like structures are filtered out. Then, based on these final HSPs and their shape tensors, a graph is formulated where each HSP defines a unique graph node, and node to node connection strengths are calculated based on the shape tensor similarity metric. Next, the user is asked to identify 2 seed positions, for artery and vein respectively (the only manual part of the algorithm). Finally, the graph is partitioned into artery and vein regions based on these seed points by max-flow/minimal cut algorithm.

(2) To segment the myocardial scar, prototype software, VPT (Siemens Healthcare), was employed. After automatically segmenting and subsequently editing the myocardial borders, a threshold was computed based on the mean plus 2 standard deviations of a manually selected region of remote myocardium. A 3D polygonal model was then created by interpolating the stack of 2D image regions classified as scar.

(3) A model-based approach was utilized to register the two datasets. Models were fit to contours delineating the left ventricular contours in the CTA and DEMR. The Iterative Closest Point method was applied to align them (please see Figure I and Figure 2 for segmentation and registration results). Data acquisition: A patient underwent DEMR (Sonata, SIEMENS) using single shot TrueFISP inversion recovery technique approximately 20 minutes after intravenous $0.2 \mathrm{mmol} / \mathrm{kg} \mathrm{Gd}$ DTPA injection. The technical data were; FOV: $285-380 \mathrm{~mm} 2$,

Figure 2 (abstract P 193)

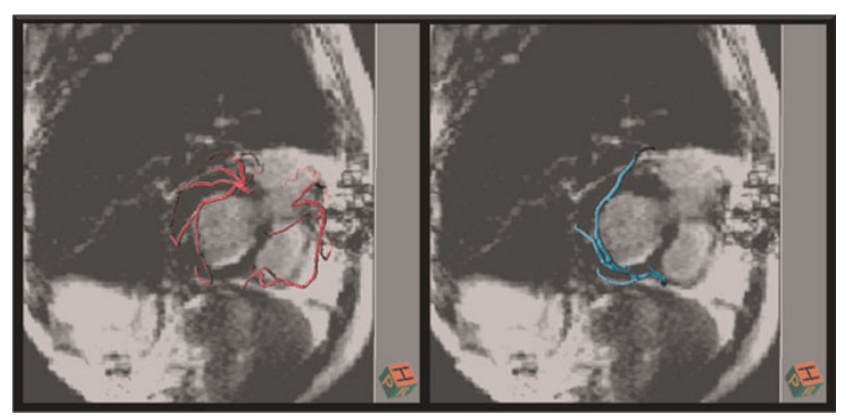


TE: I.I ms, TR: $7 \mathrm{~ms}$, Flip Angle: 50 degrees, TI: $280 \mathrm{~ms}$. For the same patient, CT (Dual Source Definition, SIEMENS) scan was performed with following parameters; Rotation Time: 335 ms, B25 Kernel (Dedicated Cardiac Kernel), Slice Thickness: 0.75 mm, Temporal Resolution: $82 \mathrm{~ms}$, Tube Current: $26 \mathrm{I} \mathrm{mAs/rot,}$ Tube Voltage: $120 \mathrm{kv}$.

Conclusion: The direct anatomic relationship between coronary arterial branches and segments of LV myocardium under evaluation for revascularization, or between coronary vein tributaries and LV myocardial regions being considered for resynchronization device placement, can be accomplished by coregistration of CTA coronary artery and/or vein maps with MR myocardial viability or scar maps.

\section{PI94}

Myocardial scar in diabetics and non-diabetics with ischemic heart disease as assessed by magnetic resonance imaging

Robert Donnino', Andrew H Nguyen', Steven P Sedlis ${ }^{2}$ and Monvadi B Srichai ${ }^{\prime}$

${ }^{I}$ NYU School of Medicine, New York, NY, USA

${ }^{2}$ Veterans Affairs NYHHCS New York Campus, New York, NY, USA

Journal of Cardiovascular Magnetic Resonance 2009, I I(SuppI I):PI94

Objective: Our objective was to determine the degree of myocardial scarring in diabetic and non-diabetic patients with ischemic heart disease (IHD).

Background: Diabetic patients with IHD are more likely to develop congestive heart failure (CHF) than non-diabetics, but the mechanism responsible for this is unclear. Recent evidence suggests that this may not be related to increased infarct size with accompanying negative remodeling and systolic dysfunction.

Methods: We retrospectively evaluated 54 consecutive patients (2I diabetics; 33 non-diabetics; mean age $69.7 \pm$ 8.3 years) who underwent both cardiac magnetic resonance (CMR) imaging and coronary angiography for evaluation of IHD between April 2006 and July 2008. Patients were evaluated for presence of CHF symptoms and degree of coronary artery disease (CAD) (measured angiographically using the modified Duke score). Myocardial scar was measured by late gadolinium enhancement on CMR for each patient using a I7-segment model of the left ventricle (LV), and was graded according to transmural extent on a semi-quantitative scale $(0=$ none, $\quad I=1-25 \%$, $2=26-50 \%, 3=51-75 \%, 4=76-100 \%)$. Total scar burden (mean grade per segment) and spatial extent (number of segments with any scar and number of segments with grades 3-4 scar) were recorded along with LV volume and ejection fraction.
Results: More diabetics than non-diabetics had CHF symptoms (76.2\% versus $45.4 \%, p<0.05$ ), and diabetics had a greater burden of CAD (Duke score $4.8 \pm 0.7$ versus 4.I $\pm 1.0, p<0.05$ ). Diabetics and non-diabetics did not differ, however, in total scar burden $(0.92 \pm 0.62$ versus $1.14 \pm 0.73, p=N S)$, number of segments with any scar $(5.6 \pm 3.4$ versus $6.7 \pm 4.6, p=N S)$, or grade $3-4$ scar $(3.7 \pm 3.0$ versus $4.1 \pm 3.1, p=N S)$. LV ejection fraction $(38.9 \pm 12.7 \%$ versus $39.6 \pm 16.0 \%, p=N S)$ and end diastolic volume (204 $\pm 86 \mathrm{ml}$ versus $212 \pm 75 \mathrm{ml}, \mathrm{p}=\mathrm{NS})$ were similar for both diabetics and non-diabetics, respectively.

Conclusion: Despite a higher incidence of $\mathrm{CHF}$ and a greater burden of CAD in diabetics, there was no significant difference in the amount of myocardial scar, LV size or ejection fraction compared to non-diabetics. These findings are consistent with recent literature suggesting that the higher incidence of $\mathrm{CHF}$ in diabetics with IHD may not be due to larger infarcts with negative remodeling and systolic dysfunction, but rather to diastolic dysfunction or other factors yet to be accounted for.

\section{PI95 \\ Optimized assessment for establishing myocardial viability prior to revascularization of a chronic total coronary occlusion using cardiac magnetic resonance imaging \\ Sharon WM Kirschbaum, Alexia Rossi, Eric Boersma, Dirk J Duncker, Martin van den Ent, Gabriel P Krestin, Patrick W Serruys, Pim J de Feyter and Robert-Jan M van Geuns \\ Erasmus MC, Rotterdam, Netherlands}

Journal of Cardiovascular Magnetic Resonance 2009, I I(SuppI I):PI 95

Introduction: Predictive value of delayed enhancement CMR is moderate especially in segments with an intermediate transmural extent of infarction (TEI).

Purpose: We sought to improve the predictive value of cardiac magnetic resonance imaging (CMR) using combined viability assessment in patients before percutaneous coronary intervention (PCl) of a chronic total coronary occlusion (CTO).

Methods: We studied patients with a successful (43/7I) and without a successful (29/7I) PCl of a CTO. Segmental wall thickening (SWT) was quantified before and after PCl. Before $\mathrm{PCl}$, using CMR, 5 viability indexes were evaluated: TEI, contractile reserve during dobutamine, end diastolic wall thickness (EDWT), unenhanced rim thickness and SWT of the unenhanced rim (SWTur). We determined predictive value for improvement in SWT>10\% after $\mathrm{PCl}$ for each viability index and for combined viability assessment.

Table I (abstract P 195) Diagnostic performance of each viability index for the prediction of improvement in SWT

\begin{tabular}{lccrrr}
\hline & Sensitivity(\%) & Specificity(\%) & PPV(\%) & NPV(\%) & Accuracy(\%) \\
\hline LDD (>7\%) & $94(88-98)$ & $77(63-87)$ & $89(82-94)$ & $87(73-95)$ & $89(83-94)$ \\
TEl $(<50 \%)$ & $89(81-94)$ & $50(36-64)$ & $79(70-85)$ & $68(51-82)$ & $76(69-83)$ \\
EDWT $(>6 \mathrm{~mm})$ & $92(84-96)$ & $27(16-41)$ & $72(64-79)$ & $61(39-80)$ & $71(63-78)$ \\
Unenhanced rim thickness $(>3 \mathrm{~mm})$ & $94(88-98)$ & $48(34-62)$ & $79(71-85)$ & $81(62-92)$ & $79(73-86)$ \\
SWTUR $(<45 \%)$ & $88(80-93)$ & $33(21-47)$ & $73(64-80)$ & $57(38-74)$ & $69(62-76)$
\end{tabular}

LDD, contractile reserve during low dose dobutamine; TEl, transmural extent of infarction; EDWT, end diastolic wall thickness; SWTur, Segmental wall thickening of the unenhanced rim; PPV, positive predictive value; NPV, negative predictive value; $95 \%$ confidence interval is presented between brackets. 
Results: Mean SWT improved significantly in patients with successful PCl $(16 \pm 19 \%$ to $39 \pm 35 \%$; $>0.0001)$, mean SWT did not improve in patients without successful PCl $(19 \pm 21 \%$ to $21 \pm 25 \% ; p=0.54)$. Predictive value for each viability index is presented in Table I. Combined viability assessment using contractile reserve during dobutamine, SWTur and TEI, improved the predictive value with a sensitivity of $92 \%(95 \% \mathrm{Cl}$ 82-97) and specificity of $83 \%(95 \% \mathrm{Cl} 70-91)$.

Conclusion: Combined viability assessment was superior to viability assessment using a single parameter. This may be useful for the selection of patients for $\mathrm{PCl}$ of a CTO.

\section{PI96}

Myocardial salvage by contrast-enhanced cine MR imaging: validation study against conventional T2 edema imaging and angiographic estimates of myocardium at risk during acute myocardial infarction

Ricardo Loureiro', Hiram G Bezerra', Joalbo M Andrade², Chun-Ho Yun', Ammar Sarwar', Michael Bolen'

and Ricardo C Cury'

'Massachusetts General Hospital - Harvard Medical School, Boston, MA, USA

${ }^{2}$ Department of Radiology - Universidade de Brasília, Brasília, Brazil

Journal of Cardiovascular Magnetic Resonance 2009, I I(SuppI I):PI 96

Purpose: To prospectively compare contrast-enhanced (CE) cine steady-state free precession (SSFP) magnetic resonance (MR) imaging to assess area at risk and myocardial salvage with T2-weighted (T2W) edema imaging and the "APPROACH angiographic score", a pathologically-proven angiographic estimate of myocardium at risk, after acute reperfused myocardial infarction (MI).

Materials and methods: The study was HIPAA compliant and was approved by the institutional review board. All participants gave written informed consent. Twenty one patients (I 9 men; mean age, $59 \pm 12$ years) were examined with T2-weighted FSE imaging, CE cineSSFP MR imaging and delayed-enhancement cardiac $M R$ imaging after a first reperfused acute $M I$. In I5 patients, a second scan was performed 3-6 months later to assess global functional recovery. A modified APPROACH score was calculated from the culprit lesion, location and severity in the angiographic tree.
Results: Myocardial regions of increased signal intensity were observed in 16 of 21 (76\%) examinations on T2W edema imaging and with CE cineSSFP MR imaging at all but one examination (20/ $21,95 \%)$. CE cineSSFP abnormality was comparable to the size of the hyperintense zone on T2W imaging $(20.6 \pm 7.0 \%$ versus $19.6 \pm 8.1 \%$ of the myocardial mass; $P=N S$ ) and to the APPROACH risk score $(23.2 \pm 12.2 \%$ of myocardial mass; $P=N S)$. The infarcted zone was significantly smaller (I $3.3 \pm 7.2$; all $P<0.05)$. Myocardial salvage, as measured by the difference of CE cineSSFP MR imaging and delayed-enhancement MRI, showed good correlation with global LV recovery and was comparable to those correlations observed for both $\mathrm{T} 2 \mathrm{~W}$ edema imaging and APPROACH risk score $(r=0.68$ for CEcineSSFP; $r=0.58$ for $\mathrm{T} 2 \mathrm{~W} ; r=0.70$ for APPROACH). While signal intensity differences between the abnormal and normal myocardium were comparable for T2W imaging and CE cineSSFP sequence, $C E$ cineSSFP MR had a better contrast-to-noise ratio (CNR) and signal-to-noise ratio (SNR) for differentiation between injured and normal regions than did T2W edema imaging $(P=0.00 \mathrm{I}$ for SNR; $P=0.02$ for CNR). The interobserver agreement (Kappa) for CE cineSSFP MR imaging (0.98) was better than that for T2W edema imaging (0.68).

Conclusion: CE cineSSFP signal abnormality depicts the area at risk in close agreement with the T2W edema imaging and the angiographic risk score, but with superior interobserver agreement and better image quality. The extent of salvaged myocardium, measured as the difference between myocardium at risk and myocardial necrosis, was associated with improvement of LVEF over time.

\section{PI97}

Steep left ventricle to aortic root angle is independently associated with dynamic left ventricular outflow tract gradient in hypertrophic cardiomyopathy: a novel association using 3-dimensional multi-modality imaging Deborah H Kwon', Nicholas G Smedira ${ }^{1,2}$,

Zoran B Popovic', Bruce W Lytle', Randolph M Setser', Marn Thamilarasan', Paul Schoenhagen', Scott D Flamm ', Harry Lever' ${ }^{\prime}$ and Milind Y Desai ${ }^{1}$

${ }^{\prime}$ Cleveland Clinic Foundation, Cleveland, $\mathrm{OH}$, USA

${ }^{2}$ Cleveland Clinic, Cleveland, $\mathrm{OH}, \mathrm{USA}$

Journal of Cardiovascular Magnetic Resonance 2009, I I(SuppI I):PI 97

Introduction: Hypertrophic cardiomyopathy (HCM) patients with similar basal septal hypertrophy (BSH) can have significantly

Table I (abstract P I97) Univariated and multivariate regression analysis testing the association between maximal LVOT gradient

\begin{tabular}{|c|c|c|c|c|}
\hline & $\begin{array}{l}\text { Univariate } \\
\text { Analysis Beta }\end{array}$ & $\begin{array}{l}\text { Univariate } \\
\text { Analysis } p \text { value }\end{array}$ & $\begin{array}{l}\text { Multivariate } \\
\text { Analysis Beta }\end{array}$ & $\begin{array}{l}\text { Multivariate } \\
\text { Analysis } \mathrm{P} \text { value }\end{array}$ \\
\hline Left ventricle to aortic root angle & -0.34 & $<0.001$ & -0.24 & 0.01 \\
\hline Age & 0.23 & 0.01 & 0.19 & 0.05 \\
\hline End-systolic volume index & -0.20 & 0.02 & -0.16 & 0.06 \\
\hline Gender & 0.05 & 0.5 & & \\
\hline Hypertension & 0.06 & 0.5 & & \\
\hline Atrial fibrillation & -0.05 & 0.5 & & \\
\hline Beta blocker use & 0.05 & 0.5 & & \\
\hline End-diastolic volume index & 0.04 & 0.7 & & \\
\hline Basal end-diastolic interventricular septal thickness & 0.02 & 0.8 & & \\
\hline
\end{tabular}


Figure I (abstract P|97)

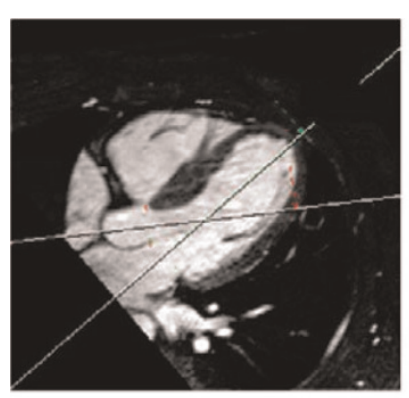

LV.Aortic Root angle $146^{\circ}$

Basal Septum $-1.9 \mathrm{~cm}$

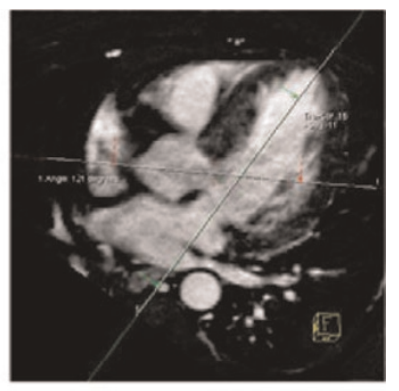

LV-Aortic Root angle $121^{\circ}$ Basal Septum $-2.3 \mathrm{~cm}$

Quantification of the LVARA in the 5 chamber view.

different degrees of dynamic obstruction, quantified by the left ventricular outflow tract gradient (LVOTG). Furthermore, an elevated LVOTG can be seen even with minimal BSH. Using advanced 3-dimensional whole-heart sequence on cardiac magnetic resonance (CMR), we observed a spectrum of acuity in the LV to aortic root angle (LVARA) in our study population of HCM patients (Figure I).

Purpose: In HCM patients, we sought to determine if a steeper LVARA was associated with an increased LVOTG, independent of BSH.

Methods: We studied 153 consecutive patients ( $\leq 65$ years) with echo-documented HCM who underwent standard CMR (I.5 T Siemens Avanto, Erlangen, Germany) along with wholeheart 3D MR angiogram which was a navigator-assisted freebreathing, ECG-triggered, fat saturated, T2-prepared, segmented 3D SSFP sequence. Imaging parameters were as follows: $\mathrm{TR}=3.8 \mathrm{~ms}, \mathrm{TE}=1.9 \mathrm{~ms}$, flip angle $=70^{\circ}$, acquired matrix $=$ 175-216 (phase direction) by 256 (readout direction) points (no interpolation), Grappa factor of 2, 24 reference lines and sampling bandwidth $= \pm 125 \mathrm{kHz}$. Typically, 60-70 slices were acquired at $1.5 \mathrm{~mm}$ thickness (interpolated) in order to cover the heart. The in-plane resolution was typically $1.3-1.6 \mathrm{~mm}$. Images were acquired during a $150 \mathrm{msec}$ data acquisition window in midto-late diastole. LVARA, LV volumes (indexed to body surface area) and BSH were measured on CMR. Maximal (resting or provocable) LVOT gradients were recorded on echocardiography. Inter and intra-observer concordance of LV-aortic root angle measurement was assessed in $14 \mathrm{HCM}$ patients using intraclass correlation coefficient (ICC).

Results: The baseline characteristics were as follows: mean age $46 \pm 14$ years, $68 \%$ male, $36 \%$ hypertensives, $73 \%$ were on beta-blockers. The mean LVARA, maximal LVOT gradient, BST, end systolic and end diastolic volume indexes were $134^{\circ} \pm 10$, $82 \mathrm{~mm} \mathrm{Hg} \pm 60,1.98 \mathrm{~cm} \pm 0.6,32 \mathrm{ml} / \mathrm{m}^{2} \pm \mathrm{II}$ and $84 \mathrm{ml} / \mathrm{m}^{2} \pm$ 16, respectively. Univariate and multivariate associations between maximal LVOT gradient and various factors are shown in Table I. Age correlated with LVARA $(r=-0.56, p<0.00 \mathrm{I})$. The ICC for intra-observer (0.9I) and inter-observer (0.88) concordance of LV-aortic root angles in the HCM group was very high (both p-values < $0.000 \mathrm{I}$ ).

Conclusion: In patients with HCM, a steeper LVARA predicts an increased LVOTG, independent of BSH. Steep LVARA, which likely represents accelerated remodelling of the LVOT, may

explain why HCM patients with similar degree of BST have different degrees of dynamic LVOT obstruction. The prognostic importance of steep LVARA remains to be determined. This study demonstrates the clinical utility of multi-modality imaging, including CMR, in further understanding the varied phenotypic presentation and complex pathophysiology found in HCM.

\section{PI98}

\section{Abstract withdrawn by author}

Journal of Cardiovascular Magnetic Resonance 2009, I I(SuppI I):PI 98

\section{PI99}

The role of cardiac magnetic resonance imaging in the assessment of systemic lupus erythematosis (SLE)

Wendy Norman', Sean G O'Neill' ${ }^{2}$, Simon Woldman ${ }^{3}$, Frederique Bailliard', Anisur Rahman ${ }^{2}$ and Andrew M Taylor'

'UCL Institute of Child Health, London, UK

${ }^{2}$ Centre for Rheumatology Research, UCL, London, UK

${ }^{3}$ The Heart Hospital, UCLH, London, UK

Journal of Cardiovascular Magnetic Resonance 2009, I I(SuppI I):PI 99

Objectives: To define the cardiac magnetic resonance (MR) appearances of cardiac disease in patients with SLE and known coronary or cerebrovascular disease, and compare theses findings with those seen on trans-thoracic echocardiography (TTE).

Background: Cardiovascular disease (CVD) is a major cause of morbidity and mortality in patients with SLE. It is increasingly recognised that accelerated atherosclerosis contributes to increased risk of myocardial ischaemia and/or stroke in this population. Autopsy and echo studies have documented numerous abnormalities in patients with SLE; however it remains

\section{Figure I (abstract PI99)}

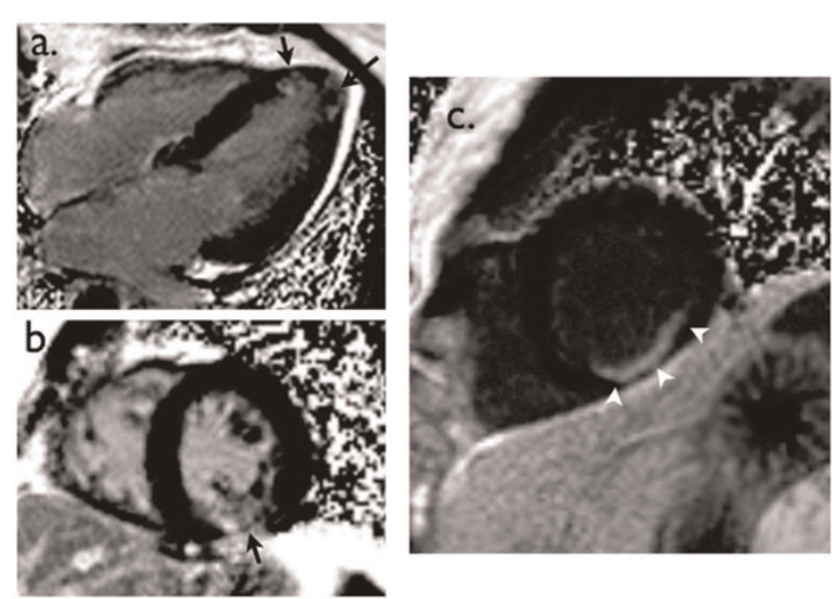

Unexpected areas of LGE: a\&b, patchy LGE in a non-coronary artery distribution pattern (arrows), consistent with possible myocarditis - a, 4-chamber view showing sptal and apical LGE and b, short-axis view showing inferior LGE; c, subendocardial LGE in a circumflex coronary artery distribution (arrowheads), consistent with silent AMI in a patient with APS. 
unclear whether these abnormalities are an important contributor to mortality and morbidity, and whether they are related to early development of coronary artery disease.

Methods: Cardiac MR assessment including late gadolinium enhancement (LGE) was performed on 22 patients meeting 4 or more of the revised ACR criteria for SLE. Imaging performed at I.5 T (Avanto, Siemens, Erlangen, Germany). II patients had previous CVD and were matched with II control patients with no history of CVD. Control patients were matched for age, gender, race and SLE disease duration. TTE was performed at the same visit. The Research Ethics Committee approved the study and all participants gave informed consent to take part.

Results: Of the subjects in the known CVD group, 5 had areas of LGE on the MR images: 4 LGE patterns were consistent with myocardial infarction, whilst in I patient (history of stroke) there was patchy LGE in a non-coronary distribution (Figure la \& $\mathrm{lb}$ ). One subject, with known antiphospholipid antibody syndrome (APS) but no history of CVD, had LGE suggestive of a previous infarct (Figure Ic). This was not evident on TTE. One patient purported to have had a myocardial infarction had no LGE. TTE myocardial abnormalities were detected on 4 of the 6 subjects that had LGE.

Conclusion: In this small study, we demonstrate that cardiac MR can detect changes in the myocardium of patients with SLE that are not evident on TTE. The 2 imaging modalities were carried out on the same day to avoid the possibility of new abnormalities developing between the dates of the MR and TTE. Three of the 22 patients we studied (14\%) had diagnostic MR scans that could potentially influence their long-term management and prognosis. Two patients with no clinical history of cardiac disease had areas of unexpected myocardial fibrosis, one with a pattern suggestive of coronary artery disease, and one with multiple areas of myocardial scarring, perhaps suggestive of myocarditis. Both these patients had normal TTE. Conversely, I patient with a supposed acute myocardial infarction had no evidence of myocardial fibrosis on MR scanning, placing this patient in a good prognostic group for the future. Further studies of cardiac MR in patients with SLE are warranted to investigate these preliminary findings.

\section{P200}

Three dimensional Phase Sensitive Inversion Recovery (PSIR) turbo FLASH for evaluation of left ventricular myocardial lesions in infiltrative and non-ischemic cardiac diseases

Aya Kino', Sven Zuehlsdorff ${ }^{2}$, Aoife Keeling', Cormac Farrelly', John Sheehan', Randall Ramsay', Renate Jerecic ${ }^{2}$ and James C Carr ${ }^{\prime}$

'Northwestern University, Chicago, IL, USA

${ }^{2}$ Siemens Medical Solutions USA, Inc., Chicago, IL, USA

Journal of Cardiovascular Magnetic Resonance 2009, I I(SuppI I):P200

Background: Delayed-enhanced MRI (DE-MRI) is typically used to assess myocardial viability by depicting regions of myocardial scarring caused by myocardial infarction. Infiltrative myocardial disease and non-ischemic cardiomyopathy demonstrate atypical patterns of enhancement on DE-MRI, producing diffuse hyperenhanced lesions throughout the myocardium. Such hyperenhanced lesions patterns may be missed by conventional 2D imaging due to non-contiguous slices and limited slice
Figure I (abstract P200)

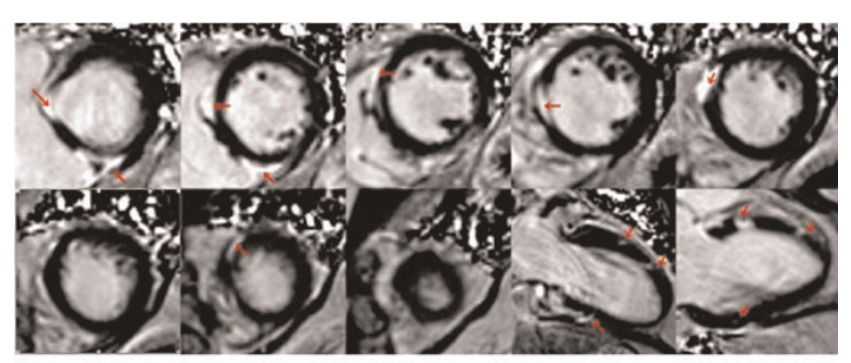

a.

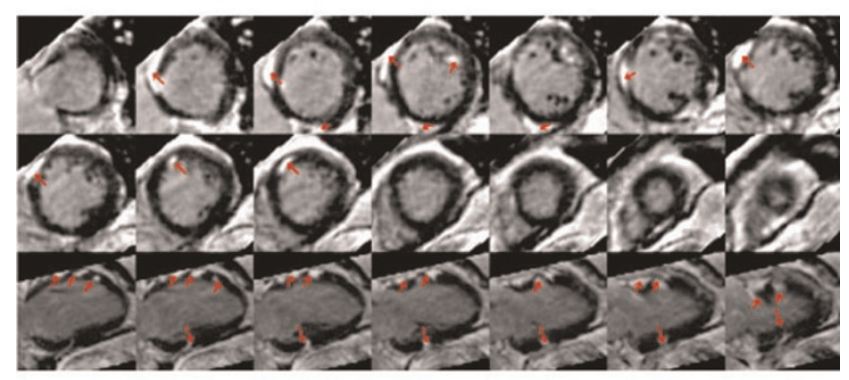

b.

(a) 2D PSIR images of the left ventricle of a 55-year-old male patient with biopsy proven sarcoidosis showing multiple scattered hyper enhanced lesions (red arrows) within the left ventricular myocardium in short axis, 2 and 3 chamber views. (b) 3D PSIR reformatted images from the same patient demonstrate more numerous lesions in a subepicardial and mid myocardial location (red arrows) compared to 2D PSIR.

coverage. 3D DE-MRI may be a more effective technique for assessing hyperenhanced lesions due to the isotropic coverage of left ventricle (LV).

Purpose: The purpose of this study was to compare a navigator gated free breathing 3D Phase Sensitive Inversion Recovery (PSIR) turboFLASH to an established 2D PSIR turboFLASH method for detecting myocardial hyperenhanced lesions caused by infiltrative myocardial disease and non-ischemic cardiomyopathy.

Materials and methods: Under an IRB approved protocol, 18 patients with suspected infiltrative myocardial heart disease and cardiomyopathy [hypertrophic cardiomyopathy (HCM) $n=9$, sarcoidosis, $n=4$, and myocarditis, $n=5$ ] were examined on a I.5 T MR scanner (MAGNETOM Avanto, Siemens AG, Erlangen, Germany) after the administration of gadopentetate dimeglumine $[0.2 \mathrm{mmol} / \mathrm{kg}$ Gadolinium-DTPA (Magnevist, Schering AG, Berlin, Germany)] using a segmented 2D PSIR turboFLASH sequence and a navigator-gated 3D PSIR turboFLASH sequence. Quantitative evaluation was carried out by measuring the volume of hyperenhaced lesions for both techniques using the VPT quantification software (Siemens AG) that considered areas with 6 standard deviations above the normal myocardial signal intensity as abnormal. Images were assessed qualitatively by 2 reviewers using the AHA 17-segment model. The presence and transmural extent of lesions were evaluated and the number of lesion per segment was counted. Image quality was scored using a four point Likert scale ( 0 -poor, non-diagnostic; I-fair, diagnostic maybe be impaired; 2-good with some artifacts and 3-excellent without artifacts). 
Results: Average total scan time for the 18 patients was 9:56 minutes for 2D PSIR and 6:54 minutes for 3D PSIR, respectively. The mean navigator efficiency was $53.2 \%$.

The image quality score did not differ significantly $(p=0.53)$ for both techniques. The total number of hyperenhanced regions detected using 3D PSIR was larger than at 2D PSIR $(P<0.05)$ (Figure I). Qualitative analysis of area of lesions $(P=0.15)$ and location $(p=0.38)$ were similar for both techniques.

Conclusion: Free breathing 3D PSIR viability imaging may be more effective than conventional 2D imaging for detecting hyperenhaced lesions associated with infiltrative heart disease and non-ischemic cardiomyopathy. The 3D approach provides an isotropic coverage of the LV and detects smaller lesions missed by $2 \mathrm{D}$ imaging.

Abstract Summary Statement: Comparison between free breathing 3D Phase Sensitive Inversion Recovery (PSIR) turboFLASH and 2D PSIR turboFLASH for detecting myocardial lesions in infiltrative and non-ischemic cardiac diseases. 3D PSIR is a promising alternative for quantification and detection of small and diffuse lesions.

\section{P20I}

Increased left ventricular torsion in hypertrophic cardiomyopathy mutation carriers with normal wall thickness

Iris Rüssel, Wessel P Brouwer, Tjeerd Germans, Tim Marcus, Marco Götte and Albert van Rossum VU Medical Center, Amsterdam, Netherlands

Journal of Cardiovascular Magnetic Resonance 2009, I I(SuppI I):P20 I

Objective: To determine the amount of left ventricular (LV) torsion in hypertrophic cardiomyopathy (HCM) mutation carriers (carriers) with normal wall thickness.

Background: Increased LV torsion has been observed in HCM and is thought to be caused by wall thickening [I]. However, structural abnormalities that precede the development of hypertrophy in HCM may also cause alterations in LV torsion in carriers with normal wall thickness [2].

Methods: Ten carriers with an LV wall thickness $<10 \mathrm{~mm}$, and ten age and gender matched controls underwent CMR cine imaging and tissue tagging. LV volumes were calculated from the cine images. Basal and apical rotations and LV torsion, defined as the circumferential-longitudinal shear angle [3], were determined from tissue tagging. Counterclockwise rotation as seen from the apex was considered positive. LV volumes, peak rotation and torsion were compared between both groups using Student's T-test. A p-value $<0.05$ was considered significant.

Results: LV end-diastolic and end-systolic volumes were not significantly different between both groups $(p=0.79$ and $p=0.36$, resp.), whereas EF was significantly larger in the carriers $(63.2 \pm 3.2 \%$ vs. $59.7 \pm 2.8 \%, p=0.02)$. Peak apical rotation and peak torsion were significantly larger in the carriers $\left(I 4.6 \pm 3.2^{\circ}\right.$ vs. $10.3 \pm 3.3^{\circ}, P=0.0 I$, and $10.2 \pm 2.3^{\circ}$ vs. $7.1 \pm 1.0^{\circ}, p=0.001$, resp.), while peak basal rotation was significantly smaller $\left(-2.5 \pm 2.2^{\circ}\right.$ vs. $\left.-4.6 \pm 1.7^{\circ}, p=0.03\right)$.

Conclusion: HCM mutation carriers with normal wall thickness demonstrate increased LV torsion. Underlying altered myocardial architecture might be responsible for this finding.

References

I. Circ 1994, 90:854-867.

2. Circ 2007, I I5:e6 |0-6 II.

3. J Cardiovasc Magn Reson 2008, 10:26.

\section{P202}

Is routine cardiac MRI justifiable in patients with non-ischemic cardiomyopathy?

Gorgi Kozeski', M Rizwan Khalid ${ }^{2}$, Fatima R Khalid ${ }^{2}$, Hormoz Kianfar', Anita Kozeska', Tarek Mousa' and Ola Akinboboye'

${ }^{I}$ New York Hospital Medical Center of Queens, Flushing, NY, USA

${ }^{2}$ Emory University, Atlanta, GA, USA

Journal of Cardiovascular Magnetic Resonance 2009, I I(SuppI I):P202

Introduction: According to the recently published appropriateness criteria, evaluation of left ventricular (LV) function in patients with heart failure is an uncertain indication for Cardiac Magnetic Resonance Imaging (CMR). However, in addition to assessment of LV function, other ancillary findings on a cardiac MRI study may alter management decisions in patients with heart

Table I (abstract P202) Description of seven patients with NICM in which CMR revealed a specific diagnosis and changed management course

\begin{tabular}{|c|c|c|c|}
\hline Patients & CMR diagnosis & Evidence of LGE & Management \\
\hline Patient I & Newly diagnosed constrictive pericarditis & Present & Surgical evaluation for pericardial stripping \\
\hline Patient 2 & Newly diagnosed cardiac sarcoidosis & $\begin{array}{l}\text { Not performed due to renal insufficiency } \\
\text { (T2 weighted imaging shows evidence of } \\
\text { increased signal intensity suggestive of } \\
\text { myocardial inflammation) }\end{array}$ & $\begin{array}{l}\text { IV Steroids Implantable cardioverter defi- } \\
\text { brillator (ICD) implantation }\end{array}$ \\
\hline Patient 3 & Newly diagnosed cardiac sarcoidosis & Present & IV steroids ICD implantation \\
\hline Patient 4 & $\begin{array}{l}\text { Newly diagnosed non-compaction cardio- } \\
\text { myopathy }\end{array}$ & Present & $\begin{array}{l}\text { Electrophysiologic evaluation and ICD } \\
\text { implantation }\end{array}$ \\
\hline Patient 5 & Newly diagnosed left ventricular mass & Present & Urgent surgical evaluation \\
\hline Patient 6 & $\begin{array}{l}\text { Newly diagnosed cardiac amyloid (AL-type } \\
\text { by kidney biopsy) Newly diagnosed large } \\
\text { bilateral pleural effusions with collapsed left } \\
\text { lung }\end{array}$ & Present & $\begin{array}{l}\text { Urgent thoracocentesis and further evalua- } \\
\text { tion of cardiac amyloidosis }\end{array}$ \\
\hline Patient 7 & Myocarditis with large right pleural effusion & Present & $\begin{array}{l}\text { Aggressive diuresis and management of } \\
\text { myocarditis }\end{array}$ \\
\hline
\end{tabular}


failure. An example is the extent of delayed enhancement, which has been shown to be a major prognostic indicator in patients with non-ischemic cardiomyopathy (NICM).

Purpose: We hypothesize that a CMR study often provides information that alters the course of management in patients with non-ischemic cardiomyopathy.

Methods: We conducted a retrospective analysis of 112 consecutive patients who underwent CMR with or without gadolinium at our center from June 2007 to April 2008. Fortythree patients were diagnosed as having a cardiomyopathy. Cardiomyopathy was defined as a left ventricular ejection fraction (LVEF) of less than $50 \%$. Twenty-four of these patients were diagnosed with NICM. All patients with NICM had previous negative ischemia evaluation.

Cardiac MRI study: After performing standard localizer images, ECG-gated breath-hold segmented k-space SSFP images were obtained in standard projections. T2-weighted and delayed enhancement images were also performed.

Results: A total of 24 patient studies were examined, including 10 males and 14 females with a mean age of $52 \pm 16$ years. Average LVEF was found to be $33 \pm 13(\%)$. Of these, 7 (29\%) had significant management changes and therapeutic interventions guided by the CMR results. Six patients had evidence of late gadolinium enhancement (LGE), and in one patient gadolinium was not given due to renal insufficiency. In this patient, T2weighted images revealed evidence of increased signal intensity suggestive of myocardial inflammation. The results of CMR in these patients and specific management changes are summarized in Table I.

Conclusion: Cardiac Magnetic Resonance Imaging is an invaluable tool in the management of patients with NICM. In our case review, approximately one-third of the patients had significant management changes guided by the CMR results, and almost all patients had presence of late gadolinium enhancement.

\section{P203}

Comparison of methods for ranking and stratification of community-dwelling adults by pericardial fat thickness measured using cardiovascular magnetic resonance imaging Michael L Chuang', Noriko Oyama', Philimon Gona ${ }^{2}$, Carol J Salton', Rahul R Jhaveri', Daniel Levy², Christopher J O'Donnell ${ }^{2}$ and Warren J Manning' 'Beth Israel Deaconess Medical Center, Boston, MA, USA ${ }^{2}$ The NHLBI's Framingham Heart Study, Framingham, MA, USA

Journal of Cardiovascular Magnetic Resonance 2009, I I(SuppI I):P203

Introduction: Pericardial adipose tissue is associated with excess cardiovascular risk factors and may itself be a marker for and modulator of cardiovascular disease, possibly through paracrine activity as pericardial adipose tissue is metabolically highly active. In many research studies pericardial fat thickness (PFT) is measured using transthoracic echocardiography from the parasternal long-axis view, from which PFT over the right ventricular (RV) free wall is determined. However, the ability of echocardiography to measure PFT is limited by acoustic window issues and potential confounding with pericardial effusion. Pericardial fat is readily seen on routine CMR imaging for ventricular function, but there is no standard approach to
Figure I (abstract P203)

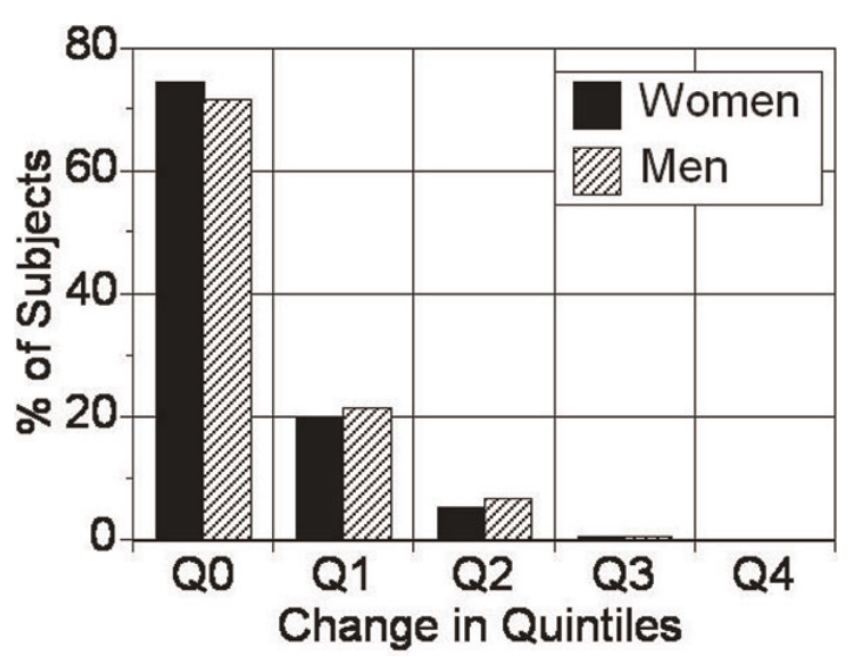

assessment of pericardial adipose burden by CMR. For simplicity and to facilitate comparison with the literature, we elected to measure RV free wall PFT by CMR from the 4-chamber view. In this abstract we sought to determine whether measurement of PFT at its maximum versus at a predefined location (mid-RV level; 4ChMID) would change stratification of subjects by PFT.

Methods: 500 adults (aged $61 \pm 9$ years, 250 women and 250 men) were randomly selected from the Framingham Offspring Cohort CMR study database. Subjects were imaged on a I.5-T scanner (Philips Medical Systems) using a cardiac array coil for RF signal reception. PFT was measured at end-diastole from the 4-chamber view using a cine SSFP sequence $(T R / T E / \theta=3.3 / 1.7 /$ $60^{\circ}, 10-\mathrm{mm}$ slice thickness, in-plane resolution $1.92 \times 1.56 \mathrm{~mm}^{2}$ ) over the mid-level right ventricle (4chMID) and at maximal thickness in the same orientation (4chMAX). Sex-specific ranking of subjects by PFT was performed for 4chMID and for 4chMAX. Changes between 4chMID and 4chMAX ranks were summarized as median [interquartile] differences. Additionally, subjects were then stratified by quintiles of PFT. We assessed concordance of individual subject rankings by Spearman correlation $\left(r_{s}\right)$ and tabulated number of across-quintile changes for each sex.

Results: PFT could be measured successfully for all subjects, with good reproduciblity between readers (interclass correlation coefficients: interobserver ICC $=0.89$, intraobserver $I C C=0.98)$. Men had significantly greater PFT than women for both $4 \operatorname{chMAX}(\mid 7.8 \pm 8.0$ vs. $\mid 4.2 \pm 6.1 \mathrm{~mm}, \mathrm{p}<0.000 \mathrm{I})$ and 4chMID $(10.7 \pm 7.2$ vs. $8.3 \pm 5.1 \mathrm{~mm}, \mathrm{p}<0.000 \mathrm{I})$. There was good correlation for ranking of PFT by $4 \mathrm{chMAX}$ and 4chMID measurements for both men $\left(r_{\mathrm{s}}=0.754\right)$ and women $\left(r_{\mathrm{s}}=\right.$ 0.763 ), both $p<0.0001$. The median [interquartile] rankdifferences were $31[14,56]$ for men and $30[15,50]$ for women. Figure I shows percentage of subjects whose quartileranking remained the same $(\mathrm{Q} 0)$ or changed by I quintile $(\mathrm{Q} I)$, or 2, 3, or 4 quintiles (Q2, Q3 and Q4 respectively).

Conclusion: Men have greater PFT than women, but concordance in ranking between 4ChMAX and 4ChMID PFT was similar for both sexes. As ranking of subjects was similar regardless of whether maximal or mid-RV PFT was used, it may be reasonable to select a standardized measurement method, i.e. mid-RV level PFT, for consistency. Further work is needed to 
determine whether PFT measured by CMR has additional prognostic value over standard clinical predictors of cardiovascular risk, but PFT can be determined reliably and reproducibly using routine SSFP sequences for assessment of ventricular function, so that PFT can be determined without increasing examination time in a standard clinical CMR examination.

\section{P204}

Late gadolinium enhancement as an independent predictor of atrial fibrillation in hypertrophic cardiomyopathy (HCM)

Rory O'Hanlon, Agata Grasso, Chiara Bucciarelli-Ducci, Meghana Kulkarni, Susan Clark, Ricardo Wage, Jessica Webb, Michael Roughton, Leena Sulaibeekh, Dana Dawson, Dudley J Pennell and Sanjay K Prasad Royal Brompton Hospital, London, UK

Journal of Cardiovascular Magnetic Resonance 2009, I I(SuppI I):P204

Introduction: Atrial fibrillation (AF) is the most common arrhythmia observed in HCM, developing in approximately $20 \%$ of all cases, with an annual incidence of I-3\% per year. The risk of AF developing is 4-6 fold greater in HCM than in the general population. Its' development is associated with an increased risk of systemic thromoboembolism, heart failure and death. Myocardial fibrosis is an important risk factor for the development of atrial fibrillation as demonstrated in post mortem studies. Cardiovascular magnetic resonance (CMR) is the gold standard imaging tool to visualise replacement myocardial fibrosis in vivo. The presence of fibrosis has been shown to be an important predictor of cardiovascular morbidity and mortality in both ischaemic and dilated cardiomyopathies, and more recently an important predictor of arrhythmia as detected by Holter monitoring in HCM.

Aims: We hypothesised that the presence of myocardial fibrosis is an independent predictor of atrial fibrillation in patients with HCM in a prospective cohort of patients.

Methods: Between 200I and 2005, I26 patients with an established diagnosis of HCM were scanned with gadolinium late enhancement. Clinical data, cardiac events, and investigations were collected prospectively to identify outcomes. Relevant data including severity of mitral regurgitation, left atrial indexed volumes, and ejection fraction were noted. The median duration of follow up was 1076 days. CMR volumes, mass, and ejection fraction were analysed using customised software (CMRTools, London). Myocardial fibrosis was assessed using standard late enhancement techniques with gadolinium-DTPA contrast agent (ADD DOSE. The amount of fibrosis was quantified from sequential short axis slices (base to apex)apex using customised software (MASS, Medis, Leiden). Myocardial epicardial and endocardial contours were delineated. Regions of normal and abnormal myocardium were identified and the total amount of fibrosis was quantified on a per segment basis using the full width half maximum technique. The total amount of fibrosis was expressed as a percentage per segment, as a total in grams, and as a percentage of the total left ventricular mass. A Cox proportional hazard model was applied to correlate the incidence of atrial fibrillation with the presence of myocardial fibrosis.
Results: Of 126 patients studied, 87 (69\%) had detectable myocardial fibrosis. In those with myocardial fibrosis the incidence of atrial fibrillation over the follow up period was $21 \%$ vs $5 \%$ for those without $(p=0.033)$. The presence of myocardial fibrosis was associated with an 8 fold increased risk of the development of atrial fibrillation (HR 8.45; Cl I.12-65.53; $P=0.038$ ). Mutivariate analysis demonstrated that fibrosis remained an independent predictor of $\mathrm{AF}$ regardless of mitral regurgitant severity, ejection fraction, and LA indexed volumes. Conclusion: The presence of LV myocardial fibrosis in HCM is an independent predictor of the development of atrial fibrillation over a median of approximately three years. This may have important implications for risk stratification, decision to anticoagulate, and maintenance of sinus rhythm in this population. A potential mechanism may relate to associated diastolic dysfunction resulting in changes to atrial architecture. It is also possible that LV changes reflect increased atrial fibrosis as part of the disease spectrum but that is more difficult to detect with present techniques.

\section{P205}

CMR evaluation in patients with high grade ventricular arrhythmias

Antonio Bernardini', Agostino Meduri ${ }^{2,3}$, Luigi Natale ${ }^{2}$, Carlo Liguori ${ }^{2}$, Riccardo Fenici ${ }^{2}$, Donatella Brisinda ${ }^{2}$ and Lorenzo Bonomo ${ }^{2}$

I “G. Mazzini” Hospital - ASL Teramo, Teramo, Italy

${ }^{2}$ Università Cattolica del Sacro Cuore - Policlinico A. Gemelli, Rome, Italy

${ }^{3}$ Università Cattolica del Sacro Cuore, Rome, Italy

Journal of Cardiovascular Magnetic Resonance 2009, I I(SuppI I):P205

Purpose: To assess by cardiac-MR the prevalence of myocardial alterations in arrhythmic patients.

Materials and methods: 43 patients with non ischemic ventricular arrhythmias. Premature ventricular complexes had left bundle branch block morphology (LBBB) in 29 cases, in 7 a right bundle branch block contour (RBBB) and 7 had polymorphic patterns(PV). US was negative in $78.4 \%$ of patients, while CMR was negative in only 13\% of patients. Studies were performed on a I.5 MR scanner with Cine sequences (Fastcard or FIESTA), bb-FSE and IR-prep FGRE I 5 minutes after injection of $0.2 \mathrm{mmol} / \mathrm{Kg}$ of Gd-DTPA.

Results: CMR found a high prevalence of morphological, signal intensity and functional myocardial abnormalities. RV dilatation was found in $85 \%$ of patients with PV arrhytmias, $48.3 \%$ of patients with LBBB morphology, $12.5 \%$ of patients with RBBB morphology. LV dilatation was present in $28.6 \%, 25 \%$ and $24.1 \%$ of patients with LBBB, PV and RBBB type arrhytmias respectively. RV wall motion abnormalities were identified in $50 \%$ and $36.7 \%$ of patients with $\mathrm{PV}$ and LBBB pattern respectively while LV motion abnormalities in $25 \%$ and $10.3 \%$. Free wall RV signal/ thickness abnormalities were found in $23.3 \%$ of patients; LV signal abnormalities were found in $11.6 \%$ of patients.

Seven patients underwent myocardial biopsy: 5 positive for myocarditis, I positive for ARVD, one had a negative biopsy.

Conclusion: In patients with primary ventricular arrhythmias MR documented high prevalence (87\%) of morphological, signal intensity and wall motion abnormalities even with negative echocardiogram. 


\section{P206}

Association between wall metrics and identification of lipid-rich necrotic core in the early-stage atherosclerosis using magnetic resonance imaging

Li Dong, Xue-Qiao Zhao, Baocheng Chu, Hunter Underhill, Thomas S Hatsukami and Chun Yuan Univerisity of Washington, Seattle, WA, USA

Journal of Cardiovascular Magnetic Resonance 2009, I I(SuppI I):P206

Introduction: Detection of early-stage of carotid atherosclerosis is important for stroke prevention. We sought to explore reliability of wall metrics measurements by high resolution $M R I$ in a population of patients with early-stage carotid atherosclerosis.

Methods: Subjects $(n=106)$ recruited from an ongoing clinical trial underwent carotid MRI scans (I.5 T GE scanner) using a high-resolution multi-sequence protocol. Artery metrics measurements, which include maximum wall thickness (MaxWT), mean wall thickness (MWT), normalized wall index $(\mathrm{NWI}=$ $\mathrm{WA} /[\mathrm{LA}+\mathrm{WA}])$, and wall area (WA), were recorded after the lumen and outer wall boundary were identified. Intraclass coefficient (ICC) was used to assess intra- and inter-reader agreements for measurement of the MaxWT, MWT, NWI, and WA. The lipid-rich necrotic core (LRNC) was identified as nonenhancing area on the contrast-enhanced $\mathrm{TI}$-weighted images with exclusion of calcification by multi-sequence images. A spearman correlation coefficient was used to correlate Occurrence of LRNC with artery metrics measurements. Prevalence of LRNC was categorized by NWI in quartiles.

Results: Intra-reader ICC's for the MaxWT, MWT, NWI, and WA are $0.92,0.94,0.95$, and 0.92 respectively. Inter-reader ICC's for measurement of the MaxWT, MWT, NWI, and WA are 0.83 , $0.80,0.85$, and 0.80 respectively. The LRNC is found in 29 arteries out of a total of 106 arteries (27.4\%). The mean area of LRNC is $3.25 \mathrm{~mm} 2$ (SD, 2.64). There are significant correlations between occurrence of LRNC and the MaxWT, MWT, NWI, and WA $(r=0.71,0.66,0.64$ and 0.42 , respectively). LRNC is not present in the first NWI quartile and $66.7 \%$ of LRNC is present in the last NWI quartile (Table I).

Discussion and conclusion: Small mean LRNC areas found in this study indicate that this study population has early-stage of carotid atherosclerosis. Excellent inter- and intra-reader ICC's suggest that MRI is reliable tool for the evaluation of carotid artery wall metrics. Good correlation between wall metrics measurements and LRNC suggests wall metrics reflect the plaque burden. In particular, NWI can be used to assess prevalence of LRNC in subjects with early-stage carotid atherosclerosis.

Table I (abstract P206) Proportion of lipid-rich necrotic core across normalized wall index

\begin{tabular}{llll}
\hline NWI & Number & Proportion & $\begin{array}{l}\text { P value } \\
\text { (comparing } \\
\text { to the } \\
\text { 4th quartile) }\end{array}$ \\
\hline Ist quartile $(\leq 0.36)$ & 24 & $0 / 24(0 \%)$ & $<0.000$ I \\
2nd quartile $(0.37-0.39)$ & 23 & $2 / 23(8.7 \%)$ & 0.0001 \\
3rd quartile $(0.40-0.45)$ & 32 & $9 / 32(28.1 \%)$ & 0.007 \\
4th quartile $(>0.45)$ & 23 & $18 / 27(66.7 \%)$ & - \\
\hline
\end{tabular}

P207

Trajectory of the time course for LVH regression and remodeling imparted by aortic valve replacement for severe aortic stenosis; a cardiovascular MRI study sponsored by the American Heart Association

Robert WW Biederman, James A Magovern, Saundra B Grant, Ronald B Williams, June A Yamrozik, Diane A Vido, Vikas K Rathi and Mark Doyle Allegheny General Hospital, The Gerald McGinnis Cardiovascular Institute, Pittsburgh, PA, USA

Journal of Cardiovascular Magnetic Resonance 2009, I I(SuppI I):P207

Background: In patients with severe aortic stenosis (AS), longterm data tracking surgically induced beneficial effects of afterload reduction via aortic valve replacement (AVR) on reverse LV remodeling are not available. Echocardiographic data is available short-term, but in limited fashion beyond one year. Cardiovascular MRI (CMR) offers the ability to track discrete changes in LV metrics with small numbers but high accuracy due to its inherent high spatial resolution and low variability.

Hypothesis: We hypothesize that progressive changes following AVR are detectable by CMR and changes in LV structure and function, triggered by AVR, continue to favorably improve over an extended period following AVR.

Methods: Ten pts $(67 \pm 12$ yrs, 6 female) with severe, but compensated, AS underwent CMR pre AVR and post AVR at $6 \pm 2$ months, I year \pm 2 month, and up to 4 yearrs \pm 5 month. LV mass index (LVMI), LV geometry, volumetrics and EF were measured (GE, EXCITE I.5 T, Milwaukee, WI). A Kruskall-Wallis one-way ANOVA was performed.

Results: Despite advanced but mostly compensated AS ((peak and mean gradient by CMR $(67 \pm 21$ and $43 \pm 10 \mathrm{mmHg}$, respectively) and by Echocardiography $(72 \pm 23$ and $44 \pm \mathrm{II}$, resepctively) ( $p=N S$ for all), all 10 pts survived $A V R$ and underwent $C M R$ at up to a 4-year time point (40 time points). LVMI markedly decreased at 6 months (I57 \pm 42 to I $34 \pm$ $32 \mathrm{~g} / \mathrm{m}^{2}, \mathrm{p}<0.005$ ), continuing to gently trend downwards at 4 yrs $(127 \pm 32 \mathrm{~g} / \mathrm{m} 2)$. Similarly, EF increased pre to post AVR $(55 \pm 22$ to $65 \pm 11 \%,(\mathrm{p}<0.05))$ and continued trending upward, remaining stable at years I-4 (66 \pm II vs. $65 \pm 9 \%)$. LVEDV index, initially high pre AVR, normalized post AVR $(83 \pm 30$ to $68 \pm 1 \mathrm{I} \mathrm{ml} / \mathrm{m} 2, \mathrm{p}<0.05)$, trending even lower by year $4\left(66 \pm 10 \mathrm{ml} / \mathrm{m}^{2}\right)$. LV stroke volume increased rapidly from pre to post AVR (40 \pm I I to $44 \pm 7 \mathrm{ml}$ ) continuing to gradually

Figure I (abstract P207)

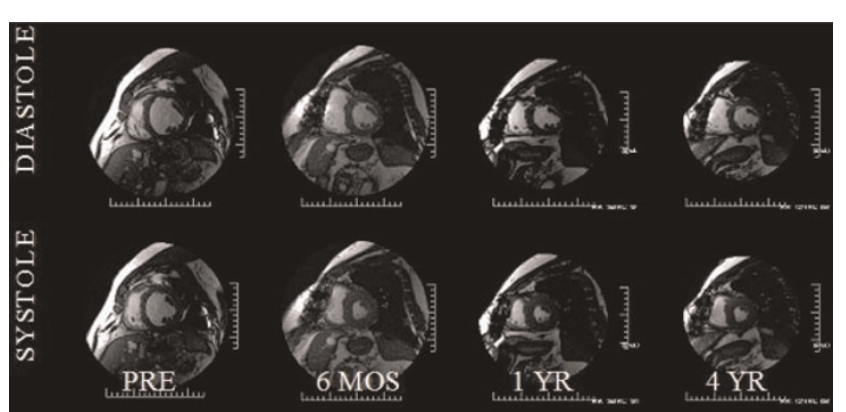


increase at 4 yrs $(49 \pm 14 \mathrm{ml}, \mathrm{p}=0.3)$. Most importantly, LVMI/ volume, a 3D measure of LV geometry, remained unchanged initially but over 4 years markedly improved $(1.07 \pm 0.2$ to $0.94 \pm 0.24, p<0.05$ ), all paralleling improvements in NYHA (3.2 \pm I.0 to I.5 \pm I.I, $\mathrm{p}<0.05)$. Figure I.

Conclusion: After the initial beneficial effects imparted by AVR in severe AS patients, there are, as expected, marked improvements in LV reverse remodeling. We have shown, via CMR, that surgically induced benefits to LV structure and function, including favorable alterations in LV geometry, are durable and, unexpectedly, show continued improvement extending through 4 years post-AVR concordant with sustained improved clinical status. The pattern of this improvement is, however, previously unrecognized. Namely, a steep trajectory of improvement early in which fully $75 \%$ of the effect that was to be present by year 4 is completed within the first 6 months. Afterwards, the slope of LV remodeling is much less steep and appears to become asymptotic.

\section{P208}

Associations of plasma C-Reactive Protein and osteopontin levels with the severities of coronary and aortic atherosclerosis

Yukihiko Momiyama', Reiko Ohmori ${ }^{2}$, Zahi A Fayad ${ }^{3}$, Teruyoshi Kihara ${ }^{4}$, Ryuichi Kato ${ }^{2}$, Hiroaki Taniguchi ${ }^{2}$, Masayoshi Nagata ${ }^{4}$, Haruo Nakamura ${ }^{2}$ and Fumitaka Ohsuzu ${ }^{2}$

INational Hospital Organization Tokyo Medical Center, Tokyo, Japan

${ }^{2}$ National Defense Medical College, Saitama, Japan

${ }^{3}$ Mount Sinai School of Medicine, New York, NY, USA

${ }^{4}$ Iruma Heart Hospital, Saitama, Japan

Journal of Cardiovascular Magnetic Resonance 2009, I I(SuppI I):P208

Introduction: Limited correlations between biomarkers (e.g. C-reactive protein (CRP) and osteopontin (OPN)) and the severity of coronary artery disease (CAD) have been reported. Recently, MRI became a useful tool for non-invasively evaluating atherosclerotic plaques in both thoracic and abdominal aortas. Using MRI, we investigated the associations of plasma CRP and OPN levels with the severities of coronary and aortic atherosclerosis.

Methods: Aortic MRI was performed on Signa I.5 T Cvi with a phased-array body coil in 136 patients undergoing coronary angiography. Transverse PDW and T2W images of thoracic descending and abdominal aortas were obtained using an ECGgated, double-inversion-recovery FSE sequence. Imaging parameters were $T R=2 R R$ intervals, $T E=10(P D W)$ and $60 \mathrm{~ms}$ (T2W), 20-cm FOV, 4-mm slice thickness, 8-mm inter-slice gap, $256 \times 256$ acquisition matrix, and 32 echo-train. For each patient, 9 slices of thoracic aorta and 9 slices of abdominal aorta were obtained at 12-mm intervals, which each covered about $10-\mathrm{cm}$ portion of thoracic aorta below the arch and $10-\mathrm{cm}$ portion of abdominal aorta above the bifurcation of iliac artery. Plaque was defined as a clearly identified luminal protrusion with focal wall thickening, and plaque extent in each slice was scored 0 to 4 points by the percentage of luminal surface involved by plaque. The severity of aortic atherosclerosis was represented as the sum of scores (plaque score). On coronary angiograms, the degree of stenosis in each segment was evaluated by 5 grades.
The severity of coronary atherosclerosis was represented as the number of $>50 \%$ stenotic vessels and the number of $>25 \%$ stenotic segments. Plasma OPN and high-sensitivity CRP levels were measured by ELISA with a commercially available kit (Human OPN assay kit, IBL) and by a BNII nephelometer (Dade Behring), respectively. Any patients with acute coronary syndromes were excluded from this study.

Results: Of the 136 patients, 53 (39\%) were on statin treatment, and 96 (7I\%) had CAD (>50\% luminal diameter stenosis) on angiograms. Thoracic and abdominal aortic plaques were found in $88(65 \%)$ and $124(91 \%)$ patients, respectively. Patients with CAD had higher OPN (562 \pm 223 vs. $445 \pm$ $234 \mathrm{ng} / \mathrm{ml}$ ) and CRP (median 0.78 vs. $0.48 \mathrm{mg} / \mathrm{l}$ ) levels than those without CAD ( $<<0.02)$. Plasma OPN levels did not correlate with CRP levels. Stepwise increase in OPN levels was found depending on the number of $>50 \%$ stenotic coronary vessels: $445 \pm 234$ (OVD), 54I \pm 239 (IVD), $556 \pm 219$ (2VD), and $604 \pm 21 \mathrm{l} \mathrm{ng} / \mathrm{ml}(3 \mathrm{VD})(\mathrm{P}<0.05)$. OPN levels correlated with the number of $>25 \%$ stenotic coronary segments $(r=0.23$ by Spearman's rank correlation test, $\mathrm{P}<0.0 \mathrm{I}$ ). Plasma CRP levels also stepwise increased depending on the number of stenotic vessels: $0.48,0.70,0.74$, and $0.88 \mathrm{mg} / \mathrm{l}(P=N S)$. CRP levels correlated with the number of stenotic segments $(r=0.2 \mathrm{I}$ by Spearman's rank correlation test, $\mathrm{P}<0.02$ ). Regarding aortic atherosclerosis, the 136 patients were divided into quartiles by plaque score. Stepwise increase in OPN levels was found depending on the quartiles of aortic plaque score: $451 \pm 165$ (QI), 47I \pm 236 (Q2), $560 \pm 232$ (Q3), and $629 \pm 252 \mathrm{ng} / \mathrm{ml}$ (Q4) $(P<0.02)$. OPN levels correlated with aortic plaque score $(r=0.26, P<0.005)$. CRP levels also stepwise increased on the quartiles: $0.40,0.56,1.08$, and $1.10 \mathrm{mg} / \mathrm{l}(P<0.00 \mathrm{I})$. CRP levels correlated with plaque score $(r=0.38, P<0.00 I)$. In multivariate analysis, aortic atherosclerosis was found to be an independent factor associated with both OPN and CRP levels, but coronary atherosclerosis was not.

Conclusion: Plasma OPN and CRP levels correlated with the severities of both coronary and aortic atherosclerosis, but these biomarkers are more likely to reflect the severity of aortic atherosclerosis than coronary atherosclerosis. MRI was useful for non-invasively evaluating atherosclerosis in thoracic and abdominal aortas.

\section{P209}

Does implantation of an Amplatzer septal occluder (ASO) device change ventricular contraction pattern? An MR tagging study

Heiko Stern and Sohrab Fratz

Deutsches Herzzentrum Munchen, München, Germany

Journal of Cardiovascular Magnetic Resonance 2009, I I(SuppI I):P209

Amplatzer occluder devices are used throughout the world for occlusion of atrial septal defects (ASD) or persistent foramen ovale (PFO). This foreign body remains in place for life time and could change the contraction pattern of the heart. The contraction pattern should be analyzed by MR tagging. In order to differentiate motion changes by unloading the right ventricle (RV) from changes by the presence of the ASO itself both patients with ASD and PFO were included, presuming that PFO occluders did not change ventricular volumes. 
Table I (abstract P209)

\begin{tabular}{|c|c|c|c|c|}
\hline & \multicolumn{2}{|l|}{ ASD } & \multicolumn{2}{|l|}{ PFO } \\
\hline & RV & LV & $\mathbf{R V}$ & LV \\
\hline Total rotation $\left({ }^{\circ}\right)$ & $-0,8$ & $-3,4 *$ & $-1,0$ & $-0,9$ \\
\hline Torsion $\left({ }^{\circ}\right)$ & $-0,4$ & 0 & 0 & $-0,3$ \\
\hline Circumferential Strain (\%) & $-2,2$ & $+0,6$ & $-1,9$ & $-1,4$ \\
\hline Radial shortening (\%) & $-2,8^{*}$ & $+0,6$ & $-1,1$ & $-1,7$ \\
\hline
\end{tabular}

$*=p<0.05$.

Patients: 16 pts with ASD and 9 pts with PFO were investigated by MR tagging before and after defect closure by ASO. None of the patients had additional heart disease or heart block.

Methods: MR tagging was performed for both RV and LV. Short axis cuts at three different levels of the ventricles were performed and an EPI-CSPAMM sequence (TE $7.5 \mathrm{~ms}$, EPI Factor II, Matrix $256 \times 128, \alpha=30^{\circ}$ ) used. Data analysis based on HARP. Rotation of both ventricles was measured during systole and diastole and rotation was summed up and given in degrees of total ventricular rotation. Torsion was defined as difference in rotation between base and apex of the heart.

Circumferential strain (CS) and radial shortening (RS) of RV and LV were measured. Ventricular volumes were calculated using contiguous short axis slices of RV and LV, stroke volumes of both ventricles were measured by MR flow measurement in aorta and pulmonary artery.

Results: $Q p / Q$ s was reduced significantly by ASO implantation in the ASD group $(p<0,002)$, but remained unchanged in PFO patients. Accordingly RV enddiastolic volume was reduced $(p<0,05)$ and LV volumes increased significantly $(p<0,005)$ by occluding the ASD, but volumes remained unchanged after implanting PFO occluders.

Absolute changes in tagging parameters are listed in Table I. Indicated are median values.

Conclusion: There were significant changes in ventricular contraction pattern after closure of the ASD by an Amplatzer occluder. This is however due to load changes in both ventricles and not due to the presence of the occluder itself. Implantation of the occluder itself, as shown in PFO patients, did not alter ventricular motion pattern, as assessed by MR tagging.

\section{P2IO}

Axial slices compared to short-axis slices for measurement of right and left ventricle volumes of patients with corrected Tetralogy of Fallot and Ebstein's anomaly by cardiac magnetic resonance Sohrab Fratz, Annika Schuhbaeck, Christina Buchner, Stefan Martinoff, John Hess and Heiko Stern Deutsches Herzzentrum München, Munich, Germany

Journal of Cardiovascular Magnetic Resonance 2009, I I(SuppI I):P2 10

Objective: To determine if axial slices or short-axis slices should be the method of choice for routine clinical measurement of right (RV) and left (LV) ventricle volumes of patients with congenital heart disease (CHD) by cardiac magnetic resonance (CMR).
Background: RV and LV volumes and function need to be measured reliably by CMR in the long-term follow-up of patients with CHD. However, no standard protocol to measure RV volumes by CMR exists. A standard protocol, LV short-axis slices, exists only for the LV. However, the basal boundary of the $\mathrm{RV}$ is difficult to trace using LV short-axis slices. Axial slices through the patient's chest have been suggested as an alternative. Axial slices are potentially beneficial compared to short-axis slices. Firstly, axial slices are very easy to plan. This is especially true in complex anatomy, as is often the case in CHD. Secondly, by using axial slices valuable morphologic information about the large vessels and atria is obtained without need of further scans. It is known that in normal individuals, RV volume measurements made from axial slices are feasible and have a superior reproducibility to conventional short-axis slices. However, using axial slices measuring RV volumes in patients with RV morphology and pathology has not been tested. Additionally, the reproducibility of measuring LV volumes from axial slices has never been studied, neither in normal individuals nor in patients with CHD. Therefore, taken together, to date the best slice orientation for the routine clinical measurement of RV and LV volumes in patients with CHD remains unknown.

Therefore, the aim of this study was to determine if short-axis or axial slices should be the method of choice for the routine clinical measurement of RV and LV volumes in patients with CHD. Methods: We studied two homogenous patient groups with RV pathology (corrected Tetralogy of Fallot, $n=46$ and Ebstein's anomaly, $n=15$ ) in which both axial slices and short-axis slices can easily be obtained by CMR. By using the f-test the inter-and intra-observer variances of both methods were compared for four volumes (enddiastolic and endsystolic RV and LV volumes) in both groups.

Results: Eight variances were significantly smaller in the axial data sets compared to the short-axis data sets. The remaining eight variances were not significantly different from another.

Conclusion: Routine clinical measurements of RV and LV volumes by CMR in patients with corrected Tetralogy of Fallot and Ebstein's anomaly should be aquired from axial slices and not short-axis slices. We furthermore suggest that axial slices should also be the method of choice for the routine clinical measurement of RV and LV volumes in all other forms of CHD.

\section{P2I I}

Computational simulations from cardiac magnetic resonance imaging reveal altered hemodynamics in coarctation patients previously treated by patch aortoplasty

John F LaDisa', Joseph Cava², Ronak Dholakia',

David C Wendell', Mary Krolikowski ${ }^{2}$

and Margaret Samyn ${ }^{2}$

'Marquette University, Milwaukee, WI, USA

${ }^{2}$ Children's Hospital of Wisconsin, Milwaukee, WI, USA

Journal of Cardiovascular Magnetic Resonance 2009, I I(SuppI I):P2 I I

Objective: The objective of this study is to quantify aortic hemodynamics in coarctation of the aorta (CoA) patients surgically treated by patch aortoplasty to determine if adverse conditions may be present in the region of the patch and, 
Figure I (abstract P2 I I)

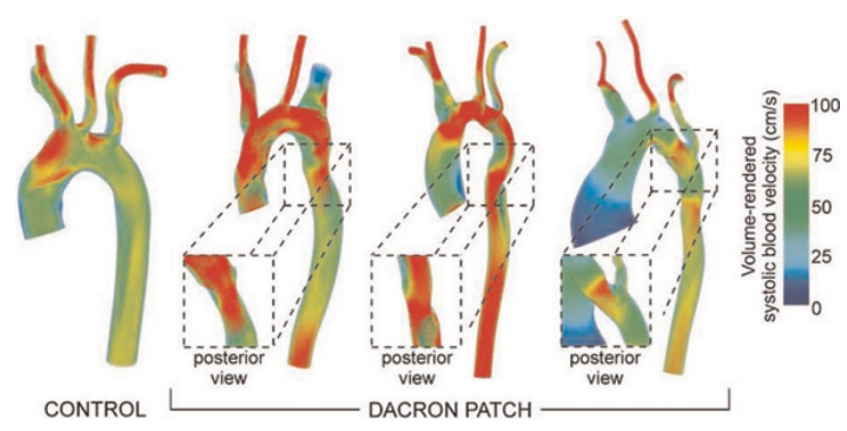

therefore, contribute to the high instance of aortic aneurysms observed in this patient population.

Background: CoA patients treated by patch aortoplasty show an increased prevalence of aneurysm development in regions associated with the patch. Altered hemodynamics may play a role in aneurysm formation and progression, especially if there is increased blood flow velocity through an associated hypoplastic transverse arch that impinges near the patch. We hypothesize that potentially adverse blood flow and wall shear stress (WSS) patterns are present in CoA patients, status post Dacron patch CoA repair.

Methods: Patients underwent clinically-indicated cardiac magnetic resonance imaging (cMRI) for anatomic definition of the aortic arch and assessment of blood flow. Gadolinium-enhanced cMRI angiography (TR/TE/FA: 4.3/I.46/25; thickness: 2 mm; FOV: $25 \times 38 \mathrm{~cm}$; acquisition matrix $384 \times 192$ ) was used to delineate the anatomy of the thoracic aorta and the innominate (IA), left carotid (LCCA), and left subclavian (LSA) arteries. Phasecontrast cMRI (PCMRI) was used to quantify blood flow just distal to the aortic valve and in the IA, LCCA, LSA and descending thoracic aorta (TR/TE/FA: 46/3.8/30; thickness: $5 \mathrm{~mm}$; FOV: $24 \times 32 \mathrm{~cm}$; acquisition matrix: $256 \times$ |44). Velocity encoding gradients were adjusted to maximize the dynamic range for each location. Patient-specific computational fluid dynamics (CFD) models were created from cMRI angiography data using cvSim software. Six data sets were obtained (25-33 yrs, mean 29) from which three models have been created to date. CFD model outlet boundary conditions that enable replication of blood flow obtained from PCMRI and cuff blood pressure measurements were applied. Simulations were performed using a stabilized finite element solver. Blood velocity and flow, pressure, and WSS were characterized and compared to a control patient (28 yr) with the same cardiac index $(2.5 \mathrm{~mL} / \mathrm{min} / \mathrm{m} 2)$.

Results: Exceptional reproduction of clinical blood pressure and flow were obtained from simulations. Time-averaged WSS in $5 \mathrm{~mm}$ bands throughout the aorta and branches was elevated in the transverse arch and repair site of Dacron CoA patients (ex. 36.7 vs $23.7 \mathrm{dyn} / \mathrm{cm} 2$ ). Descending aortic tortuosity was observed downstream of the repair site in CoA patients, leading to swirling and spatial WSS alterations relative to control (Figure I).

Conclusion: These results suggest that hemodynamic alterations, including blood flow patterns and distributions of WSS, are present in CoA patients treated by Dacron patch aortoplasty. The findings may offer insight into the high likelihood of aneurysm formation in these patients.
P2I 2 Anatomical and functional imaging of congenital
heart disease with phase contrast VIPR

Christopher J Francois, Elizabeth Nett, Kevin M Johnson, Darren Lum, Scott B Reeder and Oliver Wieben University of Wisconsin, Madison, WI, USA

Journal of Cardiovascular Magnetic Resonance 2009, I I(SuppI I):P2 12

Purpose: Demonstrate feasibility of PC VIPR for comprehensive cardiovascular imaging in congenital heart disease.

Introduction: Imaging of congenital heart disease usually requires multiple anatomical and functional scans followed by several 2D phase contrast (PC) acquisitions in a variety of oblique scan planes for flow measurements. A new acquisition technique, PC vastly undersampled isotropic projection reconstruction (PC VIPR), that allows for volumetric 3D CINE flow imaging with high spatial and temporal resolution over a large field of view in a reasonable scan time has been developed [I]. The applicability of this approach has been extended by implementing a respiratory gating scheme that allows for free breathing acquisition [2]. The high spatial resolution with isotropic voxels improves image quality compared to standard PC MR by minimizing intravoxel dephasing effects and allows for retrospective data reformatting including flow measurements in arbitrary planes.

Methods: A dual-echo PC VIPR acquisition with a PILS reconstruction and respiratory gating was implemented on our I.5 Tand $3 \mathrm{~T}$ clinical systems (GE Healthcare). To reliably achieve high quality images, several correction schemes were applied to account for the effects of TI-saturation, trajectory errors, motion, and aliasing associated with undersampling. Typical scan parameters were: $62.5 \mathrm{kHz}$ receiver bandwidth, $\left(1.0-1.25 \mathrm{~mm}^{3}\right.$ ) isotropic spatial resolution in approximately $10 \mathrm{~min}$ scan time with $50 \%$ respiratory gating efficiency, imaging volume: $32 \times 32 \times 16 \mathrm{~cm}^{3}$, VENC of $50-100 \mathrm{~cm} / \mathrm{s}$ (application specific). Cardiac gating was performed retrospectively with a temporal filter for radial acquisitions, similar to view sharing in Fourier sampling [3]. PC VIPR data were acquired after obtaining patient consent according to our IRB protocol in a total of 17 consecutive patients with a variety of pathology including aortic coarctation, Scimitar syndrome, double inlet left ventricle, and atrial septal defects, among others. The PC VIPR data were

Figure I (abstract P2 I 2)

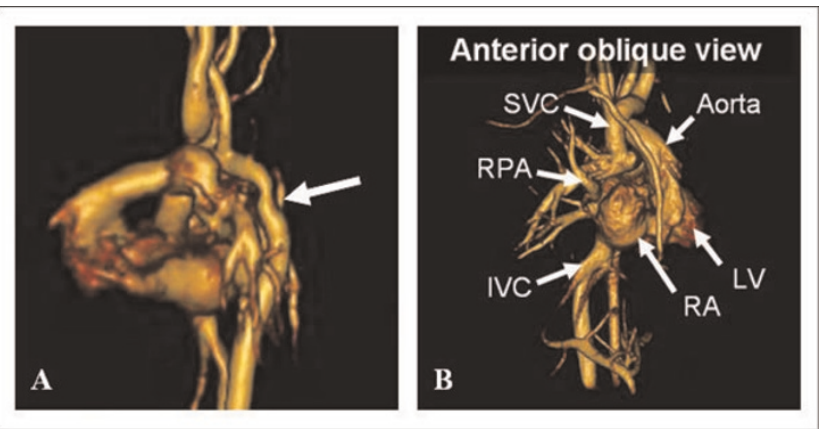

Volume rendered images in (A) 2 month-old with aortic coarctation (arrow) and (B) patient with bidirectional Glenn for DILV. 
Figure 2 (abstract P2 I 2)

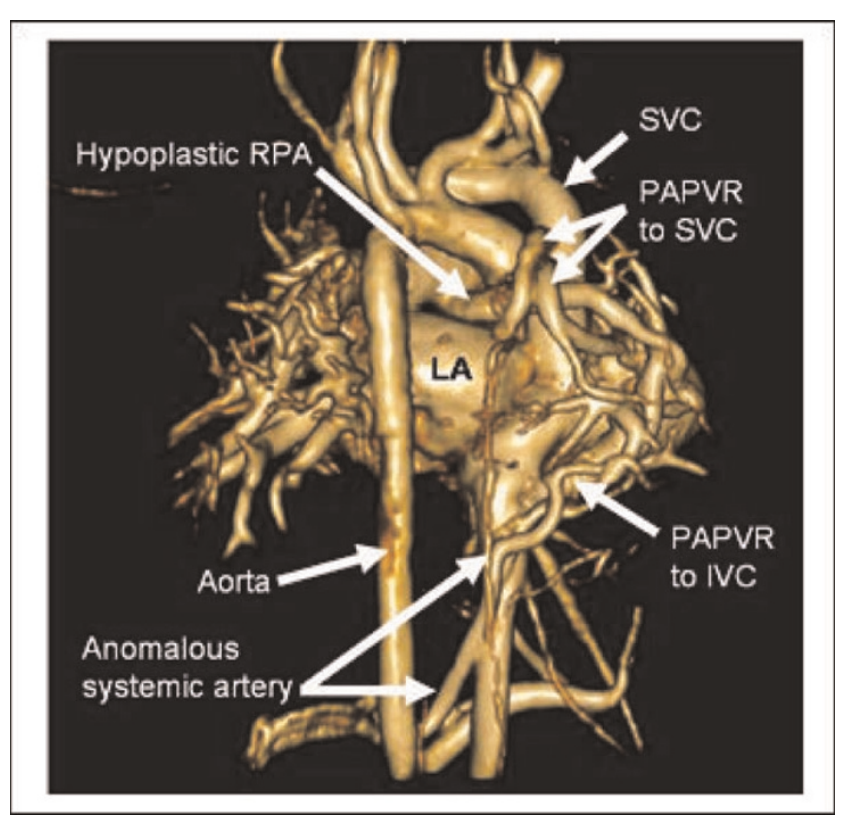

Volume rendered image in 18 month-old with pulmonary venolobar syndrome consisting of hypoplastic right pulmonary artery (RPA), partial anomalous pulmonary venous return (PAPVR) to superior vena cava (SVC) and inferior vena cava (IVC), and anomalous systemic pulmonary artery from the abdominal aorta to the right lower lobe.

reconstructed as magnitude images, velocity vector fields, and angiograms calculated similar to complex difference images. CEMRA and 2D PC images were used for comparisons when available.

Results: PC VIPR data sets were successfully acquired in all patients. MR angiograms were created using the average flow PC VIPR dataset (Figure I) to visualize cardiovascular structures. All anatomical structures visualized on CEMRA images were identified on PCVIPR images. This includes a 2 year-old with pulmonary venolobar syndrome with an anomalous pulmonary venous return to the SVC and IVC, in addition to an anomalous

Figure 3 (abstract P2| 2)

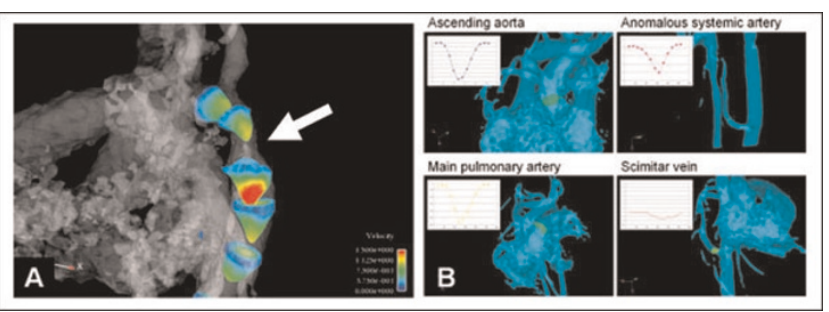

(A) Flow velocity profiles in patient shown in Fig. Ia. The highest velocity is immediately distal to coarcation (arrow). (B) Flow-time curves were measured at four different locations in patient shown in Fig. 2 using advanced visualization software. With PC VIPR, measurements can be made in any arbirtrary location after the images have been acquired. systemic artery (Figure 2). In addition, individual cardiac frames were reconstructed (with reduced SNR) and the velocity fields were visualized and analyzed with advanced software (EnSight), allowing for assessment of hemodynamics (Figure 3).

Conclusion: Comprehensive anatomical and functional cardiovascular MRI of congenital heart disease can be performed using PC VIPR, providing a powerful new tool for noninvasive diagnosis.

References

I. Gu TL, et al: AJNR 2005, 26(4):743-749.

2. Johnson KM, et al: Proc ISMRM 2008, 733.

3. Liu J, et al: IEEE TMI 2006, 25(2):|48-I57.

\section{P213}

Dependence of arterial input function on position in the left ventricle and time in the cardiac cycle Nicholas S DuRocher, Elodie Breton, Sohae Chung, Daniel Kim and Leon Axel NYU Langone Medical Center - Center for Biomedical Imaging, New York, NY, USA

Journal of Cardiovascular Magnetic Resonance 2009, I I(SuppI I):P2 I 3

Introduction: Determination of the arterial input function (AIF) is important in assessing myocardial perfusion with first pass perfusion cardiac MRI (CMR). While the signal-time curve measured at the aortic root level is likely to be the best estimate of the AIF to the left ventricle (LV) wall, because of proximity to the coronary artery openings, most studies determine the AIF from the blood pool inside the LV, since it does not require any additional data acquisition. Previous studies did not report significant differences in AIFs measured at different LV/aorta positions or at different times of the cardiac cycle $[I, 2]$. However, we have observed differences when analyzing the AIF in certain studies and sought to investigate further. In this work we investigated if there were significant differences in the measured AIF depending on where in the LV it was measured, and on when during the cardiac cycle it was measured.

Methods: First-pass perfusion MRI, using saturation-recovery TurboFLASH readouts with the following parameters: FOV = $340 \times 255 \mathrm{~mm} ;$ Matrix $=64 \times 48 ; \mathrm{TE} / \mathrm{TR}=\mathrm{I} .12 / 2.3 \mathrm{~ms}$; $\mathrm{TD}=12.4 \mathrm{~ms}$; Image Acquisition Time $=55.2 \mathrm{~ms}$; Total Acquisition Time $=76.2 \mathrm{~ms}$, was performed 3 times in a healthy volunteer in a $3 \mathrm{~T}$ whole-body MR scanner (Tim Trio, Siemens, Germany). Immediately following a $5 \mathrm{~mL}$ bolus injection of Gd-DTPA $(0.5 \mathrm{M})$ into the right antecubital vein, 8 to 10 identically positioned long axis three-chamber view images were successively acquired in every cardiac cycle. In the resulting images, regions of interest (ROIs) were manually drawn at 6 different locations in the heart: left atrium, base level close to the mitral valve, base level close to the aortic valve, total base level, apex of the left ventricle, and aortic root. The time-to-peak (TTP) of the signal intensity curve was independently assessed at every time after the QRS (cardiac phase) and in every location.

Results: Figure la shows a typical signal-time curve obtained at the base level when plotting all the cardiac phase acquisitions together. This curve shows an oscillation during the cardiac cycle superimposed on the main contrast enhancement curve. The TTP obtained for the 6 different LV areas are represented in 


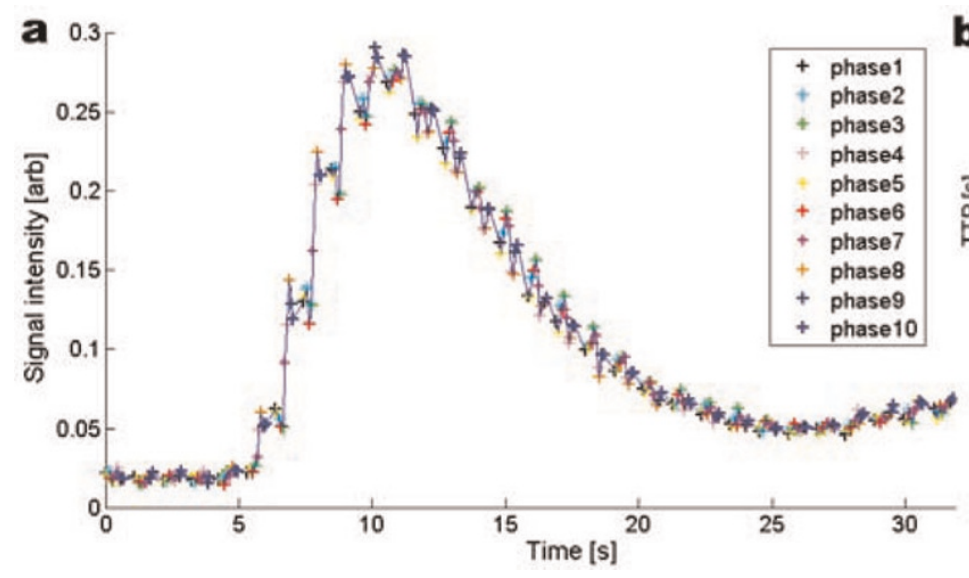

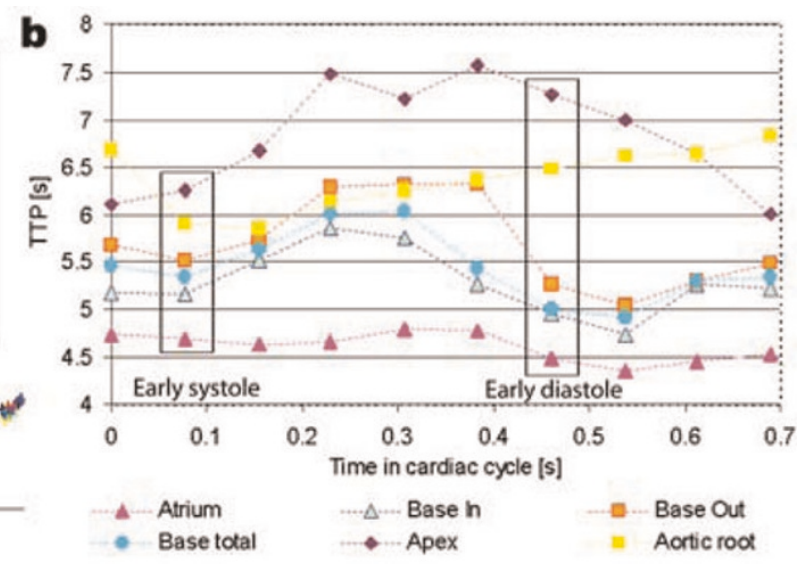

Figure Ib, separately for phase when the data have been acquired. Following the QRS complex, the heart contracts and the blood is ejected, making TTP's in the aortic root and at the base level close to the aortic valve similar in systole. During diastole, the aortic valve closes, and while the contrast levels remain relatively constant in the aortic root, the TTP inside the LV changes, reflecting ventricular filling. The TTP at the apex is approximately I s longer than the base TTP in systole, and it increases to about $2 \mathrm{~s}$ longer in early diastole.

Conclusion: In a healthy volunteer, we found that at every location considered (atrium/LV/aorta), the contrast enhancement curve fluctuates, depending on when the data have been acquired in the cardiac cycle. Moreover, the AIFs measured at the base and apex levels have significantly different time courses, as reflected in their TTP, which might result in different calculations of cardiac perfusion, depending on which is used as the AIF. We found that the signal-time curve at the base level near the aortic valve is a good approximation of the aortic root AIF in systole, but not in diastole. Further experiments are required in order to study the influence of these time differences in AIF curves, relative to the acquisition's localization and time in the cardiac cycle, on the analysis of first pass perfusion CMR.

\section{References}

I. Jerosch-Herold, et al: J Magn Reson Imaging 2004, 19:758770.

2. Ivancevic, et al: J Magn Reson Imaging 2003, I 8:372-376.

\section{P2I 4}

Use of a dark blood sequence to localize the esophagus prior to RF ablation and to assess left atrial edema post ablation

Sathya Vijayakumar, Eugene G Kholmovski,

Edward VR DiBella, Josh R Bertola

and Nassir F Marrouche

University of Utah, Salt Lake City, UT, USA

Journal of Cardiovascular Magnetic Resonance 2009, I I(SuppI I):P2 14

Aim: The aim of this study is to present an MRI technique to localize the esophagus prior to RF ablation procedure, while also tracking left atrial (LA) wall thickness as a sign of acute edema post-ablation.

Introduction: Lately, ablation targeting arrhythmogenic pulmonary vein (PV) foci for drug-refractory paroxysmal atrial fibrillation has become one of the standard methods of pulmonary vein isolation (PVI). CT or MRI is commonly performed prior to ablation for procedure guidance. Localization of the esophagus is important pre-ablation to prevent fatal atrioesophageal fistula, especially in view of a need for transmural atrial lesions. While CT is relatively established for esophageal localization [I, 2], MRI may also be useful for this purpose. Also, measuring LA edema after PVI could increase understanding of patient response to the procedure. Okada et al [3] recently reported the use of electron beam tomography to visualize LA edema after PVI. Here, we present the use of a dark blood MRI sequence that can localize the esophagus and be used to measure LA wall thickening, indicative of edema.

Methods: This study obtained and assessed data from 13 patients aged $68.8 \pm$ II.I years ranging from 49-83 years of age, 7 male and 6 female. Patients were imaged before PVI procedure, to help localize the esophagus, 24 hours, and 3 months post-procedure to assess the presence of LA edema. A respiratory navigated, black blood prepared turbo spin echo sequence was used with a TR of I RR, TE 6I ms, ETL 22, slice thickness $4 \mathrm{~mm}$, FOV $36 \mathrm{~mm}, 288 \times 288$ matrix, parallel imaging with reduction factor 2 and 44 reference lines. 18-22 continuous transaxial slices were acquired to cover the LA.

Image analysis was performed using the OsiriX Imaging Software. For localization of the esophagus with respect to PVs, the distance between the center of each PV ostium and the center of the esophagus was measured. The distance between the center

Table I (abstract P2 |4) Mean \pm SD for relative distances of the esophagus from the LIPV, RIPV and posterior wall of LA

\begin{tabular}{lll}
\hline $\begin{array}{l}\text { Distance } \\
\text { from LIPV }(\mathbf{c m})\end{array}$ & $\begin{array}{l}\text { Distance } \\
\text { from RIPV }(\mathbf{c m})\end{array}$ & $\begin{array}{l}\text { Distance from } \\
\text { posterior wall of LA }(\mathbf{c m})\end{array}$ \\
\hline $2.02 \pm 1.04$ & $3.64 \pm 0.89$ & $0.39 \pm 0.06$ \\
\hline
\end{tabular}




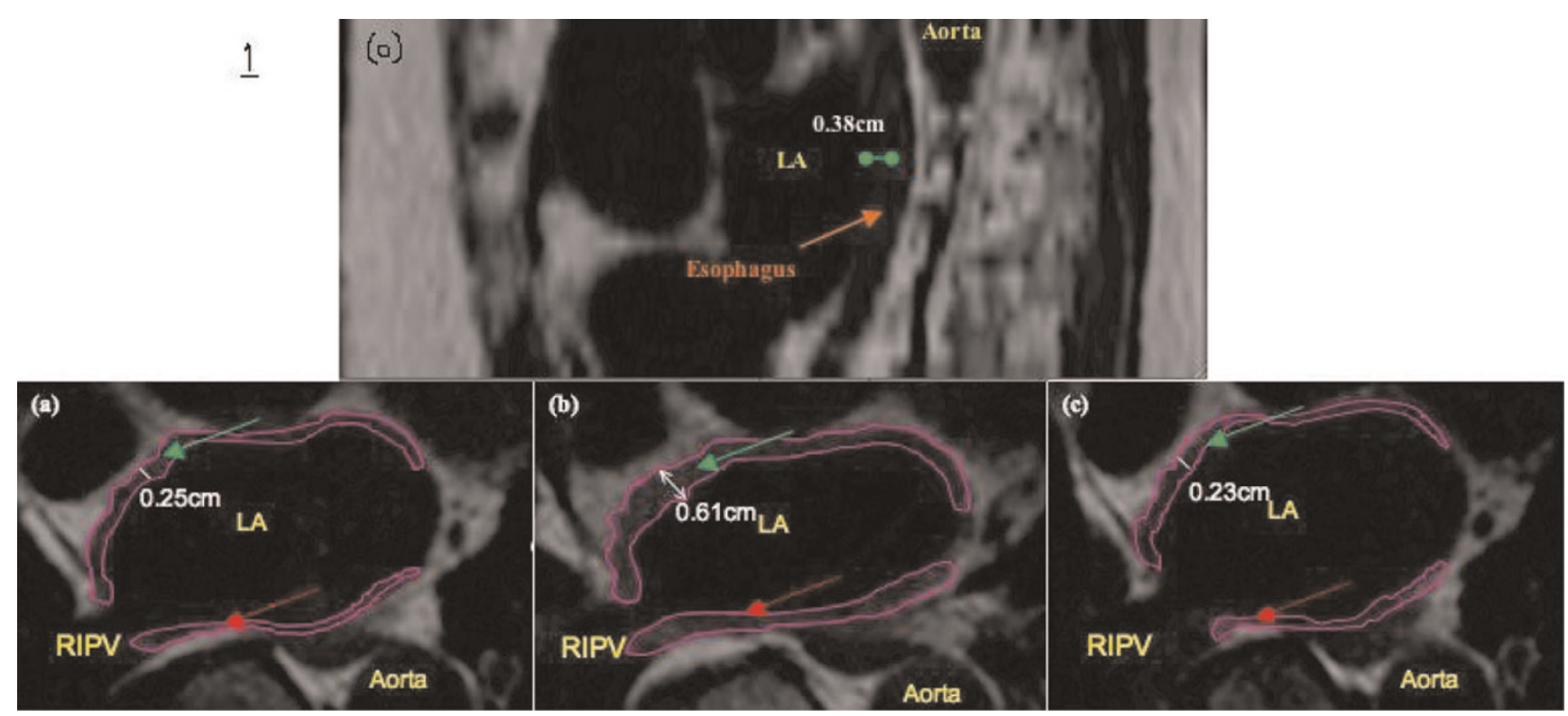

of esophagus and posterior wall of the LA was assessed in a similar way (Figure I(o)).

Figures I $(a, b, c)$ illustrate an example of how wall thickness was assessed, and how contours were drawn for measurement of areas of the anterior and posterior wall of the LA. Paired Student's t-test was used to compare the width of the LA wall, and areas of the anterior and posterior walls of the LA, at the three timepoints (pre, 24-hour and 3-month post procedure).

Results: Table I shows distances between the esophagus and PV ostia and posterior wall of LA. The mean \% increase in wall thickness and areas of posterior and anterior walls of the LA, measured 24-hours post ablation, were $53 \pm 22,51 \pm 29$ and $42.6 \pm 16$ respectively. $P<0.000$ I for all three of them implies this statistically significant increase in LA wall thickness is likely from acute edema of the LA wall [3]. It was also determined that the wall thickness, areas of posterior and anterior walls measured pre-ablation and 3-month post-ablation were not statistically significantly different $(p=0.095, p=0.5 \mathrm{I}, \mathrm{p}=0.24)$ respectively, implying that the edema visualized 24 -hour post $\mathrm{PVI}$ procedure was resolved by 3 months post-procedure.

Conclusion: This study shows that using MRI, the esophagus can be localized prior to ablation, and edema caused as a result of PVI using RF ablation can be successfully visualized. LA wall thickness changed significantly between pre and 24-hour post procedure images. With time, this edema was found to resolve itself and the measurements made 3 months postprocedure and pre-procedure were not statistically significantly different. The results obtained are comparable to those recently reported using $\mathrm{CT}$.

\section{References}

I. Monnig, et al: Europace 2005, 7:540-545.

2. Kottamp H, et al: JCEP 2005, I 6(2):|46-I50.

3. Okada T, et al: JACC 2007, 49:|436-|442.
P2I 5

Investigation of T2-weighted and

delayed enhancement magnetic resonance

imaging in reperfused acute myocardial

infarction in a porcine model

Seong Hoon Choi', Joon-Won Kang',

Sang II Choi ${ }^{2}$, Eun Ju Chun ${ }^{2}$, Byung Han Lee' and

Tae-Hwan Lim'

'Department of Radiology, Asan Medical Center,

University of Ulsan College of Medicine, Seoul,

Republic of Korea

${ }^{2}$ Department of Radiology, Seoul National

University Bundang Hospital, Seong-Nam,

Republic of Korea

Journal of Cardiovascular Magnetic Resonance 2009, I I(SuppI I):P2 I5

Introduction: It is well konwn that high signal intensity of infarcted myocardium on T2-weighted image is related to increased hydrogen density and increased T2 relaxation time. However, in recent studies, it has been reported that affected myocardium in acute or subacute myocardial infarction could be present with low signal intensity on T2-weighted image, and it is related to microvascular obstruction (MO). Intramyocardial hemorrhage can result from $\mathrm{MO}$ and may be related with the extent of MO.

Purpose: To assess the pattern of signal intensity on T2weighted image $(\mathrm{T} 2 \mathrm{WI})$ and their relation to persistent microvascular obstruction (PMO) on DE-MRI and intramyocardial hemorrhage on gross specimen in a porcine model with reperfused acute myocardial infarction.

Methods: Of the 15 pigs studied, nine underwent a 90 -minute occlusion of left anterior descending artery followed by a 90-minute reperfusion and six underwent a 180 -minute occlusion followed by a 90-minute reperfusion. ECG-triggered, 
breath-hold, turbo spin-echo T2WI was obtained along the short axis of the heart before contrast administration. Fifteen minutes after contrast administration, DE-MRI was acquired. The pattern of the signal intensity on T2WI was evaluated. The contrast ratio of the signal intensities between the infarcted and normal areas was calculated on T2WI. Visual assessment of the extent of hemorrhage on the gross specimen was performed after sacrificing the animals. Each myocardial specimen was then stained with 2,3,5-triphenyltetrazolium chloride (TTC).

Results: On T2WI, the infarcted area was shown as homogeneous high signal in eight (group $\mathrm{A}$ ) and as low or iso-signal intensity in seven pigs (group B). In group B, T2WI of the infarcted area was observed as central low signal with peripheral high signal in five pigs and as diffuse low or iso-signal intensity in the entire infarted myocardium in two pigs. The T2 contrast ratio of the infarcted area in group B was significantly lower than that in group A $(1.27 \pm 0.24$ vs. I.73 \pm 0.27 , $p<0.05)$. Myocardial infarction was seen in all fifteen pigs on DE-MRI, and PMO was observed in II out of the I5 pigs. The T2 contrast ratio of the infarcted area in pigs with PMO was significantly lower than that in pigs without PMO ( $1.38 \pm 0.25$ vs. $\mathrm{I} .89 \pm 0.3 \mathrm{I}, p<0.05)$. A strong inverse correlation was found between the extent of PMO and the T2 contrast ratio $(r=-0.8$, $p<0.05)$. The T2 contrast ratio of infarcted area in the pigs who underwent the 180-minute occlusion was significantly lower than that in those underwent who the 90-minute occlusion $(1.26 \pm 0.27$ vs. $1.68 \pm 0.28, p<0.05)$. The extent of PMO in the pigs who underwent the 180 -minute occlusion was significantly larger than that in pigs who underwent the 90 -minute occlusion $(58.6 \%$ vs. $32.1 \%, p<0.05)$. The myocardial infarcts on TTC staining were evident in all 15 pigs. On gross specimen, hemorrhage in the infarcted area was apparently visible in the pigs with PMO. A strong inverse correlation was found between the extent of hemorrhage in the infarcted area and the T2 contrast ratio $(r=-0.78, p<0.05)$. The extent of hemorrhage in the infarct area correlated well with the extent of PMO $(r=0.97, p<0.05)$.

Conclusion: From this study, we conclude that the patterns of signal intensity in infarcted myocardium on $\mathrm{T} 2 \mathrm{WI}$ were variable in a porcine model with reperfused acute myocardial infarction. The lower contrast ratio of infarcted area on $\mathrm{T} 2 \mathrm{WI}$ significantly correlates with the extent of PMO on DE-MRI and intramyocardial hemorrhage on gross specimen.

\section{P216}

T2 weighted three dimensional imaging of the whole heart

Yiu-Cho Chung', Orlando P Simonetti ${ }^{2,3}$

and Renate Jerecic ${ }^{4,5}$

'Siemens Healthcare USA, Columbus, OH, USA

${ }^{2}$ Ohio State University, Columbus, OH, USA

${ }^{3}$ The Ohio State University, Columbus, OH, USA

${ }^{4}$ Siemens Healthcare USA, Chicago, IL, USA

${ }^{5}$ Siemens Medical Solutions USA, Inc., Chicago, IL, USA

Journal of Cardiovascular Magnetic Resonance 2009, I I(SuppI I):P2 I6

Introduction: T2 weighted (T2w) dark blood turbo spin echo (DB-TSE) [I] is useful in myocardial tissue characterization such
Table I (abstract P2 I6) Optimal imaging parameters for cardiac imaging with T2w-SPACE

\begin{tabular}{llllll}
\hline $\begin{array}{l}\text { Flip angle } \\
\text { series }\end{array}$ & $\begin{array}{l}\text { Echo } \\
\text { spacing }\end{array}$ & $\begin{array}{l}\text { Echo train } \\
\text { (ETL) }\end{array}$ & $\begin{array}{l}\text { Slice turbo } \\
\text { factor }\end{array}$ & $\begin{array}{l}\text { Echo train } \\
\text { duration }\end{array}$ & TE \\
\hline VFL & $2.5 \mathrm{~ms}$ & 85 & $\mathrm{l}$ & $207 \mathrm{~ms}$ & $115 \mathrm{~ms}$ \\
CFL & $2.9 \mathrm{~ms}$ & 27 & 2 & $150 \mathrm{~ms}$ & $74 \mathrm{~ms}$ \\
\hline
\end{tabular}

as acute myocarditis and acute myocardial infarction [2, 3]. 3D acquisitions can provide high resolution images of the whole heart, allowing arbitrary views of the organ, and reducing partial volume effect. Conventional 3D TSE improves SNR but not sampling efficiency or spatial resolution. We propose a novel TSE technique for T2w 3D imaging of the whole heart that provides higher spatial resolution and reduces scan time per slice compared to 2D DB-TSE.

Purpose: To study the feasibility of a T2w 3D TSE technique for whole heart imaging with improved spatial resolution and imaging efficiency compared to 2D DB-TSE.

Methods: Sequence: The sequence (aka T2w-SPACE) uses non-selective refocusing pulses [4] to reduce echo spacing and cardiac motion sensitivity. It supports constant (CFL) and variable flip angle (VFL) refocusing pulses. VFL uses longer echo train (ETL) than CFL while maintaining good T2 weighting [4]. Optional motion sensitizing gradient (MSG) was implemented to suppress blood [5]. Navigator gating was supported. The technique was evaluated on a $1.5 \mathrm{~T}$ scanner (MAGNETOM Avanto, Siemens, Germany).

Imaging: T2w-SPACE was first optimized in 5 healthy volunteers (STIR contrast on two of them) using these parameters: inplane voxel $=1.6-1.8 \mathrm{~mm}$ isotropic, partition $=2.5-4 \mathrm{~mm}$, 28-40 partitions, 2 averages, TR $=2$ heartbeats, bandwidth/ pixel $=840 \mathrm{~Hz}, \quad 100 \%$ partition resolution. T2w-SPACE with MSG of $25 \mathrm{mT} / \mathrm{m}^{*} \mathrm{~ms}$ (3 directions) was then compared with 2D DB-TSE in 4 other healthy volunteers using the optimal parameters. SAX views were used. In the comparison, common parameters for 2D and $3 D$ acquisitions were: inplane resolution $=1.8 \mathrm{~mm}$ isotropic, fatsat, $100 \%$ phase resolution, parallel imaging rate 2 (24 reference lines), and navigator gating. 12 slices and 36 partitions (each $3.2 \mathrm{~mm}$ ) were acquired by 2D DB-TSE and T2w-SPACE respectively. 2D specific parameters were: slice $=8 \mathrm{~mm}, E T L=23$, bandwidth $/$ pixel $=300 \mathrm{~Hz}$, echo spacing $=7 \mathrm{~ms}$, TE $=56 / 76 \mathrm{~ms}$.

Figure I (abstract P2 16)

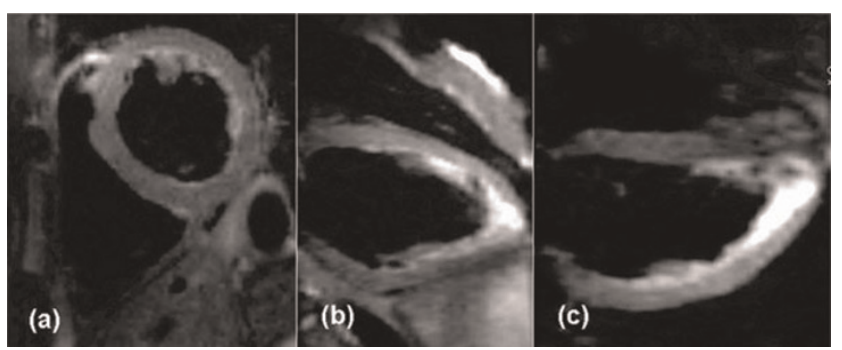

Three cardiac views of a T2w-SPACE acquisition. 
Figure 2 (abstract P2 I6)

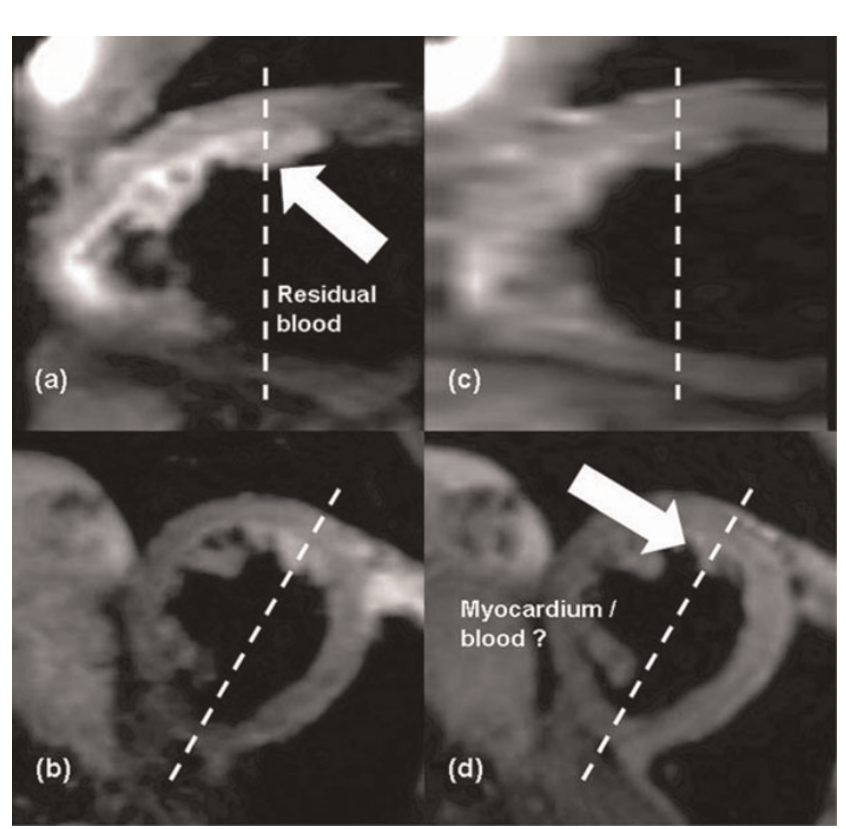

Partially suppressed blood may mimic myocardium in 2D DB-TSE. (a) VLA and (b) SAX views from T2w-SPACE, (c) VLA and (d) SAX views from 2D DB-TSE.

Results: From the 5 volunteers, VFL was preferred in subjects with slow heart rate $(R R \geq 800 \mathrm{~ms})$ while $\mathrm{CFL}\left(150^{\circ}\right)$ was suitable for subjects with fast heart rate. Scan times ranged from $2.6 \mathrm{~min}$ (VFL, $5.6 \mathrm{~s} /$ partitions, 65\% navigator efficiency) to 8.2 min (CFL, 13.7 s/partitions, $47 \%$ navigator efficiency). T2wSPACE gave consistently good image quality using parameters in Table I.

In the comparison study, all volunteers had $R R \geq 800 \mathrm{~ms}$, and VFL was used. The echo train in 2D DB-TSE was limited to $161 \mathrm{~ms}$, avoiding excessive T2 decay and signal drop-off. VLA and HLA views can be obtained from T2w-SPACE easily using MPR (Figure I). Image quality of 2D and 3D images were very comparable except that blood was better suppressed in 2D images. Figure 2 shows comparative images from T2w-SPACE and 2D DB-TSE (co-registered) from one volunteer. Note that the partially suppressed blood may mimic myocardial signal in 2D DB-TSE. Scan time per partition varied from $7.3 \mathrm{sec}$ to $13 \mathrm{sec}$ in T2w-SPACE and from $6 \mathrm{sec}$ to $30 \mathrm{sec}$ in 2D DB-TSE.

Discussion: T2w-SPACE for whole heart imaging is feasible. It requires 2 and 3 excitations/partition for the VFL and CFL mode respectively and is two times more efficient than 2D DB-TSE, sparing time for improved slice/partition resolution. In reality, heart rate and navigator efficiency determine scan times in both cases. The technique would also be relevant to myocardial tissue characterization at $3 \mathrm{~T}$ and beyond.

\section{References}

I. Simonetti OP, et al: Radiology 1996, 199(1):49.

2. Abdel-Aty H, et al: JACC 2005, 45(I I): I8I5.

3. Abdel-Aty H, et al: Circulation 2004, I 09(20):2080.

4. Mugler JP and Brookeman JR: Proc I2th ISMRM 2004, 695.

5. Koktzoglou IJ and Li D: JCMR 2007, 9(I):33.
P2 I 7

Qualitative and quantitative comparison of TGRAPPA and TSENSE real-time cine techniques during deep breathing

Mihaela Jekic', Yu Ding', Jennifer Dickerson',

Ali Merchant', YiuCho Chung ${ }^{2}$ and Orlando P Simonetti ${ }^{1}$

'Ohio State University, Columbus, OH, USA

${ }^{2}$ Siemens Medical Solutions, Columbus, OH, USA

Journal of Cardiovascular Magnetic Resonance 2009, I I(SuppI I):P2 17

Objetive: To compare the performance of real-time cine imaging using TSENSE and TGRAPPA acceleration methods during deep breathing.

Background: The rapid heart rates and heavy breathing encountered during exercise stress MRI make it difficult to achieve the necessary spatial resolution, temporal resolution, and image quality with real-time cine techniques. Parallel imaging has enabled improved temporal resolution, but at the cost of increased artifact and noise. Temporal resolution can be further improved by reducing FOV in the phase encoded direction. However, in the case of deep breathing during or after exercise, the chest wall can move in and out of the FOV causing aliasing. Surface coils can also move with deep breathing, causing a mismatch between the coil sensitivity map and the actual coil position, another potential source of artifact [I]. GRAPPA is known to be less sensitive than SENSE to these effects [2]. TSENSE [3] and TGRAPPA [I] are techniques for dynamic imaging that derive coil sensitivity maps by interleaving and averaging the undersampled dynamic frames. Our hypothesis is that TGRAPPA will perform better than TSENSE under the condition of deep breathing that causes aliasing and coil sensitivity map errors.

Methods: We acquired cine series in three views (short-axis, vertical and horizontal long-axis) in 5 healthy subjects during deep breathing using SSFP real-time cine accelerated 3-fold with TSENSE and TGRAPPA on a Siemens I.5 T Avanto, resulting in a total of 30 image series. Sensitivity maps were obtained by interleaving and averaging all undersampled frames in each series. Scan parameters were: TE/TR $0.9 / 2.2 \mathrm{~ms}$, average FOV $374 \times 299 \mathrm{~mm}$, matrix $160 \times 84$, and temporal resolution

\section{Figure I (abstract P2I7)}

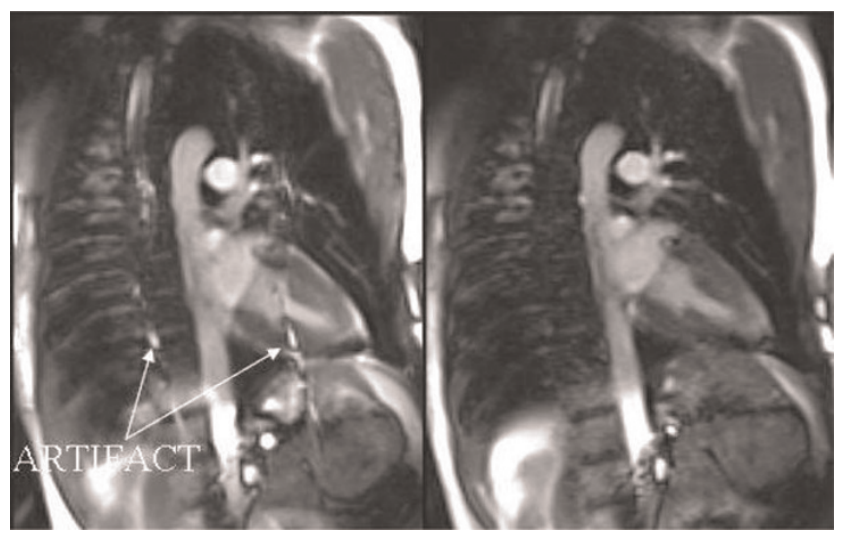

Increased artifact severity with TSENSE (left) vs. TGRAPPA (right) under deep breathing. 
$63.8 \pm 1.6 \mathrm{~ms}$. The FOV was specified immediately adjacent to the chest wall in the view in which the body appears largest in the phase encode direction (typically the VLA). Two experienced readers blinded to the study assigned qualitative scores of artifact severity: (I) none, (2) minor, (3) moderate, and (4) severe, and overall image quality: (I) excellent, (2) good, (3) diagnosis may be limited, and (4) poor, non-diagnostic. Artifacts were also quantitatively analyzed by examining peaks in the image autocorrelation function in the phase encode direction at shifts of $I / 3$ and $2 / 3 \mathrm{FOV}$, the expected locations for parallel imaging associated ghost artifacts at rate 3 acceleration. Within each series, the highest $10 \%$ of autocorrelation coefficients at I/3 FOV were averaged to define an "artifact index".

Results: TGRAPPA scores were superior to TSENSE both in terms of the physician-assessed image quality $(1.6 \pm 0.6$ vs. $2.3 \pm 0.7, p<.00 \mathrm{I})$ and artifact severity $(\mathrm{I} .7 \pm 0.6$ vs. $2.9 \pm 0.8$, $P<.001)$. The computed artifact index agreed with the qualitative artifact score, with TGRAPPA showing less artifact severity $(0.058 \pm 0.035$ vs. $0.110 \pm 0.052, p=.0036)$. Figure I illustrates the increased artifact level with TSENSE compared to TGRAPPA.

Conclusion: In the presence of deep breathing, TGRAPPA delivered superior image quality and artifact performance compared to TSENSE independent of FOV and aliasing, and exhibited less sensitivity to coil map errors. While SENSE provides an optimal reconstruction under normal conditions of breath-hold or quiet breathing, it is known to suffer from artifacts when the FOV is smaller than the object or the coil map is imperfect [2]. Our results indicate that TGRAPPA is preferred over TSENSE under conditions such as exercise stress, deep breathing, and patient motion.

References

I. Breuer FA, et al: Dynamic autocalibrated parallel imaging using temporal GRAPPA (TGRAPPA). Magn Reson Med 2005, 53:98I-985.

2. Griswold $M A$, et al: Field-of-view limitations in parallel imaging. Magn Reson Med 2004, 52: I I I8-I I 26.

3. Kellman P, et al: Adaptive sensitivity encoding incorporating temporal filtering (TSENSE). Magn Reson Med 2001, 45:846-852.

\section{P218}

Myocardial T2* mapping free of distortion using susceptibility weighted spin-echo based imaging: a feasibility study

Uwe Heinrichs', Jane F Utting', Fabian Hezel',

Gabriele A Krombach', Sebastian Kozerke ${ }^{2}$

and Thoralf Niendorf'

I RWTH Aachen, Aachen, Germany

${ }^{2}$ Institute for Biomedical Engineering, University and ETH

Zurich, Zuerich, Switzerland

Journal of Cardiovascular Magnetic Resonance 2009, I I(SuppI I):P2 I8

Introduction: Myocardial T2* mapping is of proven value for the assessment of myocardial iron content and tissue oxygenation [I, 2]. Conventionally, T2*-weighting is accomplished with gradient echo based or echo planar imaging techniques. However, the disadvantages of TI-related saturation effects, artifacts due to ventricular blood flow and image distortion must
Figure I (abstract P2 I8)

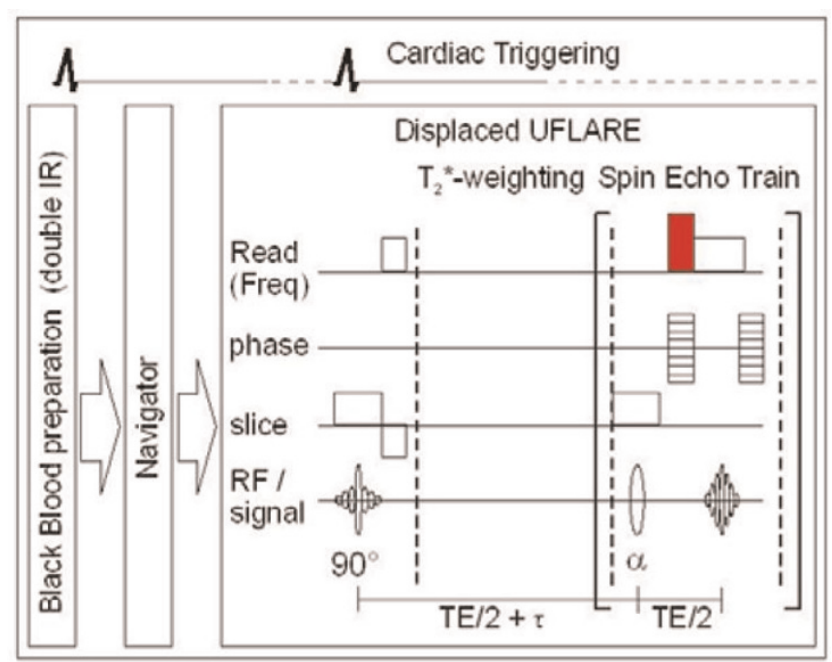

Basic scheme of cardiac and navigator gated, black blood prepared, susceptibility weighted UFLARE.

be addressed to pave the way for a broader clinical acceptance. Hence, spin-echo based acquisition strategies which generate T2* contrast free of distortion represent a valuable alternative. Purpose: This study demonstrates the promise of navigator gated, susceptibility weighted, fast spin-echo imaging in conjunction with ventricular black blood preparation, for anatomically accurate $\mathrm{T}^{*}$ mapping of the heart.

Figure 2 (abstract P2 | 8)

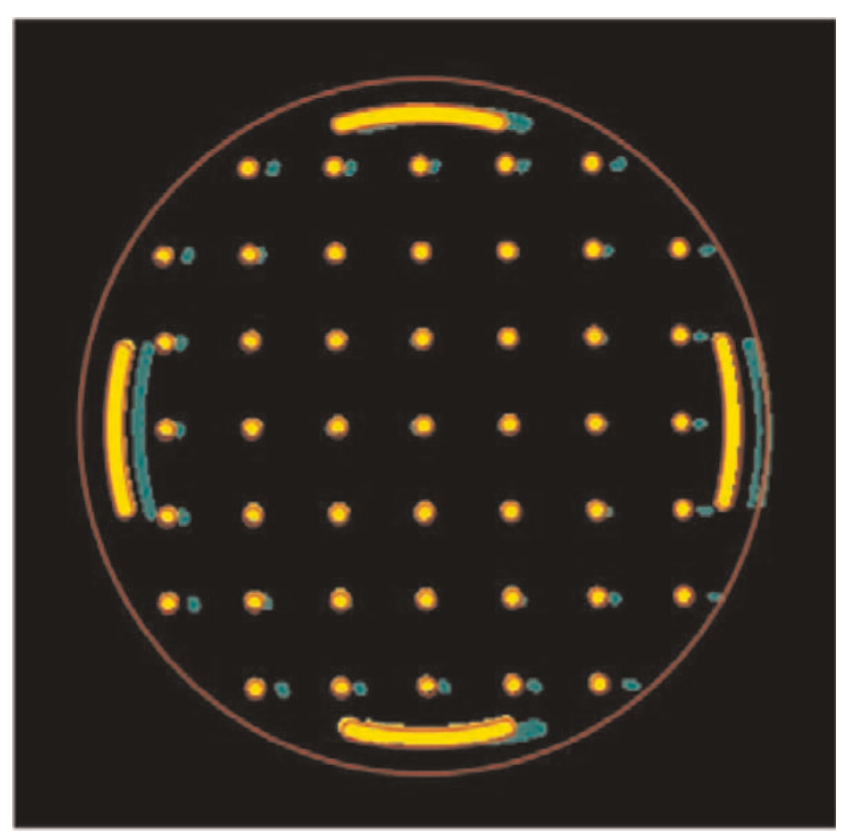

Geometrical fidelity of $T_{2} *$-weighted images overlaid on a contour plot of the test object (red): UFLARE (yellow) and EPI (green). 
Figure 3 (abstract P2 I8)
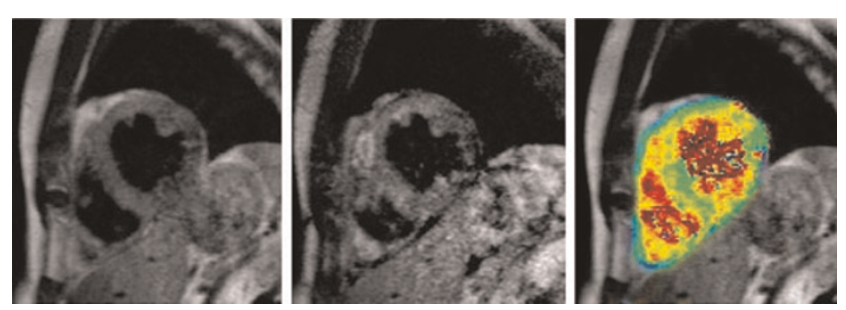

Short axis views obtained with UFLARE Left) without susceptibility weighting and middle) with strong $\mathrm{T}_{2} *$-weighting). Right) corresponding $\mathrm{T}_{2}{ }^{*}$-map calculated from multi $\tau$ UFLARE data. The color represents $T_{2}$ $*$ values ranging from $\mathrm{T}_{2} *=\mathrm{I} \mathrm{ms}$ (black) to $\mathrm{T}_{2} * \geq 85 \mathrm{~ms}$ (dark red).

Methods: The proposed cardiac triggered imaging technique is comprised of (i) a double inversion recovery module for ventricular blood suppression (ii) a navigator module for respiratory motion compensation and (iii) a fast spin echo imaging module. T2*-weighting was accomplished by implementing displaced UFLARE [3] using an extra evolution time tau between the initial excitation pulse and the first refocusing pulse (Figure I). Volunteer studies were conducted using a 5-element cardiac coil array at I.5 T (Philips Achieva, Best, Netherlands). Images were acquired with: scan matrix $192 \times 192$, FOV $250 \mathrm{~mm}$, echo train length $20, T R=2 R-R$ intervals, 3 slices with a thickness of $8 \mathrm{~mm}$ each. T2*-maps were generated from a series of images with tau ranging from 0 to $40 \mathrm{~ms}$.

Results: The images of the test object in Figure 2 demonstrate the geometrical integrity of the UFLARE image. Conversely, the EPI images revealed distortions of up to $1 \mathrm{~cm}$. Figure 3 depicts short axis views of the heart derived from a healthy subject using free breathing UFLARE in conjunction with double IR preparation without (left) and with T2*-weighting (middle) using an evolution time of tau $=20 \mathrm{~ms}$. Image quality, signal-to-noise ratio and ventricular blood suppression are suitable for clinical applications. Even for strong $\mathrm{T}_{2} *$-weighting the images are free of distortions due to $\mathrm{B} 0$-inhomogeneities and free of physiological motion artifacts. Figure 3 depicts a T2*-map (right) derived from a UFLARE data set which is superimposed to an anatomical image. T2* distribution in the myocardium is rather uniform as it is to be expected for a healthy subject. The mean T2* value of the myocardium was found to be $29.9+-6.6$ ms which matches previous reports [4].

Conclusion: The feasibility and anatomic fidelity of $\mathrm{T} 2 *$ weighted fast spin echo imaging have been demonstrated together with the image quality advantage over EPI. The proposed susceptibility spin-echo based approach promises to extend the capabilities of CVMR, including mapping and quantification of myocardial iron content, assessment of endothelial function, detection of stress induced angina pectoris, and differentiation of arteries and veins, which have all been elusive hitherto. It also holds the promise to potentially obviating the need for contrast agents for the assessment of myocardial perfusion while avoiding the drawbacks of commonly used EPI and gradient echo based approaches. In conclusion, we anticipate the extension of this work to higher field strengths to maximally exploit the SNR and contrast-to-noise (CNR) advantage and (iii) to evolve towards three-dimensional, distortion free, blood oxygen level dependent (BOLD) imaging of the heart in concert with parallel imaging.

\section{References}

I. He T, et al: Magn Reson Med 2008, 60:350.

2. Friedrich MG, et al: Circulation 2003, 108:2219-2223.

3. Niendorf T: Magn Reson Med 1999, 4I:II89-II98.

4. Messroghli DR, et al: Assessment of myocardial T2* from pixel-by-pixel maps on a clinical MR system. J Cardiovasc Magn Reson 2008, I 0(Suppl I):A240, SCMR Proceedings 2008.

\section{P219}

Evaluation of model-based contouring versus 2D segmentation for cardiac mass and volumes Andrew S Chi', Cuilian Miao', Chia-Ying Liu', Patricia A Cleary', Joao AC Lima' and David A Bluemke' IJohns Hopkins, Baltimore, MD, USA

${ }^{2}$ The George Washington University, Rockville, MD, USA

Journal of Cardiovascular Magnetic Resonance 2009, I I (Suppl I):P2 19

Objective: To compare three-dimensional, model-based contouring versus two-dimensional, semi-automatic contouring of cardiac cine MRI for determination of left ventricular mass and volumes.

Background: Traditional cardiac MRI methods have relied on slice-by-slice contouring and mathematical calculations to estimate myocardial mass and volumes. Three-dimensional model-based algorithms offer the potential advantages of faster analysis times, increased reproducibility, and physiologicallyrelevant information that includes the complex motion of the mitral valve, balanced by potential sensitivity to slice misregistration related to multiple breath-holds.

Methods and results: 100 cardiac MRI SSFP cine studies were evaluated using a model-based software (Cardiac Image Modeller (CIM)) and a semiautomatic contouring system (MASS). Parameters of cardiac function included left ventricular (LV) end diastolic mass (EDM), end diastolic volume (EDV), end systolic volume (ESV), and ejection fraction (EF). Peak ejection rate (PER) and peak filling rate (PFR) were also included. Reproducibility

\section{Figure I (abstract P2 I9)}

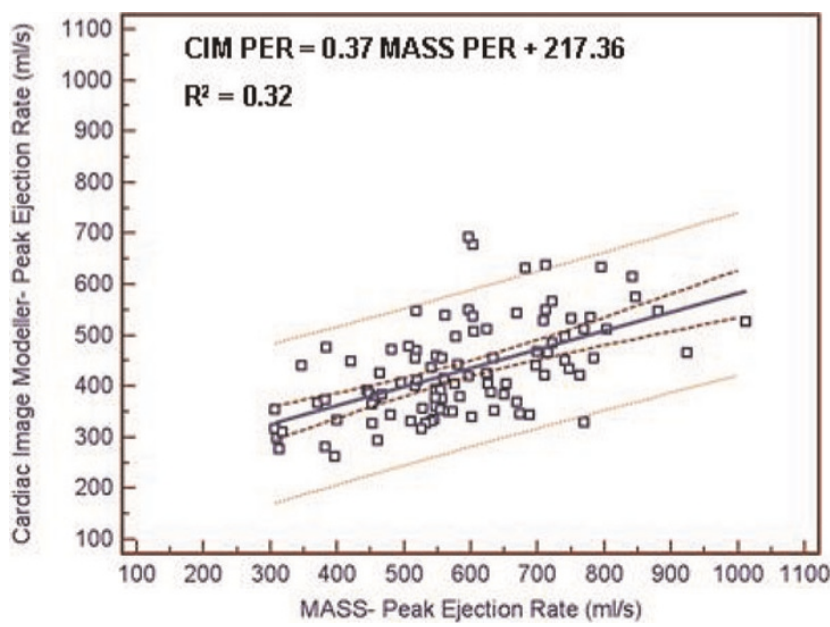

Page 252 of 316

(page number not for citation purposes) 
Figure 2 (abstract P219)

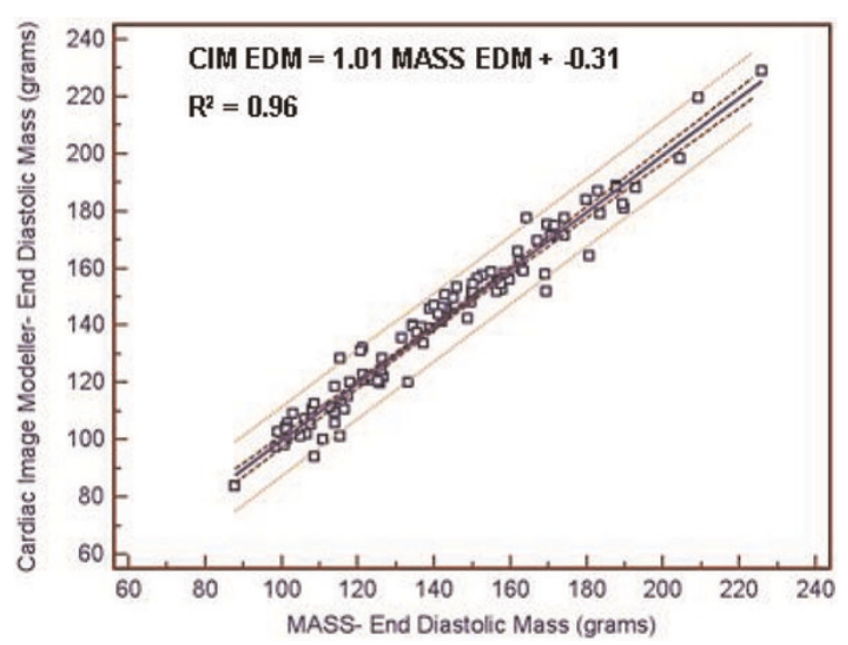

data for intra-observer variability was performed for 20 consecutive cases.

Results: Bland Altman analysis comparing CIM and MASS software demonstrated a high degree of agreement for left ventricular mass and volumes. Mean differences $( \pm$ limits of disagreement defined as $\pm 2 \mathrm{SD})$ were $-0.2 \mathrm{~g}$ (-I I.8-I I.5 g) for EDM, 0.I ml (-8.5-8.6 ml) for EDV, $-0.3 \mathrm{ml}(-6.0-5.4 \mathrm{ml})$ for ESV, and $0.3 \%(-3.6-4.2 \%)$ for EF. The relationship between CIM and MASS was linear (Figure I) and highly correlated for left ventricular end diastolic mass $\left(R^{2}=0.96\right)$ and volumes $\left(R^{2}=\right.$ 0.98). Intra-observer mean differences $( \pm 2$ SD) using the CIM software were $-2.5 \mathrm{~g}(-13.2-8.2 \mathrm{~g})$ for EDM, $3.2 \mathrm{ml}(-10.4$ $16.7 \mathrm{ml})$ for EDV, I.6 $\mathrm{ml}(-4.7-8.0 \mathrm{ml})$ for ESV and $-0.4 \%(-5.4-$ $4.6 \%$ ) for EF. Intraclass correlation coefficients for left ventricular EDM, EDV, ESV, and EF were 0.99 (0.988-0.998), 0.99 (0.97$0.99), 0.99$ (0.98-1.0), and 0.96(0.90-0.99), respectively. For filling and emptying rates (Figure 2), large variations between the two methods were identified. Mean differences ( \pm 2 SD) were $-155.2 \mathrm{ml} / \mathrm{s}(-388.5-78.1 \mathrm{ml} / \mathrm{s})$ for PER and - $109.7 \mathrm{ml} / \mathrm{s}(-357.9-$ $138.4 \mathrm{ml} / \mathrm{s}$ ) for PFR.

Conclusion: Compared to two-dimensional contouring techniques for cardiac MRI analysis, model-based contouring provides a reliable alternative for quantitative determination of myocardial mass and volumes. Characterization of systolic emptying and diastolic filling rates varies significantly between the two methods; further study is needed to understand these differences.

\section{P220}

\section{Clinical evaluation of automatic whole-heart and} coronary-artery segmentation

Marcel Breeuwer', Pierre Ermes' and Bernhard Gerber ${ }^{2}$

'Philips Healthcare, Best, Netherlands

${ }^{2}$ Louvain University, St. Luc Hospital, Brussels, Belgium

Journal of Cardiovascular Magnetic Resonance 2009, I I(SuppI I):P220

Introduction: With Steady-State Free-Precession (SSFP) MRI the heart and surrounding arteries can be imaged. The resulting image data can be used to inspect the patient-specific cardiac anatomy and to quantify aspects such as left-ventricular volume and wall mass. The data can furthermore be used to detect stenosis in the proximal and middle coronary-artery segments [I]. To simplify the visualization, quantification and stenosis detection, we have developed an almost automatic whole-heart and coronary-artery segmentation method.

Purpose: We describe the clinical evaluation of the performance of our cardiac MR whole-heart and coronary-artery segmentation method.

Methods: Our segmentation method was first developed for Computed Tomography [2] and thereafter adapted to MR [3]. The method fully automatically segments the ventricles, atria, and part of the aorta and pulmonary vessels by means of a shapeconstrained deformable model, which has been constructed from a large set of representative trainings data. Manual corrections of the segmentation are possible by single mouse clicks on the 3D segmentation surface visualization. The points where the coronary-arteries branch off the aorta (ostia) are also automatically detected. The user has to manually indicate the coronary-artery end points in the image data, these arteries are thereafter automatically tracked [4]. Total time needed for automatic segmentation/tracking is less than $2 \mathrm{~min}$ per data set (Dell 670 PC, dual-processor $3 \mathrm{GHz}, 3$ Gbyte memory).

Our method was evaluated by an experienced clinical cardiologist (third author) on whole-heart MR acquisitions from 50 patients with coronary-artery disease or heart failure (Philips

\section{Figure I (abstract P220)}

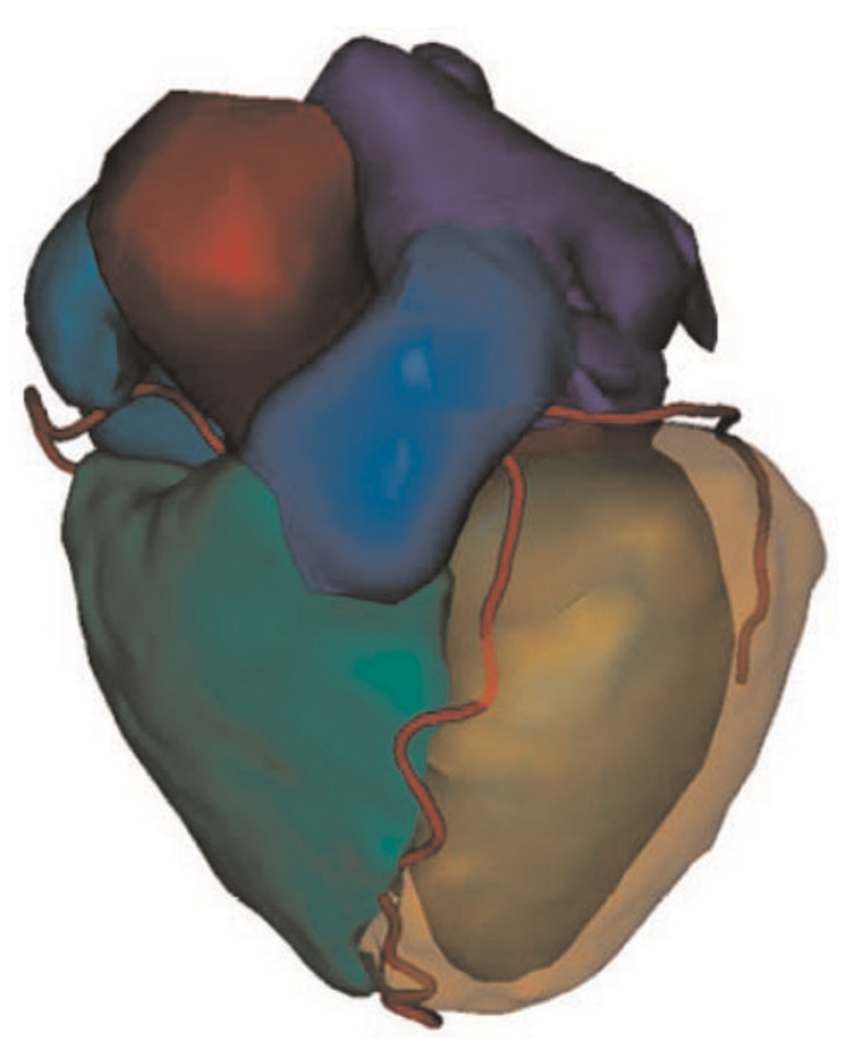

Example of whole-heart and coronary-artery segmentation (surface rendering with different colour per component). 
Figure 2 (abstract P220)

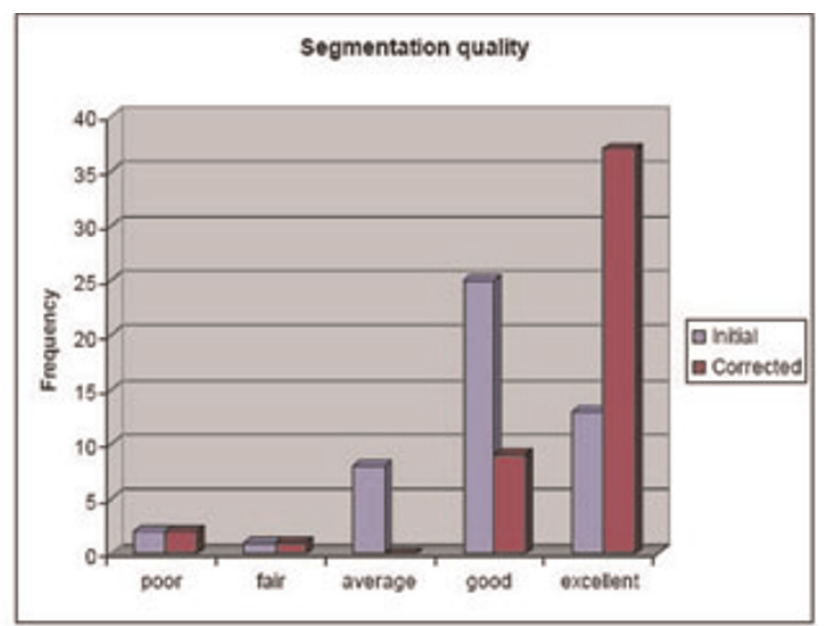

(a)

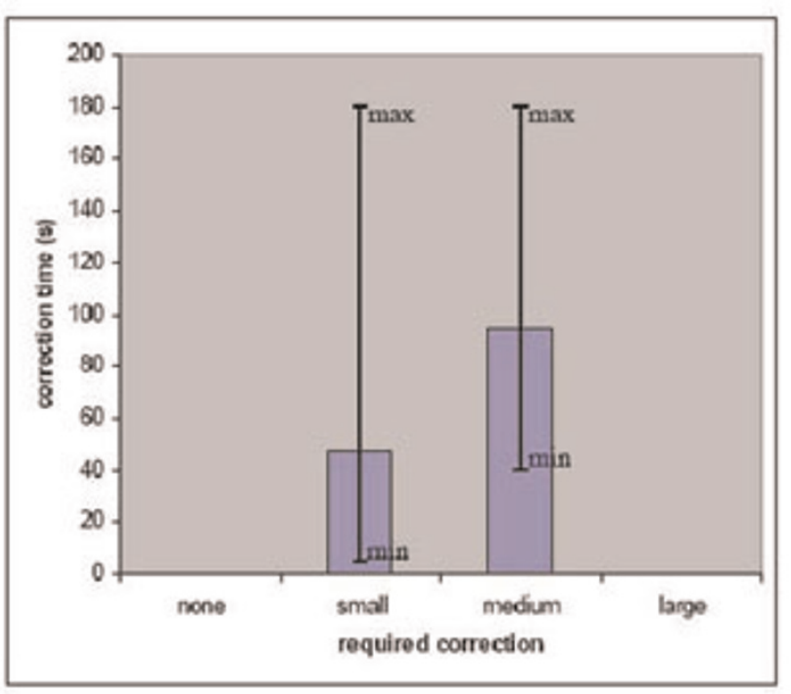

(b)

(a) Histogram of segmentation quality before and after correction (frequency $=n r$. of cases per quality category). (b) Average (blue bars), minimum and maximum (lines) correction times, for each of the categories of judged correction effort.

Achieva I.5 T, typically I 50 slices, TE $=2.14 \mathrm{~ms}, \mathrm{TR}=4.27 \mathrm{~ms}$, flip angle $=86$ degree, pixel spacing $=0.7 \mathrm{~mm}$, slice distance $=$ $0.8 \mathrm{~mm}$ ). The cardiologist visually judged the segmentation quality before and after manual correction (categories: poor, fair, average, good, excellent). The time needed to perform segmentation corrections was recorded and the cardiologist judged the required correction effort (categories: none, small, medium, large).

Results: Figure I shows an example whole-heart segmentation. Figure $2 \mathrm{a}$ shows the histogram of the quality before and after manual correction. Before correction, 47 out of 50 segmentations have an average-to-excellent overall quality. Manual correction significantly improves the quality, after correction 47 out of 50 have a good or excellent quality. Three segmentations have a poor or fair quality, manual correction does not help for these cases. Figure $2 b$ shows the time and judged effort needed to correct segmentations. Only small (average time $45 \mathrm{sec}$ ) or medium (average time $94 \mathrm{sec}$ ) correction efforts were judged to be needed. The maximum correction time $(180 \mathrm{sec})$ does however not differ for these categories.

Conclusion: For 47 out of 50 investigated cases (94\%), our automatic whole-heart and coronary-artery segmentation method in combination with small or medium correction effort is judged to result in good or excellent segmentation quality.

\section{References}

I. Yong Kim W, et al: New Engl J Med 200I, 345:1863-1869.

2. Ecabert O, et al: Proc SPIE 2007, 65 I 2: 65I20G.

3. Peters J, et al: MICCAI 2007, 402-4I0, Part II, LNCS 4792.

4. Sonnemans J: J Cardiovas Magn Reson 2008, I O(SuppI I): Proceedings SCMR 2008.

\section{P22 I}

Multi slice DENSE in a single breath hold

Andreas Sigfridsson', Henrik Haraldsson ${ }^{2}$, Tino Ebbers ${ }^{2}$, Shinichi Takase' and Hajime Sakuma'

'Mie University, Tsu, Mie, Japan

${ }^{2}$ Linköping University, Linköping, Sweden

Journal of Cardiovascular Magnetic Resonance 2009, I I(SuppI I):P22 I

Introduction: Displacement ENcoding with Stimulated Echoes (DENSE) is a method to acquire displacement maps of the myocardial motion. This can in turn be used to estimate strain which is an important measure of myocardial function. Previously, DENSE imaging has primarily been performed using multiple breath holds per slice, severely limiting its incorporation into the tight schedule of clinical routine. Strain maps are further desired throughout the left ventricle, not only in a single slice. A targeted approach, guided by results from perfusion and late

Figure I (abstract P22I)

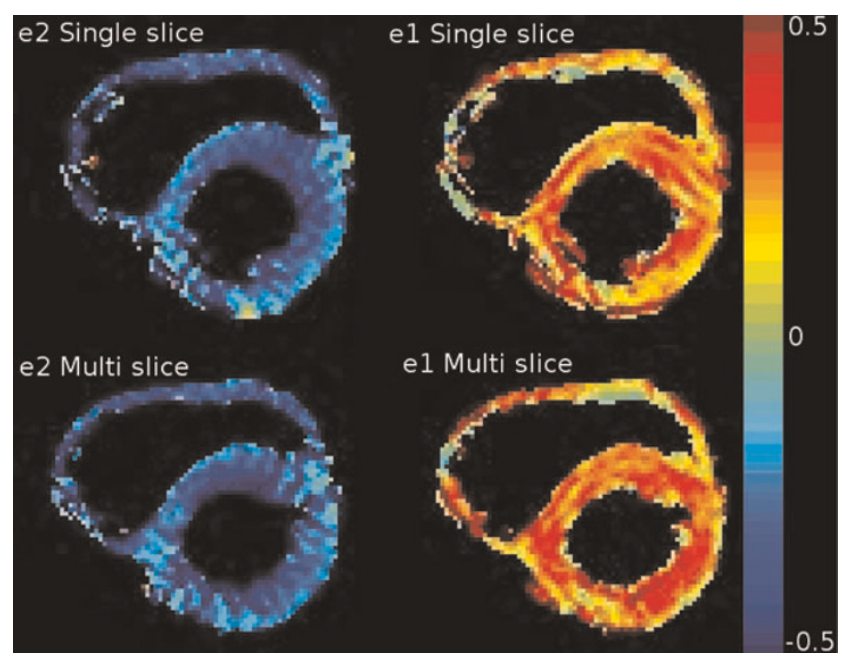


Figure 2 (abstract P22I)

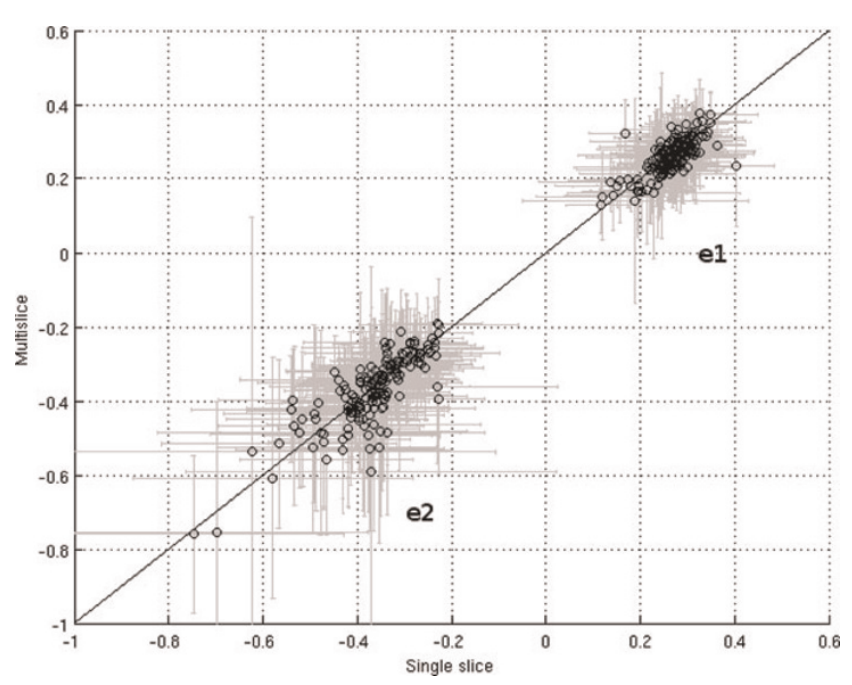

enhancement studies is tempting, but the presence of contrast agent shortens the $\mathrm{TI}$ relaxation, which hampers stimulated echo acquisitions such as DENSE. We therefore propose a multi slice acquisition for strain measurements in a single breath hold. We compare this with conventional single slice DENSE acquired in separate breath holds.

Methods: Ten healthy volunteers were imaged in a Philips Achieva I.5 T scanner, using the standard 5 channel cardiac coil. Three slices were acquired at the basal, equatorial and apical short axis levels in a single breath hold multi slice acquisition.

End systolic displacement was measured by encoding the position at the R-wave using a SPAMM I-I sequence, and decoding at end systole. The appropriate trigger delay time was determined using a long axis cine imaging sequence. The three slices were excited and read out after each other in the same cardiac cycle, with $25 \mathrm{~ms}$ separation.

Imaging parameters were: field of view $350 \mathrm{~mm}$, slice thickness $8 \mathrm{~mm}$, matrix $128 \times 1 \mathrm{I}$, SENSE factor 2, TFE-factor 3, EPIfactor 7, TR $8.9 \mathrm{~ms}$, TE $4.2 \mathrm{~ms}$. Displacement encoding strength

Figure 3 (abstract P22I)

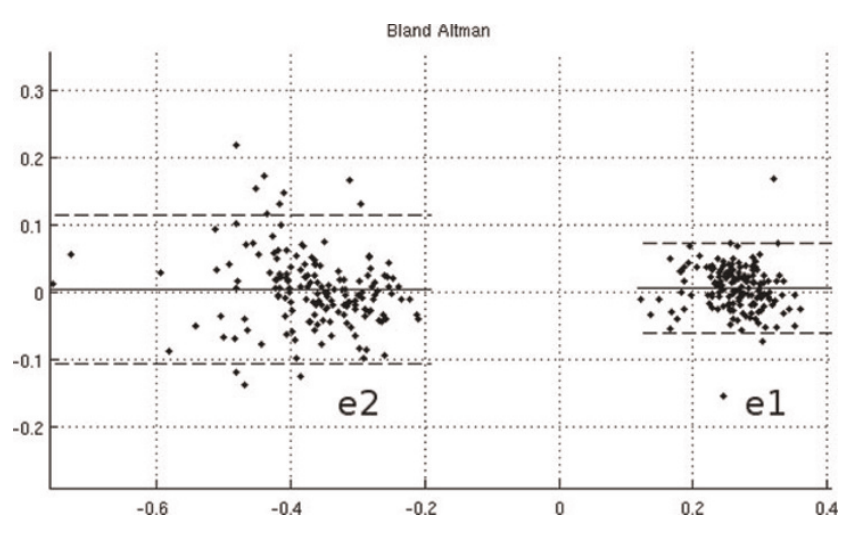

was 0.35 cycles per pixel. Flip angle was optimized to yield highest constant signal strength [I].

Both in-plane displacement directions were measured using displacement encoding in three oblique directions [2]. This allows for the subtraction of background phase errors and increases the displacement-to-noise ratio while maintaining encoding strength, and thus signal dephasing. Complementary encoding was used to suppress the TI relaxation echo[3]. Each direction was acquired in six heart beats, resulting in a total scan time of 18 heart beats.

For comparison, the three slices were also acquired separately in one 18 heart beat breath hold each. Apart from only acquiring a single slice at a time, the scan parameters were identical. The order of the single slice and multi slice acquisitions was randomized.

Eulerian strain was analysed using custom software written in MATLAB. Strain was evaluated in ROls placed manually according to the AHA 17-segment model with the apical segment excluded. Results: Strain maps of eulerian strain eigenvalues el and e2, corresponding to expansion and contraction, are shown in Figure I for single slice and multi slice acquisitions of one volunteer.

Regional strain values from all volunteers are plotted in Figure 2. Error bars indicate the standard deviation within each ROI.

A Bland-Altman analysis is shown in Figure 3. For e2 (contraction), the bias is 0.004 and limits of agreement -0.11 and 0.12. For el (expansion), the bias is 0.007 with limits of agreement -0.06 and 0.07 .

Discussion: A method for acquiring myocardial strain in the whole left ventricle in a single breath hold has been presented. It has been shown that acquiring three slices in the same breath hold results in strain values that agree well with those acquired in three separate breath holds. The small differences between the methods indicate that the slight shift in acquisition timing between the slices $(25 \mathrm{~ms})$ did not significantly influence the strain values.

Eliminating the need for multiple breath holds, multi slice DENSE extends the utility of DENSE for myocardial strain analysis.

\section{References}

I. Stuber, et al: MAGMA 1999, 9:85-91.

2. Lin, et al: MRM 2008, 60:8-13.

3. Gilson, et al: MRM 2004, 5 1:744-752.

\section{P222}

An automated method for left ventricular localization and identification of end-systolic and end-diastolic images from cine cardiac MRI Sotirios A Tsaftaris', Xiangzhi Zhou ${ }^{2,3}$, Richard Tang ${ }^{2}$, Rachel Klein ${ }^{2}$ and Rohan Dharmakumar ${ }^{2}$

'Department of Electrical Engineering and Computer Science, Northwestern University, Evanston, IL, USA

${ }^{2}$ Department of Radiology, Northwestern University, Chicago, IL, USA

${ }^{3}$ Northwestern University, Chicago, IL, USA

Journal of Cardiovascular Magnetic Resonance 2009, I I(SuppI I):P222

Introduction: A critical component in computing quantitative diagnostic metrics, such as ejection fraction, as well as image segmentation and registration is the accurate identification of the end-systolic (ES) and end-diastolic (ED) frames in cine MRI. Localization of the LV is also important, to assist further analysis 
Figure I (abstract P222)
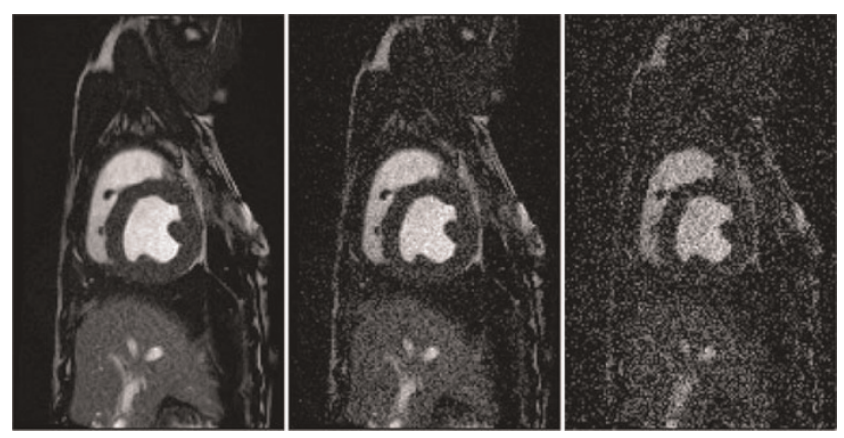

An image with various levels of noise: left no noise; middle and right additive zero mean noise of 40 and 100 standard deviation, respectively.

(ie., myocardial segmentation). Currently, these tasks are performed in a manual, semi- or fully-automated fashion. Fullyautomated methods are desirable since they can eliminate manual labor and inter- and intra-observer variability. Most methods rely on measuring the area of the blood pool in the LV chamber, but they are computationally intensive, susceptible to noise, and require prior localization and segmentation of the LV. An image-driven statistical method is presented that utilizes cross-correlation to detect ES and ED from cine MRI acquired from canines under control conditions.

Purpose: To develop a fully automated, computationally efficient, post-processing method for reliable LV localization and identification of ES and ED frames from cine cardiac MR images.

Methods: Experimental setup and imaging: Short-axis cine cardiac MR images were acquired on Siemens I.5 T scanner

\section{Figure 2 (abstract P222)}

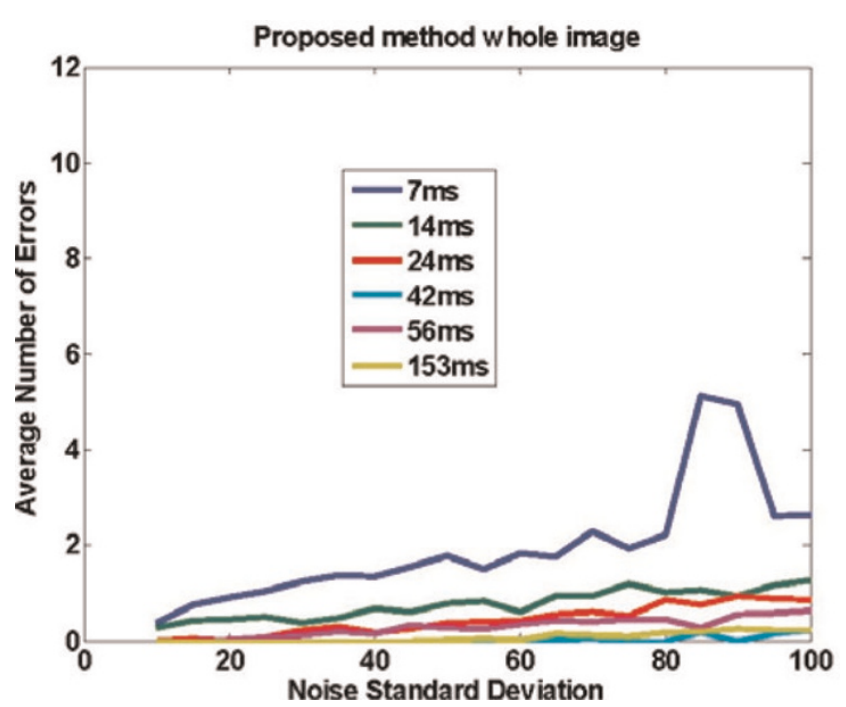

Accuarcy of the proposed method, as measured by the average number of errors, for different noise levels and for a number of different TRes, when the whole image is used.
Figure 3 (abstract P222)

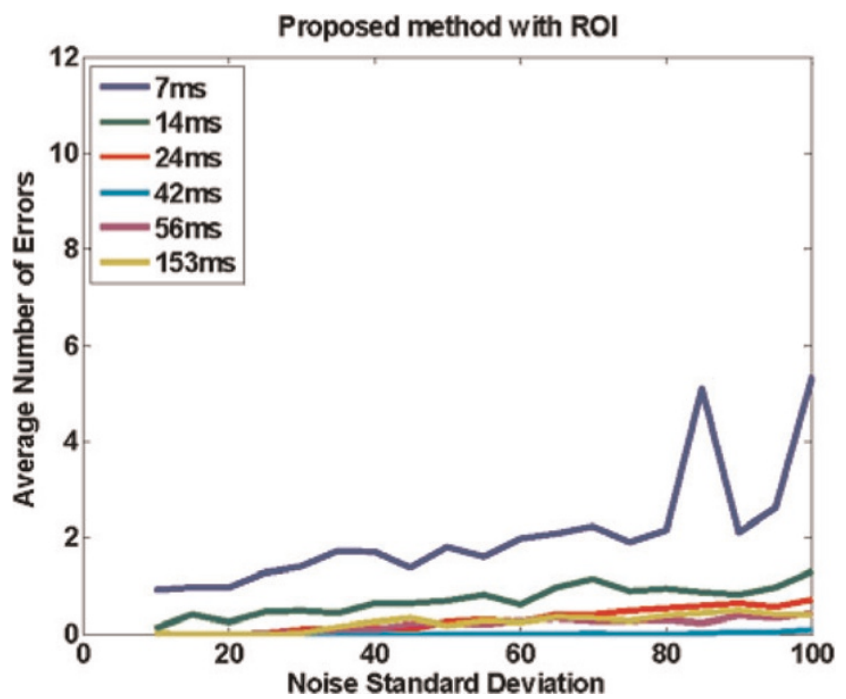

Accuracy of the proposed method with an ROI that encompasses the heart, as measured by the average number of errors, for different noise levels and for a number of different TRes. Notice that the performance is similar to Figure 2, illustrating that there is no gain in selecting an ROI, thus demonstrating that the proposed method can be used in the whole image, without needing an ROI selection.

from three canines that were sedated and mechanically ventilated. ECG-gated and breath-held SSFP acquisitions were prescribed over the mid-ventricle following scout scans at various temporal resolutions (TRes:7-153 ms). Scan parameters: voxel size $=1.2 \times 1.2 \times 6 \mathrm{~mm}^{3} ;$ flip angle $=60^{\circ} ; \mathrm{TR} / \mathrm{TE}=3.5 /$ $1.8 \mathrm{~ms}$.

Figure 4 (abstract P222)

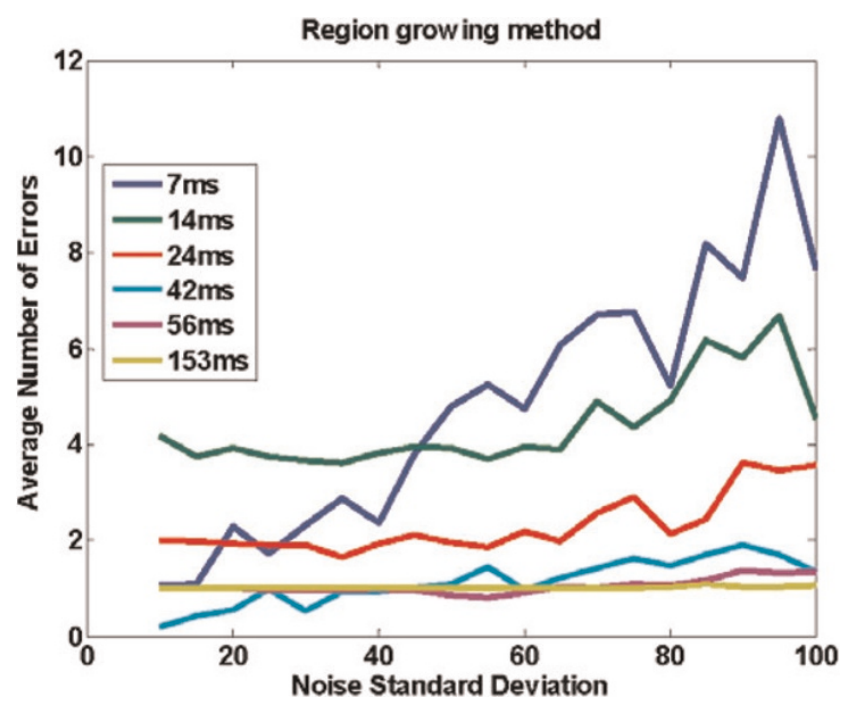

Accuracy of the seeded region growing method (SRGM), as measured by the average number of errors, for the different noise levels and for a number of different TRes. 
Figure 5 (abstract P222)

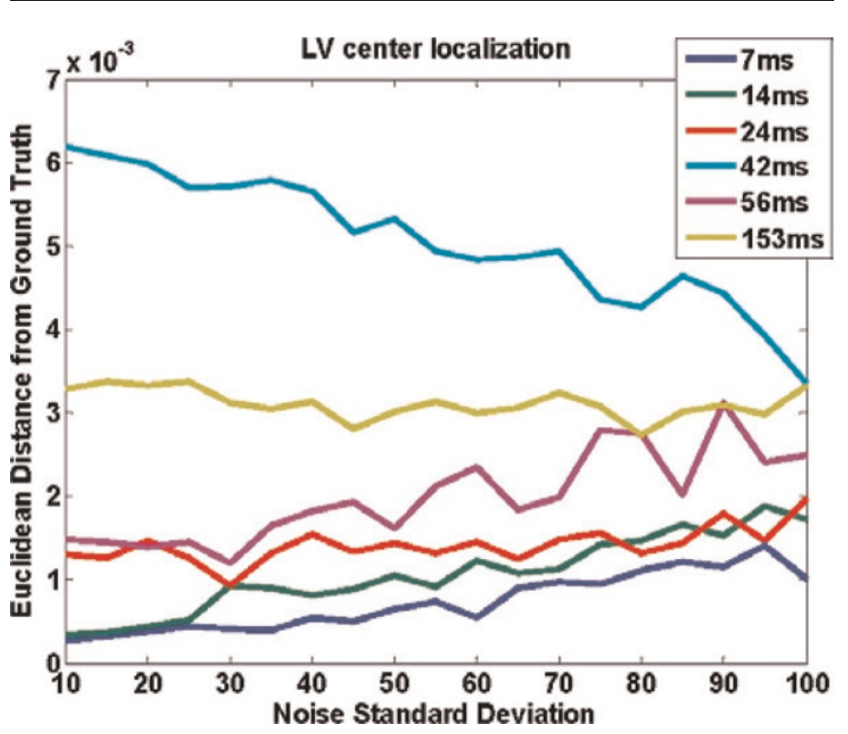

Accuracy of the proposed method in finding the center of the LV tested for different noise levels, and TRes. The accuracy is measured as the Euclidean distance from the ground truth provided by an observer. Notice that the $y$-axis is scaled at $10^{-3}$ increments.

Image processing: Each cine set was denoted as $I(x, y, t)$, where $x, y$ are pixel locations $(\mathrm{NxM})$, and $t$ denotes $I, \ldots, F$ phases. Each image was smoothed by convolution with a Gaussian filter (width $=10$ and sigma $=1$ ). The normalized cross correlation is computed between all images, giving an FxF matrix $C(i, j)$, (i, j corresponds to image pairs). The $i, j$ corresponding to the minimum of $C$ are the ES, ED images, since they are uncorrelated in the heart region (high motion). Using the same principle, the LV chamber can be localized by finding the temporal variation of $\mathrm{I}(\mathrm{x}, \mathrm{y}, \mathrm{t})$, as matrix $\mathrm{V}(\mathrm{NxM})$. $\mathrm{V}$ was then denoised using a Gaussian and binarized (Otsu's method). The centroid of the largest connected component was chosen as the LV center. The proposed identification method was compared against LV blood volume segmentation methods. A seededregion-growing method (SRGM) was chosen for segmentation instead of level-set-active-contours since it proved more robust to noise and the limited spatial resolution of the cine MRI images. Data analysis: Cine images with varying TRes were tested and additive-white-Gaussian-noise was introduced to demonstrate robustness. The seed location for SRGM was manually set inside the ventricle for each image. Average number of frames away from the true ES, ED images (manually chosen) was used as the performance metric. For each noise level, 40 trials were performed and the results were averaged.

Results: Figure I shows an image with added noise. Figures 2 to 4 demonstrate the performance of the proposed method when the whole image is used (Figure 2), and when a ROI around the heart is manually chosen (Figure 3 ) and compares it to SRGM (Figure 4). Figure 5 shows the Euclidean distance between the centers found using the proposed and the reader delineation of all cine images.

Discussion: Pre-selection of an ROI, necessary with other methods, does not offer any significant gain. SRGM underperformed in cases when image noise or TRes was high (I $4 \mathrm{~ms}$ and $24 \mathrm{~ms}$ ), did not always yield a unique global maximum and minimum, and was not robust against the appearance of papillary muscles, thus hindering the identification of ES and ED. The proposed method reliably identifies the cardiac phases, localizes the LV, without any parameterization (SRGM requires the localization of the seed and the determination of the growing threshold), is computationally elegant ( $\sim 10$ times faster than SRGM), could be easily extended to 4D MRI, and implemented in cardiac image analysis software.

\section{P223}

The use of volumetric analysis to improve cardiac magnetic resonance evaluation of left ventricular size and function

Victor Mor-Avi', Roberto M Lang', Johannes Niel², Regina Steringer-Macherbauer ${ }^{2}$, Lynn Weinert', Lissa Sugeng', Rolf Baumann ${ }^{3}$, Georg Schummers ${ }^{3}$, Amit Patel' ${ }^{\prime}$ and Hans-Joachim Nesser ${ }^{2}$

'University of Chicago, Chicago, IL, USA

${ }^{2}$ Public Hospital Elisabethinen, Linz, Austria

${ }^{3}$ TomTec Imaging Systems, Unterschleissheim, Germany

Journal of Cardiovascular Magnetic Resonance 2009, I I(SuppI I):P223

Introduction: Cardiac magnetic resonance (CMR) imaging is the current standard reference technique for left ventricular (LV) volume measurements, which are obtained from short-axis slices using the method of disks (MOD) approximation. As new threedimensional (3D) imaging and volumetric analysis techniques are developed and compared against this reference, there is growing evidence that the standard MOD-based CMR reference has limitations. Thus, the recently developed real-time 3D echocardiography was found to consistently underestimate LV volumes, among other reasons because of CMR errors at the LV base, associated with the use of frequently oblique short-axis views Purpose: The aim of this study was to test a new technique for volumetric analysis of CMR images that is free of these errors by comparing it to the standard MOD-based analysis.

Methods: Steady-state free precession dynamic gradient-echo images (Siemens MAGNETOM Sonata I.5 T scanner) were obtained in 45 patients with a wide range of LV size and function in short-axis views from LV base to apex as well as 3 long-axis planes rotated around the LV axis. Images were analyzed using the standard MOD technique and, independently, using the new volumetric approach implemented in prototype analysis software (TomTec Imaging Systems). This analysis is based on semiautomated reconstruction of endocardial surface from a combination of short- and long-axis CMR images, followed by direct quantification of LV volume confined within the endocardial surface. Both analysis techniques were used to obtain LV endsystolic and end-diastolic volumes (ESV, EDV) and ejection

Figure I (abstract P223)

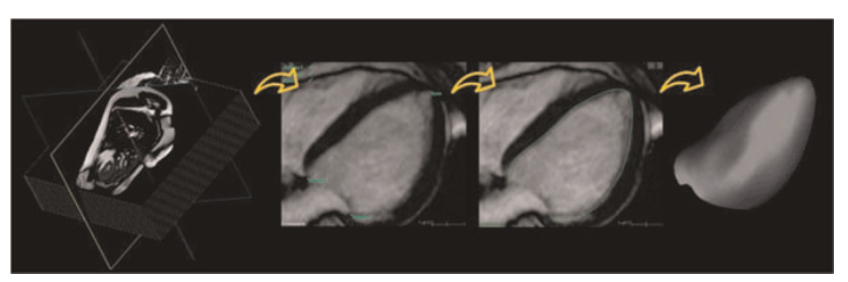


fraction (EF). Inter-technique comparisons included linear regression and Bland-Altman analyses. Reproducibility of both techniques was assessed in a subset of 10 patients using blinded repeated measurements by the same observer one week later, as well by a second independent observer.

Results: Despite the high correlation between the two CMR techniques (ESV: $r=0.99$; EDV: $r=0.98$; EF: $r=0.92$ ), the volumetric approach resulted in significantly smaller volumes, compared to the standard MOD technique (inter-technique differences: $\Delta \mathrm{ESV}=17 \pm 14 \mathrm{ml}(p<0.05) ; \Delta E D V=20 \pm 16 \mathrm{ml}$ $(\mathrm{p}<0.05) ; \Delta E F=2 \pm 6 \%$; (NS). Intra- and inter-observer variability of the MOD technique for both volumes and EF was 2 to $7 \%$ of the mean measured value, while that of the volumetric approach was 5 to $10 \%$, probably reflecting the users' learning curve with the new technique.

Conclusion: Volumetric analysis of CMR images, which does not rely on criteria for basal LV slice selection, results in smaller LV volume measurements. With its relatively high reproducibility, this approach may provide an alternative, potentially more accurate CMR reference technique for LV volume measurements, to which other $3 \mathrm{D}$ imaging modalities would be compared more fairly (Figure I).

\section{P224}

\section{Calibration of myocardial iron concentration against T2-star Cardiovascular Magnetic}

\section{Resonance}

John-Paul Carpenter', Taigang He', Paul Kirk', Lisa J Anderson ${ }^{2}$, John B Porter ${ }^{3}$, John Wood ${ }^{4}$, Renzo Galanello ${ }^{5}$, Gianluca Forni ${ }^{6}$, Gualtiero Catani ${ }^{5}$, Suthat Fucharoen ${ }^{7}$, Adam Fleming 8 , Mike House ${ }^{8}$, Greg Black ${ }^{8}$, David N Firmin', Timothy G St Pierre and Dudley J Pennell'

${ }^{I}$ Royal Brompton and Harefield NHS Trust, London, UK

${ }^{2}$ St George's Hospital NHS Trust, London, UK

${ }^{3}$ University College Hospitals NHS Trust, London, UK

${ }^{4}$ Children's Hospital, Los Angeles, Los Angeles, CA, USA

${ }^{5}$ Ospedale Regionale Microcitemie, Cagliari, Italy

${ }^{6}$ Ospedali Galliera di Genova, Genoa, Italy

${ }^{7}$ Mahidol University, Puttamonthon Nakornpathom, Thailand

${ }^{8}$ The University of Western Australia, Perth, Australia

Journal of Cardiovascular Magnetic Resonance 2009, I I(SuppI I):P224

Introduction: Heart failure secondary to myocardial siderosis remains a major problem for patients with transfusional iron overload. Direct measurement of myocardial iron concentration by biopsy is not only highly invasive but unreliable due to uneven myocardial iron deposition. T2*cardiovascular magnetic resonance (CMR) is the only non-invasive technique which is able to assess tissue iron levels. This is a rapid, robust technique with proven reproducibility and inter-site transferability which has been accepted as part of clinical practice worldwide [I]. However, little data is available on the calibration of $\mathrm{T}^{*}$ values against tissue concentration of iron [2].

Purpose: The aim of this study was to calibrate T2* cardiovascular measurements versus mycardial iron concentration.

Methods: With full ethical approval, seven whole-hearts were donated from patients with transfusion-dependent anaemia. Six of the hearts were from patients with end-stage heart failure. The
Figure I (abstract P224)

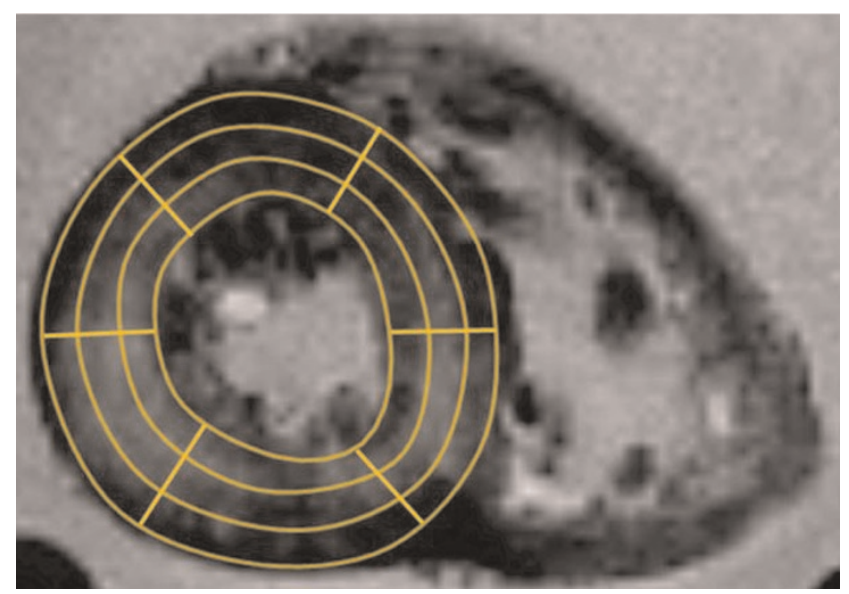

MR T2* image showing cardiac short axis slice divided into regions of interest for T2* analysis.

seventh was from a patient who died from cerebrovascular disease and had no symptoms of heart failure prior to death. 4 hearts were post-mortem specimens and 3 hearts were from patients who had cardiac transplantation for end-stage heart failure.

All hearts were formalin fixed before being sliced into 5 or 6 short axis slices (depending on the size of the organ). The apical slice was not used for analysis. A custom-made Perspex plinth was used to hold each of the slices which were then scanned immersed in water at $37^{\circ} \mathrm{C}$ using a $1.5 \mathrm{~T}$ Avanto MR scanner (Siemens, Erlangen, Germany) with a four-channel phased-array coil. A multi-echo T2-star sequence (gradient echo) was used: $\mathrm{TE}=2.47,4.48,6.49,8.5,10.6,12.7,14.8$, $16.9 \mathrm{~ms}$; field of view $150 \times 150 \mathrm{~mm}$; matrix $128 \times 128$; flip angle $35^{\circ}$; number of excitations 2; bandwidth $815 \mathrm{~Hz}$ per pixel; TR $20 \mathrm{~ms}$; slice thickness $5 \mathrm{~mm}$.

Figure 2 (abstract P224)

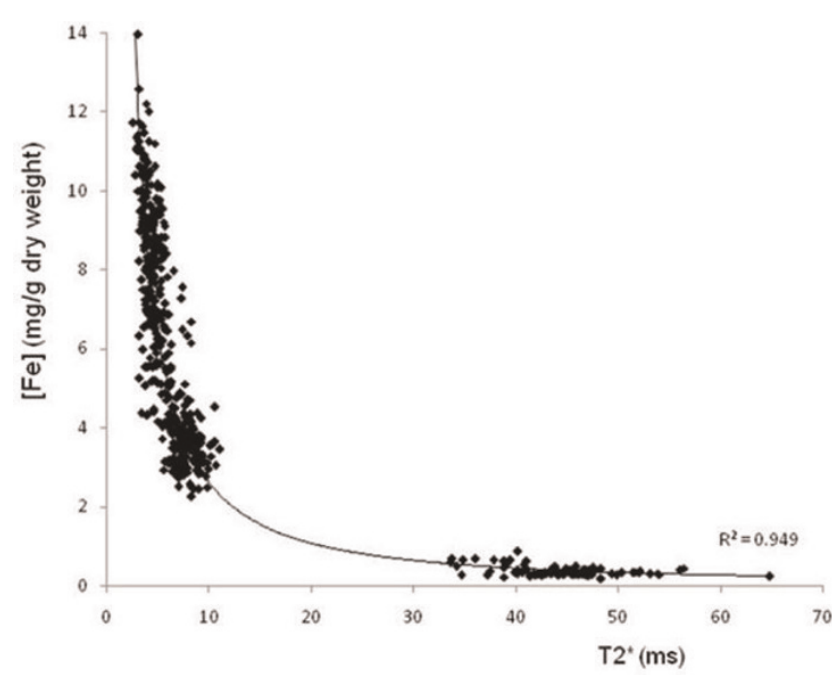

Graph of myocardial iron concentration (mg Fe/g dry weight) vs. T2-star (ms). $R^{2}=0.949$. 
Figure 3 (abstract P224)

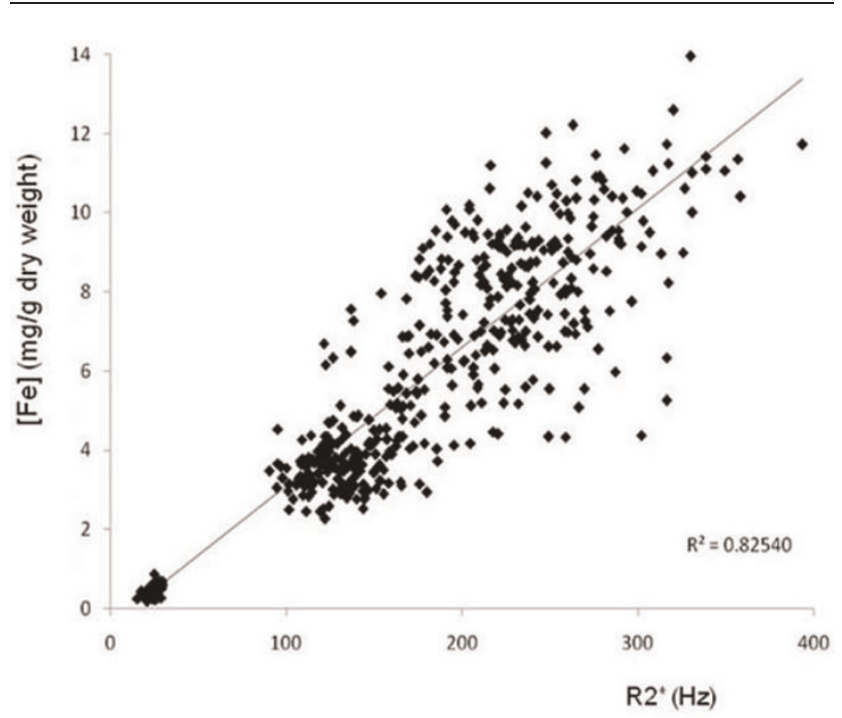

Graph of myocardial iron concentration (mg Fe/g dry weight) vs $\mathrm{R}^{2 *}(\mathrm{~Hz})$. $\mathrm{R}^{2}=0.825$

Each of the slices was subsequently divided into 6 sectors, each sector being subdivided into epicardial (outer), mesocardial (mid) and endocardial (inner) myocardial layers. Two specimens were taken from the right ventricular free wall. Analysis of myocardial iron concentration was performed using inductively coupled atomic emission spectrometry (after digestion in acid to avoid sampling errors). T2* was measured using Thalassaemia tools (a plug-in of CMRtools, Cardiovascular Imaging Solutions, London) in the corresponding regions of myocardium (see Figure I). A truncation method was used for curve-fitting as previously described [3].

Results: 31 short axis myocardial slices from the 7 hearts were analysed. A total of 558 specimen blocks (mean mass +/$\mathrm{SD}=100 \mathrm{I}+/-620 \mathrm{mg}$ ) and the corresponding region of interest (ROI) on CMR were included in the analysis. 21 (3.6\%) of the ROls were excluded from the final analysis due to technical issues (such as artefact affecting T2-star analysis). The mean wet (dry) tissue iron concentration ranged from $0.07(0.35)$ to 1.86 (8.08) $\mathrm{mg} \mathrm{Fe} / \mathrm{g}$. T2-star values ranged from 2.54 to $64.7 \mathrm{~ms}$. Iron concentration was related to T2* and R2* (Figures 2 and 3).
Conclusion: There is a linear relationship between tissue iron concentration and R2* and a non-linear relationship with T2*. The results accord with published data on myocardial iron concentration in non-iron loaded hearts, and a previous report in a single iron loaded post-mortem heart [2, 4]. In vivo T2* correlates well with that of ex-vivo formalin-fixed tissue, and therefore these data form a calibration curve for myocardial iron concentration over a wide range from normal to fatal levels [5].

\section{References}

I. Tanner MA, He T and Westwood MA, et al: Haematologica 2006, $91:$ II388-I39I.

2. Ghugre NR, Enriquez CM and Gonzalez I, et al: Magn Reson Med 2006, 56:68I-686.

3. He T, Gatehouse PD and Kirk P, et al: Magn Reson Med 2008, 60:350-356.

4. Collins W and Taylor WH: Ann Clin Biochem 1987, 24:483387.

5. He T, Carpenter JP and Jongirasiri S, et al: Can we measure iron overload in the heart using in vivo T2* MRI?. Abstract SCMR 2008.

\section{P225}

Accelerated real time 2D and segmented 3D cine imaging - whole heart approaches in a single breath hold

Yu-Po Chen', Sven Zuehlsdorff', Peter Weale ${ }^{2}$, John Sheehan', Renate Jerecic ${ }^{2}$ and James C Carr'

${ }^{1}$ Northwestern University Feinberg School of Medicine, Chicago, IL, USA

${ }^{2}$ Siemens Medical Solutions USA, Inc., Chicago, IL, USA

Journal of Cardiovascular Magnetic Resonance 2009, I I (SuppI I):P225

Introduction: The clinically well-established technique used to assess global functional cardiac parameters such as ejection fraction (EF) is a 2D cine approach in which the left ventricle (LV) is covered in multiple breath holds. The development of 32 channel receiver coils allows using high parallel acceleration factors with and increased signal to noise ratio (SNR). In this volunteer study, single breath-hold approaches have been used and the derived functional parameters (ejection fraction EF, end diastolic volume EDV, end systolic volume ESV, stroke volume SV, and myocardial mass MM) have been compared to the clinical standard technique. There is a good correlation between the standard and the single breath-hold techniques. They can

Table I (abstract P225) Protocol parameters

\begin{tabular}{|c|c|c|c|c|}
\hline & 2D Segmented (GRAPPA) & 2D (GRAPPA) & 2D (TGRAPPA) & 3D (TGRAPPA) \\
\hline TR/TE (ms) & $2.84 / 1.2$ & $2.4 / 1.04$ & $2.3 / 1$ & $2.9 / 1.44$ \\
\hline Temporal Resolution (ms) & 29.9 (interpolated) & 45.8 & 46.6 & 51.84 \\
\hline FOV & $340 \times 276$ & $340 \times 289$ & $340 \times 288$ & $380 \times 306$ \\
\hline Slice/Partition Thickness (mm) & 8 & 8 & 7 & 6 \\
\hline Voxel Size $\left(\mathrm{mm}^{3}\right)$ & $2.5 \times 1.8 \times 8.0$ & $2.3 \times 2.1 \times 8.0$ & $3.6 \times 2.4 \times 7.0$ & $3.1 \times 2.6 \times 6.0$ \\
\hline Acceleration Factor & $\begin{array}{l}\text { GRAPPA } 2 \text { (Integrated } \\
\text { Reference Lines) }\end{array}$ & $\begin{array}{l}\text { GRAPPA } 4 \text { (separate } \\
\text { reference lines and } \\
\text { shared phases) }\end{array}$ & TGRAPPA $2 \times 2$ & $\begin{array}{l}\text { TGRAPPA } 2 \times 2 \\
\text { (Non-isotropic) }\end{array}$ \\
\hline Triggering & Retrospective & Prospective & Prospective & Prospective \\
\hline Number of Breath holds & 10 & $\mathrm{I}$ & I & 1 \\
\hline
\end{tabular}


Table 2 (abstract P225) Correlation (r) of LV function parameters between the standard and each of the single breath-hold techniques

\begin{tabular}{llll}
\hline & 2D (GRAPPA) & 2D (TGRAPPA) & 3D (TGRAPPA) \\
\hline EF & 0.70 & 0.60 & 0.64 \\
EDV & 0.75 & 0.88 & 0.76 \\
ESV & 0.85 & 0.88 & 0.79 \\
SV & 0.38 & 0.62 & 0.59 \\
MM & 0.93 & 0.74 & 0.83 \\
\hline
\end{tabular}

potentially substitute conventional multi-breath hold approaches to increase patient comfort and reduce the study time.

Materials and methods: A prospectively gated multi-slice 2D real-time cine sequence was implemented on a $1.5 \mathrm{~T}$ scanner (MAGNETOM Espree, Siemens AG Healthcare Sector, Erlangen, Germany) using highly accelerated acquisition schemes. For parallel imaging, coil reference data have been derived using previously acquired reference data or a temporal method (TGRAPPA [I]), respectively. Optional, outer $k$-space data could be shared between consecutive phases to increase temporal resolution. For a segmented 3D cine approach, a TGRAPPA acquisition scheme in phase and partition encoding direction was used. Both $2 \mathrm{D}$ and $3 \mathrm{D}$ approaches allowed to acquire cine data covering the entire LV in one single breath hold using typical parameters shown in Table I. Cine data of ten healthy volunteers ( 2 male, 8 female; mean age 27.5 ; range $21-$ 40) have been acquired using a 32-channel cardiac coil (Invivo, Orlando, Florida) and the derived functional parameters have been compared using a retrospectively gated multi-breath hold protocol as clinical standard.

Functional analysis was performed using Argus (Siemens AG Healthcare Sector). Volumetric analysis was performed on all cine images; EF, EDV, ESV, SV, and MM were derived. Paired Student's t-test and linear regression were performed. A level of $\mathrm{p}<0.05$ was considered statistically significant.

Results: Linear regression showed good correlation $(r>0.75)$ between the measurements of $L V$ function parameters yielded by the standard segmented 2D protocol and those by the experimental protocols, except in measuring EF and SV.

The same analysis also showed strong correlation among the single breath-hold techniques in measuring LV parameters.

Paired t-tests indicated that there were no significant differences in measured EF and MM between the described protocols. However, significant differences were found in EDV, ESV and SV

Table 3 (abstract P225) Correlation (r) in measurements between pairs of the single breath-hold techniques

\begin{tabular}{llll}
\hline & 2D (GRAPPA)/ & 2D (GRAPPA)/ & 2D (TGRAPPA)/ \\
& 2D (TGRAPPA) & 3D (TGRAPPA) & 3D (TGRAPPA)) \\
\hline EF & 0.86 & 0.89 & 0.93 \\
EDV & 0.90 & 0.92 & 0.90 \\
ESV & 0.89 & 0.90 & 0.93 \\
SV & 0.89 & 0.89 & 0.85 \\
MM & 0.86 & 0.90 & 0.74 \\
& & & \\
\hline
\end{tabular}

Table 4 (abstract P225) Paired T-tests between the standard and each of the single breath-hold techniques (NS = no significant difference)

\begin{tabular}{llll}
\hline & 2D (GRAPPA) & 2D (TGRAPPA) & 3D (TGRAPPA) \\
\hline EF & $0.54(\mathrm{NS})$ & $0.98(\mathrm{NS})$ & $0.72(\mathrm{NS})$ \\
EDV & $\mathrm{P}<.05$ & $\mathrm{P}<.05$ & $\mathrm{P}<.0 \mathrm{I}$ \\
ESV & $\mathrm{P}<.05$ & $0.06(\mathrm{NS})$ & $\mathrm{P}<.05$ \\
SV & $\mathrm{P}<.05$ & $0.06(\mathrm{NS})$ & $\mathrm{P}<.0 \mathrm{I}$ \\
$\mathrm{MM}$ & $0.98(\mathrm{NS})$ & $0.08(\mathrm{NS})$ & $0.26(\mathrm{NS})$ \\
\end{tabular}

values; segmented $2 \mathrm{D}$ protocol consistently yielded higher values than the other protocols in these cases.

Discussion: The results of linear regression and paired t-tests suggest that the real-time 2D TGRAPPA technique can potentially substitute the standard clinical protocol. However, the data showed that the experimental protocols tended to underestimate EDV, ESV, and SV. It has been reported that prospective triggering leads to significantly lower EDV and SV values, since the late diastole is not covered by prospective triggering [2]. Since the gold standard in this study is retrospectively triggered, the difference in ECG triggering could explain the significant differences in LV volumes.

\section{References}

I. Breuer FA, et al: Dynamic autocalibrated parallel imaging using temporal GRAPPA (TGRAPPA). Magn Reson Med 2005, 53(4):98I-5.

2. Sievers B, et al: Impact of the ECG gating method on ventricular volumes and ejection fractions assessed by cardiovascular magnetic resonance imaging. J Cardiovasc Magn Reson 2005, 7(2):44I-6.

P226

Fully automatic segmentation of short and long axis cine cardiac MR

Maxim Fradkin', Cybele Ciofolo-Veit', Benoit Mory', Gilion Hautvast ${ }^{2}$ and Marcel Breeuwer ${ }^{2}$

'Philips Healthcare, Suresnes, France

${ }^{2}$ Philips Healthcare, Best, Netherlands

Journal of Cardiovascular Magnetic Resonance 2009, I I(SuppI I):P226

Introduction: Quantitative analysis of cardiac function requires delineation of the left ventricle (LV) in cine cardiac MR (CMR). Typically, this is done using short-axis (SA) images, however, acquisition of several long-axis (LA) views has become quite common. The latter can be used for the accurate and reproducible determination of the basal SA slice, known as one of the major inter-observer variability factors in SA LV measurements [I]. Since manual LV delineation is very tedious and time-consuming, automatic segmentation methods, enabling to obtain reproducible LV measurements, are highly desirable.

Purpose: We propose a fully automatic method for delineation of the endo- and epicardial contours in SA and LA cine CMR images in order to provide automatic, accurate quantitative leftventricular functional assessment. 
Figure I (abstract P226)
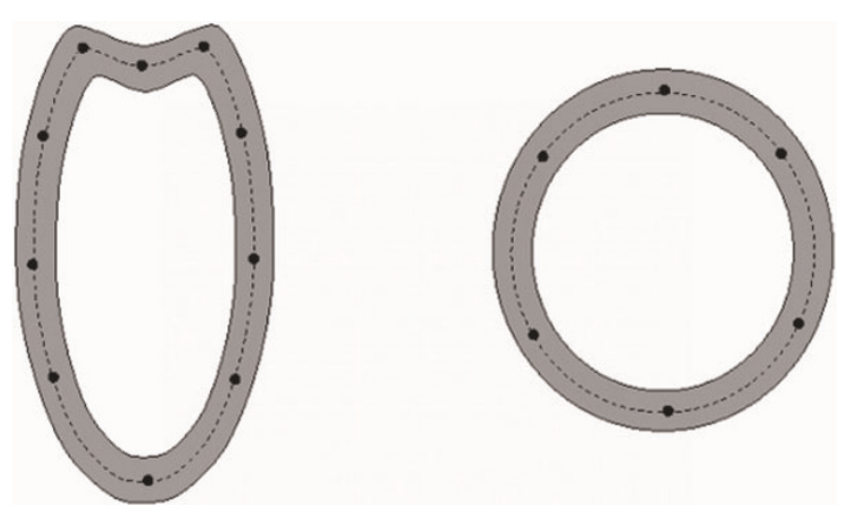

Deformable templates modeling LA (left) and SA (right): centerline (dashed) is defined by a few nodes, the template width (grey ribbon) may vary from node to node.

Methods: Our segmentation workflow consists of two steps: (I) automatic detection and delineation of the LV myocardium and valve plane in LA image(s); (2) segmentation of SA myocardial contours, using the results of step I. For modelling the LV, we use ribbon-like template, relying on interpolating splines and defined by a very few nodes; its shape is controlled by nodes' position and the template's width at each node (Figure I). The template is deformed using greedy optimization framework [2]. The optimization criterion comprises three terms, responsible for templates' shape smoothness, border contrast and region homogeneity. The optimization is done in coarse-to-fine manner, by relaxing the deformation type (from rigid to local) and adjusting the influence (weights) of different terms.

The initial position of the LA template is derived from the image acquisition geometry, while that for the SA templates is obtained by projecting the LA segmented contours on the corresponding SA slices.

We quantitatively assessed the performance of the method on a database of 35 cine CMR acquisitions (Philips Gyroscan NT Intera I.5 T, SSFP protocol, TE $=1.6 \mathrm{~ms}, \mathrm{TR}=3.3 \mathrm{~ms}$, flip angle $50^{\circ}$ for $\mathrm{SA}$ and TE $=1.5-1.7 \mathrm{~ms}, \mathrm{TR}=3.0-3.4 \mathrm{~ms}$, flip angle $55^{\circ}$ for LA). The SA ones included 9 to I 4 slices ( 325 slices in total), and the LA ones I to 2 views ( 40 views in total). The assessment

\section{Figure 2 (abstract P226)}

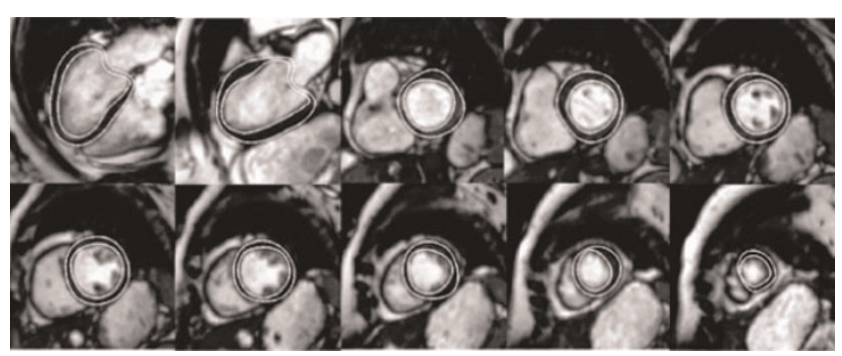

LV segmentation example for one patient: (from left to right, top to bottom): LA 4 chambers and 2 chambers; consecutive slices of SA volume from valve to apex. was made by comparing the automatically segmented contours with Gold Standard manual delineations provided by an expert. Results: For SA images, the mean positioning error (MPS) was $1.3 \pm 0.5 \mathrm{~mm}$ for the endocardium and $1.5 \pm 0.8 \mathrm{~mm}$ for the epicardium, for a pixel size of $1.4-1.8 \mathrm{~mm}$. For LA images, the MPS was $\mathrm{I} .3 \pm 0.4 \mathrm{~mm}$ (endocardium) and $\mathrm{I} . \mathrm{I} \pm 0.4 \mathrm{~mm}$ (epicardium), for a pixel size of $1.3-1.8 \mathrm{~mm}$. Both results are under one pixel accuracy and comparable with inter- and intraobserver variability. An excellent correlation was found between "manual" and "automatic" LV volumes $(r=0.98, P<0.00 \mathrm{I})$, with mean difference $2.5 \pm 7.6 \mathrm{ml}(5.5 \pm 3.9 \%)$. Note that all results were obtained without any user interaction. An example of segmentation results for one patient (8 SA slices and 2 LA views) is shown in Figure 2. We validated the method for the end diastolic (ED) phase only, since the segmentation of the whole cardiac cycle can be then obtained using automatic propagation of ED contours [3]. In earlier investigations, we already showed that propagation preserves the segmentation accuracy within acceptable ranges while being much faster than phase-by-phase segmentation.

Conclusion: We presented a robust, accurate and efficient method for the fully automatic delineation of the myocardium contours in LA + SA cine CMR images, which can be used for accurate LV functional assessment.

\section{References}

I. Weaver A, et al: J Cardiovasc Magn Reson 2006, I:82-83.

2. Williams DJ and Shah M: CVGIP: Image Understanding 1992, 55( I): |4-26.

3. Hautvast, et al: IEEE Trans Med Imag 2006, 25(I I):I4721482.

\section{P227}

Improved image reconstruction

incorporating non-rigid motion

correction for cardiac MRI using BLADE acquisition

Peter Kellman ', Magalie Viallon ${ }^{2}$, Christophe Chefd'hotel ${ }^{3}$, Alto Stemmer ${ }^{4}$ and Pierre Croisille ${ }^{5}$

${ }^{\prime} \mathrm{NIH}$, Bethesda, MD, USA

${ }^{2}$ University Hospital of Geneva, Geneva, Switzerland

${ }^{3}$ Siemens Corporate Research, Princeton, NJ, USA

${ }^{4}$ Siemens Medical Solutions, Erlangen, Germany

${ }^{5}$ University Hospital of Cardiology Louis Pradel, Lyon, France

Journal of Cardiovascular Magnetic Resonance 2009, I I(SuppI I):P227

Introduction: The application of the BLADE sequence for dark blood T2-weighted cardiac MRI has the potential for increasing

Figure I (abstract P227)

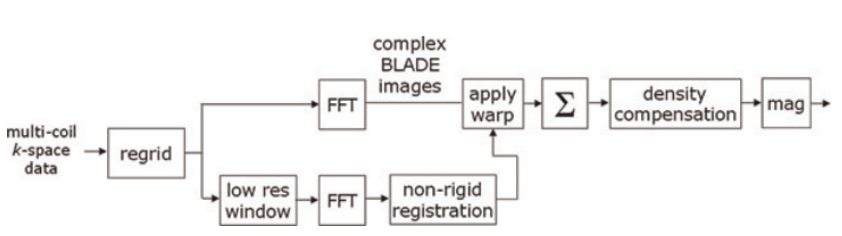

Motion corrected BLADE image reconstruction diagram (simplified). 
Figure 2 (abstract P227)

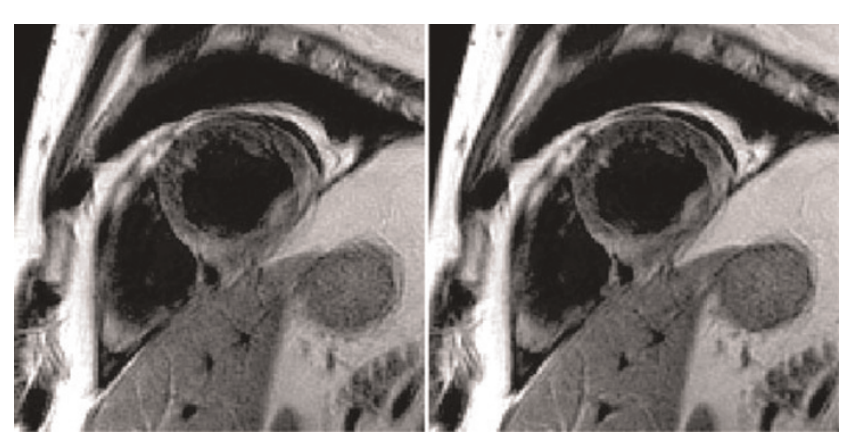

Example of a dark-blood prepared TSE BLADE image showing conventional (left) and motion corrected (right) reconstruction for a case in which there are no respiratory image artifacts.

the spatial resolution thereby improving the detailed depiction of morphology [I]. While the BLADE sequence using periodically rotating overlapping parallel lines (PROPELLER) [2] inherently provides a degree of motion tolerance, significant respiratory motion may result in image artifacts. Rigid body motion correction applied to PROPELLER MRI [2] is well suited to applications such brain imaging but is problematic in cardiac MR where the motion is not rigid. Non-rigid image registration has been successfully applied for motion corrected averaging in cardiac MR [3]. In the present study, nonrigid motion correction was applied to individual BLADE images prior to combination as a high resolution image, to migitate respiratory motion artifacts that arise despite the use of a navigator.

Purpose: To develop and evaluate an improved image reconstruction method for cardiac MRI acquired using a navigated BLADE sequence.

Methods: Dark blood prepared, navigated, ECG-gated T2weighted cardiac MR imaging was performed using a BLADE sequence with TSE readout. Typical parameters for imaging using the Siemens Magnetom AVANTO I.5 T scanner were: echo train

\section{Figure 3 (abstract P227)}

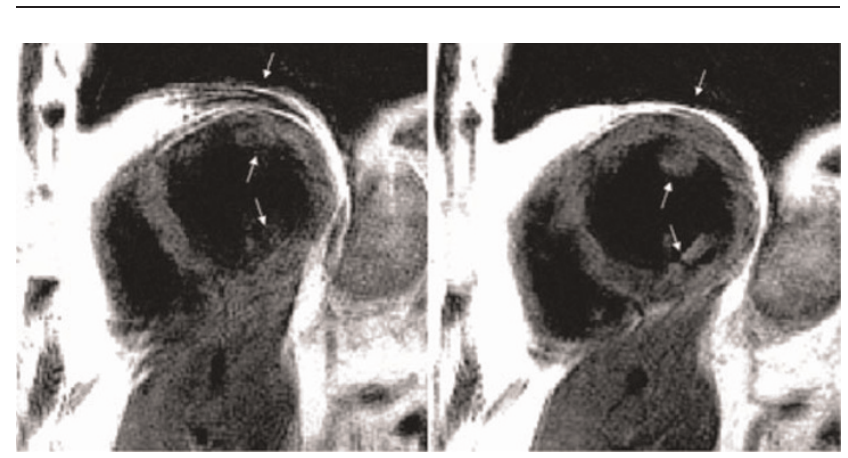

Example of a dark-blood prepared TSE BLADE image showing conventional (left) and motion corrected (right) reconstruction for a case in which there are significant respiratory image artifacts in conventional reconstruction (indicated by arrows). length $=26$, echospacing $=6 \mathrm{~ms}, \mathrm{TE}=78 \mathrm{~ms} ; \# \mathrm{BLADES}=17$, readout resolution $=256, \mathrm{PE}$ resolution $=44$ per $\mathrm{BLADE}$ after rate 2 parallel imaging using 8 central reference lines, typical FOV $320 \times 320$ with $1.25 \times 1.25 \mathrm{~mm}^{2}$ in-plane resolution with $6 \mathrm{~mm}$ slice thickness.

Imaging reconstruction (Figure I) was performed off-line from raw data using MATLAB. Sub-pixel non-rigid image registration [4] was performed on the individual low resolution BLADE magnitude images (following parallel imaging reconstruction) and the resultant motion field was applied to the full resolution complex BLADE images prior to combining as a full resolution image $(256 \times 256)$. Density correction was then applied to the full resolution image.

Results: Motion corrected and conventional BLADE reconstructions were compared for a number of slices for $\mathrm{N}=11$ patient studies. These studies included cases for which conventional reconstructions were of good quality (Figure 2) as well as several cases which exhibited respiratory motion artifacts (Figure 3). The proposed method did as well or better in all cases with significant artifact reduction in several cases.

Conclusion: The proposed approach for BLADE reconstruction using non-rigid motion correction significantly improves the image quality in cases with respiratory motion artifacts. The method is fully automatic not requiring any user interaction to define bounding regions of interest.

References

I. Viallon M, et al: Proceedings ISMRM 2008, 16: 1008.

2. Pipe J, et al: Magn Reson Med 1999, 42(5):963-969.

3. Ledesma MJ, et al: J Magn Reson Imaging 2007, 26: I84-I90.

4. Chefd'hotel C, et al: Proc IEEE ISBI 2002.

\section{P228}

Reproducibility of aortic pulse wave velocity measurements obtained with Phase Contrast Magnetic Resonance (PCMR) and applanation tonometry

Jonathan Suever', David Huneycutt ${ }^{2}$,

Enrique Rojas-Campos ${ }^{3}$, Francesca Cardarelli ${ }^{3}$,

Amol Pedneker ${ }^{4}$, Sam Fielden ${ }^{2}$, Arthur Stillman ${ }^{3}$, Paolo Raggi ${ }^{3}$ and John Oshinski ${ }^{2}$

'Biomedical Engineering, Emory University/

Georgia Institute of Technology, Atlanta,

GA, USA

${ }^{2}$ Radiology, Emory University School of

Medicine, Atlanta, GA, USA

${ }^{3}$ Cardiology, Emory University School of

Medicine, Atlanta, GA, USA

${ }^{4}$ Philips Medical Systems, Cleveland, OH, USA

Journal of Cardiovascular Magnetic Resonance 2009, I I(SuppI I):P228

Objective: To compare reproducibility of aortic pulse wave velocity (PWV) measurements obtained from phase contrast magnetic resonance (PCMR) and cross-correlation analysis to applanation tonometry.

Background: Increased pulse wave velocity (PWV) results from arterial stiffening and is commonly seen with ageing and in patients with atherosclerosis and/or hypertension [I]. Currently, applanation tonometry is the clinically accepted method to 
Figure I (abstract P228)
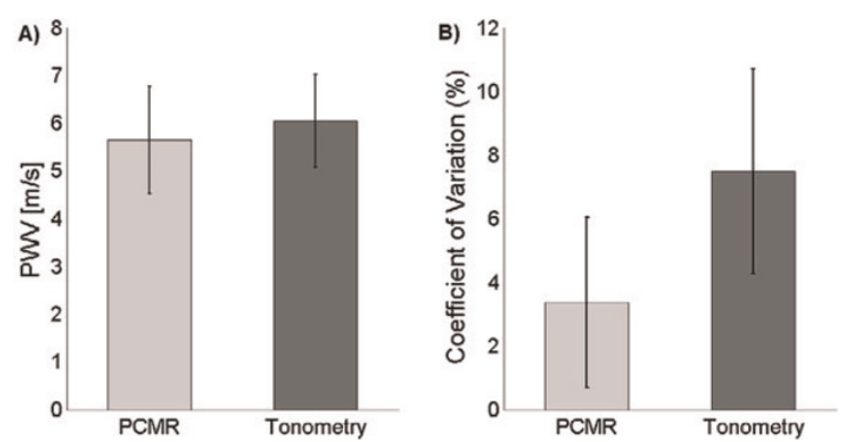

a) Average PWV for all subjects for both PCMR and tonometry $(P>0.05)$. b) Inter-scan coefficient of variation for each modality $(P<0.05)$.

quantify aortic PWV and determine aortic compliance. A direct comparison of PWV values and reproducibility between PCMR and tonometry has not been done.

Methods: PWV was measured in ten healthy volunteers (age: $32.9 \pm 8.3$ years) two times with each modality. To obtain PCMR measurements, subjects were scanned in a $1.5 \mathrm{~T}$ Philips Intera CV scanner (Philips Medical Systems, Best, The Netherlands). Oblique sagittal images in the plane of the aorta were acquired and velocity was encoded in the foot-head direction. After the first PCMR scan, subjects were removed from the scanner, repositioned, and the scan was repeated. After two PCMR scans, subjects remained in the supine position and underwent two measurements of the aortic (carotid-to-femoral) PWV by applanation tonometry using a Sphygmocor device (AtCor Medical, Sydney, Australia) in an adjacent room.

PCMR data were analyzed by examining the velocity waveforms at multiple points along the length of the thoracic and abdominal aorta throughout the cardiac cycle. Cross correlation between flow waveforms was then used to determine the transit time between two adjacent locations along the aorta [2]. A robust bisquare-weighted linear regression algorithm was used to fit a line to the arrival time/location plot, which was then used to compute PWV. Applanation tonometry data were analyzed automatically by the Sphygmocor software using a standard transfer formula.

Agreement and repeatability of PCMR and tonometry measurements were assessed by Bland-Altman statistics [3]. Inter-scan variability for each modality was evaluated with the Coefficient of Variation (CV). P-values less than 0.05 were considered statistically significant.

Results: Mean PWV values from PCMR and applanation tonometry were not statistically different (PCMR: $5.6 \pm \mathrm{I} . \mathrm{I} \mathrm{m} / \mathrm{s}$; Tonometry: $6.0 \pm 1.0, P>0.05$ ) (Figure la). The average coefficient of variation (CV) was significantly lower for PCMR compared to tonometry, $3.4 \% \pm 2.7 \%$ versus $7.5 \% \pm 3.2 \%$, respectively $(P<0.05)$ (Figure Ib). Bland Altman analysis showed that there was a $0.4 \mathrm{~m} / \mathrm{s}$ bias with tonometry being higher and a confidence interval of $\pm 1.3 \mathrm{~m} / \mathrm{s}$.

Conclusion: PWV was successfully measured by PCMR combined with cross-correlation analysis and by applanation tonometry. There was no significant difference in the PWV values; however, PCMR has a statistically significant lower intertest CV compared to tonometry. It can be concluded that PCMR and cross-correlation analysis can be combined to accurately assess PWV as an estimate of aortic compliance.

\section{References}

I. Farrar DJ, et al: Circulation 1991, 83(5): 1754-63.

2. Fielden SW, et al: J Magn Reson Imaging 2008, 27(6): | 382-7.

3. Bland JM and Altman DG: Lancet 1986, I:307-10.

\section{P229}

Non-contrast fresh-blood MRA

for assessment of abdominal endovascular stent grafts

Timothy SE Albert ', Connie Luna ${ }^{1,2}$, Patrik Zetterlund ${ }^{3}$ and Mitsue Miyazaki ${ }^{4}$

'Salinas Valley Memorial Hospital, Cardiovascular Diagnostic Center, Monterey, CA, USA

${ }^{2}$ Salinas Valley Memorial Hospital, Salina, CA, USA

${ }^{3}$ Central Coast Cardiology Research, Salinas, CA, USA

${ }^{4}$ Toshiba Medical Research Institute, Vernon Hills, IL, USA

Journal of Cardiovascular Magnetic Resonance 2009, I I(SuppI I):P229

Objective: Serial imaging of abdominal aortic endovascular stent grafts (AA-ESG) is done in order to determine: I) stent stability, 2) presence of endograft leak, and 3) residual abdominal aortic aneurysm (AAA) sac size. CT angiography (CTA) has traditionally been used for serial follow-up. Given the high incidence of renal dysfunction in patients with AAA and potential contraindications to both $\mathrm{CT}$ and $\mathrm{MR}$ contrast agents we development a non-contrast vascular imaging protocol for assessment of patients with AA-ESG.

Methods: Consecutive patients who had AAA repair with AAESG and had prior CTA for comparison were imaged with a combined black blood (BB) imaging protocol using 2D single-shot FSE for depicting vessel wall dimensions (aneurysm sac size) and a fresh-blood imaging (FBI) technique using 3D single-shot FSE for bright blood imaging of the vessel lumen (stent stability). Comparisons were made in accuracy of endovascular luminal assessment ( $\mathrm{FBI}$ vs. CTA along 3 points, see Figure I) and aneurysm wall-to-wall dimensions (BB vs. CTA). The noncontrast technique did not allow direct assessment of endograft leak. CTA was performed on a Toshiba Aquilion 64-slice CT scanner while MRI imaging was performed using a Toshiba Vantage Atlas I.5 T MRI scanner.

Results: Consecutive patients are reported on $(n=5): 73 \pm$ 4 yo, $45 \pm 16$ months since AAA repair (mean \pm SD). Aneurysm dimension at its greatest size as assessed by CTA $(3.9 \pm 0.1 \mathrm{~cm})$ was similar to that for BB imaging $(3.8 \pm 0.1 \mathrm{~cm})$ $(p=0.40)$. Endograft visualization as assessed by luminal dimensions was similar between $\mathrm{FBI}$ and CTA ( $r 2=0.96$, $n=15)$ with good visualization of the AA-ESG and surrounding arterial tree.

Conclusion: A combined approach of $2 \mathrm{D} B B$ and 3D FBI imaging of AA-ESG can accurately assess aneurysm sac size and endograft angiographic characteristics. Although this non-contrast technique cannot directly assess for endograft leak this approach may still be an acceptable alternative to contrast 
Figure I (abstract P229)

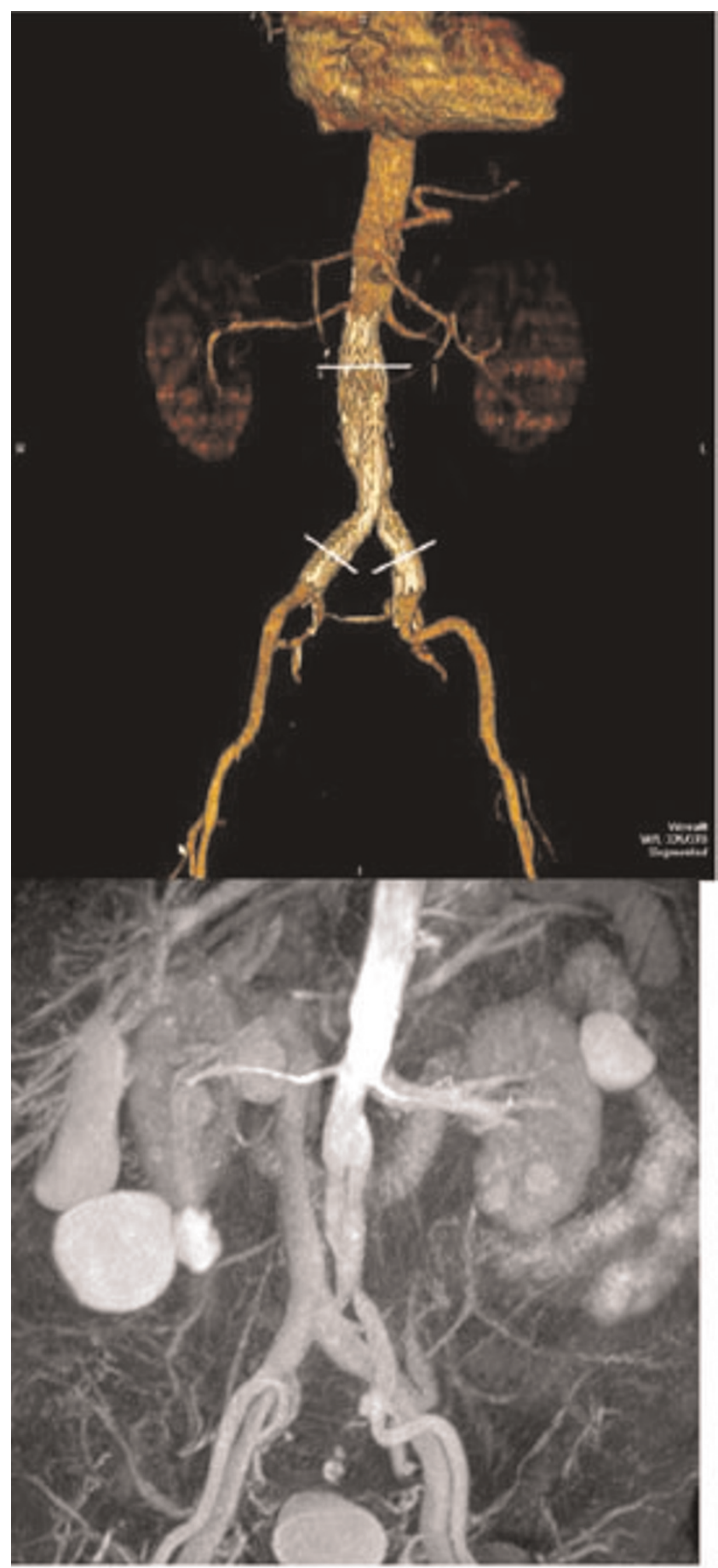

CTA (upper image) and FBI (lower image) of same patient after AA-ESG. The diastolic-triggered $\mathrm{FBI}$ image clearly depicts the lumen of the endovascular stent graft and its iliac sleeves in bright blood. The abdominal veins and large renal cysts are also well visualized.

imaging for serial follow-up of patients with renal dysfunction. Further validation of this novel technique will need to be done in a larger cohort of patients.

\section{P230}

Relationship of aortic stiffness measured by cardiovascular magnetic resonance to arterial stiffness estimates by tonometry

Ilias Kylintireas, Colin Cunnington,

Malcolm Kohler, Jonathan Diesch,

Corinne Trevitt, Robin Choudhury,

Steffen Petersen, Stefan Neubauer, Matthew Robson and Paul Leeson

University Of Oxford, Oxford, UK

Journal of Cardiovascular Magnetic Resonance 2009, I I(SuppI I):P230

Introduction: Arterial stiffness assessed by arterial tonometry - in particular pulse wave velocity and augmentation index - is associated with increased cardiovascular risk. It is now possible to use cardiovascular magnetic resonance imaging (CMR) for direct imaging of aortic function. This provides a precise assessment of the elastic properties of the aorta within distinct regions of the aorta. We therefore studied what parameters of aortic function are related to arterial tonometry-derived indices of arterial stiffness.

Purpose: We compared aortic stiffness assessed by cardiovascular magnetic resonance (CMR) pulse wave velocity (PWV) based on flow, velocity or acceleration, and aortic distensibility $(A D))$ to widely used arterial stiffness estimates provided by arterial tonometry (pulse wave velocity (PWVS) and pulse wave analysis (AI)).

Methods: Thirty young healthy volunteers (aged 23-33) underwent tonometry (Sphygmacor) for determination of carotid to femoral PWVS and radial pulse wave analysis for $\mathrm{Al}$ estimation. They then underwent CMR to assess aortic elastic properties.

Aortic distensibility was assessed from breath-hold ECG-gated, steady state free precession (SSFP) images (TR $=2.8 \mathrm{~ms}$, echo time, TE $=1.4,2 \mathrm{~mm} \times 2 \mathrm{~mm}$, slice thickness $7 \mathrm{~mm}$, temporal resolution $40 \mathrm{~ms}$ ) acquired at the level of the right pulmonary artery through the ascending and proximal descending aorta and through the distal aorta below the diaphragm to determine aortic

\section{Figure I (abstract P230)}

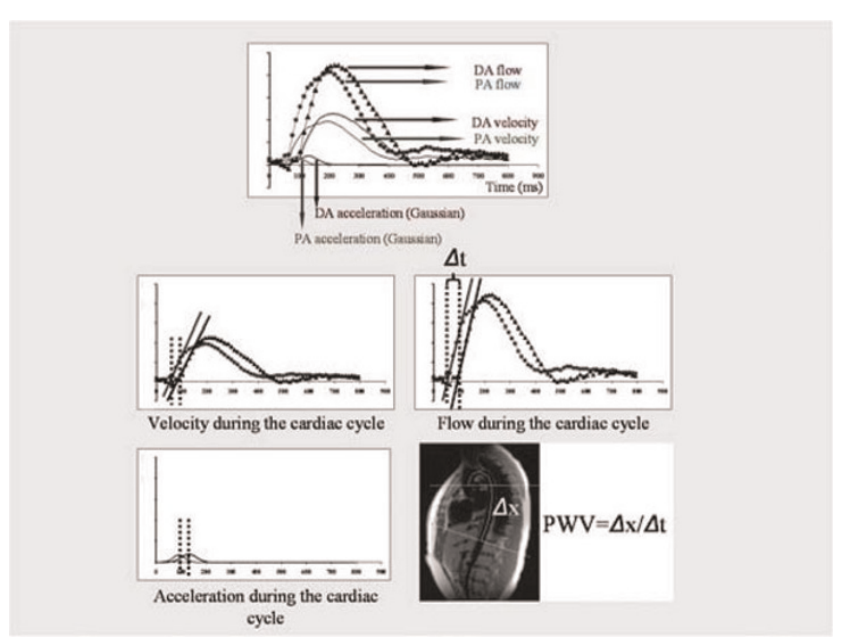




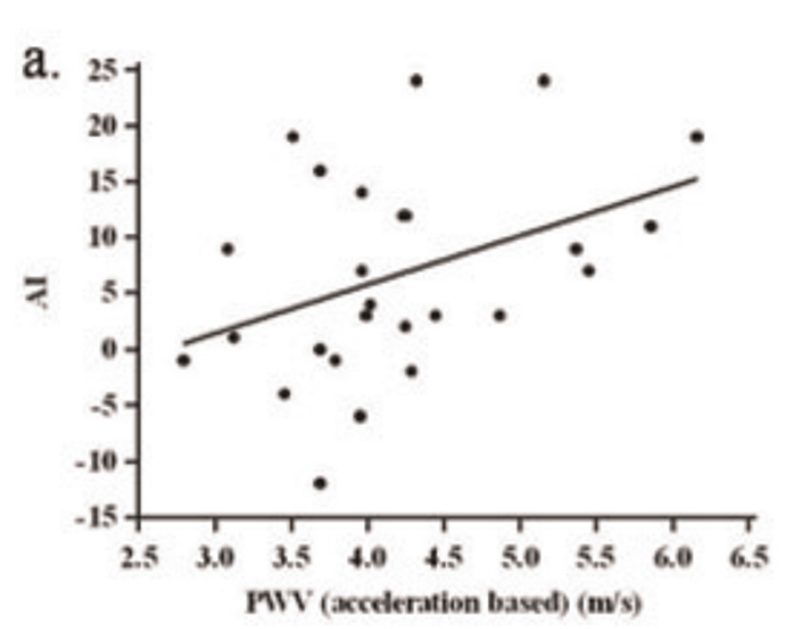

c.

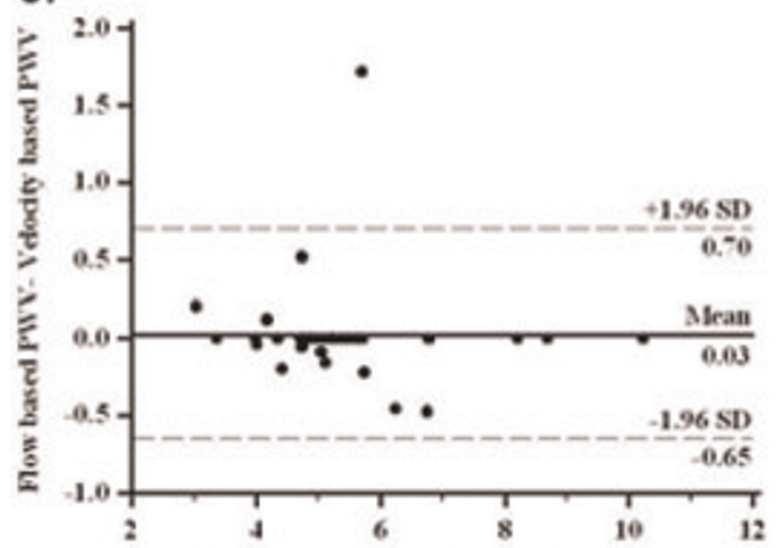

AVERIGE of How based PWV: Velody based PWV
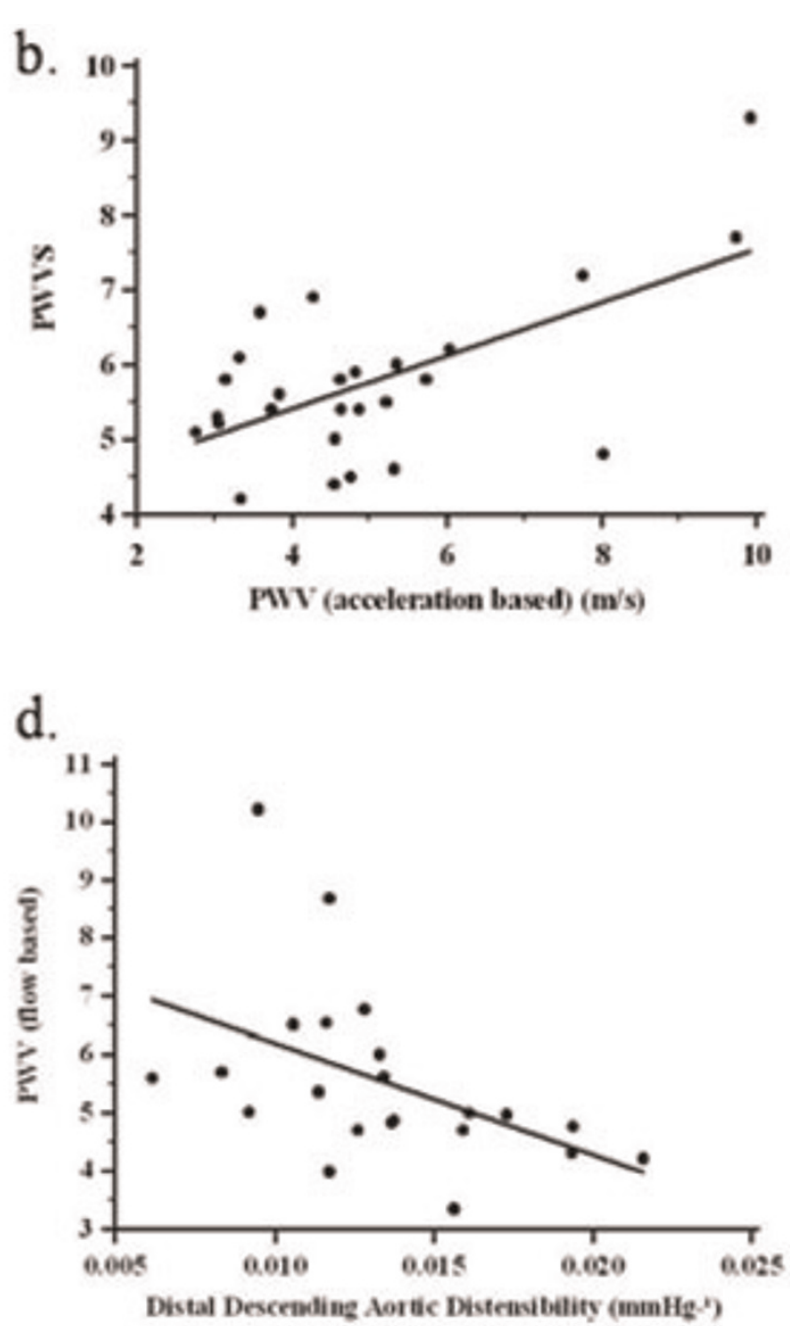

PWV over the whole length of the aorta $(r=0.615$, $P<0.001)$ (Figure $2 b)$ but also related to flow $(r=0.484$ $P<0.05)$ and velocity $(r=0.372, P<0.05)$ based MRI PWV measurements.

Aortic pulse wave velocity calculated by all three MR methods yielded an inverse correlation with descending aortic distensibility $(r=-0.521, P<0.05)$ (Figure $2 d$ ) but PWVS and Al did not correlate with aortic distensibility

Conclusion: PWVS and Al most closely reflect accelerationbased CMR PWV measurements of overall aortic stiffness. Arterial tonometry has been validated in large scale clinical studies suggesting this CMR method may be most useful for interpretation of clinical risk. Regional and overall aortic measures of elasticity were not strongly related. However, CMR PWV measures inversely correlated with aortic distensibility, suggesting that MR PWV values may provide a closer estimate of local aortic elastic properties. 
P23I

TIMI perfusion grade compared to TIMI flow in prediction of infarct size and microvascular obstruction measured by contrast-enhanced MRI Georg FK Fuernau, Kathrin Schindler, Ingo Eitel, Josef Friedenberger, Eigk Grebe, Gerhard Schuler and Holger Thiele

University of Leipzig - Heartcenter, Leipzig, Germany

Journal of Cardiovascular Magnetic Resonance 2009, I I(SuppI I):P23 I

Background: The TIMI perfusion grade (TMPG) and TIMI flow are angiographic parameters with prognostic impact in STelevation myocardial infarction (STEMI). The association of these parameters with infarct size and microvascular obstruction assessed by MRI, which also have of prognostic impact, has not been studied so far.

Methods: From 02/2006-0I/2008 280 consecutive patients with STEMI underwent primary $\mathrm{PCl}$. In an MRI study (day I-4 after $\mathrm{PCl}$ ) infarct size and microvascular obstruction in percent of left ventricular mass (\%LV) were measured by delayed enhancement. In addition, post-PCI TIMI flow and TMPG were assessed from invasive angiography. All measurements were done by blinded investigators. TMPG and TIMI flow were graded as severely impaired (0-I) or mildly impaired-normal (2-3).

Results: For post-PCI TIMI flow there was a significant difference in the extent of microvascular obstruction (TIMI $0-I: 6.6 \pm 5.1 \% \mathrm{LV}$ vs. TIMI $2-3: 3.6 \pm 4.6 \% \mathrm{LV}, \mathrm{p}=0.02 \mathrm{I})$, but no significant difference in infarct size (TIMI 0-I: $25.9 \pm 15.3 \% \mathrm{LV}$ vs. TIMI 2-3: $21.19 .3 \pm 13.4, p=0.07)$. For TMPG microvascular obstruction (TMPG 0-I: $5.3 \pm 5.2 \%$ LV vs. TMPG 2-3: $3.5 \pm 4.5 \% \mathrm{LV}, P=0.0 \mathrm{II})$ and infarct size were significantly different (TMPG $0-1$ : $25.0 \pm 13.0 \% \mathrm{LV}$ vs. $18.6 \pm 13.5 \% \mathrm{LV}$, $P=0.002$ ). In a multivariable model the strongest predictors of infarct size and microvascular obstruction were post-PCI TMPG, infarct location, Killip class, and 90 minute ST-segment resolution $(p<0.005$ for all).

Conclusion: In STEMI patients undergoing primary $\mathrm{PCl}$ the TMPG is a better indicator of angiographic success compared to TIMI flow reflected by a significant difference in infarct size measured by cardiac MRI. This might explain why the TMPG has additional prognostic impact in patients with restoration of normal TIMI flow.

\section{P232}

MRI adenosine fist-pass perfusion analysis using a SSFP sequence - are there gender differences? Nico Merkle, Markus Kunze, Volker Rasche, Matthias Kochs, Vinzenz Hombach and Jochen Woehrle University Ulm, Ulm, Germany

Journal of Cardiovascular Magnetic Resonance 2009, I I(SuppI I):P232

Purpose: Significant gender differences have been found in the performance of exercise ECG for the identification of coronary artery disease. Our aim was to evaluate possible differences in the diagnostic power of cardiac MRI using SSFP-Perfusion under adenosine related vasodilatation in both gender subgroups in a large consecutive cohort of patients with suspected CAD.

Methods: 228 (male $n=180$, female $n=48$ ) patients with suspected CAD were examined with MRI (I.5 T Intera CV) and coronary angiography. A 3-slice short axis SSFP perfusion scan with a saturation prepulse before each slice was performed during infusion of adenosine and at rest followed by myocardial scar imaging. Gadolinium - DTPA was given at $0.1 \mathrm{mmol} / \mathrm{kg}$ body weight. Images were assessed visually by two observers in a joint reading.

Results: Sensitivity, specificity and accuracy of MRI first-pass perfusion for detection of a coronary artery stenosis (>50\% luminal narrowing) on a per patient basis were $92,3 \%, 81,6 \%$ and $90.0 \%$ in the male subgroup and $97,7 \%, 94,4 \%$ and $95,8 \%$ in the female subgroup. The results for detection of a significant lesion (>70\% luminal narrowing) were $96.0 \%, 68,5 \%$ and 87,8 in the male subgroup and $96,3 \%, 80,9 \%$ and $89,6 \%$ in the female subgroup.

Conclusion: In contrast to data from stress ECG, MRI adenosine fist-pass perfusion analysis using a SSFP sequence reveals no differences regarding diagnostic performance in female compared to the male group in our cohort.

\section{P233}

Intraindividual evaluation of left ventricular function with 64-slice computed tomography, biplane cineventriculography, and two- and threedimensional transthoracic echocardiography: comparison with magnetic resonance imaging as the gold standard

Marc Dewey, Johannes Greupner, Elke Zimmermann, Andrea Grohmann, Hans-Peter Dübel, Till Althoff, Adrian Constantin Borges, Wolfgang Rutsch and Bernd Hamm

Charite-Universitätsmedizin Berlin, Berlin, Germany

Journal of Cardiovascular Magnetic Resonance 2009, I I(SuppI I):P233

Introduction: To intraindividually compare left ventricular function assessed with multislice computed tomography using 64 simultaneous detector rows (MSCT), biplane cineventriculography (CVG), and both 2D and 3D transthoracic echocardiography (2D and 3D Echo) with magnetic resonance imaging (MRI) as the gold standard as an ancillary single-center study of the multicenter trial "CorE-64".

Methods: A total of 38 patients prospectively underwent MSCT (Aquilion 64, Toshiba Medical Systems, Nasu, Japan), CVG, and MRI, and 2D as well as 3D Echo. Institutional review board approval for this prospective, cardiac function, ancillary study of a multicenter trial on coronary imaging was obtained.

Results: Regarding the ejection fraction, the agreement was significantly superior for MSCT $( \pm 13.8 \%)$ than for CVG $( \pm$ $20.4 \% ; P=0.02$; F-test $)$ and both $2 \mathrm{D}$ Echo $( \pm 19.3 \% ; P=0.049$; F-test) as well as 3D Echo ( $\pm 21.7 \% ; P=0.01 ; F$-test). MSCT (56.9 $\pm 14.7 \%, P=0.8)$, 2D Echo $(56.5 \pm 14.7 \%, P=0.9$, t-test $)$ and $3 \mathrm{D}$ Echo $(58.7 \pm 16.4 \%, P=0.34$, t-test $)$ did not significantly under- or overestimate ejection fraction in comparison to MRI (56.5 $\pm 16.0 \%)$, whereas CVG $(60.9 \pm 13.8 \%, P=0.02$, t-test) significantly overestimated ejection fraction. For the stroke volumes, the limits of agreement for CVG $( \pm 56.5 \mathrm{ml}$, $P=0.00 \mathrm{l})$ and $2 \mathrm{D}$ and $3 \mathrm{D}$ Echo $( \pm 45.4 \mathrm{ml}$ and $\pm 49.8 \mathrm{ml}$ respectively, both $P<0.05)$ were also significantly larger in comparison to MRI than for MSCT $( \pm 31.2 \mathrm{ml})$. In comparison to the reference standard MRI, CVG but not MSCT significantly overestimated the end-diastolic volume $(P<0.00 \mathrm{I})$. In contrast, both $2 D$ and $3 D$ Echo significantly underestimated the 
end-diastolic volume (both: $P<0.05$ ) in comparison to the reference standard MRI.

Conclusion: 64-slice CT appears to be significantly more accurate than CVG, 2D and 3D Echo in comparison to MRI as the reference standard. Thus, CT using 64-slice technology should allow reliable evaluation of left ventricular function in clinical practice using the same data as acquired for noninvasive coronary CT angiography.

\section{P234}

The incidence of microvascular obstruction with acute myocardial infarction and the relationship between the occurrence of microvascular obstruction and infarct size, angiographic findings and clinical background: gadolinium-contrasted MRI study

Masashi Kawade, Kunihiko Teraoka, Shintaro Kiuchi, Yoshinori Suzuki, Kenji Takazawa and Akira Yamashina Tokyo Med. University, Tokyo, Japan

Journal of Cardiovascular Magnetic Resonance 2009, I I(SuppI I):P234

Introduction: It has been shown that the presence of microvascular obstruction in humans after myocardial infarction is associated with poor prognosis and worse left ventricle remodeling. Even now it is not unclear to avoid the occurrence of $\mathrm{MO}$ as the reperfusion injury with reperfusion therapy and to treat it.

Purpose: To clarify the background of the occurrence of $\mathrm{MO}$, the incidence of $M O$ with $A M I$ pts in the acute phase was evaluated, and the relationship between the infarct size and coronary angiographic findings, and clinical backgrounds, regional wall motion were investigated.

Methods: Cardiac MR was performed in 90 patients (male/ female; 72/18, mean age was $61.3+/-11.8$ years old) within 7 days from the onset of $\mathrm{AMI}(6+/-2.4$ days). Especially 25 cases were followed and examined $2^{\text {nd }} \mathrm{CMR}$ at 6 months later. All pts were treated with $\mathrm{PCl}$ successfully and their final TIMI grade

Figure I (abstract P234)

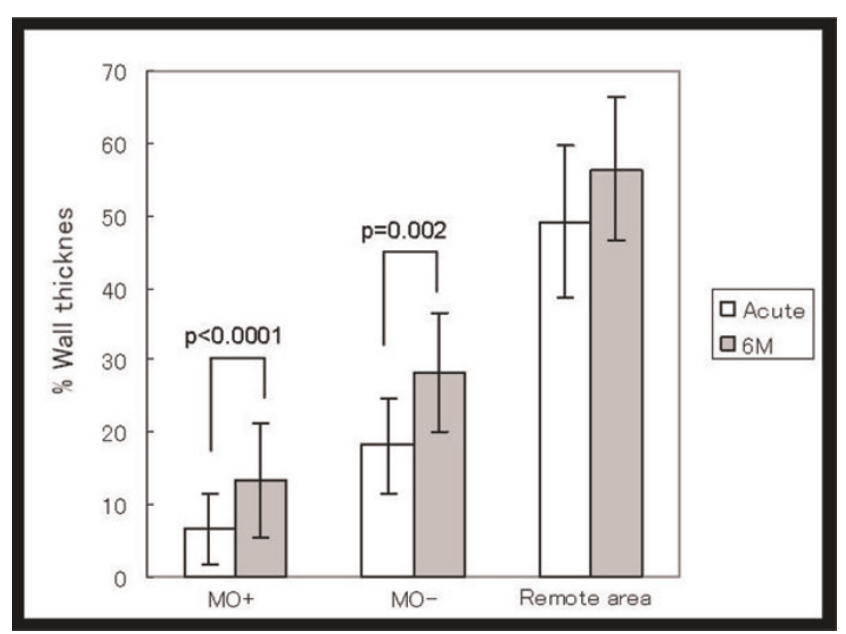

Figure 2 (abstract P234)

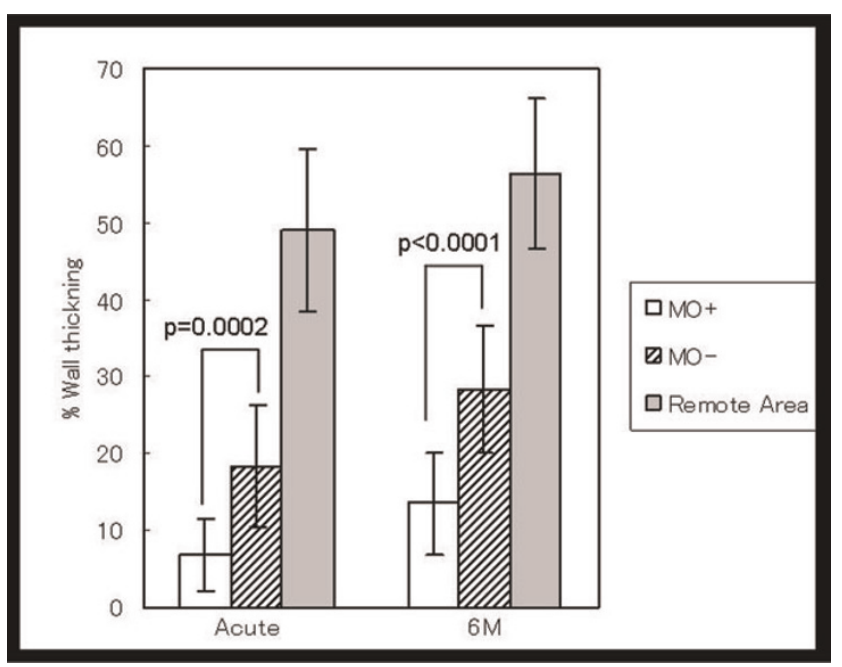

were TIMI III. The MO quantification was determined by perfusion MRI and LGE techniques.

Results: I) MO was recognized in 53 patients (58.9\%) with the both techniques. 2) Infarct area evaluated by LGE was more extended circumferentially $(P<0.000 I)$ and also more extended from endocardium to epicardium in $\mathrm{MO}$ positive patients than negatives significantly, $(P<0.000 \mathrm{I})$. 3) Peak CK level was also higher in $\mathrm{MO}$ positive patients than negatives significantly $(P<0.000 I)$. 4) The time from onset to revascularization, there was a difference in neither. But blush grade and collateral index were lower in $M O$ positive patients than negatives significantly ( $p=0.00058, p=0.046$, respectively). 5) There was higher incidence of heart failure in hospitalization of $\mathrm{MO}$ positive patients than negatives but not significantly (Fisher' test. $P=0.078) 6$ ) The segments showed $M O$ impaired segmental wall thickening (SWT) at acute stage compared to the segments without MO. The SWT remained impaired at the segments with $\mathrm{MO}$, compared to the segments without $\mathrm{MO}$ at 6 months later with 25 cases follow up (Figures I, 2).

Conclusion: In acute stage of AMI, MO was recognized in the patients having large infarction, worse angiographic findings and also the high incidence of heart failure.

\section{P235}

Single dose gadobenate dimeglumine for imaging chronic of myocardial infarction in comparison to double dose gadopentetate dimeglumine Kerstin U Bauner, Maximilian F Reiser and Armin M Huber Ludwig-Maximilian-University Munich, Campus Grosshadern, Munich, Germany

Journal of Cardiovascular Magnetic Resonance 2009, I I(SuppI I):P235

Introduction: Gadopentetate dimeglumine is a nonspecific gadolinium contrast agent, which distributes exclusively to the extracellular fluid space and is eliminated through the kidneys. Gadobenate dimeglumine is a second generation gadolinium agent that distributes extracellularly in the first minutes after 
Table I (abstract P235)

\begin{tabular}{|c|c|c|c|c|}
\hline \multicolumn{2}{|c|}{ Gadobenate dimeglumine } & \multicolumn{2}{|c|}{ Gadopentetate dimeglumine } & \multirow[b]{2}{*}{ p-value } \\
\hline \multicolumn{2}{|c|}{ Single Shot IR SSFP } & \multicolumn{2}{|c|}{ Single Shot IR SSFP } & \\
\hline TI & $246.5 \pm 37.2$ & $\mathrm{TI}$ & $262.7 \pm 25.7$ & 0.027 \\
\hline SNR infarction & $22.5 \pm 4.3$ & SNR infarction & $15.7 \pm 8.5$ & 0.042 \\
\hline SNR myocardium & $4.4 \pm 1.9$ & SNR myocardium & $3.6 \pm 2.1$ & n.s. \\
\hline SNR Ivc & $21.3 \pm 11.8$ & SNR Ivc & $14.6 \pm 11.7$ & n.s. \\
\hline CNR inf-myo & $18.1 \pm 10.1$ & CNR inf-myo & $12.1 \pm 6.7$ & 0.032 \\
\hline CNR inf-Ivc & $1.2 \pm 5.2$ & CNR inf-lvc & $1.1 \pm 6.8$ & n.s. \\
\hline SD noise & $2.3 \pm 2.0$ & SD noise & $2.3 \pm 1.9$ & n.s. \\
\hline \multicolumn{2}{|c|}{ Gadobenate dimeglumine } & \multicolumn{2}{|c|}{ Gadopentetate dimeglumine } & \\
\hline \multicolumn{2}{|l|}{ IR GRE } & \multicolumn{2}{|l|}{ IR GRE } & p-value \\
\hline TI & $244.0 \pm 29.3$ & TI & $271.2 \pm 21.6$ & 0.019 \\
\hline SNR infarction & $33.1 \pm 9.6$ & SNR infarction & $24.1 \pm 6.1$ & 0.001 \\
\hline SNR myocardium & $5.9 \pm 3.3$ & SNR myocardium & $4.4 \pm 1.6$ & n.s. \\
\hline SNR Ivc & $30.7 \pm 8.8$ & SNR Ivc & $25.1 \pm 6.9$ & $<0.001$ \\
\hline CNR inf-myo & $27.2 \pm 5.8$ & CNR inf-myo & $19.7 \pm 5.9$ & 0.005 \\
\hline CNR inf-Ive & $2.4 \pm 5.8$ & CNR inf-lvc & $5.8 \pm 7.9$ & n.s. \\
\hline SD noise & $2.5 \pm 0.7$ & SD noise & $2.3 \pm 0.7$ & n.s. \\
\hline
\end{tabular}

injection, but thereafter undergoes a dual route of elimination with approximately $96 \%$ of the injected dose excreted renally and the remainder taken up by functioning hepatocytes and excreted in the bile. It has a weak reversible binding to albumin, which results in an increase of relaxation rate, compared to gadopentetate dimeglumine.

Purpose: The two-fold higher TI relaxivity of gadobenate dimeglumine compared with gadopentetate dimeglumine and can be used for imaging delayed enhancement in the assessment of

\section{Figure I (abstract P235)}
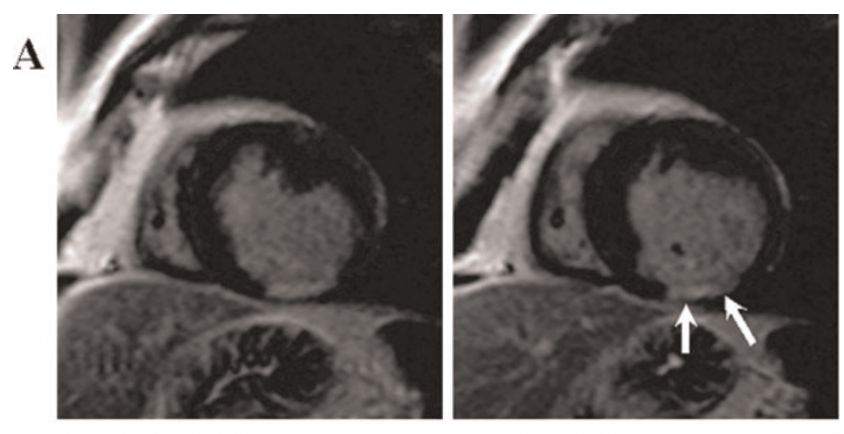

$\mathbf{B}$
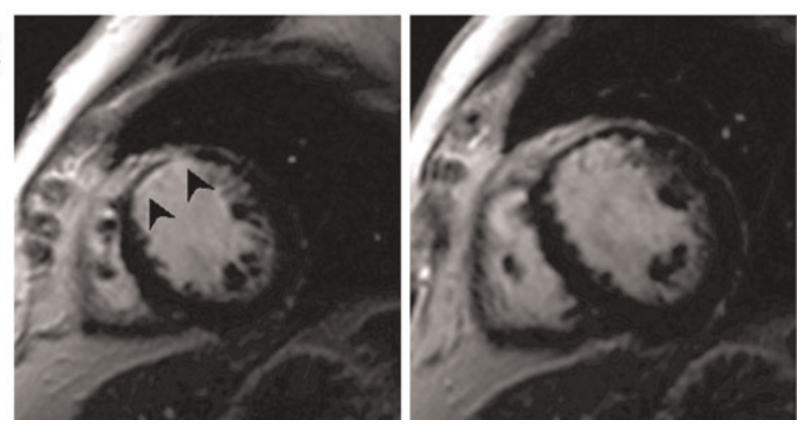

myocardial infarction. The purpose of this study was to compare $0.1 \mathrm{mmol} / \mathrm{kg}$ gadobenate dimeglumine (Gd-BOPTA, MultiHance, Bracco Imaging SpA, Milan, Italy) with $0.2 \mathrm{mmol} / \mathrm{kg}$ gadopentetate dimeglumine (Gd-DTPA, Magnevist, Bayer-Schering Pharma AG, Berlin, Germany) in cardiac MRI.

Methods: The study was performed in accordance to the institutional review board. Two groups of 20 patients underwent MR examinations for evaluation of chronic myocardial infarction on a 1.5 Tesla system. While group I received gadobenate dimeglumine at a dose of $0.1 \mathrm{mmol} / \mathrm{kg}$, group 2 received gadopentetate dimeglumine at a dose of $0.2 \mathrm{mmol} / \mathrm{kg}$. Single Shot IR SSFP and IR GRE sequences were used for imaging delayed enhancement (IR SSFP: TE/TR I.I/2.2 msec, flip 50, spatial resolution I.3 $\times 1.8 \times 8 \mathrm{~mm}^{3}$; IR GRE: TE/TR 4/II msec; flip $25^{\circ}$, spatial resolution $1.3 \times 1.8 \times 8 \mathrm{~mm}^{3}$ ). The sizes of myocardial infarctions were measured for both contrast agents in both imaging techniques by two readers. Bland-Altman analyses were performed for each sequence and gadolinium chelate. Furthermore the transmural extent of myocardial infarction was assessed by two readers according to the 17 segment model for both contrast media and both sequences and kappa-values were calculated. Signal-to-noise ratios for infarcted myocardium, normal myocardium and the left ventricular cavity were measured and the contrast-to-noise ratios of infarcted compared to normal myocardium (CNRinf-myo) and infarcted myocardium in relation to the left ventricular cavities (CNRinf-LVC) were calculated.

Results: The Bland-Altman plots in the assessment of infarction size did not reveal a systematic bias between the two readers. The mean difference between reader $I$ and 2 was less than $0.9 \mathrm{~cm}^{3}$ of mean infarction volume. Assessment of interobserver agreement regarding the transmural extent of myocardial infarction resulted in kappa-values of kappa $=0.85$ (IR SSFP) and kappa $=0.87$ (IR GRE) in gadobenate enhanced images and kappa $=0.84$ (IR SSFP) and kappa $=0.83$ (IR GRE) after administration of gadopentetate. CNRinf-normal was significantly 
higher on the images of group I (gadobenate) compared to group 2 (gadopentetate) in both sequences (Single Shot IR SSFP: $18.1 \pm 10.1$ vs. I2.I $\pm 6.7 ; p=0.032$ and IR GRE: $27.2 \pm 5.8$ vs. $19.7 \pm 5.9 ; p=0.005)($ Table I). The mean value of CNRinf-LVC for the group examined with gadobenate dimeglumine was lower, though not significantly, compared to the group examined with gadopentetate dimeglumien in IR GRE technique, while CNRinfLVC for IR SSFP resulted in equal values (single Shot IR SSFP: I. $2 \pm 5.2$ vs. I.I $\pm 6.8 ; p=$ n.s. and IR GRE $2.4 \pm 5.8$ vs. $5.8 \pm 7.9 ; \mathrm{p}=$ n.s.).

Conclusion: Low dose gadobenate dimeglumine resulted in significantly higher CNRinf-myo compared to standard dose gadopentetate dimeglumine in imaging of myocardial infarction with IR SSFP and IR GRE sequences. Demarcation of infarcted myocardium from the left ventricular cavity was equal in IR SSFP technique and not significantly different in IR GRE technique. The diagnostic value of a single dose gadobenate dimeglumine is comparable or even superior compared to a double dose gadopentetate dimeglumine for imaging myocardial infarction at least for imaging myocardial infarction in Single Shot IR SSFP technique (Figure I).

\section{P236}

Early infarct size prediction with two-dimensional speckle tracking echocardiography compared with late gadolinium enhanced CMR

Cecile Roiron', Nathan Mewton', Marie Egan², Cyril Bergerot', Pierre Yves Leroux', Pierre Croisille', Michel Ovize' and Genevieve Derumeaux'

'Hopital Cardiovasculaire Louis Pradel, Lyon, France

2INSERM U 886: "Cardioprotection 》 Université Claude

Bernard - Lyon, Lyon, France

Journal of Cardiovascular Magnetic Resonance 2009, I I(SuppI I):P236

Objective: Infarct size measurement is crucial since it predicts both viability and left ventricular remodeling. A recent technique, two dimensional speckle tracking echocardiography (STE) allows

Figure I (abstract P236)

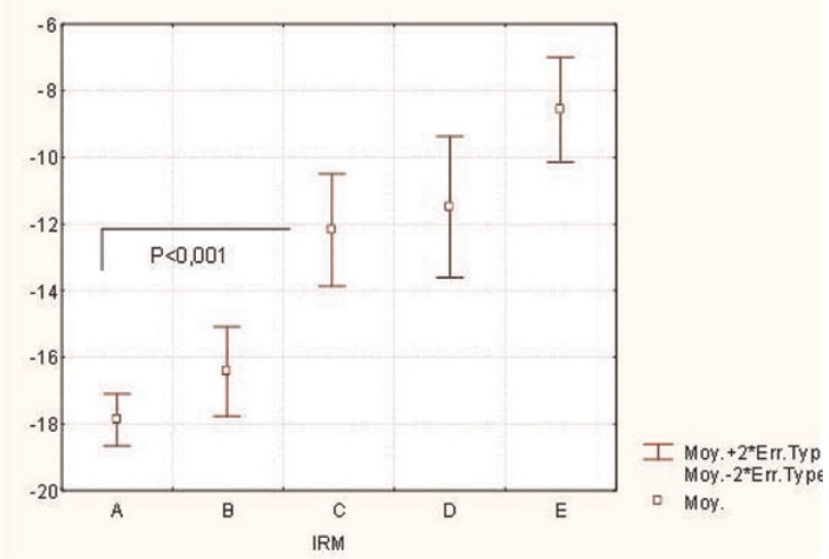

SL values plotted versus TEMI. A: normal myocardium, B: TEMI <25\%, C: TEMI 25-49\%, D: TEMI 50-74\%, E: TEMI $\geq 75 \%$. accurate evaluation of myocardial deformation indices as compared to sonomicrometry and magnetic resonance imaging (MRI).

The aim of our study was to evaluate whether peak systolic longitudinal strain (SL) measured by STE was able to quantify the transmural extent of myocardial infarction (TEMI) as assessed by late contrast enhancement MRI.

Materials and methods: 29 patients $(55,4 \pm 4,6$ years, $F$ I I/ $M$ 18) with a first reperfused myocardial infarction (MI) were included.

Echocardiography and MRI performed between day 2 and day 5 after infarction were analyzed with a 16 segment model. Segmental and global (average of segmental SL) longitudinal strain were measured by STE in apical views. Segments with $\mathrm{MI}$ were classified as non-transmural (TEMI $<25 \%$, TEMI $<50 \%$, TEMI $<75 \%$ ) and transmural $\mathrm{MI}(\mathrm{TEMI}>75 \%)$ by late gadolinium enhanced (LGE) MRI. Infarct mass was also assessed by planimetry on LGE MRI.

Statistical analysis was performed using STATISTICA ${ }^{\circ}$ software. Results: 464 segments were analyzed by MRI. Evaluation of SL by STE was feasible in $459 / 464$ segments.

STE correctly identified segmental LV dysfunction induced by MI. Mean value of LS in the transmural MI segments $(-8,56 \pm 1,22 \%)$ was significantly lower compared to normal myocardium $(-17,88 \pm$ $0,8 \%$ and to non-transmural $M I$ segments $(-16,43 \pm 1,2 \%$ in TEMI $<25 \%$ vs $-12,18 \pm 1,6 \%$ in $25-50 \%$ TEMI vs $-11,49 \pm 2,1 \%$ in 50-75\%TEMI) $(\mathrm{p}<0,00 \mathrm{I})$.

A significant relationship was found between the global longitudinal strain and the infarct mass measured by MRI $(r=0.6 \mathrm{I}$, $\mathrm{P}<0,0 \mathrm{I})$. See Figure I.

Conclusion: Early after MI, STE evaluates both the global infarct mass by global longitudinal strain and can differentiate transmural from non transmural $\mathrm{Ml}$ segments by segmental systolic longitudinal strain compared to LGE-MRI. This new technique is promising for early infarct size prediction.

\section{P237}

Is there an innate sex-based difference in the manner in which the left ventricle responds to the pressure overload of aortic stenosis?

Saundra Grant, Diane A Vido, Vikas K Rathi, June A Yamrozik, Ronald B Williams, Geetha Rayarao, Mark Doyle and Robert WW Biederman Allegheny General Hospital, The Gerald McGinnis Cardiovascular Institute, Pittsburgh, PA, USA

Journal of Cardiovascular Magnetic Resonance 2009, I I(SuppI I):P237

Introduction: Numerous investigators have suggested that women are particularly prone to develop an exaggerated LV hypertrophy $(\mathrm{LVH})$ response to increased developed pressure as compared to men. This has been best exemplified in both HTN and aortic stenosis (AS) states and has been proposed euphemistically as 'exuberant hypertrophy'. This reference implies that there is an inappropriate physiologic amplification to the typical and necessary hypertrophic response for any given blood pressure/afterload experienced by the LV. Furthermore, it suggests that there is a divergent response at the gene level to 
Figure I (abstract P237)

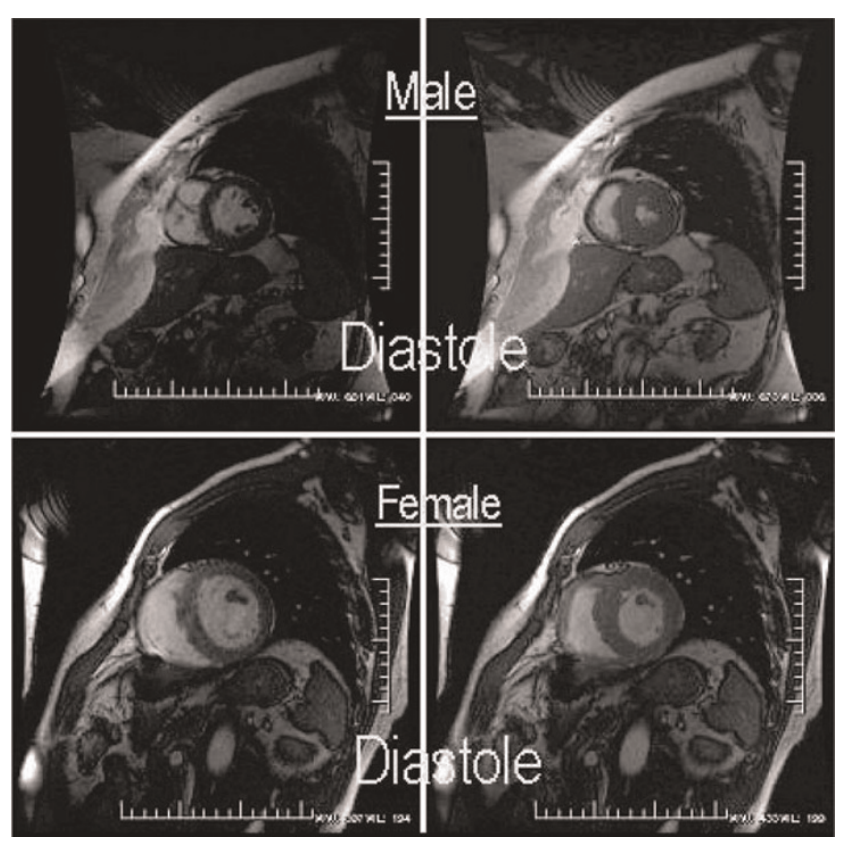

2D CVMRI images of geometry in A) 65 YO female with a small thick LV and B) 68 YO male with a larger, thinner LV, both with similar mean gradient $(52 \pm 4 \mathrm{mmHg})$, BSA $(2 . \mathrm{I} \pm \mathrm{I})$, and LVMI corrected for EDV $(\mathrm{I} .33 \pm 0.10)$ and with similar LVMI.

explain this phenomenon. The implications for this are vast, potentially calling for distinct sex-based clinical treatment programs. The societal ramifications for differential therapy are immense as our resources are limited. On the other hand, the majority of the inferences of this dichotomized observation have stemmed from 2D echocardiography and consequently may be artifactual if evaluated in a 3D CMR manner.

Hypothesis: We hypothesize that 'exuberant hypertrophy' is a misnomer and that after aortic valve replacement (AVR) there will be no sex difference in the manner or rate in which LVH regresses following afterload relief.

Methods: Following initial recruitment of 35 patients for a longitudinal 2-year CMR study, a series of patients who were alive and available were specifically contacted and re-recruited for follow-up up to 4 years from their initial AVR. All patients underwent an additional CMR for evaluation of $3 D$ volumetrics, including LV mass and EF. Using Simpson's rule, contiguous $8 \mathrm{~mm}$ slices were acquired via SSFP imaging and underwent LV endocardial and epicardial manual contouring (GE I.5 T EXCITE, Milwaukee, $\mathrm{WI})$. Pre and post-AVR LV mass and EF were compared and stratified for sex. A ' $P$ ' value of $<0.05$ was considered statistically significant.

Results: Ten (10) patients were available for CMR at $3 \pm 1$ yrs out to 4 years at follow-up post-AVR and successfully underwent CMR. Women (6; age: $68 \pm 14$ ) and men (4; age: $68 \pm 10$ ); ( $\mathrm{p}<\mathrm{NS}$ for both) were imaged. Baseline LV mass index was neither increased in females nor different between women and men $\left(75 \pm 16\right.$ vs. $\left.101 \pm 23 \mathrm{~g} / \mathrm{m}^{2}, \mathrm{p}=\mathrm{NS}\right)$. However, baseline EF was dissimilar between women and men $(67 \pm 12$ vs. $37 \pm 23 \%$, $\mathrm{p}=\mathrm{NS}$ ). Following AVR, in absolute or relative terms, LV mass index regression was not different between the sexes $(65 \pm 17$ vs. $73 \pm \mathrm{II} \mathrm{g} / \mathrm{m}^{2}$; representing a proportionate decrease in LV mass of II \pm 16 vs. $28 \pm 17 \mathrm{~g} / \mathrm{m}^{2}, p=N S$ ), (Figure I). Likewise, post-AVR, LVEF was not significantly different (68 \pm 7 vs. $6 \mathrm{I} \pm 10 \%, p=N S$, but did markedly improve in the males ( $I \pm 10$ vs. $24 \pm 20 \%, p<0.05$ ), respectively for women vs. men, again without any absolute change in LV mass index between groups.

Conclusion: While conceptually intriguing and highly controversial, the sex-based differences of exaggerated LVH formation in response to pressure afterload that are based substantially on 2D echocardiographic acquisitions appear to be artificial when based upon a 3D CMR interrogation. Specifically, after recruiting a select group of patients with AS who underwent AVR with up to 4 years of follow-up, more than sufficient time to allow for regression of LV mass to near completion (if it was going to) failed to demonstrate a significant difference as related to women vs. men. Beyond negating the 2D observation by a more sophisticated 3D CMR interrogation, the potential socio-economic repercussions are nullified should a more favorable response to afterload have been found (either at baseline or after AVR). This finding may restore the notion that there are neither phenotypic nor likely genotypic alterations in the $L V$ response to pressure afterload in women vs. men.

\section{P238}

\section{Left ventricular diastolic function assessed with cardiovascular magnetic resonance imaging and exercise capacity in patients with non-obstructive hypertrophic cardiomyopathy}

Lukasz A Malek, Jolanta Misko, Mariusz Klopotowski, Lidia Chojnowska, Mateusz Spiewak, Barbara Milosz, Renata Maczynska, Ewa Piotrowicz and Witold Ruzyllo Institute of Cardiology, Warsaw, Poland

Journal of Cardiovascular Magnetic Resonance 2009, I I(SuppI I):P238

Introduction: In patients with non-obstructive hypertrophic cardiomyopathy (HCM) and preserved left ventricular (LV) systolic function, diastolic dysfunction is one of the major factors contributing to limited exercise capacity.

Purpose: To assess the usefullness of simple cardiovascular magnetic resonance (CMR) parameters of LV diastolic function at rest and exercise capacity in patients with non-obstructive HCM and preserved LV systolic function.

Methods: The study included 13 patients who underwent cardiopulmonary exercise testing on treadmill and CMR within I month distance. Analyzed parameters of diastolic function included: atrio-ventricular plane descent (AVPD), sphericity index (SI), left ventricular mass index (LVMI), peak filling rate normalized to LV stroke volume index (PFR/LVSVI) and time from the end-systole to PFR normalized to heart rhythm (TPFR).

Results: There was a significant correlation between PFR/LVSVI at rest and peak oxygen uptake $\left(\mathrm{VO}_{2}\right.$ peak $)(r=0.64, \mathrm{p}=0.02)$. Patients with $\mathrm{V}_{2}$ peak below median $(<30 \mathrm{ml} / \mathrm{kg} / \mathrm{min}$ ) had a significantly lower PFR/LVSVI then patients with higher $\mathrm{VO}_{2}$ peak [5.12 m²/s, interquartile range (IQR) $4.16-6.82 \mathrm{vs} .7 .93 \mathrm{~m}^{2} / \mathrm{s}$, IQR 7.49-8.2I respectively, $\mathrm{p}=0.035$ ). AVPD, SI, LVMI, TPFR 
were not related to exercise capacity. There was also no correlation between $\mathrm{VO}_{2}$ peak and age $(r=-0.38, p=0.19)$, LV ejection fraction $(r=-0.36, p=0.22)$ or normalized $L V$ volume indices: LVEDVI $(r=0.09, P=0.76)$, LVESVI $(r=0.34$, $\mathrm{p}=0.26)$.

Conclusion: Assessment of LV diastolic function by peak filling rate normalized to stroke volume index with means of CMR at rest in patients with non-obstructive hypertrophic cardiomyopathy and preserved LV systolic function is a usefull predictor of exercise capacity. It can be added to CMR study protocols of HCM patients.

\section{P239}

\section{Relationship of left ventricular} non-compaction with papillary muscle insertion site and partition

Mitra Sahebazamani ', ljaz Ahmad', Geetha P Bhumireddy', Igor Klem², Joshua A Socolow', Terrence Sacchi ${ }^{1}$ and John F Heitner'

${ }^{\prime}$ New York Methodist Hospital, Brooklyn, NY, USA

${ }^{2}$ Duke Univ Medical Center, Durham, NC, USA

Journal of Cardiovascular Magnetic Resonance 2009, I I(SuppI I):P239

Introduction: Left ventricular non-compaction (LVNC) is a rare congenital morphogenetic abnormality and occurs as a result of an arrest in the compaction of the embryonic myocardium during development resulting in excessive trabeculations in the left ventricle (LV). The relationship of the papillary muscle (PM) development and LVNC has not been previously described.

Purpose: To assess the correlation between LVNC and both the degree of partitions as well as the insertion site (apical vs. mid-ventricular) of PM as compared to patients with normal compacted myocardium.

Methods: We enrolled 297 consecutive patients referred to our cardiac magnetic resonance (CMR) center for cardiac evaluation. We assessed the non-compacted (trabecular region) and compacted myocardium by drawing diameters of each at the left ventricular apex and averaging from two long axis cine views.
LVNC was defined as non-compacted to compacted ratio 2.3. The PM insertion site was determined by dividing the long axis of the LV into 3 equal regions and determining in which region (mid or apical) the papillary muscle inserted into the LV. The number of partitions of the PM was determined by counting the number of separate PM visualized $10 \mathrm{~mm}$ apical from the mid plane of the LV on short axis cine view.

Results: The mean age of the patients was 58.1 years, $60 \%$ were males. The patients were referred to CMR for the following reasons: evaluation of left ventricular function (37\%), viability (30\%), valvular diseases (21\%), and other (12\%). The average LVEF was $51.2 \pm 14.6 \%$. The mean number of PM partition in the LVNC group was 5 , and in the normal group was $6(p=0.4 I)$. The LVNC group had a significantly higher apical insertion of the PM compared to the normal group, $87 \%$ (67 of 77 patients) vs $67.3 \%$ ( 148 of 220 patients, $p<0.00$ I).

Conclusion: Patients with LVNC have a higher incidence of apical insertion of the papillary muscle into the myocardium, without a higher number of partitions.

\section{P240}

Quantification of vendor-specific relationships between fast gradient echo and steady-state free precession cine MRI for determination of myocardial mass and volumes

Andrew S Chi', W Craig Johnson ${ }^{2}$, Elzbieta Chamera', John Eng', Joan Bathon', Chia-Ying Liu', Joao AC Lima' and David A Bluemke'

Johns Hopkins, Baltimore, MD, USA

${ }^{2}$ University of Washington, Seattle, WA, USA

Journal of Cardiovascular Magnetic Resonance 2009, I I(SuppI I):P240

Objectives: To quantify the vendor-specific relationships between fast gradient echo and steady-state free precession for determination of cardiac mass and volumes.

Background: Steady-state free precession (SSFP) cine sequences have replaced fast gradient echo (FGRE) cine given the advantages of SSFP to provide faster image acquisition,

Figure I (abstract P240)

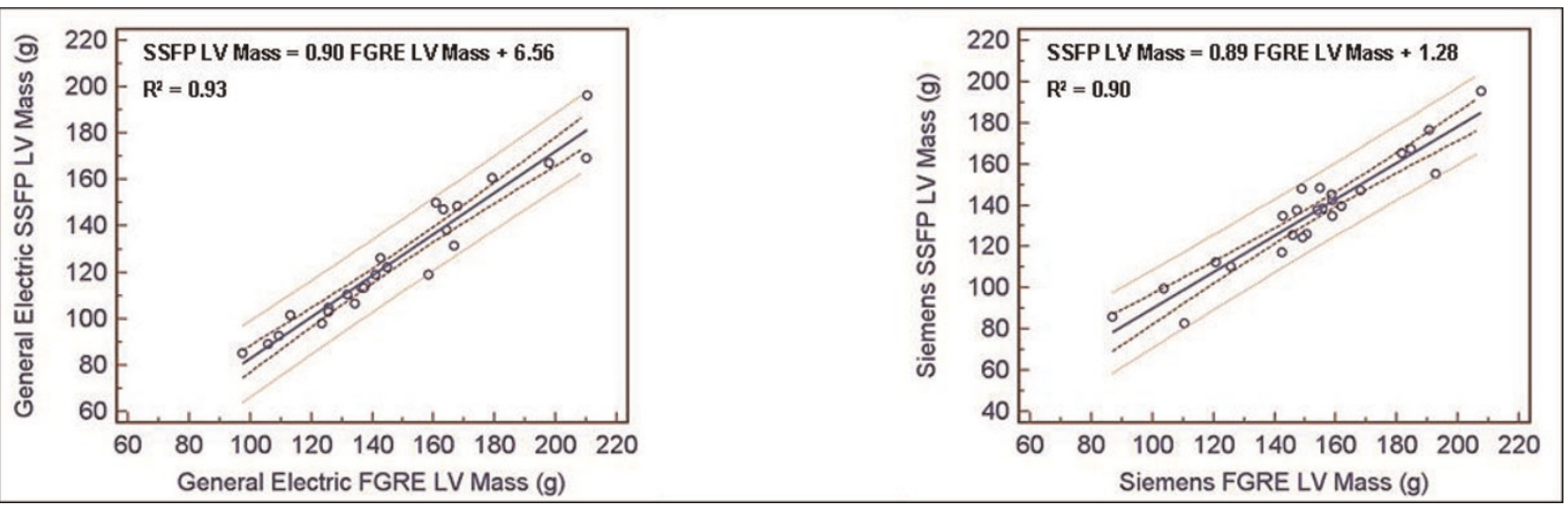


increased contrast to noise ratio, increased blood-tissue contrast, and decreased interobserver variability as compared to FGRE. Substantial differences between SSFP and FGRE for quantification of cardiac mass and volumes have been reported, but differences between vendor results using both pulse sequences has not been characterized.

Methods: 50 cardiac MRI cine studies (25 Siemens I.5 T, 25 General Electric I.5 T) were acquired using both SSFP and FGRE pulse sequences in the same study subject. Cardiac MRI analysis was performed using QMASS software to determine parameters of cardiac function including left ventricular (LV) mass, end diastolic volume (EDV), end systolic volume (ESV), ejection fraction (EF), stroke volume (SV), and cardiac output (CO). MRI analysis was first performed by a single trained reader. Interobserver variability was then assessed compared to a second reader. Results were assessed by regression and BlandAltman analyses (Figure I).

Results: Mean percentage differences between SSFP and FGRE values for LV mass, EDV, and ESV were $-10.5 \%,+17.7 \%$, and $+18.6 \%$ for Siemens scanners and $-14.9 \%,+12.8 \%$, and $+15.0 \%$ for General Electric scanners, respectively $(p<0.000$ I for all values). The relationship between SSFP and FGRE values using General Electric scanners were linear and highly correlated $(p<0.00 I)$ for LV mass, EDV, and ESV $\left(R^{2}\right.$ of $0.93,0.87$, and 0.91 , respectively). For Siemens scanners, the relationship between SSFP and FGRE was highly correlated $(p<0.001)$ for LV mass $\left(R^{2}\right.$ $=0.90)$ and moderately correlated $(p<0.001)$ for EDV $\left(R^{2}=\right.$ $0.67)$ and ESV $\left(R^{2}=0.61\right)$. The slope (intercept) for EF, SV, and CO were $0.67(22.4 \%), 0.73(29.5 \mathrm{ml})$, and $0.6 \mathrm{I}(2.25 \mathrm{l} / \mathrm{min})$ for Siemens scanners and 0.8I (I I. I\%), 0.90 (I $3.7 \mathrm{ml})$, and 0.95 (0.63 $\mathrm{I} / \mathrm{min}$ ) for General Electric scanners, respectively. Inter-observer mean differences $( \pm 2$ SD) for LV mass, EDV, and ESV using Siemens scanners were $-2.1 \mathrm{~g}(-2 \mathrm{I} .9-17.7 \mathrm{~g}),-16.5 \mathrm{ml}(-35.4-$ $2.4 \mathrm{ml})$, and $-12.1 \mathrm{ml}(-32.8-8.5 \mathrm{ml})$ for SSFP measures and $-12.1 \mathrm{~g}(-48 . \mathrm{l}-24.0 \mathrm{ml}),-18.7 \mathrm{ml}(-37.7-0.3 \mathrm{ml})$, and $-9.7 \mathrm{ml}$ $(-21.0-1.5 \mathrm{ml})$ for FGRE measures, respectively.

Conclusion: Vendor-specific differences exist when comparing the relationships between SSFP and FGRE cine MRI for parameters of cardiac function.

P24I

Prevalence and clinical correlates of miocardial fibrosis and necrosis in thalassemia major patients by CMR-DE

Alessia Pepe', Vincenzo Positano', Brunella Favilli', Daniele De Marchi', Giuseppe Rossi', Luigi Natale'2, Gennaro Restaino ${ }^{3}$, Gianluca Valeri ${ }^{4}$, Francesca Ferrara ${ }^{5}$ and Massimo Lombardi

I "G Monasterio" Foundation and Institute of Clinical Physiology, CNR, Pisa, Italy

${ }^{2}$ Policlinico "Gemelli", Roma, Italy

${ }^{3}$ Università Cattolica del Sacro Cuore, Campobasso, Italy

${ }^{4}$ Az. Osp. "Garibaldi", Ancona, ltaly

${ }^{5}$ Policlinico di Modena, Modena, Italy

Journal of Cardiovascular Magnetic Resonance 2009, I I(SuppI I):P24 I

Introduction: Cardiovascular Magnetic Resonance (CMR) by delayed enhancement (DE) has proven to visualize myocardial scarring, but no dedicated studies are available in thalassemia major.

Purpose: Aim of our study was to investigate the prevalence, extent, clinical and instrumental correlates of myocardial fibrosis or necrosis by DE CMR in thalassemia major patients.

Methods: II5 thalassemia major patients (40 men, mean age $27 \pm 8$ years) were consecutively examined at our CMR Laboratory using a I.5 T scanner (GE Excite). Steady-state free procession cines were acquired during 8-second breath holds in sequential 8-mm short axis slices from the atrio-ventricular ring to the apex to assess biventricular function. Biventricular function was quantitatively assessed using MASS $^{\circledR}$ software (Medis, Leiden, The Netherlands).

Contrast delayed enhanced images were acquired in the same view used for cine CMR from 10 to 15 minutes, after the Gadobutrol ( $1.0 \mathrm{~mol} / \mathrm{L})(0.2 \mathrm{mmoli} / \mathrm{Kg})$ intravenous administration, using a fast gradient-echo inversion recovery sequence. Inversion times were adjusted to null the normal myocardium (from $210 \mathrm{~ms}$ to $300 \mathrm{~ms}$ ) with voxel size of $1.6 \times 1.25 \times 8.0 \mathrm{~mm}$. The extent of DE areas was quantified using semi-automatic, previously validated software (HIPPO DELAY $\left.{ }^{\circledR}\right)$.

Myocardial iron overload was determined by multislice multiecho T2*. Three parallel short-axis views of the left ventricle were obtained by $\mathrm{T} 2^{*}$ gradient-echo multiecho sequence. Global LV, mid-ventricular and segmental T2* values were obtained by a dedicated software (HIPPO MIOT ${ }^{\circledR}$ ). Image analysis provided the mapping of segmental data on 16 segments of the left ventricle, according to the standard AHA/ ACC model.

A twelve lead ECG was performed within one month from CMR, in absence of clinical cardiovascular events and was read blindly by the consensus of two cardiologists who were unaware of the results of the CMR exam.

Results: DE areas were present in $28 / 115$ patients (24\%). Extent of $\mathrm{DE}$ was $3.9 \pm 2.4 \%$. In 26 patients the location of fibrosis was not specific and patchy distribution was prevalent. Two patients showed transmural DE following coronary distribution. The DE group was significantly older than the non-DE group $(31 \pm 7.7$ years versus $26 \pm 7.7$ years, $P=0.004)$. $D E$ correlated with cardiac risk factors $(P=0.0 \mathrm{I})$, history of cardiac complications $(P=0.00 \mathrm{I})$, and antiHCV antibodies $(P=0.04)$. We did not find significant relation with heart $\mathrm{T} 2 *$ values and biventricular function. Figure I shows a thalassemia major patient with no myocardial iron overload (all 16 segments T2* values > $20 \mathrm{~ms}$ ) (A) and transmural DE (black arrows) following coronary distribution in the apical region $(B, C)$.

A significant correlation was found between the presence of DE and changes in ECG (ECG abnormal in DE group 22/28 patients and in no-DE group $30 / 87$ patients; chi-square 14.9; $P=0.0001$ ).

Conclusion: In thalassemia patients the significant presence of myocardial fibrosis/necrosis seems to be a time dependent process correlating with cardiovascular risk factors and cardiac complications. HCV infection could be a causal agent in the pathogenesis of myocardial scarring. ECGchanges showed a good accuracy in predicting myocardial scarring. 
Figure I (abstract P24I)

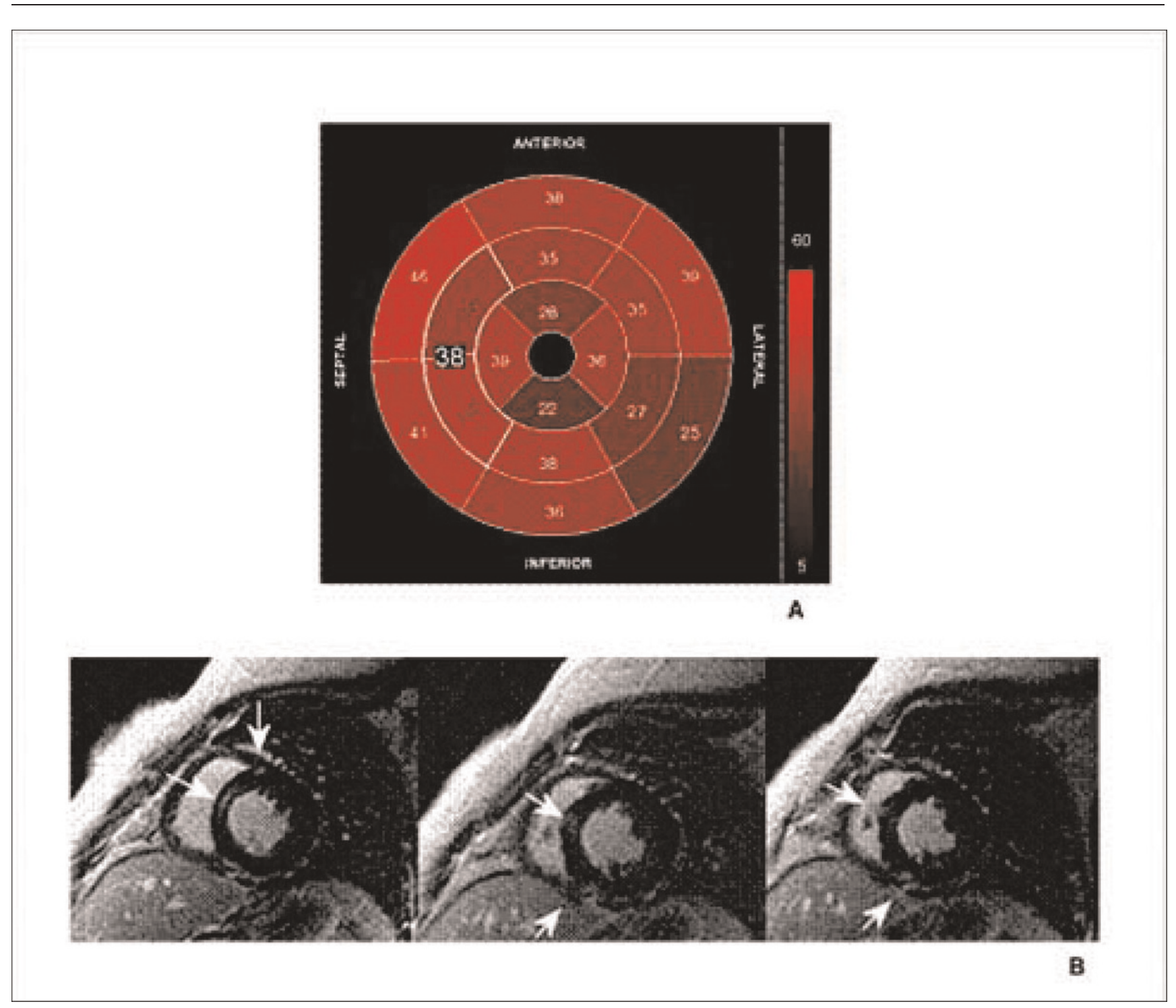

\section{P242}

Left ventricular non-compaction cardiomyopathy in adults - characterisation by cardiac magnetic resonance imaging

Constanze Merten, Stephanie Lehrke, Dirk Lossnitzer, Evangelos Giannitsis, Hugo A Katus and Henning Steen University Hospital Heidelberg, Heidelberg, Germany

Journal of Cardiovascular Magnetic Resonance 2009, I I(SuppI I):P242

Introduction: Left ventricular non-compaction (LVNC) is a congenital cardiomyopathy characterised by left ventricular hypertrabecularisation and thinning of the compacted myocardial layer. Due to its high spatial resolution and precise delineation of the non-compacted layer, cardiac magnetic resonance imaging (cMRI) is a promising method to image and diagnose LVNC cardiomyopathy.
Methods: In 19 patients who underwent cMRI for suspected cardiomyopathy LVNC was diagnosed according to diagnostic criteria such as a ratio of non-compacted to compacted myocardium (NC/C-ratio) of $>2.3$ in diastole [ $\mathrm{I}]$ and exclusion of other underlying cardiac pathology or cardiovascular risk factors. We performed cMRI using a clinical I.5 Tesla scanner (Philips Achieva). Left ventricular (LV) function, dimensions and volumes were assessed using steady-state free precession cine imaging. The NC/C-ratio was assessed in the lateral LV wall in an apical, midventricular and basal short-axis slice. Additionally, delayed contrast enhancement (DCE) imaging was performed. Results: Of the 19 patients, 6 were women (32\%), the mean age was $41.7 \pm 16.8$ years. Over the whole study group, LV function was impaired with a mean ejection fraction of $41 \pm 17 \%$. However, LV ejection fraction was normal in 6 patients (32\%). LV volumes were increased with a mean enddiastolic volume of 
$247 \pm 100 \mathrm{ml}$ and a mean endsystolic volume of $155 \pm 99 \mathrm{ml}$, resulting in mean stroke volume of $92 \pm 31 \mathrm{ml}$. Using the 17segment model, we found a mean number of II $8 \pm 2.6$ segments to be affected by LVNC with the typical predomination of the apical segments and the free lateral LV wall. The mean NC/Cratio was $5.0 \pm 1.6$ in the apical lateral wall, $3.3 \pm 0.7$ in the midventricular lateral LV wall and $2.0 \pm \mathrm{I} . \mathrm{I}$ in the basal lateral wall; the basal septum was involved in none of the patients.

DCE was present in 7 patients (37\%); in all patients non-ischemic type DCE with an intramural or spotted enhancement pattern and a predilection for the inferior right ventricular insertion zone was observed.

Conclusion: We characterised 19 patients with LVNC cardiomyopathy by cMRI. We observed an impaired LV function in the study cohort along with a dilatation of the left ventricle. The mean number of affected segments was 12 of 17. Apical and lateral segments were most frequently affected. Accordingly, the $\mathrm{NC/C}$-ratio decreased from the apical to the basal segments. Non-ischemic type DCE was observed in $37 \%$ of patients.

\section{Reference}

I. Petersen, et al: J Am Coll Cardiol 2005, 46:101-105.

\section{P243}

\section{Quantitative evaluation of the dark rim artifact} in cardiac perfusion images

Raphael D Hazel, Nathaniel Reichek and Yi Wang

St Francis Hospital, Roslyn, NY, USA

Journal of Cardiovascular Magnetic Resonance 2009, I I(SuppI I):P243

Introduction: The dark rim artifact (DRA) which appears in cardiac first pass perfusion MRI compromises both visual assessment and quantitative evaluation of ischemia. DRA signal loss in the subendocardium may be mistaken for a perfusion defect or it can overlap with a region showing a real perfusion disorder, and make it impossible to obtain an accurate assessment of myocardial blood flow. The quantitatively evaluation of the artifact will allow for precise determination of its origin, automated image assessment, and elimination of observer error from the evaluation of cardiac perfusion images. We have quantified the DRA in perfusion images from three separate studies using SSFP, TFL and EPI imaging sequences to determine the characteristics and origin of the artifact.

Methods: Retrospective quantitative analysis was done on myocardial perfusion images from 32 normal volunteers. Images from three separate studies approved by the institutional review board were analyzed for artifacts. In the first study, images were acquired in 14 normal volunteers ( 10 female) age 43.8 (s.d. 15.9) years, using a saturation recovery SSFP pulse sequence and gadodiamide $(0.05 \mathrm{mM} / \mathrm{kg})$, under adenosine $(140 \mathrm{ug} / \mathrm{kg} / \mathrm{min})$ stress followed by rest. The image parameters were as follows: body coil, TR $160 \mathrm{~ms}$, TE $1.03 \mathrm{~ms}$, FA 50, matrix $192 \times 108$; three long axis planes (HLA, VLA, and LVOT) of $8 \mathrm{~mm}$ slice thickness were recorded. In the second study, using the same imaging parameters, TFI images from 7 subjects which were acquired at rest, under cold pressor stress, and after $0.4 \mathrm{mg}$ sublingual nitroglycerin were analyzed. In the third study, images were acquired using the dual bolus approach with two doses of gadodiamide $(0.005$ and $0.05 \mathrm{mM} / \mathrm{kg})$ at rest from II normal volunteers ( 5 female) using the TFI, TFL and EPI pulse sequences in random order during a single session. Imaging parameters for each sequence were as follows: TFI (TR $199 \mathrm{~ms}$, TE I.04 ms, FA 50, matrix $192 \times 160)$ TFL (TR $163 \mathrm{~ms}$, TE $1.27 \mathrm{~ms}$, FA 10, matrix $192 \times 160)$ and EPI (TR $5.8 \mathrm{~ms}$, TE I.22 ms, FA 25, matrix $160 \times 132$ ) Three long axis and one short axis slice with thicknesses of $10 \mathrm{~mm}$ were collected. Images were analyzed for the presence of DRA using ImageJ to measure the intensity profile across the myocardium. DRA was identified as signal loss in the subendocardium occurring after the arrival of the CA in the LV with a duration coincident with the passage of the bolus through the LV.

Results: Heart motion: The signal loss per image appears to be independent of heart rate within each study. In study \#I the mean signal loss per image is $32.9 \%$ and $31.5 \%$ for rest and stress respectively, and in study \#2 the signal loss per image was $21.9 \%$, $21.3 \%$ and $20.8 \%$ for rest, cold stress and nitroglycerine respectively.

Concentration dependence: There were no artifacts in low CA dose $(0.005 \mathrm{mM} / \mathrm{kg})$ images. Only the high CA dose produced the DRA in TFI and TFL images.

Sequence dependence: DRA was found in TFI images from 8 of I I volunteers ( $4 \%$ of all images) while only 3 sets of TFL ( $2 \%$ ) images contained the DRA. EPI images did not contain the DRA. The severity of the DRA is similar for TFI and TFL images with a mean signal loss of $31.6 \%$ and $33.1 \%$ respectively. The average duration of the DRA is also similar for TFI and TFL with II.8 and 10.5 frames out of 50 respectively.

Conclusion: The signal loss in the DRA is independent of heart rate, but seems to depend on CA concentration for TFI and TFL but not EPI images. Quantitative evaluation of the DRA is feasible with first pass perfusion imaging.

\section{P244}

Are there innate differences in 3D ejection fraction between the sexes detectable by CMR? A CMR study in $\sim 4000$ patients

Robert WW Biederman, Diane A Vido, Geetha Rayarao, June A Yamrozik, Ronald B Williams, Saundra B Grant and Mark Doyle

Allegheny General Hospital, The Gerald McGinnis

Cardiovascular Institute, Pittsburgh, PA, USA

Journal of Cardiovascular Magnetic Resonance 2009, I I(SuppI I):P244

Introduction: It has long been assumed that the standard mechanical contraction parameter ejection fraction (EF) is the same in male and female hearts. Until recently, the fidelity and reproducibility of imaging techniques guaranteed virtual superimposition of EF, nullifying any gender differences were they to be present. The influence of gender on normal thresholds for EF by CV MRI is unknown.

Hypothesis: Using high resolution 3D CV MRI, we tested the hypothesis that females will have lower EF compared to males.

Methods: A database composed of consecutive patients who underwent CV MRI scanning (GE, EXCITE HD I.5 T, Milwaukee, WI) between Aug 2003 and May 2008 was interrogated to yield all patients with $\mathrm{EF}>55 \%$ and no valvular heart disease $>I+$, no evidence of CAD, HTN, or cardiomyopathy. EF was determined primarily by standard FIESTA 3D methodology or 2D when available. Patients were stratified only by gender and age. We performed two-sample $t$ tests to analyze the data and considered differences significant at $p<0.05$. 
Results: A total of 3,962 consecutive patients were evaluated, from which 1203 (30\%) with normal EF were identified. Mean age was $51.2 \pm 18.9$ years, $49.4 \%$ were males and $50.6 \%$ were females. The mean EF for males was $63.6 \pm 3.9$; range: $56-70 \%$, while for females it was $64.3 \pm 3.6$; range: $56-70 \%, p<0.00$ I. Under the assumption that EF $>55 \%$ may not be appropriate for normal thresholds, EF > $60 \%$ and $65 \%$ were also stratified but did not yield significant gender differences. Similarly, stratification by age categories (decades) did not reveal a significant difference. A subset of 150 pts with clinical CV disease, representing the entire range of $\mathrm{EF}(5 \%-81 \%)$, underwent intra and interobserver reproducibility for LVEF and was 0.13 and $0.85 \%$, respectively. Conclusion: Contrary to conventional doctrine, LV ejection performance (EF) as measured clinically, using highly reproducible and accurate 3D CV MRI, is lower for males than females as determined in the largest CV MRI database (3962 patients) to our knowledge to date examining this subject. Thus, beyond establishing normal ranges for LVEF, these observations have far reaching clinical implications in defining thresholds of normality, as well as belying potential, albeit subtle, intrinsic differences in contractile mechanisms. These results show that normal thresholds for EF using CV MRI are influenced by gender.

\section{P245}

Heterogeneity in age related central arterial stiffening: ascending aortic stiffness is a better predictor of carotid to femoral pulse wave velocity and brachial peripheral blood pressure than carotid stiffness

Alban Redheuil', Wen Chung Yu', Raymond T Yan', Elie Mousseaux ${ }^{2}$, Alain De Cesare ${ }^{2}$, David A Bluemke ${ }^{3}$ and Joao AC Lima'

IJohns Hopkins University, Baltimore, MD, USA

INSERM U678, Paris, France

${ }^{3} \mathrm{NIH}$, Bethesda, MD, USA

Journal of Cardiovascular Magnetic Resonance 2009, I I(SuppI I):P245

Introduction: Increased systolic blood pressure (SBP), pulse pressure and carotid-femoral pulse wave velocity (cfPWV) are strong predictors of cardiovascular events. The aorta and carotids are both elastic arteries susceptible to arterial stiffening and its deleterious complications. The relative importance of ascending aortic and carotid stiffness on central arterial stiffness remains unclear.

Purpose: Our aim was to study the relationship of local arterial stiffness in the carotid and ascending aorta to central arterial stiffness assessed by cfPWV and peripheral BP.

Methods: We studied 22 healthy subjects ( 12 men; mean age: 37 yrs [22-67]). Central arterial stiffness was determined using carotid and femoral tonometry and transit surface distances (cfPWV). Distensibility of the ascending aorta was calculated as the ratio of its transverse area change on MRI to the central pulse pressure from radial tonometry arterial waveforms using a transfer function. Carotid distensibility was calculated as the ratio of transverse area change by sonography to carotid pulse pressure by tonometry. Local stiffness indexes of carotid and aorta were calculated from distensibility by the reverse MoensKorteweg equation. Peripheral SBP and pulse pressure were averages of 6 brachial measurements. Linear regression was used to study correlations between aortic and carotid stiffness and cfPWV.
Results: Aortic stiffness has a significantly stronger correlation than carotid stiffness with: age $(r=0.57 ; p<0.001$ vs. 0.20 ; $P=0.02)$, peripheral SBP $(r=0.78 ; p<0.001$ vs. 0.53 ; $p<0.00 \mathrm{I})$, pulse pressure $(r=0.67$ vs. $0.47 ; p<0.00 \mathrm{I})$, and cfPWV ( $r=0.87 ; p<0.001$ vs. $0.35 ; p=0.002)$. In multivariate analysis with simultaneous adjustment for age, body mass index, SBP, pulse pressure, aortic stiffness and carotid stiffness, aortic stiffness is the strongest independent determinant of cfPWV $(p=0.0 \mathrm{I} 3), \operatorname{SBP}(p=0.005)$ and pulse pressure $(p=0.0 \mathrm{I})$.

Conclusion: Stiffness of the ascending aorta is a stronger predictor of cfPWV and peripheral SBP and pulse pressure than carotid stiffness among healthy individuals. This is consistent with heterogenous age-related stiffening of large arteries.

\section{P246}

Magnetic resonance phase contrast imaging in children with pulmonary artery hypertension

Brian Fonseca', Dunbar Ivy and Craig Lanning ${ }^{2}$

'The Children's Hospital, Denver, Denver, CO, USA

${ }^{2}$ University of Colorado, Denver, CO, USA

Journal of Cardiovascular Magnetic Resonance 2009, I I(SuppI I):P246

Objective: To compare the flow indices in the main pulmonary artery (MPA) of children with pulmonary artery hypertension $(\mathrm{PAH})$ to children without $\mathrm{PAH}$, non-invasively, with magnetic resonance phase contrast imaging.

Background: MPA flow indices obtained by magnetic resonance phase contrast imaging have recently been used to diagnose $\mathrm{PAH}$ in adults. To our knowledge these techniques have not been previously used in children.

Methods: Over a four year period, pediatric patients with $\mathrm{PAH}$ (mean PA pressure $>25 \mathrm{mmHg}$ on right heart catheterization) who had undergone cardiac MRI with phase contrast imaging of the MPA were included in the study (6 patients). Seven control patients who underwent cardiac MRI at our institution for reasons other than $\mathrm{PAH}$ were also included. There was no significant difference in age, weight or sex between the two groups.

The phase contrast images were analyzed by an observer blinded to the mean pulmonary artery pressure for: minimum and maximum MPA area; MPA area strain; maximum and mean MPA velocity; minimum, maximum, and mean flow; stroke volume; acceleration time (AT) [time from the onset of systolic flow to peak systolic flow]; ejection time (ET) [time from onset of systolic flow to the time of minimum flow]; and acceleration time to ejection time ratio (AT/ET).

Differences between the $\mathrm{PAH}$ and the control groups were calculated with an unpaired, two-tailed student $T$ test assuming equal variance.

Results: There were significant differences between the PAH and control group for minimum MPA area $\left(779 \mathrm{~mm}^{2} \pm 190\right.$ vs. $\left.500 \mathrm{~mm}^{2} \pm 128, \mathrm{p}=0.009\right)$, MPA area strain $(22 \% \pm 12 \mathrm{vs}$. $55.5 \% \pm 17, p=0.002)$, mean velocity $(7 \mathrm{~cm} / \mathrm{s} \pm 1.6 \mathrm{vs} .11 .9 \mathrm{~cm} /$ $s \pm 3.6, p=0.0 \mathrm{I})$, and ejection time $(295 \mathrm{~ms} \pm 56$ vs. 559 $m s \pm 239, p=0.02)$ There was a trend towards significance in MPA stroke volume $(43 \mathrm{ml} \pm 14 \mathrm{vs} .64 \mathrm{ml} \pm 21, \mathrm{p}=0.06)$ and acceleration time (III ms \pm 16 vs. I46 ms $\pm 37, p=0.06$ ). No significant difference was found in MPA maximum area $(945$ $\mathrm{mm} 2 \pm 226$ vs. $766 \mathrm{~mm} 2 \pm \mathrm{I77}, \mathrm{p}=0.13)$, minimum MPA flow $(-40 \mathrm{ml} / \mathrm{s} \pm 20 \mathrm{vs}$. $-29 \mathrm{ml} / \mathrm{s} \pm 24, \mathrm{p}=0.40)$, maximum MPA flow $(298 \mathrm{ml} / \mathrm{s} \pm 63 \mathrm{vs} .320 \mathrm{ml} / \mathrm{s} \pm 97, \mathrm{p}=0.64)$, mean flow $(6 \mathrm{I} \mathrm{ml} / \mathrm{s} \pm$ 
$15 \mathrm{vs.} 82 \mathrm{ml} / \mathrm{s}$ vs. $\pm 25, \mathrm{p}=0 . \mathrm{I})$, maximum velocity $(96 \mathrm{~cm} / \mathrm{s} \pm 20$ vs. $101 \mathrm{~cm} / \mathrm{s} \pm 35, \mathrm{p}=0.76)$ or $\mathrm{AT} / \mathrm{ET}$ ratio $(0.39 \pm 0.1$ vs. $0.30 \pm 0.12, p=0.17$ ).

Discussion: Our findings largely agreed with those found in adult patients with PAH. While only minimum PA diameter achieved significance, both minimum and maximum PA area were larger in the PAH group. Taken in conjunction with the significantly lower MPA strain found in the PAH group, these findings suggest lower compliance in the main MPA in children with PAH.

Although maximum MPA velocities were not significantly different between the two groups, mean MPA velocity was significantly lower in the PAH group. No significant difference was found in minimum, maximum or mean MPA flow between the two groups.

The PAH group showed a trend towards lower acceleration times, a finding that has been demonstrated in adults. Unlike adults with $\mathrm{PAH}$, the children in this study had significantly lower than normal ejection times.

Conclusion: Magnetic resonance phase contrast imaging reveals significant derangements in MPA flow dynamics in children with $\mathrm{PAH}$ which may distinguish them from children with normal pulmonary artery pressures. In contrast to findings in adults, children with PAH have significantly shorter ejection times than normal, a novel finding.

\section{P247}

\section{Complications of aortic coarctation repair assessed using cardiac magnetic resonance imaging \\ Sylvia SM Chen and Raad H Mohiaddin \\ Royal Brompton Hospital, London, UK}

Journal of Cardiovascular Magnetic Resonance 2009, I I(SuppI I):P247

Introduction: Coarctation of the aorta (CoA) accounts for up to $10 \%$ of all congenital heart defects. Open repair with surgical reconstruction has been the main form of treatment and more recently, aortic stenting has been used as a less invasive technique without the need for cardiac bypass. The long term success and impact of repair on the structure of the aorta is important with regard to morbidity and management of these patients.

Purpose: We used cardiac magnetic resonance imaging (CMR) to assess the long term success of repair of CoA with respect to structural complications and residual stenosis.

Methods: CMR studies performed in patients with repaired CoA from January 2005 to September 2008 were reviewed. Details of age at, date and type of repair were obtained from patient medical notes. Multi-slice HASTE, cine and turbo-spine echo T2 images, and aortic in-plane and through-plane flow studies were analysed for complications of repair (aneurysmal dilatation, false aneurysm, dissection) and residual stenosis. Aneurysmal dilatation was defined as localised widening at the repair site in comparison to the diameter of the pre- and postaortic segments. Residual stenosis was defined as constriction at the repair site with flow acceleration on in-plane flow analysis, \pm peak recorded velocity on through-plane measurement of $>1.5 \mathrm{~m} / \mathrm{s}$.

Results: Analyses were done on 281 studies (167 males, II4 females, aged $31 \pm 9$ years). Average time between surgical repair and balloon dilatation, and CMR imaging $18 \pm 7$ years, and between aortic stenting and CMR imaging $4 \pm 0.8$ years. Types of
Table I (abstract P247) Types of aortic coarctation repair and the main complications

\begin{tabular}{|c|c|c|c|}
\hline Repair & $\begin{array}{l}\text { Aneurysmal } \\
\text { dilatation } \\
\text { (\% within the } \\
\text { type of repair) }\end{array}$ & $\begin{array}{l}\text { Suture line } \\
\text { false aneurysm } \\
\text { (\% within type } \\
\text { of repair) }\end{array}$ & $\begin{array}{l}\text { Residual } \\
\text { stenosis } \\
\text { (\% within type } \\
\text { of repair) }\end{array}$ \\
\hline Dacron patch & $79 \%$ & $10 \%$ & $10 \%$ \\
\hline Subclavian flap & $61 \%$ & $3 \%$ & $3 \%$ \\
\hline $\begin{array}{l}\text { Resection and } \\
\text { end-to-end } \\
\text { anastomosis }\end{array}$ & $0 \%$ & $0 \%$ & $61 \%$ \\
\hline Balloon dilatation & $16 \%$ & N/A & $70 \%$ \\
\hline Aortic stenting & $2 \%$ & N/A & $13 \%$ \\
\hline Interposition graft & $0 \%$ & $9 \%$ & $13 \%$ \\
\hline Bypass graft & $0 \%$ & $25 \%$ & $0 \%$ \\
\hline
\end{tabular}

repair: resection and end-to-end anastomosis ( $n=94,83 \%)$, subclavian flap repair $(n=62,22 \%)$, aortic stenting $(n=43$, $15 \%)$, interposition graft repair $(n=31,11 \%: 22$ dacron, 9 gelseal), balloon dilatation $(n=24,9 \%)$, dacron patch repair $(n=19,7 \%)$, and bypass graft $(n=8,3 \%)$. Structural complications were most marked in patch repair (15/19 aneurysmal dilatation within the patch, $2 / 19$ suture line false aneurysms, 2/19 residual stenosis), and subclavian flap repair (38/62 aneurysmal dilatation within the flap, 2/62 suture line false aneurysms, and 2/ 62 residual stenosis). There were no direct structural complications from resection and end-to-end anastomosis, but 57/94 had residual stenosis. Balloon dilatation had 17/24 residual stenosis and mild aneurysmal dilatation in $4 / 24$. Aortic stenting showed less residual stenosis (6/43), with minimal structural complications (no dissection, and only I/43 displacement of the stent, I/43 aneurysm). Reasonably good long term results were observed in graft repairs: interposition grafts $(3 / 31$ suture line false aneurysms, 4/3I residual stenosis at arch and graft anastomosis) and bypass grafts (none with residual stenosis, and 2/8 suture line false aneurysm). Main complications of the types of repair are in Table $\mathrm{I}$.

Conclusion: Patch and flap aneurysms are frequent complications of patch and subclavian flap repair of aortic coarctation, and the rate of residual stenosis with resection and end-to-end anastomosis was high. Balloon dilatation had minimal complications, but frequent residual stenosis. Graft and bypass graft repair are the most favourable surgical repairs with limited complications and residual stenosis. Aortic stenting also appears quite successful with few complications and residual stenosis but long term follow-up is not yet available.

\section{P248}

Method for separate analysis of inflow vs. outflow regions of the right ventricle in Ebstein's anomaly Christopher Lee ${ }^{1,2}$, Florence Sheehan', Beatriz Bouzas ${ }^{3}$, Michael A Gatzoulis ${ }^{3}$ and Philip J Kilner ${ }^{3}$

'University of Washington, Seattle, WA, USA

${ }^{2}$ Georgetown University, Washingont, DC, USA

${ }^{3}$ Royal Brompton Hospital, London, UK

Journal of Cardiovascular Magnetic Resonance 2009, I I(SuppI I):P248

Background: Ebstein's anomaly (EA) is caused by underdevelopment of the inlet. However the impact of EA on regional 
Figure I (abstract P248)

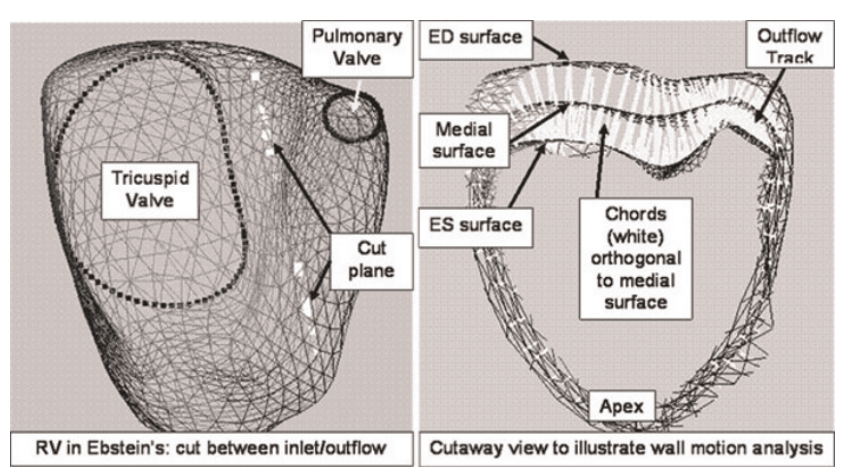

and global function in the underdeveloped inlet has been difficult to assess quantitatively.

Methods: We measured the size and shape of the right ventricle (RV) and its inlet and infundibulum (outflow) portions in 30 patients with EA aged 16-64 yrs and 9 normal subjects from $M R$ images acquired in long and short axis and oblique views. The RV was traced and reconstructed in $3 D$ as a triangular mesh using the piecewise smooth subdivision surface method. To define the volumes of the inlet and infundibulum, points were traced at the muscular ring separating the two portions, focusing on the supraventricular crest and the parietal, septal, and moderator bands. Care was taken to confirm the locations of these anatomic landmarks in intersecting views. A plane was fit to the points and used to cut the RV into inlet and infundibulum. Wall motion was measured in 13 regions by the centersurface method along chords drawn orthogonal to a surface constructed midway between the end diastolic (ED) and end systolic (ES) surfaces. The lengths of chords in each region were averaged and then normalized by dividing by the square root of body surface area. Tricuspid tilt was defined as the angle between the mitral and tricuspid annular planes, and tricuspid descent was the systolic excursion of the annulus centroid. See Figure I.

Results: The global RV ejection fraction (EF) was mildly depressed in EA patients $(45 \pm 8$ vs. $55 \pm 4 \%$ in normals, $\mathrm{P}<0.00 \mathrm{I}$ ). Their RV's were severely dilated (end diastolic volume index $185 \pm 78$ vs. $\left.82 \pm 16 \mathrm{ml} / \mathrm{m}^{2}, P<0.00 \mathrm{I}\right)$ and rounded in apical and mid RV cross sections. The tricuspid annulus was severely tilted $\left(62 \pm 26\right.$ vs. $19 \pm 9^{\circ}$ in normals, $\mathrm{P}<0.00 \mathrm{I})$. The infundibulum contained approximately $1 / 5^{\text {th }}$ of $\mathrm{RV}$ volume in both groups $(20 \pm 7$ in normals vs. $21 \pm 9 \%$ in EA, $p=N S)$. In EA patients inlet EF exceeded infundibulum EF (46 \pm 7 vs. $38 \pm 13 \%, p<0.02$ ). However the function of both inlet and infundibulum were depressed compared to normal ( $p<0.05$ for both). The function of the inlet and infundibulum differed less in normals (55 \pm 6 vs. $49 \pm 11 \%, p=N S$ ). Regional function was depressed compared to normal in all 9 inlet regions, significantly so in 4 regions. Tricuspid descent was also depressed compared to normal ( $13 \pm 5$ vs. $18 \pm 3 \mathrm{~mm}, \mathrm{p}<0.005)$ but the pattern of regional function was similar to normal with the greatest contraction occurring in basal regions. The global EF correlated with wall motion in the basal regions and inlet septum ( $r$ between 0.43 and 0.58, $p<0.05$ ) and with tricuspid annular descent $(r=0.52, p<0.005)$.
Conclusion: Despite underdevelopment of the inlet, patients with EA have relatively preserved function in this portion of the RV. Three dimensional surface reconstruction enables separate analysis of the inlet and infundibulum portions of the RV as well as detailed assessment of regional function and shape.

\section{P249}

A prospective audit of paediatric cardiac MRI under general anaesthesia; practise and problems Marina L Hughes', Emma Stockton ${ }^{1,2}$, Andrew Taylor and Angus McEwan'

${ }^{I} G r e a t$ Ormond Street Hospital for Children, London, UK

${ }^{2}$ Great Ormond Street Hospital, London, UK

Journal of Cardiovascular Magnetic Resonance 2009, I I(SuppI I):P249

Background: Cardiac MRI (CMRI) is increasingly used to assist with the surgical planning and follow up of children with congenital heart disease. In small children general anaesthesia (GA) is always required. We describe our experience of GA for paediatric CMRI, using data collected prospectively over 3 years. Methods and results: 120 patients presented for CMRI under GA from November 2005 to May 2008. These patients were aged 0 - 288 months (median 28 months), and weighed 2.8 $64 \mathrm{~kg}$ (median II.7 kg).

This cohort of patients had a large range of diagnoses, but principally comprised those with hypoplastic left heart syndrome (HLHS) and other functionally univentricular hearts for interstage imaging, patients with pulmonary atresia or Tetralogy of Fallot for surgical planning, and patients with aortic arch abnormalities.

The majority of patients (91.6\%) were admitted as a day-case to our hospital on the day of CMRI. Ten patients were imaged during an inpatient stay, three of whom (2.5\%) were inpatients on the Cardiac Intensive Care Unit (CICU) prior to their MRI.

Ten percent of patients were classified as American Society of Anaesthesiology (ASA) Class 2, 80\% ASA Class 3 and 10\% ASA Class 4.

Fifty-seven children (47.5\%) had a functionally single ventricle. Fifty patients (42\%) had normal oxygen saturations. Of the cyanosed patients, pulmonary blood flow was supplied through an aorto-pulmonary shunt in $33 \%$ of patients, and $13 \%$ had a cavopulmonary shunt. Sixteen patients (13\%) had a mixed circulation.

Six patients $(5 \%)$ had severely impaired systemic ventricular function, 10 (8.3\%) had moderately impaired function and 31 (25.8\%) had mildly impaired function. 14 patients ( $11.6 \%$ ) had at least moderate systemic outflow tract obstruction.

A senior, cardiac anaesthetist was continuously present during every case. Most patients (I00/I20,83\%) received an inhalational induction with sevoflurane. Sixteen received an intravenous induction with propofol. The anaesthetist remained in the control room with the MRI technician and cardiologist during scanning. Breath holding was achieved from within the control room by breaking the circuit and occluding the inspiratory limb of the circle system.

The majority of children (98/I20,8I.6\%) had an uneventful CMRI under GA. Nineteen (I5.8\%) patients suffered minor adverse events. One major adverse event occurred; a patient with hypoplastic left heart syndrome (HLHS) became hypotensive and had a cardiac arrest in the MRI scanner. This patient was 
successfully resuscitated. One patient with HLHS died during fasting, prior to anaesthetic, on the morning of the MRI.

Conclusion: Despite the precarious physiology of children with congenital heart disease, a GA for CMRI can be administered safely. A gas induction with sevoflurane seems safe for most children, even with impaired ventricular function. The safe provision of GA for CMRI relies on a senior multi disciplinary team working closely and regularly together. Interstage HLHS patients are particularly at risk of a serious adverse event and particular care should be taken to ensure adequate hydration before, during and after anaesthesia.

\section{P250}

Rapid cardiac cine imaging using $\mathrm{MACH}$ Mark Doyle', Geetha Rayarao,2, Diane A Vido', Vikas K Rathi', Saundra Grant', June A Yamrozik', Ronald B Williams' and Robert WW Biederman''

'Allegheny General Hospital, The Gerald McGinnis Cardiovascular Institute, Pittsburgh, PA, USA

${ }^{2}$ Allegheny General Hospital, Pittsburgh, PA, USA

Journal of Cardiovascular Magnetic Resonance 2009, I I(SuppI I):P250

Introduction: Previously, we developed a sparsely distribute k-space-time sampling approach termed BRISK, Block Regional Interpolation Scheme for K-space [I]. This approach allowed a nominal acceleration factor of 4 with good quality and low artifact. Others have developed alternative k-space-time sampling schemes, such as KT-BLAST and SLAM [2, 3]. We note that even at high acceleration factors, KT-BLAST/SLAM allowed a smooth transition from frame to frame, while BRISK experienced ringing artifacts. From these considerations we isolated key features that contribute to a successful k-space-time sparse sampling scheme: I) Update of k-space should be rapid near the center and lower near the periphery (as in BRISK) to capture highly dynamic features.

2) Update of k-space should smoothly vary over time (as in KTBLAST/SLAM) avoiding sudden transitions between k-space regions to result in smoother transition between frames.

Figure I (abstract P250)

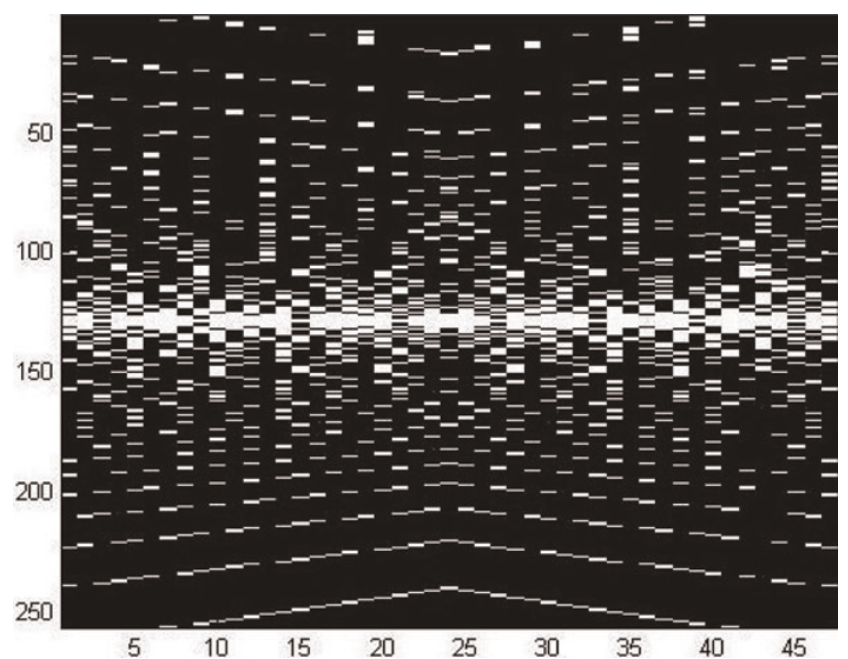

Figure 2 (abstract P250)

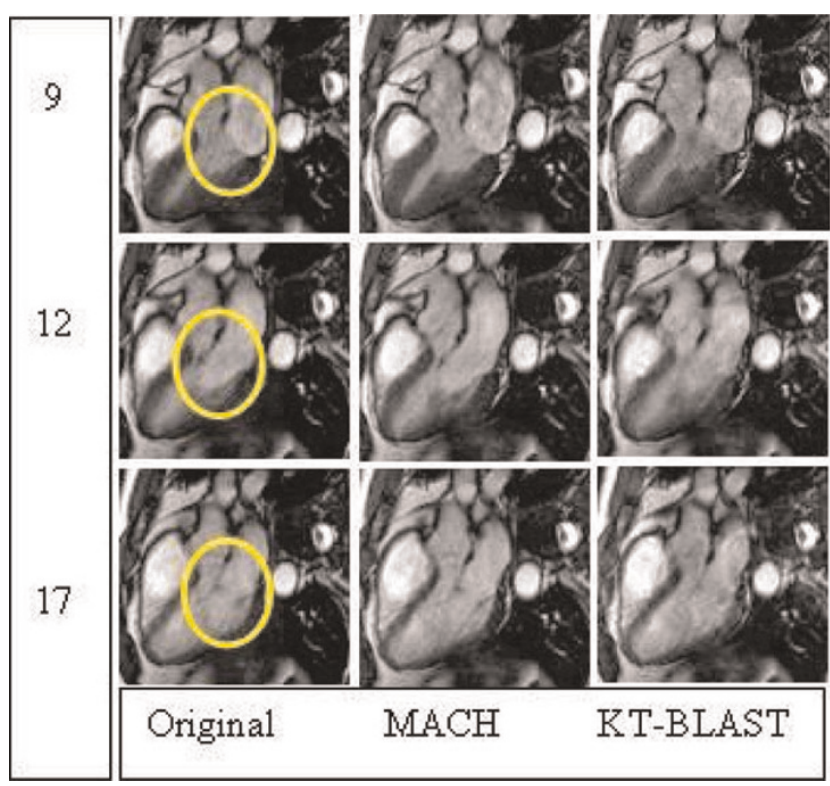

From these design principles we developed a new sparse k-spacetime sampling scheme, MACH, Multiple Acceleration Cycle Hierarchy. MACH incorporates a gradually changing rate over time, starting with the highest rate near the center of k-space and becoming progressively sparser towards the periphery, Figure I shows the k-space-time sampling scheme. In $\mathrm{MACH}$, the progressively decreasing sampling rates are not confined to integer steps, thereby providing greater opportunity for a

Figure 3 (abstract P250)
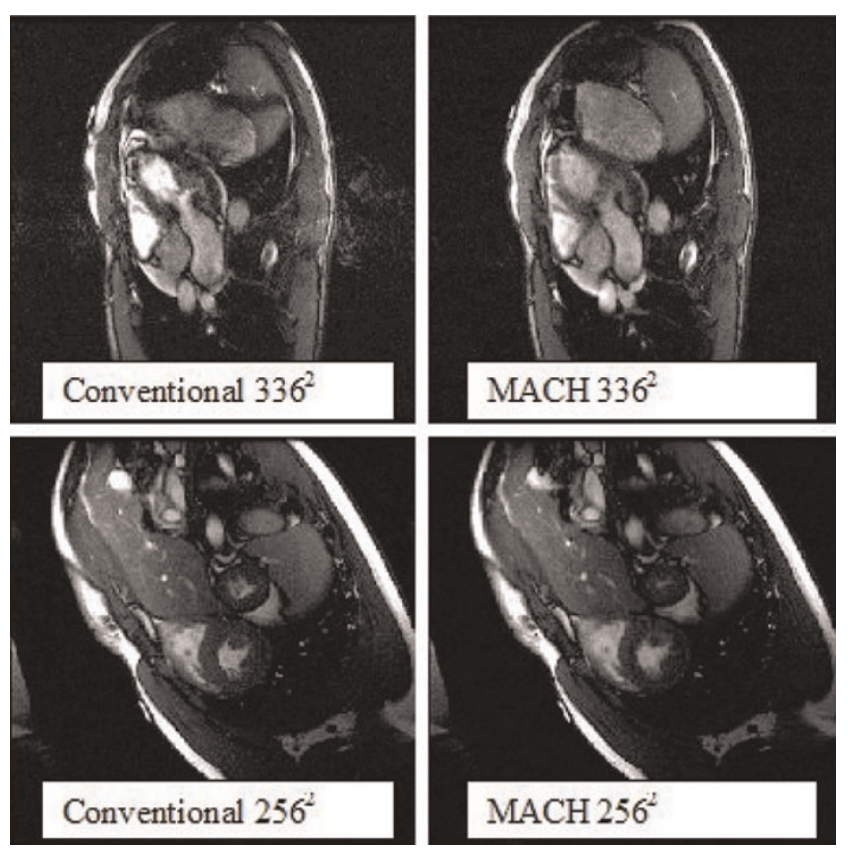
smooth transition over the k-space-time domain. Data that are not directly sampled in $\mathrm{MACH}$ are interpolated prior to applying conventional Fourier transformation to generate the series of images. Since each frame incorporates a full set of k-space data, the SNR is similar to the conventional scan.

Methods: Simulations were performed using fully acquired stead-state-free-precession (SSFP) cine image data to allow direct comparison of MACH and KT-BLAST/SLAM when using the same acceleration factors. Further, MACH was implemented on a I.5 T scanner (GE, Milwaukee, WI). Using the SSFP cine acquisition, long and short axis acquisitions were acquired using the conventional examination and $\mathrm{MACH}$ applied with a net sparse factors ranging from 2 to 5 , with matrices ranging from 224 to 336. Comparable cine acquisitions were acquired of the heart in 10 volunteers. The end-diastolic and end-systolic phases were identified and areas were planimetered and compared between the conventional and the $\mathrm{MACH}$ accelerated scans.

Results: Simulations showed that for a moderate acceleration factor of 5, KT-BLAST/SLAM represented myocardial motion well, but lost detail of the relatively fast moving valvular features, while $\mathrm{MACH}$ still represented these features. In the in vivo acquisitions, the average $\mathrm{MACH}$ acceleration factor applied was $3.5 \pm \mathrm{I} . \mathrm{I}$, end-diastolic and end-systolic ventricular chamber areas were not significantly different between the conventional and the accelerated MACH scans $(p=0.7,0.6$, respectively) and correlations were excellent at 0.99 for each. Compared to the conventional scan, there is no additional overhead with $\mathrm{MACH}$. See Figures 2 and 3.

Conclusion: In sparse k-space-time acquisition strategies, rapidly updating the central region of k-space is known to be important. We note that $\mathrm{MACH}$ achieves this condition very efficiently while also achieving a smooth transition of update rate between each region of k-space since $\mathrm{MACH}$ does not use a uniform or even a regular update rate. $\mathrm{MACH}$ was successfully implemented and shown to accurately represent cardiac regions with good fidelity.

\section{References}

I. Doyle M, Walsh EG, Blackwell GG and Pohost GM: Block Regional Interpolation Scheme for K-space (BRISK): A Rapid Cardiac Imaging Technique. Magn Reson Med 1995, 33:163-170.

2. Kozerke S, Tsao J, Razavi R and Boesiger P: Accelerating cardiac cine 3D imaging using k-t BLAST. Magn Reson Med 2004, 52(I):19-26.

3. Rehwald WG, Kim RJ, Simonetti OP, Laub G and Judd RM: Theory of high-speed MR imaging of the human heart with the selective line acquisition mode. Radiology 200 I, 220(2):540-547.

\section{P25I}

Dual-source CT angiography of the thoracic aorta using prospective cardiac gating and a low kilovoltage technique

Cormac Farrelly, Aoife Keeling, John Sheehan,

Vahid Yaghmai and James Carr

Northwestern Memorial Hospital, Chicago, IL, USA

Journal of Cardiovascular Magnetic Resonance 2009, I I(SuppI I):P25 I

Introduction: Cardiac gating leads to less artifacts and more accurate measurements of the thoracic aorta and root. Without gating the aortic root and ascending aorta are particularly prone to artifact. To date gating has lead to increased dose to patients who often have to undergo multiple studies.

Purpose: To investigate the effects of prospective cardiac gating and low kilovoltage parameters on image quality and radiation dose when acquiring $\mathrm{CT}$ angiography of the thoracic aorta (CTTA).

Methods: Dual-source CTTA was performed on 60 consecutive patients. One group of thirty were examined with retrospective gating and standard parameters ( $120 \mathrm{kV}, 340 \mathrm{mAs}$ ). The other thity were examined with prospective gating and low dosage parameters ( $100 \mathrm{kV}, 170 \mathrm{mAs})$ with the scanner in a Step and Shoot (SAS) mode that automatically recognizes and does not acquire data during ectopic heart beats. Qualitative analysis was performed by two blinded reviewers who assessed image quality and graded it on a three point scale. Sharpness of the thoracic aorta was also evaluated quantitatively at three levels by generating a line profile across the aortic vessel wall and calculating the point spread function. Both sides of the density profile were analyzed, averaged and then used to calculate sharpness (Hounsfield units/mm). Attenuation in the aorta and image noise was measured. Radiation dose was measured.

Results: Qualitative and quantitative analysis of sharpness of the thoracic aorta and image noise showed no significance difference $(p>0.05)$ between the two patient groups. Estimated effective radiation dose of the prospective low kilovoltage protocol (mean dose $<3 \mathrm{mSv}$ ) was significantly lower $(\mathrm{p}<0.0 \mathrm{I})$. Conclusion: Low dose imaging of the thoracic aorta with maintenance of image quality and sharpness is achievable using a prospective cardiac gated low kilovoltage technique.

\section{P252}

Time-resolved myocardial perfusion MRI with reduced data acquisition window, improved spatial coverage, resolution and SNR Lan $\mathrm{Ge}^{\prime}$, Aya Kino', Mark Griswold ${ }^{2}$, Charles Mistretta ${ }^{3}$, Daniel Lee ${ }^{4}$, James Carr' ${ }^{\prime}$ and Debiao $\mathrm{Li}^{\prime}$

'Northwestern University, Evanston, IL, USA

${ }^{2}$ Case Western Reserve University, Cleveland, OH, USA

${ }^{3}$ University of Wisconsin-Madison, Madison, WI, USA

${ }^{4}$ Northwestern Memorial Hospital, Chicago, IL, USA

Journal of Cardiovascular Magnetic Resonance 2009, I I (SuppI I):P252

Introduction: Perfusion MRI is a promising technique to detect ischemic heart disease. Single-shot imaging is currently used to acquire 3 slices with a temporal resolution of one time frame per cardiac cycle. However, this technique is challenged by the cardiac motion artifacts, limited spatial coverage, resolution and SNR. Reduced imaging time per slice will allow greater coverage of the heart and reduced motion artifacts, especially during stress imaging with high heart rates. Parallel imaging methods have been applied in myocardial perfusion MRI, but the acceleration factors are limited to $2-3$ because of SNR considerations. Time-resolved data acquisition with shortened data acquisition window can be performed with Conjugate-gradient Highly Constrained Backprojection reconstruction (CG-HYPR) [I, 2]. A combination of sliding composite images and CG-HYPR method allows vast undersampling, increased spatial coverage, resolution and SNR while preserving the temporal resolution of one frame per heartbeat and dynamic blood and myocardial signal intensity changes. In this work, we compared this new method with the 
Figure I (abstract P252)

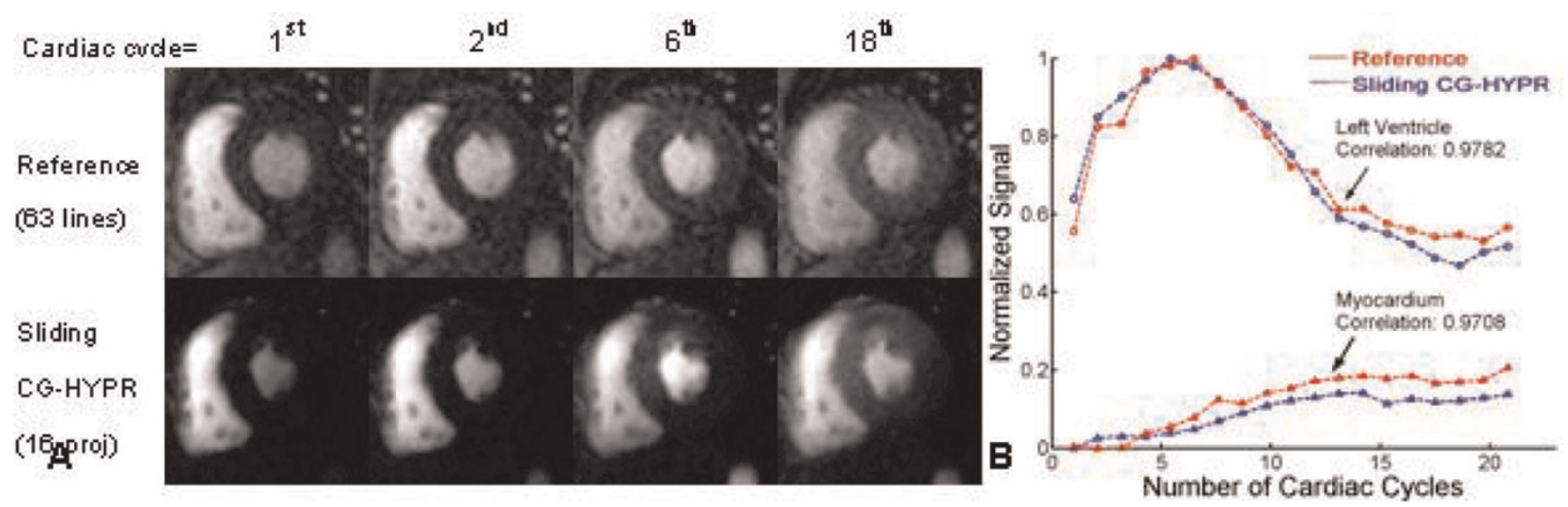

One typical example of the 6 volunteer studies. A) Comparison of the images from conventional method and sliding CG-HYPR. Blood signal change is observed in sliding CG-HYPR perfusion images, and comparable to the traditional images. B) Comparison of the left ventricle and myocardium signal changes vs. time.

conventional clinical protocol for myocardial perfusion in healthy volunteers.

Methods: An ECG-triggered, 2D multi-slice FLASH sequence with radial $k$-space sampling was used in this study. The $k$-space was acquired in a segmented interleaved fashion. The "composite images" were reconstructed by a sliding window method with full $k$-space data. CG-HYPR method was used to reconstruct timeresolved images (one image per cardiac cycle) combining signal intensity information from the undersampled radial projections, and the structural information from the sliding composite images whose center corresponds to the current cardiac cycle.

Six healthy volunteers were scanned during a single breath-hold in a $3 \mathrm{~T}$ system. The segmented SR-prepared multi-slice radial FLASH sequence was performed with the following parameters:

Figure 2 (abstract P252)

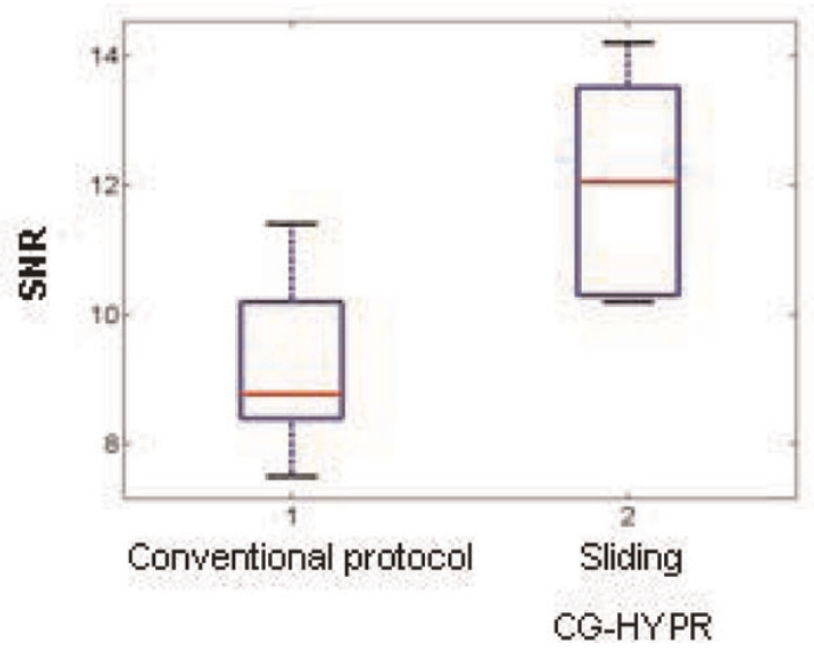

SNR comparison between images from sliding CG-HYPR and conventional protocol.
$\mathrm{TR} / \mathrm{TE} / \mathrm{flip}$-angle $=3.2 / 1.6 \mathrm{~ms} / 10^{\circ}$, matrix $=160 \times 192$, saturation pre-pulse delay $=50 \mathrm{~ms}$, number of slices $=6$, and spatial resolution $=1.4 \times 1.4 \times 8 \mathrm{~mm}$. Six datasets were collected over 60 heartbeats, with the data from 20-30 heartbeats is usable during breath-hold. Each of the sliding composite images were reconstructed over 10 cardiac cycles with 16 projections per cardiac cycle and $5 \mathrm{I}$ sliding composite images. Time-resolved images with the resolution of one image per cardiac cycle were generated after the CG-HYPR processing.

To verify the signal changes, a conventional scan was performed with the same contrast injection scheme. The imaging parameters were: TR/TE/flip-angle $=2.2 / 1.1 / 10^{\circ}$, matrix $=106 \times$ 192 (collected lines/heartbeat $=63$ ), GRAPPA factor $=2$, saturation pre-pulse delay $=40 \mathrm{~ms}$, number of slices $=3$, and spatial resolution $=3.1 \times 2.3 \times 8$. The dynamic signal changes and the SNR of images were compared between the sliding CG HYPR images and the conventional images.

Results: Although with only 16 projections per slice per cardiac cycle, the signal change distribution of the sliding CGHYPR images are similar to the reference images by conventional protocol (Figure IA), which can be further shown by the quantitative measurements (Figure IB). The mean correlation coefficients of the 6 studies for signal changes between the two methods were 0.9672 and 0.9423 for blood and myocardial signals, respectively. The averaged SNR images around blood signal peak from the two methods were calculated and compared. The CG-HYPR images had significantly higher SNR than conventional protocol ( $p<0.05$, t-test) (Figure 2$)$. The spatial coverage and spatial resolution were improved roughly by a factor of 2 .

Conclusion: This work demonstrated the feasibility of SCGHYPR for accelerated myocardial perfusion imaging. The acquisition time can be reduced dramatically, allowing an increased number of slices and reduced motion artifacts. Spatial resolution and SNR can be improved while preserving the signal change accuracy.

\section{References}

I. Mistretta CA, et al: MRM 2006.

2. Griswold MA, et al: Proc ISMRM 2007. 
P253

Mouse cardiac MRI on a 3 T clinical system using a low cost setup

Timothy F Christian', Jay Gonyea', Stephen P Bell' and Trevor Andrews ${ }^{2}$

'University of Vermont, Burlington, VT, USA

${ }^{2}$ Philips Health Care, Burlington, VT, USA

Journal of Cardiovascular Magnetic Resonance 2009, I I(SuppI I):P253

Background: Using a mouse model for cardiac imaging is advantageous due to the wide array of gene alterations possible to investigate a range of disease states. However, the physical challenges for CMRI in mice are significant (small size, high metabolic rate). In order to minimize these issues, many centers use dedicated high field small bore systems with specialized RF coils which are specific to small rodent imaging. Not all centers have the resources or volume to sustain such dedicated magnets but may still require mouse imaging studies for specific questions.

Purpose: This study describes a lower cost alternative to a complete dedicated system for mouse CMR.

Methods: The magnet is a Philips 3 TAchieva/80 $\mathrm{mt}(40 \times 2) / \mathrm{m}$ peak, $200 \mathrm{mt} / \mathrm{m} / \mathrm{ms}$ slew rate with Quasar Dual Gradients which is configured for standard clinical imaging. A mouse specific commercial MR coil for such a system costs in the range of $\$ 20,000-40,000$ to integrate into the magnet. Instead, we used a Medrad receive only, eCoil (endorectal coil) and connector box (separate purchase for prostate imaging). This is a single loop coil with a balloon covering which is removed (coil cost $=\$ 200$ ). The mouse is placed directly on the coil, serving as both a support structure and a close proximity receive coil (see Figure I). A dedicated small animal ECG/respiratory gating system (SA Instruments) was used to interface with the scanner to allow for for breathing during the acquisitions. Mice were anesthetized using $0.25-0.75 \%$ isoflurane.

Results: Short-axis ECG-gated cine gradient echo images (diastolic apical, mid and basal) are shown in Figure 2. The spatial resolution was $0.32 \times 0.32 \mathrm{~mm}$ at a slice thickness of 1.4 $\mathrm{mm}$. The temporal resolution was $24 \mathrm{~ms}$ at a heart rate of 300 bpm for a total of 8 true cardiac phases per R-R interval. Images were acquired for 4.0 NEX requiring 300-400 R-R intervals. Delayed inversion recovery imaging and $I^{\text {st }}$-pass perfusion imaging were also performed with reasonable image quality (not shown). The SNR of the blood pool to noise in figure 2 was $62.1 / 1$ and the CNR of blood pool to myocardium was $2.45 / 1$. The LVEF was $51.7 \%$ for the example shown.

Figure I (abstract P253)

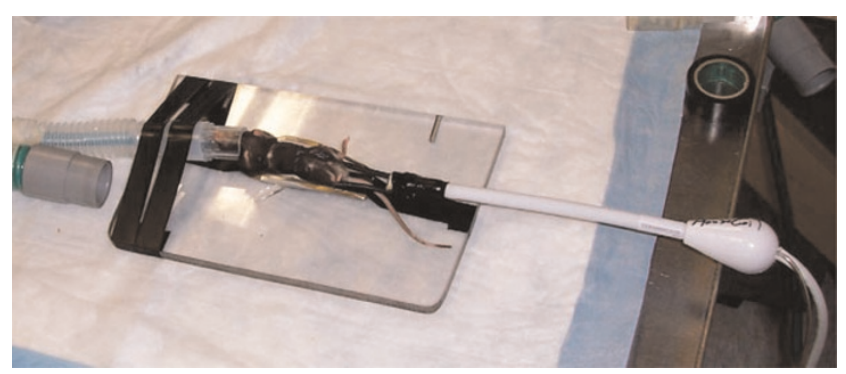

Figure 2 (abstract P253)

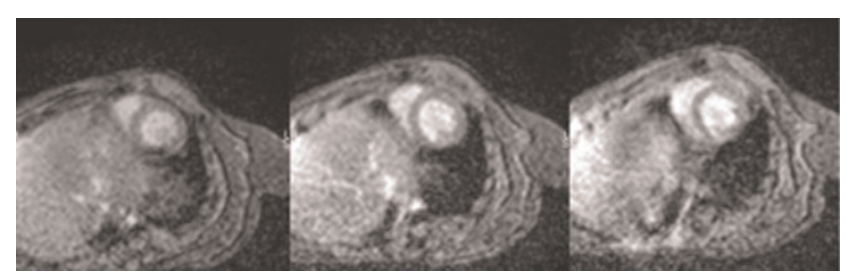

Conclusion: Use of a disposable single element endo coil allows the assessment of LV function in the mouse with reasonable temporal, spatial resolution and imaging characteristics without the purchase of a dedicated small animal system.

\section{P254}

Rapid analysis of right ventricular volumes and systolic function using Cardiovascular Magnetic Resonance Imaging

Simon C Koestner, Marco JW Götte, Robin Nijveldt, Jan GJ Groothuis, Aernout M Beek, Mark BM Hofman and Albert $C$ van Rossum

VU University Medical Center, Amsterdam, Netherlands

Journal of Cardiovascular Magnetic Resonance 2009, I I(SuppI I):P254

Introduction: The function of the right ventricle (RV) is an important prognostic factor in heart disease. Assessment of RV function using the gold standard disk-area (or Simpson) technique is time consuming and semi-quantitative evaluation (e.g. TAPSE) shows unsatisfactory correlation with quantitative analysis. The assessment of left ventricular function with a biplane area-length method has been validated for invasive ventriculography and for CMR. This method has never been used for the RV because of its particular geometry.

Purpose: This study investigates the use of an area-length technique for the rapid assessment of the volumes and function of the RV using cardiovascular magnetic resonance imaging (CMR).

Methods: 12 healthy volunteers and 25 patients with RV dilation and/or dysfunction ( 9 with pulmonary hypertension (PHT), 9 with dilated RV without PHT, 7 with ischemic heart disease) underwent cine CMR on a I.5 T scanner. RV enddiastolic volume (RVEDV), end-systolic volume (RVESV) and ejection fraction (RVEF) were calculated using a stack of shortaxis cines as the standard of reference (Simpson technique). A 4-chamber view and a perpendicular RV 2-chamber view were acquired to calculate RV volumes and RVEF using a simple arealength method $\left(A^{*} A / L\right)$ without correction coefficient in both end-diastolic and end-systolic phases with A the RV area, and $L$ the apex to base length. This was tested for both a single and a biplane long-axis view. Finally, TAPSE was measured on the 4-chamber view. Area-length methods and TAPSE were compared to the standard of reference.

Results: Analysis of the area-length method using long-axis orientations was faster $(\sim 2 \mathrm{~min})$ than the Simpson method ( $20 \mathrm{~min}$ ). The estimation of RVEF using biplane area-length method correlated well with short-axis calculation $\left(r^{2}{ }_{2 L A}=0.72\right.$, 
$\mathrm{P}<0.0 \mathrm{I})$. However, this method slightly overestimated RVEF (difference 5.5\%, $\mathrm{p}=0.045$ ). The correlation between RVEF and single long-axis analysis (4-chamber) or TAPSE was weaker $\left(r^{2}\right.$ ILA $=0.49, \mathrm{p}<0.01$ and $r^{2}{ }_{\text {TAPSE }}=0.37, \mathrm{p}<0.01$, respectively). There was a good correlation between the two orientations in RVEDV measurements $\left(r^{2}{ }_{2 L A}=0.78, p<0.01\right)$ and in ESV measurements $\left(r^{2}{ }_{2 L A}=0.82, p<0.01\right)$ using the biplane method. There was no significant volume differences between the two methods (EDV: mean $_{2 \mathrm{LA}}=196.5 \pm 92.4 \mathrm{~mL}$, mean $_{\text {SIMPSON }}=$ $208.4 \pm 78.7 \mathrm{~mL}, \mathrm{P}=0.55$; ESV: $\operatorname{mean}_{2 \mathrm{LA}}=104.2 \pm 70 \mathrm{~mL}$, mean $\left._{\text {SIMPSON }}=119.0 \pm 58.4 \mathrm{~mL}, \mathrm{p}=0.33\right)$.

Conclusion: Measurements of RV volumes and RVEF using biplane area-length method correlate well with the gold standard of contiguous short-axis measurements, despite the particular geometry of the RV. However, this method leads to a $5 \%$ overestimation of RVEF which should be taken in consideration in the RVEF calculation. As analysis time is significantly shorter, this method can be used as a rapid, quantitative screening tool.

\section{P255}

Is short-axis imaging of the LV obligatorily the most accurate method for non-invasive quantitation of the mass of the heart? Nicholas Farber, Mark Doyle, Ronald B Williams, June A Yamrozik, Rachel Paul, George J Magovern, James A Magovern, Srinivas Murali

and Robert WW Biederman

Allegheny General Hospital, The Gerald McGinnis

Cardiovascular Institute, Pittsburgh, PA, USA

Journal of Cardiovascular Magnetic Resonance 2009, I I(SuppI I):P255

Introduction: Non-invasive LV mass quantitation is an important clinical metric with considerable ramifications that include substantial morbidity and mortality. Cardiovascular MRI (CMR) has been demonstrated to be the 'gold standard' for the quantification of LV mass for almost two decades chiefly defined by its high spatial resolution affording very low variability. Over the last two decades the technique has evolved typically, into contiguous, 2D short-axis (SA) orientations as the standard for CMR cardiac mass quantitation, believed to be inherently most accurate. However, as compared to other techniques wherein accuracy suffers from inability to define endocardial/epicardial borders, any inaccuracy CMR suffers is now predominantly governed by the basal-plane acquisition whereupon exact discrimination of the AV-valve plane is arduous.

Hypothesis: We hypothesized that a systematic interrogation of alternative, non-SA imaging geometric acquisitions might now be more accurate for defining, despite lack of truly contiguous imaging, more accurate and reproducible LV mass measurements.

Methods: An agar-based gel phantom was created representative of the varied cardiac anatomy, complete with tapered apex, non-planar base, trabeculations and often asymmetrical geometry. The weight of the phantom was measured on a highperformance scale. The phantom was scanned under varied orientations: 2D short-axis, 2D long-axis, 2D radial long-axis, and $3 \mathrm{D}$ short-axis imaging. For each orientation, slice thicknesses of $5 \mathrm{~mm}^{2}$ and $10 \mathrm{~mm}^{2}$ were used, while the 3D scan used $1 \mathrm{~mm}^{3}$ and $2 \mathrm{~mm}^{3}$ thicknesses. All variations of the parameters were scanned using both steady-state free precession (SSFP) and gradient recalled-echo (GRE). Statistical analyses via Students $t$ test were performed to determine variances of the mass of the agar.

Results: The calculated mass of the agar based on the shortaxis orientation was an average of $121 \%$ of the true LV mass. The long-axis orientation gave an average of $95 \%$ of the true mass. The radial scan gave an average of $96 \%$ of the true mass. The 3D scan ( 1 or $2 \mathrm{~mm}$ slice thickness) gave an average of $98 \%$ of the true mass. Larger slice thickness caused a significant overestimation of mass in only the 2D long axis orientation. No significant difference existed between the SSFP and GRE scan sequences in this (static) phantom model.

Conclusion: By default, 3D imaging via a phantom model designed specifically to emulate the natural heterogeneity of the typical LV is inherently the most accurate, especially via thin slice imaging for quantitation of LV mass. However, scan times limit clinical practicality for optimal accuracy. Contrary to the classical thinking that has evolved over the last 2 decades, the radial longaxis orientation shows great promise, as it avoids basal-plane errors associated with the short-axis orientation and has shorter scan times. Incorporation of this strategy inherently overcomes such basal-plane registration errors which exert a much greater influence over accuracy than do contiguous slice and interpolation errors. Future validation of this proof of concept study will include ex-vivo human heart studies.

\section{P256}

The involvement of the aorta by cardiac magnetic resonance in the inflammatory process of acute coronary syndrome

Geetha P Bhumireddy', John F Heitner', Peter J Cawley ${ }^{2}$, Igor Klem², Manesh Patel ${ }^{2}$, Anna Lisa Crowley ${ }^{2}$, Jonathan W Weinsaft ${ }^{2}$, Michael Elliott ${ }^{2}$, Michele Parker², Robert M Judd ${ }^{2}$ and Raymond J $\mathrm{Kim}^{2}$

${ }^{I}$ New York Methodist Hospital, Brooklyn, NY, USA

${ }^{2}$ Duke Univ Medical Center, Durham, NC, USA

Journal of Cardiovascular Magnetic Resonance 2009, I I(SuppI I):P256

Introduction: In patients presenting with acute coronary syndrome (ACS), it has been shown that multiple coronary arteries are involved during the inflammatory process. Growing literature has also revealed that carotid and aortic vessels maybe involved in the inflammatory cascade of ACS.

Purpose: To assess the involvement of the aorta during ACS by cardiac magnetic resonance (CMR).

Methods: We prospectively evaluated 78 patients who presented to the emergency department (ED) with chest pain and were classified as either: I) ACS: if they had positive Troponin levels and typical chest pain or 2) Non-cardiac chest pain: if they had negative Troponins and a normal stress test or catheterization. We compared these 2 groups to a control group of 45 asymptomatic diabetic patients. The descending aortic wall area (AWA) and aortic wall thickness (AWT) were measured using a 
Table I (abstract P256) Clinical and aortic wall characteristics of the study population

\begin{tabular}{|c|c|c|c|c|}
\hline Characteristics & $\begin{array}{l}\text { Acute coronary } \\
\text { syndrome } n=27\end{array}$ & $\begin{array}{l}\text { Non-cardiac } \\
\text { chest pain (ED) } n=5 I\end{array}$ & $\begin{array}{l}\text { Asymptomatic } \\
\text { diabetes (Control group) } \\
n=45\end{array}$ & p-value \\
\hline Age (yrs) & $60.3 \pm 11.4$ & $53.6 \pm 10.7$ & $59.9 \pm 8$ & \\
\hline Gender (female) & $52.2 \%$ & $47 \%$ & $44.5 \%$ & \\
\hline BMI & 29.19 & 19.85 & 14.10 & \\
\hline \multicolumn{5}{|l|}{ Past medical history: } \\
\hline Smoker & $43.5 \%$ & $13.7 \%$ & $11.1 \%$ & \\
\hline Hypertension & $78.2 \%$ & $49 \%$ & $75.5 \%$ & \\
\hline Diabetes Type 2 & $21.7 \%$ & $17.7 \%$ & $100 \%$ & \\
\hline Hyperlipidemia & $30.4 \%$ & $37.3 \%$ & $73.3 \%$ & \\
\hline \multicolumn{5}{|l|}{ Family history of: } \\
\hline$C A D$ & $34.8 \%$ & $39.2 \%$ & $33.3 \%$ & \\
\hline Angina & $91.3 \%$ & $53 \%$ & 0 & \\
\hline $\mathrm{MI}$ & $4.3 \%$ & 0 & 0 & \\
\hline Coronary angiography & $4.3 \%$ & $5.9 \%$ & 0 & \\
\hline Claudication & $34.8 \%$ & 0 & $6.7 \%$ & \\
\hline Renal disease & $8.7 \%$ & $3.9 \%$ & 0 & \\
\hline CVA/TIA & $13 \%$ & $7.8 \%$ & $6.7 \%$ & \\
\hline \multicolumn{5}{|l|}{ Blood tests: } \\
\hline Troponin & $1.37 \pm 2.1$ & NA & NA & \\
\hline CKMB & $61.9 \pm 71.1$ & NA & NA & \\
\hline Creatinine & $\mathrm{I} . \mathrm{I} \pm 0.33$ & $1.1 \pm 0.9$ & $1.09 \pm 0.8$ & \\
\hline LDL & $109 \pm 46.2$ & $104 \pm 36.6$ & $107.8 \pm 36.3$ & \\
\hline HDL & $47 \pm 11.8$ & $47.5 \pm 16.2$ & $50.04 \pm 11.8$ & \\
\hline Triglycerides & $149.8 \pm 101.7$ & $149.7 \pm 76.2$ & $212.5 \pm 143$ & \\
\hline Total cholesterol & $|8| .8 \pm 51.3$ & $183.9 \pm 43$ & $|98.25 \pm 4|$ & \\
\hline CRP & $2.16 \pm 1.8$ & $0.51 \pm 0.6$ & $0.61 \pm 0.9$ & \\
\hline \multicolumn{5}{|l|}{ Medications: } \\
\hline Aspirin & $21.8 \%$ & $33.3 \%$ & $62.2 \%$ & \\
\hline Plavix & 0 & 0 & 0 & \\
\hline Beta blockers & $4.3 \%$ & $17.6 \%$ & $20 \%$ & \\
\hline ACEI & $34.8 \%$ & $23.5 \%$ & $55.5 \%$ & \\
\hline ARB & 0 & 0 & $17.8 \%$ & \\
\hline Statin & $43.4 \%$ & $27.5 \%$ & $53.5 \%$ & \\
\hline Diuretics & $26.1 \%$ & $13.7 \%$ & - & \\
\hline \multicolumn{5}{|c|}{ Aortic wall characteristics: } \\
\hline \multicolumn{5}{|l|}{ Wall thickness (mm) } \\
\hline Mean & $3.173 \pm 0.2$ & $2.576 \pm 0.1$ & $2.305 \pm 0.13$ & \\
\hline Min-Max & $2.0-5.4$ & I.I-4.0 & I.2-4.2 & $<0.001$ \\
\hline \multicolumn{5}{|l|}{ Wall area $\left(\mathrm{mm}^{2}\right)$} \\
\hline Mean & $2.114 \pm 0.2$ & $1.526 \pm 0.06$ & $1.382 \pm 0.06$ & \\
\hline Min-Max & $1.17-4.3$ & $0.77-2.7$ & $0.70-1.99$ & $<0.001$ \\
\hline \multicolumn{5}{|l|}{ Lumen area $\left(\mathrm{mm}^{2}\right)$} \\
\hline Mean & $4.501 \pm 0.248$ & $4.3076 \pm 0.136$ & $4.006 \pm 0.136$ & \\
\hline Min-Max & $2.36-8.22$ & $2.69-8.2$ & $1.58-7.22$ & 0.136 \\
\hline
\end{tabular}

Data are mean (SD) or $\mathrm{n}$ (percentage).

double inversion recovery T-2 weighted spin echo sequence by CMR with computer software analysis. See Table I.

Results: The AWA and AWT were significantly greater in patients who presented to the ED with ACS (AWA-mean $2.28 \pm 0.78 \mathrm{~mm}^{2}$; AWT-mean $3.30 \pm 0.88 \mathrm{~mm}$ ) then both patients presenting with non-cardiac CP (AWA-mean $\mathrm{I} .52 \pm 0.39 \mathrm{~mm}^{2}, \mathrm{p}<0.0 \mathrm{I}$; AWT-mean $2.53 \pm 0.64 \mathrm{~mm}$, $P<0.01$ ) and the controls (AWA-mean $1.39 \pm 0.36 \mathrm{~mm}^{2}$, $\mathrm{p}<0.0 \mathrm{l}$; AWT-mean $2.36 \pm 0.80 \mathrm{~mm}, \mathrm{p}<0.0 \mathrm{l}$ ). There was no significant difference in the patients with non-cardiac $C P$ compared to the controls.

Conclusion: Aortic wall may be involved in the inflammatory process of patients presenting with ACS.
P257

Variability of perfusion dark rim artifacts due to Gibbs ringing

Pedro Ferreira , Peter Gatehouse ${ }^{2}$, Peter Kellman³, Chiara Bucciarelli-Ducci ${ }^{1}$ and David Firmin ${ }^{1}$

I Imperial College London, London, UK

${ }^{2}$ Royal Brompton Hospital, London, UK

${ }^{3}$ National Institutes of Health, Bethesda, MD, USA

Journal of Cardiovascular Magnetic Resonance 2009, I I(SuppI I):P257

Background and aims: Gibbs ringing is a well known source of Dark Rim Artifacts (DRA) in myocardial perfusion imaging [I]. We examine the variability of this artifact. Specifically, we show 
Figure I (abstract P257)

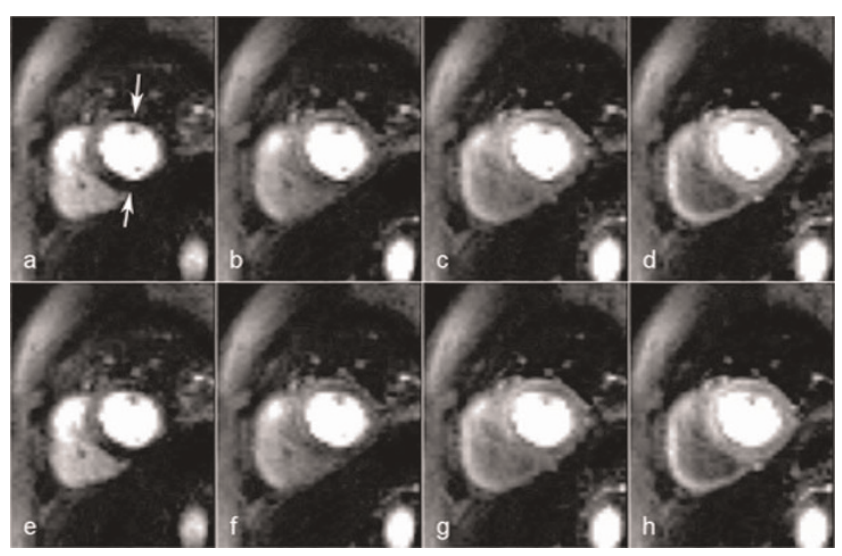

that Gibbs artifacts are highly dependent on the edge position and that sub-pixel shifts can dramatically change the appearance. Methods: Sub-pixel shifts were introduced in four in-vivo rawdata perfusion studies, where a DRA was visible. The shifts had a step of $1 / 8^{\text {th }}$ of a pixel ranging from 0.125 to 0.875 of the in-plane pixel size. The unprocessed raw-data was phase-shifted using MATLAB before reconstructing it on the scanner using the same image processing as the original data.

The original perfusion study was done on a I.5 T scanner (Avanto; Siemens): hybrid-EPI sequence with an EPI factor of 4; TR/TE of $5.1 / 1.7 \mathrm{~ms}$; base resolution 128 pixels; pixel size $2.8 \times 2.8 \times 8 \mathrm{~mm}$; flip angle $30^{\circ}$; bandwidth $1860 \mathrm{~Hz}$ pixel $^{-1}$; TI (time of inversion) of $90 \mathrm{~ms}$ using a non-selective BIR-4 saturation pulse, TSENSE with $R=2$. Perfusion was imaged during first pass of Gd-DTPA; stress induced by adenosine.

The original and shifted magnitude images were compared visually using CMRtools which applies sub-pixel interpolation in the image space.

The DRAs shown were carefully selected so as to not coincide with any known real perfusion defect.

Sub-pixel shifts in short-axis images were also simulated numerically in MATLAB. Images were also reconstructed with image based bicubic interpolation and zero filling with a factor of 4, and compared.

Results and discussion: Figure I shows 4 consecutive frames during the arrival of the CA into the myocardium of a particular patient, in the basal slice for two different sub-pixel shifts. The top row (a-d) shows DRAs in the anterior and inferior segments of the subendocardium (white arrows) for the shift where the artifacts were most prominent $(0.125$ shift). The bottom row (e-h) corresponds to the same frames, but with a half-pixel shift in the vertical direction in relation to the top row ( 0.625 shift). The DRAs in those regions are highly reduced when compared to the top row.

Figure 2a shows the numerically simulated short-axis image with Gibbs artifacts mainly in the septal, inferior, lateral and anterior regions of the subendocardium. After a diagonal shift of halfpixel, Figure $2 b$ shows a reduction of the artifacts in those regions. Figure $2 c-d$ and Figure $2 e-f$ are identical to Figure $2 a-b$, but with a bicubic image based interpolation and with zero-filling before FFT respectively. Post-FFT image interpolation shows the same dependency on the position of the edge as the non-
Figure 2 (abstract P257)
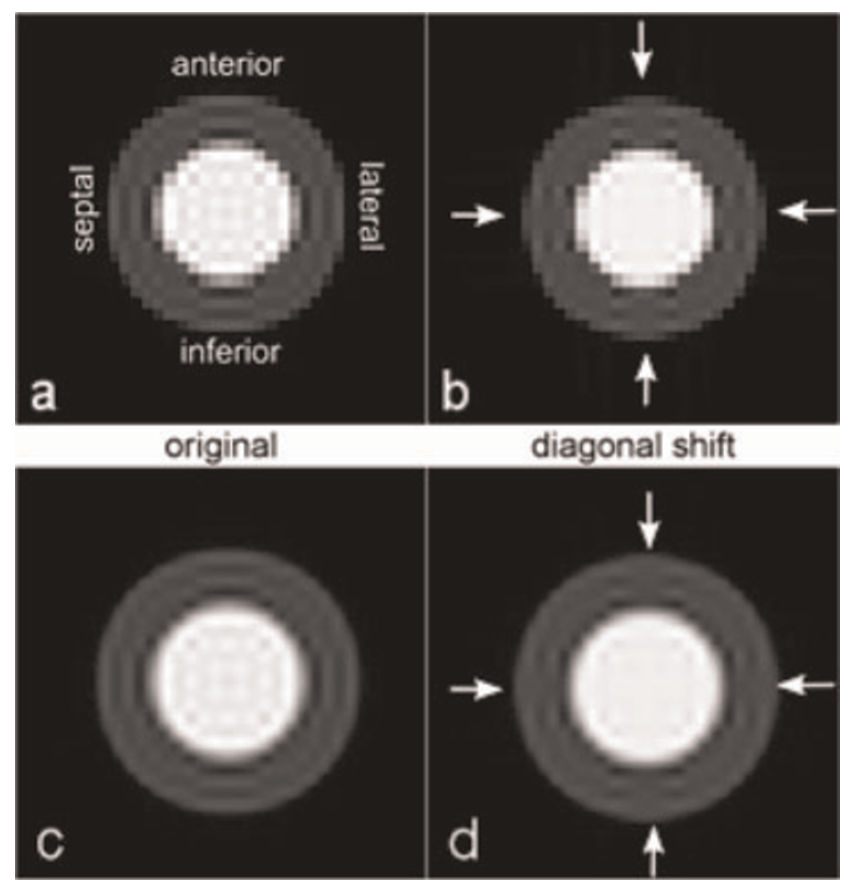

original interpolated

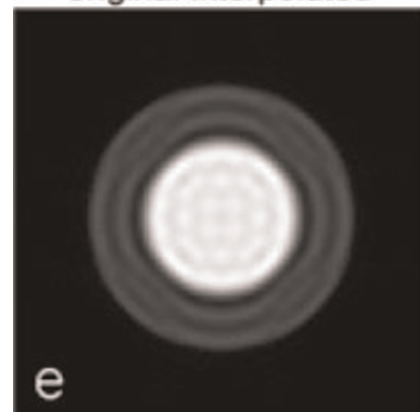

original zero filled

interpolated data. In contrast, the artifacts appear consistently without regard to the inplane offsets in the zero-filled data, in agreement with Du et al. [2].

Although the shift selected in Figure la-d was the worst-case, it should be recognised that this occurs by chance depending on image plane, in-plane offsets, and the patient's respiratory and cardiac motion. This is consistent with the random nature of DRA occurrence in clinical practice.

Conclusion: The visibility of Gibbs DRAs in perfusion studies is very dependent on the position of the subendocardial wall inside the pixel in the absence of zero-filled pre-FFT interpolation. Position variations from frame to frame in a typical gated perfusion study can explain some of the variability often seen in DRAs. Interpolation by zero-filling prior to enlarged FFT regularizes the DRA appearance.

\section{References}

I. Di Bella E, et al: MRM 2005, 54(5): 1295.

2. Du Y, et al: JMRI I994, 4(5):733. 
P258

Could Tnl level on admission predict function and infarct size in STEMI patient treated with $\mathrm{PPCl}$ CMR study

Dorota Piotrowska-Kownacka, Lukasz Kownacki, Grzegorz Opolski, Leszek Krolicki and Olgierd Rowinski Medical University of Warsaw, Warsaw, Poland

Journal of Cardiovascular Magnetic Resonance 2009, I I (SuppI I):P258

Introduction: Pain to balloon time could have significant error due to often imprecise pain onset time. We hypothesize that $\mathrm{Tnl}$ level measured on admission could be easy monitored, independent predictor of infarct size and left ventricle function Purpose: The purpose of the study was to evaluate which of the two factors better correlates with LV function and delayed enhancement volume after successfully treated first ST segment elevation myocardial infarction (STEMI).

Methods: 34 patients with first anterior STEMI who underwent successful primary PCI (TIMI III) were included into the study. Cardiovascular MRI was performed on I.5 T scanner at discharge, using 32-channel cardiac coil. Viability was evaluated on contrast-enhanced images acquired with the use of an inversion-recovery segmented gradient-echo sequence 20 minutes after contrast injection. Infarct size and LV function were analyzed quantitatively on AW workstation using MASS software. Results: Mean pain to balloon time was $3.8 \pm 2.6 \mathrm{~h}$ (ranged between I do $14 \mathrm{~h}$ ). Median Tnl level on admission was $0.6 \mathrm{ng} / \mathrm{ml}$ (ranged between 0.0 and $25.55 \mathrm{ng} / \mathrm{ml}$ ). Mean LVEF was $40.7 \% \pm 10.9$, scar size was $48 \mathrm{ml} \pm 23$ or $31 \% \pm 13.7$ as a percent of LV volume. Linear regression analyses revealed mild correlation between pain to balloon time and LV EF $(R=0.555$; $\mathrm{P}<0.00 \mathrm{I})$ and low but statistically significant with infarct size as a \% of LV volume $(R=0.34 ; p=0.048)$. No correlation was found between Tnl level on admission and infarct size or LVEF. Conclusion: Patients with longer pain to balloon time have worse LV EF at discharge.

Tnl level on admission could not be a predictor of infarct size or early ejection fraction.

\section{P259}

Comparison of Gadopentetate dimeglumine and Gadobenate dimeglumine in depiction of nonischemic fibrosis in hypertrophic cardiomyopathy Andre Rudolph, Hassan Abdel-Aty and

Jeanette Schulz-Menger

Franz-Volhard-Klinik, Kardiologie, Charité Campus Buch, Universitätsmedizin Berlin, Helios-Klinikum, Berlin, Germany

Journal of Cardiovascular Magnetic Resonance 2009, I I(SuppI I):P259

Background: There is a known link between myocardial fibrosis as defined by late gadolinium enhancement (LGE), severity of left ventricular remodeling and ventricular arrhythmias in hypertrophic cardiomyopathy (HCM). Noninvasive quantification of fibrosis may offer the possibility for risk stratification. Due to their often diffuse and blurred character these lesions remain difficult to differentiate from healthy myocardium in several cases.

Recent studies showed the superiority of Gadobenate dimeglumine (Gd-BOPTA) compared with Gadopentetate dimeglumine (Gd-DTPA) in distinguishing infarcted from viable myocardium which can be explained by the higher relaxivity of Gd-BOPTA. Therefore we hypothesized that Gd-BOPTA may have advantages over Gd-DTPA for depiction of non-ischemic fibrosis.

Methods: We prospectively enrolled eight Patients with clinically established HCM and positive LGE during clinical routine scan with $0.2 \mathrm{mmol} / \mathrm{kg}$ Gd-DTPA ( 0.5 molar $)$. They underwent a second scan at least 72 hours apart from the first CMR exam with $0.2 \mathrm{mmol} / \mathrm{kg}$ Gd-BOPTA ( $0.5 \mathrm{molar})$ using the same CMR protocol and sequence parameters. None of the patients had renal failure, coronary artery disease or general contraindications for CMR.

LGE was assessed in a short axis stack acquired 15 minutes after contrast administration by using state of the art inversion recovery gradient echo sequence (slicethickness $6 \mathrm{~mm}$, no gap, TE $5.0 \mathrm{~ms}$, FA $30^{\circ}$, matrix $256 \times 192$ ) with a Tl adjusted to null signal from normal myocardium. Positive LGE was judged positive if signal intensity was above mean +2 standard deviations of remote myocardium.

Signal intensities of injured myocardium, healthy myocardium, LV cavity and air were measured in identical locations by using anatomical landmarks in dedicated software (CMR42, circle international). Signal to noise ratio (SNR) and contrast to noise ratio (CNR) were calculated.

Results: We observed no complications relating to contrast administration. Both the SNR of injured myocardium (39.1 \pm 15.9 vs. $57.7 \pm 25.2, p=0.02)$ and the CNR between healthy and injured myocardium $(37.2 \pm 16.3$ vs. $53.0 \pm 23.5$, $P=0.021$ ) were significantly higher with Gd-BOPTA. SNR of LV cavity was significantly higher $(56.9 \pm 24.3$ vs. II $9.0 \pm 5.8$, $\mathrm{P}=0.002$ ) with Gd-BOPTA resulting in a poorer contrast between injured myocardium and blood which did not affect the diagnosis since LGE was exclusively non-subendocardial.

Conclusion: Gd-BOPTA improves the visualization of nonischemic fibrosis in HCM. This may offer the potential for a more accurate quantification of focal myocardial fibrosis in this setting.

\section{P260}

Value of late gadolinium enhancement by magnetic resonance in patients with cardiac sarcoidosis: characteristic findings and clinical utility

Eri Watanabe', Fumiko Kimura ${ }^{2}$, Michiaki Hiroe ${ }^{3}$, Takatomo Nakajima ${ }^{4}$, Ayako Nakamura ${ }^{4}$, Noriko Nishii ${ }^{4}$, Masatoshi Kawana ${ }^{5}$ and Nobuhisa Hagiwara ${ }^{4}$

'Institute of Geriatrics, Tokyo Women's Medical University, Shibuya-ku, Tokyo, Japan

${ }^{2}$ Saitama Medical University International Medical Center, Saitama, Japan

3 International Medical Center of Japan, Tokyo, Japan

${ }^{4}$ Tokyo Women's Medical University, Tokyo, Japan

${ }^{5}$ Tokyo Women's Medical University, Aoyama Hospital, Tokyo, Japan

Journal of Cardiovascular Magnetic Resonance 2009, I I(SuppI I):P260

Introduction: Although cardiac involvement is an important prognostic factor in patients with sarcoidosis, cardiac sarcoidosis (CS) is often difficult to diagnose. Cardiac magnetic resonance (CMR) imaging with late gadolinium enhancement (LGE) is considered to be useful in identifying CS early.

Purpose: We investigated LGE characteristics in patients with CS. Methods: The study included 17 patients who were diagnosed with CS by Japanese Ministry of Health and Welfare criteria and 
underwent CMR. Among the 17, 15 had LGE and were evaluated retrospectively. We obtained LGE images (short-axis, vertical longaxis, and 4-chamber views) using an inversion recovery segmented gradient-echo sequence 10 minutes after we administered gadolinium-DTPA $(0.15 \mathrm{mmol} / \mathrm{Kg}$ or $20 \mathrm{~mL})$. We analyzed patterns (patchy or band-like appearance) and locations of LGE in LV using a 17-segment model. We also assessed the extent of LGE in each segment of LV (subepicardial, midwall, subendocardial and transmural). We evaluated relationships between LGE characteristics and LV function using cine MR images.

Results: We observed band-like LGE with distinct margin in 14 patients and patchy LGE in one. The band-like pattern correlated well with pathological findings in biopsy-proven CS. Transmural LGE (T-LGE) was observed in 7 patients. All patients with T-LGE also had subepicardial lesion. In the patients without T-LGE, LGE was most common in the subepicardial layer ( $74 \%$ of enhanced segments) and most frequently observed in the basal septal wall, especially on the RV side. The number of LGE segments of the patients with T-LGE was significantly larger than that of patients without T-LGE (I $4.0 \pm 2.0$ versus $4.3 \pm 3.3$ segments, $P<0.0001)$. The ejection fraction of the LV (LVEF) of the patients with T-LGE was significantly lower than that of patients without T-LGE $(19.0 \pm 5.9 \%$ versus $50.8 \pm 6.5 \%, p<0.000 I)$, and the end-diastolic volume of the LV of the patients with T-LGE was significantly larger than that of patients without T-LGE $(236.4 \pm 50.6$ versus $96.5 \pm 9.7 \mathrm{~mL}, \mathrm{P}<0.000 \mathrm{I})$.

Conclusion: Characteristic LGE pattern and location allow accurate diagnosis of CS. CMR with LGE is useful for diagnosing CS and predicting LV function.

\section{P26I}

Imaging of aortic coarctation using Gd-DTPA and Gadofosveset: a comparative study

Gerald F Greil', Marcus R Makowski', Andrea J Wiethoff', Vicky Parish', Sergio Uribe', Christian Jansen ', Aaron Bell', Phillip Beerbaum', Martin Rohrer', Rene M Botnar', Reza Razavi ' and Tobias Schaeffter'

'Imaging Sciences Division, King's College London, St Thomas' Hospital, London, UK

${ }^{2}$ European Business Unit Diagnostic Imaging, Bayer Schering Pharma AG, Berlin, Germany

Journal of Cardiovascular Magnetic Resonance 2009, I I(SuppI I):P26I

Objective: The use of Gadofosveset in combination with a 32 channel coil and optimized image sequences allows high
Figure I (abstract P26I)

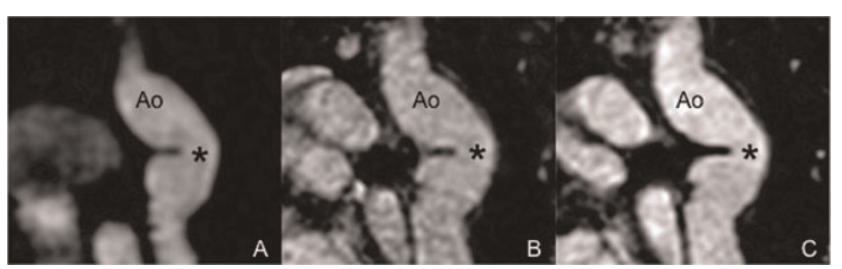

resolution free breathing and ECG triggered imaging of the aortic arch in patients with coarctation with improved imaging results compared to previous techniques.

Background: First-pass breath-hold non-ECG-triggered 3D contrast-enhanced-magnetic-resonance-angiography (CEMRA) using Gd-DTPA is commonly used for assessment of the aortic arch. However, image resolution is limited due to time constraints and vascular borders are blurred due to vascular motion and insufficient breath holds.

Methods: In 7 patients ( $30 \pm 7$ yrs) the aortic arch was imaged after surgical repair $(n=6)$ or stent implantation $(n=I)$ on a I.5 $\mathrm{T}$ clinical scanner (Philips Medical Systems). Patients were investigated twice within 7 days using Gd-DTPA (day I, 0.10$0.17 \mathrm{mmol} / \mathrm{kg}$ ) and Gadofosveset (day 2, $0.03 \mathrm{mmol} / \mathrm{kg}$ ). First pass breath hold 3D CEMRA as well as a respiratory navigator gated and end-diastolic ECG triggered 3D steady-state free precession (SSFP) sequence with a T2 prepulse were used. Gadofosveset allowed the application of an inversion recovery (IR) prepulse to suppress surrounding tissue signal. Results were compared (Table I).

Results: The navigator gated and ECG triggered 3D IR SSFP (Figure IC) sequence showed best image quality results (Table I). Cross sectional areas showed good interstudy agreement in the 3D SSFP technique without IR (Figure IB) and 3D first pass CEMRA (Figure IA) with similar image quality results using Gadofosveset and Gd-DTPA. However, these areas are smaller in end-diastolic ECG triggered respiratory gated sequences with and without IR prepulse than in breath hold 3D CEMRA (Table I, all $p<0.05$ ). Stent artifacts were similar in all sequences.

Conclusion: A respiratory-navigator-gated and ECG-triggered 3D-IR-SSFP-sequence after application of Gadofosveset allows free-breathing end-diastolic high-resolution imaging of the aortic arch in combination with a 32-channel-coil. Image

Table I (abstract P26I) Values are expressed as mean \pm standard deviation

\begin{tabular}{lllllll}
\hline $\begin{array}{l}\text { Contrast } \\
\text { Agent }\end{array}$ & Sequence & $\begin{array}{l}\text { Contrast to } \\
\text { Noise Ratio (CNR) }\end{array}$ & $\begin{array}{l}\text { Vessel Wall } \\
\text { sharpness (\%) }\end{array}$ & $\begin{array}{l}\text { Image quality } \\
\text { (mean } \pm \text { SD) }\end{array}$ & Vessel Area $\left.\mathbf{( c m}^{2}\right)$ & $\begin{array}{l}\text { Isotropic spatial } \\
\text { resolution (mm }\end{array}$ \\
\hline Gd-DTPA & CEMRA & $110 \pm 10^{\dagger}$ & $41 \pm 4^{\dagger}$ & $2.4 \pm 0.8^{\dagger}$ & $4.6 \pm 1.9^{\dagger}$ & 1.77 \\
& SSFP & $135 \pm 11^{*}$ & $48 \pm 6^{*}$ & $3.3 \pm 0.5^{*}$ & $4.1 \pm 1.7^{*}$ & 1.49 \\
Gadofosveset & CEMRA & $99 \pm 21^{\dagger}$ & $40 \pm 4^{\dagger}$ & $2.7 \pm 0.5^{\dagger}$ & $4.7 \pm 2.1^{\dagger}$ & 1.77 \\
& SSFP & $128 \pm 19^{*}$ & $46 \pm 3^{*}$ & $3.1 \pm 0.7^{*}$ & $4.1 \pm 1.6^{*}$ & 1.49 \\
& SSFP+IR & $154 \pm 14$ & $53 \pm 5$ & $3.7 \pm 0.5$ & $3.9 \pm 1.7$ & 1.49 \\
\hline
\end{tabular}

†,*, no significant differences between corresponding sequences (Gd-DTPA vs Gadofosveset). Image quality: I = non diagnostic, $2=$ diagnostic, 3 = good, 4 = excellent. 
quality is superior with slightly smaller cross sectional areas compared to first-pass CE-MRA.

\section{P262}

Aortopulmonary collaterals in single ventricle
patients
Lars Grosse-Wortmann and Shi-Joon Yoo
The Hospital for Sick Children, Toronto, ON, Canada

Journal of Cardiovascular Magnetic Resonance 2009, I I(SuppI I):P262

Introduction: Aortopulmonary collaterals (APCs) are frequent prior to and following the Fontan operation. APCs have been reported to be associated with prolonged pleural effusions and increased mortality after the Fontan operation. The degree of collateral formation is difficult to measure. A semiquantitative assessment via fluoroscopic angiography is dependent on the amount and location of contrast medium injected.

Purpose: To i) quantify APC flow in patients after bidirectional cavopulmonary connections (BCPC) and Fontan operations, using phase contrast magnetic resonance imaging (MRI), ii) assess the accuracy of flow measurements in these patients, iii) identify risk factors for the development of APCs.

Methods: $24 \mathrm{MRI}$ studies on 16 patients were analyzed retrospectively. The imaging protocol included phase contrast flow velocity mapping of the superior vena cava (SVC), right and left pulmonary arteries, all individual pulmonary veins, ascending (AAO) and descending aorta (DAO) and in the azygos/hemiazygos venous system. The patients were stratified into 2 groups: A) before and B) after their Fontan completion. APC blood flow was calculated by subtracting the volume of blood flow in the pulmonary arteries from that in the pulmonary veins.

Results: $Q p / Q$ s was $0.93 \pm 0.26$ in group $A$ and $1.27 \pm 0.16$ in group B. In groups A and B $53.3 \% \pm 16.8$ and $27.5 \% \pm 15.1$, respectively, of total pulmonary blood flow was provided by APCs. The mean inaccuracies, comparing DAO and pulmonary venous flows to that in the $A A O$ in group $A$ and the sum of SVC, DAO and collateral flows to that in the $A A O$ in group $B$, were $0.26 \pm 0.48 \mathrm{l} / \mathrm{min} / \mathrm{m}^{2}$ (corresponding to $7.9 \pm 14.5 \%$ of $A A O$ flow) and $0.18 \pm 0.43 \mathrm{l} / \mathrm{min} / \mathrm{m}^{2}$ (corresponding to $7.1 \pm 13.6 \%$ of AAO flow), respectively. For both groups combined, the degree of left to right shunting showed a positive correlation with the arterial oxygen saturations $(r=0.73, p<0.0001)$. The percentage of flow via APCs within the total pulmonary blood flow was negatively correlated with the age at the time of the BCPC ( $r=$ $-0.47, p=0.02$ ). For group $A, Q p / Q s$ and collateral flow were both correlated with a higher oxygen saturation $(r=0.59$, $p=0.02$ and $r=0.49, p=0.05$, respectively). In group $B, Q p / Q s$ correlated with the age at the time of the Fontan completion $(r=0.8 \mathrm{I}, \mathrm{p}=0.0 \mathrm{I})$.

Conclusion: APC blood flow can be quantitatively measured non-invasively, using MRI, with sufficient accuracy. The goal of volume unloading the single ventricle by creating a cavopulmonary anastomosis is counteracted by APC flow. Progression to the BCPC at a younger age is leads to a greater degree of aortopulmonary collateral artery development.
P263

Automatic segmentation of multi-echo Cardiac Magnetic Resonance images Gilion Hautvast', Yansong Zhao', Marc Kouwenhoven' and Marcel Breeuwer'

'Philips Healthcare, Best, Netherlands

${ }^{2}$ Philips Healthcare, Cleveland, $\mathrm{OH}$, USA

Journal of Cardiovascular Magnetic Resonance 2009, I I(SuppI I):P263

Introduction: Patients with thalassaemia major develop iron overloading due to regularly required blood transfusions, which is treated with iron chelation therapy. To monitor the effect of this treatment the hepatic and cardiac myocardial iron content is monitored on a regular basis. Clinical management guidelines for these patients include a yearly Cardiac Magnetic Resonance (CMR) exam [I], containing a multi-echo scan to enable T2* quantification for iron load assessment. The introduction of $\mathrm{T} 2 * \mathrm{CMR}$, alongside other improvements in clinical care, has improved survival in patients with thalassaemia major significantly [2].

Quantification of the myocardial T2* from multi-echo CMR images can be performed by fitting an exponential decay curve to the signal obtained by averaging image intensities within a region of interest in the myocardial septum in all echos [3].

Purpose: The purpose of our work is to simplify the analysis of multi-echo CMR images by developing and technically validating an automatic delineation method for the left ventricular contours in multi-echo CMR images.

Methods: The starting point for our new method was our earlier-developed method for cine CMR images [4]. Similarly to that method, our new method starts by locating the LV contours by detecting ring structures, followed by a greedy optimization of a deformable template [5] in a coarse-to-fine approach to obtain accurate myocardial contours. The new method assumes a bright myocardium and a dark blood pool. Furthermore, contour detection is performed at the first echo only, after which the contours are copied to all other echos.

We have used 28 black-blood multi-echo CMR scans from 10 patients. Each scan consisted of 4-8 echos at TE I.3-17 ms and the isotropic image resolution was $0.94-1.45 \mathrm{~mm}$. We are grateful to Children's Hospital Boston and Bioiatriki, Athens for providing us with clinical image data. Golden standard contours were defined manually on all images.

Figure I (abstract P263)

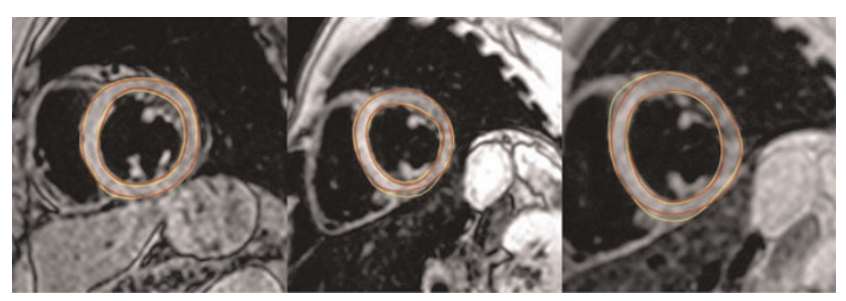

Examples of resulting LV contours (red) and the golden standard (yellow). 
Figure 2 (abstract P263)

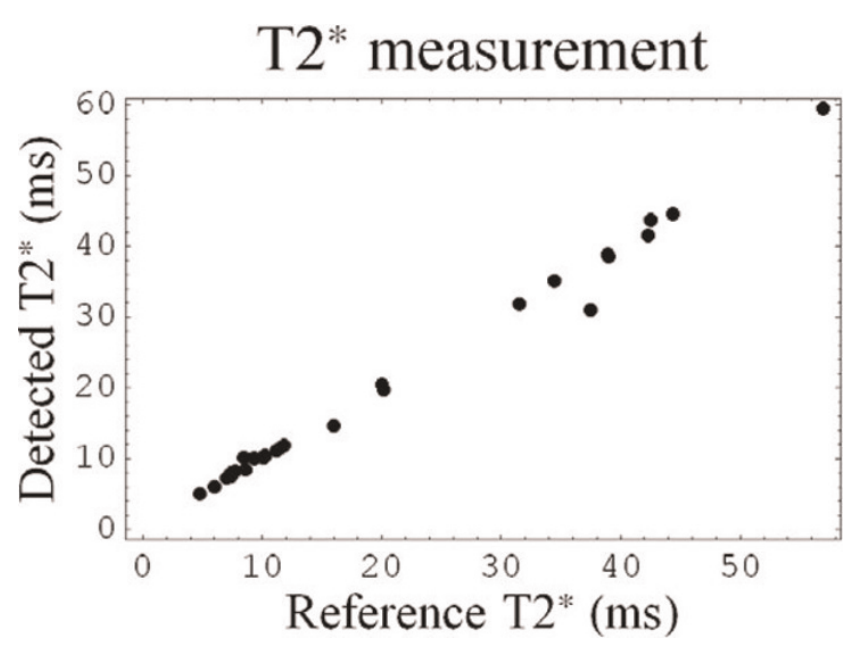

T2* from detected contours versus T2* from manual contours.

The technical validation of our method includes an a assessment of the contour quality and the effect on the $\mathrm{T} 2 *$ value. Contour quality was assessed by measuring Root-MeanSquare (RMS) contour positioning errors between the golden standard and detected contours. The effect on the T2* value was assessed by calculating the mean absolute difference and correlation coefficient between $\mathrm{T}^{*} *$ values obtained from the automatically detected contours and the golden standard contours.

To fit the exponential decay curve we have used the RatioLeast-Squares algorithm [6], in which a weighted fit is computed using decreasing weights for later echos. Especially in patients with severe iron overload, this is important as the image intensity may decrease below noise levels due to a short T2*.

Results: All images were delineated successfully (Figure I). We found RMS contour positioning errors of $1.42 \pm 0.6 \mathrm{I} \mathrm{mm}$ for the LV endocardium, and $1.36 \pm 0.96 \mathrm{~mm}$ for the LV epicardium. T2* values derived using automatically obtained contours differed on average $0.68 \mathrm{~ms}$ from T2* values derived from manually defined contours and correlated very well $\left(r^{2}=0.99\right)$ (Figure 2). Furthermore, the average deviation in $\mathrm{T} 2 *$ in iron overloaded patients $(\mathrm{T} 2 *<20 \mathrm{~ms})$ was even lower (0.36 ms).

Conclusion: We have developed and technically validated an automatic delineation algorithm for multi-echo CMR images. The resulting contours are accurate and allow for accurate T2* quantification.

References

I. "Guidelines for the Clinical Management of Thalassaemia". 2007, Thalassaemia International Federation; 2.

2. Modell B, et al: JCMR 2008, I 0:42.

3. Westwood M: “How I do Thalassaemia". SCMR Website 2008.

4. Hautvast GLTF, et al: Proc CARS 2008.

5. Amini AA, et al: IEEE TPAMI 1990, I2(9):855-867.

6. in den Kleef JJE, et al: MRM I987, 5(6):5।3-524.
P264

Ability of visual assessment of left ventricular dyssynchrony by cine-MRI to identify potential responders to cardiac resynchronization therapy Kai Muellerleile', Loant Baholli, ${ }^{1,2}$, Michael Groth', Katharina Koopmann', Achim Barmeyer', Ralf Koester', Gerhard Adam', Thomas Meinertz', Stephan Willems' and Gunnar Lund

'University Medical Center Hamburg-Eppendorf, Hamburg, Germany

${ }^{2}$ Center for Cardiology and Cardiovascular Surgery, Hamburg, Germany

Journal of Cardiovascular Magnetic Resonance 2009, I I(SuppI I):P264

Objective: To assess the ability of cine-MRI to identify potential responders to cardiac resynchronization therapy (CRT).

Methods: 40 patients with reduced ejection fraction $(28 \pm 11 \%)$, with $(n=21)$ or without $(n=18)$ left bundle branch block underwent cine-MRI and tissue-doppler imaging echocardiography (TDI). Time to peak systolic velocity (TPV) was measured by TDI for the septal, anterior, lateral and inferior left ventricle (LV). Time to peak contraction (TPC) was assessed visually for corresponding regions by cine-MRI. Left ventricular (LV) dyssynchrony was defined as the maximum delay of TPV- and TPC between opposing regions for TDI and cine-MRI, respectively. Correlation and agreement between TDI and cine-MRI were calculated. The ability of cine-MRI to identify patients with relevant echocardiographic LV dyssynchrony (defined as $>65 \mathrm{~ms}$ ) was assessed by ROC analysis.

Results: A strong correlation between LV dyssynchrony by TDI and cine-MRI was found $(r=0.84, P<0.000 I)$. Bland-Altman analysis showed a large difference of absolute values between both methods (mean difference $108 \pm 104 \mathrm{~ms}$ ). ROC analysis revealed an optimal cut-off value for cine-MRI of $>$ I $45 \mathrm{~ms}$ to identify patients with relevant echocardiographic LV dyssynchrony (sensitivity $89 \%$, specificity $95 \%$ ). The ability of cine-MRI to discriminate between patients with and without relevant echocardiographic LV dyssynchrony was excellent (area under the curve 0.98; $\mathrm{P}<0.000 \mathrm{I}$ ).

Conclusion: Visual assessment of LV dyssynchrony by cine-MRI can identify potential responders to CRT.

\section{P265}

Does the presence of $\mathbf{Q}$ waves on ECG indicate myocardial scar on cardiac MRI?

Gurjit Singh, Leonidas Tzogias, Mario Njeim, Milan V Pantelic, Khalid Nour and Mouaz H Al-Mallah Henry Ford Hospital, Detroit, MI, USA

Journal of Cardiovascular Magnetic Resonance 2009, I I(SuppI I):P265

Background: Pathologic $Q$ waves are the classic ECG sign of myocardial infarction. Prior studies have shown cardiac MRI is a more sensitive tool to detect myocardial necrosis. However, it is unclear whether pathologic $\mathrm{Q}$ waves on ECG always correlate with ischemic pattern of delayed enhancement. We tested the hypothesis that $\mathrm{Q}$ waves always indicate the presence of myocardial scaring on contrast enhanced cardiac Magnetic resonance imaging.

Methods: We included 4 I (25\% females) consecutive patients with pathologic $\mathrm{Q}$ waves on ECG who underwent contrast 
enhanced cardiac MRI (I.5 T) for various clinical reasons. Imaging protocols included cine SSFP sequence and post double dose contrast delayed imaging. $Q$ waves were considered pathologic if the total $Q$ wave voltage is more than one third of the $Q R$ voltage. Left ventricular myocardial scar was evaluated qualitatively using the AHA recommended 17 segment model by 2 readers who were blinded to the clinical and ECG data.

Results: A total of 29/4I (69\%) patients had evidence of myocardial scar in coronary pattern, 18 (44\%) of which were transmural. The presence of a $\mathrm{Q}$ only pattern (absence of $\mathrm{R}$ or $\mathrm{S}$ waves) did not improve the accuracy of ECG in identifying myocardial delayed enhancement on MRI. However, $Q$ waves only modestly localized the scar. Anterior Q waves (64\%) have the best correlation with the presence of scar in the anterior segments with lateral and inferior being $41 \%$ and $40 \%$ respectively. In addition, the $\mathrm{Q}$ only pattern did not improve the localizing accuracy of ECG.

Conclusion: The presence of pathologic $Q$ waves on ECG is not always associated with the presence of prior myocardial infarction on cardiac MRI. In addition, $Q$ waves appear to modestly correlate with the location of the prior MI. Further studies should evaluate the potential causes of pathologic $Q$ waves in patients without prior myocardial infarction.

\section{P266}

Myocardial change with microvasculer obstruction after acute myocardial infarction

Taniguchi Yasuyo, Takeshi Ishimoto, Shinitchiro Yamada,

Sachiyo Iwata, Kazuo Mizutani, Akira Shimane,

Takatoshi Hayashi and Teishi Kajiya

Himeji Cardiovasculer Center, Himeji, Japan

Journal of Cardiovascular Magnetic Resonance 2009, I I(SuppI I):P266

Introduction: Microvascular obstruction detected by Cardiovascular Magnetic Resonance (CMR) has been linked to ventricular remodeling and adverse cardiovascular events in acute myocardial infarction (AMI).

Purpose: We analyzed how the changes of myocardium with MVO in human reperfused myocardium affect left ventricular function by using two imaging modalities, CMR and cardiac nuclear imaging.

Methods: We investigated 28 patients with anterior AMI in whom primary percutaneous coronary intervention was performed successfully (mean age : 63.4 +- 8.4 year old, female $14.3 \%$ ). From nuclear imaging, ejection fraction (LVEF), left ventricular volumes, and summed defect score (SDS) 2 weeks and 3 months post-Ml were obtained by using rest gated

Table I (abstract P266) Cardiac performance

\begin{tabular}{llllll}
\hline & group M & SD & Group nM & SD & P \\
\hline & & & & & \\
EDV early (ml) & 142 & 20 & 134 & 34 & $<0.01$ \\
ESV early $(\mathrm{ml})$ & $9 \mathrm{I}$ & 17 & 86 & $4 \mathrm{I}$ & n.s. \\
EF(\%) & 37 & 5 & 40 & 13 & n.s. \\
Follow up EDV $(\mathrm{ml})$ & 149 & 30 & 128 & 47 & $<0.01$ \\
Follow up ESV $(\mathrm{ml})$ & 86 & 24 & 70 & 42 & $<0.01$ \\
EF(\%) & 44 & 7 & 46 & 10 & n.s. \\
SDS & 33 & 10 & 20 & 14 & $<0.05$ \\
\end{tabular}

technetium-99 m tetrofosmin electrocardiography-gated single photon emission computed tomography. Gdlinium contrast enhancement CMR imaging was performed within 2 weeks after AMI using a I.5 T imaging unit. The patient's characteristics and cardiac parameters were compared between two groups: with MVO (group $M, n=18$ ) and without MVO (group $n M$, $\mathrm{n}=10)$.

Results: Both groups had similar baseline characteristics about coronary risk factors. In group M, peak CK was higher (7208 IU/ $L$ and $3929 \mathrm{IU} / \mathrm{L}, \mathrm{P}<0.0 \mathrm{I})$ and reperfusion time was shorter $(4.7 \mathrm{hr}$ and $3.7 \mathrm{hr}, \mathrm{P}<0.0 \mathrm{I})$ tnan group $\mathrm{nM}$. Left ventricular enddiastolic volume (LVEDV) ( $142 \mathrm{ml}$ and I $34 \mathrm{ml}, \mathrm{P}<0.0 \mathrm{I})$ and SDS (33 and 20, $P<0.05$ ) showed significantly larger in early phase of AMI in group $M$. Although LVEDV at three months after AMI kept larger value $(149 \mathrm{ml}$ and $128 \mathrm{ml}, \mathrm{p}<0.0 \mathrm{I})$ in group M, LVEF weren't different in each group. The improvement of LVEF was also remarkably larger in group $M(21 \%$ and $9 \%)$. Any medication such as beta blocking agents and angiotensin converting enzyme inhibitors didn't affect these results. See Table I.

Conclusion: Although MVO detected by CMR predict the ventricular remodeling even in successful coronary intervention, this adaptation is thought to increase cardiac output to some extent.

\section{P267}

Regional wall motion abnormalities at rest and stress in patients with end-stage renal disease diagnosed by cardiac magnetic resonance imaging Constanze Merten, Christoph Merbach, Vedat Schwenger, Martin Zeier, Hugo A Katus, Evangelos Giannitsis and Henning Steen

University Hospital Heidelberg, Heidelberg, Germany

Journal of Cardiovascular Magnetic Resonance 2009, I I (SuppI I):P267

Introduction: Patients with end-stage renal disease (ESRD) are known to be subject to a high cardiovascular morbidity and mortality mainly due to coronary artery disease (CAD). We sought to assess the prevalence of regional wall motion abnormalities (rWMA) by cardiac magnetic resonance imaging (cMRI) in asymptomatic patients with ESRD.

Methods: We examined 77 patients with ESRD awaiting kidney transplantation by cMRI using a clinical I.5 Tesla scanner (Philips Achieva). Steady-state free precession cine images were acquired at rest and during a dobutamine stress test with four dobutamine dose steps from 10 to $40 \mu \mathrm{g} / \mathrm{kg}$ body weight over 3 minutes each. Wall motion was assessed qualitatively by two experienced observers by consensus-reading according to the 17-segmentmodel. Each segment was classified as normokinetic, hyokinetic, dyskinetic or akinetic.

Results: Of the examined patients 28 were women (36\%), the mean age was 58 years. Diabetes was known in 32 patients (42\%), a previously diagnosed CAD in 32 patients (42\%). Arterial hypertension was present in 69 patients $(90 \%)$.

At rest, left ventricular rWMA were present in 38 patients (49\%) and 123 segments, respectively. 89 segments were classified as hypokinetic, 24 as akinetic and 10 segments as dyskinetic. In 7I patients a dobutamine stress test with sufficient image quality could be performed, of those 30 patients (42\%) displayed rWMA. 63 segments with rWMA were identified at stress thereof 50 with hypokinesia, 12 with akinesia and I dyskinetic segment. 
Among patients with rWMA at rest, 12 showed a normalisation of contractility at stress, in 18 patients WMA remained unchanged during dobutamine stimulation, further 2 patients had worsening WMA at stress. In the remaining 6 patients a stress test could not be performed due to claustrophobic reactions or resulted in insufficient image quality.

In 6 patients (8.5\%) a positive stress test was observed. 4 of those patients had normal contractility at rest, in 2 patients pre-existing rWMA worsened under dobutamine stimulation. 3 patients with a positive stress test were diabetic, 2 patients had previously known CAD.

At rest and stress, the prevalence of rWMA was not different in patients with and without diabetes mellitus (47 vs. $51 \%, p=N S$ at rest and 45 vs. $40 \%$ at stress, $p=N S$ ). The presence of arterial hypertension and the myocardial mass equally had no influence of the prevalence of WMA. However, we observed significantly more $r W M A$ in patients with known CAD than in patients with a history of CAD, as well at rest (66 vs. $38 \%, p=0.016)$ as at stress (59 vs. $31 \%, p=0.02)$.

Conclusion: rWMA are e frequent finding in patients with ESRD. Patients with a known CAD have a significantly higher prevalence of rWMA. However, rWMA were also observed in about a third of patients without a history of CAD. Risk factors such as diabetes mellitus or arterial hypertension did not influence the prevalence of rWMA.

CMRI is a useful and non-invasive method to identify patients with cardiac morbidity in the high risk population of patients with ESRD.

\section{P268}

Evolution of left ventricular strain after a first time myocardial infarction. A study using velocity encoded magnetic resonance imaging

Ulrika Pahlm-Webb, Einar Heiberg, Erik Hedström and Hakan Arheden

Department of Clincial Physiology, Lund, Sweden

Journal of Cardiovascular Magnetic Resonance 2009, I I(SuppI I):P268

Purpose: To compare the evolution of regional myocardial strain in an area of myocardial infarction to remote myocardium in patients with first time myocardial infarction.

Introduction: After a myocardial infarction there is loss of function in the left ventricular region affected by ischemia. With time and treatment the function could improve. How fast the regional function improves is debated. Therefore we studied the evolution of left ventricular strain measured with velocity encoded MRI in the injured myocardium and compared it to strain in remote myocardium.

Methods: Velocity-encoded magnetic resonance images (VE$\mathrm{MRI}$ ) were acquired in 8 patients (median age: 69 years, range: 4I-74) with acute myocardial infarction and were followed with subsequent VE-MRI after I and 6 weeks, 6 and 12 months. The myocardium was manually delineated in the first time-frame and strain was automatically calculated using in house developed software http://segment.heiberg.se. Delayed enhancement images were used to determine the site of the myocardial injury. The first MRI was used as an internal control for each patient to be able to track change of regional strain over time. Strain in the injured myocardium was compared to remote myocardium over 12 months. A student t-test was used to test for significant change.
Figure I (abstract P268)

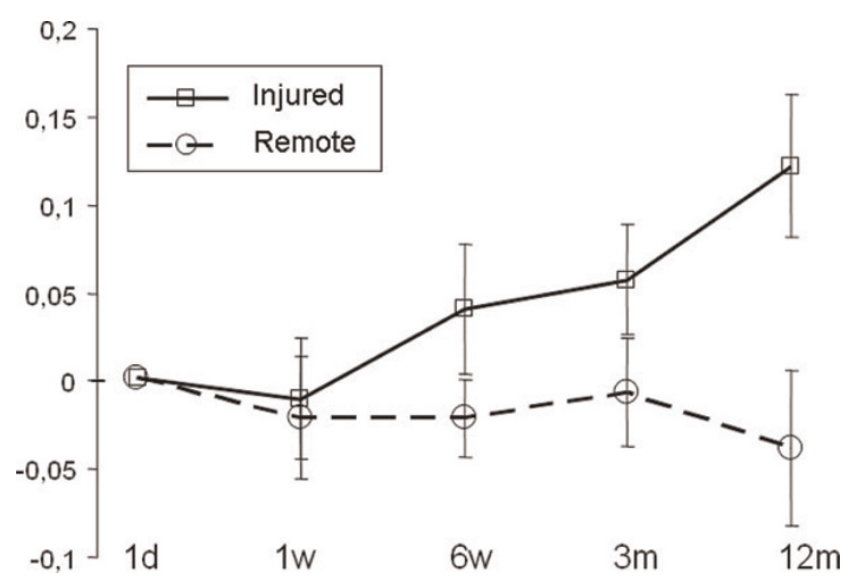

Change of strain over time at one day, one week, six weeks, three months and one year, for injured and remote myocardium, respectively.

Results: Figure I shows change of strain over time at one day, one week, six weeks, three months and one year, for injured and remote myocardium, respectively. Regional strain in the injured region increases over 12 month $(p<0.05)$ while strain in remote myocardium decreases $(p<0.05)$.

Conclusion: Regional strain in the injured region increases over 12 month while strain in remote myocardium decreases. This study shows a statistical difference in the change of strain 12 months after the infarct.

P269

Contrast-enhanced cine cardiac MR detects impairment of coronary microvascular perfusion in patients after acute myocardial infarct

Ricardo Loureiro', Hiram G Bezerra', Joalbo M Andrade², Chun-Ho Yun', Ammar Sarwar', Michael Bolen', Thomas J Brady' and Ricardo C Cury'

'Massachusetts General Hospital - Harvard Medical School, Boston, MA, USA

${ }^{2}$ Department of Radiology - Universidade de Brasilia, Brasilia, Brazil

Journal of Cardiovascular Magnetic Resonance 2009, I I(SuppI I):P269

Purpose: To prospectively compare contrast-enhanced (CE) cine steady-state free precession (SSFP) magnetic resonance imaging (MRI) to assess microvascular obstruction (MVO) with delayed-enhancement (DE) MRI, and to evaluate the association between MVO size and angiographic perfusion scores after primary percutaneous coronary angioplasty.

Materials and methods: The study was HIPAA compliant and was approved by the institutional review board. All participants gave written informed consent. Twenty one patients (I 9 men; mean age, $59 \pm 12$ years) were examined with contrastenhanced MRI after presentation and repeated at 6 months in I5 patients. TIMI Flow Grade (TFG), corrected TIMI Frame Count (cTFC), TIMI Myocardial Perfusion grade (TMPG), MVO, infarct size, and left ventricular ejection fraction (EF) were assessed. 
Results: MVO zones of decreased signal intensity were observed in 18 of 21 (86\%) examinations on CE cineSSFP MR imaging and I $7 / 2 \mathrm{I}(80 \%)$ examinations on DE MRI (P = NS). The size of CE cineSSFP hypointense zone was comparable to the MVO size measured by DE MRI $(3.3 \pm 3.1 \%$ versus $2.9 \pm 3.0 \%$ of the myocardial mass; $P=N S)$. MVO size correlated well with infarct size ( $r=0.76$ for CE cineSSFP and $r=0.83$ for DE MRI) and EF at follow-up ( $r=-0.64$ for CE cineSSFP and $r=-0.58$ for CE MRI). TFG 3 was present following percutaneous coronary intervention $(\mathrm{PCl})$ in $20 / 2 \mathrm{I}(95 \%)$ patients. Abnormal post-PCl TMPG $(0 / I / 2)$ was present in $1 \mathrm{I} / 2 \mathrm{I}(50 \%)$ of subjects and was associated with MVO size on CE cineSSFP $(3.6 \pm 2.2 \%$ with abnormal TMPG versus $0.8 \pm 0.7 \%$ of the myocardial mass (LV) with TMPG 3; $P<0.05)$ and DE MRI $(4.4 \pm 2.6 \%$ with abnormal TMPG versus $1.3 \pm 1.3 \% \mathrm{LV}$ with TMPG 3; $P<0.05)$. There was no association between abnormal post-PCI TFC $(\geq 40)$ and MVO size on CE cineSSFP $(3.5 \pm 2.5 \%$ with abnormal TFC versus $\mathrm{I} .8 \pm 2.3 \% \mathrm{LV}$ with TFC $<40, \mathrm{P}=0.26)$ and DE MRI $(2.9 \pm 2.0 \%$ with abnormal TFC versus I.5 $\pm 2.3 \%$ LV with TFC $<40$, $\mathrm{P}=0.29)$. Abnormal post-PCI TMPG was also associated with greater relative infarct size $(18.8 \%$ vs. $9.6 \%, P=0.04)$. CEcineSSFP MR had a comparable contrast-to-noise ratio (CNR) to DE MRI for depicting MVO zones ( $P=N S$ ).

Conclusion: CE cineSSFP hypointense zone depicts the microvascular obstruction region in close agreement with the DE MRI approach, and correlates with infarct size and global LV function at follow-up. TMPG seems to translate better the microvascular integrity compared to cardiac MRI measures of MVO.

\section{P270}

Comparison of left ventricle ejection fraction by echocardiography and cardiac magnetic resonance imaging in day to day clinical practice Michael A Witcik', Meghana Raghavendra', Vicki L McHugh', Jacob D Gundrum ', Kara J Kallies', Tim T Tissue ${ }^{2}$, Marvin J Shear ${ }^{2}$ and Raju G Ailiani ${ }^{2}$ ${ }^{\prime} G u n d e r s e n$ Lutheran Medical Foundation, La Crosse, WI, USA ${ }^{2}$ Gundersen Lutheran Health System, La Crosse, WI, USA

Journal of Cardiovascular Magnetic Resonance 2009, I I(SuppI I):P270

Introduction: Left ventricle ejection fraction (LVEF) is the most commonly used marker of LV function. It generally does not change over a short period of time in the absence of a major cardiovascular event or medication change. Cardiac magnetic resonance imaging (CMRI) is considered gold standard for LVEF assessment, however echocardiography is more widely available and most commonly used tool to assess LVEF.

Purpose: To evaluate the differences in clinically relevant LVEF ranges as measured by echocardiography and CMRI and to assess the reproducibility of CMRI LVEF calculations of experienced CMRI physicians compared to minimally-trained medical residents.

Methods: One hundred consecutive patients who underwent both echocardiography and CMRI from 9/01/05 to 6/18/07 at a community hospital were retrospectively reviewed. Echocardiography LVEF was derived by standard techniques. CMRI LVEF measurements were derived by standard techniques, utilizing short axis stack of TRUFISP cine images over the entire LV volume with volumetric analysis on the ARGUS work station. LVEF cutoffs for major clinical decision making were defined as
$<30 \%$ (prophylactic ICD implantation) and $<50 \%$ (diagnosis of cardiomyopathy and discontinuation of cardiotoxic chemotherapy agents), and were the basis for comparisons. Mean time frame between the echocardiogram and CMRI was 15.8 days (range 14-49 days). CMRI LVEF calculated by two internal medicine residents blinded to patient information and original CMRI was compared with experienced CMRI physician calculated LVEF. Residents received limited training in selecting appropriate CMRI images and working with ARGUS workstation. We compared physician read echocardiography and CMRI LVEF, as well as physician read and resident read CMRI derived LVEF. Results: Physician read echocardiography and CMRI LVEF comparisons divided subjects into 4 groups. Group I had LVEF $<30 \%$ on echocardiography $(n=16)$. Of these, $8(50 \%)$ had corresponding LVEF by CMRI as $>30 \%$ with differences of $5-10 \%$ in I subject $(6 \%)$ and $>10 \%$ in 7 subjects $(44 \%)$. Group 2 had LVEF $<30 \%$ on CMRI $(n=9)$. Of these, I subject $(5 \%)$ had corresponding LVEF by echocardiography $>30 \%$, with a difference of $>10 \%$. Group 3 had LVEF $\geq 30$ to $<50 \%$ on echocardiography $(n=22)$. Of these, $10(45 \%)$ had corresponding LVEF by CMRI as $>50 \%$ with differences of $5-10 \%$ in 4 subjects $(18 \%)$ and $>10 \%$ in 6 subjects $(27 \%)$. Group 4 had LVEF $\geq 30$ to $<50 \%$ on CMRI $(n=27)$. Of these, 5 subjects $(18 \%)$ had corresponding LVEF by echocardiography as $>50 \%$ with differences of $0-10 \%$ in 2 subjects $(7 \%)$ and $>10 \%$ in 3 subjects (II\%). When LVEF was assessed as $<30 \%$ by either tool, the discordance was $36 \%$; when assessed as $\geq 30$ to $<50 \%$ by either tool, the discordance was $35 \%$.

Physician read and resident read CMRI LVEF comparisons divided subjects into 2 groups. Group A had physician calculated CMRI LVEF $<30 \%(n=9)$. Of these, $8(89 \%)$ had corresponding LVEF by resident calculated CMRI as $<30 \%$. The one subject with a divergent LVEF was over read by the resident by $10.4 \%$. Group $B$ had LVEF $\geq 30$ to $<50 \%$ on CMRI $(n=27)$. Of these, $17(63 \%)$ had corresponding LVEF by resident read CMRI as $\geq 30 \%$ to $<50 \%$ with differences of $<5 \%$ in 6 subjects $(22 \%)$ and $>5 \%$ in 4 subjects (15\%). Overall discordance between physician and resident calculated CMRI LVEF for $<30 \%$ was II\%. When LVEF was assessed as $\geq 30$ to $<50 \%$, the discordance was $37 \%(22 \%$ with a difference of $<5 \%$; $15 \%$ with a difference of $>5 \%$ ).

Conclusion: Prior to making major management decisions based on echocardiography derived LVEF in clinically relevant ranges, CMRI confirmation is strongly suggested, particularly in patients with suboptimal images. These results also suggest that the analysis of CMRI data to derive accurate LVEF can be performed without extensive training and is reproducible.

\section{P27I}

Delay enhancement cardiac MR imaging for assesment of myocardial involvement in patients with systemic lupus erythematosus and clinical suspect of myocarditis

Sandra Graciela Rosales Uvera, Leticia Castellanos Cainas, Jaime Galindo Uribe, Jorge Vazquez La Madrid, Jorge Oseguera Moguel and Martha Morelos Guzman National Institute of the Medical Science and Nutrition Salvador Zubiran, Mexico D.F., Mexico

Journal of Cardiovascular Magnetic Resonance 2009, I I(SuppI I):P27 I

Introduction: The systemic lupus erythematosus (SLE) is a multiorgan inflammatory autoimmune disease mainly affecting 
Figure I (abstract P27I)

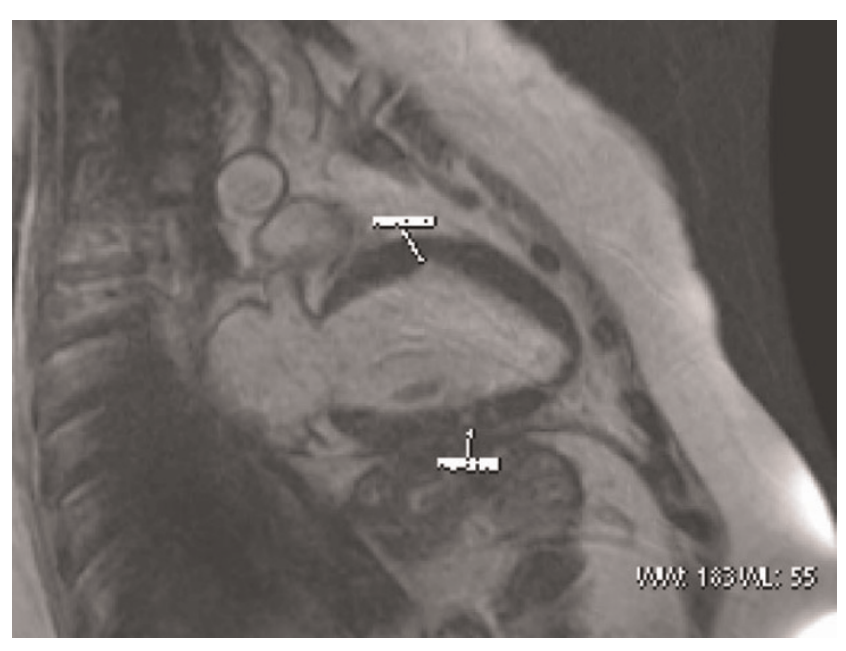

37 year old female with 3 year evolution of SLE and myocarditis, with precordial pain and dispnea, left ventricle is dilated, low ejection fraction and fibrosis with patchy pattern, other sings of damage were mitral and aortic regurgitation, biatrial enlargement and pericardial effusion.

women and is associated with high cardiovascular motality; myocarditis is a rare complication; however little knowledge about cardiac damage by myocarditis in patients with SLE. The cardiac magnetic resonance (CMR) is a useful tool for diagnosis of myocarditis and evaluate progression or regression of cardiac damage caused by SLE. See Figure I.

Purpose: The purpose of this preliminary study is to evaluate the structural and functional alterations in patients with SLE and clinical suspect of myocarditis.

Methods: 9 SLE consecutive patients (mean age 40.5 years), I man and 8 woman. Diagnosis followed the criteria of American Rheumatism Association; assessment of SLE activity was based on the European Consensus Lupus Activity Mesurement (ECLAM) Index. Underwent CMR on a GE I.5 tesla magnet for assessment of cardiac function, cine images used a balanced steady state free precession (FIESTA) technique with retrospective ECG triggering (TE = I.6 TR $=3.6$, flip angle $45=$, slice thickness $=8 \mathrm{~mm}$ ) short and long axis images were performed, matrix $224 \times 224$. Ten minutes after administration of $0.2 \mathrm{mmol} /$ $\mathrm{kg}$ of Gd-DTPA a $k$ space segmented 2D inversion-recovery gradient echo secuences (in-plane resolution $1.3 \times 1.3 \mathrm{~mm}^{2}$, slice thickeness $8 \mathrm{~mm}$ ) with complete coverage of the left ventricle (LV) myocardium in short axis slices and additional long axis view. Images were analyzed based on 17 segment model to evaluate segmental wall motions abnormalities.

Results: Mean LV end-diastolic volume $147 \mathrm{ml}$ and mean LV end-systolic volume $75 \mathrm{ml}$ with LV dilated in 2 patients, mean LV mass $109 \mathrm{gr}$, mean left ventricular ejection fraction was $5 \mathrm{I} .8 \%$, $77 \%$ have segmental wall motions abnormalities, $55 \%$ left and right atrium enlargement. Aortic valve alterations in $55 \%$, mitral valve involvement 100\%, pericardial effusion $66 \%$. Delay enhancement imaging revealed intramyocardial hyperenhancing lesions in the LV myocardium in 4 patients (44\%) without relationship with coronary artery territory.

Conclusion: Preliminary data suggest delayed enhancement CMR is avaible to detect cardiac damage caused by SLE myocarditis, besides CMR can identify valvular damage, atrial enlargement and pericardial alterations as early evolution of the disease.

\section{P272}

\section{Atrial size and function post orthotopic heart transplantation - CMR and ECHO study Nishant Kalra and Vincent L Sorrell} Sarver Heart Center, University of Arizona, Tucson, AZ, USA

Journal of Cardiovascular Magnetic Resonance 2009, I I(SuppI I):P272

Background: The conventional orthotopic heart transplantation (OHT) (biatrial) results in atrial enlargement. The variability of atrial size and function in these patients has not been previously reported.

Objectives: I. Determine the variability in the post-OHT size and function of left and right atria; 2. Analyze the relationship of donor and native atria; 3. Compare cardiac MRI (CMR) with transthoracic echocardiogram (TTE).

Methods: This is a retrospective observational study. Nine patients who had undergone OHT via biatrial anastomosis technique and had both CMR as well TTE within one month of each other were selected. Using the optimized apical 4-chamber views in both TTE and CMR cine images (SSFP), endocardial borders of the native and donor, right atrial (RA) and left atrial (LA) free wall and septum were traced from base to the valvular annulus and areas were determined in late systole when atrial volumes are maximal and late diastole when atrial volumes are minimal. RA and LA function was assessed quantitatively using percentage of change in cavity area or fractional area change (FAC). FAC was defined using the formula ([end-systolic area] [end-diastolic area])/[end-systolic area] $\times 100)$. Native and donor areas were also combined to calculate the total area of the right and left atria. Comparison was made between the TTE

Table I (abstract P272) Atrial Area (mean +/- SD) measured by CMR and TTE

\begin{tabular}{|c|c|c|c|c|}
\hline & CMR Systole & CMR Diastole & TTE Systole & TTE Diastole \\
\hline Total Left Atrial Area & $30.6+/-13.3$ & $27.7+/-13.8$ & $30.5+/-12.6$ & $22.2+1-11.1$ \\
\hline Total Right Atrial Area & $24.9+/-8.1$ & $23.6+/-7.7$ & $20.6+/-7.7$ & $18.5+/-7.5$ \\
\hline Donor Left Atrial Area & $11.9+1-3.7$ & $8.5+1-3.9$ & $|3.1+|-3.1$ & $8.3+1-2.8$ \\
\hline Donor Right Atrial Area & $12.4+\mid-3.1$ & $11.3+1-3.0$ & $11.8+1-3.4$ & $9.5+1-2.8$ \\
\hline Native Left Atrial Area & $18.7+1-10.1$ & $19.2+1-10.4$ & $17.4+/-9.8$ & $13.9+1-8.3$ \\
\hline Native Right Atrial Area & $12.5+/-5.8$ & $12.3+/-5.4$ & $8.8+/-5.1$ & $8.9+/ 4.8$ \\
\hline
\end{tabular}


Figure I (abstract P272)

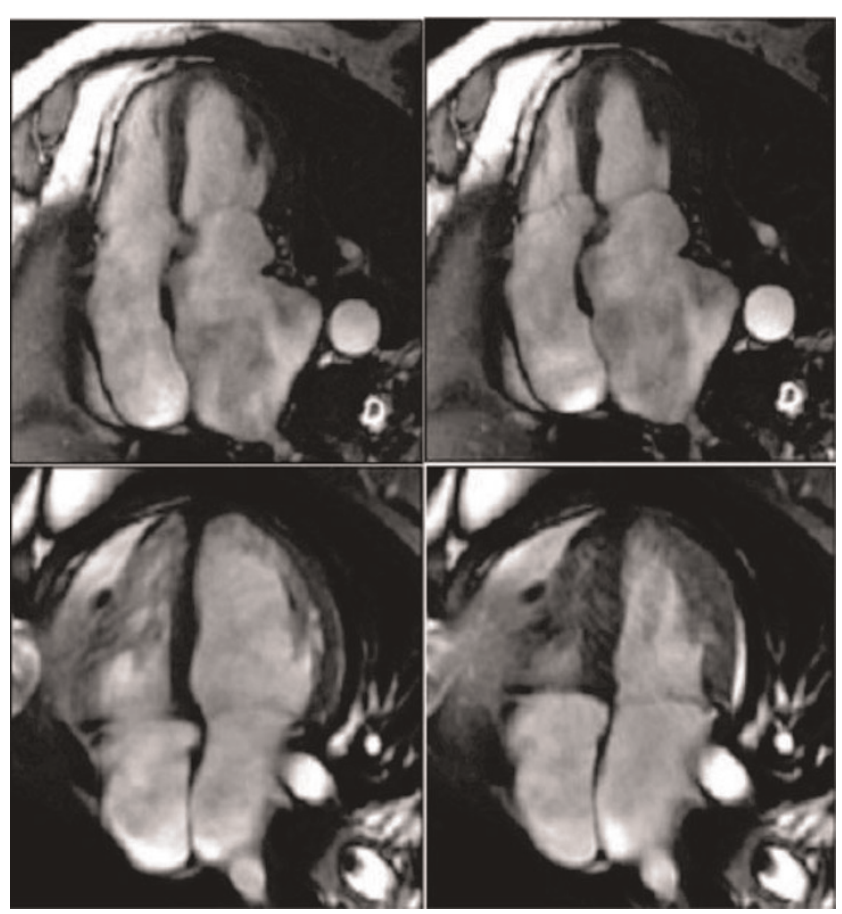

and CMR values. Data for continuous variables are expressed as mean value $+/-S D$.

Results: See Tables I and 2. TTE and CMR were performed within $6+9$ days of each other. The native atria (retained atrial cuff) are usually larger than the donor atria on the left and almost equal on right. The total LA area is usually > total RA area. 7 patients have LA total area $>24 \mathrm{~cm}^{2}$ and 4 of these patients also have RA total area $>24 \mathrm{~cm}^{2}$. TTE showed a good correlation with CMR, however within individual patients, there were both over- and under-estimation of atrial areas and TTE consistently underestimated native atrial areas. Native atrial function was consistently worse than donor atrial function. Overall total LA function was better than total RA function. TTE consistently overestimated the FAC compared to CMR in all except the native right atrial group.

Discussion: Left atrial volumes consistently predict outcome in most patient groups studied. LA size is frequently severely distorted after OHT and is dependent upon extent of retained pulmonary vein cuffs or associated donor lung preparation, individual cardiac surgeon preferences, and cardiac recipient

Table 2 (abstract P272) Fractional Area Change (mean +l- SD) measured by CMR and TTE

\begin{tabular}{lll}
\hline & CMR & TTE \\
\hline Total Left FAC & $10.3+/-8.9$ & $28.8+/ 12.2$ \\
Total Right FAC & $4.9+/-8.9$ & $10.6+/-7.8$ \\
Donor Left FAC & $29.7+/-12.8$ & $36.6+/-13.4$ \\
Donor Right FAC & $8.4+/-13.1$ & $17.6+/-16.4$ \\
Native Left FAC & $-3.9+/-13.0$ & $20.9+/-18.0$ \\
Native Right FAC & $0.6+/-10.1$ & $-8.2+/-25.1$ \\
\hline
\end{tabular}

organ status. Knowing the variations in atrial sizes may provide a new avenue for clinical investigation (Figure I). A CMR atrial area $<24 \mathrm{~cm}^{2}$ is considered normal, but rarely present in this postOHT patient subgroup.

It appears that the retained native atrium does not contribute to atrial contraction. This resulted in a negative total FAC\% in $45 \%$ (4/9) of our patients. Given the large retained native atria, and its poor contribution to atrial contraction, this report suggests that minimizing this atrial reservoir may be valuable and warrants additional study. The underestimation of native atrial size reported by TTE is most likely due to apical fore-shortening of the true long-axis and the far field ultrasound imaging.

Conclusion: This observational study suggests that despite a generally reasonable correlation between TTE and CMR measures of OHT atria, significant differences exist. Furthermore, because of suspected intra-atrial (donor versus native) mechanical dysynchrony, some patients surprisingly had a negative FAC\% suggesting that the native atria is larger during donor atrial contraction - acting as a non-functioning reservoir. The association of this to the clinical outcome has yet to be determined.

\section{P273}

Whole-body magnetic resonance angiography for the assessment of extent and severity of extra-cardiac atherosclerosis in patients with newly diagnosed coronary artery disease Stephanie Lehrke, Benjamin Egenlauf, Henning Steen, Constanze Merten, Dirk Lossnitzer, Evangelos Giannitsis and Hugo A Katus

Medizinische Universitätsklinik Heidelberg, Heidelberg, Germany

Journal of Cardiovascular Magnetic Resonance 2009, I I(SuppI I):P273

Introduction: The presence of extra- cardiac atherosclerosis is associated with an adverse prognosis in patients with coronary artery disease (CAD).

Purpose: We hypothesized that whole body magnetic resonance angiography (MRA) is a valuable tool for the non-invasive assessment of the severity of systemic atherosclerosis in patients (pts) with CAD. We further propose the use of an atherosclerosis score index as a novel marker for the severity of systemic atherosclerotic burden.

Methods: Whole-body MRA was performed on a I.5 T clinical scanner equipped with a moving table top extender (Intera Achieva ${ }^{\circledR}$, Philips Medical Systems) in 31 pts scheduled to undergo coronary angiography for suspected CAD. None of the pts had known extra-cardiac arterial disease. The arterial tree was divided into 40 segments from the carotid arteries to the arteries of the lower leg which were graded with regard to their luminal narrowing $(\leq 10 \%, \mid \mathrm{I}-25 \%, 26-50 \%, 5 \mathrm{I}-75 \%, 76-90 \%$, 91-99\%, occlusion). All segments received a score according to the severity of stenosis, ranging from I for a normal vessel to 8 for a total occlusion. The atherosclerosis score index (ASI) for each patient was calculated by dividing the sum of vessel scores by the number of analysed segments.

Results: Whole-body MRA was obtained in good quality from all pts and a total of 1088 arterial segments were analysed. Coronary angiography revealed significant CAD in 19/31 pts. These pts had a higher atherosclerosis score index (I.77 $\pm 0.1 \mathrm{I}$ 
vs $1.46 \pm 0.06, p=0.03)$ and exhibited more vessel segments with a significant $(>25 \%)$ stenosis $(2 \pm 0.48$ vs $0.83 \pm 0.34$, $p=0.05)$. ASI showed a good correlation with PROCAM $(R=0.49, P=0.0 I)$ and Framingham $(R=0.4, P=0.04)$ risk scores.

Conclusion: Our findings demonstrate that pts with newly diagnosed CAD exhibit a higher systemic atherosclerotic burden compared to pts in whom CAD was ruled out by coronary angiography. We are the first to describe the use of a whole body atherosclerosis score index as marker for systemic atherosclerotic burden which correlates well with established risk scores. This novel technique may aid in the risk-stratification in pts with suspected CAD and may aid in the evaluation of new therapies that target atherosclerotic disease.

\section{P274}

Partial anomalous pulmonary venous connection: how many vs. how much

Brian Grant, Shi-Joon Yoo, Mike T Seed and

Lars Grosse-Wortmann

Hospital for Sick Children, Toronto, ON, Canada

Journal of Cardiovascular Magnetic Resonance 2009, I I(SuppI I):P274

Introduction: The diagnosis of partial anomalous pulmonary venous connection (PAPVC) is usually made by echocardiography. However, complete anatomical delineation is often difficult. In addition, there has been controversy regarding which patients require surgical intervention. Magnetic resonance imaging (MRI) can provide comprehensive information on precise anatomy, systemic-to-pulmonary shunt (Qp:Qs) and ventricular volumes of patients with PAPVC.

Purpose: To (i) quantify the degree of left to right shunting, (ii) describe the distribution of blood flow to the right and the left lung, (iii) assess the relationship between the number of anomalously draining veins and $Q p: Q s$, (iv) assess the affect of other associated defects on Qp:Qs.

Methods: We retrospectively reviewed MRIs performed on patients with PAPVC in our institution over the last 5 years. All patients were assessed with cine imaging, contrast-enhanced angiography, and phase-contrast velocity mapping. The number of anomalous veins was recorded, and the proportion of blood flow from the anomalously draining vein relative to the total flow to that lung was measured. The proportion of anomalously drained lung volume relative to the total lung volume was estimated. Flow volumes indexed to body surface area (BSA) through the right and left pulmonary arteries and the ascending aorta were used for calculation of Qp:Qs. Ventricular end diastolic and end systolic volumes were measured and indexed to BSA.

Results: There were nineteen patients ( 13 male, mean age II.I \pm 5.8 years) with a diagnosis of PAPVC that had undergone an MRI study. Four patients were a new diagnosis made by MRI, and not detected by echocardiography. In a further 3 cases, echocardiographic suspicion was confirmed. Twelve patients had right PAPVC, 6 had PAPVC of the left upper pulmonary vein, and I patient had right-sided unilateral total anomalous pulmonary venous drainage. In 8 patients, there was an associated sinus venosus defect (SVASD). Mean Qp:Qs was $1.75 \pm 0.4$, and increased to $2.5 \pm 0.6$ in the presence of a SVASD. Qp:Qs correlated with right ventricular end diastolic volume index $(\rho=0.54 ; p=0.02)$ and the proportion of anomalous veins $(\rho=0.77 ; P=0.0 I)$. We found no increased differential pulmonary arterial blood flow to lungs with PAPVC from normal $(p=0.00 \mathrm{I})$. In individual cases, a high $Q p: Q s$ in the setting of a single anomalously draining pulmonary vein could be explained by angiographic assessment of the estimated volume of lung drained by the vein.

Conclusion: We believe that for the diagnosis of PAPVC, MRI not only allows a complete definition of the anatomy, especially in the setting of SVASD, it also details the ensuing hemodynamic consequences. Both often provide important additional information in the indication of surgery and procedure planning.

\section{P275}

Clinical implication of three-dimensional heart models for planning of surgical procedure in complex congenital heart disease

Eugenie Riesenkampff', Urte Rietdorf ${ }^{2,3}$, Ivo Wolf', Bernhard Schnackenburg ${ }^{4}$, Michael Hübler',

Vladimir Alexi-Meskishvili ${ }^{1}$, Hans-Peter Meinzer ${ }^{2}$,

Roland Hetzer', Felix Berger' ${ }^{\prime}$ and Titus Kuehne ${ }^{i}$

'German Heart Institute Berlin, Berlin, Germany

${ }^{2}$ German Cancer Research Center Heidelberg, Heidelberg, Germany

${ }^{3}$ Deutsches Krebsforschungszentrum Heidelberg, Heidelberg, Germany

${ }^{4}$ Philips Medical Systems, Hamburg, Germany

Journal of Cardiovascular Magnetic Resonance 2009, I I (SuppI I):P275

Background and aims: In patients with complex congenital heart diseases detailed three-dimensional information about intra- and extracardiac anatomy are essential for planning of surgical treatment strategies. However, it is a reality that current standard imaging techniques and their post-processing methods do not always provide the means to elucidate all required anatomic information in a comprehensive manner. We report our initial experiences with the use of realistic three-dimensional models of the heart in the clinical setting.

Methods: In II patients (age 0.7 - 27 years) with complex congenital heart disease, surgical questions were directed towards palliative or corrective surgery but consensus about the optimum treatment strategy was not reached using standard diagnostic tools including echocardiography, catheterization and conventional magnetic resonance imaging (MRI). In these patients, three-dimensional printed cast and virtual computer models of the heart were made on the basis of high-resolution whole-heart and/or cineMRI. Anatomic descriptions were compared with intraoperative findings when surgery was performed.

Results: MRI provided, independent from age related factors, sufficient image quality for producing the heart models. In all patients, the models clearly evidenced the anatomy in an unequivocal three-dimensional context. This was, as an adding to standard diagnostic imaging, invaluable information that supported surgical decision making. The anatomy as shown in the models was confirmed during surgery. Biventricular corrective surgery was planned in five patients, palliative surgery in three patients and in another 3 patients no option for surgery was seen.

Conclusion: Realistic three-dimensional models of the heart provide a new means for the assessment of complex anatomy. This method can facilitate surgical decision making and preoperative planning. 


\section{P276}

Visualizing regional myocardial oxygenation changes with statistically optimal colormaps Sotirios A Tsaftaris', Richard Tang', Rachel Klein², Debiao $\mathrm{Li}^{2}$ and Rohan Dharmakumar ${ }^{2}$

'Department of Electrical Engineering and Computer Science, Northwestern University, Evanston, IL, USA

${ }^{2}$ Department of Radiology, Northwestern University, Chicago, IL, USA

Journal of Cardiovascular Magnetic Resonance 2009, I I(SuppI I):P276

Introduction: Blood-oxygen-level dependent (BOLD) MRI may be used for detecting myocardial oxygenation (MO) changes secondary to coronary artery stenosis. Under pharmacological stress, areas in the myocardium supplied by a stenotic coronary artery are hypointense relative to healthy regions. Visualizing these changes requires manual windowing. In this paper a method for automatic visualization of myocardial signal changes reflecting the regional variations in oxygenation is presented, using images obtained from a canine study under controlled conditions. The objective of this study is to overcome the rather subjective step of windowing by establishing an optimal colormap that permits visualization of statistical changes in signal intensities between healthy and pathological cases.

Purpose: To facilitate the evaluation of myocardial BOLD images by automating the detection of regional abnormalities in $\mathrm{MO}$ under pharmacological stress in the presence of coronary artery stenosis.

Methods: Data acquisition: Breath-held and ECG-gated short-axis cardiac phase-resolved 2D SSFP-based myocardial
BOLD images were acquired in two dogs under pharmacological stress with and without left-circumflex coronary artery stenosis in a Siemens I.5 T scanner. Scan parameters: voxel size $=1.2 \times$ $1.2 \times 6 \mathrm{~mm}^{3}$; flip angle $=90^{\circ} ; \mathrm{TR} / \mathrm{TE}=5.7 / 2.9 \mathrm{~ms} ; 20$ cardiac phases.

Image processing algorithms: In order to have the greatest myocardial surface available for analysis, only the end systolic images of the two image series (adenosine without stenosis (AD) and adenosine with severe stenosis (SS)) were identified. A rectangular region of interest was chosen around the heart and a segmentation algorithm was utilized to isolate and segment the myocardium. The myocardial AD intensities (ADv) were collected and a student- $T$ distribution was fitted by maximum likelihood estimation of its parameters (mean $(\mathrm{m})$, variance (s), and degrees-of-freedom). An optimal pixel colormap was created by assigning red hues to signal values below the threshold $m$-s and yellow hues to larger values. The color-coded myocardial segments for both images were overlaid over the grayscale original images. All operations were performed in MATLAB.

Results: End-systolic $A D$ and SS images from the two canine studies are shown in Figure I with, and without, windowing. The empirical distributions for (AD and SS) of the myocardial signal intensities and the corresponding fitted student-T distributions are shown in Figure 2. Figure 3 shows the color overlays utilizing the optimized colormap.

Discussion and conclusion: As Figure I demonstrates, without proper windowing the appreciation of MO differences is cumbersome. It takes several minutes for a reader to window the images. Moreover, windowing is subjective and has large intra-

Figure I (abstract P276)

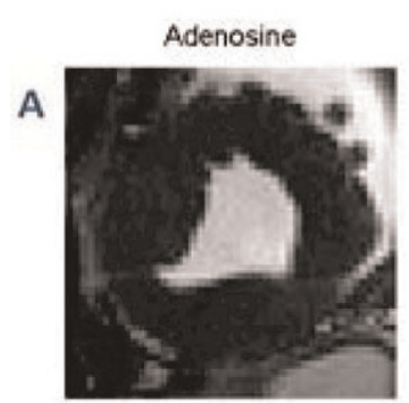

Adenosine

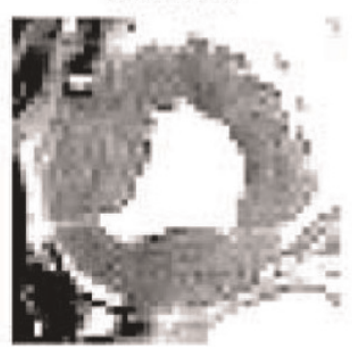

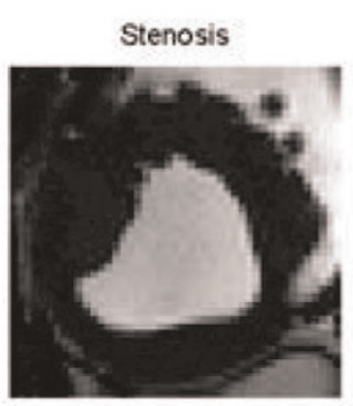

Stenosis

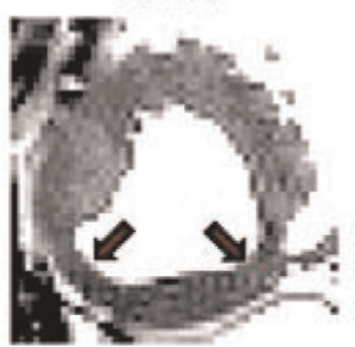

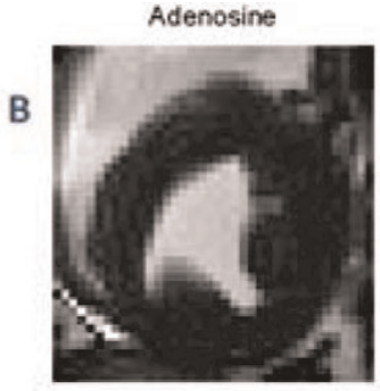

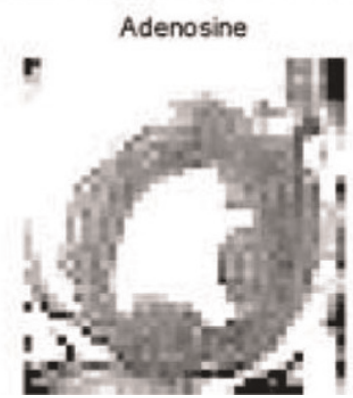

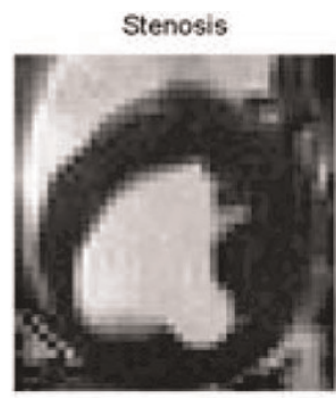

Stenosis

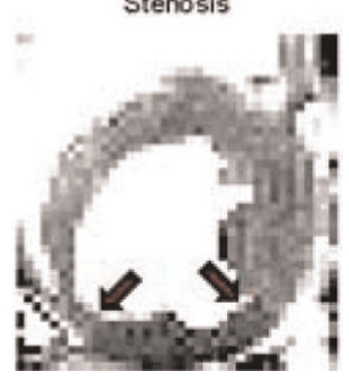

End-systolic adenosine images without (AD) and with LCX stenosis (SS) from the two controlled canine studies are shown in panels (A) and (B), respectively. The top row shows the AD and SS images without any windowing. The bottom row shows the same images but windowed the same to increase the regional myocardial contrast corresponding to oxygenation changes. Red arrows on the windowed SS images illustrate the limits of the affected myocardial segments corresponding to the LCX artery. 
Figure 2 (abstract P276)
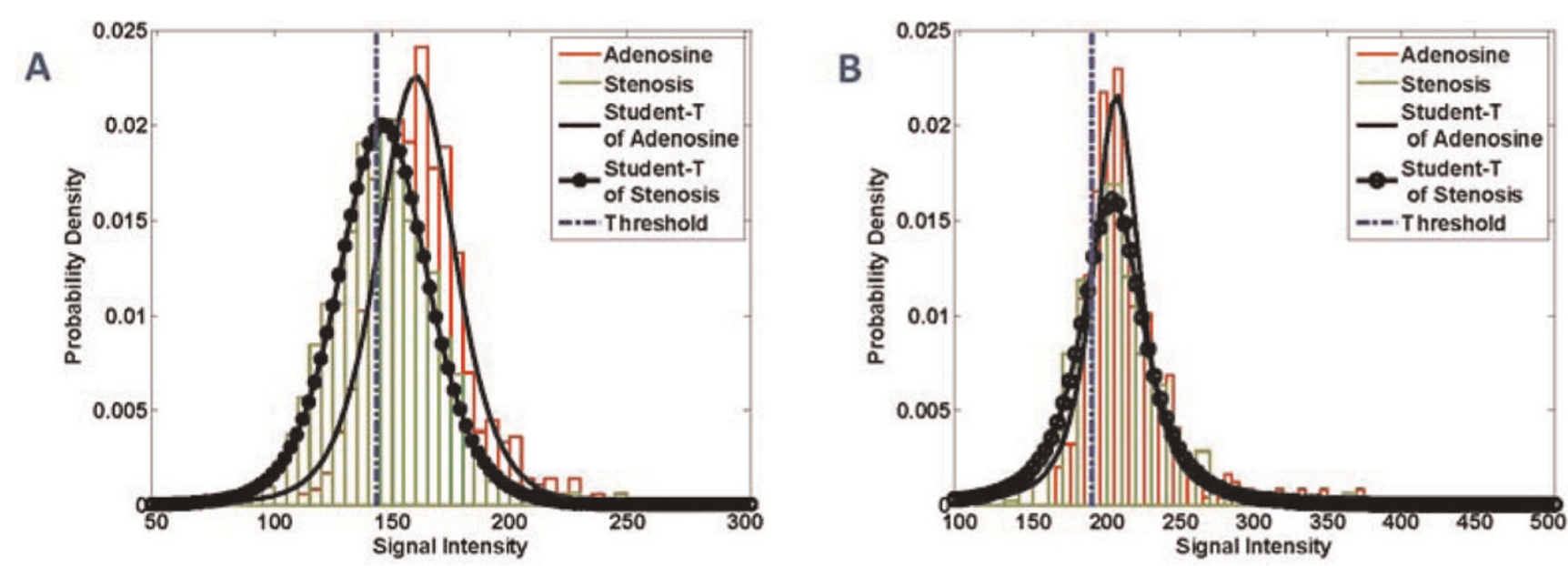

Probability density plots for the two canine studies (A) and (B) are shown. For each case the empirical densities of the adenosine and stenosis myocardial signals are plotted in red and green bars, respectively. Overlaid are the maximum likelihood estimated Student- $T$ densities for each signal. Finally, the threshold is shown in blue at 143.3 for study A, 189.7 for study B, respectively. Note that $\mathrm{m}-\mathrm{s}$ is the threshold computed from the Student-T distribution fitted to the adenosine signal as described in the text. All intensities below this threshold are color-coded in hues of red, while intesities above this threshold are color-coded in hues of yellow.

and inter-observer variability. Figure 2 illustrates that student- $T$ distribution closely approximates the intensity distribution of the myocardium. The motivation for choosing the $m-s$ as the midpoint for the colormap is to highlight intensities below this value, which are expected to have low probability of occurrence in a normal healthy case. It is expected that regions supplied by stenotic arteries will be hypointense, and will thus shift the intensity distribution towards the left. Hence, in cases where stenosis is present, the occurrences of intensities lower than $m$-s are greater, and therefore the amount of red-colored pixels are greater. Note that the contiguous red-colored region corre- sponds to the left-circumflex territory (Fig. 3). There are also mild signal changes in the AD images as well, that may be due to imaging artifacts, normal physiology changes, and/or presence of an inadvertent stenosis. Nevertheless, the presented method allows the observer to rapidly assess the presence of disease, as well as, potentially identify the section of the coronary tree that is stenotic on the basis of BOLD MRI. The proposed method forms an initial step in the development of improved visualization capabilities for myocardial BOLD MRI. The method could be fully automated method if combined with end-systolic image identification and left ventricle localization algorithms.

Figure 3 (abstract P276)
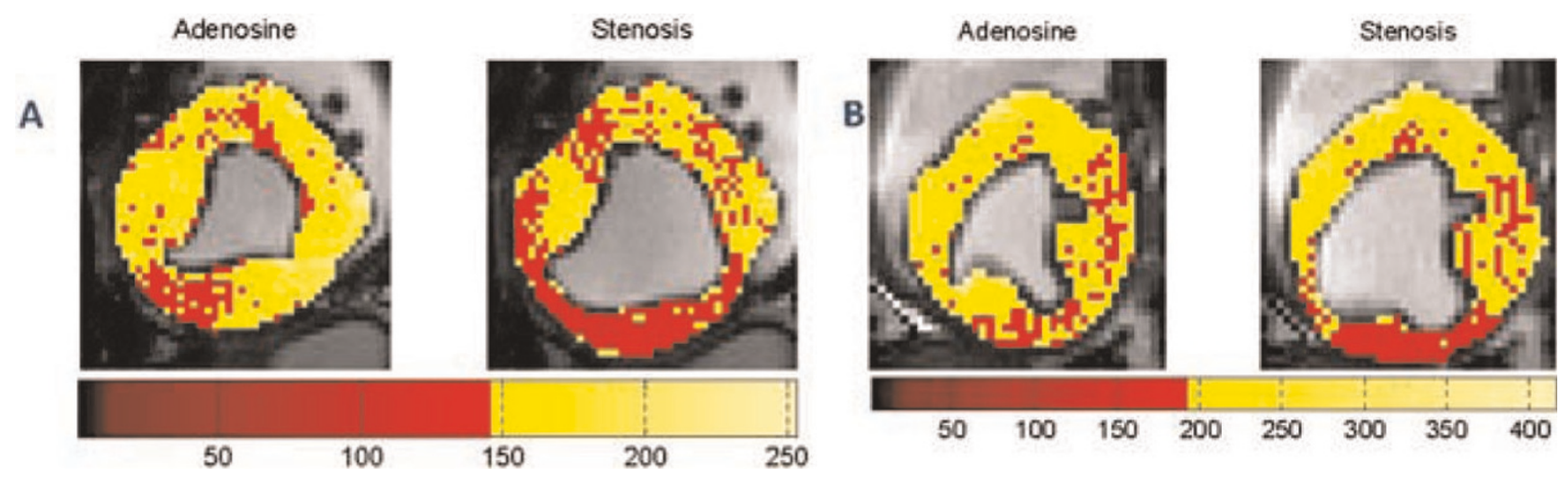

Color-coded end-systolic adenosine images without (AD) and with LCX stenosis (SS) from the two controlled canine studies are shown in panels (A) and (B), respectively. Only the myocardium was color coded. The segmentation masks were found using the algorithm in Tsaftaris, et al. ICIP 2008. The corresponding optimized colormaps are also shown. The yellow hues are for those values that are larger than the threshold as shown in Figure 2 and described in text. This method allows the observer to rapidly assess the presence of disease, as well as potentially identify the section of the coronary tree that is stenotic on the basis of BOLD MRI. 
P277

System identification theory in myocardial perfusion modeling of dynamic contrast-enhanced MRI

Jean-Paul Vallée, Marko Ivancevic, Jean-Luc Daire, Dominique Didier and Michel Kocher Radiology/Geneva University Hospital, Geneva- /4, Switzerland Journal of Cardiovascular Magnetic Resonance 2009, I I(SuppI I):P277

Introduction: Tissue perfusion and blood volume can be quantified by $\mathrm{MRI}$ after contrast media (CM) injection and a one compartment model that yields $\mathrm{KI}$, the blood to tissue transfer constant (related to myocardial blood flow MBF) and Vd, the CM distribution volume. Using system identification theory and computer simulations, the confounding effects of volume and rate of a contrast injection on the reliability of $\mathrm{KI}$ estimation was recently evaluated (Aerts MRM 2008; 59:I III). The simulations predict that the $\mathrm{KI}$ is most reliable with a high injection volume administered in a single injection, where high rates only modestly improve KI. However, the data used for the simulation were taken from a model of rectal cancer. It is not known how these simulations apply to myocardial perfusion at rest and at stress. Therefore, the aim of this study was to apply system identification theory on realistic data encountered in cardiac patients.

Methods: To evaluate the effect of the bolus shape on the model, two arterial input functions (AIF), were derived from real data (narrow AIF with a small CM volume and fast injection rate $0.035 \mathrm{mmol} / \mathrm{kg}$ at $5 \mathrm{cc} / \mathrm{sec}$ and wide AIF with a larger CM volume and slower injection rate $0.08 \mathrm{mmol} / \mathrm{kg}$ at $0.5 \mathrm{cc} / \mathrm{sec}$ as shown in Figure I) and used as input stimuli. Using constant rest and stress values for $\mathrm{KI}(0.6 \mathrm{ml} / \mathrm{ming} / \mathrm{g}$ and $2.4 \mathrm{ml} / \mathrm{min} / \mathrm{g})$ and $\mathrm{Vd}(\mathrm{I} \%)$, time transit curves (Cmyo) have been simulated by the discrete transfer function of the one compartment model derived from the Laplace transform (see Figure I). The output error (OE) method was used as a system identification method to estimate $\mathrm{KI}$ and $\mathrm{Vd}$. Finally, estimated values of $\mathrm{KI}$ and $\mathrm{K} 2$ are described for different realistic noise indices (the standard deviation of a zero mean Gaussian process varies from 0 to $10 \%$ ) and different under-sampling levels (from I to $4 \times$ ). Bias (fitted value/true value) in $\%$ and error (std dev/mean) in \% of fitted $\mathrm{KI}$ and $\mathrm{Vd}$ as well as the Bode diagram were obtained for each cases.

Figure I (abstract P277)
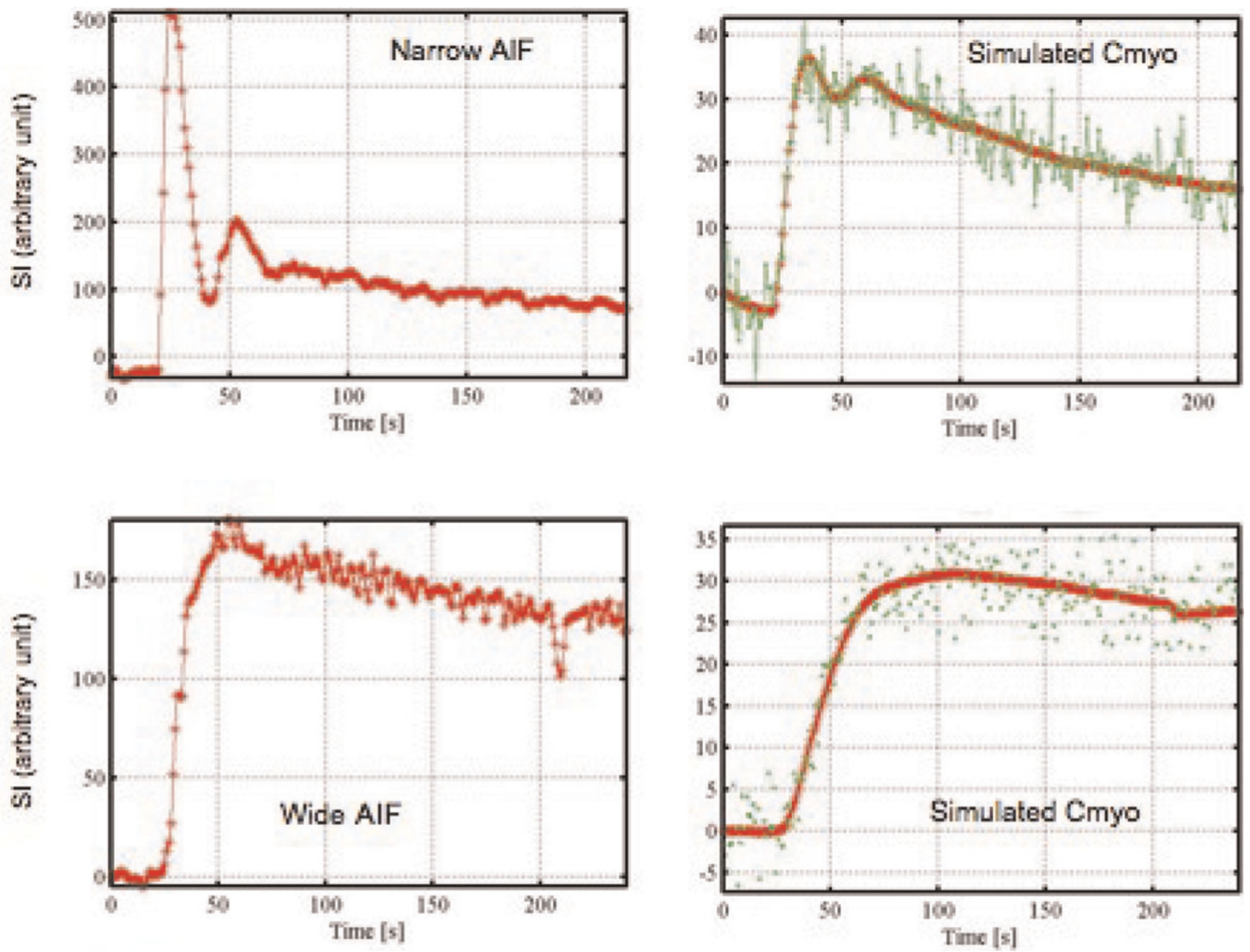
Figure 2 (abstract P277)
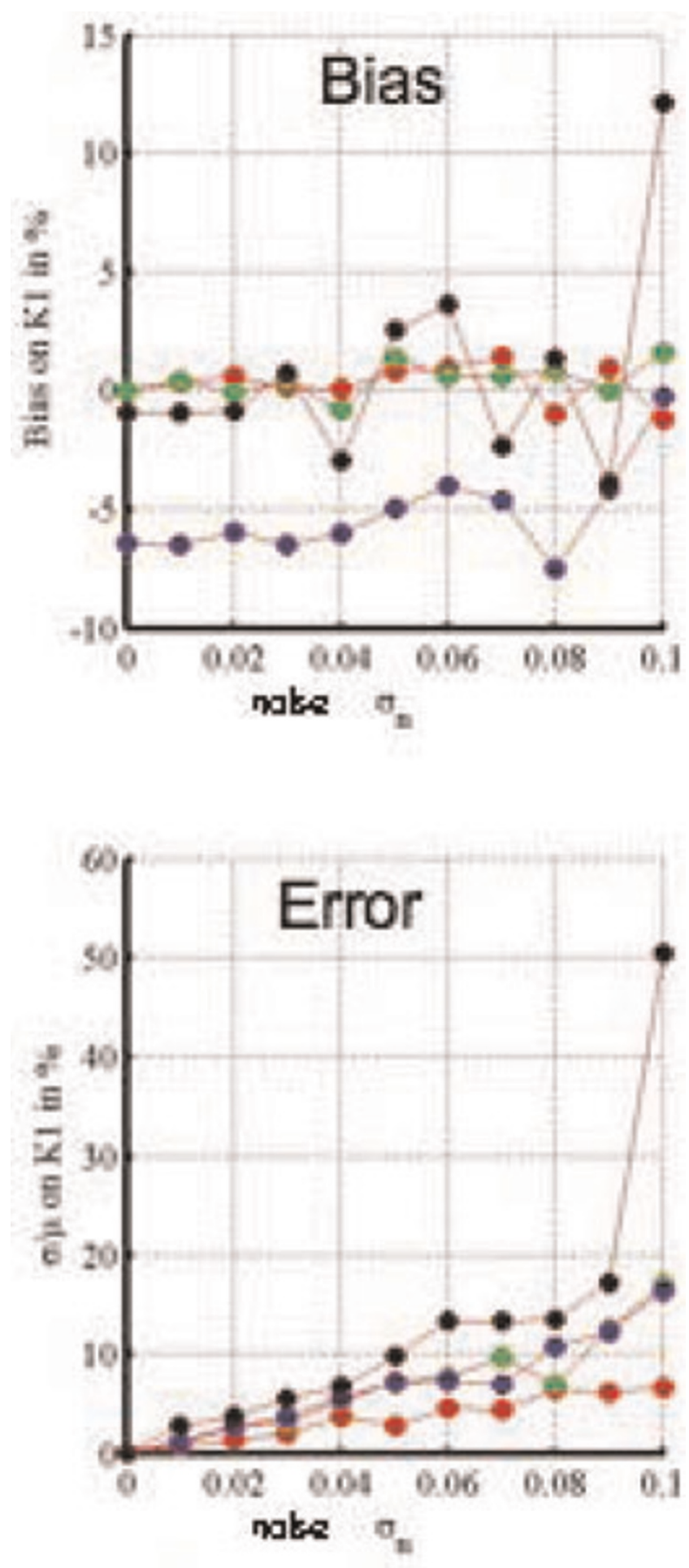

Results: At rest, no significant difference in bias and standard deviation were observed with the identification process between the two shapes of the arterial input function (narrow and wide). Under-sampled curves introduce an increase of standard deviation of rest $\mathrm{KI}$ and $\mathrm{K} 2$ but with no significant effect on the bias (<I\% for noise up to $5 \%$ ). At stress (see Figure I), there was no significant bias for both arterial input functions red and green curves of Figure I) as well as for the wide undersampled function (black curve). However, almost $5 \times$ increase of bias was observed in the undersampled narrow function (blue curve). The standard deviation was increased by undersampling as well by a slow injection rate. Regarding the distribution volume $\mathrm{Vd}$, accurate measurement was obtained for all the conditions (bias < $1 \%$ for a noise of 0.05 and standard deviation $<4 \%$ ).

The Bode diagram showed a similar pattern around the cut-off frequency for the narrow and wide AIF at rest as well as for the wide AIF at stress but not for the narrow undersampled AIF explaining the lowest performance of this AIF. See also Figure 2. Conclusion: At rest, both slow and fast injection rates as well as undersampling up to I image/4 seconds yield accurate measurements of the myocardium blood flow and distribution volume. At stress the myocardium blood flow measurement is less accurate in case of a fast injection of a small volume of CM. The simulations indicated that MBF measurements at stress can improved by slowing the injection rate for the same sampling frequency.

\section{P278}

Evaluation of 3-dimensional left ventricular velocities with cardiac MR imaging using navigator gated high temporal resolution tissue phase mapping Ion Codreanu', Cameron J Holloway', Oliver J Rider', Steffen E Petersen', Matthew D Robson', Bernd A Jung ${ }^{2}$, Stephen J Golding ${ }^{3}$, Stefan Neubauer ${ }^{\prime}$ and Kieran Clarke ${ }^{4}$ 'University of Oxford Centre for Clinical Magnetic Resonance Research, Headington, Oxford, UK

${ }^{2}$ Department of Diagnostic Radiology, Medical Physics,

University Hospital, Freiburg, Germany

${ }^{3}$ MRI Centre, Oxford Radcliffe Hospital, University of Oxford, Headington, Oxford, UK

${ }^{4}$ Department of Physiology, Anatomy and Genetics, University of Oxford, Oxford, UK

Journal of Cardiovascular Magnetic Resonance 2009, I I(SuppI I):P278

Introduction: Identification of regional myocardial dysfunction with adequate temporal and spatial resolution is important for diagnosis and management of cardiovascular disease. Assessment of regional myocardial velocities allows 3-dimensional quantification of left ventricular function and carries independent prognostic information.

Purpose: The aim of this study was to use navigator gated tissue phase mapping with high temporal resolution to obtain accurate normative data of left ventricular velocities, to assess normal patterns of left ventricular motion and to evaluate the reproducibility of this technique.

Methods: Cardiac magnetic resonance examinations were performed on a I.5 Tesla MR clinical scanner (Sonata; Siemens Medical Solutions, Erlangen, Germany). Cardiac gated, phase contrast measurements using respiratory navigator with a temporal resolution of $13.8 \mathrm{~ms}$ were obtained on two separate occasions on 13 healthy male subjects (age $22 \pm 3$ years) three weeks apart. Three short axis images were obtained for the left ventricular base, mid-ventricle and apex. Each short axis 
Figure I (abstract P278)

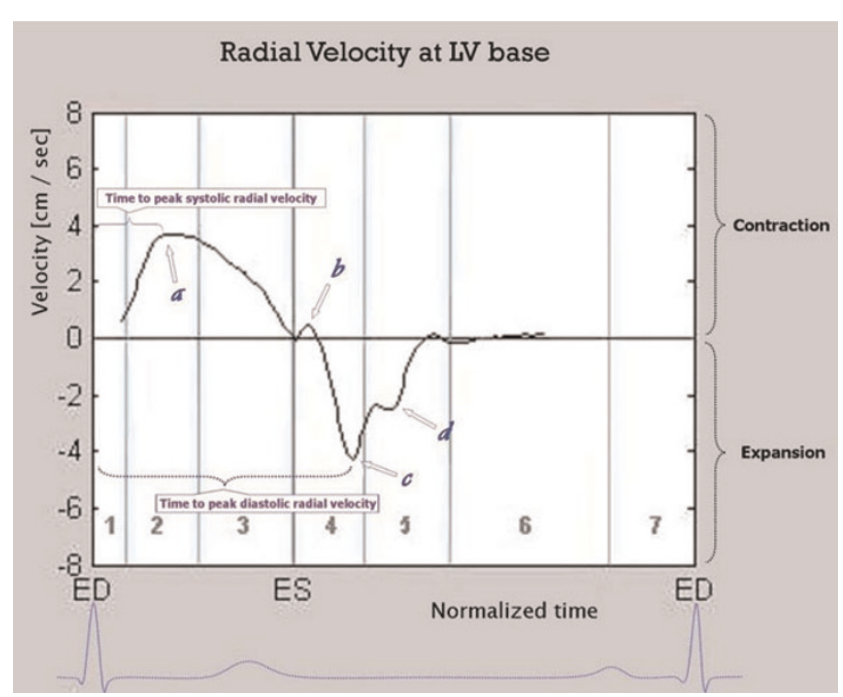

Radial velocity graph at left ventricular base. I - isovolumetric contraction, 2 - rapid ejection, 3 - reduced ejection, 4 - isovolumetric relaxation, 5 - rapid filling, 6 - diastasis, 7 - atrial contraction, ED - end diastole, ES - end systole, a - peak systolic radial velocity, b - upward directed notch in early diastole, c - peak diastolic radial velocity, $d-$ additional thrust of outward radial movement during rapid ventricular filling.

acquisition took approximately $3-5$ minutes, with an average of 60 - 70 phases per cardiac cycle. Subsequent normative velocities were obtained for radial, circumferential and longitudinal motion.

Results: High temporal resolution tissue phase mapping analysis allowed visualization of additional details of ventricular motion that have not been observed with breath-held lower temporal resolution tissue phase mapping [I]. Changes in the direction of radial, circumferential and longitudinal movement were apparent after repolarization. An additional wave of outward radial movement, corresponding to the timing of the third heart sound, was observed at the left ventricular base during rapid ventricular filling (Figure Id). An upward directed notch in early diastole (Figure Ib) similar to that on arterial pressure wave was recorded on radial and longitudinal velocity graphs. The longitudinal velocity graph showed a downward movement of the whole ventricle during rapid ejection, followed by an upward displacement after repolarization. The commencement of left ventricular untwisting was also noticed after repolarization. A normative database for radial, circumferential and longitudinal velocities for epicardial, myocardial and endocardial layers as well as for peak times was obtained using this technique. Repeated measurements showed good agreement on Bland-Altman plots.

Conclusion: Navigator gated tissue phase mapping with high temporal resolution is a reproducible technique to evaluate detailed regional myocardial wall motion and provides valuable and comprehensive information for quantifying myocardial velocities. The technique allowed us to obtain new details of left ventricular motion patterns and may prove to be a powerful tool for assessing subtle changes in myocardial function in cardiac disease.

\section{Reference}

I. Petersen SE and Jung BA, et al: Myocardial Tissue Phase Mapping with Cine Phase-Contrast MR Imaging: Regional Wall Motion Analysis in Healthy Volunteers. Radiology 2006, 238(3):816-826.

\section{P279}

Cartesian acquisition with PR-like sampling: applications to 3D contrast-enhanced MRA Stephen J Riederer and Clifton R Haider Mayo Clinic, Rochester, MN, USA

\section{Journal of Cardiovascular Magnetic Resonance 2009, I I(SuppI I):P279}

Introduction: View sharing and parallel acquisition are methods which can be used to improve the frame rate in timeresolved studies. In this work these methods are integrated and applied to contrast-enhanced MR angiography.

Purpose: CAPR for "Cartesian Acquisition with Projection Reconstruction-like sampling" integrates Cartesian k-space sampling, 2D acceleration techniques, and view sharing to provide 3D time-resolved image sequences of contrast-enhanced $M R$ angiograms. In this work we describe its development and application to multiple vascular territories.

Methods: Prior to performing in vivo studies the CAPR technique was evaluated technically. Receiver coil arrays were developed having as many as 16 elements, routinely allowing 2D SENSE acceleration of 10 or higher, with overall acceleration of 15 using 2D homodyne reconstruction. For imaging the lower legs, elements placed anterior-posterior were designed for smaller depth of response than those placed left-right so as to limit loss of SNR in 2D parallel acquisition. The portrayal of the leading edge of contrast was tested using a computer-controlled bolus phantom. This was used to evaluate edge blurring as well as absolute accuracy of position and velocity. CAPR was used to image the vasculature of the brain and the extremities at 3.0 T. For intracranial vasculature the spatial resolution is I $\mathrm{mm}$ isotropic with image update times of $\mathrm{I}-3 \mathrm{sec}$. For bilateral imaging of the lower legs, feet, and hands the parameters were adjusted for sub-mm isotropic resolution and frame times of 4-7 sec.

Results: Modified receiver coils as described can keep g-factors for the lower legs well under an absolute value of 1.5 even for accelerations as high as 8 . The phantom studies show that CAPR can effectively freeze the motion of the advancing contrast bolus, with blur of the leading edge less than $10 \%$ of the distance moved from one time frame to the next. Image reconstruction is performed immediately, with the time series of MR angiograms available for display within two minutes after complete of the acquisition. In dozens of in vivo studies CAPR has been shown to consistently provide images of high spatial resolution, with temporal resolution clearly distinguishing arterial from venous phases and with 2D SENSE accelerations of 8 or higher. For the brain the method allows I $\mathrm{mm}$ imaging of the arterial vasculature with negligible venous signals. For the lower legs the technique depicts the vasculature at the level of third order branching, even in subjects with fast arterial-to-venous flow patterns. For the feet the method routinely depicts progressive filling of the tarsal and plantar arches.

Conclusion: CAPR has been readily adaptable to all vascular areas studied thus far. It provides time-resolved 3D data sets with 
very high (I mm isotropic) spatial resolution and which clearly portray arterial and venous enhancement patterns.

\section{P280}

Contrast enhanced MRI and MR coronary angiography (MRCA) as one stop shop in patients with untreated myocardial infarction

Carlo Liguori', Luigi Natale', Agostino Meduri',

Antonio Bernardini ${ }^{2}$, Riccardo Marano'

and Lorenzo Bonomo'

'Università Cattolica del Sacro Cuore - Policlinico A. Gemelli, Rome, Italy

2“G. Mazzini” Hospital - ASL Teramo, Teramo, Italy

Journal of Cardiovascular Magnetic Resonance 2009, I I(SuppI I):P280

Purpose: To define MRI role in patients with myocardial infarction not treated by $\mathrm{PCl}$.

Methods and materials: 24 pts ( 17 males, 7 females) with myocardial infarction (7 anterior, 9 lateral, 8 inferior), untreated by $\mathrm{PCl}$, underwent $\mathrm{MRI}$ within three weeks after infarction. MRI was performed on a I.5 T scanner (GE Signa Excite) with this protocol:

I) Triple-IR FSE sequence short axis for edema;

2) Fiesta sequences on short, horizontal and vertical long axes, for regional and global systolic function;

3) FGR-ET short axis sequence for first pass;

4) IR-FGRE sequence on short, horizontal and vertical long axes, for infarct size.

5) Before delayed imaging, MRCA with breath-hold 3D Fiesta VCATS technique was obtained. MRCA was compared with the gold standard conventional coronary angiography (CA), performed within 24-48 h.

Results: MRCA was scored with a 3 points scale $(0=$ good, $\mathrm{I}$ = sufficient, 2 = poor) by 2 blinded readers; 3 exams were excluded as pts could not hold their breath. MRCA showed II severe stenoses $(>50 \%)$ and 9 occlusions, with I exam not evaluable for poor quality; CA showed severe stenoses in 14 and 7 occlusions. Collaterals and retrograde filling were present in 2 occlusions at CA; in these 2 cases DE at MRI was $\leq 50 \%$. DE and FP defects distribution always showed correlation with the diseased vessel. MRCA showed 100\% sensitivity, $78.6 \%$ specificity, $70 \%$ PPV, $100 \%$ NPV.

Conclusion: In pts not treated by primary or rescue $\mathrm{PCl}$, MRCA can rule out occlusion and/or significant stenosis; the correlation with segmental distribution of $D E$ is excellent.

\section{P28I}

\section{Repeatability of global myocardial function} parameters post pacemaker implantation Jana G Delfino', Jack A Talsma', Edward T Martin², David A Sandler ${ }^{2}$ and John N Oshinski ${ }^{3}$

'Emory University, Atlanta, GA, USA

${ }^{2}$ Oklahoma Heart Institute, Tulsa, OK, USA

${ }^{3}$ Emory University/Georgia Institute of Technology,

Atlanta, GA, USA

Journal of Cardiovascular Magnetic Resonance 2009, I I(SuppI I):P28 I

Objective: To determine the reproducibility of global myocardial function parameters in patients with implanted pacemakers.
Figure I (abstract P28I)

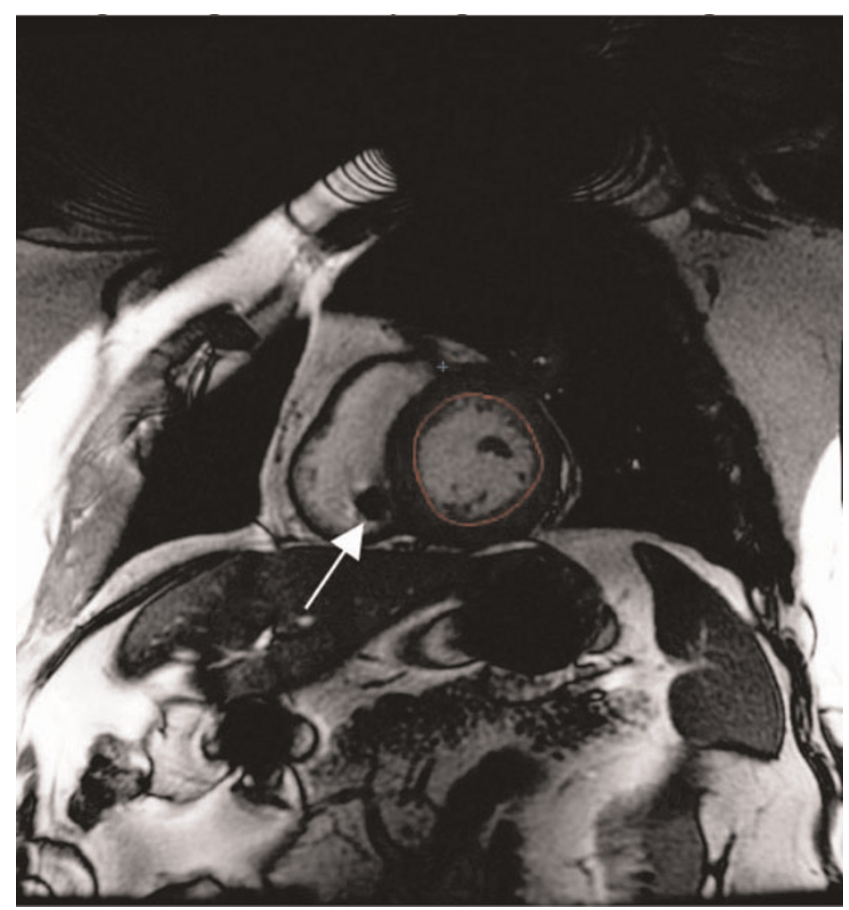

Example CMR image from a patient with an implanted dual chamber pacemaker. Although artifacts from both the pulse generator (top center) and the RV pacing lead are visible (arrow), image quality is sufficient for accurate analysis of myocardial function.

Introduction: Quantitative measurement of patient response to CRT includes reverse remodeling of the left ventricle. Usually this is measured by a $>15 \%$ reduction in end-systolic left ventricular volume at 3 months post CRT. Two-dimensional echocardiography has been the primary modality used for this endpoint. There is an interest in using MRI to evaluate response because of its superior image quality, resolution and reproducibility compared to echo. However, the image quality and reproducibility of CMR in patient with dual chamber pacemakers is unknown.

Methods: Six patients with implanted pacemakers and clinical indications for CMR were included in this study. All patients were not pacemaker dependent and had dual chamber devices $50 \%$ Medtronic Inc, 50\% Guidant Inc). Clinical indications for CMR included pericardial mass, pre-pulmonary vein ablation, pulmonary vein stenosis, myocardial fibrosis and constrictive pericarditis. MRI exams were performed on a I.5 T GE Signa Excite MRI (GE Medical Systems, Waukesha, WI). Following acquisition of scout images, cine steady state free precession (SSFP) images were acquired in the two chamber, four chamber, and short axis orientations. Image acquisition parameters were as follows: $T R=3 \mathrm{msec}, T E=1.2 \mathrm{msec}$, slice thickness $=8 \mathrm{~mm}$, FOV $=360-380 \mathrm{~mm}$, pixel size $0.7-1.5 \mathrm{~mm}$. Appropriate precautions were taken regarding pacemaker-MRI interaction including telemetry monitoring, voice and visual contact, and device interrogation pre/post MRI.

Endocardial and epicardial borders were traced on short axis cine SSFP images using MASS software (ARL, Lieden, The 
Netherlands). Global parameters of myocardial function [end diastolic volume (LVEDV), end systolic volume (LVESV), stroke volume (DV), ejection fraction (EF), and mass (LVM)] were computed from the contours. Complete border tracing and analysis was conducted by two independent observers on two separate days at least I week apart.

The mean difference between repeated measurements and the coefficient of variability (CV) were computed for each parameter to assess reproducibility.

Results: Image quality for all six patients was sufficient for analysis, Figure I. Mean difference between repeated measurements was I5.6 +/- $6.9 \mathrm{~mL}$ for LVEDV, $6.9+/-7.6 \mathrm{~mL}$ for LVESV, $8.7+/-10.3 \mathrm{~mL}$ for SV, $0.7+/-6.3 \%$ for $E F$, and $3.1+/-9.2 \mathrm{~g}$ for LVEDM. Coefficient of variability was $7.9 \%$ for LVEDV, $7.8 \%$ for LVESV, $10.0 \%$ for SV, $6.2 \%$ for EF, and $4.9 \%$ for LVEDM.

Discussion: The pulse generator and pacing leads caused signal void artifacts in the image. However, these were localized and did not interfere in identification of myocardial borders, Figure I. Image quality decreased substantially in the one patient who had two additional abandoned leads from a previous device.

Conclusion: Global measurements of myocardial function can be reliably measured with good reproducibility in patients with implanted dual chamber pacemakers.

\section{P282}

\section{Continuous table movement for peripheral MRA} with matrix coils at $3.0 \mathrm{~T}$

Harald Kramer', Peter Schmidt ${ }^{2}$,

Christian Glaser', Maximilian F Reiser'

and Karin A Herrmann'

'University Hospital of Munich, Munich, Germany

${ }^{2}$ Siemens Medical Solutions, Erlangen, Germany

Journal of Cardiovascular Magnetic Resonance 2009, I I(SuppI I):P282

Introduction: To compensate for the restricted field of view (FoV) of standard MRA systems for larger anatomical areas such as the peripheral arteries of the lower body part, a multistep approach was introduced and successfully implemented for MRA of the lower limb. However, even concepts of a sequential multi-step MRA with a double CA bolus include several disadvantages. The planning and separate data acquisition of multiple steps for both non-enhanced and contrast enhanced imaging is time consuming. To face and overcome these limitations, a novel technique for continuous table movement and data acquisition for MRA has been introduced at 3.0 Tesla.

Purpose: To compare a standard step-by-step and a newly developed continuous table movement (ct) technique for peripheral MRA at a 3.0 Tesla MR System equipped with a matrix coil system.

Methods: Peripheral MRA with continuous table movement acquires one large FoV with up to $130 \mathrm{~cm}$ in patient z-axis. Before acquiring the contrast enhanced dataset vessel scout images as well as a non contrast enhanced dataset for later subtraction are acquired. We included 14 consecutive patients referred for peripheral MRA with clinical symptoms of peripheral arterial occlusive disease (PAOD) Fontaines stage II - IV. All of them underwent both step-by-step MRA and ct-MRA in one session. Patients with impaired renal function (calculated
Figure I (abstract P282)

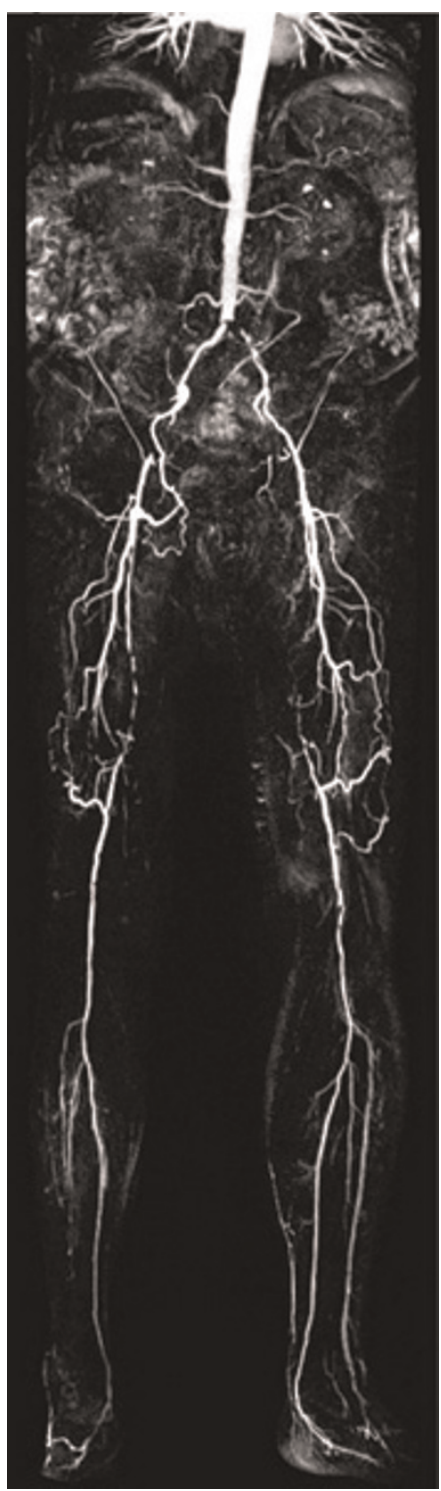

CT-MRA dataset with excellent image quality without any venous enhancement or artefacts. Clear depiction of several atherosclerotic changes like occlusion of the superficial femoral artery on both sides.

GFR $<30 \mathrm{ml} / \mathrm{min}$ ) were not included. All exams were performed on a 3.0 T MR System (Magnetom Verio). Maximal contrast agent (CA) volume was $31.5 \mathrm{ml}(1.5 \mathrm{ml}$ testbolus, $15 \mathrm{ml} / \mathrm{MRA}$ technique). For both techniques the same monophasic $C A$ injection protocol was used, $15 \mathrm{ml}$ of standard 0.5 molar CA were injected at a flow rate of $1 \mathrm{ml} / \mathrm{s}$ directly followed $25 \mathrm{ml}$ of saline also at $1 \mathrm{ml} / \mathrm{s}$. Spatial resolution of the ct-MRA datasets is technically limited to a reconstructed voxel size of $1.0 \times 1.0 \times 1.3 \mathrm{~mm}^{3}$ for the entire FoV. Acquired voxel sizes differ throughout the FoV due to a technique called "variable resolution" helping to increase resolution in the most distal part of the FoV. Step-by-step MRA reached a spatial resolution 
between $1.4 \times 1.1 \times 1.2 \mathrm{~mm}^{3}$ and $0.9 \times 0.9 \times 0.9 \mathrm{~mm}^{3}$ in the most distal calf station. First ct-MRA datasets were read and findings thereafter correlated with the step-by-step MRA datasets. See Figure I.

Results: All datasets could be evaluated. Due to the absence of multiple localizers and subtraction masks examination time was considerably shorter when using the ct-MRA technique. Relevant findings detected by step-by-step MRA were also detected by ctMRA. Different results between step-by-step MRA and ct-MRA occurred in the differentiation between no vessel-wall changes/ slight atherosclerotic changes and high grade stenosis/occlusion respectively.

Conclusion: MRA with continuous table movement is an easy applicable technique for imaging peripheral vessels without the need for planning different steps and FOV positioning, thus examination time can be reduced considerably. However, the slightly reduced spatial resolution compared to standard stepby-step MRA is a drawback especially in the most distal calf vessels.

\section{P283}

The reproducibility of cardiac T2* measurement in thalassaemia major patients using bright and black blood sequences

Gillian Smith, Taigang He, John Paul Carpenter,

Raad Mohiaddin, David Firmin and Dudley Pennell

Royal Brompton Hospital, London, UK

Journal of Cardiovascular Magnetic Resonance 2009, I I(SuppI I):P283

Introduction: Regular blood transfusions are required for the long term survival of patients with thalassaemia major (TM) but this can cause tissue iron overload. Myocardial iron overload can lead to heart failure and subsequent death. Iron chelation therapy can reduce tissue iron levels but efficacy needs to be closely monitored. Ferritin levels and hepatic iron loading do not correlate well with cardiac iron levels thus the need for a reliable, non-invasive means of follow-up. Cardiovascular magnetic resonance (CMR) using a bright blood gradient echo sequence has been validated for the quantification of tissue iron levels. Signal intensity decreases with increasing echo time and the rate of decay is proportional to iron loading. This bright blood sequence has been widely used for clinical follow-up and efficacy of pharmacological intervention. A T2* $<20 \mathrm{~ms}$ is considered indicative of cardiac iron overload. Contrast between blood pool and myocardium for this technique is however relatively low, and blood signal artifacts which propagate onto the myocardium can further reduce accuracy. A double inversion pulse black blood sequence has recently been developed to address these issues. It is of interest therefore to assess reproducibility in a larger group of patients and to compare differences according to iron overload levels.

Purpose: To evaluate the comparative reproducibility of a bright blood and recently available black blood T2* sequence for patients with different myocardial iron loading levels.

Methods: 100 consecutive TM patients, 50 with a T2* value $<20 \mathrm{~ms}$ and 50 patients with a T2* $>20 \mathrm{~ms}$ were selected (5I female, age 27 years - range II to 50) All patients were scanned using a I.5 T Siemens Sonata scanner with a 6 channel phased array cardiac coil and ECG gating. A mid ventricular short axis slice was imaged using both the bright blood and black blood sequences. Both sets of data were analysed twice by the same operator. A mono-exponential decay curve was derived using Thalassaemia tools (CMRtools, Cardiovascular Imaging Solutions, London, UK). For patients with high iron loading and longer echo time intensities points falling below the noise level were removed

Figure I (abstract P283)
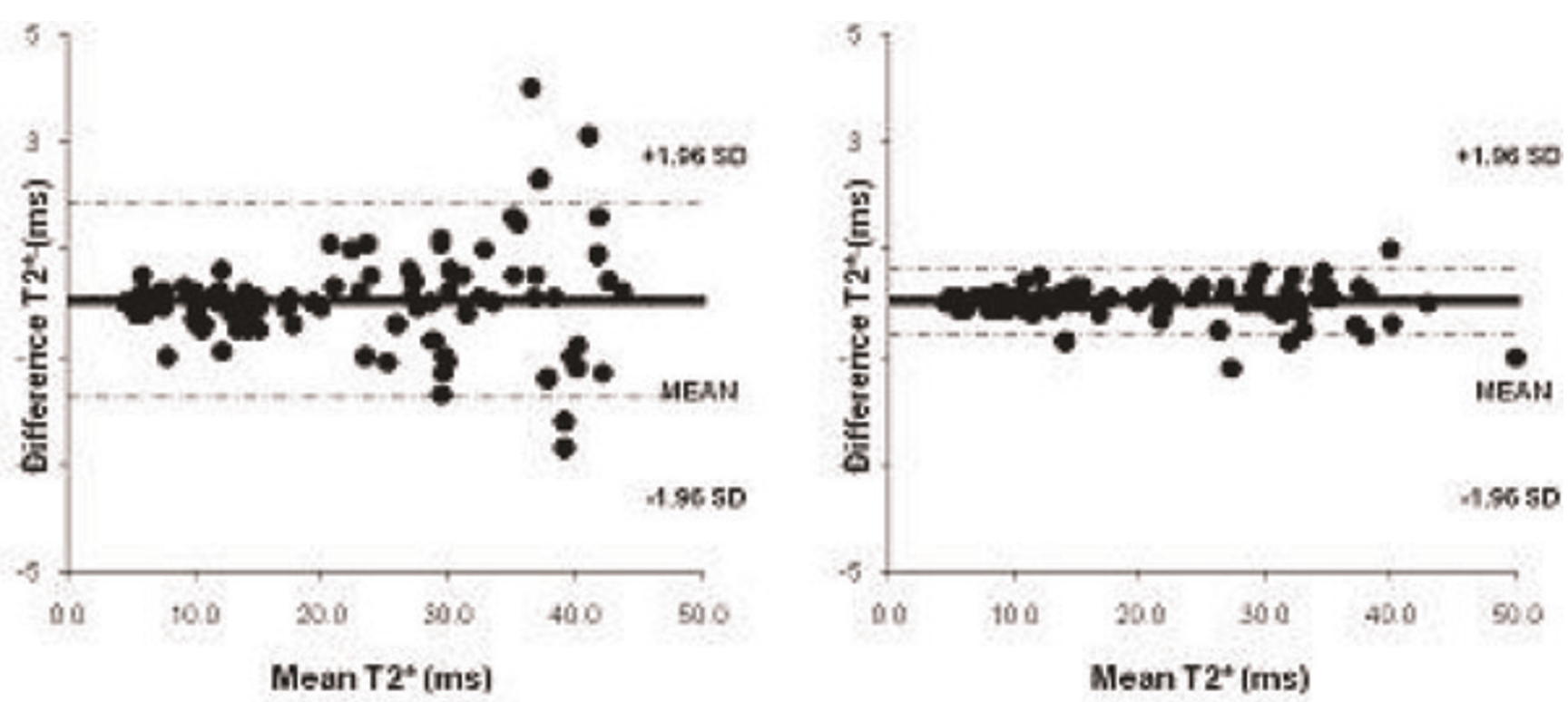

Intra-operator reproducibility of bright blood (left) and black blood (right) acquisitions for all patients. 

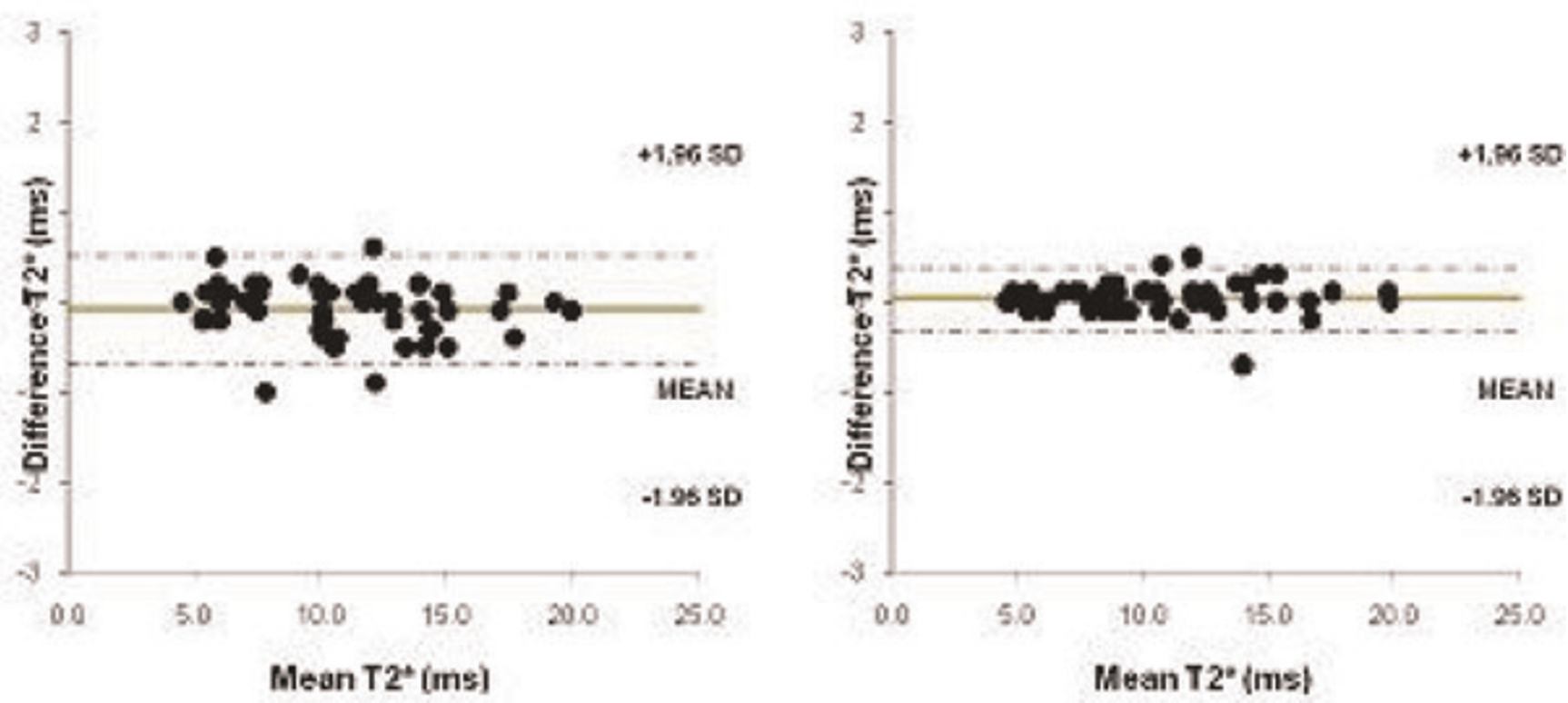

Intra-operator reproducibility of bright blood (left) and black blood (right) acquisitions for patients with T2* values $<20$ ms.

to improve the curve fit (truncation method). Bland-Altman analysis was used to show the intra-operator reproducibility for all patients and subgroups.

Results: T2* values ranged between $4.5-43.8 \mathrm{~ms}$ (mean $21.6 \pm 12 \mathrm{~ms}$ ) for all patients. For the 50 iron overloaded patients values ranged from $4.5-19.9 \mathrm{~ms}$ (mean $10.9 \pm 3.8 \mathrm{~ms}$ ) and for the patients with $\mathrm{T} 2 *$ values over $20 \mathrm{~ms}$ the range was
2I.I-43.8 (mean $32.3 \pm 6.4 \mathrm{~ms}$ ). Figure I shows the BlandAltman plot of $\mathrm{T} 2 *$ values obtained for the two measurements for the bright blood and dark blood acquisitions. The coefficient of variability was 4.23 for bright blood and I.48 for dark blood. For patients with a T2* value of $<20 \mathrm{~ms}$ the coefficient of variability was $2.8 \mathrm{I}$ (bright blood) vs 1.68 (dark blood - Figure 2) and for $>20 \mathrm{~ms} 3.88$ vs 1.29 (Figure 3 ).

Figure 3 (abstract P283)
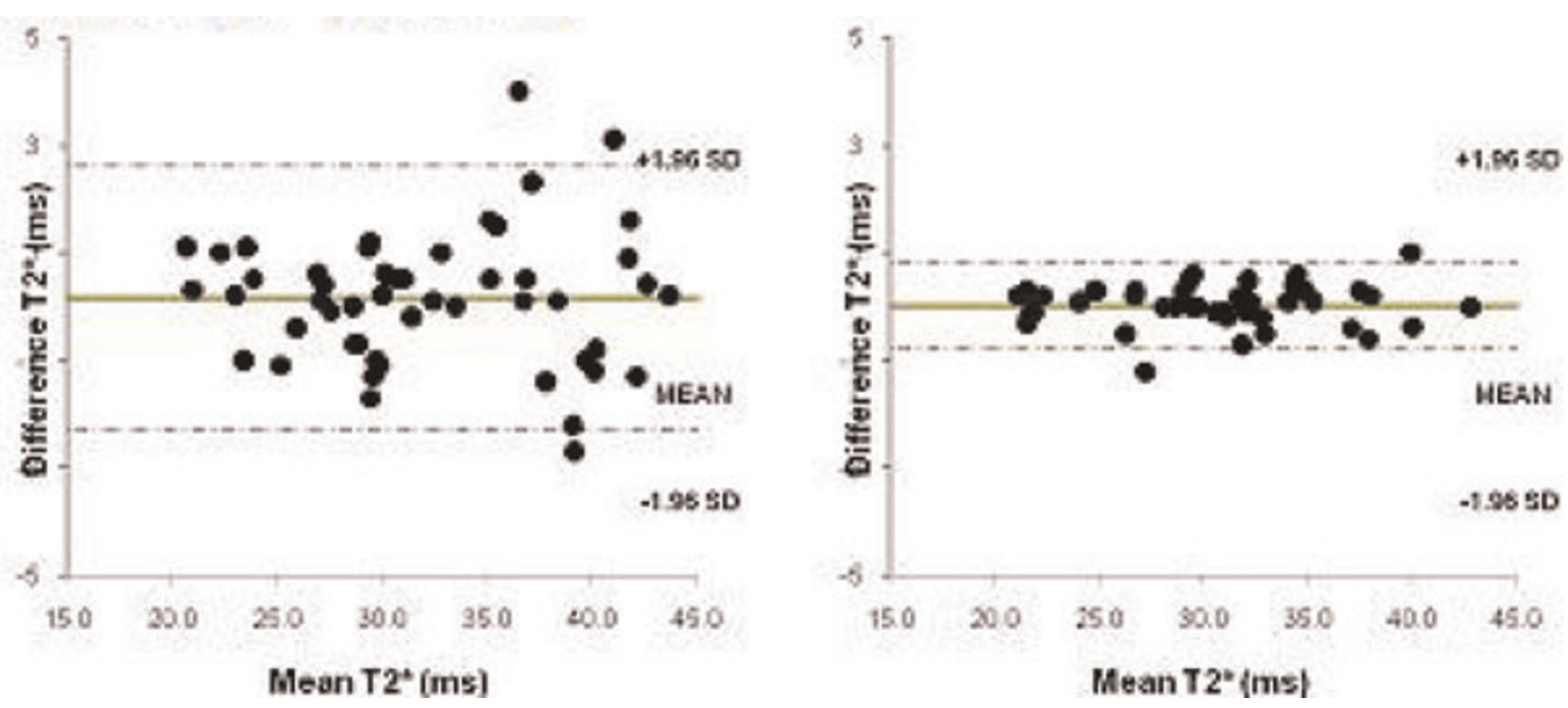

Intra-operator reproducibility of bright blood (left) and black blood (right) acquisitions for patients with T2* values $>20$ ms. 
Conclusion: This study shows improved reproducibility across all T2* values for the black blood sequence in comparison with the previously validated bright blood sequence.

\section{P284}

A new algorithm for aortic compliance evaluation in normals Yi Wang', Jianping Zhang², Edwin Estrada' and Nathaniel Reichek'

'St. Francis Hospital, Roslyn, NY, USA

${ }^{2}$ Stony Brook University, Stony Brook, NY, USA

Journal of Cardiovascular Magnetic Resonance 2009, I I(SuppI I):P284

Introduction: Aortic compliance (AC) can be evaluated noninvasively and its reduction with age in normals has been demonstrated with both MRI and Doppler echo methods. Aortic pulse wave velocity (PWV), a measurement of the flow pulse traveling along aorta as a surrogate of $A C$, can be assessed using a single breath-hold phase contrast (PC) imaging technique. Accurate determination of the time delay $(\Delta t)$ between flows in ascending and descending aortic regions is critical in PWV assessment. Various approaches have been studied for $\Delta t$, including measuring the intervals between flow onset points, between maximal flow points, and between parallel upslopes after least squares fittings. We studied a new approach using a sliding window maximal upslope approach.

Methods: Eighty healthy volunteers with informed consent (age: $59.5 \pm 13.9$ ) were screened to exclude hypertension, elevated total cholesterol and cardiovascular disease. Using the 'candy cane' view of aorta, an axial plane through the ascending and descending aorta at the pulmonary artery level was prescribed and a through-plane velocity encoded PC cine imaging was acquired with VENC of $150 \mathrm{~cm} / \mathrm{s}$, TR/TE/FA $=98 \mathrm{~ms} / 2.9 \mathrm{~ms} / 15^{\circ}$ and voxel spatial resolution $1.3 \times 2 \times 6 \mathrm{~mm}^{3}$ on a I.5 T MRI scanner. The distance traveled by the aortic pulse wave, $\Delta \mathrm{D}$, was determined as the distance along the central line between ascending and descending aorta in the 'candy cane' image. FLOW (Medis, Lunden, the Netherlands) was used to measure flow in both ascending and descending aorta

Figure I (abstract P284)

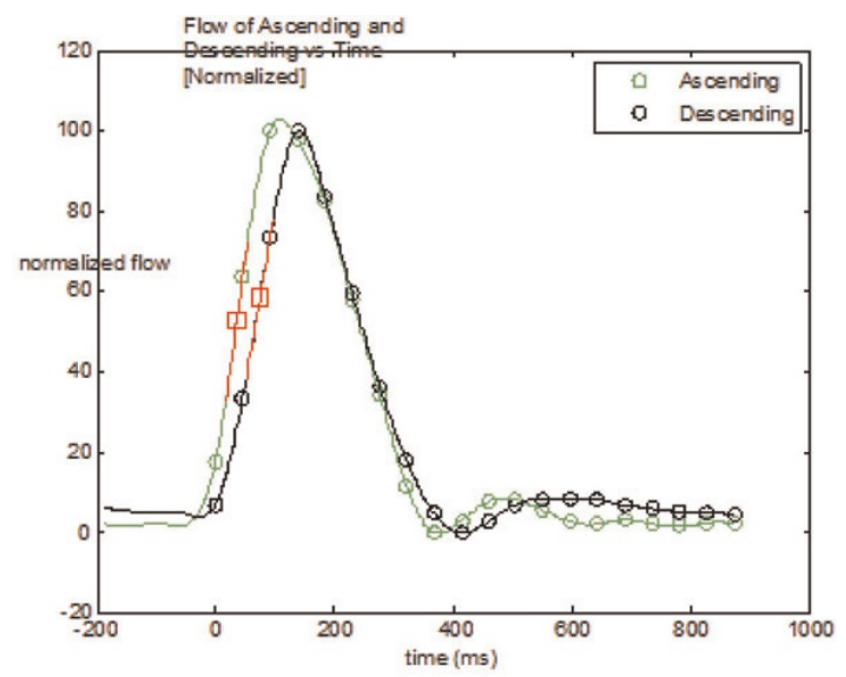

Figure 2 (abstract P284)

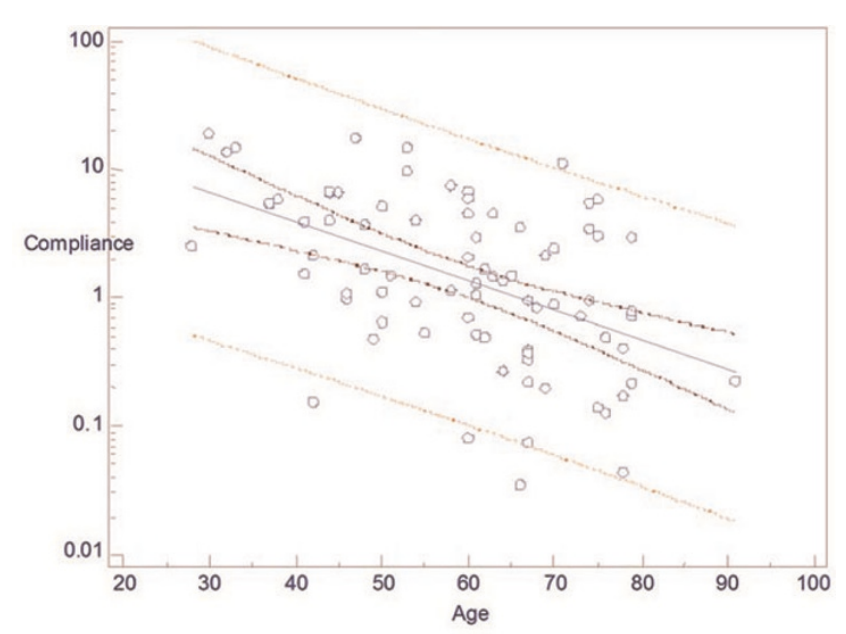

regions. For $\Delta \mathrm{t}$ assessment, a Matlab program was developed and flow upslope was calculated for each time point by taking flow values from neighboring time points using least squares means. The maximal upslope was selected as the largest upslope among all time series. We calculated $\mathrm{PWV}=\Delta \mathrm{D} / \Delta \mathrm{t}$ and aortic compliance as $A C=1 /\left(\rho^{*} P W V^{2}\right)$, where blood density $\rho=1057 \mathrm{~kg} / \mathrm{m}$. Linear regression was used to determine the relationships between $A C$ and age.

Results: Illustrative ascending (green) \& descending (black) aortic flow from ROls in the PC images with maximal upslope occurred at the time marked with red squares and at an angle in the red lines are shown in Figure I. The upslope algorithm worked well on all cases without any user interaction. The mean \pm sd of PWV and AC were II.I $\pm 9.6 \mathrm{~m} / \mathrm{s}$ and $(3.2 \pm 4.1)$ $* 10^{-5} / \mathrm{Pa}$, respectively. Linear regression of logarithm AC vs. age had an $\mathrm{R}^{2}$ of 0.25 and $\mathrm{P}<0.001, \mathrm{n}=80$ (Figure 2). The regression showed stronger correlation in females, $R^{2}=$ $0.34, \mathrm{p}<0.001, \mathrm{n}=46$; while $\mathrm{R}^{2}=0.13$ and $\mathrm{p}=0.04, \mathrm{n}=34$ in males.

Conclusion: Through-plane PC is an easy approach to evaluate aortic compliance in a single breath-hold. The aortic compliance results in normal volunteers using this imaging technique combined with the maximal upslope algorithm for $\Delta \mathrm{t}$ assessment showed a good correlation with age and has the potential to be an efficient clinical tool for assessment of vascular stiffness. Further comparison of this upslope approach to the other algorithms in different flow patterns is needed.

\section{P285}

Accuracy of gadolinium-enhanced cardiovascular magnetic resonance in the diagnosis of cardiac sarcoidosis

Leonidas Tzogias, Mario Njeim, Gurjit Singh, Claudio Schuger, Khalid Nour, Milan V Pantelic and Mouaz $\mathrm{H}$ Al-Mallah

Henry Ford Hospital, Detroit, MI, USA

Journal of Cardiovascular Magnetic Resonance 2009, I I(SuppI I):P285

Introduction: Cardiac arrhythmias are highly prevalent in cardiac sarcoidosis, however identification of cardiac 
involvement in sarcoidosis remains challenging. The role of gadolinium-enhanced cardiovascular magnetic resonance (CMR) in the diagnosis of Cardiac sarcoidosis (CS) is not well established.

Purpose: This study analyzed the accuracy of CMR in the diagnosis of CS among patients with findings suggestive of CS.

Methods: We performed gadolinium-enhanced CMR in 50 consecutive sarcoidosis patients $(63 \%$ females) who presented with symptoms suggestive of cardiac involvement. Imaging protocol included steady-state acquisition cine imaging, T2weighted black blood imaging and post-contrast delayed enhancement on a I.5-T scanner. The diagnostic accuracy of CMR for CS was determined using the Japanese criteria (JMH) as the gold standard.

Results: The diagnosis of CS was made according to JMH criteria in 9 of 50 patients (18\%). CMR revealed patchy late gadolinium enhancement (LGE), mostly involving basal and lateral segments in 12 patients. The presenting symptoms of these patients were AV block, ventricular arrhythmia and heart failure. Using the JMH as the gold standard, the sensitivity, specificity, positive predictive value, negative predictive value and overall accuracy of CMR were $89 \%, 90 \%, 67 \%, 97 \%$ and $90 \%$ respectively. The Kappa agreement between the JMH criteria and CMR was 0.70 . Interestingly, 4 patients had evidence of CS on CMR but did not meet the JMH criteria, while I patient who met JMH had no evidence of LGE.

Conclusion: In patients with sarcoidosis, CMR is a useful diagnostic tool to determine cardiac involvement, and is able to recognize an additional $11 \%$ of patients with probable cardiac sarcoidosis. JMH criteria alone may miss one third of CS cases. While this may be worse in asymptomatic patient, including CMR in the workup of CS may improve the diagnostic yield.

\section{P286}

Diffuse fibrosis in dilated cardiomyopathy results in a shorter myocardial null time

Yuchi Han, Dana C Peters and Warren J Manning BIDMC, Bston, MA, USA

Journal of Cardiovascular Magnetic Resonance 2009, I I(SuppI I):P286

Introduction: Biopsy studies have shown that $60 \%$ of dilated cardiomyopathy (DCM) patients have diffuse myocardial fibrosis. Late gadolinium enhancement cardiac MRI (LGE-CMR) has become an important tool in assessing myocardial fibrosis. Recently, studies have shown that LGE in DCM pertain a worse prognosis, including increased all-cause cardiac mortality. In these studies, LGE is considered present when the peak signal intensity is $>2$ standard deviations (SD) above the remote myocardium. However, in the presence of diffuse fibrosis, there is no remote "normal" myocardium, since the TI of the entire myocardium is decreased. Figure I shows how diffuse fibrosis, with a shorter TI than normal myocardium, will result in a shorter inversion time ( $\mathrm{TI})$ for nulling and a small difference in null points between blood and myocardium (delta$\mathrm{TI}$ ). Because of the shorter $\mathrm{TI}$ required to null diffusely fibrosed myocardium, blood signal in the LGE scan will be
Figure I (abstract P286)

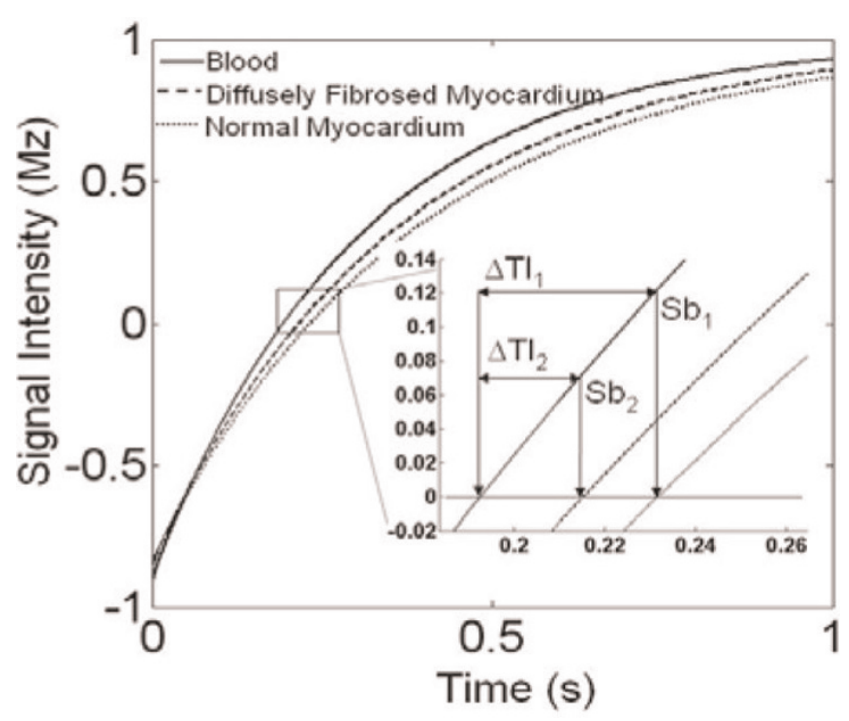

Simulation of magnetization signal intensity regrowth curves of blood $(\mathrm{TI}=300 \mathrm{~ms})$, diffuse fibrosis $(\mathrm{TI}=350 \mathrm{~ms})$ and normal myocardium $(T I=380 \mathrm{~ms})$, after a $180^{\circ}$ pulse. Zoomed view shows the difference in null times between blood, diffusely fibrosed myocardium and normal myocardium $\left(\Delta \mathrm{TI}_{1}\right.$ and $\left.\Delta \mathrm{TI}_{2}\right) . \mathrm{Sb}_{1}$ is the blood signal intensity when normal myocardium is nulled, and $\mathrm{Sb}_{2}$ is the blood signal intensity when diffusely fibrosed myocardium in nulled.

reduced. We sought to measure these effects in clinical scans of DCM patients.

Methods: Ten DCM patients (age $64 \pm 8 \mathrm{yr}, 70 \%$ male) and ten heart-rate matched healthy control subjects (age $26 \pm 9 \mathrm{yr}, 30 \%$ male) were imaged on a I.5 T Philips Achieva MR scanner (Philips HealthCare, Best, NL), equipped with a 5-element cardiac coil, using standard LGE protocol at I5-20 minutes post $0.2 \mathrm{mmol} / \mathrm{kg}$

Figure 2 (abstract P286)

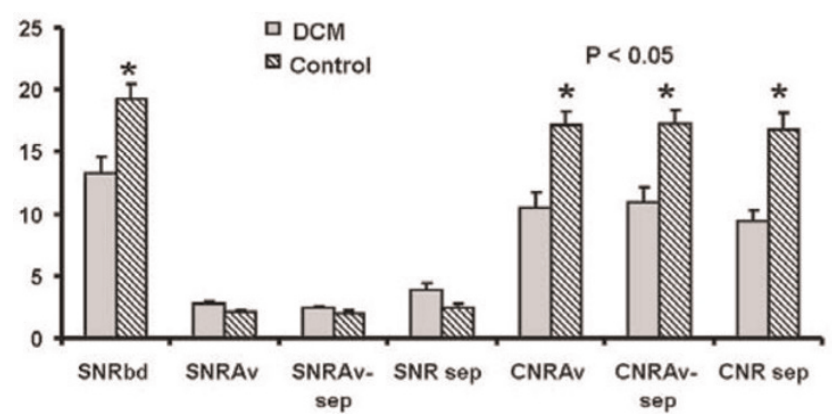

SNR and CNR of the blood and myocardium. SNRbd = SNR of the blood. SNRAv = Average SNR of the myocardium including the septum, anterior wall, lateral wall, and the inferior wall. SNRAv-sep is the average of SNR of the myocardium excluding the septum. SNR sep = SNR of the septal myocardium. CNRAv = Average CNR of the blood to myocardium including all 4 segments. CNRAv-sep $=$ CNR of the blood to mhocardium excluding the septum. CNR sep $=$ CNR of blood to septal myocardium. 
Figure 3 (abstract P286)

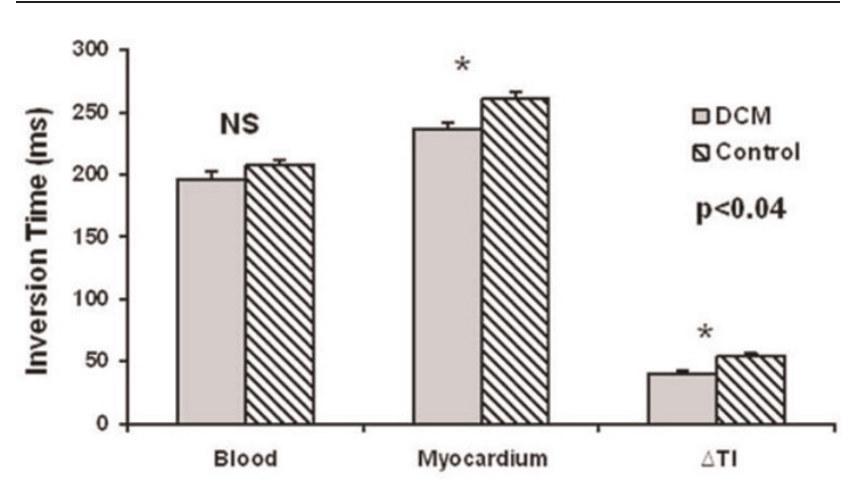

Measured optimal inversion time (TI). DCM patients have lower optimal $\mathrm{TI}$ for myocardium and a smaller difference between the blood and myocardium $\mathrm{TI}(\Delta \mathrm{TI})$ lower.

Gd-DTPA (Magnevist, Berlex, USA) injection. Imaging parameters for the 2D LGE were: 2D spoiled gradient echo inversion recovery, $160 \times 160$ matrix, $320 \mathrm{~cm}$ FOV, $8 \mathrm{~mm}$ slices with $2 \mathrm{~mm}$ gaps, TR/TE/Flip angle $=4.3 \mathrm{~ms} / \mathrm{l} .5 \mathrm{~ms} / 20^{\circ}$, partial echo, fat saturation, I RR between inversions, 2 signal averages. A Look-Locker scan was used to determine the optimal $\mathrm{TI}$ performed at 10-15 minutes post-injection with a breath-hold 2D inversion recovery multi-gradient echo sequence with echo train length of 9 views, TR/flip angle $=40 \mathrm{~ms} / / 5^{\circ}$, I RR between inversions.

Region of interests (ROIs) were used to measure signal to noise ratio and contrast to noise ratio (SNR and CNR) on the shortaxis mid-ventricular slice on the septal, anterior, inferior, and lateral walls, and in the blood pool. Noise was measured as the SD of signal in airspace anterior to the chest wall. The LookLocker data were used to estimate the true zero-crossing for the blood and myocardial signal. ROls were placed in the LV blood cavity and in the septal wall in each phase of the cardiac cycle. The zero-crossing for the signal was estimated using the linear interpolation between the two time points spanning zero signal intensity.

Results: Blood SNR and blood to myocardium CNR were significantly lower in patients with DCM compared to controls $(p<0.05)$, but not the myocardium SNR (Figure 2). CNRs of the DCM patients were all significantly lower than the controls when averaging all walls, versus only the septum, or the rest of the walls without the septum (Figure 2). Figure 3 shows that the optimal $\mathrm{TI}$ to null myocardium was significantly shorter for the DCM patients when compared to controls $(237 \pm 24 \mathrm{~ms}$ vs. $26 \mathrm{I} \pm 23 \mathrm{~ms}, \mathrm{P}=0.034)$. The delta-TI was significantly shorter $(40 \pm 10 \mathrm{~ms}$ vs. $54 \pm 13 \mathrm{~ms}, \mathrm{p}=0.017)$ in DCM patients as compared to controls (Figure 3 ).

Conclusion: DCM patients have significantly reduced blood SNR, a shorter optimal TI, and a shorter delta-TI, as predicted by the simulation in Figure I. This provides evidence for the presence of diffuse fibrosis in patients with DCM. We did not find significant difference in the CNR of the septum vs. the remaining of the walls as reported by others using the remaining walls as remote myocardium. The detection of non-discrete fibrosis is a new aradigm for LGE-CMR.
P287

Assessment of transmural perfusion effect with Blood Oxygen Level-Dependent Cardiovascular Magnetic Resonance Imaging (BOLD-CMR) Jacqueline Flewitt', Matthias Vöhringer², Jordin Green ${ }^{3}$ and Matthias Friedrich'

IStephenson CMR Centre, Calgary, AB, Canada

${ }^{2}$ Robert-Bosch-Krankenhaus, Stuttgart, Germany

${ }^{3}$ Seimens Healthcare Canada, Calgary, AB, Canada

Journal of Cardiovascular Magnetic Resonance 2009, I I (SuppI I):P287

Background: OLD-CMR has successfully been used for the diagnosis of perfusion deficits caused by coronary stenosis. New BOLD-CMR sequences show improved image quality for assessing myocardial oxygenation with excellent spatial resolution, offering a distinct advantage over other image modalities. We hypothesized that these new BOLD-CMR techniques can detect differences in oxygenation throughout the myocardial wall.

Objective: To investigate the epicardial and endocardial oxygenation with a new BOLD-CMR sequence in healthy volunteers compared to patients with suspected coronary artery disease (CAD).

Methods: II healthy volunteers (mean age $29 \pm 4$ years) and I I patients with suspected CAD (mean age $59 \pm 8$ years) who all had abnormal perfusion results on SPECT were recruited for scanning. Using a clinical I.5 T MRI system (MAGNETOM Avanto, Siemens Healthcare, Erlangen, Germany), SSFP BOLDCMR was performed on a mid left ventricular short axis slice at baseline and during adenosine infusion ( $140 \mathrm{micro}-\mathrm{g} / \mathrm{kg}$ ). Typical scan parameters were: Field-of view $193 \times 280 \mathrm{~mm}$; matrix size $106 \times 192$; slice thickness $10 \mathrm{~mm} ; \mathrm{T}_{\mathrm{R}} / \mathrm{T}_{\mathrm{E}} 5.8 / 2.9 \mathrm{~ms}$; flip angle $90^{\circ}$; typical breath-hold duration $14 \mathrm{~s}$. Images were analyzed using clinically validated software $\left(\mathrm{cmr}^{42}\right.$, Circle Cardiovascular Imaging Inc., Calgary, Canada). After automatic definition of the

\section{Figure I (abstract P287)}

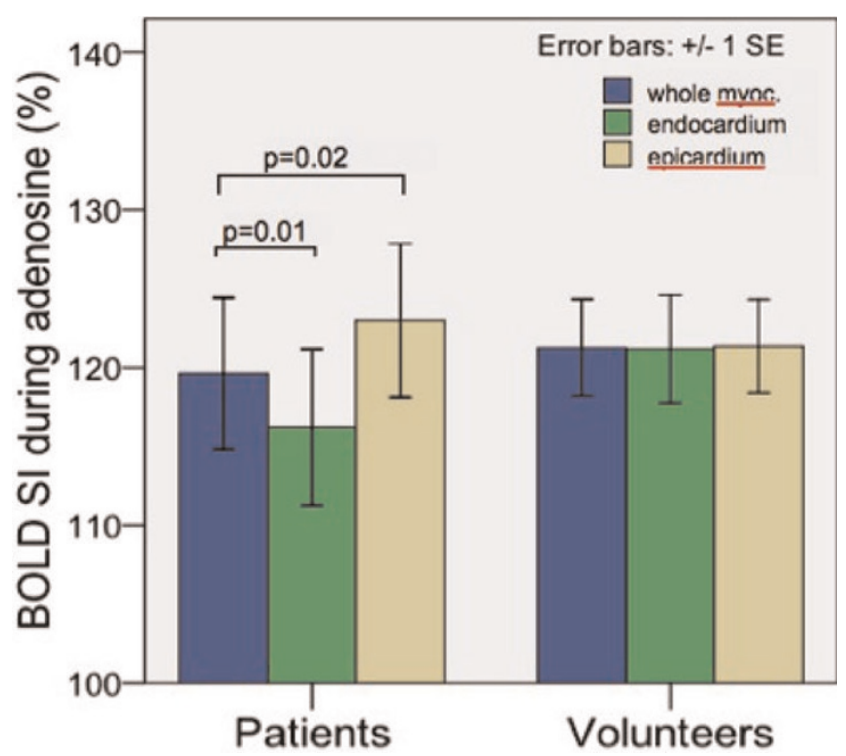

BOLD SI changes for suspected CAD patients and healthy volunteers. 
Table I (abstract P287) Adenosine induced changes in BOLD SI and HR

\begin{tabular}{|c|c|c|c|c|c|c|c|c|}
\hline & \multicolumn{2}{|c|}{$\begin{array}{l}\text { SI rel. to baseline } \\
\text { whole myocardium }\end{array}$} & \multicolumn{2}{|c|}{$\begin{array}{l}\text { SI rel. to baseline } \\
\text { endocardium }\end{array}$} & \multicolumn{2}{|c|}{$\begin{array}{l}\text { SI rel. to baseline } \\
\text { epicardium }\end{array}$} & \multicolumn{2}{|c|}{$\begin{array}{l}\text { HR rel. to } \\
\text { baseline }\end{array}$} \\
\hline & $\%$ & SE (\%) & $\%$ & SE (\%) & $\%$ & SE (\%) & $\%$ & SE $(\%)$ \\
\hline Patients with suspected CAD $(\mathrm{n}=8)$ & 119.6 & 4.8 & $116.2^{*}$ & 5.0 & $123.0 *$ & 4.9 & 148 & 8 \\
\hline Healthy volunteers $(n=11)$ & 121.2 & 3.0 & 121.3 & 3.2 & 121.0 & 2.9 & 129 & 8 \\
\hline
\end{tabular}

$*_{p}<0.05$ compared to whole myocardium.

subendocardial $50 \%$ and the subepicardial $50 \%$ of the wall thickness, the BOLD signal intensity (SI) for each was analyzed and the relative change from baseline during adenosine infusion was calculated. Late enhancement was performed to ensure that patients did not have an infarct in the selected slice for analysis. Results: Image quality was excellent in 8 patients and all healthy volunteers. Three patients were excluded due to motion artifacts on the stress portion of the exam. Results are illustrated in Figure I and summarized in Table I as means and standard error (SE). Heart rate (HR) increased from baseline with the infusion of adenosine. The mean HR increase was $48 \pm 8 \%$ for the suspected CAD patients, and $29 \pm 8 \%$ for the healthy volunteers. The increase in BOLD-SI of the whole myocardium from baseline to adenosine was similar in CAD patients $(19.6 \pm 4.8 \%)$ and in healthy volunteers $(21.2 \pm 3.0 \%)$. In healthy volunteers there was only a marginal difference between the BOLD-SI increase in the endocardium only, and epicardium only (mean difference $0.2 \pm 1.6 \%, p=0.88$ ). However, in the suspected CAD patients, the endocardial BOLD-SI increases were significantly lower than epicardial BOLD-SI increases (mean difference $5.7 \pm 2.2 \%$, $\mathrm{p}=0.03)$.

Conclusion: A state-of-the-art BOLD-sensitive MRI sequence, with its improved image quality and excellent spatial resolution, can detect differences in epicardial and endocardial myocardial oxygenation and are feasible for the clinical setting.

\section{P288}

3D peripheral subtraction MRA using flow-spoiled ECG-triggered balanced SSFP

Zhaoyang Fan', Xiaoming $\mathrm{Bi}^{2}$, John Sheehan', James Carr', Jerecic Renate ${ }^{2}$ and Debiao $\mathrm{Li}^{1}$

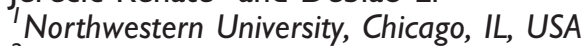

${ }^{2}$ Siemens Medical Solutions, Chicago, IL, USA

Journal of Cardiovascular Magnetic Resonance 2009, I I(SuppI I):P288

Introduction: Peripheral arterial disease (PAD) is a major cause of diminished functional capacity and quality of life in a large portion of western populations. While 3D contrastenhanced (CE) MRA is becoming a modality of choice for clinical PAD examinations, the potential for nephrogenic systemic fibrosis (NSF) in patients with renal insufficiency has triggered a renaissance of interest in non-contrast enhanced (NCE) MRA. Various NCE-MRA strategies employing 3D half-Fourier FSE [I] or balanced SSFP (bSSFP) [2] have shown great promise. Recently, flow-sensitizing dephasing-prepared (FSD) bSSFP was proposed for vessel wall imaging [3]. The present work aimed to investigate the feasibility of MRA in lower legs utilizing FSDbSSFP combined with ECG-triggering and image subtraction.
Figure I (abstract P288)

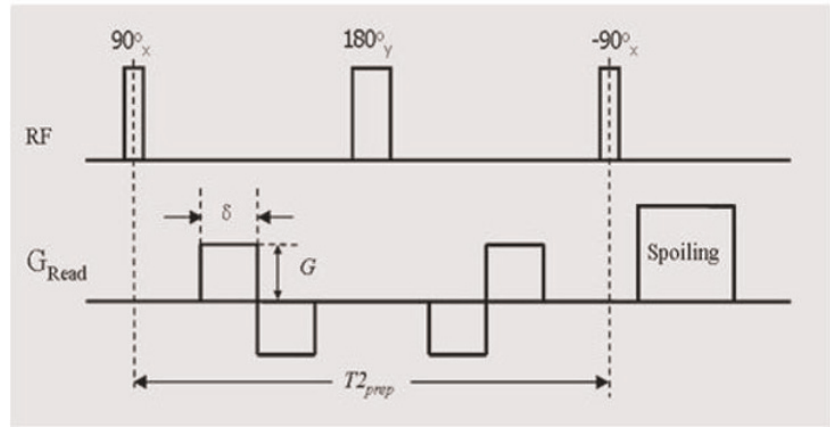

FSD module diagram.

Materials and methods: The FSD module was modified by using bipolar gradient rather than unipolar gradient before and after the center 180-RF pulse to address the artifactual issue resulted from imperfect frequency response (Figure I). Nine healthy subjects (5 M $4 \mathrm{~F}$ ) were imaged at I.5 T (Avanto, Siemens) using a peripheral phased-array coil and spine coils. Phase-contrast flow scan was first performed right above the popliteal trifurcation to derive the arterial flow peak time T. bFFSP scans were then conducted: (I) in 2 subjects, non-FSDbSSFP (bright-blood, BB) scans and FSD-bSSFP (dark-blood, DB) scans (FSD gradient strength $\mathrm{G}=10 \mathrm{mT} / \mathrm{m}$ and duration $\mathrm{d}=1.2$ $\mathrm{ms}$, applied on $\mathrm{x}$-axis) with 5 ECG-trigger delays $(0, \mathrm{I} / 2 \mathrm{~T}, \mathrm{~T}, 3 / 2$ T, 2 T), and arterial blood SNR for each leg was measured from

\section{Figure 2 (abstract P288)}

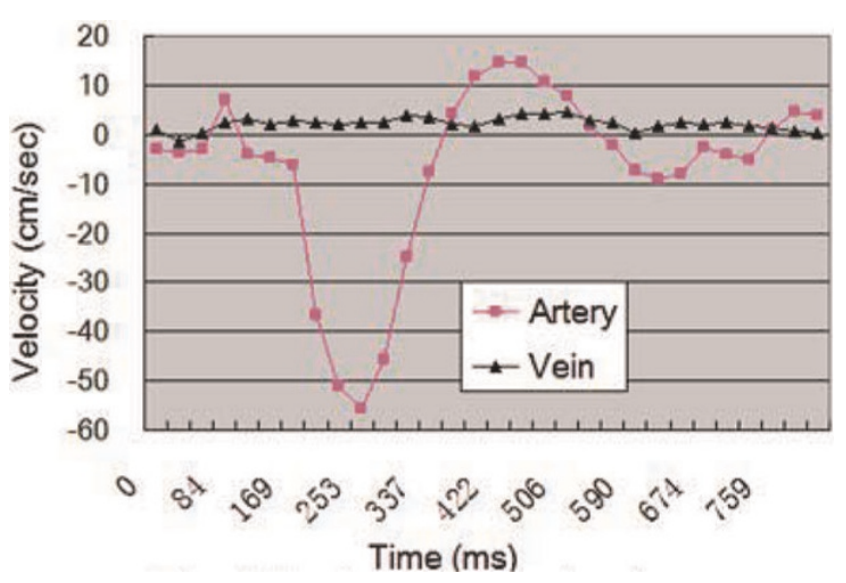

Typical blood velocity curve in one cardiac cycle. 
Figure 3 (abstract P288)

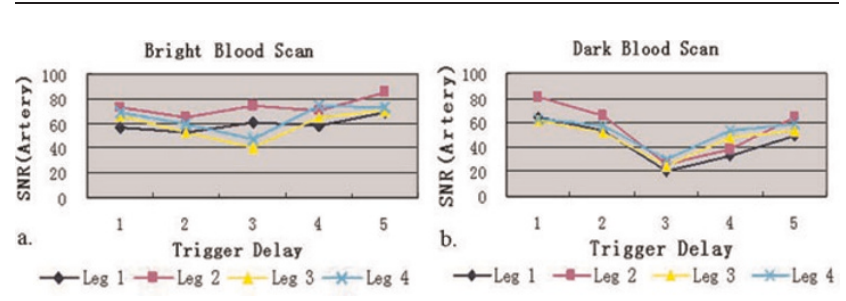

Arterial blood SNR measured from 4 legs in BB (a) and DB scans.

8 ROI's; (2) in 9 subjects, BB scans triggered at mid-diastole $(\sim 2 \mathrm{~T})$ paired with $\mathrm{DB}$ scans triggered at $\sim \mathrm{T}$ using $\mathrm{G}=5,10, \ldots$, $25 \mathrm{mT} / \mathrm{m}(\mathrm{d}=1.2 \mathrm{~ms})$, and MIP's were created from subtraction data sets and reviewed by an radiologist, using a four-grade scale (I, poor; 2, fair; 3, good; 4, excellent). bSSFP imaging parameters: $\mathrm{TE} / \mathrm{TR}=1.9 / 3.8 \mathrm{~ms}$, centric ordering, 3 shots $/$ partition, FOV = $400 \times 311 \times 67 \mathrm{~mm} 3$, matrix $=432 \times 336 \times 72$, spectral fat sat, $\mathrm{BW}=965 \mathrm{~Hz} /$ pixel, GRAPPA factor $=2$, TA $=\sim 3 \mathrm{~min} / \mathrm{scan}$.

Results: Average velocity within the lumen was considerably higher in the arteries than in the accompanying veins (Figure 2). In BB scans, higher arterial-blood SNR was achieved during diastole (Figure 3a), whereas DB scans showed superior flow void during systole (Figure $3 \mathrm{~b}$ ). On both scans, venous blood signal was barely affected. When FSD strength stepped from 5 to $25 \mathrm{mT} / \mathrm{m}$, the arterial signal on the subtraction images were generally improved but venous contamination became problematic (Figure 4). In 18 legs, the counts of score 4 for $\mathrm{G}=5$, $10, \ldots, 25 \mathrm{mT} / \mathrm{m}$ are 10(56\%), II (6I\%), 7(39\%), 0(0\%), 2(II\%), respectively.

Discussion and conclusion: In the case of a laminar flow, the faster average velocity and/or greater first gradient moment $\mathrm{ml}$ conveyed by FSD, the higher likelihood the flowing spins are dephased and thus suppressed. Hence, the arterial blood is more susceptible to FSD with a weak $\mathrm{ml}$ compared with venous blood

Figure 4 (abstract P288)

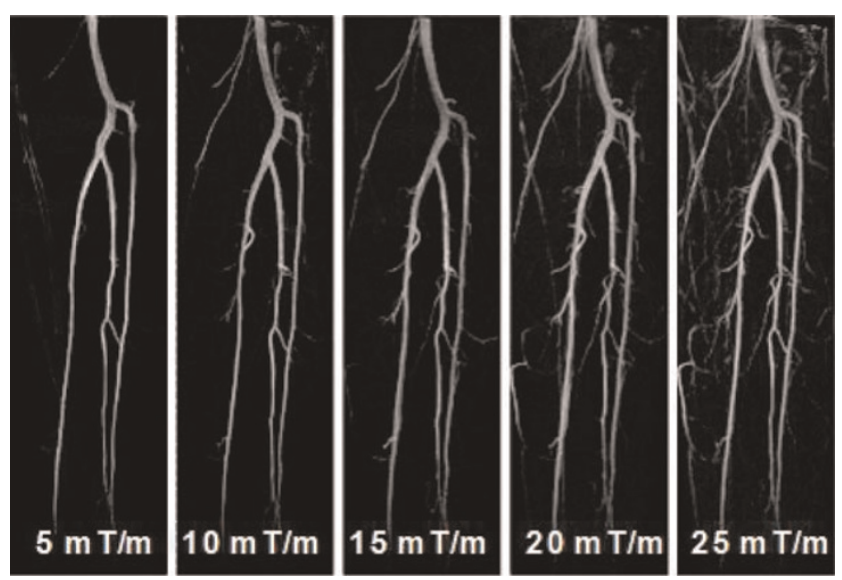

MIP images after subtraction. AS the FSD gradient increased, more arteries were shown, but venous signal might have interfered with artery visualization. during systole. Since bSSFP is not truly flow-compensated, however, bright blood scan can achieve substantially high arterial blood signal during mid-diastole. For those reasons, ECGtriggering facilitates subtraction MRA. The results indicate that FSD gradient strength, or more accurately $\mathrm{ml}$, should be controlled to a low level (for lower legs here, $G=10 \mathrm{mT} / \mathrm{m}$, $\mathrm{ml}=34.8 \mathrm{mTms} 2 / \mathrm{m}$ ) to selectively suppress arterial blood and to avoid otherwise venous contamination. The feasibility of this approach was demonstrated on healthy distal lower extremities. Further investigation on PAD patients, with CE-MRA or x-ray angiography correlation, is warranted. It is anticipated that this strategy could be applied to other vascular territories where appropriate choice of $\mathrm{ml}$ (magnitude and direction) would vary with the specific flow patterns.

\section{References}

I. Miyazaki M, et al: Radiology 2003, 227:890.

2. Stafford R, et al: MRM 2008, 59:430.

3. Koktzoglou I, et al: JCMR 2007, 9:33.

\section{P289}

Relationship between CMR and ECG-derived indices of left ventricular hypertrophy

Richard M Nethononda, Barbara Casadei, Polly Whitworth, Jane Francis, Nicola Alder, Hugh Watkins and Stefan Neubauer University of Oxford, Oxford, UK

Journal of Cardiovascular Magnetic Resonance 2009, I I(SuppI I):P289

Background: Left ventricular hypertrophy (LVH) is associated with increased risk of cardiovascular events. ECG is the most commonly used technique for detecting LVH. CMR is the gold standard for measurement of cardiac parameters including LV mass. The objective of this study was therefore to investigate the relationship between ECG- and CMR-derived indices of LVH in a cohort with substantial prevalence of LVH.

Methods: ECG and CMR data of subjects from the Oxford Family Blood Pressure cohort was evaluated. An ECG was obtained from each subject. QRS duration was measured from the beginning of the $\mathrm{Q}$ wave to the end of the $\mathrm{S}$ wave. SokolowLyon voltage was defined as SVI+RV5. Cornell voltage was defined as RaVL+SV3. The 12-lead sum voltage was the sum of QRS amplitudes in all 12 leads. These voltage indices were multiplies by the QRS duration as an expression of their time voltage index. ECG-derived LV mass was calculated according to the methods of Siegel. Short-axis end-expiration cine images of the left ventricle were acquired from each subject using a I.5 T scanner and analysed. Categorical and continuous variables were compared using Chi-squared and Student's t-test, respectively. Pearson's correlation was used to evaluate the strength of the relationship between ECG and CMR parameters. The BlandAltman method was used to evaluate agreement between LVM measured by both ECG and CMR. P value $<0.05$ was considered significant.

Results: There were 152 subjects, 83 females. Hypertension was more prevalent, and ambulatory BP as well as waist-to-hip ratios was higher amongst males. QRS duration, Sokolow-Lyon, I2-lead sum of QRS voltage and ECG-derived LVM were significantly larger in males than in females. Males had larger left ventricular mass and wall thickness. In males, there was a 
Table I (abstract P289) Correlation coefficients (r) between ECG and CMR parameters in males

\begin{tabular}{llll}
\hline & $\begin{array}{l}\text { Total } \\
\text { edwt }(\mathbf{m m})\end{array}$ & $\begin{array}{l}\text { Total } \\
\text { eswt }(\mathbf{m m})\end{array}$ & $\begin{array}{l}\text { CMR } \\
\text { LVM (g) }\end{array}$ \\
\hline QRS duration $(\mathrm{msec})$ & $0.32^{* *}$ & $0.46^{* * *}$ & $0.49^{* * *}$ \\
SL voltage duration $(\mathrm{mV} / \mathrm{msec})$ & 0.13 & $0.24 *$ & $0.35^{* *}$ \\
Cornell duration $(\mathrm{mV} / \mathrm{msec})$ & $0.26^{*}$ & $0.27^{*}$ & $0.37^{* *}$ \\
I2-lead sum duration $(\mathrm{mV} / \mathrm{msec})$ & $0.26^{*}$ & $0.35^{* *}$ & $0.43^{* * *}$ \\
ECG LVM (g) & $0.40^{* *}$ & $0.44^{* * *}$ & $0.62^{* * *}$ \\
\hline
\end{tabular}

edwt and eswt, the sum of end-diastolic and end-systolic wall thickness; CMR, cardiac magnetic resonance; LVM/I, left ventricular mass and mass index; SL, Sokolow-Lyon. ${ }^{*} p<0.05$, ${ }^{*} *_{p}<0.01$ and $* * * p<0.001$.

significant correlation between CMR LV mass and ECG indices such as QRS duration, I2-lead sum duration, Sokolow-Lyon duration and Cornell duration (Table I). QRS duration demonstrated the highest correlation with wall thickness, whilst both the 12-lead sum and Cornell voltages demonstrated marginally significant correlation with this parameter. In contrast, females (Table 2) showed no significant correlations between CMR LV mass and ECG indices such as QRS duration, 12 lead sum of voltages and Sokolow-Lyon voltages. Only Cornell voltage showed a marginally significant correlation with CMR LVM. Similarly, both QRS duration and 12-lead sum of QRS voltages showed no significant correlations with LV wall thickness. There was only marginally significant correlation between LV wall thickness and both the Sokolow-Lyon and Cornell voltage indices. The $95 \%$ limits of agreement between LV mass measured by ECG and by CMR were $-53.3 \mathrm{~g}$ to $54.7 \mathrm{~g}$ indicating that ECG overestimates LVM in those with LVM in the lower ranges and underestimates it in those with LVM in the upper ranges.

Conclusion: The lower correlations in indices of left ventricular hypertrophy measured using the two techniques possibly reflect the different components of, and physiological processes within myocardium that they each measure. ECG measures electrical activity within myocardium, whilst CMR measures total myocyte mass, non myocyte cells and interstitial components. The difference in chest geometry between genders is a possible explanation for the poor correlations of ECG vs. CMR indices of left ventricular hypertrophy in females compared to male subjects.

Table 2 (abstract P289) Correlation coefficients (r) of ECG and CMR variables in females

\begin{tabular}{llll}
\hline & $\begin{array}{l}\text { Total } \\
\text { edwt }(\mathbf{m m})\end{array}$ & $\begin{array}{l}\text { Total } \\
\text { eswt }(\mathbf{m m})\end{array}$ & $\begin{array}{l}\text { CMR } \\
\text { LVM(g) }\end{array}$ \\
\hline QRS duration (msec) & 0.09 & 0.16 & 0.21 \\
SL voltage duration $(\mathrm{mV} / \mathrm{msec})$ & 0.19 & $0.30^{* *}$ & 0.21 \\
Cornell duration $(\mathrm{mV} / \mathrm{msec})$ & $0.22^{*}$ & $0.27^{*}$ & $0.29^{*}$ \\
I2-lead sum duration $(\mathrm{mV} / \mathrm{msec})$ & 0.13 & 0.13 & 0.18 \\
ECG LVM (g) & $0.25^{*}$ & $0.34^{* *}$ & $0.35^{* *}$ \\
\hline
\end{tabular}

edwt and eswt, end-diastolic and systolic wall thickness; CMR, cardiac magnetic resonance; LVM, left ventricular mass; SL, Sokolow-Lyon. $*_{p}<0.05$ and $* *_{p}<0.01$

\section{P290}

Fast and robust visual inspection of the coronary arteries based on live path tracking

Javier Olivan Bescos, Jeroen Sonnemans

and Marcel Breeuwer

Philips Healthcare, Best, Netherlands

Journal of Cardiovascular Magnetic Resonance 2009, I I(SuppI I):P290

Introduction: A multitude of visualization techniques are available to inspect the coronary arteries in Steady-State FreePrecession (SSFP) MRI cardiac data, both 2D (planar, curved, and straightened reformatting) and $3 D$ (volume rendering and maximum intensity projection). The workflow required to generate the visualizations includes user interactions such as segmentation, path tracking, and geometry manipulation (rotating, zooming, panning, etc). When these interactions are performed manually, the workflow becomes time consuming, and the inter-user reproducibility is low.

Purpose: We describe a method that optimizes the workflow of local visual inspection of the coronary arteries by reducing the user interaction to a single mouse click. No a priori segmentation or path tracking is required, and the optimal position, orientation, and zoom factor of the views are found automatically, thereby increasing the efficiency and reducing and inter-user variability.

Methods: The method presented here should be considered as an advanced magnifying glass applied to the coronaries. When the user needs to inspect a particular location at one of the coronaries, a single mouse click locally computes a vessel centerline at the cursor position (yellow arrow in Figure I). The length of the centerline can be modified with the mouse wheel at any time. A cross section and curvilinear reformatted image and a slab volume rendering aligned with the vessel are automatically generated from the local path (Figure I). When necessary, the user can easily navigate along the selected vessel by hovering the

\section{Figure I (abstract P290)}

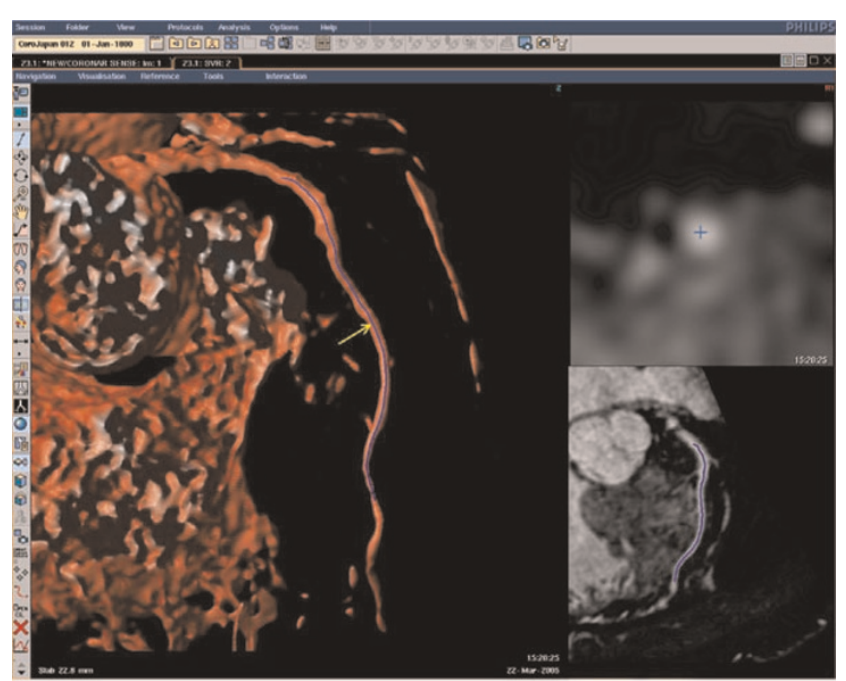

The whole image was generated from a single mouse click (yellow arrow). Based on the local center line (in blue), a slab volume rendering (left), a cross section (top right), and a curvilinear reformat (bottom right) images were generated. 
mouse along the vessel in any of the views or by pressing the up/ down keys. During navigation, the user may select different vessels with a single mouse click at any time.

Results: The accuracy of the local path tracking has been evaluated on II data sets used in the work presented in [I]. A local centerline of $5 \mathrm{~cm}$ was computed with the local path tracking algorithm at the middle point of each golden standard path. The local path was compared to the golden standard using the Repeated Averaging Algorithm [2]. The mean error was 0.32 $\mathrm{mm}$, with a standard deviation of $0.08 \mathrm{~mm}$.

Our path tracking technique is very fast, it takes only tenths of a second including the generation of the visualizations. The user thus receives live feedback about the local coronary anatomy. The fact that all visualizations are automatically properly aligned decreases the inter-user variability associated with manual interactions. When different users click at about the same location, the same renderings will be generated.

Conclusion: The method presented in this article greatly improves the workflow required to inspect the coronaries locally, while at the same time reducing the inter-user variability. A single mouse click generates optimal visualizations of the local geometry of the coronary vessels.

References

I. Sonnemans J: Proc SCMR 2008.

2. Chalana V, et al: IEEE TMI 1997, I6(5):642-652.

\section{TECHNOLOGIST PRESENTATIONS}

\section{TI}

In/opposed phase imaging effectively differentiates fat from enhanced myocardium in patients with myocardial late gadolinium enhancement

Denise Kleindienst, Hassan Abdel-Aty, Wolfgang Utz, Andre Rudolph and Jeanette Schulz-Menger Franz-Volhard-Klinik, Kardiologie, Charité Campus Buch, Universitätsmedizin Berlin, Helios-Klinikum, Berlin, Germany

Journal of Cardiovascular Magnetic Resonance 2009, I I (SuppI I):T I

Background: Suppressing fat in late gadolinium enhancement (LGE) images is a challenging task in a variety of clinical settings. Both fat and LGE appear bright in these images making it difficult to quantify the exact extent of LGE or even in some cases e.g. in myocarditis to establish the presence of late enhancement, which because of its subepicardial location may be easily confused with pericardial fat. We tested the clinical utility of a novel in/opposed phase LGE sequence in this setting.

Methods: We implemented a cardiac gated two-dimensional inversion recovery prepared pulse sequence to acquire gradient echo images (in and opp.-LGE) with two echo times (2.4 and 5.0 ms) 10 minutes after the IV injection of gadolinium DTPA (0.2 $\mathrm{mmol} / \mathrm{Kg} \mathrm{BW}$ ) in a single breath hold. This was achieved at the cost of an increase in bandwidth (I40 vs. $400 \mathrm{~Hz} / \mathrm{Px}$ ) in comparison to the standard LGE sequence. All other sequence parameters including the inversion time were kept constant (flip angle 30 , matrix I $48 \times 256$, slice thickness/gap: $7 / 3 \mathrm{~mm}$ ) Images were acquired twice once using the conventional non-fat sat LGE (con-LGE) sequence and then using the proposed in/opposed phase approach in 28 patients with positive LGE secondary to ischemic and non-ischemic conditions. Images were evaluated both quantitatively and qualitatively. Contrast to noise (CNR)
Figure I (abstract TI)

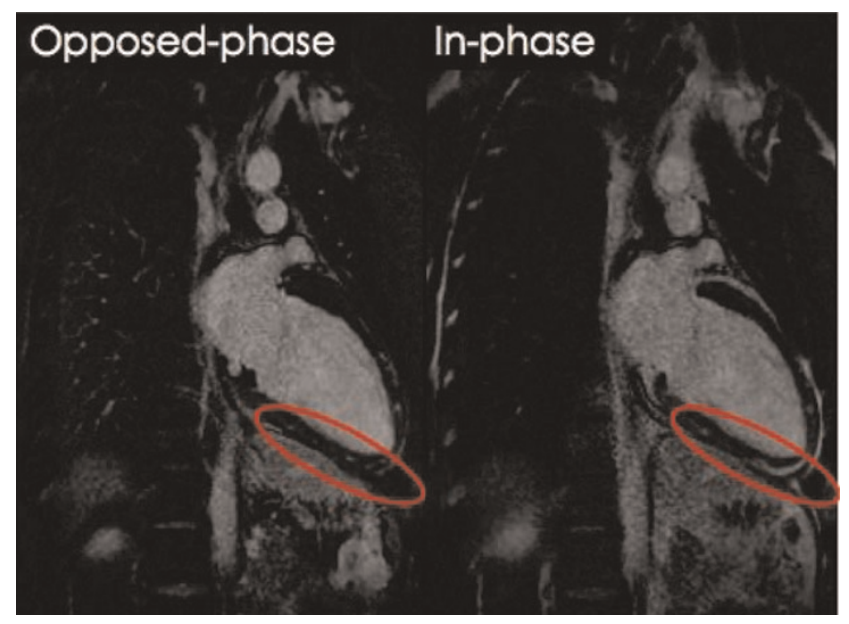

ratios between fat, normal and enhanced myocardium were measured. One independent observer visually evaluated the diagnostic quality of all images.

Results: Fat was homogenously suppressed over the entire FOV in all cases. The CNR between fat and normal myocardium decreased significantly in opp-LGE compared to con-LGE $(49.5 \pm 20$ vs. $10.6 \pm 8 ; \mathrm{p}<0.000 \mathrm{I})$ indicating effective fat suppression. Furthermore, the CNR between LGE areas and the pericardial fat increased significantly in opp-LGE compared to con-LGE ( $10.9 \pm 12$ vs. $-9.9 \pm 22$; $p<0.0001)$ implying better LGE-fat differentiation. The qualitative assessment proved the sequence robustness over a wide range of indications and that this approach could provide additional diagnostic information over that obtained from con-LGE in $2 / 28$ patients. Figure I.

Conclusion: We provide a first clinical utilization of a novel fat suppression late enhancement imaging combining in/opposed phase imaging with conventional inversion recovery gradient echo sequences. This approach enables effective differentiation between fat and enhanced myocardium, which should result in accurate LGE quantification.

\section{T2}

Is the pattern of LVH regression in patients with severe aortic stenosis altered by the sex of the patient? A 4-year pre and post aortic valve surgery study

Saundra B Grant, Diane A Vido, Vikas K Rathi, June A Yamrozik, Ronald B Williams, Geetha Rayarao, Mark Doyle and Robert WW Biederman Allegheny General Hospital, Pittsburgh, PA, USA

Journal of Cardiovascular Magnetic Resonance 2009, I I(SuppI I):T2

Introduction: Numerous investigators have suggested that woman are particularly prone to develop an exaggerated LV hypertrophy $(\mathrm{LVH})$ response to increased developed pressure as compared to men. This has been best exemplified in both HTN and aortic stenosis (AS) states and has been proposed euphemistically as 'exuberant hypertrophy'. This reference implies that there is an inappropriate physiologic amplification to the typical and necessary hypertrophic response for any given 
blood pressure/afterload experienced by the LV. Furthermore, it suggests that there is a divergent response at the gene level to explain this phenomenon. The implications for this are vast, potentially calling for distinct sex-based clinical treatment programs. The societal ramifications for differential therapy are immense as our resources are limited. On the other hand, the majority of the inferences of this dichotomized observation have stemmed from 2D Echocardiography and consequently may be artifactual if evaluated in a 3D CMR manner.

Hypothesis: We hypothesize that 'exuberant hypertrophy' is a misnomer and that after aortic valve replacement (AVR) there will be no sex difference in the manner or rate in which LVH regresses following afterload relief.

Methods: Following initial recruitment of 35 patients for a longitudinal 2-year CMR study, a series of patients who were alive and available were specifically contacted and re-recruited for follow-up up to 4 years from their initial AVR. All patients underwent an additional CMR for evaluation of $3 D$ volumetrics including LV mass and EF. Using Simpson's rule, contiguous $8 \mathrm{~mm}$ slices were acquired via SSFP imaging and underwent LV endocardial and epicardial manual contouring (GE I.5 T EXCITE, Milwaukee, WI). Pre and post-AVR LV mass and EF were compared and stratified for sex. A 'P' value of $<0.05$ was considered statistically significant.

Results: Ten (10) patients were available for CMR at $3 \pm 1$ yrs out to 4 years at follow-up post-AVR and successfully underwent CMR. Woman (6; age: $68 \pm 14$ ) and men (4; age: $68 \pm 10$ ); ( $\mathrm{P}<\mathrm{NS}$ for both) were imaged. Baseline LV mass index was not either increased in females nor different between woman and

Figure I (abstract T2)

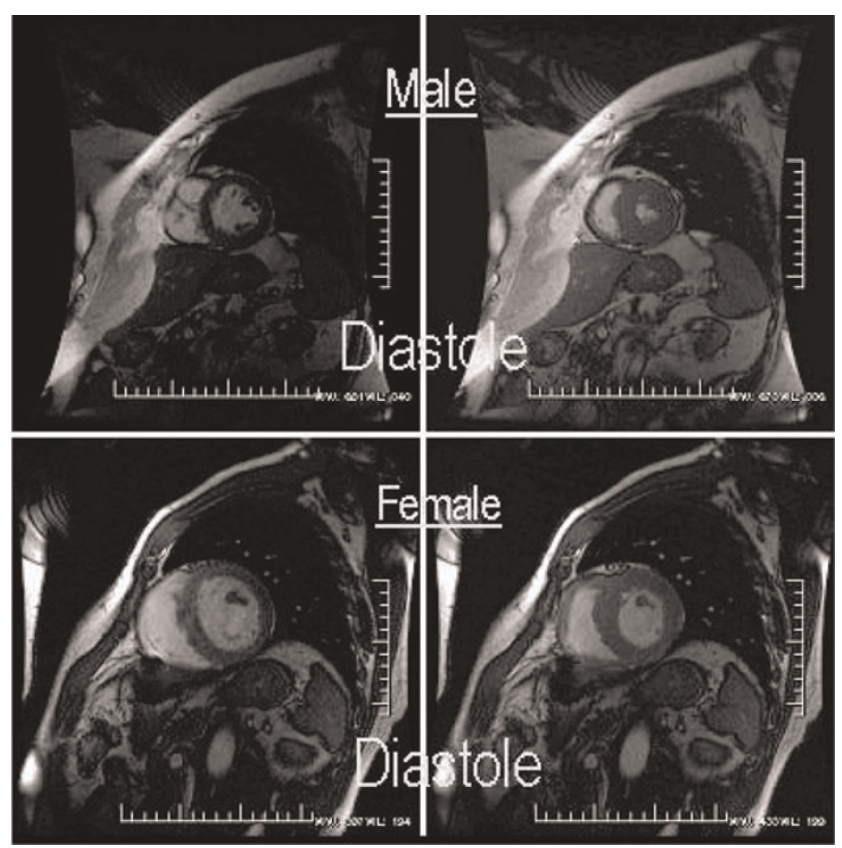

2D CVMRI images of geometry in A) 65 YO femal with a small thick LV and B) 68 YO male with a larger, thinner LV, both with similar mean gradient $(53 \pm 4 \mathrm{mmHg})$, BSA $(2.1 \pm \mathrm{I})$, and LVMI corrected for EDV $(\mathrm{I} .33 \pm 0.10)$ and with similar LVMI. men $\left(75 \pm 16\right.$ vs. $\left.101 \pm 23 \mathrm{~g} / \mathrm{m}^{2}, \mathrm{p}=\mathrm{NS}\right)$. However, baseline EF was dissimilar between woman and men $(67 \pm 12$ vs. $37 \pm 23 \%$, $\mathrm{P}=\mathrm{NS})$. Following AVR, in absolute or relative terms, $\mathrm{LV}$ mass index regression was not different between the sexes $(65 \pm 17$ vs. $73 \pm \mathrm{II} \mathrm{g} / \mathrm{m}^{2}$; representing a proportionate decrease in LV mass of $\mathrm{II} \pm 16$ vs. $28 \pm 17 \mathrm{~g} / \mathrm{m}^{2}, \mathrm{p}=\mathrm{NS}$ ), (see Figure I). Likewise, post-AVR, LVEF was not significantly different (68 \pm 7 vs. $61 \pm 10 \%, p=N S$; but did markedly improve in the males ( $1 \pm 10$ vs. $24 \pm 20 \%, p<0.05$ ), respectively for woman vs. men, again without any absolute change in LV mass index between groups.

Conclusion: While conceptually intriguing and highly controversial, the sex-based differences of exaggerated LVH formation in response to pressure afterload that are based substantially on 2D echocardiographic acquisitions appear to be artificial when based upon a 3D CMR interrogation. Specifically, after recruiting a select group of patients with AS who underwent AVR with up to 4 years of follow-up; more than sufficient time to allow for regression of LV mass to near completion if it was going to, failed to demonstrate a significant difference as related to woman vs. men. Beyond negating the 2D observation by a more sophisticated 3D CMR interrogation, the potential socio-economic repercussions, should a more favorable response to afterload have been found (either at baseline or after AVR) are nullified. This finding may restore the notion that there are neither phenotypic nor likely genotypic alterations in the LV response to pressure afterload in woman vs. men.

T3

Acquiring multiple slices in a single breath-hold. Is it practical for routine workflow?

Ricardo Wage, John-Paul Carpenter and Dudley J Pennell Royal Brompton and Harefield NHS Trust, London, UK

Journal of Cardiovascular Magnetic Resonance 2009, I I (SuppI I):T3

Introduction: The acquisition of the ventricular short axis cine stack forms the backbone of the routine cardiovascular magnetic resonance scan [I]. From this, the left and right ventricular volumes are calculated and important information is gained about wall thickness, regional function and evidence of dyssynchrony. Steady-state free precession cine (SSFP) loops can take between 8 and 12 seconds each to acquire and traditionally, a single breath-hold has been required for each slice. Parallel imaging technology has allowed reduction in the time taken for each

Figure I (abstract T3)
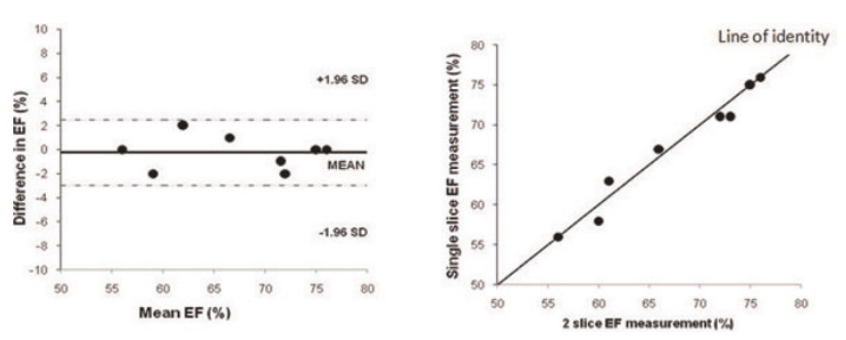

Bland-Altman plot of difference in ejection fraction (EF) and scatter plot using 2-slice versus single slice acquisition. 
Table I (abstract T3) Coefficient of variation between scan techniques for single-slice and two-slice per breath-hold acquistion.

\begin{tabular}{lllll}
\hline Parameter & $\begin{array}{l}\text { Mean } \\
\text { value }\end{array}$ & $\begin{array}{l}\text { Mean difference } \\
\text { between techniques } \\
\text { (+l- in brackets) }\end{array}$ & $\begin{array}{l}\text { Average coefficient } \\
\text { of variation (\%) }\end{array}$ \\
\hline LV end-diastolic volume (ml) & 144.7 & $3.4(6.1)$ & 4.23 & P value \\
LV end-systolic volume (ml) & 46.7 & $1.1(3.72)$ & 7.97 & 0.41 \\
Stroke volume (ml) & 97.8 & $2.4(3.5)$ & 3.58 & 0.43 \\
LV mass (g) & 133.3 & $2.2(9.4)$ & 2.04 & 0.43 \\
Ejection fraction (\%) & 67.3 & $0.25(1.39)$ & 0.45 \\
\hline
\end{tabular}

acquisition without a significant drop in signal-to-noise ratio. As a consequence, it is possible to acquire two slices for each breathhold. The scanner can be easily programmed to acquire a set of equally spaced ventricular short axis slices [2].

Purpose: The purpose of this study was to assess the practicality of using a two-slice per breath-hold ventricular short axis cine sequence in routine daily practice.

Methods: From the beginning of March 2008 to September 2008, we used a two-slice per breath-hold steady state free precession sequence to acquire the ventricular short axis stack of cines in a total of $\mathbf{4 7 8}$ patients. All patients were scanned with a I.5 T Siemens scanner (Sonata or Avanto, Siemens, Erlangen, Germany) using anterior phased-array coils and ECG gating. Sequence parameters for the SSFP cine were as follows: 2 slices ( $8 \mathrm{~mm}$ slice thickness), $25 \%$ distance factor ( $2 \mathrm{~mm}$ gap), TR $40.2 \mathrm{~ms}$, TE I.13 ms, flip angle $80^{\circ}$, base resolution 192, number of signal averages I, parallel imaging (GRAPPA; generalised autocalibrating partial parallel acquisition), bandwidth $930 \mathrm{Hx} / \mathrm{Px}$, echo spacing $2.7 \mathrm{~ms}$. Ten patients had both two-slice and single slice per breath-hold cine stack acquired. The parameters for the single slice acquisition were identical to those given above apart from using a $7 \mathrm{~mm}$ slice thickness with $3 \mathrm{~mm}$ gap.

Results: Since the beginning of the study, there have been no problems with the acquisition of the short axis stack using this technique. The images have all been suitable for analysis and calculation of ventricular volumes despite slightly increased partial volume effects towards the apex as a result of using an $8 \mathrm{~mm}$ slice thickness. In patients who had undergone both techniques, there was a low coefficient of variation between the two with no significant difference in volume or ventricular function calculations (see Figure I and Table I).

Conclusion: In conclusion, the use of a two-slice per breathhold cine acquisition is a practical method for use in daily practice. This shortens the time required for the whole ventricular short axis cine stack and allows a streamlining of workflow with overall reduction in the time taken for each CMR study. There is no significant difference in the ventricular volumes, mass or ejection fraction calculated.

References

I. Bellenger NG, Grothues F, Smith GC and Pennell DJ: Quantification of right and left ventricular function by cardiovascular magnetic resonance. Herz 2000, 25(4):392-9.

2. Hogan MC, Petersen SE and Hudsmith LE, et al: Effects of steady state free precession parameters on cardiac mass, function and volumes. Int J Cardiovasc Imaging 2007, 23:583-589.
T4

Does moving away from the center frequency resonance benefit or hurt the valvular structure evaluation? A study of on and off peak resonance Ronald B Williams, June Yamrozik, Vikas K Rathi, Geetha Rayarao, Robert WW Biederman and Mark Doyle Allegheny General Hopital, Pittsburgh, PA, USA

Journal of Cardiovascular Magnetic Resonance 2009, I I(SuppI I):T4

Introduction: Cardiac MRI (CMR) is the diagnostic modality of choice for ventricular volumes and function. The qualitative and quantitative valve function by CMR is performed by SSFP and phase contrast imaging respectively. However, often times the valve and subvalvular morphologic details are not clearly visualized in a reproducible fashion limiting the complete interrogation of the heart.

Hypothesis: We propose that adjusting the center frequency resonance (CFR) as determined by the MRI interface for each sequence may, counterintuitively, produce optimal images for valve definition. By adjusting the center frequency manually to

Figure I (abstract T4)

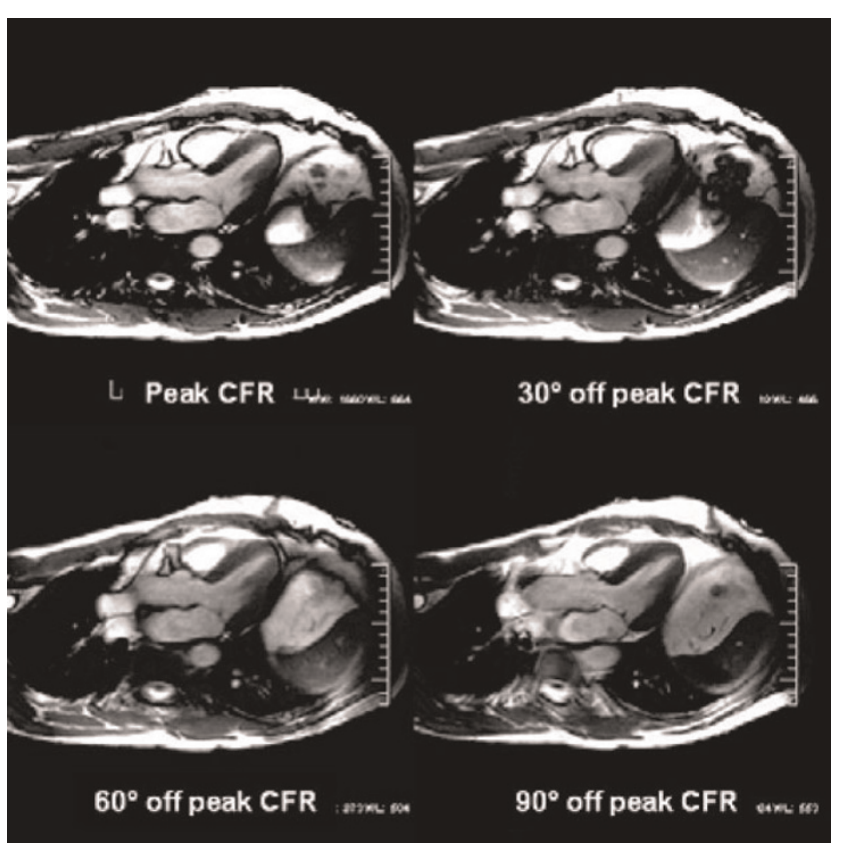


Table I (abstract T4)

\section{Subject I}

\begin{tabular}{lllrr}
\hline Series & Resonance frequency (Hertz) & Transmit gain & RI gain & R2 gain \\
\hline Peak CFR & 63868605 & 117 & 13 & 15 \\
$30^{\circ}$ off Peak CFR & 63868577 & 117 & 13 & 15 \\
$60^{\circ}$ off Peak CFR & 63858341 & 117 & 13 & 15 \\
$90^{\circ}$ off Peak CFR & 63868585 & 117 & 13 & 15
\end{tabular}

\section{Subject 2}

\begin{tabular}{llllll}
\hline Series & Resonance frequency (Hertz) & Transmit gain & RI gain & R2 gain & Tuning method \\
\hline Peak CFR & 63868553 & 128 & 13 & 15 & $\mathrm{~A}$ \\
$30^{\circ}$ off Peak CFR & 63868536 & 128 & 13 & 15 & $\mathrm{M}$ \\
$60^{\circ}$ off Peak CFR & 63868505 & 128 & 13 & 15 & $\mathrm{M}$ \\
$90^{\circ}$ off Peak CFR & 63868474 & 128 & 13 & 15 & $\mathrm{M}$ \\
\hline
\end{tabular}

Table 2 (abstract T4)

\begin{tabular}{|c|c|c|c|c|c|}
\hline \multirow{2}{*}{$\begin{array}{l}\text { Mitral valve leaflet } \\
\text { measurements }\end{array}$} & \multirow[t]{2}{*}{ Image } & \multirow{2}{*}{$\begin{array}{l}\text { SI mitral } \\
\text { leaflet }(\mathbf{m m})\end{array}$} & \multirow{2}{*}{$\begin{array}{l}\text { S3 mitral } \\
\text { leaflet }(\mathrm{mm})\end{array}$} & \multicolumn{2}{|c|}{ Difference between each degree offset } \\
\hline & & & & SI/S3 & Comparison to Peak CFR \\
\hline \multirow[t]{4}{*}{ Subject A } & Peak CFR & 2.1 & 2.2 & & \\
\hline & $30^{\circ}$ off peak CFR & 2.0 & 2.1 & $0.1 / 0.1$ & $0 / 30$ degree \\
\hline & $60^{\circ}$ off peak CFR & 2.0 & 1.9 & $0.1 / 0.3$ & $0 / 60$ degree \\
\hline & $90^{\circ}$ off peak CFR & 2.0 & 1.8 & $0.1 / 0.4$ & $0 / 90$ degree \\
\hline \multirow[t]{4}{*}{ Subject B } & Peak CFR & 2.9 & 2.9 & & \\
\hline & $30^{\circ}$ off peak CFR & 2.4 & 2.4 & $0.5 / 0.5$ & $0 / 30$ degree \\
\hline & $60^{\circ}$ off peak CFR & 2.2 & 2.4 & $0.7 / 0.5$ & $0 / 60$ degree \\
\hline & $90^{\circ}$ off peak CFR & 2.1 & 2.2 & $0.8 / 0.7$ & 0/90 degree \\
\hline
\end{tabular}

Figure 2 (abstract T4)

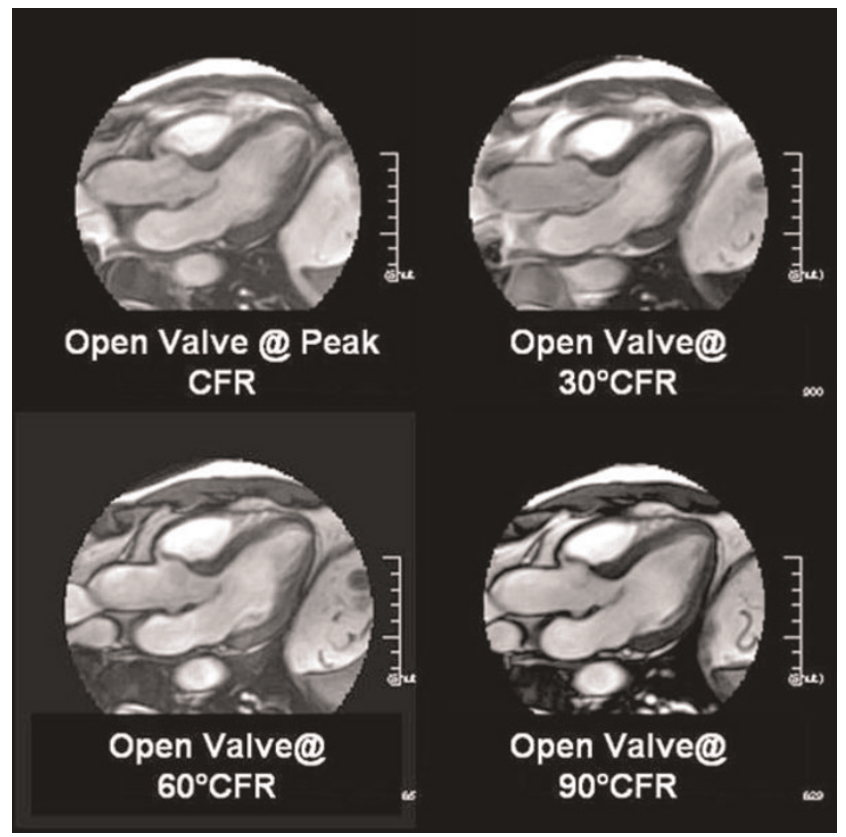

Figure 3 (abstract T4)

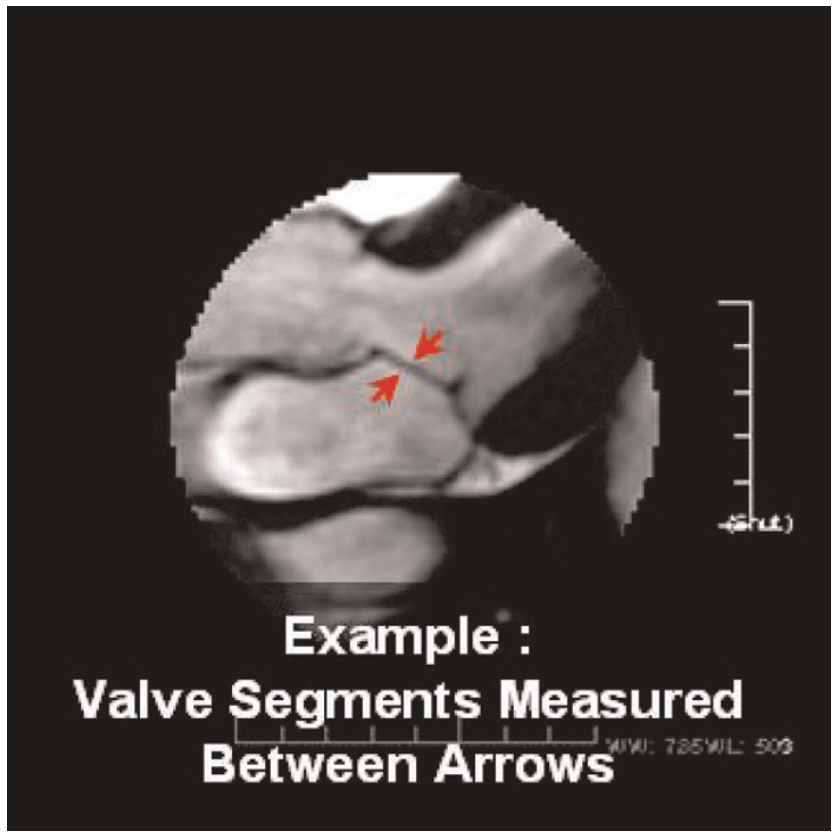


'off' resonance, we hypothesize that CFR can exaggerate the susceptibility around the valves, making the valves easier to visualize.

Methods: We scanned two healthy subjects without prior knowledge of their cardiac valvular structure or function. The imaging protocol was limited to the mitral valve and was performed on a I.5 T GE HD Excite (Milwaukee, WI), 8 channel cardiac array coil; using the SSFP sequence. The imaging parameters were: matrix: 224/224; averages: I; TR 3.285 (heart rate based); TE I.42; Tdel, temporal resolution: $27 \mathrm{msec}$. (See Table I.)

Results: The specific results are summarized in Table 2. The incremental offset of the CFR, otherwise maintaining imaging parameters, allowed improved delineation of the mitral valve leaflet, and its subvalvular apparati. Despite better visualization of the valves, the measured thickness of the valves decreased when compared to the peak CFR valve measurements (approximately 5 to $12 \%$ decline in Subject $A$ and 17 to $26 \%$ decline in subject B). We believe that this is related to the better image quality from the off resonance peak. (See Figures I, 2, 3.)

Conclusion: Off resonance CFR may assist in the diagnosis of valvular abnormalities otherwise not seen clearly with routine CMR settings which utilize on peak CFR imaging. This method for valve evaluation may be beneficial when valvular and subvalvular structure evaluation is the main aim of the imaging. Unfortunately, as the frequency is shifted, the chamber flow is exaggerated therefore leading to exaggeration of valvular regurgitation or stenosis jets. Therefore the valvular jets should only be interrogated on routine SSFP imaging parameters ('On' Peak Center Frequency).

\section{T5}

\section{Is inversion time dependent on relaxivity?}

June A Yamrozik, Mark Doyle, Vikas K Rathi, Diane A Vido, Ronald B Williams, Geetha Rayarao and Robert WW Biederman

Allegheny General Hospital, The Gerald McGinnis

Cardiovascular Institute, Pittsburgh, PA, USA

Journal of Cardiovascular Magnetic Resonance 2009, I I(SuppI I):T5

Introduction: It is known that utilizing the proper inversion time (TI) is essential when diagnosing myocardial infarction. Many factors play a role in acquiring optimal nulling of the myocardium. Recently, higher relaxivity contrast agents have been advocated for use in infarct imaging. Specifically, MultiHance ${ }^{\circledR}$ (gadobenate dimeglumine) has higher relaxivities $\left(r_{1}\right.$ and $r_{2}, 9.7^{\prime}$ and $\left.12.5^{\prime}\right)$ compared to standard gadolinium (gadopentetate dimeglumine) $\left(r_{1}\right.$ and $r_{2}, 4.9^{\prime}$ and $\left.6.3^{\prime}\right)$. Will this higher relaxivity allow a lower dosage of contrast to be used to maintain a comparable TI null time compared to the conventional gadolinium dosage?

Hypothesis: We hypothesize that a $0.15 \mathrm{mmol} / \mathrm{kg}$ dosage of MultiHance with its higher relaxivity will produce a lower TI null time in infarct patients compared to patients imaged with a standard dosage of $0.2 \mathrm{mmol} / \mathrm{kg}$ of gadolinium at 10 and 20 minutes post contrast.

Methods: A total of 52 patients with GFR $>60 \mathrm{~mL} / \mathrm{min} / 1.73 \mathrm{~m}^{2}$ were imaged. Twenty-six (26) patients (19 M, 6 F), age 42-8I years, post-myocardial infarction underwent a standard cardiac MRI (CMR) utilizing a total of $0.2 \mathrm{mmol} / \mathrm{kg}$ gadolinium dosage (Magnevist-Berlex, New Jersey, USA). This was administered as a
$0.5 \mathrm{mmol} / \mathrm{kg}$ for perfusion imaging followed by an additional 0.15 $\mathrm{mmol} / \mathrm{kg}$ for viability (DHE) imaging. Twenty-six (26) patients (2I M, $5 \mathrm{~F}$ ), age 43-83 years, post-myocardial infarction underwent a standard cardiac MRI (CMR) utilizing a total of $0.15 \mathrm{mmol} / \mathrm{kg}$ MultiHance dosage (Bracco Diagnostics, Princeton, $\mathrm{N} \mathrm{J}$, USA). This was administered as a $0.5 \mathrm{mmol} / \mathrm{kg}$ dosage for perfusion imaging followed by an additional $0.10 \mathrm{mmol} / \mathrm{kg}$ for viability imaging. The scans were acquired on a GE CV/i Excite Version 12, I.5 T system (GE, Milwaukee, WI). The sequence utilized for optimum myocardial nulling was a standard 2D Gradient Echo IRP (FGR with inversion recovery prep). Manual selection of TI was performed independent of contrast media. An 8-channel or 4-channel cardiac coil was used, again independent of the contrast agent. The sequence parameters were as follows: TE: min, FA: 20, NEX: 2 trigger delay: adjusted to onset of diastole, IRR interval and TI adjusted to null the myocardium. This sequence was performed at 10 and 20 minutes postcontrast administration.

Results: All the patients successfully completed the CMR examination without difficulty, or impairment in infarct detection. Particular attention was focused on the contrast medium utilized, dosage and its effects on the inversion time (TI) at 10 and 20 minute post-contrast. At 10 minutes post-contrast, the inversion time with Magnevist was $158.7 \pm 17.2 \mathrm{~ms}$, which was not different from that obtained with MultiHance (I54.3 \pm I3.6 $\mathrm{ms}, \mathrm{P}=0.3$ ). At 20 minutes post-contrast, the inversion time with Magnevist was $200.4 \pm 28.8 \mathrm{~ms}$, which again was not different from that obtained with MultiHance $(193.0 \pm 15.6 \mathrm{~ms}$, $\mathrm{p}=0.3$ ).

Conclusion: While the relaxivity of MultiHance compared to standard gadolinium is almost a factor of two higher, similar contrast null times are achieved by using a 75\% dosage of MultiHance as compared to gadolinium. Relaxivity alone does not predict arithmetic decreases in TI times. Thus, while this is a higher dose than expected from relaxivity considerations alone, MultiHance administration is nevertheless potentially beneficial, especially in those situations when additional imaging might be necessary, since an additional $25 \%$ of contrast is held in reserve for further administration or saved, further minimizing any potential toxicity issues.

\section{T6}

\section{Dual PARACEST and ${ }^{19} \mathrm{~F}$ MR molecular imaging of fibrin clots with targeted perfluorocarbon nanoparticles}

Kejia Cai, Lei Zhang, Jacob Myerson, Wenjing Huang,

Shelton D Caruthers, Gregory M Lanza,

Samuel A Wickline and Patrick M Winter

Washington University, School of Medicine, Saint Louis, MO, USA

\section{Journal of Cardiovascular Magnetic Resonance 2009, I I(SuppI I):T6}

Introduction: Fibrin is an abundant component of thrombi and an early marker of ruptured atherosclerotic plaques, which are the major cause of myocardial ischemia and stroke. Identification of fibrin could help detect ruptured plaques and direct therapeutic interventions to prevent or ameliorate the consequences of a heart attack or stroke. Fibrin-targeted perfluorocarbon nanoparticles provide a unique platform for molecular imaging of thrombi by MRI. The particle surface can be formulated with MRI contrast agents, such as PARACEST 
Figure 2 (abstract T6)
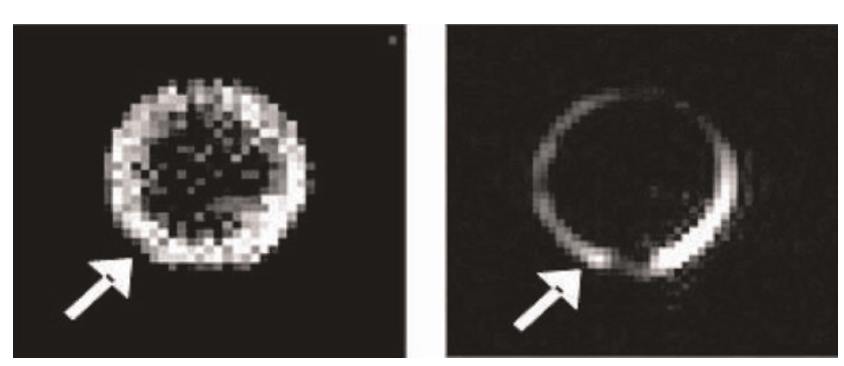

PARACEST and ${ }^{19} \mathrm{~F}$ MR imaging of a clot (Arrow) treated with fibrintargeted nanoparticles.

(PARAmagnetic Chemical Exchange Saturation Transfer) chelates that can provide "contrast on demand" by simply turning on and off a prepulse. In addition, the perfluorocarbon core can be exploited for ${ }^{19} \mathrm{~F}$ imaging. ${ }^{19} \mathrm{~F}$ offers high signal intensity and no background signal from biological tissues, yielding a unique signature.

Purpose: To combine PARACEST and ${ }^{19} \mathrm{~F}$ imaging for the corroborative detection of thrombi and quantitating the binding of targeted nanoparticles.

Methods: Fibrin-targeted PARACEST perfluorocarbon nanoparticles were prepared by incorporating a PARACEST chelate, Eu-DOTA-4AMC-benzyl-PE, and biotinylated dipalmitoylphosphatidylethanolamine (DPPE) into the lipid monolayer surface (Figure I). A phantom was prepared by diluting the particles to $0.5, \mathrm{I}, 2,4,8$ and $17 \mathrm{nM}$. Fibrin clots were prepared with dog plasma and suspended in normal saline. The targeted clots $(n=8)$ were treated with serial incubation of biotinylated antifibrin antibodies, avidin and biotinylated nanoparticles. Control clots $(n=5)$ were not incubated with nanoparticles. The phantom and clots were imaged with a II.7 T horizontal bore scanner using a single turn solenoid coil that can be manually tuned to $500 \mathrm{MHz}$ for PARACEST imaging or $470 \mathrm{MHz}$ for ${ }^{19} \mathrm{~F}$ imaging. PARACEST images were obtained with a 2 second presaturation RF pulse $(6.6 \mu \mathrm{T})$ applied at frequency offsets of $\pm 5 \mathrm{I} \mathrm{ppm}$ at a resolution of $156 \mu \mathrm{m}$ by $156 \mu \mathrm{m}$ by $4 \mathrm{~mm}$, with a $2.0 \mathrm{I}$ second TR, 2.5 millisecond TE and 8 signal averages, yielding a total imaging time of 34 minutes. ${ }^{19} \mathrm{~F}$ imaging was performed with identical settings except I second TR, 9 millisecond TE, 64 signal averages and 2 times of image matrix

Figure I (abstract T6)

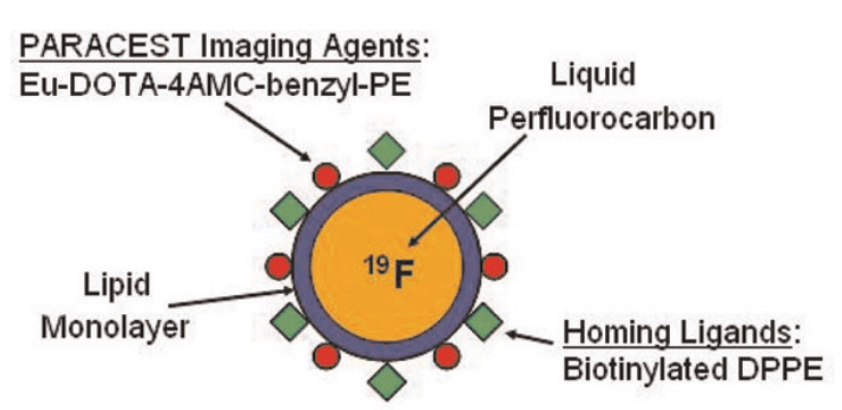

Schematic representation of fibrin-targeted perfluorocarbon nanoparticles for MR molecular imaging. interpolation. PARACEST contrast to noise ratio (CNR) and the ${ }^{19} \mathrm{~F}$ signal to noise ratio (SNR) were calculated by manual selection of regions of interest.

Results: The phantom images showed a linear increase in both PARACEST CNR and the ${ }^{19} \mathrm{~F}$ SNR with increasing nanoparticle concentrations, indicating that ${ }^{19} \mathrm{~F}$ imaging can corroborate the PARACEST signal and quantitate nanoparticle number. The 0.5 $\mathrm{nM}$ dilution showed a PARACEST CNR of 8.2, exceeding the minimum limit of detection $(C N R=5)$. Clots treated with targeted agent showed PARACEST enhancement along the clot boundary, and no enhancement in the clot interior or the surrounding saline (Figure 2). Similarly, the ${ }^{19} \mathrm{~F}$ images showed signal only at the clot surface. The PARACEST CNR was $23.6 \pm 5.5$ for the targeted clots but only $2.1 \pm 0.2$ for control clots. The ${ }^{19} \mathrm{~F} \mathrm{SNR}$ was $12.6 \pm 2.3$ for the targeted clots, but only $1.8 \pm 0.2$ for the control clots. The ${ }^{19} \mathrm{~F}$ signal suggests that the nanoparticle concentration on the clot surface was $2.5 \mathrm{nM}$. Interestingly, the phantom experiment predicts that $2.5 \mathrm{nM}$ of nanoparticles would only produce a PARACEST CNR of 15.6, which indicates that binding the nanoparticles to a target actually improves the PARACEST mechanism. This could result from slowing of the water exchange rate with the PARACEST chelate. Conclusion: Dual PARACEST and ${ }^{19} \mathrm{~F}$ MR molecular imaging of fibrin with targeted PARACEST perfluorocarbon nanoparticles was demonstrated at II.7 T. Phantoms showed the detection limit of the PARACEST nanoparticles was $<500$ pM. The PARACEST enhancement observed on the clot surface was higher than the expected value based on the ${ }^{19} \mathrm{~F}$ signal, suggesting that binding the nanoparticles to a target improves the efficacy of the contrast mechanism.

\section{T7}

A case of cardiac langerhans histiocytosis

Ashley Simmons and Thomas Rosamond

University of Kansas Medical Center, Kansas City, KS, USA

Journal of Cardiovascular Magnetic Resonance 2009, I I(SuppI I):T7

Introduction: Langerhans histiocytosis (eosinophilic granuloma) is a disease characterized by proliferation of specialized bone marrow-derived Lnagerhans cells and mature eosinophils. The disease is rare and typically found in children. There is debate over whether the disease is neoplastic or reactive. To date, there have been no cases of Langerhans cell histiocytosis involving the myocardium.

Case report: A 44 year old female with a past medical history of hypertension presented to the emergency department with complaints of chest pain. Her troponin was elevated and peaked at 3.76. She underwent cardiac catheterization which showed a normal left ventricular ejection fraction and no significant obstructive coronary artery disease. Chest CT showed nodular and cystic opacities within the upper lobe suggestive of Langerhans cell histiocytosis. There was no evidence of pulmonary embolism or aortic dissection, no lymphadenopathy. Cardiac MRI was performed and showed a focal area of increased TI signal intensity, decreased perfusion and heterogeneous transmural delayed hyperenhancement in the posterior basal segment of the left ventricle. A diagnosis of fibroxanthomatous infiltrative myocardial disease due to Langerhans Histiocytosis was made on the basis of cardiac MR images and CT chest images. Transbronchial biopsy and broncho-alveolar lavage 
showed no evidence of histiocytosis. The differential diagnosis remains focal eosinophilic granuloma versus viral myocarditis.

Conclusion: Cardiac MRI can help with the differential diagnosis of infiltrative myocardial diseases with delayed hyperenhancement techniques.

T8

The role of CMR in the diagnosis of systemic vasculitis: a case study

Phillip Andrews

University Hospital of North Staffordshire, Stoke-on-Trent, UK

Journal of Cardiovascular Magnetic Resonance 2009, I I (SuppI I):T8

An 18 yr old caucasian man presented with neurological symptoms and breathlessness. Echocardiography demonstrated a left ventricular abnormality of unclear aetiology. The patient was referred onwards to our unit for further assessment by cardiac magnetic resonance. Imaging was performed on a I.5 T Philips Intera Acheiva, using a five element phased-array surface coil.

On steady state free precession (SSFP) cine images the appearances were of marked left ventricular hypertrophy with partial cavity obliteration. Left ventricular cavity volumes were reduced and ejection fraction was $54 \%$. Early post contrast inversion recovery gradient echo (IRGE) images showed an area of low signal within the LV cavity distinct from the myocardium. The mass was markedly hypointense to both myocardium and the blood pool. Delayed IRGE sequences 10 minutes following the administration of contrast demonstrated widespread subendocardial hyperenhancement in all myocardial segments sparing only the basal septum. CMR was able to confidently establish the diagnosis of endomyocardial fibrosis with a large left ventricular thrombus burden.

The patient had no history of foreign travel or malnutrition, making a vasculitis the most likely aetiology.

Baseline blood testing demonstrated a mild eosinophilia of $17 \%$, a $C$ reactive protein of $27 \mathrm{mg} / \mathrm{L}$ (normal $0-5$ ) and an erythrocyte sedimentation rate of $27 \mathrm{~mm} / \mathrm{h}$ (normal 0-20). Further imaging demonstrated normal renal arteries on MR angiogram. However CT thorax demonstrated pulmonary haemorrhage and MRI brain demonstrated white matter abnormalities in the cerebral cortex. These findings were in keeping with the clinical diagnosis of vasculitis, Although autoantibodies were all normal, the most likely process is a variant of Churg Strauss.

Cardiac MR was able to establish the diagnosis of endomyocardial fibrosis which was pivotal in identifying the underlying vasculitic disease process. 